UC-NRLF

|| || ||||||||||||||||||||||||||||||||||||||

40

B 4 106 438 

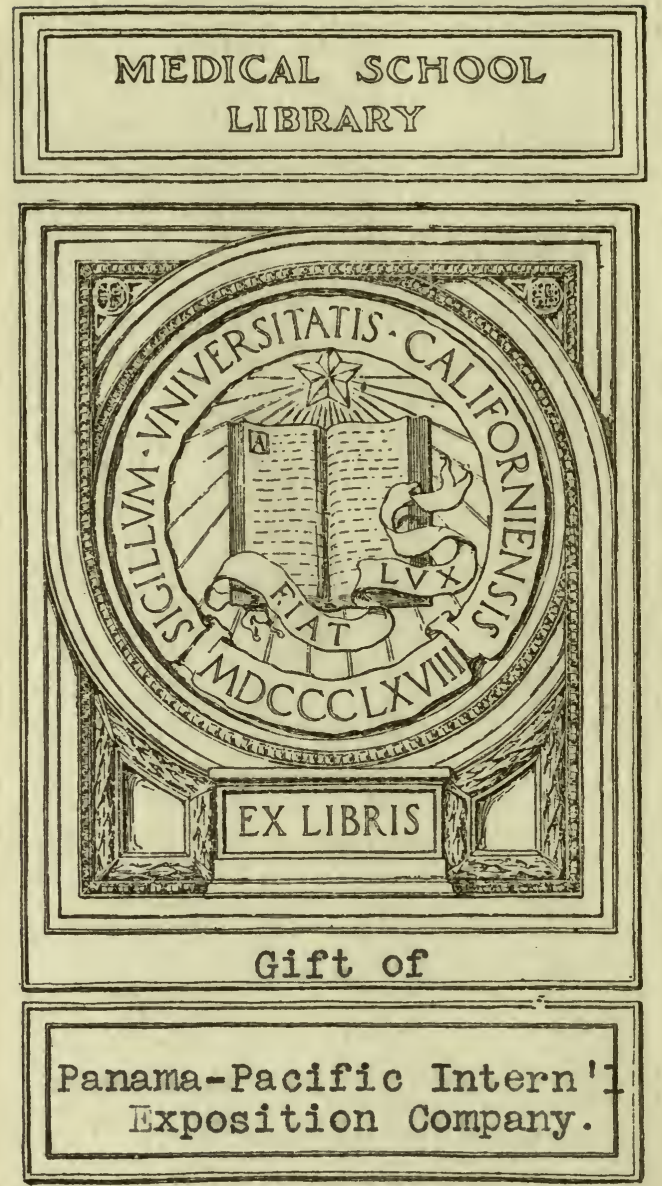


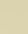


Digitized by the Internet Archive in 2007 with funding from Microsoft Corporation 



\section{HALLIBURTON'S}

\section{HANDBOOK OF PHYSIOLOGY}





\section{HANDBOOK OF P H Y S I O L O G Y}

B Y W. D. H A L L I B U R T O N, M.D. LL.D., F.R.C.P., F.R.S.

PROFESSOR OF PHYSIOLOGY, KING'S COLLEGE, LONDON

ELEVENTH EDITION ${ }^{i \varphi}$

(BEING THE TWENTY-FOURTH EDITION OF KIRKES' PHYSIOLOGY) $=$

WITH NEARLY SIX HUNDRED ILLUSTRATIONS IN THE TEXT, MANY OF WHICH ARE COLOURED, AND THREE COLOURED PLATES

\section{COMPLIMENTS OF}

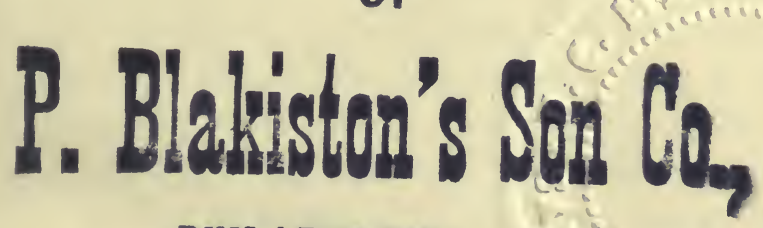
PMIIAELPPIA, PA.

PHILADELPHIA

P. BLAKIS'TON'S SON \& CO. 1012. WALNUT STREET 



\section{PREFACE TO THE ELEVENTH EDITION}

ONCE more this book has been thoroughly revised. The sections dealing with salivary secretion, the formation of urine, and much of the chapter on respiration have been almost entirely rewritten. New matter on the process of conduction in the heart, on the applications of physical chemistry to physiology, on so-called vitamines, and several other subjects has been introduced. The concluding chapter on reproduction and development is a new one, and replaces the two last chapters in previous editions.

I am again indebted to several kind friends for assistance in carrying out my task. I would especially thank Mr Barcroft, F.R.S., for his valuable help in the chapters that deal with saliva, respiration, and the kidney; Dr C. S. Myers, who has kindly revised the parts which deal with the special senses; Dr Hertz of Guy's Hospital, for useful hints in bringing the chapter on the movements of the stomach and intestines up to date; and Dr F. H. A. Marshall, for similar kind offices in relation to the subject of reproduction.

Fin.-I think, in conclusion, I may congratulate my readers that, although the present edition contains so much that is new both in text and illustrations, I have been successful in introducing this additional information without increasing the bulk of the volume. As a matter of fact, this edition is one page shorter than the last.

W. D. HALLIBURTON.

King's College, London, 1913. 



\title{
CON TENTS
}

\author{
CHAPTER I
}

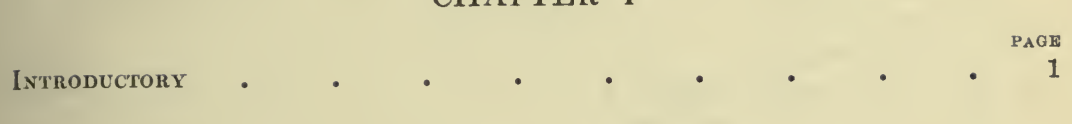

CHAPTER II

Tue Animal Cell

\section{CHAPTER III}

Epithelium

\section{CHAPTER IV}

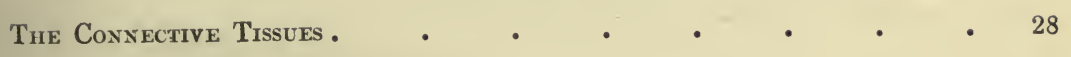

\section{CHAPTER V}

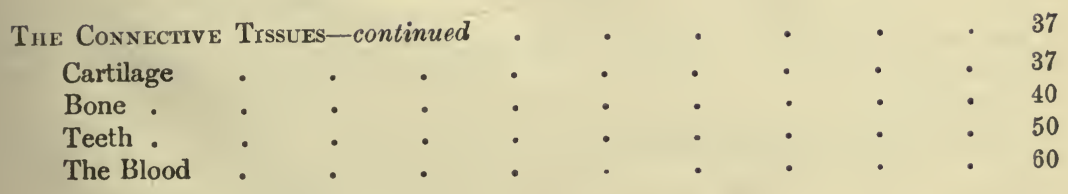

\section{CHAPTER VI}

Muscular Tissue. 


\section{CHAPTER VII}

NERVE

CHAPTER VIII

IRritability and Contractility

\section{CHAPTER IX}

Change in Form in a Muscle when it Contracts . $.4 . \quad$. 87

Instruments used . $\quad . \quad$. $\quad . \quad$. . 87

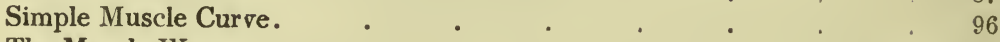

The Muscle-Wave $\quad$ • $\quad$. $\quad$. $\quad$. $\quad$. $\quad . \quad 100$

Effect of two Successive Stimuli . $\quad$. $\quad . \quad$. $\quad . \quad$. 101

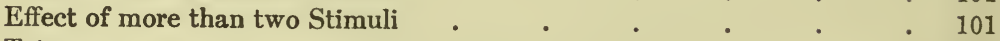

Tetanus

Voluntary Tetanus

CHAPTER X

Extensibility, Elasticity, and Work of Muscle . . . . 108

\section{CHAPTER XI}

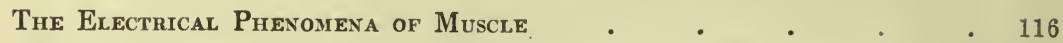

\section{CHAPTER XII}

Thermal and Chemical Changes in Muscle . $\quad . \quad$. $\quad . \quad$. 129

Fatigue

Rigor Mortis .

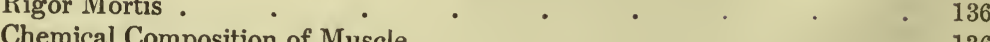

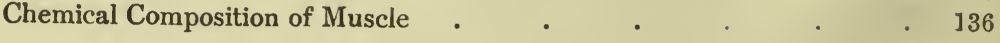

CHAPTER XIII

Comparison of Voluntary and Involuntary Muscle 


\section{CHAPTER XIV}

Physiology of NeRve .

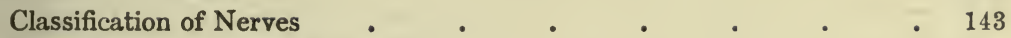

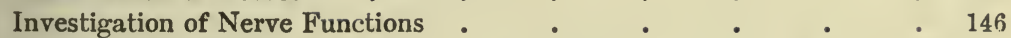

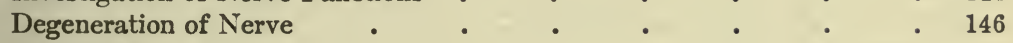

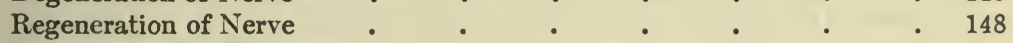

Roots of the Spinal Nerves . $\quad$ - $\quad$. $\quad$. $\quad$. $\quad . \quad 155$

Changes in Nerve during Activity . $\quad$. $\quad$. $\quad$. $\quad$. 158

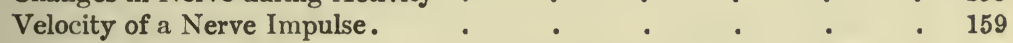

Direction of a Nerve Impulse $\quad$. $\quad$. $\quad$. $\quad$. $\quad$. $\quad 160$

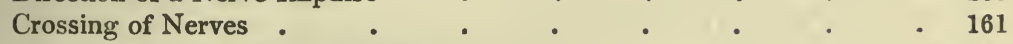

The Nature of the Nerve Impulse . $\quad$. $\quad$. $\quad$. $\quad$. 163

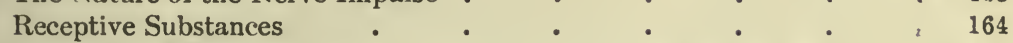

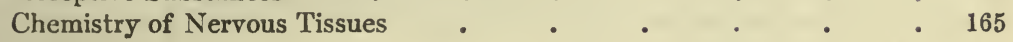

CHAPTER XV

Electrotonus

CHAPTER XVI

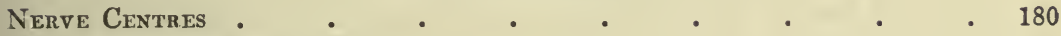

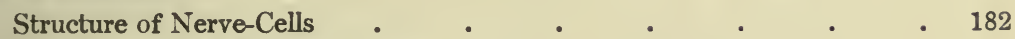

The Law of Axipetal Conduction . $\quad$. $\quad$. $\quad$. $\quad . \quad$. 191

The Significance of Nissl's Granules $\quad$ - $\quad$. $\quad$ • 192

Classification of Nerve-Cells according to their Function . . . 194

\section{CHAPTER XVII}

The Autonomic Nervous System

196

\section{CHAPTER XVIII}

Trophic Nerves. 206

CHAPTER XIX

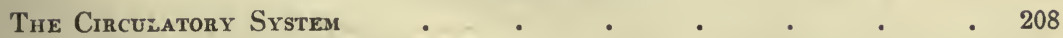

The Heart . . . . . . . . . 208

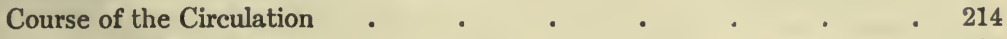

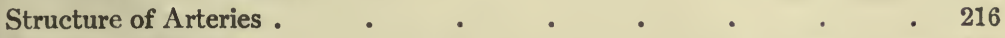

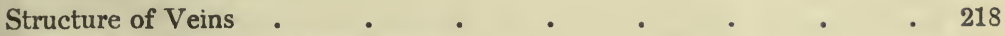

Structure of Capillaries and Sinusoids _ $\quad$. $\quad$. $\quad$. $\quad$. 222

Structure of Lymphatic Vessels $\quad$. $\quad$. $\quad$. $\quad . \quad 223$

CHAPTER XX

The Circulation of the Brood 


\section{CHAPTER XXI}

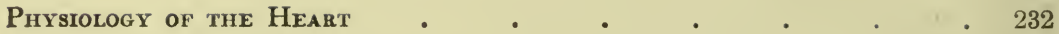

The Cardiac Cycle • • • • • • . 232

Action of the Valves of the Heart . $\quad$. . . . . . 234

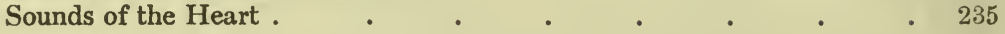

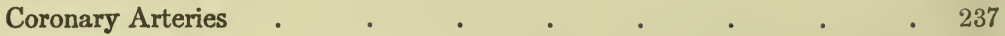

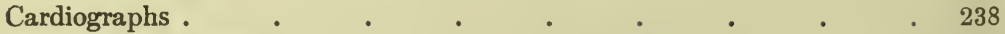

Intracardiac Pressure $\quad . \quad$. $\quad$. $\quad$. $\quad$. $\quad . \quad 240$

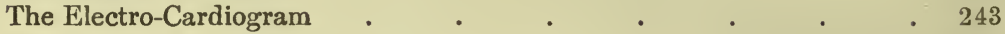

Frequency of the Heart's Action . . . . . . . . 247

Work of the Heart . . . . . . . . 247

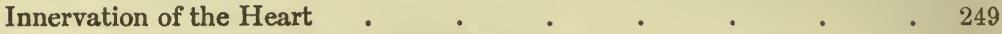

Rhythm, Conduction, etc., in Heart Muscle _ . . . . 254

The Isolated Heart . $\quad$. $\quad$. $\quad$. . . 260

CHAPTER XXII

The Crrculation in the Blood-vessels . . . . . . 263

Use of the Elasticity of the Vessels . . . . . . . 265

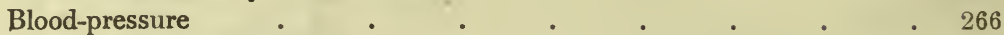

Velocity of the Blood-flow . $\quad . \quad$. $\quad . \quad$. $\quad . \quad 281$

The Time of a Complete Circulation $\quad$. $\quad$. $\quad$. $\quad . \quad 289$

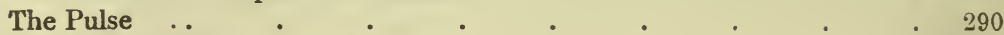

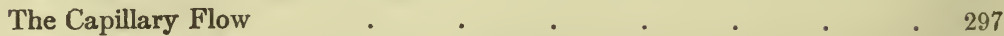

The Venous Flow $\quad$. $\quad$. $\quad$. $\quad$. $\quad . \quad 299$

The Vaso-motor Nervous System . . . . . . 300

Plethysmography . . . . . . . . . 308

Pathological Conditions _. . . . . . . . 311

Local Peculiarities of the Circulation _ . . . . $\quad$. 312

\section{CHAPTER XXIII}

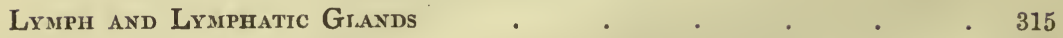

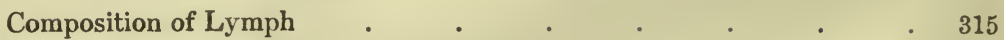

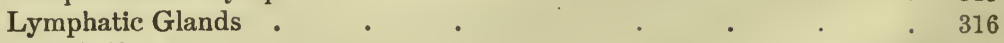

Lymph Flow . $\quad$. $\quad$. . . . . 318

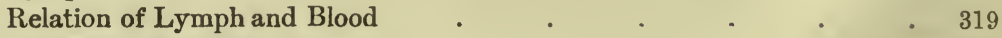

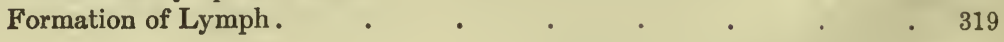

CHAPTER XXIV

Physical Chemistry and its bearing on Physiological Problems. 


\section{CHAPTER XXV}

Tine Ductless Grayds

PAGE

331

Spleen

Hæmolymph Glands .

Thymus

Thyroid

Parathyroids .

Supra-renal Capsules .

Pituitary Body

Pineal Gland .

Coccygeal and Carotid Glands

332

336

336

337

339

340

343

345

345

\section{CHAPTER XXVI}

Respiration

Respiratory Apparatus

Respiratory Mechanism

Graphic Record of Respirations

The Gases of the Blood

Solution of Gases in Water

Dalton-Henry Law

Tension of Gases in Fluids

Measurement of Quantity of Gas in Fluids

Relation between Quantity and Tension

The Mechanism of Gaseous Exchange in the Lung

1. Oxygen

2. Carbonic Acid

Cause and Regulation of Respiration

1. The Respiratory Centre

2. The Nervous Factor in Respiration

3. The Chemical Factor in Respiration

Special Respiratory Acts

Artificial Respiration.

Ventilation

\section{CHAPTER XXVII}

The Relation of Respiration to other Processes in the Body

Effect of Respiration on the Circulation . . . . . $\quad 385$

Asphyxia

Relation of Respiration to Nutrition

Oxygen Want

Mountain Sickness

Respiration at High Pressures

Carbon Monoxide Poisoning .

Cheyne-Stokes Respiration.

Diabetic Coma 


\section{CHAPTER XXVIII}

The Chemical Compostur or page

Carbohydrates

Fats

Proteins

Lipoids

Enzymes

\section{CHAPTER XXIX}

\section{THE BLood}

Coagulation of the Blood

The Plasma and Serum

The Blood-Corpuscles

Development of the Blood-Corpuscles

Chemistry of the Blood-Corpuscles .

Hæmoglobin .

Immunity

\section{CHAPTER XXX}

Food

Dietaries

Milk

The Mammary Glands

Eggs .

Meat .

Flour .

Bread .

Cooking of Food

Accessories to Food .

Unknown but Essential Constituents of Food

\section{CHAPTER XXXI}

The Alimentary Canal; Secreting Glands .

\section{CHAPTER XXXII}

\section{Saliva}

The Salivary Glands .

The Secretion of Saliva

Secretory Nerves of Salivary Glands

The Saliva 


\section{CHAPTER XXXIII}

The Gastric Juice

Innervation of the Gastric Glands .

Action of Gastric Juice

\section{CHAPTER XXXIV}

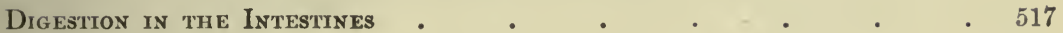

The Pancreas $\quad$. $\quad$. $\quad$. $\quad$. 517

Composition and Action of Pancreatic Juice ․ . $\quad$. $\quad$. 518

The so-called Peripheral Reflex Secretion of the Pancreas _ . 520

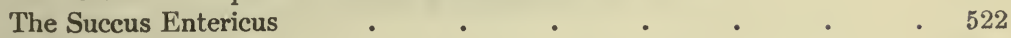

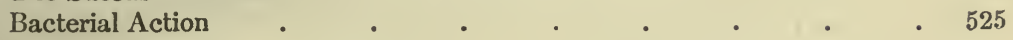

\section{CHAPTER XXXV}

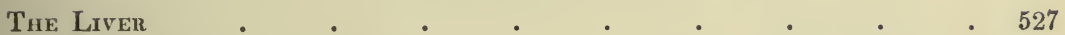

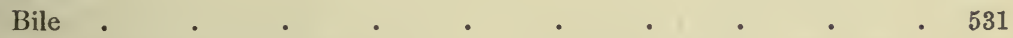

Glycogenic Function of the Liver . $\quad . \quad$. $\quad . \quad$. $\quad$. 536

Diabetes $\quad . \quad$. $\quad . \quad$. $\quad . \quad$. 538

The Liver and Fat Metabolism $\quad$. $\quad$. $\quad$. $\quad$. $\quad$. 541

CHAPTER XXXVI

The Absorption of Food

543

\section{CHAPTER XXXVII}

The Mechanical Processes of Digestion . . . . . 550

Mastication . . . . . . . . . 550

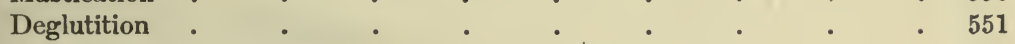

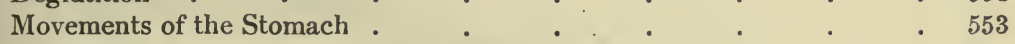

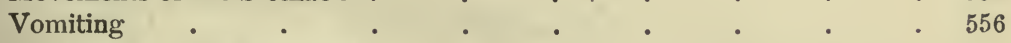

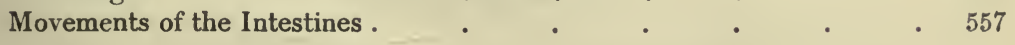

CHAPTER XXXVIII

The Urinary Apparatus

The Nerves of the Kidney . . . . . $\quad$. 571

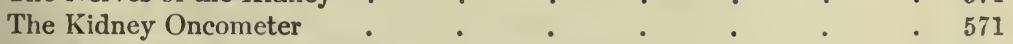

The Functions of the Kidney $\quad$. $\quad$. $\quad$ : $\quad$ • $\quad$. $\quad$. 572

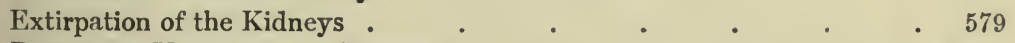

Passage of Urine into the Bladder . . . . . . 580

Micturition 


\section{CHAPTER XXXIX}

The URINe

Urea.

Ammonia

Creatine and Creatinine

Uric Acid

Hippuric Acid

Inorganic Constituents of Urine

Urinary Deposits

Pathological Urine

CHAPTER XL

The Skin and Its Appendages .

\section{CHAPTER XLI}

\section{General Metabolism}

Balance in Health

Metabolism of Carbohydrates

Metabolism of Fat

Metabolism of Protein

Growth and Maintenance

Inanition or Starvation

CHAPTER XLII

The Conservation of Energy .

\section{CHAPTER XLIII}

Temperature

\section{CHAPTER XLIV}

The Central Nervous System .

\section{CHAPTER XLV}

\section{CHAPTER XLVI}

Structure of the Bulb, Pons, and Mid-Brain 


\section{CHAPTER XLVII}

Structure of the Cerebellum .

\section{CHAPTER XLVIII}

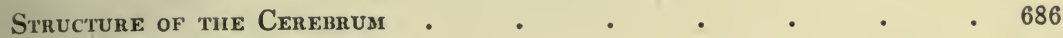

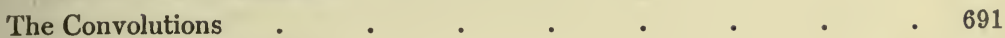

Histology of the Cortex $\quad$. $\quad$. $\quad$. $\quad$. $\quad$. $\quad$. $\quad$. 695

The White Matter $\quad$. $\quad$. $\quad$. $\quad$. $\quad . \quad 698$

\section{CHAPTER XLIX}

Functions of the Spinal Cord .

The Cord as an Organ of Conduction

Reflex Action of the Cord

Reflex Action in Man

The Principle of the Common Path .

Reaction Time

Spinal Visceral Reflexes

CHAPTER L

Functions of the Cerebruar

Removal of the Cerebrum

Localisation of Cerebral Functions

Function and Myelination

Association Fibres and Centres

Electrical Variation .

Sleep and Narcosis

$$
\begin{aligned}
& \text {. } . \quad . \quad .704
\end{aligned}
$$

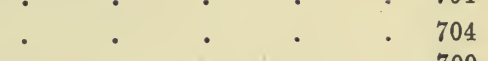

$$
\begin{aligned}
& \begin{array}{r}
709 \\
\hline
\end{array} \\
& \text { - } 711 \\
& \text { - } 716 \\
& \text { - } 720 \\
& \text { - } 721
\end{aligned}
$$

\section{CHAPTER LI}

Functions of the Ceremeluur.

The Semicircular Canals

\section{CHAPTER LII}

The Physiologr of Conscrous States .

\section{CHAPTER LIII}

Cutaneous Sensatrons .
Tactile End-Organs

Localisation of Tactile Sensations

Varieties of Cutaneous Sensations 


\section{CHAPTER LIV}

Motorial and Visceral Sensations

CHAP'TER LV

Taste and Syell.

Taste

Smell .

CHAPTER LVI

Hearing

Anatomy of the Ear .

Physiology of Hearing

CHAPTER LVII

Vorce and Speech

Anatomy of the Larynx

Movements of the Vocal Cords

The Voice

Speech

Defects of Speech

\section{CHAPTER LVIII}

\section{The Eye and Vision}

The Eyeball .

The Eye as an Optical Instrument

Accommodation

Defects in the Optical Apparatus

The Skiascope

Functions of the Iris .

Functions of the Retina

The Ophthalmoscope

The Perimeter

Visual Sensations and Colour Vision

Changes in the Retina during Activity

Movements of the Eyeballs .

Nervous Paths in the Optic Nerves .

Visual Judgments 


\section{CHAPTER LIX}

Reproduction, Development, Growtir And Death
The New-born Child and its Development
The Male Reproductive Organs

\section{COLOURED PLATES}

Varietues of Colourless Corpuscles .

to face page 456

Plate of Absorption Spectra.

, , $\quad 466$

Simultaneous and Successive Contrast

, , , 845 



\section{FAHRENHEIT and CENTIGRADE SCALES.}

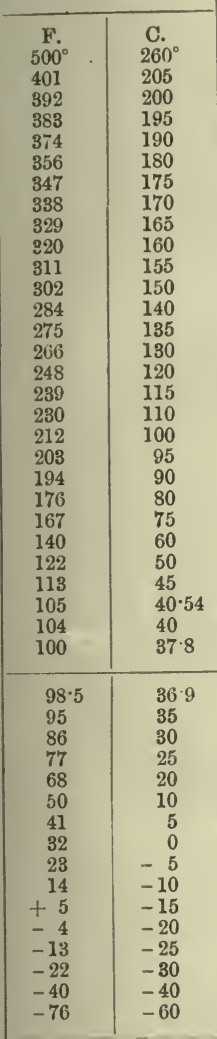

1 deg. F. $=54^{\circ} \mathrm{C}$. $1.8,=1^{\circ} \mathrm{C}$. Freezing point $0^{\circ} \mathrm{C}$ $=32^{\circ} \mathrm{F}$.

Boiling point $100^{\circ} \mathrm{C}$. $=212^{\circ} \mathrm{F}$.

To convert de. grees $\mathrm{F}$. into degrees C., subtract 32 , and multiply by $\frac{5}{9}$.

To convert degrees C. into degrees F., multiply by 8 , and add $32^{\circ}$.

\section{MEASUREMENTS.}

\section{INGTH.}

1 metre $)=39.37$ English 10 decimetres $\quad$ inches 100 centimetres $\}$ (or 1 yard and $3 \frac{1}{3}$ in.)

1 decimetre $\}=3.937$ inches $\left.\begin{array}{r}10 \text { centimetres } \\ 100 \text { millimetres }\end{array}\right\}$ (or nearly 4 inches)

1 centimetre $\quad=\cdot 3937$ or about 10 millimetres (nearly $\frac{2}{5}$ inch). 1 millimetre $=$ nearly ${ }_{2}^{2} 6$ inch. Or,

ONe Metre $=39 \cdot 37079$ inches.

(It is the ten-millionth part of a quarter of the meridian of the earth.)

1 Decimetre $=4$ in.

1 Centimetre $=\frac{4}{10}$ in.

1 Millimetre $=$ in

Decametre $=32.80$ feet.

Hectometre $=109.36 \mathrm{yds}$.

Kilometre $=0.62 \mathrm{mile}$.

One inch $=2.539$ Centimetres.

One foot $=3.047$ Decimetres.

One yard $=0.91$ of a Metre.

One mile $=1.60$ Kilometre.

\section{WEIGHT.}

(One gramme is the weight of a cubic centimetre of water at $4^{\circ} \mathrm{C}$. at Paris.)

1 gramme

10 decigrammes $=15.432349 \mathrm{grs}$. 100 centigrammes $\left\{\right.$ (or nearly $15 \frac{1}{2}$ ). 1000 milligrammes

$\left.\begin{array}{rl}1 & \text { decigramme } \\ 10 & \text { centigrammes }\end{array}\right\}$ = rather more 100 milligrammes $\}$ than $1 \frac{1}{2}$ grain.

1 centigramme $\}$ = rather more 10 decigrammes $\}$ than $\frac{3}{20}$ grain.

1 milligramme $\begin{gathered}=\text { rather more } \\ \text { than }{ }_{2 \pi}^{3} \text { grain. }\end{gathered}$

1 Decagramme $=2 \mathrm{dr} .34 \mathrm{gr}$.

1 Hectogrm. = $3 \frac{1}{2}$ oz. (Avoir.)

1 Kilogrm. = $21 \mathrm{~b} .3 \mathrm{oz} .2 \mathrm{dr}$. (Avoir.)
A grain equals about $1 \cdot 16$ gram., a Troy Oz, about 31 grams.

a 1b. Avoirdupois about $\frac{1}{2}$ Kilogrm., and $1 \mathrm{cwt}$. about 50 Kilogrms.

\section{CAPACITY}

1,000 cubic decimetres $\}=1$ cubic $1,000,000$ cubic centimetres $\}$ metre.

1 cubic decimetre

1000 cubic centimetres \}$=1$ litre. Or,

ONe Litre $=1$ pt. $15 \mathrm{oz} .1 \mathrm{dr} .40$.

(For simplicity, Litre is used to signify 1 cubic decimetre, a little less than 1 English quart.)

Decilitre (100 c.c.)

$=2 \frac{6}{6} \mathrm{~d} \mathbf{r}$.

Millilitre (1 c.c.) $\quad=17 \mathrm{~m}$.

Decalitre $=2 \frac{1}{5}$ gals.

Hectolitre $=22$ gals

Kilolitre (cubic metre) $=27 \frac{1}{2}$ bushels.

A cubic inch $=16.38$ c.c.; a cubic foot $=28.315$ cubic dec., and a gallon $=$ $4 \cdot 54$ litres.

\section{CONVERSION SCALE.}

To convert Grammes to OUNCES avoirdupois, multiply by 20 and divide by 567 . To convert Kiloorammes to Pounds, multiply by 1000 and divide by 454 .

To convert Litres to Gallons, multiply by 22 and divide by 100 .

To convert Litres to PINTs, multiply by 88 and divide by 50 .

To convert Mullimetres to INCHes, multiply by 10 and divide by 254 .

To convert METRES to YARDS, multiply by 70 and divide by 64 .

\section{SURFACE MEASUREMENT.}

1 square metre $=$ about $1550 \mathrm{sq}$. inches (or 10,000 sq. centimetres, or $10.75 \mathrm{sq}$. ft.) $1 \mathrm{sq}$. inch $=$ about $6.4 \mathrm{sq}$. centimetres. 1 sq. foot = " 930 ," "s

\section{FNERGY MEASURE.}

1 kilogrammetre $=$ about $7 \cdot 24 \mathrm{ft}$. pounds.

1 foot pound = , $\quad 1381 \mathrm{kgm}$.

1 foot ton $=", 310 \mathrm{kgms}$.

HEAT EQUIVALENT.

1 kilocalorie $=425.5$ kilogrammetres.

\section{ENGLISH MEASURES.}

Apothecaries Weight.

7000 grains $=1 \mathrm{lb}$.

Or,

437.5 grains $=1 \mathrm{oz}$.
Avoirdupois Weight.

16 drams $=1 \mathrm{oz}$.

$16 \mathrm{oz} . \quad=1 \mathrm{lb}$.

28 lbs. = 1 quarter.

4 quarters $=1$ ewt.

20 cwt. $=1$ ton.

Measure of 1 decimetre, or 10 centimetres, or 100 millimetres

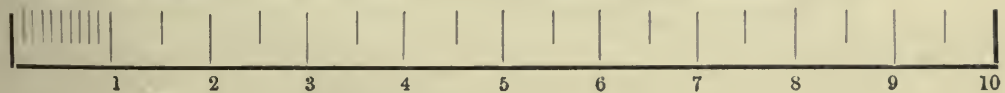





\title{
HANDBOOK OF PHYSIOLOGY
}

\author{
C H A P TE R I
}

\section{INTRODUCTORY}

BIOLOGY is the science that treats of living things, and it is divided into two main branches, which are called respectively Morphology and Physiology. Morphology is the part of the science that deals with the form or structure of living things, and with the problems of their origin and distribution. Physiology, on the other hand, treats of their functions, that is, the manner in which their individual parts carry out the processes of life. To take an instance: the eye and the liver are two familiar examples of what are called organs; the morphologist or anatomist studies the structure of these organs, their shape, their size, the tissues of which they are composed, their position in the body, and the variations in their structure met with in different parts of the animal kingdom. The physiologist studies their uses, and seeks to explain how the eye fulfils the function of vision, and how the liver forms bile, and ministers to the needs of the body in other ways.

Each of these two great branches of biological science can be further subdivided according as to whether it deals with the animal or the vegetable kingdom; thus we get vegetable physiology and animal physiology. Human physiology is a large and important branch of animal physiology, and to the student of medicine is obviously the portion of the science that should interest him most. In order to understand morbid or pathological processes it is necessary that the normal or physiological functions should be learnt first. Physiology is not a study which can be put aside and forgotten when a certain examination has been passed; it has a most direct and intimate bearing in its application to the scientific and successful investigation of disease. It will be my endeavour throughout the subsequent pages of this book to point out from time to time the practical relationships between physiology and pathology. 
Human physiology will be our chief theme, but it is not a portion of the great science that can be studied independently of its other portions. Thus, many of the experiments upon which our knowledge of human physiology rests have been performed principally on certain of the lower animals. In order to obtain a wide view of vital processes it will be occasionally necessary to go still further afield, and call the science of vegetable physiology to our assistance.

The study of physiology must go hand in hand with the study of its sister science anatomy, but the sciences of chemistry and physics must also be considered. Indeed, physiology has been sometimes defined as the application of the laws of chemistry and physics to life. That is to say, the same laws that regulate the behaviour of the mineral or inorganic world are also to be found operating in the region of organic beings. If we wish for an example of this we may again go to the eye; the branch of physics called optics teaches us, among other things, the manner in which images of objects are produced by lenses; these same laws regulate the formation of the images of external objects upon the sensitive layer of the back of the eye by the series of lenses in the front of that organ. An example of the application of chemical laws to living processes is seen in digestion; the food contains certain chemical substances which are acted on in a chemical way by the various digestive juices in order to render them of service to the organism.

The question arises, however, is there anything else? Are there any other laws than those of physics and chemistry to be reckoned with? Is there, for instance, such a thing as "vital force"? It may be frankly admitted that physiologists at present are not able to explain all vital phenomena by the laws of the physical world; but as knowledge increases it is more and more abundantly shown that the supposition of any special or vital force is unnecessary; and it should be distinctly recognised that when, in future pages, it is necessary to allude to vital action, it is not because we believe in any specific vital energy, but merely because the phrase is a convenient one for expressing something that we do not fully understand, something that cannot at present be brought into line with the physical and chemical forces that operate in the inorganic world.

But just as there is no hard-and-fast line between physiology and its allies pathology, anatomy, physics, and chemistry, so also there is no absolute separation between its three great divisions; physical, chemical, and so-called vital processes have to be considered together.

Physiology is a comparatively young science. Though Harvey more than three hundred years ago laid the foundation of our science by his discovery of the circulation of the blood, it is only during the last sixty or seventy years that active growth has occurred. The 
reasons for this recent progress come under two headings: those relating to observation and those relating to experiment.

The method of observation consists in accurately noting things as they occur in nature; in other words, the knowledge of anatomy must be accurate before correct deductions as to function are possible. The instrument by which such correct observations can be made is, par excellence, from the physiologist's standpoint, the microscope, and it is the extended use of the microscope, and the knowledge of minute anatomy resulting from that use, which has formed one of the greatest. stimuli to the successful progress of physiology during recent times.

But important as observation is, it is not the most important method; the method of experiment is still more essential. This consists, not in being content with mere reasonings from structures or occurrences seen in nature, but in producing artificially changed relationships between the structures, and thus causing new combinations that if one had waited for Nature herself to produce might have been waited for indefinitely. Anatomy is important, but mere anatomy has often led people astray when they have tried to reason how an organ works from its structure only. Experiment is much more important; that is, one tests one's theories by seeing whether the occurrences actually take place as one supposes; and thus the deductions are confirmed or corrected. It is the universal use of this method that has made physiology what it is. Instead of sitting down and trying to reason out how the living machine works, physiologists have actually tried the experiment, and so learnt much more than could possibly have been gained by mere cogitation. Many experiments involve the use of living animals, but the discovery of anæsthetics, which renders such experiments painless, has got rid of any objection to experiments on the score of pain.

The adult body consists of a great number of different parts; and each part has its own special work to do. Such parts of the body are called organs. Each organ does not only its own special work, but acts in harmony with other organs. This relationship between the organs enables us to group them together into what are termed systems. Thus, we have the circulatory system, that is, the group of organs (heart, arteries, veins, etc.) concerned in the circulation of the blood; the respiratory system, that is, the group of organs (air passages, lungs, etc.) concerned in the act of breathing; the digestive system, which deals with the digestion of food; the excretory system, with the getting rid of waste products; the muscular system, with movement; and the skeletal system, with the support of the softer parts of the body. Over and above all these is the nervous system (brain, spinal cord, nerves), the great master system of the body wbish presides over, controls, and regulates the functions of the other systems.

If we proceed to make an anatomical analysis, and take any 
organ, we see that it consists of various textures, or, as they are called, elementary tissues. Just as one's garments are made up of textures (cloth, lining, buttons, etc.), so each organ is composed of corresponding tissues. The elementary tissues come under the following four headings:-

1. Epithelial tissues.

2. Connective tissues.

3. Muscular tissues.

4. Nervous tissues.

Each of these is again divisible into subgroups.

If we continue our anatomical analysis still further, we find

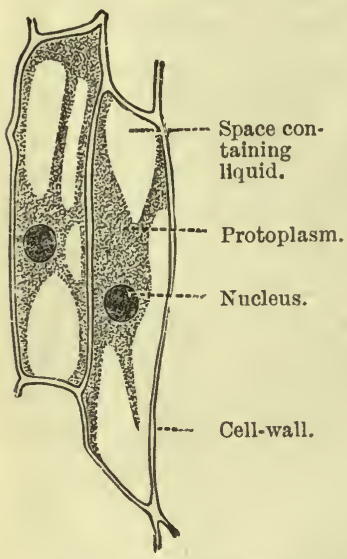

Fro. 1.-Vegetable cells. that the individual tissues are built up of structures which require the microscope for their accurate study. Just as the textures of a garment are made up of threads of various kinds, so also in many of the animal tissues we find threads or fibres, as they are called. But more important than the threads are little masses of living material. Just as the wall of a house is made up of bricks united by cement, so the body walls are built of extremely minute living bricks, united together by different amounts of cementing material. Each one of these living units is called a cell.

Some of the tissues already enumerated consist of cells with only very little cement material binding them together; this, for instance, is seen in the epithelial tissues;

but in other tissues, particularly the connective tissues, which are not so eminently living as the rest, the amount of cement or intercellular material is much greater, and in this it is that the fibres are developed that confer the necessary strength upon these binding. tissues.

If, instead of going to the adult animal, we look at the animal in its earliest stage of development, the ovum, we find that it consists of a single little mass of living material, a single cell. As development progresses it becomes an adherent mass of cells. In the later stages of development various tissues become differentiated from each other by the cells becoming grouped in different ways, by alterations in the shape of the cells, by deposition of intercellular matter between the cells, and by chemical changes in the living matter of the cells themselves. Thus in some situations the cells are grouped into the various epithelial linings; in others the cells become elongated and form muscular fibres; and in others, as in the connective tissues, there is a preponderating amount of intercellular 
material which may become permeated with fibres, or be the seat of the deposition of calcareous salts, as in bone. Instances of chemical changes in the cells themselves are seen on the surface of the body, where the superficial layers of the epidermis become horny; in the mucous glands, where they become filled with mucin, and in adipose tissue, where they become charged with fat.

The term cell was first used by botanists; in the popular sense of the word a cell is a space surrounded by a wall, as the cell of a prison, or the cell of a honeycomb. In the vegetable cell there is a wall made of the starch-like material called cellulose; within this is the living matter, and a number of large spaces or vacuoles filled with a watery fluid. The use of the term cell by botanists was therefore completely justified.

But the animal cell is different; as a rule, it has no obvious cellwall, and vacuoles are not conspicuous. It is just a little naked lump of living material. This living material is jelly-like in consistency, possessing the power of movement, and the name protoplasm has been bestowed on it.

Somewhere in the protoplasm of all cells, generally near the middle in animal cells, is a roundish structure of more solid consistency than the rest of the protoplasm, called the nucleus.

An animal cell may therefore be defined as a mass of protoplasm containing a nucleus.

The simplest animals, such as amœbæ, consist of one cell only; the simplest plants, such as bacteria, torulæ, etc., consist of one cell only.
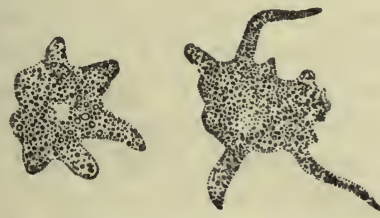

Fia. 2.-Amoebæ; unicellular animals.

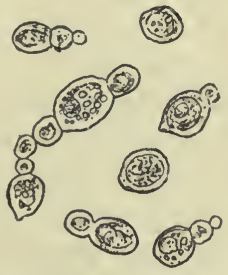

Fia. 3.-Cells of the yeast plant in process of bud. ding; unicellular plants.

These organisms are called unicellular. In the progress of their life history the cell divides into two; and the two new cells separate and become independent organisms, to repeat the process later on.

The higher animals and plants are always unicellular to start with, but on dividing and subdividing the resulting cells stick together and subsequently become differentiated and altered in the manner already indicated. In spite of these changes, the variety of which produces the great complexity of the adult organism, 
there are certain cells which still retain their primitive structure; notable among these are the white corpuscles of the blood.

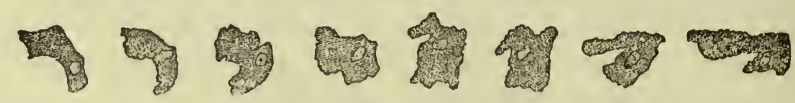

FIG. 4. - Human colourless blood-corpuscle, showing its successive changes of outline within ten minutes when kept moist on a warm stage. (Schofield.)

It would appear at first sight an easy problem to distinguish between a living thing, and one which is not living. The principal signs of life are the following:-

1. Irritability; that is the property of responding by some change under the influence of an external agent or stimulus. The most obvious of these changes is movement (amœboid movement, ciliary movement, muscular movement, etc.).

2. Power of assimilation, that is, ability to convert into protoplasm the nutrient material or food which is ingested.

3. Power of growth; this is a natural consequence of the power of assimilation.

4. Power of reproduction; this is a variety of growth.

5. Power to excrete; to give out waste materials, the products of other activities.

It should, however, be recognised that certain of these five characteristics may be absent or latent, and yet the object may be living. For instance, power of movement is absent in many vegetable structures; certain seeds and spores can be dried and kept for many years in an apparently dead condition, and yet will sprout and grow when placed in appropriate surroundings.

Of all the signs of life, those numbered 2 and 5 in the foregoing list are the most essential. Living material is in a continual state of unstable chemical equilibrium, building itself up on the one hand, breaking down on the other; the term used for the sum total of these intra-molecular rearrangements is metabolism. The chemical substances in the protoplasm which are the most important from this point of view are the complex nitrogenous compounds called Proteins. So far as is at present known, protein material is never absent from living substance, and is never present in any thing else but that which is alive or has been formed by the agency of living cells. It may therefore be stated that Protein Metabolism is the most essential characteristic of vitality. 


\section{CHAPTER II}

\section{THE ANIMAL CELL}

AN animal cell is usually of microscopic dimensions, in the human body varying from $\frac{1}{300}$ to $\frac{1}{3000}$ of an inch in diameter.

It consists of-

1. Protoplasm. This makes up the main substance of the cell.

2. Nucleus: a vesicular body within the protoplasm, generally situated near the centre of the cell.

3. Centrosome and attraction sphere: these are contained within the protoplasm, near the nucleus.

These three portions demand separate study.

\section{Protoplasm.}

Protoplasm is a soft jelly-like material; it usually contains minute particles or granules floating in it which are more solid in consistency, or globules (vacuoles) containing a watery fluid may be present. There is considerable difference of opinion between histologists as to whether, apart from this, protoplasm exhibits any structure or is a homogeneous jelly. With high powers of the microscope it can, however, be demonstrated that in many cells the protoplasm can be differentiated into two parts, a fine network of fibrillæ in which the more fluid and apparently structureless portion of the protoplasm is contained. This view of the structure of protoplasm is shown diagrammatically in the accompanying figure (fig. 5).

This theory of protoplasmic structure was advanced by Leydig, and has in more recent years been adopted by Schäfer, who speaks of the network or spongework as the

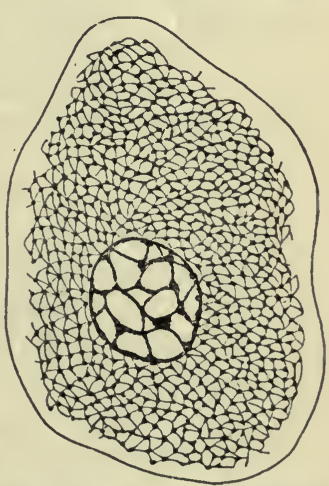

Fig. 5.-Diagram of an animal cell consisting of fibrillated protoplasm, containing a nuclous.

reticulum or spongioplasm, and the more fluid portion in its meshes as the enchylema or hyaloplasm. Bütschli, on the other 
hand, regards the spongioplasm as the optical effect of a honeycomb or froth-like structure, whereas other observers regard all such appearances as artifacts, that is, as produced artificially by methods of fixing and staining. Hardy, in particular, has shown that a film of any colloidal substance such as gelatin will, when it "sets," present the appearance of a network, and he regards it as probable that the network seen in cells may be due to a similar setting or coagulation of the protoplasm which occurs either when the cell dies or is fixed by hardening reagents. Schäfer, however, has quite recently been successful in obtaining instantaneous photographs of white blood-corpuscles in the living condition entirely untreated by any reagents, and these distinctly show the presence of a fine fibrillar network in the greater extent of their protoplasm. The spongioplasm-hyaloplasm theory is therefore now in a safer position than it occupied previously, and the adoption of this view renders more intelligible, as we shall see later, the phenomena of protoplasmic movement.

If we adopt this hypothesis, the granules seen in protoplasm may be in part thickened portions of the spongioplasm, but there is no doubt that the majority of them are freely floating in the protoplasm. Some of these are fatty in nature (staining black with osmic acid), some are composed of the substance called glycogen or animal starch (staining reddish-brown with iodine), and sometimes in a few unicellular animals they consist of inorganic (calcareous) matter. But by far the most constant and abundant of the granules are, like the main substance of the protoplasm, protein or albuminous in composition; by some observers these protein granules are regarded as essential constituents of the protoplasm. Substances stored within the protoplasm, such as pigment granules, fat globules, fluid in vacuoles, and glycogen, are spoken of as cell-contents or paraplasm.

The chemical structure of protoplasm can only be investigated after the protoplasm has been killed. The substances it yields are (1) Water ; at least three-quarters of the weight, often more, consist of water. (2) Proteins. These are the most constant and abundant of the solids. A protein or albuminous substance consists of carbon, hydrogen, nitrogen, oxygen, with sulphur and phosphorus in small quantities only. In nuclein, a complex material found in the nuclei of cells, phosphorus is more abundant. The protein obtained in greatest abundance in the cell protoplasm is called a nucleo-protein ; that is to say, it is a compound containing varying amounts of this material nuclein with protein. White of egg is a familiar instance of an albuminous substance or protein, and the fact (which is also familiar) that this sets into a solid on boiling will serve as a reminder that the greater number of the proteins found in nature have a 
similar tendency to coagulate under the influence of heat and other agencies. (3) Lipoids. These are so called because they resemble fats in their solubilities; they are present usually only in small quantities, and those which most constantly occur are phosphorised fats (such as lecithin) and cholesterin, a monatomic alcohol. (4) Inorganic salts, especially phosphates and chlorides of calcium, sodium, and potassium.

\section{The Nucleus.}

In form the nucleus is generally round or oval, but it may have in some cases an irregular shape, and in other cases there may be more than one nucleus in a cell.

The nucleus exercises a controlling influence over the nutrition and subdivision of the cell; any portion of a cell cut off from the nucleus undergoes degenerative changes.

A nucleus consists of four parts-

1. The nuclear membrane, which encloses it.

2. A network of fibres in appearance like the spongioplasm of the protoplasm, but on a larger scale; that is to say, the threads of which it is composed are much coarser and much more readily seen. The name chromoplasm has been given to this network.

3. The nuclear sap or matrix, a semi-fluid and homogeneous substance which occupies the interstices of the spongework of chromoplasm.

4. Nucleoli; - these are of two principal varieties; some are knots or thickened portions of the network (pseudo-nucleoli), and others, the true nucleoli, lie in the nuclear sap.

These four parts of the.nucleus are represented in the next diagram.

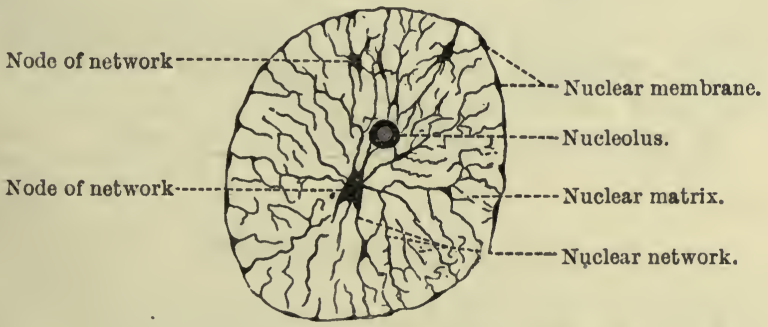

Fic. 6.-The nucleus-diagrammatic. (Waldejer.)

In the investigation of microscopic objects, a histologist is nearly always obliged to use staining agents; the extremely thin objects he examines are so transparent that, without such stains, much of the 
structure would be invisible. If such dyes as hæmatoxylin or safranin are employed, it is the nucleus which becomes most deeply stained, and thus stands out on the lighter background of the protoplasm.

But the whole nucleus does not stain equally deeply; it is the chromoplasmic filaments and the nucleoli which have most affinity for the stain, while the nuclear sap is comparatively unaffected. Hence the terms chromatin and achromatin originally introduced by Fleming. The membrane, the network, and the nucleoli are composed of chromatic substance or chromatin; it is so called not because it has any colour in the natural state, but because it has an affinity for colours artificially added to it. For a corresponding reason, achromatin or

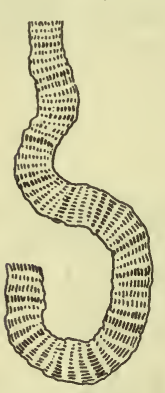

FiG. 7. - Part of a chromoplasmic filament, greatly magni. fied. (Carnoy.) achromatic substance is the name given to the substances which make up the nuclear sap.

Balbiani showed that the chromoplasmic filaments are apparently transversely marked into alternate dark and light bands ; this is due to the existence of minute highly refracting particles imbedded in regular series in a clear homogeneous and unstainable matrix (see fig. 7). The term chromatin should properly be restricted to these particles. These particles have special affinity for basic dyes, such as methylene blue and safranin.

Coming next to the chemical composition of the nucleus, it is found to consist principally of protein and protein-like substances. The nuclei of cells may be obtained by subjecting the cells to the action of artificial gastric juice; the protoplasm is nearly entirely dissolved, but the nuclei resist the solvent action of the juice. No doubt the nuclei contain several chemical compounds, but the only one of which we have any accurate knowledge has been termed nuclein, and this is identical with the substance called chromatin by histologists. It is soluble in alkalis, but precipitated by acids; it is different from a simple protein, as it contains in addition to carbon, nitrogen, oxygen, hydrogen, and sulphur, a large quantity ( 7 to 8 per cent. or even more) of phosphorus in its molecule. In many cases nucleins contain iron also.

\section{The Attraction Sphere.}

In addition to the nucleus and protoplasm, all living cells contain another structure, namely, a minute particle called a "centrosome," which has an attractive influence on protoplasmic fibrils and granules in its neighbourhood, the whole appearance produced being called an attraction sphere (fig. 8).

It is most prominent in cells which are dividing or about to 
divide. The centrosome, and then the attraction sphere, become double (fig. 9). In all probability the centrosome gives the primary

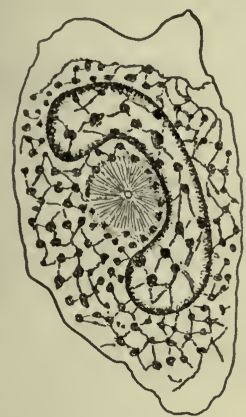

Fig. 8. - A cell (semi-diagrammatic) showing its attraction sphere. In this, as in most cases, the attraction sphere lies near the nucleus. (Schäfer.)

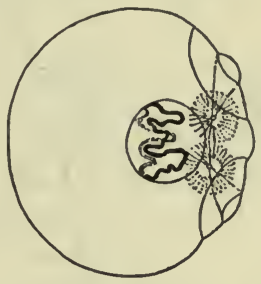

Fig. 9.-Orum of the worm Ascaris, showing a twin attraction sphere. The nucleus with its contorted filament of chromoplasm is repre. sented, but the protoplasm of the cell is not filled in. ( $v$. Beneden.)

impulse to cell-division. Some cells, for instance, the giant cells of red marrow, contain numerous centrosomes.

\section{Protoplasmic Movement.}

A cell possesses the power of breathing, that is, taking in oxygen; of nutrition, of building itself up from food materials; and of excretion, or the getting rid of waste material. But the most obvious characteristic of most cells is their power of movement.

When an amœba is observed with a high power of the microscope, it is found to consist of an irregular mass of protoplasm containing one or more nuclei, the protoplasm itself being more or less granular and vacuolated. If watched for a minute or two, an irregular projection is seen to be gradually thrust out from the main body and retracted; a second mass is then protruded in another direction, and gradually the whole protoplasmic substance is, as it were, drawn into it. The
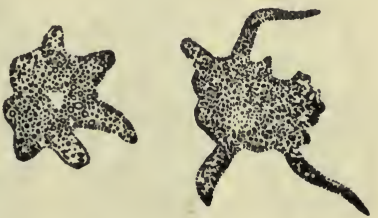

Fra. 10.-Amcbæ. Amœba thus comes to occupy a new position, and when this is repeated several times we have locomotion in a definite direction, together with a continual change of form. These movements, when observed in other cells, such as the colourless blood-corpuscles of higher animals (fig. 11), in the branched cells of the cornea and elsewhere, are hence termed amceboid. The projections which are alternately protruded and retracted are called pseudopodia. 
A streaming movement is not infrequently seen in certain of the protozoa, in which the mass of protoplasm extends long and fine processes, themselves very little movable, but upon the surface
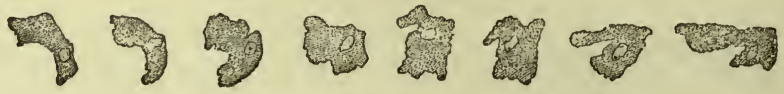

F1G 11.-Human colourless blood-corpuscle, showing its successive changes of ontline within ten minutes when kept moist on a warm stage. (Schoficld.)

of which freely-moving or streaming granules are seen. A gliding movement has also been noticed in certain animal cells; the motile part of the cell is composed of protoplasm bounding a central mass; by means of the free movement of this layer, the cell may be observed to move along.
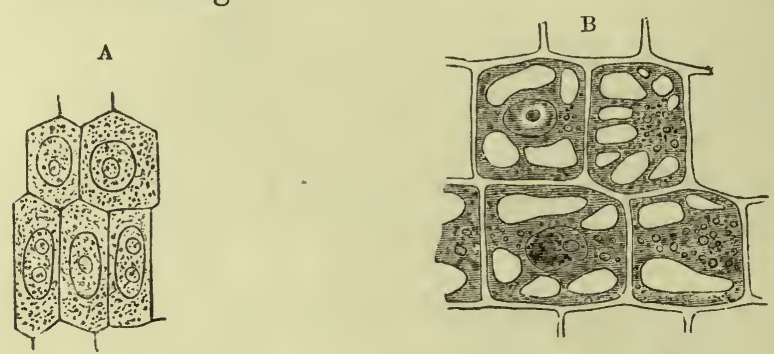

F1G. 12.-(A) Young vegetable cells, showing cell-cavity entirely filled with granular protoplasm enclosing a large oval nucleus, with one or more nucleoli.

(в) Older cells from same plant, showing distinct cellulose-wall and vacuolation of protoplasm.

In vegetable cells the protoplasmic movement can be well seen in the hairs of the stinging-nettle and Tradescantia and the cells of
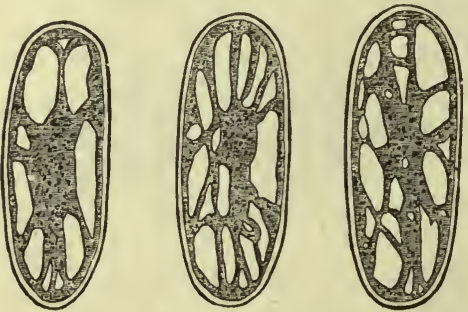

Fic. 13.-Cell of Tradescantia drawn at successive intervals of two minutes.-The cellcontents consist of a central mass connected by many irregular processes to a peripheral film, the whole forming a vacuolated mass of protoplasm, which is continually changing its shape. (Schofield.) its shape. (schofield.) towards or away from the irregular mass in the centre of the cell-cavity. Many of these streams of protoplasm run together into larger ones and are lost in the by the movement of the granules nearly always imbedded in it. For example, if part of a hair of Tradescantia (fig. 13) is viewed under a high magnifying power, streams of protoplasm containing crowds of granules hurrying along, like the foot-passengers in a busy street, are seen flowing steadily in definite directions, some coursing round the film which lines the interior of the cell-wall, and others flowing

Vallisneria and Chara ; it is marked 
central mass, and thus ceaseless variations of form are produced. The movement of the protoplasmic granules to or from the periphery is called circulation, whereas the movement of the protoplasm round the interior of the cell is called rotation.

The first account of the movement of protoplasm was given by Rösel in 1755 , as occurring in a small Proteus, probably a large freshwater amoba. His description was followed twenty years later by Corti's demonstration of the rotation of the cell sap in Characeæ, and in the earlier part of last century by Meyer in Vallisneria, 1827, and by Robert Brown, 1831, in "Staminal Hairs of Tradescantia." Then came Dujardin's description of the granular streaming in the pseudopodia of Rhizopods; movements in other animal cells were described somewhat later (Planarian eggs, v. Siebold, 1841; colourless blood-corpuscles, Wharton Jones, 1846).

There is no doubt that protoplasmic movement is essentially the same thing in both animal and vegetable cells. But in vegetable cells the cell-wall obliges the movement to occur in the interior, while in the naked animal cells the movement results in an external change of form.
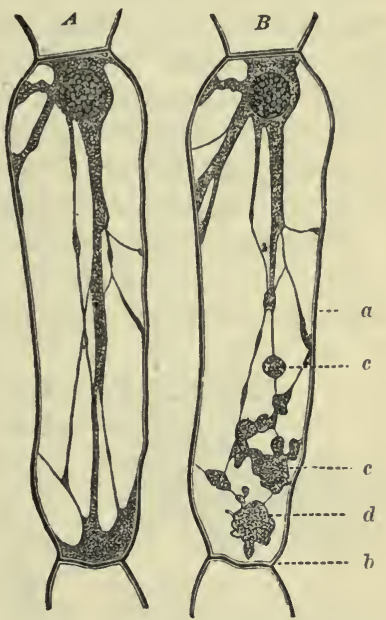

Fio. 14.-Cells from the staminal hairs of Tradescantia. $A$, fresh in water; $B$, the same cell after slight electrical stimulation; $a, b$, region of stimulation; $c$, $d$, clumps and knobs of contracted protoplasm. (Kithne.)

Although the movements of amœboid cells may be loosely described as spontaneous, yet they are produced and increased under the action of external agencies which excite them, and which are therefore called stimuli, and if the movement has ceased for the time, as is the case if the temperature is lowered beyond a certain point, movement may be set up by raising the temperature. Again, contact with-foreign bodies, gentle pressure, certain salts, and electricity, produce or increase the movement in the amcba. The protoplasm is, therefore, sensitive or irritable to stimuli, and shows its irritability by movement or contraction of its mass. The effects of some of these stimuli may be thus further detailed:-

a. Changes of temperature.-Moderate heat acts as a stimulant: the movement stops when the temperature is lowered near the freezing-point or raised above $45^{\circ}$ C. $\left(113^{\circ} \mathrm{F}\right.$.); between these two points the movements increase in activity; the optimum temperature is about $37^{\circ}$ to $38^{\circ} \mathrm{C}$. Though cold stops the movement of protoplasm, exposure to a temperature even below $0^{\circ} \mathrm{C}$. does not prevent 
its reappearance if the temperature is raised; on the other hand, prolonged exposure to a temperature of $42^{\circ}-45^{\circ} \mathrm{C}$. altogether kills the protoplasm and causes it to enter into a condition of heat rigor. This is due to the coagulation of the proteins present.

b. Chemical stimuli.-Distilled water first stimulates then stops amœboid movement, for by imbibition it causes great swelling and finally bursting of the cells. In some cases, however (myxomycetes), protoplasm can be almost entirely dried up, but remains capable of renewing its movement when again moistened. Dilute salt solution and very dilute alkalis stimulate the movements temporarily. Acids or strong alkalis permanently stop the movements : ether, chloroform, veratrine and quinine also stop it for a time.

Movement is suspended in an atmosphere of hydrogen or carbonic acid, and resumed on the admission of air or oxygen; complete withdrawal of oxygen will after a time kill protoplasm.

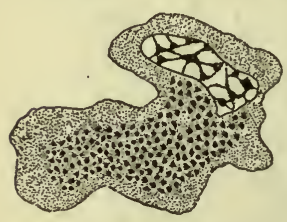

Fia. 15.-An amœboid corpuscle of the newt killed by instantaneous appli. cation of steam, showing the appearance of the pseudopodia. (After Schäfer, "Quain's Ana. tomy.")

c. Electrical.-Weak currents stimulate the movement, while strong currents cause the cells to assume a spherical form and to become motionless.

The amœboid movements of the colourless corpuscles of the blood may be readily seen when a drop of blood from the finger is mixed with salt solution, and examined on a warm stage with the microscope. If a pseudopodium of such a corpuscle is observed under a high power, it will be seen to consist of hyaloplasm, which has flowed out of its spongy home, the reticulum. Later, however, a portion of the reticular part of the protoplasm may enter the pseudopodium. The cells may be fixed by a jet of steam allowed to play for a moment on the surface of the cover glass. Fig. 15 illustrates one fixed in this way.

If, therefore, we adopt Schäfer's views on the structure of protoplasm we see that the essential act in the protrusion of a pseudopodium is the flowing of the hyaloplasm out of the spongioplasm; the retraction of the pseudopodium is a return of the hyaloplasm to the spongioplasm. The spongioplasm has an irregular arrangement with openings in all directions, so that the contractility of undifferentiated cells may exhibit itself towards any point of the compass.

The relation of cells to various forms of stimulus has been recently very extensively studied. Various forms of unicellular organisms have been used in these experiments, and the stimuli employed have been chemical, thermal, light, electric currents, and so forth. If the cell moves towards the source of attraction, the term positive taxis is employed; if it is repelled, negative taxis. The words, chemo-taxis, thermo-taxis, photo-taxis, galvano-taxis, etc., indicate the kind of stimulusi nvestigated. 


\section{Cell Division.}

A cell multiplies by dividing into two; each remains awhile in the non-dividing condition, but later it grows and subdivides, and the process may be repeated indefinitely.

The supreme importance of the cell, the growth of the body from cells, and the fact that cells are the living units of the organism, were first established in the vegetable world by Schleiden, and extended to the animal kingdom by Theodor Schwann. The ideas of physiologists depending on this idea are grouped together as cellular physiology, which under the guidance of Virchow was extended to pathology also: Virchow expressed the doctrine now so familiar as to be almost a truism in the terse phrase omnis cellula $e$ cellula (every cell from a cell).

The division of a cell is preceded by division of its nucleus. Nuclear division may be either (1) simple or direct, which consists in the simple exact division of the nucleus into two equal parts by constriction in the centre, which may have been preceded by division of the nucleolus; or (2) indirect, which consists in a series of changes which goes on in the arrangement of the nuclear reticulum, resulting in the exact division of the chromatic fibres into two parts, which form the chromoplasm of the daughter nuclei.

The changes in the nucleus during indirect division constitute

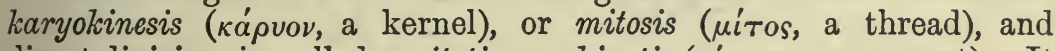

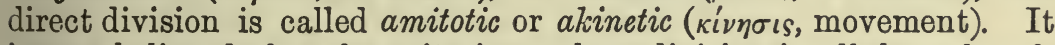
is now believed that the mitotic nuclear division is all but, though not quite, universal. Somewhat different accounts of the stages of the nuclear division have been given by different authorities, according to the kind of cell in which the nuclear changes have been studied; but, speaking generally, the process may be divided into the following stages :-

1. The non-dividing nucleus (fig. 16).

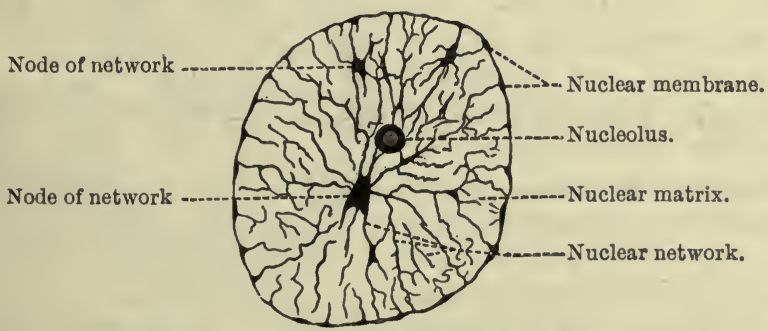

Fra. 16.-The non-dividing nucleus. (Waldeyer.)

2. The spirem or skein stage: the nucleoli dissolve, and the nuclear filaments form loops which run from one pole of the nucleus 
to the other (fig. 17). In some cells there is at first one long, much twisted thread, which subsequently breaks up into segments. The loops are called chromosomes.

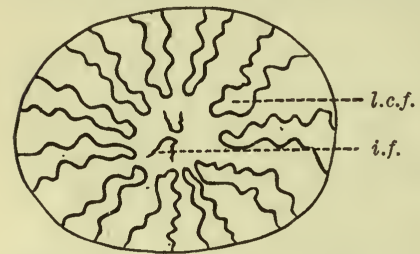

Fro. 17.-Early condition of the skein stage viewed at the polar end. l.c.f. Looped chromatic filament; i.f., irregular filament. (Rabl.) commencement of changes in the nucleus; the two attraction spheres become prominent in cell division, and the connecting achromatic
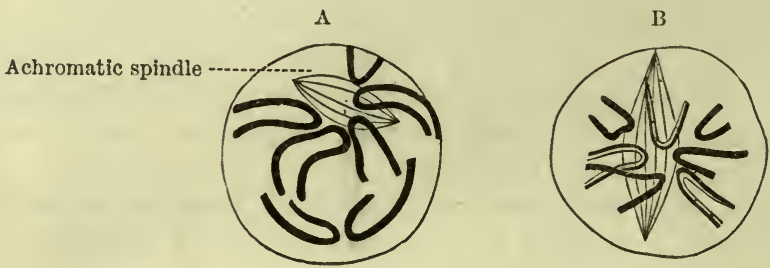

F10. 18.-Later condition of the skein stage in karyokinesis, A, The chromosomes become less con. voluted and the achromatic spindle appears. $\mathbf{B}$, The chromosomes split into two and the achpo. matic spindle becomes longitudinal. (Waldeyer.)

spindle is probably also formed from them or from the achromatic material of the nucleus.

At this stage the nuclear membrane is lost, and thus cell protoplasm and nuclear sap become continuous; the protoplasmic granules are arranged radially from the polar corpuscles. The star-like arrangement of these granules is much better marked in embryonic cells, indeed the lines present very much the appearance of fibrils (see fig. 20).

The $\mathrm{V}$-shaped chromosomes sink to the equator of the spindle, and arrange themselves so as to project horizontally from it.

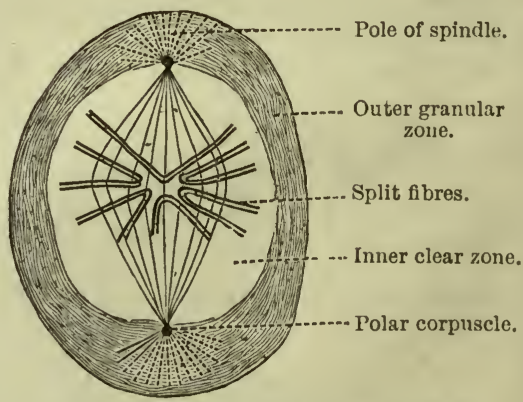

FiG. 19.-Monaster stage of karyokinesis. (Waldeyer.)

so as to project horizontally 
In cells which are the result of the sexual process, the number of chromosomes is always even, an equal number being contributed by each sex.

The number of chrornosomes varies with the species from four to twenty-four; in man the number is sixteen.

5. The stage of metakinesis. The sister threads separate, one set going towards one pole, and the other to the other pole of the spindle (fig. 21): these form the two daughter nuclei. The chromosomes are probably pulled into their new position by the contraction of the spindle fibres attached to them.

6. Each daughter nucleus goes backwards through the same series of changes; the

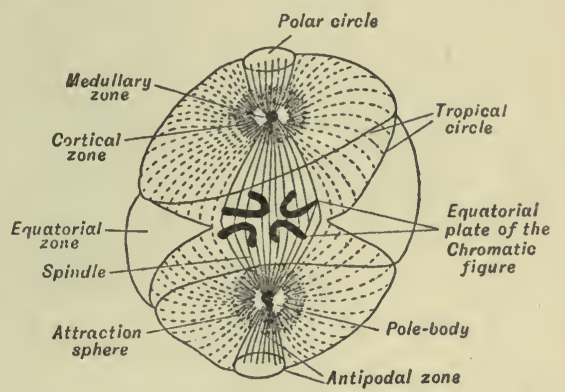

Fra. 20.-Ovum of the worm Ascaris in process of division. The attraction spheres are at opposite ends of the ovum; at the equator of the spindle which unites them, four chromosomes are seen. The protoplasm of the ovum, except in the equatorial zone of the cell, is arranged in lines radiating from the centre (centrosome) of the attraction sphere. (Waldeyer.) diaster or double star is followed by the dispirem or double skein, until at last two resting nuclei are obtained (fig. 22).

A new membrane forms around each daughter nucleus, the spindle atrophies, and the attraction sphere becomes less prominent. The
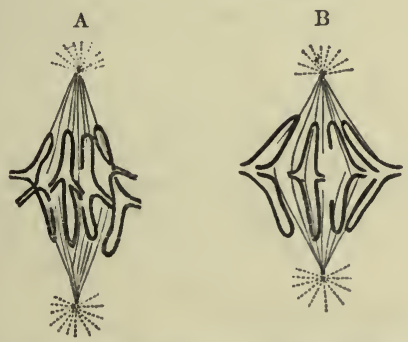

C

Fro. 21.-Metakinesis. A, Early stage. B, Later stage. c, Latest stage-formation of diaster. A and B show how the sister threads disentangle themselves from one another. (Waldeyer.)

division of the protoplasm into two parts around the nuclei begins in the diaster stage, and is complete in the stage represented in fig. 22 .

The karyokinetic process has been watched in all its stages by more than one observer. The time occupied varies from half an hour to three hours; the details, however, must be studied in hardened and appropriately stained specimens. They are most readily seen in cells with large nuclei, such as occur in the epidermis of 
amphibians, in the egg cells of certain worms, or in the growing tips of plants.

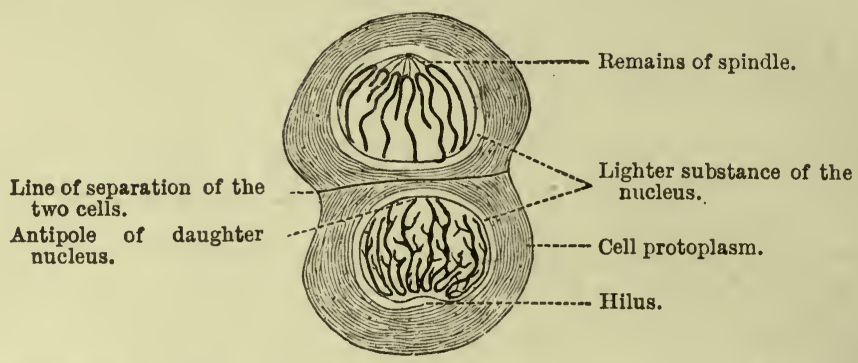

Fre. 22.-Final stages of karyokinesis. In the lower daughter nucleus the changes are still more advanced than in the upper. (Waldeyer.)

The phases may be summarised in a tabular way as follows:-

Network or Reticului . . . 1. Resting condition of mother nucleus (fig. 16).

Skein or SpIrem . . . $\left\{\begin{array}{l}\text { 2. Close skein of fine convoluted filaments } \\ \text { (fig. 17). } \\ 3 . \begin{array}{c}\text { Open skein of thicker filaments. Spindle } \\ \text { appears (fig. 18 A). }\end{array}\end{array}\right.$

Cleavage . . . . . . 4. Movement of $V$-shaped chromosomes to middle of nucleus, and each splits into two sister threads (fig. $18 \mathrm{~B}$ ).

STAR or Monaster . . . 5. 5tellate arrangement of $V$ filaments at

equator of spindle (fig. 19).

Divergence or Metakinesis - . 6. Separation of cleft filaments and movement along fibres of spindle (fig. $21 \mathrm{~A}$ and $\mathrm{B}$ ).

Dourle Star or Diaster - . 7. Conveyance of $V$ filaments towards poles of spindle (fig. $21 \mathrm{c}$ ).

Double Skein or Dispirem . $\quad \cdot\left\{\begin{array}{l}\text { 8. Open skein in daughter nuclei. } \\ 9 \text {. Close skein in daughter nuclei (fig. 22). }\end{array}\right.$

Network or Reticulus . . . 10. Resting condition of daughter nuclei (fig. 22).

\section{The Ovum.}

The ovary (see Chapter LIX.) is an organ which produces ova. An ovum is a simple animal cell; its parts are seen in the next diagram.

It is enclosed in a membrane called the zona pellucida. The body of the cell is composed of protoplasm loaded with granules of food material, called the yolk or vitellus. The nucleus and nucleolus are sometimes still called by their old names, germinal vesicle and germinal spot respectively. The attraction sphere is not shown in the diagram.

The ovum first discharges from its interior a portion of its nucleus, which forms two little globules upon it called the polar globules. 
Fertilisation then occurs; that is to say, the head or nucleus of a male cell called a spermatozoon penetrates into the ovum, and becomes fused with the remains of the female nucleus.

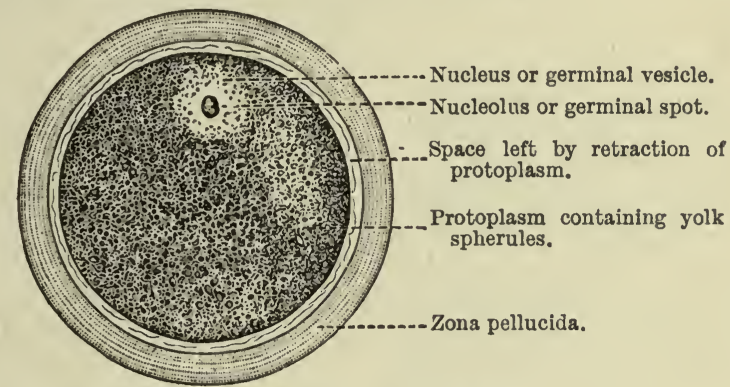

Fic. 23.-Representation of a human ovum. (Cadiat.)

Cell division or segmentation then begins, and the early stages are represented in the next figure.
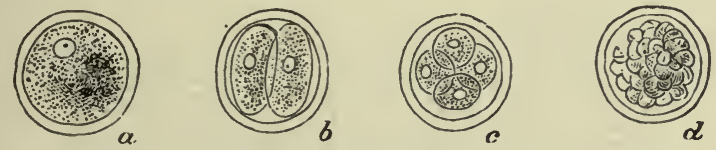

Fio. 24.-Diagram of an ovum (a) undergoing segmentation. In (b) it has divided into two, in (c) into four; and in $(d)$ the process has resulted in the production of the so-called "mulberry-mass." (Frey.)

Fluid discharged from the cells accumulates within the interior of the mulberry mass seen in fig. $24 d$, and later, if a section is cut through it, the cells will be found arranged in three layers.

The outermost layer is called the epiblast. The middle layer is called the mesoblast. The innermost layer is called the hypoblast.

From these three layers the growth of the rest of the body occurs, nutritive material being derived from the mother in mammals by means of an organ called the placenta.

The epiblast forms the epidermis, the outermost layer of the adult. It also forms the nervous/system.

The hypoblast, the innermost layer of the embryo, forms the lining epithelium of the alimentary (except that of the mouth and anus, which are involutions from the epiblast) and respiratory tracts, that is, the innermost layer of the adult. It also forms the cellular elements in the large digestive glands, such as the liver and pancreas, which are originally, like the lungs, outgrowths from the primitive digestive tube.

The mesoblast forms the remainder, that is, the great bulk of the body, including the muscular, osseous, and other connective tisswes ; the circulatory and urino-genital systema 


\section{CHAPTER III}

\section{EPITHELIUM}

WE have seen in the introductory chapter that the elementary tissues of which the organs of the body are built up may be arranged into four groups: epithelial, connective, muscular, and nervous. The first of these, the epithelial tissues, follows naturally on a study of the animal cell, as an epithelium may be defined as a tissue com-

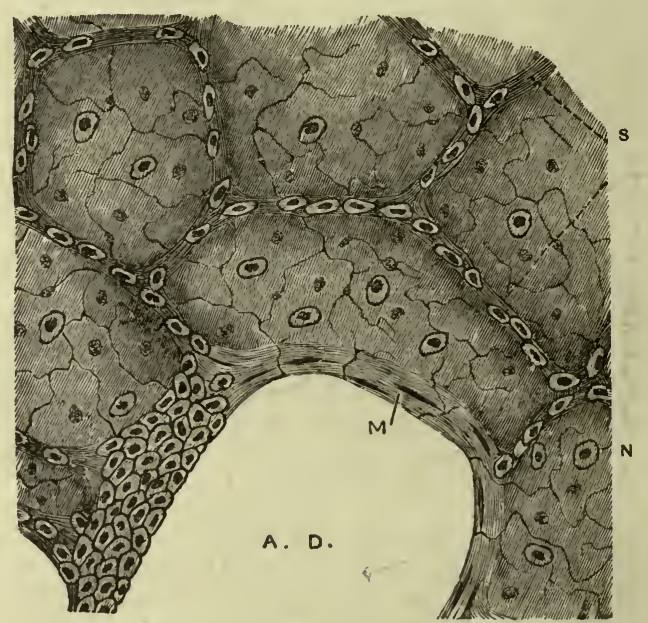

F10. 25.-From a section of the lung of a cat, stained with silver nitrate. N. Alveoli or air-cells, lined with large flat, nucleated cells, with some smaller polyhedral nucleated cells. (Klein and Noble Smith.)

posed entirely of cells united by a minimal amount of cementing material. As a rule, an epithelium is spread out as a membrane, covering a surface or lining the cavity of a hollow organ.

Epithelia may be grouped into two great classes, each of which may be again subdivided according to the shape and arrangement of the cells of which it is composed. 
Class 1.-Simple epithelium; that is, an epithelium consisting of only one layer of cells. Its subgroups are:-

a. Pavement epithelium. This consists of a layer of thin cells arranged in the form of an accurately fitting mosaic; this is typically seen in the epithelium that lines the air-sacs of the lungs (fig. 25). The endothelium found in the interior of the blood and lymph vessels and serous sacs is very similar in structure, but differs from other epithelia in being the only one of mesoblastic origin (fig. 26).

b. Cubical and columnar epithelium. Here the cells, as their names imply, are thicker. Cubical epithelium is found in the alveoli of the thyroid, in the tubules of the testis, and in the ducts of many glands. Columnar epithelium lines the alimentary canal from the stomach to the anus.

The four figures (figs. 27-30) present the very typical columnar cells, each with a bright striated border, which arc found lining the intestine. Fig. 28 shows how they are arranged on the surface of a villus, one of the numerous little projections found in the small intestine. The gaps seen there are due to the formation of what are called goblet cells. In some of the columnar cells a formation of granules occurs; these consist of a substance called mucigen; these run together and are discharged from the cell as a brightly refracting globule of mucin, leaving the cell with open mouth like a goblet, the nucleus being sur-

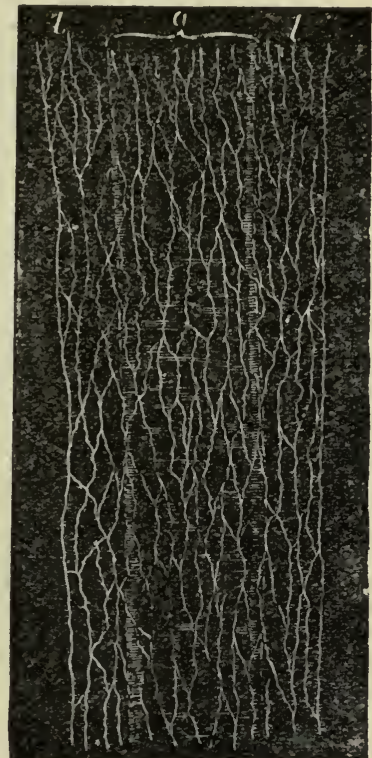

FIG. 26.-Surface view of an artery from the mesentery of a frog, ensheathed in a perivascular lymphatic vessel. $a$, The artery, with its circular muscular coat (media) indicated by broad transverse markings, with an indication of the adventitia outside. $l$, Lymphatic vessel ; its wall is a simple endothelial membrane. (Klein and Noble Smith.) rounded by the remains of the protoplasm in the narrow stem (fig. 30). This transformation is a normal process continually going on throughout life, the discharged mucin being the chief constituent of phlegm or mucus. The cells themselves may recover their original shape after discharge and repeat the process later on.

c. Ciliated epithelium; this form of epithelium presents so many points of physiological interest, that a separate section will be devoted to it later in this chapter.

Cuass 2.-Compound Epithelium; that is, an epithelium consisting of more than one layer of cells. It contains two subgroups.

a. Transitional epithelium found lining the bladder and ureters. 
It consists of three or four layers of large cells, the most typical of which are pear-shaped (fig. 31 ).

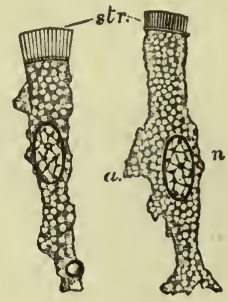

1.

FIG, 27.-Columnar epithelium cells of the rabbit's intestine. The cells have been isolated after maceration in very weak chromic acid. The cells are much vacuolated, and one of them has a fat globule near its attached end. The striated border (str.) is well seen, and the bright disc separating it from the cell protoplasm. $n$, nucleus with intra-nuclear network; $a$, a thinned-out winglike projection of the cell which probably fitted between two adjacent cells. (Schäfer.)

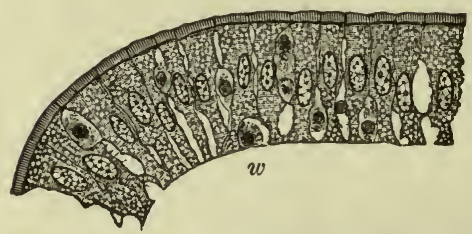

FIG. 29.-A row of columnar cells from the rabbit's intestine. Smaller cells are seen between the epithelium cells; these are lymph-corpuscles. (Schäfer.)

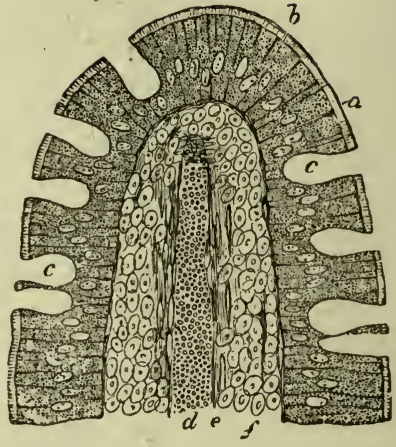

Fic. 28.-Vertical section of an intestinal villus of a cat. $a$, The striated border of the eji. thelium; $b$, columnar epithelium; $c$, goblet cells ; $d$, central lymph-vessel ; $c$, unstriped muscular fibres; $f$, adenoid stroma of the villus in which are contained lymph-cor. puscles. (Klein.)
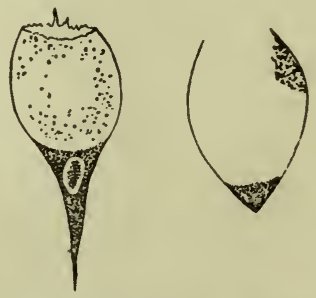

FIQ. 30.-Goblet cells. (Kloin.)
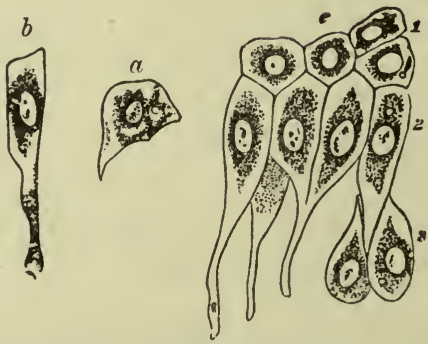

Fia. 31.-Epithelium of the bladder. $a$, One of the cells of the first row ; $b$, a cell of the second row ; $c$, cells in situ, of first, second, and deepest layers. (Obersteiner.)

b. Stratified epithelium. Here the cells are arranged in numerous layers. It is found composing the epidermis, and the linings of the 
various orifices of the body. It lines the upper end of the alimentary canal from the mouth to the point where the cesophagus or gullet enters the stomach. The deepest layers are columnar or cubical in shape, and the surface layers are composed of flattened scales, their

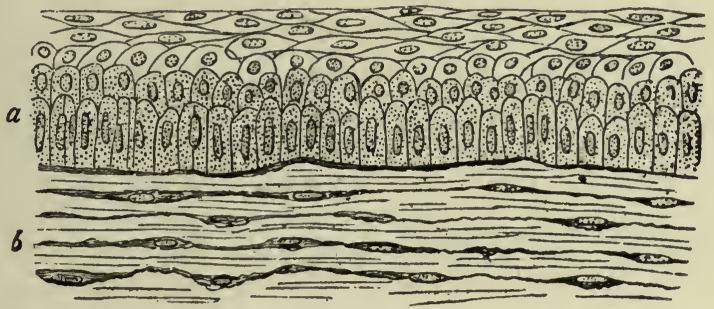

F10. 32.-Vertical section of the stratified epithelium of the rabbit's cornea. $a$, Anterior epithelium, showing the different shapes of the cells at various depths from the free surface; $b, a$ portion of the substance of cornea. (Klein.)

protoplasm being replaced by horny material or keratin. Covering the front of the cornea of the eye is a typical form of stratified epithelium (fig. 32), but the number of layers is not so great as it is in the majority of such epithelia.

\section{Ciliated Epithelium.}

The cells of ciliated epithelium are generally of columnar shape (fig. 33), but they may occasionally be spheroidal (fig. 34).

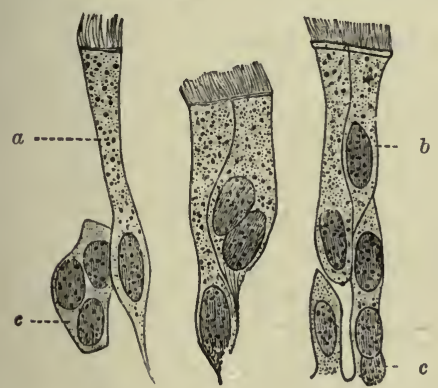

Fro. 33.-Ciliated epithelium from the human trachea. $a$, Large fully-formed cell ; $b$, shorter cell ; $c$, developing cells with more than one nucleus. (Cadiat.)

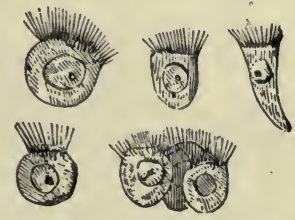

F1a. 34.-Spheroidal ciliated cells from the mouth of the frog. $\times 300$ diame. ters. (Sharpey.)

Each cell is surmounted by a bunch of fine tapering filaments. They were originally called cilia because of their resemblance in shape to eyelashes. They differ from eyelashes in being extremely small, 
and in not being stiff; they are in fact composed of protoplasm. During life these move to and fro, and so produce a current of

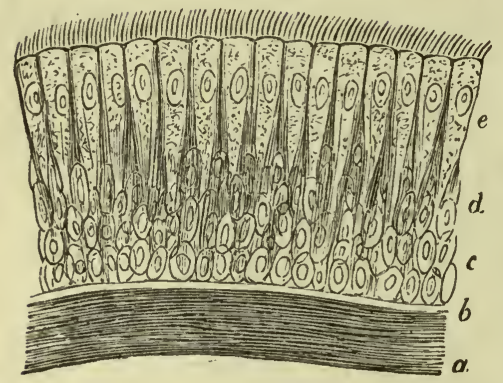

FIu. 35.-Ciliated epithelium of the human trachea. $a$, Layer of longitudinally arranged elastic fibres; $b$, basement membrane; $c$, deepest cells, circular in form; $d$, intermediate elongated cells; $e$, outermost layer
of cells fully developed and bearing cilia. $\times 350$. (Kölliker.) fluid over the surface they cover. Like columnar cells, they may form goblet cells and discharge mucin.

In the larger ciliated cells, it will be seen that the border on which the cilia are set is bright, and composed of little knobs, to each of which a cilium is attached; in some cases the knobs are prolonged into the cell protoplasm as filaments or rootlets (fig. 36). According to some observers these rootlets are outgrowths from the multiplied centrosome of the cell.

The bunch of cilia is homologous with the striated border of columnar cells.

Ciliated epithelium is found in the human body, (1) lining the air passages, but not in the alveoli of the lungs; these are lined by pavement epithelium; (2) in the Fallopian tubes and upper part of the uterus; (3) in the ducts of the testis known as the vasa efferentia and coni vasculosi; here the cilia are the longest found in the body; (4) in the ventricles of the brain and central canal of the spinal cord; (5) the tail of a spermatozoon may also be regarded as a long cilium.

In other animals cilia are found in other parts; for instance, in the frog the mouth and gullet are lined by ciliated cells; in the tadpole, the whole surface of the body and especially the gills are covered with cilia. Among the invertebrates one finds many protozoa completely covered with cilia ; in many embryos the cilia are arranged in definite bands round the body; in the rotifers or wheel animalcules, a ring of cilia round the mouth gives the name to this particular group. The gills of many animals are covered with

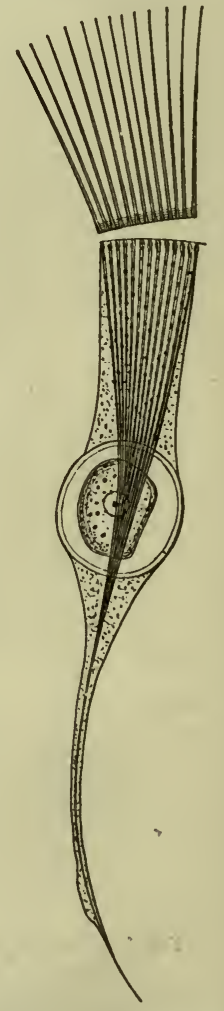

Frg. 36.-Ciliated cell from the intestine of a mol. lusc. (Engelmann.) cilia; and the cells of portions of the kidncy tubules in some animals are ciliated. 


\section{Ciliary Motion.}

Ciliary motion reminds one of amoboid movement, but it is much more rapid, and more orderly. It consists of a rhythmical movement of the cilia, a bending over, followed by a lessening of the curvature, repeated with great frequency.

When living ciliated epithelium, e.g., from the gill of a mussel, or from the mouth of the frog, is examined under the microscope in a drop of 0.9 per cent. solution of common salt (normal saline solution), the cilia are seen to be in constant rapid motion, each cilium being fixed at one end, and swinging or lashing to and fro. The general impression given to the eye of the observer is very similar to that produced by waves in a field of corn, or swiftly running and rippling water, and the result of their movement is to produce a continuous current in a definite direction, and this direction is the same on the same surface, being usually in the case of a cavity towards the external orifice.

There is not only rhythmicality in the movement of a single cilium, but each acts in harmony with its fellows in the same cell, and on neighbouring cells.

The uses of cilia can from the above be almost guessed; in the respiratory passages they create a current of mucus with entangled dust towards the throat; in the Fallopian tube or oviduct they assist the ovum on its way to the uterus; in the gullet of the frog they act downwards and assist swallowing; in the ciliated protozoa they are locomotive organs. Over the gills of marine animals they keep up a fresh supply of water, and in the case of the rotifers, which are fixed animals, the current of water brings food to the mouth.

Ciliary motion is independent of the will, and of the influence of the nervous system. It may continue for many hours after death or removal from the body, provided the portion of tissue under examination be kept moist. Its independence of the nervous system is shown also in its occurrence in the lowest invertebrate animals, which are unprovided with anything analogous to a nervous system. The vapour of ether or chloroform and carbon dioxide arrest the motion, but it is renewed on the discontinuance of the application. The movement ceases when the cilia are deprived of oxygen, although it may continue for a time in the absence of free oxygen, but is revived on the admission of this gas. The contact of various substances, e.g., bile, strong acids, and alkalis, will stop the motion altogether; but this depends on destruction of the delicate substance of which the cilia are composed. Temperatures above $45^{\circ} \mathrm{C}$. and near $0^{\circ}$ C. stop the movement, whereas moderate heat and dilute alkalis are favourable to the action, and revive the movement after temporary cessation. The exact explanation of ciliary movement is not known; 
whatever may be the precise cause, the movement must depend upon some changes going on in the cell to which the cilia are attached, as when the latter are cut off from the cell the movement ceases, and when severed so that a portion of the cilia are left attached to the cell, the attached and not the severed portions continue the movement. It has been suggested by Engelmann that the contractile part of the protoplasm is only on the concave side of a curved cilium, and that when this contracts the cilium is brought downwards; when relaxation occurs, the cilium rebounds by the elastic recoil of the convex border.

Schäfer has suggested that the flow of hyaloplasm backwards and forwards will explain ciliary as it will amoboid movement. In an amcboid cell, the spongioplasm is irregular in arrangement, hence an outflow of hyaloplasm from it can occur in any direction. But in the curved projection called a cilium, the hyaloplasm can obviously flow in only one direction into the cilium and back again. The flow of hyaloplasm into the cilium will raise the pressure there and cause it to straighten; a movement in the reverse direction will cause the cilium to curve.

The action of dilute alkalis and acids on cilia is interesting. Dilute acids stop ciliary motion; and cilia, if allowed to act in salt solution for a time, get more and more languid, and finally cease acting; in popular language they become fatigued. Now we shall find in muscle that fatigue is largely due to the accumulation of the acid products of muscular activity; remove the sarco-lactic acid and fatigue passes off. It is probable that the same occurs in other contractile tissues; the cilia gradually stop, due to acid products of their activity collecting around them; when these are neutralised with dilute alkali the cilia resume activity.

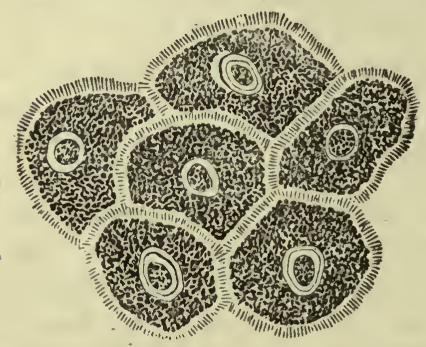

FIG. 37.--Jagged cells from the middle layers of stratified epithelium, from a vertical section of the gum of a newborn infant. (Klein.)

\section{Nutrition of Epithelium.}

Epithelium has no blood-vessels; it is nourished by lymph. When the blood is circulating through the thinwalled small blood-vessels in the tissues beneath the epithelium, some of its fluid constituents escape. This fluid is called lymph; it penetrates to all parts of the cellular elements of tissues and nourishes them. In the thicker varieties of epithelium, the presence of the irregular minute channels between the cells (fig. 37) enables the lymph to soak more readily between the cells than it would otherwise be able to do. Epithelium is also destitute of 
nerves as a rule. But in stratified epithelium, particularly that covering the cornea at the front of the eye and in the deeper layers of the epidermis, a plexus of nerve-fibrils is found.

\section{Chemistry of Epithelium.}

There is not much to add to what has been already stated concerning cells; protoplasm and nucleus have the same chemical composition as has been already mentioned in Chapter II. Two new substances have, however, been mentioned in the foregoing chapternamely, mucin and keratin.

Mucin.-This is a widely distributed substance occurring in epithelial cells or shed out by them (see goblet cells, fig. 30 ). It also forms the chief constituent of the cementing substance between epithelial cells. We shall again meet with it in the intercellular substance of the connective tissues. The mucins obtained from different sources varies somewhat in composition and reactions, but they all agree in the following points:-

(a) Physical character: viscid and tenacious.

(b) Precipitability from solutions by acetic acid. They all dissolve in dilute alkalis, such as lime-water.

(c) They are all compounds of protein, with a carbohydrate material; by treatment with mineral acid this is hydrolysed into a reducing but non-fermentable sugar-like substance.

The substance mucin, when it is formed within cells (goblet cells, cells of mucous glands), is preceded in the cells by granules of a substance which is not mucin, but is readily changed into mucin. This precursor, or mother-substance of mucin, is called mucigen or mucinogen.

Keratin, or horny material, is the substance found in the surface layers of the epidermis, in hairs, nails, hoofs, and horns. It is very insoluble, and chiefly differs from other proteins in its high percentage of sulphur.

The silver nitrate reaction of cementing substance. The principal chemical reaction which is employed by histologists for demonstrating the cement or intercellular substance which binds epithelial cells together was formerly supposed to be due to the formation of a silver-protein compound which was reduced by sunlight. Macallum has conclusively shown that this is not the case, but that it is an inorganic reaction. Cementing material is specially rich in chlorides (mainly sodium chloride); the addition of silver nitrate leads to the formation of silver chloride, and it is this which is reduced by light. The silver reaction obtained in other tissues is similarly explained: in fact silver nitrate is a micro-chemical reagent for detecting the localities in the body where chlorides occur. According to Macallum the nuclci of all cells are entirely free from chlorides. 


\section{CHAPTER IV}

\section{THE. CONNECTIVE TISSUES}

THE connective tissues are the following :-

1. Areolar tissue.

2. Fibrous tissue.

6. Jelly-like tissue.

3. Elastic tissue.

7. Cartilage.

4. Adipose tissue.

8. Bone and dentine.

5. Retiform and lymphoid tissues.

At first sight these numerous tissues appear to form a very heterogeneous group, including the most solid tissues of the body (bone, dentine) and the most fluid (blood).

But on examining a little more deeply, one finds that the grouping of these apparently different tissues together depends on a number of valid reasons, which may be briefly stated as follows:-

1. They all resemble each other in origin. All are formed from the mesoblast, the middle layer of the embryo.

2. They resemble each other structurally; that is to say, the cellular element is at a minimum, and the intercellular material at a maximum.

3. They resemble each other functionally; they form the skeleton, and act as binding, supporting, or connecting tissues to the softer and more vital tissues.

An apology is sometimes made for calling the blood a tissue, because one's preconceived idea of a tissue or texture is that it must be something of a solid nature. But all the tissues contain water. Muscular tissue contains, for instance, at least three-quarters of its weight as water. Blood, after all, is not much more liquid than muscle. Blood, moreover, contains cellular elements analogous to the cells of other tissues, but separated by large quantities of a fluid intercellular material called blood-plasma.

Blood is also mesoblastic, and thus the two first characteristics of a connective tissue are present. It does not fulfil the third condition by contributing to the support of the body as part of the skeleton, but it does so in another sense, and serves to support the body by conveying nutriment to all parts. 


\section{Areolar Tissue.}

This is a very typical connective tissue. It has a wide distribution, and constitutes the subcutaneous, subserous, and submucous tissues. It forms sheaths (fasciæ) for muscles, nerves, blood-vessels, glands, and internal organs, binding them in position and penetrating into their interior, supports and connects their individual parts.

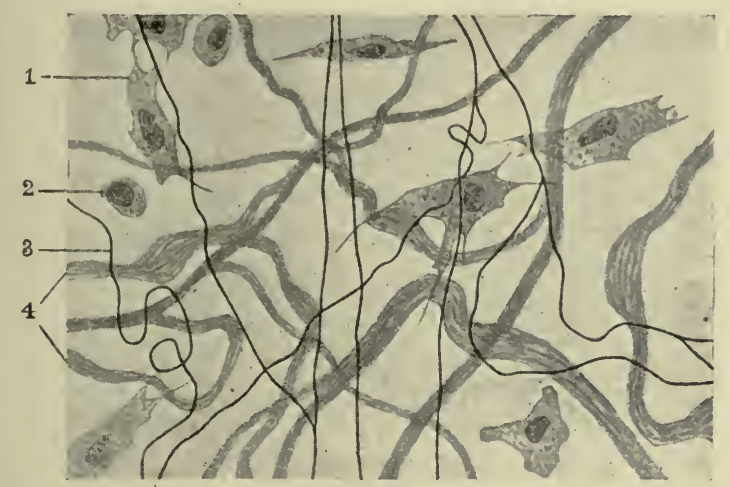

Fia. 38. -Loose areolar tissue from the subcutaneous tissue of the cat. $\times 300$. This shows the two varieties of libres, and two of the varieties of connective-tissue"corpuscles, namely, the lamellar cells or fibroblasts, and a wander cell. 1, Lamellar cell; 2, wander cell; 3 , elastic fibres; 4, bundles of white fibres. (After Szymonowicz.)

On microscopic examination it is seon that this typical connective tissue consists of four different kinds of material, or, as they may be termed, histological elements. They are:-

(a) Cells, or connective-tissue corpuscles.

(b) A homogeneous matrix, ground substance, or intercellular material.

(c) White fibres

(d) Yellow or elastic fibres $\{$ These are deposited in the matrix.

In considering these four histological elements we may first take the fibres, because they are the most obvious and abundant of the structures observable.

The white fibres. These are exquisitely fine fibres collected into bundles which have a wavy outline. The bundles run in different directions, forming an irregular network, the meshes between which are called areolce; hence the name areolar.

They are composed of the chemical substance called collagen. On boiling they yield gelatin; some chemists regard collagen as the anhydride of gelatin; but whether this is so or not, the gelatin is undoubtedly derived from the collagen. Gelatin is a protein though 
it has certain characters which distinguish it from most members of the large protein family. Its most characteristic property is its power of jellying or gelatinising; that is, it is soluble in hot water, and on cooling the solution it sets into a jelly.

The yellow or elastic fibres. These are seen readily after the white fibres are rendered almost invisible by treatment with dilute acetic

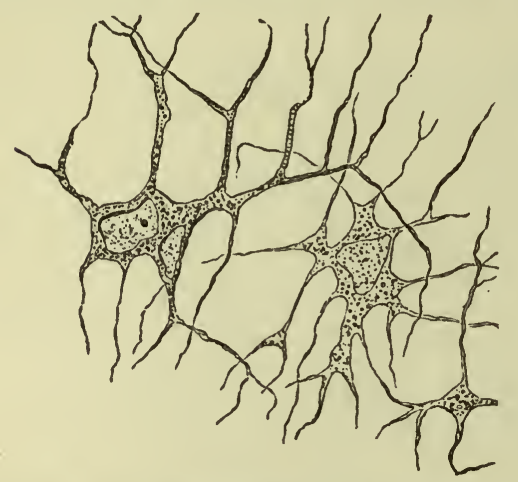

FIG. 39.-Horizontal preparation of the cornea of frog, stained with gold chloride; showing the network of branched corneal corpuscles. The ground substance is completely colourless. $\times 400$. (Klein.)

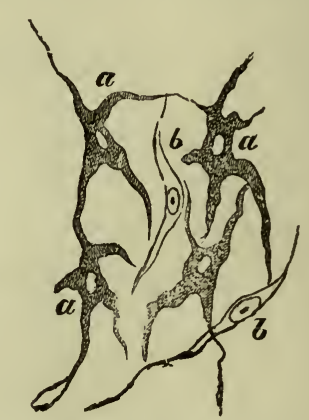

Fra. 40.-Ramified pigment cells, from the tissue of the choroid coat of the eye. $\times 350$. $a$, Cell with pigment; $b$, colourless fusiform cells. (Kölliker.)

acid, or after staining with such dyes as magenta and orcein, for which elastic fibres have a great affinity. They are bigger than the white fibres, have a distinct outline, and a straight course; they run singly, branch, and join neighbouring fibres.

The material of which the elastic fibres are composed is called elastin, another somewhat exceptional protein. It is unaltered, as we have seen, by dilute acid. It also resists the action of very strong acid, and is not affected by boiling water.

Connective-tissue corpuscles. These are the cells of connective tissue: the following are the varieties most frequently seen.

1. Lamellar cells (fig. 38). These are branched, and the branches of neighbouring cells may unite as in the cornea (fig. 39); they were formerly called fibroblasts from the mistaken idea that they gave rise to the formation of fibres.

2. Pigment cells. These are lamellar cells laden with a brown or black pigment. They are seen in the subcutaneous tissues of many animals, e.g., the frog, and in the choroid coat of the eyeball (fig. 40).

3. Mast-cells. These are usually unbranched, and their protoplasm is crowded with albuminous granules which are stained deeply by gentian-violet and other basic dyes. 
They are most abundant in the neighbourhood of bloodvessels (fig. 41). The name was given to them by Ehrlich, who erroneously believed that they multiplied on a rich diet. Certain cells called clasmatocytes by Ranvier are very like the mast-cells, but their protoplasm branches.

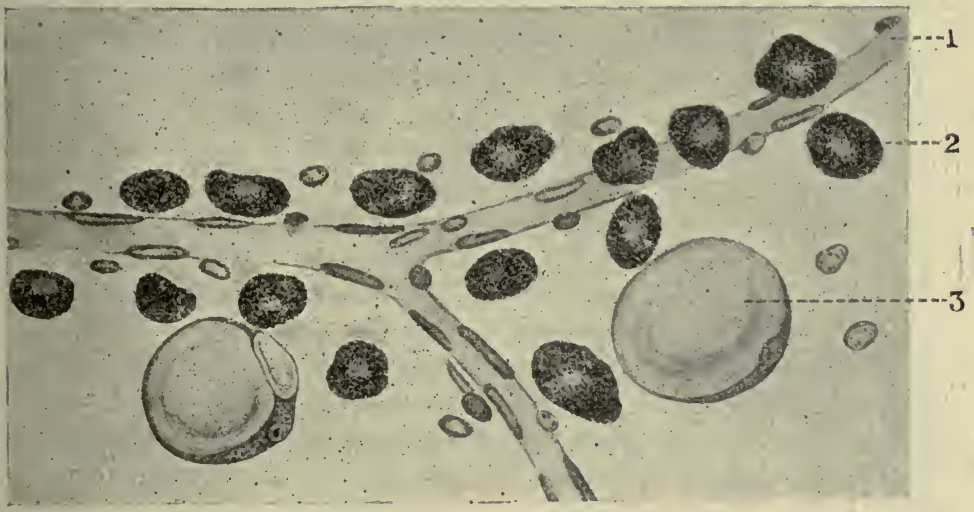

FiG. 41. - Subcutaneous areolar tissue of the rat, showing a small blood-vessel, with numerous mastcells in the neighbourhood, also two fat-cells. $\times 540$. 1, Small blood-vessel; 2, mast-cell; 3, fat-cell. (After Szymonowicz.) *

4. Wander cells. White blood-corpuscles which have emigrated from the neighbouring blood-vessels.

The ground-substance. This is represented in fig. 38 by the shaded background.

It may be readily demonstrated in a silver nitrate preparation (fig. 42); for the intercellular material has the same property of reducing silver salts in the sunlight that the cement-material of epithelium has (see p. 27). It becomes in consequence dark brown, with the exception of the spaces occupied by the corpuscles.

The spaces intercommunicate like the cells, and being considerably larger than the cells form a ramifying network of irregular channels, which were first termed by v. Recklinghausen the Saft Kanälchen, or little juice canals. Areolar tissue is certainly pro-

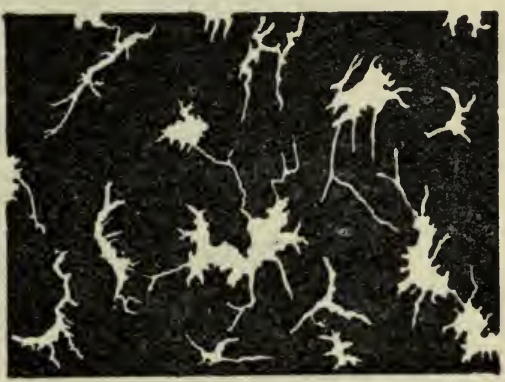

Fug. 42.-Ground-substance of connective tissue, stained by silver nitrate. The cell spaces are left white. (After Schäfer.) vided with blood-vessels, but the tissue elements are, as in all tissues, provided with nutriment by the exudation from the blood called 
lymph. The Saft Kanälchen enable the lymph to penetrate to every part of the areolar tissue.

\section{Fibrous Tissue.}

This is a kind of connective tissuo in which the white fibres predominate; it is found in tendons and ligaments, in the periosteum,

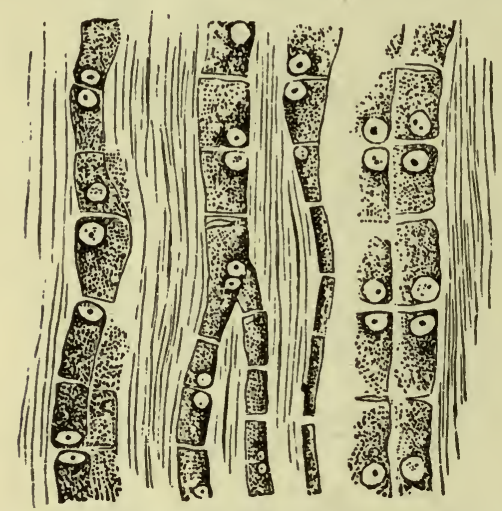

Fic. 43.-Caudal tendon of young rat, showing the arrangement, form, and structure of the tendon cells. The bundles of white fibres between which they lie have been rendered transparent and indistinct by the application of acetic acid. $\times$ 300. (Klein.)

The cell spaces in which the cells lie are in arrangement like the cells; they can be brought into relief by staining with silver nitrate (see fig. 44).

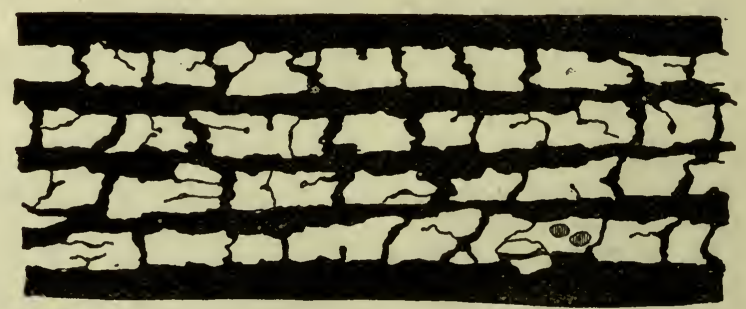

FIG. 44.-Cell spaces of tendon, brought into view by treatment with silver nitrate. (After Schäfer.)

\section{Elastic Tissue.}

This is a form of connective tissue in which the yellow or elastic fibres predominate. The yellow fibres are larger than those found in areolar tissue (see fig. 45), and are bound into bundles by areolar 
tissue. It is found in the ligamentum nuchæ of the $\mathrm{ox}$, horse, and many other animals; in the ligamenta subflava of man; in the arteries and veins, constituting the fenestrated coat of Henle; in the lungs and trachea; in the stylo-hyoid, thyro-hyoid, and cricothyroid ligaments; and in the true vocal cords.

Elastic tissue, being extensible and elastic (i.e., recoiling after it has been stretched), has a most important use in assisting muscular

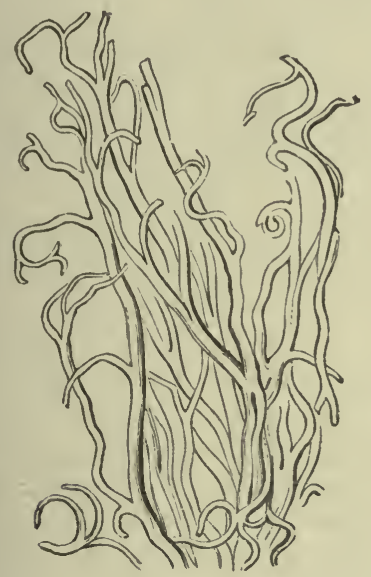

Fig. 45.-Elastic fibres frum the ligamenta subflava. $\times 200$. (Sharpey.)

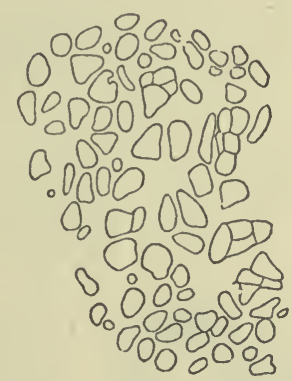

Fig. 46.-Transverse section of a portion of lig. nuchæ, showing the angular out. line of the fibres. (After Stöhr.)

tissue in a mechanical way, and so lessening the wear and tear of such an important tissue as muscle. Thus, in the ligamenta subflava of the human vertebral column it assists in the maintenance of the erect posture; in the ligamentum nuchæ in the neck of quadrupeds it assists in the raising of the head and in keeping it in that position. In the arterial walls, and in the air tubes and lungs, it has a similar important action, as we shall see when discussing the subjects of the circulation and respiration.

\section{Adipose Tissue.}

In almost all regions of the human body a larger or smaller quantity of adipose or fatty tissue is present; the chief exceptions are the subcutaneous tissue of the eyelids, penis and scrotum, the nymphr, and the cavity of the cranium.

Adipose tissue is developed in connection with areolar tissue, and forms in its meshes little masses of unequal size and irregular shape, to which the term lobules is applied. 
Under the microscope each lobule is found to consist of little vesicles or cells which present dark, sharply-defined edges when viewed with transmitted light: they are about $\frac{1}{400}$ or $\frac{1}{500}$ of an inch in diameter; each consists of a structureless and colourless membrane or bag formed of the remains of the original protoplasm of the cell, filled with fatty matter, which is liquid during life, but is in part solidified (or sometimes crystallised) after death. A nucleus is always present in some part or other of the cell protoplasm, but it is not easily visible unless the tissue is stained.

The oily matter contained in the cells is composed of the compounds of fatty acids with glycerin, which are named olein, stearin, and palmitin. On the addition of osmic acid, fat-cells are stained black; this is due to the olein present, which reduces the osmium tetroxide to a lower oxide, which has a black colour. Fat is stained deep yellow by Sudan III. and red by Scharlach R.

Fat-cells are developed from connective-tissue corpuscles; these colls may be found exhibiting every intermediate gradation between

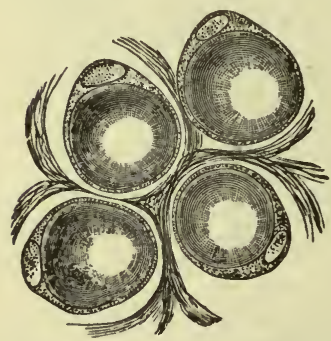

Fig. 47.-Fat-cells from the omentum of a rat. (Klein.) an ordinary corpuscle and a mature fatcell. The process of development is as follows: a fow small drops of oil make their appearance in the protoplasm, and by their confluence a larger drop is produced: this gradually increases in size at the expense of the original protoplasm of the cell, which becomes correspondingly diminished in quantity till in the mature cell it only forms a thin film, with a flattened nucleus imbedded in its substance (figs. 41 and 47 ).

A large number of blood-vessels are found in adipose tissue, which subdivide until each lobule of fat contains a fine meshwork of capillaries ensheathing each individual fat-cell.

Among the uses of adipose tissue these are the chiof:-

a. It serves as a store of combustible matter which may be reabsorbed into the blood when occasion requires, and, being used up in the metabolism of the tissues, helps to preserve the heat of the body.

b. The fat which is situated beneath the skin must, by its want of conducting power, assist in preventing undue waste of the heat of the body by escape from the surface.

c. As a packing material, fat serves very admirably to fill up spaces, to form a soft and yielding yet elastic material wherewith to wrap tender and delicate structures, or form a bed with like qualities on which such structures may lie, not endangered by pressure. As examples of situations in which fat serves such purposes may bo 
mentioned the palms of the hands, the soles of the feet, and the orbits.

\section{Retiform Tissue.}

Retiform or reticular tissue is a kind of connective tissue in which the ground-substance is of more fluid consistency than elsewhere.

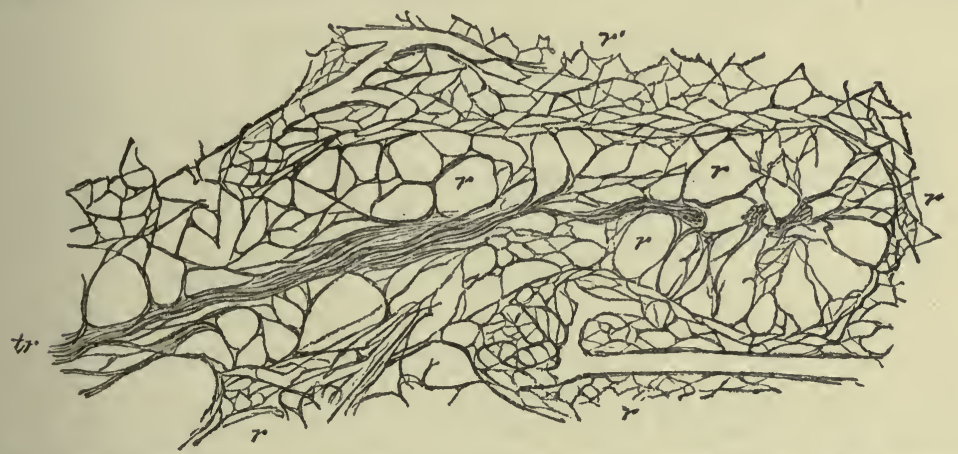

Fia. 48.-Retiform tissue from a lymphatic gland, from a section which has been treated with dilute potash. (Schäfer.)

There are few or no elastic fibres in it, and the white fibres run in very fine bundles forming a close network. The bundles are covered and concealed by flattened connective-tissue corpuscles. When these are dissolved by dilute potash, the fibres are plainly seen (fig. 48).

The statement has been made that the fibres of retiform tissue are chemically different from those of areolar tissue, in spite of the fact that they are indistinguishable microscopically, and in many places continuous with each other. Mrs Rosenheim has conclusively proved that chemical differences do not exist between the two groups of fibres; both are made of collagen, and the substance termed reticulin by Siegfried is an artifact; it is merely collagen which has been rendered resistant and insoluble by the reagents (alcohol, ether) used in its preparation.

\section{Adenoid or Lymphoid Tissue.}

This is retiform tissue in which the meshes of the network are largely occupied by lymph corpuscles. These are in certain foci

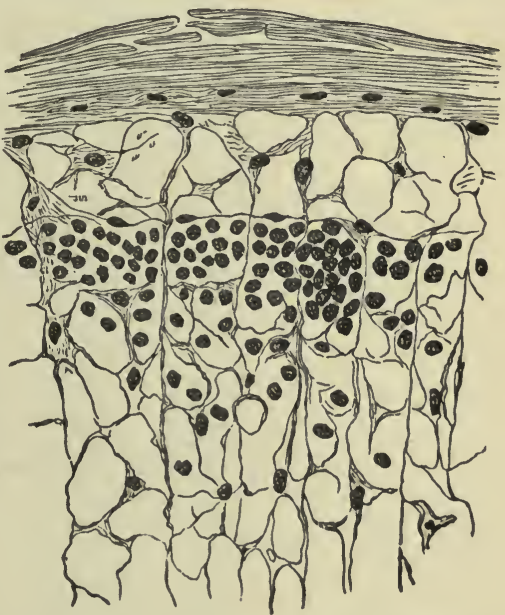

Fra. 49.-Part of a section of a lymphatic gland, from which the corpuscles have been for the most part removed, showing the supporting retiform tissue. (Klein and Noble Smith.)

actively multiplying; they get into the lymph stream, which washes them into the blood, where they become the variety of colourless 
corpuscles called lymphocytes. It is found in the lymphatic glands, the thymus, the tonsils, in the follicular glands of the tongue, in Peyer's patches, and in the solitary glands of the intestines, in the Malpighian corpuscles of the spleen, and under the epithelium of many mucous membranes.

\section{Jelly-like Connective Tissue.}

We have now considered connective tissues in which fibres of one or the other kind predominate, and some in which the cells are in preponderance. We come lastly to a form of connective tissue in which the ground-substance is in excess of the other histological elements. This is called jelly-like connective tissue. The cells and fibres scattered through it are few and far between. It is found largely in the embryo, notably in the Whartonian jelly, which surrounds and protects the blood-vessels of the umbilical cord. In the adult it is found in the vitreous humour of the eye.

The occurrence of large quantities of ground-substance in such tissues has enabled physiologists to examine its chemical nature. Its chief constituents are water, and one or more varieties of mucinlike substances termed mucoids and mineral salts (especially sodium chloride). 


\section{CHAPTER V}

THE CONNECTIVE TISSUES (continued)

Cartilage, Bone, Teeth, Blood

\section{Cartilage.}

CARTILAGE is popularly termed gristle. It may be divided into two chief kinds: Hyaline cartilage; here the matrix or ground-substance is clear and free from fibres: Fibro-cartilage; here the matrix is per-

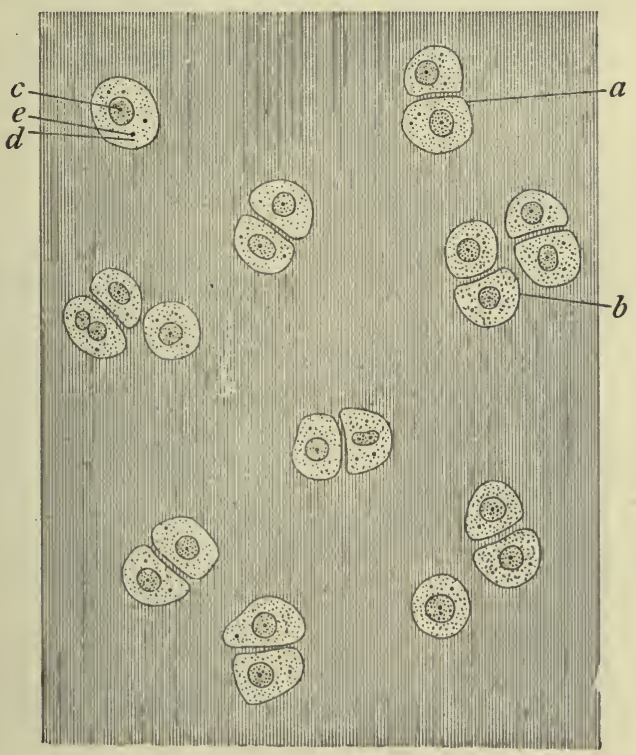

Fio. 50. Section of articular cartilage. $a$, Group of two cells ; $b$, group of four cells ; $d$, protoplasm of cell with $e$, fatty granules; $c$, nucleus. (After Schäfer.)

vaded with connective-tissue fibres; when these are of the white variety, the tissue is white fibro-cartilage; when they are of the yellow or elastic variety, the tissue is yellow or elastic fibro-cartilage. 
Hyaline Cartilage is found in the following places:-

1. Covering the articular ends of bones; here it is called articular cartilage (fig. 50).

2. Forming the rib-cartilages; here it is called costal cartilage.

3. The cartilages of the nose, of the windpipe, of the external auditory meatus, and the greater number of the laryngeal cartilages.

4. Temporary cartilage: rods of cartilage which prefigure the majority of the bones in process of development.

Hyaline cartilage in many situations (costal, laryngeal, tracheal) shows a tendency to become calcified late in life.

On boiling, the ground-substance of cartilage yields a material called chondrin. This resembles gelatin very closely, and the differences in its reactions are due to the fact that chondrin is not a chemical individual, but a mixture of gelatin with varying amounts of mucoid substances.

\section{White Fibro-Cartilage occurs-}

1. As inter-articular fibro-cartilage-e.g., the semilunar cartilages of the knee-joint.

2. As circumferential or marginal cartilage, as on the edges of the acetabulum and glenoid cavity.

3. As connecting cartilage-e.g., the inter-vertebral discs.

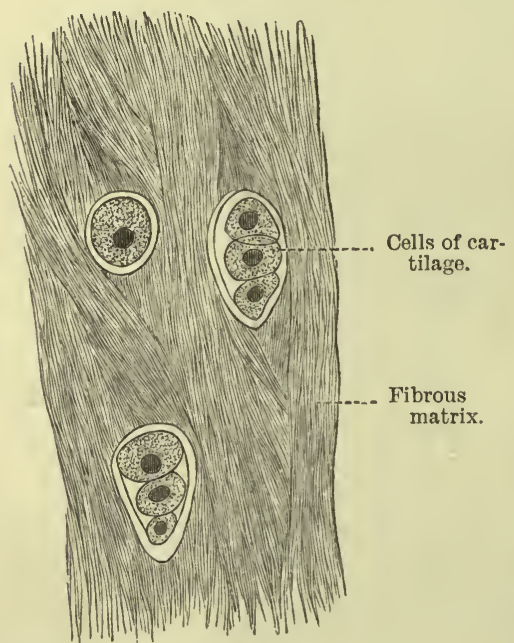

FIG. 51.-White fibro-cartilage. (Cadiat.)

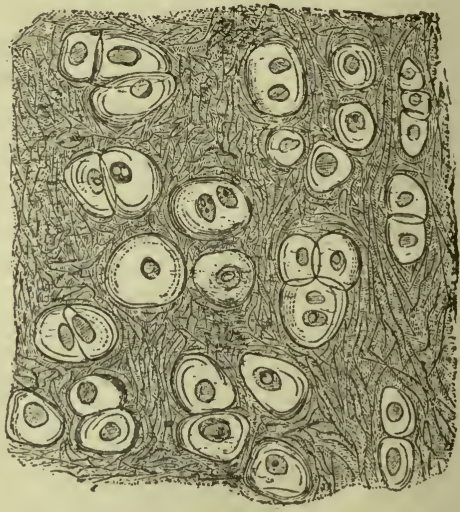

FIG. 52.-Yellow or elastic fibro-cartilage. (Cadiat.)

White fibro-cartilage (fig. 51) is composed of cells and a matrix. The latter is permeated by fibres of the white variety.

In this kind of fibro-cartilage it is not unusual to find portions so 
densely fibrous that no cells can be seen; but in other parts continuous with these, cartilage-cells are freely distributed.

Yellow or Elastic Fibro-Cartilage is found in the pinna of the external ear, in the epiglottis and cornicula laryngis, and in the Eustachian tube.

The cells in this variety of cartilage are rounded or oval, with well-marked nuclei and nucleoli (fig. 52). The matrix in which they are seated is pervaded in all directions by fine elastic fibres, which form an intricate interlacement about the cells : a small and variable quantity of non-fibrillated hyaline intercellular substance is present around the cells.

Development of Cartilage.-Like other connective tissues, cartilage originates from mesoblast; the cells are unbranched, and the

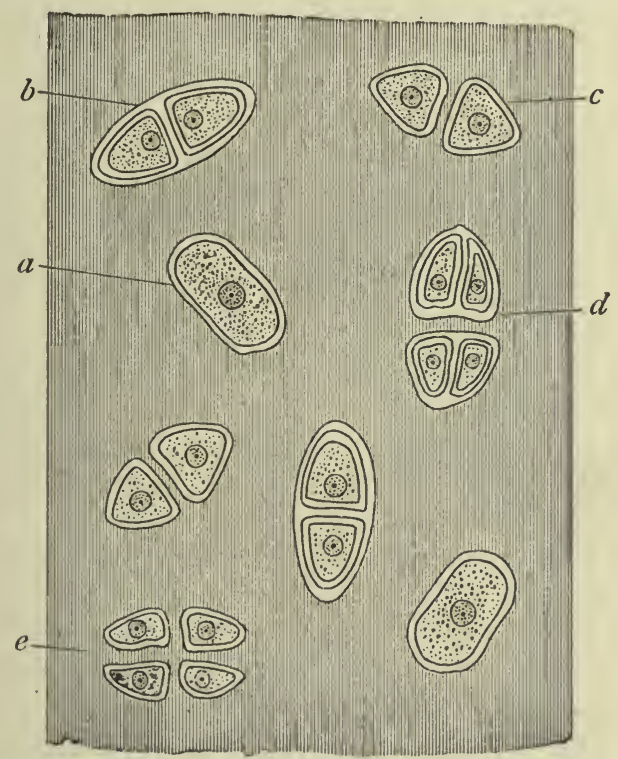

F1G. 53. - Plan of multiplication of cells in cartilage. $a$, Cell in its capsule; $b$, divided into two, each with a capsule; $c$, primary capsule disappeared, secondary capsules coherent with matrix; $d$, tertiary division; $e$, secondary capsules disappeared, tertiary coherent with matrix. (After Sharpey.)

disposition of the cells in fully formed cartilage in groups of two, four, etc., is due to the fact that each group has originated from the division of a single cell, first into two, each of these again into two, and so on. This process of cell division is accompanied with the usual karyokinetic changes.

Each cell deposits on its exterior a sheath or capsule; on division each of the daughter-cells deposits a new capsule within this, and the process may be repeated (see fig. 53). 
Thus the cells get more and more separated. The fused capsules form a very large part of the matrix, and indications of their previous existence may sometimes be seen in fully formed cartilage by the presence of faint concentric lines around the cells.

In a variety of cartilage found in the ears of rats and mice, called cellular cartilage, the cells never multiply to any great extent, and they are only separated by their thickened capsules.

But in most cartilages the cell-capsules will not explain the origin of the whole matrix, for intercellular material accumulates outside the capsules and still further separates the cells.

By certain methods of double staining, this twofold manner of formation may be shown very markedly. We have seen that chondrin obtained by boiling cartilage is really a mixture of two substances; one is a mucoid material, and comes from the capsules ; the other is gelatin, which comes from the rest of the groundsubstance which is collagenous. In hyaline cartilage, however, the collagen does not become precipitated to form fibres, but in white fibro-cartilage it does. In yellow fibro-cartilage the matrix is pervaded by a deposit of elastin, which results in the formation of a network of elastic fibres.

\section{Bone.}

Bone contains nearly 50 per cent. of water; the solid material is composed of earthy and animal matter in the proportion of about 67 per cent. of the former to 33 per cent. of the latter. The earthy matter is composed chiefly of calcium phosphate, but besides this, there is a small quantity (about 11 of the 67 per cent.) of calcium carbonate, calcium fluoride, and magnesium phosphate.

The animal matter is chiefly collagen, which is converted into gelatin by boiling.

The animal and earthy constituents of bone are so intimately blended and incorporated the one with the other, that it is only by severe measures, as for instance by a white heat in one case and by the action of concentrated acids in the other, that they can be separated. Their close union, too, is further shown by the fact that when by acids the earthy matter is dissolved out, or on the other hand when the animal part is burnt out, the shape of the bone is alike preserved.

The proportion between these two constituents of bone varies slightly in different bones in the same individual and in the same bone at different ages.

To the naked eye there appear two kinds of structure in different bones, and in different parts of the same bone, namely, the dense or compact, and the spongy or cancellous tissue. Thus, in making a longitudinal section of a long bone, as the humerus or femur, the 
articular extremities are found capped on their surface by a thin shell of compact bone, while their interior is made up of the spongy or cancellous tissue. The shaft, on the other hand, is formed almost entirely of a thick layer of the compact bone, and this surrounds a central canal, the medullary cavity - so called from its containing the medulla or marrow.

In the flat bones, as the parietal bone or the scapula, the cancellous structure (diploë) lies between two layers of the compact tissue, and in the short and irregular bones, as those of the carpus and tarsus, the cancellous tissue fills the interior, while a thin shell of compact bone forms the outside.

Marrow.-There are two distinct varieties of marrow-the red and yellow.

Red marrow is the connective tissue which occupies the spaces in the cancellous tissue; it is highly vascular, and thus maintains the
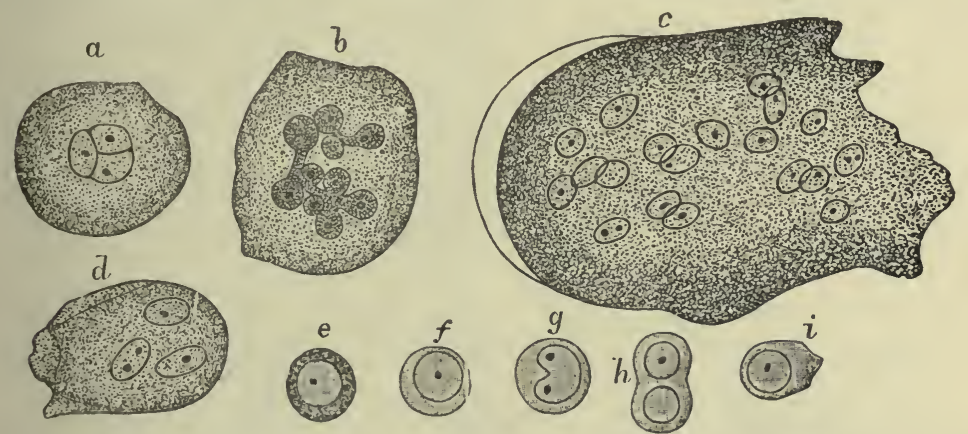

FI. 54.-Cells of the red marrow of the guinea-pig, highly magnified. $a, \mathrm{~A}$ large cell, the nucleus of which appears to be partly divided into three by constrictions ; $b$, a cell, the nucleus of which shows an appearance of being constricted into a number of smaller nuclei; $c$, a so-called giant cell or nyeloplaxe, with many nuclei; $d$, a smaller myeloplaxe, with three nuclei; $e-i$, proper cells of the marrow. (E. A. Schäfer.)

nutrition of the spongy bone, the interstices of which it fills. It contains a fow fat-cells and a large number of marrow-cells. The marrow-cells are amcboid, and resemble large leucocytes; the granules of some of these cells stain readily with acid and neutral dyes, but a considerable number have coarso granules which stain readily with basic dyes like methylene blue. Among the cells are some smaller nucleated cells of the same tint as coloured bloodcorpuscles. These are termed erythroblasts. From them the coloured corpuscles of the blood are developed. There are also a few large cells with many nuclei, termed giant cells or myeloplaxes (fig. 54).

Yellow marrow fills the medullary cavity of long bones, and consists chiefly of fat-cells with numerous blood-vessels; many of its cells also are the colourless marrow-cells just mentioned. 
Periosteum and Nutrient Blood-vessels.-The surfaces of bones, except the part covered with articular cartilage, are clothed by a tough, fibrous membrane, the periosteum; and it is from the blood-vessels which are distributed in this membrane, that the bones, especially their more compact tissue, are in great part supplied with nourishment; minute branches from the periosteal vessels enter the little foramina on the surface of the bone, and find their way to the Haversian canals, to be immediately described. The long bones are supplied also by a proper nutrient artery which, entering at some part of the shaft so as to reach the medullary cavity, breaks up into branches for the supply of the marrow, from which again small vessels are distributed to the interior of the bone. Other small blood-vessels pierce the articular extremities for the supply of the cancellous tissue.

Microscopic Structure of Bone.-Notwithstanding the differences of arrangement just mentioned, the structure of all bone is found under the microscope to be essentially the same.

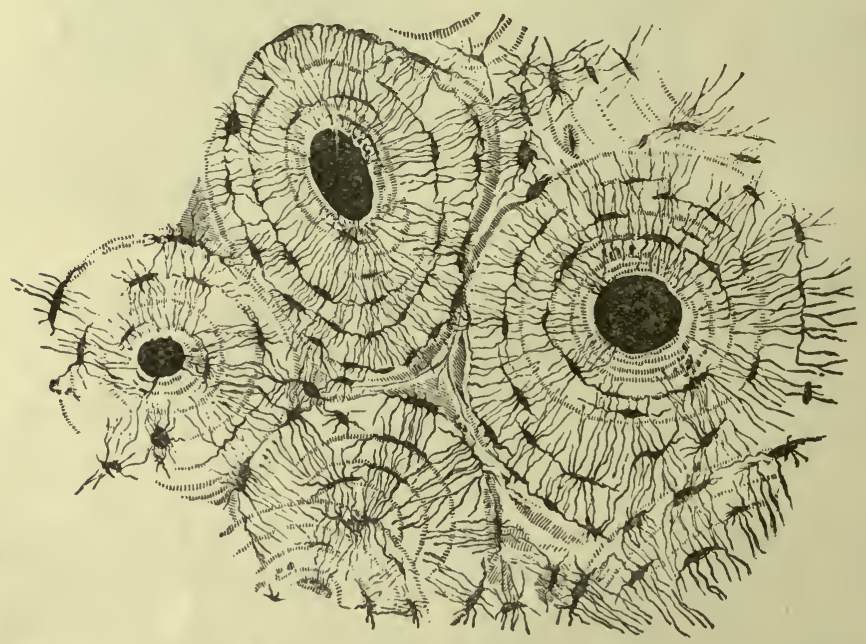

Eid. 55.-Transverse section of compact bony tissue (of humerus). Three of the Haversian canals are seen, with their concentric rings; also the lacunæ, with the canaliculi extending from them across the direction of the lamellæ. The Haversian apertures were filled with air and débris in grinding down the section, and therefore appear black in the figure, which represents the object as viewed with transmitted light. The Haversian systems are so closely packed in this section, that scarcely any interstitial lamellæ are visible. $\times 150$. (Sharpey.)

Examined with a rather high power its substance is found to contain a multitude of small irregular spaces, approximately fusiform in shape, called lacunce, with very minute canals or canaliculi leading from them, and anastomosing with similar little prolongations from other lacunæ (fig. 55). In very thin layers of bone, no 
other canals but these may be visible; but on making a transverse section of the compact tissue as of a long bone, e.g., the humerus or ulna, the arrangement shown in fig. 55 can be seen.

The bono is mapped out into small circular districts, at or about the contre of each of which is a hole, around which is an appearance as of concentric layers; the lacunce and canaliculi follow the same concentric plan of distribution around the small hole in the centre, with which indeed they communicate.

On making a longitudinal section, the central holes are found to be simply the cut extremities of small canals which run lengthwise through the bone, anastomosing with each other by lateral branches (fig. 56); these canals are called Haversian canals, after the name

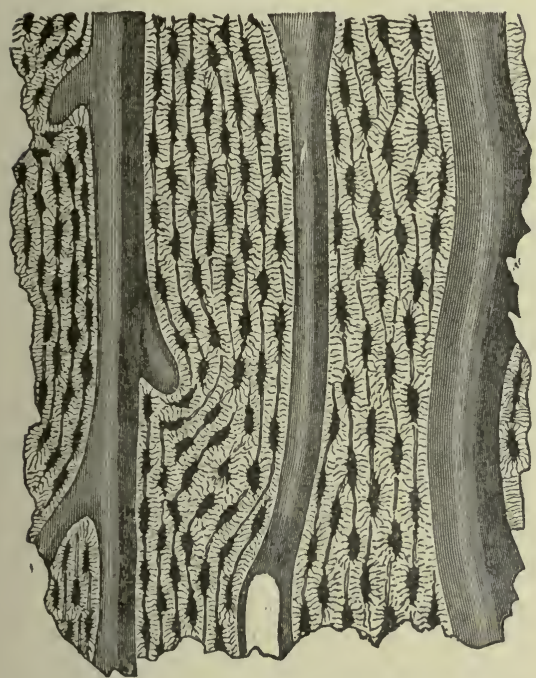

Fio. 56.-Longitudinal section from the human ulna, showing Haversian canals, lacunæ, and canaliculi. (Rollett.)

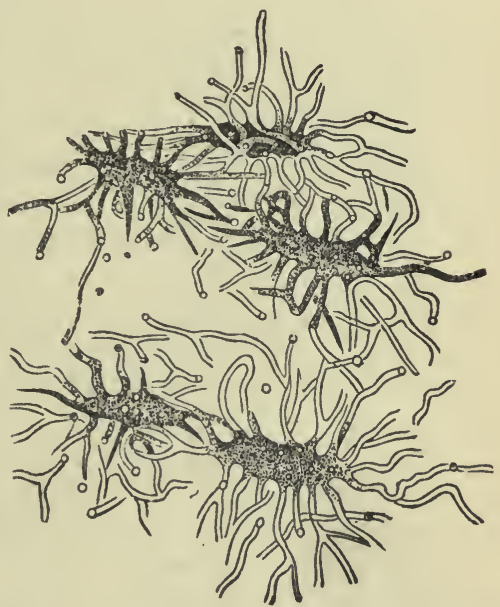

Fic. 57.-Bone-corpuscles with their processes as seen in a thin section of human bone. (Rollett.)

of the physician, Clopton Havers, who first accurately described them. They are occupied by blood-vessels.

The lacunæ are occupied by branched cells, which are called bone-cells, or bone-corpuscles (fig. 57); these closely resemble ordinary branched connective-tissue corpuscles. Bone is thus essentially connective tissue, the ground-substance of which is impregnated with lime salts. The bone-corpuscles with their processes, occupying the lacunæ and canaliculi, correspond exactly to the connective-tissue corpuscles lying in branched spaces. The connection of the lacunæ by the canaliculi allows the nutrient lymph to pass from place to place. 
Lamellæ of Compact Bone.-In the shaft of a long bone three distinct sets of lamellæ can be clearly recognised.

1. Circumferential lamellæ; these are concentrically arranged just beneath the periosteum, and around the medullary cavity.

2. Haversian lamellæ; these are concentrically arranged around the Haversian canals to the number of six to eighteen around each.

3. Interstitial lamellæ; these connect the systems of Haversian lamellæ, filling the spaces between them, and consequently attaining their greatest development where the Haversian systems are few, and vice versa.

The ultimate structure of the lamellæ is fibrous. If a thin film be peeled off the surface of a bone, from which the earthy matter has

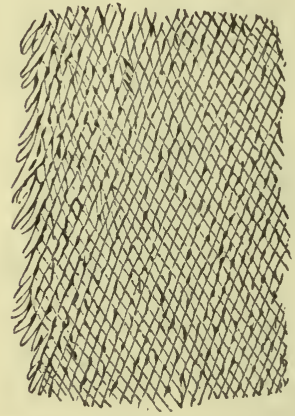

Fig. 58.-Thin layer peeled off from a softened bone. This figure, which is in. tended to represent the reticular structure of a lamella, gives a better idea of the object when held rather farther off than usual from the 'eye. $\times 400$. (Sharpey.)

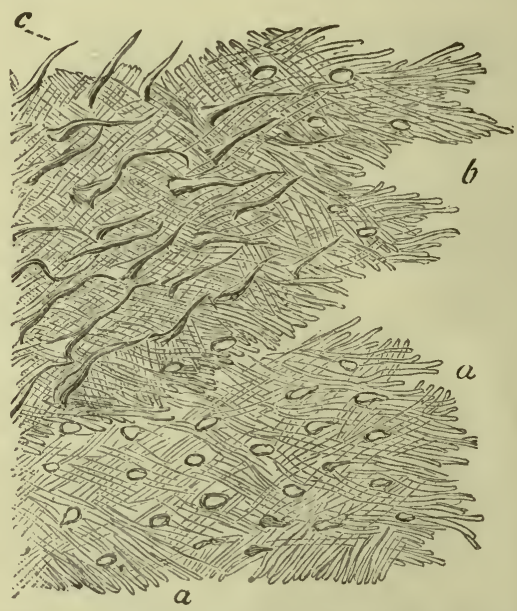

Fig. 59.-Lamellæ torn off from a decalcified human parietal bone at some depth from the surface. $a, a$, Lamellæ, showing intercrossing fibres; $b$, darker part, where several lamellæ are superposed; $c$, perforating fibres. Apertures, through which perforating fibres had passed, are seen especially in the lower part, $a, a$, of the figure. (Allen Thomson.)

been removed by acid, and examined with a high power of the microscope, it will be found composed of very slender fibres decussating obliquely, but coalescing at the points of intersection, as if here the fibres were fused rather than woven together (fig. 58). These are called the intercrossing fibres of Sharpey; they correspond to the white fibres of connective tissue, and form the source of the gelatin obtained by boiling bone.

In many cases, as in the parietal bone, the lamellæ are perforated by tapering fibres called the perforating fibres of Sharpey, resembling in character the ordinary white or more rarely the elastic fibres, 
which bolt the neighbouring lamellæ together, and may be drawn out when the latter are torn asunder (fig. 59). These perforating fibres originate from ingrowing processes of the periosteum, and in the adult still retain their connection with it.

Development of Bone.-From the point of view of their development, all bones may be subdivided into two classes:-

(a.) Those which are ossified directly or from the first in a fibrous membrane afterwards called the periosteum-e.g., the bones forming the vault of the skull, parietal, frontal, and a certain portion of the occipital bones.

(b.) Those whose form, previous to ossification, is laid down in hyaline cartilage - e.g., humerus, femur.

The process of development, pure and simple, may be best studied in bones which are not preceded by cartilage; and without a knowledge of this process (ossification in membrane), it is impossible to understand the more complex series of changes through which such a structure as the cartilaginous femur of the fœtus passes in its transformation into the bony femur of the adult (ossification in cartilage).

Ossification in Membrane.-The membrane, afterwards forming the periosteum, from which such a bone as the parietal is developed, consists of two layers - an external fibrous, and an internal cellular or osteogenetic.

The external layer is made up of ordinary fibrous tissue. The internal layer consists of a network of fine fibrils with a large number of nucleated cells (osteoblasts), some of which are oval, others drawn out into long branched processes: it is more richly supplied with capillaries than the outer layer. It is this portion of the periosteum which is immediately concerned in the formation of bone.

In such a bone as the parietal, ossification is preceded by an increase in the vascularity of this membrane, and then spicules, starting from a centre of ossification near the centre of the future bone, shoot out in all directions towards the periphery. These primary bone spicules consist of fibres which are termed osteogenetic fibres; they are composed of a soft, transparent substance called osteogen, around and between which calcareous granules are deposited. The fibres in their precalcified state are likened to bundles of white fibrous tissue, to which they are similar in chemical composition, but from which they differ in being stiffer and less wavy. The deposited granules after a time become so numerous as to imprison the fibres, and bony spicula result. By the junction of the osteogenetic fibres and their resulting bony spicules a meshwork of bone is formed. The osteogenetic fibres, which become indistinct as calcification proceeds, persist in the lamellæ of adult bone as the intercrossing fibres of Sharpey. The osteoblasts, being in part retained within the bony layers thus 
produced, form bone corpuscles. On the bony trabeculæ first formed, layers of osteoblastic cells from the osteogenetic layer of the periosteum repeat the process just described; and as this occurs in several thicknesses, and also at the edges of the spicules previously formed, the bone increases, both in thickness, length and breadth. The process is not completed by the time the child is born; hence the fontanelles or still soft places on the heads of infants. Fig. 60 represents a small piece of the growing edge of a parietal bone.

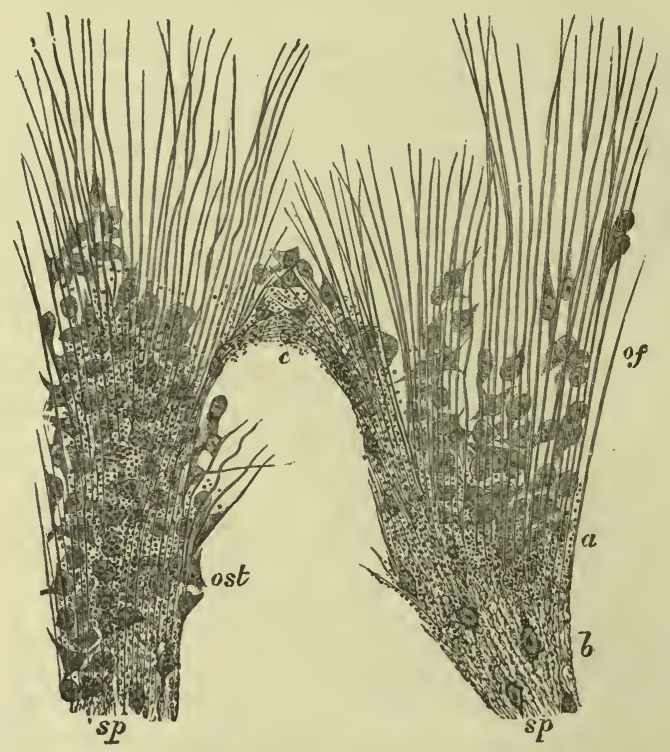

FIG. 60.-Part of the growing edge of the developing parietal bone of a foetal cat. $s p$, Bony spicules with some of the osteoblasts imbedded in them, producing the lacunr; of, osteogenetic fibres prolonging the spicules with osteoblasts (ost') between them and applied to thein. (Schäfer.)

The bulk of the primitive spongy bone is in time converted into compact bony tissue, with Haversian systems. Those portions in the interior not converted into bone become filled with the red marrow of the cancellous tissue.

Ossification in Cartilage.-Under this heading, taking the femur or any other long bone as an example, we have to consider the process by which the solid cartilaginous rod which represents the bone in the fœtus is converted into the hollow cylinder of compact bone with expanded ends formed of cancellous tissue of which the adult bone is made up. We must bear in mind the fact that this fotal cartilaginous femur is many times smaller than even the medullary cavity of the shaft of the mature bone, and, therefore, that not a trace of the: 
original cartilage can be present in the femur of the adult. Its purpose is indeed purely temporary; and, after its calcification, it is gradually and entirely absorbed.

The cartilaginous rod which forms the precursor of a fœtal long bone is sheathed in a membrane termed the perichondrium, which exactly resembles the periosteum just described.

Between the cartilaginous prefigurement of which the fotal long bone consists and the adult bone there are several intermediate stages.

The process may, however, be most conveniently described as occurring in three principal stages.

The first stage consists of two sets of changes, one in the cartilage, the other under the perichondrium. These take place side by side. In the cartilage the cells in the middle $*$ become onlarged and separated from one another. The cartilage-cells on each side get arranged in rows in the direction of the extremities of the cartilaginous rod. If at this stage one cuts the little embryonic bone with a knife, the knife encounters resistance, and there is a sensation of grittiness. This is due to the fact that calcareous particles are deposited in the matrix; and in consequence of this the matrix stains differently with histological reagents from the unaltered matrix. Simultaneously with this, the periosteal tissue is forming layer

* This is the case in nearly all the long bones, but in the terminal phalanges the change occurs first, not in the middle but at their distal extremities.

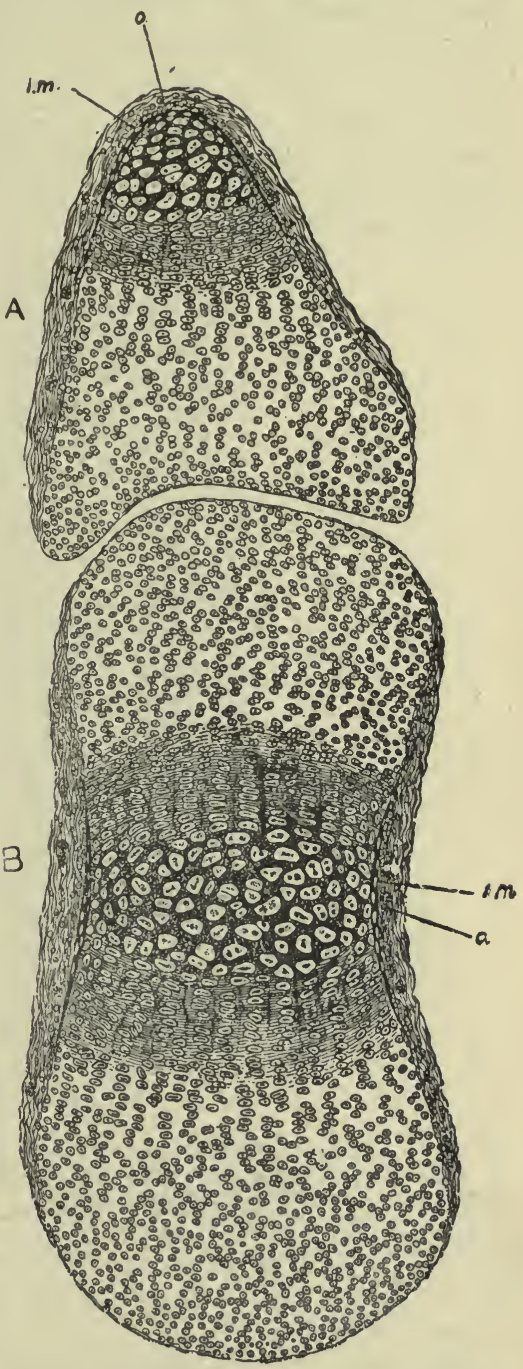

Fia. 61.--Section of two foetal phalanges ; the carti. lage-celis in the centre of $B$ are enlarged and separated from one another by calcified matrix. im, Layer of bone deposited under the perios. teum; 0 , layer of osteoblasts by which this layer was formed. The rows of cartilage-cells are seen on each side of the centre of calcification. In $\mathbf{A}$, the terminal phalanx, the changes begin at the tip. (After Dixey.) 
after layer of true bone; this is formed exactly in the same way as in such a bone as the parietal; by the agency of the osteoblasts, osteogenetic fibres, and then spicules of bone, are formed by deposit of calcareous matter. As the layers are formed, some of the osteoblasts get walled in between the layers and become bone-cells.

In the later part of this stage the calcareous deposit between the cartilage-cells cuts them off from nutrition, and they in consequence waste, leaving spaces that are called the primary areoloc. The calcareous deposit creeps up between the rows of cartilage-cells,

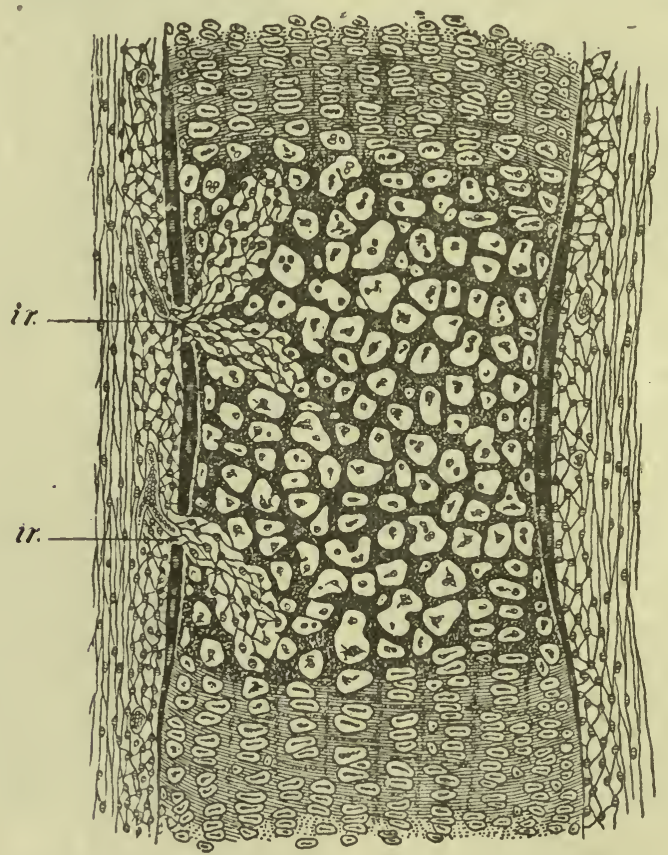

FiG. 62.--Ossification in cartilage showing stage of irruption. The shrunken cartilage-cells are seen in the primary areolæ. At ir an irruption of the subperiosteal tissue has penetrated the sub. periosteal bony crust. (After Lawrence.)

enclosing them in calcified boxes containing one, two, or more cells each. The wasting of the cells leads here also to the formation of primary areolæ.

We may roughly compare the two sets of cells engaged in the process to two races of settlers in a new country. The cartilage-cells constitute one race, and so successfully build for themselves calcareous homes as to be completely boxed up; so they waste and disappear, leaving only the walls of their homes enclosing the spaces called primary areolæ. The osteoblasts, the other race of cells under the 
perichondrium, are forming layers of true bone in that situation. Some, it is true, get walled in in the process, and become bonecorpuscles, but the system of intercommunicating lacunæ and canaliculi maintains their nutrition.

These two races are working side by side, and at first do not interfere with each other. But soon comes a declaration of war, and we enter upon the second stage of ossification, which is very appropriately called the stage of irruption (fig. 62). Breaches occur in the bony wall which the osteoblasts have built like a girdle round the calcifying cartilage, and through these the perichondrial tissue pours an invading army into the calcified cartilage. This consists of osteoblasts, the bone-formers; osteoclasts, or the bone-destroyers; the latter are large cells, similar to the myeloplaxes found in marrow (fig. 54). There are also a fow fibres, and a store of nutrient supply in the shape of bloodvessels.

Having got inside, the osteoclasts set to work to demolish the homes of the cartilage-cells, the walls of the primary areolæ, and thus large spaces are formed, which are called the secondary areoloe, or the medullary spaces. On the ruins of the calcified cartilage, the osteoblasts proceed to deposit true bone in layers, just as they were wont to do in their own country, under the periosteum.

The third stage of ossification is a repetition of these two stages towards the extremities of the cartilage. The cartilage-cells get flattened and arranged in rows; calcareous deposit occurs around these, and primary areolæ result; then follows the advance of the subperiosteal

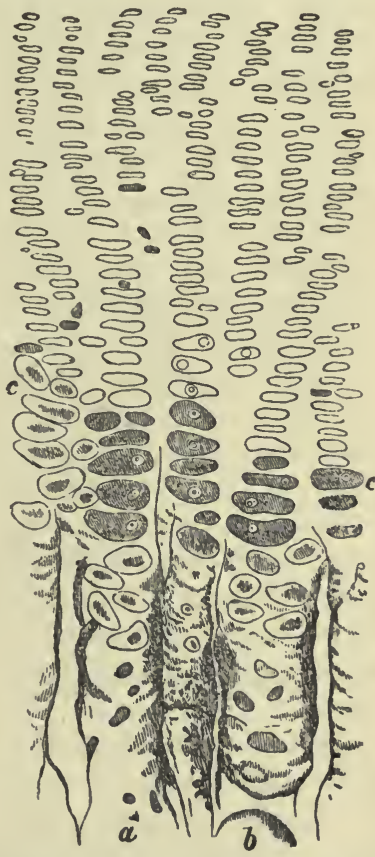

Fig. 63.-Longitudinal section of ossifying cartilage. Calcified trabeculæ are seen extending between the columns of cartilage-cells. c, Cartilage-cells; $a, b$, secondary areolæ. $\times 140$. (Sharpey.) tissue, the demolition of the primary areolæ, the formation of secondary areolæ, and the deposit of true bone. At the same time, layer upon layer is still being deposited beneath the periosteum, and these, from being at first a mere girdle round the waist of the bone, now extend towards its extremities.

The next figure (fig. 63) is a magnified view of the line of advance.

The bone which is first formed is less regularly lamellar than that of the adult. The lamellæ are not deposited till after birth and 
their formation is preceded by a considerable amount of absorption. To carry our simile further, the osteoblasts are not satisfied with the rough constructions that they were first able to make, but having exterminated the cartilage, they destroy (again through the agency of the regiment of giant osteoclasts) their first work, and build regular lamellæ, leaving lacunæ for the accommodation of those who desire to retire from active warfare.

About this time, too, the marrow cavity is formed by the absorption of the bony tissue that originally occupied the centre of the shaft. Here the osteoclasts have again to do the work, and, with this final act of destruction, all remains of any calcified cartilage of the fœtal bone entirely disappear.

The formation of a so-called cartilage bone is thus, after all, a formation of bone by subperiosteal tissue, just as it is in the so-called membrane bone.

After a time the cartilage at the ends of the shaft begins to ossify independently, and the epiphyses are formed. They are not joined on to the shaft till late in life, so that growth of the bone in length can continue till union takes place.

Bone grows in width by the deposition of layers under the periosteum, like successive rings formed under the bark of a growing tree. This was shown long before the histological details which we have described were made out by Sharpey. Silver rings were placed by Duhamel around the bones of young pigeons. When killed later, the rings were completely covered in by bone; and in the animals killed last, were even found in the central cavity. Another series of experiments was performed upon pigs. The young animals were fed alternately on ordinary food and food dyed by the red pigment madder. The new bony tissue acts like what dyers called a "mordant": it fixes the dye, and the rings of bone deposited during the madder periods were distinctly red in colour.

The importance of the periosteum in bone formation has always been recognised by surgeons. When removing a piece of bone they are careful, if possible, to leave the periosteum behind: this leads to regeneration of the lost bone. If it is absolutely necessary to remove the periosteum, successful cases have occurred in which the living periosteum from an animal has effectively been transplanted. MacEwen has recently shown that minute fragments of living bone are even more efficacious in virtue of the bone-cells they contain.

\section{The Teeth.}

During the course of his life, man, in common with most other mammals, is provided with two sets of teeth; the first set, called the temporary or milk teeth, makes its appearance in infancy, and is in 
the course of a few years shed and replaced by the second or permanent set.

The temporary or milk teeth are ten in number in each jaw, namely, on either side from the middle line two incisors, one canine, and two deciduous molars, and are replaced by ten permanent teeth. The number of permanent teeth in each jaw is, however, increased to sixteen by the development of three molars on each side of the jaw, which are called the permanent or true molars.

The following tables show the average times of eruption of the Temporary and Permanent teeth. In both cases the eruption of any given tooth of the lower precedes, as a rule, that of the corresponding tooth of the upper jaw.

\section{Temporary or Milk Teeth.}

The figures indicate in months the age at which each tooth appears.

\begin{tabular}{|c|c|c|c|}
\hline INCISORS. & $\begin{array}{c}\text { DECIDUOUS } \\
\text { FIRST } \\
\text { MOLARS. }\end{array}$ & CANINES. & $\begin{array}{c}\text { DECIDUOUS } \\
\text { SECOND } \\
\text { MOLARS. }\end{array}$ \\
\hline 6 & 12 & 18 & 24 \\
\hline
\end{tabular}

\section{Permanent Teeth.}

The age at which each tooth is cut is indicated in this table in years.

\begin{tabular}{|c|c|c|c|c|c|c|c|}
\hline $\begin{array}{c}\text { FIRST } \\
\text { MOLARS. }\end{array}$ & \multicolumn{2}{|c|}{ INCISORS. } & \multicolumn{2}{c|}{$\begin{array}{c}\text { BICUSPIDS OR PRE- } \\
\text { MOLARS. }\end{array}$} & CANINES. & $\begin{array}{c}\text { SECOND } \\
\text { MOLARS. }\end{array}$ & $\begin{array}{c}\text { THIRD } \\
\text { MOLARS OR } \\
\text { WISDOMS. }\end{array}$ \\
\hline 6 & 7 & 8 & 9 & 10 & 11 & 12 & 17 to 25 \\
\hline
\end{tabular}

The times of eruption given in the above tables are only approximate: the limits of normal variation are tolerably wide. Certain diseases affecting the bony skeleton, e.g. Rickets, retard the eruptive period considerably.

It is important to notice that it is a molar which is the first tooth to be cut in the permanent dentition, not an incisor as in the case of the temporary set, and also that it appears behind the last deciduous molar on each side.

The third molars, of ten called $W$ isdoms, are sometimes unerupted through life from want of sufficient jaw space and the presence of the other teeth; cases of whole families in which their absence is a characteristic feature are occasionally met with. 
When the teeth are fully erupted it will be observed that the upper incisors and canines project obliquely over the lower front teeth, and the external cusps of the upper bicuspids and molars lie outside those of the corresponding teeth in the lower jaw. This arrangement allows to some extent of a scissor-like action in dividing and biting food in the case of incisors; and a grinding motion in that of the bicuspids and molars when the side to side movements of the lower jaw bring the external cusps of the lower teeth into direct articulation with those of the upper, and then cause them to glide down the inclined surfaces of the external and up the internal cusps of these same upper teeth during the act of mastication.

The work of the canine teeth in man is similar to that of his incisors. Besides being a firmly implanted tooth and one of stronger substance than the others, the canine tooth is important in preserving the shape of the angle of the mouth, and by its shape, whether pointed or blunt, long or short, it becomes a character tooth of the dentition as a whole in both males and females.

Another feature in the fully developed and properly articulated set of teeth is that no two teeth oppose each other only, but each tooth is in opposition with two, except the upper Wisdom, usually a small tooth. This is the result of the greater width of the upper incisors, which so arranges the "bite" of the other teeth that the lower canine closes in front of the upper one.

Should a tooth be lost, therefore, it does not follow that its former opponent remaining in the mouth is rendered useless and thereby liable to be removed from the jaw by a gradual process of extrusion commonly seen in teeth that have no work to perform by reason of absence of antagonists.

\section{Structure of a Tooth.}

A tooth is generally described as possessing a crown, neck, and root.

The crown is the portion which projects beyond the level of the gum. The neck is that constricted portion just below the crown which is embraced by the free edges of the gum; and the root includes all below this.

On making longitudinal and transverse sections through its centre (figs. 64,65), a tooth is found to be composed of a hard material, dentine or ivory, which is moulded around a central cavity which resembles in general shape the outline of the tooth; the cavity is called the pulp cavity from its containing the very vascular and sensitive pulp.

The tooth-pulp is composed of loose connective tissue, blood-vessels, nerves, and large numbers of cells of varying shapes; on the surface in close connection with the dentine is a specialised layer of 
cells called odontoblasts, which are elongated columnar cells with a large nucleus at the tapering ends farthest from the dentine.
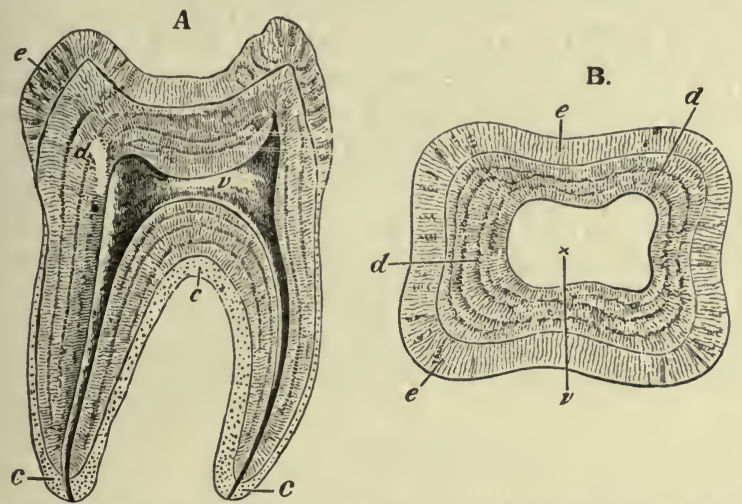

FIG. $64 .-\mathbf{A}$, longitudinal section of a human molar tooth; $c$, cement; $d$, dentine; $e$, enamel; $v$, pulpcavity. (Owen.)

$\mathbf{B}$, transverse section. The letters indicate the same as in $\mathbf{A}$.

The blood-vessels and nerves enter the pulp through a small opening at the apical extremity of each root. The nerves terminate

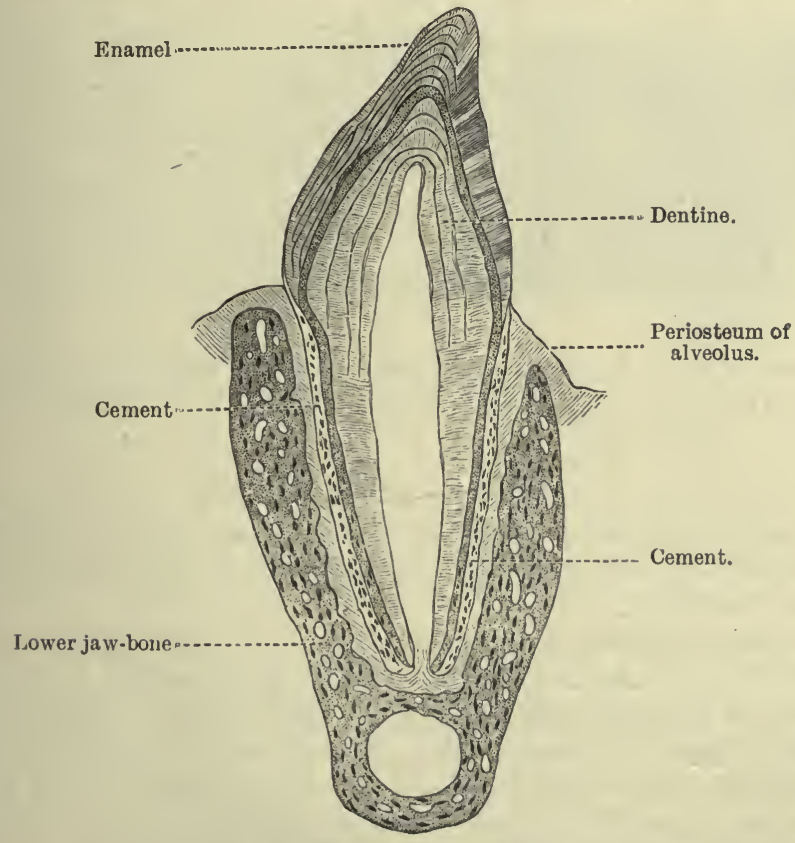

Fig. 65.-Premolar tooth of cat in situ. 
by branching into fine fibrilla which enter the dentinal tubes. No lymphatics have been seen in the pulp.

A layer of very hard calcareous matter, the enamel, caps that part of the dentine which projects beyond the level of the gum; while sheathing the portion of dentine which is beneath the level of the gum, is a layer of true bone, called the cement or crusta petrosa.

At the neck of the tooth, where the enamel and cement come into contact, each is reduced to an exceedingly thin layer; here the cement overlaps the enamel, and is prolonged over it. On the surface of the crown of the tooth, when it first comes through the jaw, is a thin membrane called Nasmyth's membrane, or the cuticle of the tooth. The covering of enamel becomes thicker towards the crown, and the cement towards the lower end or apex of the root.

\section{Dentine or Ivory.}

Dentine closely resembles bone in chemical composition. It contains, however, only 10 per cent. of water. The proportion in a hundred parts of the solids is about twenty-eight animal to seventytwo of earthy matter. The former, like the animal matter of bone,

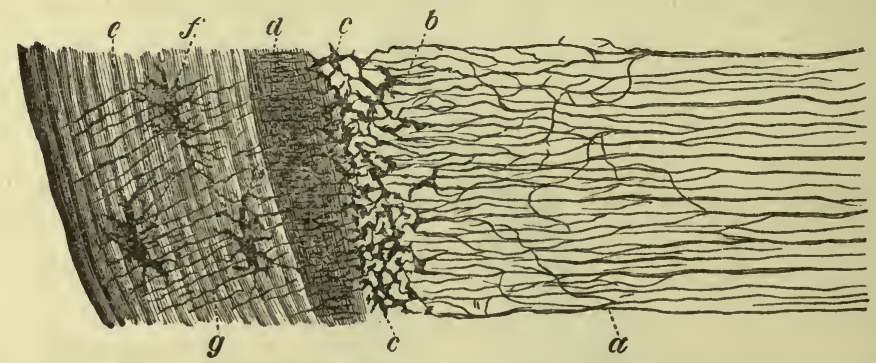

FIG. 66.-Section of a portion of the dentine and cement from the middle of the root of an incisor tooth. $a$, Dentinal tubules ramifying and terminating, some of them in the interglobular spaces $b$ and $c ; d$, inner layer of the cement with numerous closely set canaliculi ; $e$, outer layer of cement; $f$, lacunie; $g$, canaliculi. $\times 350$. (Kölliker.)

may be converted into gelatin by boiling. It also contains a trace of fat. The earthy matter is made up chiefly of calcium phosphate, with a small portion of the carbonate, and traces of calcium fluoride and magnesium phosphate.

Under the microscope dentine is seen to be finely channelled by a multitude of delicate tubes, which by their inner ends communicate with the pulp-cavity, and by their outer extremities come into contact with the under part of the enamel and cement, and sometimes even penetrate them for a greater or less distance (figs. 66, 68 ). The matrix in which these tubes lie is composed of "a reticulum of fine fibres of connective tissue modified by calcification, and where 
that process is complete, entirely hidden by the densely deposited lime salts" (Mummery).

The tubules of the dentine, the average diameter of which at their inner and larger extremity is $\frac{1}{4500}$ of an inch, contain fine prolongations from the tooth-pulp which are processes of the odontoblasts, the columnar cells lining the pulp-cavity together with nervefibrils; the relation of these processes to the tubules in which they lie is precisely similar to that of the processes of the bone-corpuscles to the canaliculi of bone. The outer portion of the dentine, underlying the cement, and the enamel to a much lesser degree, forms a more or less distinct layer termed the granular or interglobular layer (fig. 66). It is characterised by the presence of a number of irregular minute cavities. The explanation of these will be seen when we study the development of a tooth.

\section{Enamel.}

Enamel is by far the hardest tissue in the body; it is composed of the same inorganic compounds that enter into the composition of dentine and bone. According to Tomes, it contains no animal matter at all, and only 2 or 3 per cent. of water. Gelatin is a characteristic

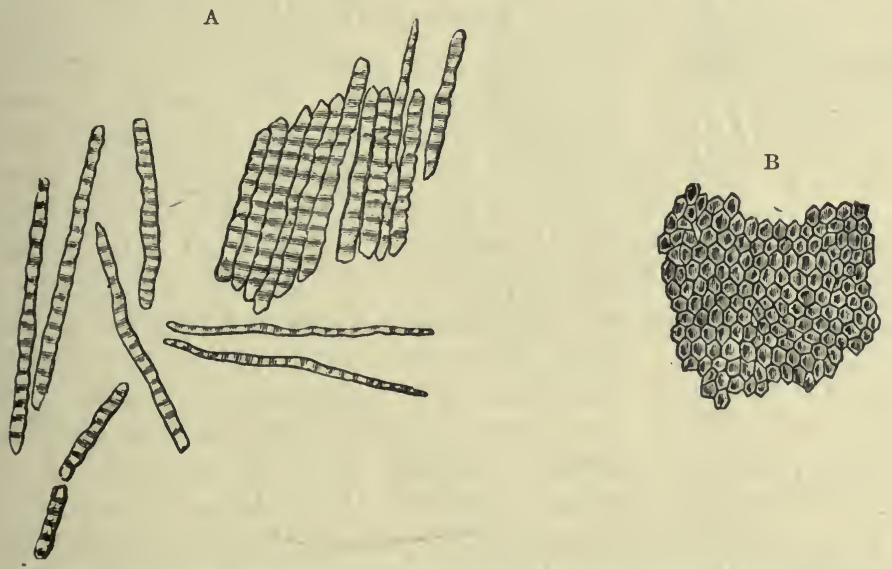

Fio. 67.-Enamel prisms. A, fragments and single prisms of the transversely -striated enamel, isolated by the action of hydrochloric acid. B, surface of a small fragment of enamel, showing the hexagonal ends of the fibres with darker centres. $\times 350$. (Kölliker.)

product of connective tissue, and enamel is not a connective tissue, but is epithelial in origin.

Examined under the microscope, enamel is found composed of sixsided prisms (figs. 67,68$) \frac{1}{5000}$ of an inch in diameter, which are set on end on the surface of the dentine, and fit into corresponding depressions in the same. 


\section{Crusta Petrosa.}

The crusta petrosa or cement (fig. $66, e, d$ ) is composed of true bone, and in it are lacunæ $(f)$ and canaliculi $(g)$, which sometimes com-

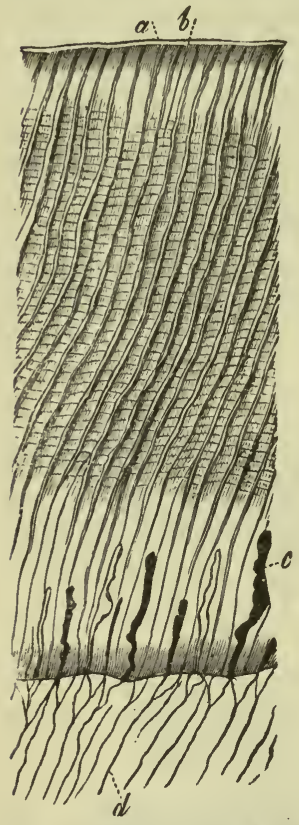

FIG. 68.-Thin section of the enamel, and a part of the dentine. $a$, Cuticular pellicle of the enamel (Nasmyth's membrane); $b$, enamel columns with fissures between them and cross striæ; $c$, larger cavities in the enamel, communicating with the extremities of some of the dentinal tubules $(d)$. $\times 350$. (Kölliker.) municate with the outer finely branched ends of the dentinal tubules, and generally with the interglobular spaces. Its laminæ are bolted together by perforating fibres like those of ordinary bone (Sharpey's fibres). Cement differs from ordinary bone in possessing no Haversian canals, or, if at all, only in the thickest part. Such canals are more. often met with in teeth with the cement hypertrophied than in the normal tooth.

\section{Development of the Teeth.}

The first step in the development of the teeth consists in a downward growth (fig. 69, 1) from the deeper layer of stratified epithelium of the mucous membrane of the mouth, which becomes thickened in the neighbourhood of the maxillæ or jaws now in the course of formation. This process passes downward into a recess of the imperfectly developed tissue of the embryonic jaw. The downward epithelial growth forms the common enamel or dental germ, and its position is indicated by a slight groove in the mucous membrane of the jaw. After this there is an increased development at certain points corresponding to the situations of the future milk-teeth. The common enamel germ thus becomes extended by further growth into a number of special enamel germs (fig. 70) corresponding to each of the milk-teeth, and connected to the common germ by a narrow neck $(f)$. Each tooth is thus placed in its own special recess in the embryonic jaw.

As these changes proceed, there grows up from the underlying connective tissue into each enamel germ (fig. $71, p$ ), a distinct vascular papilla (dental papilla), and upon it the enamel germ becomes moulded, and presents the appearance of a cap of two layers of epitheliam separated by an interval (fig. $71, f^{\prime}$ ). Whilst part of the subepithelial tissue is elevated to form the dental papilla, the part which bounds the embryonic teeth forms the dental 
sac (fig. $71, s$ ); and the rudiment of the jaw sends up processes forming partitions between the teeth. In this way small chambers are produced in which the dental sacs are contained, and thus the sockets of the teeth are formed. The papilla is composed of nucleated cells arranged in a meshwork of connective tissue, the outer or peripheral part being covered with a layer of columnar nucleated cells called odontoblasts.

These cells, either by secretion, or as some think by direct transformation of the outer part of each, form a layer of dentinal matrix on the apex of the papilla, or if the tooth has more than one cusp, then at the apex of each cusp. This layer is first uncalcified (odontogen), but globules of calcareous matter soon appear in it. These, becoming more numerous, blend into the first cap of dentine. In the meanwhile the odontoblasts have formed a second layer of odontogen within this (fig. 72 ), and this in turn becomes calcified; thus layer after layer is formed, each extending laterally further than its predecessor; the layers blend except in some places; here portions of odontogen remain, which in a tooth macerated for histological purposes get destroyed, and appear as the interglobular spaces (fig. 66),

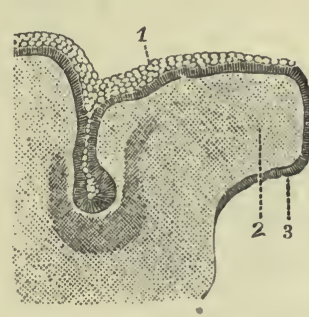

Fra. 69 .

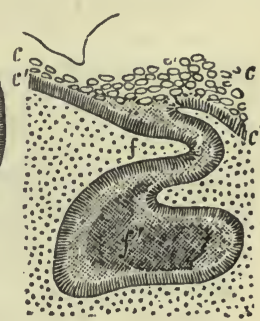

Fig. 70.

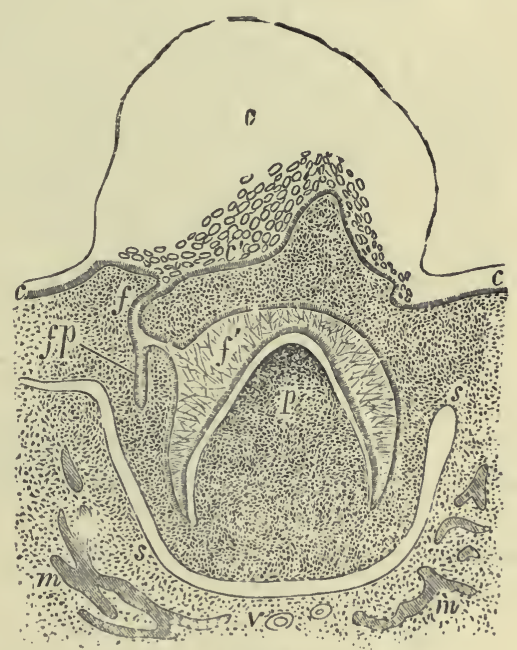

Fic. 71.

Fros. 69, 70, 71.-Sections of the upper jaw of a foetal sheep. FIG. 69.-1, common enamel germ dipping down in to the mucous membrane; 2 , palatine process of jaw ; 3, Rete Malpighi. Fig. 70.-Here the section passes through one of the special enamel germs which is becoming flask-shaped; $c, c^{\prime}$, epithelium of mouth; $f$, neck; $f^{\prime}$, body of special enamel germ. FIa.71.-A laterstage; $c$, outline of epithelium of gum; $f$, neck of enamel germ; $f^{\prime}$, enamel organ; $p$, papilla; $s$, dental sac forming; $f p$, the enamel germ of permanent tooth; $m$, bone of jaw ; $v$, vessels cut across. (Waldeyer and Kölliker.) so called because bounded by the deposit of calcareous salts, which occurs, as we have already seen, in the form of globules.

As the odontoblasts retire towards the centre, depositing layer after layer of dentine, they leave behind them long flaments of their 
protoplasm around which the calcareous deposit is moulded; thus the dentinal tubules occupied by the processes of the odontoblasts are formed.

The other cells of the dental papilla form the cells of the pulp.

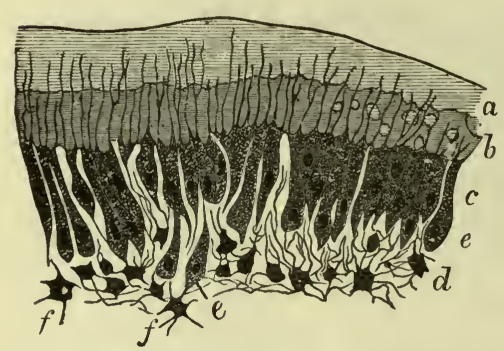

Fic. 72.-Part of section of developing tooth of a young rat; showing the mode of deposition of the dentine. Highly magnified. $a$, Outer layer of fully formed dentine; $b$, uncalcified matrix with one or two nodules of calcareous matter near the calcified parts; $c$, odontoblasts sending processes into the dentine; $d$, pulp; $e$, fusiform or wedge-shape cells found between odontoblasts; $f$, stellate cells of pulp in fibrous connective tissue. The section is stained with carmine, which colours the uncalcified matrix but not the calcified part. (E. A. Schäfer.)

\section{Formation of the enamel.-} The portion of the enamel or dental germ that covers the dental papilla is at this stage called the enamel organ. This consists of four parts (see figs. 73 and 74).

1. A layer of columnar epithelium cells in contact with the dentine. These are called the enamel cells, adamantoblasts, or ameloblasts.

2. Two or three layers of smaller polyhedral nucleated cells, the stratum intermedium of Hannover.

3. A matrix of non-vascular jelly-like tissue containing stellate cells.

4. An outer membrane of several layers of flattened epithelium cells.

The first three layers on an enlarged scale are seen in fig. 74 .

The enamel prisms are formed by the agency of the ends of the adamantoblasts which abut on the dental papilla. Each forms a fine deposit of globules staining with osmic acid and resembling keratin in its resistance to mineral acid. At one time it was believed that each adamantoblast was itself calcified and converted into an enamel 
prism, but this view has been disproved by recent research. The layer of keratin-like material is outside the bodies of the cells, although a process of each adamantoblast extends into it as a tapering fibre (process of Tomes), and it is usually produced simultaneously with the first layer of uncalcified dentine; when it undergoes calcification, the first layer of enamel is complete. The adamantoblasts then repeat the process, first causing a deposition of keratin-like material, and this in turn is calcified, and so on. During the formation of layer after layer of enamel, the adamantoblasts retire. By the time the enamel is approaching completion the other layers of the enamel organ have almost disappeared, and they entirely

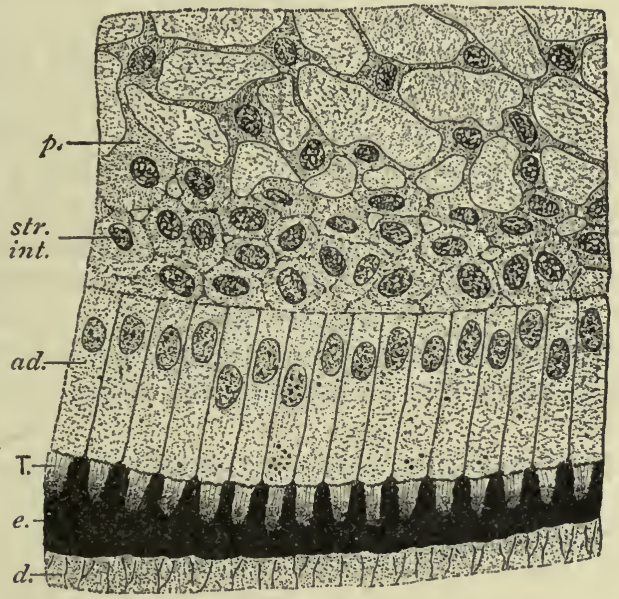

Fig. 74.-Highly magnified view of a piece of the enamel organ in a kitten's canine. $d$, Superficial layer of dentine. $e$, Newly formed enamel stained black by osmic acid. T, Tomes' processes from the adamantoblasts, ad.; str. int., stratum intermedium of the enamel organ. $p$, Branched cells of the enamel pulp. (After Röse.)

disappear when the tooth emerges through the gum. But for some little time there is a somewhat more persistent membrane covering the crown; this is Nasmyth's membrane, or the enamel cuticle; this is the last-formed keratinous layer of enamel which has remained uncalcified.

As with the dentine, the formation of enamel appears first on the apex of each cusp.

The cement or crusta petrosa is formed from the internal tissue of the tooth sac, the structure and function of which are identical with those of the osteogenetic layer of the periosteum; or, in other words, ossification in membrane occurs in it.

The outer layer or portion of the membrane of the tooth sac forms the dental periosteum. 
This periosteum, when the tooth is fully formed, is not only a means of attachment of the tooth to its socket, but also in conjunction with the pulp is a source of nourishment to it. Additional laminæ of cement are added to the root from time to time during the life of the tooth (as is especially well seen in the abnormal condition called an exostosis), by the process of ossification taking place in the periosteum. On the other hand, absorption of the root (such as occurs when the milk-teeth are shed) is due to the action of the osteoclasts of the same membrane.

In this manner the first set, or milk-teeth, are formed; and each tooth, as it grows, presses at length on the wall of the sac enclosing it, and, causing its absorption, is cut, to use a familiar phrase.

The temporary or milk teeth are later replaced by the growth of the permanent teeth, which push their way up from beneath them.

Each temporary tooth is replaced by a tooth of the permanent set which is developed from a small sac which was originally an offshoot from the sac of the temporary tooth which precedes it, and called the cavity of reserve (fig. $71, f p$ ). Thus the temporary incisors and canines are succeeded by the corresponding permanent ones, the temporary first molar by the first bicuspid; the temporary second molar develops two offshoots, one for the second bicuspid, the other for the permanent first molar. The permanent second molar is budded off from the first permanent molar, and the wisdom from the permanent second molar.

The development of the temporary teeth commences about the sixth week of intra-uterine life, after the laying down of the bony structure of the jaws. Their permanent successors begin to form about ten weeks later. The second permanent molars originate about the third month after birth, and the wisdom teeth about the third year.

The Blood.

A full consideration of the blood will come later, so here we shall only rapidly and briefly enumerate its principal characters.

The blood is a fluid which holds in suspension large numbers of solid particles which are called the corpuscles. The fluid itself is called the plasma or liquor sanguinis. It is a richly albuminous fluid; and one of the proteins in it is called fibrinogen.

After blood is shed it rapidly becomes viscous, and then sets into a jelly. The jelly contracts and squeezes out of the clot a strawcoloured fluid called serum, in which the shrunken clot then floats.

The formation of threads of a solid protein called fibrin from the soluble protein called fibrinogen is the essential act of coagulation; this, with the corpuscles it entangles, forms the clot. Serum is plasma minus the fibrin which it yields. The following scheme shows the relationships of these constituents at a glance:- 


\section{Blood $\left\{\begin{array}{l}\text { Plasma } \\ \text { Corpuscles }\end{array} \quad\left\{\begin{array}{l}\text { Serum } \\ \text { Fibrin }\end{array}\right\}\right.$ Clot.}

The corpuscles are of two chief kinds, the red and the white. The white corpuscles are typical amœboid, nucleated cells.

The red corpuscles are much more numerous than the white, averaging in man 5,000,000 per cubic millimetre, or 400 to 500 red to each white corpuscle. It is these red corpuscles that give the red colour to the blood. They vary in size and structure in different groups of the vertebrates. In mammals they are biconcave (except in the camel tribe, where they are biconvex) non-nucleated discs, in man $\frac{1}{3200}$ inch in diameter; during fœtal life nucleated red corpuscles are, however, found. In birds, reptiles, amphibians and fishes they are biconvex oval dises with a nucleus: they are largest in the amphibia. The most important and abundant of the constituents of the red corpuscles is the pigment which is called hoemoglobin. This is a protein-like substance, but is remarkable as it contains a small amount of iron (about 0.4 per cent.).

The blood during life is in constant movement. It leaves the heart by the vessels called arteries, and returns to the heart by the vessels called veins; the terminations of the arteries and the commencements of the veins are, in the tissues, connected by the thinwalled microscopic vessels called capillaries. In the capillaries, leakage of the blood-plasma occurs; this exuded fluid (lymph) carries nutriment from the blood to the tissue-elements, and removes from them the waste products of their activity. The lymph is collected by lymphatic vessels, which converge to the main lymphatic, called the thoracic duct. This opens into the large veins near to their entrance into the heart; and thus the lymph is returned to the blood.

But blood is also a carrier of oxygen, and it is the pigment hæmoglobin which is the oxygen carrier; in the lungs the hæmoglobin combines with the oxygen of the air, and forms a loose compound of a bright scarlet colour called oxyhocmoglobin. This arterial or oxygenated blood is taken to the heart and thence propelled by the arteries all over the body, where the tissues take the respiratory oxygen from the oxyhæmoglobin, and this removal of oxygen changes the colour of blood to the darker tint it has in the veins. The veins take the blood (minus a large quantity of oxygen and plus a large quantity of carbonic acid received in exchange from the tissues) to the heart, which sends it to the lungs to get rid of its surplus carbonic acid, and replenish its store of oxygen; then the same round begins over again. It should, however, be noted that hæmoglobin is not a carrier of carbonic acid; that gas is carried mainly as carbonates in the blood-plasma. 


\section{CHAPTER VI}

\section{MUSCULAR TISSUE}

Muscle is popularly known as flesh. It possesses the power of contraction, and is, in the higher animals, the tissue by which their movements are executed. The muscles may be divided from a physiological standpoint into two great classes - the voluntary muscles, those which are under the control of the will, and the involuntary muscles, those which are not. The contraction of the involuntary muscles is, however, controlled by the nervous system, only by a different part of the nervous system from that which controls the activity of the voluntary muscles.

When muscular tissue is examined with the microscope, it is seen to be made up of small, elongated, thread-like structures, which are called muscular fibres; these are bound into bundles by connective tissue, and in the involuntary muscles there is in addition a certain amount of cement substance, stainable by nitrate of silver, between the fibres.

The muscular fibres are not all alike; those of the voluntary muscles are seen by the microscope to be marked by alternate dark and light stripings or striations; these are called transversely striated muscular fibres. The involuntary fibres have not got these markings as a rule. There is one important exception to this rule, namely, in the case of the heart, the muscular fibres of which are involuntary, but transversely striated. There are, however, histological differences between cardiac muscle and the ordinary voluntary striated muscles. The unstriated involuntary muscular fibres found in the walls of the stomach, intestine, bladder, blood-vessels, uterus, and other contractile organs are generally spoken of as plain muscular fibres.

From the histological standpoint there are, therefore, three varieties of muscular fibres found in the body of the higher animals: two of them are transversely striated, and one is not. The relationship of this histological classification to the physiological 
classification into voluntary and involuntary is shown in the following table:-

1. Transversely striated muscular fibres :

a. In skeletal muscle .

b. In cardiac muscle

2. Plain muscular fibres : In blood-vessels, intestine, uterus, bladder, etc.

\section{Voluntary.}

INVOLUNTARY.

All kinds of muscular tissue are therefore composed of fibres, but the fibres are essentially different from those we have hitherto studied in the connective tissues. There the fibres are developed in the intercellular material; here, in muscle, the fibres are developed from the cells; that is, the cells themselves become elongated to form the muscular fibres.

\section{Voluntary Muscle.}

The voluntary muscles are those which are sometimes called skeletal, constituting the whole of the muscular apparatus attached to the bones.*

The fibres vary in thickness and length a good deal, but they average $\frac{1}{500}$ inch in diameter, and about 1 inch in length. Each

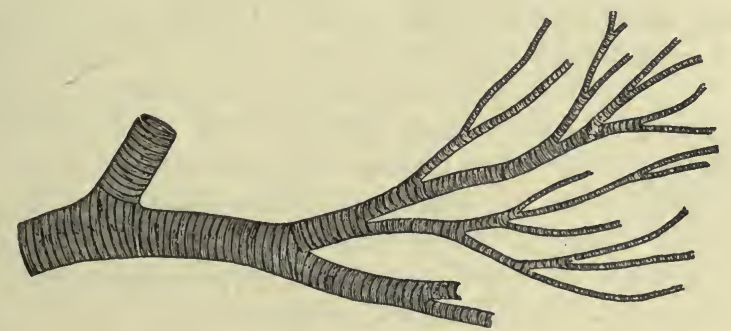

Fıc. 75. - A branched muscular fibre from the frog's tongue. (Kölliker.)

fibre is cylindrical in shape, with rounded ends; many become prolonged into tendon bundles by which the muscle is attached to bone. As a rule they are unbranched, but the muscle fibres of the face and tongue divide into numerous branches before being inserted to the under surface of the skin, or mucous membrane (fig. 75). The fibres in these situations are also finer than in the majority of the voluntary muscles.

Each fibre consists of a sheath, called the sarcolemma, enclosing a soft material called the contractile substance. The sarcolemma is

* The muscular fibres of the pharynx, part of the oesophagus, and of the muscles of the external ear, though not under the control of the will, have the same structure as the voluntary muscular fibres. 
homogeneous, elastic in nature, and especially tough in fish and amphibia. It may readily be demonstrated in a microscopic preparation of fresh muscular fibres by applying gentle pressure to the cover slip; the contractile substance is thereby ruptured, leaving the sarcolemma bridging the space (fig. 76). To the sarcolemma are seen adhering some nuclei.

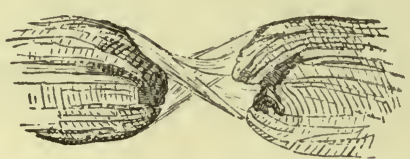

Fig. 76.-Muscular fibre torn across, the sarcolemma still connecting the two parts of the fibre. (Todd and Bow. man.)

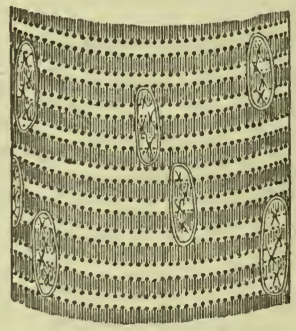

Fio. 77.-Muscular fibre of a mammal highly mag. nified. The surface of the fibre is accurately focussed. (Schäfer.)

The contractile substance within the sheath is made up of alternate discs of dark and light substance.

Muscular fibres contain oval nuclei. In mammalian muscle these are situated just beneath the sarcolemma; but in frog's muscle they

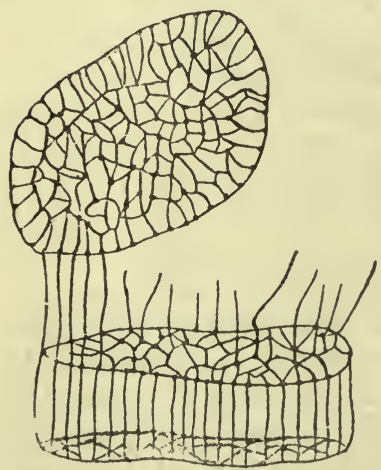

Fig. 78.-Portion of muscle-fibre of water-beetle, showing network very plainly. One of the transverse networks is split off, and some of the longitudinal bars are shown broken off. (After Melland.) composed of an interstitial substance lying between the essentially contractile portions of the muscle. A muscular fibre is thus made cular fibre. The chromoplasm of the nucleus has generally a spiral arrangement, and often there is a little granular protoplasm (well seen in the muscular fibres of the diaphragm) around the poles of each nucleus.

If the surface of a fibre is carefully focussed with a high power, rows of apparent granules are seen lying at the boundaries of the light streaks, and fine longitudinal lines passing through the dark streaks may be detected uniting the apparent granules (fig. 77).

In specimens treated with dilute acids or gold chloride, the granules are seen to be connected side by side, or transversely also. This reticulum (fig. 78), with its longitudinal and transverse meshes, is 
up of what are called fibrils or sarcostyles; and the longitudinal interstitial substance with cross networks comprising the reticulum just referred to is called sarcoplasm. By the use of certain reagents, such as osmic acid or alcohol, the fibrils may be completely separated from one another.

A transverse section of a muscular fibre (fig. 79) shows the sections of these fibrils; the interstitial sarcoplasm is represented as white in the drawing. The angular fields separated by sarcoplasm may still be called by their old name, areas of Cohnheim.

If, instead of focussing the surface of a fibre, it is observed in its depth, a fine dotted line is seen bisecting each light stripe; this has been variously termed Dobie's line, or Krause's membrane (fig. 80). At one time this was believed to be

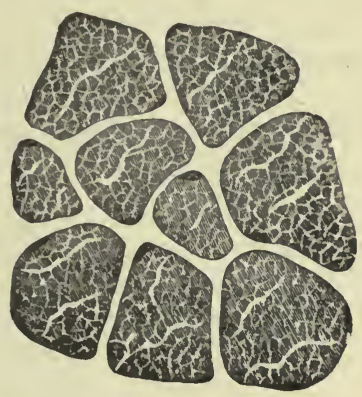

Fig. 79.-Transverse section through muscular fibres of human tongue. The nuclei are deeply stained, situated at the inside of the sar. colemma. Each muscle fibre shows "Cohnheim's areas." $\times 450$. (Klein and Noble Smith.) an actual membrane continuous with the sarcolemma. It is probably very largely an optical effect, caused by light being transmitted between discs of different refrangibility.

If cross membranes do exist they are not very resistant; this was well shown by an accidental observation first made by Kühne, and subsequently seen by others. A minute thread-worm, called the Myorectes, was observed crawling up the interior of the contractile substance of a muscular fibre; it crawled without any opposition from membranes, and the track it left, closed up slowly behind it without interfering with the normal cross-striations of the contractile substance. This observation strikingly illustrates the fact that the contractile substance in a muscular fibre is fluid, but only semi-fluid, for the closing of the thread-worm's track occurred slowly as a hole always closes in a viscous material.

Another appearance which is sometimes seen is a fine clear line running across the fibre in the middle of each dark band. It is called Hensen's line or disc.

A muscular fibre may not only be broken up into fibrils, but under the influence of some reagents, such as dilute hydrochloric acid, it can be broken up into discs, the cleavage occurring in the centre of each light stripe. Bowman, the earliest to study muscular fibres with profitable results, concluded that the subdivision of a fibre into fibrils was a phenomenon of the same kind as the cross cleavage into discs. He considered that both were artificially produced by a separation in one or the other direction of particles of the fibre he called "sarcous elements." The cleavage into discs is how- 
ever much rarer than the separation into fibrils; indeed, indications of the fibrils are seen in perfectly fresh muscle before any reagent has been added, and this is markedly evident in the wing muscles of many insects. It is now believed that cleavage into discs is a purely artificial phenomenon.

Haycraft, who has also investigated the question of muscular structure, concludes that the cross-striation is entirely due to optical

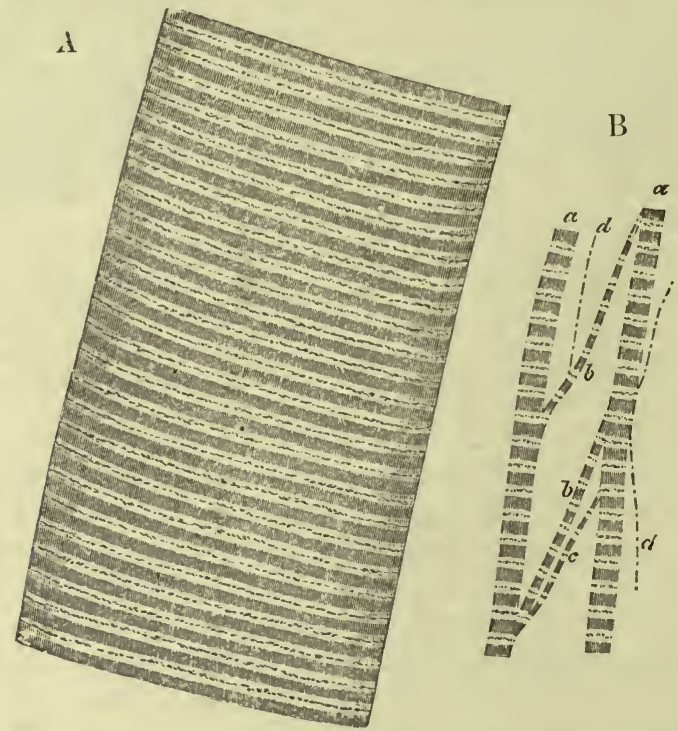

Fig. 80.-A. Portion of a human muscular fibre. $\times 800$. B. Separated bundles of fibrils equally magnified; $a$, $a$, larger, and $b, b$, smaller collections; $c$, still smaller; $d, d$, the smallest which could be detached, possibly representing a single series of sarcous elements. (Sharpey.)

phenomena. The fibrils are varicose, and where they are enlarged different refractive effects will be produced from those caused by the intermediate narrow portions. This view he has very ingeniously supported by taking negative casts of muscular fibres by pressing them on to the surface of collodion films. The collodion cast shows alternate dark and light bands like the muscular fibres.

Most histologists have rejected this view, for the behaviour of the dark stripes to various micro-chemical and staining reagents, and to polarised light, is different from that of the light stripes. The difference is therefore not merely one of diameter, but of chemical composition.

The rapidity of muscular contraction seems to be proportional to the clearness of the cross-striation, and insects' muscles which are 
remarkable for perfection of mechanism have consequently been the subject of many researches. In the wing muscles of these animals the sarcostyles are separated by a considerable quantity of interstitial sarcoplasm, which may be of nutritive importance; at any rate it allows the intimate structure of the individual sarcostyles to be worked out very thoroughly. As the result of such work, Schäfer has arrived at the following conclusions:-

Each sarcostyle is subdivided in the middle of each light stripe by transverse lines (membranes of Krause) into successive portions, which may be termed sarcomeres. Each sarcomere is occupied by a portion of the dark stripe of the whole fibre; this portion of the dark stripe may be called a sarcous element.* The sarcous element

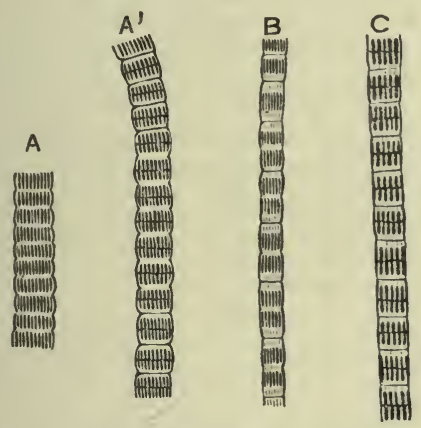

F1G. 81.-Sarcostyles from the wing-muscles of a wasp.

A, A', Sarcostyles showing degrees of contraction.

B, A sarcostyle extended with the sarcous elements separated into two parts.

c) Sarcostyles moderately extended (semidiagrammatic). (E. A. Schäfer.)
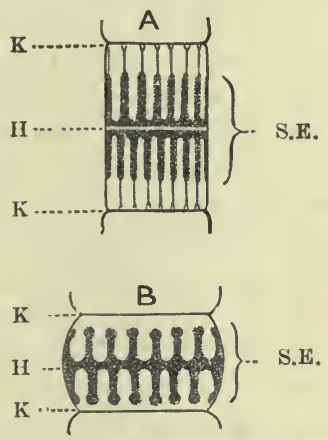

F1G. 82.-Diagram of a sarcomere in a moderately extended condition, $A$, and in a contracted condition, B.

к, K, Krause's membranes ; II, plane of Hensen; s.E. poriferous sarcous element. (E. A. Schäfer.)

is really double, and in the stretched fibre (fig. 81, B) separates into two at the line of Hensen. At either end of the sarcous element is a clear interval separating it from Krause's membrane; this clear interval is more evident in the extended sarcomere (fig. 81, B), and diminishes on contraction (fig. 81, A). The cause of this is to be found in the structure of the sarcous element. It is pervaded with longitudinal canals or pores open towards Krause's membrane, but closed at Hensen's line. In the contracted muscle the clear part of the muscle substance passes into these pores, disappears from view to a great extent, swells up the sarcous element, widens it, and shortens the sarcomere. In the extended muscle, on the other hand, the clear substance passes out from the pores of the sarcous element, and lies

* Notice that this expression has a different meaning from what it originally had when used by Bowman. 
between it and the membrane of Krause; this lengthens and narrows the sarcomere.* This is shown in the diagrams (fig. 82). It may be added that the sarcous element does not lie free in the middle of the sarcomere, but is attached at the sides to a fine enclosing envelope, and at either end to Krause's membrane by fine lines running through the clear substance (fig. 82, A).

This view is interesting, because it brings into harmony amœeboid, ciliary, and muscular movement. In all three instances we have protoplasm composed of two materials, spongioplasm and hyaloplasm. In amœboid movement the irregular arrangement of the spongioplasm allows the hyaloplasm to flow in and out of it in any direction. In ciliary movement the flow is limited by the arrangement of the spongioplasm to one direction; hence the limitation of the movement in one direction (see p. 26). In muscle, also, the definite arrangement of the spongioplasm (represented by the sarcous element) in a longitudinal direction limits the movement of the hyaloplasm (represented by the clear substance of the light stripe), so that it must flow either in or out in that particular direction. The muscular fibre is made up of sarcostyles, and the sarcostyle of sarcomeres. The contraction of the whole muscle is only the sum total of the contraction of all the constituent sarcomeres.

In an ordinary muscular fibre it is stated that when it contracts, not only does it become thicker and shorter, but the light stripes become dark and the dark stripes light. This again is only an optical illusion, and is produced by the alterations in the shape of the sarcostyles, affecting the sarcoplasm that lies between them. When the sarcous elements swell during contraction, the sarcoplasm accumulates opposite the membranes of Krause, and diminishes in amount opposite the sarcous elements; the accumulation of sarcoplasm in the previously light stripes makes them appear darker by contrast than the dark stripes proper. This is shown in fig. 83. There is no true reversal of the stripings in the fibrils themselves.

That this is the case can be seen very well when a muscular fibre is examined with polarised light. A polarising microscope contains a Nicol's prism beneath the stage of the microscope which polarises the light passing through the object placed on the stage. The eyepiece contains another Nicol's prism, which detects this fact. If the two Nicols are parallel, the light passing through the first passes also through the second; but if the second is at right angles to the first, the light cannot traverse it, and the field appears dark. If an object on the microscope stage is doubly refracting it will appear bright in this dark field; if it remains dark it is singly refracting. The sarco-

* The existence of open pores is not admitted by all observers. These regard the passage of fluid in and out of the sarcous element as due to diffusion through its membrane. 
plasm is singly refracting or isotropous; it remains dark in the dark field of the polarising microscope. The fibrils or sarcostyles are in great measure doubly refracting or anisotropous, and appear bright in the dark field of the polarising microscope. The sarcostyle, however, is not wholly doubly refracting; the sarcous elements

C

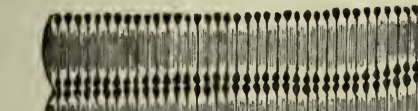

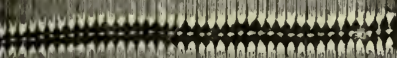

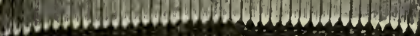

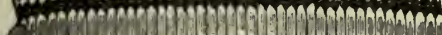

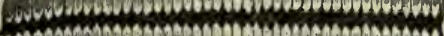

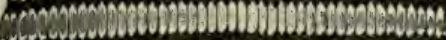

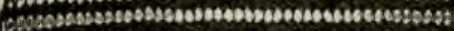

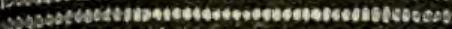

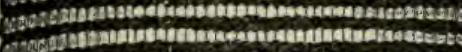

I

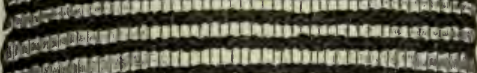

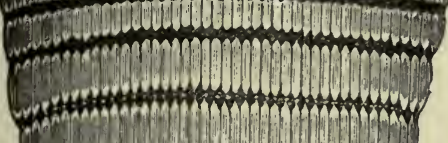

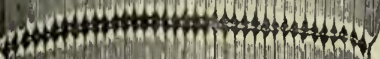

\section{(t)}

R

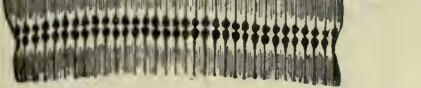

Fia. 83.-Wave of contraction passing over a muscular fibre of water-beetle. R, R, Portions of the fibre at rest ; c, contracted part ; I, I, intermediate condition. (Schäfer.)

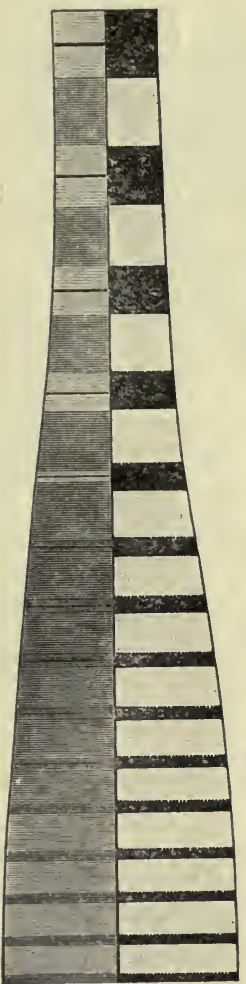

Fig. S4.-This figure (after Engelmamn) illustrates the appearance of a muscular fibre as examined in ordinary light (left-hand side) and in polarised light (right-hand side). In the upper part of the diagram the fibre is not contracted, in the lower part it is contracted. The (1ark bands are seen to be bright by polarised light, owing to their being doubly refracting or anisotropous; during contraction, fluid passes from the singly refracting or isotropous light band into the doubly refracting dark band, which, in consequence, becomes widened out.

are doubly refracting, and the clear intervals are singly refracting. On contraction there is no reversal of these appearances, though of course the relative thickness of the singly refracting intervals varies inversely with that of the doubly refracting sarcous elements (see fig. 84).

The meaning and causation of the optical appearances of striated 
muscle fibres, and the changes they undergo during contraction have been the subjects of numerous hypotheses. One of two only of

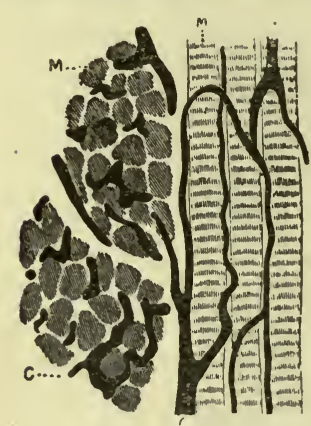

Fic. 85.-Three muscular fibres running longitudinally, and two bundles of fibres in trans. verse section, $\mathbf{M}$, from the tongue. The capillaries, $\mathrm{c}$, are injected. $\times 150$. (Klein and Noble Smith.) these theories have been mentioned in the preceding paragraphs, and it should be recognised that even experts are content to leave the matter very largely an open question at present.

Blood-vessels of Muscle.-The arteries break up into capillaries, which run longitudinally in the intervening connective tissue, transverse branches connecting them (fig. 85). No blood-vessels ever penetrate the sarcolemma. The muscular fibres are thus, like other tissues, nourished by the exudation from the blood called lymph.

The motor nerves of voluntary muscle pierce the sarcolemma, and terminate in expansions called end-plates, to be described on p. 78 .

The sensory nerves of voluntary muscle terminate in structures known as the neuro-muscular spindles, which will be dealt with in the chapter on Motorial Sensations.

\section{Red Muscles.}

In many animals, such as the rabbit, and some fishes, most of the muscles are pale, but some few (like the diaphragm, crureus, soleus, semi-membranosus, in the rabbit) are red. These muscles contract more slowly than the pale muscles, and their red tint is due to hæmoglobin contained within their contractile substance.

In addition to these physiological distinctions, there are histological differences between them and ordinary striped muscle, viz. :-

1. Their muscular fibres are thinner.

2. They have more sarcoplasm.

3. Longitudinal striation is therefore more distinct.

4. Transverse striation is more irregular than usual.

5. Their nuclei are situated not only under the sarcolemma, but also in the thickness of tho fibre.

6. The transverse loops of the capillary network are dilated into little reservoirs, far beyond the size of ordinary capillaries.

\section{Cardiac Muscle.}

The muscular fibres of the heart, unlike those of other involuntary muscles, are striated; but although in this respect they resemble the skeletal muscles, they have distinguishing characteristics of their own. The fibres which lie side by side are united at 
frequent intervals by short branches (fig. 86). The fibres are smaller than those of the ordinary striated muscles, and their transverse striation is less distinct. No sarcolemma can be discerned. Each fibre has only one nucleus which is situated in the middle of its substance. At the junctions of the fibres there is a certain amount of cementing material, stainable by silver nitrate. This is bridged across by fine fibrils from cell to cell.

The above is a general description of the fibres in the great mass of the cardiac musculature. But immediately beneath the lining membrane of the ventricles, and in the main connecting strand which links the auricles to the ventricles (the auriculo-ventricular bundle) are found peculiar fibres known after their discoverer as Purkinje's fibres; these are large, clear,

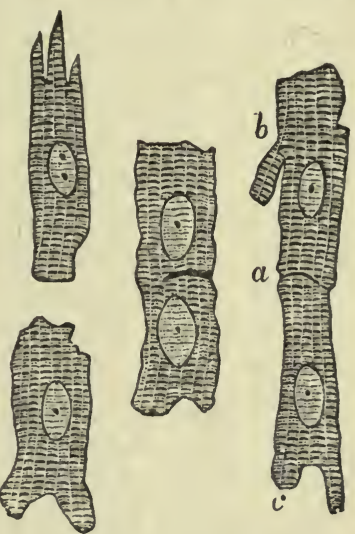

Fig. 86.-Muscular fibre-cells from the heart. (F. A. Schäfer.) quadrangular cells with granular protoplasm containing several nuclei, and striated only on their margins. The special meaning and function of these fibres will be described in the chapter on the heart.

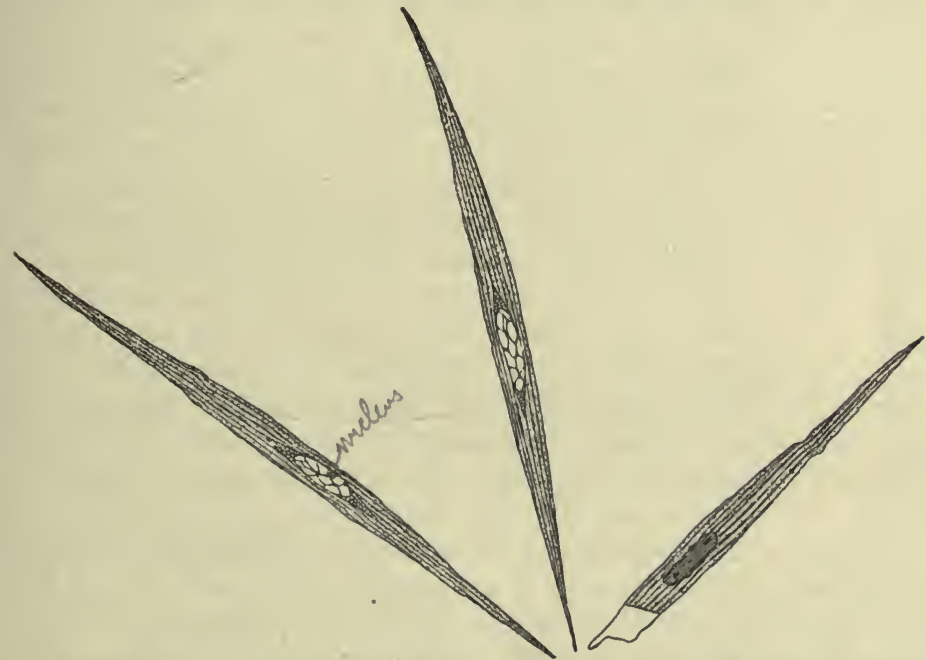

Fig. 87. - M uscular fibre-cells from the muscular coat of intestine-highly magnified. Note the longitudinal striation, and in the broken fibre the sheath is visible.

Plain Muscle.

Plain muscle forms the proper muscular coats (1.) of the digestive 
canal from the middle of the œsophagus to the internal sphincter ani; (2.) of the ureters and urinary bladder; (3.) of the trachea and bronchi; (4.) of the ducts of glands; (5.) of the gall-bladder; (6.) of the vesiculæ seminales; (7.) of the uterus and Fallopian tubes; (8.) of blood-vessels and lymphatics; (9.) of the iris, and ciliary muscle of the eye. This form of tissue also enters largely into the composition (10.) of the tunica dartos, the contraction of which is the principal cause of the contraction of the scrotum on exposure to cold. It occurs also in the skin generally, being found surrounding the secreting part of the sweat glands and in small bundles attached to the hair follicles; it also occurs in the areola of the nipple. It is composed of long, fusiform cells (fig. 87), which are not as a rule more than $\frac{1}{6} 0$ inch long. Each cell has an oval or rod-shaped nucleus. The cell substance is longitudinally but not transversely striated. Each cell or fibre, as it may also be termed, has a delicate sheath. The fibres are united by cementing material, which can be stained by silver nitrate, and is bridged across by fine filaments passing from cell to cell.

The nerves in involuntary muscle (both cardiac and plain) do not terminate in end-plates, but by plexuses or networks, which ramify between and around the muscular fibres.

\section{Development of Muscular Fibres.}

All muscular fibres (except those of the sweat glands, which are epiblastic) originate from the mesoblast. The plain fibres are simply elongated cells in which the nucleus becomes rod-shaped. In cardiac muscle, the likeness to the original cells from which the fibres are formed is not altogether lost, and this is specially the case for the Purkinje's fibres alluded to on the preceding page.

Voluntary muscular fibres are developed from cells which become elongated, and the nuclei of which multiply. In most striated muscle fibres the nuclei ultimately take up a position beneath the cell-wall or sarcolemma which is formed on the surface. Striations appear first along one side, and extend round the fibre (fig. 88), then they extend into the centre.

During life new fibres appear to be formed in part by a longitudinal splitting of pre-existing fibres; this

FIG. 88. - Develop. ing muscular fibre from foetus of two months. (Ran. vier.) is preceded by a multiplication of nuclei; and in part by the lengthening and differentiation of embryonic cells (sarcoplasts) found between the fully formed fibres.

In plain muscle, growth occurs in a similar way: this is well illustrated in the enlargement of the uterus during pregnancy. After parturition the fibres shrink to their original size, but many disappear and are removed by absorption. 


\section{CHAPTER VII}

\section{NERVE}

Nervous tissue is the material of which the nervous system is composed. The nervous system is composed of two parts, the central nervous system, and the peripheral nervous system. The central nervous system consists of the brain and spinal cord; the peripheral nervous system consists of the nerves, which conduct the impulses to and from the central nervous system, and thus bring the nerve-centres into relationship with other parts of the body.

Some of the nerves conduct impulses from the nerve-centres and are called efferent; those which conduct impulses in the opposite direction are called afferent. When one wishes to move the hand, the nervous impulse starts in the brain and passes down the efferent or motor nerve-tracts to the muscles of the hand, which contract; when one feels pain in the hand, afferent or sensory nerve-tracts convey an impulse to the brain which is there interpreted as a sensation. If all the nerves going to the hand are cut through, all communication with the nerve-centres is destroyed, and the hand loses the power of moving under the influence of the will, and the brain receives no impulses from the hand, or, as we say, the hand has lost sensibility.

This distinction between efferent and afferent nerves is a physiological one, which we shall work out more thoroughly later on. No histological distinction can be made out between motor and sensory nerves, and it is histological structure which we wish to dwell upon in this chapter.

Under the microscope nervous tissue is found to consist essentially of nerve-cells and their branches. The nerve-cells are contained in the brain and spinal cord, and in smaller collections of cells on the course of the nerves called ganglia. The part of the nervecentres containing cells is called grey matter.

Long branches of the nerve-cells are known as nerve-fibres. These become sheathed in a manner to be immediately described, and are contained in the nerves, and in the white matter of brain and 
spinal cord. The bodies of nerve-cells differ in size, shape, and arrangement, and we shall discuss these fully when we get to the

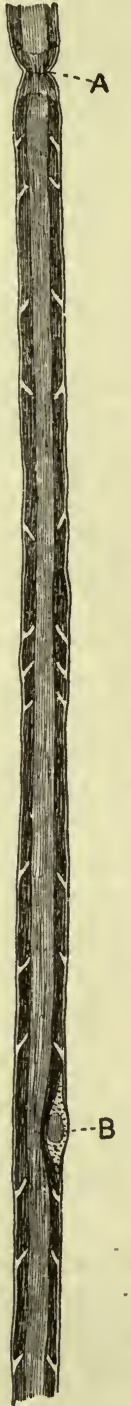

Fig, 89. - Nervefibre stained with osmic acid. A, node; $\mathrm{B}$, nucleus. (Key and Retzius.) nerve-centres. For the present it will be convenient to confine ourselves to the nerve-fibres as they are found in a nerve.

Nerve-fibres are of two histological kinds, medullated and non-medullated. Medullated nerve-fibres are found in the white matter of the nerve-centres and in the nerves originating from the brain and spinal cord. Non-medullated nerve-fibres occur in the sympathetic nerves.

The medullated or white fibres are characterised

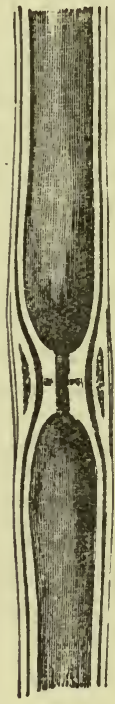

Fic. 90.-A node of Ranvier in a medullated nerve-fibre, viewed from above. The medullary sheath is in. terrupted, and the primi. tive sheath thickened. Copied from Axel Key and Retzius. $\times 750$. (Klein and Noble Smith.)

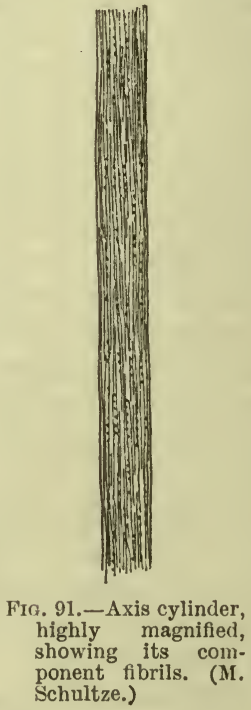

by a sheath of white colour, fat-like in nature, and stained black by osmic acid; it is called the medullary sheath or white substance of Schwann; this sheathes the essential part of the fibre which is a process from a nerve-cell, and is called the axis cylinder. Outside the medullary sheath is a thin homogeneous membrane of elastic nature called the primitive sheath or neurilemma. 
The axis cylinder is a soft transparent thread in the middle of the fibre; it is made up of exceedingly fine fibrils (fig. 91) which stain readily with gold chloride. The medullary sheath gives a characteristic double contour and tubular appearance to the fibre. It is interrupted at regular intervals known as the nodes of Ranvier. The stretch of a nerve-fibre between two nodes is called an inter-node, and in the middle of each inter-node is a nucleus which belongs to the primitive sheath. Besides these interruptions, a variable number of oblique clefts are also seen dividing the sheath into medullary segments (fig. 89); but most if not all of these are produced artificially in the preparation of the specimen.

The medullary sheath also contains a horny substance called neurokeratin: the arrangement of this substance is in the form of a network or reticulum holding the fatty matter of the sheath in its meshes. The occurrence of horny matter in the epidermis, in the development of the enamel of teeth, and in nerve, is an interesting chemical reminder that all these tissues originate from the same embryonic layer, the epiblast. The fat-like matter consists largely of cholesterin, a monatomic alcohol, and phosphorised fats, such as lecithin.

Near their terminations the nerve-fibres branch: the branching occurs at a node (fig. 92).

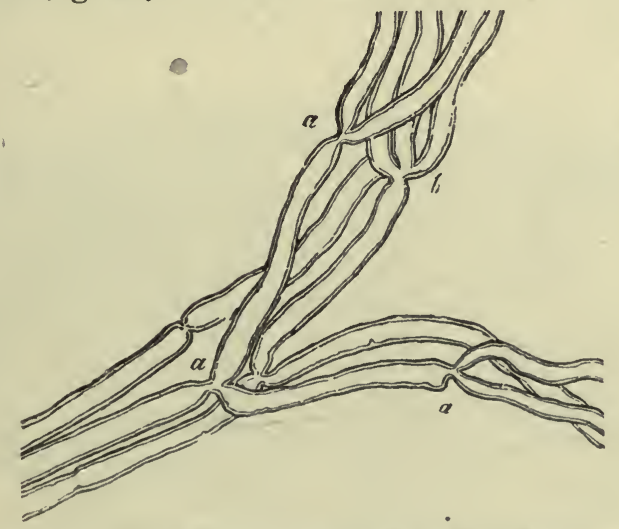

Fio. 92.-Small branch of a muscular nerve of the frog, near its termination, showing division of the fibres $-a$, into two; $b$, into three. $\times 350$. (Kölliker.)

Staining with silver nitrate produces a peculiar appearance at the nodes, forming what is known as the crosses of Ranvier.

One limb of the cross is produced by the dark staining of cement substance which occurs between the segments of the neurilemma; the other limb of the cross is due to the staining of a number of minute transverse bands in the axis cylinder (Fromann's lines), which is here 
not closely invested by the medullary sheath (fig. 93). Macallum has shown that this appearance of transverse striping is an artifact

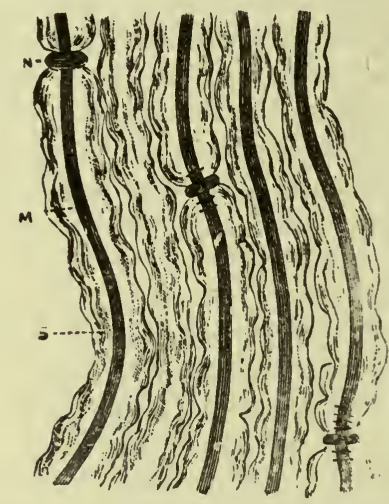

F10. 93.- Several fibres of a bundle of medullated nerve-fibres acted upon by silver nitrate to show behaviour of nodes of Ranvier, M, towards this reagent. The silver has penetrated at the nodes, and has stained the axis-cylinder, $M$, for a short distance. $\mathrm{S}$, the white substance. (Klein and Noble Smith.)

and can be obtained in any exposed portion of an axis cylinder, that is, wherever the silver nitrate can penetrate to it.

The arrangement of the nerve-fibres in a nerve is best seen in a transverse section.

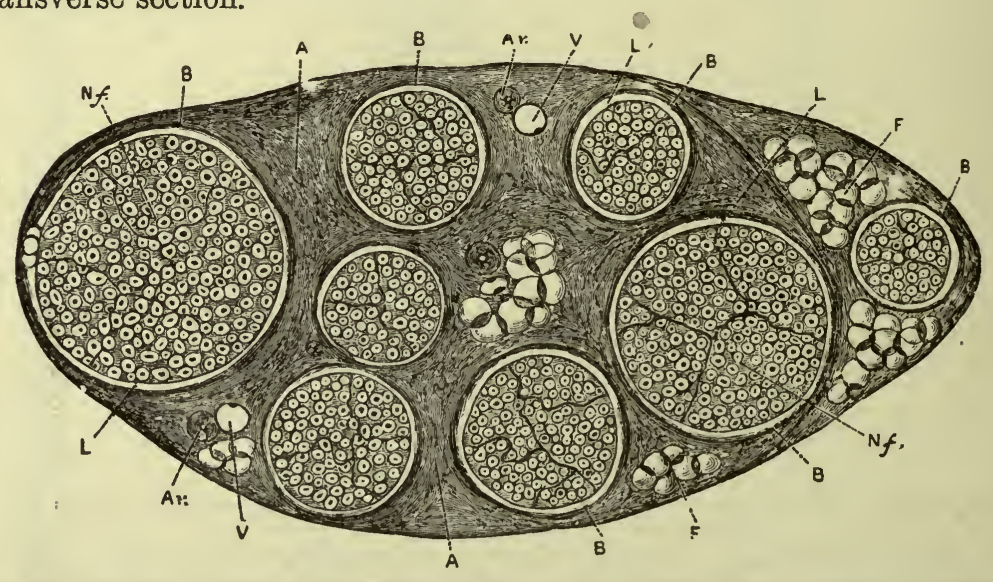

Fio. 94.--Transverse section of the sciatic nerve of a cat about $\times 100$.- It consists of bundles (funiculi) of nerve-fibres ensheathed in a fibrous sheath, epineurium, $\mathbf{A}$; each bundle has a special sheath (not sufficiently marked out from the epineurium in the figure) or perineurium $\mathbf{B}$; the nerve-fibres $\mathrm{N} f$ are separated from one another by endoneurium; L, lymph spaces; $\mathrm{Ar}$, artery ; $\mathrm{V}$, vein; F, fat. Somewhat diagrammatic. (V. D. Harris.)

The nerve is composed of a number of bundles or funiculi of nervefibres bound together by connective tissue. The sheath of the whole 
nerve is called the epineurium; that of the funiculi the perineurium; that which passes between the fibres in a funiculus, the endoneurium (fig. 94). Single nerve-fibres passing to their destination are surrounded by a prolongation of the perineurium, known as the Sheath of Henle. The nerve trunks themselves receive nerve-fibres which ramify and terminate as end-bulbs in the epineurium.

The size of the nerve-fibres varies; the largest fibres are found in the spinal nerves, where they are 14.4 to $19 \mu$ in diameter.* Others mixed with these measure 1.8 to $3.6 \mu$. These small nervefibres are the visceral nerves; they pass to collections of nerve-cells called the sympathetic ganglia, whence they emerge as non-medullated fibres, and are distri-

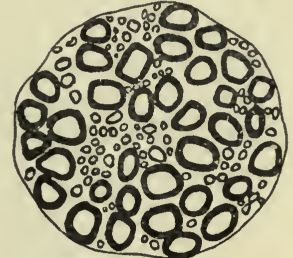

FIG. 95.-Section 8cross a nerve bundle in the second thoracic anterior root of the dog, stained with osmic acid. (Gaskell.) buted to involuntary muscle. They are well seen in sections stained by osmic acid, the black rings being the stained medullary sheaths (fig. 95).

The non-medullated fibres or fibres of Remak have no medullary sheath, and are therefore devoid of the double contour of the medullated fibres, and are unaffected in appearance by osmic acid. They

A

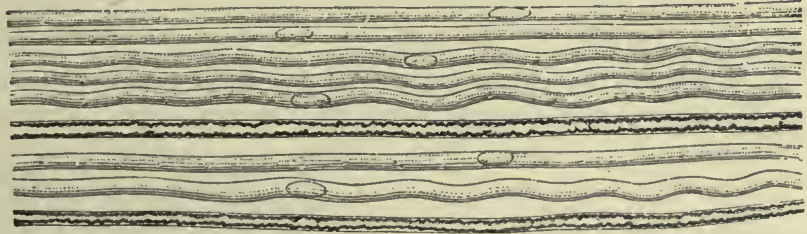

B

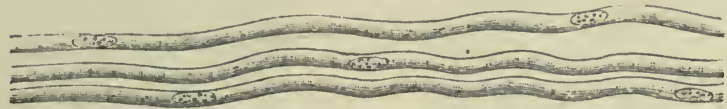

Fia. 96.-Grey, or non-medullated nerve-fibres. A. From a brancli of the olfactory nerve of the sheep; two dark-bordered or white fibres from the fifth pair are associated with the pale olfactory fibres. B. From the sympathetic nerve. $\times 450$. (Max Schultze.)

consist of an axis cylinder covered by a nucleated sheath. They branch frequently.

$* \mu=$ micro-millimetre $=\frac{1}{1000}$ millimetre. 


\section{Termination of Nerves in Muscle.}

In the voluntary muscles the motor nerve-fibres have special end-organs called end-plates (fig. 97). The fibre branches two or three times, and each branch goes to a muscular fibre. Here the

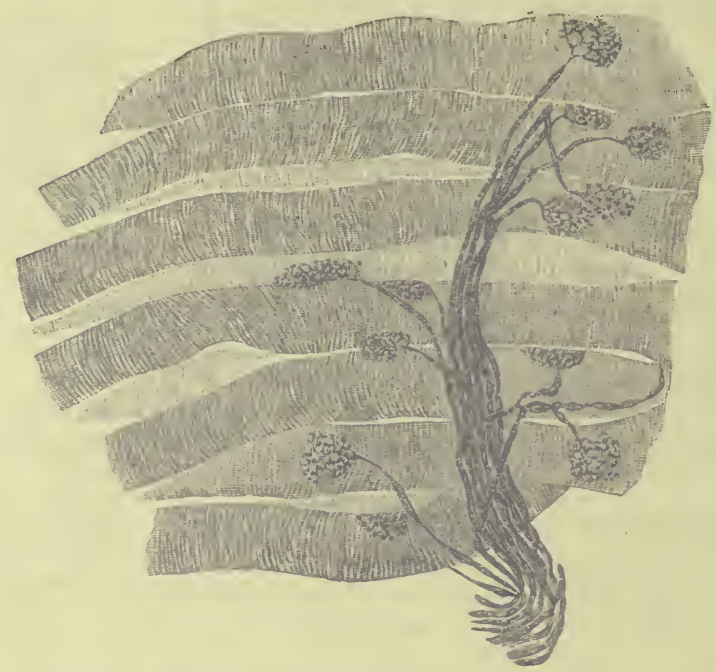

Fia. 97.-End-plates; chloride of gold preparation to show the axis cylinders and their final ramifications of fibrillæ. $\times 170$. (Szymonowicz.)

neurilemma becomes continuous with the sarcolemma, the medullary sheath stops short, and the axis cylinder branches several times.

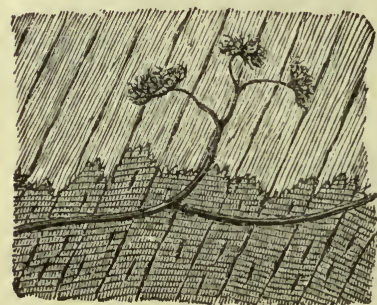

Fig. 98.-Termination of medullated nerve-fibres in tendon near the mus. cular insertion. (Golgi.)

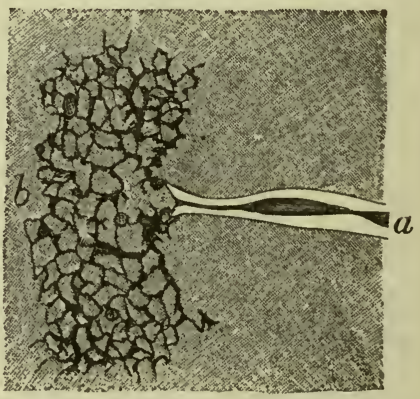

F10. 99.-One of the reticulated end-organs of fig. 98, more highly magnified. Medullated nerve-fibre; $b$, reticulated end-organ. (Golgi.)

This ramification is imbedded in a layer of granular protoplasm containing numerous nuclei. Considerable variation in shape of the 
end-plates occurs in different parts of the animal kingdom. Somewhat similar nerve-endings are seen in tendon; these, however, are doubtless sensory (figs. 98, 99).

In the involuntary muscles, the fibres, which are for the most part non-medullated, form complicated plexuses near their termination. The plexus of Auerbach (fig. 100) between the muscular coats of the intestine is a typical case. Groups of nerve-cells will be noticed at the junctions of the fine nervous cords. From these plexuses fine branches pass off and bifurcate at frequent intervals, until at last ultimate fibrillæ are reached. These subdivisions of the axis cylinders do not anastomose with one another, but they come into close relation-

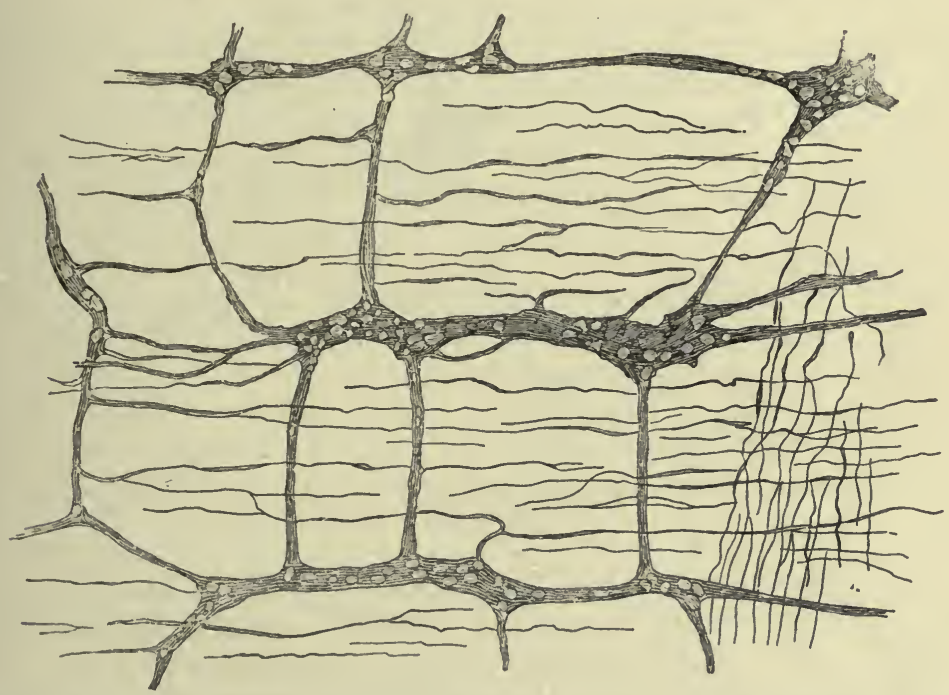

Fic. 100.-Plexus of Auerbach, between the two layers of the muscular coat of the intestine. (Cadiat.)

ship with the involuntary muscular fibres; though some histologists have stated that they end in the nuclei of the muscular fibres, it is now believed that they do not pass into their interior.

The terminations of sensory nerves are in some cases plexuses, in others special end-organs. We shall deal with these in our study of sensation.

\section{Development of Nerve-fibres.}

A nerve-fibre is primarily an outgrowth from a nerve-cell, as is shown in the accompanying diagram (fig. 101). A nerve-cell, though it may have many branches, only gives off one process which becomes the axis cylinder of a nerve-fibre. This acquires a medullary sheath 
when it passes into the white matter of the brain or spinal cord, and a primitive sheath when it leaves the nerve-centre and gets into the nerve. But at first the axis cylinder is not sheathed at all.

The formation of the sheaths is still a matter of doubt, but the generally accepted opinion is that the primitive sheath is formed by mesoblastic cells which become flattened out and wrapped round the fibre end to end. These are separated at the nodes by intercellular or cement substance stainable by silver nitrate (fig. 93). The medullary sheath is formed, according to some, by a fatty change occurring in the parts of these same cells which are nearest to the axis cylinder,

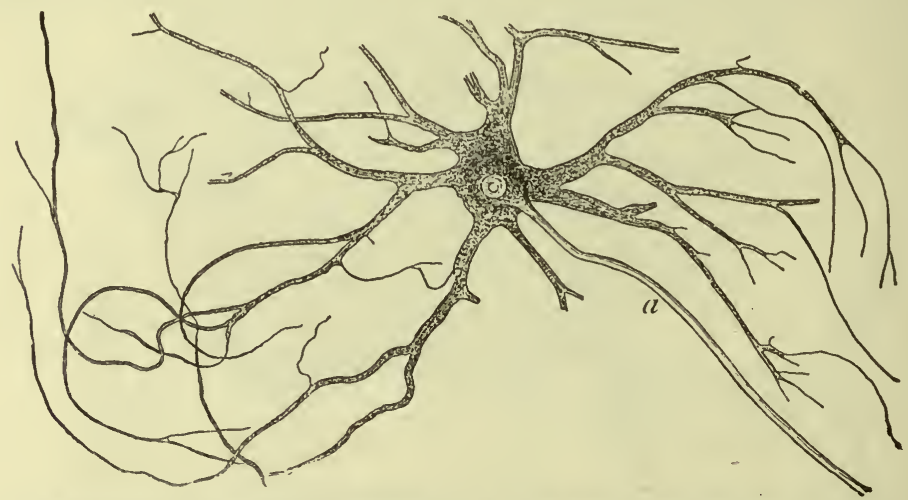

FIG. 101.-Multipolar nerve-cell from anterior horn of spinal cord; $a$, axis cylinder process. (Max Schultze.)

but it is much more probable that it is formed from the peripheral layer of the axis cylinder; the presence of neurokeratin in it distinctly points to an epiblastic origin. The fact also that, in the nerve-centres, the medullated nerve-fibres have no primitive sheath, and the phenomena of Wallerian degeneration, to be described later, all tend to confirm the same view. 


\section{CHAPTER VIII}

\section{IRRITABILITY AND CONTRACTILITY}

Irritability or Excitability is the power which certain tissues possess of responding by some change (transformation of energy) to the action of an external agent. This external agent is called a stimulus.

Undifferentiated cells such as white blood-corpuscles are irritable; when stimuli are applied to them they execute the movements we have learnt to call amœboid.

Ciliated epithelium cells and muscular fibres are irritable; they also execute movements under the influence of stimuli.

Nerves are irritable; when they are stimulated, a change is produced in them; this change is propagated along the nerve, and is called a nervous impulse; there is no change of form in the nerve visible to the highest powers of the microscope; much more delicate and sensitive instruments than a microscope must be employed to obtain evidence of a change in the nerve; it is of a molecular nature. But the irritability of nerve is readily manifested by the results the nervous impulse produces in the organ to which it goes; thus the stimulation of a motor nerve produces a nervous impulse in that nerve which, when it reaches a muscle, causes the muscle to contract: stimulation of a sensory nerve produces a nervous impulse in that nerve which, when it reaches the brain, causes a sensation.

Secreting glands are irritable; when stimulated they secrete.

The electrical organs found in many fishes such as the electric eel, and torpedo ray, are irritable; when they are stimulated they give rise to an electrical discharge.

Contractility is the power that certain tissues possess of responding to a stimulus by change of form. Contractility and irritability do not necessarily go together; thus both muscle and nerve are irritable, but of the two, only muscle is contractile.

Some movements visible to the microscope are not due to contractility; thus granules in protoplasm or in a vacuole may often be seen to exhibit irregular, shaking movements due simply to vibrations transmitted to them from the outside. Such movement is known as Brownian movement. 
Instances of contractility are seen in the following cases:-

1. The movements of protoplasm seen in simple animal and vegetable cells have been already described on pp. 11 to 14 .

2. The movements of pigment cells. These are well seen under the skin of such an animal as the frog; under the influence of electricity and of other stimuli, especially of light, the pigment granules are massed together in the body of the cell, leaving the processes quite transparent (fig. 102). If the stimulus is removed the granules gradually extend into the processes again. Thus the skin of the frog is sometimes uniformly dusky, and sometimes quite light coloured. The chameleon is an animal which has become almost proverbial, since it possesses the same power to a marked degree. This function is a protective one ; the animal approximates in colour that of its surroundings, and so escapes detection.

In the retina we shall find a layer of pigment cells (fig. 103), the
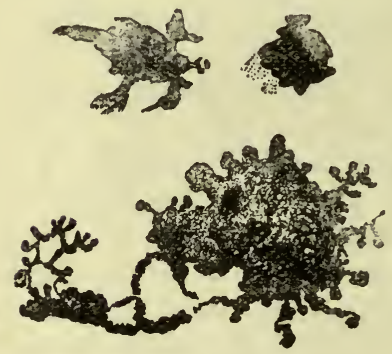

FIG. 102.-Frog's pigment cells.

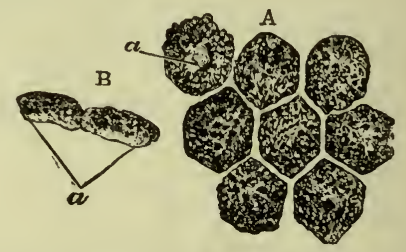

Fig. 103.-Pigment cells from the retina. A. Cells still cohering, seen on their surface; $\alpha$, nucleus indistinctly seen. In the other cells the nucleus is concealed by the pigment granules. B. Two cells seen in profile; $a$, the outer or posterior part containing scarcely any pig. ment. $\times 370$. (Henle.)

granules in which are capable of moving in the protoplasm in a somewhat similar way; the normal stimulus here also is light.

3. Ciliary movement; here we have a much more orderly movement which has already been described (see p. 25).

4. In Vorticellæ, a spiral thread of protoplasm in their stalk enables them by contracting it to lower the bell at the end of the stalk.

5. In certain of the higher plants, such as the sensitive and carnivorous plants, movements of the stalks and sensitive hairs of the leaves occur under the influence of stimuli.

6. Muscular movement. This for the student of human physiology is the most important of the series; it is by their muscles that the higher animals (man included) execute most of their movements.

If we contrast together amœboid, ciliary, and muscular movement, we find that they differ from each other very considerably. Amœboid movement can occur in any part of an amoboid cell, and in any direction. Ciliary and muscular movement are limited to one direc- 
tion; but they are all essentially similar, consisting of the movement of hyaloplasm in and out of spongioplasm; it is the arrangement of the spongioplasm that limits and controls the movement of the hyaloplasm (see also p. 68).

Rhythmicality.-In some forms of movement there is not only order in direction, but order in time also. This is seen in ciliary movement, and in many involuntary forms of muscular tissue, such as that of the heart. Here periods of contraction alternate with periods of rest, and this occurs at regular intervals. Under the influence of certain saline solutions,* voluntary muscles may be made artificially to exhibit rhythmic contractions.

A familiar instance of rhythmic movement in the inorganic world is seen in a water-tap nearly turned off but dripping; water accumulates at the mouth of the tap till the drop is big enough to fall; it falls, and the process is repeated. If, instead of water, gum or treacle, or some other viscous substance is watched under similar circumstances, the drops fall much more slowly; each drop has to get bigger before it possesses enough energy to fall. Thus we may get different rates of rhythmic movement. So in the body, during the period of rest, the cilium or the heart is accumulating potential energy, till, as it were, it becomes so charged that it discharges; potential energy is converted into kinetic energy or movement.

When contraction travels as a wave along muscular fibres, or from one fibre to another, the term peristalsis is employed. These waves are well seen in such a muscular tube as the intestine, and are instrumental in moving its contents along. The heart's contraction is a more complicated peristalsis occurring in a rhythmic manner.

The question may be first asked, what evidence there is of irritability in muscle? May not the irritability be a property of the nerve-fibres which are distributed throughout the muscle and terminate in its fibres? The doctrine of independent muscular irritability was enunciated by Haller more than a century ago, and was afterwards keenly debated. It was finally settled by the following experiment of Claude Bernard.

If a frog is taken and its brain destroyed by pithing, it loses consciousness, but the circulation goes on, and the tissues of its body retain their vitality for a considerable time. If now a few drops of a solution of curare, the South American arrow poison, are injected with a small syringe under the skin of its back, it loses in a few minutes all power of movement. If next the sciatic or any other nerve going

* Biedermann's fluid has the following composition:-Sodium chloride 5 grammes, alkaline sodium phosphate 2 gr., sodium carbonate $0.5 \mathrm{gr}$., water 1 litre. If one end of the sartorius of a curarised frog is dipped into this fluid, it contracts rhythmically in a manner analogous to the heart. A solution of pure sodium chloride $(0.65$ per cent.) has a similar action. 
to muscle is dissected out and stimulated, no movement occurs in the muscles to which it is distributed. Curare paralyses the end-plates, so that nervous impulses cannot get past them and cause any effect on the muscles. But if the muscles are stimulated themselves, they contract.

Another proof that muscle possesses inherent irritability was adduced by Kühne. In part of some of the frog's muscles (e.g. part of the sartorius) there are no nerves at all; yet these parts are irritable and contract when stimulated.

The evidence of the statement just made that the poisonous effect of curare is on the end-plates is the following:-The experiment described proves it is not the muscles that aro paralysed. It must therefore be either the nerves, or the links between the nerve-fibres and the muscular fibres. By a process of exclusion we arrive at the conclusion that it is these links, for the following experiment shows it is not the nerves. The frog is pithed as before, and then one of its legs is tightly ligatured so as to include everything except the sciatic nerve of that leg. Curare is injected and soon spreads by the circulating blood all over the body except to the leg protected by the ligature. It can get to the sciatic nerve of that leg because that was not tied in with the rest. The sciatic nerve of the other leg is now dissected out; when the muscles supplied by it cease to contract when the nerve is stimulated, the frog may be considered to be fully under the influence of the drug. But on stimulating the sciatic nerve of the protected limb, the muscles respond normally; this shows that the nerve which has been exposed to the action of the poison has not been affected by it.

\section{Varieties of Stimuli.}

The normal stimulus that leads to muscular contraction is a nervous impulse; this is converted into a muscular impulse (visible as a contraction) at the end-plates. This nervous impulse starts at the nerve-centre, brain, or spinal cord, and travels down the nerve to the muscle. In a reflex action the nervous impulse in the nervecentre is started by a sensory impulse from the periphery; thus when one puts one's hand on something unpleasantly hot, the hand is removed; the hot object causes a nervous impulse to travel to the brain, and the brain reflects down to the muscles of the hand another impulse by the motor nerves which causes the muscles to contract in such a manner as to move the hand out of the way.

But the details of muscular contraction can be more readily studied in misscles removed from the body of such an animal as the frog, and made to contract by artificial stimuli. When we have considered these, we can return to the lessons they teach us about the normal contractions in our own bodies. 
The first thing to do is to make from a pithed frog a muscle-nerve preparation; the muscle usually selected is the gastrocnemius, the large muscle of the calf of the leg, with the sciatic nerve attached. For some experiments the sartorius or gracilis may be used; but nearly all can be demonstrated on the gastrocnemius.

The tendon of the gastrocnemius may be tied to a lever with a flag at the end of it, and thus its contractions rendered more evident; the bone at the other end is fixed in a clamp. Stimuli may be applied either to the nerre or to the muscle. If the stimulus is applied to the nerve, it is called indirect stimulation; the stimulus starts a nervous impulse which travels to the muscle; the muscle

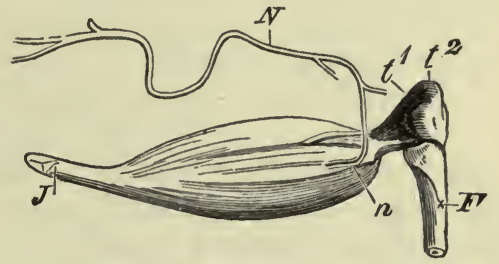

Fig. 104.-Muscle-nerve preparation. F, Femur; $\mathrm{N}$, nerve; $\mathrm{J}$, tendo Achillis. (M'Kendrick.)

is thus stimulated as it is in voluntary contraction by a nervous impulse. Stimulation of the muscle itself is called direct stimulation. These stimuli may be:

1. Mechanical ; for instance a pinch or blow.

2. Chemical; for instance salt or acid sprinkled on the nerve or muscle.

3. Electrical; the constant or the induced current may be used.

In all cases the result of the stimulation is muscular contraction. Of all methods of artificial stimulation, the electrical is the one most generally employed, because it is more under control and the strength and duration of the stimuli (shocks) can be regulated easily. We shall therefore have to study some electrical apparatus.

Chemical stimuli are peculiar, for some which affect muscle do not affect nerve, and vice versd; thus glycerin stimulates nerve, but not muscle; ammonia stimulates muscle, but not motor nerves.

We may regard stimuli as liberators of energy; muscle and nerve and other irritable structures undergo disturbances in consequence of a stimulus. The disturbance is some form of movement, visible movement in the case of muscle, molecular movement in the case of nerve. A stimulus may be regarded as added motion. Sir William Gowers compares it to the blow that causes dynamite to explode, or the match applied to a train of gunpowder. A very slight blow will explode a large quantity of dynamite; a very small spark will fire a long train of gunpowder. So in muscle or nerve the effect is often out of all proportion to the strength of the stimulus; a light touch on the surface of the body may elicit very forcible nervous and muscular disturbances; and moreover, the effect of the stimulus is propagated along the nerve or muscle without loss. 


\section{Contraction of Muscle.}

Muscle undergoes the following changes when it contracts :-

1. Changes in form.

2. Changes in extensibility and elasticity.

3. Changes in temperature.

4. Changes in electrical condition.

5. Chemical changes.

In brief, each of these changes is as follows:-

1. Changes in form. - The muscle becomes shorter, and at the same time thicker. The amount of shortening varies so that the length of the muscle when contracted is from 65 to 85 per cent. of what it was originally. Up to a certain point, increase of the strength of the stimulus increases the amount of contraction. Fatigue diminishes, and up to about $33^{\circ} \mathrm{C}$. the application of heat increases the amount of contraction. Beyond this temperature the muscular substance begins to be permanently contracted, and a condition called heat rigor, due to coagulation of the muscle proteins, sets in a little over $40^{\circ} \mathrm{C}$.

What the muscle loses in length it gains in width; there is no appreciable change of volume.

Among the changes in form must also be mentioned those changes in the individual muscular fibres which require a microscope for their investigation; these have been already considered (see p. 68).

2. Changes in elasticity and extensibility.-The contracted muscle is more stretched by a weight in proportion to its length than an uncontracted muscle with the same weight applied to it; the extensibility of contracted muscle is increased; its elasticity is diminished.

3. Changes in temperature.-When muscle is at work or contracting, more energetic chemical changes are occurring than when it is at rest; more heat is produced, and its temperature rises.

4. Changes in electrical condition.-A muscle when it contracts undergoes a variation in its electrical condition.

5. Chemical changes.- These consist in an increased consumption of oxygen, and an increased output of waste materials such as carbonic acid, and sarcolactic acid. After prolonged contraction the muscle consequently acquires an acid reaction.

These five sets of changes will form the subjects of the following five chapters. 


\section{CHAPTER IX}

CHANGE IN FORM IN A MUSCLE WHEN IT CONTRACTS

Though it has been known since the time of Erasistratus (B.C. 304) that a muscle becomes thicker and shorter when it contracts, it was not until the invention of the graphic method by Ludwig and Helmholtz, about sixty years ago, that wo possessed any accurate knowledge of this change. The main fact just stated may be seen by simply looking at a contracting muscle, such as the biceps of one's own arm; but more elaborate apparatus is necessary for studying the various phases in contraction and the different kinds of contraction that may occur.

These may be readily demonstrated on the ordinary muscle-nerve preparation (gastrocnemius and sciatic nerve) from a frog. By the graphic method, one means that the movement is recorded by a writing. We shall find that the same method is applied to the heart's movements, respiratory movements, blood pressure, and many other important problems in physiology. The special branch of the graphic method we have now to study is called myography; the instrument for writing is called a myograph; the writing itself is called a myogram. Put briefly, a myograph consists of a writing point at the end of a lever attached to the muscle, and a writing surface which travels at a uniform rate, on which the writing point inscribes its movement.

The first thing, however, that is wanted is something to stimulate the muscle and make it contract; the stimulus is usually applied to the nerve, and the form of stimulus most frequently employed is electrical.

The galvanic battery in most common use is the Daniell cell. It consists of a well-amalgamated zinc rod immersed in a cylinder of porous earthenware containing 10 per cent. sulphuric acid; this is contained within a copper vessel (represented as transparent for diagrammatic purposes in fig. 105) filled with saturated solution of copper sulphate. Each metal has a binding screw attached to it, to which wires can be fastened. The zinc rod is called the positive element, the copper the negative element. The distal ends of the wires 
attached to these are called poles or electrodes, and the pair of electrodes may be conveniently held in a special form of holder. The electrode attached to the positive element (zinc) is called the negative pole or

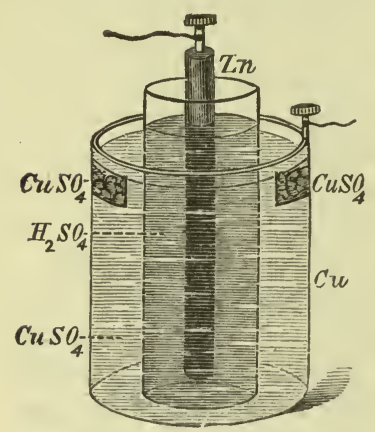

FIG. 105.-Diagram of a Daniell's kathode; that attached to the negative element (copper) is called the positive pole or anode. If now the two electrodes are connected together, an electrical, galvanic, or constant current flows from the copper to the zinc outside the battery, and from the zinc to the copper through the fluids of the battery; if the electrodes are not connected the circle is broken, and no current can flow at all. If now a nerve or muscle is laid across the two electrodes the circuit is completed, and it will be noticed at the moment of completion of the circuit the muscle enters into contraction; if the muscle is lifted off the electrodes, another contraction occurs at the moment the circuit is broken. The same thing is done more conveniently by means of a key : figs. 106 and 107 represents two common forms of key. A key is a piece of apparatus by which the current

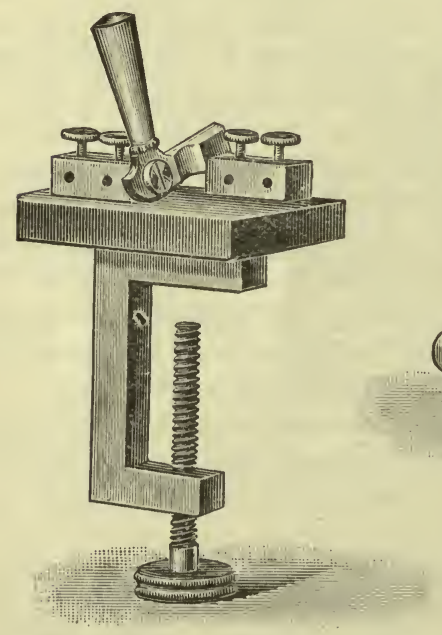

Fra. 106.-Du Bois Reymond's Key.

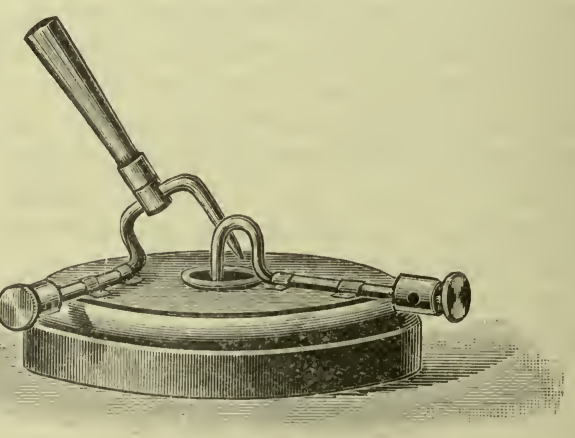

Fis. 107.-Mercury Key.

can be allowed to pass or not through the nerve or muscle laid on the electrodes. When the key is open the current is broken, as in the next figure (fig. 108); when it is closed the current is allowed to pass. The opening of the.key is called break; the closing of the key is called make. A contraction occurs only at make and break, not while the current is quietly traversing the nerve or muscle. 
But it will be seen in the Du Bois Reymond key (fig. 106) that there are four binding screws. This key is used as a bridge or short circuiting key, and for many reasons this is the best way to use it. The next diagram (fig. 109) represents this diagrammatically. The two wires from the battery go one to each side of the key; the electrodes come off one from each side of the key. When the key is open no current can get across it, and therefore all the current has to go to the electrodes with the nerve resting on them; but when the key is closed, the current is cut off from the nerve, as then practically all of it goes by the metal bridge, or short cut, back to the battery. Theoretically a small amount of current goes through the nerve; but the resistance of animal tissues to electrical currents is enormous as compared to that of metal, and the amount of electricity that flows through a conductor is inversely proportional to the resistance; the resistance in the metal bridge is so small that for all practical purposes, all the current passes through it.

Another form of electrical stimulus is the induced current, produced in an induction coil.

In a battery of which the metals are connected by a wire, we have
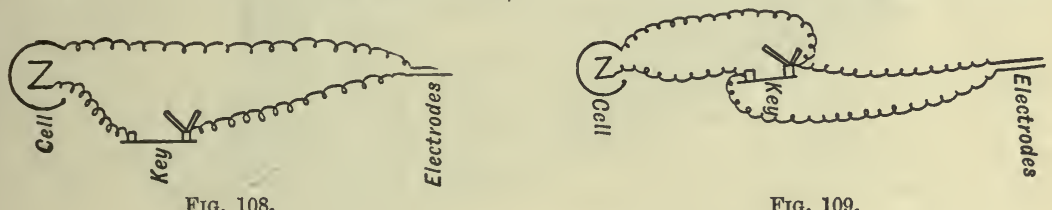

Frg. 109.

seen that the current in the wire travels from the copper to the zinc ; if we have a key on the course of this wire the current can be made or broken at will. If in the neighbourhood of this wire we have a second wire forming a complete circle, nothing whatever occurs in it while the current is flowing through the first wire, but at the instant of making or breaking the current in the first or primary wire, a momentary electrical current occurs in the secondary wire, which is called an induced current; and if the secondary wire is not a complete circle, but its two ends are connected by a nerve, this induction shock traverses the nerve and stimulates it; this causes a nervous impulse to travel to the muscle, which in consequence contracts.

If the first and second wires are coiled many times, the effect is increased, because each turn of the primary coil acts inductively on each turn of the secondary coil.

The direction of the current induced in the secondary coil is the same as that of the current in the primary coil at the break; in the opposite direction at the make. The nearer the secondary coil is to the primary, the stronger are the currents induced in the former. 
Fig. 110 represents the Du Bois Reymond coil, the one generally employed in physiological experiments; $c$ is the primary coil, and $d$ and $d^{\prime}$ its two ends, which are attached to the battery, a key being interposed for making and breaking; $g$ is the secondary coil, the two terminals of which are at its far end; to these the electrodes to the nerve are attached; the distance between the two coils, and so the strength of the induction currents, can be varied at will. It is only when the primary current is made or broken, or its intensity increased or diminished, that induction shocks occur in the secondary circuit which stimulate the nerve. When one wishes to produce a rapid succession of make and break shocks the automatic interrupter or

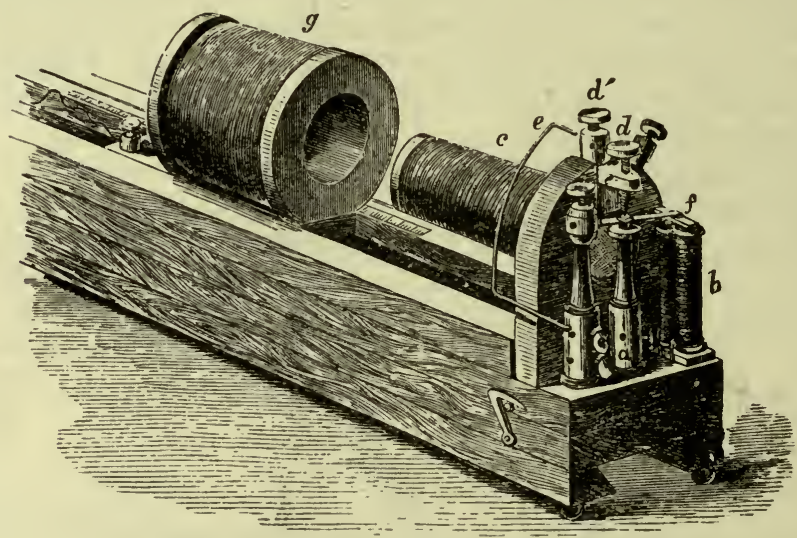

Fia. 110.-Du Bois Reymond's Induction Coil.

Wagner's hammer seen at the right-hand end of the diagram is included in the circuit.

The next thing to be noticed is that the break effects are stronger than the make effects; this is easily felt by placing the electrodes on the tongue. This is due to what is called Faraday's extra current. This is a current produced in the primary coil by the inductive influence of contiguous turns of that wire on each other; its direction is against that of the battery current at make, and so the make shock is lessened. At the break the extra current is of such short duration (because when the circuit is broken there can be no current at all) that for all practical purposes it may be considered as non-existent.

The same difference of strength occurs alternately in the repeated shocks produced by Wagner's hammer. Helmholtz, to obviate this, introduced a modification now known after him. It consists in bridging the current by a side wire, so that the current never 
entirely ceases in the primary coil, but is alternately strengthened and weakened by the rise and fall of the hammer; the strengthening corresponds to the ordinary make, and is weakened by the make extra current, which occurs in the opposite direction to the battery current; the break is also incomplete, and so it is weakened by the break extra current, which being in the same direction as the battery current impedes its disappearance.

The two next diagrams show the way the interrupter acts. We are supposed to be looking at the end of the primary coil; the battery wires are attached to the binding screws $\mathrm{A}$ and $\mathrm{E}$ (fig. 111). The current now passes to the primary coil by the

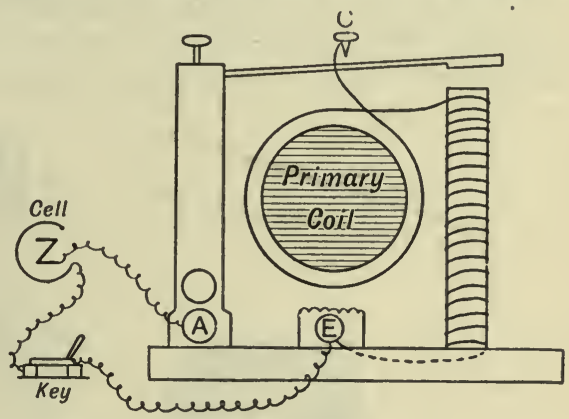

Fia. 111. pillar on the left and the spring or handle of the hammer as far as the screw (C); after going round the primary coil, one turn only of which is seen, it twists round a pillar of soft iron on the right-hand side, and then to the screw $\mathrm{E}$ and back to the battery; the result of a current going around a bar of soft iron is to make it a magnet, so it attracts the hammer, and draws the spring away from the top screw $\mathrm{C}$, and thus breaks the current; the current ceases, the soft

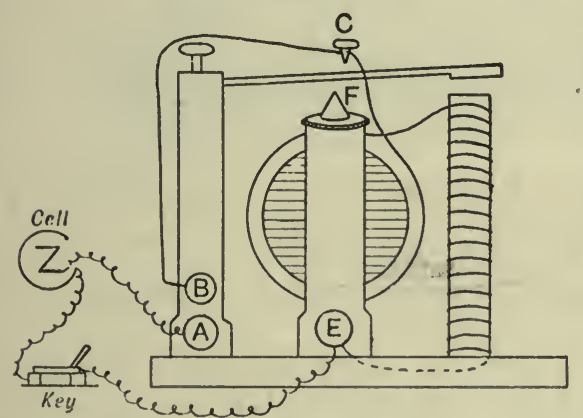

Fig. 112. iron is no longer a magnet, so it releases the hammer, and contact is restored by the spring; then the same thing starts over again, and so a succession of break and make shocks occurs alternately and automatically.

In Helmholtz's modification (fig. 112) the battery wires are connected as before. The interrupter is bridged by a wire from $\mathrm{B}$ to $\mathrm{C}$ (also shown in fig. $110, e) . \mathrm{C}$ is raised out of reach, and the lower screw $\mathrm{F}$ is brought within reach of the spring. Owing to the wire $\mathrm{BC}$, the vibration of the hammer never entirely breaks the current.

Instead of Wagner's hammer a long vibrating reed constructed on the same principle is often used. This has the advantage that the rate of vibration can be varied at will by means of a sliding 
clamp which fixes the reed, so that different lengths of it can be made to vibrate. If a long piece of reed vibrates, it does so slowly, and thus successive induction shocks at long intervals can be sent into the nerve. But if one wishes to stimulate a nerve more rapidly, the length of reed allowed to vibrate can be shortened.

In Ewald's modification of the coil there is another simple method of modifying the rate of the interrupter. But an hour spent in the laboratory with an induction coil and cell will teach the student

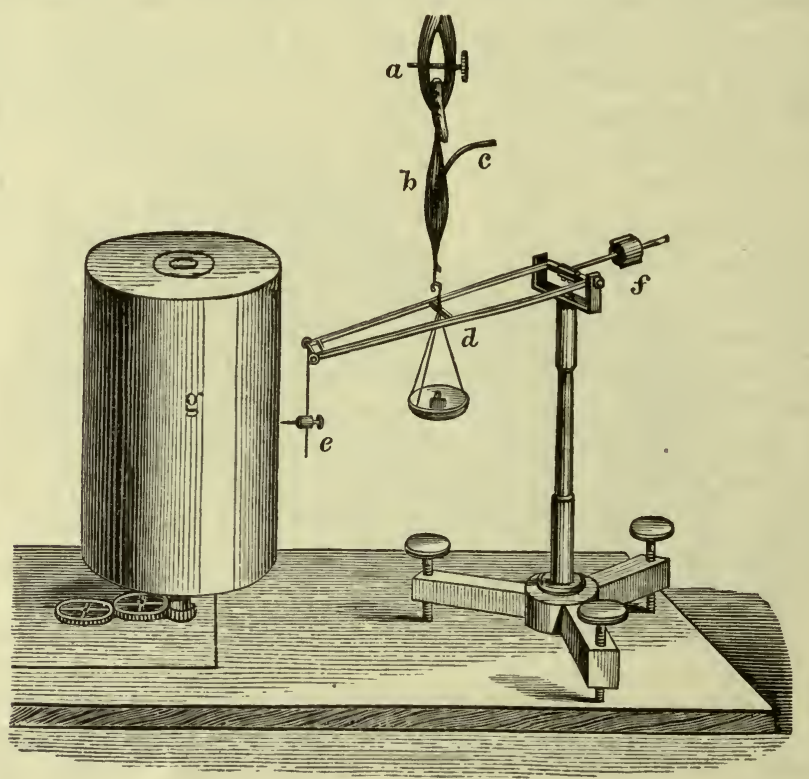

Fic. 113.-Myograph of von Helmholtz, shown in an incomplete form. $a$, Forceps for holding frog's femur; $b$, gastrocnemius ; $c$, sciatic nerve; $d$, scale-pan; $e$, marker recording on cylinder; $f$, counterpoise. (M'Kendrick.)

much more easily all these facts than any amount of reading and description.

We can pass now to the myograph. There are many different forms of this instrument. Fig. 113 shows Helmholtz's instrument.

The bony origin of the gastrocnemius is held firmly by forceps, and the tendo Achillis tied to a weighted lever; the end of the lever is provided with a writing-point such as a piece of pointed parchment; when the muscle contracts it pulls the lever up, and this movement is magnified at the end of the lever. The writing-point scratches on a piece of glazed paper covered with a layer of soot; the paper is wrapped round a cylinder. When the lever goes up the writing-point will mark an upstroke; when it falls it will mark a 
downstroke, and if the cylinder is travelling, the downstroke will be written on a different part of the paper than the upstroke; thus a muscle curve or myogram is obtained. The paper may then be removed, varnished, and preserved.

Fig. 114 shows a somewhat different arrangement.

The muscle is fixed horizontally on a piece of cork, $\mathrm{B}$, one end being fixed by a pin thrust through the knee-joint into the cork; the

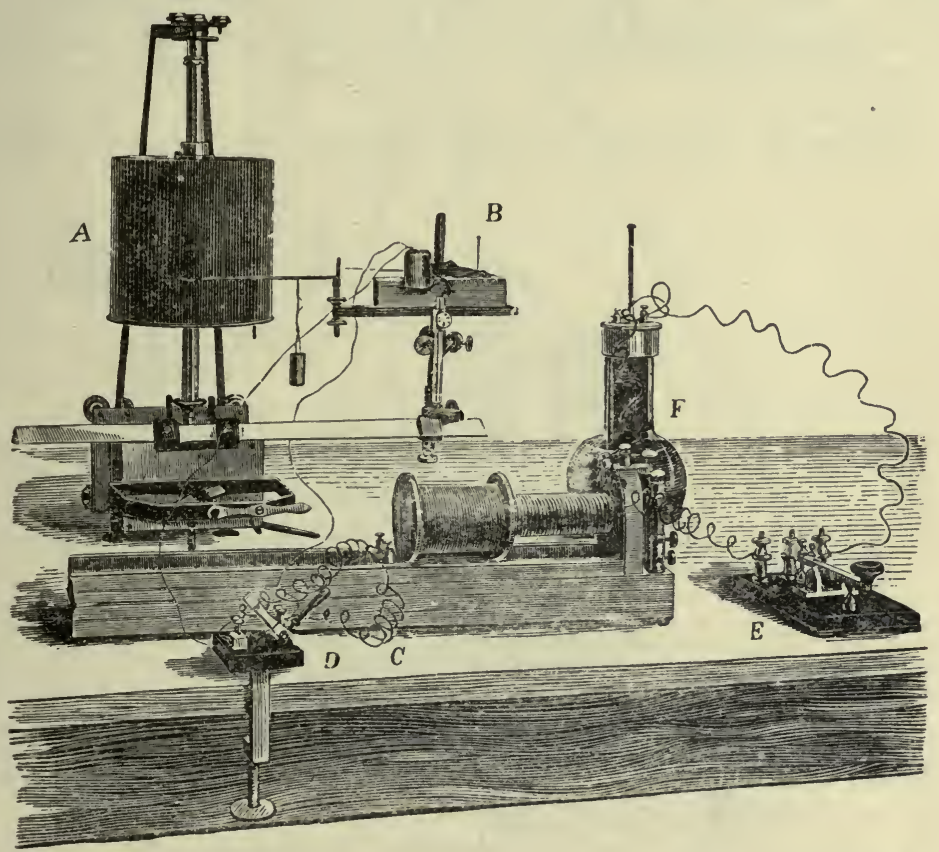

Fig. 114.-Arrangement of the apparatus necessary for recorling muscle contractions with a revolving cylinder carrying smoked paper. A, revolviug cyliuder; B, the muscle arranged upon a corkcovered board which is capable of being raised or lowered on the upright, which also can be moved along a solid triangular bar of metal attached to the base of the recording apparatus-the tendon of the gastrocnemius is attached to the writing lever, properly weighted, by a ligature. The electrodes from the secondary coil pass to the nerve-being, for the sake of convenience, first of all brought to a short-circuiting key, D (Du Bois Reymond's); C, the induction coil; F, the battery (in this fig. a bichromate one); $\mathrm{E}$, the key (Morse's) in the primary circuit.

tendo Achillis is tied to a lever which is weighted near its fulcrum: the lever is so arranged that it rests on a screw till the muscle begins to contract; the muscle therefore does not feel the weight till it begins to contract, and gives a better contraction than if it had been previously strained by the weight. This arrangement is called afterloading.

The writing surface is again a travelling cylinder tightly covered with smoked glazed paper. The rest of the apparatus shows how 
cell, coil, keys, and electrodes are applied with the object of stimulating the nerve.

The key $\mathrm{E}$ makes and breaks the primary circuit, but the effect is only felt by the muscle-nerve preparation when the short-circuiting key $\mathrm{D}$ in the secondary circuit is opened.

Instead of the key $\mathrm{E}$ it is better to have what is called a "kickover" key, which the cylinder by means of a bar projecting from it knocks over and so breaks the primary circuit during the course of a revolution. The exact position of the writing-point at the moment of break, that is the moment of excitation, can then be marked on the blackened paper.

Besides the travelling cylinder there are other forms of writing

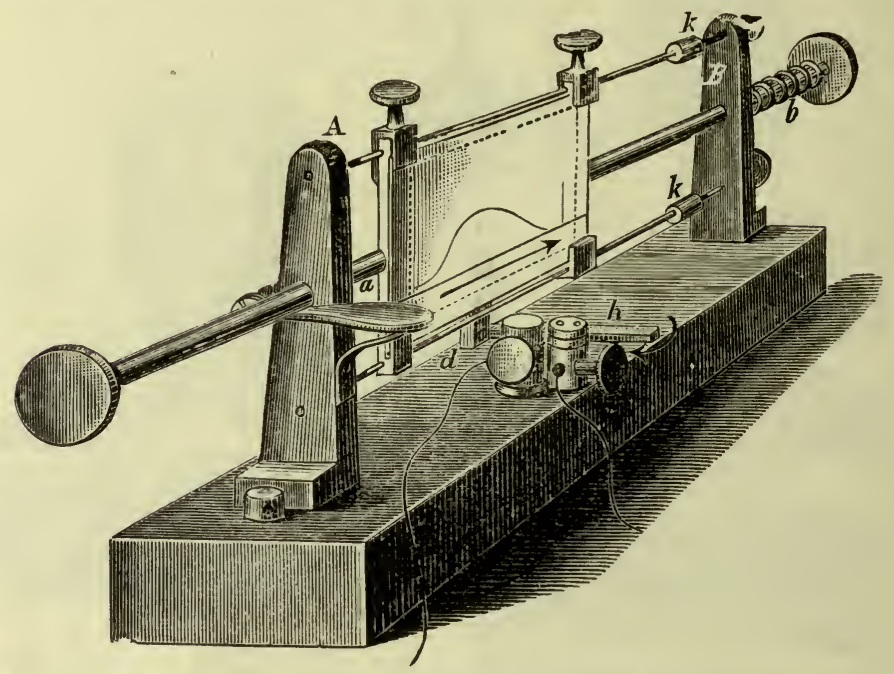

Fia. 115.-Du Bois Reymond's Spring Myograph. (M'Kendrick.)

surface. Thus fig. 115 represents the spring myograph of $\mathrm{Du}$ Bois Reymond. Here a blackened glass plate is shot along by the recoil of a spring; as it travels it kicks uver a key, and the result of this, the muscular contraction, is written on the plate.

The pendulum myograph (fig. 116) is another form. The pendulum carries a smoked glass plate upon which the writing-point of the muscle lever is made to mark. The break shock is sent into the muscle-nerve preparation by the pendulum in its swing opening a key in the primary circuit. This key is shown on an enlarged scale in $\mathrm{BC}$ (fig. 116).

To keep the preparation fresh during an experiment, it should be covered with a glass shade, the air of which is kept moist by means 
of wet blotting-paper. One form of moist chamber is shown in fig. 117.

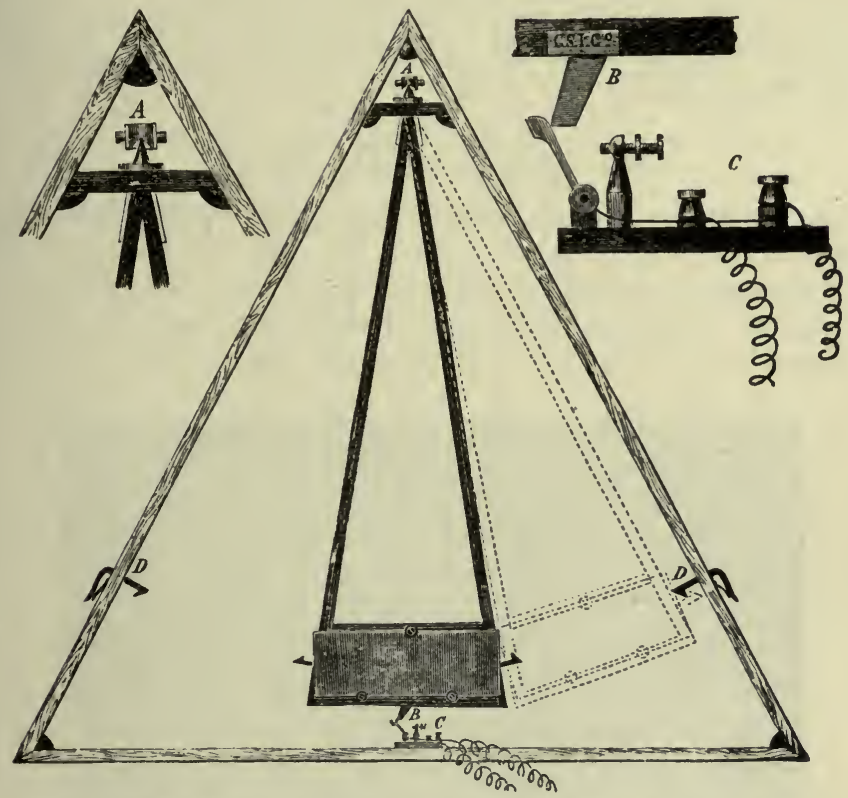

Fig. 116.-Pendulum Myograph and accessory parts (Fick's pattern). A, pivot upon wlich pendulum swings; $\mathrm{B}$, catch on lower end of myograph opening the key, C, in its swing; D, a spring-catch which retains myograph, as indicated by dotted lines, and on pressing down the handle of which the pendulum swings along the arc to $\mathrm{D}$ on the left of figure, and is caught by its spring.

The last piece of apparatus necessary is a time-marker, so that the events recorded in the myogram can be timed. The simplest

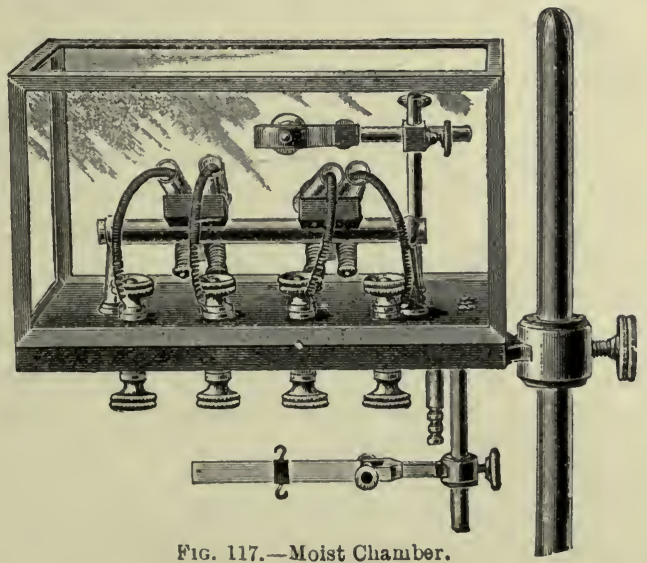


time-marker is a tuning-fork vibrating 100 times a second. This is struck, and by means of a writing-point fixed on to one of the prongs of the fork, these vibrations may be written beneath the myogram. More elaborate forms of electrical time-markers or chronographs are frequently employed.

\section{The Simple Muscle Curve.}

We can now pass on to results, and study first the result of a single instantaneous stimulus upon a muscle. This causes a single or simple muscular contraction, or, as it is often called, a twitch. The graphic record of such a contraction is called the simple muscle curve. One of these is shown in the accompanying figure (fig. 118).

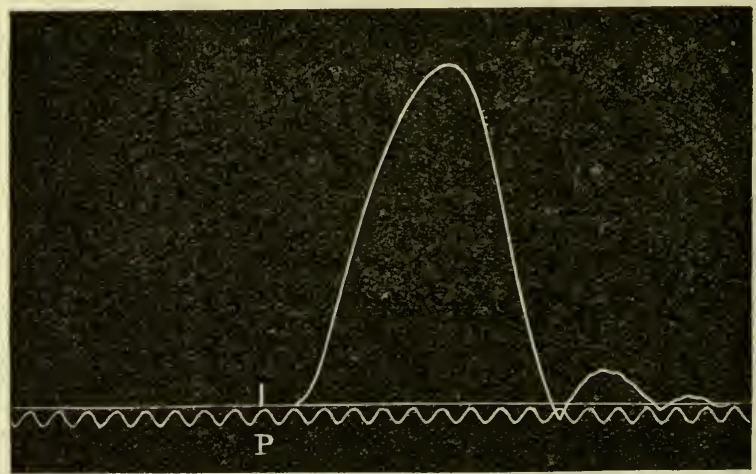

FIG. 118.-Simple muscle curve.

The muscle was stimulated by a single induction-shock, at the instant marked $\mathrm{P}$ upon the base-line. The lower wavy line is traced by a tuning-fork vibrating 100 times a second, and serves to measure the time occupied in each part of the contraction.

It will be observed that after the stimulus has been applied there is an interval before the contraction commences. This interval, termed the latent period, when measured by the tuningfork tracing is seen to be about $\frac{1}{100}$ sec. During the latent period there is no apparent change in the muscle.

The second part is the stage of contraction proper. The lever is raised by the shortening of the muscle. The contraction is at first very rapid, but then progresses more slowly to its maximum.

The next stage is the stage of elongation. After reaching its highest point, the lever descends in consequence of the elongation of the muscle. The small waves which follow the main curve are simply due to the elasticity of the muscle and recording apparatus, and are most marked when the contraction is rapid and vigorous. 
The whole contraction occupies about $\frac{1}{10}$ of a second. With regard to the latent period, it should be pointed out that if the muscle is stimulated indirectly, i.e., through its nerve, some of the apparent lost time is oceupied in the propagation of the nervous impulse along the nerve. To obtain the true latent period, this must be deducted. Then there is latency in the apparatus (friction of the lever, etc.) to be taken into account. This can be got rid of by photographing the contracting muscle, on a sensitive photographic plate travelling at an accurately-timed rate. By such means it is found that the true latent period is much shorter than was formerly supposed. It is only $\frac{1}{400}$ of a second. In red muscles it is longer.

We now come to the action of various factors in modifying the character of the simple muscle curve.

1. Influence of strength of stimulus. - A minimal stimulus is that which is just strong enough to produce a contraction. If the strength of stimulus is increased the amount of contraction as measured by the height of the curve is increased, until a certain point is reached (maximal stimulus), beyond which increase in the stimulus produces no increase in the amount of contraction. The latent period is shorter with a strong than with a weak stimulus.

2. Influence of load.-Increase of load decreases the amount of contraction, until at last a weight is reached which the muscle is unable to lift. The latent period is somewhat longer with a heavy load than with a light one.

3. Influence of fatigus. - This can be very well illustrated by letting the muscle write a curve with every revolution of the cylinder, until it ceases to contract at all. Fig. 119 shows the result. At first the contractions improve, each being a little higher than the preceding; this is known as the beneficial cffect of contraction, and the graphic record is called a staircase. Then the contractions get less and less. But what is most noticeable is that the curves are much more prolonged; the latent period gets longer; the period of contraction gets longer; and the period of relaxation gets very much longer; this condition is known as contracture, so that the original base-line is not reached by the time the next stimulus arrives. In the last stages of fatigue, contracture passes off. Contracture is often absent in fatigue of mammalian muscle.

4. Effect of temperature.-Cold at first increases the height of contraction, then diminishes it; otherwise the effect is very like that of fatigue, increasing the duration of all stages of the curve.

Moderate warmth increases the height and diminishes the duration of all stages of the curve, latent period included. This may be readily shown by dropping salt solution * at different tempera-

* Physiological saline solution used for bathing living tissue is a 0.9 per cent. solution of sodium chloride in ordinary tap water. 


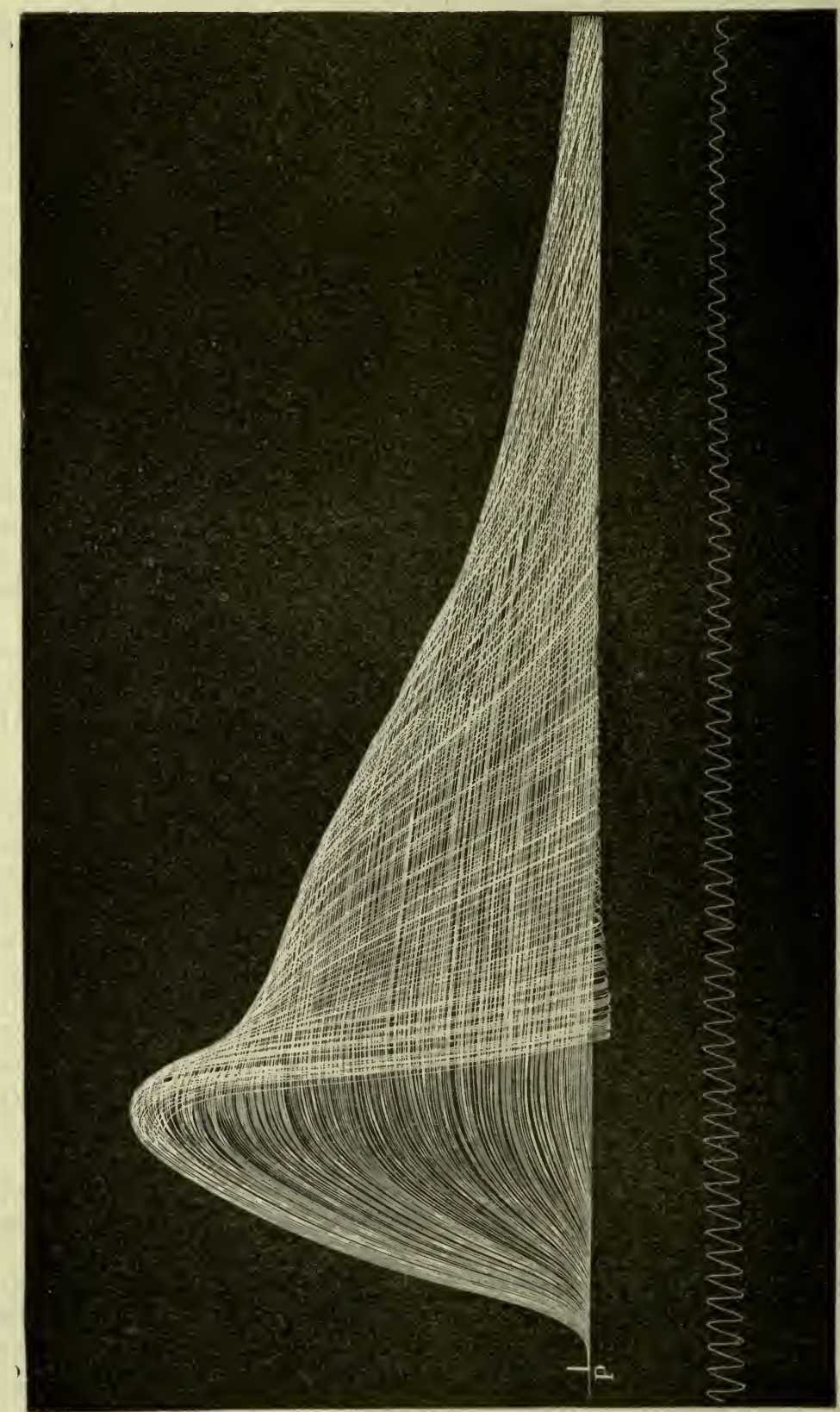


tures on to the muscle before taking its curve. Fig. 120 shows the result of such an experiment. Too great heat (above $42^{\circ} \mathrm{C}$.) induces heat rigor, due to the coagulation of the muscle proteins.

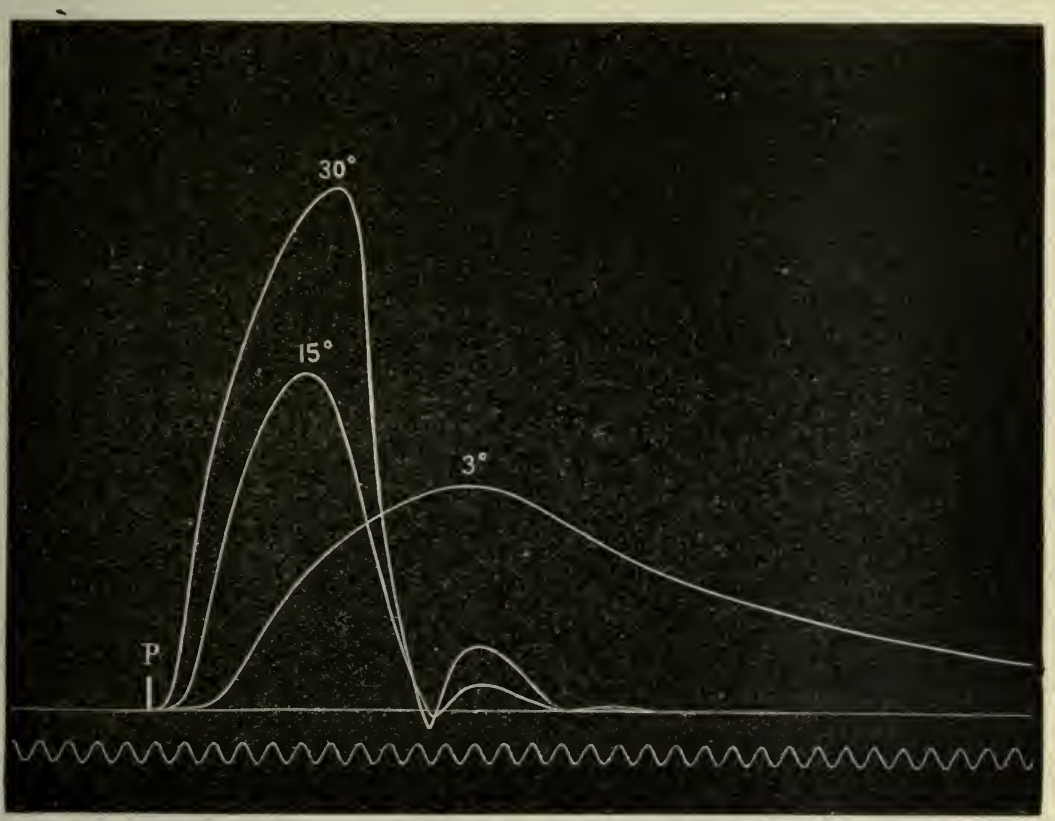

FIG. 120.-Effect of temperature on the simple muscie curve. The variuus temperatures are marked on the curves. $\mathbf{P}$ is the puint of stmulation; and the time-tracing again indicates hundredths of a second.

5. Effect of veratrine.-If this is injected into the frog before the muscle-nerve preparation is made, tho very remarkable result seen

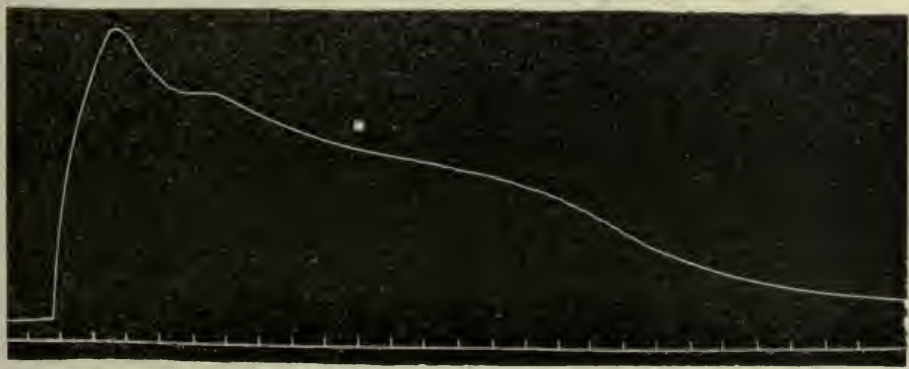

F10. 121.-Veratrine curve, taken on a very slowly travelling cylinder; the time-tracing indicates seconds, not hundredths of a second as in the previous diagrams.

in fig. 121 is produced on stimulation; there is an enormous 
prolongation of the period of relaxation; marked by a secondary rise, and sometimes by tremors. The second rise has received various explanations, none of which can be regarded as satisfactory. After repeated stimulation the veratrine effect passes off, but returns after a period of rest.

\section{The Muscle-Wave.}

The first part of a muscle which contracts is the part where the nerve-fibres enter; the nerve impulses, however, are so rapidly carried to all the fibres that for practical purposes they all contract together. But in a nerveless muscle, that is one rendered physiologically nerveless by curare, if one end of the muscle is stimulated, the contraction travels as a wave of thickening to the other end of the muscle, and the rate of propagation of this wave can be recorded graphically. The next figure (fig. 122) represents one of the numerous methods

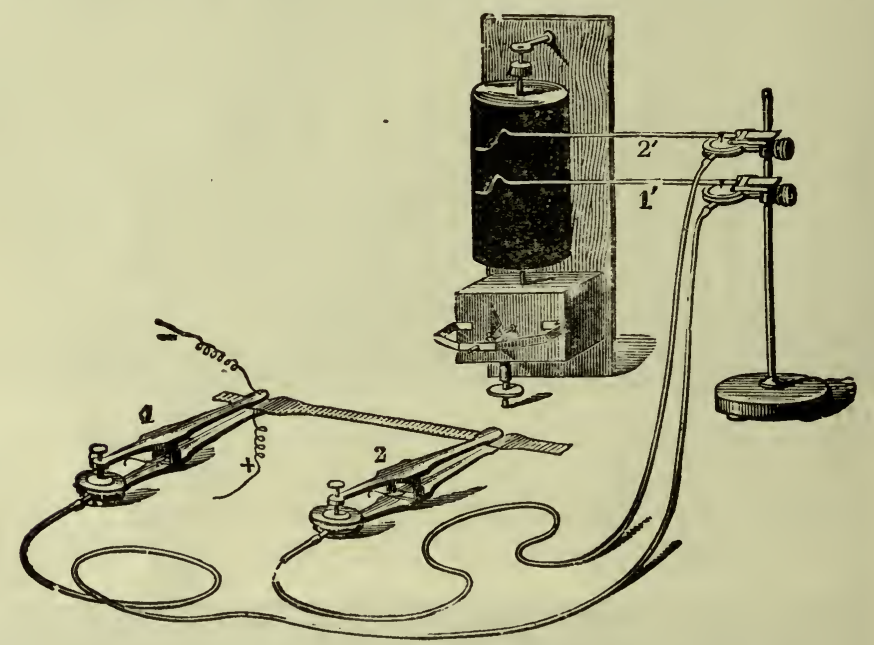

Fig. 122.-Arrangement for tracing the muscle-wave. (M'Kendrick.)

that have been devised for this purpose. A muscle with long parallel fibres, like the sartorius, is taken; it is represented diagrammatically in the figure. It is stimulated at the end, where the two wires, + and -, are placed; it is grasped in two places by pincers, which are opened by the wave of thickening; the opening of the first pair of pincers (1) presses on a drum or tambour connected to a second tambour with a recording lever $\left(1^{\prime}\right)$, and this lever goes up first; the lever $\left(2^{\prime}\right)$ of the tambour connected with the second pair of pincers (2) goes up later. If the length of muscle between the pairs of pincers is measured, and by a time-tracing the delay in the raising 
of the second lever is ascertained, we have the arithmetical data for calculating the rate of propagation of the muscle-wave. It is about 3 metres per second in frog's muscle, but is hastened by warmth and delayed by cold and fatigue.

\section{The Effect of Two successive Stimuli.}

If a second stimulus follows the first stimulus at a sufficient interval of time, each will cause a twitch and two simple muscle curves will be written (fig. 123, A); the second is a little bigger than the first (beneficial effect of contraction). If the second stimulus arrives before the muscle has finished contracting under the influence of the first, a second curve will be added to the first, as shown in fig. 123, B. This is called superposition, or summation of effects.

If the two stimuli are in such close succession that the second occurs during the latent period of the first, the result will differ according as the stimuli are maximal or submaximal. If they are maximal, the second stimulus is without effect; but if submaximal, the two stimuli are added together, and though producing a simple muscle curve, produce one which is bigger than either would have produced separately. This is called summation of stimuli (fig. 123, C).

\section{Effect of More than Two Stimuli.}

If a succession of stimuli are sent into a muscle, or its nerve, the results obtained depend on the rate at which the stimuli follow one another. If the time intervals between the stimuli are sufficiently great, each stimulus will produce a simple muscular contraction, and one records a succession of twitches, and the beneficial effect of previous action is exhibited in what is known as a staircase (fig. 124, $\mathrm{A}$ and $\mathrm{B})$.

If the induction shocks follow each other more rapidly, the effect is a continuation of the superposition curve already described in connection with two successive stimuli. Just as a second stimulus adds its curve to that written as the result of the first, so a third stimulus superposes its effect on the second; a fourth on the third, and so on. Each successive increment is, however, smaller than the preceding, and at last the muscle remains at a maximum contraction, till it begins to relax from fatigue.

A succession of stimuli may be sent into the nerve of a nervemuscle preparation by means of the Wagner's hammer of a coil, or the vibrating reed previously mentioned (p. 92). This method of stimulation is called faradisation. Fig. 124, C to F, shows the kind of tracings one obtains. The number of contractions corresponds to the number of stimulations; the condition of prolonged contraction 
A

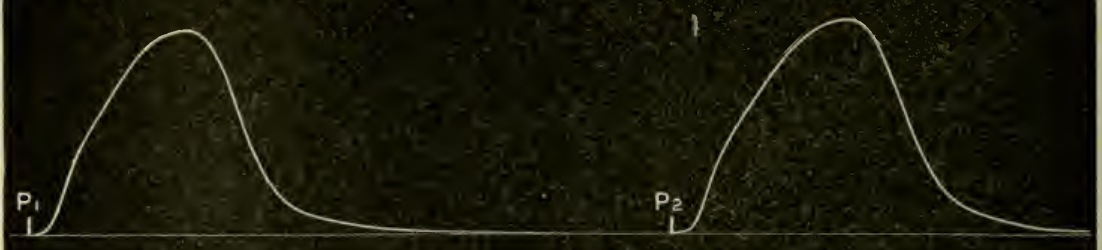

B

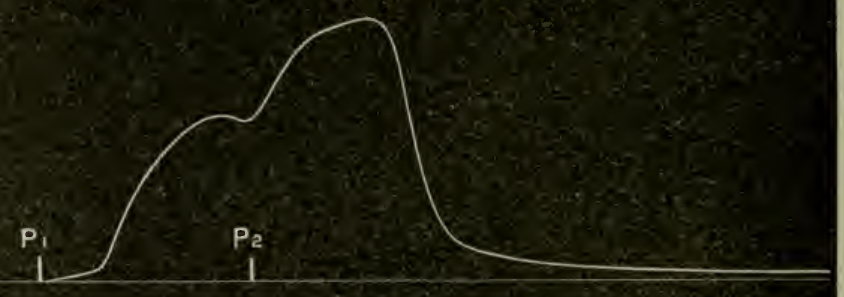

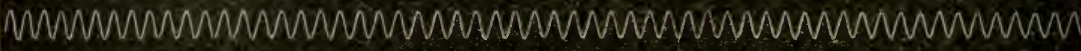

C

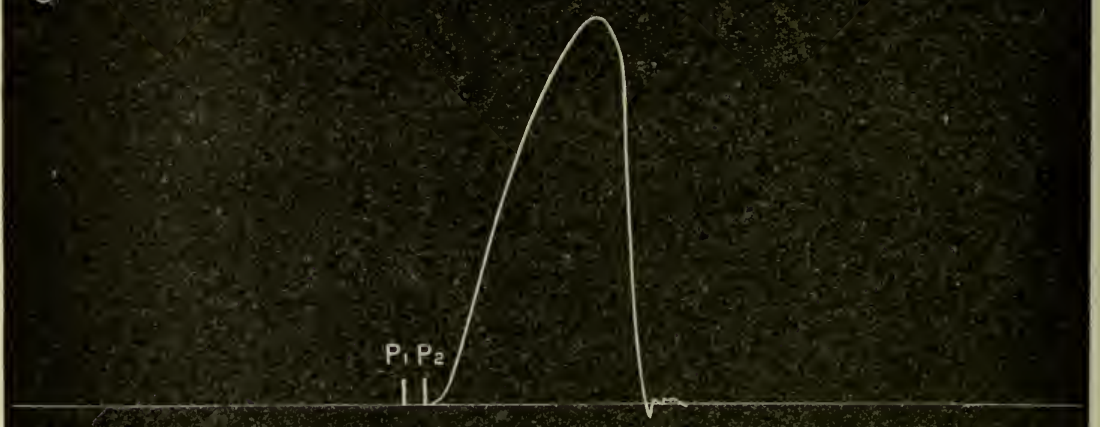

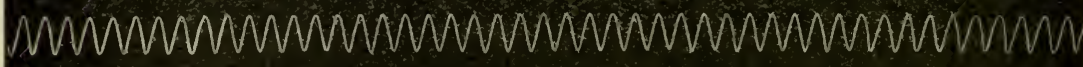

FIG. 123.-Etfect of two successive excitations. The two points of excitation $\left(P_{1}\right.$ and $\left.P_{2}\right)$ are marked in each case on the base-line. In $\mathbf{A}, \mathrm{P}_{1}$ and $\mathrm{P}_{2}$ are sufficiently far a part to give separate curves. In $B$ they are nearer together, and superposition is seen. In $C$ they are sufficiently near to give summation of stimuli. Submaximal stimnli were used throughout; and the time-tracing -in each case shows hundredths of a second. 


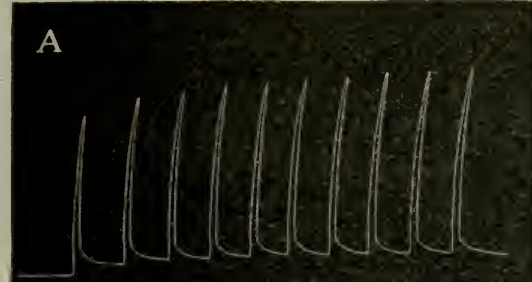

\section{B}

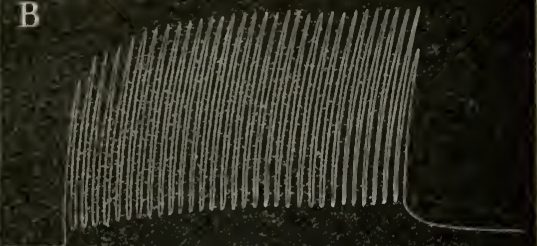

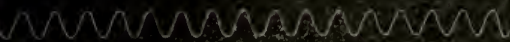
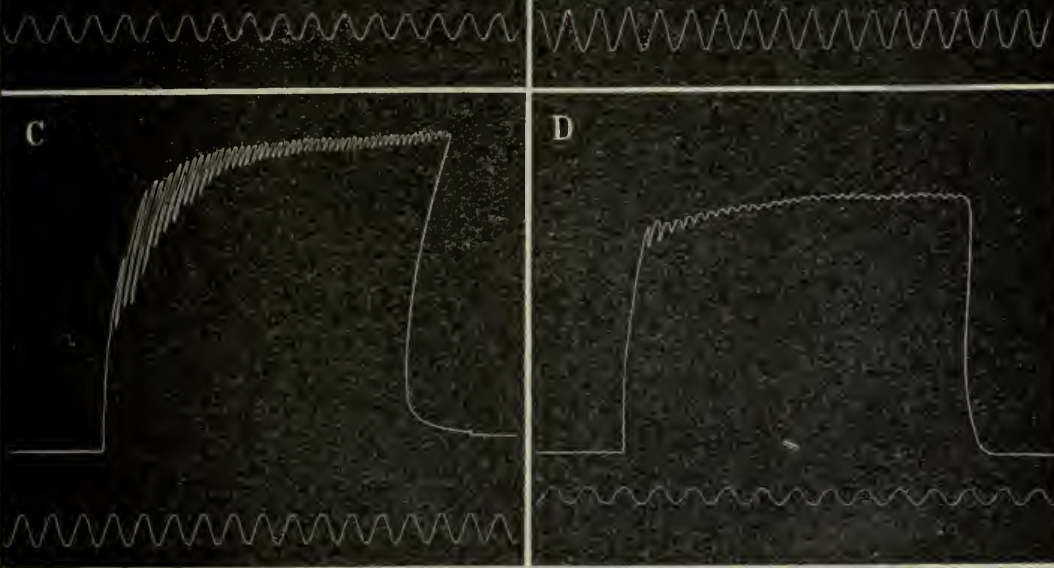

D

E

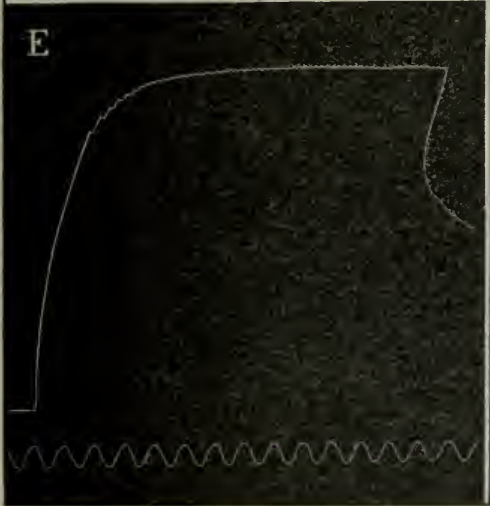

F
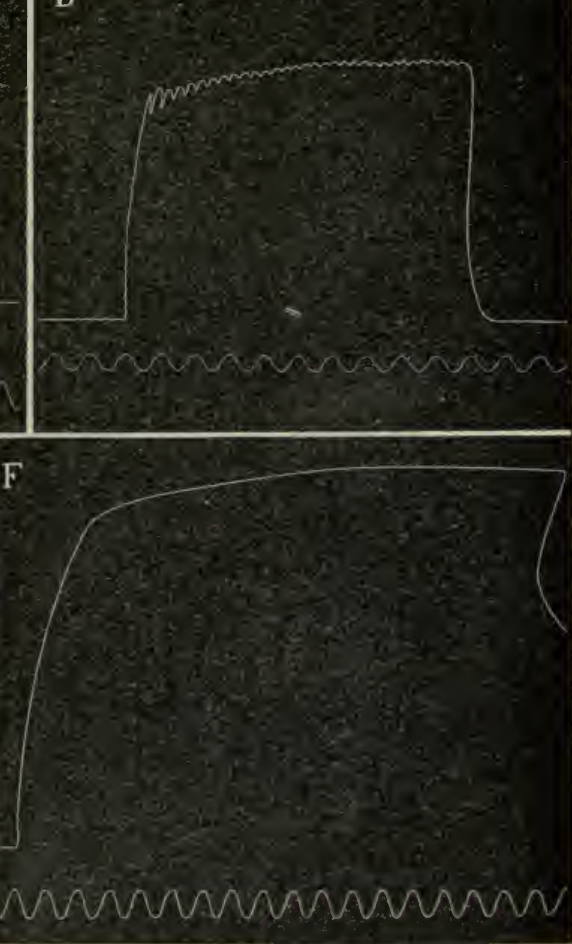

F11. 124.-Composition of tetanus. These six tracings were obtained on a slowly moving drum from a frog's gastrocnemius, which was excited by a succession of induction slocks. By a mechanical contrivance the rate of the vibrating hammer which interrupted the primary circuit of the inductorium could be easily varied; and the rate of the hammer was increased from about 1 per second in $\mathbf{A}$ to 30 per second in $\mathbf{F}$. In $\mathbf{A}$, separate twitches are seen; in $\mathbf{B}$, the rate was still insufficient to cause fusion; in both $\mathrm{A}$ and $\mathrm{B}$, the staircase effect is well seen. In $\mathrm{C}$ and $\mathrm{D}$, the rate was sufficiently great to cause incomplete tetanus; in $\mathrm{E}$, tetanus was nearly complite, and in $\mathrm{F}$ it is complete. The time-tracing in each case shows half-seconds. 
so produced, the muscle never relaxing completely between the individual contractions of which it is made up, is called tetanus: incomplete tetanus, when the individual contractions are discernible (fig. 124, C, D, and E); complete tetanus, as in fig. 124, F, when the contractions are so rapid as to be completely fused to form a continuous line without waves.

The rate of faradisation necessary to cause complete tetanus varies a good deal; for frog's muscle it averages 15 to 20 per second; for the pale muscles of the rabbit, 20 per second; for the more slowly contracting red muscles of the same animal, 10 per second; and for the extremely slowly contracting muscles of the tortoise 2 per second is enough. With fatigue as the period of relaxation becomes prolonged, the rate necessary to produce complete tetanus is diminished.

\section{Voluntary Tetanus.}

We have seen that voluntary muscles under the influence of artificial stimuli may be made to contract in two ways: a single excitation causes a single contraction; a rapid series of excitations causes a series of contractions which fuse to form tetanus.

We now come to the important question, in which of these two ways does voluntary muscle ordinarily contract in the body? The answer to this is, that voluntary contraction resembles, though it is not absolutely identical with, tetanus artificially produced. It is certainly never a twitch. The nerve-cells from which the motor fibres originate do not possess the power of sending isolated impulses to the muscles; they send a series of impulses which result in a muscular tetanus, or voluntary tetanus, as it may conveniently be termed.

If a stethoscope is placed over any contracting muscle of the human body, such as the biceps, a low sound is heard. The tone of this sound, which was investigated by Wollaston, and later by Helmholtz, corresponds to thirty-six vilurations per second; this was regarded as the first overtone of a note of eighteen vibrations per second, and for a long time 18 per second was believed to be the rate of voluntary tetanus.

The so-called "muscle sound" is, however, no indication of the rate of muscular vibration. Any irregular sound of low intensity will produce the same note; it is, in fact, the natural resonance-tone of the meinbrana tympani of the ear, and, therefore, selected by the organ of hearing when we listen to any irregular mixture of faint, low-pitched tones and noises.

A much more certain indication of the rate of voluntary tetanus is obtained by the graphic method. The myographs hitherto described are obviously inapplicable to the investigation of such a 
problem in man. The instrument employed is termed a transmission myograph. The next figure shows the recording part of the apparatus.

It is called a Marey's Tambour. It consists of a drum, on the membrane of which is a metallic disc fastened near one end of a lever, the far extremity of which carries a writing point. The interior of the drum is connected by an india-rubber tube (seen at the righthand end of the drawing) to a second tambour called the receiving

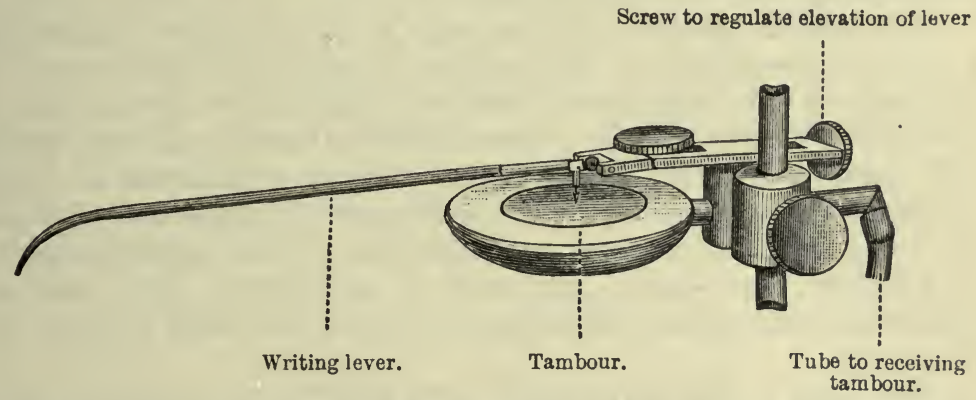

Fig. 125.-Marey's Tambour, to which the movement of the column of air in the first tambour is conducted by a tube, and from which it is communicated by the lever to a revolving cylinder, so that the tracing of the movement is obtained.

tambour, in which the writing lever is absent. Now if the receiving tambour is held in the hand, and the thumb presses on the metallic disc on the surface of its membrane, the air within it is set into vibrations of the same rate as those occurring in the thumb muscles; and these are propagated to the recording tambour and are written in a magnified form by the end of the lever on a recording travelling surface.

The tracing obtained is that of an incomplete tetanus, which by a

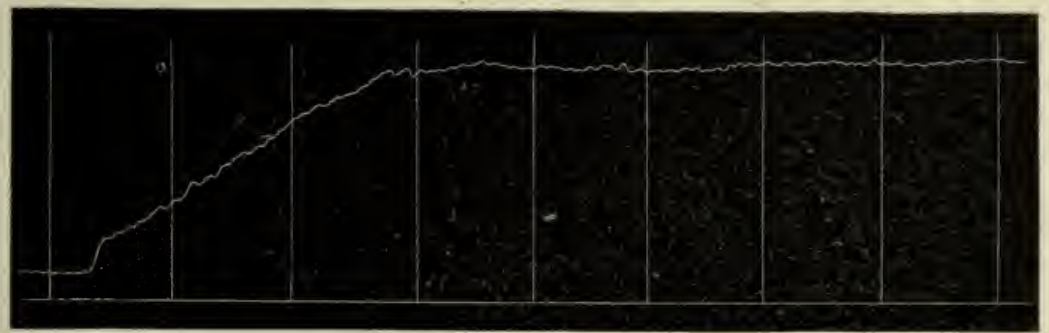

FiG. 126.-Tracing of a voluntary contraction of the opponens pollicis on a slowly moving drum, by means of the transmission myograph. The vertical lines indicate seconds. (Schäfor, Canney, and Tunstall.)

time-marker can be seen to be made up of 10 to 12 vibrations a second. A typical tracing is shown in the above figure (fig. 126). 
In some diseases these tremors are much increased, as in the clonic convulsions of epilepsy, or those produced by strychnine poisoning, but the rate is the same.

Similar tracings can be obtained in an anæsthetised animal by strapping the receiving tambour on the surface of a muscle, and causing it to contract by stimulating the brain or spinal cord. The rate of stimulation makes no difference; however slow or fast the stimuli occur, the nerve-cells of the central ncrvous system give out impulses at their own normal rate.

The same is seen in a reflex action. If a tracing is taken from a frog's gastrocnemius, the muscle being left in connection with the rest of the body, its tendon only being severed and tied to a lever, and if the sciatic nerve of the other leg is cut through, and the end attached to the spinal cord is stimulated, an impulse passes up to the cells of the cord, and is then reflected down to the gastrocnemius, under observation. The impulse has thus to traverse nerve-cells; the rate of stimulation then makes no difference; the reflex contraction occurs at the same rate, 10 or 12 per second.

But now a difficulty arises; if a twitch only occupics $\frac{1}{10}$ of a second, there would be time for ten complete twitches in a second; they would not fuse to form even an incomplete tetanus. There must be some means by which each individual contraction can bo lengthened till it fuses with the next contraction, or else each component part of the contraction is itself composite; recent experiments by Piper indicate that the latter explanation is the true one; he found that each wave of the curve obtained by the graphic method is really itself due to fusion of contractions occurring at a more rapid rate. The method he employed was to count the number of electrical variations which accompany a voluntary contraction, on the assumption that each fundamental unit of the contraction has an electrical change as its concomitant. This can be accomplished by the use of a very delicate galvanometer (the string galvanometer, p. 121), the movements of which can be photographed on a rapidly travelling plate. The number of electrical variations is then found to be a fixed one for each muscle, but to vary in different muscles. Various spinal and cranial motor centres have thus different rhythms, and of those hitherto studied the cells of the motor fibres of the fifth cranial nerve have the highest rate of discharge, 86 to 100 per second. In muscles supplied by spinal nerves the rate is lower, 40 to 60 .

Lever Systems.-The arrangement of the muscles, tendons, and bones presents examples of the three systems of levers which will be known to anyone who has studied mechanics; the student of anatomy will have no difficulty in finding examples of all three systems in 
the body. What is most striking is that the majority of cases are levers of the third kind, in which there is a loss of the mechanical power of a lever, though a gain in the rapidity and extent of the movement.

Most muscular acts involve the action of several muscles, often of many muscles. The acts of walking and running are examples of very complicated muscular actions in which it is necessary not only that many muscles should take part, but also must do so in their proper order and in due relation to the action of auxiliary and antagonistic muscles. This harmony in a complicated muscular action is called coördination.

By the device of taking instantaneous photographs at rapidly repeated intervals during a muscular act, the details of different modes of locomotion in man and other animals have been very thoroughly worked out. With this branch of research the name of Prof. Marey is intimately associated. 


\section{CHAPTER X}

\section{EXTENSIBILITY, ELASTICITY, AND WORK OF MUSCLE}

MUSCLE is both extensible and elastic. It is stretched by a weight, that is, it possesses extensibility; when the weight is taken off, it returns to its original length, that is, it possesses elasticity. The two properties do not necessarily go together; thus a piece of putty is very extensible, but it is not elastic; a piece of steel or a ball of ivory are only slightly extensible, but after the stretching force has been removed they return to their original size and shape very perfectly.

A substance is said to be strongly elastic, when it offers a great resistance to external forces; steel and ivory are strongly elastic.

A substance is said to be perfectly elastic, when its return to its original shape is absolute; again steel and ivory may be quoted as examples.

Muscle is very extensible, i.e., it is easily stretched; it is feebly elastic, i.e., it opposes no great resistance to external force; it is, however, perfectly elastic; that is, it returns to its original shape very exactly after stretching. This is true in the case of living muscle within the body, but after very great stretching even in the body, and still more so after removal from the body, when it begins to undergo degenerative changes culminating in death, its elasticity is less perfect.

The cohesion of muscular tissue is less than that of tendon. E. Weber stated that a frog's muscle one centimetre square in transverse section will support a weight of a kilogramme (over 2 lbs.) without rupture, but this diminishes as the muscle gradually dies.

The extensibility of any material may be studied and recorded by measuring the increase of length which occurs when that material is loaded with different weights. In Helmholtz's myograph (fig. 113), different weights may be placed in the scale-pan beneath the muscle, and the increase of length recorded on a stationary blackened cylinder by the downward movement of the writing-point; the cylinder may 
then be moved on a short distance, more weight added, and the additional increase of length similarly recorded, and so on for a succession of weights.

If this experiment is done with some non-living substance, like a steel spring or a piece of india-rubber, instead of a living muscle, it is found that the amount of stretching is proportional to the weight; a weight $=2$ produces an extension twice as great as that produced by a weight $=1$; in this way one obtains a tracing like that seen on the left hand of figure 127, and the dotted line drawn through the lowest points of the extensions is a straight one.

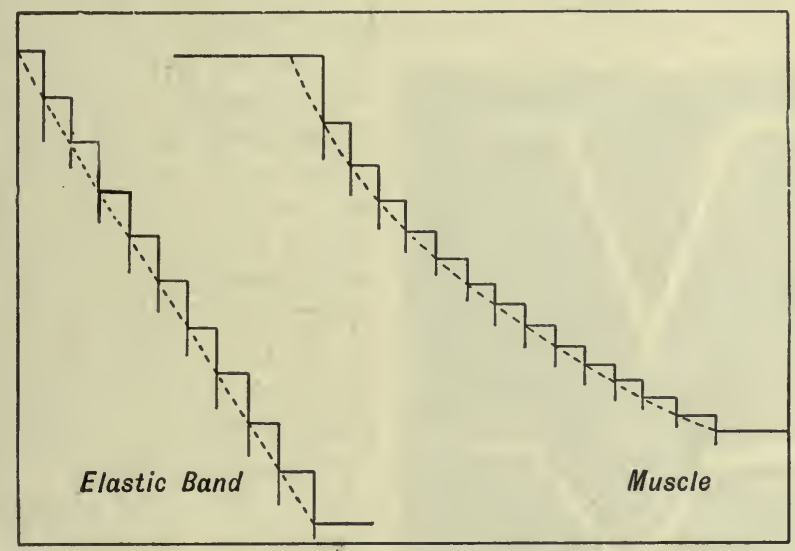

Fig. 127.-(After Waller.)

With muscle, however, this is different; each successive addition of the same weight produces smaller and smaller increments of extension, and the dotted line obtained is a curve.

A continuous curve of extensibility may be obtained by placing a gradually and steadily increasing force beneath the muscle instead of a succession of weights added at intervals. The most convenient way of doing this is to use a steel spring, which is gradually and steadily extended; and the writing-point connected to the muscle inscribes its excursion on a slowly moving cylinder. If, then, after the muscle has been stretched, the steel spring is gradually and steadily relaxed, the muscle retracts and again writes a curve now in the reverse direction, until it regains its original length.* But in muscles removed from the body, unless they are very slightly loaded, the return to the original length is never complete; the muscle is

* A mathematical examination of these curves shows that they are not rectangular hyperbolas as they were once considered. They are very variable in form, and cannot be identified with any known mathematical curve. 
permanently longer to a slight extent, which varies with the amount of the previous loading.

If the muscle is slowly loaded and slowly unloaded, the curvature of its tracing is much more marked than if the experiment is done rapidly.

The folluwing three tracings are reproduced from some obtained by Dr Brodie. In the method used, the records are not complicated by the curve of a lever, but the movement was simply magnified by a beam of light falling on a mirror attached to the end of the muscle, and reflected on to a travelling photographic plate. Each tracing is to be read from right to left; the first one (A) shows the result of stretching a steel spring by a steadily increasing force; the end of

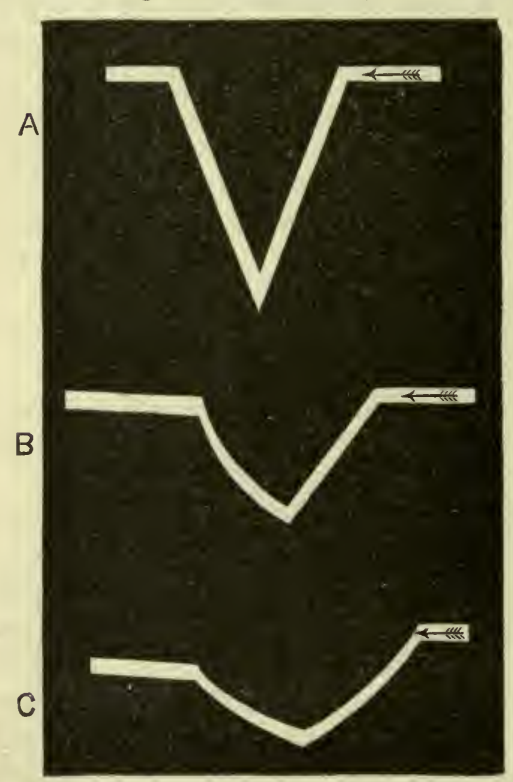

FI(i. 12S.-Curves of extensibility. (Brodie.) the spring grets lower and lower, and describes a straight line; at the apex of the tracing unloading began and went on steadily till the spring once more regained its initial length. The upstroke, like the downstroke, is a straight line. In $\mathrm{B}$ and $\mathrm{C}$ muscles were used; it will be noticed that the muscle does not regain its original length after unloading is completed, and the upward tendency of the tracing beyond this point represents afterretraction. In $\mathrm{B}$, the extension was applied rapidly, the tracing is almost a straight line; in $C$, the extension was brought about more slowly, and the tracing is a curve; in both cases the tracing of the period of unloading shows more curvature.

This introduces us to what is called after-extension and afterretraction. That is to say, after a muscle is weighted there is an immediate elongation, followed by a gradual elongation which continues for some time; or if a muscle has been weighted and is then unloaded there is an immediate slackening, followed by a gradual after-retraction.

This may be shown by looking at the graphic records shown in the next diagram. It will be noticed that the extension is greatest when the muscle is in a contracted condition, and smallest when it is dead (in rigor). In fatigue the after-extension is very marked, and the return after unloading very imperfect. 
We may now give the results of an actual experiment; a muscle was loaded with successive weights of 50, 100, 150, etc., grammes, and its length carefully measured in centimetres.

\begin{tabular}{|c|c|c|c|c|c|c|}
\hline Load extension & $\begin{array}{l}50 \\
3 \cdot 2\end{array}$ & $\begin{array}{l}100 \\
6\end{array}$ & $\begin{array}{l}150 \\
8 \\
?\end{array}$ & $\begin{array}{l}200 \\
9 \cdot 5 \\
1 \cdot 5\end{array}$ & $\begin{array}{c}250 \\
10 \\
0\end{array}$ & $\begin{array}{c}300 \\
10 \cdot 3 \\
0.3\end{array}$ \\
\hline Increment of extension & & $2 \cdot 8$ & 2 & $1 \cdot 5$ & $0 \cdot \bar{z}$ & \\
\hline
\end{tabular}

Figure 129 shows that the contracted muscle is more extensible than the uncontracted muscle. This may be still further illustrated by an example given on the following page in the form of a diagram.

The thick lines represent the contracted muscle, the thin ones the uncontracted. It is represented as being stretched by different weights indicated along the top line; and the lengths under the influence of these weights are separated by equal distances. Thus A C represents the length of the uncontracted muscle, A B of the contracted muscle when unloaded. $\mathrm{A}^{\prime} \mathrm{C}^{\prime}$ and $A^{\prime} B^{\prime}$ the same under the influence of a weight of 50 grammes, and so on.

The curve connecting the ends of the lengths of the contracted muscle falls faster than that obtained from the uncontracted one, until at the point $\mathrm{P}$ under the influence of a weight of 250 grammes, the two curves meet; that is to say, 250 grammes is the weight which the muscle was just unable to lift. Suppose a muscle has to lift the weight of 200 grammes, it begins with a length $A^{\prime \prime} C^{\prime \prime}$, but when it contracts it has is length $A^{\prime \prime} B^{\prime \prime}$, that is, it has contracted a distance of $\mathrm{B}^{\prime \prime} \mathrm{C}^{\prime \prime}$, which is very small; when it has to lift a less weight it shortens more,

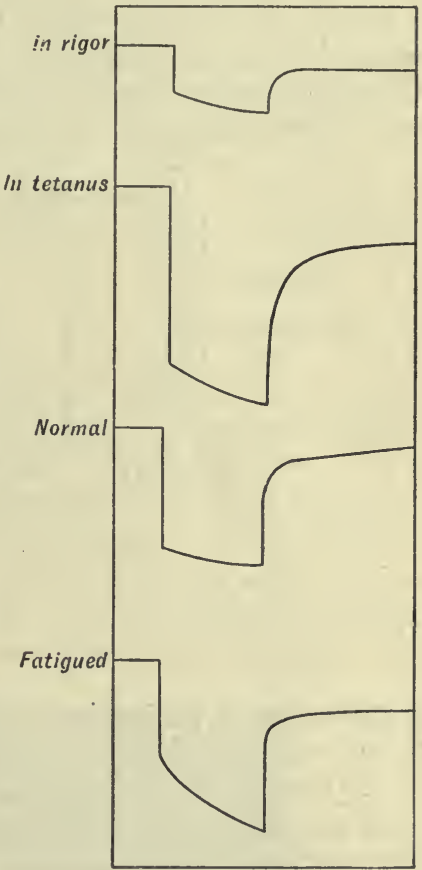

Fia. 129.- Extensibility of muscle in diflerent states; tested by 50 grammes applied for short yeriods. Tracings to be read from left to right. (After Waller.) when a greater weight it shortens less; till when it shortens least it lifts the greatest weight.

This experiment illustrates tho general truth that when a muscle is contracted it is more extensible. At the point $\mathrm{P}$ the energy tencling to shorten the muscle (its contractile power) is exactly equal to the energy tending to lengthen it against its elastic force. Thus wo have the apparent paradox at this point, that a muscle when 
contracted has exactly the same length as when uncontracted; but this is a matter of everyday experience; if one tries to lift a weight beyond one's strength, one fails to raise it, but nevertheless one's muscles have been contracting in the effort; they have not contracted in the restricted sense of becoming shorter, but that is not the only change a muscle undergoes when it contracts; the other changes, electrical, thermal, chemical, etc., have taken place, as evidenced in one's own person by the fact that the individual has got warm in his efforts, or may even feel fatigue afterwards.

But the paradox does not end here, for if diagram 130 is again looked at, it will be seen that beyond the point $\mathrm{P}$ the two curves cross; in other words, the muscle may even elongate, due to increase of extensibility, when it contracts. This is known after its discoverer as Weber's paradox. The increase of extensibility of muscle during

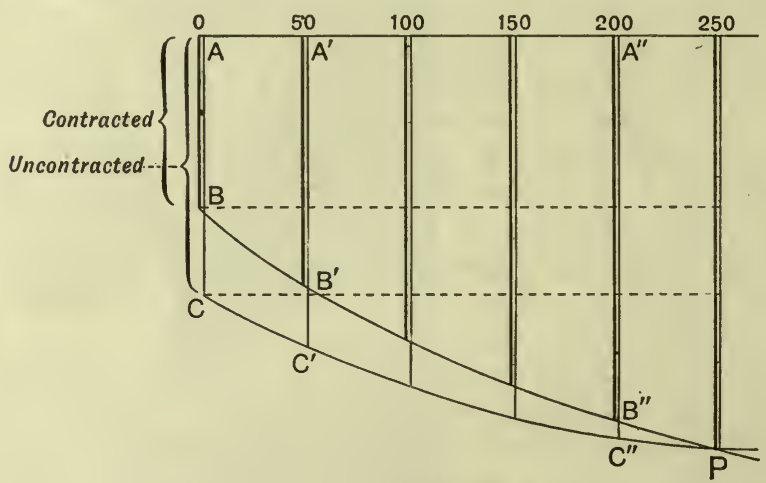

Fig. 130.

contraction is protective and tends to prevent rupture in efforts to raise heavy woights.

Influence of Temperature on Extensibility.-If a piece of iced india-rubber is taken and stretched by a weight, its retractility when the weight is removed is very small. If, now, when the weight is on it, it is warmed at one point, as by placing the hand on it, its retractility is increased and it contracts, raising the weight. Some physiologists have considered that muscular contraction can be explained in this way; they have supposed that the heat formed in muscular contraction acts like warmth as applied to india-rubber. This view is, however, incorrect. It is much more probable that there is no causal relationship between the temperature-change and the extensibility-change which occur when muscle contracts; both are simultaneously produced by the stimulus.

Moreover, the influence of heat on muscle is by no means the same as that on india-rubber. This influence is not invariable, and 
at certain temperatures near the freezing-point, and under the influence of certain weights, actual elongation may occur when the temperature is raised.

\section{Muscular Tonus.}

In the living animal, muscles are more or less stretched, but never taut between their two attachments. They are in a state of tonicity or tonus, and when divided they contract and the two parts separate. Thus a muscle, even at rest, is in a favourable condition to contract without losing time or energy in taking in slack.

Muscular tonus is under the control of the nervous system (on the reflex character of this control, see later, under Tendon Reflexes); the muscles lengthen when their nerves are divided, or when they are rendered physiologically nerveless by curare. Besides the nervous system, the state of muscular nutrition dependent on a due supply of healthy blood must also be reckoned as important in maintaining muscular tonus.

\section{Work of Muscle.}

The question of muscular work is intimately associated with that of elasticity. In a technical sense, work (W) is the product of the load $(l)$ and the height $(k)$ to which it is raised. $\mathrm{W}=l \times h$.

Thus in fig. 130, when the muscle is unloaded the work done is nil: $\mathrm{W}=\mathrm{BC} \times \mathrm{O}=\mathrm{O}$. When the load is 250 , again the work done is nil, because then $h=0$. With the load $50, \mathrm{~W}=\mathrm{B}^{\prime} \mathrm{C}^{\prime} \times 50$.

If the height is measured in feet and the load in pounds, work is expressed in terms of foot-pounds. If the height is measured in

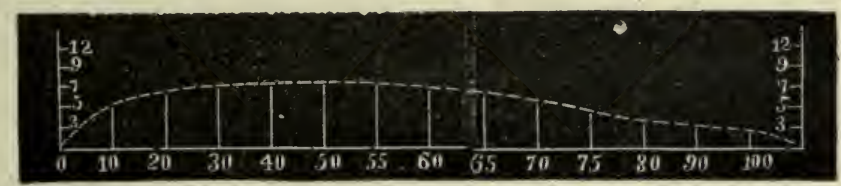

FIG. 131.-Diagram to show the mode of measuring muscle work. (M'Kendrick.)

millimetres or metres, and the load in grammes, the work is expressed in gramme-millimetres or gramme-metres respectively.

This may be shown diagrammatically by marking on a horizontal base line or abscissa, distances proportionate to different weights, and vertical lines (ordinates) drawn through these represent the height to which they are lifted (see fig. 131).

In the diagram (fig. 131) the figures along the base line represent grammes, and the figures along the vertical line represent millimetres. The work done as indicated by the first line is $10 \times 5=50$ 
gramme-millimetres, the next $20 \times 6=120$ gramme-millimetres, and so on, while the last on the right, $100 \times 3=300$ gramme-millimetres. It is thus seen that the height of a muscle-curve is no measure of the work done by the muscle unless the weight lifted is taken into account as well.

The following figures are taken from an actual experiment done with the frog's gastrocnemius (Weber):-

\begin{tabular}{|c|c|c|c|c|c|}
\hline \multicolumn{2}{|c|}{ Weight lifted. } & \multicolumn{2}{|c|}{ Height. } & \multicolumn{2}{|c|}{ Work done. } \\
\hline \multicolumn{2}{|c|}{5 grammes } & $27 \cdot 6$ & imetres & 138 & -millimetres \\
\hline 15 & , & & , & 376 & , , \\
\hline & ," & $11 \cdot 45$ & ", & 286 & ," \\
\hline 30 & ", & $7 \cdot 3$ & , & 219 & ,", \\
\hline
\end{tabular}

The work increases with the weight up to a certain maximum, after which a diminution occurs, more or less rapidly, according as the muscle is fatigued.

Similar experiments have been made in human beings, weights being lifted by the calf muscles, or elbow muscles, leverage being

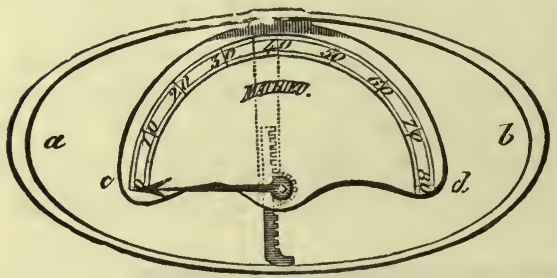

FIc. 132.-Dynamometer. allowed for. In the higher animals the energy so obtained compared with the frog is about twice as great for the same volume of muscular tissue.

Fig. 132 represents a common form of dynamometer for clinical use, employed in testing the muscles of the arms and hands. It is squeezed by the hand, and an index represents kilogrammes of pressure.

The muscle, regarded as a machine, is sometimes compared to artificial machines like a steam-engine. A steam-engine is supplied with fuel, the latent energy of which is transformed into work and heat. The carbon of the coal unites with oxygen to form carbonic acid, and it is in this process of combustion or oxidation that heat and work are liberated. Although the analogy between muscle and a steam-engine is by no means an exact one (see more fully p. 397), nevertheless it may stand for our present purpose. In a steamengine a good deal of fuel is consumed, but there is great economy in the consumption of the living muscular material. Take the work done by a gramme (about 15 grains) of muscle in raising a weight of 4 grammes to the height of 4 metres (about 13 feet); in 
doing this work probably less than a thousandth part of the muscle has been consumed.

Next let us consider the relationship between the work and the heat produced. An ordinary locomotive wastes about 96 per cent. of its available energy as heat, only 4 per cent. being represented as work. In the best triple-expansion steam-engine the work done rises to 12.5 per cent. of the total energy.

In muscle, various experimenters give different numbers. Thus, Fick calculated that 33 per cent. of the mechanical energy is available as work; later he found this estimate too high, and stated the number as 25; Chauveau gives 12 to 15 ; M'Kendrick 17. Thus inuscle is a little more economical than the best steam-engines; but the muscle has this great advantage over any engine, for the heat it produces is not wasted, but is used for keeping up the body temperature, the fall of which below a certain point would lead to death, not only of the muscles but of the body generally.

So far wc have been speaking as though the only active phase of muscular contraction is the period of shortening. It is, however, extremely probable that lengthening is also an active process. This was originally mooted by Fick, who pointed out that the fall of a muscle lever during the relaxation period is of variable speed, and is obviously not due to the passive elongation of the muscle by gravity ; the way in which this part of the curve is varied by such agencies as temperature, and drugs like veratrine, also indicates that relaxation is an independent process.

Isotonic and Isometric Curves. - If, in recording the contraction of a muscle, the load is applied vertically under the muscle, its pull upon the muscle varies during the successive stages of a single contraction, owing to the inertia of the load. In order to avoid this variation in tension, it is usual to apply the weight at a point close to the fulcrum of the recording lever, so that when the lever is raised, the weight remains practically stationary, and thus the error due to its inertia is avoided. In order to apply the necessary tension to the muscle, the weight hanging on the lever must be increased in the ratio of the distances of the muscle and weight from the fulcrum. A twitch recorded under such circumstances is called isotonic, i.e., one in which the tension remains constant throughout. If, on the other hand, the muscle is fixed at both ends, and then excited, the resulting activity expresses itself in a phase of increasing tension followed by one of decreasing tension. If the alterations of tension are recorded, we obtain what is called an isometric curve. This curve is obtained by making the muscle pull against a spring which is so strong that the muscle can only move it to a very slight extent. This slight movement is then highly magnified. The curve thus obtained resembles in its main features an isotonic contraction, but its maximum is reached earlier, and it returns to the zero position sooner. The flat top of the isometric curve described by the earlier observers was due to the imperfection of the instruments employed. The tracings of muscle curves given in previous illustrations (see figs. 118 to 124) were obtained by the isotonic method, but it is probable that the isometric curve is a more faithful record of the variations in the intensity of the contraction process than that yielded by the isotonic method. The momentum or swing of a light lever such as is used for obtaining isotonic curves will no doubt account for the extra upward movement it executes. The whole matter has been keenly discussed, and the foregoing view is that expressed by Kaiser. Schenk, on the other hand, maintains what appears to be an improbable idea, that there are really two kinds of change in muscle, which account for the difference obtained by the two methods. 


\section{CHAPTER XI}

THE ELECTRICAL PHENOMENA OF MUSCLE

WE have seen that the chemical processes occurring in muscular contraction lead to a transformation of energy into work and heat. These changes are accompanied by electrical disturbances also.

The history of animal electricity forms one of the most fascinating of chapters in physiological discovery. It dates from 1786, when Galvani made his first observations. Galvani was Professor of Anatomy and Physiology at the University of Bologna, and his wife was one day preparing some frog's legs for dinner, when she noticed that the apparently dead legs became convulsed when sparks were emitted from a frictional electrical machine which stood by. Galvani then wished to try the effect of lightning and atmospheric electricity on animal tissues. So he hung up some frogs' legs to the iron trelliswork round the roof of his house by means of copper hooks, and saw that they contracted whenever the wind blew them against the iron. He imagined this to be due to electricity secreted by the animal tissues, and this new principle was called Galvanism. But all his contemporaries did not agree with this idea, and most prominent among his opponents was Volta, Professor of Physics at another Italian university, Pavia. He showed that the muscular contractions were not due to animal electricity, but to artificial electricity produced by contact with different metals.

The controversy was a keen and lengthy one, and was terminated by the death of Galvani in 1798. Before he died, however, he gave to the world the experiment known as "contraction without metals," which we shall study presently, and which conclusively proved the existence of animal electricity. Volta, however, never believed in it. In his hand electricity took a physical turn, and the year after Galvani's death he invented the Voltaic pile, the progenitor of our modern batteries. Volta was right in maintaining that galvanism can be produced independently of animals, but wrong in denying that electrical currents could be obtained from animal tissues. Galvani was right in maintaining the existence of animal electricity, but 
wrong in supposing that the contact of dissimilar metals with tissues proved his point.

This conclusion has been arrived at by certain new methods of investigation. In 1820 Oersted discovered electro-magnetism: that is, when a galvanic current passes along a wire near a magnetic needle, the needle is deflected one way or the other, according to the direction of the current. This led to the invention of the astatic needle and the galvanometer, an instrument by which very weak electrical currents can be detected. For a long time the subject of animal electricity, however, fell largely into disrepute, because of the quackery that grew up around it. It is not entirely free from this evil nowadays; but the scientific investigation of the subject has led to a considerable increase of knowledge, and among the names of modern physiologists associated with it must be particularly mentioned those of $\mathrm{Du}$ Bois Reymond and Hermann.

Before we can study these it is, however, necessary that we should understand the instruments employed.

The Galvanometer.-The essential part of a galvanometer is a magnetic needle suspended by a delicate thread; a wire coils round

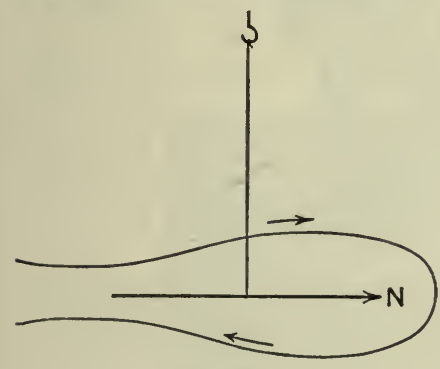

Fig. 133.

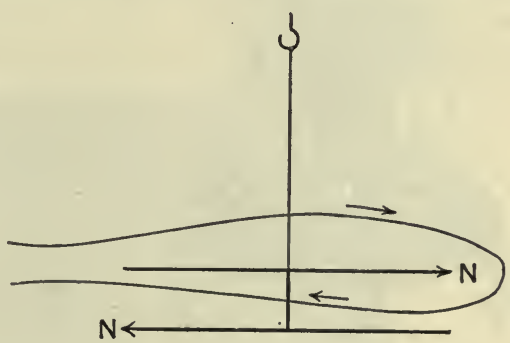

FrG. 134

it; and if a current flows through the wire, the needle is deflected. Suppose a man to be swimming with the current with his face to the needle, the north-seeking pole is turned to the left hand. But such a simple instrument as that shown in fig. 133 would not detect the feeble currents obtained from animal tissues. It is necessary to increase the delicacy of the apparatus, and this is done in several ways. In the first place, the needle must be rendered astatic, that is, independent of the earth's magnetism. The simplest way of doing this is to fix two needles together (as shown in fig. 134), the north pole of one pointing the same way as the south pole of the other. The current is led over one needle and then over the other; the effect is to produce a deflection in each in the same direction, and so the sensitiveness of the instrument is doubled. If now the wire is coiled not only once, but twice or more in the same position, each coil has its effect 
on the needles; the multiplication of the effect of a weak current in this way is accomplished in actual galvanometers by many hundreds of turns of fine wire.

Fig. 135 illustrates the best galvanometer of this type: that of

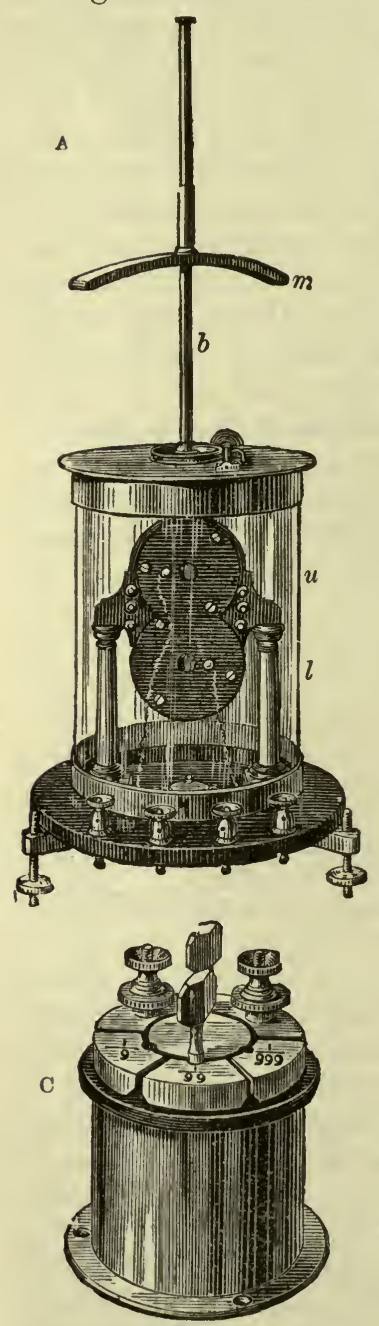

Sir William Thomson (afterwards Lord Kelvin). It is called a reflecting galvanometer, because the observer does not actually watch the moving needle, but a spot of light reflected on to a scale from a little mirror, which is attached to and moves with the needle. A very small movement of the needle is rendered evident, because the movement of the spot of light being, as it were, at the end of a long lever-namely, the beam of light, magnifies it.

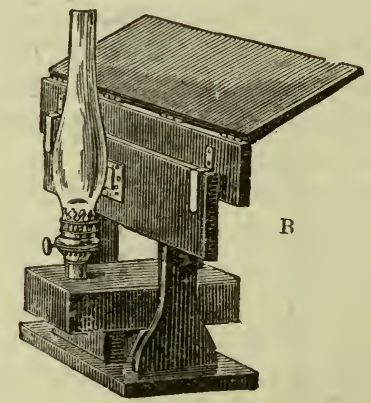

Fir. 135.-Reflecting galvanometer. (Thomson.) A. The galvanometer consists of two systenis of small astatic needles suspended by a fine hair from a support, so that each set of needles is within a coil of fine insulated copper wire, that forming the lower coil being wound in an opposite direction to the upper. Attached to the upper set of needles is a small mirror about $\frac{1}{4}$ inch in diameter; the light from the lamp at B is thrown upon this little mirror, and is reflected upon the scale on the other side of $B$, not shown in the figure. The coils $u l$ are arranged upon brass uprights, and their ends are carried to the binding screws. The whole apparatus is placed upon a vulcanite plate capable of being levelled by the screw supports, and is covered by a brassbound glass shade, the cover of which is also of brass, and supports a brass rod $b$, on which moves a weak curved magnet $m$. $\mathbf{C}$ is the shunt by means of which the amount of the current sent into the galvanometer may be regulated. When in use the scale is placed about three feet from the galvanometer, which is arranged east and west, the lamp is lighted, the mirror is made to swing, and the light from the lamp is adjusted to fall upon it, and it is then regulated until the reflected spot of light from it falls upon the zero of the scale. The wires from the non-polarisable electrodes touching the muscle are attached to the outer binding screws of the galvanometer, a key intervening for short circuiting, or if a portion only of the current is to pass into the galvanometer, the shunt should intervene as well with the appropriate plug in. When a current passes into the galvanometer the needles and, with them, the mirror, are turned to the right or left according to the direction of the current. The amount of the deflection of the needle is marked on the scale by the spot of light travelling along it.

Non-polarisable Electrodes,-If a galvanometer is connected 
with a muscle by wires which touch the muscle, electrical currents are obtained in the circuit which are set up by the contact of metal with muscle. The currents so obtained form no evidence of electromotive force in the muscle itself. It is therefore necessary that the wires from the galvanometer should have interposed between them and the muscle some form of electrodes which are non-polarisable. Fig. 136 shows one of the earliest non-polarisable electrodes of $\mathrm{Du}$ Bois Reymond. It consists of a zinc trough on a vulcanite base. The inner surface of the trough is amalgamated and nearly filled with a saturated solution of zinc sulphate. In the trough is placed a cushion of blotting-paper, which

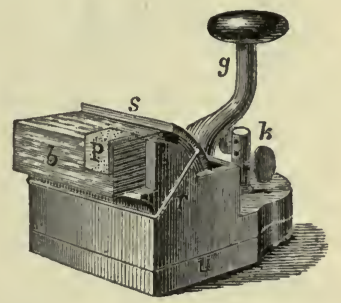

FiG. 136.-Non-polarisable elec. trode of Du Bois Reymond. (M'Kendrick.) projects over the edge of the trough; on it there is a pad of china clay or kaolin, moistened with physiological salt solution $(0.9$ per cent. sodium chloride); on this pad one end of the muscle rests. The binding screw $(k)$ connects the instrument to the galvanometer; the other end, or some other part of the same muscle, is connected by another non-polarisable electrode in the same way to the other side of the galvanometer. If there is any electrical difference of

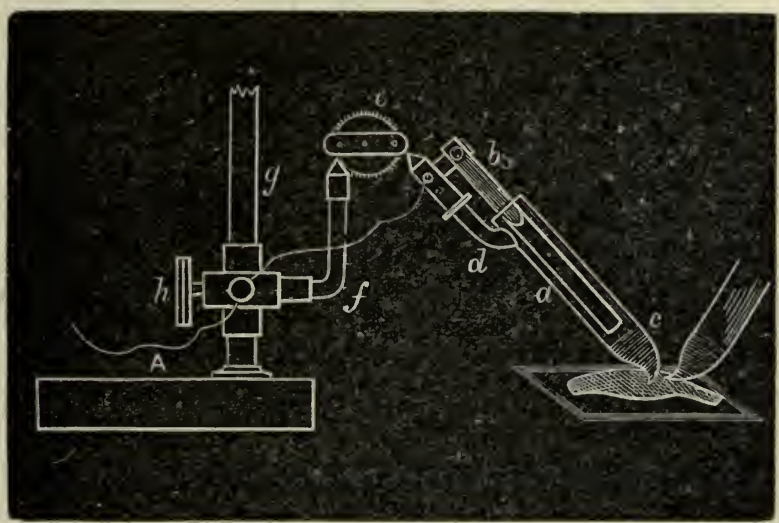

FIG. 137.-Diagram of Du Bois Reymond's non-polarisable:electrodes. $a$, Glass tube filled with a saturated solution of zinc sulphate, in the end, $c$, of which is china clay drawn out to a point; the clay is moistened with physiological salt solution; in the solution of zinc sulphate a well amalgamated zinc rod is immersed and connected, by means of the wire $\mathbf{A}$, with the galvanometer. The remainder of the apparatus is simply for convenience of application. The muscle and the end of the second electrode are to the right of the figure.

potential (that is, difference in amount of positive or negative electricity) between the two parts of the muscle thus led off, there will be a swing of the galvanometer needle; the galvanometer detects the existence and direction of any current that occurs. 
Fig. 137 shows a more conveniont form of non-polarisable electrodes.

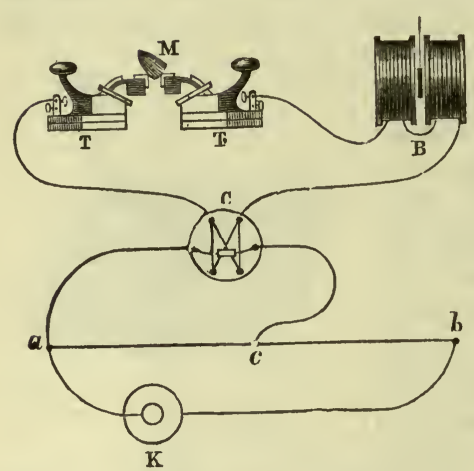

Fia. 138.-Arrangement for measuring the electromotive force of muscle.

In order to measure the strength (electromotive force) of such currents, the mere amount of swing of the needle is only a very rough indication, and in accurate work the arrangement shown in fig. 138 must be used. The electromotive force is usually measured in terms of a standard Daniell cell. The two surfaces of the muscle (M) are led off to a galvanometer (B); the needle swings, and then a fraction of a Daniell cell is introduced in the reverse direction so as to neutralise the muscle current, and bring back the needle to rest. From the Daniell cell K, wires pass to the ends $a, b$ of a long platinum wire of high resistance, called the compensator; $c$ is a slider on this wire; $a$ and $c$ are connected to the galvanometer, the commutator $\mathrm{C}$ enabling the observer to ensure that the current from the Daniell passes in the opposite direction to that produced by the muscle. If the slider $c$ is placed at the end $b$ of the compensator, the whole strength of the Daniell will be sent through the galvanometer and will more than
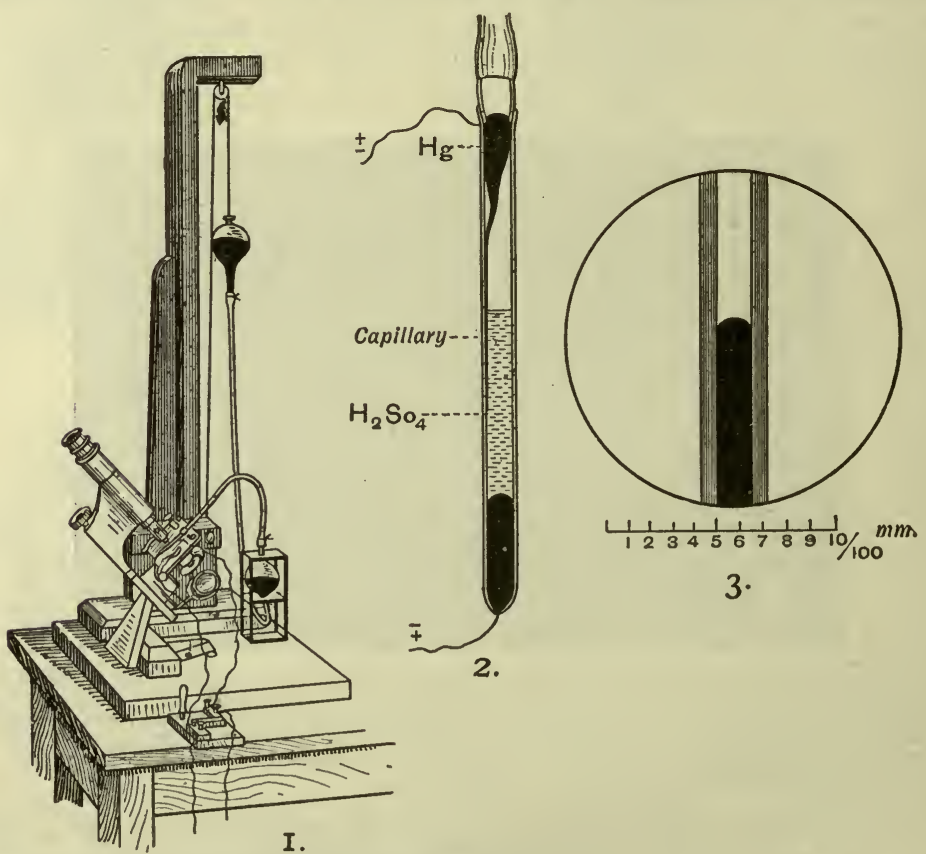

3 .

Fig. 139.-Lippmann's Capillary Electrometer. (After Waller.)

1. Pressure apparatus and microscope on stand of which the capillary tube is fixed. 2. Capillary tube, fixed in outer tube containing 10 per cent. sulphuric acid; the platinum
wires are also shown.

3. Capillary and column of mercury as seen in the field of the microscope. 
neutralise the muscle current; if $c$ is half way between $a$ and $b$, half the Daniell's strength will be sent in; but this is also too much; $a c$ will be found to be only quite a small fraction of $a b$; and this fraction will correspond to a proportional fraction of the electromotive force of the Daniell cell.

Lippmann's Capillary Electrometer.-This instrument is often used instead of the galvanometer. It consists of a glass tube drawn out at one end to a fine capillary and filled with mercury. It is connected to an apparatus by which the pressure on this mercury can be lowered or increased. The open capillary tube is enclosed within another tube filled with 10 per cent. sulphuric acid. Two platinum wires fused through the glass, pass respectively into the mercury and the acid, and the other ends of these wires are connected by electrodes to two portions of the surface of a muscle. The capillary tube is observed by a microscope (see fig. 139). The surface of the mercury is in a state of tension which is easily increased or diminished by variations of electrical potential, and the mercury moves in the direction of the negative pole.

If the shadow of the mercurial column is thrown upon a travelling sensitive photographic plate, photographs are obtained which show the electrical variations in a living tissue in a graphic manner. The instrument is exceedingly sensitive, and its indications are practically instantaneous. The instrument has been much used in investigating the electrical changes of the heart, as will be more fully discussed when we are considering that organ.

The String Galvanometer.-This is even more sensitive than the capillary electrometer, and we owe its introduction to Professor Einthoven of Leiden. In the ordinary galvanometer, as we have seen, the current passes through a fixed coil of wire, and deflects a small magnet suspended in the centre. This arrangement can be inverted, the magnet being made large and fixed and the coil small and movable. The Einthoven galvanometer is a development of this type. The magnet is large, and shaped so as to give a very intense field, and the "coil " is reduced to a single thread of quartz, silvered on the surface so as to conduct the current. The movements of this thread are too small to be followed by the unaided eye, so the poles of the magnet are pierced by a hole in which a microscope magnifying 600 diameters is arranged, so as to cast the image of the string on a moving photographic plate. We have already seen one use of this instrument in the study of voluntary muscular contraction (see p. 106); we shall have to return to it in our study of the heart, and respiration, in later chapters.

\section{We can now pass on to a consideration of results.}

In muscles that are removed from the body, it is found that on leading off two parts of their surface to a galvanometer, the galvanometer needle generally swings. The most marked result is obtained with a piece of muscle in which the fibres run parallel to one another, and the longitudinal surface is connected with one of the cut ends by a wire ( 2 in fig. 140).

On the course $0^{\prime}$ 'he wire a galvanometer indicates that a current flows from the centre to the cut end outside the muscle, and from the cut end to the centre inside the muscle. If, now, the muscle is thrown into tetanic contraction, the needle returns more or less completely to the position of rest.

Du Bois Reymond, who first described these facts, called the first current the current of rest, and the second current the current of action; the change in direction is indicated by the expression negative variation; this means that the current of action is in the opposite direction to the current of rest, and therefore lessens or neutralises it. The word negative is therefore used in its arithmetical, 
not its electrical sense. Du Bois Reymond explained this by supposing that a muscular fibre is built up of molecules, each of which

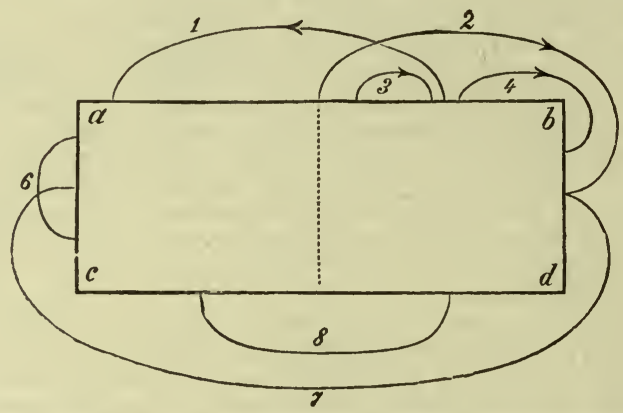

FIG. 140.-Diagram of the currents in a muscle prism. (Du Bois Reymond.)

is galvanometrically positive in the centre and galvanometrically negative at both ends. So when a muscle is cut across, a number of the galvanometrically negative ends of these molecules is exposed. On contraction the difference between the centre and ends of each molecule is lessened, and the resultant effect on the whole muscle (made up of such molecules) is similar.

In the foregoing sentence I have employed the rather cumbrous adjectives, galvanometrically positive and galvanometrically negative, instead of the terms positive and negative which are usually employed by physiologists.

If we take a Daniell cell and connect it to a galvanometer, the zinc, as we have seen, is the electro-positive element, and the copper the electro-negative element, but the ends of the wires which connect these metals to the galvanometer have the reverse names; the kathode or negative pole is connected to the zinc or positive metal ; the anode or positive pole is connected to the copper or negative metal. The current enters the galvanometer by the anode, and leaves it on its way back to the zinc by the kathode. Therefore, although the copper is electronegative, it may be spoken of as galvanometrically positive, and the zinc though electro-positive, as galvanometrically negative.

If we apply this to a muscle, we have seen that the current flows (in the wire that connects the uninjured longitudinal surface to the cut end) from the longitudinal surface to the cut end ; the longitudinal surface thus corresponds to the copper of the Daniell cell, and is therefore electro-negative, though galvanometrically positive; similarly the cut end corresponds to the zinc, and is electro-positive though galvanometrically negative.

The omission of the qualifying prefix to positive and negative has led to a good deal of confusion in physiological writings. A physicist uses the terms positive and negative as meaning electro-positive and electro-negative respectively, and as Dr Waller has pointed out, it is time that physiologists adopted the same nomenclature. In what now follows, I propose to adopt Dr Waller's suggestion.

There is no doubt about the facts as described by $\mathrm{Du}$ Bois Reymond. We now adopt, however, an entirely different view of their meaning: in causing this revolution of ideas the principal part has been played by Hermann. Hermann showed that the so-called 
current of rest does not exist; it is really a current produced by injury, and is now generally called a dcmarcation current: the more the ends of the muscle are injured the more positive they become; and when they are connected to the uninjured centre, a current naturally is set up as described by Du Bois Reymond. If a muscle is at rest and absolutely uninjured it is iso-electric; that is, it gives no current at all when two parts of it are connected together by a wire.

Since Du Bois Reymond's researches, the electrical changes which occur during a single twitch have been studied. also, and before we can understand the "negative variation" of tetanus, it is obviously necessary to consider the electrical variation which takes place during a twitch, for tetanus is made up of a fused series of twitches.

The electrical change during a twitch is called a diphasic variation. The contracting part of a muscle becomes first more positive than it was before; it then rapidly returns to its previous condition. The increase of positivity indicates a disturbance of the stability of the tissue; the disappearance of this increased positivity is the result of a return of the muscular tissue to a state of rest. If the muscle is stimulated at one end, a wave of contraction travels along it to the other end. This muscle-wave (see p. 100) may be most readily studied in a curarised muscle, that is, in a muscle which is physiologically nerveless. The electrical variation travels at the same rate as the visible contraction, but precedes it.

Suppose two points $(p$ and $d$ ) of the muscle (fig. 141) are connected by nonpolarisable electrodes to a galvanometer, and that the muscle-wave is started by a single stimulus applied at A; as soon as the wave reaches $p$ this point be-

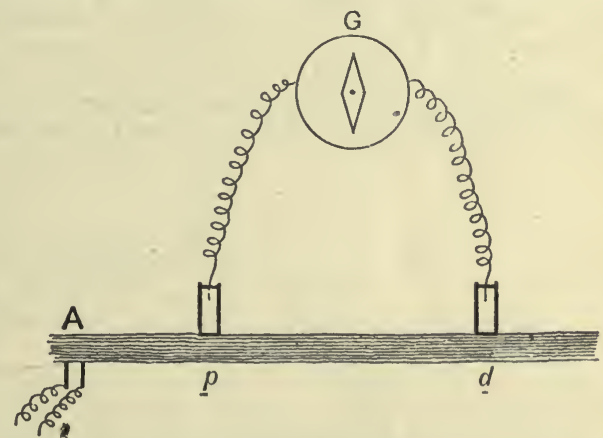

FIa. 141. comes positive to $d$, and therefore a current flows from $d$ to $p$ through the galvanometer G. A moment later the two points are equi-potential and no current flows; a minute fraction of a second* later this balance is upset, for when the wave reaches the point $d$, that point is positive to $p$, and the galvanometer needle moves in the opposite direction. 
The galvanometer is not the best instrument to employ to demonstrate these facts; the inertia of the needle may be so great that it is impossible for it to catch and respond to the two phases. Wedenski has made extensive use of the telephone instead, and the

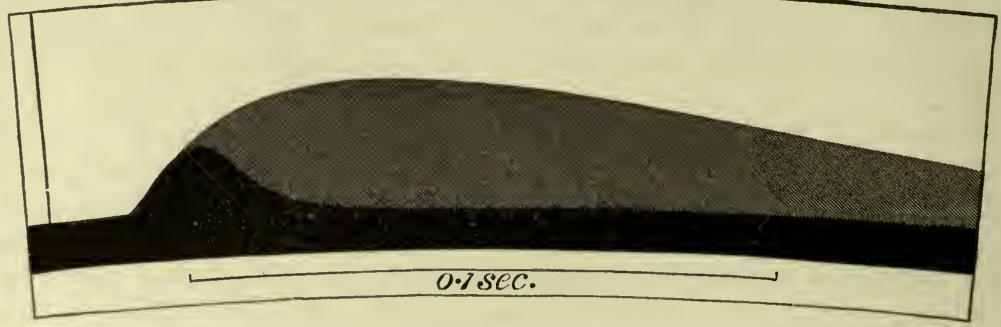

FiG. 142.-Diphasic curve (black) of the normal sartorius. The grey curve is the monophasic curve of the same muscle when one electrometer contact was placed on the injured end. The two photographic curves are placed one over the other so that the beginnings coincide. (Burdon Sanderson.)

sounds produced in it by the electrical changes in the muscle are distinctly audible. An appeal to the eye, however, is generally regarded as more satisfactory than one to the ear, and for this purpose the capillary electrometer is the instrument most frequently employed, as its responses are immediate; the mercury moves first in one direction, and then in the other. The deep black curve in the next figure (fig. 142) shows the record obtaining by photographing the movement of the column of mercury on a rapidly travelling photographic plate.

The capillary electrometer has the advantage of giving us the means of measuring the time of onset and duration of the electrical disturbance, and experiments

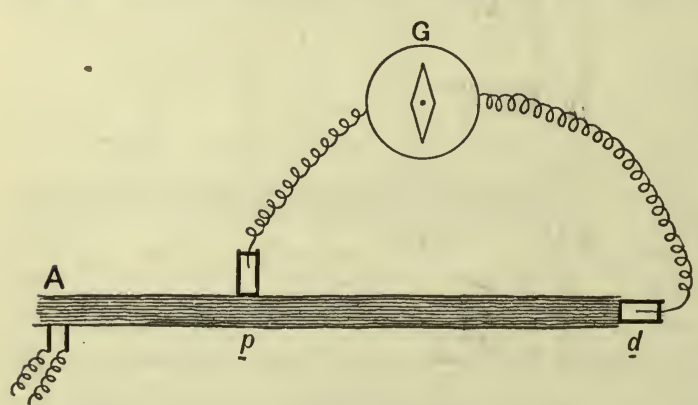

FIG. 143. made with this instrument show that the change only lasts a few thousandths of a second, and is over long before the other changes in form, etc., are completed. Sir J. Burdon Sanderson gives the following numbers from experiments with the frog's gastrocnemius. When the muscle was excited through its nerve the electrical response began $\frac{4}{1000}$ and the change of form $\frac{8}{1000}$ second after the stimulation; the second phase of the electrical response began $\frac{11}{100}$ second after excitation. When the muscle was directly excited, the latent period was much shorter, the change in form beginning $\frac{4}{1000}$ and the electrical change in less than $\frac{x}{1000}$ second after excitation.

If, however, instead of examining the electrical change in the muscle in the manner depicted in fig. 141, one electrode is placed on 
the uninjured surface and the other on the cut end (see fig. 143), the electrical response is a different one.

Under these circumstances, the electrical change is a monophasic variation, for when the muscle-wave reaches $d$, this part of the muscle, owing to its injured state, does not respond to the excitatory condition, and the electrical response is also extinguished.

The grey curve in figs. 142 and 144 is the graphic record of the change as revealed by the capillary electrometer. It will be seen that the ascending limb of the curve is identical in the two cases, but that the second phase is absent. From the point at which the diphasic curve approaches its culmination the injury curve diverges from it, continuing to ascend; the line soon after becomes horizontal, and then begins slowly to decline. This long tail denotes only the gradual disappearance of polarisation of the mercury meniscus.

The meaning of such photographic records becomes clear by testing the electrometer with known differences of potential, and from such data it is possible to construct what may be called an interpretation diagram (fig. 144). The horizontal line is that of equipotentiality of the two surfaces of contact $p$ and $d$. The curve $\mathrm{P}^{\prime}$ expresses the relative positivity of the surface $p$; the curve $\mathrm{D}^{\prime}$, the corresponding relative positivity of the surface $d$. $\mathbf{S}^{\prime}$ is a curve of which the ordinates are the algebraic sums of the corresponding ordinates of $\mathrm{P}^{\prime}$ and $\mathrm{D}^{\prime} . \mathrm{S}$ is the photographic curve which expresses $S^{\prime} ; P$ is the photographic curve which expresses $\mathrm{P}^{\prime}$ (monophasic variation). The numbers under the horizontal line indicate hundredths of a second; the distance $t t^{\prime}$ expresses the time taken by the wave in its progress from $p$ to $d$.

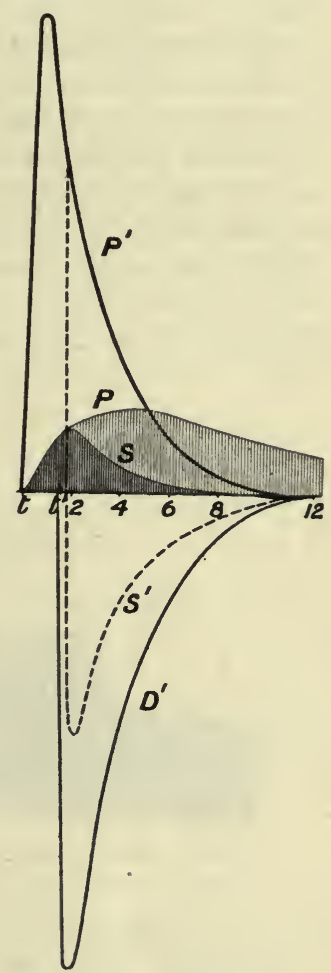

Fig. 144.-Interpretation dia. gram. (Burdon Sanderson.)

From these considerations we can now pass to study what occurs when the muscle enters into tetanus. The simplest case is that which was first observed by $\mathrm{Du}$ Bois Reymond. He placed his nonpolarisable electrodes in the positions indicated in fig. 143, one $(p)$ on the comparatively uninjured surface, the other $(d)$ on the devitalised cut end. He sent in the tetanising series of shocks at A. The electrical response is under these circumstances a summation of the individual electrical responses evoked by instantaneous stimuli; and the monophasic character of the single response explains easily what occurs during tetanus; the centre of the muscle becomes more positive than it was before, and so the electrical difference of potential 
between the centre and the injured end is lessened. But with regard to uninjured muscle the problem is not so easy. It is at first sight difficult to see why the summed effects of a series of diphasic variations should take the direction of the first phase, as was found to be the case by Du Bois Reymond in experiments with the frog's gastrocnemius. One would have anticipated that "negative" variation in the arithmetical sense would be absent altogether, and this is the case in absolutely normal muscles; Hermann has shown that it is so during tetanus of the human forearm. But a muscle removed from an animal's body cannot be considered absolutely normal, and if the two contacts be placed on the comparatively uninjured longitudinal surface, as in fig. 142, a negative variation is observed, each excitatory phase becoming weaker as it progresses, and the second phase of each diphasic effect is weaker than the first. The following figure (fig. 145) illustrates the record obtained by the capillary electrometer

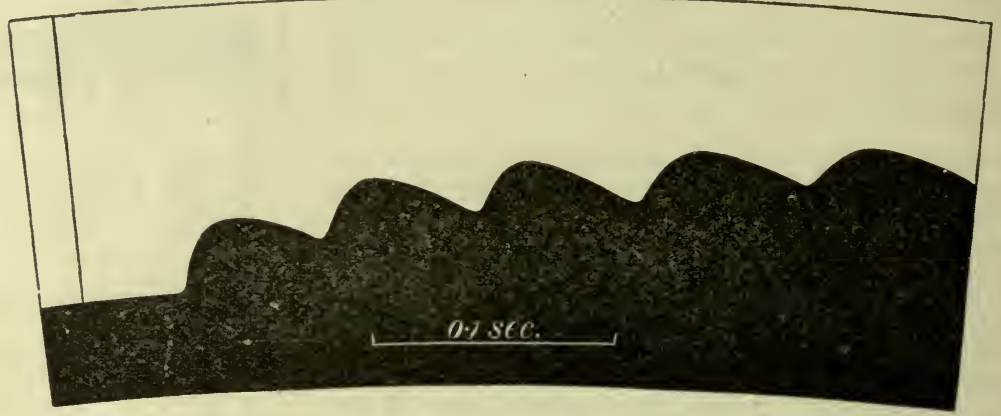

Fia. 145.--Electrometer record of injured sartorius during tetanus. (Burdon Sanderson.)

from an injured sartorius excited 14 times a second; each oscillation represents a single monophasic variation. The individual oscillations can, however, be seen when the excitations follow one another more rapidly, even up to 80 or 100 per second.

Muscle is not the only tissue which exhibits electrical phenomena. A nerve which is uninjured is iso-electric; injury causes a demarcation current; activity is accompanied with a similar diphasic wavo travelling along the nerve simultaneously with the nervous impulse. The activity of secreting glands, vegetable tissues, retina, etc., is accompanied with somewhat similar electrical changes, which we shall study in detail later.

But the most prominent exhibition of animal electricity is seen in the electric organs of electric fishes. In some of these fishes the electric organ is modified muscle, in which a series, as it were, of hypertrophied end-plates correspond to the plates in a voltaic pile. 
In other fishes the electric organ is composed of modified skin glands. But in each case the electric discharge is the principal phenomenon that accompanies activity.

\section{The Rheoscopic Frog.}

The electrical changes in muscle can be detected not only by the galvanometer and electrometer, but also by what is known as the physiological rheoscope; this consists of an ordinary muscle-nerve preparation from a fresh and vigorous frog. The nerve is stimulated by the electrical changes occurring in muscles, and the nervous impulse so generated causes a contraction of the muscles of the rheoscopic preparation. The following are the principal experiments that can be shown in this way:-

1. Contraction without metals. If the nerve of a nerve-muscle preparation A is dropped upon another muscle B (fig. 146) or upon

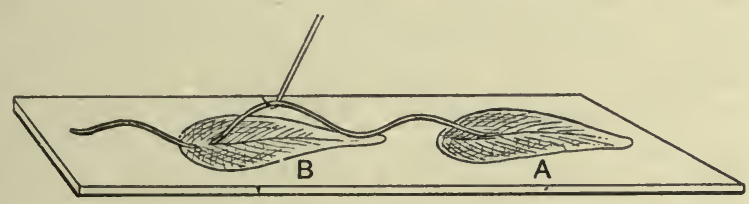

FrG. 146.-Galvani's experiment without metals.

its own muscle, it will be stimulated by the injury current of the muscle on which it is dropped, and this leads to a contraction of the muscle (A) which it supplies. The experiment succeeds best if the nerve is dropped across a longitudinal surface and a freshly made transverse section.

2. Secondary contraction. This is caused by the current of

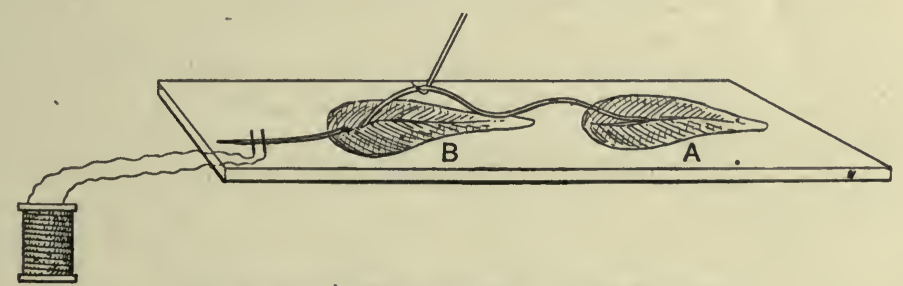

Fig. 147.-Secondary contraction. (After Waller.)

action. If, while the norre of $\mathrm{A}$ is resting on the muscle $\mathrm{B}$ (fig. 147), the latter is made to contract by the stimulation of its nerve, the nerve of $\mathrm{A}$ is stimulated by the electrical variation which accompanies the contraction of the muscle B, and so a contraction of muscle $\mathrm{A}$ is produced. This is called secondary con- 
traction. It may be either a secondary twitch or secondary tetanus, according as to whether the muscle $B$ is made to contract singly or tetanically.

3. Secondary contruction from the heart. If an excised but still beating frog's heart is used instead of muscle B, and the nerve of A laid across it, each heart's beat, accompanied as it is by an electrical variation, will stimulate the nerve and cause a twitch in the rheoscopic muscle A. 


\section{CHAPTER XII}

\section{THERMAL AND CHEMICAL CHANGES IN MUSCLE}

IN muscular contraction there is a transformation of the potential energy of chemical affinity into other forms of energy, especially molar motion and heat. Heat is a form of motion in which there is movement of molecules; in molar motion there is movement of masses. The fact that when a blacksmith hammers a piece of iron it becomes hot is a familiar illustration of the transformation of one mode of movement into the other. Heat is measured in heat-units or calories. One calorie is the energy required to raise the temperature of 1 gramme of water from $0^{\circ}$ to $1^{\circ} \mathrm{C}$.; and this in terms of work is equal to 425.5 gramme-metres, that is, the energy required to raise the weight of 425.5 grammes to the height of 1 metre.

A muscle when uncontracted is nevertheless not at absolute rest. We have already seen that it possesses tonus or tone; it also possesses what we may call chemical tone; that is, chemical changes are occurring in it, and consequently heat is being produced. But when it contracts, the liberation of energy is increased; work is done, and more heat is produced; the heat produced represents more of the energy than the work done. The more resistance that is offered to a muscular contraction, the more is the work done relatively increased and the heat diminished. The amount of heat produced is increased by increasing the tension of the muscle. It diminishes as fatigue comes on. On increasing the strength of the stimulus the amount of heat increases faster, proportionately, than the work performed.

If work is done by a few large contractions, more heat is produced than if the same work is done by a larger number of smaller contractions; that is, more chemical decomposition occurs, and fatigue ensues more rapidly in the first case. This fact is within the personal experience of everyone. If one ascends a tower, the work done is the raising of the weight of one's body to the top of the tower. If the staircase in the tower has a gentle slope, each step being low; far less fatigue is experienced than if one ascended to the same height by a smaller number of steeper steps. 
On a cold day one keeps oneself warm by muscular exercise; this common fact is confirmed by more accurate experiments on isolated muscles, the heat produced being sufficient to raise temporarily the temperature of the muscle. This can be shown in large animals by inserting a thermometer between the thigh muscles and stimulating the spinal cord. The rise of temperature may amount to several degrees.

In the case of frog's muscles, Helmholtz found that, after tetanising them for two or three minutes, the temperature rises $0.14^{\circ}$ to $0.18^{\circ} \mathrm{C}$; ; and for each single twitch Heidenhain gives a rise of temperature of from $0.001^{\circ}$ to $0.005^{\circ} \mathrm{C}$.

For the detection of such small rises in temperature, a thermopile, and not a thermometer, is employed.

A thermopile consists of a junction of two different metals; the metals are connected by wires to a galvanometer. If the junction is heated an electrical current passes round the circuit, and is detected by the galvanometer. The metals usually employed are

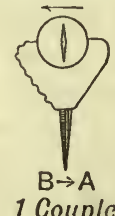

1 Couple.

FIG. 148

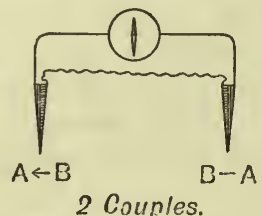

Fia. 149.

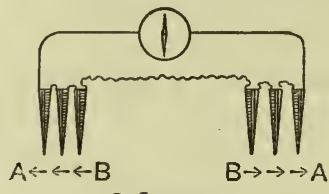

3 Couples.

Fig. 150.

FIGs. 14S-150.-Schene of thermo-electric couples. (After Waller.)

iron and German silver, or antirnony and bismuth. If the number of couples in the circuit is increased, each is affected in the same way, and thus the electrical current is increased through the galvanometer. The arrangement is shown in the fig. 148, which also indicates the direction of the currents produced, the metals employed being antimony and bismuth. By using 16 couples of this kind Helmholtz was able to detect a change of $\frac{1}{4000}$ of a degree Centigrade.

Within certain limits, the strength of the current is directly proportional to the rise of temperature at the junction.

If two couples are in circuit, as shown in fig. 149, and they are heated equally, no current will pass through the galvanometer, the current through one couple being opposed by the current through the other. But if the two couples are heated unequally, the direction of swing of the galvanometer needle indicates which is the warmer. To apply this to the frog's gastrocnemius, plunge several needle-shaped couples (fig. 150) into a frog's gastrocnemius of one side and the same number of couples into the gastrocnemius of the other side, and then excite first one then the other sciatic nerve; a deflection of the galvanometer will be observed first in one, then in the other direction, indicating the production of heat first on one side, then on the other. 


\section{Chemical Changes in Muscles.}

The chemical changes which are normally occurring in a resting muscle are much increased when it contracts. Waste products of oxidation are discharged, and the most abundant of these is carbonic acid. Sarcolactic acid is also produced, and the alkaline reaction of a normal muscle is replaced by an acid one. The muscles of animals hunted to death are acid; the acid reaction to litmus paper of a frog's gastrocnemius can be readily shown after it has been tetanised for 10 to 15 minutes.

When a muscle contracts, the quantity of oxygen consumed is increased, and the discharge of carbonic acid is increased also. This is the cause of the rise in heat-production just described. It is important to note, however, that this change follows rather than accompanies the increase of work, as we shall see more at length when we are studying tissue-respiration (p. 397).

For a certain time after its removal from the body, an excised muscle can be made to contract and give off oxidation products such as carbonic acid in an atmosphere containing no oxygen at all. The oxygen used has thus been stored up in the muscle previously. The oxygen is not, however, present in the free state, for no oxygen can be detected in the gases obtained from muscles by means of an airpump. Excised muscles, however, must be regarded as partially asphyxiated, for their individual fibres are largely cut off from that ready supply of oxygen which normally reaches them by the blood. During life (and the living condition can be imitated by placing an excised muscle in an atmosphere of pure oxygen) the muscular substance breaks down into a number of simpler substances; one of these is carbonic acid. The others, however, or some of them, are at once built up again with the inclusion of oxygen and some carbon-containing substance, perhaps sugar, into living material. The muscle, therefore, does not contain any of the by-products of its own metabolism. In excised muscle, when the oxygen supply is deficient the by-products accumulate, as a result of which very striking alterations take place:-(1) The reaction of the muscle changes and the phenomena of fatigue and functional death set in. (2) The proteins become coagulated, and this is the physical basis of rigor mortis.

There are other chemical changes in the muscle when it contracts, for instance, a change of glycogen into sugar. The question whether nitrogenous waste is increased during muscular activity has been a much debated one. It has now, however, been finally proved that an increased consumption of carbon (in large measure derived from the carbohydrate stored in the muscle) is a marked and immediate feature of muscular activity. Any increase in the consumption of 
nitrogen is negligible, and only occurs when the muscles do not receive a due share of non-nitrogenous food. (See more fully chapter on Metabolism.)

\section{Fatigue.}

If the nerve of a nerve-muscle preparation is continually stimulated, the muscular contractions become more prolonged (see p. 97), smaller in extent, and finally cease altogether.

The muscle is said to be fatigued: this is due to the consumption of the substances available for the supply of energy in the muscle, but more particularly to the accumulation of waste products of contraction; of these, sarcolactic acid is an important one. Fatigue may be artificially induced in a muscle by feeding it on a weak solution of lactic acid, and then removed by washing out the muscle with salt solution containing a minute trace of an alkali. If the muscle is left to itself in the body, the blood-stream washes away the accumulation of acid products, and fatigue passes off.

The question next presents itself, where is the seat of fatigue? Is it in the nerve, the muscle, or the end-plates? If, after fatigue has ensued and excitation of the nerve of the preparation produces no more contractions, the muscle is itself stimulated, it contracts; this shows it is still irritable, and, therefore, not to any great extent the seat of fatigue.

If an animal is poisoned with curare, and it is kept alive by artificial respiration, excitation of the peripheral end of a motor nerve produces no contraction of the muscles it supplies. If one goes on stimulating the nerve for many hours, until the effect of the curare has disappeared, the block at the end-plates* is removed and the muscles contract: the seat of exhaustion is therefore not in the nerves. By a process of exclusion it has thus been localised in the nerve-endings.

When the muscle is fatigued in the intact body, there is, however, another factor to be considered beyond the mere local poisoning of the end-plates. This is the effect of the products of contraction passing into the circulation and poisoning the central nervous system. This aspect of the question has been specially studied by Waller and by Mosso. Mosso devised an instrument called the ergograph, which is a modification of Waller's dynamograph invented many years previously. The arm, hand, and all the fingers but one are fixed in a suitable holder; the free finger repeatedly lifts a weight over a pulley, and the height to which it is raised is registered by a marker on a blackened surface.

By the use of this and similar instruments it has been shown that the state of the brain and central nervous system generally is a

* Another convenient block which is sometimes used is to throw à-constant current into the nerve between the point of excitation and the muscles. This prevents the nerve impulses from reaching the muscles. 
most important factor in fatigue, and that the fatigue products produced in the muscles during work cause part of their injurious effects by acting on the central nervous system and diminishing its power of sending out impulses.

One of the most striking of Mosso's experiments illustrates in a very forcible manner the fact that the central nervous system is more easily fatigued than the nerve-endings in muscle. A person goes on lifting the weight until, under the influence of the will, he is unable to raise it any more. If then without waiting for fatigue to pass off, the nerves going to the finger muscles are stimulated artificially by induction shocks, they once more enter into vigorous contraction.

Mosso has also shown that the introduction of the blood of a fatigued animal into the circulation of a normal one will give rise in the latter to all the symptoms of fatigue. The blood of the fatigued animal contains the products of activity of its muscles, but still remains alkaline; the poisonous substance cannot therefore be free lactic acid. Lactic acid is doubtless one only of the products of muscular activity; we have at present no accurate knowledge of the chemical nature of the others.

It should, however, be mentioned that some physiologists (Lee, Ioteyko, etc.), still regard the effect on the end plates as the most important factor in fatigue.

The statement that nerves are not fatiguable, does not mean that the nervefibres undergo no metabolic changes when transmitting a nerve impulse, but that the change is so slight, and the possibilities of repair so great, that fatigue in the usual acceptation of the term cannot be demonstrated. Waller made the interesting but tentative suggestion that the medullary sheath is a great factor in repair, or, in his own words, "the active grey axis both lays down and uses up its own fatty sheath, and it is inexhaustible not because there is little or no expenditure, but because there is an ample re-supply."

A year or two after these words were written, Miss Sowton, at Dr Waller's suggestion, undertook a piece of work in order to test the truth of this hypothesis. If the absence of fatigue is due to the presence of the fatty sheath, fatigue ought to be demonstrable in nerve-fibres in which the fatty sheath is absent. She selected the olfactory nerve of the pike as the non-medullated nerve with which to try the experiment, and her results confirmed Dr Waller's expectation; the galvanometric replies of the nerve became somewhat feebler after repeated stimulation.

It appeared to me advisable to test the question in another way. The splenic nerves seemed to be the most convenient large bundles of non-medullated fibres for the purpose. Dr T. G. Brodie was associated with me in carrying out the investigation. A dog is anæsthetised, the abdomen opened, the spleen exposed, and the splenic nerves which lie by the side of the main splenic artery are laid bare. It is quite easy to dissect out a length of nerve sufficient for the experiment (11 $1 \frac{1}{2}$ to 2 inches). The nerve is then cut as far from the spleen as possible, and the spleen is enclosed in an air oncometer connected to the bellows volume recorder invented by Dr Brodie. On stimulating the nerve with a weak faradic current the organ contracts, and the recording lever falls. The diminution of the size of the spleen is quite visible to the naked eye, however, without the use of any apparatus. The next thing to do is to put a block on the course of the nerve, which will prevent the nerve impulses from reaching the spleen. Here we met with some difficulty. Curare and atropine are both ineffective : the constant current has a great disadvantage; non-medullated nerves are so much affected that very feeble constant 
currents will completely block the transmission of impulses, and not only that, but the nerve remains blocked after the current is removed. After the current has been allowed to flow for two minutes the nerve remains impassable to nerve impulses for an hour or more, and then slowly recovers. If, therefore, faradic excitation of the nerve is kept up all this time and fails to excite the contraction of the spleen after the removal of the constant current, it is impossible to say whether

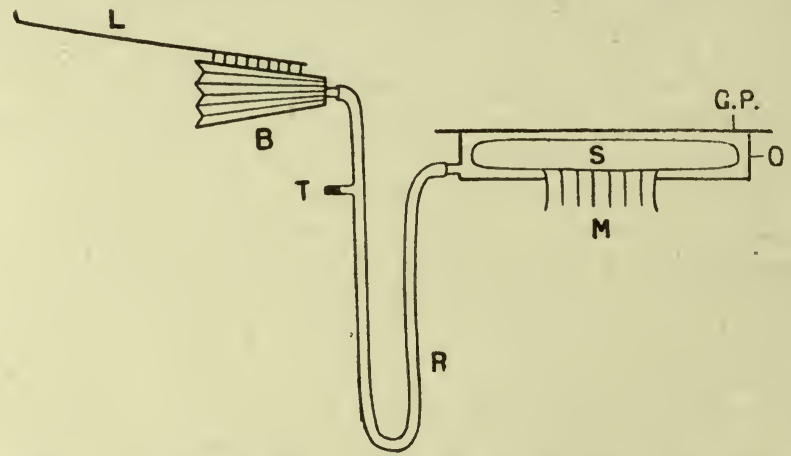

FIG. 151.-Apparatus for obtaining splenic curves. s, Spleen in oncometer o, which is made of gutta percha, and covered with a glass plate (G.P.) luted on with vaseline. $M$ is the splenic mesentery containing vessels and nerves; this passes through a slit in the base of the oncometer which is made air-tight with vaseline. The oncometer is connected to the flexible bellows ( $\mathrm{B}$ ) by the india-rubber tube $(R)$, the side tube $(T)$ being closed during an experiment by a piece of glass rod. The recording lever (L) writes on a revolving drum.

this is due to fatigue of the nerve-fibres on the proximal side of the block, or whether it may not be due to the fact that the block (due to electrolytic changes caused constant current) is still effective.

Our best results were obtained by using cold instead of a constant current as our blocking agent.

Fig. 151 is an outline drawing of the apparatus used, and fig. 152 shows the

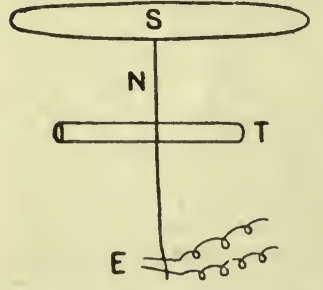

Fia. 152.-Arrangement of apparatus in connection with the splenic nerve. $\mathrm{s}$ is the spleen, and $\mathrm{N}$ the main bundle of nerves. The nerve rests on the metal tube (T) through which fluid at the required temperature is kept flowing, and on the electrodes (E) which come from the secondary coil of an inductorium.

excitation the nerve is just as excitable as it was at the start, and a full splenic contraction is obtained when the cold block is removed. arrangement adopted in connection with the nerve. The nerve $(\mathrm{N})$ rests on a metal tube ( $\mathrm{T})$ through which water can be kept flowing. $\mathrm{E}$ is the situation of the electrodes. If the nerve is excited, the spleen contracts, and the recording lever (in fig. 151) falls. If now brine at 0 to $2^{\circ} \mathrm{C}$. is kept flowing through $\mathrm{T}$, the nerve impulses are blocked by the cold, and cannot reach the spleen. Immediately the cold brine is replaced by warm water at $30^{\circ} \mathrm{C}$., the nerve again becomes passable by nerve impulses, and the spleen contracts once more.

While the fluid in $\mathrm{T}$ is kept at the low temperature mentioned, the nerve is excited with strong induction shocks all the time, and the spleen remains irresponsive; the nerve impulses are able to reach $\mathrm{T}$ but not to pass it. If then warm water is passed through $\mathrm{T}$, and the block produced by the cold is thus removed, and the spleen continues to be irresponsive, we have a proof that the piece of nerve between $\mathrm{E}$ and $\mathrm{T}$ has been fatigued. But our experiments have shown us that non-medullated nerve is just as difficult to fatigue as medullated nerve. Even after six hours' continuous 
We have made similar experiments with vaso-motor nerves, such as the cervical sympathetic nerve in the rabbit, the splanchnic nerve of the dog, and the sciatic nerve in a curarised dog, and have obtained corresponding results. This confirms the work previously published by Eve. Eve excited the cervical sympathetic for twelve hours, and found no loss of excitability at the end of that time. Eve stimulated the nerve below the upper cervical ganglion, and the main object of his work was to ascertain whether any histological evidence of fatigue could be found in the cells of the ganglion. The only change he could find there was a somewhat diffuse staining of the cells by methylene blue, which he attributes to the formation of acid substances in the cells. A blue stain of similar appearance may be induced in the motor cells of the spinal cord, after exhaustion is produced in them by giving strychnine. In such experiments the spinal cord becomes, as a rule, distinctly acid to litmus paper. Max Verworn has more recently employed strychnine as a means of producing fatigue. He considers that the only specific effect of this alkaloid is increase of reflex activity, and he attributes the subsequent paralysis to vascular conditions and the accumulation of fatigue products, among which he places carbon dioxide in the first rank. Eve, on the contrary, did not find that carbonic acid alone produces the effects.

We must conclude from such experiments that Dr Waller's theory is unproved, and that while fatigue is demonstrable in nerve-cells, it cannot be shown to occur in nerve-fibres of either the medullated or non-medullated variety by these methods.

In carrying out these experiments we noticed that though no functional fatigue can be demonstrated, there is noticeable, especially in vaso-motor nerves, a phenomenon which Howell terms stimulation fatigue; this means that the actual spot of nerve stimulated becomes after a time less excitable, and finally, inexcitable, though it will still transmit impulses, if the excitation is applied above the spot originally stimulated. We think that the use of the term "fatigue" in this connection is a mistake; the prolonged electrical excitation causes injurious polarisation (due to electrolytic changes) of the nerve, which renders it less excitable. This view has been confirmed by Prof. Gotch by means of experiments with the capillary electrometer. This so-called "stimulation fatigue" was not excluded in Miss Sowton's experiments, and will possibly explain her results. The splenic nerves, curiously enough, do not exhibit this phenomenon in any marked degree, and so were peculiarly well adapted to test the question of functional fatigue. On $\dot{a}$ priori grounds we should hardly expect non-medullated nerves to be peculiarly susceptible of real fatigue, when one considers that many of them, such as the vaso-constrictors, are in constant action throughout life.

It should be clearly understood that all these experiments prove only that nerve-fibres are not fatiguable under ordinary conditions of stimulation. If we assume that nerve is entirely "unfatiguable," we must assume also that its activity is not associated with the consumption of material and the production of waste products. This would render nerve unique among all the other tissues of the body, and is, moreover, contradicted by recent discoveries of evidence of metabolic changes in a nerve during its activity. We are therefore driven to the conclusion that repair is excecdingly rapid and perfect, although it is impossible to agree with Waller that the repairing process is definitely associated with the presence of a medullary sheath. The interval between successive induction shocks is certainly short, but it is apparently long enough to allow the nerve to recover completely before the next stimulus arrives. If, however, the interval between two successive stimuli is made very brief indeed $(0.006 \mathrm{sec}$.), the second stimulus is ineffective because of the fatigue due to the first. If the irritability of the nerve is depressed by cold, by asphyxia, or by an anæsthetic (such as yohimbine), the irresponsive period may be lengthened to as much as one to two tenths of a second.

In one of the foregoing paragraphs the following sentence occurs : "fatigue is demonstrable in nerve-cells." M'Dougall has recently adduced evidence that fatigue in the central nervous system has its seat not so much in the bodies of the nerve-cells as in their synaptic junctions, which are the points of highest resistance (that is, where impulses pass with greatest difficulty) in all mental and other operations in which the brain and spinal cord share, even when no fatigue exists. 


\section{Rigor Mortis.}

After death, the muscles gradually lose their irritability and pass into a contracted condition. This affects all the muscles of the body, and usually fixes it in the natural posture of equilibrium or rest. The general stiffening thus produced constitutes rigor mortis or postmortem rigidity.

The cause of rigor is the coagulation of the muscle-plasma, which is more fully described in the next section. This coagulation results in the formation of myosin, and is gradual in onset. Simultaneously the muscles (a) become shortened and opaque, (b) heat is evolved, (c) they give off carbonic acid, and (d) become acid in reaction; this is due in part to the formation of sarcolactic acid, and in part to the formation of acid phosphates.

After a varying interval, the rigor passes off, and the muscles are once more relaxed. This sometimes occurs too quickly to be caused by putrefaction, and there is very little doubt that it is really the first stage in the self-digestion or autolysis which occurs in all tissues after death, owing to the presence of intracellular enzymes or ferments. It is known that a pepsin-like or proteolytic enzyme is present in muscle, as in many other animal tissues, kidney, spleen, etc. (Hedin), and that such enzymes act best in an acid medium. The conditions for the solution of the coagulated myosin are therefore present, as the reaction of rigored muscle is acid.

Order of Occurrence.-The muscles are not affected simultaneously by rigor mortis. It affects the neck and lower jaw first; next, the upper extremities, extending from above downwards; and lastly, reaches the lower limbs; in some rare instances it affects the lower extremities before, or simultaneously with, the upper extremities. It usually ceases in the order in which it begins: first at the head, then in the upper extremities, and lastly in the lower extremities. It seldom commences earlier than ten minutes, or later than seven hours after death; and its duration is greater in proportion to the lateness of its accession.

The occurrence of rigor mortis is not prevented by the previous existence of paralysis in a part, provided the paralysis has not been attended with very imperfect nutrition of the muscular tissue.

In some cases of sudden death from lightning, violent injuries, or paroxysms of passion, rigor mortis has been said not to occur at all; but this is not always the case. It may, indeed, be doubted whether there is really a complete absence of the post-mortem rigidity in any such cases; for the experiments of Brown-Séquard make it probable that the rigidity may supervene immediately after death, and then pass away with such rapidity as to be scarcely observable.

\section{Chemical Composition of Muscle.}

The phenomena of rigor mortis will be more intelligible if we consider the chemical composition of muscle. 
The connective tissue of muscle resembles connective tissue elsewhere; the gelatin and fat obtained in analyses of muscle are derived from this tissue. The sarcolemma is composed of a substance which resembles elastin in its solubilities.

The contractile substance within the muscular fibres is, during life, of semi-liquid consistency, and contains a large percentage of proteins and smaller quantities of extractives and inorganic salts. By the use of a press this substance can be squeezed out of perfectly fresh muscles, and it is then called the muscle-plasma.

After death, muscle-plasma, like blood-plasma, coagulates (thus causing the stiffening known as rigor mortis). The solid clot corresponding to the fibrin from blood-plasma is called myosin, and the liquid residue is called the muscle-serum.

Pursuing the analogy further, it is found that the coagulation of both muscle-plasma and blood-plasma can be prevented by cold, by strong solutions of neutral salts, and by potassium oxalate, which precipitates, as the insoluble oxalate of calcium, the lime salts essential for the coagulation process. In both cases the clotting is produced by the action of an enzyme developed after death. In both cases the precursor of the solid clot is a protein of the globulin class which previously existed in solution.

Fibrin in the blood-clot is formed from the previously soluble fibrinogen of the blood-plasma. Myosin in the muscle-clot is formed from the previously soluble myosinogen* of the muscle-plasma. When the blood-clot contracts it squeezes out blood-serum; when the muscle-clot contracts it squeezes out muscle-serum. The muscleserum contains small quantities of albuminous material, together. with the extractives and salts of the muscle. The origin of the sarcolactic acid is a controversial question: some believe it originates from the carbohydrate (glycogen and sugar); others think it comes from the protein molecules in the muscle.

The general composition of muscular tissue is the following:-

Water :
Solids :
Proteins :
Gelatin :
Fat
Extractives
Inorganic salts

The proteins, as already stated, chiefly pass into the clot: very little is found in the muscle-serum.

The extractives comprise a large number of organic substances, all present in small quantities, some of which are nitrogenous, like creatine, creatinine, xanthine, and hypoxanthine: the rest are nonnitrogenous-namely, fats, glycogen, sugar, inosite, and the variety

\footnotetext{
* For further details see small text at the end of this chapter.
} 
of lactic acid known as sarcolactic acid. The inorganic salts are chiefly salts of potassium, especially potassium phosphate.

The condition of dead muscle reminds one somewhat of contracted muscle. Indeed, the similarity is so striking that Hermann propounded the idea that contracted muscle is muscle on the road to death, the differences between the two being of degree only. $\mathrm{He}$ considers that, on contraction, a hypothetical material termed inogen is broken up into carbonic acid, sarcolactic acid, and myosin; on death the same change occurs, only to a much more marked extent.

This idea is a far-fetched one, but it is a useful reminder of the similarities of the two cases. In chemical condition, contracted and dead muscle are alike, so far as the formation of acid products is concerned; there is, however, no evidence of any formation of a muscle-clot (myosin) during the contraction of living muscle, as there is in dead muscle. Then heat is produced in both cases, and in both cases also the muscle is electro-positive to uncontracted muscle.

Here, however, the analogy must end: for living contracted muscle is irritable, dead muscle is not. Living contracted muscle is more extensible than uncontracted muscle; muscle in rigor mortis is not so (see fig. 129, p. 111). The contraction of living muscle is favoured by feeding it with a solution of dextrose, while the process of rigor is hindered by the same solution. (F. S. Lee.)

Our correct knowledge of the proteins of muscle and of the phenomena of rigor mortis date from the year 1864, when Kuihne obtained muscle-plasma by subjecting frozen frog's muscle to strong pressure. A good many years later I was successful in repeating these experiments with mammalian muscle. By fractional heat coagulation, and by thcir varying solubilities in neutral salts, I was able to separate four different proteins in the muscle-plasma.

1. A globulin precipitable by heat at $47^{\circ} \mathrm{C}$. This is analogous to the cellglobulin found in most protoplasmic structures. I gave it the name paramyosinogen.

2. A proteid with many of the characters of a globulin, coagulable by heat at $56^{\circ} \mathrm{C}$.; and this I termed myosinogen.

3. A globulin (myoglobulin), precipitable by heat at $63^{\circ} \mathrm{C}$.

4. An albumin similar in its properties to serum albumin is also present; but this and the myoglobulin only occur in quite small amounts.

In addition to these, there is a small quantity of nucleo-protein from the nuclei, and in the red muscles hæmoglobin is present; the normal pigment of the so-called pale muscles is termed myohomatin by MacMunn, and this is doubtless a derivative of hæmoglobin.

The two most abundant and important proteins are the first two in the list, namely, paramyosinogen and myosinogen. They occur in the proportion of about 1 to 4 , and both enter into the formation of the muscle-clot (myosin). The myoglobulin is probably derived from the adherent connective tissue and the albumin from adherent blood and lymph.

In 1895 v. Fürth took up the subject. On the main question we are in substantial agreement, namely, that in the muscle-plasma there are the two proteins just alluded to, and that these both contribute to the formation of the muscle-clot. The main points of difference between us are in the names of the proteins. He uses physiological saline solution to extract the muscle-plasma, and this extract coagulates spontaneously on standing; he is doubtful whether a specific myosin-ferment brings about the change. Paramyosinogen he terms myosin, and this passes directly into 
the clotted condition ; but myosinogen, called myogen by v. Fürth, first passes into a soluble condition (congulable by heat at the remarkably low temperature of $40^{\circ} \mathrm{C}$.) before it clots : the soluble stage he calls soluble myogen-fibrin; a better name is soluble myosin.

We may put this in a diagrammatic way as follows:-

Proteins of the living muscle.

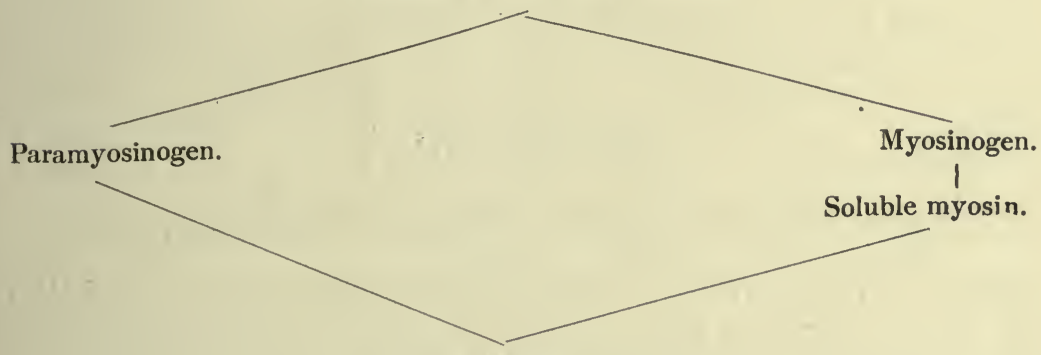

Myosin

(the protein of the Muscle-clot).

V. Fürth also calls attention to some characters of myosinogen which separate it from the typical globulins; e.g., it is not precipitable by dialysing the salts away from its solutions. It may be therefore called an atypical globulin.

In mammalian muscle, soluble myosin is only found as a stage in the process of rigor mortis, but in the muscles of the frog and other amphibia it is present as such in the living muscle.

'The muscle-plasma from fishes' muscle contains another protein termed myoprotein by v. Fürth. It is precipitable by dialysis, but not coagulable by heat.

Brodie, and later, Vernon, did some interesting experiments on heat rigor. When a muscle is heated above a certain temperature it becomes contracted and stiff, losing its irritability completely. This is due to the coagulation of the muscle proteins. If a tracing is taken of the contraction, it is found to occur in a series of steps : the first step in the shortening occurs at the coagulation temperature of the paramyosinogen $\left(47^{\circ}-50^{\circ} \mathrm{C}\right.$.), and if the heating is continued, a second shortening occurs at $56^{\circ} \mathrm{C}$, the coagulation temperature of myosinogen. If, however, a frog's muscle is used, there are three steps, namely, at $40^{\circ}$ (coagulation temperature of soluble myosin), $47^{\circ}$, and $56^{\circ}$. This work of Brodie's is especially valuable because it teaches us that the proteins in muscle-plasma, or in saline extracts of muscle, are present also in the actual muscle-substance. He also made clear another important point, namely, that the irritability of the muscle is lost after the first step in the shortening has occurred. In other words, in order to destroy the vitality of muscular tissue, it is not necessary to raise the temperature sufficiently high to coagulate all its proteins, but that when one of the muscular proteins has been coagulated, the living substance as such is destroyed; the proteins of muscle cannot therefore be regarded as independent units; the unit is protoplasm, and if one of its essential constituents is destroyed, protoplasm as such ceases to live.

Hans Przibram has attempted to classify the dnimal kingdom on the basis of the muscle-proteins ; his conclusions are based on the examination of only thirty species of animals, and may require revision in the future, but such as they are, they are as follows :-

Invertebrates : paramyosinogen present; myosinogen absent.

Vertebrates : paramyosinogen and myosinogen both present.

Fishes : in addition to these two principal proteids, soluble myosin and myoprotein (in large quantities) occur.

Amphibians : like fishes, except that myoprotein is only present in traces.

Reptiles, birds, mammals : myoprotein is absent, and soluble myosin is only present when rigor mortis commences. 


\section{CHAPTER XIII}

\section{COMPARISON OF VOLUNTARY AND INVOLUNTARY MUSCLE}

THe main difference between voluntary and involuntary muscle is the difference expressed in their names. Voluntary muscle is under the control of that portion of the central nervous system the activity of which is accompanied by volition. Involuntary muscle, on the other hand, is, as a rule, also under the control of the central nervous system, but of a portion of the central nervous system the activity of which is independent of volition. There appear, however, to be exceptions to this rule, and the involuntary muscle executes its contractions independently of nervous control; that is to say, it is sometimes in the truest sense of the term really involuntary. This is very markedly seen in the developing heart of the embryo, which begins to beat before any nerve-fibres have grown into it from the central nervous system.

Another characteristic of involuntary muscle is a tendency to regular alternate periods of rest and activity, or rhythmicality. This is best exemplified in the heart, but it is also seen in the lymphatic vessels, especially the lymph hearts of the frog, and the mesenteric lymphatic vessels (lacteals) of many animals. It is seen in the veins of the bat's wing, and in the muscular tissue of the spleen, stomach, intestine, bladder, and other parts.

A third characteristic of involuntary muscle is peristalsis. If any point of a tube of smooth muscle such as the small intestine is stimulated, a ring-like constriction is produced at this point. After lasting some time at this spot it slowly passes along the tube at the rate of 20 to 30 millimetres per second. This advancing peristaltic wave normally takes place in only one direction, and so serves to drive on the contents of the tube.

Involuntary muscle nearly always contains numerous plexuses of non-medullated nerve-fibres with ganglion cells; so that much discussion has taken place on the question whether the phenomena of rhythmicality and peristalsis are properties of the muscular tissue itself or of the nerves mixed with it. The evidence available (namely, 
that portions of muscular tissue entirely free from nerves act in the same way as those that possess nerves) indicates that it is the muscular rather than the nervous tissues that possess these properties; though it can hardly be doubted that under normal conditions the contraction of involuntary muscle is influenced and controlled by nervous agency.

As instances of nerveless involuntary muscles which possess the property of rhythmical action, we may take the ventricle apex of the frog's or tortoise's heart. If this is cut off and fed with a suitable nutritive fluid at considerable pressure it will beat rhythmically (Gaskell). The middle third of the ureter is another instance of muscular tissue free from nerves, but which nevertheless executes peristaltic movements. Perhaps, however, the most striking example is that of the fœtal heart, which begins to beat directly it is formed, long before any nerves have grown into it.

The artificial stimuli employed for involuntary are the same as those used for voluntary muscle; single induction shocks are, however, often ineffectual to produce contraction, but the make, and to a less extent the break, of a constant current will act as a stimulus.

The faradic current is a good stimulus, but it never throws involuntary muscle into tetanus; in the heart, strong stimulation will sometimes effect a partial fusion of the beats, but never complete tetanus. The rate of stimulation makes no difference; in fact, very often a rapid rate of stimulation calls forth less rapidly occurring contractions than a slow rate.

It is possible to render the frog's heart quiescent by tying a ligature tightly around the junction of the sinus with tho right auricle, but the heart can be made to contract on stimulating it. It is then found that the latent period is much longer, than in voluntary muscle; if a series of stimuli are applied, say, at intervals of a second or two, each produces a single heart-beat; the successive contractions so obtained show a well-marked staircase (beneficial effect of contraction, see p. 101). The strength of the stimulus in such an experiment does not matter; a minimal stimulus elicits a maximum effect ("all or nothing"-Waller).

The contraction of smooth muscle is so sluggish that the various stages of latent period, shortening and relaxation, can be followed with the eye; the latent period often exceeds half a second in duration. It does not obey the "all or nothing" law.

The normal contraction of voluntary muscle is a kind of tetanus (see p. 104); the normal contraction of cardiac and plain muscle is a much prolonged single contraction. A very valuable piece of evidence in this direction is seen in the experiment on the heart with the physiological rheoscope (see p. 128). Each time the heart contracts the rheoscopic preparation executes a single twitch. 
not a tetanus. This is an indication that the electrical change is a single one, and not a succession of changes such as occurs in tetanus.

But though involuntary muscle cannot be thrown into tetanus, it has the property of entering into a condition of sustained contraction called tonus. We shall have to consider this question again in connection with the plain muscular tissue of the arterioles.

Involuntary muscle when it contracts undergoes thermal and chemical changes similar to those we have dealt with in the case of the voluntary muscles.

Involuntary muscle is usually supplied with two sets of nerves, one of which (accelerator) increases and the other of which (inhibitory) decreases its activity. The nerve-endings in involuntary muscle require a much larger dose of curare to affect them than the endplates in voluntary muscle.

The phenomena of rigor mortis in involuntary muscle have not been so fully studied as in the case of voluntary muscle. It has, however, been shown that the chemical composition of involuntary muscle differs in no noteworthy manner from that of voluntary muscle, and on death the muscle becomes acid; such products as carbonic acid and sarcolactic acid are formed. In the heart, stomach, uterus, and rectum, post-mortem rigidity has been noted, and it probably occurs in all varieties of plain muscle.

Swale Vincent has shown that the characteristic proteins (paramyosinogen and myosinogen) occur in both striped and unstriped muscle, and the heat rigor curves of involuntary muscle are practically identical with those obtained by Brodie (see p. 139). He is inclined to think that the two proteins are formed by the breaking down of a compound protein which in living muscle mainly coagulates at $47^{\circ} \mathrm{C}$. This view is taken by Stewart in reference to striped muscle also, but has been very seriously questioned by $\nabla$. Fürth. The most striking chemical difference between unstriped and striped muscle is seen in the amount of nucleo-protein which they contain. Plain muscle contains six to eight times the amount found in voluntary muscle ; cardiac muscle contains an intermediate quantity. 


\section{CHAPTER XIV}

\section{PHYSIOLOGY OF NERVE}

MANY points relating to the physiology of nerve have been already studied in connection with muscle. But there still remain further questions upon which we have hardly touched as yet.

\section{Classification of Nerves.}

The nerve-fibres which form the conducting portions of the nervous system may be classified into three main groups, according to the direction in which they normally conduct nerve impulses. These three classes are:-

1. Efferent nerve-fibres.

2. Afferent nerve-fibres.

3. Inter-central nerve-fibres.

1. Efferent or centrifugal nerves are those which conduct impulses from the central nervous system (brain and spinal cord) to other parts of the body. When, for instance, there is a wish to move the hand, the impulse starts in the brain, and travels a certain distance down the spinal cord; it leaves the spinal cord by one or more of the spinal nerves, and so reaches the muscles of the hand, which are thrown into contraction. Such nerves are called motor. A list of the classes of efferent nerves is as follows:-
a. Motor.
b. Accelerator.
c. Inhibitory.
d. Secretory.
e. Electrical.
$f$. 'Trophic.

a. Motor nerves. Some of these go to voluntary muscles; others to involuntary muscles, such as the vaso-motor nerves which supply the muscular tissue in the walls of arteries. 
b. Accelerator nerves are those which produce an increase in the rate of rhythmical action. An instance of these is seen in the sympathetic nerves that supply the heart.

c. Inhibitory nerves are those which cause a slowing in the rate of rhythmical action, or it may be its complete cessation. Inhibitory nerves are found supplying many kinds of involuntary muscle; a very typical instance is found in the inhibitory fibres of the heart which are contained within the trunk of the vagus nerve.*

d. Secretory nerves are found supplying many secreting glands, such as the salivary glands, gastric glands, and sweat glands. The impulse which travels down a secretory nerve stimulates secretion in the gland it supplies.

e. Electrical nerves are found in the few fishes which possess electrical organs. The impulse which travels down these nerves causes the electrical organ to be thrown into activity.

f. Trophic nerves are those which control the nutrition of the part they supply.

2. Afferent or centripetal nerves are those which conduct impulses in the reverse direction, namely, from all parts of the body to the central nervous system. When one feels pain in the finger, the nerves of the finger are stimulated, an impulse travels up the nerves to the spinal cord, and then to the brain. The mental process set up in the brain is called a sensation; the sensation, however, is referred to the end of the nerve where the impulse started, and the sensation of pain does not appear to occur in the brain, but in the finger. This is an instance of a sensory nerve; and the terms afferent and sensory may often be used synonymously. The nerves of sensation may be grouped as follows:-

a. The nerves of special sense; such as, of sight, hearing, taste, smell, and touch.

b. The nerves of general sensibility; that is, of a vague kind of sensation not referable to any of the special senses; as an instance, we may take the vague feelings of comfort or discomfort in the interior of the body.

c. Nerves of pain. It is a moot point whether these are anatomically distinct from the others, but there is some evidence that this is the case (see more fully chapters on Sensation)

The words "sensory" and "afferent," however, are not quite synonymous. Just as we may have efferent impulses leaving the brain for the heart or blood-vessels of which we have no con-

* The question has been much debated whether voluntary muscle is provided with inhibitory nerves; they do, however, appear to be present in certain nerves supplying the muscles of the claws of lobsters and similar crustaceans. 
scious knowledge, so also afferent impulses may travel to the central nervous system which excite no conscious feelings. The afferent nerve-tracts to the cerebellum form a very good instance of these.

Then, too, the excitation of many afferent nerves will excite what are called reflex actions. We are very often conscious of the sensations that form the cause of a reflex action, but we do not necessarily have such sensations. Many reflex actions, for instance, occur during sleep; many may be executed by the spinal cord even after it has been severed from the brain, and so the brain cannot be aware of what is occurring.

A reflex action is an action which is the result of an afferent impulse. Thus a speck of dust falls into the eye, and causes movements of the eyelids to get rid of the offending object. The dust excites the sensory nerve-endings in the conjunctiva, an impulse travels to the centre of this nerve in the brain, and from the brain a reflected impulse travels to the muscles of the eyelid. As an instance of a reflex action in which secretion is concerned, take the watering of the mouth which occurs when food is seen or smelt. The nerves of sight or smell convey an afferent impulse to the brain, which reflects, down the secretory nerves, an impulse which excites the salivary glands to activity.

These, however, are instances of reflex action which are accompanied with conscious sensation, but like all pure reflex actions are not under the control of the will.

An instance of a reflex action not accompanied with consciousness is seen in a man with his spinal cord cut across or crushed, so that any communication between his brain and his legs is impossible. $\mathrm{He}$ cannot move his legs voluntarily, and is unconscious of any feelings in them. Yet when the soles of his feet are tickled he draws his legs up, the centre of reflex action being in the grey matter of the lower region of the spinal cord.

For a reflex action, three things are necessary: (1) an afferent nerve, (2) a nerve-centre consisting of nerve-cells to receive the afferent impulse and send out the efferent impulse, and (3) an efferent nerve along which the efferent impulse may travel. If the reflex action is a movement, the afferent nerve is called excito-motor; if it is a secretion, the afferent nerve is called excito-secretory; and similarly, afferent nerves may also be excito-accelerator, excito-inhibitory, etc.

3. Inter-central nerve-fibres are those which connect nervecentres together; they connect different parts of brain, and of the cord to one another, and we shall find in our study of the nervecentres that they are complex in their arrangement. 


\section{Investigation of the Functions of a Nerve.}

There are always two main experiments by which the function of a nerve may be ascertained. The first is section, the second is stimulation.

Section consists in cutting the nerve and observing the loss of function that ensues. Thus, if a motor nerve is cut, motion of the muscles it supplies can no longer be produced by activity of the nerve-centre; the muscle is paralysed. If a sensory nerve is cut, the result is loss of sensation in the part it comes from.

Stimulation of the cut nerve is the opposite experiment. When a nerve is cut across, one piece of it is still connected with the brain or spinal cord; this is called the central end; the other piece, called the peripheral end, is still connected with some peripheral part of the body. Both the central and the peripheral end should be stimulated; this is usually done by means of induction shocks. In the case of a motor nerve, stimulation of the central end produces no result; stimulation of the peripheral end produces a nervous impulse which excites the muscles to contract. In the case of a sensory nerve, stimulation of the peripheral end has no result, but stimulation of the central end causes a sensation, usually a painful one, and reflex actions, which are the result of the sensation.

When a nerve is cut across, there are other results than the loss of function just mentioned; for even though the nerve is still left within the body with a normal supply of blood, it becomes less and less irritable, till at last it ceases to respond to stimuli altogether. This diminution of excitability starts from the point of section and travels to the periphery, but is temporarily preceded by a wave of increased excitability travelling in the same direction (Ritter-Valli law).

This loss of excitability of nerve is accompanied with degenerative changes which are of so great importance as to demand a separate section.

\section{Degeneration of Nerve.}

Suppose a nerve is cut right across, the piece of the nerve left in connection with the brain or spinal cord remains approximately healthy both in structure and functions; but the peripheral piece of the nerve loses its functions and undergoes what is generally called, after the discoverer of the process, Wallerian degeneration. A nerve is made up of nerve-fibres, and each nerve-fibre is essentially a branch of a nerve-cell; when the nerve is cut, the axis cylinders in the peripheral portion are separated from the cells of which they are branches and from which they have grown. These portions of the 
axis cylinders which are cut off from their parent cells die, breaking up into fragments; the medullary sheath of each undergoes a process of disintegration into droplets of myelin, which are ultimately absorbed and removed by the lymphatics. At the same time there is a multiplication of the nuclei of the primitive sheath. This degenerative process begins to be visible microscopically two or three days

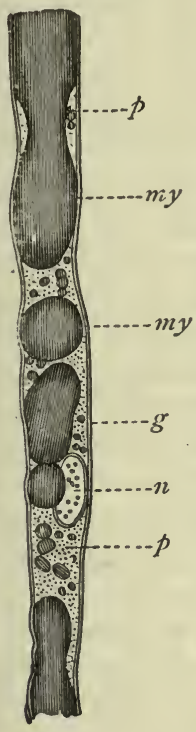

A

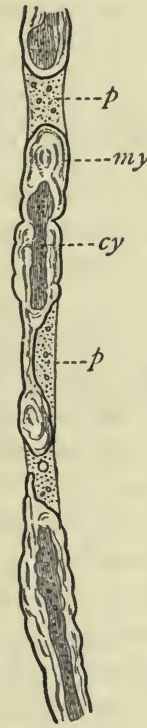

B
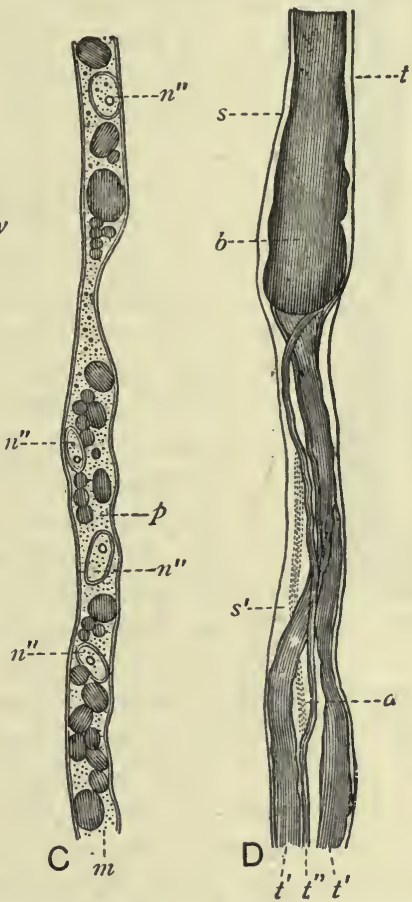

Fia. 153,-Degeneration and regeneration of nerve-fibres. A, nerve-fibre, fifty hours after operation. $m y$, Medullary sheath breaking up into myelin drops. $p$, Granular protoplasm. $n$, Nucleus. $g$, Primitive sheath or neurilemma. B, nerve-fibre after four days. cy, Axis cylinder partly broken up and enclosed in portions of myelin. C, a more advanced stage in which the medullary sheath has almost disappeared. Numerous nuclei, $n^{\prime \prime}$, are seen. $\mathrm{D}$, commencing regeneration; several fibres $\left(t^{\prime}, t^{\prime \prime}\right)$ have sprouted from the somewhat bulbous cut end $(b)$ of the nerve-fibre. $a$, An axis cylinder which has not yet acquired its medullary sheath. $s, s^{\prime}$, Primitive sheath of the original fibre. (Ranvier.)

after the section has been made In the case of the non-medullated fibres, there is no medullary sheath to exhibit the disintegrative changes just alluded to; and the nuclei of the sheath do not multiply; there is simply death of the axis cylinder. The degeneration occurs simultaneously throughout the whole extent of the nerve. Ranvier's original diagram is reproduced in fig. 153. Fig. 154 is drawn from a specimen of degenerated fibres stained by osmic acid: the myelin droplets are coloured black by this method. 
A great amount of attention has been directed to this process of degeneration, because it has formed a valuable method of research in

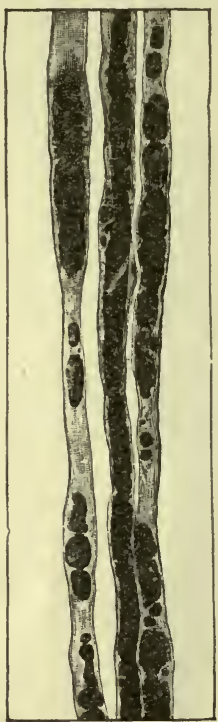

Fı. 154.-Degenerated nervefibres at an early stage, where the fragmentation of the medullary sheath into myelin droplets is well shown. Stained by osmic acid. (S. Martin.) tracing nervous tracts, and ascertaining the nerve-cells from which they originate. It must not, however, be regarded as an isolated phenomenon in physiology; it is only an illustration of the universal truth that any portion of a cell (in this case the axis-cylinder process) cut off from the nucleus of the cell degenerates and dies.

\section{Regeneration of Nerve-Fibres.}

If a nerve is cut and allowed to heal, restoration of function occurs after the lapse of a variable time, which can be shortened if the cut ends of the nerve are sutured together. This surgical assistance is of special importance when the nerve is a large one, and the formation of dense cicatricial tissue between the ends is thus minimised. The restoration of function is due to regeneration of nervefibres, which sprout out from the central end of the cut nerve and grow distalwards, replacing those which have degenerated. The new fibres, which are the earliest to appear, are of a much narrower diameter than those they replace; this is illustrated in fig. 153, D. Later the new fibres are larger. It is obvious that a mass of dense scar tissue will hinder the successful growth of the nerve-fibres. When regeneration does not take place, the central ends of the cut fibres and the cells from which they originate undergo slow atropic changes (disuse atrophy).

The view that in the embryo each nerve-fibre develops as an outgrowth from a nerve-cell, and grows in a distal direction, finally becoming united to other tissues in the periphery of the body, is associated especially with the name of His, and has been accepted by the majority of embryologists. There have been other views held, but it will be sufficient to mention only one of these, for it is the one which, next to that of His, has been favoured by investigators. Briefly it is as follows: the nerve-fibre is not a secondarily formed bridge between the central nervous system and the peripheral organs, but exists from the very first, and in subsequent development it merely undergoes elaboration, and increases in bulk and in length as the distance from the central nervous system and 
the periphery increases with the increasing size of the developing animal.

I shall not fully discuss the pros and cons of this controversy, but only say that the available evidence appears to $m e$ strongly in favour of the first of the two views, and it has within the last fow years been supported by a very remarkable ocular demonstration of its truth. Mr Ross Harrison of the Johns Hopkins University, Baltimore, has actually seen the fibres growing outwards in embryonic structures. Pieces of the primitive nervous tube which forms the central nervous system were removed from frog embryos, and kept alive in a drop of lymph for a considerable time; the cilia of the neighbouring epidermic cells remained active for a week or more; embryonic mesoblastic cells in the vicinity were seen to become transformed into striated muscular fibres, and there was therefore no doubt that even under artificial conditions of this kind-rendered necessary for microscopic purposes-life and growth were continuing. From the primitive nerve-cells, and from these alone, nerve-fibres were observed growing and extending into the surrounding parts. Each fibre shows faint fibrillation, but its most remarkable feature is its enlarged end, which exhibits a continual change of form. This amœboid movement is very active, and it results in drawing out and lengthening the fibre to which it is attached, and the length of the fibre increases at the rate of about a micro-millimetre in one or two minutes. Similar observations have since this been made in the embryos of other animals.

I think these observations show beyond question that the nervefibre develops by the overflowing of protoplasm from the central cells, and thus give us direct evidence in favour of the view which most embryologists previously held mainly as the result of circumstantial evidence. Such, then, being the general state of our knowledge regarding the way in which nerve-fibres grow in the developing animal, it is not surprising to find that the prevalent idea regarding their regeneration after injury follows the same lines. The original teaching of the elder Waller (1852), that regeneration occurs by fibres growing out from the central stump into the peripheral segment of the nerve, was formulated at a time when the relationship of nerve-fibres to nerve-cells was not so fully recognised as it is at present; and the Wallerian doctrine may be accepted with confidence to-day. It has, however, been questioned from time to time, and the earliest to hold an opposite view was Vulpian. Vulpian, working with Philippeaux, cut nerves in young animals, excising long portions so as to prevent the two ends uniting. Some months later they were surprised to find that a number of new perfectly formed nerve-fibres had appeared in the peripheral segment, and that this segment possessed the physiological properties of being excitable and capable of conducting nerve impulses. To this 
phenomenon they gave the name of "autogenetic regeneration." The publication of these results provoked a long controversy, which lasted from 1859 to 1874 , and was closed at the latter date by Vulpian withdrawing his new idea. He did so because in the meanwhile he had repeated his experiments more carefully, and so discovered that, although the ends of the divided nerve had not joined up, connection with the central nervous system had nevertheless been re-established by means of fibres growing into the peripheral segment from other nerves cut through in skin and muscle in the course of the operation.

The controversy has been revived within the last few years, and the position of the disputants has been almost exactly the same as that occupied by Waller and Vulpian half a century ago. Modern investigators have, however, the advantage of being able to apply new methods of research, and are provided with many histological reagents of which the older workers were destitute. It is, however, never safe to argue entirely from microscopic appearances, for nervefibres may be simulated by non-nervous structures, and a strand that looks like a nerve-fibre is not really such unless it can be experimentally shown to be both excitable and capable of conducting nerve impulses.

Vulpian's old doctrine of auto-regeneration has been revived in this country by Ballance and Purves Stewart, and in Scotland by Kennedy. The most prominent and persistent supporter of the autogenetic theory is, however, a German neurologist named Bethe. But none of these investigators have excluded the fallacy which underlay the work of Vulpian and Philippeaux, as has been recently pointed out by Langley and Anderson. These two workers at first thought they also had obtained evidence of purely peripheral regeneration, and it was not until they carried out careful dissections that they convinced themselves that union with the central nervous system had really occurred. The new nerve-fibres which grow into the peripheral segment from other nerves divided in the operation, often do so by a devious and contorted course. If the number of medullated nerve-fibres in the peripheral end is small, then the connection with central fibres was found to be slight; and in cases where no connection occurred then medullated nerve-fibres were entirely absent. Bethe admits a variability in the number of medullated fibres, and this, though easily explicable on the view that such fibres come by accident from the central ends of divided nerves, is not accounted for at all by the autogenetic theory.

Bethe's views have been contested not only by Langley and Anderson, but also by Lugaro, by Kölliker, by Cajal, by Marinesco, by Mott, and Edmunds in conjunction with myself, and by numerous others. 
I may mention a few of the experimental results which have come out of the renewed work elicited by the promulgation of the autogenetic theory.

(1) It is possible entirely to prevent reunion with the central ends of divided nerves. In our own work we accomplished this by removing a long stretch of the main nerve experimented with, by making the skin incision as small as possible, and by inclosing the top end of the peripheral segment in a cap of sterilised gutta-percha. Under such circumstances no auto-regeneration occurs.

(2) Pieces of nerve may be transplanted under the skin, and in time a few fully formed medullated fibres appear within the degener. ated bundle of fibres. This is adduced by Kennedy as undoubted evidence of auto-genesis, but, again, is easily explicable on the hypothesis that the new fibres had wandered in from cutaneous nerves divided in the course of the operation, and we showed that if this fallacy is excluded by transplanting the nerve, not into the subcutaneous tissues, but on to the stomach wall in a sheath of peritoneum, where invasion by nerves is practically impossible, no regeneration occurs at all.

(3) The late appearance of the medullary sheath in those portions of the regenerating fibres which are most distant from the place where the nerve is originally cut and sutured, is a conclusive piece of evidence that the new nerve-fibres grew from the central end in a peripheral direction.

(4) After regeneration has occurred, the nerve may be again cut across, either on the central side of the original point of section (as in Langley and Anderson's work), or on the peripheral side of the original seat of operation (as in our own work). In the former case Wallerian degeneration occurs in all the new fibres, showing that they were all under the nutritive control of the cells of the central nervous system In the latter case the degeneration took place solely on the peripheral side of the second cut. The direction of degeneration is always the direction of growth, so this experiment shows that the growth of the new fibres had not started from the periphery centralwards, but in the reverse direction. On looking up the literature of the subject, I found that Vulpian also did this experiment with the same result, and it can hardly be doubted that this formed one of the factors that later led him to abandon the autogenetic theory. An experiment on somewhat the same lines has been carried out recently by Lugaro: he has shown that regeneration of the cut nerves connected with the lower part of the spinal cord does not occur after that part of the spinal cord has been extirpated. This is a very striking piece of evidence, showing the dependence of the growth of fibres on the activity of the cells of the central nervous system with which they are originally connected. 
Cajal, by the help of his new silver method, has come to the conclusion that the new formation of nerve axons in the peripheral stump is exclusively due to growth from the central end. $\mathrm{He}$

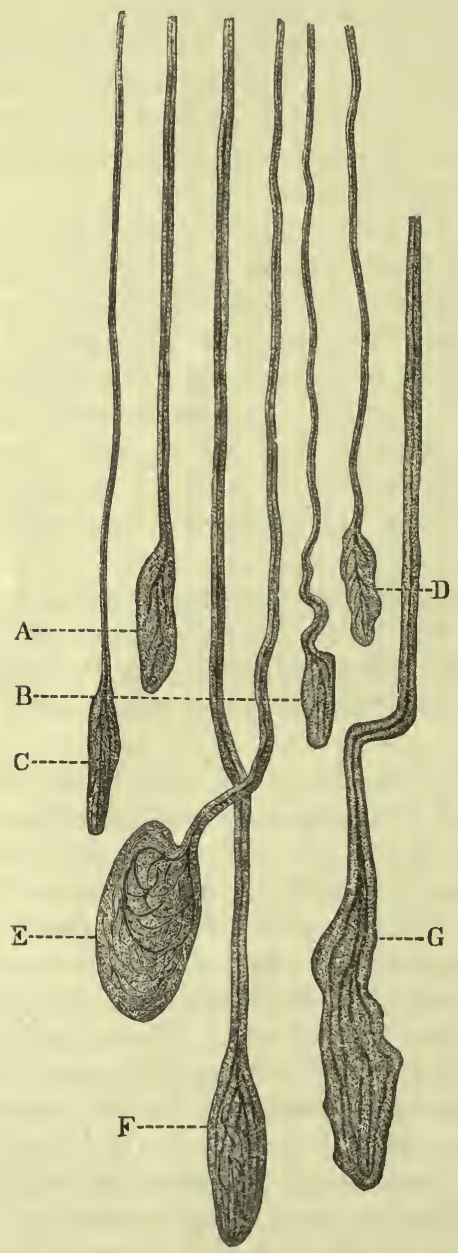

FIG. 155.- Olive-shaped swellings at the ends of nerve-tibres growing distalwards from the central ends twenty-one days after the nerve had been divided. (Marinesco.)

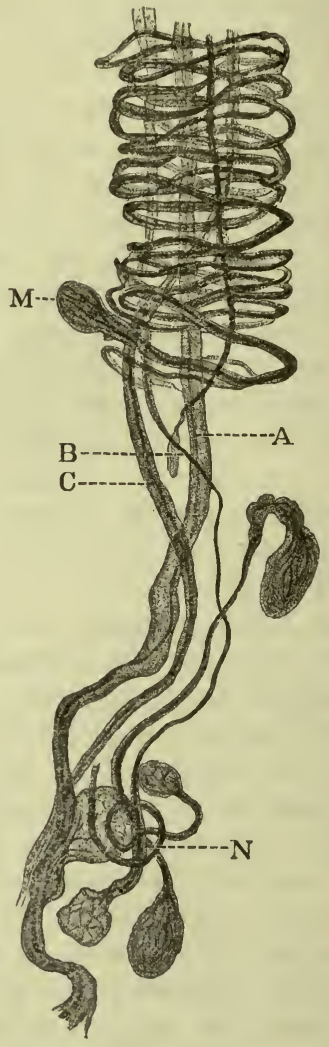

FIG. 156. - Spiral forms often seen in regenerating nerve-fibres: the fibres are seen to be of varying thickness, and each is provided with a terminal swelling. (Marinesco.)

figures the long and often contorted course of these growing fibres in the swelling at the cut central end, and shows that they ultimately reach their goal-the peripheral segment-in time and in spite of all hindrances. The greater the obstacles interposed the later does the 
union and consequent regeneration in the peripheral end occur. $\mathrm{He}$ also draws attention to the olive-shaped swelling at the free end of each growing axis-cylinder. These are also figured by Marinesco, who calls attention to the fact that these terminal swellings, although they may roughly be described as olive-shaped, vary a good deal in external form; this is shown in the accompanying drawings (figs. 155 and 156), and is quite intelligible now that we have Ross Harrison's description of the constant changes of form they exhibit in embryonic history.

The two next figures (157 and 158) are drawn from preparations stained by Cajal's new method, and they require but little comment.

They show the now fibres penetrating the cicatricial tissue of the junction from the central end in a peripheral direction; they show the absence of any new axons developed autogenetically in the peripheral end. Such preparations ought to carry conviction to those who have any lingering belief in auto-regeneration, that the Wallerian view is the only possible one to adopt.

It must not, however, be supposed that the peripheral end is entirely inactive; for while degeneration is progressing in the axons and their fatty sheath, an active multiplication of the cells of the primitive sheath or neurilemma is taking place. These neurilemmal cells probably play a nutritive action towards the more important structures within them, and Graham Kerr, in a recent study of nerve growth in the fish Lepidosiren, has supported in a very conclusive and entirely independent way the view that Mott and I advanced some years ago of the value of the neurilemma in maintaining the nutrition of the axis cylinder. There is but little doubt also that these cells act as phagocytes in the removal of the degenerated products of the other portions of the nerve-fibre. But after this is accomplished they elongate and unite into long chains. It is this appearance that has led some observers into regarding them as true nerve-fibres; they have jumped to the conclusion that the neurilemmal cells are also able to form a conducting core, and so have regarded auto-regeneration as a histological possibility. But all recent observations by the best methods, as I have already stated, have failed to discover either an axial core or a fatty sheath in these "embryonic nbres," as they have been termed. Howell and Huber put it very well twenty years ago, when they said the peripheral structures are able to prepare the scaffolding, but the axon, the essential conducting core of the fibre, has an exclusively central origin.

The change in the neurilemmal cells which occurs in the peripheral segment is even more vigorous at the central termination of the cut nerve; here its nutritive function (or apotrophic function, as Marinesco calls it) is effective, and provides for the nourishment of the actively lengthening axis cylinders. At the peripheral end, 


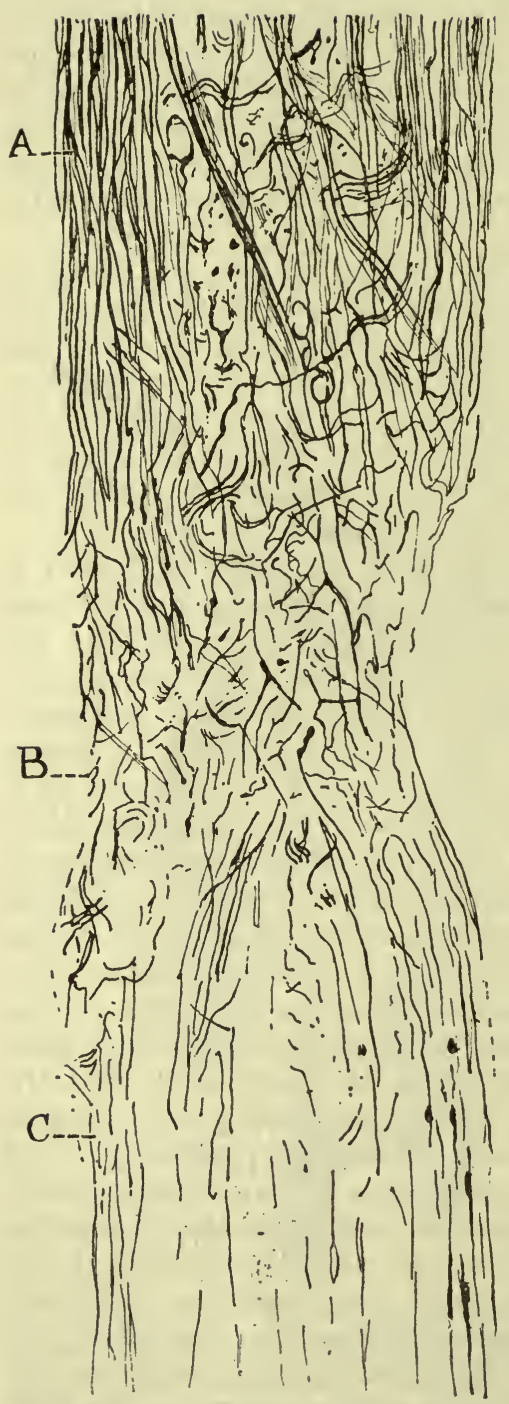

Fra. 157.-Longitudinal section of sciatic nerve of new-born dog eight days after the nerve had been divided. Union had already taken place. At the extremity of the central end (A) growing fibres are seen running in various directions towards the cicatrix (B); $\mathrm{C}$ is the peripheral end, into which a few fibres are already penetrating. (Marinesco.)

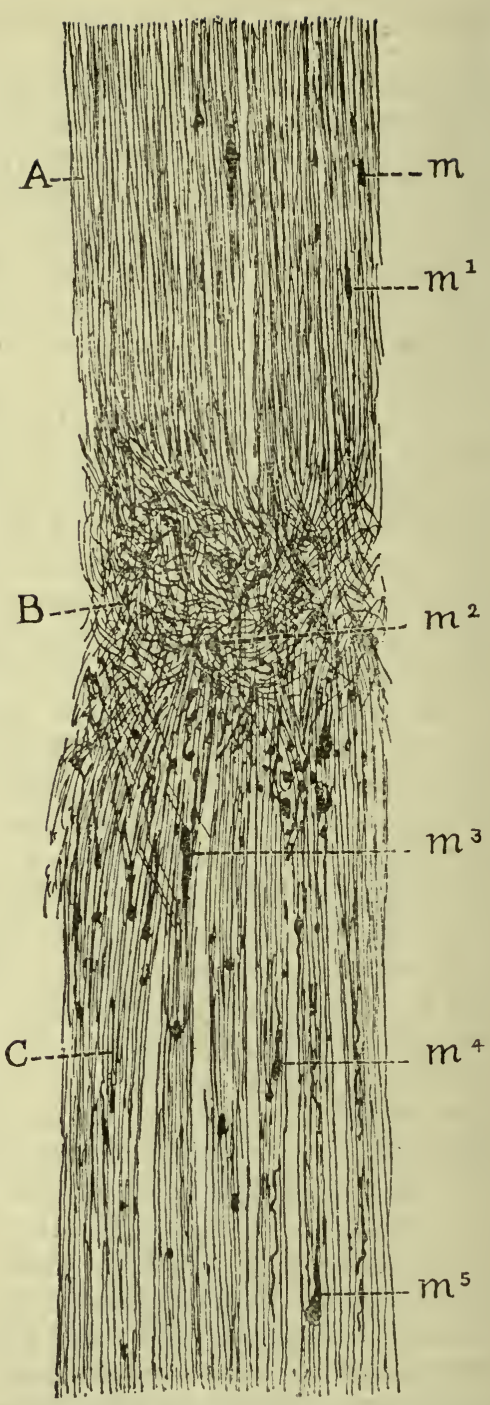

Fig. 158.-Longitudinal section of dog's nerve, twenty-one days after the nerve had been divided. A more advanced condition is seen than that shown in fig. 157. A is the central end; $\mathrm{B}$ the cicatrix of union, where the nervefibres are seen in great numbers taking an irregular course. Into $C$, the peripheral end, many fibres have already successfully pene. trated; $m, m^{\prime}, m^{\prime \prime}$, etc., are the terminal swellings of fibres growing from the central stump and directed towards the peripliery, except in the case of $m^{\prime \prime}$, where the twisting of the new fibre in its efforts to grow had, at the time when the animal was killed, directed the termination backwards. (Marinesco,) 
unless the axons reach it, it is ineffective in so far as any real new formation of nerve-fibres is concerned. If, however, the axons reach the peripheral segment, the work of the neurilemmal cells has not been useless, for they provide the supporting and nutritive elements necessary for their continued and successful growth. Moreover, the neurilemmal activity appears to be essential. In the white fibres of the central nervous system the neurilemma is absent; in this situation not only is the removal of the products of degeneration a very slow process, but regeneration does not occur.

\section{Functions of the Roots of the Spinal Nerves.}

The general truths enunciated in the two preceding sections are well illustrated by the experiments made to determine the functions of the roots of the spinal nerves. Each spinal nerve originates from the spinal cord by two roots. One of these is called the anterior or ventral root: it consists of nerve-fibres which originate from the large multipolar cells in that portion of the grey matter in the interior of the spinal cord which we shall presently learn to call the anterior horn. These nerve-fibres are all medullated; the large ones join up with the posterior root to form the spinal nerve; the small nerve-fibres leave the root and pass to the sympathetic chain, which then distributes non - medullated

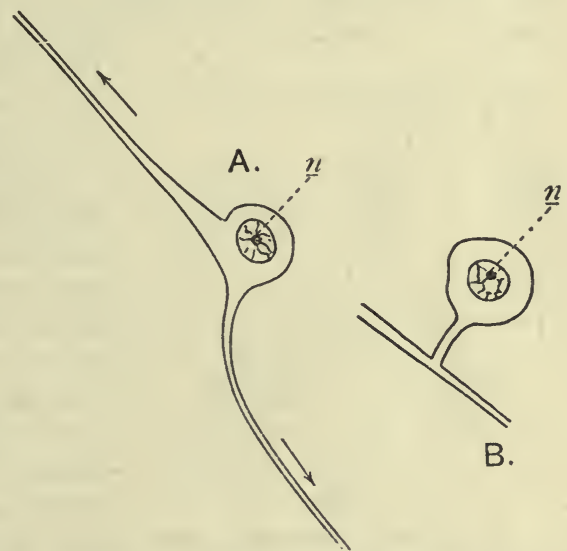

Fra. 159.-A, bipolar cell from spinal ganglion of a $4 \frac{1}{2}$ weeks embryo. $n$, Nucleus; the arrows indicate the direction in which the nerve processes grow, one to the spinal cord, the other to the periphery. B, a cell from the spinal ganglion of the adult; the two processes have coalesced to form a T-shaped junction. (Diagrammatic.)

fibres to the involuntary fibres of the blood-vessels and viscera.

The other root, the posterior or dorsal root, has upon it a collection of nerve-cells forming the spinal ganglion. Each nerve-cell is enclosed within a nucleated sheath of connective-tissue, and it is from these nerve-cells that the fibres of the posterior roots grow. In the embryo, each nerve-cell has two processes (fig. 159, A), one of which grows to the spinal cord, where it terminates by branching around the multipolar cells of the grey matter; the other process grows outwards to the periphery. In the adult mammal (not in fishes) the two processes coalesce in the first part of their course, forming a T-shaped junction (fig. 159, B). 
The great discovery that the anterior roots are motor and the posterior sensory is usually attributed to Sir Charles Bell (1811); but an examination of his writings shows that the deductions he drew were incorrect. It was Magendie (1822) who solved this fundamental problem, and Herbert Mayo, the first Professor of Physiology at King's College, London, who elucidated similar facts in relation to the cranial nerves which supply motion and sensation in the face region. Magendie found that on section of the anterior roots there resulted paralysis of the muscles supplied by the nerves; on section of the posterior roots there was loss of sonsation. These experiments clearly pointed to the conclusion that the anterior roots contain the efferent (motor) fibres; and the posterior roots the afferent (sensory) fibres. This conclusion was confirmed by the experiment of stimulation. Stimulation of the peripheral end of the cut anterior root caused muscular movement; of the central end, no effect. Stimulation of

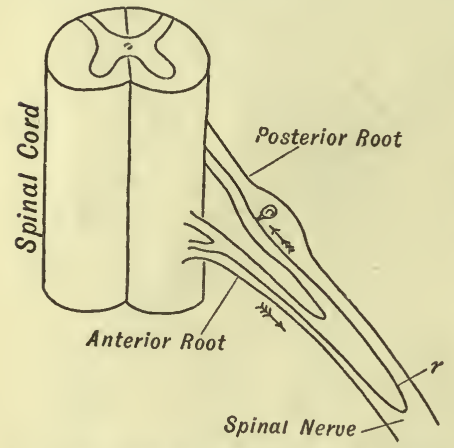

Fra. 160.-Diagram to illustrate recurrent sensibility.

of the sensory fibres. They often, however, run down the mixed nerve a considerable distance before returning to the posterior roots.

The diagram on this page (fig. 160) illustrates the course of one of these recurrent fibres $(r)$; the arrows represent the direction in which it conveys impulses.

Degeneration of roots. - The facts in connection with this subject were made out by Waller, and may be best understood by referring to the next diagram (fig. 161).

A represents a section of the mixed nerve beyond the union of the roots; the whole nerve beyond the section degenerates, and is consequently shaded black in the figure.

$B$ represents the result of section of the anterior root; only the anterior root-fibres degenerate; the sensory fibres of the posterior root remain intact. The small medullated nerve-fibres (not shown in the diagram) also degenerate as far as the ganglion cells of the 
sympathetic system with which they communicate. The recurrent sensory fibres in this root do not degenerate with the others, but are found degenerated in the part of the anterior root attached to the spinal cord.

Section of the posterior root always produces the same physiological effect (loss of sensation)* wherever the section is made, but the degeneration effect is different according as the section is made on the proximal or distal side of the ganglion. If the section is made beyond the ganglion, the degeneration occurs as shown in $\mathrm{C}$ beyond the section in the peripheral portion of

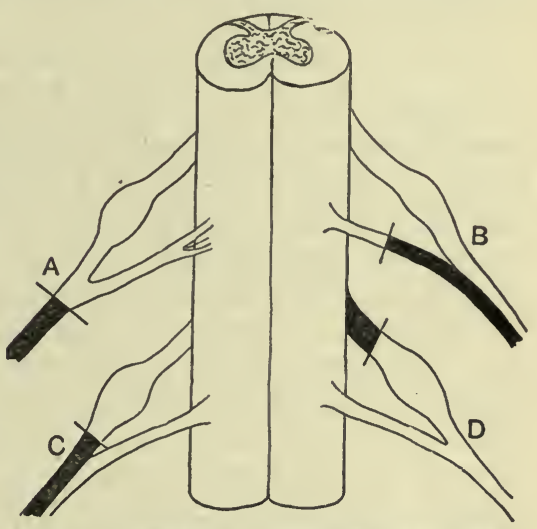

Fig. 161.-Diagram to illustrate Wallerian degene. ration of nerve-roots. the posterior root-fibres; the anterior root remains intact except for

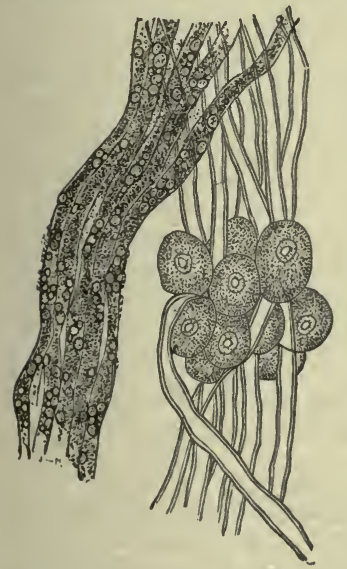

Fig. 162.-Groups of fibres from the anterior and posterior roots several days after sec. tion of both roots close to the cord; the anterior fibres are degenerated; the posterior, being still in connection with the nerve-cells from which they grew, are normal. the recurrent sensory fibres which it contains. If the section is made as in $\mathrm{D}$, between the ganglion and the cord, the only piece that degenerates is the piece severed from the ganglion and running into the cord; these fibres may be traced up in the posterior column of the spinal cord until they terminate in grey matter, which they do at different levels. The whole of the sensory fibres, including the recurrent ones which are still attached to the ganglion, remain histologically healthy.

The accompanying figure (fig. 162) is one of the original illustrations made by $\mathrm{Dr}$ Waller, and I am indebted to the present Dr Waller for permission to reproduce it.

These facts of degeneration teach us, what we also learn from the study of embryology, that the nerve-fibres of the anterior root are connected to the nerve-cells within the spinal cord, while the posterior root-fibres are connected to the cells of the spinal ganglia; or, to put it another way, the trophic centres which

* In order to obtain any appreciable loss of motion or sensation, it is necessary to divide several roots (anterior or posterior as the case may be), as there is a good deal of overlapping in the peripheral distribution of the fibres. 
control the nutrition of the fibres are situated within the cord for the anterior roots, and within the spinal ganglia for the posterior roots.

\section{Changes in a Nerve during Activity.}

When a nerve is stimulated, the change produced in it is called a nervous impulse; this change travels along the nerve, and the propagation of some change is evident from the effects which follow: sensation, movement, secretion, etc.; but in the nerve itself very little change can be detected. There is no change in form; the most delicate thermopiles have failed to detect any production of heat, and we are almost completely ignorant of any chemical changes. The only alteration which can be readily detected as evidence of this molecular change in a nerve is the electrical one. Healthy nerve is iso-electric, but during the passage of a nervous impulse along it there is a very rapid diphasic variation, which travels at the same rate as the nervous impulse. This is similar to the diphasic change in muscle, and can be detected and measured in the same way.

Waller regards the current of action of any excitable tissue as an index of the magnitude of action, and records the movement of the galvanometer by photographing the excursion of the spot of light on a moving photographic plate. He has in this way obtained records from muscle, nerve, retina, skin, plant tissues, etc. He points out that the only available index of action within the nerve itself is this electrical sign of activity, whereas in muscle the mechanical action can be compared with its accompanying electrical changes. The amount of contraction in a muscle caused by excitation of its nerve is only a very rough, or even a fallacious, indication of the excitability of the nerve, because the nerve is connected to the muscle by motor end-plates, and these, as we have already seen, are fatigued long before the nerve shows any sign of fatigue.

Using this method, Waller has obtained a number of interesting results on the variation in nerve action produced by drugs and other agents. He finds that the effect of carbonic acid is to cause a diminution, and finally disappearance of the galvanometric response; when this gas is replaced by air the nerve recovers, and the action-currents increase. Ether acts similarly; but with chloroform recovery is difficult to obtain. Small doses of carbonic acid increase the action-currents, and Waller considers that the staircase effect in muscle (p. 101), and the similar progressive increase noted in the action-currents of nerve as the result of repeated stimulation are due to the evolution of this gas during activity.

This hypothesis has been recently confirmed by Bæyer and Fröhlich. They have shown that peripheral nerves participate in respiratory exchanges, using up oxygen and producing carbonic acid in minute but measurable amounts. In the absence of oxygen, stimulation ceases after some hours to evoke the activity of a nerve, but on readmission of the gas recovery is almost instantaneous. The store of oxygen so obtained will again keep up nervous activity for a considerable time even although no fresh oxygen is supplied. This illustrates the great power nerve has in repairing itself and in storing oxygen.

There can be no doubt that the existence of the electrical variation is as a rule the index of the excitatory alteration in a nerve. But in the present state of our knowledge we are not justified in assuming that it gives an absolutely faithful record. The electrical variation can be detected in a nerve for many days after its removal from the body; although the electrical change is a concomitant of the real excitatory process, the former may be therefore perceptible when other evidence of the existence of the latter fails. Moreover, Gotch and Burch have obtained further evidence of the dissociation of the electrical response from the excitatory process. In the frog's sciatic nerve, it is possible with two stimuli in rapid suc- 
cession to obtain only one electrical response near the seat of excitation which has been cooled, while two such responses occur in a more peripheral warmer region.

Excitability and conductivity. - It is necessary to distinguish between these two properties of nerve. Changes in excitability, and in the power of conducting nerve impulses, do not necessarily go together, as shown in the following experiment :The nerve of a frog's leg is led through a glass tube, the ends of which are sealed with clay, care being taken that the nerve is not compressed. The tube is provided with an inlet and outlet, so that gases may be passed through it. Two pairs of electrodes are arranged, so that the nerve can be stimulated either within or outside the little gas chamber. If carbon dioxide or ether vapour is passed through the tube, both excitability and conductivity are in time abolished, but excitability disappears first; at this stage, if the nerve is stimulated by an induction shock inside the tube, the muscle does not respond, but on stimulating the nerve at the end distant from the muscle and outside the tube, the muscle contracts. The nerve, therefore, is not excitable, though it will conduct impulses. At a later stage shocks administered by either pair of electrodes provoke no contraction. When the poisonous vapour is replaced by air, the nerve recovers, and conductivity returns before excitability. If alcohol vapour is used conductivity is stated to vanish before excitability.

Gotch has shown that cold applied to a nerve acts very much like carbonic acid. Intense cold will cause disappearance of both excitability and conductivity; but cold of such a degree which abolishes the excitability of the nerve to induction shocks, increases its excitability to the constant current, and also to mechanical stimuli.

\section{Velocity of a Nerve Impulse.}

This may be measured, as was first done by Helmholtz, in motor nerves as follows: a muscle-nerve preparation is made with as long a nerve as possible; the nerve is stimulated first as near to the muscle, and then as far from the muscle, as possible. The moment of stimulation and the moment of commencing contraction is measured by taking muscle-tracings on a rapidly moving surface in the usual way, with a time-tracing beneath. The contraction ensues later, when the nerve is stimulated at a distance from the muscle, than in the other case, and the difference in the two cases gives the time occupied in the passage of the impulse along the piece of nerve, the length of which can be easily measured.

A similar experiment can be performed on man by means of the transmission myograph (see p. 105). If a tracing of the contraction of the thumb muscles is taken, the two stimuli may be successively applied through the moistened skin, first at the brachial plexus below the clavicle; and secondly, at the median nerve at the bend of the elbow.

The same method may be employed in man for determining the rate of transmission in sensory nerves. A man is told to make a given signal, such as to open a key in an electrical circuit, when he receives a stimulus such as an induction shock applied to one of his toes; the time between the excitation and the reply is easily measured. A second experiment is then performed in the same way, except that the stimulus is applied to another part of his body; for instance, his knee. The time interval is again measured, and found 
to be shorter; the difference between the time intervals in the two experiments will obviously measure the time occupied by the impulse in traversing a stretch of nerve equal to the distance between his toe and his knee.

Another method, largely employed by Bernstein, is to take the electrical change as the indication of the impulse. A stimulus is applied to one end of a long nerve, and the change in the electrical condition of the nerve is recorded by a galvanometer connected to the other end of the nerve. The time between the application of the stimulus and the galvanometric reply is measured.

The velocity of the nerve impulse has by such experiments been found to vary with temperature, and to be approximately the same in both motor and sensory nerves. In cold-blooded animals it is thus slower than in warm-blooded animals. In the frog, for instance, at ordinary room temperature it averages 27 metres per second. In man, at normal body temperature it is 120 metres per second. In the case of non-medullated fibres the velocity is much slower; these observations have been chiefly made on invertebrate animals; in the non-medullated nerves of the lobster it is 6 , and in the octopus only 2 metres per second, and values lower than these have been recorded in other cases.

\section{Direction of a Nerve Impulse.}

Nerve impulses are conducted normally in only one direction: in efferent nerves from, in afferent nerves to, the nerve-centres. But there are some experiments which point to the conduction occurring under certain circumstances in both directions.

Thus, in the galvanometer experiment just described, if the nerve

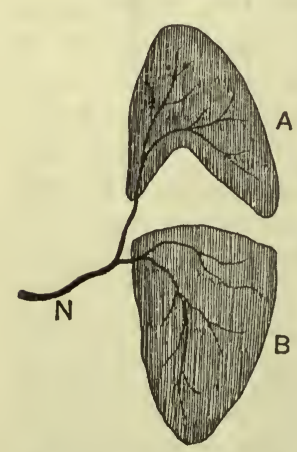

FIG. 163.-Gracilis of frog. (After Waller.) is stimulated in the middle instead of at one end, the electrical change (the evidence of an impulse) is found to be conducted towards both ends of the nerve.

Kühne's gracilis experiment proves the same point. The gracilis muscle of the frog (fig. 163 ) is in two portions, with a tendinous intersection, and supplied by nerve-fibres that branch into two bundles; excitation strictly limited to one of these bundles, after division of the tendinous intersection, causes both portions of the muscle to contract.

Another striking experiment of the same kind can be performed with the nerve that supplies the electrical organ of Malapterurus. This nerve consists of a single axis cylinder and its branches; stimulation of its posterior free end causes the "discharge " of the electrical 
organ, although the nervous impulse normally travels in the opposite direction.

\section{Crossing of Nerves.}

Some experiments designed to prove the possibility of nervous conduction in both directions were performed many years ago by Paul Bert. He grafted the tip of a rat's tail either to the back of the same rat, or to the nose of another. When union had been effected, the tail was amputated near its base. After a time, irritation of the end of the trunk-like appendage on the back or nose of the rat gave rise to sensation. The impulse thus passed from base to tip, instead of from tip to base, as formerly. This experiment does not, however, prove the point at all; for all the original nerve-fibres in the tail must have degenerated, and the restoration of sensation was due to new fibres, which had grown into the tail. Exactly the same objection holds to another series of experiments, in which the motor and sensory nerves of the tongue were divided and united crosswise. Festoration of both movement and sensation does occur, but is owing to new nerve-fibres growing out from the central stumps of the cut nerves.

Though these experiments do not prove what they were intended to, they are of considerable interest in themselves. Dr R. Kennedy has recently carried out a very careful piece of work on this question of nerve-crossing. He cut in a dog's thigh the nerves supplying the flexor and the extensor muscles, and sutured them together crosswise. Regeneration of structure and restoration of function occurred equally quickly, as in those cases in which the central ends had been united to the peripheral ends of their own proper nerves. On examining the cortex of the brain in those animals in which nerve-crossing had been accomplished, it was found that stimulation of the region which in a normal animal gave flexion, now gave extension of the limb, and vice versa.

A series of equally important experiments have also been carried out by Langley, in which he shows that the same facts are true for the nerves that supply involuntary muscle. These nerve-fibres will under certain experimental conditions terminate by arborising around other nerve-cells than those which they normally form connections (synapses)* with. It will be sufficient to give one typical experiment. If the vagus nerve is cut across in the neck, its peripheral end degenerates downwards; if the cervical sympathetic is cut across below the superior cervical ganglion, its peripheral end degenerates upwards, as far as the ganglion. If subsequently the central end of the cut vagus is united to the peripheral end of the

* The meaning of the term "synapse" is fully explained in Chapter XVI. (p. 188). 
cut sympathetic, in the course of some weeks the vagus fibres grow into the sympathetic and form synapses around the cells of the superior cervical ganglion, and stimulation of the united nerve now produces such effects as are usually obtained when the cervical sympathetic is irritated; for instance, dilatation of the pupil, raising of the upper eyelid, and constriction of blood-vessels of the head and neck: (See accompanying diagram, fig. 164.)

Such experiments as these are important because they teach us that though the action of nerves may be so different in different cases (some being motor, some inhibitory, some secretory, some

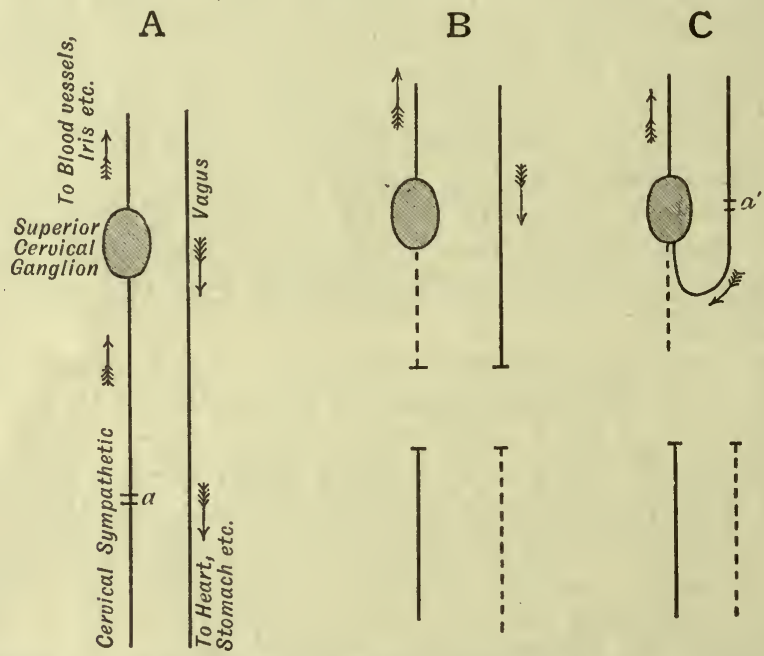

Fig. 164.-Diagram to illustrate Langley's experiment on vagus and cervical sympathetic nerves. In A, the two nerves are shown intact; the direction of the impulses they normally carry is shown by arrows, and the names of some of the parts they supply are mentioned. In B, both nerves are cut through. The degenerated portions are represented by discontinuous lines. In $\mathbf{C}$, the union described in the text has been accomplished, and stimulation at the point $a^{\prime}$ now produces the same results as were in the intact nerves $(\mathrm{A})$ produced by stimulation at $a$.

sensory, etc.), after all what occurs in the nerve trunk itself is always the same; the difference of action is due to difference either in the origin or distribution of the nerve-fibres. If we remember the familiar illustration in which nerve trunks are compared to telegraph wires, we may be helped in realising this. The destination of a certain group of telegraph wires may be altered, and the alteration may produce different consequences at different places; the electric change, however, in the wires would be the same in all cases. So the nerve impulse going along a nerve is always the same sort of molecular disturbance; if it is made as in the experiment just described, to go by a wrong channel, it produces just the same results as though the impulse had reached its destination by the usual channel. 


\section{The Nature of the Nerve Impulse.}

What is the nature of this change which we have provisionally been alluding to as a molecular disturbance? The ancients imagined the nerves were tubes along which a flow of a spiritual essence (animal spirits) took place. We know that this is not the case, but we do not know anything else about it for certain. Theories there are in plenty, but none of them are adequate to explain the phenomenon. The theories fall under two main headings, chemical and physical. In a chemical theory we may compare the transmission of the impulse to the propagation of a flame along a train of gunpowder; but such an analogy is very imperfect, for the gunpowder is entirely consumed, and has not the power to repair itself as a nerve has. Nevertheless there are certain facts which make a chemical theory acceptable; these are :-

(1) Analogy with muscle, where the propagation of the muscular impulse is undoubtedly largely due to the propagation of chemical disturbances.

(2) Evidence that the nerve does undergo metabolic changes, as shown by the necessity for oxygen, and the production of minute amounts of carbon dioxide.

(3) Arrhenius and van 't Hoff showed that a rise of $10^{\circ}$ in temperature increases the velocity of a chemical reaction to two or three times its original rate. Purely physical changes are not accelerated nearly so greatly by the same rise of temperature. Maxwell's recent experiments show that a rise of $10^{\circ} \mathrm{C}$. approximately doubles the velocity of nerve conduction, and the conclusion is drawn that, therefore, the nerve impulse is a chemical phenomenon. Keith Lucas confirmed this observation. Woolley obtained the same figure from the influence of temperature on the rate of conduction in muscle, so probably the conduction process is of a similar nature in both tissues.

The physical theories in relation to this question compare the nerve impulse to the way in which an electrical change is propagated along a wire. When the electrical accompaniment of nervous activity was first discovered this view was unhesitatingly accepted by many physiologists, and the current of action was regarded not as an accidental concomitant of the impulse, but as the change which really constitutes the essence of the impulse, and which serves to excite the chemical and other changes in the tissues to which the nerve is distributed. Two facts, however, stood out at once which rendered the adoption of this simple view difficult; one of these is the slow rate of conduction in nerve; and the other is the phenomenon of inhibition; it is quite conceivable that an electrical disturbance, foeble though it be, can fire off an excitable tissue and lead 
to increase in its activity; it is much more difficult to understand how it can possibly produce a lessening of action such as occurs in inhibition. .Nevertheless the "discharge hypothesis," as it used to be called, has been revived of late in modified form, and electrolytic changes with liberation of ions occurring between the fibrils and the interfibrillar material are supposed to constitute the main feature of the impulse. Macdonald considers that the potassium salts in organic combination within the axis cylinder are the principal materials that undergo the change which is propagated along the nerve; he thus reduces the phenomenon of nervous conduction to electrolytic dissociation and association of inorganic ions. The comparatively slow rate at which the change is propagated must, if this is so, be due to admixture or combination of the salt with the less mobile colloid substances of the conducting core. It is interesting to state, if only in outline, the kind of theories which are in the air at present. We must await with patience to see whether they or any of them contain a germ of truth, or whether, like so many theories in the past, they will be forgotten in the future.

\section{Receptive Substances.}

Langley, as a result of the study of certain poisons on various tissues and organs, has made the interesting suggestion that in all cell-protoplasm two classes of constituents at least are present: (1) a chief substance or substances concerned with the main function of the cell; and (2) receptive substances which may be acted upon by chemical materials, or in certain cases by nervous stimuli. The receptive substance affects, or can affect, the metabolism of the chief substance. A cell, for instance, can contain a motor receptive substance, or an inhibitory receptive substance, or both, and the effect of a nerve impulse will then depend on the proportion of the two kinds of receptive substance which is affected by the impulse.

Receptive substances are at present entirely hypothetical, and we have no knowledge of their chemical composition. The assumption that they exist does, however, explain certain difficulties, particularly in the action of such drugs as nicotine and curare, which are agents that act on nerve-endings in muscle. If the receptive substances really exist, the drugs mentioned probably act on them and not on the nerve-endings proper.

In support of the new theory, Dixon has shown that chemical substances are produced in the heart during inhibition which can be dissolved out by alcohol, and then used to produce inhibition in another heart.

The theory is an attractive one, but is not much more than a theory at present. If, however, a muscle is rendered active by the 
production of a chemical material which plays the part of a stimulus, and if it is rendered inactive by the production of chemical changes of an opposite kind, we really only throw the difficulty further back; for we do not know how it is that the nervous impulses produce these chemical effects on the receptive substance or substances.

\section{Chemistry of Nervous Tissues.}

Fresh nervous tissue is alkaline to litmus, but like most other living structures, it turns acid after death; this change is more rapid in grey than in white matter. The acidity is due to sarcolactic acid.

Nervous tissues contain a high percentage of water; the following table gives the mean of a large number of analyses I have made:-

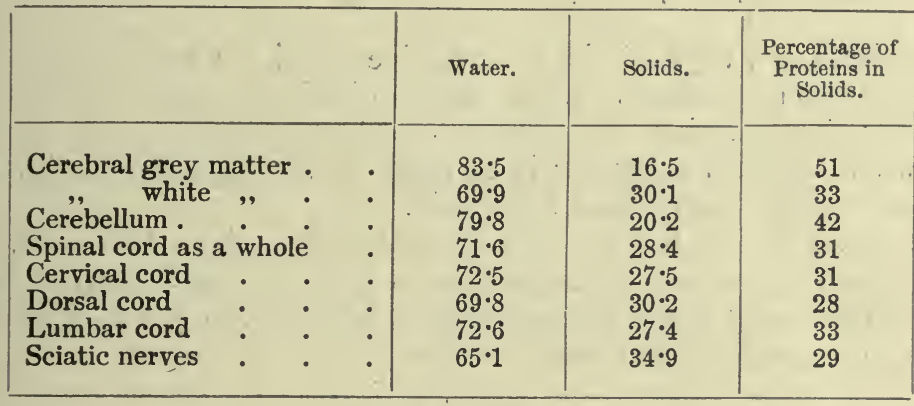

Proteins. The above table shows also the high percentage of protein which is present. In grey matter where the cells are prominent structures this is most marked, protein here comprising more than half of the solids present.

The most abundant protein is nucleo-protein, and micro-chemical observations have shown that the granules in nerve-cells (Nissl's granules) which stain readily with methylene blue and other basic dyes are nucleo-protein in nature. There is also a certain amount of globulin, which, like the paramyosinogen of muscle, is coagulated by heat at a low temperature (in mammals $47^{\circ} \mathrm{C}$ ). Neurokeratin, which is especially abundant in white matter, is also present.

A nerve, or a strip of the central nervous system, shortens when it is heated; this "heat contraction" occurs in a series of steps, which, as in the case of muscle, take place at the coagulation temperatures of the proteins present. The first step in the shortening occurs in the frog at about $40^{\circ}$, in the mammal at about $47^{\circ}$, and in the bird at about $52^{\circ} \mathrm{C}$. The nerve is killed at the same temperatures.

Lipoids. These are also abundant constituents of nervous tissue; 
they will be more fully studied in Chapter XXVIII. ; we will therefore for the present merely state that they comprise:-

1. Phosphatides, or phosphorised fats. Of these lecithin is the best known; kephalin and sphingornyelin are others.

2. Galactosides; these are nitrogenous glucosides free from phosphorus; they yield on hydrolysis the reducing sugar galactose.

3. Cholesterin or cholesterol, a crystalline monatomic alcohol of the terpene series. Its formula is $\mathrm{C}_{27} \mathrm{H}_{45} \mathrm{OH}$.

The following are some recent analyses of nerve by Falk, the numbers given are percentages of the total solids :-

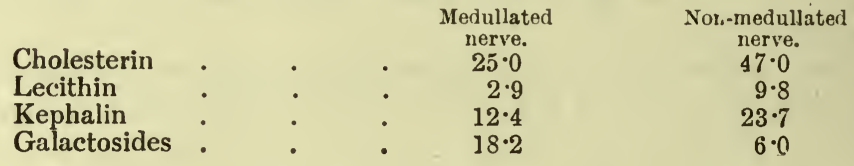

Lecithin is a type of the phosphatides, and we may contrast its decomposition products with those obtained from a fat. An ordinary fat contains the elements carbon, hydrogen, and oxygen, and when it takes up water it is split or hydrolysed into its constituent parts, glycerin and fatty acid.

Lecithin $\left(\mathrm{C}_{42} \mathrm{H}_{84} \mathrm{NPO}_{9}\right)$ contains not only carbon, hydrogen, and oxygen, but nitrogen and phosphorus as well. When it is hydrolysed, it yields not only glycerin and a fatty acid, but also phosphoric acid, and a nitrogenous base termed choline.

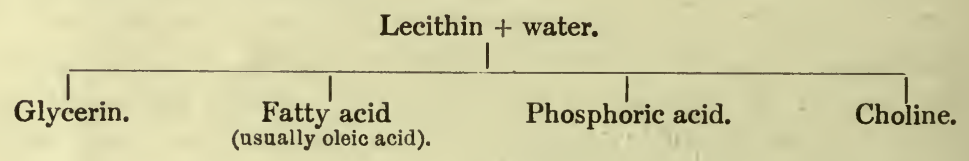

Choline is an ammonium-like base, which contains three methyl $\left(\mathrm{CH}_{3}\right)$ groups. Its formula is $\mathrm{N}\left(\mathrm{CH}_{3}\right)_{3} \mathrm{CH}_{2} \cdot \mathrm{CH}_{2}(\mathrm{OH})_{2}$, and when it breaks up, trimethylamine $\mathrm{N}\left(\mathrm{CH}_{3}\right)_{3}$ is one of its decomposition products.

Extractives. Small quantities of numerous other organic substances are included under this general term ; creatine, xanthine, hypoxanthine, inosite, lactic acid, uric acid, and urea have been identified.

Inorganic salts. The proportion of mineral salts amounts to a little more than 1 per cent. of the total solids. Potassium salts are the most abundant. We have already noted that Macdonald attributes many of the phenomena of nervous action to electrolytic changes in these potassium salts, though his views on that question should for the present be accepted with caution (p. 164).

Macallum uses for the micro-chemical detection of potassium an acid solution of cobalt nitrite, and precipitates in silu the yellow hexanitrate of cobalt and potussium, 
which is turned black on the addition of ammonium sulphide. His principal results are :-potassium is found in cell protoplasm, but more abundantly in intercellular material; in striped muscle it is limited to the dark bands, and in pancreatic cells to the granular zone. It is not discoverable in any nuclei, nor in nerve-cells, but in nerve-fibres is found in patches external to the axis cylinder. Macdonald points out that these are spots which have bcen injured, and it is apparently only on injury that the potassium is liberated in a form which renders it detectable by Macallum 's reagent.

Chemical changes in nervous tissues during activity. This is an almost unknown field. No change of reaction can be detected in nerves after the most prolonged stimulation. The only thing known for certain is that oxygen is essential, especially for the activity of grey matter; cerebral anæmia is rapidly followed by loss of consciousness and death. The slight respiratory changes which can be detected in peripheral nerves have already been considered on p. 158 . It can hardly be doubted that the phosphatides, which are extremely labile substances, participate in metabolism; and Hans Meyer has pointed out that anæsthetics such as chloroform and ether are soluble in lipoids, and that this interaction may by lessening oxidative processes lead to the production of unconsciousness.

Chemical changes in degenerative conditions. In Wallerian degeneration of nerve, several investigators have attempted to discover how the degenerated nerve differs from a healthy nerve. Little or no change in the peripheral end can be detected up to about three days after a nerve has been divided, and the nerve-fibres remain excitable up to that time. They then show a progressive increase in the quantity of water, and a corresponding decrease in the proportion of solids. The percentage of phosphorus also decreases, and it entirely disappears in a little more than three weeks after the nerve is cut. When regeneration occurs, the nerves return approximately to their previous composition.

It has also been shown that in spinal cords in which a unilateral degeneration of the pyramidal tract has been produced by a lesion in the opposite cerebral hemisphere, there is a similar increase of water and diminution of phosphorus on the degenerated side. Further, in a divided nerve Noll has shown that the phosphorised material also diminishes somewhat in the central end, due to "disuse atrophy."

This disappearance of phosphorus must be due to the break-up of phosphatides, and the liberation of phosphoric acid which is carried away as phosphates by the lymph and blood.

The staining reactions of a degenerated nerve also indicate that the appearances are not only due to a breakdown in an anatomical sense, but in a chemical sense also. Of these staining reactions the one most often employed is that which is associated with the name of Marchi. This is the black staining which the medullary sheaths 
of degenerated nerve-fibres show when after being hardened in Müller's fluid they are treated with Marchi's reagent, a mixture of Müller's fluid, and osmic acid. Healthy nerve-fibres are not blackened by this reagent, because the more rapidly penetrating chromic acid of the Müller's fluid has already supplied the unsaturated oleic acid radical in the lecithin and other phosphatides with all the oxygen they can take up. But when the nerve is degenerated, the oleic acid is either increased in amount, or so liberated from its previous combination in the lecithin molecule, that it is then able also to take oxygen from osmic acid and reduce it to a lower black oxide. In the later stages of degeneration the Marchi reaction is not obtained, because the fat globules have then been absorbed.

In certain diseases of the central nervous system, such as general paralysis of the insane, degeneration occurs on a large scale, and the products of the chemical disintegration of the cerebral tissue have been sought for in the blood, but with more profitable results in the cerebro-spinal fluid. This fluid under those circumstances shows an excess of protein which is mainly nucleo-protein; cholesterin can also be usually detected in the fluid, and so also can choline or some similar base which originates from the decomposition of phosphatides. Although many physiologists have taken up the choline question and the methods for identifying this base, it must be admitted that the tests hitherto devised are not absolutely conclusive, 'for sufficient of the base cannot be collected for a complete analysis. The base which is present if not choline is a nearly related substance, perhaps a derivative of choline, and according to the latest researches the questionable material is trimethylamine, which we have already seen is a cleavage product of choline.

Cerebro-spinal fluid. This plays the part of the lymph of the central nervous system, but it differs considerably from all other forms of lymph. It is a very watery fluid, containing besides some inorganic salts similar to those of the blood a trace of protein matter (globulin) and a small amount of sugar. Normally it contains neither nucleo-protein, cholesterin, or choline, and practically no cells. Colourless corpuscles, however, occur in it in inflammatory conditions. There is no doubt that the fluid is a true secretion, and that the cells which secrete it are the cubical epithelial cells which cover the choroid plexuses. The choroid structure may indeed be spoken of as the choroid gland; only it differs from other glands in having the secreting epithelium on its outer surface. Injection of an extract of the choroid plexuses into the circulation causes a very rapid increase in the flow of the cerebro-spinal, fluid which can be collected from a cannula thrust into the subcerebellar space, or into the lumbar region of the spinal canal. 


\section{CHAPTER XV}

\section{ELECTROTONUS}

WHEN a constant current is thrown into a nerve, there is an excitation which leads to a nervous impulse, and this produces a contraction of the muscle at the end of the nerve. Similarly, there is another contraction when the current is taken out. While the current is flowing through the nerve, the muscle is quiescent. But while the current is flowing there are changes in the nerve, both as regards its electrical condition and its excitability. These changes are summed up in the expression electrotonus.

In the investigation of this subject the instruments employed are the same as those already studied, with the addition of two others that it will be convenient to describe before passing on to the study of electrotonus itself. These are the reverser or commutator, and the rheochord.

Pohl's commutator is the form of reverser generally employed. It consists of a block of ebonite provided with six pools of mercury,
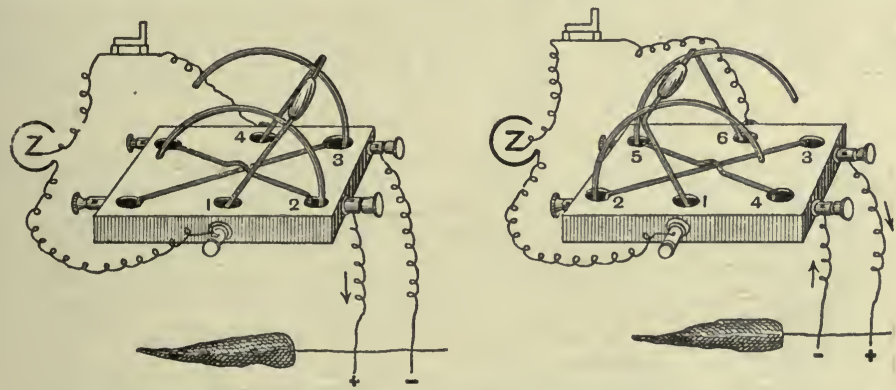

Fic. 165.-Pohl's Commutator, with cross wires. (After Waller.)

each of which is provided with a binding screw. The corner pools are connected by diagonal cross wires, and. by a cradle consisting of an insulating handle fixed to two arcs of copper wire which can be tilted so that the two middle pools can be brought into communication with either of the two lateral pairs of pools. Fig. 165 shows how, by 
altering the position of the cradle, the direction of the current from one electrode to the other is reversed. The numbers 1,2 , 3 , etc., indicate the path of the current in the two cases.

Sometimes the reverser is used without the cross wires for a different purpose. The battery wires are connected as before with the middle mercury pools. Each lateral pair of pools is connected by wires to a pair of electrodes. 'The two pairs of electrodes may be applied to two portions of a nerve, or to two different nerves, and by tilting the cradle to right or left the current can be sent through one or the other pair of electrodes.

The rheochord is an instrument by means of which the strength of a constant current passed through a nerve may be varied. It consists of a long wire $(r, r, r)$ of high resistance stretched on a board. This is placed as a bridge on the course of the battery current. (See fig. 166.) The current is thus divicled into two parts: one part through

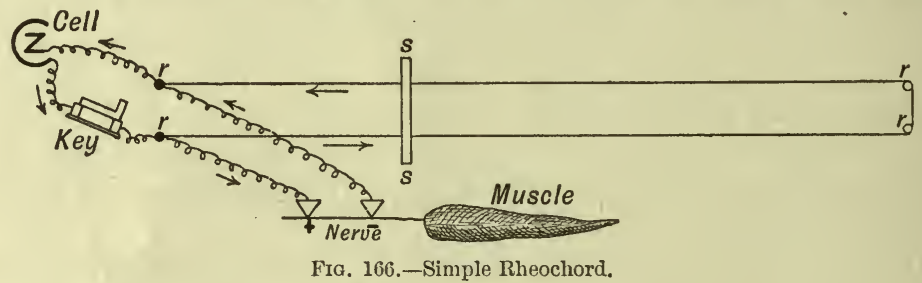

the bridge, the other through the nerve, which is laid across the two non-polarisable electrodes at the ends of the wires. The resistance through the bridge is varied by the position of the slider $(s s)$. The farther the slider is from the battery end of the instrument the longer is the bridge, and the higher its resistance, so that less current goes that way and more to the nerve.

The next figure shows the more complicated form of rheochord invented by Poggendorf. The number of turns of wire is greater, so

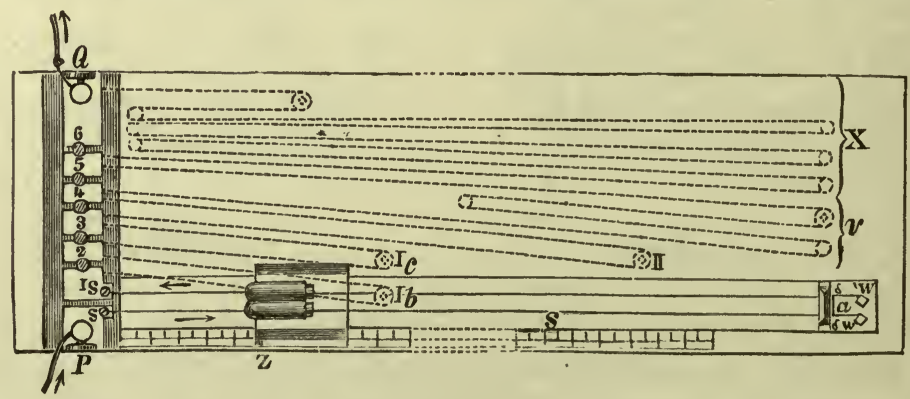

Fig. 167.-Poggendorf's Rheochord.

that the resistance can be varied to a much greater extent than in the simpler form of the instrument. 
The term "electrotonus" includes two sets of changes in the nerve; first an electrical change, and secondly changes in excitability and conductivity. We will take the electrical change first.

Electrotonic currents.-The constant current is passed through the nerve from a battery, non-polarisable electrodes being used; it is called the polarising current. If portions of the nerve beyond the electrodes are connected ("led off") as in the diagram (fig. 168) by non-polarisable electrodes to galvanometers, a current will in each case be indicated by the swing of the galvanometer needles. The electrotonic current in the neighbourhood of the negative pole or kathode is called the katelectrotonic current; and that in the neighbourhood of the anode is called the anelectrotonic current. In both cases the electrotonic current has the same direction as the polarising current. These currents are dependent on the physical integrity of medullated

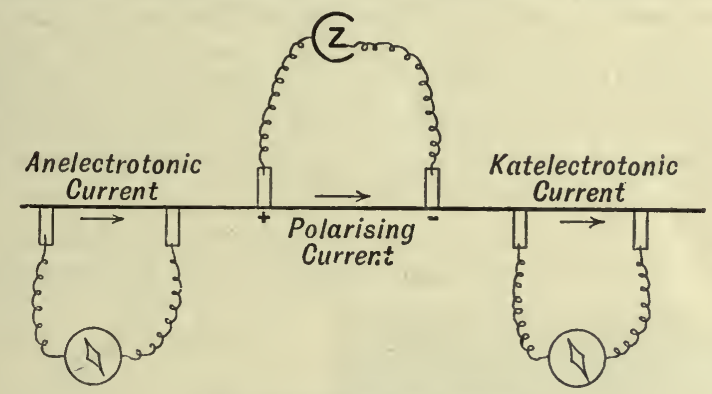

F1c. 168.-Electrotonic currents.

nerve; they are not found in muscle, tendon, or non-medullated nerve; they are absent or diminished in dead or degenerated nerve. They can, however, be very successfully imitated in a model made of zinc wire encased in cotton soaked with salt solution. The electrotonic currents must be carefully distinguished from the normal current of action, which is a momentary change rapidly propagated with a nervous impulse which may be produced by any method of stimulation. The electrotonic currents are produced only by an electrical (polarising) current; they vary in intensity with the polarising current, and last as long as the polarising current passes through the nerve.

After the polarising current is removed, after-electrotonic currents occur in different directions in the three regions tested.

(a) In the intrapolar region, the after-current is opposite in direction to the original polarising current; unless the polarising current is strong and of short duration, when it is in the same direction.

(b) In the katelectrotonic region, the after-current has the same direction as the katelectrotonic current.

(c) In the anelectrotonic region, the after-current has at first the same, then the opposite direction to the anelectrotonic current. 
The experiment known as the paradoxical contraction depends upon electrotonic currents. The sciatic nerve of the frog divides in the lower part of the thigh into two parts. If one division is cut across, and its central end stimulated electrically (the spinal cord having been previously destroyed), the muscles supplied by the other branch contract, because the nerve-fibres in this branch have been stimulated by the electrotonic variation in the divided branch.

This experiment must be carefully distinguished from Kühne's gracilis experiment described on p. 160. In the gracilis experiment the nerve-fibres themselves branch, and any form of stimulation applied to one branch will cause contraction of both halves of the muscle. In the paradoxical contraction, the bundles of nerve-fibres are merely bound side by side in the sciatic trunk; there is therefore no possibility of conduction of a nerve impulse in both directions; the stimulus, moreover, must be an electrical one.

Electrotonic alterations of excitability and conductivity.When a constant current is passed through a nerve, the excitability and conductivity of the nerve are increased in the region of the kathode, and diminished in the region of the anode. When the current is taken out these properties are temporarily increased in the neighbourhood of the anode, and diminished in that of the kathode.

This may be shown in the case of a motor nerve by the following experiment. The next diagram represents the apparatus used.

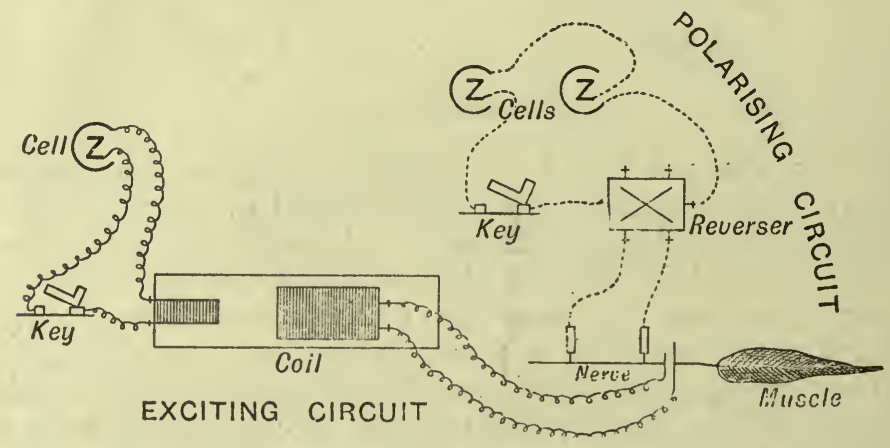

Fro. 169.-Diagram of apparatus used in testing electrotonic alterations of excitability.

An exciting circuit for single induction shocks is arranged in the usual way, the exciting electrodes being placed on the nerve near the muscle. A polarising circuit is also arranged, and includes a battery, key, and reverser; the current is passed into the nerve by means of non-polarisable electrodes. When the polarising current is thrown into the nerve, or taken out, a contraction of the muscle occurs, but these contractions may be disregarded for the present.

The exciting circuit is arranged with the secondary coil so far from the primary that the muscle responds to break only, and the tracing 
may be recorded on a stationary blackened cylindèr. The cylinder is moved on a short distance, and this is repeated. The height of the lines drawn may be taken as a measure of the excitability of the nerve. The polarising current is then thrown in, in a descending direction (i.e. towards the muscle); the kathode is thus the non-polarisable electrode near to the exciting electrodes. While the polarising current is flowing, take some more tracings by breaking the exciting current. The increase in the excitability of the nerve is shown by the much larger contractions of the muscle; probably a contraction will be obtained now at both make and break of the exciting current. After removing the polarising current, the contractions obtained by exciting the nerve will be for a short time smaller than the normal, but soon return to their original size.

Exactly the reverse occurs when the polarising current is ascending, i.e. from the muscle towards the spinal cord. The non-polarisable electrode near the exciting electrodes is now the anode. While the polarising current is passing, the excitability of the nerve is diminished so that induction shocks which previously produced contractions of a certain size, now produce smaller contractions, or none at all. On removing the polarising current, the after-effect is increase of excitability.

The following figure is a reproduction of a tracing from an actual experiment. The after-effects are not shown. $\mathrm{N}$ represents a series of contractions obtained when the nerve is normal, $K$ when it is katelectrotonic, $A$ when it is anelectrotonic.

Exactly similar results are obtained if one uses mechanical stimuli instead of induction shocks. The best mechanical form of stimulus is to allow drops of mercury to fall on the nerve.

The same is true for chemical stimuli. If the exciting electrodes are removed, and salt sprinkled on the

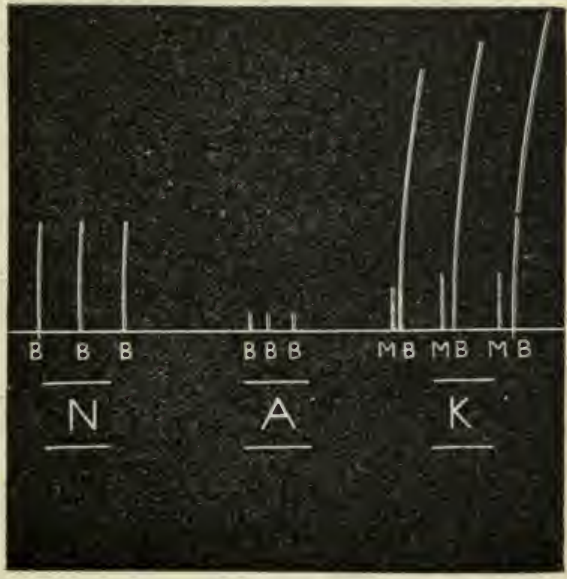

Fio. 170.-Electrotonus. M, make. B, break. nerve near the muscle, the latter soon begins to quiver; its contractions are increased by throwing in a descending and diminished by an ascending polarising current.

The increase in irritability is called katelectrotonus, and the decrease is called anelectrotonus. The accompanying diagram (fig. 
171) shows how the effect is most intense at the points $(a, k)$ where the electrodes are applied, and extends in gradually diminishing intensity on each side of them. Between the electrodes the increase shades off into the decrease, and it is evident that there must be a

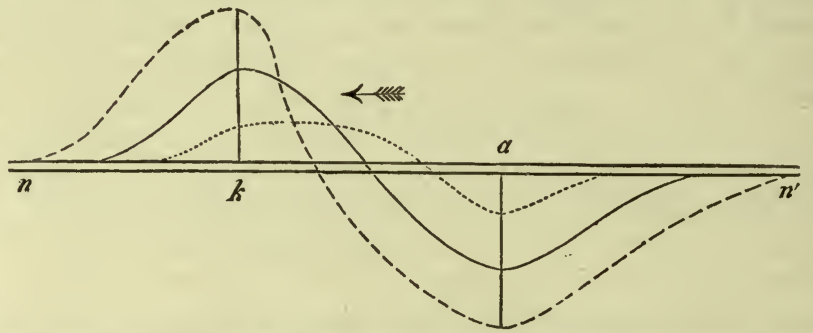

FIG. 171.-Diagram illustrating the effects of various intensities of the polarising current. $n, n^{\prime}$, Nerve, $a$, anode $; k$, kathode ; the curves above indicate increase, and those below decrease of irritability; and when the current is small the increase and decrease are both small, with the neutral point near $a$, and as the current is increased in strength, the changes in irritability are greater, and the neutral point approaches $k$.

neutral point where there is neither increase nor decrease of irritability. The position of this neutral point is found to vary with the intensity of the polarising current-when the current is weak the point is nearer the anode, when strong nearer the kathode.

Pflüger's law of contraction.- The constant current sometimes causes a contraction both at make and break, sometimes at make only, sometimes at break only. The difference depends on the strength and direction of the current; and follows from the electrotonic changes of excitability and conductivity we have been studying. Increase of excitability acts as a stimulus; so that at the make the kathode is the stimulating electrode, and at the break the anode is the stimulating electrode.

The facts may be demonstrated in the following way (fig. 172);

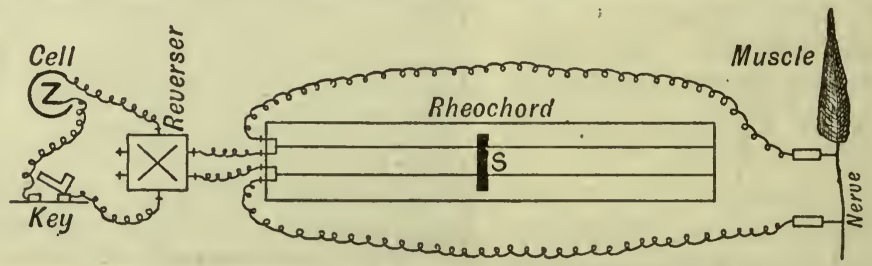

Fia. 172.-Arrangement of apparatus for demonstrating Pfliiger's law.

from a battery lead the wires to the middle screws of a reverser (with cross wires), interposing a key; from one pair of end screws of the reverser lead wires to the binding screws of the rheochord; from these same screws of the rheochord the non-polarisable electrodes lead to the nerve of a nerve-muscle preparation. The strength of the current 
is varied by the slider $\mathrm{S}$. The nearer $\mathrm{S}$ is to the binding screws tho less is the resistance in the rheochord circuit, and the less the current through the nerve. With a weak current, a contraction occurs at make only. With a stronger current (ascending or descending) contraction occurs both at make and break. With a very strong current (six Groves), the contraction occurs only at make with a descending current; and only at break with an ascending current.

The contractions produced in the muscle of a nerve-muscle preparation by a constant current have been arranged in a table which is known as Pflüger's Law of Contraction.

\begin{tabular}{|c|c|c|c|c|c|}
\hline \multirow{2}{*}{\multicolumn{2}{|c|}{$\begin{array}{l}\text { STRENGTH OF } \\
\text { CURRENT USED. }\end{array}$}} & \multicolumn{2}{|c|}{ DesCENDING CURRENT. } & \multicolumn{2}{|c|}{ ASCENDING CURRENT. } \\
\hline & & Make. & Break. & Make. & Break. \\
\hline $\begin{array}{l}\text { Weak . } \\
\text { Moderate } \\
\text { Strong. }\end{array}$ & $\dot{.}$ & $\begin{array}{l}\text { Yes. } \\
\text { Yes. } \\
\text { Yes. }\end{array}$ & $\begin{array}{l}\text { No. } \\
\text { Yes. } \\
\text { No. }\end{array}$ & $\begin{array}{l}\text { Yes. } \\
\text { Yes. } \\
\text { No. }\end{array}$ & $\begin{array}{l}\text { No. } \\
\text { Yes. } \\
\text { Yes. }\end{array}$ \\
\hline
\end{tabular}

The increase of irritability at the kathode when the current is made is greater, and so more potent to produce a contraction than the rise of irritability at the anode when the current is broken; and so with weak currents the only effect is a contraction at the make. But when the strength of the current is increased the rise of excitability is in all cases sufficient to provoke a contraction (moderate effect in above table). The alteration in conductivity is not sufficient to prevent the impulses being propagated to the muscle.

With strong currents the case is a little more complicated, because here the diminution of conductivity is so. great that certain regions of the nerve become impassable by nerve impulses. When the current has an ascending direction, the impulse at the break is started at the anode, and as this is next to the muscle there is no hindrance to the propagation of the impulse, but at the make the impulse started at the kathode is blocked by the extreme lowering of conductivity at the anode. When the current is descending the kathode is near the muscle, and so the impulse at make reaches the muscle without hindrance; but at the break, the impulse started at the anode has to traverse a region of nerve, the conductivity of which is so lessened that the excitation is not propagated to the muscle.

G. N. Stewart has stated in opposition to the foregoing statements that at the make conductivity is most lowered at the kathode, and at the break at the anode. In other words, conductivity and excitability vary in opposite directions. His results have, however, not been accepted by other physiologists, and are due to a complex set of excitatory and polarisation changes produced by the galvanometric methods he adopted. Gotch's much more trustworthy experiments with the 
electrometer are directly opposed to those of Stewart. , The following simple experiment devised by Gotch appears to be quite conclusive that conductivity like excitability is lessened at the anode when the current is made. Three non-polarisable electrodes are employed (fig. 173), the current is first closed from $\mathrm{A}_{2}$ to $\mathrm{K}$, and the time which intervenes before the muscle contracts is measured; it is then closed from $A_{1}$ to $K$, and the time again measured. In both cases, excitation occurs at $K$, but the time of response in the second case $\left(A_{1}\right.$ to $\left.K\right)$ is longer, because in that case the nerve impulse has to traverse a region of nerve at $A_{1}$ in which the power of conduction is lessened.

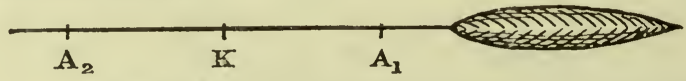

Fig. 173.-Diagram to illustrate Gotch's experiment with triple electrodes.

Sometimes (when the preparation is specially irritable) instead of a simple contraction a tetanus occurs at the make or break of the constant current. This is due to chemical (electrolytic) changes produced by the current, and is liable to occur at the break of a strong ascending current which has been passing for some time into the preparation, or at the make of a strong descending current; both being conditions which increase the excitability of the piece of nerve nearest to the muscle; this is called Ritter's tetanus, and may be stopped in the first case by throwing in the current in the same direction, or in the second case by throwing in a current in the opposite direction, i.e., by conditions which lessen the irritability of this piece of nerve.

The same general laws hold for muscle as well as for nerve, but are more difficult to demonstrate; the main fact, however, that the kathode is the stimulating electrode at the make, and the anode at the break, may be easily shown by the following experiment: if a curarised, that is, a physiologically nerveless muscle, is arranged, as in the experiment, for demonstrating the muscle-wave (see fig. 122, p. 100), and a non-polarisable electrode placed at each end, the musclewave at the make of a constant current starts at the kathode and at the break it starts at the anode.

An induced current in the secondary circuit of an inductorium may be regarded as a current of such short duration that the opening and closing are fused in their effects. This is true for all induction currents, whether produced by the make or break of the primary circuit. The kathode will always be the more effective in causing contraction.

\section{Response of Human Muscles and Nerves to Electrical STIMULATION.}

Perhaps the most important outcome of this study of the response of muscle and nerve to electrical stimulation is its application to the muscles and nerves of the human body, because here it forms a most valuable method of diagnosis in cases of disease. 
In the normal state, nerves can be stimulated through the moistened skin either by induction shocks, or by the make and break of a constant current. In the case of the motor nerves this is shown by the contraction of the muscles they supply; and in the case of the sensory nerves by the sensations that are produced. In the case of the sensory nerves, the sensation produced by the constant current is most intense at the instant of make and break, or when the strength of the current is changed in the direction either of diminution or increase; but there is a slight sensation due doubtless to the electrotonic alterations in excitability which we have been studying, during the whole time that the current is passing.

When the nutrition of the nerves is impaired, much stronger currents of both the induced and constant kinds are necessary to evoke muscular contractions than in the normal state. When the nerves are completely degenerated (as, for instance, when they are cut off from the spinal cord, or when the cells in the cord from which they originate are themselves degenerated, as in infantile paralysis) no muscular contraction can be obtained on stimulating the nerves even with the strongest currents.

The changes in the excitability of the muscles are less simple, because in them there are two excitable structures, the terminations of the nerves, and the muscular fibres themselves. Of these, the nerve-fibres are the more sensitive to induction currents, and the faradic stimulation of a muscle under normal circumstances is by means of these motor nerve-endings. Thus we find that its excitability corresponds in degree to that of the motor nerve supplying it. The muscular fibres are, even in the normal state, less sensitive to faradism (that is, a succession of induction shocks) than the nerve, because they are incapable of ready response to stimuli so very short in duration as are the shocks of which a faradic current consists. The proof of this consists in the fact that under the influence of curare, which renders the muscle practically nerveless, the muscle requires a much stronger faradic current to stimulate it than in the normal state. When the nerve is degenerated, the make or break of the constant current stimulates the muscle as readily as in the normal state; but the contraction is propagated more slowly than that which occurs when the nerve-fibres are intact, and is due to the stimulation of the muscular fibres themselves. The fact that, under normal circumstances, the contraction which is caused by the constant current is as quick as that produced by an induction shock, is ground for believing that in health the constant, like the induced current, causes the muscle to contract chiefly by exciting the motor nerves within it.

When the motor nerve is degenerated, and will not respond to any form of electrical stimulation, the muscle also loses all its power of 
response to induction shocks. The nerve-degeneration is accompanied by changes in the nutrition of the muscular fibres, as is evidenced by their rapid wasting, and any power of response to faradism they possessed in the normal state is lost. But the response of the muscle to the constant current remains, and is indeed more ready than in health, doubtless in consequence of nutritive changes which develop what the older pathologists called, truly enough, "irritable weakness." There is, moreover, a qualitative as well as a quantitative change. In health the first contraction to occur on gradually increasing the strength of the current is at the negative pole, when the circuit is closed (see Pflüger's law), and a stronger current is required before closure-contraction occurs at the positive pole. But in the morbid state we are discussing, closure-contraction may occur at the positive pole as readily as at the negative pole. This condition is called the "Reaction of Degeneration."

Suppose a patient comes before one with muscular paralysis. This may be due to disease of the nerves, of the cells of the spinal cord, or of the brain. If the paralysis is due to brain disease, the muscles will be slightly wasted owing to disuse, but the electrical irritability of the muscles and nerves will be normal, as they are still in connection with the nerve-cells of the spinal cord that control their nutrition. But if the paralysis is due to disease either of the spinal cord or of the nerves, this nutritive influence can no longer be exercised over the nerves or muscles. The nerves will degenerate; the muscles waste rapidly; the irritability of the nerves to both forms of electrical stimulation will be lost; the muscles will not respond to the faradic current, but in relation to the constant current they will exhibit what we have called the "reaction of degeneration."

This illustrates the value of the electrical method as a means of diagnosis, that is, of finding out what is the matter with a patient. It is also a valuable means of treatment; by making the muscles contract artificially, their nutrition is kept up until restoration of the nerves or nerve-centres is brought about. Another illustration will indicate that the facts regarding electrotonic variation of excitability are true for sensory as well as for motor nerves; in a case of neuralgia, relief will often be obtained by passing a constant current through the nerve; but the pole applied to the nerve must be the anode which produces diminution of excitability, not the kathode which produces the reverse.

Waller has pointed out that Pflüger's law of contraction, as formulated for frogs' muscles and nerves, is true for human muscles and nerves in the main, but there are certain discrepancies. These arise from the method necessarily employed in man being different from those used with a muscle-nerve preparation. In a muscle-nerve preparation the nerve is dissected out, the two electrodes placed on it, and the current has of necessity to traverse the piece of nerve between the two electrodes. In man, the current is applied by means of electrodes or rheophores 
which consist of metal discs covered with wash leather, and soaked in brine. One of these is placed on the moistened skin over the nerve, and the other on some indifferent point, such as the back. The current finds its way from one electrode to the other, not necessarily through the nerves to any great extent (though it will be concentrated at the nerve as it leaves the anode or reaches the kathode), but diffuses widely through the body, seeking the paths of least resistance. Thus it is impossible to get pure anodic or kathodic effects. If the anode is applied over the nerve, the current enters by a series of points (polar zone), and leaves by a second series of points (peripolar zone). The second series of points is very close to the first, as the current leaves the nerve as soon as possible, seeking less resistant paths. The polar zone will be in the condition of anelectrotonus, the peripolar in that of katelectrotonus, so that although the former effect will predominate, the points being more concentrated, the latter effect may prevent a pure anelectrotonic effect being observed (fig. 174).

Pflüger's law of contraction according to which excitation occurs at the kathode on the make of a constant current, and at the anode on the break, holds good for all excitable tissues. The excitation at the break is probably really due to the make of a polarisation current having its kathode at the former anode, and is therefore fundamentally of the same nature as the make contraction ; or, in general
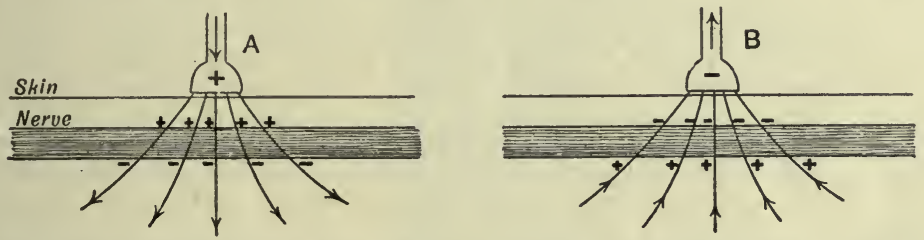

Fia. 174.-Electrodes applied to the skin over a nerve-trunk. In $\mathbf{A}$ the polar area is anelectrotonic, and the peripolar katelectrotonic. The former condition, therefore, preponderates, since the current is more concentrated. In $\mathrm{B}$ the conditions are reversed, the polar zone corresponding here to the kathode. (After Waller.)

terms, excitation occurs only at the place where a current leaves the excitable tissue. No doubt the effect is determined by the electrolytic changes occurring at the point of entry and exit of the current; the development of kat-ions must therefore be the chemical change that results in excitation. It is difficult to imagine that in a degenerated muscle there should be a reversal of such a fundamental law, and that excitation should be associated with the development of an-ions. Yet this is supposed to occur in the qualitative change known as the "reaction of degeneration." Page May investigated this question afresh, and found that the reversal of the law is only apparent, not real, and is due to the imperfect method which clinical observers must necessarily employ when testing the electrical reaction of muscles through the skin. By the use of appropriate electrodes on the degenerated muscles of animals, it is possible to detect the source of error. Let us substitute a muscle for a nerve in the diagrams of fig. 174 ; the current enters a few fibres at the anode, then spreads in all directions, and leaves the muscle by a number of diffused kathodic points. If the muscle is degenerated, its excitability is high, and the ready response at the anode when the current is made does not really occur at the actual anode, but in the neighbouring and more widespread peripolar kathodes. In other words, degenerated muscle obeys the general law of excitable tissues, and excitation occurs only at the situation where the current leaves the muscle. At the actual anode there is relaxation or absence of effect; this is obviously not observable through the human skin because the change is very limited in extent; it can be actually seen in the exposed muscles of an animal. 


\section{CHAPTER XVI}

\section{NERVE-CENTRES}

THE nerve-centres consist of the brain and spinal cord; they are characterised by containing nerve-cells, from which the nerve-fibres of the nerves originate. Small collections of nerve-cells are found also in portions of the peripheral nervous system, where they are called ganglia. The spinal ganglia on the posterior roots of the spinal nerves, and the sympathetic ganglia are instances of these.

The general arrangement of the cerebro-spinal axis is given in the accompanying diagram. The nerves which take origin from the brain are called cranial nerves; there are twelve pairs of these; some of them, such as the olfactory, optic, and auditory nerves, are nerves of special sense; others supply the region of the head with motor and sensory fibres. One pair (the tenth), called the pneumogastric or vagus nerves, are mainly distributed to the viscera of the thorax and abdomen, and a part of another pair (the eleventh), called the spinal accessory nerves, unites with the vagus prior to such distribution. We shall in our subsequent study of the heart, lungs, stomach, and other organs have frequently to allude to these nerves. The first two pairs of cranial nerves (the olfactory and the optic) arise from the cerebrum. The remaining ten pairs are connected with the district of grey matter called the floor of the fourth ventricle or its immediate neighbourhood; this tract of grey matter is situated at the lower part of the brain where it joins the spinal cord; this portion of the brain is called the Bulb or Medulla oblongata.

The spinal nerves are arranged in pairs, 31 in number. Their general structure and functions we have already studied (pp. 155-158).

The more intimate structure of the brain and spinal cord we shall consider at length in subsequent chapters. For the present we shall deal with some of the general aspects of the nerve-centres, both as regards structure and function.

The brain and spinal cord consist of two kinds of tissue, easily distinguishable by the naked eye. They are called respectively white matter and grey matter. 
White matter is composed of medullated nerve-fibres, which differ in structure from the medullated fibres of nerve by having no primitive sheath (neurilemma).

Grey matter is the true central material so far as regards function; that is to say, it is the part which receives and sends out nervous impulses; it is characterised by containing the bodies of the nervecells.

In the brain the grey matter is chiefly situated on the surface, forming what is called the cortex; the white matter and certain subsidiary masses of grey matter are in the interior.

In the spinal cord, the grey matter is in the interior, the white matter outside.

In both grey and white matter the nerve-cells and nerve-fibres are supported by a peculiar tissue which is called neuroglia. It is composed of cells and fibres, the latter being prolonged from the cells. Some of the fibres are radially arranged. They start from the outer ends of the ciliated epithelium cells that line the central canal of the spinal cord and the ventricles of the brain, and diverge constantly branching towards the surface of the organ, where they end by slight enlargements attached to

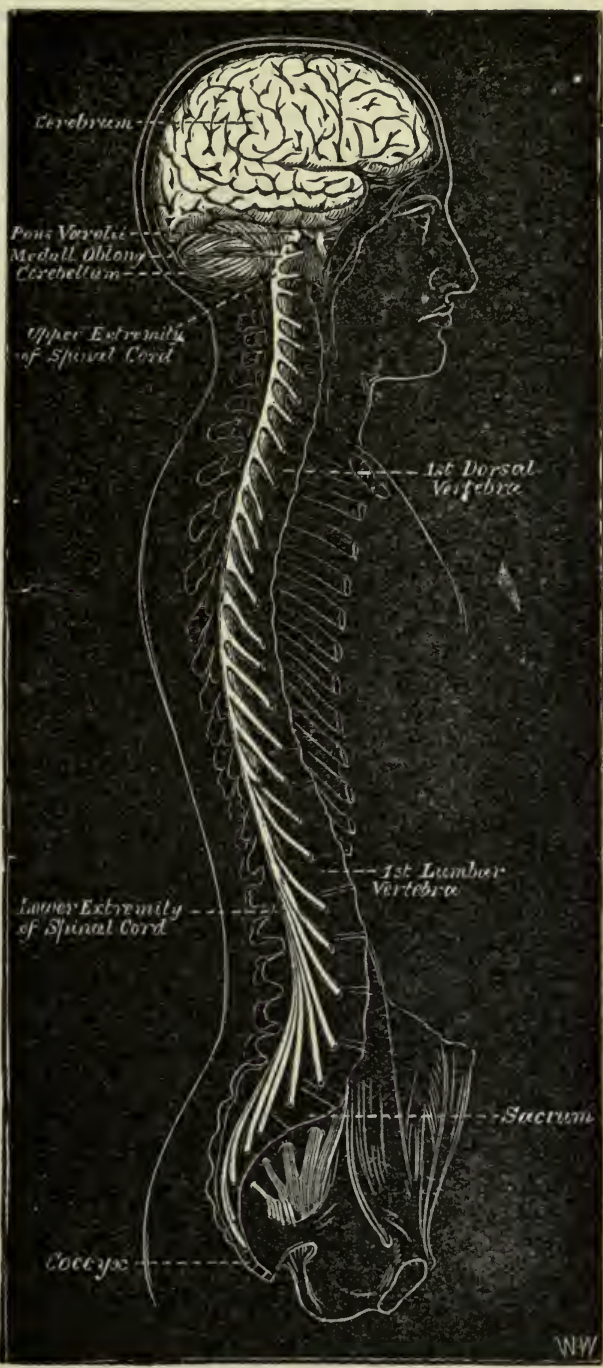

Fia. 175.-View of the cerebro-spinal axis of the nervous system. The right half of the cranium and trunk of the body has been removed by a vertical section; the membranes of the brain and spinal cord have also been removed, and the roots and first part of the fifth and ninth cranial, and of all the spinal nerves of the right side, have been dissected out and laid separately on the wall of the skull and on the several vertebræ opposite to the place of their natural exit from the cranio-spinal cavity. (After Bourgery.) 
the pia mater. The other fibres of the tissue are cell processes of the neuroglia or glia cells proper, or spider cells as they are sometimes termed (see fig. 176).

Neuroglia is thus a connective tissue in function, but it is not one in origin. Like the rest of the nervous system, it originates from the outermost layer of the embryo, the epiblast. All true connective tissues are mesoblastic.

Chemically, it is very different from connective tissues. It con-

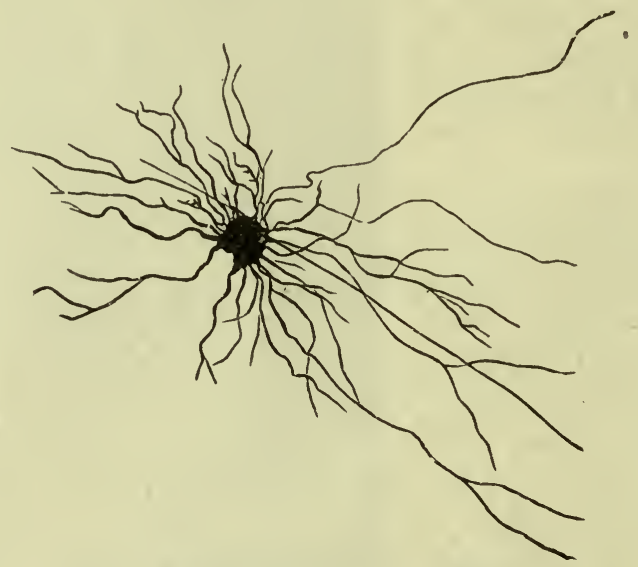

FIG. 176.-Branched neuroglia-cell. (After Stöhr.)

sists of an insoluble protein material called neuro-keratin, or nervehorn, similar to the horny substance, keratin, which is found in the surface layers of the epidermis.

\section{Structure of Nerve-Cells.}

Nerve-cells differ a good deal both in shape and size.

In the early embryonic condition, the future nerve-cell is a small nucleated mass of protoplasm without processes. As development progresses branches grow, and by this means it is brought into contact with the branches of other nerve-cells. When the nerve-cells degenerate, as they do in some cases of brain and cord disease, there is a reversal of this process; just as in a dying tree the terminal branches, those most distant from the seat of nutrition, are the first to wither, so it is in the degenerating nerve-cell. If one traces the structure of nerve-cells throughout the zoological series, there is also seen an increase in their complexity, and the number of points of contact, produced by an increase in the number and complexity of the branches, multiplies (fig. 177). 
The simplest nerve-cells known are termed bipolar. In the lower animals the two processes come off from the opposite ends of the cells ; the cell, in other words, appears as a nucleated enlargement on the course of a nerve-fibre. Fig. 178 (A) shows one of these nervecells from the Gasserian ganglion of the pike. The cells of the Gasserian and spinal ganglia in the mammalian embryo are also bipolar, but as development progresses, the two branches become fused for a considerable distance, so that in the fully formed animal each cell appears to be unipolar. This is shown in a more diagrammatic way in fig. 159, p. 155 . The bifurcation of the nerve-fibre is
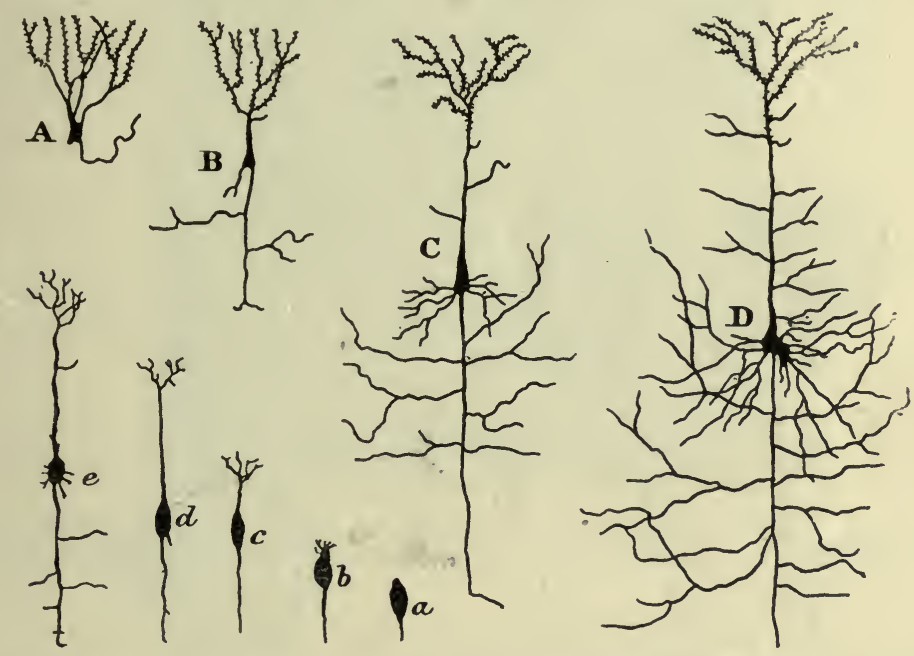

Fic. 177.-Diagram after Ramon y Cajal to show the ontogenetic (or embryological) and phylogenetic (i.e. in the animal series) development of a neuron. A, cerebral cell of frog; B, newt; C, mouse ; $\mathrm{D}$, man. As the place in the zoological series rises, the neuron increases in complexity and in the number of points of contact; this is produced partly by an increase of the dendrons, partly by an inerease in the side branches or collaterals of the axon. $a, b, c, d, e$, show the early stages in the development of a similar cell in the human embryo ; the first branch of the cell to appear (in $a$ ) is the axon; the dendrons are later outgrowths. The reversal of this process takes place in primary degeneration.

spoken of as a $\mathrm{T}$-shaped junction. As will be seen in fig. $178(\mathrm{C})$, the nerve process has a convoluted course on the surface of the cell before it bifurcates. In these ganglia it should be also noted that each cell is enclosed within a connective tissue sheath, and the nuclei seen are those of the connective tissue corpuscles.

The majority of nerve-cells found in the body are multipolar. Here the cell becomes angular or stellate. Fig. 179 shows the usual form of cell present in sympathetic ganglia. From the angles of the cell, branches originate; the majority of these branches divide and subdivide until each ends in an arborescence of fine twigs or fibrils; 
but one process, and one process only, of each cell becomes the axis cylinder of a nerve-fibre.

Passing next to the central nervous system, we here again find the multipolar cell is the principal kind present.

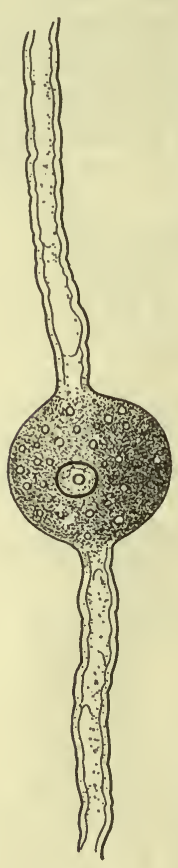

A

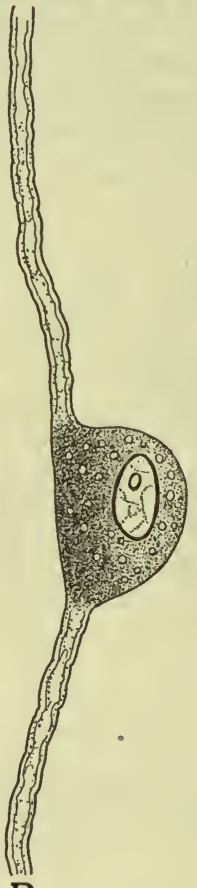

B

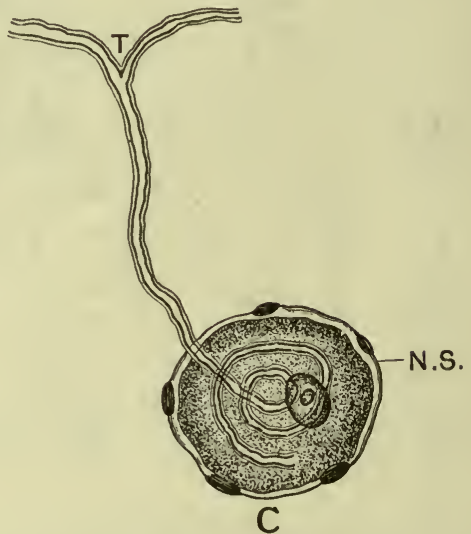

FIG. 178.-Bipolar nerve-cells. A. From the Gasserian ganglion of the pike (after Bidder). B. From a spinal ganglion of a $4 \frac{1}{2}$ weeks' human embryo (after His). C. Adult condition of the mammalian spinal ganglion cell : $\mathrm{N}$. S. nucleated sheath ; only the nuclei seen in profile are represented. T. is the $T$-shaped junction (after Retzius).

Fig. 180 shows one of the typical multipolar cells of the spinal cord. Here again, only one process $(a)$ becomes the axis cylinder of a nerve-fibre, and the others break up into arborisations of fibrils. The cells have a finely fibrillar structure, and the fibrils can be traced into the axis cylinder process and the other branches of the cell. Between the fibrils the protoplasm of the cell contains a number of angular or spindle-shaped masses, which have a great affinity for basic aniline dyes like methylene blue. They are known as Nissl's granules. These nerve-cells often contain, especially in the adult, granules of pigment, usually yellow, the nature of which has not been determined. 
In preparations made by Golgi's chromate of silver method, the cells and their processes are stained an intense black by a deposit of

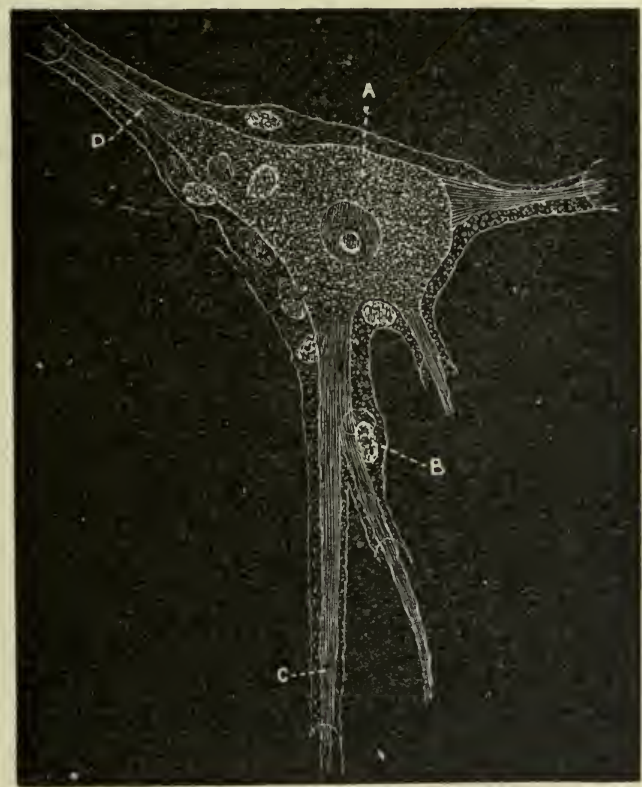

FIG. 179. - An isolated sympathetic ganglion cell of man, showing sheath with nucleated cell lining, $B$. A. Ganglion cell, with nucleus and nucleolus. C. Branched process. D. Axis cylinder process (Key and Retzius.) $\times 750$.

silver. The various structures in the cells (nucleus, granules, fibrils, etc.), are not visible in such preparations, but the great advantage of

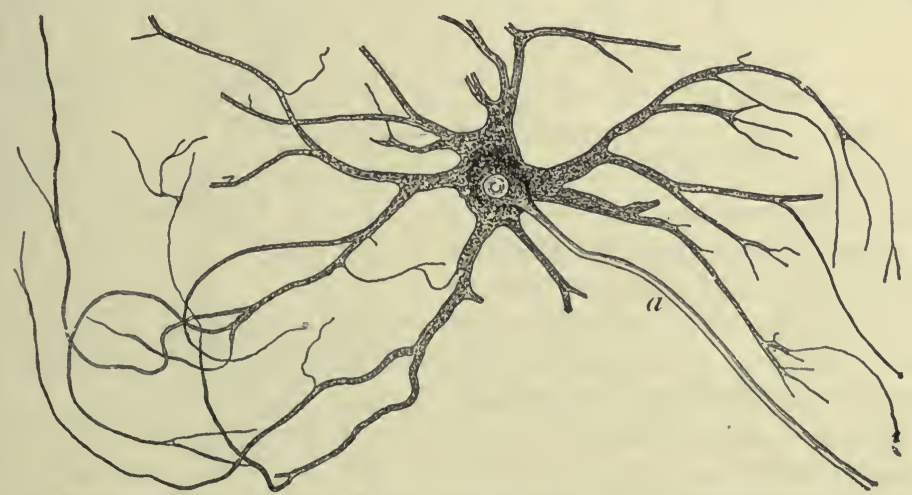

FiG. 180.-Multipolar nerve-cell from anterior horn of spinal cord; $a$, axis cylinder process. (Max Schultze.) 
the method is that it enables one to follow the branches to their finest ramifications. It is thus found that the axis cylinder process is not

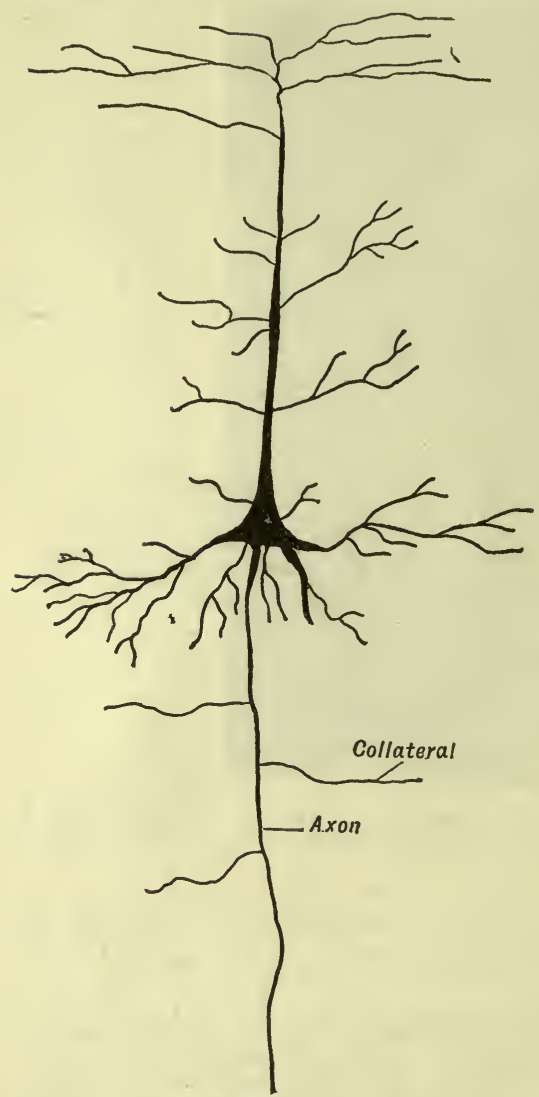

Fig. 181.-Pyramidal cell of human cerebral cortex. Golgi's method.

passes away from the nerve-centre, and gets bound up with other similarly sheathed axis cylinders to form a nerve; but all ultimately terminate in an arborescence of fibrils in various end - organs (end-plates, muscle spindles, etc.). unbranched, as represented in fig. 180 , but invariably gives off side-branches, which are called collaterals; these pass into the adjacent nerve-tissue. The axis cylinder then acquires the sheaths, and thus is converted into a nerve-fibre. This nervefibre sometimes, as in the nervecentres after a more or less extended course, breaks up into a terminal arborescence enveloping other nerve-cells; the collaterals also terminate in a similar way. The longest type of axis cylinder is that which

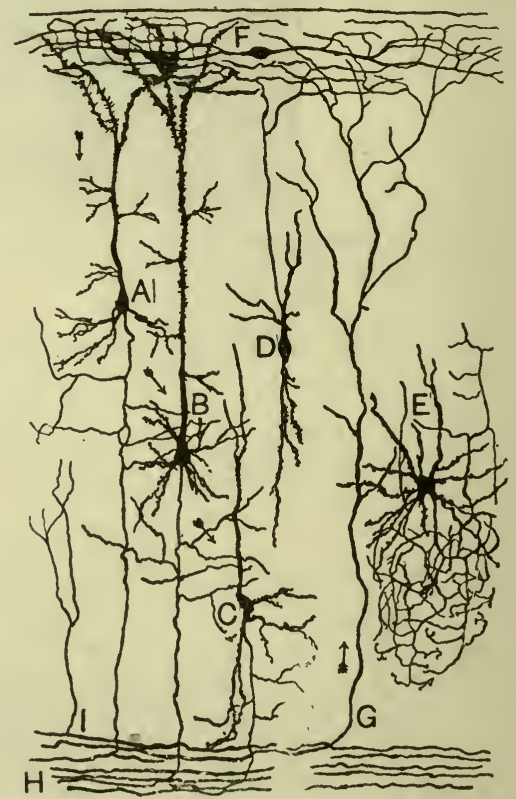

FIG. 1S2.-Cerebral cortex of mammal, prepared by Golgi's method. A, B, C, D, F, nervecells; E, neuroglia-cell. (Ramon y Cajal.)

In the grey matter of the cerebrum the nerve-cells are various in shape and size, but the most characteristic cells are pyramidal in 
shape. They are especially large and numerous in what are called the motor areas of the brain. The apex of the cell is directed to the surface; the apical process is long and tapering, and finally breaks up into fibrils that lie parallel to the surface of the brain (tangential fibres). From the lower angles and other parts branching processes originate; the axis cylinder comes off from the base of the pyramid. (See figs. 181, 182).

The grey matter of the cerebellum contains a large number of small nerve-cells, and one layer of large cells. These are flask-shaped, and are called the cells of Purkinje. The neck of the flask breaks up

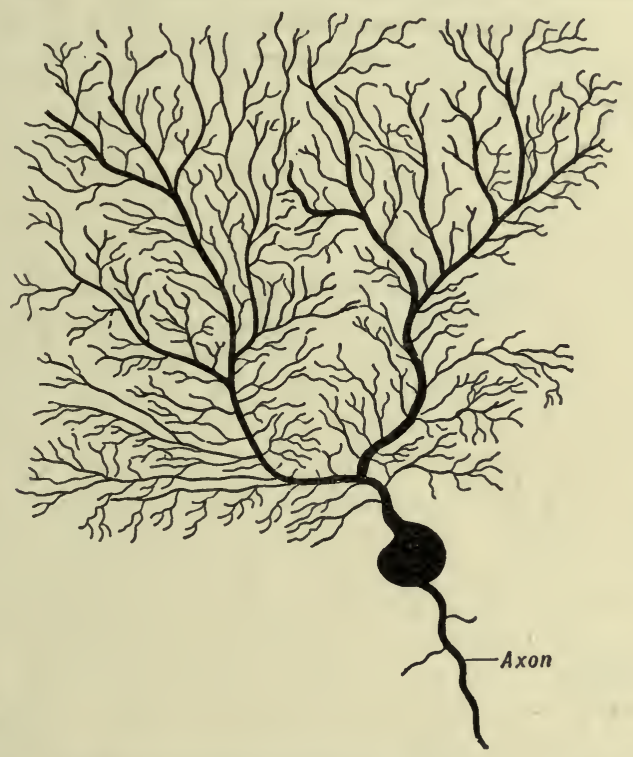

Fig. 183.-Cell of Purkinje from the human cerebellum. Golgi's method. (After Szymonowicz.)

into branches, and the axis cylinder process comes off from the base of the flask (fig. 183).

The whole nervous system consists of nerve-cells and their branches, supported by neuroglia in the central nervous system, and by connective tissue in the nerves. Some of the processes of a nerve-cell break up almost immediately into smaller branches ending in arborescences of fine twigs; these branches, which used to be called protoplasmic processes, are now termed dendrons. One branch becomes the long axis cylinder of a nerve-fibre, but it also ultimately terminates in an arborisation; it is called the axis cylinder process, or, more briefly, the axon. The term neuron or neurone is applied to 
the complete nerve-unit, that is, the body of the cell, and all its branches. The fibrils of the axon may be traced through the body of the cell from the dendrons.

The next idea which it is necessary to grasp is, that each nerveunit (cell plus branches of both kinds) is anatomically independent of every other nerve-unit. There is no true anastomosis of the branches from one nerve-cell with those of another; the arborisations interlace and intermingle, and nerve impulses are transmitted from one nerve-unit to another, through contiguous, but not through continuous structures. A convenient expression for the intermingling of arborisations is synapse (literally, a clasping).

Fig. 184 is a diagram of the nervous path in a spinal reflex action. Excitation occurs at S, the skin or other sensory surface, and the

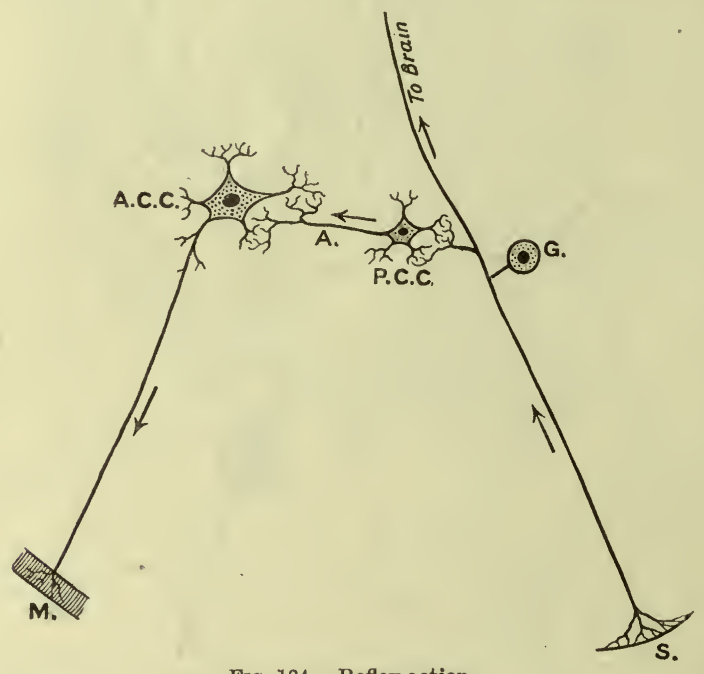

FIG. 184.--Reflex action.

impulse is transmitted by the sensory nerve-fibre to the central nervous system. It does not become anatomically connected to any of the cells of the central nervous system. The only cell-body in actual continuity with the sensory nerve-fibre is the one in the spinal ganglion $(G)$ from which it grew. On entering the spinal cord, the main fibre conveys impulses upwards which ultimately reach the brain, but in the spinal cord it gives off fine side branches or collaterals which terminate in branches that arborise around one or more cell bodies and their dendrons; these cells are small ones situated in the posterior cornu of the spinal grey matter; one only (P.C.C.) is shown in the diagram. The short axon of this cell similarly terminates by a synaptic junction with one or more of the large 
multipolar cells of the anterior cornu of the spinal grey matter; one of these shown in the figure is labelled A.C.C. This motor-cell is thus stirred up to action and sends an impulse by its axon to the muscular fibres (M) it supplies. Thus excitation of the skin will cause, by this spinal reflex arc, the contraction of muscles. In some cases severe excitation will cause contraction of the muscles of the opposite side of the body (crossed reflex); under such circumstances the intermediary neuron (P.C.C.) sends its axon to the anterior horn-cells of the opposite side. The synaptic junctions are naturally the places which the impulse has the greatest difficulty in traversing; and some observers believe that at the points of contact there is a kind of undifferentiated interstitial protoplasm which the impulse has to get through.

This example illustrates a most important general truth, namely, that a nervous impulse does not necessarily travel along the same nerve-fibre all the way, but there is what we may term a system of relays. The nervous system is very often compared to a telegraphic system throughout a country. The telegraph offices represent the nerve-centres, the afferent nerve-fibres correspond to the wires that carry the messages to the central offices, and the efferent nervefibres are represented by the wires that convey messages from the central offices to more or less distant parts of the country. This illustration will serve us very well for our present purpose, provided that it is always remembered that a nervous impulse travels more slowly than electricity. Suppose, now, one wishes to send a message from the metropolis, which will represent the brain, to a distant house, say in the Highlands of Scotland. There is no wire straight from London to that house, but the message ultimately reaches the house; one wire takes the message to Edinburgh; another wire carries it on to the telegraph station in the town nearest to the house in question; and the last part of the journey is accomplished by a messenger on foot or horseback. There are at least two relays on the journey.

We may take another illustration of this. Suppose one wishes to move the arm; the impulse starts in the nerve-cells of the brain, but there are no fibres that go straight from the brain to the muscles of the arm. The impulse travels down the spinal cord, by what are called pyramidal fibres, which form synapses with the nerve-cells of the spinal cord, and from these cells fresh nervefibres pass on the impulse to the arm-muscles. This is shown in the accompanying diagram (fig. 185). The cell of the cerebral grey matter is represented by C.C., and its axon (pyramidal fibre) by P.F. This passes into the white matter of the brain, and in the medulla oblongata it crosses over, and then travels down the opposite side of the spinal cord. It enters the grey matter in the 
part of the cord which controls the arm movements, and terminates by arborising around small cells at the base of the posterior cornu

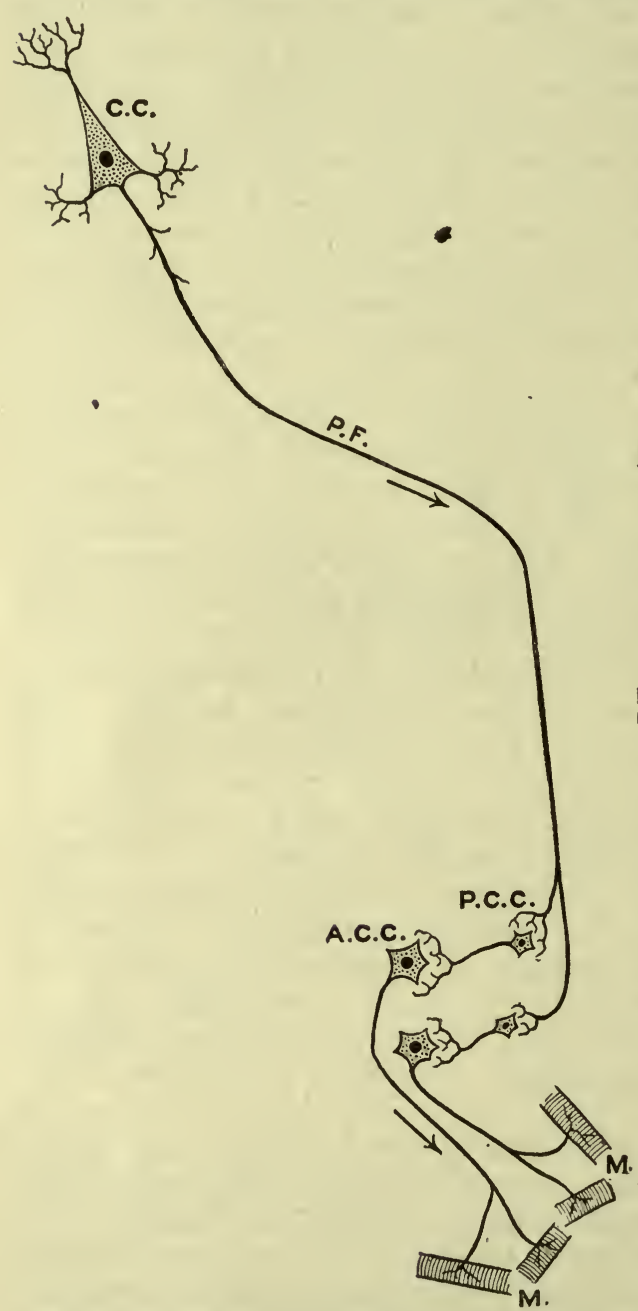

FIG. 185.-Diagram of the neurons of the motor path. (P.C.C.); thence the impulse is transferred to the large motor cells of the anterior cornu (A.C.C.), and the final link in the chain is formed by the motor nerve-fibres to the muscular fibres (M). The spinal cord cells are thus surrounded by arborisations (synapses), derived not only from the sensory Enerves but by fibres from the upper part of the nervous system. We now see how it is possible that reflex actions in the cord may be controlled by impulses from the brain.

The system of relays is still more complicated in the case of sensory impulses, as we shall see later on; the same is true for the motor path to involun. tary muscle, accessory cellstations being situated in the sympathetic ganglia.

We may now return for a moment to the subject of degeneration. If the nervefibre is cut off from its connection with the spinal nerve-cell, the peripheral end degenerates as far as the muscle.

Suppose, now, the pyramidal fibre were cut across, the piece still attached to the brain-cell would remain in a comparatively normal condition, but the peripheral end would degenerate as far as the next synapse. We can thus use the degeneration method to trace out tracts of nerve-fibres in the white matter of the central nervous system. The histological change in the fibres is here the same 
as that already described in the nerves, except that, as there is no primitive sheath, there can be no multiplication of its nuclei; there is instead an overgrowth of neuroglia. Degenerated tracts consequently stain differently from healthy white matter, and can by this means be easily detected.

Another method of research which leads to the same results as the degeneration method is called the embryological method. The nerve-fibres which grow from different groups of nerve-cells become fully developed at different dates, and so, by examining brains and cords of embryos of different ages, one is able to make out individual tracts before they have blended in the general mass of white matter.

We shall, however, return to this subject when later on we are studying the physiology of the central nervous system in detail.

\section{The Law of Axipetal Conduction.}

A general law has been laid down by van Gehuchten and Cajal, that all nerve impulses are axipetal, that is, they pass towards the attachment of the axon, by which they leave the body of the cell. In other words, the direction of an impulse is towards the body of the cell in the dendrons, and away from it in the axon. When we further consider that every nervous pathway is formed of a chain of cells, and that the impulse always takes the "forward direction," we see that there is what we may compare to a valved action which permits the passage of impulses in one direction only. The synapses are the situations of these so-called valves.

On the onward propagation of a nerve impulse through a chain of neurons, its passage is delayed at each synapse, hence there is additional "lost time" at each of these blocks. The relative number of the blocks furnishes a key to the differences found in reaction time for different refiexes and psychical processes. This we may illustrate by two examples, one taken from the frog, the other from man.

1. If a frog's posterior root is stimulated, the time lost in the spinal cord when the gastrocnemius of the same side contracts is $0.008 \mathrm{sec}$; if the opposite gastrocnemius contracts, the additional lost time is 0.004 sec. If we assume that in the latter case, two extra synapses have to be jumped, the delay at each is 0.002 sec.

2. In the case of the eye and ear in man the total length of the pathway to the brain is approximately the same, and so the reaction times inight be expected to be equal; but this is not the case; the reaction time in response to a sudden sound is $0.150 \mathrm{sec}$., in response to a sudden flash of light $0.195 \mathrm{sec}$. The greater delay in response to a visual stimulus directly corresponds to the greater number of 
synapses through which it has to travel (see later, on the structure of the visual and auditory mechanisms).

The valved condition of nervous paths also explains another difficulty. We have seen on p. 160 that under certain circumstances a nervous impulse will travel in both directions along a nerve. Yet when we stimulate the motor fibres in an anterior spinal root, the only effect is a contraction of muscles; there is no effect propagated backwards in the spinal cord. No doubt a nervous impulse does travel backwards to the anterior horn cells, but it is there extinguished, it cannot jump the synapses backwards, and there is no negative variation to be detected in a galvanometer connected to the pyramidal tracts in the cord.

The law of axipetal conduction is no doubt true for the majority of neurons. But there is at any rate one very striking exception, namely, in the neurons of the spinal ganglia; here the impulse passes to the body of the cell by one axon from the periphery, and away from it to the spinal cord by the other. To say, as some do, that the peripheral process is really a dendron because it conducts impulses centripetally, is simply arguing in a circle.

\section{The Significance of Nissl's Granules.}

If portions of the brain or spinal cord are fixed in absolute alcohol, and sections obtained from the hardened pieces are stained by means of methylene blue, the nerve-cells exhibit a characteristic appearance. The nucleus and nucleolus take up the blue stain, but the total amount of chromatin present in the nucleus is not large, except in embryonic nerve-cells; throughout the cell body a number of angularshaped masses, which are termed Nissl's granules, are also stained blue. These extend some distance into the dendrons, but not into the axon. The substance of which they are composed is termed chromatoplasm, or chromophilic material. The existence of granules in cells which have an affinity for basic dyes such as methylene blue is not at all common; the granules in the majority of the white bloodcorpuscles, for instance, have an affinity for acid dyes. Microchemical methods have shown that the main constituent of the Nissl granules is an iron-containing nucleo-protein. The name kinetoplasm has been given to it by Marinesco in order to express the idea that it forms a source of energy to the cell. It can hardly be denied that the substance of which the granules are composed, forming as it does so large a proportion of the cell-contents, and made of a material in which nuclein forms an important constituent, is intimately related to the nutritional condition of the neuron. Some have even compared it to the granular material which is present in secreting cells; in these cells before secretion occurs, the granules accumulate, 
and during the act of secretion they are discharged and converted into constituents of the secretion. It is stated by some observers that the Nissl granules are used up during the discharge of energy from nerve-cells, and it certainly is the case that if the cells are examined after an epileptic fit, in which there has been a very massive discharge of impulses, the Nissl granules have disappeared, or at least broken up into fine dust-like particles, so that the cell presents a more uniform blue staining. This is called chromatolysis (see fig. 186). It is, however, doubtful whether this is due to a transformation associated with intense activity, or whether it may not be
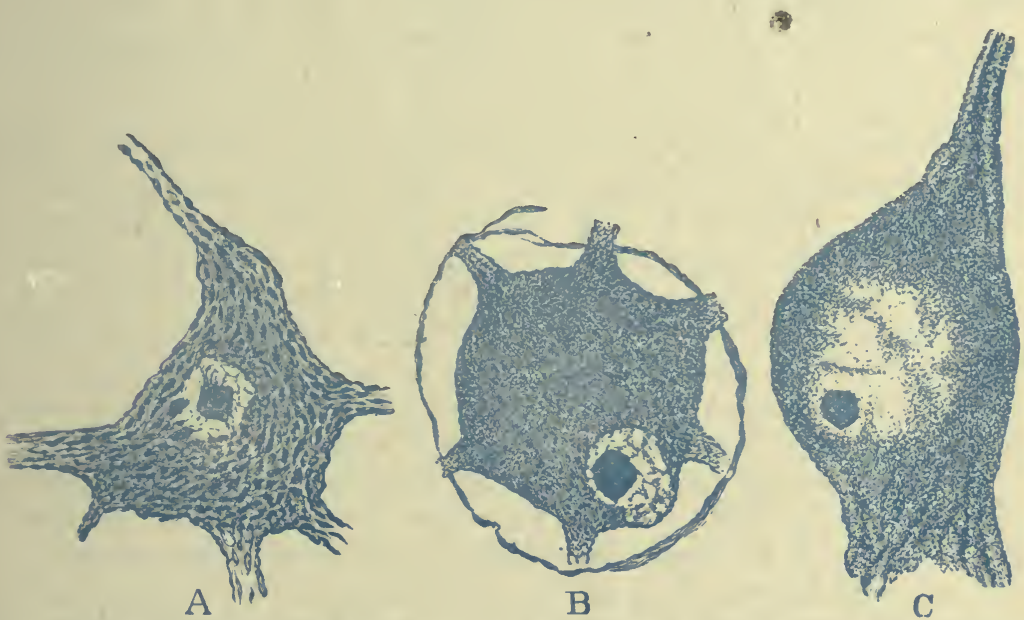

Fia. 186.-Nissl's granules. A. Normal pyranidal cell of human cerebral cortex. B. Swollen oede. matous pyramidal cell from a case of status epilepticus. Notice diffuse staining, and absence of Nissl's granules; the nucleus is enlarged and eccentric. The lymph space around the cell is dilaterl. C. Pyramidal cell of dog after ligature of vessels going to brain and consequent anæmia. Notice great swelling of the nucleus, and advanced chromatolysis, most marked at the periphery of the cell. 700 diameters. (After Mott.)

caused by venosity of the blood.- The cells are very sensitive to altered vascular conditions; anæmia, for instance, produces a similar change accompanied with swelling of the cell, and swelling and in extreme cases extrusion of the nucleus.

The most convincing observations in reference to the influence of fatigue in producing chromatolysis have been made on bees; their nerve-cells are rich in chromophilic material when they are about to leave the hive in the morning. In the evening, after a hard day's work, this material is much reduced in quantity.

By this sensitive method neurologists have been able to identify changes in the cells which could not be detected by the previous methods of staining. Thus the cells have been examined in various diseases, or after being subjected to the action of certain poisons, and 
valuable results have been obtained. We will, however, be content with alluding to only one pathological condition, namely, that produced by extremely high fever (hyperpyrexia); in this condition chromatolysis is very marked and is produced by the coagulation of the proteins of the cell-protoplasm by the high temperature.

The question has arisen whether the Nissl granules are present as such in the living cell, or whether they are artifacts produced by the fixative action of strong alcohol. But, whichever view is correct, the method is a valuable one, and Nissl's views on this question appear to be indisputable: they are briefly as follows:-Healthy cells fixed and stained in a constant manner will appear the same under constant optical conditions, and the appearances then seen form the equivalent of such healthy cells during life. It follows that if the cells prepared by the same method and examined under the same conditions show a difference from the equivalent or symbol of healthy cells, the difference is the measure of some change that has occurred during life. The view most generally held is that the granules are artifacts, and that the actual Nissl substance in the living nerve-cell is a fluid plasm of rich nutritive value to the fibrils.

Chromatolysis alone is not indicative of cell destruction, and a cell may recover its function afterwards. The integrity of the nucleus and of the fibrils between which the Nissl substance lies is much more important to the actual vitality of the cell.

When a nerve-fibre is cut across, the distal segment undergoes Wallerian degeneration; this is an acute change. But the nerve-cell and the piece of the nerve-fibre still attached to it do not remain unaffected. If regeneration of the fibre, and restoration of function takes place, no change is observable. But if regeneration does not occur (and it never takes place in the central nervous system), the cell and its processes undergo a slow chronic wasting; one of the earliest signs of this disuse atrophy is chromatolysis.

\section{Classification of Neurons according to their Function.}

In addition to the anatomical classification of the nerve-cells already given, they may be grouped into four chief classes on a physiological basis:-

1. Afferent neurons.

2. Efferent neurons.

3. Intermediary neurons.

4. Distributing neurons.

1. Afferent neurons.-Originally the cell bodies are situated at the periphery, and are connected with a process or afferent fibre which passes to and arborises among the nerve-cells of the central nervous 
system. This primitive condition is well seen in the earthworm, and persists in the olfactory cells of all vertebrates.

As evolution progresses, the peripheral cell sinks below the integument, leaving a process at the surface; this is seen in the worm Nereis (see fig. 187). Ultimately in the vertebrates the body of the
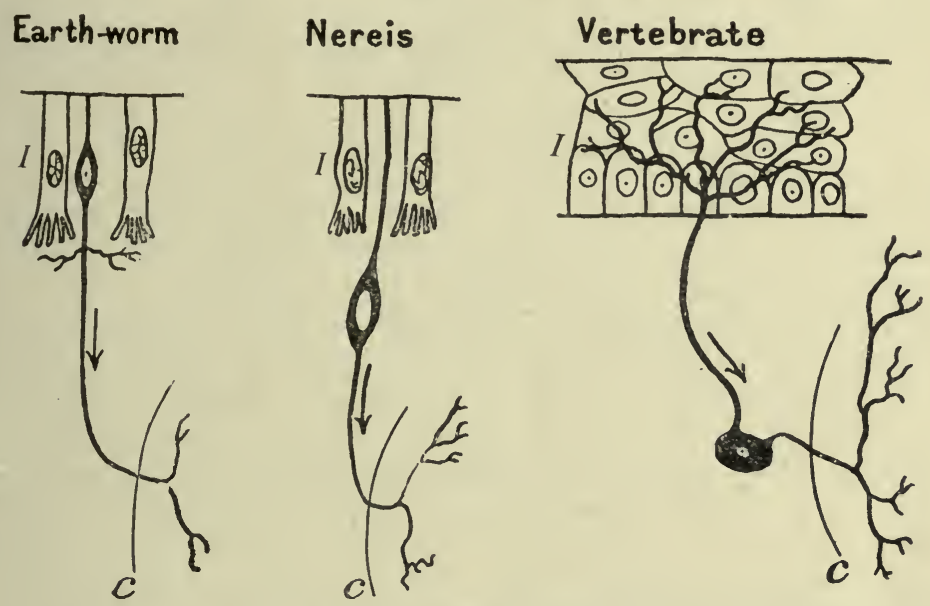

F19. 187.-Diagram to illustrate the primitive conditions of the afferent nerve-cell, and the manner in which it becomes altered in the process of evolution. (After Retzius.) I, integument; C, central nervous system; the arrows show the direction in which the impulse passes.

cell approaches close to the central nervous system, in the spinal ganglion of the posterior root, and the peripheral sensory nerve-fibre is correspondingly longer.

The afferent neurons such as those of the spinal ganglia and the corresponding ganglia of the cranial nerves, are peculiar in possessing no dendrons.

2. Efferent neurons.-The anterior horn-cells of the spinal cord are instances of these; their axons go directly to muscle fibres.

3. Intermediary neurons. - These receive impulses from afferent cells, and transmit them either directly or indirectly through other intermediary cells to efferent cells. The majority of the cells of the brain and cord come under this heading; they serve the purposes of association and coördination, and thus their activity underlies psychical phenomena.

4. Distributing neurons.-These are the cells of the sympathetic ganglia; they are situated outside the central nervous system; they receive impulses from efferent cells in the central nervous system, and distribute them to involuntary muscles and secreting glands. 


\section{CHAPTER XVII}

THE AUTONOMIC NERVOUS SYSTEM

Aluusion has frequently been made in the foregoing chapters to sympathetic nerves. These nerves govern the processes in the body over which we have no voluntary control. They innervate cardiac muscle, the plain muscle in the walls of blood-vessels, and in the walls of other contractile viscera such as the stomach and intestine, the bladder, and the organs of generation. Secretory nerve-fibres also come into the same category. In the chapters which immediately follow this one, we shall be studying such organs, organs which carry on the vegetative functions of life as it was formerly the custom to call them. It is therefore desirable that, at the outset, we should obtain some general idea of the nervous mechanism involved in controlling and regulating these functions.

The sympathetic system proper consists of a chain of ganglia or collections of nerve-cells, situated on each side of the vertebral column. These ganglia correspond roughly with the spinal segments; the uppermost is called the superior cervical ganglion, and the next the inferior cervicul ganglion; these are the only two ganglia in the cervical region in the dog; in man there is a middle cervical ganglion in addition. The inferior cervical ganglion is connected to the first thoracic ganglion (a large ganglion sometimes called the ganglion stellatum) by fibres, some of which go in front of, and others behind the subclavian artery; this ring around the artery is called the aninulus of Vieussens; after this the correspondence of the ganglia to the spinal nerve roots is more exact, and we finally reach the ganglion at the end of the chain, the ganglion coccygeum.

All these ganglia (with the possible exception in some animals of the inferior cervical ganglion) send bundles of nerve-fibres to the spinal nerves, and the communicating strands between the ganglia and the spinal nerves are termed the rami communicantes. The rami communicantes are divided into white and grey. The white rami consist of medullated fibres of small diameter; the grey rami consist mainly of non-medullated nerve-fibres.

The sympathetic chain, then, is a system of ganglia longitudinally 
arranged, and these ganglia are called the vertebral or the lateral gunglia.

In connection with the lateral chain are other outlying ganglia, such as the semilunar ganglion, from which the coliac plexus takes origin; the superior mesenteric ganglion, and the inferior mesenteric ganglion, from which the hypogastric nerve takes origin. These outlying ganglia are called the collateral or the prevertebral ganglia. These differ from the lateral ganglia in not sending any fibres back to the spinal nerves; their fibres pass onwards to the thoracic, abdominal, and pelvic viscera.

Finally, there are ganglia situated in the walls of the organs themselves, as, for instance, those in the heart wall, and those in the plexuses of the wall of the alimentary canal (the plexuses of Auerbach and of Meissner). By some, these ganglia are included with the collateral ganglia, but it appears better for descriptive purposes to speak of them as Gaskell does, as a third group, and name them the terminal ganglia.

The sympathetic system thus consists of three sets of ganglia with strands connecting them together, and all come into ultimate connection with fibres which leave the spinal cord.

There is, however, another set of ganglia which are related anatomically in a similar way to some of the cranial nerves, and physiologically with the involuntary muscles and glands in the head region as well as with some of the thoracic and abdominal organs. Thus we have the ciliary ganglion in connection with the third cranial nerve; then there are such ganglia as the spheno-palatine, otic, and submaxillary, in connection with other cranial nerves.

It has been considered wise not to extend the term sympathetic to these, but to include both them and the sympathetic system under one common term, and Langley's suggestion that this word should be autonomic has been very generally adopted. The word indicates that they possess a certain power of self-government, and are to some extent, at any rate, independent of the central nervous system.

The impulses that pass to the involuntary musculature of the body arise in the central nervous system, and travel to the ganglia of the autonomic system by means of fine medullated nerve-fibres; the diameter of these fibres varies from 1.8 to $3.6 \mu$; the fibres therefore contrast with the motor fibres which pass to voluntary muscles; the diameter of these being 14 to $19 \mu$ (see fig. 95, p. 77). There is a further contrast in the two cases; the motor fibres to voluntary muscles pass uninterruptedly from the brain or cord until they terminate in the end-plates of the voluntary muscles. The autonomic fibres, on the other hand, terminate by arborising around cells in one or other of the autonomic ganglia, and from the ganglion cells a fresh relay of nerve-fibres carries on the impulse to the 
involuntary muscles. There is thus an extra cell-station or synaptic junction outside the central nervous system altogether. The autonomic path, in other words, consists of two neurons; one from the central nervous system to the ganglion, and a second from the ganglion to the peripheral tissue. The first axon is termed the preganglionic fibre; the second, the post-ganglionic fibre. The preganglionic fibres are fine medullated ones, and the post-ganglionic fibres are usually non-medullated, but there are exceptions to this rule.

The small medullated or pre-ganglionic fibres arise from the following four regions of the central nervous system.

1. From the mid-brain, issuing therefrom by the third cranial nerve (motor oculi).

2. From the medulla oblongata, issuing therefrom in the seventh (facial), ninth (glosso-pharyngeal), tenth (vagus), and eleventh (spinal accessory) nerves.

3. From the thoracic region of the spinal cord, issuing therefrom in the anterior roots of the spinal nerves and passing from these by the white rami communicantes to the sympathetic ganglia. These occur in all the nerves from the first or second thoracic, as far down as the second, third, or fourth lumbar nerves.

4. From the sacral region of the spinal cord, issuing therefrom by the anterior roots of the second, third, and fourth sacral nerves, and thence passing by white rami communicantes to sympathetic ganglia; these fibres supply the descending colon, rectum, anus, and urino-genital organs in the pelvis, and they constitute the nervi erigentes.

It will be noticed that in the spinal district white rami communicantes only occur in certain regions: but all the spinal nerves have grey rami which consist of post-ganglionic fibres returning to the spinal nerves for distribution to the blood-vessels of the body wall, to the muscles which erect the hairs (pilo-motor nerves), and to the sweat glands of the skin.

The general arrangernent of such nerves is represented in fig. 188.

The cell-station of any particular pre-ganglionic fibre is not necessarily situated in the first ganglion to which it passes; the fibres of the white ramus communicantes of the second thoracic nerve, for instance, do not all have their cell-stations in the second thoracic ganglion, but may pass upwards or downwards in the chain to a more or less distant ganglion before they terminate by arborising around its cells. It therefore follows that fibres that leave any given spinal nerve by its white ramus, do not necessarily return as post-ganglionic fibres by the grey ramus to the same spinal nerve, although, for the sake of simplifying the diagram, they are represented as doing so in fig. 188. 


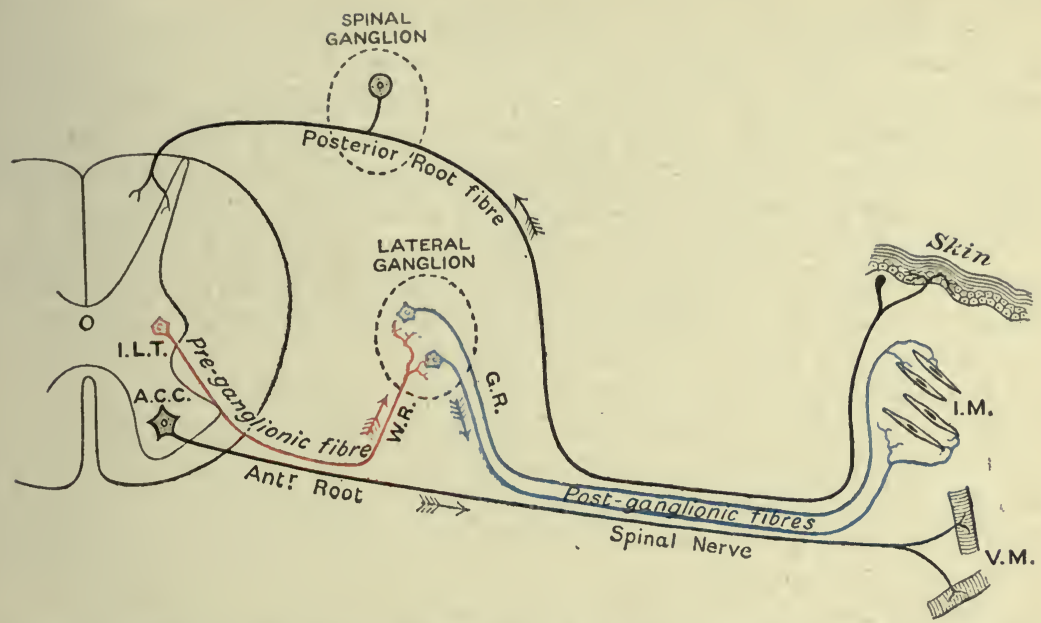

Fic. 188.-Diagram of the autonomic path in the spinal region. A.C.C. anterior cornual cell giving rise to a large motor nerve-fibre which is distributed to voluntary muscle (V.M.). I.L.T. a small cell of the intermedio-lateral tract giving rise to a small medullated nerve-fibre which leaves the cord by an anterior root, and leaves the anterior root by the white ramus (W.R.); it terminates by arborising around cells in a ganglion of the sympathetic chain. From these cells fresh non-medullated axons continue the impulse, and return to the spinal nerve by the grey ramus (G.R.) being finally distributed to involuntary muscular fibres (I.M.). The pre-ganglionic path is coloured red, the post-ganglionic blue. To complete the diagram, a posterior root-fibre is also shown with its parent cell in a spinal ganglion.
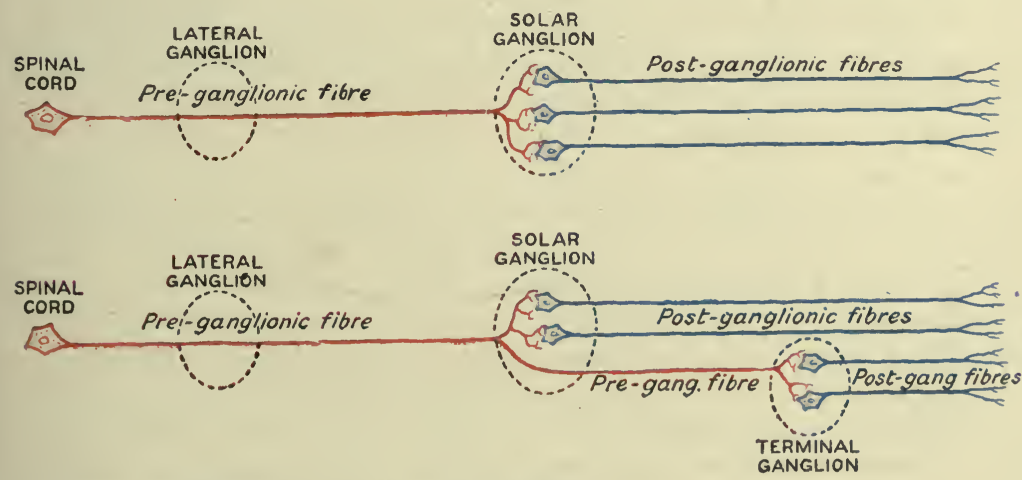

B.

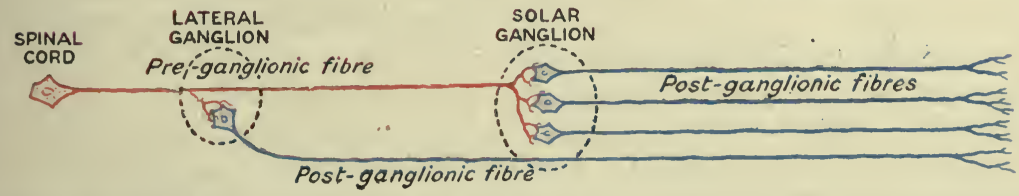

C.

Fio 189.-Arrangement of pre- and post-ganglionic fibres in splanchnic anil inferior splanchnic nerves. (After Langley.) 
Furthermore, there are many fibres of the white rami which enter the lateral chain of ganglia and pass through them without communicating with their cells at all, and never return to the spinal nerves by grey rami. They pass out of the lateral chain either to collateral or even terminal ganglia before reaching their cell-stations, whence they emerge as post-ganglionic fibres. This is the case for the sympathetic supply of the blood-vessels of and involuntary muscular fibres of the thoracic, abdominal, and pelvic viscera, and is therefore true for such important nerves as the cardiac accelerators, the splanchnics, and the nervi erigentes.

Fig. 189 shows the course of the splanchnic fibres, and will assist the student in grasping this method of distribution.

The great majority are arranged as in $A$, that is to say they have their cell-stations in the solar ganglion. Comparatively few are arranged as in B, where some fibres do not reach their cell-stations until they arrive at the terminal ganglion situated in the walls of the viscus (for instance, the pancreas) to which they are distributed. A few possibly and occasionally are arranged as in C, with a cell-station for some of their branches in the lateral sympathetic chain.

It will be noticed that if any post-ganglionic fibre is traced backwards, there is one and only one cell-station between the central nervous system and the ultimate distribution of the nerve fibrils.

The next question that arises is, how have all these facts been ascertained; for it is obviously impossible to follow the individual fibres with the microscope, and still less with the naked eye. The method above all others which has proved successful in solving the problem is the, nicotine method, originally introduced by Langley and Dickinson, and employed since by Langley mainly in conjunction with H. K. Anderson.

The Nicotine Method.-Nicotine in small doses paralyses nervecells, but not nerve-fibres. Before the paralytic effect of nicotine comes on, it excites the nerve-cells, and this in the case of the bloodvessels causes a general constriction of the arterioles, and a consequent rise of arterial pressure. It is still a matter of uncertainty whether the drug produces these effects on the nerve-cells themselves or on the terminal arborisations (synapses) of the fibres that surround them, or on receptive substances (see p. 164) either in the cells or present at the synaptic junctions. But whichever of these modes of action is the correct one, the main result is the same; a nervous impulse which reaches a ganglion by a pre-ganglionic fibre cannot get across to the corresponding post-ganglionic fibres if the animal is poisoned with nicotine. Stimulation of the anterior nerveroots, or of the white rami no longer produces movements of the involuntary muscular tissues, because the paralysed cell-stations act as blocks to the propagation of the impulses. If, however, post- 
ganglionic fibres are stimulated, the usual effects (for instance, constriction of blood-vessels, erection of the hairs, etc.) take place. If instead of injecting nicotine into the circulation, and so producing a general effect, the nicotine is painted over one or more ganglia, there will be a block in those fibres only which have their cellstations in those particular ganglia. By patiently examining all the ganglia in this way in turn, stimulating the fibres that enter it and those that leave it, Langley and his colleagues, after years of work, have been successful in localising the cell-stations on most of the autonomic paths in the body.

We shall in later chapters be considering the autonomic nerve supply of the individual organs, but it will be convenient here to state in a general way the main course of the distribution of these nerves; we have seen that the outflow from the central nervous system occurs in four regions, and therefore we may take these seriatim.

1. The autonomic nerve-fibres which arise from the mid-brain.These emerge by the third nerve; the pre-ganglionic fibres pass to the ciliary ganglion; the post-ganglionic arising from the cells of this ganglion run in the short ciliary nerves to supply the intrinsic muscles of the eyeball (sphincter iridis and ciliary muscle).

2. The autonomic nerve-fibres which arise from the medulla oblongata. - These emerge by the following nerves:-

(a) Seventh and ninth nerves. These supply the blood-vessels with vaso-dilator fibres and also the secreting glands in the nose and mouth region. Many of these fibres (for instance, those in the chorda tympani) get bound up with branches of the fifth nerve, and are distributed with them. The ganglia on the course of these fibres are the spheno-palatine, otic, submaxillary, and sublingual ganglia.

(b) Tenth and eleventh nerves. These are distributed by the branches of the tenth or vagus nerve to the œsophagus, stomach, and part of the intestine, to the bronchial muscles, to the heart, and to the gastric and pancreatic secretory mechanism. Here our knowledge of the localisation of the cell-stations is not so exact as it is in other parts; some of the fibres appear to have their cell-stations in the ganglion on the trunk of the vagus, but in most cases they do not become post-ganglionic until the terminal ganglia in the walls of the various organs mentioned are reached.

3. The autonomic fibres which arise from the thoracic region of the spinal cord.-These constitute the best known of the autonomic fibres, and we may describe them according to their distribution under the following two headings:-

(a) The white rami leave the spinal nerves and find their cellstations in lateral ganglia, returning by the grey rami for distribution 
to the involuntary muscular tissue and glands in the body walls and skin.

Thus in the lateral chain of ganglia we find the cells on the course of the pilo-motor nerves, of the nerves to the sweat glands, possibly of the splenic nerves, and last but not least, of the vasoconstrictors of the head, limbs, and body wall. Indeed, at one time, Gaskell suggested that the lateral chain should be called the chain of vaso-motor ganglia. In general terms the cell-stations are situated in ganglia that correspond with the various spinal segments; those for the lower limbs, for instance, being further down the chain than those for the trunk and upper limb. The vaso-constrictor fibres destined for the head, ascend the cervical sympathetic and do not reach their cell-station until they arrive at the superior cervical ganglion.

(b) The pre-ganglionic fibres traverse the lateral ganglia, and emerge still as pre-ganglionic fibres, which find their cell-stations in more or less outlying ganglia (collateral or terminal). We have already taken the splanchnic nerve as one example of this mode of distribution; the nerve contains inter alia the vaso-constrictor fibres, and viscero-inhibitory fibres of the abdominal organs. The hypogastric nerve arises in a similar way from the inferior mesenteric ganglion and joins the pelvic plexus. The medullated preganglionic fibres of this nerve arise from the upper lumbar nerveroots.

4. The autonomic nerve-fibres which arise from the sacral region of the spinal cord.-The pre-ganglionic fibres emerge in the white rani of the second, third, and fourth sacral nerves. They pass through the sacral ganglia of the lateral chain without forming connections with any cells there, and they pass on as the nervus erigens, or pelvic nerve, to join the pelvic plexus. The fibres of this nerve supply vasodilator fibres to the external generative organs (whence its name), to the rectum and anus, and motor fibres to the musculature of the descending colon and rectum, and have their cell-stations in the small scattered ganglia of the pelvic plexus, or in terminal ganglia in the walls of the viscera they supply.

Looking at the involuntary muscles for a moment from a rather different point of view, we see that they (or most of them) differ from the voluntary muscles in being supplied by two sets of nerve-fibres with opposite functions. In the case of the heart, we have an accelerator set which course through the sympathetic, and an inhibitory set which course through the vagus. In the case of the blood-vessels, we have an accelerator set called vaso-constrictors, and an inhibitory set called vaso-dilators. In the case of the contractile viscera we have also viscero-accelerator and viscero-inhibitory, which respectively hasten and lessen their peristaltic movements. 
Adopting Gaskell's nomenclature, we may term the accelerator groups of nerves katabolic, because they increase the activity of the muscles they supply, bringing about an increase of wear and tear and an increase in the discharge of waste materials, the products of their activity. The inhibitory nerves, on the other hand, are anabolic, as they produce a condition of rest in the tissues they supply, and so give an opportunity for repair, or constructive metabolism.

As a general rule, though there are exceptions to it, the cellstations of the anabolic nerves are in collateral or terminal ganglia, whereas the cell-stations for katabolic nerves are in the lateral chain, or in some cases in collateral ganglia.

Our descriptions and diagrams have further shown us that postganglionic fibres are more numerous than pre-ganglionic fibres, and this brings us to the main object served by the ganglia on the autonomic nerves. Nature has, as it were, before her the problem of supplying with nerves the vast mass of muscles in the body, and the space at her command in the various exits from the cranium and spinal canal does not allow of more than a comparatively small outflow from the central nervous system.

The difficulty is met to some extent by the branching of the outflowing nerve-fibres, and in the case of the voluntary muscles this appears to be sufficient. The most striking example of this can be seen in the electrical organ of the Malapterurus, where the millions of its subdivisions on each side of the body are all supplied by the branches of a single axis cylinder process originating from a single giant nerve-cell in the brain.

But in the case of the involuntary muscular tissue there is an additional means of distribution, for each fibre that leaves the central nervous system arborises around a number of cells in the autonomic ganglia, and thus the impulse is transferred to a large number of new axis cylinder processes.

The name sympathetic was originally bestowed on the system of nerves we are considering, because the ganglia were believed to be the centres for reflex actions, or sympathetic actions as they were formerly designated.

During their work on autonomic nerves Langley and Anderson have once more investigated this ancient question, but the only instances where such a thing seemed possible were the following:When all the nervous connections of the inferior mesenteric ganglion are divided except the hypogastric nerves, stimulation of the central end of one hypogastric causes contraction of the bladder, the efferent path to which is the other hypogastric nerve. But the action is not truly reflex; it is caused by the stimulation of the central ends of motor-fibres which issue from the spinal cord, and which after passing through the ganglion send branches down each 
hypogastric nerve. The experiment is in fact similar to Kühne's gracilis experiment (p. 160). They also observed an apparent reflex excitation of certain nerves supplying the erector muscles of the hairs (pilo-motor nerves) through other sympathetic ganglia; but this is explicable in the same way.

It certainly is the case that under normal circumstances the centres for reflex action are in the central nervous system. But there do appear to be some conditions in which it is possible for ganglia to assume this function. The recovery of vaso-motor tone, and of tone in certain viscera after destruction of extensive tracts of the spinal cord, or the persistence of peristaltic action in the intestine after cutting through all its nerves, are cases in point. (See further, under Intestinal Movements, and Spinal Visceral Reflexes.) Such action forms, in fact, the chief justification for the adoption of the new term, autonomic.

\section{Afferent Nerves of the Autonomic System.}

Up to the present point, we have only considered the efferent fibres of the autonomic nerves. No survey of the autonomic system will, however, be complete which does not include an account of the afferent fibres. This will not occupy much space, because our information on this side of the subject is so scanty.

The "vegetative" functions of the body are carried out independently of volition, and under normal circumstances they also cause no sensations. In pre-anæsthetic days, surgeons discovered that the viscera possess no sensibility in the ordinary sense; they may be handled and cut without producing pain; and, with the exception of the esophagus, they are insensitive also to heat and cold.

Still, under abnormal conditions we become conscious of their activity, especially if it is excessive, as for instance in the very severe pain which the various forms of colic give rise to. But even under these circumstances there is great difficulty in accurately localising the pain.

'The afferent or sensory fibres are much less numerous than those which are efferent. This has been ascertained by cutting the anterior nerve roots which communicate with an autonomic nerve; the efferent fibres will degenerate peripherally, but the sensory fibres will remain intact, and the relative number of healthy and degenerated fibres can then be counted. Thus in the splanchnic and hypogastric nerves about one-tenth of the fibres are found to be sensory; and in the pelvic nerve about one-third of the total fibres are sensory.

The grey rami contain few if any sensory fibres; excitation of 
their central ends produces neither pain nor reflex action; and the same is true for the cervical sympathetic.

On the other hand, excitation of the central ends of the white rami prorluces reflex movements, especially in involuntary muscles, as is evidenced by a rise of blood-pressure due to constriction of peripheral arteries; this is especially the case with white rami connected with thoracic and abdominal viscera.

It is therefore deduced from this that the sensory autonomic fibres enter the central nervous system by the white rami, but whether they come into relationship with the cells of either sympathetic or spinal ganglia is very uncertain. Possibly the cells of origin are within the spinal cord itself. In the cranial region we have some information especially in connection with the vagus nerves of the existence of afferent fibres, which we shall be studying in detail in connection with the heart and the lungs.

Referred Pain.-Localisation of painful or uncomfortable feelings arising from disorders of internal organs is always very difficult. But they are associated with pains in the skin, and this referred pain, as it is called, often plays an important part in ascertaining the position of internal maladies. Pains arising from intestinal irritation are referred to the skin of the lumbar region in the area supplied by the lower thoracic nerves; pains originating in the stomach are referred to an area of skin above this at the lower margin of the ribs, those from the heart to the shoulder region, and so forth.

Each viscus appears to be correlated with a definite patch or band of skin; this may even be tender on pressure. Ross's suggestion that the pain in such case is referred by sensory cutaneous fibres ending in the same segments of the cord as do the afferent fibres from the viscera in question, has been placed beyond doubt by the subsequent work of Mackenzie and of Head. 


\title{
CHAPTER XVIII
}

\author{
TROPHIC NERVES
}

NERVES exercise a trophic or nutritive influence over the tissues and organs they supply; for when a nerve going to an organ is cut, the wasting or degenerative process continues beyond the nerve; the muscles it supplies waste also, and waste much more rapidly than can be explained by simple disuse. The same is seen in the testicle after section of the spermatic cord; and in the disease of joints called Charcot's disease, the trophic changes are to be explained by disease of the nerves supplying them. After the division of the fifth cranial nerve there is loss of sensation in the corresponding side of the face: the cornea in two or three days begins to get opaque, and this is followed by a slow inflammatory process which may lead to a destruction not only of the cornea, but of the whole eyeball. The same is seen in man; when the fifth nerve is diseased or pressed upon by a tumour beyond the Gasserian ganglion, the result is loss of sensation in the face and conjunctiva, an eruption (herpes) appears on the face, and ulceration of the cornea leading in time to disintegration of the eyeball may occur too. In disease such as hæmorrhage in the spinal ganglia there is a similar herpetic eruption on the skin (shingles).

In the case of the fifth nerve the evidence that there are special nerve-fibres to which these trophic changes are due, is an experiment by Meissner and Büttner, who found that division of the most internal fibres is most potent in producing them.

Those, however, who do not believe in special trophic nerves, attribute the changes in the eyeball to its loss of sensation. Dust, etc., is not felt by the cornea, and is therefore allowed to accumulate and set up inflammation. This is supported by the fact that if the eyeball is protected by sewing the eyelids together the trophic results do not ensue. On the other hand, in paralysis of the seventh nerve, the eyeball is much more exposed, and yet no trophic disorders follow.

Others have attributed the change to increased vascularity due to disordered vaso-motor changes; against this is the fact that in 
disease of the cervical sympathetic, the disordered vaso-motor phenomena which ensue do not lead to the disorders of nutrition we have described. Nevertheless in trophic disorders, it is very difficult to be sure that the disordered metabolism is not in part due to vascular disturbances.

There can, therefore, be but little doubt that we have to deal with the trophic influence of nerves; * but the dust, etc., which falls on the cornea must be regarded as the exciting cause of the ulceration. The division or disease of the nerve acts as the predisposing cause. The eyeball is more than usually prone to undergo inflammatory changes, with very small provocation.

The same explanation holds in the case of the influence of the vagi on the lungs. If both these nerves are divided, the animal usually dies within a week or a fortnight from a form of pneumonia called vagus pneumonia, in which gangrene of the lung substance is a marked characteristic. Here the predisposing cause is the division of the pneumogastric nerves; the exciting cause is the entrance of particles of food into the air passages, which on account of the loss of sensation in the larynx and neighbouring parts are not coughed up. Another trophic disturbance that follows division of the vagi is fatty degeneration of the heart.

Many bedsores are due to prolonged confinement in bed with bad nursing; these are of slow onset. But there is one class of bedsores which are acute; these are especially met with in cases of paralysis, due to disease of the spinal cord; they come on in three or four days after the onset of the paralysis in spite of the most careful attention; they cannot be explained by vaso-motor disturbance, nor by loss of sensation; the nutrition of the skin is so greatly impaired that the mere contact of it with the bed for a few days is sufficient to act as the exciting cause of the sore.

It will be noticed that in some instances of trophic disorder the nerves which are injured are efferent; the muscular wasting that occurs when a muscular nerve is cut is the best marked example of this. In nerve itself Wallerian degeneration follows the direction of growth, which, as a rule, is the direction in which the nerve transmits impulses. The acute Wallerian change does not actually leap synapses, still the trophic influence of one set of neurons upon a second set among which the axons of the first set terminate is shown by a slow wasting process, of which chromatolysis is an early visible sign. In the peripheral axons of the cells of the spinal and corresponding cranial ganglia, the trophic disorder follows a peripheral direction, while impulses are carried in the opposite or afferent direction. 'The trophic influence here travels against the stream of impulse. It cannot fail to be a striking fact that the most marked trophic disorders with which we are acquainted, herpes, acute bedsores, Charcot's disease, eye changes after division or injury to the fifth nerve, vagus pneumonia, etc., are due to interference with sensory channels. Loss of sensation is the great predisposing cause of nutritive mischief.

* The proof, however, that there are distinct nerve-fibres anatomically is not very conclusive. 


\section{CHAPTER XIX}

\section{THE CIRCULATORY SYSTEM}

THE circulatory system consists of the heart, the arteries, or vessels that carry the blood from the heart to other parts of the body, the veins, or vessels that carry the blood back to the heart again, and the capillaries, a network of minute tubes which connect the terminations of the smallest arteries to the commencements of the smallest veins. We shall also have to consider in connection with the circulatory system, the lymphatics, which are vessels that convey back the lymph (the fluid which exudes through the thin walls of the bloodcapillaries) to the large veins near to their entrance into the heart, and the large lymph spaces contained in the serous membranes.

\section{The Heart.}

This is the great central pump of the circulatory system. It lies in the chest between the right and left lungs (fig. 190), and is enclosed in a covering called the pericardium. The pericardium is an instance of a serous membrane. Like all serous membranes it consists of two layers; each consists of fibrous tissue; one layer envelops the heart and forms its outer covering or epicardium; this is the visceral layer of the pericardium; the other layer of the pericardium, called its parietal layer, is situated at some little distance from the heart, being attached below to the diaphragm, the partition between the thorax and the abdomen. The visceral and parietal layers are continuous for a short distance along the great vessels at the base of the heart, and so form a closed sac. This sac is lined by endothelium; in health it contains just enough lymph (pericardial fluid) to lubricate the two surfaces and enable them to glide over each other smoothly during the movements of the heart. The presence of numerous elastic fibres in the epicardium enables it to follow without hindrance the changing shape of the heart itself; but the parietal layer of the pericardium appears to be inextensible, and so it limits the dilatation of the heart. 
The pericardium is a comparatively simple serous membrane, because the organ it encloses is a single one of simple external form. All serous membranes are of similar structure; thus the pleura which encloses the lung, and the peritoneum which encloses the abdominal viscera differ from it only in anatomical arrangement. The great complexity of the peritoneum is due to its enclosing so many organs. Every serous membrane consists of a visceral layer applied to the organ or organs it encloses; and a parietal layer continuous with this in contiguity with the parietes or body-walls.

The Chambers of the Heart.-The interior of the heart is divided by a longitudinal partition into two chief cavities-right and left. Each of these chambers is again subdivided transversely into an upper and a lower portion, called respectively, auricle and ventricle, which freely communicate one with the other; the aperture of communication, however, is guarded by valves, so disposed as to allow blood to pass freely from the auricle into the ventricle, but not in the opposite direction. There are thus four cavities in the heart-the auricle and ventricle of one side being quite separate from those of the other (figs. 191, 192).

The right auricle is a thin-walled cavity of quadrilateral shape, prolonged at one corner into a tongueshaped portion, the right auricular appendix, which slightly overlaps the exit of the aorta, from the heart.

The interior is smooth, being lined with the general lining of the heart, the endocardium, and into it

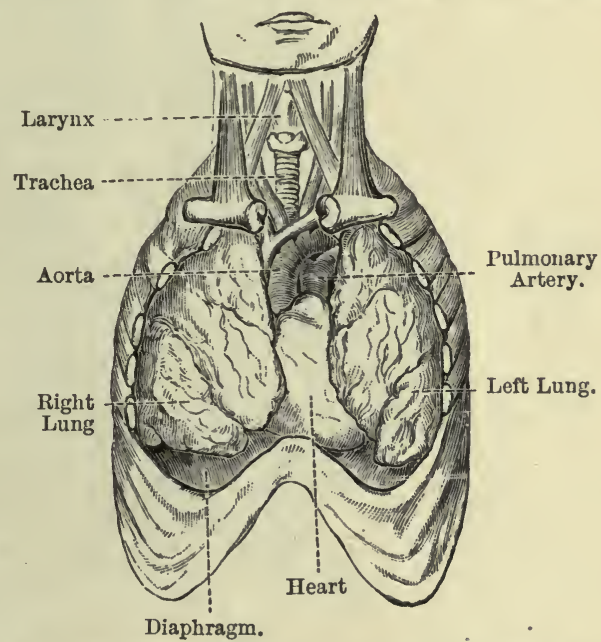

F1a. 190.-View of heart and lungs in situ. The front portion of the chest-wall and the outer or parietal layers of the pleurre and pericardium have been removed. The lungs are partly collapsed. open the superior and inferior-venæ cavæ, or great veins, which convey the blood from all parts of the body to the heart. The opening of the inferior vena cava is protected and partly covered by a membrane called the Eustachian valve. In the posterior wall of the auricle is a slight depression called the fossa ovalis, which corresponds to an opening between the right and left auricles which exists in fœtal life. The coronary sinus, or the dilated portion of the coronary vein, also opens into this chamber.

The right ventricle occupies the chief part of the anterior surface of the heart, as well as a small part of the posterior surface. It takes no part in the formation of the apex. On section after death 
its cavity, in consequence of the encroachment upon it of the septum ventriculorum, is crescentic (fig. 193); it has two openings, one communicating with the right auricle, and the other with the pulmonary artery; both orifices are guarded by valves, the former called tricuspid and the latter semilunar.

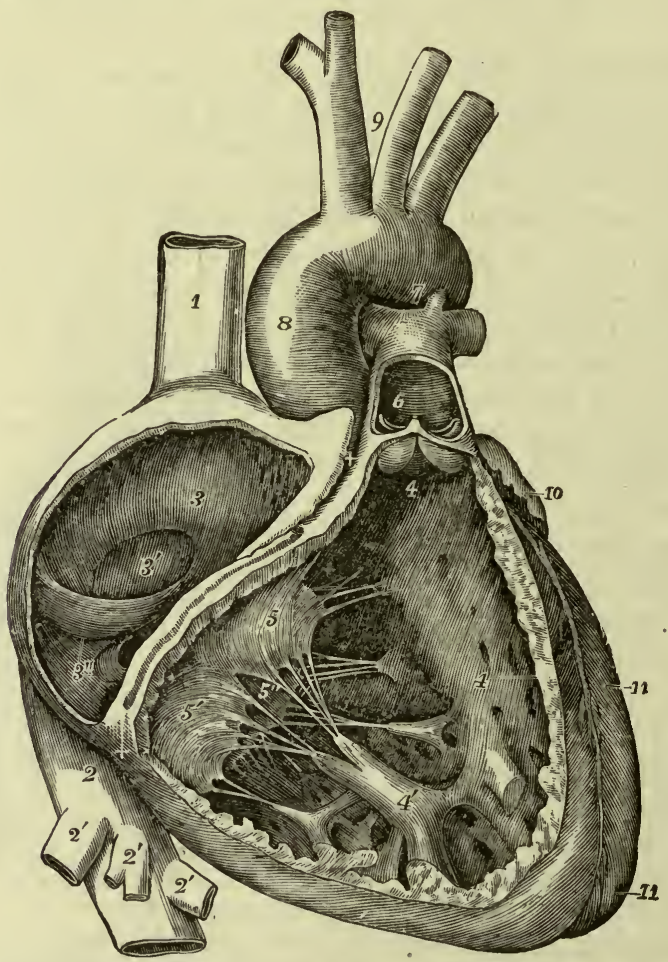

Fic. 191.-The right auricle and ventricle opened, and a part of their right and anterior walls removed, so as to show their interior. $\frac{1}{2} .-1$, superior vena cava; 2 , inferior vena cava; 2 ', hepatic veins cut short; 3 , right auricle; $3^{\prime}$, placed in the fossa ovalis, below which is the Eustachian valve; $3^{\prime \prime}$, is placed close to the aperture of the coronary vein $;++$, placed in the auriculo-ventricular groove, where a narrow portion of the adjacent walls of the auricle and ventricle has been preserved ; 4,4 , cavity of the right ventricle, the upper figure is immediately below the semilunar valves; $4^{\prime}$, large columna carnea or musculus papillaris ; $5,5^{\prime}, 5^{\prime \prime}$, tricuspid valve; 6 , placed in the interior of the pulmonary artery, a part of the anterior wall of that vessel having been removed, and a narrow portion of it preserved at its commencement, where the semilunar valves are attached; 7 , concavity of the aortic arch close to the cord of the ductus arteriosus; 8, ascending part or sinus of the arch covered at its commencement by the auricular appendix and pulmonary artery; 9 , placed between the innominate and left carotid arteries; 10 , appendix of the left auricle; 11, 11, the outside of the left ventricle, the lower figure near the apex. (Allen Thomson.)

The left auricle is best seen from behind. It receives on either side two pulmonary veins. The left auricle is somewhat thicker than the right. The left auriculo-ventricular orifice is oval, and a little smaller than that on the right side. 
The left ventricle occupies the chief part of the posterior surface. In it are two openings very close together, viz., the auriculo-ventri-

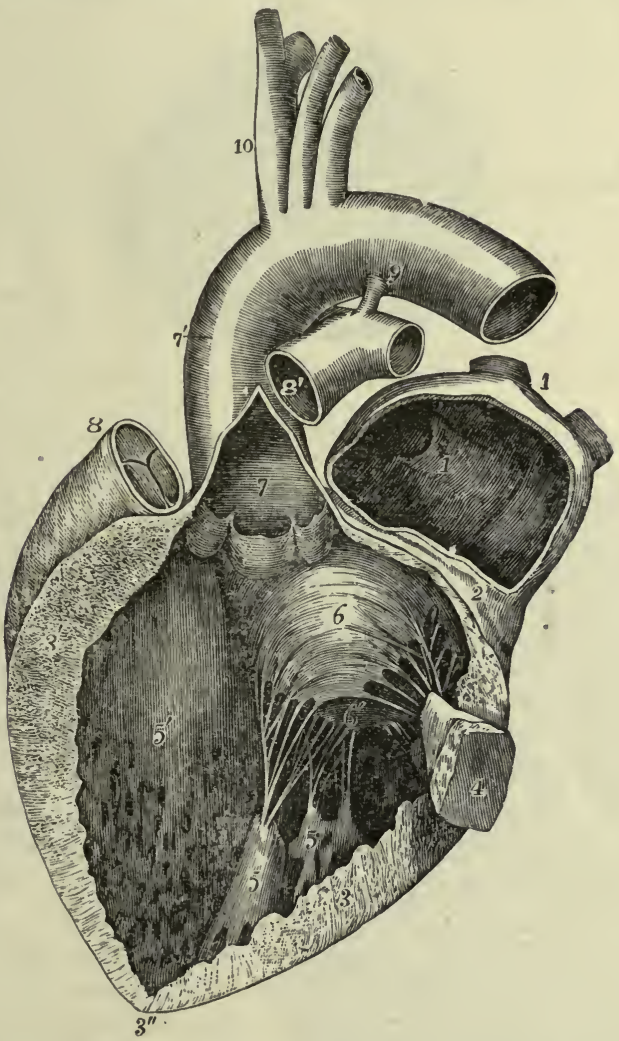

Fig. 192. -The left auricle and veutricle opened and a part of their anterior and left walls removed. $\frac{1}{2}$. -The pulmonary artery has been divided at its commencement; the opening into the left ventricle is carried a short distance into the aorta between two of the segments of the semilunar valves; and the left part of the auricle with its appendix has been removed. The right auricle is out of view. 1 , the two right pulmonary veins cut short; their openings are seen within the auricle; $1^{\prime}$, placed within the cavity of the auricle on the left side of the septum and on the part which forms the remains of the valve of the foramen ovale, of which the crescentic fold is seen towards the left hand of $1^{\prime} ; 2$, a narrow portion of the wall of the auricle and ventricle preserved round the auriculoventricular orifice; $3,3^{\prime}$, the cut surface of the walls of the ventricle, seen to become very much thinner towards $3^{\prime \prime}$, at the apex; 4, a small part of the anterior wall of the left ventricle which has been preserved with the principal anterior columna carnea or musculus papillaris attached to it; 5,5 , musculi papillares; $5^{\prime}$, the left side of the septum, between the two ventricles, within the cavity of the left ventricle; $6,6^{\prime}$, the mitral valve; 7 , placed in the interior of the aorta, near its commencement and above the three segments of its semilunar valve which are hanging loosely together; $7^{\prime}$, the exterior of the great aortic sinus; 8 , the root of the pulmonary artery and its semilunar valves; $8^{\prime}$, the separated portion of the pulmonary artery remaining attached to the aorta by 9 , the cord of the ductus arteriosus; 10 , the arteries rising from the summit of the aortic arch. (Allen Thomson.)

cular and the aortic, guarded by the valves corresponding to those of the right side of the heart, viz., the bicuspid or mitral and the semi- 
lunar. The walls of the left ventricle, which in man are nearly half an inch in thickness, are about three times as thick as those of the right.

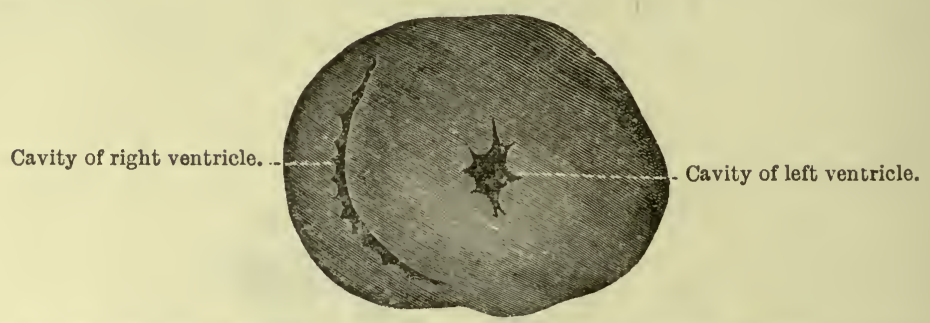

FIG. 193.-Transverse section of bullock's heart in a state of cadaveric rigidity. (Dalton.)

Capacity of the Chambers.-During life each ventricle is capable of containing about three ounces of blood. "The capacity of the auricles is rather less than that of the ventricles: the thickness of their walls is considerably less. The latter condition is adapted to the small amount of force which the auricles require in order to empty themselves into their adjoining ventricles; the former, to the circumstance that the ventricles are partly filled with blood before the auricles contract.

Size and Weight of the Heart.-The heart is about 5 inches long (about $12.6 \mathrm{~cm}$.), $3 \frac{1}{2}$ inches $\left(8 \mathrm{~cm}\right.$.) greatest width, and $2 \frac{1}{2}$ inches $(6.3 \mathrm{~cm}$.) in its extreme thickness. The average weight of the heart in the adult is from 9 to 10 ounces (about 300 grms.); its weight gradually increases throughout life till middle age; it diminishes in old age.

Structure.-The main thickness of the heart-wall is composed of muscular tissue; but a ring of connective tissue, to which many of the muscular fibres are attached, lies between each auricle and ventricle at the auriculo-ventricular orifice. The embryonic origin of the heart from a single tube is indicated by the fact that the superficial layers of muscle of the auricles and also of the ventricles encircle the chambers of the two sides; this ensures their simultaneous contraction. Very varying accounts are given of the arrangement of the layers of fibres especially in the ventricles, but the best appears to be the following: in the interior we have muscular fibres which are thrown into the columnæ carneæ, and papillary muscles; it may be called the papillary layer. Next come fibres arranged circularly, some round the left, and others round the right ventricle; this layer called the circular layer comprises the main thickness of the ventricular wall, and the act of propelling the blood into the arteries is chiefly performed by it. External to this, arranged in three principal bands, are fibres which spirally encircle one ventricle, and 
then pass by the septum around the other ventricle, and finally terminate at the base of the heart: this is called the spiral layer.

In the amphibian heart there is an obvious muscular connection between the auricle and ventricle. A similar muscular link mingled with non-medullated nerve-fibres also occurs in the mammal. This bundle passes down from the interauricular septum to the interventricular septum, where it divides into two bundles, one for each ventricle: its fibres are peculiar in structure and are known as Purkinje's fibres (p. 71). It is called the auriculoventricular bundle. At the junction of the superior vena cava and the right auricle is a focus of tissue known as the sino-auricular node which corresponds to the sinus venosus of the embryo and lower mammals. The special significance of these structures will be discussed later (see p. 257).

Valves.-The arrangement of the heart's valves is such that the blood can pass only in one direction (fig. 194).

The tricuspid valve (5, fig. 191) presents three principal cusps or subdivisions, and the mitral or bicuspid valve has two such portions (6, fig. 192). But in both valves there is between each two principal portions a smaller one: so that more properly, the tricuspid may be described as consisting of six, and the mitral of four, portions. Each portion is of triangular form. Its base is continuous with the bases of the neighbouring portions, so as to form an annular membrane around the auriculoventricular opening, and is

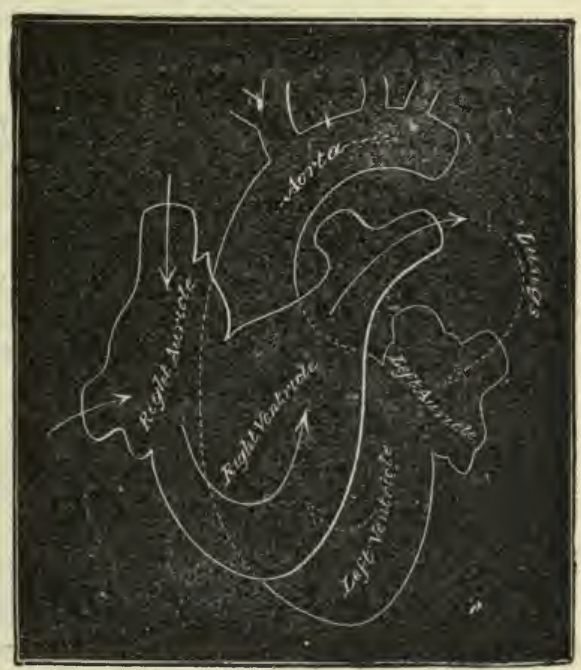

Fio. 194.-Diagrem of the circulation through the heart. (Dalton.). fixed to the tendinous ring which encircles the orifice.

While the bases of the cusps of the valves are fixed to the tendinous rings, their borders are fastened by slender tendinous fibres, the chordoc tendinece, to the internal surface of the walls of the ventricles, the muscular fibres of which project into the ventricular cavity in the form of bundles or columns-the columnce carnece. These columns are not all alike, for while some are attached along their whole length on one side, and by their extremities, others are attached only by their extremities; and a third set, to which the 
name musculi papillares has been given, are attached to the wall of the ventricle by one extremity only, the other projecting, papillalike, into the cavity of the ventricle (5, fig. 192), and having attached to it chordæ tendineæ. Of the tendinous cords, besides those which pass to the margins of the valves, there are some of especial strength, which pass to the edges of the middle and thicker portions of the cusps. The ends of these cords are spread out in the substance of the valve, giving its middle part its peculiar strength and toughness. Moreover, the musculi papillares are so placed that, from the summit of each, tendinous cords proceed to the adjacent halves of two of the principal divisions, and to one intermediate or smaller division, of the valve.

The preceding description applies equally to the mitral and tricuspid valve; but it should be added that the mitral is considerably thicker and stronger than the tricuspid, in accordance with the greater force which it is called upon to resist.

The semilunar valves guard the orifices of the pulmonary artery and of the aorta. They are nearly alike on both sides of the heart; but the aortic valves are more strongly constructed than the pulmonary valves, in accordance with the greater pressure which they have to withstand. Each valve consists of three parts which are of semilunar shape, the convex margin of each being attached to a fibrous ring at the place of junction of the artery to the ventricle, and the concave or nearly straight border being free, so as to form a little pouch like a watch-pocket (7, fig. 192). In the centre of the free edge of the pouch, which contains a fine cord of fibrous tissue, is a small fibrous nodule, the corpus Arantii, and from this and from the attached border fine fibres extend into every part of the mid substance of the valve, except a small lunated space just within the free edge, on each side of the corpus Arantii. Here the valve is thinnest, and composed of little more than the endocardium. Thus constructed and attached, the three semilunar pouches are placed side by side around the arterial orifice of each ventricle; they are separated by the blood passing out of the ventricle, but immediately afterwards are pressed together so as to prevent any return. Opposite each of the semilunar cusps, both in the aorta and pulmonary artery, there is a bulging outwards of the wall of the vessel: these bulgings are called the sinuses of Valsalva.

\section{Course of the Circulation.}

The blood is conveyed away from the left ventricle (as in the diagram, fig. 195) by the aorta to the arteries, and returned to the right auricle by the veins, the arteries and veins being continuous with each other at the far end by means of the capillaries.

From the right auricle the blood passes to the right ventricle, then 
by the pulmonary artery, which divides into two, one for each lung, then through the pulmonary capillaries, and through the pulmonary veins (two from each lung) to the left auricle. From here it passes into the left ventricle, which brings us back to where we started from.

The complete circulation is thus made up of two circuits, the one, a shorter circuit from the right side of the heart to the lungs and back again to the left side of the heart; the other and longer circuit, from the left side of the heart to all parts of the body and back again to the right side. The circulations through the lungs and through the system generally are respectively named the Pulmonary

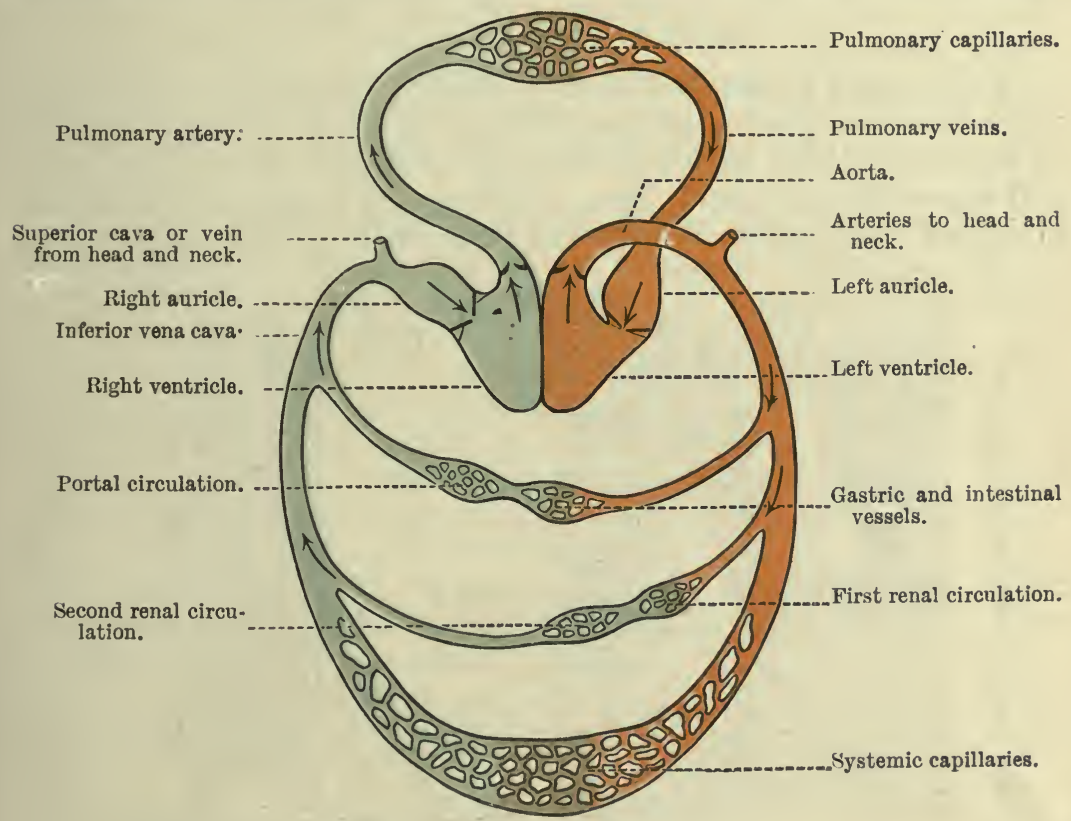

FIG. 195.-Diagram of the circulation.

and Systemic or lesser and greater circulations. It will be noticed also in the same figure that a portion of the stream of blood having been diverted once into the capillaries of the intestinal canal, and some other abdominal organs, and gathered up again into a single stream, is a second time divided in its passage through the liver, before it finally reaches the heart and completes a revolution. This subordinate stream through the liver is called the Portal circulation. A somewhat similar accessory circulation is that through the kidneys, called the Renal circulation. The difference of colours in fig. 195 indicates roughly the difference between arterial and venous blood. 
The blood is oxygenated in the lungs, and the formation of oxyhæmoglobin gives to the blood a bright red colour. This oxygenated or arterial blood (contained in the pulmonary veins, the left side of the heart, and systemic arteries) is in part reduced in the tissues, and the deoxygenated hæmoglobin is darker in tint than the oxyhæmoglobin; this venous blood passes by the systemic veins to the right side of the heart and pulmonary artery to the lungs, where it once more receives a fresh supply of oxygen.

N.B.-It should, however, be noted that the lungs, like the rest of the body, are also supplied with arterial blood, which reaches them by the bronchial arteries.

\section{The Arteries.}

The arterial system begins at the left ventricle in a single large trunk, the aorta, which almost immediately after its origin gives off in the thorax three large branches for the supply of the head, neck, and upper extremities; it then traverses the thorax and abdomen, giving off branches, some large and some small, for the supply of the

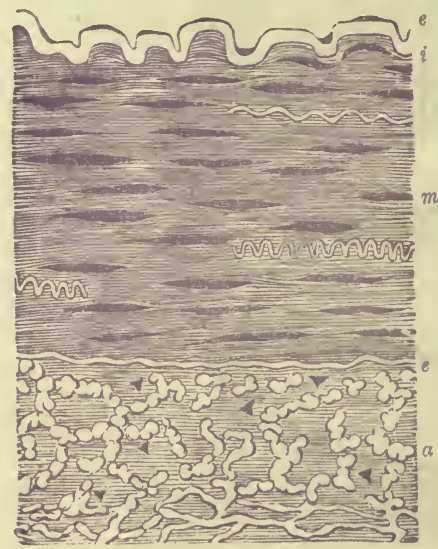

FIG. 196.-Transverse section through a large branch of the inferior mesenteric artery of a pig. e, Endothelial mem. brane; $i$, tunica elastica interna, no subendothelial layer is seen; $m$, mus cular tunica media, containing only a few wavy elastic fibres; $e, e$, tunica elastica externa, dividing the media from the connective-tissue adventitia, a. (Klein and Noble Smith.) $\times 350$.

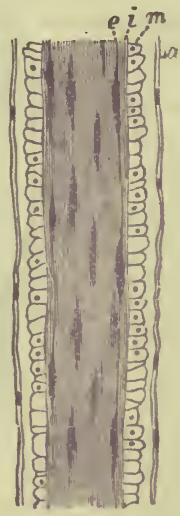

Fig. 197.-Minute artery viewed in longitudinal section. $e$, Nucleated endothelial membrane, with faint nuclei in lumen, looked at from above; $i$, elastic membrane; $m$, muscular coat or tunica media; $a$, tunica adventitia. (Klein and Noble Smith.) $\times 250$.

various organs and tissues it passes on its way. In the abdomen it divides into two chief branches, for the supply of the lower extremities. The arterial branches wherever given off divide and subdivide, until the calibre of each subdivision becomes very minute, and 
these minute vessels lead into capillaries. Arteries are, as a rule, placed in situations protected from pressure and other dangers, and are, with few exceptions, straight in their course, and frequently communicate (anastomose or inosculate) with other arteries. The branches are usually given off at an acute angle, and the sum of the sectional areas of the branches of an artery generally exceeds that of the parent trunk; and as the distance from the origin is increased, the area of the combined branches is increased also. After death, arteries are usually found dilated (not collapsed as the veins are) and empty, and it was to this fact that their name ( $\alpha \rho \tau \eta \rho i a$, the windpipe) was given them, as the ancients believed that they conveyed air to the various parts of the body. As regards the arterial system of the lungs, the pulmonary artery is distributed much as the arteries belonging to the general systemic circulation.

Structure.-The arterial wall is composed of the following coats:-

(a) The external coat or tunica adventitia (figs. 196 and 197, a), the strongest part of the wall of the artery, is formed of areolar tissue, with which is mingled throughout a network of elastic fibres. At the inner part of this outer coat the elastic network forms, in some arteries, so distinct a layer as to be sometimes called the external elastic coat (fig. 196, e).

(b) The middle coat (fig. 196, $m$ ) is composed of both muscular and elastic fibres, with a certain proportion of areolar tissue. In the larger arteries (fig. 196) its thickness is comparatively as well as absolutely much greater than in the small ones; it constitutes the greater part of the arterial wall. The muscular fibres are unstriped (fig. 198), and are arranged for the most part transversely to the long axis of the artery; while the elastic element, taking also a transverse direction, is disposed in the form of closely interwoven and branching fibres, which intersect in all parts the layers of muscular fibres. In arteries of various
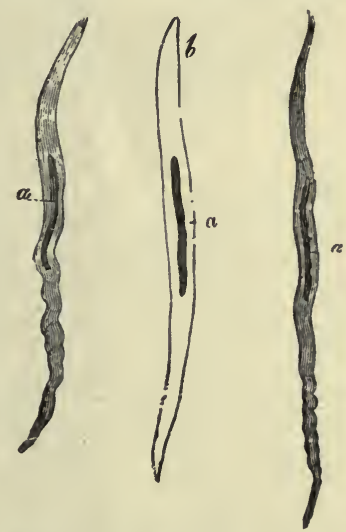

Fig. 198.-Muscular fibre-cells from human arteries, magni. fled 350 diameters. (Kölliker.) $a$. Nucleus. b. A fibre-cell treated with acetic acid. sizes there is a difference in the proportion of the muscular and elastic element, elastic tissue preponderating in the largest arteries, and unstriped muscle in those of medium and small size.

(c) The internal coat is formed by a layer of elastic tissue, called the fenestrated membrane of Henle. Its inner surface is lined with a delicate layer of elongated endothelial cells (fig. 196, e), which make it smooth, so that the blood may flow with the smallest possible 
amount of resistance from friction. Immediately external to the endothelial lining of the artery is fine connective tissue (subendothelial layer) with branched corpuscles. Thus the internal coat consists of three parts, $(a)$ an endothelial lining, $(b)$ the subendothelial layer, and $(c)$ elastic layer.

Vasa Vasorum.-The walls of the arteries are, like other parts of the body, supplied with little arteries, ending in capillaries and veins, which, branching throughout the external coat, extend for some distance into the middle, but do not reach the internal coat. These nutrient vessels are called vasa vasorum.

Nerves.-Most of the arteries are surrounded by a plexus of sympathetic nerves, which terminate in a plexus between the muscular fibres.

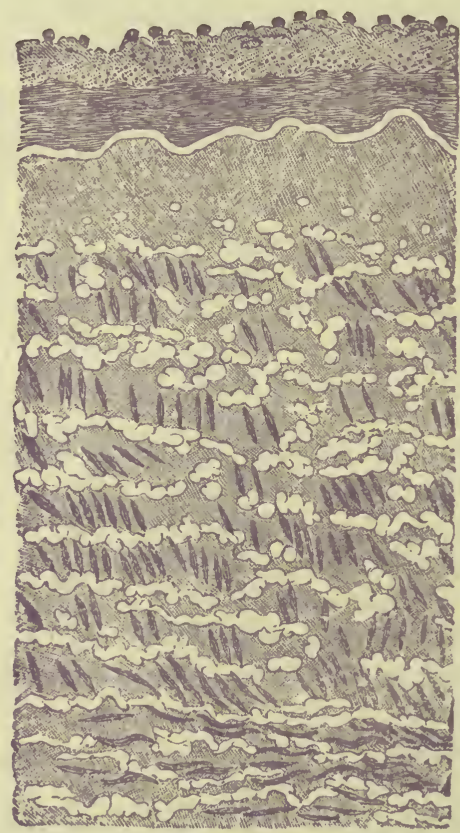

Endothelium.

Subendothelial layer.

Elastic layer.

Middle coat.

Fig. 199 - Transverse section of aorta through the internal and about half the middle coat.

\section{The Veins.}

The venous system begins in small vessels which are slightly larger than the capillaries from which they spring. Thèse vessels are gathered up into larger and larger trunks until they terminate (as regards the systemic circulation) in the two venæ cavæ and the 
coronary veins, which enter the right auricle, and (as regards the pulmonary circulation) in four pulmonary veins, which enter the left auricle. The total capacity of the veins diminishes as they approach the heart; but, as a rule, their capacity is two or three times that of the corresponding arteries. The pulmonary veins, however, are an exception to this rule, as they do not exceed in capacity the pulmonary arteries. The veins are found after death more or less collapsed, owing to their want of elasticity. They are usually distributed in a superficial and a deep set which communicate frequently in their course.

Structure.-In structure the coats of veins bear a general resemblance to those of arteries (fig. 200). Thus, they possess outer, middle, and internal coats.

(a) The outer coat is constructed of areolar tissue like that of the arteries, but it is thicker. In some veins it contains muscular fibres, which are arranged longitudinally.

(b) The middle coat is considerably thinner than that of the arteries; it contains circular unstriped muscular fibres, mingled with a few elastic fibres and a large proportion of white fibrous tissue. In the large veins, near the heart, namely, the vence cavoe and pulmonary veins, the middle coat is replaced, for some distance from the heart, by circularly arranged striped musculàr fibres, continuous with those of the auricles. The veins of bones, and of the central nervous system and its membranes have no muscular tissue.

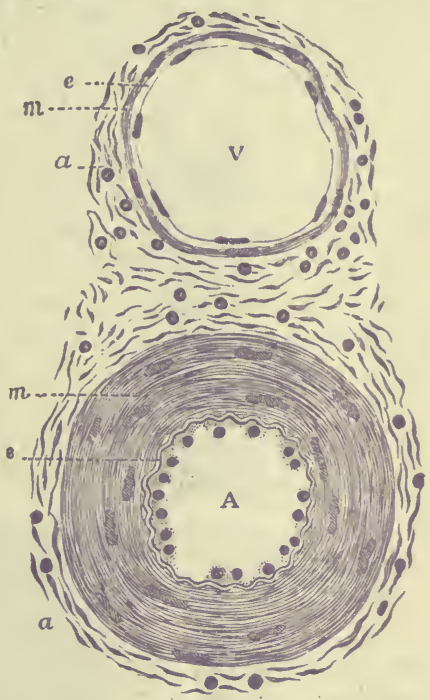

Fia. 200.-Transverse section through a small artery and vein of the mucous membrane of a child's epiglottis; the artery is thick-walled and the vein thinwalled. A. Artery, the letter is placed in the lumen of the vessel. $e$, Endothelial cells with nuclei clearly visible; these cells appear very thick from the contracted state of the vessel. Outside it a double wavy line marks the elastic layer of the tunica intima. $m$, Tunica media, consisting of unstriped muscular fibres circularly arranged; their nuclei are well seen. $a$, Part of the tunica adventitia showing bundles of connective-tissue fibre in section, with the circular nuclel of the connective-tissue corpuscles. This coat gradually merges into the surrounding connective tissue. $v$. In the lumen of the vein. The other letters indicate the same as in the artery. The muscular coat of the vein $(m)$ is seen to be much thinner than that of the artery. $\times 350$. (Klein and Noble Smith.)

(c) The internal coat of veins has a very thin fenestrated membrane, which may be absent in the smaller veins. The endothelium is made up of cells elongated in the direction of the vessel, but wider than in the arteries.

Valves.-One main distinction between arteries and veins is the presence of valves in the latter vessels. The general construc- 
tion of these valves is similar to that of the semilunar valves of the aorta and pulmonary artery, already described; but their free margins are turned in the opposite direction, i.e., towards the heart, so as to prevent any movement of blood backward. They are commonly placed in pairs, at various distances in different veins, but almost uniformly in each (fig. 201). In the smaller veins single valves

A.

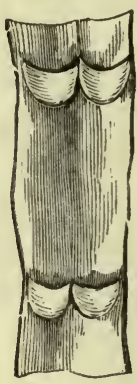

B

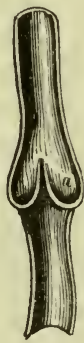

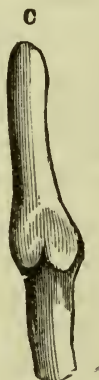

FiG. 201. - Diagram showing valves of veins. A, part of a vein laid open and spread out, with two pairs of valves. B, longitudinal section of a vein, showing the apposition of the edges of the valves in their closed state. C, portion of a distended vein, exhibiting a swelling in the situation of a pair of valves.

are often met with; and three or four are sometimes placed together, or near one another, in the largest veins, such as the

A.

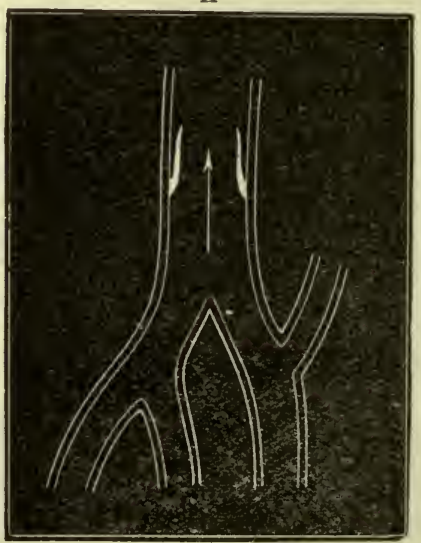

$\mathrm{B}$

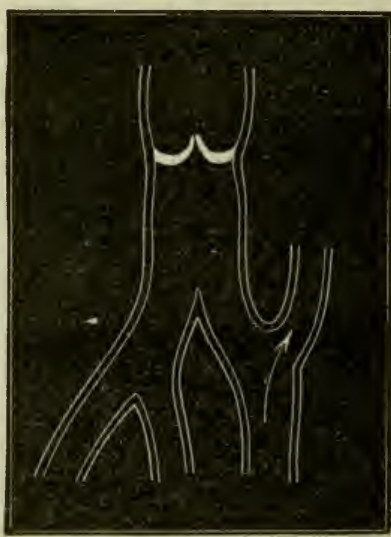

FIG. 202.-A, vein with valves open. B, with valves closed; stream of blood passing off by lateral channel. (Dalton.)

subclavian, at their junction with the jugular veins. They are composed of an outgrowth of the subendothelial tissue covered with 
endothelium. Their situation in the superficial veins of the forearm is readily discovered by pressing along their surface, in the direction opposite to the venous current, i.e. from the elbow towards the wrist; when little swellings (fig. 201, c) appear in the position of each pair of valves. These swellings at once disappear when the pressure is removed.

Valves are not equally numerous in all veins, and in many they are absent altogether. They are most numerous in the veins of the extremities, and more so in those of the leg than the arm. They are commonly absent in veins of less than a line in diameter, and, as a general rule, there are few or none in those which are not subject to muscular pressure. Among those veins which have no valves may be mentioned the superior and inferior vena cava, the pulmonary veins, the veins in the interior of the cranium and vertebral canal, the veins of bone, and the umbilical vein. The valves of the portal tributaries are very inefficient.

Lymphatics of Arteries and Veins.-Lymphatic spaces are present in the coats of both arteries and veins. In the external coat of large

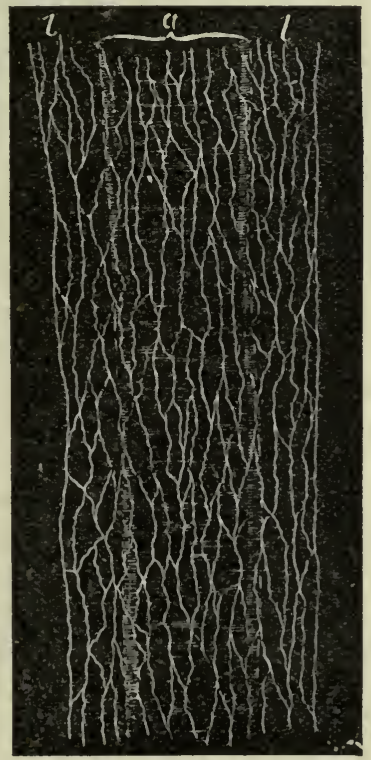

Fig. 203.- Surface view of an artery from the mesentery of a frog, ensheathed in a perivascular lym. phatic vessel. a, The artery, with its circular muscular coat (media) indicated by broad transverse markings, with an indication of the adventitia outside. $l$, Lymplatic vessel; its wall is a simple endothelial membrane. (Klein and Noble Sn ith.)

vessels they form a plexus of more or less tubular vessels. In smaller vessels they appear as spaces lined by endothelium. Sometimes, as 
in the arteries of the omentum, mesentery, membranes of the brain, lung, liver and spleen, the spaces are continuous with vessels which distinctly ensheath them-perivascular lymphatics (fig. 203).

\section{The Capillaries.}

In most cases the blood finds its way from the small arteries to the small veins through a network of minute cylindrical vessels called capillaries. But in certain cases (parathyroid, spleen, the thyroid of some animals, erectile tissue, the placenta, and the embryonic liver and kidney) the connecting system of vessels are larger and have an irregular shape; these vessels are termed sinusoids.

The, walls of both capillaries and sinusoids are composed of endothelium-a single layer of elongated flattened and nucleated cells, so joined and dovetailed together as to form a continuous

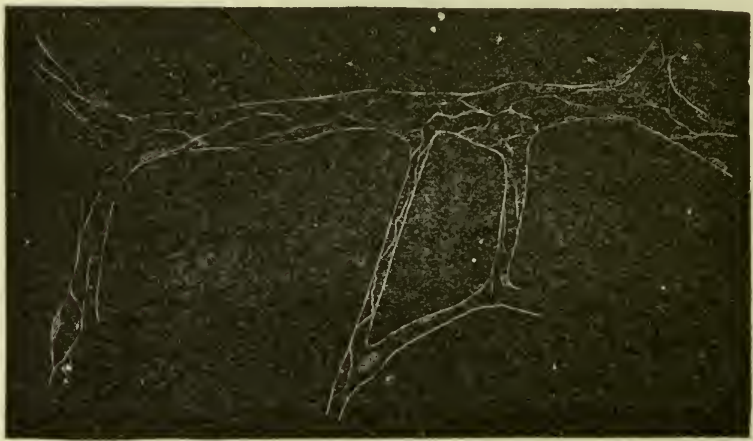

FiG. 204.-Capillary blood-vessels from the omentum of rabbit, showing the nucleated endothelial membrane of which they are composed. (Klein and Noble Smith.)

transparent membrane (fig. 204). Here and there the endothelial cells do not fit quite accurately; the space is filled up with cement material; these spots are called pseudo-stomata.

The diameter of the capillary vessels varies somewhat in the different tissues of the body, the most common size being about $\frac{1}{2000}$ th of an inch $(12 \mu)$. Among the smallest may be mentioned those of the brain, and of the follicles of the mucous membrane of the intestines; among the largest, those of the skin, lungs, and especially those of the medulla of bones.

The size of capillaries varies necessarily in different animals in relation to the size of their blood-corpuscles: thus, in the Proteus, the capillary circulation can just be discerned with the naked eye.

The form of the capillary network presents considerable variety in the different tissues of the body: the varieties consist principally of modifications of two chief kinds of mesh, the rounded and the elongated. That kind in which the meshes or interspaces have a 
roundish or polygonal form is the most common, and prevails in those parts in which the capillary network is most dense, such as the lungs (fig. 205), most glands and mucous membranes, and the cutis. The capillary network with elongated meshes is observed in parts in which the vessels are arranged among bundles of fine tubes or fibres, as in muscles and nerves. In such parts, the meshes form parallelograms (fig. 206), the short sides of which may be from three

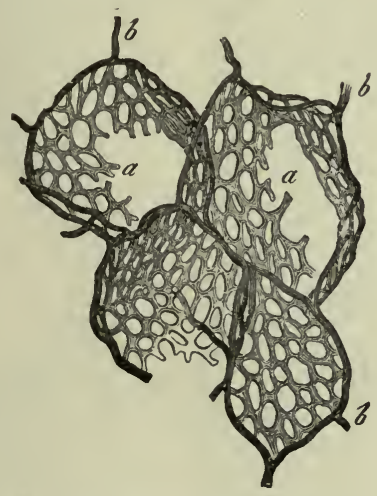

FIG. 205.-Network of capillary vessels of the air-cells of the horse's lung magnified. $a, a$, Capillaries proceeding from $b$, $b$, terminal branches of the pulmonary artery. (Frey.)

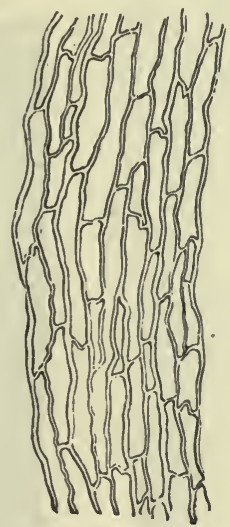

Fia. 206.-Injected capillary vessels of muscle seen with a low mag. nifying power.

(Sharpey.)

to eight or ten times less than the long ones; the long sides are more or less parallel to the long axis of the fibres.

The number of the capillaries and the size of the meshes in different parts determine in general the degree of vascularity of those parts. The capillary network is closest in the lungs and in the choroid coat of the eye.

It may be held as a general rule, that the more active the functions of an organ are, the more vascular it is. Hence the narrowness of the interspaces in all glandular organs, in mucous membranes, and in growing parts, and their much greater width in bones, ligaments, and other comparatively inactive tissues.

\section{Lymphatic Vessels.}

The blood leaves the heart by the arteries; it returns to the heart. by the veins; but this last statement requires modification, for in the capillaries some of the blood-plasma escapes into the cell spaces of the tissues and nourishes the tissue-elements. This fluid, which is 
called lymph, is gathered up and carried back again into the blood by a system of vessels called lymphatics.

The principal vessels of the lymphatic system are, in structure, like small thin-walled veins, provided with numerous valves. The beaded appearance of the lymphatic vessels shown in figs. 208 and 209 is due to the presence of these valves. They commence in fine microscopic lymph-capillaries, in the organs and tissues of the body,

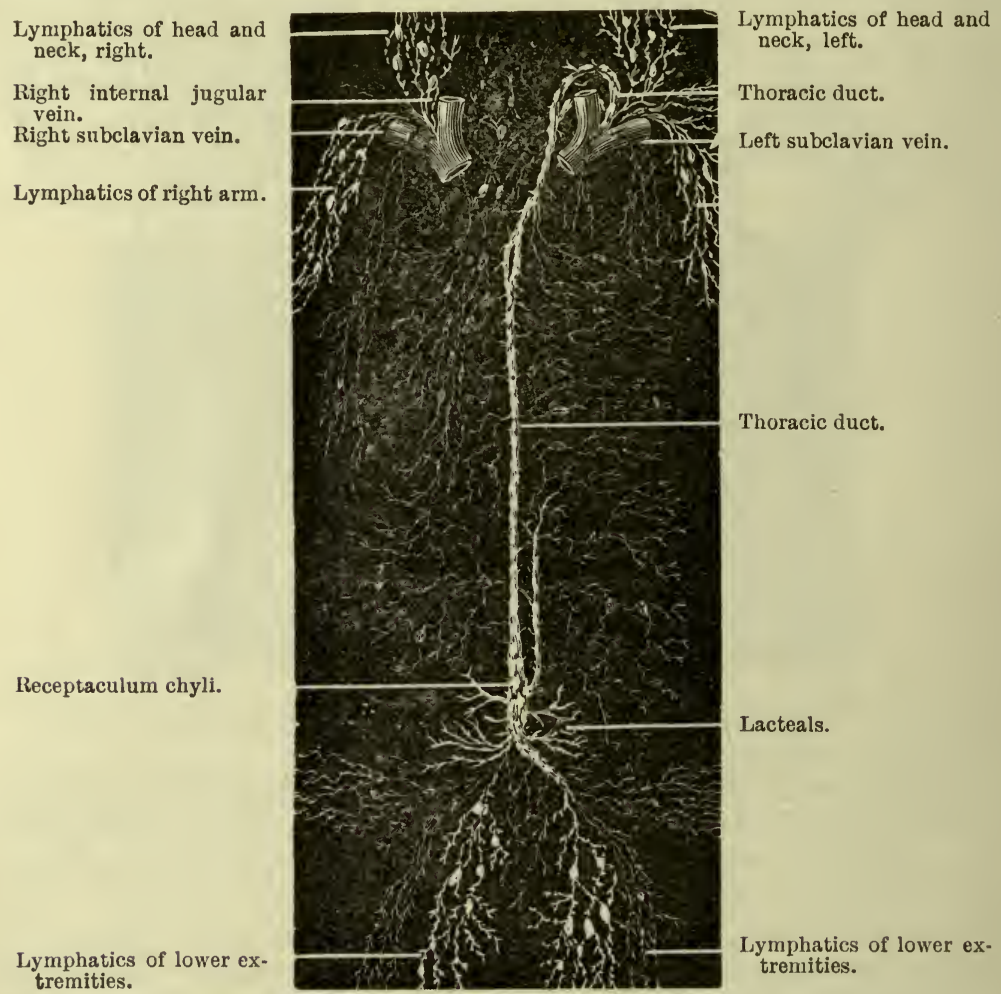

Fig. 207.-Diagram of the principal groups of lymphatic vessels. (From Quain.)

and they end in two trunks which open into the large veins near the heart (fig. 207). The fluid which they contain, unlike the blood, passes only in one direction, namely, from the fine branches to the trunk, and so to the large veins, on entering which it is mingled with the stream of blood. In fig. 207 the greater part of the contents of the lymphatic system of vessels will be seen to pass through a comparatively large trunk called the thoracic duct, which finally empties its contents into the blood-stream, at the junction of the internal 
jugular and subclavian veins of the left side. There is a smaller duct on the right side. The lymphatic vessels of the intestinal canal

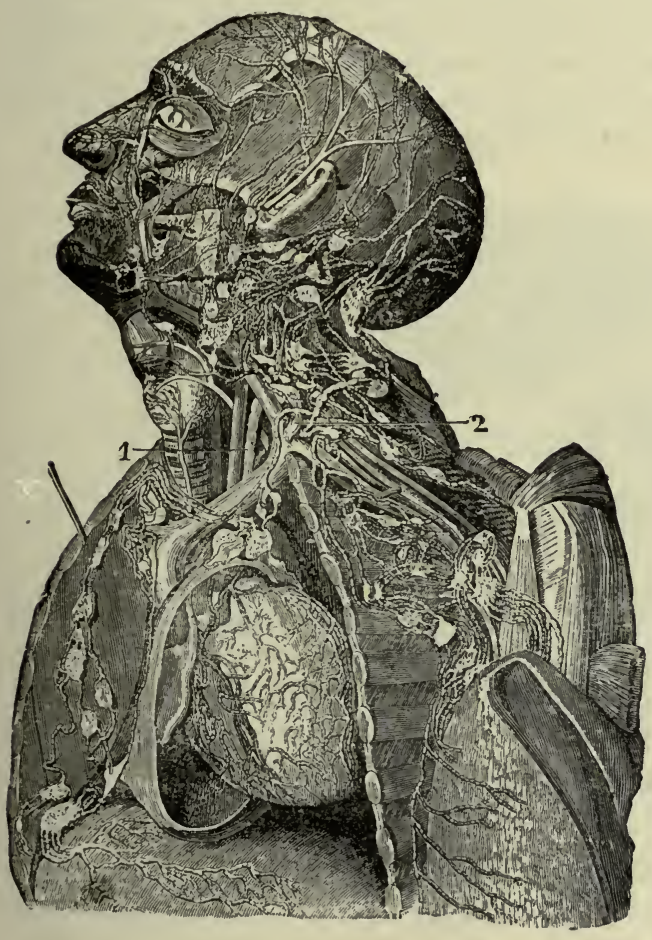

Fig. 208.-Lymphatic vessels of the head and neck and the upper part of the trunk (Mascagni). fo-The chest and pericardium have been opened on the left side, and the left mamma detached and thrown outwards over the left arm, so as to expose a great part of its deep surface. The principal lymphatic vessels and glands are shown on the side of the head and face and in the neck, axilla, and mediastinum. Between the left internal jugular vein and the common carotid artery, the upper ascending part of the thoracic duct marked 1, and above this, and descending to 2 , the arch and last part of the duct. The termination of the upper lymphatics of the diaphragm in the medias. tinal glands, as well as the cardiac and the deep mammary lymphatics, is also shown.

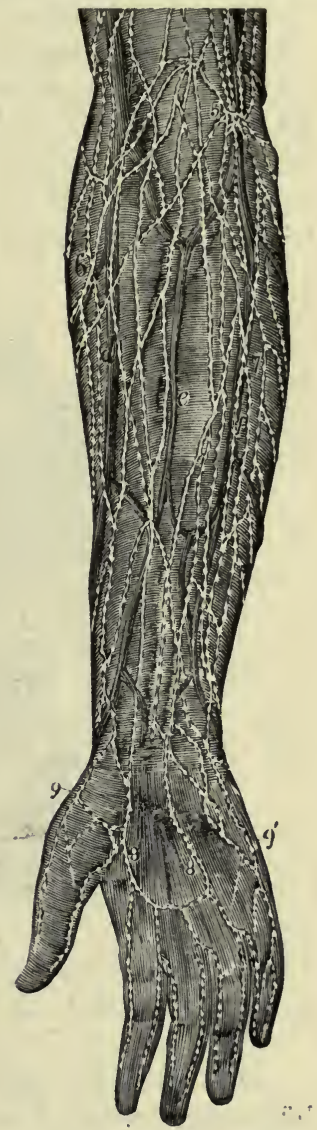

Fig. 209.-Superficial lymphatics of the forearm and palin of the hand. $f_{0}-5$. Two small glands at the bend of the arm. 6. Radial lymphatic vessels. 7. Ulnar lymphatic vessels, $8,8^{\prime}$. Palmar arch of lymphatics. 9, 9 . Outer and inner sets of vessels. $b$. Cephalic vein. $d$, Radial vein. $e$, Median vein. $f$, Ulnar vein. The lymphatics are represented as lying on the deep fascia. (Mascagni.)

are called lacteals, because during digestion (if the meal contains fat) the fluid contained in them resembles milk in appearance; and the lymph in the lacteals during the period of digestion is called chyle. 
Chyle is lymph containing finely divided fat-globules. In some parts of its course the lymph-stream passes through lymphatic glands, to be described later on.

Origin of Lymph Capillaries.-The lymphatic capillaries commence most commonly either $(a)$ in closely-meshed networks (see fig. 210), or (b) in irregular lacunar spaces, lined by endothelium, between the various structures of which the different organs are composed. These spaces freely communicate with the cell spaces (Saft Kanälchen, see p. 31) of the tissues.

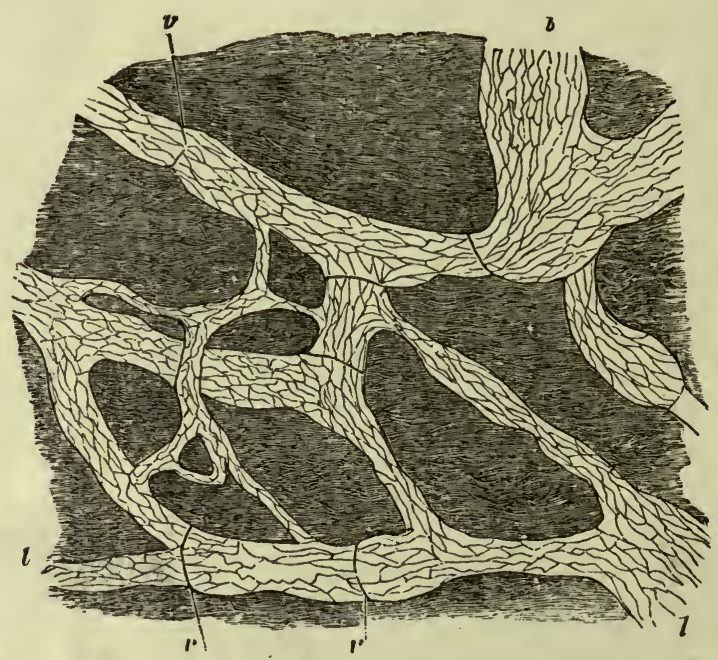

FIG. 210.-Lymphatics of central tendon of rabbit's diaphragm, stained with silver nitrate. The shaded background is composed of bundles of white fibres, between which the lymphatics lie. $l$, Lymphatics lined by long narrow endothelial cells, and showing $v$ valves at frequent intervals. (schofield.)

The lacteals offer an illustration of another mode of origin, namely, as blind dilated extremities in the villi of the small intestine (see fig. 28, p. 22).

The structure of lymphatic capillaries is very similar to that of blood capillaries; their walls consist of a single layer of elongated endothelial cells with sinuous outline, which cohere along their edges to form a delicate membrane. They differ from blood capillaries mainly in their larger and very variable calibre, in the presence of valves, and in their numerous communications with the spaces of the tissues.

In certain parts of the body, stomata exist, by which lymphatic capillaries directly communicate with parts formerly supposed to be closed cavities. They have been found in many serous membranes; a serous cavity thus forms a large lymph-sinus or widening out of the lymph-capillary system with which it directly communicates. 


\section{CHAPTER XX}

\section{THE CIRCULATION OF THE BLOOD}

WE have now to approach the physiological side of the subject, and study the means by which the blood is kept in movement, so that it may convey nutriment to all parts, and remove from those parts the waste products of their activity.

Previous to the time of Harvey, the vaguest notions prevailed regarding the use and movements of the blood. The arteries were supposed by some to contain air, by others to contain a more subtle essence called animal spirits; the animal spirits were supposed to start from the ventricles of the brain, and they were controlled by the soul which was situated in the pineal gland. How the animal spirits got into the arteries was an anatomical detail which was bridged across by the imagination.

There was an idea that the blood moved, but this was considered to be a haphazard, to-and-fro movement, and confined to the veins. The proofs that the movement is in a circle were discovered by William Harvey, and to this eminent discoverer also belongs the credit of pointing out the methods by which every physiological problem must be studied. In the first place there must be correct anatomical knowledge, and in the second there must be experiment, by which deductions from structure can be tested; moreover, this second method is by far the more important of the two. Harvey's proofs of the circulation came under both these heads. The structural or anatomical facts upon which he relied were the following:-

1. The existence of two distinct sets of tubes in connection with the heart, namely, the arteries and the veins.

2. The existence in the heart and also in the veins, of valves which would only allow the passage of the blood in one direction.

His experimental data were the following:-

3. That the blood spurts with great force and in a jerky manner from an artery opened during life, each jerk corresponding with a beat of the heart.

4. That if the large veins near the heart are tied, the heart 227 
becomes pale, flaccid, and bloodless, and on removal of the ligature the blood again flows into the heart.

5. If the aorta is tied, the heart becomes distended with blood, and cannot empty itself until the ligature is removed.

6. The preceding experiments were performed on animals, but by the following experiment he showed that the circulation is a fact in man also; if a ligature is drawn tightly round a limb, no blood can enter it, and it becomes pale and cold. If the ligature is somewhat relaxed so that blood can enter but cannot leave the limb, it becomes swollen. If the ligature is removed, the limb soon regains its normal appearance.

7. Harvey also drew attention to the fact that there is general constitutional disturbance resulting from the introduction of a poison at a single point, and that this can only be explained by a movement of the circulating fluid all over the body.

8. If an artery is wounded, hæmorrhage may be stopped by pressure applied between the heart and the wound; but in the case of a wound in a vein, the pressure must be applied beyond the seat of injury.

Since Harvey's time many other proofs have accumulated; for instance:-

9. If a substance which, like ferrocyanide of potassium, can be readily detected, is injected at a certain point into a blood-vessel, it will after the lapse of a short interval have entirely traversed the circulation and be found in the blood collected from the same point.

10. Perhaps the most satisfactory proof of the circulation is one now within the reach of every student, though beyond that of Harvey. It consists in actually seeing the passage of the blood from small arteries through capillaries into veins in the transparent parts of animals, such as the tail of a tadpole or the web of a frog's foot. Harvey could not follow this part of the circulation, for he had no lenses sufficiently powerful to enable him to see it. Harvey's idea of the circulation here was that the arteries carried the blood to the tissues, which he considered to be of the nature of a sponge, and the veins collected the blood again, much in the same way as drainage pipes would collect the water of a swamp. The discovery that the ends of the arteries are connected to the commencements of veins by a definite system of small tubes we now call capillaries, was made by Malpighi, in the year 1661. He first observed them in the tail of the tadpole, and Leeuwenhoek, seven years later, saw the circulation in the lung of the frog.

We can now proceed to study some of the principles on which the circulation depends:-

The simplest possible way in which we could represent the circulatory system is shown in fig. 211 A. Here there is a closed 
ring containing fluid, and upon one point of the tube is an enlargement $(\mathrm{H})$ which will correspond to the heart. It is obvious that if such a ring made of an ordinary Higginson's syringe and a tube were placed upon the table, there would be no movement of the fluid in it; in order to make the fluid move there must be a difference of pressure between different parts of the fluid, and this difference of pressure is caused in the fluid by the pressure on it of the heart walls. If, for instance, one takes the syringe in one's hand and squeezes it, one imitates a contraction of the heart: if the syringe has no valves, the fluid would pass out of each end of it in the direction of the two arrows placed outside the ring. When the pressure on the syringe is relaxed (this would correspond to the interval between the heart beats), the fluid would return into the heart again in the direction of the two arrows placed inside the ring. This, however, would be merely a to-and-fro movement, not a circula-
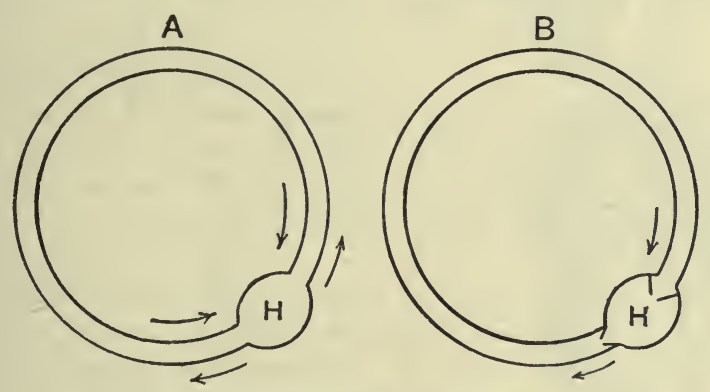

FTa. 211.-Simple schema of the circulation.

tion. Fig. $211 \mathrm{~B}$ shows how this to-and-fro movement could, by the presence of valves, be converted into a circulation; when the heart contracts the fluid could pass only in the direction of the outer arrow; when the heart relaxes it could pass only in the direction of the inner arrow; the direction of both arrows is the same, and so if the contraction and relaxation of the heart are repeated often enough the fluid will move round and round within the tubular ring.

The main factor in the circulation is difference of pressure. In general terms fluid flows from where the pressure is high to where it is lower. This difference of pressure is produced in the first instance by the contraction of the heart, but we shall find in our study of the vessels that some of this pressure is stored up in the elastic arterial walls, and keeps up the circulation during the periods that the heart is resting.

Coming to different groups in the animal kingdom we may take the crayfish or the lobster as instances of animals which possess a hæmolymph system, that is, there is no distinction between blood 
and lymph. The heart pumps the circulating fluid along a system of vessels which distribute it over the body; there are no capillaries, and the hæmolymph is discharged into the tissue spaces; it is thence drained into channels which convey it to the gills, and after it is aërated there in a set of irregular vessels, it is returned to the pericardium. It is sucked from the pericardium into the heart during diastole, through five small orifices in the cardiac wall; during systole these are closed by valves. In these animals the rate of flow of hæmolymph is necessarily slow.

In worms, the circulatory system is almost as simple as in the

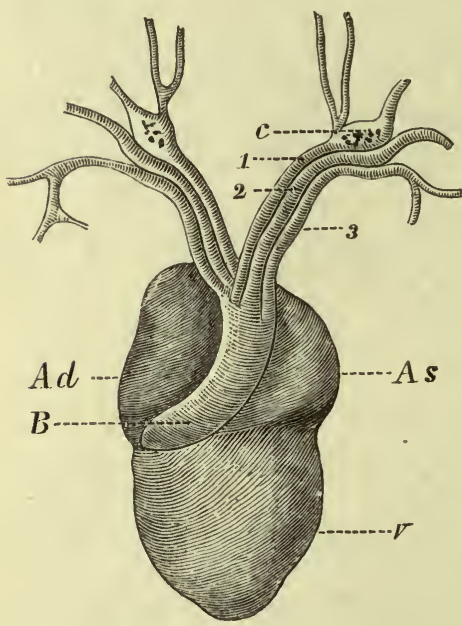

Fic. 212.-The heart of a frog (Rana esculenta) from the front. $V$, ventricle; $A d$, right auricle; $A s$, left auricle; $B$, bulbus arteriosus, dividing into right and left aortæ. (Ecker.) last of all is the aortic bulb. From the bulb, branches pass to the gills, where they break up into capillaries, and the blood is aërated: it then once more enters larger vessels which unite to form the dorsal aorta, whence the blood is distributed by arteries to all parts of the body; here it passes into the systemic capillaries, then into the veins which enter the sinus (whence we started) by a few large trunks.

Taking the frog as an instance of an amphibian, we find the heart more complex, and the simple peristaltic action of the heart muscle as we have described it in the hearts of worm and fish, becomes correspondingly modified. There is only one ventricle, but there are two auricles, right and left.

The ventricle contains mixed blood, since it receives arterial 
blood from the left auricle (which is the smaller of the two), and venous blood from the right auricle; the right auricle receives the venous blood from the sinus, which in turn receives it from the systemic veins. The left auricle, as in man, receives the blood from the pulmonary veins.

When the ventricle contracts, it forces the blood onward into the aortic bulb which divides into branches on each side for the supply of the head (fig. 212, 1), lungs and skin (fig. 212, 3), and the third branch (fig. 212 , 2), unites with its fellow of the opposite side to form the dorsal aorta for the supply of the rest of the body.

Passing from the amphibians to the reptiles, we find the division of the

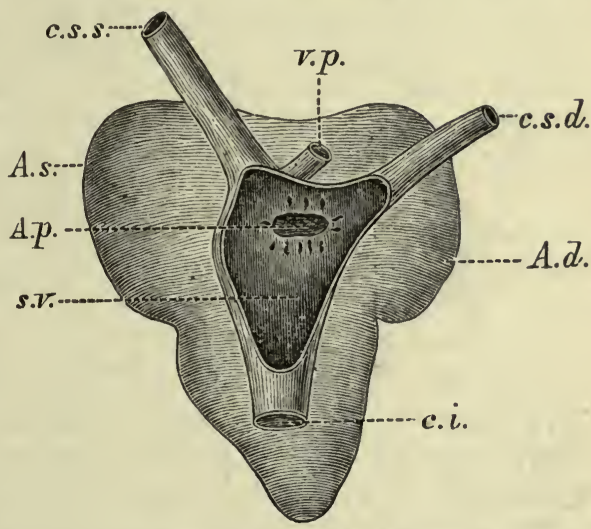

F1G. 213.-The heart of a frog (Rana esculenta) from the back, s. v, Sinus venosus opened; c,ss, , left vena crva superior; c.s.d.; right vena cava superior; c.i., vena cava inferior; v.p., vena pulmonalis; $A . d$. ., right auricle; A.s., left auricle; $A . p$. , opening of communication between the right auricle and the sinus venosus. $\times 21-3$. (Ecker.)

ventricle into two beginning, but it is not complete till we reach the birds. The heart reaches its fullest development in mammals, and we have already described the human as an example of the mammalian heart. The sinus venosus is not present as a distinct chamber in the mammalian heart (except in a very early fotal stage), but is represented by that portion of the right auricle at which the large veins enter. 


\section{CHAPTER XXI}

\section{PHYSIOLOGY OF THE HEART}

\section{The Cardiac Cycle.}

The series of changes which occurs in the heart constitutes the cardiac cycle. This must be distinguished from the course of the circulation. The term cycle indicates that if one observes the heart at any particular moment, the heart from that moment onwards undergoes certain changes until it once more assumes the same condition that it had at the moment when the observation commenced, when the cycle is again repeated, and so on. This series of changes consists of alternate contraction and relaxation. Contraction is known as systole, and relaxation as diastole.

The contraction of the two auricles takes place simultaneously, and constitutes the auricular systole; this is followed by the simultaneous contraction of the two ventricles, ventricular systole, and that by a period during which the whole of the heart is in a state of relaxation or diastole; then the cycle again commences with the auricular systole.

Taking 72 as the average number of heart beats per minute, each cycle will occupy $\frac{1}{72}$ of a minute, or a little more than 0.8 of a second. This may be approximately distributed in the following way:-

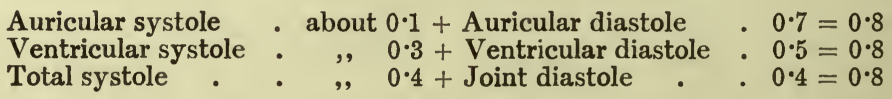

If the speed of the heart is quickened, the time occupied by each cycle is diminished, but the diminution affects chiefly the diastole. These different parts of the cycle must next be studied in detail.

The Auricular Diastole.-During this time, the blood from the large veins is flowing into the auricles, the pressure in the veins though very low being greater than that in the empty auricles. The blood expands the auricles, and during the last part of the auricular 
diastole it passes on into the ventricles. The dilatation of the auricles is assisted by the elastic traction of the lungs. The lungs being in a closed cavity, the thorax, and being distended with air, are in virtue of their elasticity always tending to recoil and squeeze the air out of their interior; in so doing they drag upon any other organ with which their surface is in contact: this elastic traction will be greatest when the lungs are most distended, that is during inspiration, and will be more felt by the thin-walled auricles than by the thick-walled ventricles of the heart.

The Auricular Systole is sudden and very rapid; by contracting, the auricles empty themselves into the ventricles. The contraction commences at the entrance of the great veins, and is thence propagated towards the auriculo-ventricular opening. The reason why the blood does not pass backwards into the veins, but onward into the ventricles, is again a question of pressure; the pressure in the relaxed ventricles, which is so small as to exert a suction action on the auricular blood, is less than in the veins. Moreover, the auriculo-ventricular orifice is large and widely dilated, whereas the mouths of the veins are constricted by the contraction of their muscular coats. Though there is no regurgitation of the blood backwards into the veins, there is a stagnation of the flow of blood onwards to the auricles. The veins have no valves at their entrance into the auricles, except the coronary vein, which does possess a valve; there are valves, however, at the junction of the subclavian and internal jugular veins.

Ventricular Diastole; during the last part of the auricular diastole and the whole of the auricular systole, the ventricles have been relaxed and then filled with blood. The dilatation of the ventricles is chiefly brought about in virtue of their elasticity; this is particularly evident in the left ventricle, with its thick muscular coat. It is equal to $23 \mathrm{~mm}$. of mercury, and is quite independent of the elastic traction of the lungs, which, however, in the case of the thinner-walled right ventricle comes into play.

The Ventricular Systole; this is the contraction of the ventricles, and it occupies more time than the auricular systole; when it occurs the auriculo-ventricular valves are closed and prevent regurgitation into the auricles, and when the force of the systole is greatest, and the pressure within the ventricles exceeds that in the large arteries which originate from them, the semilunar valves are opened, and the ventricles empty themselves, the left into the aorta, the right into the pulmonary artery. Each ventricle ejects about 3 ounces of blood with each contraction; the left in virtue of its thicker walls acts much more forcibly than the right. The greater force of the left ventricle is necessary, as it has to overcome the resistance of the small vessels all over the body; whereas the right 
ventricle has only to overcome peripheral resistance in the pulmonary district.

The shape of both ventricles during systole has been described as undergoing an alteration, the diameters in the plane of the base being diminished, and the length of the ventricles slightly lessened. The whole heart, moreover, moves towards the right and forwards, twisting on its long axis and exposing more of the left ventricle anteriorly than when it is at rest. These movements, which were first described by Harvey, have been since Harvey's time believed to be the cause of the cardiac impulse or apex beat which is to be felt in the fifth intercostal space about three inches from the middle line. It has, however, been shown by Haycraft that these changes only occur when the chest walls are open. When the heart contracts in a closed thorax it undergoes no rotation, and the contraction is concentric, that is, equal in all directions. The diminution of the heart's volume which occurs in systole cannot be the cause of the apex beat; it would rather tend to draw the chest wall inwards than push it outwards.

The apex beat is caused by two changes in the physical condition of the heart. In the first place, on systole the heart becomes hard and tense, and secondly, its attachment to the aorta becomes rigid instead of being flexible as it is in diastole. Thus, in systole, the heart becomes rigidly fixed to the aorta, and, as this vessel is curved, it tends to open out into a straight line, but is prevented by the counterresistance at the two ends of the arch. These are $(a)$ the resistance of the chest wall against the heart, and (b) that of the vertebræ and ribs against the thoracic aorta. The pressure of the heart against the chest wall is confined to a small area, situated in the fifth intercostal space, because the heart surface is much more curved than the internal thoracic wall. The forward movement this pressure causes is the apex beat. It must be noted that this movement is not over the actual apex of the heart, but is communicated from an area on the anterior cardiac surface.

\section{Action of the Valves of the Heart.}

1. The Auriculo-Ventricular.-The distension of the ventricles with blood continues throughout the whole period of their diastole. The auriculo-ventricular valves are gradually brought into place by some of the blood getting behind the cusps and floating them up; by the time that the diastole is complete, the valves are in apposition, and they are firmly closed by the reflux current caused by the systole of the ventricles. The diminution in the size of the auriculo-ventricular rings which occurs during systole, renders the auriculo-ventricular valves competent to close these openings. The margins of the cusps of the valves are still more secured in apposition with one another, by the simultaneous contraction of the musculi papillares, whose chordæ tendineæ have a special mode of attachment for this object. The cusps of the auriculo-ventricular valves meet not by their edges only, but. by the opposed surfaces of their thin outer borders.

The musculi papillares prevent the auriculo-ventricular valves from being everted into the auricle. For the chordæ tendineæ might allow the valves to be pressed back into the auricle, were it not that when the wall of the ventricle is brought by its contraction nearer to the auriculo-ventricular orifice, the musculi papillares more than compensate for this by their own contraction; they hold the cords 
tight, and, by pulling down the valves, add slightly to the force with which the blood is expelled.

These statements apply equally to the auriculo-ventricular valves on both sides of the heart; the closure of both is generally complete every time the ventricles contract. But in some circumstances the tricuspid valve does not completely close, and a certain quantity of blood is forced back into the auricle. This has been called its safetyvalve action. The circumstances in which it usually happens are those in which the vessels of the lung are already completely full when the right ventricle contracts, as, e.g., in certain pulmonary diseases, and in very active muscular exertion. In these cases, the tricuspid valve does not completely close, and the regurgitation of the blood may be indicated by a pulsation in the jugular veins synchronous with that in the carotid arteries.

2. The Semilunar Valves.-The commencement of the ventricular systole precedes the opening of the aortic valves by a fraction of a second, as is proved by examining records of the intraventricular and aortic pressure curves taken simultaneously. The first result of the contraction of the ventricles is the closure of the auriculo-ventricular valves, and as soon as this has been effected the intraventricular pressure begins to rise. It quickly reaches a point at which it equals the aortic pressure, and then exceeds it, and as soon as this pressure difference has been established the aortic valves are opened and blood flows from the ventricle into the aorta. The valves are kept open as long as the intraventricular pressure exceeds the aortic, but as soon as the heart has emptied itself, the ventricle begins to relax, its internal pressure consequently begins to fall, and an instant is quickly reached at which it is exceeded by the aortic. The blood, therefore, tends to flow back from the aorta, and in so doing fills up the pockets of the semilunar valves, which have always remained partly filled, and brings them together with a sharp movement. The movements of the valves are therefore effected by the occurrence of differences of pressure upon their two faces. When they meet they completely close the orifice, because their inner edges, which are thinner than the rest of the valves, are brought into apposition and held so by the high pressure acting on their aortic surfaces only.

\section{The Sounds of the Heart.}

When the ear is placed over the region of the heart, two sounds may be heard at every beat of the heart, which follow in quick succession, and are succeeded by a pause or period of silence. The first or systolic sound is dull and prolonged; its commencement coincides with the impulse of the heart against the chest wall, and it lasts during the greater part of the ventricular systole; it 
just precedes the pulse at the wrist. The second or diastolic sound is shorter and sharper, with a somewhat flapping character, and follows the end of ventricular systole, and is audible just after the radial pulse is felt. The sounds are often but somewhat inaptly compared to the syllables, lübb-dǔp.

Causes.-The exact cause of the first sound of the heart is a matter of discussion. Two factors probably enter into it, viz., first, the vibration of the auriculo-ventricular valves and the chordoe tendineor. This vibration is produced by the increased intraventricular pressure set up when the ventricular systole commences, which puts the valves on the stretch. It is not unlikely, too, that the vibration of the ventricular walls themselves, and of the aorta and pulmonary artery,

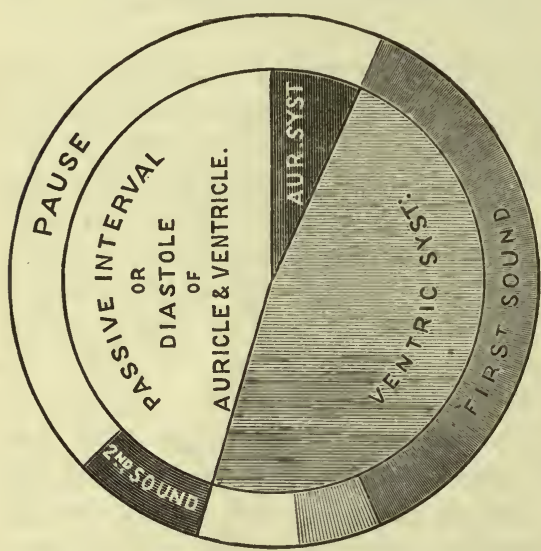

Fra. 214.-Scheme of cardiac cycle. The inner circle shows the events which occur within the heart; the outer the relation of the sounds and pauses to these events. (Sharpey and Gairdner.) all of which parts are suddenly put into a state of tension at the moment of ventricular contraction, may have some part in producing the first sound. The second factor is a muscular sound produced by contraction of the mass of muscular fibres which forms the ventricle. Looking upon the contraction of the heart as a single contraction and not as a series of contractions or tetanus, it is at first sight difficult to see why there should be any muscular sound at all when the heart contracts, as a single muscular contraction does not produce sound. It has been suggested, however, that it arises from the repeated unequal tension produced when the wave of muscular contraction passes along the very intricately arranged fibres of the ventricular walls. Many regard the valvular element as the more important of the two factors, because the sound is loudest at first, when the vibration of the valves commences, and fades away as the vibrations cease. If the sound was mainly muscular, it would be loudest when the muscular contraction was most powerful, which is approximately about the middle of the ventricular systole. The facts of disease lend support to the theory that the first sound is mainly valvular; for when the valves are incompetent, the first sound is largely replaced by a murmur due to regurgitation of blood into the auricle. After the removal of the heart from the body, the muscular contribution to the first sound is audible, but it is very faint. It is stated to have a somewhat lower pitch than the valvular sound. 
There is, on the other hand, much to be said against the view that the cause of the first sound is entirely due to vibration of the auriculo-ventricular valves. Any sound produced by the valves must be very quickly damped by the high pressure acting on their ventricular surfaces only. The sustained character of the sound (throughout practically the whole of the ventricular systole) is on the other hand exactly what is to be expected if it is of muscular origin. The argument that the extent to which the muscle sound contributes to the production of the first sound can be judged from the sound heard in an isolated and empty heart is quite fallacious, since under these conditions the muscle is contracting against no resistance.

The cause of the second sound is more simple than that of the first. It is entirely due to the vibration consequent on the sudden stretching of the semilunar valves when they are pressed down across the orifices of the aorta and pulmonary artery. The influence of these valves in producing the sound was first demonstrated by Hope, who experimented with the hearts of calves. In these experiments two delicate curved needles were inserted, one into the aorta, and another into the pulmonary artery, below the line of attachment of the semilunar valves, and, after being carried upwards about half an inch, were brought out again through the coats of the respective vessels, so that in each vessel one valve was included between the arterial walls and the wire. Upon applying the stethoscope to the vessels, after such an operation, the second sound ceased to be audible. Disease of these valves, when sufficient to interfere with their efficient action, also demonstrates the same fact by modifying the second sound or destroying its distinctness.

The contraction of the auricles is inaudible.

The first sound is heard most distinctly at the apex beat in the fifth interspace; the second sound is best heard over the second right costal cartilage-that is, the place where the aorta lies nearest to the surface. The pulmonary and aortic valves generally close simultaneously. In some cases, however, the aortic may close slightly before the pulmonary valves, giving rise to a "reduplicated second sound." The pulmonary contribution to this sound is best heard over the second left cartilage.

\section{The Coronary Arteries.}

The coronary arteries are the first branches of the aorta; they originate from the sinuses of Valsalva, and are destined for the supply of the heart itself; the entrance of the coronary vein, into the right auricle, we have already seen (p. 209).

Ligature of the coronary arteries causes almost immediate 
death; the heart, deprived of its normal blood-supply, beats irregularly, goes into fibrillary twitchings, and then ceases to contract altogether.

In fatty degeneration of the heart in man, sudden death is by no means infrequent. This is in many cases due to a growth in thickness of the walls of the coronary arteries called atheroma, which progresses until the lumen of these arteries is obliterated, and the man dies almost as if they had been ligatured.

Self-steering Action of the Heart.-This expression was originated by Bricke. He supposed that the semilunar valves closed the orifices of the coronary arteries during the systole of the heart. Unlike all the other arteries of the body, the coronary arteries would therefore fill only during diastole, and this increased fulness of the vessels in the heart walls during diastole would assist the ventricle to dilate. This, however, is incorrect; the valves do not cover the mouths of the arteries; and at the beginning of systole the velocity and pressure in the coronary arteries increase ; but later on during systole the ventricular wall is so strongly contracted that the muscular tension becomes greater than the coronary pressure, and so the coronary arteries and their branches are compressed, and the blood driven back into the aorta; the coronary arteries are then again filled with the commencing diastole. Self-steering action of the heart therefore exists, but it is brought about in a different way from what Brücke supposed.

\section{Cardiographs.}

A cardiograph is an instrument for obtaining a graphic record of the heart's movements. In animals the heart may be exposed, and levers connected to its various parts may be employed to write on a revolving blackened surface.

A simple instrument for the frog's heart is the following :-

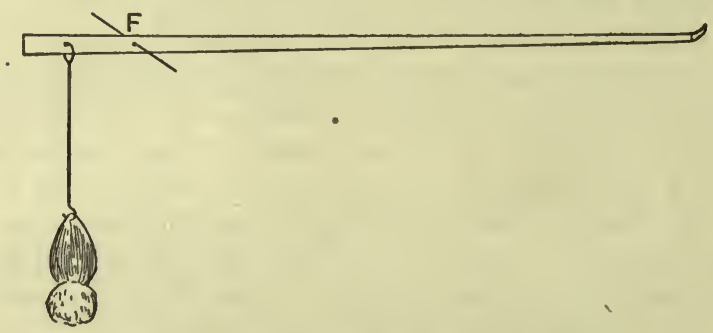

Fig. 215.-Simple Cardiograph for frog's heart.

The sternum of the frog having been removed, the pericardium opened, and the frænum (a small band from the back of the heart to the pericardium) divided, the heart is pulled through the opening, a minute hook placed in its apex, and this is fixed by a silk thread to a lever pivoted at $\mathrm{F}$ as in the figure. The cardiac wave of contraction starts at the sinus, this is followed by the auricular systole, and that by the ventricular systole and pause. This is 
recorded as in the next figure (fig. 216) by movements of the writing point at the end of the long arm of the lever. Such apparatus is, however, not applicable to the human heart, and all the various forms of cardiograph devised for this purpose are modifications of Marey's tambours. One of those most frequently used is depicted in the next two diagrams.

It (fig. 217) consists of a cup-shaped metal box over the open front of which is stretched an elastic india-rubber membrane, upon which is fixed a small knob of hard wood or ivory. This knob, however, may be attached, as in the figure, to the side of the box by means of a spring, and may be made to act upon a metal disc attached to the elastic membrane.

The knob is for application to the chest wall over the apex beat. The box or tambour communicates by means of an air-tight tube with the interior of a second tambour, in connection with which is a long and light lever. The shock of the heart's

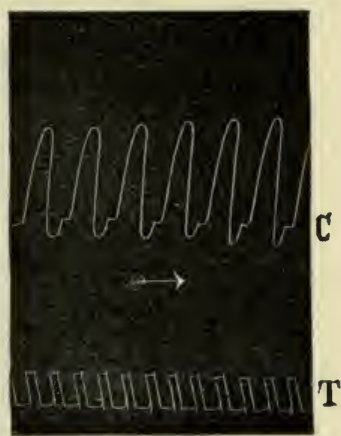

FIG. 216.-Cardiogram of frog's heart C, showing auricular, followed by ventricular beat; $\mathrm{T}$, time in half seconds. impulse being communicated to the ivory knob and through it to the first tambour, the effect is at once transmitted by the column of air in the elastic tube to the interior of the second tambour (fig. 218), also closed, and through the elastic and movable lid of the latter to the lever, which is placed in connection with a registering apparatus, which consists of a cylinder covered with

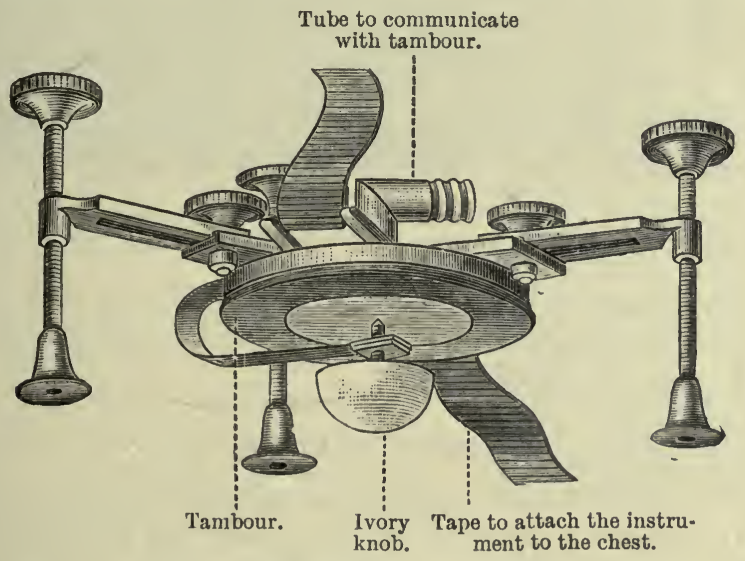

F10. 217.-Cardiograph. (Sanderson's.)

smoked paper, revolving with a definite velocity. The point of the lever writes upon the paper, and a tracing of the heart's impulse or cardiogram is thus obtained.

Fig. 219 represents a typical tracing obtained in this way. The first small rise of the lever is caused by the auricular, the second larger rise by the ventricular systole; the downstroke represents the 
pause, the tremors at the commencement of which are partly instrumental and partly caused by the closure of the semilunar valves.

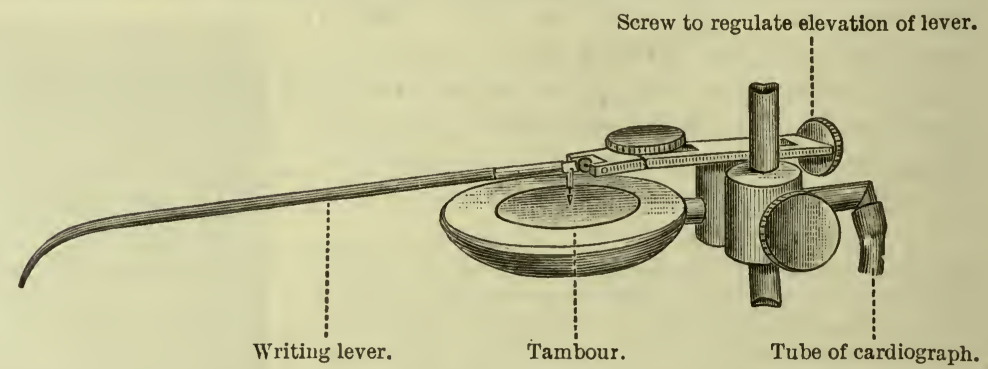

FIG. 218.-Marey's Tambour, to which the movement of the column of air in the first tambour is conducted by a tube, and from which it is communicated by the lever to a revolving cylinder, so that the tracing of the movement of the impulse beat is obtained.

Another method of obtaining a tracing from one's own heart consists in dispensing with the first tambour, and placing the tube of the recording tambour in one's mouth, and holding the breath

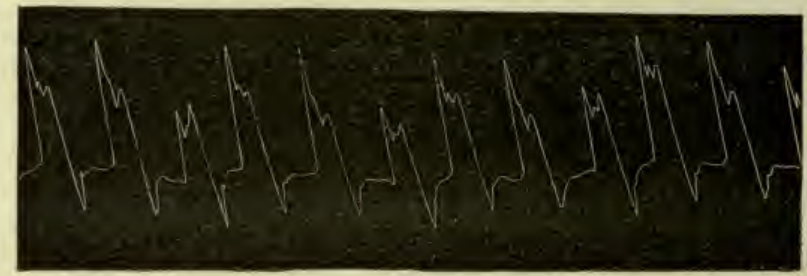

Fig. 219.-Cardiogram from human heart. The variations in the individual beats are due to the influence of the respiratory movements on the heart. To be read from left to right.

though keeping the glottis open. The chest then acts as the first tambour, and the movements of the lever (cardio-pneumatogram) may be written in the usual way.

\section{Intracardiac Pressure.}

The tracings of the cardiograph are, however, very variable, and their interpretation is a matter of discussion. A much better method of obtaining a graphic record of the events of the cardiac cycle consists in connecting the interior of an animal's heart with recording apparatus. There are several methods by which the intracardiac pressure may be recorded.

By placing two small india-rubber air-bags or cardiac sounds down the jugular vein into the interior respectively of the right auricle and the right ventricle, and a third in an intercostal space in front of the heart of a living animal (horse), and placing these bags, by means of 
long narrow tubes, in communication with three tambours with levers, arranged one over the others in connection with a registering apparatus (fig. 220), Chauveau and Marey were able to record and

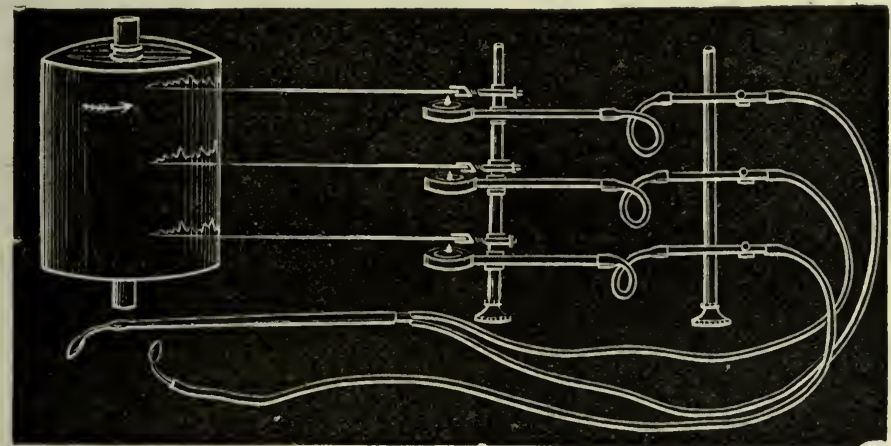

FiG. 220.-Apparatus of MM. Chauveau and Marey for estimating the variations of endocardiac pressure, and the production of the impulse of the heart.

measure the variations of the intracardiac pressure and the comparative duration of the contractions of the auricles and ventricles. By means of the same apparatus, the synchronism of the impulse with the contraction of the ventricles is also shown.

In the tracing (fig. 221), the intervals between the vertical lines represent periods of a tenth of a second. The parts on which any given vertical line falls represent simultaneous events. It will be seen that the contraction of the auricle, indicated by the marked curve at $\mathbf{A}$ in the first tracing, causes a slight increase of pressure in the ventricle, which is shown at $\mathbf{A}^{\prime}$ in the second tracing, and produces also a slight impulse, which is indicated by $\mathrm{A}^{\prime \prime}$ in the third tracing. The large curve of the ventricular and the impulse tracings, between $\mathrm{A}^{\prime}$ and $\mathrm{D}^{\prime}$, and $\mathrm{A}^{\prime \prime}$ and $\mathrm{D}^{\prime \prime}$, are caused by the ventricular contraction; while the smaller undulations, between $\mathrm{B}$ and $\mathrm{C}, \mathrm{B}^{\prime}$ and $\mathrm{C}^{\prime}, \mathrm{B}^{\prime \prime}$ and $\mathrm{C}^{\prime \prime}$, are caused

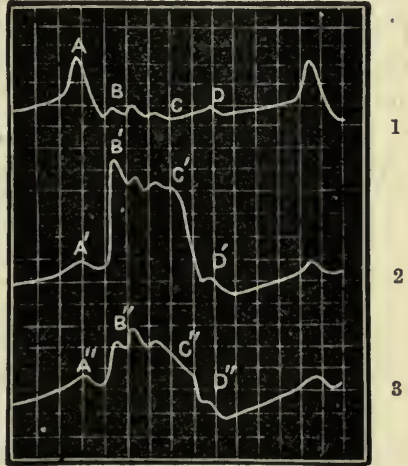

FIG. 221.-Tracings of (1), Intra-auricular, and (2), Intraventricular pressure, and (3), of the impulse of the heart; to be read from left to right; obtained by Chauveau and Marey's apparatus.

by the vibrations consequent on the tightening and closure of the auriculo-ventricular valves.

Much objection has, however, been taken to this method of investigation. First, because it does not admit of both positive and negative pressure being recorded. Secondly, because the method 
is only applicable to large animals, such as the horse. Thirdly, because the intraventricular changes of pressure are communicated to the recording tambour by a long elastic column of air; and fourthly, because the tambour arrangement has a tendency to record inertia vibrations. Rolleston reinvestigated the subject with a more suitable but rather complicated apparatus. The principle of his method consisted in placing the cavity of a heart-chamber in communication with a recording apparatus by means of a tube containing saline solution. His recording apparatus consisted of a lever connected to a piston; the upward and downward movements of the piston-rod were due to the varying pressures exerted on the blood by the contraction and dilatation of the heart.

Another and still better method of overcoming the imperfections of Marey's tambour is by the use of Hürthle's manometer (fig. 222).

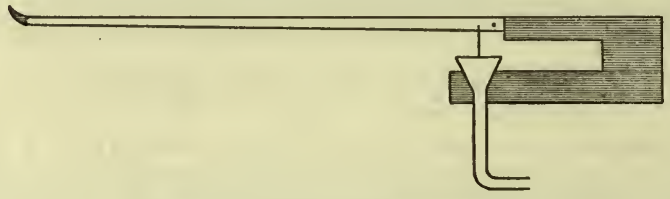

Fic. 222.-Hürthle's Manometer.

In this the tambour is very small, the membrane is made of thick rubber, and the whole, including the tube that connects it to the heart, is filled with a strong saline solution (saturated solution of sodium sulphate).

The tracing obtained by this instrument, when connected with the interior of the ventricle, is represented in the next figure.

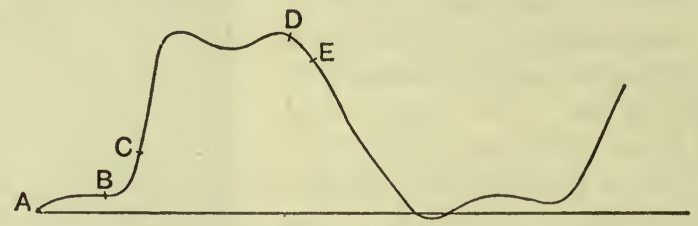

Fia. 223.-Curve of intraventricular pressure. (After Hürthle.)

The auricular systole causes a small rise of pressure (A B); it lasts about 05 second. It is immediately followed by the ventricular contraction, which lasts from $\mathrm{B}$ to $\mathrm{D}$. From $\mathrm{B}$ to $\mathrm{C}$ the ventricle is getting up pressure, so that at $\mathrm{C}$ it equals the aortic pressure. This takes 02 to 04 second. Just beyond $\mathrm{c}$ the aortic valves open, and blood is driven into the aorta; the outflow lasts from C to D ( 2 second). At $\mathrm{D}$ the ventricle relaxes. The flat top of the curve is spoken of as the systolic plateau, and according to the state of the 
heart and the peripheral resistance may present a gradual ascent or descent; it occupies about 18 second. Almost immediately after the relaxation begins the intraventricular pressure falls below the aortic, so that the aortic valves close near the upper part of the descent at $\mathbf{E}$.

The actual amount of pressure in the heart is measured by a mercurial manometer, which is connected to the heart by a tube containing a valve. This was first used by Goltz and Gaule. If the valve permits fluid to go only from the heart, the manometer will indicate the maximum pressure ever attained during the cycle. If it is turned the other way, it will indicate the minimum pressure. The following are some of the measurements taken from the dog's heart in terms of millimetres of mercury :-

\begin{tabular}{|c|c|c|c|c|}
\hline Left ventricle & & & $\begin{array}{r}\text { Maximum } \\
\text { pressure. } \\
140 \mathrm{~mm} .\end{array}$ & $\begin{array}{c}\text { Minimum } \\
\text { pressure. } \\
-30 \text { to } 40 \mathrm{~mm} .\end{array}$ \\
\hline Right ventricle & • & - & . $\quad 60 \mathrm{~mm}$. & $-15 \mathrm{~mm}$. \\
\hline Right auricle. & . & • & . $20 \mathrm{~mm}$. & -7 to $8 \mathrm{~mm}$. \\
\hline
\end{tabular}

By a negative (-) pressure one means a pressure less than that of the atmosphere, so that the mercury is sucked up in the limb of the manometer towards the heart.

Another valuable instrument introduced by Hürthle is called the differential manometer. In this instrument, two cannulæe are brought into connection with tambours ( $A$ and $B$ ) which work on points of a lever at equal distances from and on

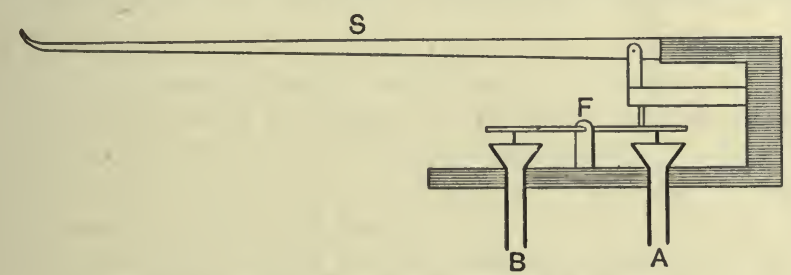

FIG. 224.-Diagram of Hürthle's differential Manometer.

opposite sides of its fulcrum (r). The lever sets in motion a writing style (s). This instrument enables us to determine the relations of the pressure changes in any two cavities. For instance, suppose $\mathrm{A}$ is connected to the left ventricle, and $\mathrm{B}$ to the aorta; when the pressure in the ventricle is greater than that in the aorta, the writing style will be raised; when the pressure in the aorta is greater than that in the ventricle, the style will fall; when the two pressures are equal, it will be in the zero position.

\section{The Electro-Cardiogram.}

The muscular tissue of the heart gives rise on action to an electrical disturbance which is in all essential features the same as the diphasic variation we have already studied in Chapter XI. in connection with voluntary muscle. The excised beating heart of a frog call be roadily connected either to a galvanometer or 
electrometer, and the different phases of the action current can in the former case be ascertained by watching the movements of the magnetic needle, and in the latter case by watching under the microscope the movements of the meniscus of mercury in the capillary tube. If the eyepiece of the microscope is removed, and the image of the mercurial column allowed to fall on a moving photographic plate, a graphic record is obtained, in which the to-and-fro movements of the mercury are shown as waves. Such a graphic record is termed an electro-cardiogram, and one of these is shown in the next figure (fig. 225).

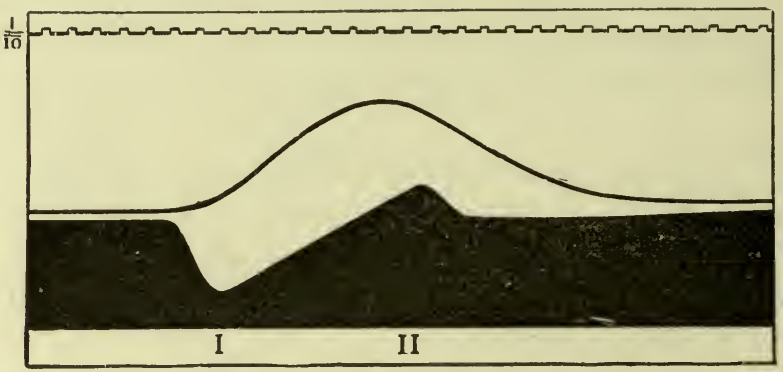

FIa. 225.-Electru-cardiugram frum frog's ventricle. Diphasic variation. Simultaneuus photograph of a single beat (upper black line), and the accompanying electrical change indicated by the level of the black area, which shows the varying level of mercury in a capillary electrometer. The time. tracing at the top marks tenths of a second. (Waller.)

It is, however, possible (as Waller first demonstrated) to obtain an electro-cardiogram in the intact animal, and even in man. If a dog is placed with a fore paw in a basin of salt solution, and a hind paw in another, and the two basins are led off to the electrometer, the electrical changes produced by the beating heart will be conducted through the body of the animal and through the electrometer, and the movements of the mercury can be watched with a microscope or recorded on a travelling photographic plate. By the use of this method Miss Buchanan has succeeded in performing what otherwise would have been the impossible task of counting the heart rate of small mammals such as mice. The photographic plate must travel at great speed, and the notches in the shadow of the mercurial column, which correspond to the heart-beats, were found in the mouse to occur at the rate of 700 per minute. In a corresponding way the human electro-cardiogram can be registered, as shown in fig. 226. In that particular experiment, the "lead-offs" were from mouth and left foot. It is more usual to employ one hand and one foot.

We must, however, recognise that the heart muscle is not a simple longitudinal strip like a sartorius, but is arranged in a complex way, and in the mammalian heart is arranged around four 
chambers, and that the left and right sides are contracting simultaneously. One would therefore anticipate that there would be a corresponding complexity in the electrical record of the intact organ. This expectation has been verified by later work in which investigators have used more sensitive instruments. But there was some indication of this even in the records of the earlier workers who employed the capillary electrometer. Thus Bayliss and Starling described in the mammalian heart a triphasic variation, and Gotch by means of careful experiments on both cold and warm blooded animals, has shown that this is explicable in the following

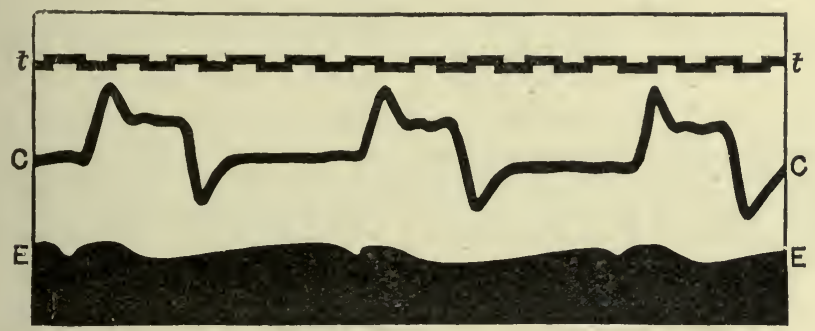

Fre. 226.-Human heart. Electro-cardiogram, EE, and simultaneous cardiogram, CC. Time, $t$, is marked in thth second. The lead-offs to the capillary electrometer were from the mouth to the sulphuric acid, and from the left foot to the mercury. (Waller.)

way. Leaving out of account complications due to auricular activity, he has shown that the contraction process in each ventricle and its electrical concomitant commences at the part of the base of the ventricle where it is continuous with its respective auricle; the contraction wave travels to the apex and returns to the part of the base from which the aorta on one side and the pulmonary artery on the other side arise. An electrode placed on the base will therefore record the increased positivity at the beginning and the end of the ventricular contraction; the electrode on the apex will record the middle phase when the contraction wave reaches that point, and causes an increase of positivity there.

By far the most delicate instrument now in use is Einthoven's String Galvanometer (see p. 121), and during the last few years it has been much employed, not only by Einthoven himself, but by numerous other observers, including Dr T. Lewis, in this country. The excursions of the thread or string can be photographed, and the following diagram is a cardio-electrogram obtained from the human heart during a single beat, the electrodes being connected with the right and left hands.

It will be seen that there is a small movement due to the auricular systole, and several large ones which accompany ventricular contraction. The exact meaning of these different waves is still far 
from clear, and the extent of each varies considerably even in health, but in heart disease the electro-cardiogram shows very marked differences from the normal, especially in cases of "heart-block." When in the future the meaning of each part of the record is under stood, the physician will be provided with a new help to diagnosis.

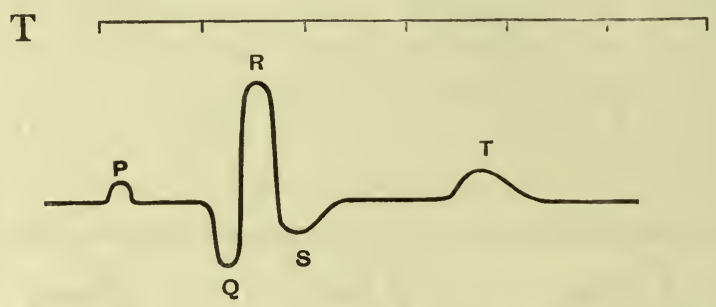

FIG. 227.-Electro-cardiogram obtained by photographing the movements of the thread of a String Galvanometer. The electrodes were connected to a man's right and left hands. Waves upwards indicate that the base (right ventricle) is galvanometrically negative to the apex (left ventricle); downward waves have the opposite meaning. Wave $P$ accompanies auricular systole; waves $\mathrm{Q}, \mathrm{R}, \mathrm{S}$, and T occur during ventricular systole. The time-tracing (T) shows tenths of a second. (After Einthoven.)

The Cardiophonogram.-An interesting extension of this work consists in the registration of the heart sounds. This was first done by Hürthle some years ago, but Einthoven's string galvanometer, as an instrument of precision far exceeding these previously used, has enabled him to repeat this work with much greater accuracy. A stethoscope is placed over the chest and connected to a microphone, which magnifies the heart sounds; the vibrations in the microphone are communicated as electrical changes by a transformer to the string galvanometer, the movements of the quartz fibre being finally photographed on a travelling plate. If simultaneously an electrocardiograrn is taken, the simultaneity of the first heart sound with the ventricular systole, and of the second heart sound with the commencement of ventricular diastole, are very conclusively demonstrated. In heart disease where the adventitious sounds called murmurs are present, their time relationships in the cardiac cycle are most clearly seen.

Einthoven has further found the presence of a third heart sound, which is inaudible to the unaided ear, although it was first described by Dr A. G. Gibson of Oxford, in a patient in whom it was very pronounced, by means of ordinary auscultation. It seems, however, to be present in all human hearts in varying degrees of intensity when the cardiophonogram is examined. It occurs during diastole, and follows the second sound after a short pause. It is not due to a reduplication of the second sound, nor is it a presystolic murmur such as can be heard in man when there is obstruction at the auriculo-ventricular orifices. Einthoven adduces evidence against both these views, and believes it is produced at the aortic orifice; the semilunar valves and the neighbouring portion of the aortic wall being thrown for a second time and for a short period into vibration by the changes in the aortic pressure which occur during diastole. 


\section{Frequency of the Heart's Action.}

The heart of a healthy adult man contracts about 72 times in a minute; but many circumstances cause this rate to vary even in health. The chief are age, temperament, sex, food and drink, exercise, time of day, posture, atmospheric pressure, temperature. Some figures in reference to the influence of age are appended.

The frequency of the heart's action gradually diminishes from the commencement to near the end of life, thus :-

Before birth the average number of pulsations per minute is

Just pulsations per minute is 150

During the first year : from 140 to 130

During the second year ,", 115 to 100
About the seventh year . from 90 to 85

About the fourteenth

year . . , , 85 to 80

In adult age : . ., 80 to 70

In old age : $\quad . \quad$, 70 to 60

In health there is a uniform relation between the frequency of the heart-beats and of the respirations; the proportion being 1 respiration to 3 or 4 beats. The same relation is generally maintained in cases in which the action of the heart is naturally accelerated, as after food or exercise; but in disease this relation may cease.

\section{Work of the Heart.}

Waller compares the work performed by the heart in a day to that done by an able-bodied labourer working hard for two hours. The heart's work consists in discharging blood against pressure, and in imparting velocity to it. It is therefore necessary to know how much blood is expelled from the heart at each beat, the time occupied in such expulsion, the velocity with which the blood is expelled, and the pressure against which the heart has to act. Without going into the somewhat elaborate calculations obtained from these and other data, it will be sufficient to say that about $\frac{1}{40}$ of the total energy of the heart is used in imparting velocity to the blood, but when the blood reaches the aorta the velocity is checked, and that the kinetic energy of the blood in the aorta is only about $\frac{1}{800}$ of the total energy imparted to the blood by the heart.

The Output of the Heart.-Direct measurements of the heart's output have been made by Stolnikow and Tigerstedt. The former cut off the whole of the systemic circulation in the dog, and then measured the amount of blood passing through the simplified circulation, which consisted only of the pulmonary and coronary vessels by means of a cylinder interposed on the course of the vessels, (see fig. 228). Tigerstedt made his observations by means of a Stromuhr (see next chapter) inserted into the aorta. Severe operative measures of this kind, however, interfere with the circulation a good deal.

G. N. Stewart has introduced an ingenious method, the principle of which is the following:-A solution of an innocuous substance, which can be easily recognised and estimated, is allowed to flow for a definite time and at a uniform rate into the heart; the substance selected was sodium chloride. This mingles with the 
blood and passes into the circulation. At a convenient point of the vascular system, a sample of blood is drawn off just before the injection, and an equal amount during the passage of the salt; the quantity of the sodium chloride solution which must be added to the first sample in order that it may contain as much as the second sample is determined. This determination gives the extent to which the salt solution has been mixed with the blood in the heart,

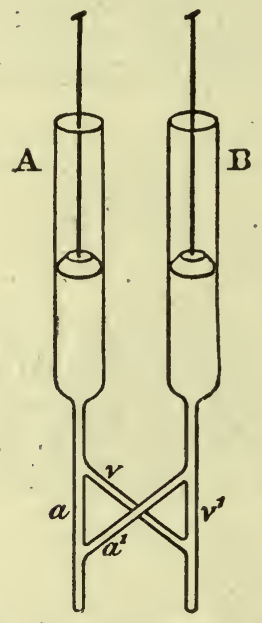

Fig. 228,-Stolnikow's apparatus. A and B are two cylinders titted with floats provided with writing-points at their upper ends. The tube from the lower end of each bifurcates into two, $a$ and $v$ from $\mathrm{A} ; a^{\prime}$ and $v^{\prime}$ from B. $a$ and $a^{\prime}$ are nnited together and enter the right carotid artery $; v$ and $v^{\prime}$ unite and are inserted into the superior vena cava. The remaining branches of the aorta and the inferior vena cava are tied. $B$ is first filled with defibrinated blood, which passes down $v^{\prime}$ into the right auricle, thence to the right ventricle, lungs (where it is oxygenated), and then enters the left side of the heart; the left ventricle expels it by the tube $a$ into $A$, so that the float in $\mathbf{A}$ rises while that in $B$ falls. As soon as B is empty the tubes $v$ and $a^{\prime}$ which were previously clamped are released, and $v^{\prime}$ and $a$ are clamped instead. The left ventricle now expels its blood by the tube $a^{\prime}$ into the cylinder B ; simultaneously A empties itself through $v$ into the right side of the heart. Zigzag lines are thus traced by the writing-points on the top of the floats, and their frequency enables one to estimate the output of the left ventricle in a given time. (After Starling.) drawn from the tambour to the cardi-
ometer; when the heart expands, the air will move in the reverse direction.
These movements are written by the end of the lever of the tambour, and variations in the excursions of this lever correspond with variations in the amount of blood expelled from or drawn into the heart with systole and diastole respectively. By calibrating the instrument the actual volume of the blood expelled can be ascertained. tion which has run into the heart, the output in a given time can be calculated.

All these experiments have been on animals. The results obtained necessarily vary with the size of the animal used, and with the rate at which the heart is beating. If the same relationship holds for man as for animals, Stewart calculates that in a man weighing 70 kilos the output of each ventricle per second is less than 0.002 of the body weight, i.e., about 105 grammes of blood per second, or 87 grammes (about 80 c.c.) per heart-beat with a pulse rate of 72 .

An instrument called the cardiometer was invented by Roy for registering the output of the heart. His instrument was made of metal, and oil was used as the transmitting medium in its interior. A simple modification of this applicable to the heart of a small mammal like a cat has been devised by Barnard. It consists of an india-rubber tennis ball with a circular orifice cut in one side of it large enough to admit the heart; a glass tube is securely fixed into a small opening on the opposite side of the ball. The animal is anæsthetised, and its thorax opened. The animal is kept alive by artificial respiration. The pericardium is then opened by a crucial incision, the heart is slipped into the ball ; the pericardium overlaps the outside of the ball, and the apparatus is rendered air-tight by smearing the edges of the hole with vaseline. The four corners of the pericardium are then tightly tied by ligatures round the glass tube just mentioned. This tube is connected by a stout india-rubber tube to a Marey's tambour or a piston-recorder, the writing-point of which is applied to a moving blackened cylinder. When the heart contracts, air will be withdrawn from the tambour to the cardi- 


\section{Innervation of the Heart.}

The nerves of the heart, which under normal circumstances control its movements, are:-

1. Cardiac branches of the vagus (inhibitory fibres).

2. Cardiac branches of the sympathetic (augmentor and accelerator fibres).

These pass to the heart and terminate in certain collections of ganglion cells in its wall (terminal ganglia); from these cells postganglionic fibres are distributed among the muscular fibres. In addition to these nerves, which are efferent, we have to mention:-

The sensory or afferent nerves of the heart, the best known of which is called the depressor nerve. This nerve, starting from the cardiac tissue, joins the vagus trunk; it passes to the bulb, especially to the vaso-motor centre. We shall therefore postpone its study until we are considering the vaso-motor nerves.

The Vagus. - The ninth, tenth, and eleventh cranial nerves arise close together from the grey matter in the floor of the fourth ventricle, and leave the bulb by a number of rootlets. These rootlets are divided by Grossmann into three groups, $a, b$, and $c$; there is a good deal of blending of the rootlets before they ultimately emerge from the skull, but the a (the uppermost) group corresponds fairly well with the fibres of the glossopharyngeal, $b$ with those of the vagus, and $c$ (the lowermost) with those of the spinal accessory. The rootlets of the tenth nerve pass through two ganglia called respectively the jugular ganglion, and the ganglion trunci vagi. The fibres of the spinal accessory nerve which join the vagus are chiefly motor, especially to the larynx, but some go to the heart. The vagus gives off branches to many organs, pharynx, larynx, heart, lungs, cesophagus, and various abdominal organs. We have, however, in this place only to deal with its cardiac fibres. It has been known since the experiments of the Brothers Weber in 1845 that stimulation of one or both vagi produces slowing or stoppage of the beats of the heart. It has since been shown that in all vertebrate animals, this is the normal result of vagus stimulation; the phenomenon is called inhibition, and the nerve-fibres cardio-inhibitory. Section of one vagus produces slight acceleration of the heart; this result is better marked when both vagi are divided. This shows that the restraining influence of the vagus is being continuously exercised; it is, however, found that the amount of vagus control varies a good deal in different animals. The most potent artificial stimulus which can be applied to the vagus nerve to produce inhibition of the heart is a rapidly interrupted induction current; severe mechanical stimuli have a slight effect, but chemical and thermal stimuli are ineffective. 
A certain amount of confusion has arisen as to the effect of vagus stimulation, because so many experiments have been made on the frog. In this animal the sympathetic fibres join the vagus after it leaves the skull, and so what is usually called the vagus in this animal should more properly be termed the vago-sympathetic. It will readily be understood that by stimulating a mixed nerve, one obtains

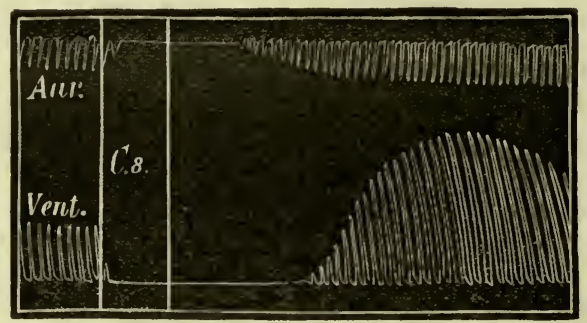

Fio. 229.-Tracing showing the actions of the vagus on the heart. Aur., auricular; Vent., ventricular tracing. The part between the perpendicular lines indicates the period of vagus stimulation. C.8 indicates that the secondary coil was $8 \mathrm{c.m}$. from the primary. The part of the tracing to the left shows the regular contractions of moderate height before stimulation. During stimulation, and for some time after, the beats of auricle and ventricle are arrested. After they commence again they are small at first, but soon acquire a much greater amplitude than before the application of the stimulus. (From Brunton, after Gaskell.)

an intermixture of effects. If, however, one stimulates the intracranial vagus before the sympathetic blends with it, a pure inhibitory effect is obtained. Figs. 229 and 230 show the common effect of stimulating the mixed trunk; the inhibitory effect is usually manifested first, and this is followed by the augmentor effect due to

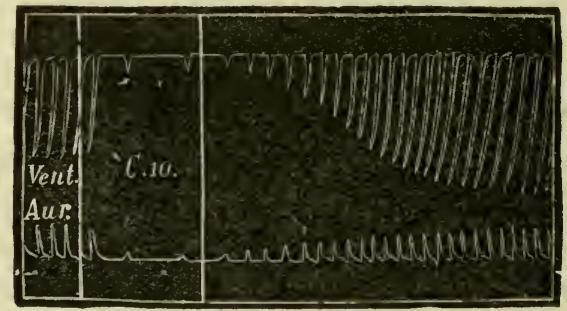

FIG. 230.-Tracing showing diminished amplitude and slowing of the pulsations of the auricle and ventricle without complete stoppage during stimulation of the vagus. (From Brunton, after Gaskell.)

sympathetic action. But it is by no means infrequent to obtain the phenomena in the reverse order. It is often stated that the right nerve contains more inhibitory fibres than the left, but this is by no means a constant rule. One can always obtain good inhibition if the stimulus is applied to the wall of the sinus; here one stimulates the post-ganglionic fibres which originate from the nerve-cells in the sinus ganglion around which the vagi terminate. 
The effect of the stimulus is not immediately seen; one or more beats may occur before stoppage of the heart takes place, and slight stimulation may produce only slowing and not complete stoppage of the heart (fig. 230). The stoppage may be due either to prolongation of the diastole, as is usually the case, or to diminution of the systole. Vagus stimulation lessens the conductivity of the cardiac tissue, but it does not abolish the irritability of the heart-muscle, since direct mechanical stimulation may bring out a beat during the standstill caused by vagus stimulation. The inhibition of the beats varies in duration, but if the stimulation is a prolonged one, the beats reappear before the current is shut off. This is known as "vagus escape," and is probably due to fatigue of the vagal endings.

The Sympathetic.-The influence of the sympathetic is the reverse of that of the vagus. Stimulation of the sympathetic produces acceleration of the heart-beats, and as a rule, section of the nerve produces slowing. Hence the nerve is also in constant action like the vagus. The acceleration produced by stimulation of the sympathetic fibres is accompanied by increased force, and so the action of the nerve is also termed augmentor. It is probable that the augmentor fibres are distinct from the accelerator fibres, because in mammals one or two of the small nerves leaving the stellate ganglion on stimulation produce augmentation without acceleration.

The fibres of the sympathetic system which influence the heart-beat in the frog, leave the spinal cord by the anterior root of the third spinal nerve, and pass by the ramus communicans to the third sympathetic ganglion,

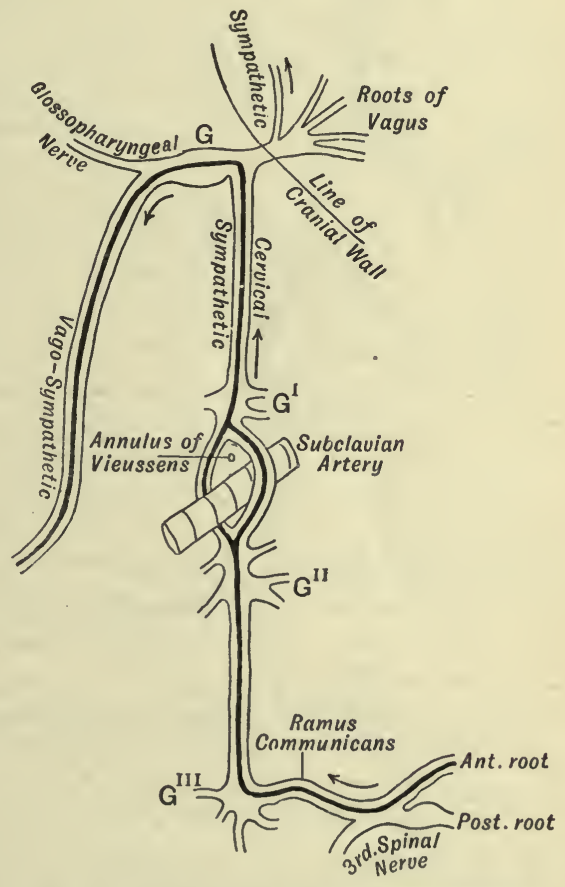

FIa. 231.-Heart nerves of frog. (Diagrammatic.) then to the second sympathetic ganglion, then by the annulus of Vieussens (round the subclavian artery) to the first sympathetic ganglion, and finally in the main trunk of the sympathetic, to near the exit of the vagus from the cranium, where it joins that nerve 
and runs down to the heart within its sheath, forming the joint vagosympathetic trunk. These fibres are indicated by the dark line in fig. 231. The fibres of the sympathetic seen running up into the skull are for the supply of blood-vessels there. It should be noted that the frog has no spinal accessory nerve.

In the mammal the sympathetic fibres leave the cord by the

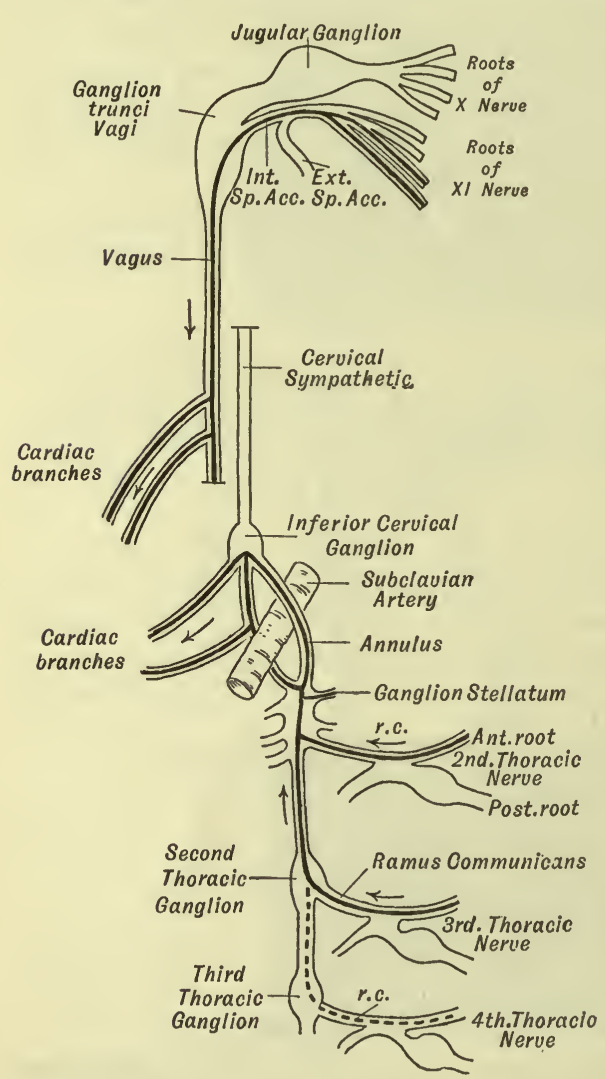

FIG. 232.-Heart nerves of mammal. (Diagrammatic.) second and third dorsal nerves, and possibly by anterior roots of two or more lower nerves; they pass by the rami communicantes to the ganglion stellatum, or first thoracic ganglion, and thence by the annulus of Vieussens to the inferior cervical ganglion of the sympathetic; fibres from the annulus, or from the inferior cervical ganglion, proceed to the heart (see fig. 232).

In man, the cardiac branches of the sympathetic travel to the heart from the annulus of Vieussens and cervical sympathetic in superior, middle, and lower bundles of fibres. These pass into the cardiac plexus, and surrounding the coronary vessels ultimately reach the heart.

By stimulating each rootlet in his three groups, Grossmann found the cardio-inhibitory fibres in the lower two or three rootlets of group $b$ and the upper rootlet of group $c$. There are probably differences in different animals. In the cat and dog Cadman finds that the rootlets in the $a$ group are respiratory and afferent inhibitory, and that all the efferent inhibitory fibres are in group $c$.

The inhibitory fibres are medullated, and only measure $2 \mu$ to $3 \mu$ in diameter; they pass to the heart and have their cell-stations in the ganglia of that organ. Some of the sympathetic fibres, on the other hand, reach the heart as nonmedullated fibres; having their cell-stations in the sympathetic (inferior cervical and first thoracic) ganglia; but the majority do not reach their cell-stations until they reach terminal ganglia in the heart wall. The augmentor and accelerator centres in the central nervous system have not yet been accurately localised. 
Influence of Drugs.-The question of the action of drugs on the heart forms a large branch of pharmacology. We shall be content here with mentioning two only, as they are largely used for experimental purposes by physiologists. Atropine produces considerable augmentation of the heart-beats by paralysing the inhibitory mechanism. Muscarine (obtained from poisonous fungi) produces marked slowing, and in larger doses temporary stoppage of the heart. Its effect is a prolonged inhibition, and can be removed by the action of atropine. The action of atropine cannot, however, be easily antagonised by muscarine ; a large dose is necessary. That these drugs act on the nerves, and not the muscular substance of the heart, is shown by the fact that in the hearts of early embryos, so early that no nerves have yet grown to the heart, these drugs have little or no effect. (Pickering.)

Reflex Inhibition.-Thus there is no doubt that the vagi nerves are simply the media of an inhibitory or restraining influence over the action of the heart, which is conveyed through them from the centre in the medulla oblongata, which is always in operation. The restraining influence of the centre in the medulla may be reflexly increased by stimulation of many afferent nerves, particularly those from the nasal mucous membrane, the larynx, and the lungs. A blow on the abdomen causes inhibition and fainting; a blow on the larynx, even a moderate one, will kill. There is no comparison between the ease with which stimulation of the laryngeal or pulmonary fibres produces inhibition, as compared to the difficulty of obtaining inhibition from the alimentary tract. Tobacco smoke in some people and animals, by acting on the terminations of the vagi or their branches in the respiratory system, may also produce reflex inhibition of the heart. Some very remarkable facts concerning the readiness by which reflex inhibition of the fish's heart may be produced were made out by M'William; any irritation of the tail, gills, mucous membrane of mouth and pharynx, or of the parietal peritoneum, causes the heart to stop beating.

In connection with the subject of reflex inhibition, it may be mentioned in conclusion that though we have no voluntary control over the heart's movements, yet cerebral excitement will produce an effect on the rate of the heart, as in certain emotional conditions.

Action of Chloroform on the Cardiac Mechanism.-The mammalian heart is more difficult to stop by stimulation of the vagus than the frog's heart; commonly it is only slowed, and the amplitude of the beat reduced, yet it is most important for the student of medicine to recollect that vagus inhibition may have far-reaching results. One of the most familiar causes of heart stoppage in surgical practice is that produced by chloroform; chloroform acts directly on the cardiac tissue when it is administered incautiously, or in too large 
doses over long periods of time; the term inhibition is not applicable in this case, and the effects of the poisonous action of chloroform on the heart itself can be avoided by keeping the proportion of chloroform in the inspired air at 2 per cent. or less. But in other cases which are seen both in animals and human beings who may be peculiarly susceptible to the influence of chloroform, heart stoppage occurs during the onset of anæsthesia long before the percentage of chloroform in the blood has reached a value which is toxic to the heart. Some have considered that death during the induction of chloroform anæsthesia is due to the vapour irritating the vagal terminations in the lung, and so leading to reflex inhibition of the heart. Embley's experiments, however, lead to the conclusion that the chloroform acts on the vagus centre in the medulla oblongata. In animals, cutting the vagi immediately sets the heart going again. In man this operation cannot be performed, and it is therefore a wise precaution, whenever it is necessary to administer chloroform, to give beforehand a small dose of atropine under the skin so as to temporarily paralyse the vagus endings in the heart.

Gaseous Exchanges in the Heart.-The using up of oxygen by the living heart was well illustrated by an old experiment of Yeo's. He passed a weak solution of oxyhæmoglobin through an excised beating frog's heart, and found that after it had passed through the heart, the solution became less oxygenated and venous in colour.

This is still better shown by the following numbers, obtained by Barcroft and Dixon by estimating the gases in the blood entering and leaving the coronary vessels of a cat. It will be seen that the metabolism in the heart tissue is reduced during inhibition; this is followed by increased metabolism during the subsequent period, which corresponds with the increase of visible activity which then occurs, and which is seen in the tracings given in figs. 229 and 230 .

\begin{tabular}{|c|c|c|c|c|}
\hline & & Normal Heart. & $\begin{array}{l}\text { During Vagus } \\
\text { Inhibition. }\end{array}$ & $\begin{array}{l}\text { After Vagus } \\
\text { Inhibition. }\end{array}$ \\
\hline $\begin{array}{l}\text { Oxygen used up per minute } \\
\text { Carbonic acid given out per minute }\end{array}$ & $\dot{.}$ & $\begin{array}{l}0.21 \text { c.c. } \\
0.45 \text { c.c. }\end{array}$ & $\begin{array}{l}0.13 \text { c.c. } \\
0.07 \text { c.c. }\end{array}$ & $\begin{array}{l}0.34 \text { c.c. } \\
0.22 \text { c.c. }\end{array}$ \\
\hline
\end{tabular}

\section{Rhythm, Conduction, etc., in Cardiac Muscle.}

In one time, the rhythm which cardiac muscle exhibits was supposed to be due to the action upon it of the nerves which are present. We now know that the property of rhythmical peristalsis resides in the muscular tissue itself, though normally during life it is controlled and regulated by the nerves that supply it. This conclusion may be expressed by saying that cardiac rhythm is myogenic, not 
neurogenic. There are still a few physiologists who maintain the older neurogenic theory, but these are mainly those whose chief work has been performed on the hearts of invertebrate animals, and it is quite possible that the mechanism there is a different one. But so far as the vertebrate heart is concerned, the myogenic theory is now held, because (1) the fœtal heart manifests rhythm long before any nerves reach it; (2) the apex of the ventricle of such animals as frogs and tortoises can be made to beat rhythmically by perfusing it with suitable fluids at high pressure; and this part of the heart has few nerves and no ganglion cells; and (3) the rate of conduction of the peristaltic wave is slow, and corresponds to the rate of muscular rather than of nervous conduction.

The older observers, who first made the striking observation that a heart will continue to beat after its removal from the body for a considerable period, and who at the same time were imbued with the neurogenic theory, naturally placed the seat of rhythm in the intracardiac ganglia. They were not at the time aware of the general arrangements of autonomic nerves, and therefore did not recognise that the ganglia were terminal cell-stations on the course of the nerves which reach the heart via vagus and sympathetic.

The intracardiac nerves have been chiefly studied in the frog; in this animal the two vago-sympathetic nerves terminate in various groups of ganglion-cells; of these the most important are Remak's ganglion, situated at the junction of the sinus with the right auricle; and Bidder's ganglion, at the junction of the auricles and ventricle. A third collection of ganglion cells (von Bezold's ganglion) is situated in the interauricular septum. From the ganglion-cells, postganglionic fibres spread out over the walls of the sinus, auricles, and upper part of the ventricle. Remak's ganglion used to be called the local inhibitory centre of the heart; it is really the chief cell-station of the inhibitory fibres, and stimulation of the heart at the sinoauricular junction is the most certain way of obtaining stoppage of the heart. Bidder's ganglion was called the local accelerator centre for a corresponding reason.

The intracardiac ganglia have been examined in a few other coldblooded animals (for instance the tortoise), but any precise knowledge of their arrangement and position in the mammalian and human heart is unfortunately lacking.

Conduction in the Heart.-As already stated, the slow rate of propagation of the wave points to the link being muscular rather than nervous, and histology lends support to this view, the muscular fibres being connected to each other by intercellular bridges of protoplasm (see p. 71). An experimental proof of the same view is the following: if a strip of the heart wall is taken and a number of cuts going nearly completely across it, be made first from one side, 
then from the other, all the nerves must be cut through at least once, and the only remaining tissue not severed is muscular, yet the strip still continues to beat; in other words, the propagation is myodromic. The passage of the wave from one chamber to another is also myodromic. The slow rate of propagation indicates that this is so, and the view has been fully proved by the discovery of muscular strands, passing across from one chamber to the next.

It appears that under normal conditions the wave of contraction in the heart starts at the sinus, and travels over the auricles to the ventricle; the irritability of the muscle and the power of rhythmic contractility is greatest in the sinus, less in the auricles, and still less in the ventricles. Under ordinary conditions the apical portion of the ventricles exhibits very slight power of spontaneous contraction. The importance of the sinus as the starting-point of the peristalsis can be shown by warming it. If a frog's heart is warmed by bathing it in warm salt solution at about body temperature, it beats faster; this is due to the sinus starting a larger number of peristaltic waves; that this is the case may be demonstrated by warming localised portions of the heart by a small galvano-cautery; if the sinus is warmed the heart beats faster, but if the auricles or ventricles are warmed there is no alteration in the heart's rate. The sinus in the frog's heart, and that portion of the right auricle in the mammal's heart which corresponds to the sinus, is always the last portion of the heart to cease beating on death, or after removal from the body (ultima moriens, Harvey). This is an additional proof of the superior rhythmical power which it possesses. The fœetal remnant of the sinus in the mammal's heart has been called the sino-auricular node (Keith and Flack), and this has been believed to act as the "pace-maker" of the heart. But after its removal other parts in the same region which contain no Purkinje's fibres have been found quite capable of maintaining the ordinary cardiac rhythm. The sino-auricular node cannot therefore be the specific or exclusive pace-maker, and some observers regard the final part of the large veins outside the heart altogether as more important from this point of view.

It is supposed that the wave starting at the sinus is more or less blocked by a ring of lower irritability at its junction with the auricle; again, the wave in the auricle is similarly delayed in its passage over to the ventricle by a ring of lesser irritability, and thus the wave of contraction is delayed at its entrance into both auricular and ventricular tissue. By an arrangement of ligatures, or, better, of clamps, one part of the heart may be isolated from the other portions, and the contraction when aroused by an induction shock may be made to stop in the portion of the heart muscle in which it begins. It is not unlikely that the contraction of one portion of the heart acts as a stimulus to the next portion, and that clamps and ligatures prevent or "block" 
this normal propagation of stimuli. It must not, however, be thought that the wave of contraction is incapable of passing over the heart in any other direction than from the sinus onwards; for it has been shown that by the application of appropriate stimuli at appropriate instants, the natural sequence of beats may be reversed, and the contraction starting at the arterial part of the ventricle may pass to the auricles and then to the sinus. If clamps or ligatures are not applied sufficiently tightly one often sees partial blocking; a few waves get through but not all; or if the ventricular wall is left connected with other parts of the heart by only a small portion of undivided muscular tissue, the effect is much the $\operatorname{same} \theta$, the wave is only able to pass the block every second or third beat.

Heart-Block.-The phenomena of blocking just described were made out by Gaskell many years ago in his study of the hearts of cold-blooded animals. But the same sort of thing occurs also in the mammalian heart. The starting-point in our knowledge of this branch of the subject was the discovery by Stanley Kent of bands of muscular tissue passing across from auricles to ventricles, and the principal one was subsequently and independently re-discovered by His, and is known as Kent's bridge or the Bundle of His, or better as the auriculo-ventricular bundle. It arises in close connection with the fibres of the interauricular septum, and with the tissue of the sino-auricular node at the junction of the superior vena cava and right auricle. It comes also into relationship with another similar mass or fœtal residue known as the auriculoventricular node which lies at the base of the auricular septum on the right side below the coronary sinus. From here the bundle runs along the top of the intraventricular septum and divides into right and left sub-divisions which run down to their respective ventricles along the septum which separates them, and into the papillary muscles arising from the septum. From the papillary muscles fine strands run to other parts of the ventricle immediately under the endocardium, and finally reach the apex. All these structures are made of the peculiar muscular fibres known as Purkinje's fibres (see p. 71). There is no doubt that this is the main conducting path from auricles to ventricles, and although there are nerve-fibres mingled with the muscular fibres of Purkinje there is no reason for modifying the view already expressed that conduction is myodromic.

The conclusion that the auriculo-ventricular bundle is the important link which propagates the rhythmic wave has been reached first by experiments on animals, and secondly, by observations in disease in man. In animals, cutting through the bundle abolishes the ordinary sequence of cardiac events. The auricles go on beating, but the ventricles stop altogether, and later when they 
do begin to beat again, the ventricular beat is much less frequent than the auricular, and bears no relationship to it. This lack of correspondence in auricular and ventricular rhythm is known as arhythmia.

When the bundle is destroyed by disease in man (Stokes-Adams' disease) there is a similar dissociation between the auricular and ventricular rhythm, the ventricles beating slowly and the auricles rapidly. Sometimes the "heart-block" is incomplete. This is seen in the early stages of the disease; then one out of every two or three auricular waves gets over to the ventricle, just as in Gaskell's experiments on the frog's heart when the clamp is not sufficiently tight.

These recently discovered facts throw a good deal of light on the propagation of the normal heart wave. It starts in the sinoauricular node, and spreads thence to both auricles; it also travels to the ventricles by the auriculo-ventricular bundle, reaching first the papillary muscles, and thence the rest of the heart until it arrives at the apex, and finally returns by the spiral fibres (see p. 212) to the base of the heart in the region of the origin of the pulmonary artery, which is the representative of the bulbus aortæ of the primitive heart. It is therefore not surprising that the electro. cardiogram (p. 246) is complex. The wave $\mathrm{P}$ due to auricular activity is followed by a pause before the waves which accompany ventricular systole occur. During this pause it is supposed that the excitatory wave is travelling along the auriculo-ventricular bundle, the mass of which is too small to affect the galvanometer. The pause which intervenes after the wave $R$, which is the sign of commencing ventricular activity, is supposed to be due to a state of equipotentiality when the great mass of the circular muscles of the ventricle are contracting; the final wave $T$ indicates the arrival of the contraction wave at the base by the spiral fibres.

The Stannius Experiment.-This consists in applying a tight ligature to the heart between the sinus and the right auricle; the sinus continues to beat, but the rest of the heart is quiescent. The quiescent parts of the heart may be made to contract in response to mechanical or electrical stimulation. If a second ligature is applied to the junction of the auricles with the ventricle, the ventricle begins to beat again; the auricles may also beat, but they usually do not. According to Gaskell, the effect of the first ligature is simply an example of blocking; it is, however, difficult to wholly accept this view, for if instead of applying a ligature at the sino-auricular junction, the heart wall is simply cut through at this spot, the auricles and ventricle are not thereby always rendered quiescent. It appears probable, therefore, that there is some truth in the older view that the ligature acts as a stimulus irritating the vagal terminations in Remak's ganglion, and so eliciting a condition of prolonged 
inhibition; this, however, passes off after a variable time, and the auricles and ventricle once more beat rhythmically. It is impossible to explain the effect of the second Stannius ligature except on the hypothesis that it acts as a stimulus, and there is no $\dot{a}$ priori reason why the two ligatures should act in opposite ways.

The fact that the Stannius heart is quiescent has enabled physiologists to study the effects of stimuli upon heart muscle. A single stimulus produces a single contraction, which has a long latent period, is slow, and propagated as a wave over the heart at the rate of $\frac{2}{5}$ to $\frac{3}{5}$ inch, or $10-15 \mathrm{~mm}$. a second. A second stimulus causes a rather larger contraction, a third one larger still, and so on for some four or five beats, when the size of the contraction becomes constant. This staircase phenomenon, as it is called, is also seen in voluntary muscle, but it is more marked in the heart. The accompanying tracing (fig. 233) shows the result of an actual experiment.

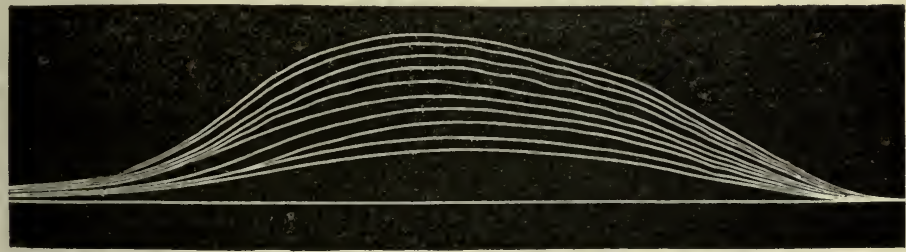

FIs. 233. - Staircase from frog's heart. This was obtained from a Stannius preparation; an induction shock being sent into it with every revolution of the cylinder (rapid rate). The contractions became larger with every beat. To be read from right to left.

There are, however, more marked differences than this between voluntary and heart muscle. The first of these is, that the amount of contraction does not vary with the strength of the stimulation. A stimulus strong enough to produce a contraction at all brings out as big a beat as the strongest. The second is, that the heart muscle has a long refractory period; that is to say, after the application of a stimulus, a second stimulus will not cause a second contraction until after the lapse of a certain interval called the refractory period. The refractory period lasts as long as the contraction period. The third difference depends on the second, and consists in the fact that the heart-muscle can never be thrown into complete tetanus by a rapid series of stimulations; with a strong current there is a partial fusion of the beats, but this is entirely independent of the rate of faradisation. Indeed, as a rule, the heart responds by fewer beats to a rapid than to a slow rate of stimulation.

Although nearly all our information on this subject is derived from the examination of the hearts of cold-blooded animals, and mainly from the heart of the frog, there is no reason to suppose that what is true for one vertebrate is not true for all; such differences 
as do occur are differences of degree and detail rather than of kind, and Wooldridge, many years ago, succeeded in performing the Stannius experiment on the heart of a mammal.

\section{The Isolated Heart.}

If a frog's heart is simply excised and allowed to remain without being fed, it ceases to beat after a time varying from a few minutes to an hour or so. But if it is fed with a nutritive fluid, it will continue to beat for many hours. Other substances such as drugs may bo added to the perfusion fluid, and their effects noted. The fluid may be passed through the heart, and the apparatus employed may be exemplified by the following diagram of Schäfer's heart-plethysmograph (fig. 234). A frog's heart is tied on to the end of a perfusion

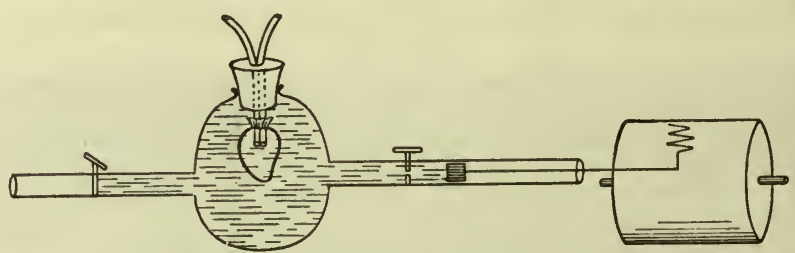

FiG. 234.-Schäfer's Heart-plethysmograph.

cannula, one tube of which serves for the fluid to enter, the other for it to leave. The end of the cannula projects into the ventricle; the frog's heart, it should be remembered, possesses no coronary vessels; the spongy texture of the cardiac tissue enables it to take up what it requires from the blood in its interior.

The cannula passes through the well-fitting stopper of an air-tight vessel containing oil. On one side of the vessel is a tube, in which a lightly moving piston is fitted; to this a writing-point is attached. The piston is moved backwards and forwards by the changes of volume in the heart causing the oil to alternately recede from and pass into this side tube. The corresponding tube on the other side can be opened and the tube with the piston closed when one wishes to cease recording the movements. It is with instruments of this kind that, a vast amount of valuable work was performed, and the name of the late Dr Sydney Ringer is specially connected with investigations of drug action by means of this method.

The best nutritive fluid to employ is undoubtedly the natural fluid, the blood. But in order to use blood there are practical difficulties; it is difficult, for instance, to obtain much blood from a frog; it is difficult to prevent it from clotting, and if agents are added to check clotting, such agents usually act deleteriously in the cardiac tissue. The blood of another animal may not be altogether 
innocuous, and this is specially the case if that blood has been previously whipped, and the fibrin removed. Physiologists therefore owe Dr Ringer a deep debt of gratitude for his discovery of the solution now known as Ringer's solution. This is physiological saltsolution to which minute quantities of calcium and potassium salts have been added. In other words, the inorganic salts in the proportion occurring in the blood will maintain cardiac activity for a long time without the addition of any organic material. These salts are not nutritive in the strict sense, but they constitute the stimulus for the heart's action. Howell of Baltimore has shown that such an inorganic mixture is especially efficacious in throwing the sinus or venous end of the heart into rhythmical action. The normal stimulus for the starting of the heart-beat is thus to be sought in the mineral constituents of the blood. These mineral compounds in solution are broken up into their constituent ions; and of these, sodium ions are the most potent in maintaining the osmotic conditions that lead to irritability and contractility. A solution of pure sodium chloride, however, finally throws the heart into a condition of relaxation; hence it is necessary to mix with it small amounts of calcium ions to restrain this effect. Potassium is not absolutely necessary, but it also favours relaxation during diastole. Calcium, on the other hand, is the element which produces contraction, and if present alone or in excess, will produce an intense condition of tonic contraction known as calcium rigor.

Some physiologists have manifested a hesitation in accepting the simple view that the various kations mentioned actually originate the heart-beat, and have advanced the hypothesis that they influence a mysterious factor they have named the inner stimulus. What this inner stimulus is, is entirely unknown, and whether or not it is connected with one or more of Langley's receptive substances is equally a matter of speculation. If it exists it is not able to originate cardiac rhythm in the absence of the appropriate inorganic salts.

The Excised Mammalian Heart.-During the past few years it has been shown that the mammalian heart can also be kept alive and active after it has been excised. Valuable as the results have been from a study of the frog's heart, one can hardly doubt that those which one hopes to obtain in the future from a similar study of the mammal's heart will be still more important, and still more trustworthy for the drawing of deductions useful to man. Already the new method has shown its usefulness not only in reference to the metabolism occurring during normal cardiac activity, but also from the pharmacological point of view.

In order to maintain the action of the excised mammalian heart certain precautions must be taken-

1. The perfusion fluid must be maintained at or about body temperature $\left(37^{\circ} \mathrm{C}\right.$.). 
2. It must circulate through the coronary vessels.

3. It must be well oxygenated.

As before, living blood is the ideal fluid for perfusion, but the practical difficulties in its use are so great, that a modification of Ringer's fluid is usually employed. On this fluid the heart will continue to beat for many hours, but it will beat longer (sometimes several days) if a little dextrose is added to the solution. We owe this addition, and the oxygenation alluded to above, to Dr Locke; and the perfusion fluid now universally employed is consequently called Locke's solution. This has the following composition:-

Pure distilled water

Sodium chloride

Potassium chloride

Calcium chloride

Sodium bicarbonate

Dextrose

$\begin{array}{ll}100 \text { c. c. } & \\ 0.9 \text { grammes. } \\ 0.042 \quad, \\ 0.048 \quad, \\ 0.02 \quad, \\ 0.2\end{array}$

Dr Locke has tried other sugars besides dextrose, but no other has the same favourable effect; lævulose is better than most other sugars, but not nearly so good as dextrose. Locke and Rosenheim have further shown that the dextrose is used up during cardiac activity, and this lends support to the view already expressed on the importance of this kind of sugar as a source of muscular energy (see p. 131).

A mammal such as a cat or rabbit is killed by bleeding or pithing. The heart enclosed in the pericardium is quickly cut out, and gently kneaded to free it from blood, in some warm Ringer's solution. The pericardium is then dissected off, and a cannula tied into the aorta; this is connected to a burette which is kept full of Locke's solution. The solution must be maintained at body temperature, by a warm water-jacket, and must be well oxygenated by letting oxygen bubble through it. The fluid is then allowed to flow, and its pressure closes the aortic valves, and so the fluid enters the coronary arteries, and escapes from the right auricle, which should be freely opened. Under these circumstances the heart will continue to beat for many hours. A graphic record may be obtained by putting a small hook into the apex, and attaching this by a thread to a recording lever beneath it. A very good illustration of the usefulness of the method for demonstrating the action of drugs consists in adding a small amount of chloroform to the circulating fluid, and one notices its immediate depressant effect; on the other hand, a minute dose of adrenaline markedly increases the rate and force of the heart. 


\section{CHAPTER XXII}

\section{THE CIRCULATION IN THE BLOOD-VESSELS}

The movement of the blood from the heart through the arteries, capillaries, and veins back to the heart again, depends on a number of physical factors; and in the consideration of this important subject we shall have to take into account the general laws which regulate the movement of fluids in tubes, as well as their special application to the flow of the blood in the blood-vessels.

The contraction of the heart is the primary propelling force, and the increase of pressure which is thus communicated to the blood it contains causes that blood to enter the arteries; the arterial bloodpressure is higher than that in the capillaries, and the capillary pressure is higher than that in the veins; the venous pressure gradually falls as we approach the heart; it is lowest of all in the heart cavities during diastole; fluid moves in the direction of lower pressure, hence the flow of blood is from the heart through the vessels back to the heart again.

The vessels are not rigid tubes, but possess marked elasticity; it is owing to this that the intermittent force of the heart is modified in such a way that the stream of blood in the capillaries is a constant one, and under norrnal circumstances exhibits no pulsation; the pulse is one of the main characters of the arterial flow. A further complication is due to the fact that the vessels through which the blood flows are of varying calibre, and this is the main factor in determining its velocity. Every time an artery divides, the united sectional area of its branches is greater than that of the parent artery, although, of course, each of the individual branches is of smaller calibre. The total bed of the stream is thus becoming greater, until when we reach the capillaries the bed is increased suddenly and enormously, being several hundred times greater than that of the aorta from which they all ultimately spring. In the case of the veins the same is true in the reverse direction; the sectional area of a vein is less than that of the total sectional area of its tributaries; hence as we approach the heart the total bed of the stream is becoming continually 
smaller, but never so small as in the corresponding arteries; a vein is always twice the size, often more than twice the size, of the corresponding artery. Velocity of flow varies inversely with the bed of the stream; the velocity is therefore greatest in the aorta, slows down in the small arteries, and becomes slowest of all in the capillaries where the total bed is widest; we may compare the combined capillaries to a vast lake into which the arterial river flows. On leaving the capillaries, the blood, in traversing the veins once more, becomes accelerated because the bed of the stream becomes narrower, but its speed in a vein is only about half that in the corresponding artery because the bed is twice as great.

In connection with the variation in the bed of the stream we must also consider the question of resistance. If the increase in sectional area took place without division of the stream into numerous branches, the main effect would be to lower resistance to the flow of fluid; but the friction-lowering effect of increased area is much more than counterbalanced by the increased surface of the numerous branches, and there is increased friction on this account. The resistance of the capillaries would be large even for a stream of water, and when we consider that the blood is much more viscid than water, we see the effect must be much greater. The resistance to the flow of fluid along a small tube is in inverse proportion to the fourth power of the diameter, i.e., if the diameter of the tube is halved, the resistance is increased sixteen-fold. Between the arteries and the capillaries are the small arteries or arterioles; these vessels are always in a state of moderate or tonic constriction; they may roughly be compared to narrow inlets into the wide capillary lake. The main resistance to the passage of blood through the tissues is situated in the arterioles, and not in the capillaries; this is usually spoken of as the peripheral resistance, and it is variable by alterations in the calibre of the arterioles, their muscular tissue being under the control of nerves which are termed vaso-motor.

The main resistance is in the arterioles and not in the capillaries for the following reason: each individual capillary is small, and its resistance therefore great, but their number is so immense, and the total bed so large, that the resultant resistance offered is comparatively small. This is well brought out by a comparison of the velocity in the two cases; in the arterioles the velocity has to be high in order to supply with blood the large capillary areas springing from them; in the capillaries, as we have already seen, the velocity is low.

After this general account of the main features of the circulation, we can now pass to a detailed description of the various points raised. 


\section{Use of the Elasticity of the Vessels.}

If a pump is connected to a rigid tube, such as a glass tube, filled with water, and a certain amount of water is forced into the tube, an exactly equal amount of water is driven out from the open end. During the intervals of pumping the flow ceases, accurately at the instant the inflow stops. If in the next place the open orifice is constricted and the pumping continued as before, the outflow is still restricted to the time during which water is being driven into the tube. The only difference is that a greater force of pumping will be required if the pump is to empty itself in the same time as before, and the force required will increase in proportion to the degree of constriction of the orifice, until with a fairly considerable constriction the force required will be enormous.

If the rigid tube is replaced by an elastic one with a wide free opening, the outflow will again be intermittent but not quite restricted to the time of the pumping. This latter difference is because the elastic wall of the tube will stretch a little at each output of the pump, and this continues after the pump has ceased to discharge, and will then recover, at the same time driving out the extra small amount of fluid it contained, after the pump has ceased to act. The flow will thus be intermittent, but the outflow will last for a short time longer than the inflow. If now the orifice be diminished, the duration of the outflow will begin to increase still further, and, as the constriction is iricreased more and more, will gradually extend over the diastolic period of the pumping. The amount of work required to drive the fixed volume of fluid through the constricted orifice is the same with a rigid and with an elastic tube. In the former case, however, the duration of the outflow is of necessity the same as that of the inflow, whereas in the second case this time is prolonged. If the constriction of the orifice of the elastic tube is sufficiently increased, a point is at last reached at which the outflow lasts throughout the whole cycle of the pump, and here therefore some of the energy imparted to the fluid by the pump is converted into a pressure energy represented by the tension of the elastic walls of the tube, and this energy is given out again after the fluid has ceased to enter the tube and is just sufficient to exactly drive out the stored fluid during the resting period, and a point will ultimately be reached at which the outflow will become not only continuous but also constant. The degree of constriction necessary to produce this effect will depend upon the distensibility of the elastic tube. The more distensible this is, the earlier will this stage be reached, and the lower will be the mean pressure. This is the condition we find in the circulatory system.

Let us now apply this to the body. 
At each beat the left ventricle forces about thrce ounces of blood into the already full arterial system. The arteries are elastic tubes, and the amount of elastic tissue is greatest in the large arteries. The first effect of the extra three ounces is to distend the aorta still further; the elastic recoil of the walls drives on another portion of blood, which distends the next section of the arterial wall, and this distension is transmitted as a wave along the arteries, but with gradually diminishing force as the total arterial stream becomes larger. This wave constitutes the pulse-wave. Between the strokes of the pump, or, in other words, during the periods of diastole, the energy imparted to the elastic arterial walls by the heart, and which has produced distension of the arteries, comes into play; their recoil drives the blood onwards and the arteries return to their original size. The flow, therefore, does not cease during the heart's inactivity, so that although the force of the heart is an intermittent one, the flow through the capillaries and the veins beyond is a constant one, all trace of pulsation having disappeared. The peripheral resistance which keeps up the blood-pressure in the arteries, and like the constriction at the end of our india-rubber tube, assists in the conversion of the intermittent into a continuous and constant stream, is to. be found in the arterioles or small arteries, just before the blood passes into what we have termed the vast capillary lake. These small arteries with their relative excess of muscular tissue, are in health always in a state of moderate tonic contraction.

The large arteries contain a considerable amount of muscular as well as elastic tissue. This co-operates with the elastic tissue in adapting the calibre of the vessels to the quantity of blood they contain. For the amount of blood in the vessels is never quite constant, and were elastic tissue only present, the pressure exercised by the walls of the containing vessels on the contained blood would be sometimes very small, sometimes too great. The presence of a contractile element, however, provides for a certain uniformity in the amount of pressure exercised. There is no reason to suppose that the muscular coat assists in propelling the onward current of blood, except in virtue of the fact that muscular tissue is elastic, and therefore co-operates in the large arteries with the elastic tissue in keeping up the constant flow in the way already described.

The contractility of the arterial walls fulfils a useful purpose in checking hæmorrhage should a small vessel be cut, as it assists in the closure of the cut end, and this in conjunction with the coagulation of the blood arrests the escape of blood.

\section{Blood-pressure.}

The circulation of the blood depends on the existence of different degrees of pressure in different parts of the circulatory system; 
there is a diminution of pressure from the heart onwards through arteries, capillaries, and veins, back to the heart again.

Fig. 235 represents roughly the fall of pressure along the systemic vascular system.

It falls slowly in the great arteries and manifests oscillations corresponding with the alternate systole and diastole of the heart; at the end of the arterial system it falls suddenly and extensively in the course of the arterioles; it again falls gradually through the capillaries and veins till in the large veins near the heart it is negative. Such a diagram of blood-pressure is thus very different from one of velocity; the velocity like the pressure falls from the arteries to the capillaries, but unlike it, rises again in the veins.

We must now study the

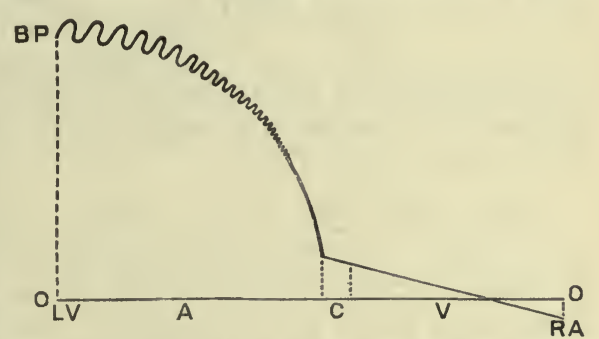

Fı. 235.-Height of blood-pressure (BP) in LV, left ventricle. A, arteries; C, capillaries; V, veins ; $\mathrm{RA}$, right auricle; 00 , line of no pressure.

methods by which blood-pressure is measured and recorded, and the main causes that produce variations in its amount.

In order that we may understand the methods that are used for this purpose, it will be first necessary for us to consider some of the general laws of fluid pressure, and then to study the methods that are employed in an artificial schema of the circulation.

Fluid pressure is a different thing from the pressure of a solid, and is exercised equally in all directions. If a cylindrical vessel, placed vertically, is filled with a cylinder of ice, the pressure of the ice will be exercised on the bottom of the cylinder, but not on its sides. When the ice melts, the water presses on the sides also, and if a hole is made in the cylinder below the level of the upper surface of the water, the water will flow out of the hole, and the force with which it escapes will be proportional to the depth of the hole beneath the surface. If we take a square centimetre as the unit of area, the actual pressure exerted on it is $h \times d \times g$, where $h$ is the height of the free surface above the level where we are measuring the pressure, $d$ the density of the fluid, and $g$ the acceleration of gravity (981). Suppose a gramme of water to flow out, we may consider that this gramme has fallen through a height or head $h$ in centimetres from the free surface to the opening; it comes practically from the top, because it is there that the liquid disappears from inside the vessel. In falling the height $h$, it gives out $h g$ ergs of work.

The unit of force is called a dyne; a moving body is said to possess momentum: this is measured by the product of its mass and its velocity; thus the effective quantity of motion of a body may be large on account of its having a large 
mass (for instance, a heavy waggon rolling down a hill), or large velocity (for instance, a bullet speeding through the air). A force continuously applied to a moving mass produces a continuous increase in its rate of movement; this is termed acceleration, and force may be defined as the rate of change of momentum ; it can be measured, therefore, by observing the amount of momentum it generates in a measured time, and dividing by that time. If a gramme is taken as the unit of mass, a centimetre as the unit of length, and a second as the unit of time, the unit of force

$$
\begin{aligned}
& =\frac{\text { momentum }}{\text { Time. }}=\frac{\text { gramme-centimetre per second }}{\text { Time in seconds. }} \\
& =\text { gramme-centimetre per second, per second }=1 \text { dyne. }
\end{aligned}
$$

The unit which corresponds to the dyne in the measurement of work is called an erg, that is, the work done in lifting a gramme weight through the height of one centimetre; the weight of a gramme is 981 dynes, and the work done in lifting it one centimetre is 981 ergs.

The kinetic energy of a body moving with velocity $v$ is $\frac{1}{2} \times$ mass $\times v^{2}$, or for one gramme $\frac{1}{2} v^{2}$; hence if all the work that liquid can do is spent in giving kinetic energy to it, the velocity with which it will flow out is given by putting the kinetic energy = work done. In . other terms :-

$$
\frac{1}{2} v^{2}=g h ; \text { hence } v=\sqrt{2 g h} \text { or } h=\frac{v^{2}}{2 g}
$$

A liquid, however, has not necessarily a free surface, but may be completely enclosed, as is the water in a system of hydraulic pressure mains, or the blood in the circulatory system. The pressure in such a system at any point may be measured by inserting at that point a vertical tube at right angles to the blood-vessel; the blood would rise in it to a point, and would form a free surface a certain distance up this tube; the head $h$ in the above calculation must be reckoned from this free surface downwards. If, instead of using a tube of fine bore for this purpose, we employ a wider tube, say of ten times greater area, the height or head to which the fluid rises will be the same as in the narrow tube, though naturally the actual weight of fluid supported will be ten times greater; but the weight per unit of area is the same in both cases. When, therefore, we measure the pressure of fluid in terms of the height of a column of fluid, such as mercury, which it will balance, we really mean that the force of the blood is equal to the weight of the mercury it supports per unit of area, and this will naturally be proportional to the height of the column.

Let us next consider the simple case of a fluid flowing from a reservoir, $\mathrm{R}$ (fig. 236), along a tube, which we will imagine is open at the other end.

In the course of the tube we will suppose three upright glass tubes $(A, B$, and $D)$ are inserted at equal distances. Between $B$ and $\mathrm{D}$ there is a bladder, which may be divided into a number of channels by packing it with tow to represent the capillaries, and between $B$ and 
C, a clip E, which can be tightened or loosened at will, and which will roughly represent the peripheral resistance produced by the arterioles. The far end of the tube is provided with a stop-cock. If this stop-cock is closed there will naturally be no flow of fluid, and the fluid will rise to equal heights indicated by the dotted line in all the upright tubes. This shows that the pressure in all parts of the tube is the same. The upright tubes which measure the lateral pressure exerted by the fluid on the wall of the main tube, are called pizometers, manometers, or pressure measurers.

If now the stop-cock is opened, the fluid flows on account of the difference of pressure brought about by gravitation; the height of the

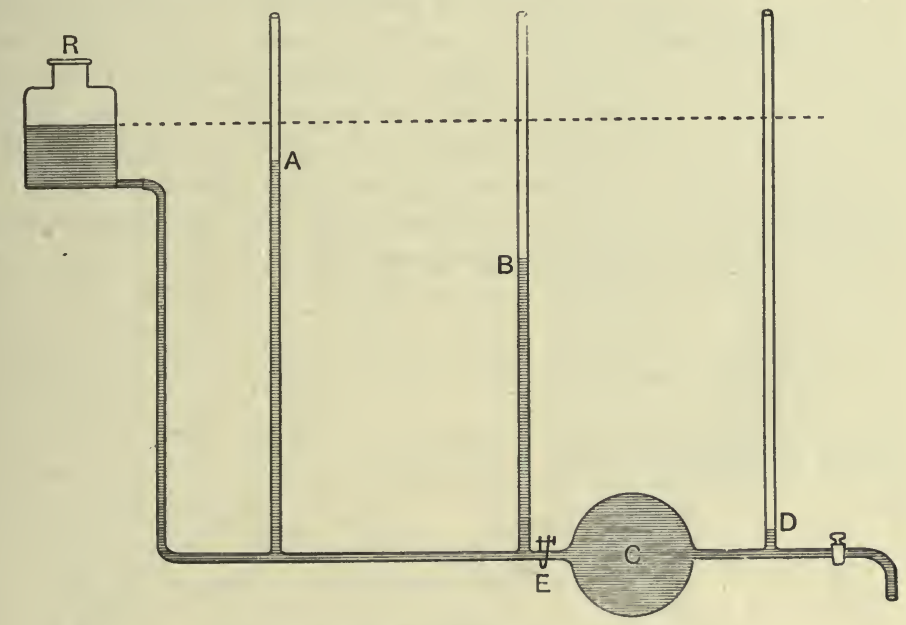

FIG. 236.-Schema to illustrate blood-pressure.

fluid in the manometers indicates that the pressure is greatest in $R$, less in $A$, less still in $B$, and least of all in D.

On account of the peripheral resistance of the arterioles and capillaries, the pressure is very small in the veins, as indicated by the height of the fluid in the manometer $\mathrm{D}$. The difference between $\mathrm{D}$ and $\mathrm{B}$ is much more marked than the difference between $\mathrm{B}$ and $\mathrm{A}$. If the fluid which flows out of the end of the tube is collected in a jug and poured back into $R$, we complete the circulation. But the schema is an extremely rough one, and is especially faulty in that the pressure which starts at $R$ is nearly constant and not intermittent. This may be remedied by taking $R$ in the hand, and raising and lowering it alternately. The fluid in the manometers bobs up and down with every rise and fall of $\mathrm{R}$ : this is least marked in $\mathrm{D}$. The greater and the faster the movement of $R$, the greater is the rise of arterial pressure. This is a rough illustration of the fact that increase in 
the force and frequency of the heart's beat causes a rise of arterial pressure.

Again, if more fluid is poured into $R$, there is a corresponding rise in fluid in the manometers. This illustrates the rise of pressure produced by an increase in the contents of the vascular system.

And this schema, rough though it is, also serves to illustrate the third important factor in the maintenance of the blood-pressure, namely, the peripheral resistance. This is done by means of the clip $\mathrm{E}$; if the clip is tightened, one imitates increased constriction of the arterioles; if it is loosened, one imitates dilatation of the arterioles. If it is closed entirely, the fluid in A and B rises to the same level as that in $R$; the pressure of $R$ is not felt at all by $C$ and $D$, which empty themselves, and the flow ceases. If the clip $\mathrm{E}$ is only tightened so as not to be quite closed, the arterial pressure (in $\mathrm{A}$ and $\mathrm{B}$ ) rises, and the venous pressure (in D) falls; if the clip is freely opened, the arterial pressure falls, and the venous pressure rises.

These same facts can be demonstrated by a more perfect circulation schema, such as is represented in fig. 237.

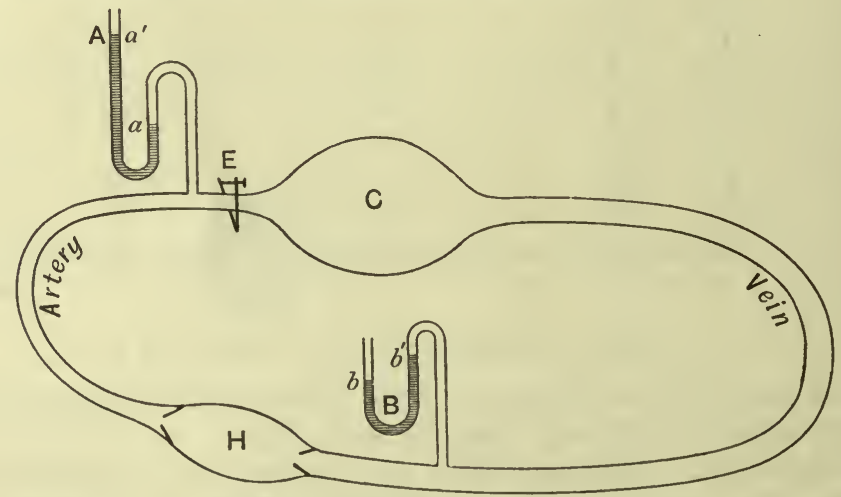

Fig. 237. - Schema of the circulation.

The heart $(\mathrm{H})$ is represented by a Higginson's syringe, which is worked with the hand; the tube from it represents the arterial system, the clip $\mathrm{E}$ the resistance of the arterioles; $\mathrm{C}$ is the capillary lake, from which the vein (larger than the artery) leads back to the heart $\mathrm{H}$. $\mathrm{A}$ and $\mathrm{B}$ are two manometers which respectively indicate arterial and venous pressures. Only in place of straight tubes mercurial manometers are used. Each of these is a $U$-tube about half filled with mercury, and united to the artery or vein by a tube containing fluid. If the mercury in the two limbs of the $U$ is at the same level, the pressure of the fluid in connection with one limb is exactly equal to 
that exerted by the atmospheric pressure on the other. The mercury, however, is pushed up in the far limb of the manometer connected to the artery, the pressure there being greater than that of the atmosphere; this is therefore called positive pressure, and the total amount of pressure is measured by the difference between the levels $a$ and $a^{\prime}$. The manometer $\mathrm{B}$ attached to the vein, however, indicates a negative pressure $\left(b b^{\prime}\right)$, that is a pressure less than that of the atmosphere, so that the mercury in the limb nearest the vein is sucked up.

Anderson Stuart's kymoscope (fig. 238) is a more complete schema. It consists of a long leaden tube filled with fluid, the two ends of which are connected by an india-rubber tube on which is a valved syringe to represent the heart On the course of the tube are a large number of openmouthed upright manometers which indicate the pressure when the syringe is worked, and confer on the tube the elasticity nocessary to cause the disapperrance of the pulso in the middle region which represents the capillaries. The long leaden tube is twisted round a cylinder, so that the manometers are placed closely side by side.

We can now pass on to the methods adopted in the investigations of bloodpressure in animals.

The fact that the blood exerts considerable pressure on the arterial walls may bo readily shown by puncturing any artery; the blood is propelled with great force through the opening, and the jet rises to a considerable height; in the case of a small artery, where the pressure is

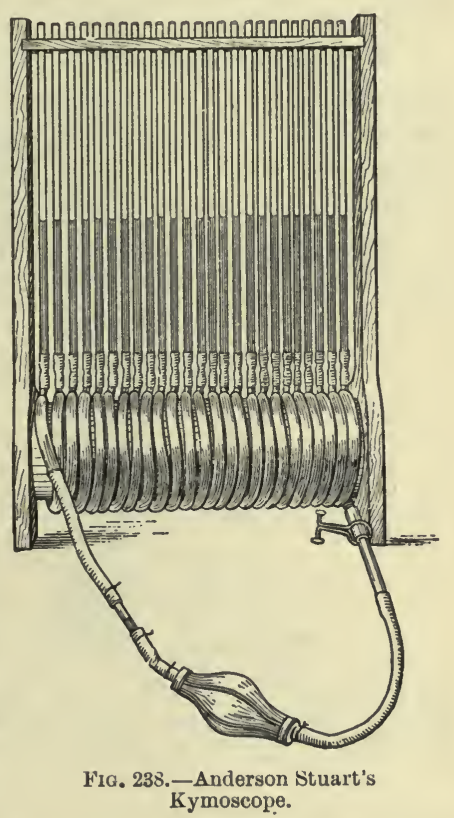
lower, the jet is not so high as in a large artery: the jerky character of the outflow due to the intermittent action of the heart is also seen. If a vein is similarly injured, the blood is expelled with much less force, and the flow is continuous, not intermittent.

The first to make an advance on this very rough inethod of demonstrating blood-pressure was the Rev. Stephen Hales, vicar of Teddington (1702). He inserted, using a small brass tube as a cannula, a glass tube at right angles to the femoral artery of a horse, and noted the height to which the blood rose in it. This is a method like that which we used in the first schema described (fig. 236). The blood rose to the height of about 8 feet, and having reached its highest 
point, it oscillated with the heart-beats, each cardiac systole causing a rise, each diastole a fall. Hales also noted a general rise during each inspiration. The method taught Hales these primary truths in connection with arterial pressure, but it possesses many disadvantages ; in the first place, the blood in the glass tube very soon clots, and in the second place, a column of liquid 8 feet high is an inconvenient one to work with.

The first of these disadvantages was overcome to a great extent by Vierordt, who attached a tube filled with saturated solution of sodium carbonate to the artery, and the blood-pressure was measured by the height of the column of this saline solution which the blood would support.

The second disadvantage was overcome by Poiseuille, who introduced the heavy liquid, mercury, as the substance on which the blood exerted its pressure; and the U-shaped mercurial manometer was connected to the artery by a tube filled with sodium carbonate solution to delay clotting.

The study of blood-pressure cannot, however, be considered to have been in a satisfactory condition until the introduction by Carl

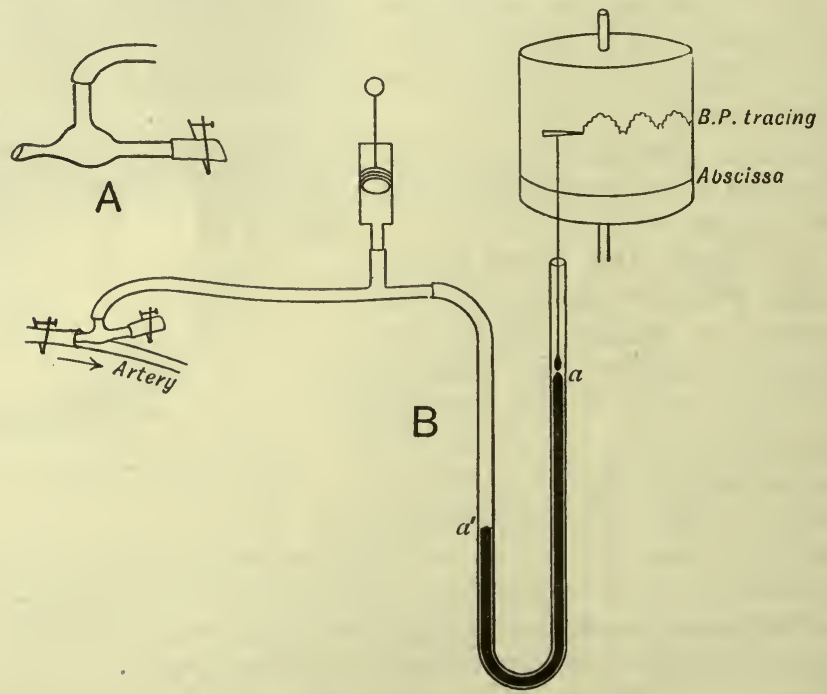

FiG. 239.-Diagram of mercurial Kymograph.

Ludwig of the Kymograph; that is to say, Poiseuille's homodynamometer was combined with apparatus for obtaining a graphic record of the oscillations of the mercury. The name kymograph or wavewriter, we shall see immediately, is a very suitable one.

A skeleton sketch of the apparatus is given in fig. 239. 
The artery is exposed and clamped, so that no hæmorrhage occurs; it is then opened, and a glass cannula is inserted and firmly tied in. The form of cannula usually employed (François Franck's) is shown on a larger scale at $A$; the narrow part with the neck in it is tied into the artery towards the heart; the cross piece of the $\mathrm{T}$ is united to the manometer; the third limb is provided with a short piece of india-rubber tubing which is kept closed by a clip and only opened on emergencies, such as to clear out a clot with a feather should one form in the cannula during the progress of an experiment.

The tube by means of which the cannula is united to the man-

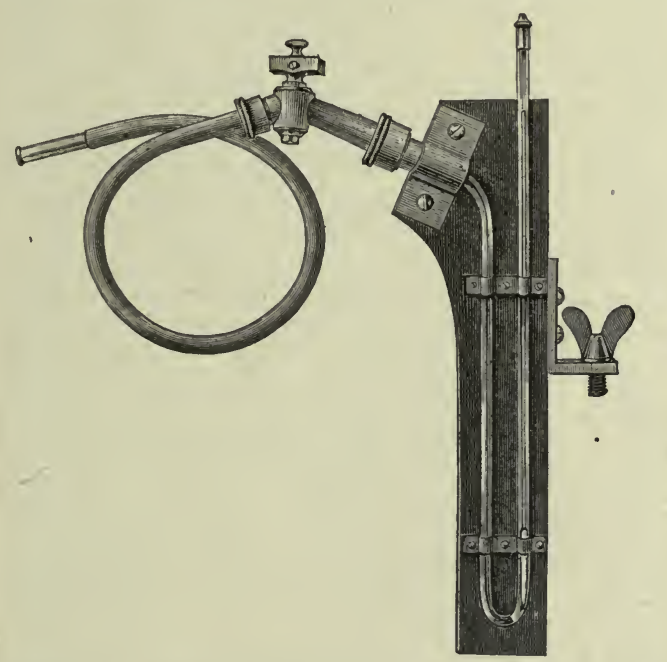

FIa. 240.-The manometer of Ludwig's Kymograph. It is also shown in fig. 241, D, C, E. The nercury which partially fills the tube supports a float in the form of a piston, nearly filling the tube; a wire is fixed to the float, and the writing style or pen fixed to the wire is guided by passing through the brass cap of the tube; the pressure is communicated to the mercury by means of a flexible metal tube filled with fluid.

ometer is not an elastic one, but is made of flexible metal or thick rubber, so that none of the arterial force may be wasted in expanding it. The tube, cannula, and proximal limb of the manometer are all filled with a saturated solution of sodium carbonate, sodium sulphate, or other salt which will mix with blood and delay its clotting. Before the clip is removed from the artery, the pressure is first got up by a syringe (or pressure bottle containing the same saline solution suspended at a good height above the apparatus and connected to it by a tube), so that the mercury rises in the distal limb to a height greater than that of the anticipated blood-pressure; this prevents blood passing into the cannula when the arterial clip is removed.

In the distal limb of the U-tube, floating on the surface of the 
mercury, is an ivory float, from which a long steel wire extends upwards, and terminates in a stiff piece of parchment or a bristle which writes on a moving surface covered with smoked paper. When the two limbs of the mercury are at rest, the writing-point inscribes a base-line or abscissa on the travelling surface; when the pressure is got up by the syringe it writes a line at a higher level. When the arterial clip is removed it writes waves as shown in the diagram

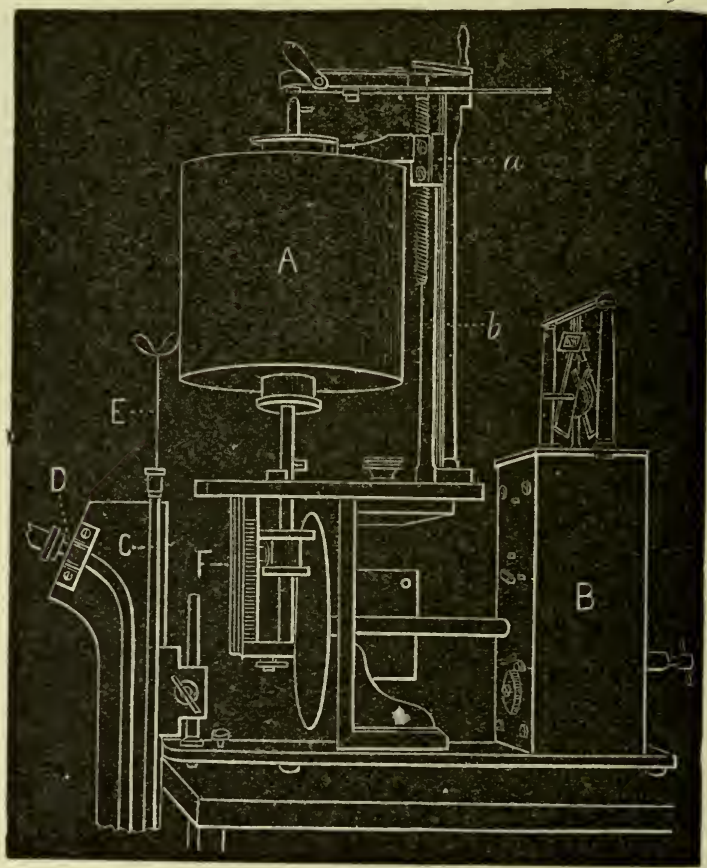

Fig. 241.-Diagram of mercurial Kymograph. A, revolving cylinder, worked by a clockwork arrangement contained in the box (B), the speed being regulated by a fan above the box; the cylinder is supported by an upright $(b)$, and is capable of being raised or lowered by a screw $(a)$, by a handle attached to it; D, C, E, represent the mercurial manometer, which is shown on a larger scale in fig. 240 .

(fig. 239), the large waves corresponding to respiration (the rise of pressure in most animals accompanying inspiration), ${ }^{*}$ the smaller ones to the individual heart-beats. The blood-pressure is really twice as great as that indicated by the height of the tracing above the abscissa, because if the manometer is of equal bore throughout, the mercury falls in one limb the same distance that it rises in the other; the true pressure is measured by the difference of level between $a$ and $\alpha^{\prime}$ (fig. 239).

* The explanation of the respiratory curves on the tracing is postponed till after we have studied Respiration. 
Fig. 240 shows a more complete view of the manometer, and fig. 241 is a diagram of the arrangement by means of which it is made into a kymograph.

Fig. 242 shows a typical normal arterial blood-pressure tracing on a larger scale.

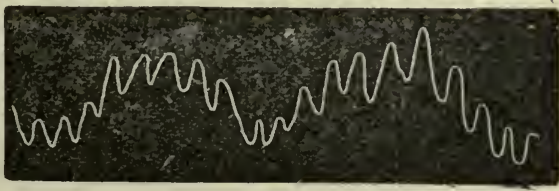

Fig. 242.-Normal tracing, somewhat magnified, of arterial pressure in the rabbit obtained with the mercurial kymograph. The smaller undulations correspond with the heart-beats, the larger curves with the respiratory movements. The abscissa or base-line, which on this scale would be several inches below the tracing, is not shown. (Burdon-Sanderson.)

In taking a tracing of venous blood-pressure, the pressure is so low and corresponds to so few millimetres of mercury, that a saline solution is usually employed instead of mercury. If the vein which

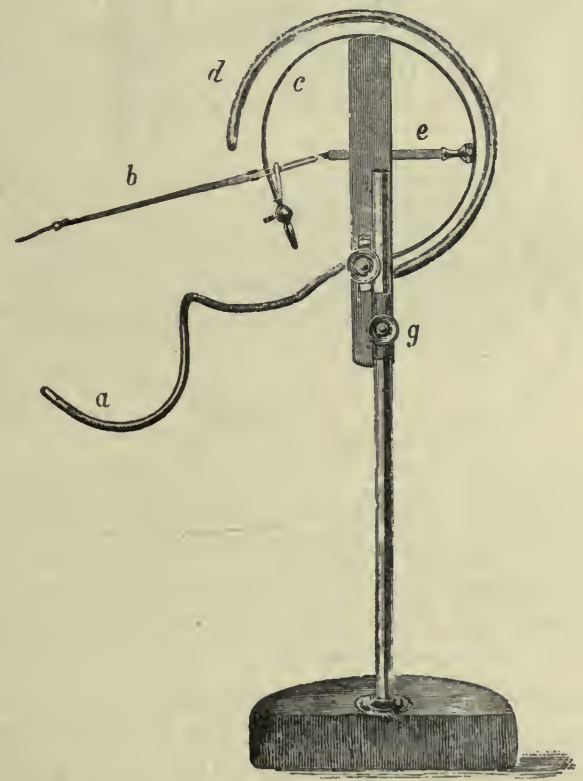

F1G. 243. - A form of Fick's Spring Kymograph. $a$, Tube to be connected with artery ; c, hollow spring, the movement of which moves $b$, the writing lever; $e$, screw to regulate height of $b ; d$, outside protective spring; $g$, screw to fix on the upright of the support.

is investigated is near the heart, a venous pulse is exhibited on the tracing, with small waves as before corresponding to heart-beats, and 
larger waves to respiration, only the respiratory rise in pressure now accompanies expiration.

The capillary pressure is estimated by the amount of pressure necessary to blanch the skin; this has been done in animals and men (v. Kries, Roy and Brown).

Other manometers are often employed instead of the mercurial one. Fick's is one of these. The blood-vessel is connected as before with the manometer, and the pressure got up by the use of a syringe

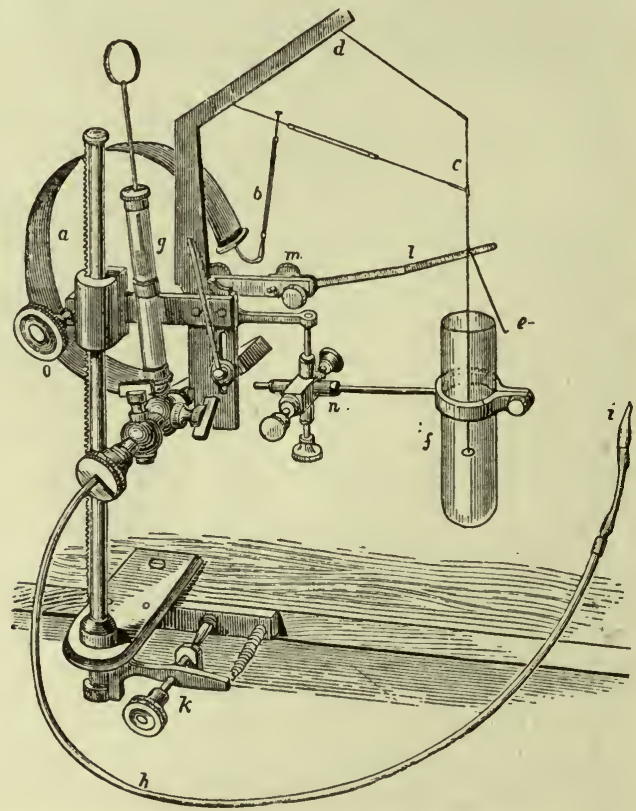

FıG. 244.-Fick's Kymograph, improved by Hering (after M'Kendrick). a, Hollow spring filled with alcohol, bearing lever arrangement $b, d, c$, to which is attached the marker $e$; the rod $c$ passes downwards into the tube $f$, containing castor oil, which offers resistance to the oscillations of $c$; $g$, syringe for filling the leaden tube $h$ with saturated sulphate of sodium solution, and to apply sufficient pressure as to prevent the blood from passing into the tube $h$ at $i$, the cannula inserted into the vessel; $l$, abscissa-marker, which can be applied to the moving surface by turning the screw $m ; k$, screw for adjusting the whole apparatus to the moving surface; 0 , screw for elevating or depressing the Kymograph by a rack-and-pinion movement; $n$, screw for adjusting the position of the tube $f$.

(which is seen in fig. 244,g), before the clip is removed from the artery. The manometer itself is a hollow C-shaped spring filled with liquid; this opens with increase, and closes with decrease of pressure, and the movements of the spring are communicated to a lever provided with a writing-point.

Hürthle's manometer (see p. 242) is also very much used. The advantage of these forms of manometer is that the character and extent of each pressure change is much better seen. In a mercury 
manometer the inertia is so great that it cannot respond to the very rapid variations in pressure which occur within an artery during each cardiac cycle. If Fick's or Hürthle's manometer is employed, and the surface travels sufficiently fast, these can be recorded (see fig. 245). These instruments, though useful for recording the complete

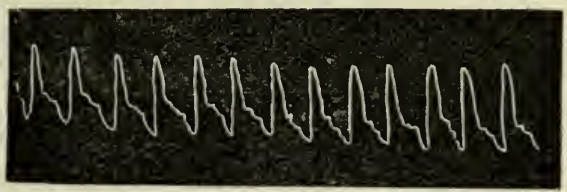

Fig. 245.-Normal arterial tracing obtained with Fick's Kyıograph in the dog. (Burdon-Sanderson.)

changes in pressure, require calibration : that is, the extent of movement that corresponds to known pressures must be ascertained by actual experiment. They teach us that the highest pressure reached during systole may be twice or thrice the lowest attained during diastole. .

The following table gives the probable average height of bloodpressure in various parts of the vascular system in man. They have been very largely inferred from experiments on animals:-

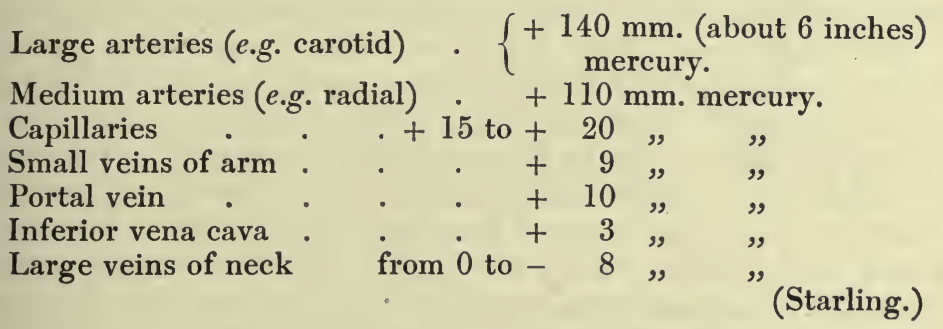

These pressures are, however, subject to considerable variations; the principal factors that cause variation are the following:-

Increase of arterial blood-pressure is produced by-

1. Increase in the rate and power of the heart-beat.

2. Increase in the contraction of the arterioles.

3. Increase in the total quantity of blood (plethora, after a meal, after transfusion).

Decrease in the arterial blood-pressure is produced by-

1. Decrease in the rate and force of the heart-beat.

2. Decrease in the contraction of the arterioles.

3. Decrease in the quantity of blood (e.g. after hæmorrhage).

The above is true for general arterial pressure; but if we are investigating local arterial pressure in any organ, the increase or 
decrease in the size of the arterioles of other areas may make its effect felt in the special area under investigation.

Venous pressure varies directly with the volume of the blood; in the arteries the effect of increase of fluid is slight and temporary, owing to the rapid adaptability of the peripheral resistance; the excess of fluid collects in and distends the easily dilatable venous reservoir. With regard to the first and second factors in the foregoing table, venous pressure varies in the opposite way to arterial pressure.

It is easy to understand how this is; when the rate of the heart increases, the total volume of blood discharged into the aorta per second is increased; similarly, an increase in the force of the beat also results in an increase in the cardiac output, and in both cases a more rapid and complete emptying of the auricle is produced. This is felt throughout the whole of the pulmonary circulation, and the accelerated flow therefore causes a fall in the venous pressure. If, however, the rise of pressure is due to a contraction of the arterioles, a stage may be reached in which the heart is no longer able to overcome the high pressure produced. It then fails to empty itself, and the bloow is dammed up on the venous side, i.e. the venous pressure rises.

With regard to the arterioles, contraction means a rise in arterial pressure, because while the amount sent into the arteries remains the same the outflow into the capillaries is cut down. More blood is therefore retained in the arteries; they become more distended and the pressure rises. The first effect of this upon the venous pressure will be to diminish it, because if more blood is retained in the arteries there is less for the veins and capillaries. The flow into the veins is thus decreased, and the venous pressure therefore falls. Moreover, the heart usually responds to a rise in pressure by increasing its force and rate, and consequently more blood is taken from the veins near the heart. For both reasons, then, the venous pressure will fall, but that fall is limited, as pointed out above, to such an increase only as the heart is capable of overcoming successfully.

Capillary pressure is increased by-

1. Dilatation of the arterioles; the blood-pressure of the large arteries is then more readily propagated into them.

2. The size of the arterioles remaining the same, increase of arterial pressure from any other cause will produce a rise of capillary pressure.

3. By narrowing the veins leading from the capillary area; complete closure of the veins may quadruple the capillary pressure. This leads secondarily to an increased formation of lymph (dropsy); as when a tumour presses on the veins coming from the legs.

4. Any circumstance that leads to increased pressure in the veins 
will act similarly; this is illustrated by the effects produced by gravity on the circulation, as in alterations of posture.

Capillary pressure is decreased by the opposite conditions.

Capillary pressure is much more influenced by changes in the venous pressure, than by changes in the arterial pressure, since there is between the arteries and capillaries the variable and usually. unknown peripheral resistance of the arterioles.

Effect of gravity on the circulation.-The main effect of gravity is that the veins are filled with blood in the part which is placed down. Thus, if an animal is placed suddenly with its legs hanging down, less blood will go to the heart, and the blood-pressure in the arteries will fall temporarily in consequence. This hydrostatic effect of gravity is soon overcome by an increased constriction of the vessels of the splanchnic area, when the vaso-motor mechanism is working normally. The efficient action of the "respiratory pump" is also of importance in counteracting gravity.

A very striking illustration of the effect of gravity on the circulation can be demonstrated on the eel. The animal is anæsthetised, and a small window is made in the body wall to expose the heart. If the animal is then suspended tail downwards, the beating heart is seen to be empty of blood; all the blood accumulates in the tail and lower part of the body; the animal has no "respiratory pump," such as a mammal possesses, to overcome the effects of gravity. If, however, the animal, still with its tail downwards, be suspended in a tall vessel of water, the pressure of the water outside its body enables it to overcome the hydrostatic effect of gravitation, and the heart-cavities once more fill with blood during every diastole. Another experiment was originally performed by Salathe on a "hutch" rabbit. If the animal is held by the ears with its legs hanging down, it soon becomes unconscious, and if left in that position for about half an hour it will die. This is due to anæmia of the brain; the blood accumulates in the very pendulous abdomen which such domesticated animals acquire, and the vaso-motor mechanism of the splanchnic area is deficient in tone, and cannot be set into such vigorous action as is necessary to overcome the bad effects of gravity. Consciousness is, however, soon restored if the animal is placed in a horizontal position, or if while it is still hanging vertically the abdomen is squeezed or bandaged. A wild rabbit, on the other hand, suffers no inconvenience from a vertical position; it is a more healthy animal in every respect; its abdomen is not pendulous, and its vaso-motor power is intact.

We shall, a few pages later, be considering the methods by which blood-pressure may be estimated in man. The effects of gravity on the pressure in various parts can be well shown by alterations of posture. This is an important practical question, especially during 


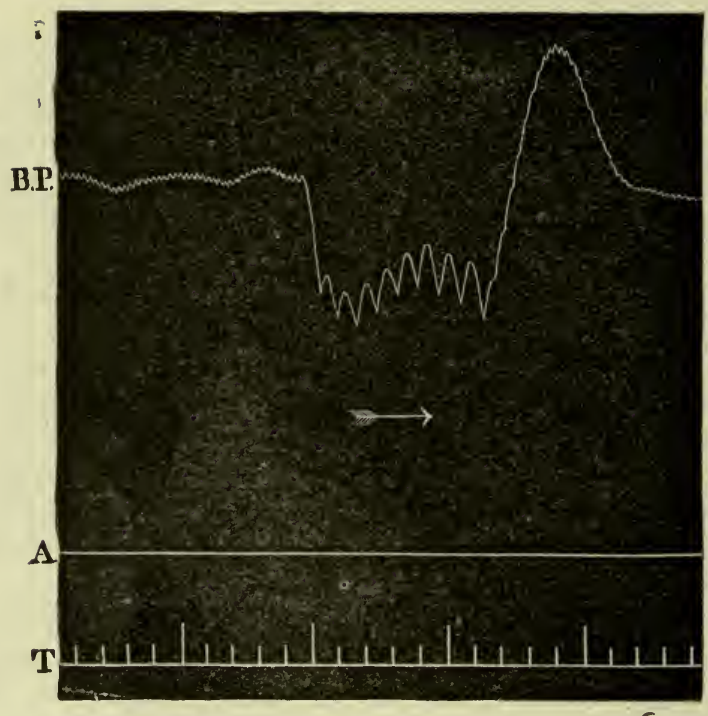

FiG. 246. - Effect of weak stimulation of the peripheral end of vagus on arterial blood-pressure (earotid of rabbit). BP, blood-pressure; A, abscissa or base-line; T, time in seconds. Note fall of bloodpressure and slow heart-beats.

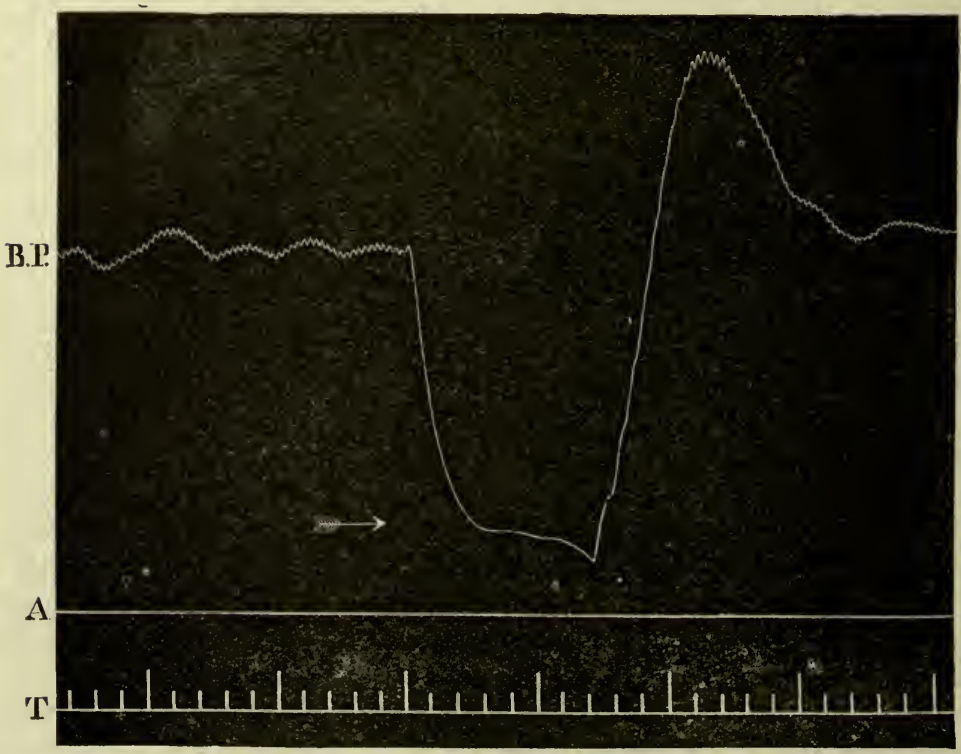

Fra. 247.- Effect of strong stimulation of the peripheral end of vagus on arterial blood-pressure (carot of rabbit). Note stoppage of heart and fall of blood-pressure nearly to zero; after the recommenc ment of the heart, the blood-pressure rises, as in fig. 246 , above the normal for a short time. 
anæsthesia, when the forces which counteract the bad effects of gravity may not be working efficiently; if the legs are hanging down, the result may be serious.

The pressure in the Pulmonary Circulation varies from $\frac{1}{3}$ to $\frac{1}{8}$ (mean $\frac{1}{6}$ ) of that in the systemic vessels.

The influence of the Cardiac Nerves on blood-pressure. The importance of the heart's action in the maintenance of blood-pressure is well shown by the effect that stimulation of the vagus nerve has on the blood-pressure curve. If the vagus of an animal is exposed and cut through, and the peripheral end stimulated, the result is that the heart is slowed or stopped; the arterial blood-pressure consequently falls; the fall is especially sudden and great if the heart is completely stopped. There is a rise in venous pressure. The effect on arterial pressure is shown in the two accompanying tracings ; fig. 246 represents the effect of partial, and fig. 247 of complete stoppage of the heart; in both cases the animal used was a rabbit, and the artery the carotid.

On stimulating the cardiac sympathetic (accelerator and augmentor fibres) the increased action of the heart causes a rise of arterial pressure.

The effects of stimulating the central end of the vagus and other nerves cannot be understood until we have studied the vaso-motor nervous system.

\section{The Velocity of the Blood-flow.}

We have already seen that the velocity of the current of blood is inversely proportional to the sectional area of the bed through which it flows. The flow is therefore swiftest in the aorta and arteries, and slowest in the capillaries. In very round numbers, the rate is about a foot per second in the aorta, and about an inch per minute in the capillaries. The capacity of the veins is about twice or thrice that of the arteries; so the velocity in the veins is from a half to a third of that in the corresponding arteries. The rate in the veins increases as we approach the heart, as the total sectional area of the venous trunks becomes less and less.

The question of velocity is one of great importance, for it is on velocity that the actual amount of blood supplying the tissues mainly depends. In the capillaries the rate can be measured by direct microscopic investigation of the transparent portions of animals. E. H. Weber and Valentin were among the earliest to make these measurements in the frog, and the mean of their estimates gives the velocity as $25 \mathrm{mms}$. per minute in the systemic capillaries. In warm-blooded animals the velocity is somewhat greater; in the dog it is $\frac{1}{50}$ to $\frac{1}{100}$ inch $(0.5$ to $0.75 \mathrm{~mm}$.) per second. It must be remembered that the total length of capillary vessels through which any given portion of 
blood has to pass probably does not exceed from $\frac{1}{50}$ to $\frac{1}{30}$ inch $(0.5 \mathrm{~mm}$.), and therefore the time required for each quantity of blood to traverse its own appointed portion of the general capillary system will scarcely amount to a second. It is during this time that the blood does its duties in reference to nutrition.

In the larger vessels direct observations of this kind are not possible, and it is necessary to have recourse to some instrumenital method.

Volkmann was the first to make more or less accurate measurements by introducing a long $U$-shaped glass tube into the course of an artery. A diagram of this hoemodromometer, as it was termed, is shown in tho accompanying diagram (fig. 248); this is filled with

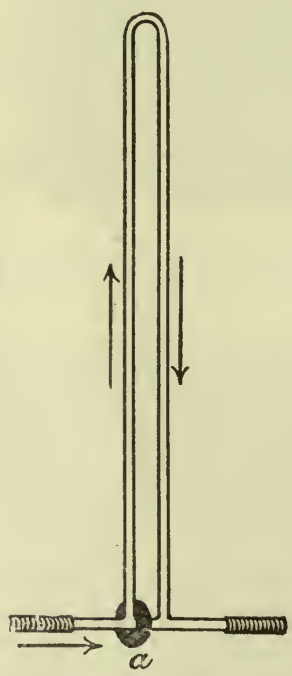

Fro. 248.-Volkmann's Hæmodromom ter.

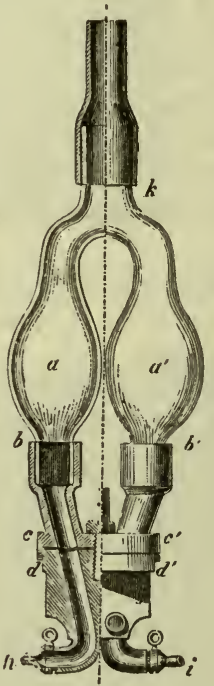

Fı. 249.-Ludwig's Stromuhr.

salt solution, and provided with a stop-cock $a$; this tap is so arranged that the blood can flow straight across from one section of the artery to the other; then at a given instant it is turned into the position shown in the diagram, and the blood has to traverse the long U-tube, and the time that it takes to traverse the tube, the length of which is known, is accurately observed. If the sectional area of the tube is the same as that of the artery, the velocity is obtained without further correction; but the difficulty of obtaining glass tubes with the exact calibre of every blood-vessel which one desires to experiment with led to the abandonment of this method, and Ludwig's Stromuhr (literally stream-clock) took its place. This consists of a 
$U$-shaped glass tubo dilated at $\alpha$ and $\alpha^{\prime}$, the ends of which, $h$ and $i$, are of known calibre. The bulbs can be filled by a common opening at $k$. The instrument is so contrived that at $b$ and $b^{\prime}$, the glass part is firmly fixed into metal cylinders, attached to a circular horizontal table $c c^{\prime}$, capable of horizontal movement on a similar table $d d^{\prime}$, about the vertical axis marked in the figure by a dotted line. The openings in $c c^{\prime}$, when the instrument is in position, as in fig. 249, corresponds exactly with those in $d d^{\prime}$; but if $c c^{\prime}$ is turned at right angles to its present position, there is no communication between $h$ and $a$ and $i$ and $a^{\prime}$, but $h$ communicates directly with $i$; and if turned through two right angles $c^{\prime}$ communicates with $d$, and $c$ with $d^{\prime}$, and there is no direct communication between $h$ and $i$. The experiment is performed in the following way:-The artery to be investigated is divided and connected with two cannulæ and tubes which fit it accurately with $h$ and $i ; h$ is the central end, and $i$ the peripheral; the bulb $a$ is filled with olive oil up to a point rather lower than $k$, and $a^{\prime}$ and the remainder of $a$ is filled with defibrinated blood; the tube on $k$ is then carefully clamped; the tubes $d$ and $d^{\prime}$ are also filled with defibrinated blood. When everything is ready, the blood is allowed to flow into $a$ through $h$, thus driving the oil over into $a^{\prime}$ and displacing the defibrinated blood through $i$ into the peripheral end of the artery; $a^{\prime}$ is then full of oil; when the blood reaches the former level of the oil in $a$, the disc $c c^{\prime}$ is turned rapidly through two right angles, and the blood flowing through $d$ into $a^{\prime}$ again displaces the oil, which is driven into $a$. This is repeated several times, and the duration of the experiment noted. The capacity of $a$ and $a^{\prime}$ is known; the diameter of the artery is then measured, and as the number of times $a$ has been filled in a given time is known, the velocity of the current can be calculated.

We may take an example to illustrate this :-

$$
\text { Velocity }=\frac{\text { volume per second }}{\text { sectional area }}=\frac{\mathrm{V}}{\mathrm{S}} \text {. }
$$

If the capacity of the bulb is 5 c.c., and it required 100 seconds to fill it 10 times, then the amount of blood passing through the instrument would be 50 c.c. in 100 seconds, or 0.5 c.c. in 1 second. Next, suppose the diameter of the artery is $4 \mathrm{~mm}$. The sectional area is $\pi r^{2} ; r$ is the radius $(2 \mathrm{~mm}$.), and $\pi=3 \cdot 1416$. From these data we get

Velocity $=\frac{\mathrm{V}}{\mathrm{S}}=\frac{0 \cdot 5 \text { c.c. }}{3 \cdot 1416 \times 2^{2}}=\frac{500 \text { cubic millimetres }}{3 \cdot 1416 \times 4}=39 \cdot 8 \mathrm{~mm}$. per sec.

Many modifications of Ludwig's original instrument have been devised. Fig. 250 shows Tigerstedt's.

The tubes $\mathrm{A}$ and $\mathrm{B}$ are placed in connection with the two ends 
of the cut artery as before; there is also a turntable arrangement at $\mathrm{F}$, by means of which the two upper tubes $\mathrm{C}$ and $\mathrm{D}$ may be connected as in the figure; or by twisting it through two right angles, $D$ can be

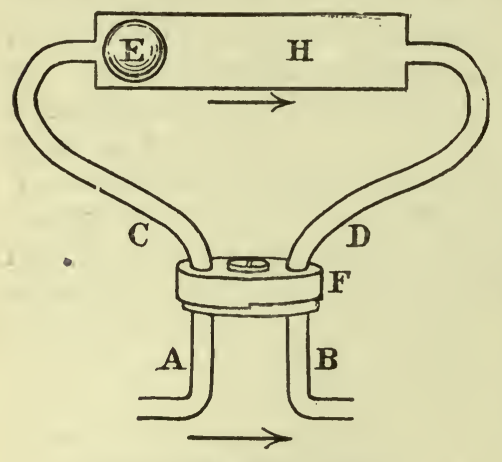

FiG. 250.-Tigerstedt's Stromuhr. made to communicate with $A$, and $\mathrm{C}$ with $\mathrm{B}$. In place of the two bulbs of Ludwig's instrument there is a glass cylinder $\mathrm{H}$ which contains a metal ball $\mathrm{E}$. The whole instrument is washed out with oil to delay clotting, and filled with defibrinated blood. As soon as blood is allowed to flow from the artery, the ball $\mathrm{E}$ is driven over by the current till it reaches the other end of the cylinder; the instrument is then rapidly rotated through two right angles, and once more the ball is driven to the opposite end. This is repeated several times, and the number of revolutions during a given period is noted. The capacity of the cylinder minus that of the ball is ascertained, and the velocity is calculated by the same formula as that already given.

The Stromuhr has one advantage over the hæmodromometer, in that it enables one to note changes in mean velocity during the course of an experiment. The mean velocity varies very greatly even during a short experiment. Thus, in the carotid artery of a dog, the velocity of the stream varied from 350 to $730 \mathrm{~mm}$. per second in the course of eighty seconds; in the same artery of the rabbit the variations were still more extensive (94 to $226 \mathrm{~mm}$. per second-Dogiel).

Other instruments have been devised which give the variations in the velocity during the phases of the heart-beat; and some of these lend themselves to the graphic method, and give tracings of what is called the velocity pulse. Before we can understand these, it is necessary first to study the relationship of velocity to blood-pressure. Mere records of blood-pressure give us no indication of the velocity of the blood-stream; the latter depends, not on the absolute amount of pressure, but on the differences of pressure between successive points of the vascular system. When a fluid is in movement along a tube the forces maintaining the flow are two in number, the one hydrokinetic, the other hydrostatic. Thus, if we consider the flow from one point in the tube to another (say, for example, at $1 \mathrm{~cm}$. distance), the forces producing the flow are (1) the kinetic energy possessed by the blood when it enters the first spot (i.e. $\frac{m v^{2}}{2}$ dynes, 
or $\frac{n v^{2}}{2 g}$ gramme-centimetres); and (2) the difference between the two lateral pressures at the two points in question. The important point to remember with respect to the part the pressure plays, is that the actual value of the lateral pressure does not matter, but that the resulting velocity, so far as pressure is concerned, depends only upon the fall of pressure between the two points. Therefore, the measurement to be determined is the rate of fall of pressure, or, as it is usually expressed, the pressure gradient. The steeper this gradient is, the more rapid is the flow. Thus, if an artery is suddenly cut across, the blood will spurt out at a far greater velocity than it possessed when flowing along the intact artery, because the pressure gradient has been enormously increased in steepness. If, on the other hand, we suddenly cut across a vein along which the blood had been flowing at the same pace as in the intact artery first investigated, the flow will not be markedly accelerated, because the change in pressure gradient has not been increased to nearly so great an extent.

Again, the flow along a vein is just as rapid as along an artery of the same size, for although the actual pressure in the vein is much less, the pressure gradient is just as steep.

The influence of the kinetic factor is also of great importance in the consideration of the flow of blood along the arteries and veins. In the first place, it is obviously possible for the blood to flow from one point to another at a higher pressure if the kinetic energy at the first point is more than enough to compensate for the pressure increase. Under such circumstances the velocity at the second point must of course be less than that at the first. This implies, therefore, that the bed of the stream has widened, and under such circumstances the blood could actually flow uphill. In the case of the veins, as we have previously seen, the bed continuously narrows, so that this cannot take place; still it is possible to conceive such a condition to occur as that in which the blood from a well-filled vein empties into a more collapsed larger vein situated at a higher level. The one instance in which this effect is produced, and is of great importance, is in the filling of the auricles and ventricles. As these cavities fill, the blood comes to rest and so loses all kinetic energy; consequently the whole of the kinetic energy possessed by the blood flowing in the veins is converted into static energy, that is, into a pressure-head; in this way the cavities are distended to a much higher pressure than that in the great veins. The acute distension of the right auricle which follows any sudden failure of the right ventricle is brought about chiefly in this way.

It is usual to speak of the lateral pressure of the blood on the 
vessel wall as the pressure-head, and of the kinetic energy measured in terms of a pressure as the velocity-head. We could then say that the velocity between any two points is determined by the difference between the two pressure-heads plus the velocity-head at the first point. One method of recording the velocity-head is by the use of a tube (Pitot's tube) shaped as in the accompanying figure (fig. 251). The blood is made to enter at $\mathrm{A}$, and leave through $\mathrm{B}$; in the same straight line as $A$ is a tube $C$, and a second tube $D$ is placed at right angles to the tube $\mathrm{B}$. If the tubes $\mathrm{C}$ and $\mathrm{D}$ are placed vertically and were sufficiently long, the blood would flow up C until it

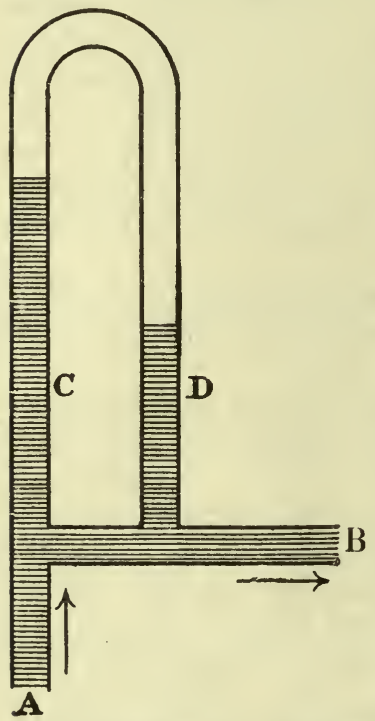

FIG. 251.-Diagram to illustrate the principle of Pitot's Tube and Cybulski's Photo-hæmatachometer. to determine the velocity in absolute measures, the instrument must be previously calibrated by passing through it fluids flowing at known rates. It will be sufficient to give the results of one experiment; in the carotid artery during the ventricular systole the flow was at the rate of 238-248 $\mathrm{mm}$. per second; during the diastole it sank to $127-156$; in the femoral artery of the same animal, these numbers were 356 and 177 respectively.

To determine the pressure gradient in arteries, simultaneous measurements of the lateral pressures in two vessels at different distances from the heart must be recorded.

It has been found that the diastolic pressures in the crural and 
carotid are practically identical, but that the maximum systolic pressure is actually higher in the crural than in the carotid; in the dog the difference may amount to as much as $60 \mathrm{~mm}$. mercury. This difference is partly to be explained in that the carotid arises from the aorta at a right angle, and therefore gives the true pressurehead, while the crural, to a considerable extent, faces the stream, and therefore gives both pressure-head and velocity-head.

Unfortunately, at present no really satisfactory measurements are at hand from which the pressure gradient can be determined.

Cybulski's instrument is not the only one we possess for obtaining records of the velocity-pulse. Vierordt invented a hæmo-tachometer, employing the principle of the hydrometric pendulum; his instrument was improved by Chauveau. Chauveau's instrument is shown in fig. 252 .

It consists of a thin brass tube, $a$, in one side of which is a small perforation closed by thin vulcanised india-rubber. Passing through the rubber is a fine lever, one end of which, slightly flattened, extends into the lumen of the tube, while the other moves over the face of a dial. The tube is inserted into the interior of an artery, and ligatures

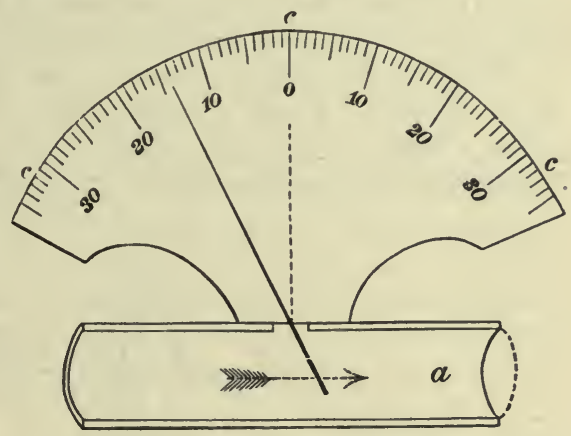

FiG. 252.-Diagram of Chauveau's Dromograph. $a$, Brass tube for introduction into the lumen of the artery, and containing an index needle, which passes through the elastic membrane in its side, and moves by the impulse of the blood-current; $c$, graduated scale, for measuring the extent of the oscillations of the needle. applied to fix it, so that the "velocity pulse," i.e., the change of velocity with each heart-beat, may be indicated by the movement of the outer extremity of the lever on the face of the dial.

In order to obtain the actual value of these movements in terms of velocity, the instrument must be calibrated beforehand. The next diagram, fig. 253, shows how the instrument may be adapted to give

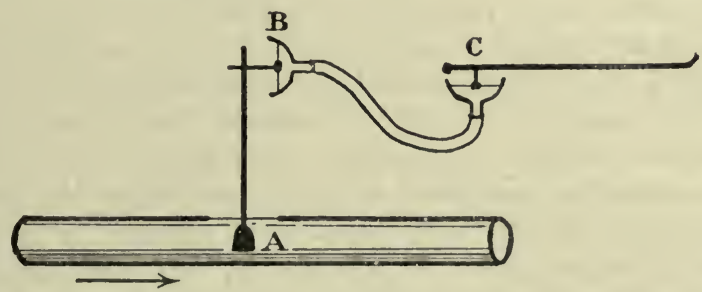

F10. 253.-Chauveau's Dromograph connected with tambours to give a graphic record.

a graphic record. The movements of the pendulum are brought to bear upon a tambour, $B$, which communicates by a tube with the 
recording tambour C. If the mass of the pendulum is small, the accuracy of the instrument is considerable. Fig. 254 shows the tracing obtained from the carotid artery of the horse. The pressure curve is placed below it for purpose of comparison. The tracing shows the effects during the time corresponding to one cardiac cycle. On both curves the upstroke is the effect of the ventricular systole; this terminates at the apex of the first small curve (between the vertical lines 3 and 4 ) on the downstroke of the pressure curve, the rest of the downstroke until the commencement of the next systole (line 5) corresponds with the ventricular diastole. Beyond the line 4 is a larger secondary wave, which is known as the dicrotic wave; the smaller post-dicrotic waves are due to elastic vibrations. We shall be studying all these points more in detail when we come to the pulse. When we compare the two curves together we note that the velocity curve reaches its maximum before the pressure curve; this

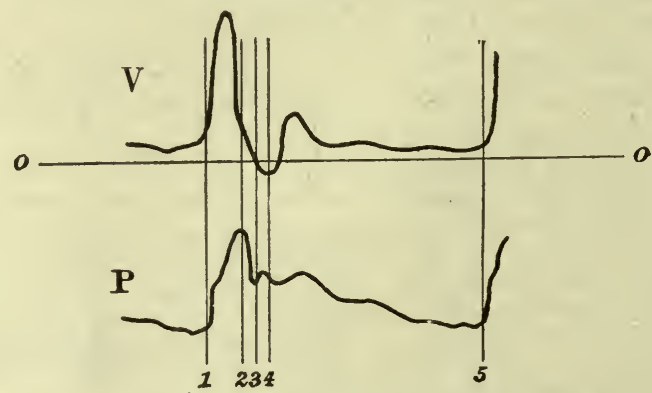

FIG. 254.-Velocity curve (V), and pressure curve (P) from the carotid artery of the horse; 00 , abscissa of velocity curve; $1,2,3,4$ show simultaneous points on both curves. (Chauveau and Marey.)

is because, as the arteries become overfilled, the heart cannot maintain the initial velocity of output. The blood is thus forced along the arteries; then comes the diastole, and the recoil of the elastic arteries not only forces the blood onwards, but also produces a back-swing against the closed aortic valves; this produces the notch before the dicrotic wave; the blood is reflected from the aortic valves, once more producing a positive wave (the dicrotic wave). This affects both speed and pressure. It will be noticed that during the dicrotic notch the pressure falls comparatively little, but in the velocity curve the fall is considerable, and the curve may sink below the base line 00 . This negative effect is naturally much more marked in the aorta and its first large branches than in situations farther from the heart.

In actual values Chauveau found that the velocity in the horse's carotid reached $520 \mathrm{~mm}$. per second during systole; it sank to 220 at the time of the dicrotic wave, and to 150 during diastole.

The effect on the blood-flow of functional activity or vaso-motor 
changes has also been observed. Thus Lortet found that the carotid flow is five or six times greater when the horse is actively masticating than when it is at rest. After section of the cervical sympathetic, the lessening of the peripheral resistance raised the velocity from 540 to $750 \mathrm{~mm}$. per second.

\section{The Time of a Complete Circulation.}

Among the earliest investigators of the question how long an entire circulation takes, was Hering. He injected a solution of potassium ferrocyanide into the central end of a divided jugular vein, and collected the blood either from the other end of the same vein, or from the corresponding vein of the other side. The substance injected is one that can be readily detected by a chemical test (the prussian blue reaction). Vierordt improved this method by collecting the blood as it flowed out, in a rotating disc divided into a number of compartments. The blood was tested in each compartment, and the ferrocyanide was discovered in one which in the case of the horse received the blood about half a minute after the injection had been made. The experiment was performed in a large number of animals, and the following were a few of the results obtained:-

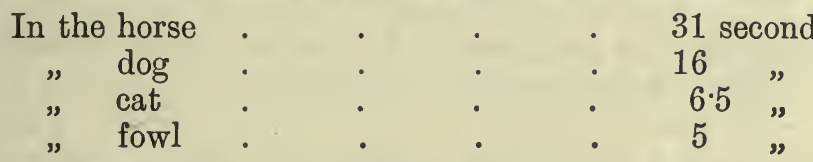

At first sight these numbers show no agreement, but in each case it was found that the time occupied was 27 heart-beats. The dog's heart, for instance, beats twice as fast as the horse's, and so the time of the entire circulation only occupies half as much time.

The question has recently been reinvestigated by Stewart by improved methods, which have shown that the circulation time is considerably less than was found by the researches of Hering and Vierordt. The great objection to the older method is the fact that hæmorrhage is occurring throughout the experiment, and this would materially weaken the heart and slow down the circulation. Stewart has employed two methods. In the first, the carotid artery is exposed, and non-polarisable electrodes applied to it. These are placed in circuit with a cell, a galvanometer, and one arm of a Wheatstone's bridge. After the resistances in the bridge have been balanced, and the galvanometer needle brought to rest, a small quantity of strong sodium chloride solution is injected into the opposite carotid. As soon as the salt reaches the carotid artery, the resistance of the blood is altered, the balance of the Wheatstone's bridge is upset, and the galvanometer needle moves. The period between the injection and the swing of the needle is accurately noted. 
The second method used is even simpler, and gives practically the same results; a solution of methylene blue is injected into a vessel. The corresponding vessel on the opposite side is exposed, placed upon a sheet of white paper, and strongly illuminated. The time is noted between the injection and the moment when the blue colour is seen to appear in the vessel under observation. Stewart has applied these methods also for determining the time occupied by the passage of blood through various districts of the circulation; the longest circulation times were found in the portal system and the lower limbs. He calculates that the total circulation time in man is about 15 seconds.

None of these methods, however, give the true time of the entire circulation; they give merely the shortest possible time in which any particle of bloud can travel through the shortest pathway. The blood that travels in the axial current, or which takes a broad pathway through wide capillaries, will arrive far more speedily at its destination than that which creeps through tortuous or constricted vessels. The direct observations of Tigerstedt on the output of the left ventricle show that the circulation time of the whole blood is at least three times as long as the period arrived at by the Hering method. It is therefore fallacious to use the circulation times arrived at by Hering's or Stewart's mothods as a basis for calculating the total amount of the blood in the body.

\section{The Pulse.}

This is the most characteristic feature of the arterial flow. It is the response of the arterial wall to the changes in lateral pressure caused by each heart-beat.

A physician usually feels the pulse in the radial artery, since this is near the surface, and supported by bone. It is a most valuable indication of the condition of the patient's heart and vessels. It is necessary in feeling a pulse to note the following points:-

1. Its frequency; that is the number of pulse-beats per minute. This gives the rate of the heart-beats.

2. Its strength; whether it is a strong, bounding pulse, or a feeble beat; this indicates the force with which the heart is beating.

3. Its regularity or irregularity; irregularity may occur owing to irregular cardiac action either in force or in rhythm.

4. Its tension; that is the force necessary to obliterate it. This gives an indication of the state of the arterial walls and the peripheral resistance.

In disease there are certain variations in the pulse, of which we shall mention only two; namely, the intermittent pulse, due to the 
heart missing a beat every now and then; and the water-hammer pulse, due either to aortic regurgitation or to a loss of elasticity of the arterial walls; either of these circumstances diminishes the

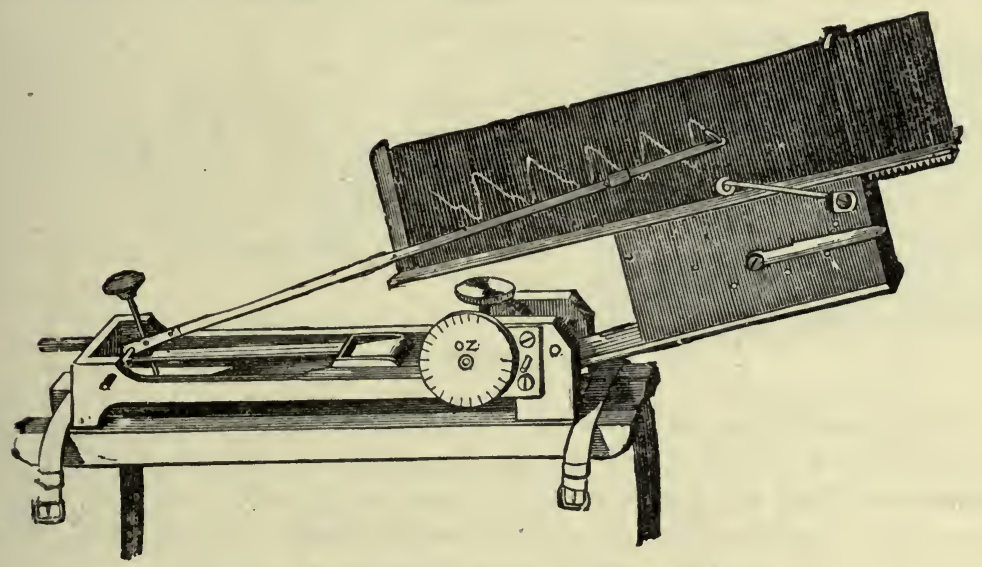

FIG. 255.-Marey's Sphygmograph, modified by Mahomed.

unward flow of blood during the heart's diastole, and thus the suddenness of the impact of the blood on the arterial wall during systole is increased. When this condition is due to arterial disease, such as atheroma or calcification, this sudden pulse, combined with the decreased extensibility of the arteries, may lead to rupture of the walls, and this is especially serious if it occurs in the arteries of the brain (one cause of apoplexy).

In order to study the pulse more fully, it is necessary to obtain

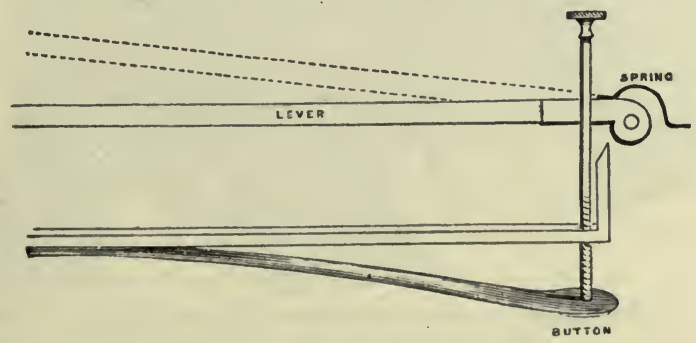

Fra. 256.-Diagram of the lever of the Sphygmograph.

a graphic record of the pulse-beat, and this is accomplished by the use of an instrument called the sphygmograph. This instrument consists of a series of levers, at one end of which is a button placed over the artery; the other end is provided with a writing-point to 
inscribe the magnified record of the arterial movement on a travelling surface.

The instruments most frequently used are those of Marey, ono of the numerous modifications of which is represented in figures 255 , 256 , and 257, and of Dudgeon (fig. 258).

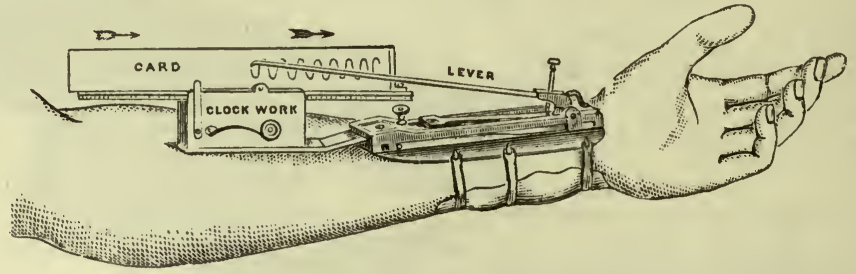

Fia. 257.-The Sphygmograph applied to the arm

Each instrument is provided with an arrangement by which the pressure can be adjusted so as to obtain the best record. The measurement of the pressure is, however, rough, and both instruments have the disadvantage of giving oscillations of their own to the

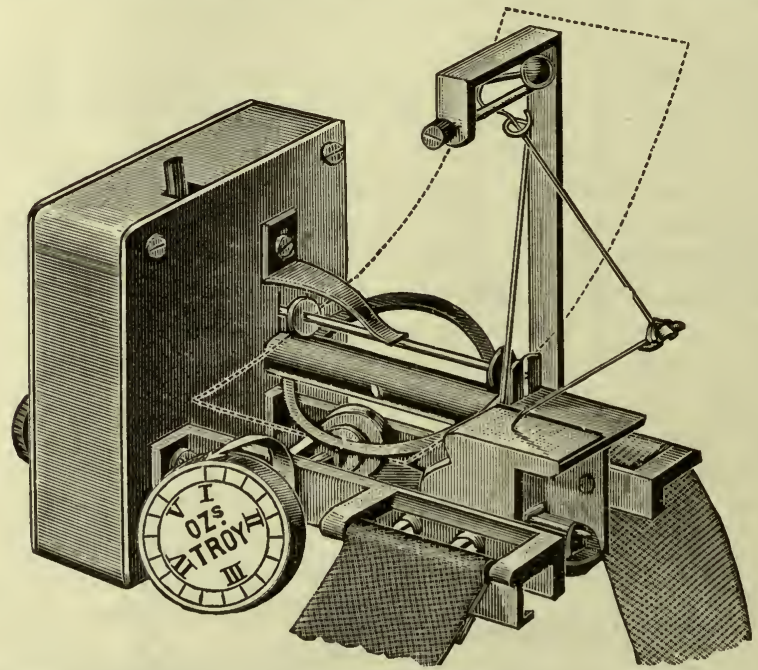

Fig. 258.-Dudgeon's Sphygmograph. The dotted outline represents the piece of blackened paper on which the sphygmogram is written.

sphygmogram; this is specially noticeable in Dudgeon's sphygmograph. But these defects may be overcome by the use of some form of sphygmometer. (See later, p. 295.) It is also important to remember that the pad or button placed upon the artery rests partly on the venæ comites, so that not only arterial tension but any 
turgidity arising from venous congestion, will affect the height and form of the sphygmographic record.

Fig. 259 represents a typical sphygmographic tracing obtained from the radial artery. It consists of an upstroke due to the expansion of the artery, and a downstroke due to its retraction. The descent is more gradual than the upstroke, because the elastic recoil acts more constantly and steadily than the heart-beat. On the descent are several secondary (katacrotic) elevations.

$A$ is the primary, or percussion wave; $\mathrm{C}$ is the pre-dicrotic, or tidal wave; $\mathrm{D}$ is the dicrotic wave, and $\mathrm{E}$ the post-dicrotic wave, and of these

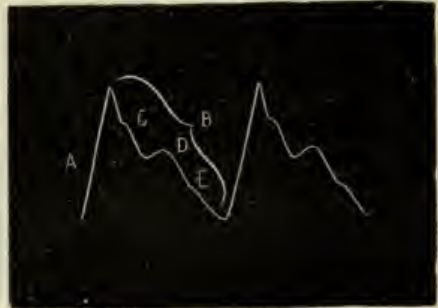

Fia. 259.-Diagram of pulse-tracing. A, upstroke; B, downstroke; C, pre-dicrotic wave; $\mathrm{D}$, dicrotic; $\mathrm{E}$, post-dicrotic wave. there may be several. In some rare cases there is a secondary wave on the upstroke, which is called an anacrotic wave (fig. 260).

These various secondary waves have received different interpretations, but the best way of explaining them is derived from information obtained by taking simultaneous tracings of the pulse, aortic pressure, apex beat, and intraventricular pressure, as in the researches of Hürthle. By this means it is found that the percussion and tidal waves occur during the systole of the heart, and the other waves during the diastole. The closure of the aortic valves occurs just before the dicrotic wave. The secondary waves on the downstroke other than the dicrotic are due to the elastic tension of the

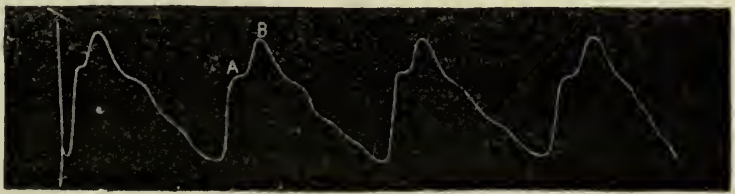

Fic. 260.-Anacrotic pulse.

arteries, and are increased in number when the tension of the arteries is greatest. Some of the post-dicrotic waves are also doubtless instrumental in origin. The dicrotic wave has a different origin. It was at one time thought that this wave was due to a wave of pressure reflected from the periphery, but this view is at once excluded by the fact that wherever we take the pulse-tracing, whether from the aorta, carotid, radial, dorsalis pedis, or elsewhere, this secondary elevation always follows the percussion wave after the same interval, showing that it has its origin in the commencement of the arterial system. 
Moreover, a single pressure-wave reflected from the periphery would be impossible, as such a wave reflected from one part would be interfered with by those froin other parts; moreover, a dicrotic elevation produced by a pressure-wave reflected from the periphery, would be increased by high peripheral resistance, and not diminished, as is actually the case.

The primary cause of the dicrotic wave is the closure of the semilunar valves; as already explained when we were considering the

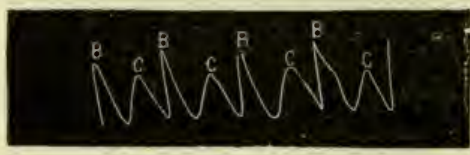

Fig. 261.-Dicrotic pulse velocity pulse (p. 288), the inflow of blood into the aorta suddenly ceases, and the blood is driven back against the closed aortic doors by the elastic recoil of the aorta; tho wave rebounds from these and is propagated through the arterial system as the dicrotic elevation. The production of the dicrotic wave is favoured by a low blood-pressure when the keart is beating forcibly, as in fever. Such a pulse is called a dicrotic pulse (fig. 261), and the second beat can be easily felt by the finger on the radial artery.

The percussion wave is produced by the ventricular systole expanding the artery. The sharp top at its summit is due to the sudden upward spring of the light lever of the sphygmograph. If it were possible to obtain a true record of what really occurs, we should doubtless have a tracing as shown by the continuous line in the accompanying figure (fig. 259). The apex of the tidal wave, B, marks the end of the ventricular systole.

In our study of intracardiac pressure, we saw that the systolic plateau sometimes has an ascending, sometimes a descending, slope (see

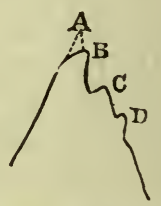

FIG. 262. - Diagram of pulse-tracing; A, percussion; $\mathrm{B}$, tidal ; $\mathrm{C}$, dicrotic ; and $D$, post. dicrotic waves. p. 243); we now come to the explanation of this fact. If after the first sudden rise of pressure in the aorta the peripheral resistance is low, and the blood can be driven on from the aorta more rapidly than it is thrown in, the plateau will sink. If, on the other hand, the peripheral resistance is high, the aortic pressure will rise as long as the blood is flowing in, and we get an ascending systolic plateau and an anacrotic pulse. Thus an anacrotic pulse is seen in Bright's disease, where the peripheral resistance is very high.

If a long pulse-tracing is taken, the effect of the respiration can be seen causing an increase of pressure, and a slight acceleration of the heart's beats during inspiration.

The main waves of the pulse can be demonstrated without the 
use of any instrument at all, by allowing the blood to spurt from a cut artery on to the surface of a large sheet of white paper travelling past it. We thus obtain what is very appropriately called a homautograph (fig. 263).

A distinction must be drawn between the pulse as felt at any one spot in the course of an artery, and the pulse-wave which is propagated throughout the arterial systom. This wave of expansion travels along the arteries, and is started by the propulsion of the contents of the left ventricle into the already full arterial system. The more distant the artery from the heart, the longer the interval that elapses between the ventricular beat and the arrival of the pulse-wave. Thus it is felt in the carotid earlier than in the radial artery, and is still later in the dorsal artery of the foot. The difference of time is, however, very slight; it is only a small fraction of a second; the wave travels at the rate of from 5 to 10 metres a second, that is twenty to thirty times the rate of the blood current.

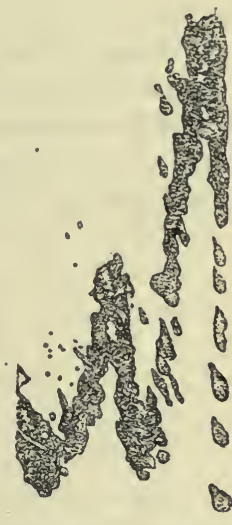

FiG. 263.-Hæmauto. graph, to be read from right to left.

The Rate of Propagation of the Pulse-Wave.-The method of ascertaining this may be illustrated by the use of a long elastic tube into which fluid is forced by the sudden stroke of a pump. If a series of levers are placed along the tube at measured distances, those nearest the pump will rise first, those farthest from it last. If these are arranged to write on a revolving cylinder under one another, this will be shown graphically, and the time-interval between their movements can be measured by a time-tracing. 'The same principle is applied to the arteries of the body; a series of Marey's tambours are applied to the heart and to various arteries at known distances from the heart; their levers are arranged to write immediately under one another, as in fig. 220 , p. 241. The difference in time between the commencement of their upstrokes is measured by a time-tracing in the usual way.

The tracing taken with a sphygmograph is that of the pressure pulse; we may regard it as a blood-pressure tracing without a base line. The actual measurement of the blood-pressure in the human subject is effected by instruments which may be applied to the vessels without any dissection.

These instruments are termed sphygmometers, and the best of them are modifications of one originally introduced by Riva Rocci. C. J. Martin's pattern consists of a four-sided elastic bag about four and a half inches wide, and long enough to encircle the arm. It is wrapped round the arm, and outside of it a cuff of strong canvas is firmly strapped. Air is forced into the bag by a tube leading from a ball syringe; this tube is also connected by a side branch to a mercury manometer. As one continues to pump and distend the bag, the pulse-beats are transmitted to the mercury which is seen to rise in the manometer and oscillate with the pulse-beats. As the 
pressure rises the oscillations become more pronounced, and at a certain point they exhibit a greater excursion than they do at any other height; beyond this point of maximal pulsation, the oscillations diminish in amplitude, and as the distension of the bag is increased still more, the pressure is at last reached, when it is sufficient to obliterate the pulse, and the oscillations of the mercurial column cease, and the pulse is no longer to be felt at the wrist. The pressure necessary to do this is equal to the systolic pressure, and the height of the mercurial column should be noted when the pulse just disappears. The point of maximal pulsation gives a reading of the

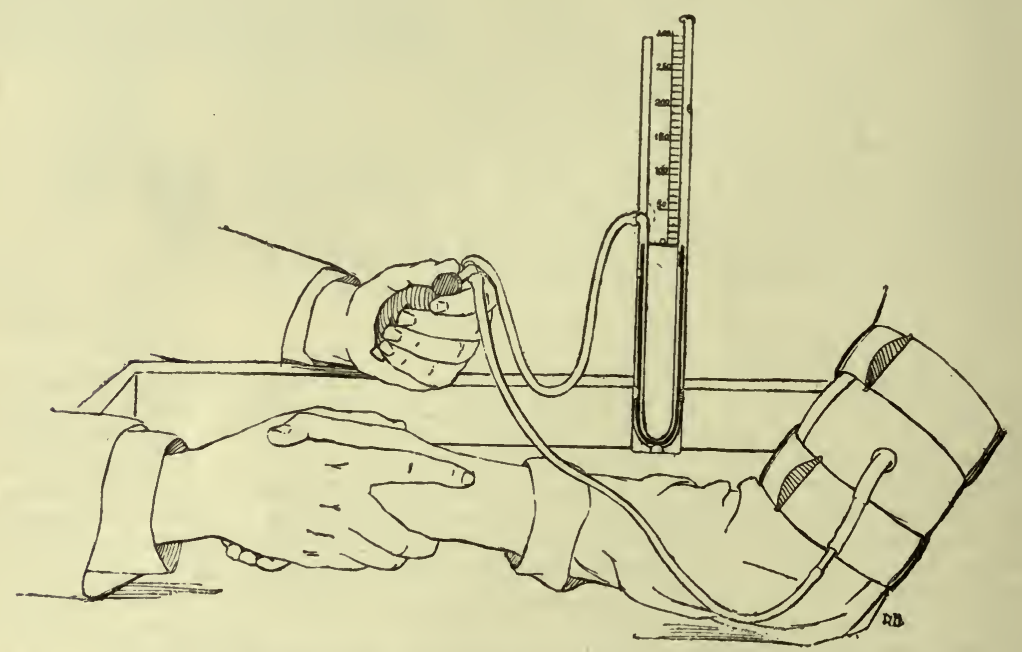

F17. 264.-Martin's Sphygmometer (made by Hawskley, 357 Oxford Street).

diastolic pressure. The systolic pressure is more easily read than the diastolic pressure, for it is by no means easy to judge accurately where the pulsations are greatest. Moreover, the amount of systolic pressure gives one more useful information of the condition of the circulation than does the diastolic pressure.

The normal pressure in the radial artery of healthy young adults is 110 to $120 \mathrm{~mm}$. Hg. It appears to be as constant as the body temperature. In the recumbent posture the pressure is slightly lower than in the erect position. This relation is reversed in conditions of exhaustion. The pressure in the lower limbs is greater than that in the upper limbs in the standing posture owing to the effect of gravity. During muscular exertion the pressure is raised, while in the subsequent period of rest it is subnormal. Mental work raises the pressure; during rest and sleep it is lowered. The taking 
of food produces no noteworthy effect. In disease there are naturally great variations, and the study of these has yielded valuable results.

\section{The Capillary Flow.}

When the capillary circulation is examined in any transparent part of a living animal by means of the microscope the blood is seen to flow with a constant equable motion; the red blood-corpuscles move along, mostly in single file, and bend in various ways to accommodate themselves to the tortuous course of the capillary, but instantly recover their normal outline on reaching a wider vessel.

At the circumference of the stream in the larger capillaries, and in the small arteries and veins, there is a layer of blood-plasma in contact with the walls of the vessel, which moves more slowly than the blood in the centre. Anyone who has rowed on a river will know that the swiftest current is in the middle of the stream. The red corpuscles occupy the middle of the stream and move with comparative rapidity; the colourless corpuscles run much more slowly by the walls of the vessel; while next to the wall there is a transparent space in which the fluid is at comparative rest (the so-called "still layer"); if any of the corpuscles happen to be forced within it, they move more slowly than before, rolling lazily along the side of the vessel, and often adhering to its wall. Sometimes, when the motion of the blood is not strong, many of the white corpuscles collect in a capillary vessel, and for a time entirely prevent the passage of the red corpuscles.

When the peripheral resistance is greatly diminished by the dilatation of the small arteries, so much blood passes on from the arteries into the capillaries at each stroke of the heart, that there is not sufficient remaining in the arteries to distend them. Thus, the intermittent current of the ventricular systole is not converted into a continuous stream by the elasticity of the arteries before the capillaries are reached; and so intermittency of the flow occurs both in capillaries and veins, and a pulse is produced there. The same phenomenon may occur when the arteries become rigid from disease, and when the beat of the heart is so slow or so feeble that the blood at each cardiac systole has time to pass on to the capillaries before the next stroke occurs; the amount of blood sent out at each stroke is then insufficient to properly distend the elastic arteries.

It was formerly supposed that the occurrence of any transudation from the interior of the capillaries into the midst of the surrounding tissues was confined, in the absence of injury, strictly to the fluid part of the blood; in other words, that the corpuscles could not escape from the circulating stream, unless the wall of the containing blood-vessel was ruptured. Augustus Waller affirmed, in 1846, that 
he had seen blood-corpuscles, both red and white, pass bodily through the wall of the capillary vessel in which they were contained; and that, as no opening could be seen before their escape, so none could be observed afterwards - so rapidly was the part healed. But these observations did not attract much notice until the phenomenon was rediscovered by Cohnheim in 1867 .

Cohnheim's experiment was performed in the following manner: A frog is anæsthetised; and the abdomen having been opened, a portion of small intestine is drawn out, and its transparent mesentery spread out under a microscope. After a variable time, occupied by dilatation, following contraction of the minute vessels and accompanying

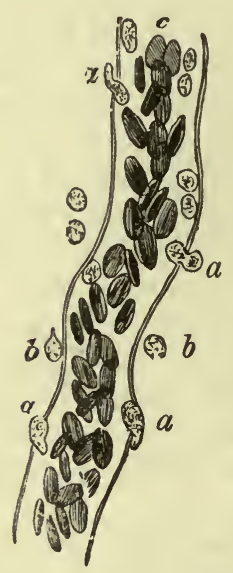

FIG. 265.-A large capillary from the frog's mesentery eight hours after irrita. tion had been set up, showing emigration of leucocytes. $a$, Cells in the act of traversing the capillary wall; $b$, some already escaped. (Frey.) quickening of the blood-stream, there ensues a retardation of the current, and blood-corpuscles begin to make their way through the capillaries and small vessels.

Diapedesis, or emigration of the white corpuscles, occurs to a small extent in health. But it is much increased in inflammation, and may go on so as to form a large collection of leucocytes (i.e. white corpuscles) outside the vessels.

The emigration of red corpuscles is only seen in inflammation, and is a passive process; it occurs when the holes made by the emigrating leucocytes do not close up immediately, and so the red corpuscles escape too.

The real meaning of the process of inflammation is a subject which is being much discussed now, but it may be interesting to state briefly the views of Metschnikoff, who has in recent years been a prominent investigator of the subject. Even if these views do not represent the whole truth, it can hardly be doubted that the phenomena described play a very important part in the process. Metschnikoff teaches that the vascular phenomena of inflammation have for their object an increase in the emigration of leucocytes, which have the power of devouring the irritant substance, and removing the tissues killed by the lesion. They are therefore called phagocytes (devouring or scavenging corpuscles). It may be that the microbic influence, or the influence of the chemical poisons they produce, is too powerful for the leucocytes; then they are destroyed, and the dead leucocytes become pus corpuscles; but if the leucocytes are successful in destroying the foreign body, micro-organisms, and disintegrated tissues, they disappear, wandering back to the blood-vessels, and 
the lost tissue is replaced by a regeneration of the surrounding tissues.*

The circulation through the capillaries must, of necessity, be largely influenced by that which occurs in the vessels on either side of them in the arteries or the veins; their intermediate position causes them to feel at once any alteration in the size, rate, or pressure of the arterial, and more especially of the venous blood-stream. The apparent contraction of the capillaries, on the application of certain irritating substances, and during fear, and their dilatation in blushing, may be referred primarily to the action of the small arteries.

\section{The Venous Flow.}

The blood-current in the veins is maintained primarily by the vis a tergo, that is, the force behind, which is the blood-pressure transmitted from the heart and arteries; but very effectual assistance to the flow is afforded by the action of the muscles capable of pressing on the veins with valves, as well as by the suction action of the heart, and the aspiratory action of the thorax (vis a fronte).

The effect of muscular pressure upon the circulation may be thus explained. When pressure is applied to any part of a vein and the current of blood in it is obstructed, the portion behind the seat of pressure becomes swollen and distended as far back as the next pair of valves, which are in consequence closed (fig. 202, B, p. 220). Thus, whatever force is exercised by the pressure of the muscles on the veins, is distributed partly in pressing the blood onwards in the proper course of the circulation, and partly in pressing it backwards and closing the valves behind.

The circulation might lose as much as it gains by such an action, if it were not for the numerous communications which the veins make with one another; through these, the closing up of the venous channel by the backward pressure is prevented from being any serious hindrance to the circulation, since the blood, the onward course of which is arrested by the closed valves, can at once pass through some anastomosing channel, and proceed on its way by another vein. Thus, the effect of muscular pressure upon veins which have valves, is turned almost entirely to the advantage of the circulation.

In the web of the bat's wing, the veins are furnished with valves, and possess the remarkable property of rhythmical contraction and dilatation, whereby the current of blood within them is distinctly accelerated (Wharton Jones). The contraction occurs, on an average,

* This question is closely related to that of immunity, which is discussed in the chapter on the Blood (Chapter XXIX.). 
about ten times in a minute; the existence of valves prevents regurgitation, so the entire effect of the contractions is auxiliary to the onward current of blood. Analogous phenomena are occasionally found in other animals.

A venous pulse is observed under the conditions previously described (p. 297), when the arterioles are dilated so that the arterial pulse passes through the capillaries to the veins.

A venous pulse is also seen in the superior and inferior vena cava near to their entrance into the heart; this corresponds to variations of the pressure in the right auricle. When the ventricle is contracting there is a slow rise, due to the fact that the blood cannot get into the ventricle, and so distends the auricle; a second short, sharp elevation of pressure is produced by the auricular systole. Alterations of venous pressure are also produced in the great veins by the respiratory movements, the pressure sinking during inspiration, and rising during expiration.

\section{The Vaso-Motor Nervous System.}

The vaso-motor nervous system consists of the vaso-motor centre situated in the bulb, of certain subsidiary vaso-motor centres in the spinal cord, and of vaso-motor nerves, which are of two kinds-(a) those the stimulation of which causes constriction of the vessels; these are called vaso-constrictor nerves; $(b)$ those the stimulation of which causes dilatation of the vessels; these are called vaso-dilator nerves.

The muscular structure of arteries was first described by Henle in 1841 ; but it was not until twelve years later that the nerves supplying this muscular tissue were discovered. The names of Claude Bernard, Brown-Séquard, and Schiff are specially connected with this discovery.

These nerves exert their most important action in the vessels which contain relatively the greatest amount of muscular tissue, namely, the small arteries or arterioles.

Under ordinary circumstances, the arterioles are maintained in a state of moderate or tonic contraction, and this constitutes the peripheral resistance, the use of which is to keep up the arterial pressure, which must be high enough to force the blood through the capillaries and veins in a continuous stream back to the heart.

Another function which is served by this muscular tissue is to regulate the amount of blood which flows through the capillaries of any organ in proportion to its needs. During digestion, for instance, it is necessary that the digestive organs should be supplied with a large quantity of blood: for this purpose the arterioles of the splanchnic area are relaxed, and there is a vast amount of blood in this 
area, and therefore a correspondingly small amount in other areas, such as the skin; this accounts for the sensation of chilliness experienced after a full meal. The skin vessels form another good example; one of the most important uses of the skin is to get rid of the heat of the body in such a way that the body temperature shall remain constant; when excess of heat is produced there is also an increase in the loss of heat; the skin vessels are then dilated, and so more blood is exposed on the surface, and thus an increase in the radiation of heat from the surface is brought about. On the other hand, when it is necessary that the heat produced should be kept in the body, the loss of heat is diminished by a constriction of the skin vessels, as in cold weather. The alteration of the calibre of the vessels is brought about by the action of the vaso-motor nervous system on the muscular tissue of the arterioles.

There are certain organs of the body in which the necessity for alterations in their blood-supply does not exist. Such organs are, heart itself, the lungs, and the brain. It is in the vessels of these organs that the influence of vaso-motor nerves is at a minimum. The pulmonary vessels are stated by Bradford and Dean to be supplied by nerves which leave the cord in the upper thoracic region; but on stimulating these the rise of pressure produced is extremely small; it is very doubtful if the fibres in question are really vasoconstrictors; the small rise observed may be partly or even wholly due to the acceleration of the heart, which is another result of stimulating these nerve-roots.

The vaso-motor centre lies in the grey matter of the floor of the fourth ventricle; it is a few millimetres in length, reaching from the upper part of the floor to within about $4 \mathrm{~mm}$. of the calamus scriptorius. The position of this centre has been discovered by the following means: when it is destroyed the tone of the small vessels is no longer kept up, and in consequence there is a great and universal fall in arterial blood-pressure; when it is stimulated there is an increase in the constriction of the arterioles all over the body, and therefore a rise of arterial blood-pressure. Its upper and lower limits have been determined in the following way: a series of animals is taken, and the central nervous system divided in a different place in each; the cerebrum and cerebellum may be cut off without affecting blood-pressure, the vaso-motor centre must therefore be below these; if the section is made just above the medulla, the blood-pressure still remains high, and it is not till the upper limit of the centre is passed that the blood-pressure falls. Similarly, in another series of animals, if the cervical cord is cut through, and the animal kept alive by artificial respiration, there is an enormous fall of pressure due to the influence of the centre being removed from the vessels; in other experiments the section is made 
higher and higher, and the same result noted, until at last the lower limit of the centre is passed, and the fall of pressure is less and less marked the higher one goes there, until in the animal in which the section is made at the upper boundary of the centre the bloodpressure is not affected at all, and the centre can be influenced reflexly by the stimulation of afferent nerves, the pressor and depressor nerves, which we shall be considering immediately.

After the destruction of the vaso-motor centre in the bulb, there is a fall of pressure. If the animal is kept alive, the vessels after a time recover their tone, and the arterial pressure rises; it rises still more on stimulating the central end of a sensory nerve; this is due to the existence of subsidiary vaso-motor centres in the spinal cord; for on the subsequent destruction of the spinal cord the vessels again lose their tone and the blood-pressure sinks.

The exact position of the vaso-motor centre in the bulb is far from clear; there is no special group of cells there which an anatomist can point to as exercising this function, in the same way as he can point to the respiratory or the cardioinhibitory centre. Possibly the cells are scattered over a large area and do not occur in definite groups.

The fibres that leave these cells to pass down the spinal cord probably travel along the lateral columns; but here again exact information is lacking, and we do not know whether or not they decussate in the bulb or elsewhere. They terminate by arborising around the cells in the grey matter of the subsidiary vaso-motor centres, the anatomical position of which is probably in the cells of the intermedio-lateral tract. From these cells fresh axis-cylinder processes originate, which pass out as the small medullated nervefibres in the anterior roots of the spinal nerves.

The general arrangement of the vaso-motor nerves will have been already gathered from our description of the Autonomic Nervous System (Chapter XVII.); but we may briefly recapitulate the main facts.

The vaso-constrictor nerves for the whole body leave the spinal cord by the anterior roots of the spinal nerves from the second thoracic to the second lumbar, both inclusive. They leave the roots by the white rami communicantes, and pass into the ganglia of the sympathetic chain, which lies on each side of the vertebral column. That is to say, the small medullated or pre-ganglionic nerve-fibres terminate by arborising around the cells of these ganglia, and a fresh relay of axis-cylinder processes from these cells carry on the impulses.

Those which are destined for the supply of the vessels of the head pass into the ganglion stellatum or first thoracic ganglion, thence through the annulus of Vieussens to the inferior cervical ganglion, and thence along the sympathetic trunk to their destination. Their cell-station is in the superior cervical ganglion. 
The new fibres which arise in the ganglia are-usually non-medullated, and are termed post-ganglionic. Those for the body wall and limbs pass back from the sympathetic ganglia to the spinal nerves by the grey rami communicantes, and are distributed with the other spinal nerve-fibres. The cell-stations for the upper limb fibres are in the ganglion stellatum, and for the lower limb fibres in the lower lumbar and upper sacral ganglia.

Those for the interior of the body pass into the various plexuses of sympathetic nerves in the thorax and abdomen, and are distributed to the vessels of the thoracic and abdominal viscera. This set includes the most important vaso-motor nerves of the body, the splanchnics. Their cell-stations are situated in the various collateral ganglia.

The vaso-dilator nerves have been stated to accompany those just described, but they are not limited to the outflow from the second thoracic to the second lumbar. Thus, the nervi erigentes originate as white rami communicantes from the second and third sacral nerves, and the chorda tympani, another good example of -a vaso-dilator nerve, is a branch of the seventh cranial nerve.

Our knowledge of vaso-dilator nerves is limited, except in such instances as the two nerves just mentioned. Equally deficient is our information concerning vaso-dilator centres in the central nervous system. W. M. Bayliss, in his search for vaso-dilator fibres in the dog, was not successful in finding any for the hind limb in the abdominal sympathetic chain; but the only fibres, excitation of which produced vascular dilatation there, are contained in the posterior roots. He also found fibres in the posterior roots of the 12th and 13th thoracic nerves, which act as vaso-dilators of the small intestine. Not only is vaso-dilatation the result of mechanical, or electrical stimulation of these roots, but experiments are adduced which show that in normal reflexes, such as occur when the depressor nerve is stimulated, the dilator impulses travel by the same route. This raises the question whether the posterior roots contain true efferent fibres. The facts of degeneration show that they do not. Bayliss is therefore driven to the conclusion that the same nerve terminations in the periphery serve both to take up sensory impressions and to convey inhibitory impulses to the muscular structures in which they end. In other words, we have here another example which may be added to those previously mentioned (p. 160), that nerve-fibres may convey impulses in both directions. The term antidromic is used by Bayliss to express the fact that impulses may travel in the reverse direction to that in which they usually pass.

The Vaso-motor centre can be excited directly by induction currents; the result is an increase of arterial blood-pressure owing to an increase of the contraction of the peripheral arterioles. 


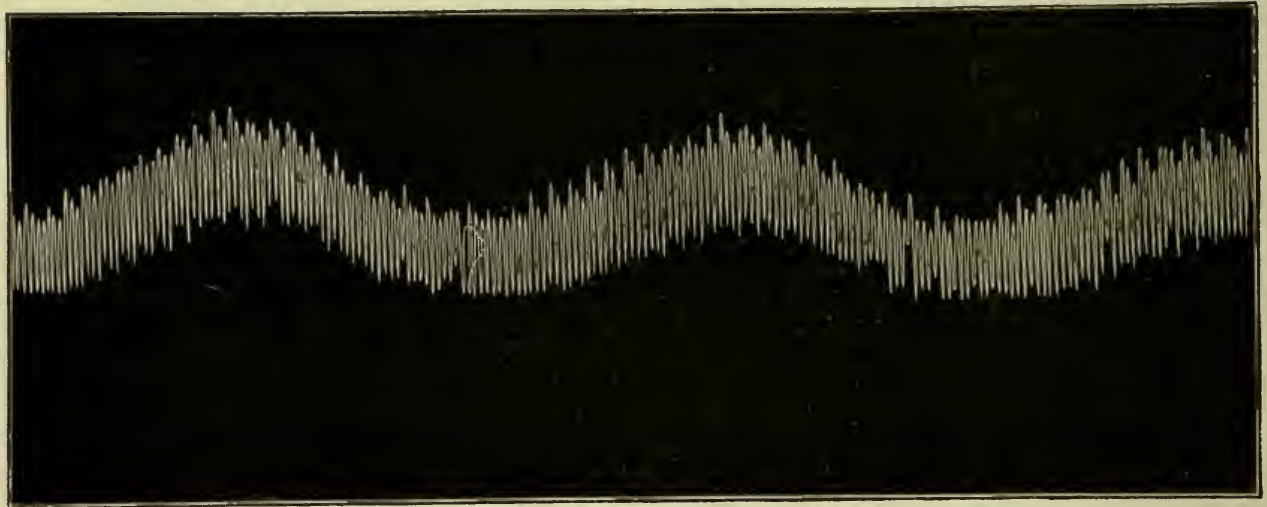

FIG. 266.-Arterial blood-pressure tracing from dog showing Alayer waves. (Sherrington.)

B.P.

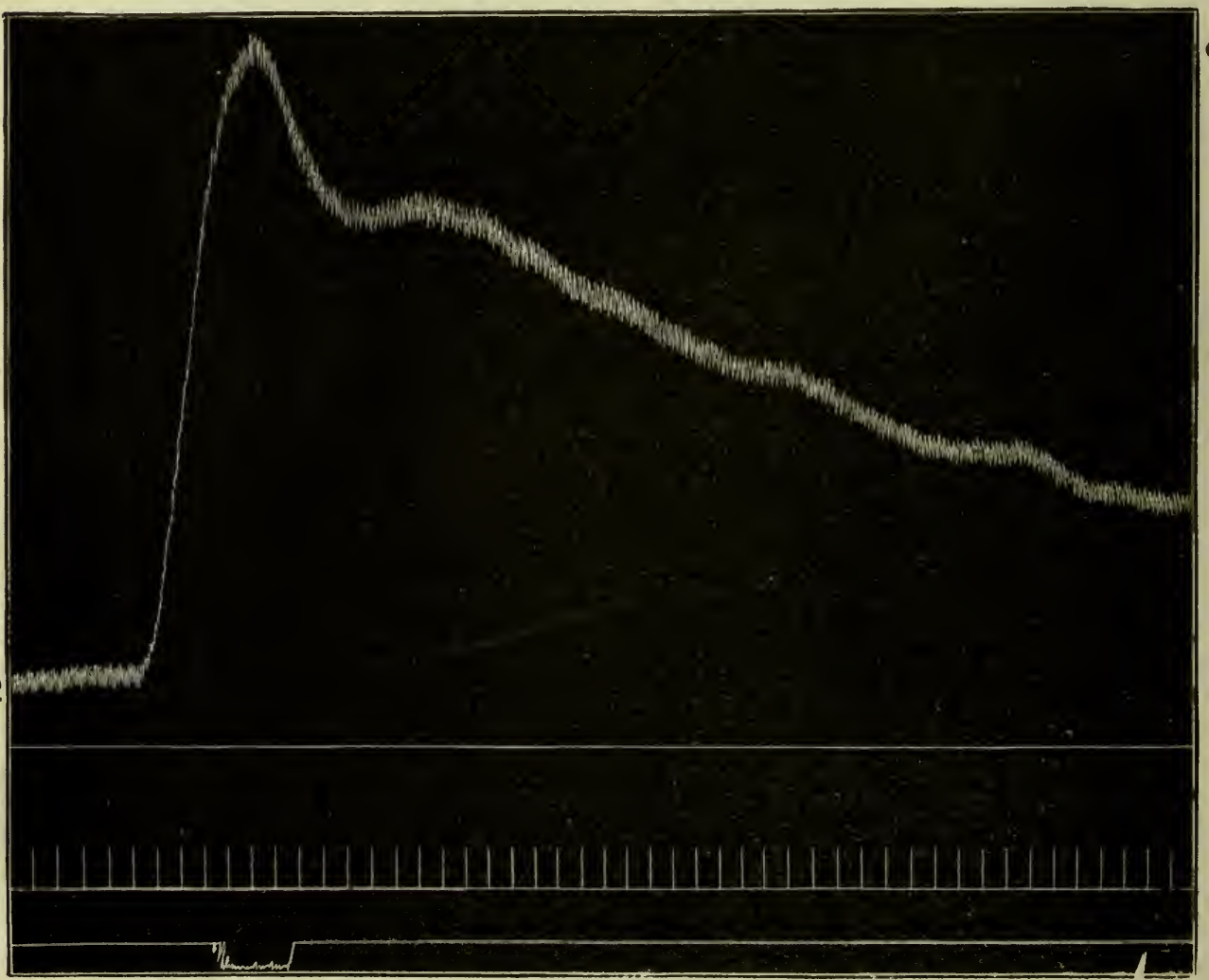

Fic. 267.-Rise in arterial blood-pressure produced by stimulating the central end of a sensory nerve (external propliteal) in a cat under the influence of morphine and curare. BP, blood-pressure; A, abscissa or base-line; $T$, time intervals of 5 seconds; $\mathbf{E}$, signal line, the lowering of which indicates the period of stimulation of the nerve. The size of the figure is slightly reduced in reproduction. (Sherrington.) 
It can also be excited by the action of poisons in the blood which circulates through it; thus, strophanthus or digitalis causes a marked rise of general arterial pressure due to the constriction of the peripheral vessels brought about by impulses from the centre.

It is also excited by venous blood, as in asphyxia; the rise of blood-pressure which occurs during the first part of asphyxia is due to constriction of peripheral vessels; the fall during the last stage of asphyxia is largely due to heart failure. When asphyxia is brought about by the cessation of artificial respiration in a curarised animal, waves are often observed on the blood-pressure curve synchronous with the normal rate of respiration. The respiratory centre is making ineffectual efforts to produce breathing movements, and thus it affects its neighbour, the vaso-motor centre, in a parallel manner. Such waves are known as Traube-Hering wavcs. One, however, frequently sees in tracings of blood-pressure in anæsthetised animals larger waves which arise from a slow rhythmic action of the vasomotor centre, and which are much slower in their rhythm than those due to respiration. Fig. 266 represents a tracing from a dog which shows these waves (Mayer's uaves). The tracing shows three sets of waves, first the oscillations due to the heart-beats, next in size those produced by the respiratory movements, which in their turn are superposed on the prolonged Mayer waves.

The Vaso-motor centre may be excited reflexly.-The afferent impulses to the vaso-motor centre may be divided into pressor and depressor.

Most sensory nerves are pressor nerves. The sciatic or the vagus nerves may be taken as instances; when they are divided and their central ends stimulated, the result is a rise of blood-pressure due to the stimulation of the vaso-motor centre, and a consequent constriction of the arterioles all over the body, but especially in the splanchnic area. Fig. 267 shows the result of such an experiment. It is necessary in performing the experiment to administer curare as well as an anæsthetic to the animal, in order to obviate reflex muscular struggles, which would themselves produce a rise in arterial pressure.

Many sensory nerves also contain depressor fibres; these produce the opposite effect. The most marked bundle of these is known as the depressor nerve. In most animals this is bound up in the trunk of the vagus; but in some, such as the rabbit, cat, and horse, the nerve runs up as a separate branch from the heart (or, according to some recent observations, from the commencement of the aorta), and joins the vagus or its superior laryngeal branch, and ultimately reaches the vaso-motor centre. When this nerve is stimulated (the vagi having been previously divided to prevent reflex inhibition of the heart), a marked fall of arterial blood-pressure is produced (see fig. 268). 
Stimulation of this nerve affects the vaso-motor centre in such a way that the normal constrictor impulses that pass down the vaso-constrictor nerves are inhibited. The fall of pressure is very slight after section of the splanchnic nerves, showing that the splanchnic area is the part of the body most affected. The normal function of this

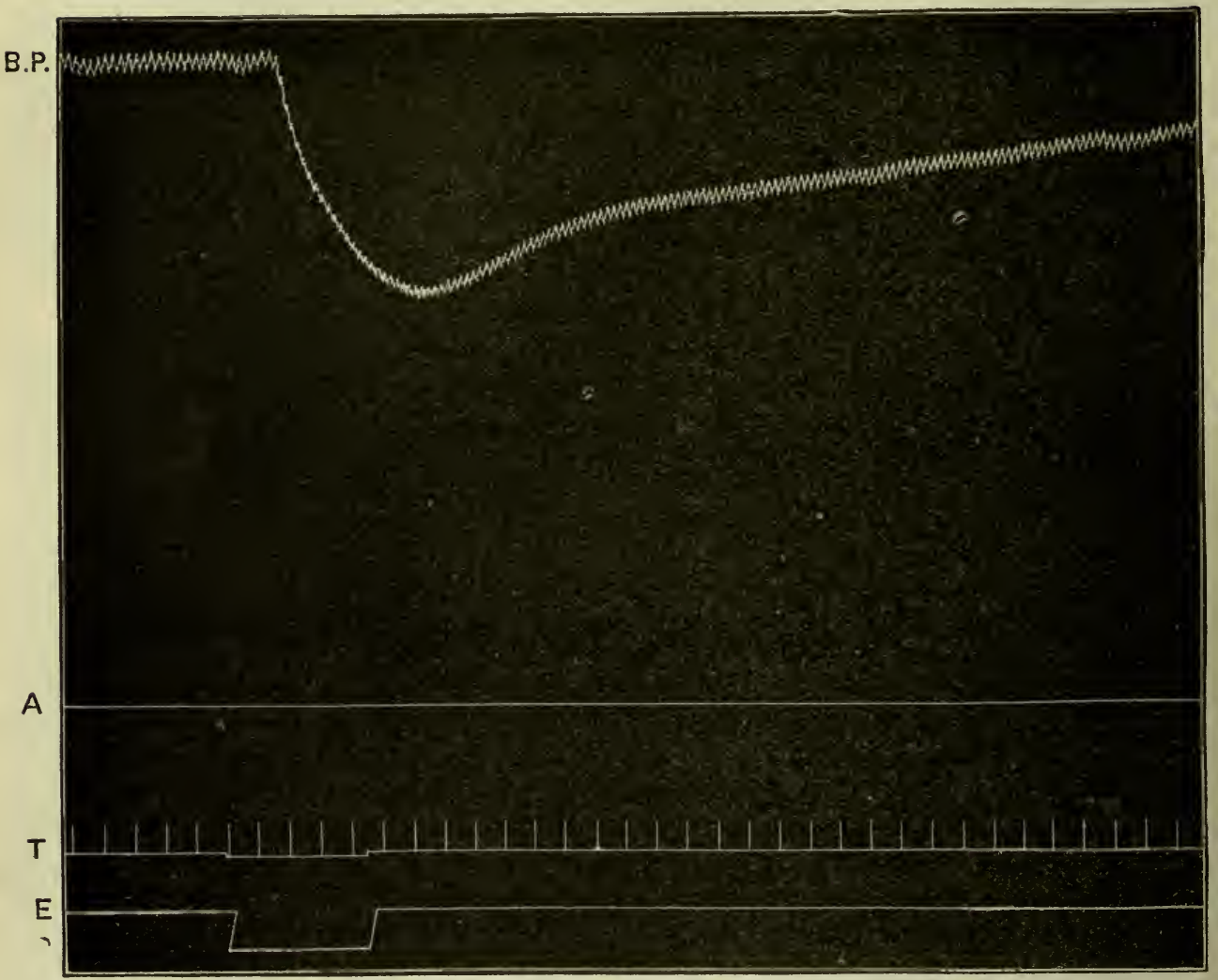

FIG. 263.-Tracing of arterial blood-pressure showing the effect of stimulating the central end of the Depressor nerve in a cat. The letters prefixed to the vartous lines have the same meaning as in fig. 267. (Sherrington.)

nerve is to adapt the peripheral resistance to the heart's action: if the constriction of the arterioles is too high for the heart to overcome, an impulse by this nerve to the vaso-motor centre produces reflexly a lessening of the peripheral resistance.

N.B.-The term depressor should be carefully distinguished from inhibitory; stimulation of the peripheral end of the vagus produces a fall of blood pressure due to inhibition (slowing or stoppage) of the heart (see figs. 24.6 and 247); stimulation of the central end of the 
depressor nerve produces a lowering of blood-pressure for a different reason, namely, a reflex relaxation of the splanchnic arterioles.

Experiments on Vaso-motor nerves. - The experiments on the vaso-motor nerves are similar to those performed on other nerves when one wishes to ascertain their functions. They consist of section and excitation.

Section of a vaso-constrictor nerve, such as the splanchnic, causes a loss of normal arterial tone, and consequently the part supplied by the nerve becomes flushed with blood. Stimulation of the peripheral end causes the vessels to contract and the part to become comparatively pale and bloodless. This can be very readily demonstrated on the ear of an anæsthetised rabbit. This is a classical experiment associated with the name of Claude Bernard. Division of the cervical sympathetic produces an increased redness of the side of the head, and looking at the ear, the transparency of which enables one to follow the phenomena easily, the central artery with its branches is seen to become larger, and many small branches not previously visible come into view. The ear feels hotter, though this effect soon passes off as the exposure of a large quantity of blood to the air causes a rapid loss of heat. On stimulating the peripheral end of the cut nerve, the ear resumes its normal condition, and then becomes paler than usual owing to excessive constriction of the vessels.

Section of a vaso-dilator nerve, such as the chorda tympani, produces no effect on the vessels, but stimulation of its peripheral end causes great enlargement of all the arterioles, so that the submaxillary gland and the neighbouring parts supplied by the nerve become red and gorged with blood, and the pulse is propagated through to the veins; the circulation through the capillaries may be so rapid that the blood is arterial in colour in the veins. Another effect, free secretion of saliva, we shall study in connection with that subject.

Other examples of vaso-dilator nerves are the nervi erigentes to the erectile tissue of the penis, etc., and of the lingual nerve to the vessels of the tongue.

It is, however, probable that all the vessels of the body receive both constrictor and dilator nerves. But the presence of the latter is difficult to determine unless they are present in excess; if they are not, stimulation affects the constrictors most. The effect of section is also inconclusive; for if a mixed nerve is cut, the only effect observed is a dilatation due to removal of the tonic constrictor influence.

To solve this difficult problem, three methods are in use :-

1. The method of degeneration.-If the sciatic nerve is cut, the vessels of the limb dilate. This passes off in a day or two. If the peripheral end of the nerve is then stimulated, the vessels are dilated, as the constrictor fibres degenerate earliest, and so one gets a result due to the stimulation of the still intact dilator fibres, 
2. The method of slowly interrupted shocks.-If a mixed nerve is stimulated with the usual rapidly interrupted faradic current, the effect is constriction; but if the induction shocks are sent in at long intervals (e.g., at intervals of a second), vaso-dilator effects are obtained. This can be readily demonstrated on the kidney vessels by stimulation of the anterior root of the eleventh thoracic nerve in the two ways just indicated.

By studying the rate of flow of the blood through the submaxillary gland, in which the vaso-constrictor and dilator fibres run separate courses, it has been shown that if both sets of fibres are simultaneously excited, constriction is produced during the stimulation, while marked dilatation follows after the stimulation has ceased. Excitation of the constrictors alone is not followed by dilatation. These results explain the mode of action of slowly interrupted shocks, for with each there will only be a very slight constriction, while the dilator effects which run a much slower course will be summed up to produce a marked effect.

3. The influence of temperature.-Exposure to a low temperature depresses the constrictors more than the dilators. If the leg is placed in ice-cold water, stimulation of the sciatic, even if it has only been recently divided, produces a flushing of the skin with blood.

\section{Plethysmography.}

The action of vaso-motor nerves can be studied in another way than by the use of various forms of manometer, which is the only

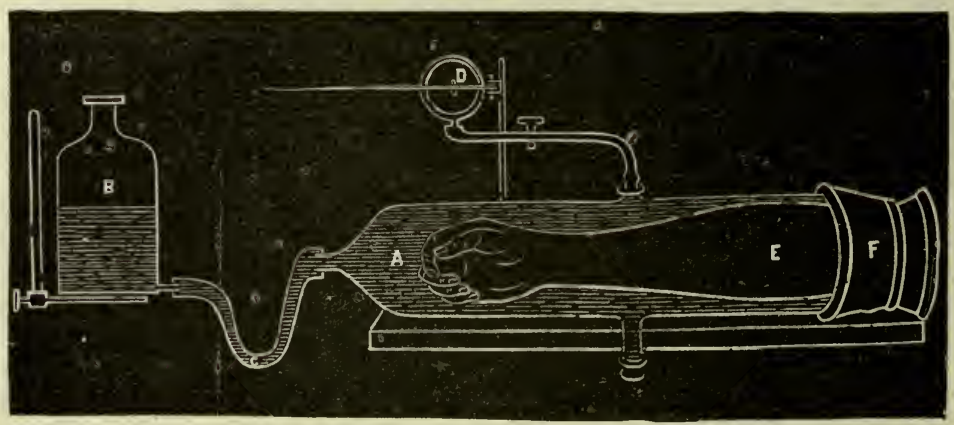

Fia. 269.-Plethysmograph. By means of this apparatus, the alteration in volume of the arm $\mathrm{s}$, which is enclosed in a glass tube A, filled with fluid, the opening through which it passes being firmly closed by a thick gutta-percha band $\mathrm{F}$, is communicated to the lever $\mathrm{D}$, and registered by a recording apparatus. The fluid in A communicates with that in $\mathrm{B}$, the upper limit of which is above that in A. The alterations in volume are due to alterations in the blood contained in the arm. When the volume is increased, fluid passes out of the glass cylinder, and the lever D also is raised, and when a decrease takes place the fluid returns again from $B$ to $A$. It will therefore be evident that the apparatus is capable of recording alterations of the volume of blood in the arm.

method we have considered so far. The second method, which is often used together with the manometer, consists in the use of an instrument which records variations in the volume of any limb, or 
organ of an animal. Such an instrument is called a plethysmograph. One of these instruments applied to the human arm is shown in the accompanying figure (fig. 269).

Every time the arm expands with the heart's systole, a little of the fluid in the plethysmograph is expelled and raises the lever. Variations in volume due to respiration are also seen in the tracing. An air plethysmograph connected to a sensitive recorder gives equally good results.

A study of the volume pulse shows it to possess the same main characters (for instance, a dicrotic wave on the downstroke) which we have already described in connection with the velocity pulse, and the pressure pulse (see p. 288).

When the same method in a modified form is applied to such viscera as the kidney or spleen, the instrument is generally called an oncometer. The earliest oncometers were made by Roy.

Roy's oncometer (figs. 270 and 271) consists of a metal capsule, of shape suitable to enclose the organ: its two halves are jointed together, and fit accurately except at one opening which is left for the vessels of the organ. A delicate membrane is attached to the rim of each half, the space between which and the metal is filled with warm oil. The tube from the oncometer is connected to the oil-containing cavity of the recorder by a tube also containing oil. An increase in the volume of the organ squeezes the oil out of the oncometer into the recorder, and so produces a rise of its piston and lever; a contraction of the organ produces a fall of the lever.

These elaborate instruments have now been entirely superseded by air oncometers, and Schäfer was the first to employ an air oncometer in his work on the spleen.

If now we are investigating the action of the anterior root of eleventh thoracic nerve on the vessels of the kidney, a tracing is taken simultaneously of the arterial blood-pressure in the carotid, and of the volume of the kidney by the oncometer. On stimulating the nerve rapidly, there is a slight rise of arterial pressure, but a large fall of the recording lever, showing that the kidney has diminished in volume. It is evident that there must be an active contraction of the arterioles of the kidney, causing it to diminish in size, for the blood-pressure tracing (which is taken as a control to be sure the changes are not otherwise produced) shows that there is no failure of the heart's activity to account for it.

We shall return to the subject of the oncometer in connection with the spleen and the kidney. We may, however, say in passing what a very important experimental method plethysmography is becoming. Since the introduction of air oncometers, the method is remarkably easy to apply, and it is now part of the routine practice of physiologists, when they are investigating the action of a drug, 
or of a nerve, on any organ, to record its volume changes by the plethysmographic method. Thus, the salivary glands, lobes of the

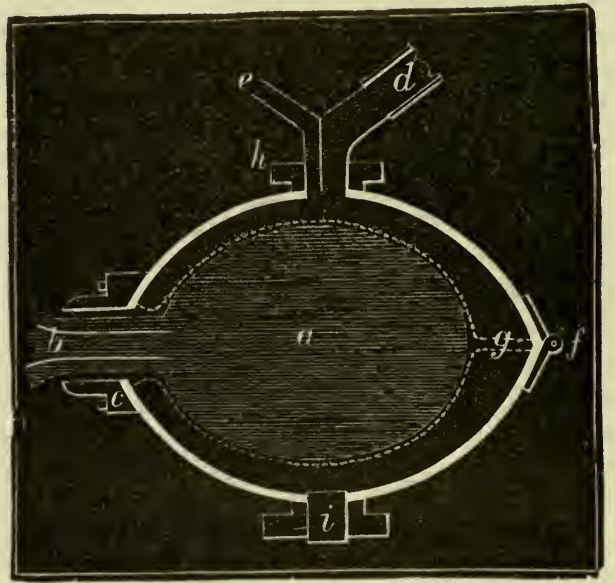

Fig. 270.-Diagram of Roy's Oncometer. a represents the kidney enclosed in a metal box, which opens by hinge $f: b$, the renal vessels and duct. Surrounding the kidney are two chambers formed by membranes, the edges of which are firmly fixed by being clamped between the outside metal capsule, and one (not represented in the figure) inside, the two being firmly screwed together by screws at $h$, and below. The membranous chamber below is filled with a varying amount of warm oil, according to the size of the kidney experimented with, through the opening, then closed with the plug ? After the kidney has been enclosed in the capsule, the membranous chamber above is filled with warm oil through the tube $e$, which is then closed by a tap (not represented in the diagram); the tube $d$ communicates with a recording apparatus, and any alteration in the volume of the kidney is communicated by the oil in the tube to the chamber $d$ of the Oncograph, fig. 271.

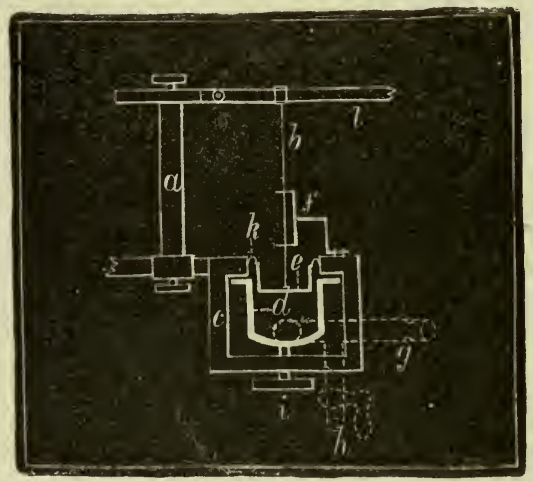

FiG. 271.-Roy's Oncograph, or apparatus for recording alterations in the volume of the kidney, etc., as shown by the oncometer-a, upright, supporting recording lever $l$, which is raised or lowered by needle $b$, which works through $f$, and which is attached to the piston $e$, working in the chamber $d$, with which the tube from the oncometer communicates. The oil is prevented from being squeezed out as the piston descends by a membrane, which is clamped between the ring-shaped surfaces of cylinder by the screw $i$ working upwards; the tube $h$ is for filling the instrument.

liver or lung, the limbs, the kidney, spleen, a coil of intestine, etc., can each be easily enclosed in an appropriately shaped gutta-percha 
box, covered with a glass plate made air-tight with vaseline. There are always two openings to such a box, one to allow the vessels and nerves to enter (leakage of air around these is prevented by packing with cotton-wool soaked in vaseline); the other opening is filled up with a piece of glass tubing which is connected by an india-rubber tube to the recording apparatus (see fig. 272). The most delicate of

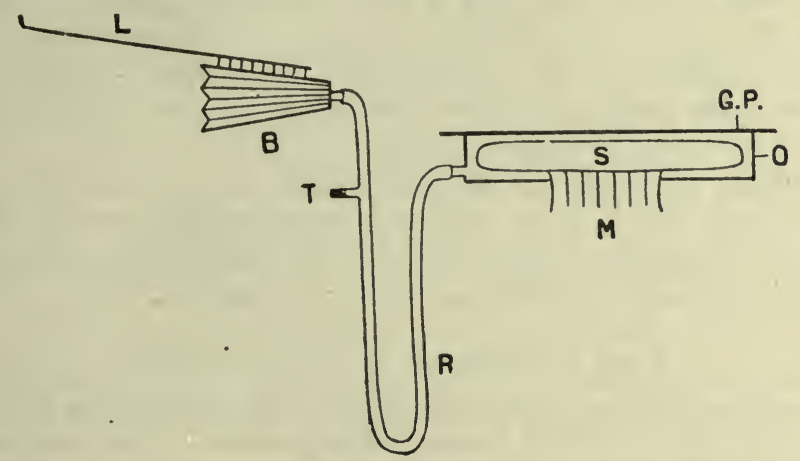

Fı. 272.-Apparatus for obtaining spleuic curves. s, Spleen in oncometer o, which is made of gutta. percha, and covered with a glass plate (G.P.) luted on with vaseline. M is the splenic mesentery containing vessels and nerves; this passes through a slit in the base of the oncometer which is made air-tight with vaseline. The oncometer is connected to the flexible bellows (B) by the india. rubber tube $(R)$, the side tube $(T)$ being closed during an experiment by a piece of glass rod. The recording lever $(\mathrm{L})$ writes on a revolving drum.

the volume recorders is the bellows-recorder of Brodie or the piston recorder of Hürthle.

Of all the oncometers, I am inclined to believe that the intestinal oncometer is the most instructive, because the coil of intestine under observation gives a truer record of what is occurring in that important area called the splanchnic area, than any other abdominal organ.

\section{Pathological Conditions.}

The vaso-motor nervous system is influenced to some extent by conditions of the cerebrum, some emotions, such as fear, causing pallor (vaso-constriction), and others causing blushing (vasodilatation).

It is almost impossible to over-estimate the importance of the study of vaso-motor phenomena as a means of explaining certain pathological conditions; our knowledge of the processes concerned in inflammation is a case in paint.

Disorders of the vessels due to vaso-motor disturbances are generally called angio-neuroses. Of these we may mention the following:-

Tache cérebrale is duo to abnormal sensitiveness of the vascular 
nerves; drawing the finger-nail across the skin causes an immediate wheal, or at least a red mark that lasts a considerable time. At one time this was considered characteristic of affections of the cerebral meninges such as tubercular meningitis, and was consequently called the " meningeal streak." It, however, occurs in a variety of pathological conditions of the nervous system, both cerebral and spinal.

In certain conditions which lead to angina pectoris the pain in the heart is in part due to its being unable to overcome an immense peripheral resistance, and the condition is relieved by the administration of such drugs as amyl-nitrite or nitroglycerin, which relax the vessels and cause universal blushing.

Raynaud's disease is one in which there is a localised constriction of the vessels which is so effectual as to entirely cut off the blood supply to the capillary areas beyond, and if this lasts any considerable time may lead to gangrene of the parts in question.

\section{Local Peculiarities of the Circulation.}

The most remarkable peculiarities attending the circulation of blood through different organs are observed in the cases of the brain, erectile organs, lungs, liver, spleen, and kidneys.

In the Brain.-The brain must always be supplied with blood, for otherwise immediate loss of consciousness would follow. Hence, to render accidental obliteration almost impossible, four large arteries are supplied to the brain, and these anastomose together in the circle of Willis. The two vertebral arteries are, moreover, protected in bony canals. Two of the brain arteries can be tied in monkeys, and three or even all four in dogs, without the production of serious symptoms. In the last case enough blood reaches the brain by branches from the superior intercostal arteries to the anterior spinal artery. The sudden obliteration of one carotid artery in man may in some cases produce epileptiform spasms ; the sudden occlusion of both occasions loss of consciousness. Uniformity of supply is further ensured by the arrangement of the vessels in the pia mater, in which, previous to their distribution to the substance of the brain, the large arteries break up and divide into innumerable minute branches ending in capillaries, which, after frequent communication with one another, enter the brain and carry into nearly every part of it uniform and equable streams of blood. The arteries are enveloped in a special lymphatic sheath. The arrangement of the veins within the cranium is also peculiar. The large venous trunks or sinuses are formed so as to be scarcely capable of change of size; and composed, as they are, of the tough tissue of the dura mater, and, in some instances, bounded on one side by the bony cranium, they are not compressible by any force which the fulness of the arteries might exercise through the substance of the brain; nor do they admit of distension when the flow of venous blood from the brain is obstructed. No valves are placed between the vertebral veins and the vena cava; the vertebral veins anastomose with the cerebral sinuses. Hence on squeezing the thorax and abdomen, venous blood can be pressed from those parts out of any opening made into the longitudinal sinus. Expiration acts in the same way; it raises the cerebral venous pressure; if the skull wall is defective the brain expands owing to the distension of its capillaries during the expiratory act. The exposed brain also expands with each systole of the heart. Owing to the fact that the brain lies enclosed in the cranium, the arterial pulse is transmitted through the brain substance to the cerebral veins, and so the blood issues from these in pulses.

Since the brain is enclosed in the rigid cranium the volume of blood in the cerebral vessels cannot alter unless the volume of the other cranial contents alters in the opposite sense.

These conditions of the brain and skull led Monro and Kellie many years ago 
to advance the opinion that the quantity of blood in the brain must be the same at all times. This doctrine has been frequently disputed, and many have advanced the theory that increase or diminution of the blood is accompanied with simultaneous diminution or increase of the cerebro-spinal fluid, so that the contents of the cranium are kept uniform in volume. But the recent work of Leonard Hill has shown that the Monro-Kellie doctrine is in the main true. Histological evidence has recently been obtained of the existence of nerve plexuses round the pial arteries. The arteries are muscular, and the nerves therefore are most probably vaso-motor in function. Experimental evidence so far, however, has not established that the action of these nerves is a marked one; the cerebral circulation passively follows the slightest changes in aortic and, more especially, vena cava pressure, and no active vaso-motor change has been conclusively proved. The velocity of blood-flow through the brain is thus influenced markedly by the condition of the vessels of the splanchnic area. If these are unduly dilated, the bloodflow through the brain may be so reduced as to lead to fainting. Thus, to restore a fainting person the head must be lowered between the knees. Muscular exercise, by returning blood to the heart from the veins of the lower parts of the body, conduces to the maintenance of an efficient cerebral circulation.

It is not the volume of the blood but the velocity of flow which is altered in the brain by changes in the general circulation. The brain with its circulating blood almost entirely fills the cranial cavity in the living animal; that is, there is no more cerebro-spinal fluid there than is sufficient to moisten the membranes. Cerebro-spinal fluid escapes into the lymphatics, and possibly also into the veins, at any pressure above the cerebral venous pressure; the tension of this fluid and the pressure in the veins are therefore always the same. The fluid is secreted by the epithelium covering the vascular fringes of the choroid plexuses in the ventricles of the brain (see p. 172), and is absorbed mainly by the lymphatics. There is not enough of this absorbable fluid present to allow of more than a slight increase of the volume of blood in the brain. If the aortic pressure rises and the vena cava pressure remains constant, the conditions in the brain are as follows :-

More blood in the arteries, less in the veins, increased velocity of flow.

While if the aortic pressure remains constant and the vena cava pressure rises, the conditions are :-

Less blood in the arteries, more in the veins, diminished velocity of flow.

The brain presses against the cranial wall with a pressure equal to that in the cerebral capillaries. A foreign body introduced within the cranium, such as a blood-clot or depressed bone, produces local anæmia of the brain, by occupying the room of the blood. So soon as the capillaries are thus obliterated the pressure is raised to arterial pressure. This local increase of cerebral tension cannot be transmitted by the cerebro-spinal fluid, because this fluid can never be retained in the meningeal spaces at a tension higher than that of the cerebral veins, but is immediately reabsorbed. The anatomical arrangements of the tentorium cerebelli and the falciform ligaments are such as to largely prevent the transmission through the brain-substance of a local increase of pressure. There is complete pressure discontinuity between the cranial and vertebral cavities. The serious results that follow cerebral compression are primarily due to obliteration of the blood-vessels, and consequent anæmia of the brain. A very small foreign body will, if situated in the region of the bulb, produce the gravest symptoms. For the centres which control the vascular and respiratory systems are rendered anæmic thereby. The cerebral hemispheres may, on the other hand, be compressed to a large extent without causing a fatal result. The major symptoms of compression arise as soon as any local increase of pressure is transmitted to the bulb and causes anæmia there.

In Erectile Structures. - The instances of greatest variation in the quantity of blood contained, at different times, in the same organs, are found in certain structures which, under ordinary circumstances, are soft and flaccid, but, at certain times, receive an unusually large quantity of blood, become distended and swollen by it, and pass into the state which has been termed erection. Such structures are the corpora cavernosa and corpus spongiosum of the penis in the male, and the clitoris in the female; and, to a less degree, the nipple of the mammary gland in 
both sexes. The corpus cavernosum penis, which is the best example of an erectile structure, has an external fibrous membrane or sheath ; and from the inner surface of the latter are prolonged numerous fine lamellæ which divide its cavity into small compartments. Within these is situated the plexus of veins upon which the peculiar erectile property of the organ mainly depends. It consists of short veins which very closely interlace and anastomose with each other in all directions, and admit of great variations of size, collapsing in the passive state of the organ, but capable of an amount of dilatation which exceeds beyond comparison that of the arteries and veins which convey the blood to and from them. The strong fibrous tissue lying in the intervals of the venous plexuses, and the external fibrous membrane or sheath with which it is connected, limit the distension of the vessels, and during the state of erection, give to the penis its condition of tension and firmness. The same general condition of vessels exists in the corpus spongiosum urethræ, but around the urethra the fibrous tissue is much weaker than around the body of the penis, and around the glans there is none. The venous blood is returned from the plexuses by comparatively small veins. For all these veins one condition is the same; namely, that they are liable to the pressure of muscles when they leave the penis. The muscles chiefly concerned in this action are the erector penis and accelerator urinæ. Erection results from the distension of the venous plexuses with blood. The principal exciting cause in the erection of the penis is nervous irritation, originating in the part itself, and derived reflexly from the brain and spinal cord. The nervous influence is communicated to the penis by the pudic nerves, which ramify in its vascular tissue; and after their division the penis is no longer capable of erection.

Erection is not complete, nor maintained for any time except when, together with the influx of blood, the muscles mentioned contract, and by compressing the veins, stop the efflux of blood, or prevent it from being as great as the influx.

The circulation in the Lungs, Liver, Spleen, and Kidneys will be described in our study of those organs. 


\section{CHAPTER XXIII}

\section{LYMPH AND LYMPHATIC GLANDS}

As the blood circulates through the capillary blood-vessels, some of its liquid constituents exude through the thin walls of these vessels, carrying nutriment to the tissue elements. This exudation is called lymph; it receives from the tissues the products of their activity, and is collected by the lymph channels, which converge to the thoracic duct-the main lymphatic vessel-and thus the lymph once more re-enters the blood-stream near to the entrance of the large systemic veins into the right auricle.

Lymph is a fluid, which comes into much more intimate relationship with metabolic processes in the tissues than the blood; in fact, there is only one situation - the spleen-where the blood comes into actual contact with the elements-that is, cells, fibres, etc.-of a tissue.

\section{Composition of Lymph.}

Lymph is alkaline; its specific gravity is about 1015, and after it leaves the vessels it clots, forming a colourless coagulum of fibrin. It is like blood-plasma in composition, only diluted so far as its protein constituents are concerned. This is due to the fact that proteins do not pass readily through membranes. The proteins present are called fibrinogen, serum globulin, and serum albumin; these we shall study with the blood-plasma. The salts are similar to those of blood-plasma, and are present in about the same proportions. Chlorides, however, are more abundant in lymph than in blood. The waste products, such as carbonic acid and urea, are also more abundant. The amount of solids dissolved in lymph is about 6 per cent., more than half of which is protein in nature.

When examined with the microscope the transparent lymph is found to contain colourless corpuscles, which are called lymphocytes; these are cells with large nuclei and comparatively little protoplasm. They pass with the lymph into the blood, and constitute there one of the varieties of colourless blood corpuscles.

All the lymphatics pass at some point of their course through 
lymphatic glands, which are the factories of these corpuscles. Lymphocytes also pass into the lymph-stream wherever lymphoid tissue is found, as in the tonsils, thymus, Malpighian bodies of the spleen, Peyer's patches, and the solitary glands of the intestine. The lymph that leaves these tissues is richer in lymph-cells than that which enters them.

When lymph is collected from the thoracic duct after a meal containing fat, it is found to be milky. This is due to the presence in the lymph of minutely subdivided fat particles absorbed from the interior of the alimentary canal. The lymph is then called chyle. The fat particles constitute what used to be called the molecular basis of chyle. If the abdomen is opened during the process of fat absorption, the lymphatics are seen as white lines, due to their containing this milky fluid. They are consequently called lacteals.

The structure and arrangement of the lymphatic vessels are given in Chapter XIX., and we have now to study the structure of

\section{The Lymphatic Glands.}

Lymphatic glands are round or oval bodies varying in size from a hemp-seed to a bean, interposed in the course of the lymphatic

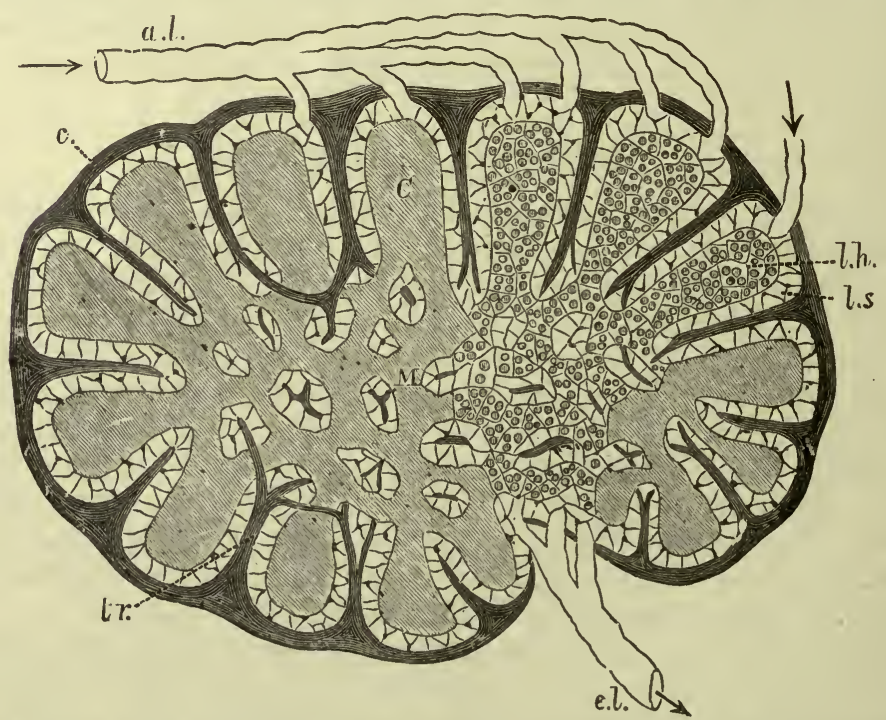

F'I. 273.-Diagrammatic section of lymphatic gland. a.l., Afferent; e.l., efferent lymphatics; $C$, cortical substance; l.h., lymphoid tissue; l.s., lymph-path; c., fibrous capsule sending in trabeculæ $t r$. into the substance of the gland. (Sharpey.)

vessels, and through which the lymph passes in its course to be discharged into the blood-vessels. They are found in great numbers in 
the mesentery, and along the great vessels of the abdomen, thorax, and neck; in the axilla and groin; a few in the popliteal space, but not further down the leg, and in the arm as far down as the elbow.

A lymphatic gland is covered externally by a capsule of connective tissue, generally containing some unstriped muscle. At the inner side of the gland, which is somewhat concave (hilus), the capsule sends inwards processes called trabeculoe in which the bloodvessels are contained, and these join with other processes prolonged from the inner surface of the part of the capsule covering the convex or outer part of the gland; they have a structure similar to that of the capsule, and entering the gland from all sides, and freely communicating, form a fibrous scaffolding. The interior of the gland is seen on section, even when examined with the naked eye, to be made up of two parts, an outer or cortical, which is light coloured, and an inner or medullary portion of redder appearance (fig. 273). In the cuter part, or cortex, of the gland the intervals between the trabeculæ are large and regular; they are termed alveoli; whilst in the more central or medullary part is a finer meshwork formed by an irregular anastomosis of the trabecular processes. Within the alveoli of the cortex and in the meshwork formed by the trabeculæ in the medulla, is contained lymphoid tissue; this occupies

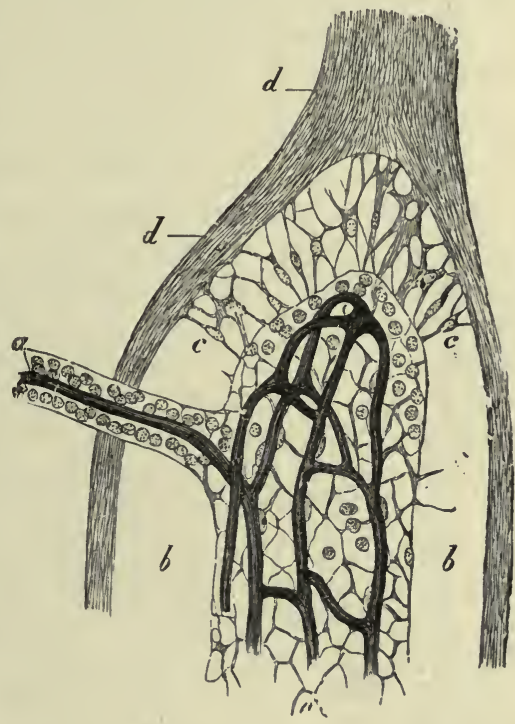

Fig. 274. - A small portion of medullary substance from a mesenteric gland of the ox. $d, d$, Trabeculæ; $a$, part of a cord of lymphoid tissue from which all but a few of the lymph-corpuscles have been washed out to show its supporting meshwork of retiform tissue and its capillary blood-vessels (which have been injected, and are dark in the figure); $b, b$, lymph-path, of which the retiform tissue is represented only at $c, c . \times 300$. (Kölliker.) the central part of each alveolus; but at the periphery, surrounding the central portion and immediately next the capsule and trabeculæ, is a more open meshwork of retiform tissue constituting the lymph-path, and containing but few lymph-corpuscles. At the inner part of the alveolus, the central mass divides into two or more smaller rounded or cord-like masses which, joining with those from the other alveoli, form a much closer arrangement than in the cortex; spaces (fig. 274b), are left within those anastomosing cords, in which are found portions of the trabecular meshwork and the continuation of the lymph-path. 
The lymph enters the gland by several afferent vessels, which pierce the capsule and open into the lymph-path; at the same time they lay aside all their coats except the endothelial lining, which is continuous with the lining of the lymph-path. The efferent vessels begin in the medullary part of the gland, and are continuous with the lymph-path here as the afferent vessels are with the cortical portion.

The efferent vessels leave the gland at the hilus, and either at once, or very soon after, join together to form a single vessel.

Blood-vessels which enter and leave the gland at the hilus are freely distributed to the trabecular and lymphoid tissues.

\section{The Lymph Flow.}

The flow of the lymph towards the point of its discharge into the veins is brought about by several agencies. With the help of the valvular mechanism all occasional pressure on the exterior of the lymphatic and lacteal vessels propels the lymph onward; thus muscular and other external pressure accelerates the flow of the lymph as it does that of the blood in the veins. The action of the muscular fibres of the small intestine, and the layer of unstriped muscle present in each intestinal villus, assist in propelling the chyle; in the small intestine of many animals the chyle has been seen moving with intermittent propulsions that correspond with the peristaltic movements of the intestine. For the general propulsion of the lymph and chyle, it is probable that, in addition to external pressure, some of the force is derived from the contractility of the vessel's own walls. The respiratory movements, also, favour the current of lymph through the thoracic duct as they do the current of blood in the thoracic veins.

Lymph-Hearts. - In amphibia, reptiles and some birds, an important auxiliary to the movement of the lymph and chyle is supplied in certain muscular sacs, named lymph-hearls, and it has been shown that the caudal heart of the eel is a lymphheart also. The number and positions of these organs vary. In frogs and toads, there are usually four, two anterior and two posterior. Into each of these cavities several lymphatics open, the orifices of the vessels being guarded by valves, which prevent the retrograde passage of the lymph. From each heart a single vessel proceeds, and conveys the lymph directly into the venous system. Blood is prevented from passing into the lymphatic heart by a valve at its orifice.

The muscular coat of these hearts is of variable thickness; in some cases it can only be discovered by means of the microscope ; but in every case it is composed of striped fibres. The contractions of the hearts are rhythmical, occurring about sixty times in a minute. The pulsations of the cervical pair are not always synchronous with those of the pair in the ischiatic region, and even the corresponding sacs of opposite sides are not always synchronous in their action.

Unlike the contractions of the blood-heart, those of the lymph-heart appear to be directly dependent upon a certain limited portion of the spinal cord. For Volkmann found that so long as the portion of spinal cord corresponding to the third vertebra of the frog was uninjured, the cervical pair of lymph-hearts 
continued pulsating after all the rest of the spinal cord and the brain were destroyed ; while destruction of this portion, even though all other parts of the nervous centres were uninjured, instantly arrested the hearts' movements. The posterior, or ischiatic, pair of lymph-hearts were found to be governed, in like manner, by the portion of the spinal cord corresponding to the eighth vertebra. Division of the posterior spinal roots did not arrest the movements; but division of the anterior roots caused them to cease at once.

Innervation of the Thoracic Duct.-By determining the rate of outflow of a fluid at constant pressure passing through the thoracic duct, Camus and Gley have obtained evidence of the presence of nerves, causing both dilatation and constriction of the duct. These are contained in the sympathetic chain below the first thoracic ganglion. The effect of stimulation is principally dilatation.

\section{Relation of Lymph and Blood.}

The volume of blood in the body remains remarkably constant. If the amount is increased by injection of fluids, at first its specific gravity is lessened, but in a short time, often in a few minutes, it returns to the normal. The excess of fluid is got rid of in two ways : (1) by the kidneys, which secrete profusely; and (2) by the tissues, which become more watery in consequence. After the renal arteries are ligatured, and the kidney is consequently thrown out of action, the excess of water passes only into the tissues.

On the other hand, a deficiency of blood (for instance, after hæmorrhage) is soon remedied by a transfer of water from the tissues to the blood through the intermediation of the lymph.

In severe hæmorrhage life has often been saved by transfusion of blood from another person. The transfer of the blood of another animal to the human vascular system is usually dangerous, especially if the blood has been defibrinated, for the serum of one animal is usually poisonous to another, producing various changes, of which a breakdown of the corpuscles (hæmolysis) is the most constant sign. It is, however, quite unnecessary to use blood at all for this. purpose; saline (preferably Ringer's) solution should be used instead.

\section{Formation of Lymph.}

Carl Ludwig taught that the lymph flow is conditioned by two factors : first, differences in the pressure of the blood in the capillaries and of the fluid in the tissue spaces, giving rise to a filtration of fluid through the capillary walls; and secondly, chemical differences between these two Hluids, setting up osmotic interchanges through the wall of the blood-vessel. (See further, next chapter.)

If the lymph is produced by a simple act of filtration, then the amount of lymph must rise and sink with the value of $\mathrm{D}-d ; \mathrm{D}$ representing the capillary blood-pressure, and $d$ the pressure in the tissue spaces.

In support of this mechanical theory, various workers in Ludwig's 
laboratory showed that increased capillary pressure due to obstruction of the venous outflow increases the amount of lymph formed; and that diminution of the pressure in the lymph spaces, by squeezing out the lymph previously contained in them, leads to an increase in the transudation.

On the other hand, there were some facts which could not be well explained by the filtration theory, among which may be mentioned the action of curare in causing an increase of lymph flow.

Heidenhain was the first to fully recognise that the laws of filtration and osmosis as applied to dead membranes may be considerably modified when the membranes are composed of living cells ; and he considered that the formation of lymph is due to the selective or secretory activity of the endothelial walls of the capillaries. This so-called vital action of the endothelial cells is seen in the fact that after the injection of sugar into the blood, in a short time the percentage of sugar in the lymph becomes higher than that in the blood. There must, therefore, be some activity of the endothelial cells in picking out the sugar from the blood and passing it on to the lymph. The excess of chlorides in lymph is also in favour of the same view.

Heidenhain was the inventor of the term lymphagogues (literally, lymph drivers). These are substances like curare, which have a specific action in causing an increased lymph flow. Heidenhain considered that the majority of these act by stimulating the endothelial cells to activity. This conclusion, however, has been subjected to much criticism. In this country the question has been taken up by Starling, who has shown that the influence of vital action is not so marked as Heidenhain supposed it to be, but that most of the phenomena in connection with lymph formation can be explained by the simpler mechanical theory. Starling considers that the amount of lymph produced in any part depends on two factors:-

1. The pressure at which the blood is flowing through the capillaries. Heidenhain took the arterial pressure in his experiments as the measure of the capillary pressure; Starling points out, very justly, that this is incorrect, as there is between the arteries and the capillaries the unknown peripheral resistance in the arterioles.

2. The permeability of the capillary wall. This varies enormously in different regions; it is greatest in the liver, so that an intracapillary pressure which would cause lymph to flow here is without effect on the production of lymph in the limbs. Liver lymph is also richer in protein than lymph from the limbs.

The flow of lymph may therefore be increased in two ways:-

1. By increasing the intracapillary pressure. This may be done locally by ligaturing the veins of an organ; or generally by injecting a large amount of fluid into the circulation, or by the injection of 
such substances as sugar and salt (Heidenhain's second class of lymphagogues) into the blood. These attract water from the tissues into the blood, and thus increase the volume of the circulating fluid and raise the intracapillary pressure.

2. By increasing the permeability of the capillary wall by injuring its vitality. This may be done locally by scalding a part; or generally, by injecting certain poisonous substances, such as peptone, leech extract, decoction of mussels, etc. (Heidenhain's first class of lymphagogues). These act chiefly on the liver capillaries; curare acts chiefly on the limb capillaries. There is no doubt that in pathological conditions which lead to the production of a great increase of lymph (dropsy) this second factor is the more important of the two; the increased permeability of the capillaries may be the result of malnutrition, or due to the action of poisons produced by the disease.

In reference to the action of the endothelial cells, it is necessary to recognise that they are alive, and are therefore capable of exerting a selective action which may mask or counteract or assist purely physical processes. If the action of poisons was simply to injure the vessel wall and increase its permeability, the amount of lymph should be proportional to the intensity of the injury; but this is not always found to be the case. Lymph formation is doubtless mainly influenced by the physical conditions present, for the action of such thin cells as those of the capillary wall cannot be sufficiently great to entirely counteract these conditions; at the same time it is impossible to deny that there is some such action as may be described by the terms "selective" or "secretory." The question is closely related to that of absorption from the alimentary canal, and we shall find in studying that subject that there has been a similar difference of opinion, but that recent research has confirmed the theory of selective activity of the absorptive epithelium.

Dr Martin H. Fischer has advanced a new theory of dropsy or œdema within the last few years. He believes that circulatory conditions are of minor importance, but that the main factor leading to transudation of water into the tissues is to be found in the tissues themselves. He finds that colloids imbibe more water from an acid solution than under other conditions. He therefore believes that it is the accumulation of acid products (such as lactic acid) in the tissues that determines their increased affinity for water, and thus they attract it out of the blood. The theory has met with much adverse criticism, and it is too early at present to state whether Fischer or his critics are correct. 


\section{CHAPTER XXIV}

\section{PHYSICAL CHEMISTRY AND ITS BEARING ON PIIYSIOLOGICAI, PROBLEMS}

THE investigations of physical chemists during recent years have given us new conceptions of the nature of solutions, and these have important bearings on the explanation of osmotic phenomena, and so are interesting to the physiologist.

Water is the fluid in which soluble materials are usually dissolved, and at ordinary temperatures it is a fluid the molecules of which are in constant movement ; the hotter the water the more active are the movements of its molecules, until when at last it is converted into steam, the molecular movements become much more energetic. Perfectly pure water consists of molecules with the formula $\mathrm{H}_{2} \mathrm{O}$, and these molecules undergo practically no dissociation into their constituent ions, and it is for this reason that pure water is not a conductor of electricity.

If a substance like sugar is dissolved in the water, the solution still remains incapable of conducting an electrical current. The sugar molecules in solution are still sugar molecules; they do not undergo dissociation.

But if a substance like salt is dissolved in the water, the solution is then capable of conducting electrical currents, and the same is true for most acids, bases, and salts. These substances do undergo dissociation, and the simpler materials into which they are broken up in the water are called ions. Thus, if sodium chloride is dissolved in water a certain number of its molecules become dissociated into sodium ions, which are charged with positive electricity, and chlorine ions, which are charged with negative electricity. Similarly a solution of hydrochloric acid in water contains free hydrogen ions and free chlorine ions. Sulphuric acid is decomposed into hydrogen ions and ions of $\mathrm{SO}_{4}$. The term ion is thus not equivalent to atom, for an ion may be a group of atoms, such as $\mathrm{SO}_{4}$, in the example just given.

Further, in the case of hydrochloric acid, the negative charge of the chlorine ion is equal to the positive charge of the hydrogen ion; but in the case of the sulphuric acid, the negative charge of the $\mathrm{SO}_{4}$ ion is equal to the positive charge of two hydrogen ions. We can thus speak of monovalent, divalent, trivalent, etc., ions.

Ions positively charged are called kat-ions because they move towards the kathode or negative pole ; those which are negatively charged are called an-ions because they move towards the anode or positive pole. The following are some examples of each class :-

Kat-ions. Monovalent:- $\mathrm{H}, \mathrm{Na}, \mathrm{K}, \mathrm{NH}_{4}$, etc.

Divalent :- $\mathrm{Ca}, \mathrm{Ba}, \mathrm{Fe}$ (in ferrous salts), etc.

Trivalent:- $\mathrm{Al}, \mathrm{Bi}, \mathrm{Sb}, \mathrm{Fe}$ (in ferric salts), etc.

An-ions. Monovalent :- $\mathrm{Cl}, \mathrm{Br}, \mathrm{I}, \mathrm{OH}, \mathrm{NO}_{3}$, etc.

Divalent :-S, $\mathrm{Se}, \mathrm{SO}_{4}$, etc.

Roughly speaking, the greater the dilution the more nearly complete is the dissociation, and in a very dilute solution of such a substance as sodium chloride we may consider that the number of ions is double the number of molecules of the salt present.

The ions liberated by the act of dissociation are, as we have seen, charged with electricity, and when an electrical current is led into such a solution, it is conducted 322 
through the solution by the movement of the ions. Substances which exhibit the property of dissociation are known as electrolytes.

The liquids of the body contain electrolytes in solution, and it is owing to this fact that they are able to conduct electrical currents.

This conception of electrolytes which we owe to Arrhenius is extremely important in view of the question of osmotic pressure, because the act of dissociation increases the number of particles moving in the solution, and so increases the osmotic pressure, for in this relation an ion plays the same part as a molecule.

Another physiological aspect of the subject is seen in a study of the actions of mineral salts in solution on living organisms and parts of organisms. Many years ago Ringer showed that contractile tissues (heart, cilia, etc.) continue to manifest their activity in certain saline solutions.

Loeb and his fellow-workers have confirmed these statements, but interpret them now as ionic action. Contractile tissues will not contract in pure solutions of nonelectrolytes (such as sugar, urea, albumin). But different contractile tissues differ in the nature of the ions which are most favourable stimuli. Thus cardiac muscle, cilia, amœeboid movement, karyokinesis, cell division, are all alike in requiring a proper adjustment of ions in their surroundings if they are to continue to act, but the proportions must be different in individual cases.

Loeb at one time considered that the process of fertilisation was mainly ionic action, but since then he has modified his views; the action of ions is only one of many factors. Howell's work, however, on the action of ions in the causation of the heart-beat (see p. 261) may be taken as one of the best.proved instances of the importance of this branch of study.

Gramme-molecular Solutions. - From the point of view of osmotic pressure a convenient unit is the gramme-molecule. A gramme-molecule of any substance is the quantity in grammes of that substance equal to its molecular weight. A gramme-molecular solution is one which contains a gramme-molecule of the substance per litre. Thus a gramme-molecular solution of sodium chloride is one which contains 58.46 grammes of sodium chloride $(\mathrm{Na}=23 \cdot 00: \mathrm{Cl}=35.46)$ in a litre. A gramme-molecular solution of grape sugar $\left(\mathrm{C}_{6} \mathrm{H}_{12} \mathrm{O}_{6}\right)$ is one which contains 180 grammes of grape sugar in a litre. A gramme-molecule of hydrogen $\left(\mathrm{H}_{2}\right)$ is 2 grammes by weight of hydrogen, and if this was compressed to the volume of a litre, it would be comparable to a gramme-molecular solution. It therefore follows that a litre containing 2 grammes of hydrogen contains the same number of molecules of hydrogen in it as a litre of a solution containing 58.46 grammes of sodium chloride, or one containing 180 grammes of grape sugar, has in it of salt or sugar molecules respectively. To put it another way, the heavier the weight of a molecule of any substance, the more of that substance must be dissolved in the litre to obtain its gramme-molecular solution. Or still another way: if solutions of various substances are made all of the same strength per cent., the solutions of the materials of small molecular weight will contain more molecules of those materials than the solutions of the materials which have heavy molecules. We shall see that the calculation of osmotic pressure depends upon these facts.

Diffusion, Dialysis, Osmosis. - If two gases are brought together within a closed space, a homogeneous mixture of the two is soon obtained. This is due to the movements of the gaseous molecules within the confining space, and the process is called diffusion. In a similar way diffusion will effect in time a homogeneous mixture of two liquids or solutions. If water is carefully poured on to the surface of a solution of salt, the salt or its ions will soon be equally distributed throughout the whole. If a solution of albumin or any other colloidal substance is used instead of salt in the experiment, diffusion will be found to occur much more slowly. If, instead of pouring the water on to the surface of a solution of salt or sugar, the two are separated by a membrane made of such a material as parchment paper, a similar diffusion will occur, though more slowly than in cases where the membrane is absent. In time, the water on each side of the membrane will contain the same quantity of sugar or salt. Substances which pass through such membranes are called crystalloids. Substances which have such large molecules (starch, protein, etc.) that they will not pass through such membranes are called colloids. Diffusion of substances in solution which have to deal with an intervening membrane 
is usually called dialysis. The process of filtration (i.e., the passage of materials througl the pores of a membrane under the influence of mechanical pressure) may

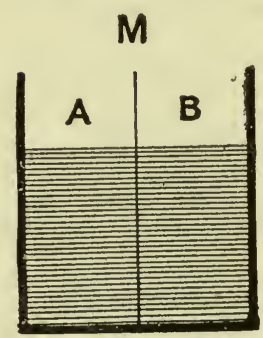

FIG. 275 . be excluded in such experiments by placing the membrane (M) vertically as shown in the diagram (fig. 275), and the two fluids $A$ and $B$ on each side of it. Diffusion through a membrane is not limited to the molecules of water, but it may occur also in the molecules of certain substances dissolved in the water. But very few or no membranes are equally permeable to water and to molecules of the substances dissolved in the water. If in the accompanying diagram the compartment $\mathrm{A}$ is filled with pure water, and B with a sodium chloride solution, the liquids in the two compartments will ultimately be found to be equal in bulk as they were at the start, and each will be a solution of salt of half the strength of that originally in the compartment B. But at first the volume of the liquid in compartment $B$ increases, because more water molecules pass into it from A than salt molecules pass from B to $\mathbf{A}$. The term osmosis is generally limited to the stream of water molecules passing through a membrane, while the term dialysis is applied to the passage of the molecules in solution in the water. The osmotic stream of water is especially important, and in connection with this it is

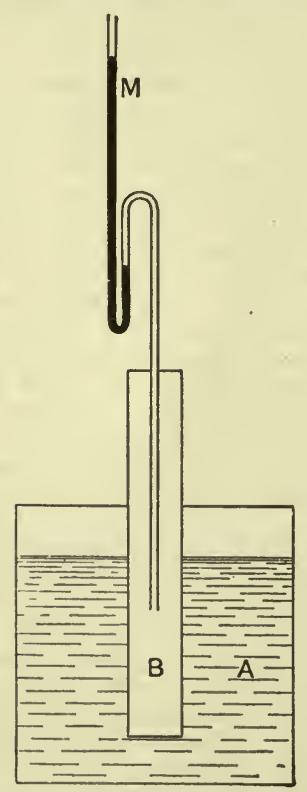

FIG. 270.-A, outer vessel, con. taining distilled water; B, inner semi-permeable vessel, containing 1 per cent. salt solution; $\mathbf{M}$, mercurial manometer. (After Starling.) diffuses in ; and (2) because the membrane of the bladder leaks; that is, permits of filtration when the pressure within it has attained a certain height. first, then, osmosis (the diffusion of water) is more rapid than the dialysis (the diffusion of the salt molecules or ions). The older explanation of this was that salt attracted the water, but we now express the fact differently by saying that the salt in solution exerts a certain osmotic pressure : the result of the osmotic pressure is that more water flows from the water side to the side of the solution than in the contrary direction. The osmotic pressure varies with the amount of substance in solution, and is also altered by variations of temperature occurring more rapidly at high than at low temperatures.

If we imagine two masses of water separated by a permeable membrane, as many water molecules will pass through from one side as from the other, and so the volumes of the two masses of water will remain unchanged. If now we imagine the membrane $M$ is not permeable except to water, and the compartment A contains water, and the compartment B contains a solution of salt or sugar ; under these circumstances water will pass through into $B$, and the volume of $\mathbf{B}$ will increase in proportion to the osmotic pressure of the sugar or salt in solution in $\mathrm{B}$, but no molecules of sugar or salt can get through into A from B, so the volume of fluid in A will continue to decrease, until at last a limit is reached. The determination of this limit, as measured by the height of a column of fluid or mercury which it will support, will give us a measurement of the osmotic pressure.

If a bladder containing strong salt solution is placed in a vessel of distilled water, water passes into the bladder by osmosis, so that the bladder is swollen, and a manometer connected with its interior will show a rise of pressure (osmotic pressure). But the total rise of pressure cannot be measured in this way for two reasons : (1) because the salt diffuses out as the 
It is therefore necessary to use a membrane which will not allow salt to pass out either by dialysis or filtration, though it will let the water pass in. Such membranes are called semi-permeable membranes, and one of the best of these is ferrocyanide of copper. This may be made by taking a cell of porous earthenware and washing it out first with copper sulphate and then with potassium ferrocyanide. An insoluble precipitate of copper ferrocyanide is thus deposited in the pores of the earthenware.

If such a cell is arranged as in fig. 276 , and filled with a 1 per cent. solution of sodium chloride, water diffuses in, till the pressure registered by the manometer reaches the enormous height of $5000 \mathrm{~mm}$. of mercury. If the pressure in the cell is increased beyond this artificially, water will be pressed through the semi-permeable walls of the cell and the solution will become more concentrated.

In other words, in order to make a solution of sodium chloride of greater concentration than 1 per cent., a pressure greater than $5000 \mathrm{~mm}$. of mercury must be employed. The osmotic pressure exerted by a 2 per cent. solution would be twice as great.

Though it is theoretically possible to measure osmotic pressure by a manometer in this direct way, practically it is hardly ever done, and some of the indirect methods of measurement described later are used instead. The reason for this is that it has been found difficult to construct a membrane which is absolutely semipermeable; they are nearly all permeable in some degree to the molecules of the dissolved crystalloid. In course of time, therefore, the dissolved crystalloid will be equally distributed on both sides of the membrane, and osmosis of water will cease to be apparent, since it will be equal in both directions.

Many explanations of the nature of osmotic pressure have been brought forward, but none is perfectly satisfactory. The following simple explanation is perhaps the best, and may be rendered most intelligible by an illustration. Suppose we have a solution of sugar separated by a semi-permeable membrane from water; that is, the membrane is permeable to water molecules, but not to sugar molecules. The streams of water from the two sides will then be unequal; on one side we have water molecules striking against the membrane in what we may call normal numbers, while on the other side both water molecules and sugar molecules are striking against it. On this side, therefore, the sugar molecules take up a certain amount of room, and do not allow the water molecules to get to the membrane; the membrane is, as it were, screened against the water by the sugar, therefore fewer water molecules will get through from the screened to the unscreened side than vice versâ. This comes to the same thing as saying that the osmotic stream of water is greater from the unscreened water side to the screened sugar side than it is in the reverse direction. The more sugar molecules that are present, the greater will be their screening action, and thus we see that the osmotic pressure is proportional to the number of sugar molecules in the solution, that is, to the concentration of the solution.

Osmotic pressure is, in fact, equal to that which the dissolved substance would exert if it occupied the same space in the form of a gas (Van't Hoff's hypothesis). The nature of the substance makes no difference; it is only the number of molecules which causes osmotic pressure to vary. The osmotic pressure, however, of substances like sodium chloride, which are electrolytes, is greater than what one would expect from the number of molecules present. This is because the molecules in solution are split into their constituent ions, and an ion plays the same part as a molecule, in questions of osmotic pressure. In dilute solutions of sodium chloride ionisation is more complete, and as the total number of ions is then nearly double the number of original molecules, the osmotic pressure is nearly double what would have been calculated from the number of molecules.

The analogy between osmotic pressure and the pressure of gases is very complete, as may be seen from the following statements :-

1. At a constant temperature osmotic pressure is proportional to the concentration of the solution (Boyle-Mariotte's law for gases).

2. With constant concentration, the osmotic pressure rises with and is proportional to the temperature (Gay-Lussac's law for gases).

3. The osmotic pressure of a solution of different substances is equal to the sum 
of the pressures which the individual substances would exert if they were alone in the solution (Henry-Dalton law for partial pressure of gases).

4. The osmotic pressure is independent of the nature of the substance in solution, and depends only on the number of molecules or ions in solution (Avogadro's law for gases).

Calculation of Osmotic Pressure. - We may best illustrate this by an example, and to simplify matters we will take an example in the case of a non-electrolyte such as sugar. We shall then not have to take into account any electrolytic dissociation of the molecules into ions. We will suppose we want to calculate the osmotic pressure of a 1 per cent. solution of cane sugar.

One gramme of hydrogen at atmospheric pressure and $0^{\circ} \mathrm{C}$. occupies a volume of 11.2 litres; two grammes of hydrogen will therefore occupy a volume of 22.4 litres. A gramme-molecule of hydrogen-that is, 2 grammes of hydrogen-when brought to the volume of 1 litre, will exert a gas pressure equal to that of 22.4 litres compressed to 1 litre-that is, a pressure of 22.4 atmospheres. A gramme-molecular solution of cane sugar, since it contains the same number of molecules in a litre, must therefore exert an osmotic pressure of 22.4 atmospheres also. A gramme-molecular solution of cane sugar $\left(\mathrm{C}_{12} \mathrm{H}_{22} \mathrm{O}_{11}\right)$ contains 342 grammes of cane sugar in a litre of water. A 1 per cent. solution of cane sugar contains only 10 grammes of cane sugar in a litre; hence the osmotic pressure of a 1 per cent. solution of cane sugar is $\frac{10}{342} \times 22.4$ atmospheres, or 0.65 of an atmosphere, which in terms of a column of mercury $=760 \times 0.65=494 \mathrm{~mm}$.

It would not be possible to make such a calculation in the case of an electrolyte, because we should not know how many molecules had been ionised. In the liquids of the body, both electrolytes and non-electrolytes are present, and so a calculation is here also impossible.

We have seen that for such liquids the osmotic pressure is seldom directly measured by a manometer, because of the difficulty in obtaining perfect semipermeable membranes; we now see that mere arithmetic often fails us; and so we come to the question to which we have been so long leading up, viz., how osmotic pressure is actually determined.

Determination of Osmotic Pressure by means of the Freezing-point. This is the method which is almost universally employed. A very simple apparatus (Beckmann's differential thermometer) is all that is necessary. The principle on which the method depends is the following :-The freezing-point of any substance in solution in water is lower than that of water; the lowering of the freezing-point is proportional to the molecular concentration of the dissolved substance, and that, as we have seen, is proportional to the osmotic pressure.

When a gramme-molecule of any substance is dissolved in a litre of water, the freezing-point is lowered by $1.87^{\circ} \mathrm{C}$., and the osmotic pressure is, as we have seen, equal to 22.4 atmospheres, that is, $22.4 \times 760=17,024 \mathrm{~mm}$. of mercury.

We can, therefore, calculate the osmotic pressure of any solution if we know the lowering of its freezing-point in degrees Centigrade; the lowering of the freezing-point is usually expressed by the Greek letter $\Delta$.

$$
\text { Osmotic pressure }=\frac{\Delta}{1.87} \times 17,024 .
$$

For example, a 1 per cent. solution of sugar would frecze at $-0.052^{\circ} \mathrm{C}$; its osmotic pressure is therefore $\frac{.052 \times 17,024}{1.87}=473 \mathrm{~mm}$., a number approximately equal to that we obtained by calculation.

Mammalian blood serum gives $\Delta=0.56^{\circ} \mathrm{C}$. A 0.9 per cent. solution of sodium chloride has the same $\Delta$; hence serum and a 0.9 per cent. solution of common salt have the same osmotic pressure, or are isotonic. The osmotic pressure of blood serum is $\frac{.56 \times 17,024}{1.87}=5000 \mathrm{~mm}$. of mercury approximately, or a pressure of nearly 7 atmospheres.

The osmotic pressure of solutions may also be compared by observing their effect on red blood-corpuscles, or on vegetable cells such as those in Tradescantia. 
If the solution is hypertonic, i.e., has a greater osmotic pressure than the cell contents, the protoplasm shrinks, and loses water, or if red corpuscles are used, they become crenated; if the solution is hypotonic, i.e., has a snialler osmotic pressure than the material within the cell-wall, no shrinking of the protoplasm in the vegetable cell takes place; and if red corpuscles are used they swell and liberate their pigment. Isotonic solutions, such as physiological salt solution, produce neither of these effects, because they have the same molecular concentration and osmotic pressure as the material within the cell-wall.

Physiological Applications. - It will at once be seen how important all these considerations are from the physiological standpoint. In the body we have aqueous solutions of various substances separated from one another by membranes. Thus we have the endothelial walls of the capillaries separating the blood from the lymph ; we have the epithelial walls of the kidney tubules separating the blood and lymph from the urine; we have similar epithelium in all secreting glands; and we have the wall of the alimentary canal separating the digested food from the blood-vessels and lacteals. In such important problems, then, as lymph-formation, the formation of urine and other excretions and secretions, and absorption of food, we have to take into account the laws which regulate the movements both of $w$ ater and of substances which are held in solution by the water. In the body osmosis is not the only force at work, but we have also to consider filtration, that is, the forcible passage of materials through membranes, due to differences of mechanical pressure. Further complicating these two processes we have to take into account another force, namely, the secretory or selective activity of the living cells of which the membranes in question are composed. This is sometimes called by the name vital action, which is an unsatisfactory and unscientific expression. The laws which regulate filtration, inbibition (or adsorption), and osmosis are fairly well known and can be experimentally verified. But we have undoubtedly some other force, or some other manifestation of force, in the case of living membranes. It probably is some physical or chemical property of living matter which has not yet been brought into line with the known chemical and physical forces which operate in the inorganic world. We cannot deny its existence, for it sometimes operates so as to neutralise the known forces of osmosis and filtration.

The more one studies the question of lymph-formation, the more convinced one becomes that mere osmosis and filtration will not explain it entirely. The basis of the action is no doubt physical, but the living cells do not behave like the dead membrane of a dialyser; they have a selective action, picking out some substances and passing them through to the lymph, while they reject others.

The question of gaseous interchanges in the lungs is another of a similar kind. Some maintain that all can be explained by the laws of diffusion of gases ; others have asserted that the action is wholly or partly vital. We shall, however, find that recent accurate work has shown that the main facts are explicable on a physical basis. Take again the case of absorption. The object of digestion is to render the food soluble and diffusible; it can hardly be supposed that this is useless; the readily diffusible substances will pass more easily through into the blood and lymph : but still, as Waymouth Reid has shown, if the living epithelium of the intestine is removed, absorption comes very nearly to a standstill, although from the purely physical standpoint removal of the thick columnar epithelium would increase the facilities for osmosis and filtration.

The osmotic pressure exerted by crystalloids is very considerable, but their ready diffusibility limits their influence on the flow of water in the body. Thus if a strong solution of salt is injected into the blood, the first effect will be the setting up of an osmotic stream from the tissues to the blood. The salt, however, would soon diffuse out into the tissues, and would now exert osmotic pressure in the opposite direction. Moreover, both effects will be but temporary, because excess of salt is soon got rid of by the excreting organs.

Osmotic Pressure of Proteins. - It has been generally assumed that proteins, the most abundant and important constituents of the blood, exert little or no osmotic pressure. Starling, however, has claimed that they have a small osmotic pressure; if this is so, it is of importance, for proteins, unlike salt, do not diffuse readily, and their effect therefore remains as an almost permanent factor in the 
blood. Starling gives the osmotic pressure of the proteins of the blood-plasma as equal to $30 \mathrm{~mm}$. of mercury. We should from the theoretical standpoint find it difficult to imagine that a pure protein can exert more than a minimal osmotic pressure. It is made up of such huge molecules that, even when the proteins are present to the extent of 7 or 8 per cent., as they are in blood-plasma, there are comparatively few protein molecules present, and these are in a state of colloidal solution, not true solution. Still, by means of this weak but constant pressure it is possible to explain the fact that an isotonic or even a hypertonic solution of a diffusible crystalloid may be completely absorbed from the peritoneal cavity into the blood.

The functional activity of the tissue elements is accompanied by the breaking down of their protein constituents into such simple materials as urea (and its precursors) sulphates and phosphates. These materials pass into the lymph, and increase its molecular concentration and its osmotic pressure; thus water is attracted (to use the older way of putting it) from the blood to the lymph, and so the volume of the lymph rises and its flow increases. On the other hand, as these substances accumulate in the lymph they will in time attain there a greater concentration than in the blood, and so they will diffuse towards the blood, by which they are carried to the organs of excretion.

But, again, we have a difficulty with the proteins ; they are most important for the nutrition of the tissues, but they are practically indiffusible. We must therefore assume that their presence in the lymph is due to filtration from the blood. The plasma in the capillaries is under a somewhat higher pressure than the lymph in the tissues, and this tends to squeeze the constituents of the blood, including the proteins, through the capillary walls. I have, however, already indicated that the question of lymph-formation is one of the many physiological problems which await solution by the physiologists of the future.

Waymouth Reid finds that absolutely pure proteins exert no osmotic pressure; the pressure observed is due to saline and other materials from which it is difficult to disentangle the protein.* Hæmoglobin is an exception to this rule; it exerts a small osmotic pressure and forms a true solution with water.

Colloidal Solutions.-The study of colloids is important, seeing how many important physiological substances belong to this class; for instance, the proteins, starches, and soaps. Their main characters are, that they do not pass the membrane of a dialyser, their solutions are opalescent, they crystallise with difficulty if at all, they have a tendency to form jellies (as in the case of gelatin), or to coagulate under the influence of heat and other agents (as in the case of most proteins), and they exert a low osmotic pressure. Inorganic substances (e.g., several metals, and compounds such as silicic acid) may also assume a colloidal condition; these are in an unstable physical condition. passing from the "sol " (or soluble) to the "gel " (or jelly-like) condition under slight provocation. This confers upon them their power to act as catalysts.

The solutions formed by colloidal materials are not true solutions, even although the highest powers of the microscope reveal no visible particles. Nevertheless efficient filters made of gelatin will not allow these substances to pass through them. Colloidal solutions also show what is known as the Tyndall phenomenon ; that is, the particles, though invisible, will nevertheless scatter light, just as minute dust particles in the air are lit up by a beam of sunlight. This test forms the basis of the instrument known as the ultra-microscope. Such observations show that colloidal solutions are really suspensions of extremely minute particles.

Reaction Velocity.-Most reactions in Inorganic Chemistry take place between electrolytes substances which are good conductors of the electric current. These may be considered as reactions between ions, and Ionic Reactions occur at

* Bayliss has shown that the saline constituents found in a native protein are not mechanically mixed with it, and are also not in true chemical combination with it, but are in a condition intermediate between these two extremes, to which the term adsorption is applied. Many dyes used for staining fabrics and histological preparations are also adsorbed. 
such enormous velocity as to be practically instantaneous. Ionic reactions take place between the inorganic constituents of living cells, but such reactions occurring as they do in a colloidal medium are somewhat slowed down, but even so are completed in an immeasurably short time. The most important substances (fats, carbohydrates, proteins) in living tissues are, however, not electrolytes, and reactions between them are spoken of as molecular reactions, and occur so slowly that it is possible to ascertain the rate at which they take place. Reaction $V e l o c i t y$ is defined as the quantity of the substance transformed, measured in gramme-molecules per litre, which disappears in the unit of time (one minute). When starch is transformed into sugar, or protein into amino-acids, there is only one substance transformed, and such reactions which compose the majority of the reactions in living cells are called unimolecular reactions, or reactions of the first order. When, for instance, starch is changed into sugar by the action of an acid, it is the starch alone which is altered; the acidity undergoes no diminution. Similarly when the change is brought about by an enzyme, the starch only is changed; the enzyme is still present in its original quantity. Reaction velocity is thus of special importance in a study of the changes produced by enzymes, and these are the most frequent of all changes in living structures.

Since the quantity of the substance acted upon is continually diminishing, the velocity of the reaction cannot remain the same throughout, but must diminish in a certain ratio. Suppose 20 parts out of 100 are transformed in the first minute, there will be only 80 parts remaining at the commencement of the second minute :-

$$
100-\frac{100}{5}=80
$$

Similarly at the commencement of the third minute we have only 64 left, 16 having disappeared :-

$$
80-\frac{80}{5}=64
$$

In the fourth minute, $12 \cdot 8$ disappears and $51 \cdot 2$ is left :-

$$
64-\frac{64}{5}=51 \cdot 2
$$

and so on.

In order to express this in general terms, we may label the original concentration 100 by the symbol $\mathrm{C}_{0}$, and for $80,64,51 \cdot 2$, etc., use the terms $\mathrm{C}_{1}, \mathrm{C}_{2}, \mathrm{C}_{3}$, etc. .... $\mathrm{C}_{t}$. The constant figure in the above example is $\frac{1}{5}$ or 0.2 . This may be represented by $k$. The equations then run :-

$$
\begin{array}{lrl} 
& \mathrm{C}_{0}-\mathrm{C}_{0} k=\mathrm{C}_{1}, \text { or } \mathrm{C}_{0}(1-k)=\mathrm{C}_{1} \\
\text { Further } & \mathrm{C}_{0}(1-k)-\mathrm{C}_{0}(1-k) \times k=\mathrm{C}_{2} ; \\
\text { or } & \mathrm{C}_{0}(1-k)^{2}=\mathrm{C}_{2^{\circ}} \\
\text { Further } & \mathrm{C}_{0}(1-k)^{3}=\mathrm{C}_{3^{*}} \\
\text { Finally } & \mathrm{C}_{0}(1-k)^{t}=\mathrm{C}_{t^{*}}
\end{array}
$$

If this is plotted out in the form of a curve, we obtain the curve known as a logarithmic curve.

In other cases the law is a different one, and we find that the reaction velocity is not directly proportional to the quantity of reacting substance, but to the square of this quantity. In all such cases, two substances are simultaneously changed in their concentration. Such a process takes place in the decomposition of esters (compounds of organic acids and alcohols), under the influence of an alkali; here not only is the amount of ester becoming less, but the alkali is also used up in the formation of salts of the organic acid. Such reactions are called bimolecular reactions, or reactions of the second order. Certain reactions in living cells are of this order, but reactions of higher orders still are not as yet known in living cells.

We shall again have to return to the study of molecular reactions when speaking of the action of enzymes. It will then be more convenient to study one of the peculiarities they possess, namely, the phenomenon known as reversibility. 
Surface Tension. - The surface layer of a liquid possesses certain properties which are not shared by the rest of it, for in the interior the arrangement of matter is symmetrical round any point, whereas on the surface the surroundings consist of liquid on one side only, while on the other side is solid, or gas, or it may be another liquid. In a gas, the molecules are free from one another's attractive influence and fly about freely with high velocity, producing pressure on the walls of the containing vessel; in a liquid, the mutual attractions of the molecules are great enough to keep the substance together in a definite volume; in order to separate the molecules and convert the liquid into gas a large amount of energy is requiredthe so-called latent heat of evaporation. The molecular attractions in a liquid are thus very great, so that a molecule of the surface layer is strongly pulled in wards, and this layer constitutes a stretched elastic skin, and the power thus exerted is spoken of as surface tension. The effect of surface tension is most simply seen in a free drop of liquid, such as a rain-drop, or a drop of oil immersed in a mixture of alcohol and water of the same density. There is then nothing to prevent the tension in the surface layer from contracting as much as possible, and the drop will therefore assume a form in which its volume will have the smallest surface, that is, the drop will assume the form of a sphere.

Now animal cells are liquid, and when they are at rest, other forces being absent, they also are spherical, and although they do not possess, as a rule, a definite wall of cellulose or other hard substance such as vegetable cells have, nevertheless the surface film, exercising the force called surface tension, plays the part of an elastic skin, and is termed the plasmatic membrane. This membrane plays an important physiological rôle. In the projection of pseudoporlia, for instance, variations in the surface tension must occur in different parts of the circumference of the cell. Protoplasm, however, is not a simple liquid, but contains substances of varying chemical composition, and substances which have the power of diminishing surface tension always show a tendency to accumulate at the surface. Hence the fats and lipoids which are powerful depressants of surface tension are found probably in a state of an extremely fine emulsion more abundantly in the plasmatic membrane than elsewhere in the cell. The interstitial spaces between the fat globules are filled up with a watery colloid solution, namely a protein solution. The theory of diffusion of dissolved substances through membranes as applied to cells has been profoundly influenced by the discovery of the composition of the plasmatic membrane. At one time it was believed that diffusion of a colloid material was prevented by the pores of the membrane being too small to allow large molecules to get through them; it was considered to act as a sort of sieve. But this cannot be the whole explanation, and it is now held that solution affinities play the most important part; that is to say, a membrane is permeable to substances which are soluble in the material of the membrane. Such solubility may imply the formation of actual chemical unions, or more frequently the process is one of adsorption; this latter process comes specially into play when nutritive materials are assimilated by the cell by means of the protein solution which occupies the interstices between the fat globules. On the other hand, the permeability of the plasmatic membrane by substances such as alcohol, chloroform, and ether, is mainly determined by the solubility of these materials in the fatty or fat-like components of the membrane, and this consideration is the foundation of the Meyer-Overton theory of the narcotic effect on cells which these volatile anæsthetics exercise. 


\section{CHAPTER XXV}

\section{THE DUCTLESS GLANDS}

Tre ductless glands form a heterogeneous group of organs, most of which are related in function or development with the circulatory system. The function of a gland that has a duct is a comparatively simple physiological problem, but the use of ductless glands was for long a puzzle to investigators. Recent research has, however, shown that most of, if not all, the ductless glands do form a secretion, and this internal secretion, as it is termed, leaves the gland by the venous blood or lymph, and thus is distributed and ministers to the needs of parts of the body elsewhere. Many of the glands which possess ducts and form an external secretion, form an internal secretion as well. Among these, the liver and pancreas may be mentioned. The term internal secretion was first used by Claude Bernard in relation to the liver.

In many cases the internal secretion is essential for life, and removal of the gland that forms it, leads to a condition of disease culminating in death. In other cases the internal secretion is not essential, or its place is taken by that formed in similar glands in other parts of the body.

The body is a complex machine; each part of the machine has its own work to do, but must work harmoniously with other parts. Just as a watch will stop if any of its numerous wheels get broken, so the metabolic cycle will become disarranged or cease altogether if any of the links in the chain break down.

In unravelling the part which the ductless glands play in this cycle, it is at present impossible in many cases to state precisely what the particular function of each is; all one can say is, when the gland is removed or its function interfered with, that the metabolic round is broken somehow, and that this upsets the rest of the machinery of the body. The difficulty of investigating this subject is increased by the fact that it is impossible to get the internal secretion in a state of purity and examine it; it is always 
mixed with, and masked by, the lymph or blood into which it is poured.

In spite of this, however, our knowledge in this branch of physiology is increasing, particularly in connection with some of these ductless glands. The methods of investigation which have been employed are the following:-

1. Extirpation.-The gland in question is removed, and the effect of the absence of the internal secretion noted.

2. Disease.-In cases where the function of the gland is in abeyance, owing to its being diseased, the symptoms are closely observed.

3. Injection of Extracts. - The gland is taken in a fresh condition; an extract is made of it, and this is injected into the circulation of healthy animals, and into that of those animals from which the gland has been previously removed, and the effects watched.

4. Transplantation.-After the gland is removed and the usual effect produced, the same gland from another animal is transplanted into the first animal, and restoration of function looked for.

The case of the lymphatic glands we have already studied; they furnish the blood with a supply of a certain kind of colourless corpuscle. Removal of lymphatic glands is not fatal, as the other lymphatic glands and other collections of lymphoid tissue that remain behind carry on the work of those that are removed.

The internal secretion theory of the ductless glands is that which is most in vogue at present. It should be mentioned, however, that there is another theory, which may be called the auto-intoxication theory. According to this view the gland is excretory (i.e., gets rid of waste and harmful materials) rather than secretory (i.e., production of something useful to the organism). When the gland is removed, the waste products therefore accumulate and produce harmful results. It is possible that as our knowledge increases, it may be found in certain cases that both these theories may be in part true.

\section{The Spleen.}

The Spleen is the largest of the ductless glands; it is situated to the left of the stomach. It is of a deep red colour and of variable shape. Vessels enter and leave the gland at a depression on the inner side called the hilus. The spleen is covered externally almost completely by a serous coat derived from the peritoneum, while within this is the proper fibrous coat or capsule of the organ. The latter contains numerous elastic fibres and a large amount of unstriated muscular tissue. Prolonged from its inner surface are fibrous processes or trabeculoe, containing much unstriated muscle, which enter the interior of the organ, and, dividing and anastomosing in all parts, form a supporting framework in the interstices of which the proper substance of the spleen (spleen-pulp) is contained. 
The spleen-pulp, which is of a dark red or reddish-brown colour, is composed chiefly of cells, imbedded in a network formed of fibres, and the branchings of large nucleated cells. The network so formed is thus very like a coarse kind of retiform tissue. Some of the cells in the meshes of the network are granular corpuscles resembling the lymph-

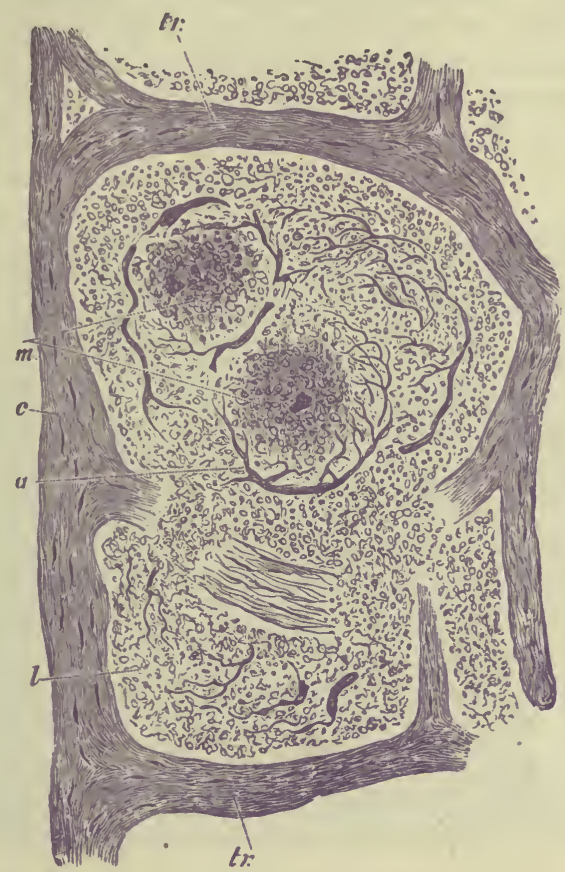

F'ı. 277.-Section of injected dog's spleen. c, Capsule; $t r$, trabeculæ; $m$, two Malpighian bodies with numerous small arteries and capillaries; $a$, artery ; $l$, lymphoid tissue, consisting of closely packed lymphoid cells supported by very delicate retiform tissue; a light space unoccupied by cells is seen all round the trabeculæ, which corresponds to the "lymph-path" in lymphatic glands. (Schofield.)

corpuscles, both in general appearance and in being able to perform amœboid movements; others are red blood-corpuscles of normal appearance or variously changed; while there are also large cells containing either a pigment allied to the colouring matter of the blood, or rounded corpuscles like red corpuscles.

The splenic artery, after entering the spleen divides into branches, which soon leave the trabeculæ, with which at first they are sheathed, and their outer coat is then replaced by one of lymphoid tissue; they end in an open brushwork of capillaries, the endothelial cells of which become continuous with those of the rete of the spleen-pulp. The veins begin by a similar open set of capil- 
laries from the large blood spaces of the pulp. The veins soon pass into the trabeculæ, and ultimately unite to form the splenic vein. This arrangement readily allows lymphoid and other corpuscles to be swept into the blood-current.

On the face of a section of the spleen can be seen usually readily with th, naked eye, minute, scattered, rounded or oval whitish spots, mostly from $\frac{1}{30}$ to $\frac{1}{60}$ inch $\left(\frac{5}{6}\right.$ to $\frac{2}{5} \mathrm{~mm}$.) in diameter. These are the Malpighian corpuscles of the spleen, and are situated on the sheaths of the minute splenic arteries. They are in fact outgrowths of the outer coat of lymphoid tissue just referred to (see fig. 27i). Blood capillaries traverse the Malpighian corpuscles and form a plexus in their interior. The structure of a Malpighian corpuscle of the spleen is practically identical with that of a lymphoid nodule.

The spleen has the following functions:-

(1.) The spleen, like the lymphatic glands, is engaged in the formation of colourless blood-corpuscles. For it is quite certain, that the blood of the splenic vein contains an unusually large proportion of white corpuscles; and in the disease termed leucocythomia, in which the white corpuscles of the blood are remarkably increased in number, there is found a hypertrophied condition of the spleen, especially of the Malpighian corpuscles. The white corpuscles formed in the spleen also doubtless partly leave that organ by lymphatic vessels.

By stimulating the spleen to contract in a case of splenic leucocythæmia by means of an electric current applied over it through the skin, the number of leucocytes in the blood is alnost immediately increased.

Removal of the spleen is not fatal; but after its removal there is an overgrowth of the lymphatic glands to make up for its absence.

(2.) It forms coloured corpuscles, at any rate, in some animals; in these animals, cells are found in the spleen similar to those we have described in red marrow, and called homatoblasts. In these animals, if the spleen is removed, the red marrow hypertrophies.

(3.) There is reason to believe that in the spleen many of the red corpuscles of the blood, those probably which have discharged their office and are worn out, undergo disintegration; for in the coloured portions of the spleen-pulp an abundance of such corpuscles, in various stages of degeneration, are found, and in those cases of disease in which the destruction of blood-corpuscles is increased (pernicious anæmia), iron accumulates in the spleen as in the liver. It was formerly supposed that the spleen broke down the corpuscles and liberated hæmoglobin, which, passing in the blood of the splenic vein to the liver, was discharged by that organ as bile-pigment. But this is not the case; the disintegration does not proceed so far as to 
actually liberate hæmoglobin; there is no free hæmoglobin in the blood-plasma of the splenic vein.

(4.) The spleen participates in nitrogenous metabolism, especially in the formation of uric acid (see Uric Acid formation in Chapter on Urine).

(5.) Besides these direct offices, the spleen fulfils some purpose in regard to the portal circulation with which it is in close cunnection. From the readiness with which it admits of being distended, and from the fact that it is generally small while gastric digestion is going on, and enlarges when that act is concluded, it is supposed to act as a kind of vascular reservoir, or diverticulum to the portal

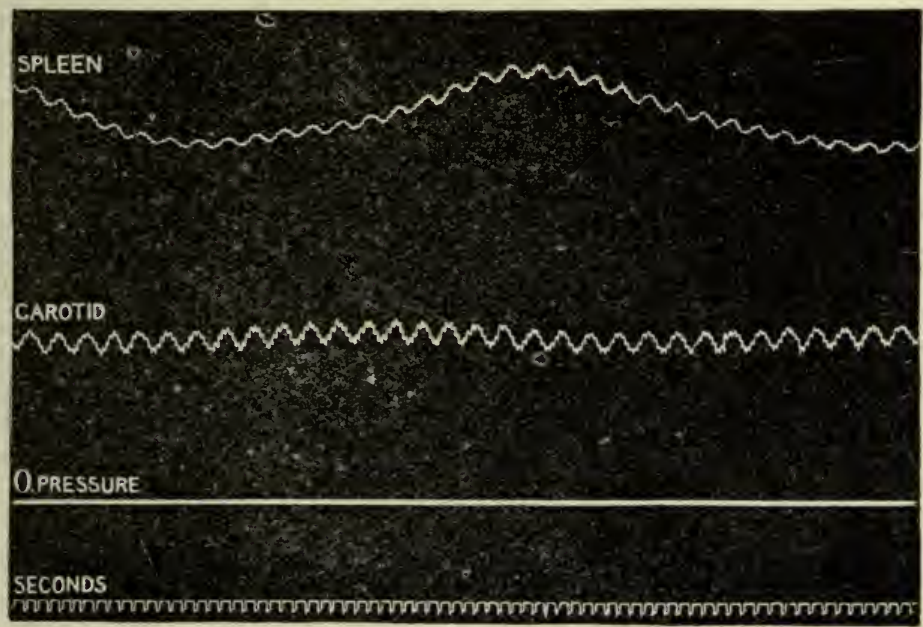

F1G. 278.-The upper tracing is the spleen recurd; the next is carotid blood-pressure taken with a mercurial kymograph. The straight line beneath this is the abscissa of the arterial pressure ; and the lowest tracing is the time in seconds.

system, or more particularly to the vessels of the stomach. This mechanical influence on the circulation, however, can hardly be supposed to be more than a very subordinate function. The main use of the contractility of the spleen is to assist the passage of the blond through itself.

It has been found by experiment that when the splenic nerves are cut the spleen enlarges, and that contraction can be brought about by stimulation of the peripheral ends of the divided nerves. If the splenic nerves are not cut, contraction is produced by (1) stimulation of the spinal cord; (2) reflexly by stimulation of the central stumps of certain divided nerves, e.g., vagus and sciatic; (3) by local stimulation by an electric current; (4) by the administration of quinine and some other drugs. 
It has been shown by the oncometer (see p. 309) that the spleen undergoes rhythmical contractions and dilatations, due to the contraction and relaxation of the muscular tissue in its capsule and trabeculæ. A tracing also shows waves due to the rhythmical alterations of the general blood-pressure. Fig. 278 is a typical tracing obtained by Schäfer's air oncometer from a dog's spleen.

It shows, first, the large waves occurring about once a minute, due to the splenic systole and diastole; secondly, smaller waves on this, due to the effect of respiration on the blood-pressure; and on these, smaller waves still, corresponding with the individual heartbeats. The large waves due to the splenic contractility still go on after the division of all the splenic nerves. These nerve-fibres leave the spinal cord in numerous thoracic anterior roots; they have cellstations in the sympathetic chain (Schäfer) or semi-lunar ganglia (Langley).

\section{Hæmolymph Glands.}

The existence of glands which partake of the nature both of the spleen and of lymphatic glands, has long been known. They have been recently more fully investigated by T. Lewis. He finds them in most mammals, and they can be readily distinguished from ordinary lymphatic glands by their red colour. He divides them into (1) homai glands, which are characterised by the fact that the sinuses contain blood only; the spleen is in fact a large hæmal gland; and (2) hoemal lymphatic glands, in which the sinuses are filled by a mixture of blood and lymph.

\section{The Thymus.}

This gland attains its greatest size soon after birth, and after the second year it gradually diminishes, until in adult life hardly a vestige remains; it is then replaced by adipose and connective tissue. This, at any rate, has been the general belief until the last fow years. Some recent observations, however, appear to show that the thymus persists longer, and may grow until puberty; and that some true thymus tissue may persist throughout life.

The gland is surrounded by a fibrous capsule, which sends in processes, forming trabeculæ, that divide the gland into lobes, and carry the blood-and lymph-vessels. The large trabeculæ branch into small ones, which divide the lobes into lobules. The lobules are further subdivided into follicles by fine connective tissue. A follicle is polyhedral in shape, and consists of cortical and medullary portions, both of which are composed of adenoid or lymphoid tissue, but in the medullary portion the matrix is coarser, and is not so filled up with lymphoid corpuscles as in the cortex. Scattered in the lymphoid tissue of the medulla are the concentric corpuscles of 
Hassall (fig. 279), which are nests or islands of epithelial cells cut off from the epithelium of the pharynx in process of development.

The functions of the thymus are very obscure. It has generally been assumed that the lymphoid tissue of which it is composed form colourless corpuscles; but Stöhr asserts that it is not true lymphoid tissue.

It has been stated that in hibernating animals, in which it undoubtedly persists throughout life, that as each hibernating period approaches the gland enlarges, and its cells become laden with fat. In this case, the store of fat will serve to maintain combustion processes during the winter sleep.

Removal of the gland in the frog is stated

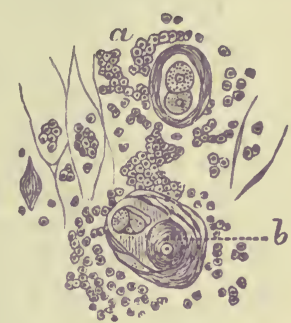

F'IG. 279.-The reticulum of the thymus. $a$, Lymph cells ; $b$, corpuscles of Hassall. (Cadiat.) to be followed by muscular weakness, paralysis, and finally death; but later observations have failed to confirm this result, either in frogs or mammals. Intravenous injection of extracts of thymus lowers arterial blood-pressure and accelerates the heart, but extracts of most organs produce similar effects, especially on the blood-pressure.

Lately it has been suggested that there is some relationship between the thymus and the generative organs; and this view is supported by the circumstance that castration retards the atrophy of the thymus, whilst removal of the thymus hastens the growth of the testes.

\section{The Thyroid.}

The thyroid gland is situated in the neck. It consists of two lobes, one on each side of the trachea; these lobes are connected across the middle line by a middle lobe or isthmus. It is highly vascular, and varies in size in different individuals.

The gland is encased in a capsule of dense areolar tissue. This sends in strong fibrous trabeculæ, which enclose the thyroid vesicleswhich are rounded or oblong sacs, consisting of a wall of thin hyaline membrane lined by a single layer of short cylindrical or cubical cells. These vesicles are filled with transparent colloid nucleo-protein material. The colloid substance increases with age, and the cavities appear to coalesce. In the interstitial connectivetissue is a round meshed capillary plexus, and a large number of lymphatics. The nerves adhere closely to the vessels.

In the vesicles there are sometimes, in addition to the yellowish glassy colloid material, epithelium cells, colourless blood-corpuscles, and also coloured corpuscles undergoing disintegration.

It is difficult to state definitely the function of the thyroid body; it is one of those organs of great importance in the metabolic round; and its removal or disease is followed by general disturbances. It no 
doubt forms an internal secretion; to this the colloid material mentioned contributes, as it is found in the lymphatic vessels of the organ.

When the gland is diseased in children and its function obliterated, a species of idiocy is produced called cretinism.

The same condition in adults is called myxoedema; the most marked symptoms of this condition are slowness, both of body and mind, usually associated with tremors and twitchings. There is also a peculiar condition of the skin leading to the overgrowth of the subcutaneous tissues, which in time is replaced by fat; the hair falls off, the hands become spade-like; the whole body is unwieldy and clumsy like the mind.

A similar condition occurs after the thyroid is completely removed

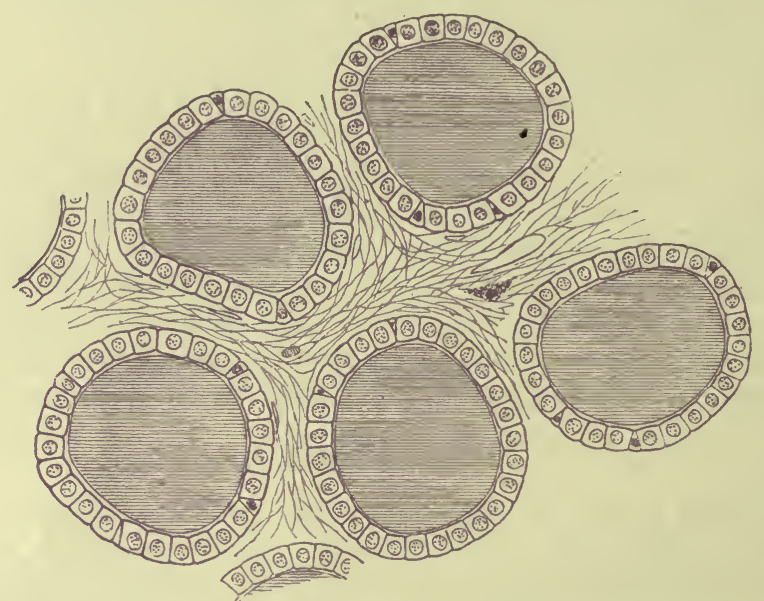

FIG. 250.-Section of human thyroid; the few vesicles shown are lined by cubical epithelium, and contain a colloid material. (After Scliafer.)

surgically; this is called cachexia strumipriva; this operation, which was performed previous to our knowledge of the importance of the thyroid, is of course not performed nowadays.

Lastly, in many animals removal of the thyroid produces analogous symptoms, in the overgrowth of the connective tissues especially under the skin, and in the nervous symptoms (twitchings, convulsions, etc.).

The term Myxœdema was originally given under the erroneous idea that the swelling of the body is due to mucin. In the early stages of the disease there is a slight increase of mucin, because all new connective tissues contain a relatively large amount of ground substance, the most abundant constituent of which, next to water, is mucin. But there is nothing characteristic about that. 
The discovery of the relationships between the thyroid and these morbid conditions is especially interesting, because important practical results in their treatment have followed close on the heels of experimental investigation. The missing internal secretion of the thyroid may be replaced in these animals and patients by grafting the thyroid of another animal into the abdomen; or more simply by injecting thyroid extract subcutaneously; or even by feeding on the thyroid of other animals. This treatment, which has to be kept up for the rest of the patient's life, is entirely successful. Chemical physiologists have been diligently searching to try and discover what the active material in thyroid extract is which produces such marvellous results; the view at present held is that the efficacy of thyroid extract is mainly though not entirely due to a substance which Baumann separated from the gland, and which stands almost unique among physiological compounds by containing a large percentage of iodine in its molecule. Thyro-iodin or Iodo-thyrin, as this substance has been called, is present in combination with protein matter in the colloid substance.

Intravenous injection of thyroid extract in a normal animal lowers blood-pressure; but in an animal from which the thyroid has been removed it stimulates the heart and raises blood-pressure.

In healthy animals and men, administration of thyroid produces an increase in nitrogenous metabolism.

\section{Parathyroids.}

These are small bodies, usually four in number, situated near or imbedded in the substance of the thyroid. They are made up of elongated groups of polyhedral cells, bound together by connective tissue, and well supplied with blood-vessels. Some have supposed that parathyroid is only immature thyroid tissue, but a study of development shows that the parathyroids have a different embryonic origin from the thyroid, and in the lower vertebrates the two organs are entirely distinct. It is only in the mammals that they are so closely associated anatomically. They are probably associated physiologically also, and it has been by no means easy to determine the rôle of each. Most of the facts described in the preceding section on the thyroid were discovered previous to the recognition of the parathyroids, and since then the view has been advanced that in removing the thyroid it is really the simultaneous removal of the parathyroids which causes the nervous symptoms. Certainly the most prominent symptom after extirpation of the parathyroids is tetany (muscular spasms and twitchings).

The parathyroids contain no iodine, and it is doubtful if they form an internal secretion. If they do not, their function must be to neutralise poisonous substances formed elsewhere, and 
the symptoms after extirpation will then be due to the toxic effect of an accumulation of the poison. It has further been shown that tetany produced in animals and men by parathyroidectomy can be relieved by the administration of calcium salts, and so it is possible that the parathyroids may be related in some way to calcium metabolism.

\section{The Suprarenal Capsules.}

These are two triangular or cocked-hat-shaped bodies, each resting by its lower border upon the upper border of the kidney.

The gland is surrounded by an outer sheath of connective tissue, which sends in fine prolongations forming the framework of the gland. The gland tissue proper consists of an outside firmer cortical portion and an inside soft, dark medullary portion.

(1) The cortical portion is divided into (fig. 281) columnar groups

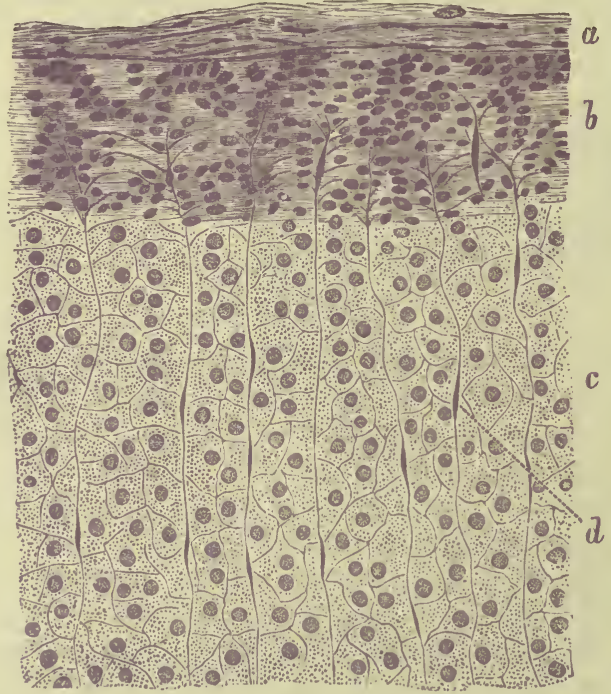

FIG. 281.-Vertical section through part of the cortical portion of suprarenal of guinea-pig. $a$, Cap. sule; $b$, zona glomerulosa ; $c$, zona fasciculata ; $d$, connective tissue supporting the columns of the cells of the latter, and also indicating the position of the blood-vessels. (S. K. Alcock.)

of cells (zona fasciculata). Immediately under the capsule, however, the groups are more rounded (zona glomerulosa), while next to the medulla they have a reticular arrangement (zona reticularis). The cells themselves are polyhedral, each with a clear round nucleus, and contain lipoid globules.

(2) The medullary substance consists of a coarse rounded or irregular meshwork of fibrous tissue, in the alveoli of which are 
masses of multinucleated protoplasm (fig. 282), numerous blood-vessels (sinusoids, see p. 222), and an abundance of nerve-fibres and cells.

The cells of the medulla are characterised by the presence of certain reducing substances. One of these takes a brown stain with chromic acid, and gives other colour reactions; it is, therefore, called a chromogen. Another is similar in many of its characters to jecorin, a lecithin-like substance united to glucose also found in the liver, spleen, and other organs.

The immense importance of the suprarenal bodies was first indicated by Addison, who, in 1855, pointed out that the disease now known by his name is associated with pathological alterations of these

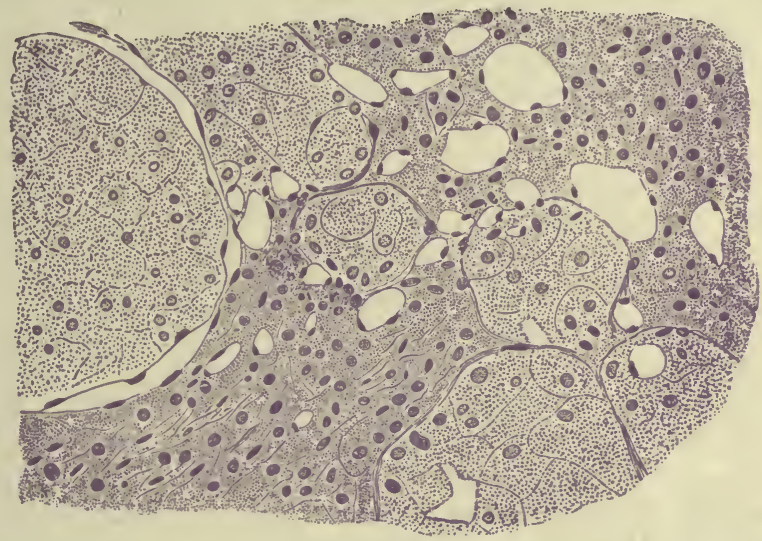

FIG. 232.-Section through a portion of the medullary part of the suprarenal of guinea-pig. The vessels are very numerous, and the fibrous stroma more distinct than in the cortex, and is, moreover, reticulated. The cells are irregular and larger, clear, and free from oil globules. (S. K. Alcock.)

glands. This was tested experimentally by Brown-Séquard, who found a fow years later that removal of the suprarenals in animals is invariably and rapidly fatal. The symptoms are practically the same (although more acute) as those of Addison's disease, namely, great muscular weakness, loss of vascular tone, and nervous prostration. The pigmentation (bronzing) of the skin, however, which is a marked symptom in Addison's disease, is not seen in animals. The experiments of Brown-Séquard attracted much attention at the time they were performed, but were almost forgotten for many years, until they were confirmed by Abelous, Langlois, Schäfer, and others. The effects on the muscular system are the most marked results both after removal of the capsules and after injection of an extract of the glands. The effect of injecting such an extract on the voluntary muscles is to increase their tone, so that a tracing obtained from them resembles that produced by a small dose of veratrine, namely, a pro- 
longation of the period of relaxation. The effect on involuntary muscle is even more marked; there is an enormous rise of arterial bloodpressure due chiefly to a contraction of the arterioles. This is produced by the direct action of the extract on the arterioles, not an indirect one through the vaso-motor centre.* The substance in the extract that produces the effect is known as adrenaline; it is confined to the medulla of the capsules, and is absent in cases of Addison's disease.

The suprarenal bodies, therefore, form something which is distributed to the muscles and is essential for their normal tone; when they are removed or diseased, the effects seen are the result of the absence of this internal secretion.

Adrenaline has received various names from the different chemists (Abel, v. Fürth, Takamine, etc.), who have isolated it. It is very powerful; solutions of one part in a million will produce physiological effects. Its composition is shown by the following formula :-

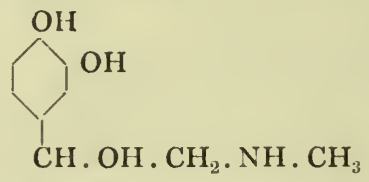

and it is therefore a methyl-amino derivative of catechol (Pauly, Jowett). Recently, compounds closely allied to it in composition and action have been made synthetically (Stolz, Friedmann, Dakin).

Whether this discovery will lead to the same kind of results, as in the case of the thyroid, must be left to the future to decide. There is already some evidence to show that injection of suprarenal extract is beneficial in cases of Addison's disease. The discovery of adrenaline itself is, however, one of immense practical importance. Its action on the small blood-vessels is so powerful that quite weak solutions applied locally will subdue the congestion of inflammation and even arrest hæmorrhage.

The splanchnic nerves have been shown to contain secretory fibres which control the amount of adrenaline poured into the circulation.

The use of the suprarenal cortex is still unknown. It has been suggested that it has some effect on the development of the organs of generation, but these views are quite hypothetical. The cortex, however, contains large quantities of lipoid material (cholesterin, lecithin, and similar substances), and the droplets seen in the fresh cells consist of these compounds; the suggestion that the suprarenal cortex plays a part in the metabolism of these substances appears to be the only feasible one at present.

* It is the sympathetic nerve terminals which are really affected. Any-muscular tissue supplied by sympathetic nerves contracts under the influence of adrenaline. Vessels destitute of constrictor fibres (coronary, pulmonary and cerebral) are not contracted by adrenaline. 
There are some points of interest in the development and comparative physiology of the suprarenals. In mammals the medullary portion is developed in connection with the sympathetic, and is at first distinct and outside the cortical portion which is developed in connection with the upper part of the Wolffian body; it gradually insinuates itself within the cortex (Mitsukiri). In Elasmobranch fishes the suprarenals consist throughout life of separate portions; one, the inter-renal body, is median in position and single; this corresponds to the cortex of the mammalian suprarenal; extracts of this are inactive, and in the Teleostean fishes, where it is the sole representative of the suprarenal, it may be removed without any harm to the animal. The other portion of the Elasmobranch suprarenal is paired, and derived from the sympathetic ganglia. This corresponds to the medulla; it contains the same chromogen as the medulla of the mammalian suprarenal, and extracts of it have the same physiological action (S. Vincent).

The tissue of the suprarenal medulla is often called chromophil tissue, on account of the ready way in which it stains with chromic salts. Such tissue is, moreover, not confined to the suprarenal, but is found in scattered patches in the retro-peritoneal region and in many sympathetic ganglia, especially in the abdomen. The histological resemblance is accentuated by the presence of numerous sympathetic cells in the suprarenal medulla. The chromophil tissue wherever found always yields adrenaline.

\section{The Pituitary Body.}

This occupies the sella turcica of the sphenoid bone. It may be divided into three parts, which show developmental, structural and functional differences.

(1) The anterior lobe is developed as a tubular prolongation from the epiblast of the buccal cavity, but the growth of intervening tissue soon cuts off all connection with the mouth. It consists of large granular cells and numerous blood-vessels. Its precise function is undetermined, although probably it is a vascular gland pouring an internal secretion into the blood, which influences growth. Abnormal hypertrophy of the pituitary produces the condition known as acromegaly, in which the bones of the face and limbs hypertrophy; and if the view advanced above of the anterior lobe is correct, the condition is caused by an increase of the internal secretion. Feeding young animals and children in the anterior lobe hastens the growth of their skeletal tissues.

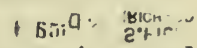

(2) The pars intermedia.-This lies between the anterior and posterior lobes, and forms a closely fitting investment of the latter lobe. It is developed in association with the anterior lobe, and consists of finely granular cells arranged in layers closely applied to the body and neck of the posterior lobe and the under surface of adjacent parts of the brain. Colloid material occurs between the 
cells, which passes into the adjacent nervous substance, to be absorbed by lymphatics which carry it to the cavity of the posterior lobe, and so into the third ventricle of the brain. The existence of colloid cysts in the pituitary closely resembling those of the thyroid has led many observers to the conclusion that the function of the two glands is similar, and that after removal of the thyroid the pituitary may take on its work vicariously. After extirpation of the thyroid gland, the cells of the pars intermedia do manifest increased activity, and the colloid matter increases but this is all that can be said at present in favour of such a view; the removal of the two organs produces very different symptoms; injection of extracts produces different effects; moreover, the pituitary contains no iodine, therefore the colloid material is a different substance in the two cases.

(3) The posterior lobe.-This is connected to the floor of the third ventricle, of which it forms a developmental outgrowth; in some animals (cat) it remains hollow throughout life, in others (dog) the neck alone remains hollow, and in most (including man) both body and neck are solid, with traces of a cavity in the neck. Though developed from the brain, it contains in the adult no nerve cells, but consists mainly of neuroglia. It is surrounded and invaded by the epithelium cells and colloid matter derived from the pars intermedia. It plays the part of a brain gland in virtue of these epithelial cells. What the use of the secretion into the third ventricle may be is far from clear. P. T. Herring, to whom we owe many of the facts already given, suggests that disturbances of the posterior lobe may be responsible for the diabetic condition so frequently seen in cases of acromegaly. Whether this is so or not, injections of aqueous extracts :of the gland have pronounced physiological effects, and these may be boiled without losing their activity. Although we do not know the precise nature of the active substance (provisionally called pituitrin) in the posterior lobe, we can at any rate say, therefore, that it is not protein.

Intravenous injection of such extracts produces:-

1. A temporary rise of arterial blood-pressure; this is not due to the presence of adrenaline, for a second injection following the first produces no such effect, whereas the rise of pressure produced by adrenaline may be repeated time after time. The second and following injections of pituitary extract, unless they occur at much prolonged intervals, produce only a slight fall of pressure, which is the effect produced by most tissue extracts. The rise of pressure which occurs at the first injection is, however, like that of adrenaline, produced mainly by constriction of peripheral arterioles. Slowing of the heart may occasionally also be produced. Pituitrin causes àlso contraction of other forms of involuntary muscle, for instance the uterus.

2. The extract has a specific effect on the kidney, and causes 
there not constriction but dilatation of the blood-vessels, which persists for a very long time. Adrenaline, on the other hand, constricts the kidney arterioles. This dilatation is accompanied with pronounced diuresis. It can hardly be doubted that this is no mere accident, but that there is some definite relationship between the activity of the posterior lobe of the pituitary and the kidney function. It also stimulates milk secretion (Ott, Schäfer). Extracts of the anterior lobe produce neither a rise of blood-pressure nor any effect upon the kidney or mammary gland.

The pituitary body is essential for life. Paulesco, and later Harvey Cushing and Horsley, found that total removal of the organ is fatal in a few days. The same result follows entire removal of the anterior lobe. On the other hand, removal of the posterior lobe produces no such effect. Partial removal of the anterior lobe produces a condition known as hypopituitarism, in which adiposity accompanied by (or secondary to) atrophy of the organs of generation are the most marked signs. If the operation is done before adolescence, there is a persistence of sexual infantilism. The transplantation of the organ from another animal, or injection of anterior lobe extracts, prolongs the life of animals after total extirpation, or relieves the symptoms after partial extirpation. In many of Horsley's experiments no such symptoms occurred, even although only minute portions of the anterior lobe were left behind.

\section{The Pineal Gland.}

This gland, which is a small reddish body, is placed beneath the corpus callosum, and rests upon the corpora quadrigemina. It is composed of tubes and saccules lined and sometimes filled with epithelial cells, and containing deposits of earthy salts (brain sand). A few small atrophied nerve-cells without axons are also seen.

In certain lizards, such as Hatteria, and in certain fishes such as the lamprey, the pineal outgrowth is better developed and may be paired. One division corresponds to the pineal gland; the other becomes developed into an eye-like structure connected by nervefibres to the habenular ganglion; this third eye is situated centrally on the upper surface of the head, but is covered by skin.

\section{The Coccygeal and Carotid Glands.}

These are situated, the one in front of the tip of the coccyx and the other at the point of bifurcation of the common carotid artery. They are made up of a plexus of small arteries, and are enclosed and supported by fibrous tissue. They contain also polyhedral cells collected into spheroidal clumps (carotid gland) or irregular nodules (coccygeal gland). Some of the cells of the carotid gland stain brown with chromic acid like those of the suprarenal medulla. 


\section{CHAPTER XXVI}

\section{RESPIRATION}

THE respiratory apparatus consists of the lungs and of the air-passages which lead to them. In marine animals the gills fulfil the same functions as the lungs of air-breathing animals. The muscles which move the thorax and the nerves that supply them must also be included under the general heading Respiratory System; and, using this expression in the widest sense, it includes practically all the tissues of the body, since they are all concerned in the using up of oxygen and the production of waste materials, such as carbonic acid.

Essentially a lung or gill is constructed of a thin membrane, one surface of which is exposed to the air or water, as the case may be, while, on the other is a network of blood-vessels - the only separation between the blood and aërating medium being the thin wall of the blood-vessels, and the fine membrane on one sicle of which vessels are distributed. The difference between the simplest and the most complicated respiratory membrane is one of degree only.

The lungs in the mammalia are only the medium for the exchange, on the part of the blood, of carbonic acid for oxygen. They are not the seat, in any special manner, of those combustion-processes of which the production of carbonic acid is the final result. These processes occur in all parts of the body in the substance of the tissues.

\section{The Respiratory Apparatus.}

The lungs are contained in the chest or thorax, which is a closed cavity having no communication with the outside except by means of the respiratory passages. The air enters these passages through the nostrils or through the mouth, whence it passes through the larynx into the trachea or windpipe, which about the middle of the chest divides into two tubes, bronchi, one to each (right and left) lung.

The Larynx is the upper part of the passage, and will be described in connection with the voice. 
The Trachea and Bronchi.-The trachea extends from the cricoid cartilage, which is on a level with the fifth cervical vertebra, to a

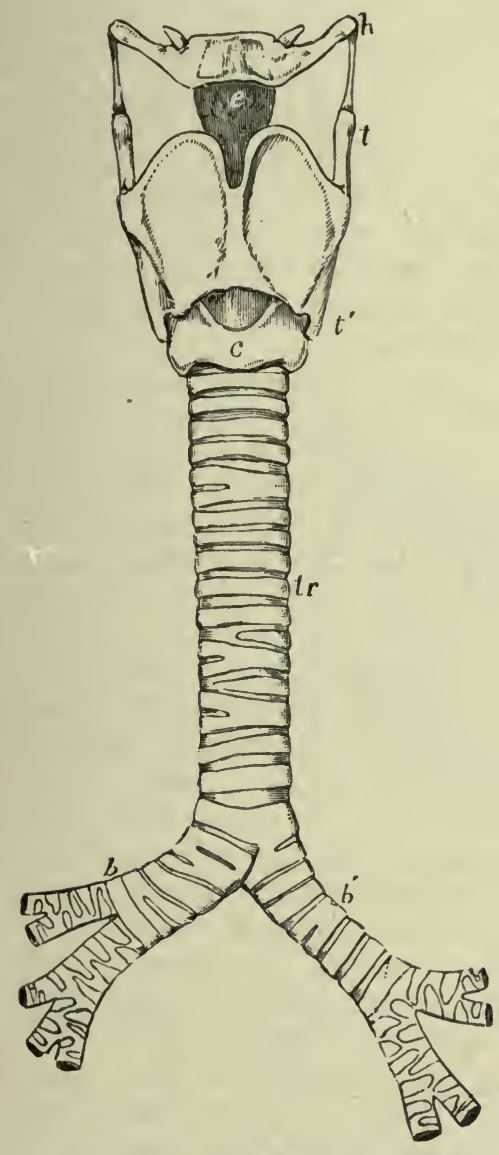

Fio. 2S3A.-Outline showing the general form of the larynx, trachea, and bronchi, as seen from the front. $h$, The great cornu of the hyoid bone: $e$, epiglottis; $t$, superior, and $t^{\prime}$, inferior cornu of the thyroid cartilage; $c$, middle of the cricoid cartilage: $t r$, the trachea, showing sixteen cartila: ginous rings; $b$, the right, and $b^{\prime}$, the left bronchus. (Allen Thomson.)

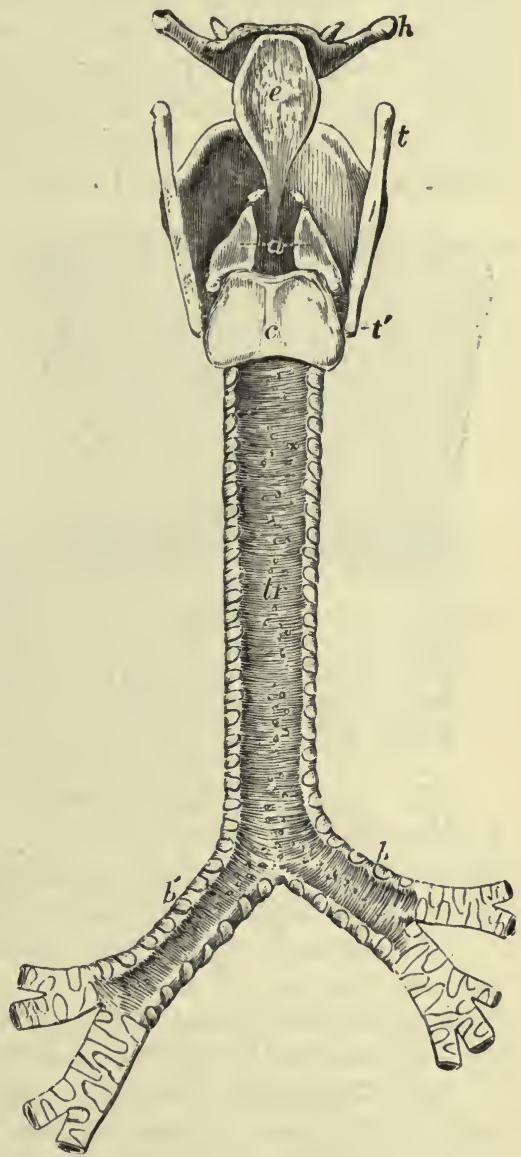

F10. 283B,-Outline showing the general forn of the larynx, tracliea, and bronchi, as seen from behind. $h$, Great cornu of the hyoid bone; $t$, superior, and $t^{\prime}$, the inferior cornu of the thyroid cartilage; $e$, epiglottis ; $a$, points to the back of both the arytenoid cartilages, which are surmounted by the cornicula; $c$, the middle ridge on the back of the cricoid cartilage; $t r$, the posterior membranous part of the trachea; $b, b^{\prime}$, right and left bronchi. (Allen Thomson.)

point opposite the third dorsal vertebra, where it divides into the two bronchi, one for each lung (fig. 283). It measures, in man, about four or four and a half inches in length and from three-quarters of 
an inch to an inch in diameter, and is essentially a tube of fibro-elastic membrane, within the layers of which are imbedded a series of cartilaginous rings, from sixteen to twenty in number. These rings extend only around the front and sides of the trachea (about two-thirds of its circumference) and are deficient behind; the interval between their posterior extremities is bridged over by a continuation of the fibrous membrane in which they are enclosed (fig. 283B). The inner surface of the trachea is lined with ciliated epithelium; this,

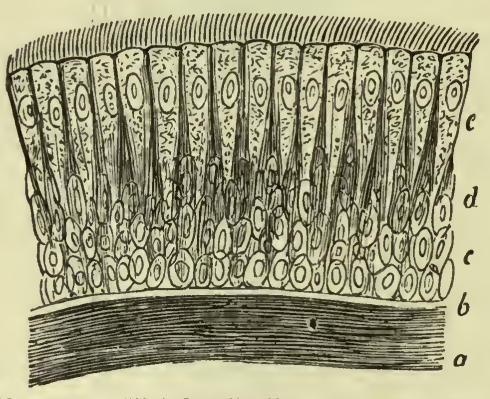

FIc. 284.-Ciliated epithelium of the human traches. $a$, Layer of longitudinally arranged elastic fibres; $b$, basement membrane; $c$, deepest cells circular in form; $d$, inter. mediate elongated cells; $e$, outermost layer of cells fully developed and bearing cilia. $\times$ 350. (Kölliker.)

The two bronchi into which together with the basement membrane on which it rests, and a deeper layer of connective tissue, forms the mucous membrane of the trachea.

Numerous mucous glands are situated in the substance of the mucous membrane; their ducts perforate the various structures which form the wall of the trachea, and open through the mucous membrane into the interior. A layer of unstriped muscle is situated beneath the mucous membrane at the back of the tube where the cartilaginous rings are absent. the trachea divides, resemble the trachea in structure, with the difference that in them there is a distinct layer of unstriped muscle arranged circularly beneath the mucous membrane, forming the muscularis mucosa.

The bronchi divide and subdivide, in the substance of the lungs, into a number of smaller and smaller branches (bronchial tubes), which penetrate into every part of the organ, until at length they end in the smaller subdivisions of the lungs called lobules.

All the larger branches have walls formed of fibrous tissue, containing portions of cartilaginous rings, by which they are held open, and unstriped muscular fibres, as well as longitudinal bundles of elastic tissue. They are lined by mucous membrane the surface of which, like that of the trachea, is covered with ciliated epithelium, but the several layers become less and less distinct until the lining consists of a single layer of short columnar cells covered with cilia (fig. 285). The mucous membrane is abundantly provided with mucous glands.

As the subdivisions become smaller and smaller, and their walls thinner, the cartilaginous rings become scarcer and more irregular, until, in the smaller bronchial tubes, they are represented only by minute and scattered cartilaginous flakes. When the bronchial tubes, 
by successive branchings, are reduced to about $\frac{1}{40}$ of an inch $(6 \mathrm{~mm}$.) in diameter they lose their cartilaginous element altogether, and their walls are formed only of a fibrous elastic membrane with circular muscular fibres; they are still lined, however, by a thin mucous membrane with ciliated epithelium, the length of the cells bearing the cilia having become so far diminished that the cells are now cubical. In the smaller bronchial tubes the muscular fibres are relatively more abundant than in the larger ones, and form a distinct circular coat.

Most of the structures which have been described are of some clinical importance. The secretion of the mucous glands, for instance, may be greatly increased in the condition known as catarrh of the mucous membrane. The secretion, or phlegm, is worked up to

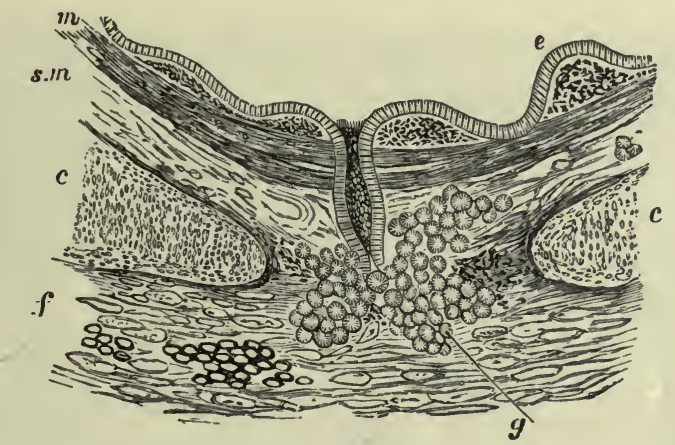

Fig. 285.-Transverse section of a bronchial tube, about $\frac{1}{2}$ inch in diameter. $e$, Epithelium (ciliated), immediately beneath it is the corium of the mucous membrane, of varying thickness; $m$, muscular layer; $s . m$, submucous tissue; $f$, fibrous tissue; $c$, cartilage enclosed within the layers of fibrous tissue; $g$, mucous glands. (F. E. Schulze.)

the larynx by the ciliated epithelium. Its presence irritates the very sensitive surface of that organ, and induces a cough by which the phlegm is expelled from the respiratory passages into the mouth.

The whole inner surface of the bronchi may become inflamed and filled with fluid, through which the air has to be forced at each respiration (bronchitis).

A disorder of another nature, bronchial asthma, is caused by undue contraction of the circular muscles of the bronchi. The passages are thus rendered too narrow for the necessary volume of air to pass conveniently, and as a result the respiration becomes forced. The bronchial muscles are supplied by the vagus nerve, and relaxation of them may be brought about by drugs which prevent the passage of impulses along the vagus.

The Lungs and Pleurce.-The lungs occupy the greater portion of 
the thorax. They are of a spongy elastic texture, and are composed of numerous minute air-sacs, and on section every here and there the air-tubes may be seen cut across. Any fragment of lung (unless from a child that has never breathed, or in cases of disease in which the lung is consolidated by inflammation or pneumonia) floats in water; no other tissue (except fat) does this.

Each lung is enveloped by a serous membrane-the pleura, one layer of which adheres closely to its surface, and provides it with its smooth and slippery covering, while the other adheres to the inner surface of the chest-wall. The continuity of the two layers, which form a closed sac, as in the case of other serous membranes, will be best understood by reference to fig. 286. The appearance of a space, however, between the pleura which covers the lung (visceral layer)

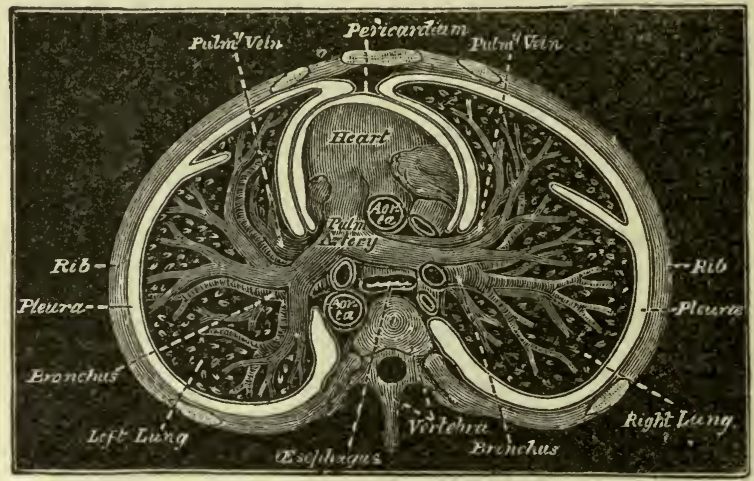

Fra. 286.-Transverse section of the chest.

and that which lines the inner surface of the chest (parictal layer) is inserted in the drawing only for the sake of distinctness. It does not really exist. The layers are, in health, everywhere in contact one with the other; and between them is only just so much fluid as will ensure the lungs gliding easily, in their expansion and contraction, on the inner surface of the parietal layer, which lines the chestwall.

If, however, an opening is made so as to permit air or fluid to enter the pleural sac, the lung, in virtue of its elasticity, recoils, and a considerable space is left between it and the chest-wall. In other words, the natural elasticity of the lungs would cause them at all times to contract away from the ribs were it not that the contraction is resisted by atmospheric pressure which bears only on the inner surface of the air-tubes and air-sacs. On the admission of air into the pleural sac, atmospheric pressure bears alike on the inner and 
outer surfaces of the lung, and their elastic recoil is no longer prevented.

The pleura, like other sorous sacs, is frequently tho seat of inflammatory changes (pleurisy); the pleural cavity then becomes enlarged by an increase in the amount of fluid lymph which it contains. The increase is accompanied by corresponding collapse of the lungs. A formation of fibrin may take place in the exuded fluid; this adheres to the pleura and causes its surfaces, originally smooth, to become rough, and painful friction between the two surfaces, or even their adhesion to one another, may supervene.

Each lung is partially subdivided into separate portions called lobes; the right lung into three lobes, and the left into two. Each of these lobes, again, is composed of a large number of minute parts, called lobules. Each pulmonary lobule may be considered to be a lung in miniature, consisting, as it does, of a branch of the bronchial tube, of airsacs, blood-vessels, nerves, and lymphatics, with a sparing amount of areolar tissue.

On entering a lobule, the small bronchial tube, the structure of which has just been described (fig. 287) divides and subdivides; its walls at the same time become thinner and thinner, until at length they are formed only of a thin membrane of areolar, muscular, and elastic tissue, lined by a layer of pavement epithelium not provided with cilia. At the same

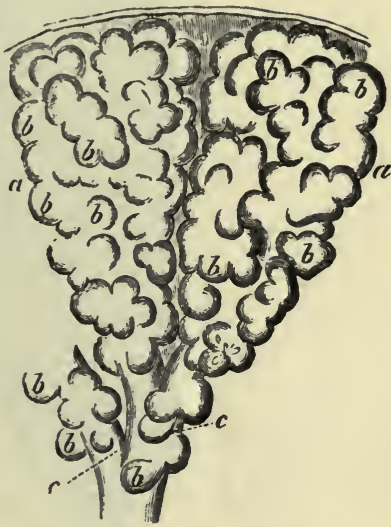

Fig. 287.-Two small infundibula or groups of air-sacs, $a$, with air-sacs, $b \quad b$, and the ultimate bronchial tubes, $c c$, with which the air-sacs communicate. From a newborn child. (Kölliker.) time they are altered in shape; each of the minute terminal branches widens out funnel-wise, and its walls are pouched out irregularly into small saccular dilatations, called air-sacs. Such a funnel-shaped terminal branch of the bronchial tube, with its group of pouches or air-sacs, is called an infundibulum, and the irregular oblong space in its centre, with which the air-sacs communicate, an intercellular passage.

The air-sacs, or air-vesicles, may be placed singly, like recesses from the intercellular passage, but more often they are arranged in groups, or even in rows, like minute sacculated tubes; so that a short series of vesicles, all communicating with one another, open by a common orifice into the tube. The vesicles are of various forms, according to the mutual pressure to which they are subject; their 
walls are nearly in contact, and they vary from $\frac{1}{50}$ th to $\frac{1}{70}$ th of an inch ( 5 to $\cdot 3 \mathrm{~mm}$.) in diameter. Their walls are formed of fine membrane, like those of the intercellular passage; this membrane is folded on itself so as to form a sharp-edged border at each circular orifice of conmunication between contiguous air-vesicles, or between the vesicles and the bronchial passages. Numerous fibres of elastic tissue are spread out between contiguous air-sacs, and many of these are attached to the outer surface of the fine membrane of which each sac is composed, imparting to it additional strength and the power of recoil after distension. The vesicles are lined by a layer of pavement epithelium (fig. 288). Outside the air-vesicles a network of pulmonary

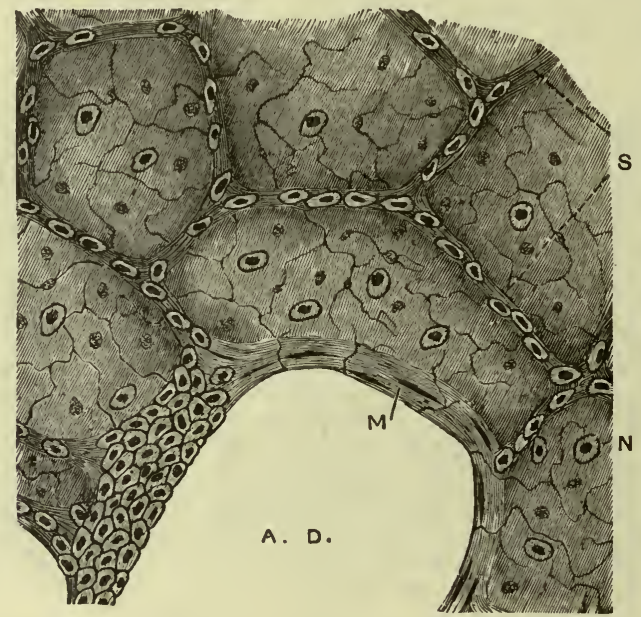

FIG. 288. - Section of lung stained with silver nitrate. A. D., alveolar duct or intercellular passage; $\mathrm{S}$, alveolar septa; $\mathrm{N}$, alveoli or air-sacs, lined with large flat cells, with some smaller polyhedral cells; M, plain muscular fibres surrounding the alveolar duct. (Klein and Noble Smith.)

capillaries is spread out so densely (fig. 289) that the interspaces or meshes are even narrower than the vessels, which are, on an average, $\frac{1}{3000}$ th of an inch $(8 \mu)$ in diameter. Between the air in the sacs and the blood in these vessels nothing intervenes but the thin walls of the air-sacs and of the capillaries; and the exposure of the blood to the air is the more complete, because the folds of membrane between contiguous air-sacs, and often the spaces between the walls of the same, contain only a single layer of capillaries, both sides of which are thus at once exposed to the air. The arrangement of the capillaries is shown on a larger scale in fig. 205 (p. 223).

Area of the Surface of the Lung.-The object of the complicated structure of the lung is to provide a very large surface, for the 
interchange of gases, in a compact organ. The total surface of the inside of the lung has been variously calculated, but it may be taken to be about 90 square metres in the adult, or about the size of a carpet necessary to cover the floor of a good-sized room (10 yards by 12 ).

The vesicles of adjacent lobules do not communicate; so that, when any bronchial tube is closed or obstructed, the supply of air is lost for all the sacs opening into it or its branches.

Blood-supply. - The lungs receive blood from two sources, $(a)$ the pulmonary artery, $(b)$ the bronchial arteries. The former conveys venous blood to the lungs to be arterialised. The branches of the bronchial arteries convey arterial blood from the aorta for the

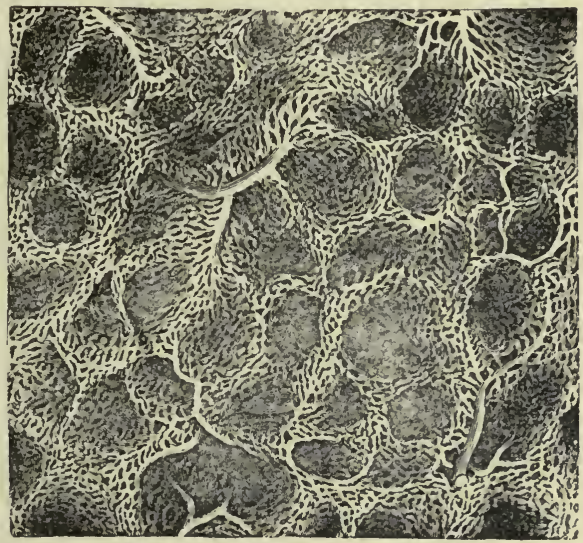

FIG. 289.-Capillary network of the pulmonary blood-vessels in the human lung. $\times 60$. (Kölliker.)

nutrition of the walls of the bronchi, of the larger pulmonary vessels, of the interlobular connective tissue, etc.; the blood of the bronchial vessels is returned chiefly through the bronchial and partly through the pulmonary veins.

Lymphatics.-The lymphatics consist of irregular lacunæ in the walls of the air-sacs, in the walls of the bronchial tubes, and in the pulmonary pleura. The lymphatic vessels from all these irregular sinuses pass in towards the root of the lung to reach the bronchial lymphatic glands.

Nerves.-The nerves of the lung contained in the anterior and posterior pulmonary plexuses are formed by branches of the vagus and sympathetic. They follow the course of the vessels and bronchi, and in the walls of the latter many small ganglia are situated. 


\section{The Respiratory Mechanism.}

Respiration consists of the alternate expansion and contraction of the thorax, by means of which air is drawn into or expelled from the lungs. These acts are called Inspiration and Expiration respectively.

For the inspiration of air into the lungs a movement of the sidewalls and floor of the chest takes place, so that the capacity of the interior is enlarged. By such increase of capacity there will be a diminution of the pressure of the air in the lungs, and a fresh quantity will enter through the larynx and trachea to equalise the pressure on the inside and outside of the chest.

For the expiration of air, the opposite movement diminishes the capacity of the chest; the pressure in the interior will be thus increased, and air will be expelled, until the pressure within and without the chest are again equal. In both cases the air passes through the trachea, there being no other communication with the exterior of the body; and the lung remains, under all circumstances, closely in contact with the walls and floor of the chest. The movements of the lung are therefore passive, not active, and depend on the changes of shape of the closed cavity in which they are contained. A perforation of the chest-wall would mean that the lung on that side would no longer be of use; a similar injury on the other side (double pneumothorax) would cause death. If the two layers of the pleura were adherent, those portions of the lung would be expanded most where the movements of the chest are greatest. The existence of the two layers prevents this, and thus the lung is equally expanded throughout.

Inspiration.-This is a muscular act; the effect of the action of the inspiratory muscles is an increase in the size of the chest-cavity in the vertical, the lateral, and antero-posterior diameters.

The vertical diameter of the chest is increased by the contraction and consequent descent of the diaphragm; at rest, the diaphragm is dome-shaped with the convexity upwards; the central tendon forms a slight depression in the middle of this dome. On contraction the muscular fibres shorten, and so the convexity of the double dome is lessened. The central tendon is drawn down a certain distance, but the chief movement is at the sides. For the effective action of this muscle, its attachment to the lower ribs is kept fixed by the contraction of the quadratus lumborum. The diaphragm is supplied by the phrenic nerves.

The increase in the lateral and antero-posterior diameters of the chest is effected by the raising of the ribs, the upper ones being fixed 
by the scaleni. The greater number of the ribs are attached very obliquely to the spine and sternum.

The elevation of the ribs takes place both in front and at the sides - the hinder ends being prevented from performing any upward, movement by their attachment to the spine. The movement of the front extremities of the ribs is of necessity accompanied by an upward and forward movement of the sternum to which they are attached, the movement being greater at its lower than at its upper end.

The muscles by which the ribs are raised, in ordinary quiet inspiration, are the external intercostals, and that portion of the internal intercostals which is situated between the costal cartilages; and these are assisted by the levatores costarum, and the serratus posticus superior.

In extraordinary or forced inspiration, additional muscles are pressed into service, such as the sternomastoid, the serratus magnus, the pectorales, and the trapezius. Laryngeal and face muscles also come into play.

The expansion of the chest in inspiration presents some peculiarities in different persons. In young children, it is effected chiefly by the diaphragm. The movement of the abdominal walls being here more manifest than that of any other part, it is usual to call this the abdominal type of respiration. In men, together with the descent of the diaphragm, and the pushing forward of the front wall of the abdomen, the chest and the sternum are subject to a wide movement in inspiration (inferior costal type). In women, the movement appears less extensive in the lower, and more so in the upper, part of the chest (superior costal type).

Expiration.-From the enlargement produced in inspiration, the chest and lungs return, in ordinary tranquil expiration, by their elasticity to their previous condition; the force employed by the inspiratory muscles in distending the chest and overcoming the elastic resistance of the lungs and chest-walls, is returned as an expiratory effort when the muscles are relaxed. This elastic recoil of the chest and lungs is sufficient, in ordinary quiet breathing, to expel air from the lungs in the intervals of inspiration, and no muscular power is required. In all voluntary expiratory efforts, however, as in speaking, singing, blowing, and the like, and in many involuntary actions also, as sneezing, coughing, etc., something more than merely passive elastic power is necessary, and the proper expiratory muscles are brought into action. The chier of these are the abdominal muscles, which, by pressing on the viscera of the abdomen, push up the floor of the chest formed by the diaphragm, and by thus making pressure on the lungs, expel air from them through the trachea and larynx. All muscles, however, which depress 
the ribs, must act also as muscles of expiration, and therefore we must conclude that the abdominal muscles are assisted in their action by the interosseous part of the internal intercostals, the triangularis sterni, the serratus posticus inferior, and quadratus lumborum. When by the efforts of the expiratory muscles, the chest has been squeezed to less than its average size, it again, on relaxation of the muscles, returns to the normal dimensions by virtue of its elasticity. The construction of the chest-walls, therefore, admirably adapts them for recoiling against and resisting undue contraction as well as undue dilatation.

\section{Graphic Record of Respiratory Movements.}

Among numerous methods which have been described for recording the respiratory movements the simplest in the case of the human

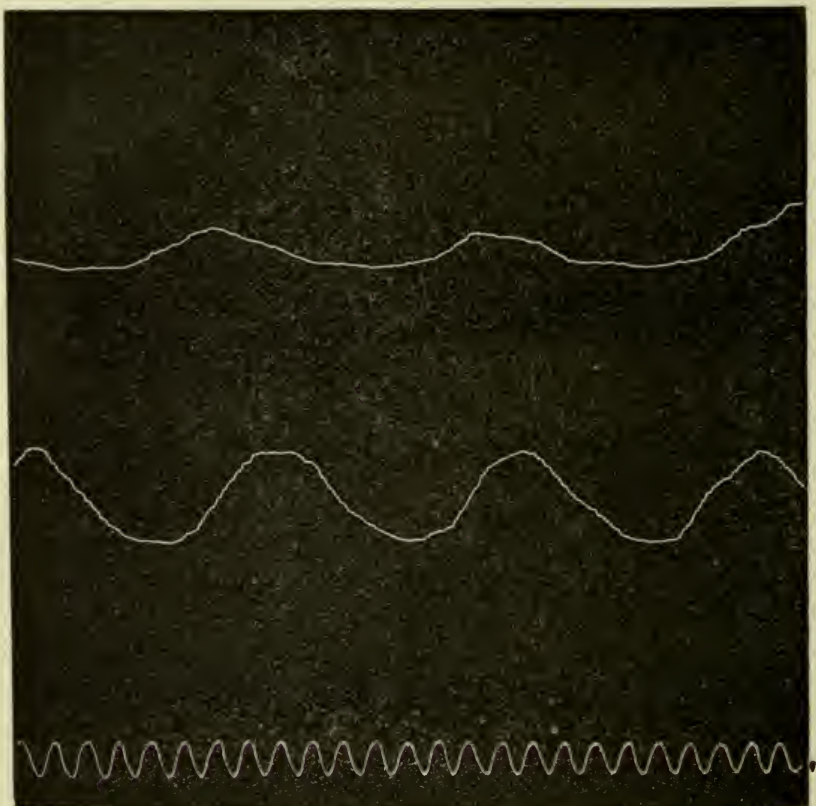

FiG. 290.- Stethograph tracings from the human subject. Each upstroke is due to inspiration; each downstroke to expiration. The lowest line is a time-tracing marking half-seconds.

subject, especially if he be a patient in bed, is to fasten a bandage loosely round the chest. Between the bandage and the chest-wall a flexible hollow rubber ball is placed. This ball communicates by a rubber tube with a recording tambour. All such appliances are 
called Stethographs. In tracings taken with a stethograph applied to the chest-wall of man or animals, the large up-and-down strokes due to respiration have smaller tremors upon them, due to the heartbeats.

The tracings in fig. 290 were obtained by applying a stethograph to a man's chest. During the tracing shown at the top, he was breathing quietly; during the tracing shown on the next line, he was breathing deeply.

It is also possible to record the diaphragmatic movements by the insertion of an elastic bag connected with a tambour into the

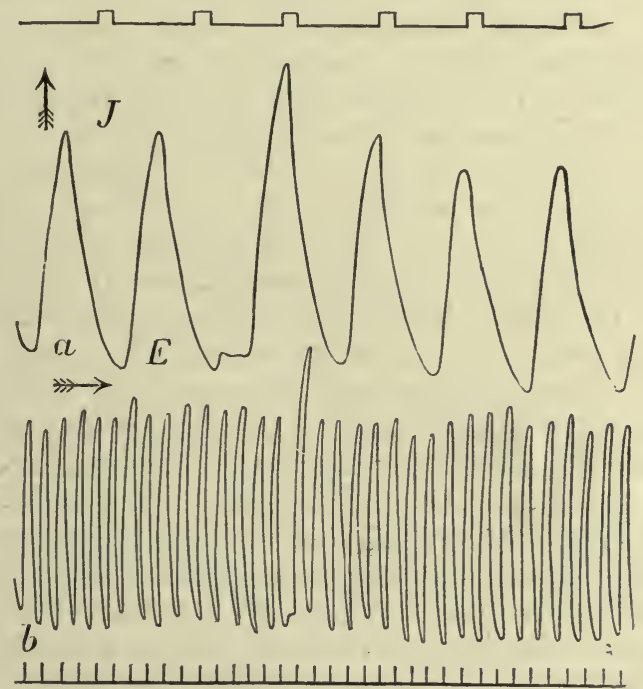

FIG. 291.-Tracing of the normal diaphragm respirations of rabbit. a, With quick movement of drum ; $b$, with slow movement. The upstrokes represent inspiration; the downstrokes, expiration. To be read from left to right. The time tracing in each case represents seconds. (Marckwald.)

abdomen below it (plirenograph), by the insertion of needles into different parts of its structure, or by recording the contraction of isolated strips of the diaphragm. Such a strip attached in the rabbit to the xiphisternal cartilage may be detached, and attached by a thread to a recording lever; this strip serves as a sample of the diaphragm.

Fig. 291 shows a tracing obtained in this way.

The variations of intrapleural pressure may be recorded by the introduction of a cannula into the pleural cavity, which is connected with a mercurial manometer.

The act of inspiring air, especially in women and children, is a little shorter than that of expelling it, and there is commonly a very 
slight pause between the end of expiration and the beginning of the next inspiration.

If the ear is placed in contact with the wall of the chest, or is separated from it only by a good conductor of sound or stethoscope, a faint respiratory or vesicular murmur is heard during inspiration. This sound varies somewhat in different parts-being loudest or coarsest in the neighbourhood of the trachea and large bronchi (tracheal and bronchial breathing), and fading off into a faint sighing as the ear is placed at a distance from these (vesicular breathing). It is best heard in children, and in them a faint murmur is heard in expiration also. The cause of the vesicular murmur has received various explanations; but most observers hold that the sound is produced by the air passing through the glottis and larger tubes, and that this sound is modified in its conduction through the substance of the lung. The alterations in the normal breath sounds, and the various additions to them that occur in different diseased conditions, can only be properly studied at the bedside.

During the action of the muscles which directly draw air into the chest, those which guard the opening through which it enters are not passive. In hurried breathing the instinctive dilatation of the nostrils is well seen, although under ordinary conditions it may not be noticeable. The opening at the upper part of the larynx or rima glottidis is slightly dilated at each inspiration for the more ready passage of air, and becomes smaller at each expiration ; its condition, therefore, corresponds during respiration with that of the walls of the chest. There is a further likeness between the two acts in that, under ordinary circumstances, the dilatation of the rima glottidis is a muscular act and its narrowing chiefly an elastic recoil.

Terms used to express Quantity of Air breathed.--a. Tidal air is the quantity of air which is habitually and almost uniformly changed in each act of breathing. In a healthy adult man it averages about 500 c.c., or rather more than 30 cubic inches, according to the recent measurements made by Haldane. This will be expanded at body temperature to 600 c.c. This amount of air is not sufficient to fill the lungs. Haldane gives the capacity of the upper air-passages and bronchial tubes as 200 c.c., and therefore about a third of the tidal air is required to fill this dead space. At the end of an expiration, however, the tubes and alveoli are not empty of air, and the sudden inrush of atmospheric air during the next inspiration effects a complete mixture of this air with that left in the air-passages; the air in the axial stream of the current will penetrate as far as the alveoli, but what is sucked into the alveoli is mainly some of the mixture from the bronchial passages, and that in turn is derived from the mixture (containing more atmospheric air in proportion) in the upper air-cavities. During expiration 
the air which leaves the lungs will come in part from the alveoli, but the effect of the stream of outgoing air is mainly as before, to effect a thorough admixture of the air in the intermediate air-passages; thus the alveolar air will become mixed with that in the bronchial tubes, and that in turn will be mixed with that in the upper air-chambers. In a succession of alternate ordinary inspirations and expirations adequate ventilation is secured, but obviously the composition of the expired air is not the same as that of alveolar air, for the latter, though it is ultimately breathed out, is diluted on its upward journey by mixture with the bronchial air, and that in its turn with the air of the upper air-chambers ; in other words, the expired air is alveolar air (rich in carbon dioxide) diluted with bronchial air (richer in oxygen) and with atmospheric air (still richer in oxygen).

b. Complemental air is the quantity over and above this which can be drawn into the lungs in the deepest inspiration; its amount averages 100 cubic inches, or about 1600 c.c.

c. Reserve or supplemental air.-After an ordinary expiration, such as that which expels the tidal air, a further quantity of air, about 100 cubic inches (1600 c.c.) can be expelled by a forcible deep expiration. This is termed reserve or supplemental air. The last portion of the air thus expelled will consist of air from the alveoli.

d. Residual air is the quantity which still remains in the lungs after the most violent expiratory effort. Its amount depends in great measure on the absolute size of the chest, but may be estimated at about 100 cubic inches, or about 1600 c.c.

The total quantity of air which passes into and out of the lungs of an adult, at rest, in 24 hours, varies from 400,000 (Marcet) to 680,000 (Hutchinson) cubic inches. This quantity, however, is increased, and may be more than doubled by exertion.

e. Respiratory or Vital Capacity.-The vital capacity of the chest is indicated by the quantity of air which a person can expel from his lungs by a forcible expiration after the deepest inspiration possible. The average capacity of an adult, at $154^{\circ} \mathrm{C}$. $\left(60^{\circ} \mathrm{F}\right.$.), is about 225 to 250 cubic inches, or 3500 to 4000 c.c. It is the sum of the complemental, tidal, and supplemental air.

The respiratory capacity, or as John Hutchinson called it, vital capacity, is usually measured by a modified gasometer or spirometer, into which the experimenter breathes,-making the most prolonged expiration possible after the deepest possible inspiration. The quantity of air which is thus expelled from the lungs is indicated by the height to which the air-chamber of the spirometer rises; and by means of a scale placed in connection with this, the number of cubic inches is read off. Haldane measures the volume of air expired by the reduction which takes place in the volume of the body when placed within a plethysmograph large enough to take a man, with the exception of his head.

The number of respirations in a healthy adult person usually ranges from 14 to 18 per minute. It is greater in infancy and childhood. 
It varies also much according to different circumstances, such as exercise or rest, health or disease, etc. Variations in the number of respirations correspond ordinarily with similar variations in the pulsations of the heart. In health the proportion is about 1 to 4 , or 1 to 5, and when the rapidity of the heart's action is increased, that of the chest movement is commonly increased also; but not in every case in equal proportion. It happens occasionally in disease, especially of the lungs or air-passages, that the number of respiratory acts increases in quicker proportion than the beats of the pulse; and, in other affections, much more commonly, that the number of the pulse-beats is greater in proportion than that of the respirations.

The Frorce of Inspiratory and Expiratory Muscles.-The force with which the inspiratory muscles are capable of acting is greatest in individuals of the height of from five feet seven inches to five feet eight inches, and will elevate a column of nearly three inches (about $60 \mathrm{~mm}$.) of mercury. Above this height the force decreases as the stature increases; so that the average of men of six feet can elevate only about two and a half inches of mercury. The force manifested in the strongest expiratory acts is, on the average, one-third greater than that exercised in inspiration. But this difference is in great measure due to the power exerted by the elastic reaction of the walls of the chest; and it is also much influenced by the disproportionate strength which the expiratory muscles attain, from their being called into use for other purposes than that of simple expiration. The force of the inspiratory act is, therefore, better adapted than that of the expiratory for testing the muscular strength of the body. (John Hutchinson.)

In ordinary quiet breathing, there is a negative pressure of only $1 \mathrm{~mm}$. during inspiration, and a positive pressure of from 2 to $3 \mathrm{~mm}$. mercury during expiration.

The instrument used by Hutchinson to gauge the inspiratory and expiratory power was a mercurial manometer, to which was attached a tube fitting the nostrils, and through which the inspiratory or expiratory effort was made.

The greater part of the force exerted in deep inspiration is employed in overcoming the resistance offered by the elasticity of the lungs.

In man the pressure exerted by the elasticity of the lungs alone is about $6 \mathrm{~mm}$. of mercury. This is estimated by tying a manometer into the trachea of a dead subject, and observing the rise of mercury that occurs on puncture of the chest-walls. If the chest is distended beforehand so as to imitate a forcible inspiration, a much larger rise $(30 \mathrm{~mm}$.) of the mercury is obtained. During life this elastic force is assisted by the contraction of the plain muscular fibres - of the alveoli and bronchial tubes, the pressure of which probably does not exceed 1 or $2 \mathrm{~mm}$. Hutchinson calculated that the total force to be 
overcome by the muscles in the act of inspiring 200 cubic inches of air is more than $450 \mathrm{lbs}$.

It is possible that the contractile power which the bronchial tubes and air-vesicles possess, by means of their muscular fibres, may assist in expiration; but it is more likely that the chief purpose of this muscular tissue is to regulate and adapt, in some measure, the quantity of air admitted to the lungs, and to each part of them, according to the supply of blood: the muscular tissue also contracts upon and gradually expels collections of mucus, which may have accumulated within the tubes, and which cannot be ejected by forced expiratory efforts, owing to collapse or other morbid conditions of the portion of lung connected with the obstructed tubes (Gairdner).

\section{The Gases of the Blood.}

Before the student can study either the chemistry of respiration or its regulation, which is in part a chemical process, it is necessary that he should have an adequate conception of the fundamental laws which regulate the retention of oxygen and carbonic acid in the blood; and as the blood presents many complications, it will be best at the outset to consider the solution of gases in such a simple medium as water.

\section{Solution of Gases in Water.}

If water is shaken up with oxygen, a certain definite amount of oxygen will become dissolved in the water. Under the same conditions the same quantity of oxygen would always be dissolved, and in the following argument it is assumed throughout that the temperature remains constant. The amount dissolved depends then upon two circumstances, each of which can be measured. The first is the pressure of the oxygen to which the water is exposed when shaken; the second is a property of the oxygen itself, namely, its solubility in water. The solubilities of different gases differ very much; some (for instance, oxygen) are not readily soluble in water, whilst others, such as carbonic acid, are very soluble.

If a cubic centimetre of water was introduced into a large airtight bottle containing pure oxygen at the atmospheric pressure, and another cubic centimetre of water was similarly placed in a bottle containing pure carbonic acid at the same pressure, the former would be found to have dissolved 0.04 c.c. of oxygen, the latter 1 c.c. of carbonic acid. These figures represent the degrees to which the two gases are soluble in water under similar circumstances, and are called their coefficients of solubility. The coefficient of solubility of gas in a liquid is therefore the volume of gas which 1 c.c. of the liquid will dissolve at $760 \mathrm{~mm}$. of mercury, that is, atmospheric pressure. 
The quantity of gas which a liquid will dissolve depends not only on the solubility of the gas, but upon the pressure of the gas to which the liquid is exposed. Thus, in the instance given above, if the oxygen had been rarefied in the bottle until it only exerted a pressure of one-fifth of an atmosphere, the water would have taken up not 0.04 c.c. of oxygen, but only one-fifth of that amount, 0.008 c.c. To take another example, 1 c.c. of water shaken up with pure nitrogen at $760 \mathrm{~mm}$. pressure will dissolve 0.02 c.c. ; but suppose the pressure to be reduced to four-fifths of the atmospheric pressure the water will dissolve $.02 \times \frac{4}{5}=.016$ c.c. If we represent the coefficient of solubility of a gas by $\mathrm{K}$, and the pressure of the gas to which the liquid is exposed by $\mathrm{P}^{\prime}$, and the atmospheric pressure by $\mathrm{P}$; then the quantity (Q) of the gas dissolved by 1 c.c. of the liquid may be obtained by the following formula-

$$
\mathrm{Q}=\mathrm{K} \times \frac{\mathrm{P}^{\prime}}{\mathrm{P}}
$$

\section{Dalton-Henry Law.}

What has been said above is as true of gases which are mixed together as of pure gases. For instance, we have seen that a cubic centimetre of water shaken up with oxygen at one-fifth of an atmosphere $\left(153 \mathrm{~mm}\right.$. pressure) will absorb $.04 \times \frac{1}{5}=.008$ c.c. ; or if shaken with nitrogen at a pressure of four-fifths of an atmosphere, it will dissolve $.02 \times \frac{4}{5}=.016$ c.c. If now a c.c. of water be shaken with air (a mixture of one part of oxygen to four of nitrogen), it will have absorbed .008 c.c. of oxygen and .016 c.c. of nitrogen. This fact has been stated as the Dalton-Henry Law in the following words:When two or more gases are mixed together, each of them produces the same pressure as if they separately occupied the entire space and the other gases were absent. The total pressure of the mixture is the sum of the partial pressures of the individual gases in the mixture.

\section{The Tension of Gases in Fluids.}

In the cases which have been discussed up to this point, a condition of equilibrium exists between the gas dissolved in the fluid and the gas in the atmosphere to which the fluid is exposed, so that as many molecules of the gas leave the surface of the fluid as enter it. The gas dissolved in the fluid therefore exercises a pressure which is the same as that of the gas in the atmosphere when equilibrium exists. For the sake of convenience the word Tension is applied to the pressure of the gas in the fluid.

Definition of Tension.- The tension of a gas dissolved in-a fluid is equal to the pressure of the same gas in an atmosphere with which the gas in the fluid would be in equilibrium. Above, wo have called 
the pressure which the gas exerts on the liquid, $\mathrm{P}^{\prime}$. If we call the tension of the gas in the liquid, T, we find that when equilibrium exists, $\mathrm{P}^{\prime}=\mathrm{T}$. In the case of all true solutions, therefore, we may replace $\mathrm{P}^{\prime}$ in our previous equation by $\mathrm{T}$; therefore, $\mathrm{Q}=\mathrm{K} \times \frac{\mathrm{T}}{\mathrm{P}}$. We thus arrive at a relation between two separate things, which must be most carefully distinguished from one another-the quantity of the gas dissolved in the liquid and its tension.

Measurement of Tension.- Numerous instruments, called tonometers, exist for measuring the tension of gases in fluids. Of these, the instrument which has given the most trustworthy measurements of the oxygen and carbonic acid tensions in circulating blood is that invented by Krogh (fig. 292).

A T-shaped cannula (A) is introduced into the blood-vessel, say the carotid artery; the blood fills the cavity $\mathrm{B}$ and leaves it at $\mathrm{C}$, so that a constant stream of blood is kept

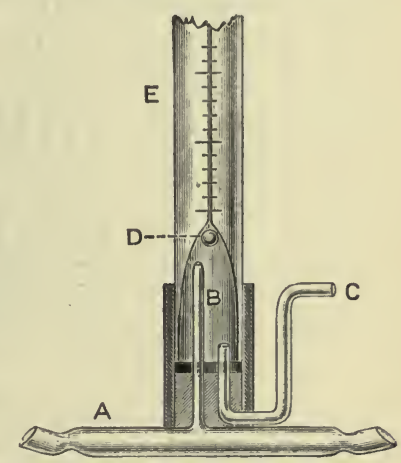

FIG. 292.-Krogh's Tonometer. flowing. Into it a small bubble of air (D) is introduced. Exchange of gases takes place between the bubble and the blood, and the former very soon gets into equilibrium with the latter. When it has done so, the bubble is withdrawn up the capillary tube $\mathrm{E}$, taken away, and analysed.

As an example, suppose the bubble on analysis proved to consist of 4 per cent. carbonic acid and 12 per cent. oxygen, together with nitrogen and aqueous vapour. The gas in the instrument was compressed by the pressure of the arterial blood (say $120 \mathrm{~mm}$. of mercury) in addition to the atmospheric pressure of $760 \mathrm{~mm}$. of mercury, and therefore the total pressure was $120+760=880 \mathrm{~mm}$. of mercury. Four per cent. of this would have been due to the carbonic acid; 4 per cent. of 880 is 35.2 . Twelve per cent. would have been due to the oxygen; 12 per cent. of 880 is 105.6 . That is, the carbonic acid and oxygen tensions would have been in round figures 35 and $106 \mathrm{~mm}$. of mercury respectively.

\section{Measurement of the Quantity of the Gases of the Blood.}

This may be done by means of an air-pump, or by a chemical method. (1) The extraction of the gases from the blood by means of the mercurial air-pump depends upon the fact that blood gives off all its gases when it is boiled in a vacuum. The total gas obtained is first measured; then the carbon dioxide is removed by caustic 
potash, and the gas that remains consists of oxygen and nitrogen; the oxygen is then removed by pyrogallic acid, and the residual gas

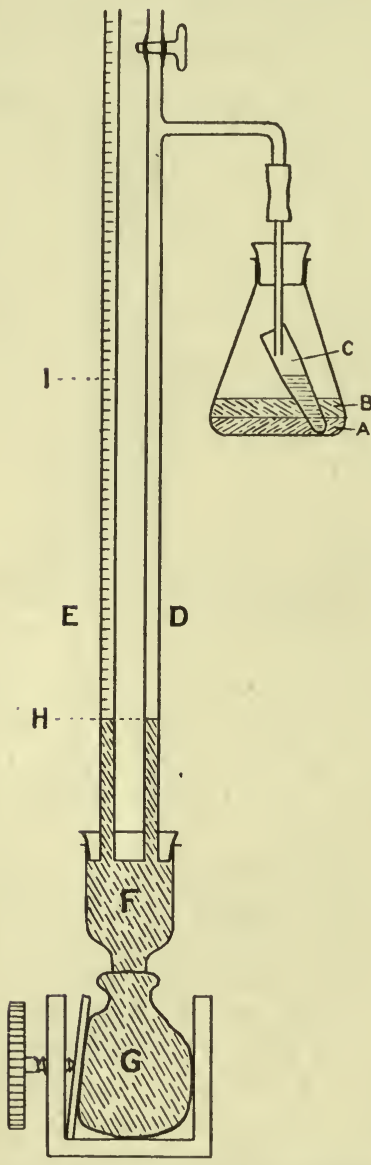

Fig. 293.-Apparatus for blood-gas analysis. is nitrogen. (2) The chemical method * adopted is as follows:-

When a solution of oxyhæmoglobin is shaken with potassium ferricyanide, it yields the same amount of oxygen to the air as it would if boiled in a vacuum. In much the same way urea when treated with sodium hypobromite yields up all its nitrogen, and the apparatus used for determining the oxygen in blood is very similar to a Dupré's urea apparatus (see Chapter XXXIX.). The blood (5 c.c.) is placed in the large bottle (fig. 293, A) underneath a layer of dilute ammonia solution (B). The blood is thus protected from the air whilst the apparatus becomes equal in temperature to the bath in which it is placed. The blood is shaken with the ammonia solution, which lakes it thoroughly; the ferricyanide solution is then spilt into the laked blood from the tube $\mathrm{C}$, and the oxygen is shaken out of the solution. When the oxygen has been determined the bottle is opened and tartaric acid is placed in the small tube $\mathrm{C}$; this is subsequently spilt into the mixture of blood, ammonia, and ferricyanide; it liberates the carbonic acid which is also shaken out of the fluid. The carbonic acid does not come completely out, however, and a correction has to be introduced for the quantity which remains in solution. The gas (oxygen or carbonic acid, as the caise may be) passes over into the tube D, which was previously filled up to the zero mark with water, and connected to a reservoir (F); this would drive water out of $\mathrm{F}$

* The apparatus figured is one of the earliest, and many modifications have been invented since which are in use in different laboratories. The differences are, however, in detail only; the principle throughout is the same. The details of the mercury pump I have considered it best to omit. They are very complicated, and the forms of pump used vary greatly in different laboratories; the method can only be learnt by actually using the instrument. 
into the open tube $\mathrm{E}$, and the water will therefore rise in $\mathrm{E}$; but in practice it is convenient to keep the gas always at the same volume; this may be done by raising the pressure in the open limb $(\mathrm{E})$ of the pressure gauge by squeezing some of the water, with which the gauge is filled, out of a rubber reservoir $(G)$ which forms the base of the gauge, thus the level of the water in $\mathrm{D}$ is maintained at the zero mark, while that in $\mathrm{E}$ rises from $\mathrm{H}$ to $\mathrm{I}$. The actual measurement then is the increase of pressure (i.e., the height of the column of water H I) which is necessary to keep the gas at the same volume after the oxygen or carbonic acid has been shaken off as it previously occupied. From this the quantity coming off can be calculated.

The chemical method is not quite so accurate as the vacuum pump, but it is much more convenient for the study of many problems, as it requires less blood, and, owing to its simplicity, a great number of observations can be made upon a single animal, or it can be used for the systematic investigation of the blood in man.

\section{Relation between Quantity and Tension of Gases in Blood.}

In the preceding paragraphs the methods of measuring the tension and the quantity of gas in a given sample of blood have been described. It is now necessary to consider the relationship between them.

On page 363 we have seen that for gases in solution in water, $\mathrm{Q}=\mathrm{K} \times \frac{\mathrm{T}}{\mathrm{P}}$ where $\mathrm{Q}$ is the quantity of gas dissolved, $\mathrm{T}$ the tension, $\mathrm{K}$ the coefficient of solubility, and $\mathrm{P}$ the atmospheric pressure. Since $K$ and $P$ are constant, it follows that $Q$ varies directly in proportion to $\mathrm{T}$; that is to say, if the tension is doubled, the quantity of gas dissolved is also doubled; if the tension is trebled, the quantity of gas is trebled, and so on. These results might be plotted out on a curve in which the quantities are placed on the ordinate and the tension on the abscissa. Such a curve would give the quantity of gas dissolved at any given tension, and in the case of water the curve would turn out to be a straight line.

But in the case of both the oxygen and the carbonic acid in blood, the curve is not a straight line.

Oxygen in Blood.-From every 100 c.c. of arterial blood, about 20 c.c. of oxygen can be removed by the air-pump. Nearly all of this oxygen is chemically combined with hæmoglobin; the amount in actual solution in the blood is 0.7 c.c. for every 100 c.c. of blood. The quantity of oxygen which 100 c.c. of blood takes up is called the "oxygen capacity," which should not be confounded with the "specific oxygen capacity" defined below. In normal human blood, the figure is 18.5 c.c., and this forms the basis of standardisation of hæmoglobinometers (p. 445). 
Hæmoglobin owes its value as a respiratory pigment to two principal facts. (1) It can unite with a large quantity of oxygen, and therefore blood can carry about thirty times as much oxygen to the tissues as plasma would under the same circumstances. (2) The interaction between hæmoglobin and oxygen is a reversible one; the two unite in the lungs, where the pressure of oxygen is high; but when oxygen is absent or at a low pressure, as in the tissues, the hæmoglobin parts with its store of oxygen.

The reaction between hæmoglobin and oxygen is a chemical one. At most, one gramme of hæmoglobin can unite with 1.34 c.c. of oxygen. This figure is not quite constant, probably on account of slightly different forms of globin (the protein constituent of hæmoglobin) united with the hæmatin (the iron containing constituent) in different animals. The relation between the respiratory oxygen and the iron of the hæmoglobin is, however, quite constant, and is called the "specific oxygen capacity." Each gramme of iron in hæmoglobin unites with 400 c.c. of oxygen; these figures are in the relation of one atom of iron to two atoms of oxygen. The reversible nature of the reaction may therefore be expressed by the equation $\mathrm{Hb}+\mathrm{O}_{2} \rightleftarrows \mathrm{HbO}_{2}$. A reversible reaction means that it will go in either direction according to the concentration of the substances present; thus if the concentration of oxygen in solution is increased,

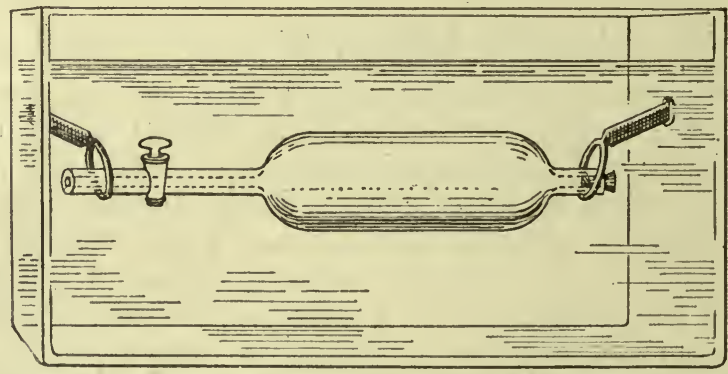

Fig. 294.-Barcroft's Tonometer, suspended horizontally in warm bath in which it is rotated.

more of the hæmoglobin will become oxyhæmoglobin; and if it is diminished, oxyhæmoglobin will break up into hæmoglobin (sometimes called reduced hæmoglobin) and oxygen.

The reader must be clear that when we speak of the concentration of oxygen in solution, we mean in physical solution, that is, not united chemically with the hæmoglobin. This quantity varies in the direct ratio of the oxygen pressure to which the hæmoglobin solution is exposed; therefore the problem before us is to determine the relative quantities of $0 x y-$ and reduced hæmoglobin when a hæmoglobin solution is shaken up with oxygen at different pressures. 
Suppose that we have six vessels similar to that in fig. 294 (Barcroft's Tonometer), and each contains a few c.c. of a solution of hæmoglobin and gases of the following composition:-

No. 1. Nitrogen and no oxygen.

No. 2. Nitrogen and enough oxygen to give $5 \mathrm{~mm}$. oxygen pressure.

No. 3.

No. 4 .

No. 5 .

No. 6.

", , ,

$\begin{array}{rr}\text {, } & 10 \\ \text {, } & 20 \\ \text {, } & 50\end{array}$

10
20
50
100

",

,

,

,

Each tonometer is rotated round and round in a bath at body temperature until the hæmoglobin and the oxygen are in equili-

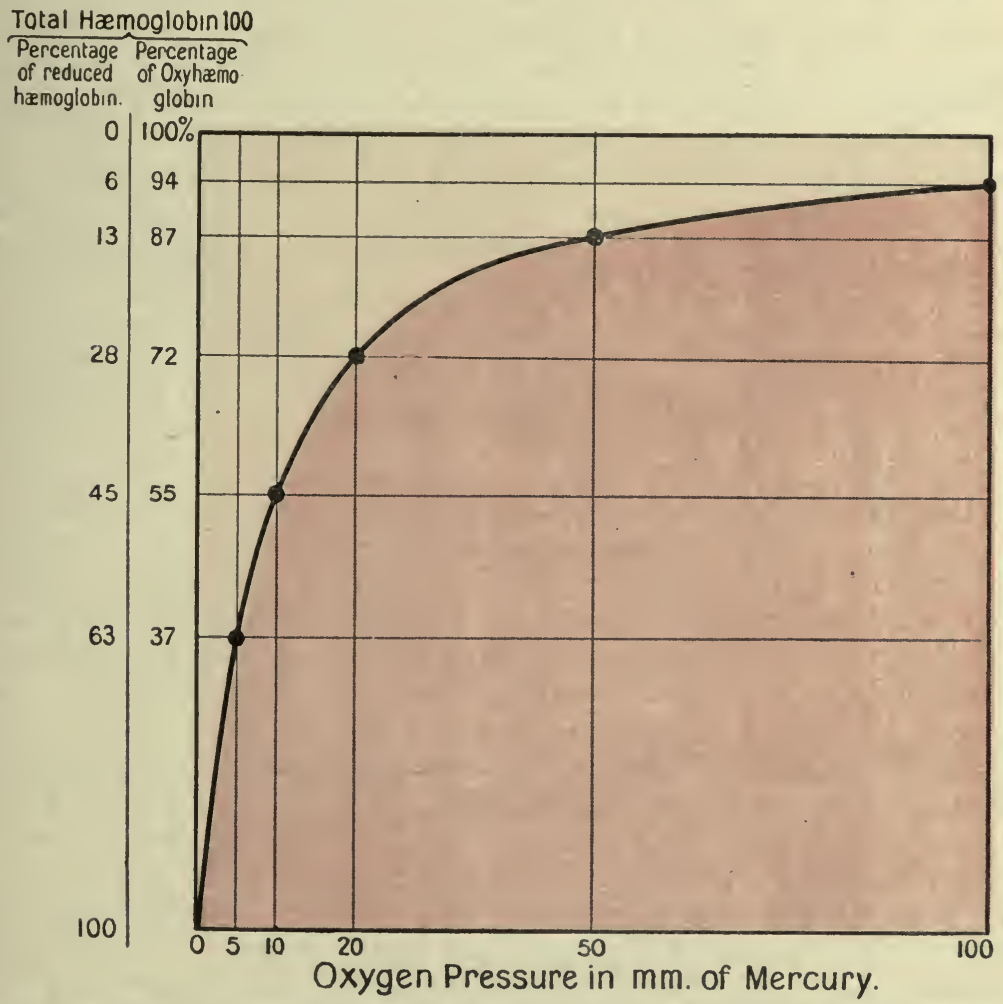

F1G, 295.-Dissociation curve of hæmoglobin solution at $37^{\circ} \mathrm{C}$. Purple, reduced hæmoglobin; red, oxyhæmoglobin.

brium; this will take about a quarter of an hour; the solution is then withdrawn and examined in order to ascertain the relative quantities of oxy-and reduced hæmoglobin in the six vessels. 
The figures for a pure solution of hæmoglobin would be:-

\begin{tabular}{lrrrrrrr} 
& No. 1. & No. 2. & No. 3. & No. 4. & No. 5. & No. 6. \\
Percentage of hæmoglobin & 100 & 63 & 45 & 28 & 13 & 6 \\
Percentage of oxyhæmoglobin & 0 & 0 & 37 & 55 & 72 & 87 & 94 \\
\hline & $\frac{100}{100}$ & $\frac{10}{100}$ & $\frac{10}{100}$ & $\frac{10}{100}$ & $\frac{100}{100}$
\end{tabular}

The same answer may be expressed graphically; if the pressures of oxygen are plotted horizontally, and the percentages of oxyand reduced hæmoglobin in the solution are plotted vertically, we get the curve shown in the accompanying diagram (fig. 295), which is called the dissociation curve of homoglobin.

In a reversible reaction such as the one under consideration, the molecules are continually forming and breaking up again, and the rate at which this occurs is influenced by various conditions. Among the conditions of importance in the body are (1) temperature; (2) the presence of salts; and (3) the presence of acids, especially carbonic acid. These factors tend to make the oxyhæmoglobin molecules break down more rapidly and form more rapidly. From the point of view of the body's needs, it is clearly necessary, not only that hæmoglobin should acquire oxygen at the pressure of that gas in the lungs, and part with it at the diminished oxygen pressure in the tissues, but that the two processes should occur at about the same rate, that is, within one second, which is about the time occupied by any given portion of blood in travelling along the capillaries (see p. 282).

It would be futile to have an oxygen carrier in the blood which took a fraction of a second to acquire its oxygen, and a fraction of an hour to release it. Yet a solution of pure hæmoglobin is just such a substance, for its power of acquiring oxygen is very great, and its power of releasing it is very small. Happily, however, a hæmoglobin solution and blood are two very different things. In the red corpuscles, the hæmoglobin is dissolved in a medium containing various salts of which those of potassium are most prominent; these salts confer on hæmoglobin the property of giving up its oxygen much more readily when exposed to low concentrations of oxygen such as are present in the capillaries of the tissues, while at the same time it acquires oxygen more readily at higher oxygen pressures such as blood is exposed to in the lungs. But the mere presence of potassium salts is not in itself capable of rendering the hæmoglobin an efficient oxygen carrier; two other factors come into play in the body - one is the high temperature, and the second is the presence of carbonic acid. This is illustrated in the curves depicted in fig. 296. - To obtain the rate of oxidation, oxygen at alveolar pressure, mixed with nitrogen, was passed through reduced blood; to obtain the rate of reduction, nitrogen, free from oxygen, was passed through 
oxygenated blood. The vertical figures represent the percentage saturation of blood with oxygen; the horizontal figures represent time in minutes.

It will be seen that at room temperature (A), the rate of oxidation is rapid, but the rate of reduction is very slow. At body temperature the rate of reduction is enormously increased (B); but at body temperature in the presence of carbonic acid the two rates are practically equal $(\mathrm{C})$; the amount of carbonic acid employed was the same as that which pervades the body, viz., at a tension of $40 \mathrm{~mm}$. of mercury. This quantity is also approximately equal to that present
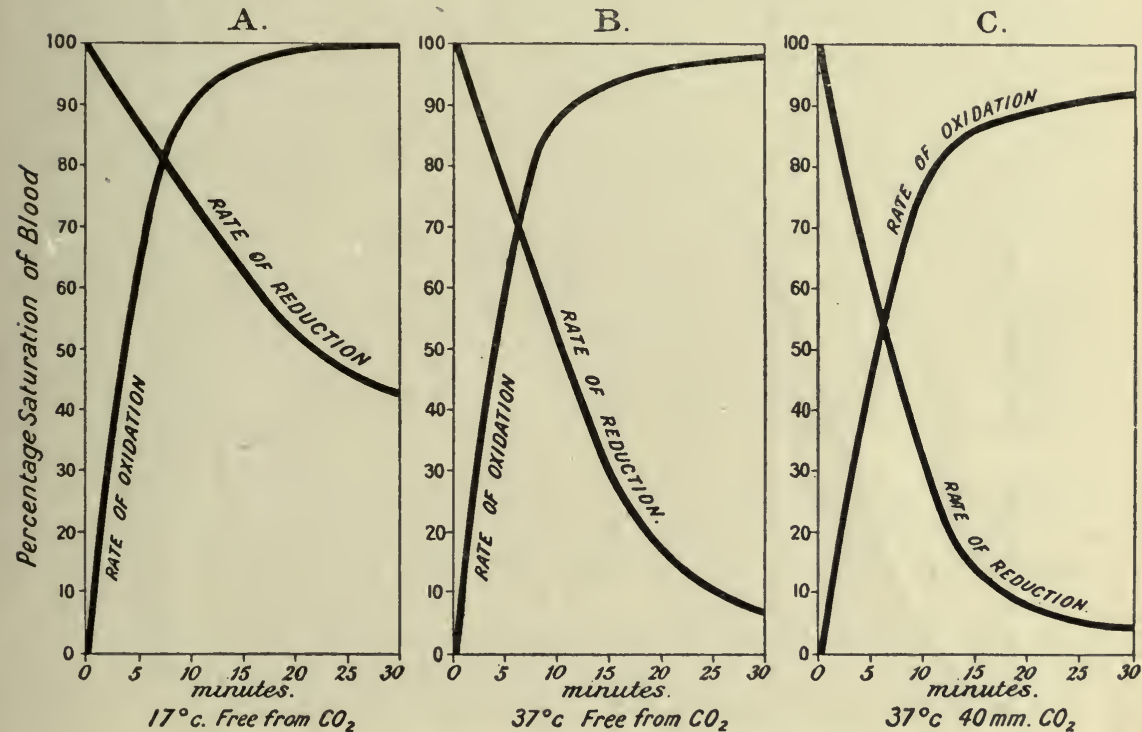

FIG. 296.-Curves showing rate of oxidation and reduction in blood under the conditions described in the text; A, at room temperature $\left(17^{\circ}\right.$ C. $) ; B$, at body temperature $\left(37^{\circ}\right.$ C. $)$; C, at body temperature when carbon dioxide at $40 \mathrm{~mm}$. pressure was added to the nitrogen.

in the alveolar air. The curves show that the rate of reductiou is increased, but the rate of oxidation is a little lessened. The two curves in $\mathrm{C}$ present an extraordinary degree of symmetry, so wonderfully has nature adapted the conditions of life in order that the needs of the body may be served by a substance hæmoglobin which by itself is ill adapted for the purpose of oxygen transport.

In order therefore to understand the dissociation of oxygen from hæmoglobin in the body, we must study it, not in a pure solution, but under the more complex conditions actually existing in the body. 
Under these conditions, viz., in the presence of potassium salts, at $37^{\circ} \mathrm{C}$, and in the presence of a carbonic acid pressure of $40 \mathrm{~mm} . \mathrm{Hg}$, the dissociation curve is that shown in the next diagram (fig. 279).

The two coloured figures (295 and 297) should be carefully compared; they present to the eye graphically the superiority of hæmoglobin as an oxygen carrier when it is present in the actual blood, over that which it possesses in a pure solution. In the second

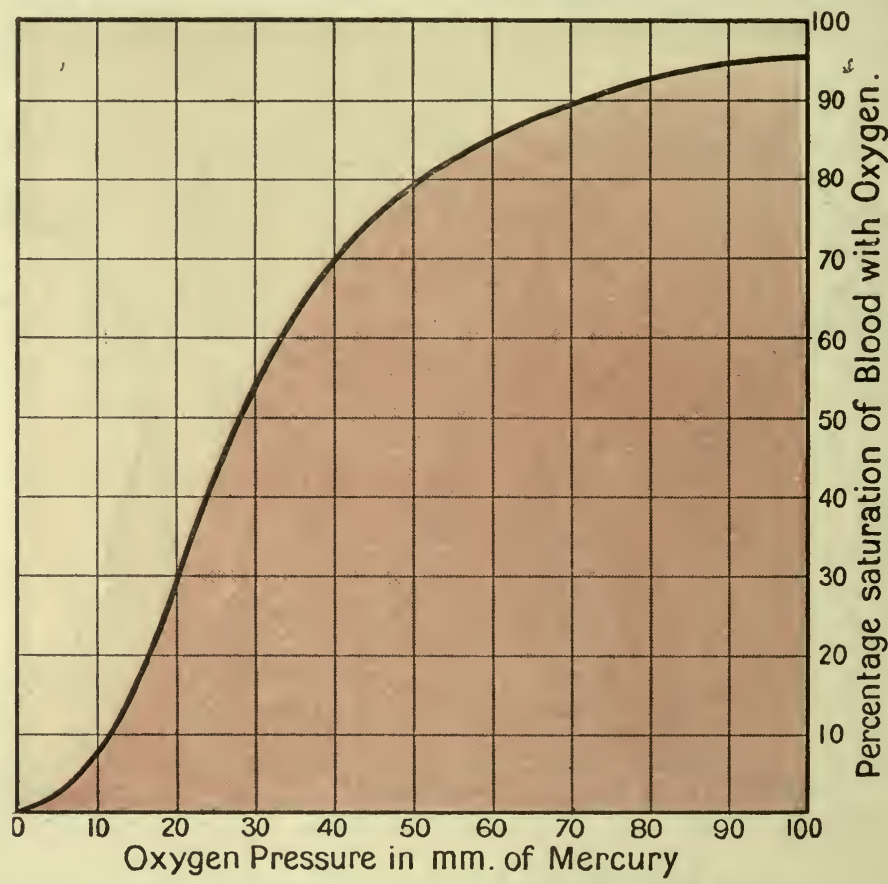

Fig. 297.-Dissociation curve of hæmoglobin in the actual blood. Purple, reduced hæmoglobin; red, oxyhæmoglobin.

curve, that of the blood itself, it will be seen that at an oxygen pressure of over $60 \mathrm{~mm}$. of mercury (the pressure in the lung alveoli is about 100) the blood will nearly saturate itself with oxygen, and that at prossures below 50 the blood loses its oxygen rapidly, whilst at $10 \mathrm{~mm}$. pressure it is nearly completely reduced. As the rate at which oxygen can diffuse out of the capillaries into the surrounding tissues depends upon the pressure it exerts in the plasma, it is important that the hæmoglobin of the blood should be capable of a 
considerable degree of reduction when it is in contact with fluid containing oxygen at a pressure of 20 to $30 \mathrm{~mm}$. of mercury.

Carbonic Acid in Blood.-If blood is divided into plasma and corpuscles, it will be found that both yield carbonic acid, but the yiold from the plasma is the greater. If we place blood in a vacuum it bubbles, and gives out all its gases; addition of a weak acid causes no further liberation of carbonic acid. When plasma or serum is similarly treated the gas also comes off, but about 5 per cent. of the carbonic acid is fixed-that is, it requires the addition of some stronger acid, such as phosphoric or tartaric acid, to displace it.

One hundred volumes of venous blood contain about forty-six volumes of carbonic acid. Whether this is in solution or in chemical combination is determined by ascertaining the tension of the gas in the blood. One hundred volumes of blood plasma would dissolve about an equal volume of the gas from an atmosphere of carbonic acid, if its solubility in plasma were equal to that in water. If, then, the carbonic acid were in a state of solution, its tension would be very high, but, as we have seen, it proves to be only equal to about 5 per cent. of an atmosphere (that is approximately equal to $40 \mathrm{~mm}$. of mercury). This means that when venous blood is brought into an atmosphere containing 5 per cent. of carbonic acid, the blood neither gives off any carbonic acid nor takes up any from that atmosphere. Hence the remainder of the gas, 41 per cent., is in a condition of chemical combination.

Of the carbonic acid present in chemical composition, the greater part is present as sodium bicarbonate $\left(\mathrm{NaHCO}_{3}\right)$. About a third is in loose combination with the proteins of the blood, and a small quantity exists as normal sodium carbonate $\left(\mathrm{Na}_{2} \mathrm{CO}_{3}\right)$.

Differences between Arterial and Venous Blood.-The average quantity of gas that can be extracted from arterial and venous blood respectively is :-

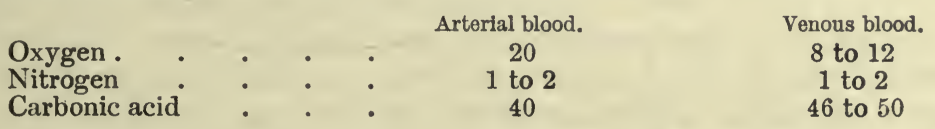

It will be noticed that the amount of nitrogen which is simply dissolved in the blood from the air is small in amount. It has no physiological significance, and is the same in both varieties of blood. The important distinction between arterial and venous blood is in the other two gases, and as the table shows, on the average every 100 c.c. of blood which pass through the lungs gain 8 c.c. of oxygen and lose 6 c.c. of carbonic acid. We will now study the mechanism by which this gaseous interchange is effected. 


\section{The Mechanism of Gaseous Exchange in the Lung.}

\section{Oxygen.}

The simplest explanation of the passage of oxygen from the alveolar air into the blood is that the process is one of diffusion. This view (which is generally regarded as adequate in the case of normal respiration) can be maintained if it can be proved that the pressure of oxygen in the alveolar air is as great or greater than the tension. of oxygen in the arterial blood, and therefore $a$ fortiori greater than that of oxygen in the venous blood.

The conception of respiration based upon this view would be that the pressure of oxygen in the air of the alveoli though less than that in the atmosphere, is greater than that in venous blood; hence oxygen passes from the alveolar air into the blood plasma; the oxygen immediately combines with the hæmoglobin, and thus leaves the plasma free to absorb more oxygen; and this goes on until the hæmoglobin is entirely, or almost entirely, saturated with oxygen. The reverse change occurs in the tissues when the partial pressure of oxygen is lower than in the plasma, or in the lymph that bathes the tissue elements; the plasma parts with its oxygen to the lymph, the lymph to the tissues; the oxyhæmoglobin then undergoes dissociation to supply more oxygen to the plasma and lymph, and thus in turn to the tissues once more. This goes on until the oxyhæmoglobin loses on the average about half of its store of oxygen; 1 c.c. of arterial blood contains 0.2 c.c. oxygen; 1 c.c. of venous blood contains 0.1 c.c. oxygen.

Haldane and Priestley introduced a very simple method of collecting alveolar air which has the advantage of being applicable to man. A piece of rubber tubing is taken about 1 inch in diameter and about 4 feet long. A mouthpiece is fitted into one end. About 2 inches from the mouthpiece a small hole is made into which is inserted the tube of a gas-receiver, or sampling-tube, as in the figure (fig. 298). The gas-receiver is fitted at the upper end with a three-way tap, and the lower end is also closed by a tap. Before it is used, it is filled with mercury. The subject of the experiment breathes normally through the tube for a time, and then, at the end of a normal inspiration, he expires quickly and very deeply through the mouthpiece and instantly closes it with his tongue. The lower tap of the receiver is then turned, and as the mercury runs out, a sample of the air takes its place and fills the receiver; this sample is then analysed. A second experiment is then done, in which the subject expires deeply at the end of a normal expiration, and another sample obtained. The mean 
result of the two analyses represents the mean composition of the alveolar air. Since the gaseous interchange between the blood and the alveolar air is going on continuously, it is evident that at the end of inspiration there will be a maximum percentage of oxygen, and a minimum percentage of carbonic acid; the converse obtains at the end of expiration. These observers proved by other considerations which it is unnecessary to go into, that the air obtained was really the alveolar or residual air unmixed with any of the air of the "dead space" of the respiratory passages.

From the analysis of this air, they arrived at the conclusion that the normal oxygen pressure in it is 13 per cent. of an atmosphere (that is approximately equal to $100 \mathrm{~mm}$. of mercury).

The other important measurement, namely that of the tension of oxygen in the blood, is made by Krogh's tonometer (p. 363), and the experiments show that diffusion is quite sufficient to account

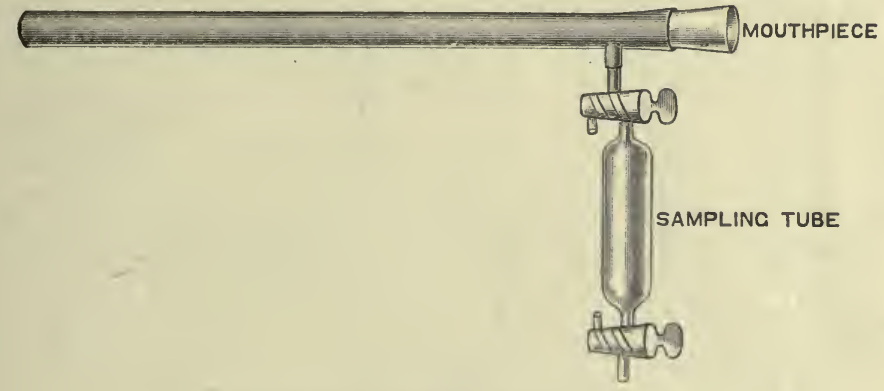

Fic. 298.-Apparatus for obtaining alveolar air.

for the passage of oxygen from the alveoli to the blood. The following experiment may be cited as an example, and will be best understood by the next diagram (fig. 299).

It shows the relations between the pressures of oxygen and carbonic acid in the air of the lungs and the blood respectively. The pressures of gas are measured vertically, and expressed as percentages of an atmosphere; the horizontal measurements are those of time. It will be seen that the experiment consisted of three periods, separated by the vertical lines A and B. During the first and last periods the animal breathed a mixture consisting of 14.7 per cent. oxygen, and the remainder nitrogen; in the middle period ordinary air containing nearly 21 per cent. of oxygen was breathed. It will be seen that the oxygen pressure of the alveolar air (dotted line) is always higher than the oxygen tension of the arterial blood (continuous black line); as the former rises the latter rises, and vice versa. The lower part of the diagram shows the 
relationships of the carbon dioxide; the alveolar tension of this gas (dotted line) is always lower than that of the arterial blood (continuous black line). It will further be noticed that the pressure differences are less in the case of carbonic acid than in that of oxygen; this coincides with the ease with which carbonic acid passes out through the membrane which separates the blood from the air.

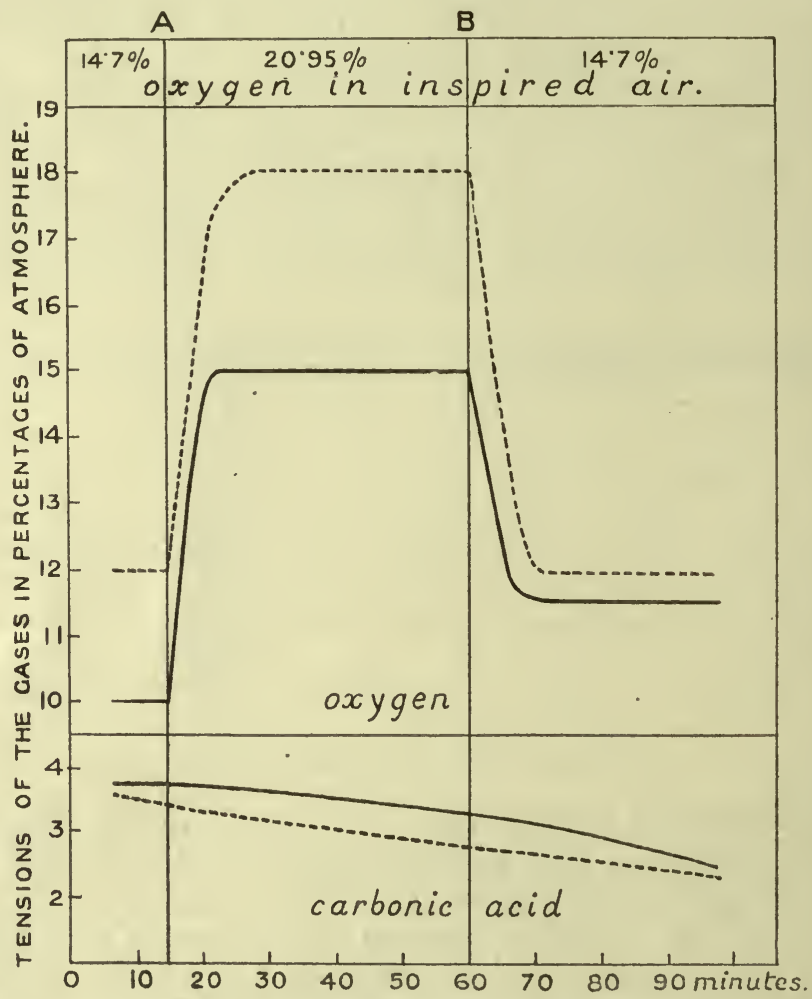

Fra. 299.-Diagram to represent the relationship between the tensions of oxygen and carbon dioxide in blood and alveolar air in rabbit (after Krogh).

Some authorities consider that in cases of definite oxygen want, such as during violent muscular exercise, or on the tops of high mountains, the lining epithelium of the pulmonary alveoli can, by a process of active secretion, transfer oxygen from the alveolar air to the blood. In the case of exercise the observations made by different workers are at variance, whilst at high altitudes they are so few as to make further work desirable before physiologists generally can accept the possibility of the secretion of oxygen by the lung. That 
secretion is not impossible is shown by the fact that a similar secretion of oxygen is known to occur in the swim-bladder of certain fishes. The swim-bladder corresponds morphologically with the lungs of a mammal, and the oxygen stored in it is far in excess of anything that can be explained by mere diffusion from the sea-water. This storage of oxygen, moreover, ceases when the vagus nerves which supply the swim-bladder are divided.

\section{Carbonic Acid.}

The tension of carbonic acid in the alveolar air is measured, like that of oxygen, by the method of Haldane and Priestley, whilst the tension in the blood is measured by the tonometer.

The tension of the carbonic acid in the tissues is high, but one cannot give exact figures; we can measure the tension of the gas in certain secretions: in the urine it is 9 , in the bile 7 per cent. The tension in the cells themselves must be higher still.

The following figures (from Fredericq) give the tension of carbonic dioxide in percentages of an atmosphere:-

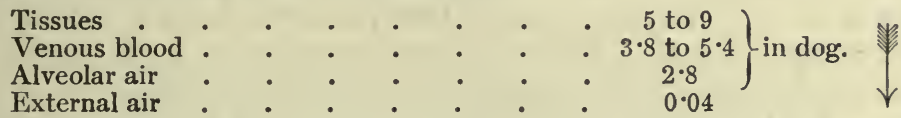

The arrow indicates the direction in which the gas passes, namely, in the direction of pressure from the tissues to the atmosphere.

In view of the above figures and of such experiments as thit of Krogh, cited on the opposite page, and having regard to the very slight changes in the tension of carbonic acid in the alveolar air, which are capable of affecting the respiratory centre (a subject we shall immediately pass to), we shall adhere to the view that diffusion explains the passage of that gas from the blood to the alveolar air, and that it is unnecessary to call to our assistance the hypothesis that secretory activity of the alveolar epithelium is at work.

\section{Cause and Regulation of Respiration.}

There are three factors, each of which plays a part in maintaining and regulating the rhythmic movements of respiration. They are the respiratory centre, the vagus nerves, and the chemical condition of the blood.

\section{The Respiratory Centre.}

In the central nervous system there is a specialised small district called the respiratory centre. This gives out impulses which travel down the spinal cord to the centres of the spinal nerves that innervate the muscles of respiration. It also receives various afferent fibres, the most important of which are contained in the trunk of the 
vagus. The vagus is chiefly an afferent nerve in relation to respiration. It, however, also is in a minor degree efferent, for it supplies the muscular tissue of the lungs and bronchial tubes, and exercises a trophic influence on the lung.

The respiratory centre was discovered by Flourens; it is situated at the tip of the calamus scriptorius, and coincides in position with the sensory centre of the vagus. The existence of subsidiary respiratory centres in the spinal cord has been mooted, but the balance of experimental evidence is against their existence. Flourens found that when the respiratory centre is destroyed, respiration at once ceases, and the animal dies. He therefore called it the "vital knot" (nœud vitale).

The centre is affected not only by the afferent impulses which reach it by such nerves as the vagus, but also by those from the cerebrum; so that we have a limited amount of voluntary control over the respiratory movements.

The respiratory centre is probably twofold, consisting of an inspiratory and an expiratory centre. Of these two the inspiratory centre is so much the more active that its importance is a subject of universal agreement; whereas, the existence of an expiratory centre is doubted by some physiologists, who regard expiration as a mere cessation of the active process of inspiration, and a mechanical falling back of the tissues into their places.

\section{The Nervous Fuctor in Respiration.}

During normal respiration, as opposed to forced respiration, an impulse passes from the lung to the respiratory centre during each complete respiration. This has been discovered by placing the vagus on non-polarisable electrodes connected to a galvanometer, and observing the current of action which accompanies each impulse. The action-current takes place at the height of each inspiration.

The currents that occur in the vagus during respiration can be investigated with the capillary electrometer, as was done by Alcock and Seemann; they can still be more accurately studied by the use of Einthoven's string galvanometer (see p. 121). The accompanying figures (fig. 300) are reproduced from Einthoven's work on the subject. They were obtained from a dog.

In fig. $300 \mathrm{~A}$, normal respiration was taking place, and the line $\mathrm{R}$ is a tracing of the respiratory movements; the lowermost line $(H)$ is a tracing of the heart-beats. The top tracing $(\mathrm{E})$ is a photographic record of the movement of the quartz fibre in the galvanometer, which was connected by electrodes to the vagus nerve. The vagoelectrogram, as we may term it, shows large waves, which indicate the changes in the activity of the nerve in reference to respiration; the 
smaller waves upon these are due to its activity in reference to the heart.*

In fig. $300 \mathrm{~B}$, a condition of apnœa was produced so that the dog did not breathe for a certain time. The vagogram therefore shows no respiratory waves; and the variations due to cardiac action are the only waves seen.

During normal respiration, then, it seems that the inspiratory centre alone is active, and that after the inspiration has reached a

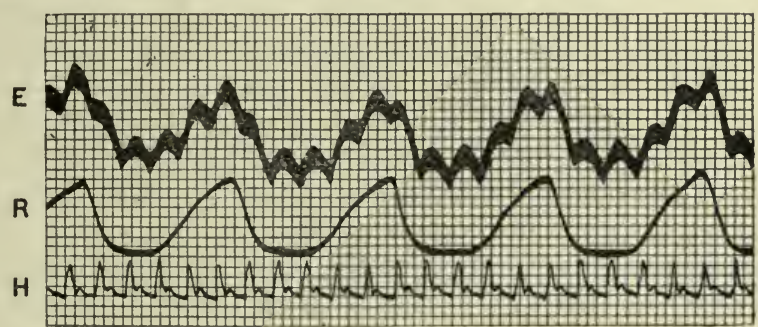

A

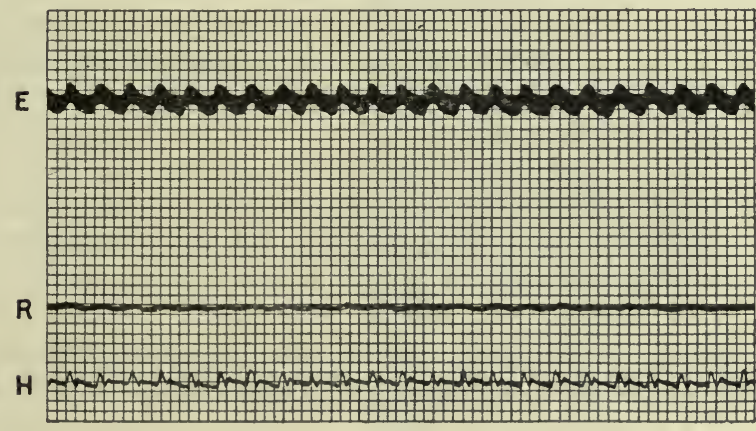

B

Fra. 300.-A, Upper line (E) is the electro-vagogram; the middle tracing $R$ is that of the respiratory movements; the lowermost line $(H)$ is a tracing of the heart-beats. In $B$, apnoa was produced, and the electro-vagogram shows only the electrical variations in the vagus, which are due to cardiac action. (Einthoven.)

certain point, it is checked by an impulse (inhibitory) coming from the lung along the vagus.

A theoretical question arises at this point: Supposing no inhibitory impulse came up the vagus, would the inspiration ever cease of itself ? In answer to this question we may say at once that when both vagi are divided, the respirations become much slower and deeper, but they do not entirely cease. If this were the whole case, we should conclude that the respiratory centre had a slow

* Einthoven regards these as mainly due to afferent (depressor) impulses; whether this is so or not is immaterial to the main question discussed above. 
inherent rhythm, which was quickened by the vagus impulses; but it is claimed that when all impulses, both from the brain above and the sensory nerves below, are cut off from the respiratory centre, the respiratory rhythm ceases. The operation is, however, a very severe one, and therefore inconclusive.

Leaving the question of normal respiration, we may proceed to consider the impulses passing up the vagus during forced respiration. The presence of impulses in these nerves can again be best detected by their action-currents, and in forced inspiration the same actioncurrent is shown by the galvanometer as we have just mentioned occurs during normal breathing; it can also be induced by artificial inflation of the lung. When the lung is alternately and deeply inflated and deflated, a small electrical variation frequently appears also in the vagus nerve during each deflation. We have, therefore, evidence that a nervous impulse is passing up the vagus during this period, but whether this impulse of the expiratory period is inhibitory to an expiratory centre, or a stimulus to an inspiratory centre, is very difficult to decide. The following experiments of Head, however, suggest the existence of a double centre.

His method of recording the movements was by means of that convenient slip of the diaphragm which is found in rabbits (see p. 357).

His method of dividing the vagus was by freezing it; he laid it across a copper wire, the end of which was placed in a freezing mixture. This method is free from the disadvantage which a cut with a knife or scissors possesses, namely, a stimulation at the moment of section. On dividing one vagus, respiration became slightly slower and deeper; on dividing the second nerve, this effect was much more marked.

On exciting the central end of the divided nerve, inspiratory efforts increased until at last the diaphragm came to a standstill in the inspiratory position. But if a weak stimulus was employed, the reverse was the case; the expiratory efforts increased, inspiration becoming weaker and weaker, until at last the diaphragm stopped in the position of expiration.

These facts were known previously, but the interpretation of them, in the light of further experiments now to be described, is the following:-

There are in the vagus two sets of fibres, one of which produces an increased activity of the inspiratory part of the respiratory centre, and the other an increased activity of the expiratory part of that centre. Stimulation of the first stops expiration and produces inspiration; stimulation of the second does the reverse.

The question now is, What is it that normally produces this alternate stimulation of the two sets of fibres? If we discover this we shall discover the prime moving cause in the alternation of the 
inspiratory and expiratory acts. It was sought and found in the alternate distension and contraction of the air-vesicles of the lungs where the vagus terminations are situated.

In one series of experiments positive ventilation was performed; that is, air was pumped repeatedly into the lungs, and so increased their normal distension; this was found to decrease the inspiratory contractions of the diaphragm, until at last they ceased altogether, and the diaphragm stood still in the expiratory position (fig. 301, A).

In a second series of experiments, negative ventilation was performed ; that is, the air was pumped repeatedly out of the lungs, and a condition of collapse of the air-vesicles produced. This was found to increase the inspiratory contractions of the diaphragm, expiration became less and less, and at last the diaphragm assumed the position of inspiratory standstill (fig. 301, B).

Head regards ordinary respiration as an alternate positive and negative ventilation, though not so excessive as in the experiments just described. Inspiration is positive ventilation, and so provides the nervous mechanism of respiration with a stimulus that leads to expiration. Expiration is a negative ventilation, and so provides the stimulus that leads to inspiration.

We must naturally be on our guard against regarding the forcible inflations and deflations produced by a pump as completely analogous to the changes produced in the lungs by ordinary breathing; nevertheless, the two sets of impulses are undoubtedly called into action if the respiratory processes are sufficiently energetic, and of the two sets of impulses, those

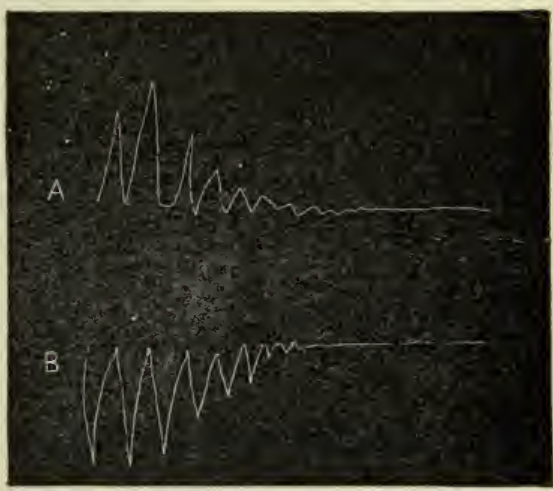

Frg. 301.-Tracings of diaphragm. The upward movements of the tracings represent inspiration; the downward movements, expiration. A, result of positive, B, of negative ventilation. (After Head.) which are started by the inspiratory movement play a more active part in the regulation of respiration than those started by the expiratory movement, so much so that in unlaboured breathing they alone need be considered.

Apnœa.-If positive and negative ventilation are used together rapidly and alternately at a rate quicker than the respiratory rhythm, both inspiratory and expiratory processes are inhibited, and the respiration ceases for a short time. This follows naturally from the experiments previously described. This can be done on an animal with a pair of bellows fixed to a tube in the trachea; or voluntarily by one- 
self taking a number of deep breaths rapidly. This condition, called apnoea, is not due, as at one time supposed, to over-oxygenation of the blood, but is, according to Head, produced reflexly; for under normal circumstances arterial blood is almost fully oxygenated. Apnœa is observed if inert gases, such as nitrogen or hydrogen, are used instead of air. The pause, however, is then shorter, as the blood becomes venous, and in a short time stimulates the respiratory centre to activity.

Under abnormal circumstances, namely, after division of the vagi, apnce obviously cannot be due to such reflex action. Fredericq holds that even ordinary apncea has a chemical rather than a nervous origin. He attributes it, however, not to over-oxygenation, but to a lessening of the carbonic acid in the blood.

\section{The Chemical Factor in Respiration.}

A consideration of apnœa thus leads us to the study of the chemical stimuli that play their part in the respiratory process. Their importance has been recently demonstrated by Haldane and Priestley.

In the first place, they introduced the new and simple method of obtaining the composition of the air in the alveoli, described on p: 372 . They found that, under constant atmospheric pressure, in man the alveolar air contains a nearly constant percentage of carbon dioxide in the same person. In different individuals this percentage varies somewhat, but averages $5 \cdot 1$ per cent. of an atmosphere in men, and $4 \cdot 7$ in women and children.

With varying atmospheric pressures, the percentage varies inversely as the atmospheric pressure, so that the pressure or tension of the carbon dioxide remains constant. The oxygen pressure, however, varies widely under the same conditions.

These observations and the next to be immediately described furnish the chemical key to the cause of the amount of pulmonary ventilation, and play an important part in conjunction with the respiratory nervous system in the regulation of breathing. For the respiratory centre is not only affected by the impulses reaching it by the vagi and other afferent nerves, but it is also very sensitive to any rise in the tension of carbon dioxide in the blood that supplies it. The changes in the tension of this gas in the arterial blood are normally proportional to the changes in the carbon dioxide pressure in the alveoli, and the changes in the lung alveoli are transmitted to the respiratory centre by the blood. They found that a rise 0.2 per cent. in the alveolar carbon dioxide pressure is sufficient to double the amount of alveolar ventilation during rest. During sudden muscular work the alveoiar carbon dioxide pressure increases slightly, and the pulmonary ventilation is consequently increased. Most 
physiologists consider that the action of carbonic acid is not specific, but that other acids can act in the same way. As the result of prolonged exercise, the carbonic acid pressure in the alveoli falls greatly; nevertheless there is increased pulmonary ventilation. This is because lactic and perhaps other acids are thrown into the blood, which raise its total acidity (hydrogen ion concentration), see p. 395 .

Changes in the oxygen pressure within wide limits have no such influence; the normal chemical stimulus to respiration is, therefore, presence of an increase of carbon dioxide, and not diminution of oxygen. If these limits are exceeded, as when the oxygen in the atmosphere falls below 13 per cent., the respiratory centre begins to be excited by want of oxygen. It is probable that other fatigue products, such as sarcolactic acid, assist the carbon dioxide in stimulating the respiratory centre.

In connection with the relative importance of the nervous and chemical factors in breathing, F. H. Scott has shown that the principal respiratory nerves (the pneumogastrics) regulate the rate or rhythm of the respiratory movements, whilst the chemical factor specially regulates the amount of pulmonary ventilation, that is, the depth of the individual respiratory efforts; for when these nerves are divided, a rise in the alveolar tension of carbon dioxide (or great diminution of the oxygen in the respired air) increases the depth, but not the rate of breathing.

To recapitulate:-In a normal respiration the chemical and nervous factors would, therefore, appear to be related somewhat as follows:-The inspiratory centre makes an effort, the degree of exaltation of the centre, and therefore, the magnitude of the effort, more especially in the matter of depth, is governed by the tension of carbonic acid in the blood, but it is cut short by an inhibitory impulse passing up the vagus, only to begin again when the effects of this inhibitory impulse are removed.

During fotal life the need of the embryo for oxygen is small, and is amply met by the transference of oxygen from the maternal blood through the thin walls of the fœetal capillaries in the placenta. But when the child is born, this source of oxygen is no longer available, and the increasing venosity of the blood stimulates the respiratory centre to action, and is the essential cause of the first inspiratory efforts the new-born child makes to obtain the oxygen it requires. It is said that if the placental circulation is stopped while the child is still in utero, respiratory efforts are also made. Some regard the action of the air on the body surface as an accessory cause of the first respirations, and it is the practice to increase this in feeble children by stimulating the cutaneous nerves by the application of cold water to the skin. Such treatment always causes deep inspirations, even in the adult. There are other nerves stimulation of which influences the respiratory act; for instance, stimulation of the central end of the glossopharyngeal inhibits the respiratory movements for a short period; this accounts for the very necessary cessation of breathing during swallowing. Stimulation of the central end of the cut superior laryngeal nerve, or of its terminations in the mucous membrane of the larynx, as when a crumb is "swallowed the wrong way," produces an increase of expiratory efforts, culminating in coughing. 


\section{Special Respiratory Acts.}

Coughing.-In the act of coughing there is first of all a deep inspiration, followed by an expiration; but the latter, instead of being easy and uninterrupted, as in normal breathing, is obstructed, the glottis being momentarily closed by the approximation of the vocal cords. The abdominal muscles, then strongly acting, push up the viscera against the diaphragm, and thus make pressure on the air in the lungs until its tension is sufficient to noisily open the vocal cords which oppose its outward passage. In this way considerable force is exercised, and mucus or any other matter that may need expulsion from the air-passages is quickly and sharply expelled by the outstreaming current of air. The act is a reflex one, the sensory surface which is excited being the mucous membrane of the larynx, and the superior laryngeal nerve is the afferent nerve; stimulation of other parts of the respiratory mucous membrane will also produce cough, and the point of bifurcation of the trachea is specially sensitive. Other sensory surfaces may also act as the "signal surface" for a cough. Thus, a cold draught on the skin, or tickling the external auditory meatus, in some people will set up a cough.

Sneezing.-The same remarks that apply to coughing are almost exactly applicable to the act of sneezing; but, in this instance, the blast of air, on escaping from the lungs, is directed, by an instinctive contraction of the pillars of the fauces and descent of the soft palate, chiefly through the nose, and any offending matter is thence expelled.

The "signal surface" is usually the nasal mucous membrane, but hore, as in coughing, other causes (such as a bright light) will sometimes set the reflex going.

Hiccough is an involuntary sudden contraction of the diaphragm, causing an inspiration which is suddenly arrested by the closure of the glottis, causing a characteristic sound. It arises from gastric irritation.

Snoring is due to vibration of the soft palate.

Sobbing consists of a series of convulsive inspirations at the moment of which the glottis is partially closed.

Sighing and Yawning are emotional forms of inspiration, the latter associated with stretching movements of jaws and limbs. They appear to be efforts of nature to correct, by an extra deep inspiration, the venosity of the blood due to inactivity produced by ennui or grief. Their contagious character is due to sympathy.

There are many other abnormalities of the respiratory mechanism which will become familiar to the student of medicine during his clinical studies. We may mention as examples: laryngismus stridulus (the spasmodic croup of children); this is a nervous affection due to increased reflex irritability of the laryngeal mechanism; the 
fits of suffocation are produced by tonic spasm of the adductor muscles of the glottis. Asthma is another nervous affection, and has been already briefly referred to on p. 349 . Whooping-cough is an infectious disease, the poison of which also acts on the nervous respiratory system.

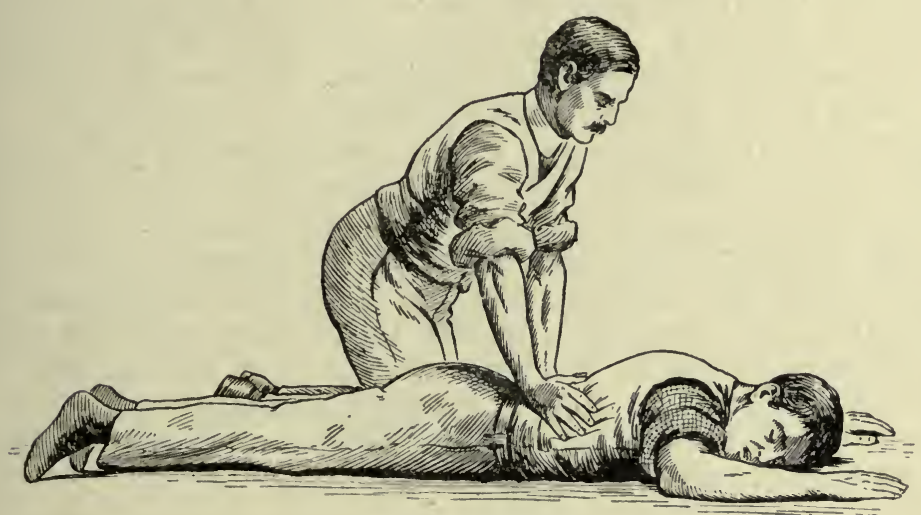

A

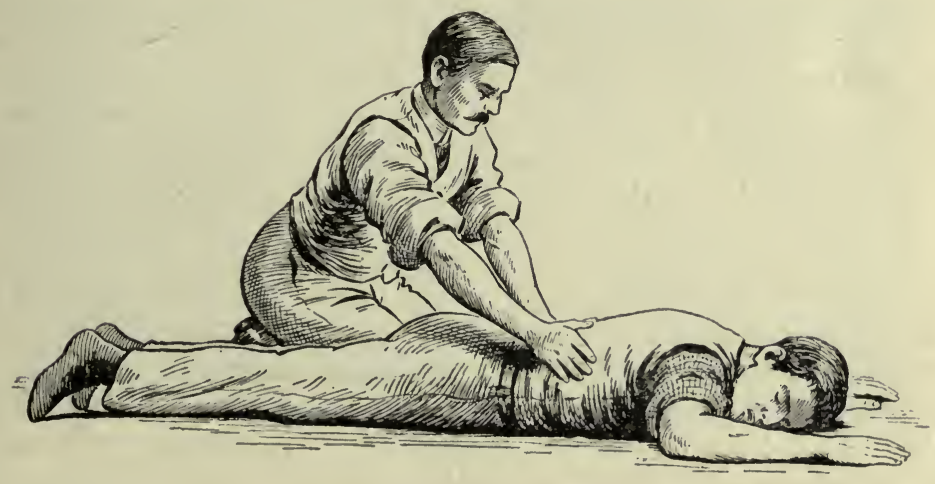

B

Fı. 302.-This illustrates the two principal positions $A$ and $B$ in performing Schäfer's method of artificial respiration.

\section{Artificial Respiration.}

In experiments on animals in which it is necessary to open the chest, life can be maintained by pumping air into the lungs; this is done by means of some form of pump or bellows, the delivery tube of which is connected to the trachea by a cannula, a side hole in 
which provides for the escape of the expired air. A bottle containing the anæsthetic is placed on the course of the delivery tube.

Artificial respiration is sometimes necessary in man to restore normal breathing, as for instance in those who are apparently dead from drowning. In such cases speed in commencing the artificial breathing, and perseverance in continuing the process are essential. Many have been restored to life after the efforts have been continued for an hour or more. It is now recognised that of the numerous methods for performing artificial respiration, that recently introduced by Schäfer is the simplest, least injurious, and most effective. The subject is laid on the ground in the prone position, with a thick folded garment under his chest. The operator kneels by his side or athwart him facing his head, and places his hands on each side over the lower ribs. He slowly throws the weight of his body forwards, and thus presses upon the thorax of the subject, and forces air out of the lungs (fig. 302, A); he then gradually relaxes the pressure by bringing his body up again, but without removing his hands (fig. 302, B). This is repeated regularly at the rate of twelve to fifteen times a minute until normal respiration begins, or until all hope of restoration is given up.

\section{Ventilation.}

Some observers have stated that certain noxious substances are ordinarily contained in expired air which are much more poisonous than carbonic acid, but more careful researches have failed to substantiate this. If precautions be taken by absolute cleanliness to prevent admixture of the air with exhalations from skin, teeth, and clothes, the expired air only contains one noxious substance, and that is carbonic acid.

Absolute cleanliness is, however, not the rule; and the air of rooms becomes stuffy when the amount of expired air in them is just so much as to raise the percentage of carbonic acid to 0.1 per cent. An adult gives off about 0.6 cubic feet of carbonic acid per hour, and if he is supplied with 1000 cubic feet of fresh air per hour, he will add 0.6 to the 0.4 cubic feet of carbonic acid it already contains; in other words, the percentage of that gas will be raised to 0.1. An hourly supply of 2000 cubic feet of fresh air will lower the percentage of carbonic acid to 0.07 , and of 3000 cubic feet to 0.06 , and this is the supply which is usually recommended. In order that the air may be renewed without giving rise to draughts, each adult should be allotted sufficient space in a room, at least 1000 cubic feet.

Leonard Hill has recently stated that the effects of bad ventilation are not so much due to changes in the chemical composition of the air, as to the absence of movement in the air; moving air has a stimulating, and still air a depressing effect. 


\section{CHAPTER XXVII}

\section{THE RELATION OF RESPIRATION TO OTHER PRGCESSES IN THE BODY}

We shall in this chapter treat of the relationship botween respiration and the circulation, and between respiration and metabolism, and in conclusion deal with certain pathological conditions, which are important for the light they throw upon physiological processes.

\section{The Effect of Respiration on the Circulation.}

The main effect of respiration on the circulation is shown in the accompanying figure (fig. 303). It will be noticed that the arterial

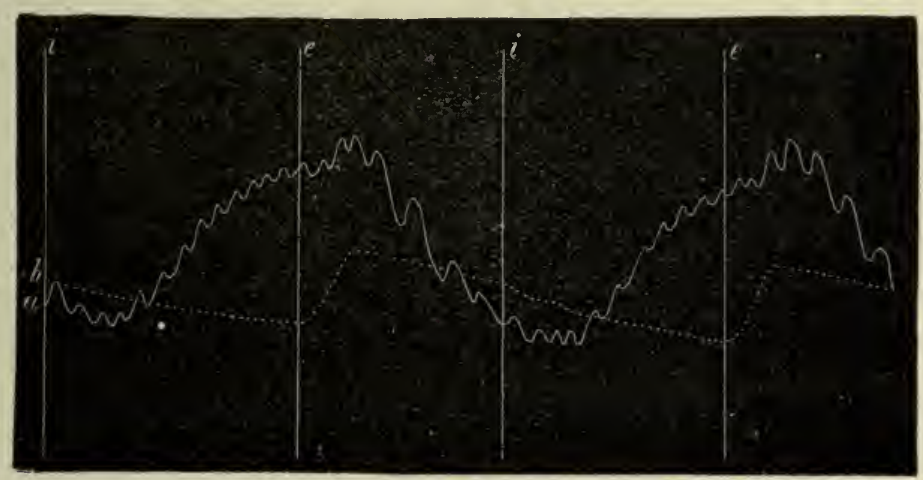

Fı. 303.-Comparison of blood-pressure curve with curve of intra-thoracic pressure. (To be read from left to right.) $a$ is the curve of blood-pressure with its respiratory undulations, the slower beats on the descent being very marked; $b$ is the curve of intra-thoracic pressure obtained by connecting one limb of a manometer with the pleural cavity. Inspiration begins at $i$ and expiration at $e$. The intra-thoracic pressure rises very rapidly after the cessation of the inspiratory effort, and then slowly falls as the air issues from the chest; at the beginning of the inspiratory effort the fall becomes more rapid. (M. Foster.)

pressure rises with inspiration and falls with expiration, but that the two events are not quite synchronous, the rise of pressure beginning a little later than the inspiratory act, and the fall a little later than the expiratory act.

It will also be seen that the heart beats more rapidly during tho 
rise of blood-pressure than during the fall. This difference disappears when the vagi are cut. Respiratory undulations, however, are still present, though not so marked as before; hence the cardiac variations are not their sole cause. They are chiefly the result of the mechanical conditions dependent on the lungs and heart with its large vessels being contained within the air-tight thorax. When the capacity of the chest is increased in inspiration, the tension of the lung tissue due to its greater expansion is increased; hence the difference between the intra-pleural pressure and that in the lungs (which is atmospheric) becomes more marked, for the difference of pressure is to be measured by the elastic force of the lung tending to produce its collapse. If the intra-thoracic pressure is measured, it is found that it varies from -5 to $-7 \mathrm{~mm}$. of mercury at the end of expiration to - 30 at the end of a deep inspiration; that is to say, from 5 to 7 to $30 \mathrm{~mm}$. less than the atmospheric pressure (760 mm. of mercury). The pressure outside the heart and large thoracic vessels is correspondingly diminished during inspiration to the same extent, and produces its main effect (distension) upon the veins because they are never fully distended, and because the pressure within them is low. This increase in the "pressure gradient" (i.e., the rate of fall of pressure) between the intra- and extra-thoracic great veins results in a proportionately more rapid flow of blood into the thorax, and therefore into the right side of the heart; for within certain limits the right heart can be easily expanded more fully if a greater supply of blood is provided. Consequently, the output from the right side of the heart increases, and thus via the pulmonary circuit the inflow into the left side of the heart is increased; in its turn, therefore, the output from the left ventricle rises, and so the aortic pressure is raised. This effect would be counteracted if the aorta and its branches within the thorax were as easily affected by changes of the intra-thoracic pressure as are the thin-walled and easily distensible veins; the thick wall of the aorta and its branches, however, prevents them from undergoing much change of this kind during ordinary breathing. The conditions in the veins are reversed when, with the expiratory act, the thorax returns to its former size; therefore the arterial blood-pressure falls.

The effect of inspiration on arterial blood-pressure is at first assisted by the pressure of the diaphragm, as it descends, on the abdominal veins, and blood is thus sent upwards into the chest by the vena cava inferior. On the other hand, this is to some extent counterbalanced by the obstruction in the passage of the blood downwards in the abdominal aorta, and upwards from the veins of the lower extremities, but again the veins are the vessels more easily influenced by moderate changes in external pressure.

We thus see that these various physical conditions produce during inspiration an increased flow of blood into the right heart; this 
increased supply of blood is then passed via the pulmonary circuit to the left heart; this takes a little time; hence it is that the effect of inspiration in raising arterial pressure is not seen at the very commencement of the inspiration. In fact, in some animals which normally breathe very quickly (for instance, the rabbit), inspiration is over, and the next expiration has begun before the rise of bloodpressure occurs. By making a rabbit breathe slowly (Fredericq accomplished this by cooling the medulla oblongata), the tracing obtained is similar to that which is got from an animal like a dog, which normally breathes slowly.

The delay which occurs in the inspiratory rise of arterial blood-pressure has been attributed by some to an increase of the capacity of the pulmonary capillaries brought about by the distension of the chest; this sudden increase in the bed of the stream would temporarily retard the rate of flow through the pulmonary circuit. Recent research has, however, shown that even considerable changes in the capacity of the blood-vessels of the lung, as, for instance, by shutting off the entire circulation of one lung (Tigerstedt), have little or no influence on the systemic pressure; it is therefore extremely doubtful whether small changes such as would be produced in ordinary breathing can have any effect on the inflow into the left auricle.

When the chest of an animal is freely opened, and artificial respiration performed in order to keep it alive, respiratory undulations on the arterial pressure-curve are still seen, but they are in the reverse direction. These obviously cannot be produced in the mechanical way just described. The forcible inflation with air at first squeezes more blood out of the alveolar capillaries, that is, the capacity of these vessels is diminished, and this theoretically might increase the quantity of blood thrown into the left ventricle, and so cause a rise of arterial pressure. But the main effect of increased intra-alveolar pressure is to produce an increased resistance to the pulmonary circulation, and the rate of flow into the left side consequently falls ; the aortic pressure therefore falls, while the pressure in the pulmonary artery rises. If the high positive intrapulmonary airpressure persisted, a condition would soon be reached, in which the increased blood-pressure in the pulmonary artery would lead to a greater flow, and the aortic blood-pressure would remain constant; this, however, has been shown to take a much longer time than an ordinary respiration period. Hence the effect of inflations of the lungs at the ordinary respiration rate is to diminish the aortic blood-pressure; this rises again, for the opposite reasons, in the intervals of deflation which correspond to expiration.

If artificial respiration is performed while the thorax is not opened, a further complication arises from the fact that the increased intrapleural pressure decreases the rate of flow of blood into the thorax, and under these conditions the blood-pressure in the pulmonary artery falls, and in consequence the fall in the aortic blood-pressure 
becomes more marked with each inflation than it does when the thorax is open.

The last point of detail we have to consider is the cause of the greater frequency of the heart during the inspiratory phase, a phenomenon which is evidently due to lessening of vagus action, since the inequality of the heart-rate disappears whon the vagi are cut. The question before us is, What is the cause of the rhythm in the activity of the vagus centre? There appear to be two factors concerned in its causation: one is a reflex action, the other is what may be termed a central overflow. We will consider these separately.

1. The reflex. Stimulation of the pulmonary branches of the vagus by electrical stimuli, or of their terminations in the alveoli by certain irritating vapours such as bromine, causes a reflex inhibition of the heart; great distension of the alveoli has a similar effect, but moderate distension, such as occurs in an ordinary inspiration, has the opposite reflex effect, causing the heart to beat more rapidly. The afferent fibres from the pulmonary alveoli enter the bulb by the upper set of the rootlets of the combined glossopharyngeal-vagus-spinal accessory nucleus (the $a$ group, p. 249). Sometimes the rootlets of this group are three in number, sometimes two. When there are two, the lower rootlet, when there are three, the lower two rootlets, contain the fibres in question (Cadman).

2. The overflow. The respiratory centre exhibits alternate phases of activity, or what is termed a rhythmical action. It is in close anatomical connection with two other important centres in the bulb, namely, the cardio-inhibitory and the vaso-motor centres. Considering how closely these three centres are connected by association fibres, it is not surprising that the cells of the two latter centres should be affected by the rhythm of the cells of the respiratory centre, and the term overflow is an expression that roughly indicates what occurs. This overflow from the respiratory centre affects its. two neighbours in the same way. During inspiration the activity of both the cardio-inhibitory centre and of the vaso-motor centre is diminished, hence the heart beats faster. The factor which we have termed the overflow is more important than that which we have described as the reflex.

These facts show us that the parallelism of the respiratory and arterial pressure-curves is not merely the result of the mechanical conditions already described, though these are the most important. But in the normal condition with the thorax closed, and the vagi uncut, certain nervous factors come also into play.

Valsalva's Experiment.-In speaking of the effects of expiration, we have considered only ordinary quiet expiration. With forced expiration, there is considerable impediment to the circulation; this is markedly seen in what is called Valsalva's experiment. This con- 
sists in making a forced expiratory effort with the mouth and noso shut; the effects are most marked in people with an easily compressible thorax. By such an act the intrathoracic and abdominal pressures rise so greatly that the outlets of the veins of the limbs, head, and neck into the thorax are blocked. At first, the blood in the abdominal veins is drawn on into the right heart; this produces a slight rise of arterial pressure; but soon, if the effort is continued, the lungs are emptied of blood, the filling of the right heart is opposed, and the blood is dammed back in the peripheral veins, where the pressure rises to mean arterial pressure. The arterial pressure begins then to fall; but before any considerable fall occurs, the expiratory effort ceases from exhaustion of the subject of the experiment, and a deep inspiration is taken. . During this inspiration, the blood delivered by the right heart is all used in the filling of the comparatively empty pulmonary vessels; thus several beats of the left ventricle become abortive, and produce no effect on the radial artery; the face blanches, and the subject becomes faint from cerebral anæmia.

\section{Asphyxia.}

Asphyxia may be produced in various ways: for example, by the prevention of the due entry of oxygen into the blood, either by direct obstruction of the trachea or other part of the respiratory passages, or by introducing instead of ordinary air a gas devoid of oxygen, or by interference with the due interchange of gases between the air and the blood.

The symptoms of asphyxia may be roughly divided into three stages: (1) the stage of exaggerated breathing; (2) the stage of convulsions; (3) the stage of exhaustion.

In the first stage the breathing becomes more rapid, and at the same time deeper than usual, inspiration at first being especially exaggerated and prolonged. The muscles of extraordinary inspiration are called into action, and the effort to respire is laboured and painful. This is soon followed by a similar increase in the expiratory efforts, which become excessively prolonged, being aided by all the muscles of extraordinary expiration. During this stage, which lasts a varying time from a minute upwards, according as the deprivation of oxygen is sudden or gradual, the lips become blue, the eyes are prominent, and the expression intensely anxious. The prolonged respirations are accompanied by a distinctly audible sound; the muscles attached to the chest stand out as distinct cords. This stage includes the two conditions hyperpnoea (excessive breathing) and dyspnoea (difficult breathing), which follows later. It is due to the increasingly powerful stimulation of the respiratory centre by the increasingly venous blook. 
In the second stage, which is not marked by any distinct line of demarcation from the first, the violent expiratory efforts become convulsive, and then give way, in men and other warm-blooded animals, to general convulsions, which arise from the further stimulation of the centres in brain and cord by venous blood. Spasms of the muscles of the body in general occur, and not of the respiratory muscles only. The convulsive stage is a short one, and lasts less than a minute.

The third stage, or stage of exhaustion. In it the respirations all but cease, the spasms give way to flaccidity of the muscles, there is insensibility, the conjunctivæ are insensitive and the pupils are widely dilated. Every now and then a prolonged sighing inspiration takes place, at longer and longer intervals, until breathing ceases altogether, and death ensues. During this stage the pulse is scarcely to be felt, but the heart may beat for some seconds after the respiration has stopped. The condition is due to the gradual paralysis of the centres by the prolonged action of the venous blood. This stage may last three minutes and upwards.

After death from asphyxia it is found in the great majority of cases that the right side of the heart, the pulmonary arteries, and the systemic veins are gorged with dark, almost black, blood, and the left side of the heart, the pulmonary veins, and the arteries are empty. The explanation of these appearances may be thus summarised: when oxygenation ceases, venous blood at first passes freely through the lungs to the left heart, and so to the great arteries. Owing to the stimulation of the vaso-motor centres by the venous blood, the arterioles, particularly those of the splanchnic area, are constricted; the arterial blood-pressure therefore rises, and the left side of the heart becomes distended. The highly venous blood passes through the arterioles, and, favoured by the laboured respiratory movements, arrives at the right side of the heart, which it fills and distends; the right side of the heart is becoming feebler at the same time, and therefore unable to effectively discharge its blood through the pulmonary circuit. Simultaneously the left ventricle is also becoming weakened, and therefore its suction action diminishes. In this way the blood is dammed back in the right heart and veins, and the left side of the heart therefore gets into the empty condition in which it is found after death. Some consider that the early onset of rigor mortis in the left ventricle may be in part a cause of its contracted and empty condition.

In the first and second stages of asphyxia, the arterial pressure rises above the normal; this is due to the constriction of the arterioles which is in part produced by the suprarenal glands pouring out more adrenaline into the circulation, owing to their being stimulated by the excess of carbonic acid in the blood. The fall of pressure in the 
last stage is mainly due to heart failure. If the vagi are not divided previously, the rise of pressure is much less, and the heart beats very slowly: this enables the heart to last longer, and is due to excitation

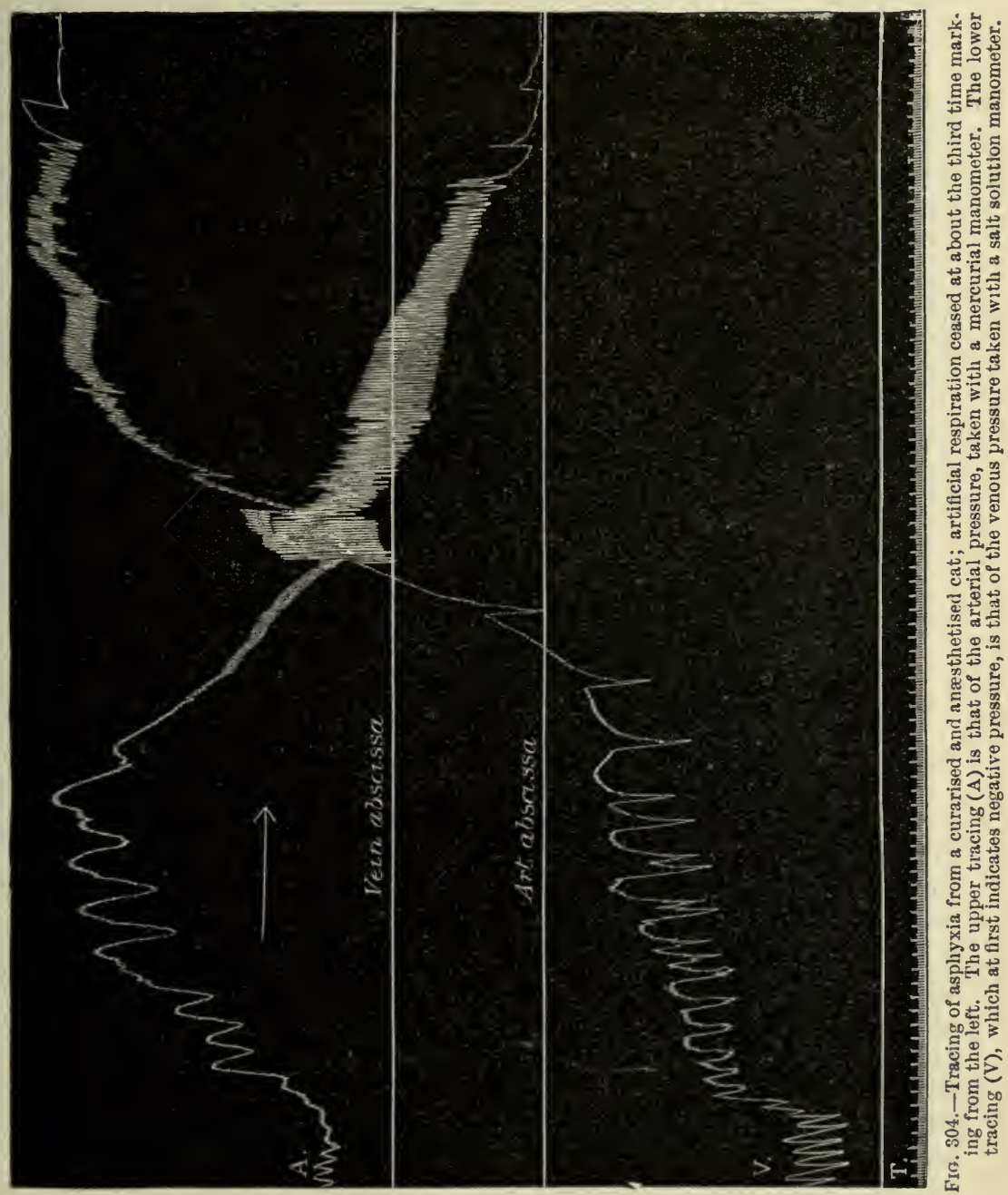

of the cardio-inhibitory centre by venous blood. The accompanying photograph of a tracing (fig. 304), which I owe to Dr C. J. Martin, shows these effects; it has been somewhat reduced in size for purposes of reproduction. The lower tracing is that of venous 
pressure taken with a salt solution manometer from the jugular vein. It will be noticed that the fall of arterial pressure is accompanied with a great rise of venous pressure due to the venous congestion just described.

\section{The Relation of Respiration to Nutrition.}

The gaseous intorchanges in the lungs constitute what is frequently termed external respiration. Oxygen obtains an entrance into the blood, and is carricd to the tissues in the looso compound known as oxyhæmoglobin. In the tissues, this compound is dissociated, and the respiratory oxygen is utilised by the tissuo elements for the combustion processes which occur consequent on their activity. Of the ultimate products, carbonic acid and a portion of the water find an outlet by the lungs, to which they are transported by the venous blood. The gaseous interchanges in the tissues constitute what is known as internal or tissue respiration.

Inspired and Expired Air.-We may comparo the composition of the inspired or atmospheric air with that of the expired air in the following table:-

\begin{tabular}{|c|c|c|c|}
\hline & & Inspired air. & Expired air. \\
\hline $\begin{array}{l}\text { Oxygen . } \\
\text { Nitrogen } \\
\text { Carbonic acid } \\
\text { Watery vapour } \\
\text { Temperature. }\end{array}$ & • & $\begin{array}{c}20.96 \text { vols. per cent. } \\
79.04 \text { ", ", } \\
\text { variable ", }\end{array}$ & $\begin{array}{c}16.03 \text { vols. per cent. } \\
79 \\
4.4 \quad ", \\
\text { saturated" } \\
\text { that of body }\left(37^{\circ} \mathrm{C} \text {. }\right)\end{array}$ \\
\hline
\end{tabular}

The chief change is in the proportion of oxygen and carbonic acid. The loss of oxygen is about 5 , the gain in carbonic acid about $4 \cdot 5$. If the inspired and expired airs are carefully measured at the same temperature and barometric pressure, the volume of expired air is thus found to be rather less than that of the inspired.* The conversion of oxygen into carbonic acid would not cause any change in the volume of the gas; for a molecule of oxygen $\left(\mathrm{O}_{2}\right)$ would give rise to a molecule of carbonic acid $\left(\mathrm{CO}_{2}\right)$ which would occupy the same volume (Avogadro's law). It must, however, be remembered that carbon is not the only element which is oxidised. Fat and protein contain a number of atoms of hydrogen, which, during metabolism, are oxidised to form water; a small amount of oxygen is also used in the formation of urea. Carbohydrates contain sufficient oxygen in their own molecules to oxidise their hydrogen; hence the apparent loss of oxygen is least when a vegetable diet (that is, one consisting largely of starch

* This diminution of volume will cause a slight rise in the proportionate volume of nitrogen per cent. 
and other carbohydrates) is taken, and greatest when much fat and protein are eaten. The quotient $\frac{\mathrm{CO}_{2} \text { given off }}{\mathrm{O}_{2} \text { absorbed }}$ is called the respiratory quotient. Normally it is $\frac{4.5}{5}=0.9$, but it varies considerably with diet, as just stated.

The amount of respiratory interchange of gases is estimated by enclosing an animal in an air-tight chamber, except that there is a tube entering and another leaving it; by one tube oxygen or air can enter, and is measured by a gas-meter as it passes in. The air is drawn through the chamber, and leaves it by the other tube; this air has been altered by the respiration of the animal, and in it the carbonic acid and water are estimated; it is drawn into bottles containing a known amount of an alkali; this combines with the carbonic acid and is increased in weight: the increase in weight gives the amount of carbonic acid ; the alkali used in Regnault and Reiset's apparatus was potash; Pettenkofer used baryta water; Haldane recommends soda-lime. The water is estimated in bottles containing pumice moistened with sulphuric acid.

The accompanying (lrawing (fig. 305) shows the essential part of

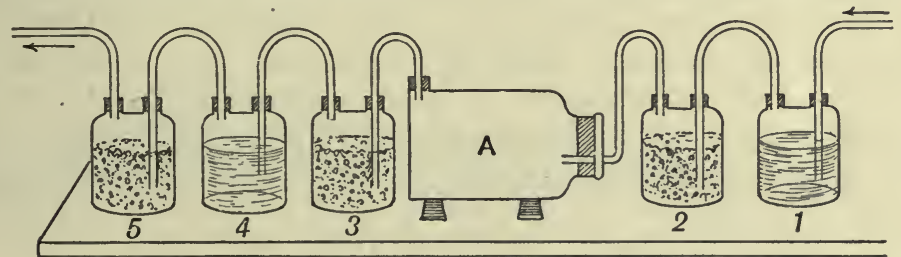
Frc. 305.-Haldane's apparatus for estimating the carbonic acid and aqueous vapour given off by an
animal.

the simple but effective apparatus used by Haldane. The animal is placed in the vessel A; air is sucked through the apparatus (which must be perfectly air-tight) by a water-pump at a suitable rate. The arrows indicate the direction in which the air passes. It goes first through two Woulff's bottles, 1 and 2. No. 1 contains soda-lime, which frees the air from carbonic acid; No. 2 contains pumice-stone moistened with sulphuric acid, which frees the air from aqueous vapour. The pure, dry air next reaches the animal chamber, and the animal gives off to it carbonic acid and aqueous vapour. It passes then through the three bottles, 3,4 , and 5. No. 3 contains pumice and sulphuric acid, which removes the water; No. 4 contains soda-lime, which absorbs the carbonic acid; and No. 5 contains pumice and sulphuric acid, which absorbs any water carried over from bottle 4 . The increase of weight in bottle 3 at the end of a given time (e.g. an hour) is the weight of water given off by the animal in that time; the in- 
crease of weight in bottles 4 and 5 weighed together gives the amount of carbonic acid produced by the animal in the same time.

Ranke gives the following numbers from experiments made on a man, who was taking a mixed diet consisting of 100 grammes of protein, 100 of fat, and 250 of carbohydrate in the twenty-four hours. The amount of oxygen absorbed in the same time was 666 grammes; of which 560 passed off as carbonic acid, 9 in urea, 19 as water formed from the hydrogen of the protein, and 78 from that of the fat.

Vierordt from a number of experiments on human beings gives the following average numbers: the amount of oxygen absorbed in the twenty-four hours is 744 grammes; this leads to the formation of 900 grammes of carbonic acid (this contains about half a pound of carbon) and 360 grammes of water.

The respiratory interchange is lessened during sleep. It is especially small in the winter sleep of hibernating animals. During hibernation the respiratory quotient sinks to 0.5 , so that the animals actually gain weight from retention of oxygen. This aspect of respiration is essentially so much a part of "metabolism" that it will be dealt with more in detail in the chapters which deal with that subject. (See especially the respiration calorimeter in Chap. XLII.)

Tissue Respiration.-As has been already stated, respiration may be divided into internal or tissue respiration and external or pulmonary respiration. External respiration is much the less obscure, and we have treated of it at considerable length, not only on this account, but also on account of the very frequent impairments of the pulmonary mechanism which are met with in disease. It must be borne in mind, however, that pulmonary respiration is but the means, and tissue respiration is the end.

Tissue respiration consists in the passage of oxygen from the blood of the capillaries to the cells of the tissues, and the passage of carbonic acid in the reverse direction. This gaseous interchange is no doubt brought about by a simple process of diffusion. The oxygen passes out of the plasma

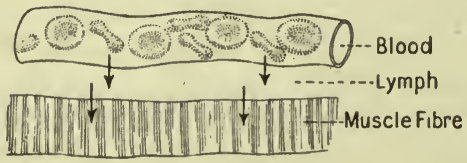

FiG. 306. of the blood through the capillary wall, and then through the lymph until it reaches the cell in which it is going to be used, which we will suppose is a muscle fibre (fig. 306). In order that a constant stream of oxygen may pass from the blood to the fibre, there must be a difference of oxygen pressure between the oxygen dissolved in the plasma, and that dissolved in the lymph, and the latter must be at a greater pressure than that dissolved in the muscle fibre. The amount of oxygen which passes will, other things being equal, be directly proportional 
to these pressure differences, and as the amount varies greatly at different times, it is obvious that the pressure differences vary greatly also. When the muscle is at rest, the oxygen pressure in the capillaries is very near to that in the muscle fibre; when the muscle is active and using large quantities of oxygen, the intracapillary oxygen pressure is much greater than the intra-muscular oxygen pressure. Such a change might be brought about by a rise in the intra-capillary oxygen pressure, or a fall in the intra-muscular oxygen pressure, or by both taking place simultaneously. Let us therefore enquire what is known about these quantities.

The tension of oxygen in muscle has recently been calculated as being at most equal to $19 \mathrm{~mm}$. of mercury; from this it may vary down to zero. Within these limits the conditions for diffusion can be increased by a drop in the intra-muscular oxygen pressure.

There is, in addition, a mechanism for raising the intra-capillary oxygen pressure. This is the increased quantity of acid (carbonic and sarco-lactic acids) which is thrown into the blood as the result of muscular metabolism. The following diagram (fig. 307) shows the extent, both in degree and time, of this pouring of acid into the blood as the result of a tetanic contraction of a muscle lasting only a few seconds.

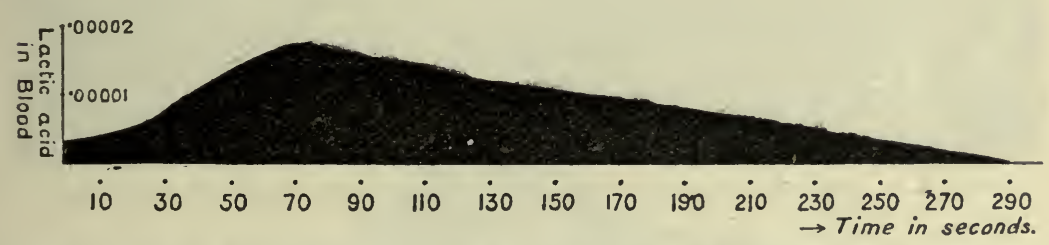

Fra. 307.-The black area represents the lactic acid thrown into the blood during the time following a tetanus that lasted 84 seconds; the work done by the muscle was 70 gramme-centimetres; the total quantity of lactic acld formed was 0.003 grammes. The figures on the vertical line represent fractions of a gramme of lactic acid per second.

In glandular structures the oxygen pressure is higher than in muscle; probably owing to the relatively more copious bloodsupply of glands, equilibrium is more readily established between the blood and the gland cells, the oxygen pressure in the cells being almost that present in venous blood.

The quantity of oxygen used by different tissues varies not only with the degree of their activity, but also with the nature of the tissues. On the whole it may be said that, weight for weight, glandular tissue uses most oxygen; next in order come the muscular tissues, and last of all, the connective tissues. There are some important tissues, notably the nervous system, about which little is known in this connection. The amount of oxygen used by 
an organ or tissue per gramme per minute is called its coefficient of oxidation.

Measurement of Coefficient of Oxidation.-The method adopted depends upon whether the observer desires to ascertain the coefficient at a particular moment of time, or its average value over a considerable period. If the former is desired, it is necessary:(1) to estimate the gases in the blood going to and emerging from the organ; this is performed by the chemical method of expelling the oxygen and carbonic acid from samples of the arterial and venous blood by means of potassium ferricyanide and tartaric acid respectively (see p. 364); (2) to determine the amount of blood passing through the organ in a given time, say one minute; and (3) at the conclusion of the experiment the organ is weighed, so that the gaseous exchange per gramme can be calculated.

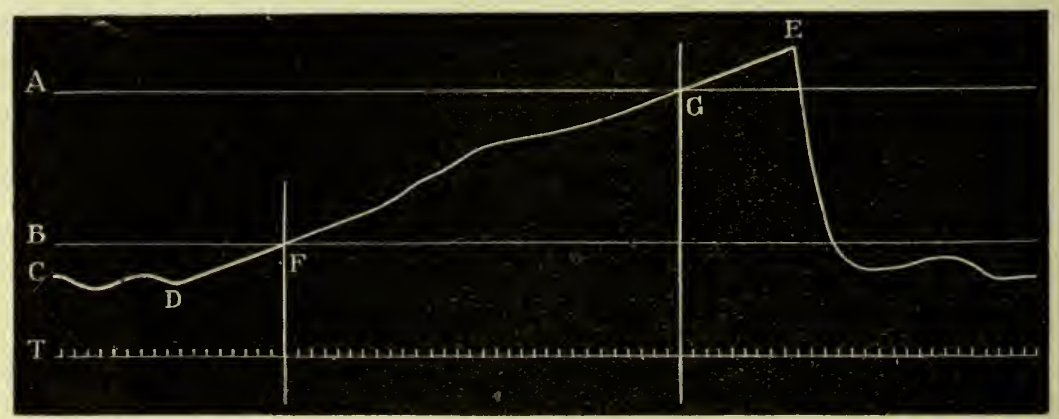

FIG. 308.-Tracing to illustrate Brodie's method of ascertaining the rate of blood-flow through an organ. For explanation see text.

The rate of the flow of blood through an organ may be ascertained, either by directly measuring the venous blood as it emerges from the organ, or by a very simple method introduced by Brodie; the organ is enclosed in an oncometer connected to a bellows recorder; the issuing vein is compressed for about a second, and the blood flowing into the organ causes it to expand; the lever of the recorder rises quickly or slowly according to the rate at which the blood is flowing into the organ. The recorder is first calibrated by injecting half a cubic centimetre of water into the tubing leading to it, and the position of the lever at rest, and that which it occupies when the oncometer is distended by half a cubic centimetre of water are marked continuously by two fixed writing-points. In the accompanying figure, obtained from an experiment on a kidney, these two lines are lettered $\mathrm{A}$ and $\mathrm{B}$. The surface travels at a quick rate, and the time-tracing $\mathrm{T}$ shows thirtieths of a second. The line $\mathrm{C}$ is traced by the oncometer lever. 
At the point $\mathrm{D}$ the renal vein was compressed, and at the point $\mathrm{E}$ the compression ceased. From $\mathrm{D}$ to $\mathrm{E}$ the inflowing blood caused the kidney to expand and the recording lever to rise. It crosses the two horizontal lines at $\mathrm{F}$ and $\mathrm{G}$ respectively. During this time ( $F$ to $G$ ), therefore, half a cubic centimetre of blood entered the kidney, and this time was thirty-three vibrations of the time-marker, that is 1.1 second. Hence the rate of blood-flow would be $\frac{0.5 \times 60}{1.1}=27.3$ c.c. of blood per minute.

In order to measure the gaseous exchange of an organ over a long period the organ is supplied with blood which alternately traverses the organ and aerates itself in a closed chamber. The amount of oxygen in the chamber is kept constant by the addition of that gas to the air of the chamber at the same rate at which the circulation acquires it. The amount of oxygen so added is measured. The method has recently been applied with conspicuous success to the gaseous exchange of the heart.

\section{Relation of Tissue Respiration to Functional Activity.}

In all organs, so far as is known, increased activity is accompanied by increased oxidation.

Much interest centres about the question of the order of time in which these events take place. This matter has been investigated

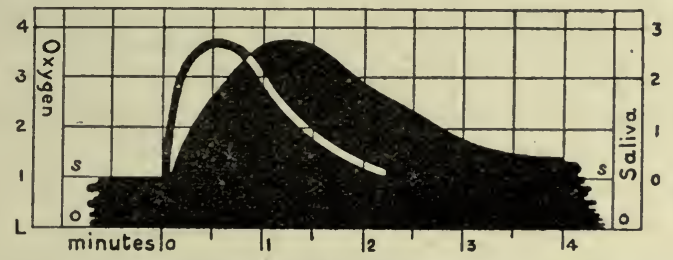

FiG. 309. -Tise black-white line represents rate of salivary secretion in c.c. per minute. $S-S=$ base line for saliva. Black area $=0 x y g e n$ used by the gland. $0-0=0 x y g e n$ base line.

in the case of skeletal muscle and the submaxillary gland (fig. 309), both of which organs can be thrown into profound activity for a short, space of time; in each caso most of the oxidation follows upon the activity, and not the activity upon the oxidation. The important inference is drawn that the contraction or secretion, as the case may be, is not caused by the oxidation in the sense that the machinery of a locomotive is driven by the energy derived from the oxidation of the coal; rather is the mechanism like that of a spring which is liberated at the moment of doing the work, and has to be rewound subsequently; the process of rewinding involves oxidation. In the case of muscle, the heat-formation which occurs in the period 
following activity only takes place if the muscle is supplied with oxygen. The output of carbonic acid, in its turn, follows upon the intake of oxygen. The order of events is therefore (1) increase of functional activity, (2) increase of heat formation and oxygen taken in, and (3) increase of carbonic acid put out.

The following table shows the coefficients of oxidation for resting organs, and the extent to which they are incręased in activity.

\begin{tabular}{|c|c|c|c|c|}
\hline Organ. & Condition of Rest. & $\begin{array}{l}\text { Oxygen used } \\
\text { per minute } \\
\text { per gramme } \\
\text { of organ. }\end{array}$ & $\begin{array}{l}\text { Condition of } \\
\text { Activity. }\end{array}$ & $\begin{array}{l}\text { Oxygen used } \\
\text { per minute } \\
\text { per gramme } \\
\text { of organ. }\end{array}$ \\
\hline $\begin{array}{l}\text { Voluntary } \\
\text { muscle. }\end{array}$ & $\begin{array}{l}\text { Nerves cut. } \\
\text { Tone absent. }\end{array}$ & 0.003 c.c. & $\begin{array}{l}\text { Tone existing in rest. } \\
\text { Gentle contraction. } \\
\text { Active contraction. }\end{array}$ & $\begin{array}{l}0.006 \text { c.c. } \\
0.020 \text { c.c. } \\
0.080 \text { c.c. }\end{array}$ \\
\hline $\begin{array}{l}\text { Unstriped } \\
\text { muscle. }\end{array}$ & Resting: & 0.004 c.c. & Contracting. & 0.007 c.c. \\
\hline Heart. & $\begin{array}{l}\text { Very slow and } \\
\text { feeble con- } \\
\text { tractions. }\end{array}$ & 0.007 c.c. & $\begin{array}{l}\text { Normal contractions. } \\
\text { Very active. }\end{array}$ & $\begin{array}{l}0.05 \text { c.c. } \\
0.08 \text { c.c. }\end{array}$ \\
\hline $\begin{array}{l}\text { Submaxillary } \\
\text { gland. }\end{array}$ & Nerves cut. & 0.03 c.c. & Chorda stinulation. & 0.10 c.c. \\
\hline Pancreas. & Not secreting. & 0.03 c.c. & $\begin{array}{l}\text { Secretion after injection } \\
\text { of secretin. }\end{array}$ & 0.10 c.c. \\
\hline Kidney. & Scanty secretion. & 0.03 c.c. & $\begin{array}{l}\text { After injection of } \\
\text { diuretic. }\end{array}$ & 0.10 c.c. \\
\hline Intestines. & Not absorbing. & 0.02 c.c. & Absorbing peptone. & 0.03 c.c. \\
\hline Liver. & $\begin{array}{l}\text { In fasting } \\
\text { animal. }\end{array}$ & $\begin{array}{l}0.01 \text { to } \\
0.02 \text { c.c. }\end{array}$ & In fed animal. & $\begin{array}{l}0.03 \text { to } \\
0.05 \text { c.c. }\end{array}$ \\
\hline $\begin{array}{l}\text { Suprarenal } \\
\text { gland. }\end{array}$ & Normal. & $\cdot 045$ c.c. & $\ldots$ & ... \\
\hline
\end{tabular}

In many cases the quantitative relationships have not been worked out.

Recent research has shown that in the case of the heart, if $\mathrm{N}$ is the number of beats per minute, $\mathrm{T}$, the maximum blood-pressure which is attained at each beat, and $\mathrm{O}$, the amount of oxygen used; then $\frac{\mathrm{N} \times \mathrm{T}}{\mathrm{O}}$ is a constant quantity, unless the cardiac muscle is itself 
rendered less efficient, as may be done by the use of drugs. This is in agreement with a series of researches on the heat given out by frogs' muscle, which shows that the heat given out in a single contraction varies directly with the tension in the muscle. The efficiency of the heart regarded as a machine seems to be low (about 2 per cent.), owing perhaps to the anatomical disposition of the strands of fibres.

Intensity of Respiration.-Most of the figures. relating to gaseous metabolism given in the preceding paragraphs were obtained from the examination of the tissues and organs of the dog. If all the tissues were examined in turn, and their relative weights known, an average might be struck which would give the gaseous metabolism for the body taken as a whole, and this might be expressed as the amount of oxygen used per minute per gramme of bodyweight. An easier and more practicable method, however, is to weigh the animal, and then from the composition of the inspired and expired air and the amount of oxygen taken in and given out, calculate how much is retained and utilised. In the dog, the amount is about 0.016 c.c. of oxygen per minute per gramme of body-weight. This figure, however, is not the same in all animals, and the size of the figure will indicate what we may term the intensity of respiration. Thus in cold-blooded animals, especially fishes with their small supply of oxygen, the figure is very much smaller. Among warm-blooded animals great variations are also seen; the intensity of respiration, for instance, is much greater in birds than in mammals. Among the mammals, the intensity of respiration varies, roughly, inversely with the size of the animal; thus, in the mouse, an animal that breathes with extreme rapidity, the intensity is probably ten to fifteen times greater than in the $\mathrm{dog}$, and in the elephant very much less. In man, the average is about half that in the dog, that is, 0.008 c.c. of oxygen per gramme of body-weight per minute.

\section{Oxygen want.}

The balance between the demand for oxygen and the supply may be upset either by increasing the demand or decreasing the supply.

Oxygen want may therefore take place either as the result of violent exercise or of diminished oxygen pressure. Normally a man takes into his blood about 400 c.c. of oxygen per minute, in sleep about half this, and in such exercise as is afforded by the pushing of a motor bicycle up a steep hill, 3000 c.c. per minute or more are absorbed by the blood. During the violent contraction of a muscle, the blood-flow through it is momentarily retarded, though over a considerable interval of time it is much increased. Nevertheless 
the momentary retardation leads to a certain amount of oxygen want, and this is probably the cause of the lactic acid output, to which we have drawn attention (see pp. 381 and 395). Lactic acid production in muscle indicates incomplete oxidation. The acid itself probably increases the blood-supply to the muscle automatically by relaxing the arteriole walls.

The symptoms produced by exercise and by decreased pressure (as in mountain sickness) are not the same, probably because the former is accompanied by an increase in the production of carbonic acid and of heat. Indeed most of the symptoms which are the ordinary effects of exercise are due probably to these causes, the actual oxygen supply to the brain being adequate during exercise.

\section{Mountain Sickness.}

The symptoms of mountain sickness differ in different persons as do those of other forms of intoxication. In one person vomiting will be the prominent feature, in another, absence of self-restraint; in a third, inability to perform any task, such as arithmetic, involving accurate cerebral processes. There is no doubt that the cause is want of oxygen. This lack of oxygen causes the production of the poisonous materials, which affect the brain and produce the symptoms. These toxic substances are no doubt products of incomplete oxidation; it is not clear how far these are produced in the brain, and how far they are carried to it in the blood.

In considering what is the lowest pressure of oxygen consistent with normal function, it must be borne in mind that with lungs of a given size this limiting pressure varies directly with the number of c.c. of oxygen which must be absorbed (Bohr).

The effect of exercise is to increase the amount of oxygen required by the tissues. The amount of oxygen required by the same person under different circumstances varies very much. Thus Zuntz resting and fasting on Monte Rosa required only 259 c.c. of oxygen per minute, whilst in the act of climbing he wanted 1329 c.c. of oxygen per minute.

In the former condition he could have remained in comfort at an altitude at which the pressure of oxygen in his alveoli was very low, but as soon as he made any effort he realised that this pressure of oxygen was insufficient to allow him to do muscular work.

Now the alveolar pressure depends partly on the barometric pressure, and partly on the nature of the respiration. The deeper the respiration the more nearly does the pressure of oxygen in the alveoli approximate to that in the atmosphere (i.e. the higher is the alveolar pressure of oxygen). Thus it happens that men who take deep respirations can tolerate altitudes which are impossible for 
shallow breathers. This is illustrated by the following figures obtained from observations on three different men :-

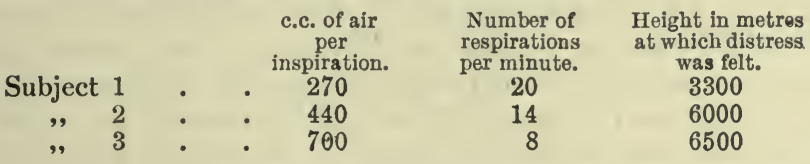

Mountain sickness therefore attacks different persons at different altitudes. If no form of adaptation took place, the following would be the approximate pressures of oxygen in the pulmonary alveoli at the following altitudes:-

$$
\begin{aligned}
& \text { Sea level . } 100 \mathrm{~mm} \text {. } \\
& 10,000 \text { feet . . } 59 \mathrm{~mm} \text {. } \\
& 15,000 \text { feet . . } 38 \mathrm{~mm} \text {. }
\end{aligned}
$$

It is known that at alveolar pressures of $60 \mathrm{~mm}$. or thereabouts the symptoms of oxygen want begin in an artificial chamber. Inspection of the dissociation curve of human blood, p. 370, will show that at $59 \mathrm{~mm}$. the arterial blood might be upwards of 85 per cent. saturated with oxygen, but at $38 \mathrm{~mm}$. it would be only 66 per cent. saturated. This condition of the blood would account for the symptoms.

Methods of adaptation to high altitudes. - Apart from the question of "training" which will be dealt with below, adaptation takes place along two lines, each of which tends to bring more oxygen into a given quantity of blood. This is accomplished (1) by raising the alveolar pressure of oxygen higher than the figures given above; and (2) by increasing the quantity of hæmoglobin in the blood.

The rise in alveolar oxygen pressure is brought about by more rapid breathing. The more rapid breathing in turn is the result of the respiratory centre being hustled by acid formation if the respiration slows down. There is therefore more oxygen and less carbonic acid in the alveolar air and in the blood than there otherwise would be. Actually, the oxygen pressures in the alveolar air were found to be:-

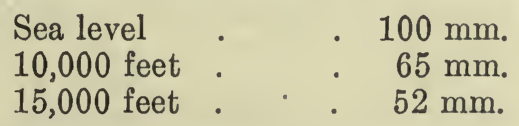

The chief difference is naturally at the greatest altitude. Here, instead of the alveolar oxygen pressure being $38 \mathrm{~mm}$. it is $52 \mathrm{~mm}$.; the blood could therefore become about 80 per cent. instead of 66 per cent., saturated with oxygen - a very material increase. Moreover, since the rate at which the blood becomes oxygenated is directly proportional to the oxygen pressure, the difference between $38 \mathrm{~mm}$. and $52 \mathrm{~mm}$. is most beneficial. The figures for the percentage 
saturation are obtained by employing the dissociation curve for blood at $40 \mathrm{~mm}$. carbonic acid pressure. The legitimacy of this may be questioned, since the carbonic acid pressure in the blood at an elevation of. 15,000 feet is only about $25 \mathrm{~mm}$.* The curve is, however, true for the blood at all altitudes since the reaction of the blood remains the same (or may shift a trifling amount to the acid side), some other less volatile acid making up for the deficiency in carbonic acid.

Increase of hoemoglobin in the blood.-At high altitudes the number of corpuscles per cubic millimetre in human blood is said to be excessive. Some caution is necessary in attributing physiological significance to such statements, for they may be in part illusory. In balloon ascents, for instance, the number of corpuscles in blood may increase very rapidly. This is not due to fresh formation of blood, but to the excessive secretion of lymph, which leads to concentration of the blood. Nevertheless, in animals kept at high altitudes for some days there is an actual increase in the formation of corpuscles, as shown by the appearance of nucleated corpuscles thrust into the blood by the bone-marrow, and an increase in the amount of iron in the blood at the expense of the quantity of that element in the liver.

Training. - We have seen that a factor in the onset of mountain sickness is the amount of oxygen which passes through the lung epithelium. It is a matter of experience that from various causes an individual can do the same amount of muscular work, such as performing the same mountain-climb, with a less degree of metabolism after training. This is in part due to the fact that the trained person has lost unnecessary weight, and in part because he uses his muscles to better advantage. Thus every unit of energy spent in work demanded a total expenditure of energy of 7 . units in the case of an untrained town-dweller, 5 units in the case of a partially trained tourist, and 3.3 units in the case of an Alpine porter. Therefore, in the same climb, the Alpine carrier would only. suffer half the increase of metabolism that the town-dweller would. He would therefore need correspondingly less oxygen, and he could reach a height at which he would have a correspondingly lower alveolar oxygen tension.

\section{Respiration at High Pressures.}

Prolonged exposure to pressures of oxygen, equal to 1300 to 1400 $\mathrm{mm}$. of mercury, induces pneumonia, and death rapidly follows. It is

* The diminution of carbonic acid tension in alveolar air and blood is termed acapnia, and Thorso attributed mountain sickness to this condition. Although this view is incorrect, it is necessary to remember that carbonic acid is not a mere waste product, but performs certain duties in the body (stimulating respiration and assisting the dissociation of oxyhæmoglobin); in certain conditions, as in shock, acapnia is an important factor. 
not possible, therefore, for men to work in air which is compressed to the extent of producing so great a pressure of oxygen.

Caisson diseuse.-In the boring of tunnels and in carrying out operations in the beds of rivers, it is usual to sink an iron tube in which the men work. This tube or caisson is closed except at the end at which the work is progressing, and the water is prevented from inundating it by pumping air into it at a pressure higher than that of the water. The men enter through a chamber with double doors or "air-lock." In this chamber the pressure can be raised or lowered. The pressure in the caisson rarely exceeds 4 atmospheres, which corresponds to about $600 \mathrm{~mm}$. of oxygen; at this pressure the workers do not suffer whilst they are in the caisson, but grave symptoms may take place shortly after they have come out. Similar symptoms are experienced by divers who come to the surface from great depths. The symptoms may take the form of paralysis, vomiting, severe abdominal pain, vertigo, etc. They are due to the fact that the plasma, and indeed all the fluids which permeate the organs of the body, become saturated with oxygen and nitrogen at the pressure of the caisson, and therefore when the pressure is suddenly removed, minute bubbles form throughout the body and injure such tissues as the spinal cord, or produce blockage of the vessels. Short hours are necessary for caisson workers, for then the body has not time to get saturated with air at the caisson pressure, and in all cases "decompression" must be gradual and slow; this gradual release from pressure is accomplished in the "air-lock." The dangers we have mentioned then cease to exist.

The atmospheric gases are specially soluble in fat; fat people are therefore very susceptible to caisson disease, and should, in fact, be prohibited from labour in caissons.

\section{Carbon Monoxide Poisoning.}

The fatal effects often produced by this gas (as in accidents from burning charcoal stoves in small close rooms, or where there is an escape of coal gas), are due to its entering into combination with the hæmoglobin of the blood-corpuscles, and thus hindering their oxygencarrying function. In an atmosphere containing both oxygen and carbon monoxide, the relative quantities of the two gases which the hæmoglobin will absorb varies with the partial pressure of the gases. The affinity of hæmoglobin for carbon monoxide is, however, much greater than its affinity for oxygen, and the compound formed-carboxyhæmoglobin-is much more stable than oxyhæmoglobin is. If, therefore, any considerable quantity of carbon monoxide is present in the air, the hæmoglobin will be almost completely charged with carboxyhæmoglobin, and asphyxia would follow. If the patient is given pure oxygen to breathe even at a late stage, 
two things will happen:-(1) The blood will take up in simple physical solution about seven times as much oxygen as when exposed to air, and this may be sufficient to carry on life; (2) as regards the saturation of the hæmoglobin, the balance is now in favour of the oxygen, weak as its affinity for hæmoglobin is, and the carbon monoxide gradually works its way out of the body.

\section{Cheyne-Stokes Respiration.}

This is a condition in which the breathing waxes and wanes to a remarkable degree (fig. 310). It is an exaggeration of the type of respiration which is often seen during sleep in perfectly healthy people. The condition was first observed by the two Dublin physicians whose names it bears. It may be induced in normal persons if they make themselves pant violently for 1-2 minutes. If then respiration is allowed to take its own course, there will first

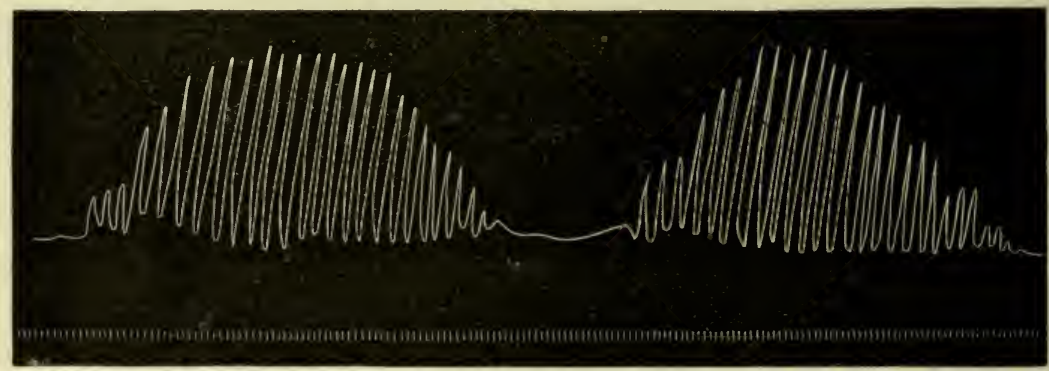

FIG. 310.-Stethograph tracing of Cheyne-Stokes respiration in a man. The time is marked in seconds. (Pembrey and Allen.)

be a pause (apnœa), then Cheyne-Stokes respiration will be set up. The groups will become less and less distinct, and respiration will ultimately become normal. The explanation of this phenomenon is as follows:-

The panting causes an undue amount of carbonic acid to leave the body, with the result that the carbonic acid tension in the blood and in the tissues sinks to perhaps a quarter or a third of its usual value. Already we have seen that carbonic acid is an active stimulant to the respiratory centre, and its removal causes respiration to cease, hence the apnœa. But during the apnœic period the arterial blood becomes less and less oxygenated, with the result that lactic acid formation (a constant result of oxygen want) takes place in the tissues ; the lactic acid, like carbonic acid, stimulates the respiratory centre, which remains active till the advent of oxygen causes oxiatation of the acid; there is then another pause, and so on. Cheyne-Stokes breathing is dependent, then, on oxygen want. 
"If from any cause, such as cerebral hæmorrhage or circulatory failure, the circulation through the respiratory centre is interfered with, or if the absorption of oxygen is interfered with by such causes as diminished barometric pressure or pathological conditions in the lungs, the occurrence of periodic or Cheyne-Stokes breathing becomes easily intelligible."-(Haldane and Douglas).

Pathological Cheyne-Stokes respiration may be removed by administration either of oxygen or of carbonic acid.

Pembrey and Pitts have also taken tracings of the same condition in the hibernating dormouse, hedgehog, marmot, and bat. In some cases the respiration has the typical Cheyne-Stokes character, with a gradual waxing and waning. In other cases periods of respiratory activity alternate with periods of apnœa, but all the respiratory efforts are about equal in force. This is known as Biot's respiration,

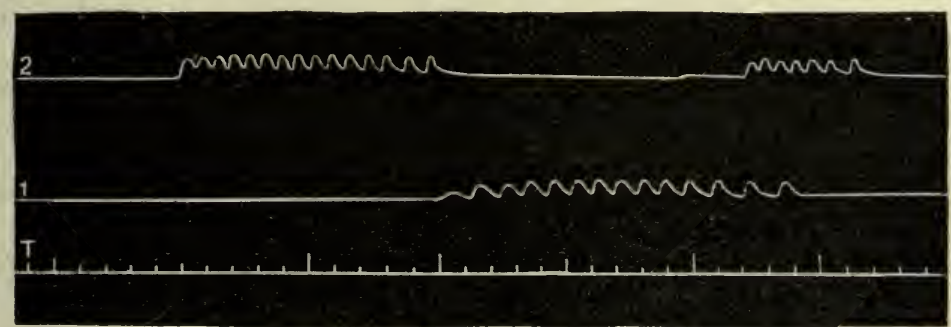

Fra. 311.-Biot's respiration in hibernating dormouse. The line marked $T$ gives time in seconds. Line 1 gives the tracing of a respiratory group which occurred once every 80 seconds, the tempera. ture of the animal being $11^{\circ} \mathrm{C}$. On warming the animal to $13^{\circ} \mathrm{C}$. the respiratory groups becanie more frequent (line 2). On warming the animal still further it awakened, and breathing, at first accompanied by shivering, became continuous. (Pembrey and Pitts.)

and is illustrated by the accompanying illustration taken from a hibernating dormouse.

\section{Diabetic Coma.}

In the preceding chapter conditions have been considered which depend upon the stimulus given to the respiratory centre by the condition of the gases in the blood. Carbonic acid is, however, not the only body capable of affecting the respiratory centre; lactic acid, as we have just seen, will stimulate it, and probably all other acids in solution will do the same. If, therefore, other acid radicals are thrown into the blood, the respiration will increase till the amount of carbonic acid in the blood is proportionally decreased. It is the total concentration of such radicals, or rather of the hydrogen ions which they carry in their wake, that remains a constant factor. In diabetic coma other acids (e.g., hydroxybutyric) make their appearance, and the respiration is exaggerated till only a small amount of carbonic acid remains. 


\section{CHAPTER XXVIII}

\section{THE CHEMICAL COMPOSITION OF TIE BODY}

THE elements found in the body are carbon, nitrogen, hydrogen, oxygen, sulphur, phosphorus, fluorine, chlorine, iodine, silicon, sodium, potassium, calcium, magnesium, lithium, iron, and occasionally traces of manganese, copper, and lead.

Of these, very few occur in the free state. Oxygen (to a small extent) and nitrogen are found dissolved in the blood; hydrogen is formed by putrefaction in the alimentary canal. With some few exceptions such as these, the elements enumerated above are found combined with one another to form compounds.

The compounds, or, as they are frequently termed in physiology, the proximate principles, found in the body are divided into-

(1) Mineral or inorganic compounds.

(2) Organic compounds, or compounds of carbon.

The inorganic compounds present are water, various acids (such as hydrochloric acid in the gastric juice), ammonia (as in the urine), and numerous salts, such as calcium phosphate in bone, sodium chloride in blood and urine, and many others.

The organic compounds are more numerous; they may be subdivided into-

$$
\begin{array}{ll}
\text { Nitrogenous } & \left\{\begin{array}{l}
\text { Proteins-e.g., albumin, myosin, casein, gelatin, etc. } \\
\text { Simpler nitrogenous bodies-e.g., lecithin, urea, } \\
\text { creatine. }
\end{array}\right. \\
\text { Non-nitrogenous }\left\{\begin{array}{l}
\text { Fats-e.g., butter, fats of adipose tissue. } \\
\text { Carbohydrates-e.g., sugar, starch. } \\
\begin{array}{c}
\text { Simpler organic bodies-e.g., cholesterin, lactic } \\
\text { acid. }
\end{array}
\end{array}\right.
\end{array}
$$

The subdivision of the organic proximate principles into proteins, fats, and carbohydrates forms the starting-point of chemical physiology.

\section{Carbohydrates.}

The Carbohydrates are found chiefly in vegetable tissues, and many of them form important foods. Some carbohydrates are, however, found in or formed by the animal organism. The most important 
of these are glycogen, or animal starch; dextrose ; and lactose, or milk sugar.

The carbohydrates may be conveniently defined as compounds of carbon, hydrogen, and oxygen, the two last-named elements being in the proportion in which they occur in water. But this definition is only a rough one, and if pushed too far would include many substances such as acetic acid, lactic acid, and inosite, which are not carbohydrates. Research has shown that the chemical constitution of the simplest carbohydrates is that of an aldehyde, or a ketone, and that the more complex carbohydrates are condensation products of the simple ones. In order, therefore, that we may understand the constitution of these substances, it is first necessary that we should understand what is meant by the terms aldehyde and ketone.

A primary alcohol is one in which the hydroxyl $(\mathrm{OH})$ and two hydrogen atoms are attached to the same carbon atom; it, therefore, contains the group $\mathrm{CH}_{2} \mathrm{OH}$. Thus the formula for common alcohol (primary ethyl alcohol) is

$$
\mathrm{CH}_{3} \cdot \mathrm{CH}_{2} \mathrm{OH} \text {. }
$$

The formula for the next alcohol of the same series (primary propyl alcohol) is

$$
\mathrm{CH}_{3} \cdot \mathrm{CH}_{2} \cdot \mathrm{CH}_{2} \mathrm{OH} \text {. }
$$

If a primary alcohol is oxidised, the first oxidation product is called an aldehyde; thus ethyl alcohol yields acetic aldehyde:-

$$
{ }_{[\text {[Ethyl alcohol.] }}^{\mathrm{CH}_{3} \cdot \mathrm{CH}_{2} \mathrm{OH}}+\mathrm{O}=\underset{\text { [Acetic aldehyde }]}{\mathrm{CH}_{3}} \cdot \mathrm{COH}+\mathrm{H}_{2} \mathrm{O} \text {. }
$$

The typical group $\mathrm{COH}$ of the aldehyde is not stable, but is easily oxidisable to form the group $\mathrm{COOH}$ (carboxyl), and the compound so formed is called an acid; in this way acetic aldehyde forms acetic acid :-

$$
\underset{\text { [Acetic aldehyde.] }}{\mathrm{CH}_{3}} \cdot \mathrm{COH}+\mathrm{O}=\underset{\text { [Acetic acid.] }}{\mathrm{CH}_{3}} \cdot \mathrm{COOH} \text {. }
$$

The majority of the simple sugars are aldehydes of more complex alcohols than this; they are spoken of as aldoses. The readiness with which aldehydes are oxidisable renders them powerful reducing agents, and this furnishes us with some of the tests for the sugars.

Let us now turn to the case of the ketones. A secondary alcohol is one in which the $\mathrm{OH}$ group and one hydrogen atom are attached to the same carbon atom; thus secondary propyl alcohol has the formula

$$
\mathrm{CH}_{3} \cdot \mathrm{CHOH} \cdot \mathrm{CH}_{3} \text {. }
$$

Its typical group is therefore $\mathrm{CHOH}$. When this is oxidised, the first oxidation product is called a ketone, thus :-

$$
\underset{\text { [Secondary propyl alcohol.] }}{\mathrm{CH}_{3}} \cdot \mathrm{CHOH} \cdot \mathrm{CH}_{3}+\mathrm{O}=\underset{\text { [Propyl ketone or acetone.] }}{\mathrm{CH}_{3}} \cdot \mathrm{CO} \cdot \mathrm{CH}_{2} \mathrm{O} \text {. }
$$

It therefore contains the group CO.

Some of the sugars are ketones of more complex alcohols; these 
are called ketoses. The only one of these which is of physiological interest is lævulose.

The alcohols of which we have already spoken are called monatomic, because they contain only one $\mathrm{OH}$ group. Those which contain two $\mathrm{OH}$ groups (such as glycol) are called diatomic; those which contain three $\mathrm{OH}$ groups (such as glycerin) are called triatomic; and so on. The hexatomic alcohols are those which contain six $\mathrm{OH}$ groups. Three of these hexatomic alcohols with the formula $\mathrm{C}_{6} \mathrm{H}_{8}(\mathrm{OH})_{6}$ are of physiological interest; they are isomerides, and their names are sorbite, mannite, and dulcite. By careful oxidation their aldehydes and ketones can be obtained; these are the simple sugars; thus, dextrase is the aldehyde of sorbite; mannose is the aldehyde of mannite; lævulose is the ketone of mannite; and galactose is the aldehyde of dulcite. These sugars all have the empirical formula $\mathrm{C}_{6} \mathrm{H}_{12} \mathrm{O}_{6}$. They furnish an excellent example of what is called stereochemical isomerism; that is, the position of the atoms or groups of atoms in space within the sugar molecule varies. The constitutional formulæ of three important simple sugars are shown below. The six carbon atoms in each case form an open chain, but the way in which the hydrogen and hydroxyl atoms are linked to them differs.<smiles>OC[C@H](O)[C@H](O)[C@H](O)[C@H](O)CO</smiles>

[Dextrose.]

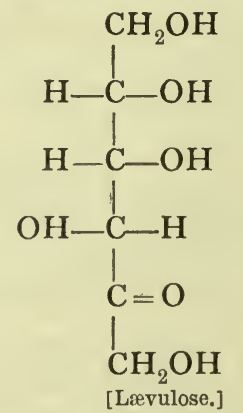

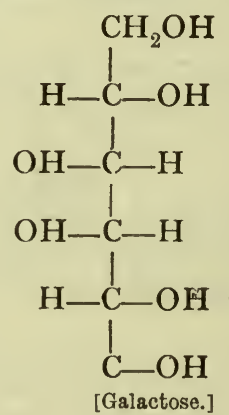

The aldehyde constitution of dextrose and galactose is at once evident, the typical aldehyde group $(\mathrm{COH})$ being at the end of the chain, whereas the ketone constitution of lævulose is shown by the typical ketone group ( $\mathrm{CO}$ ) not at the end of the chain.

By further oxidation, the sugars yield acids with various names.

If we take such sugars as typical specimens, we see that their general formula is

$$
\mathrm{C}_{n} \mathrm{H}_{2 m} \mathrm{O}_{m}
$$

and as a general rule $n=m$; that is, the number of oxygen and carbon atoms are equal. This number in the case of the sugars already mentioned is six. Hence they are called hexoses.

Sugars are known to chemists, in which this number is $3,4,5,7$, etc., and these are called trioses, tetroses, pentoses, heptoses, etc. The majority of these 
have no physiological interest. It should, however, be mentioned that a pentose has been obtained from the certain nucleic acids presently to be described (see $p$. 431) which are contained in animal organs (pancreas, liver, etc.), and in plants (for instance, yeast). If the pentoses which are found in various plants are given to an animal, they are excreted in great measure unchanged in the urine.

The hexoses are of great physiological importance. The principal ones are dextrose, lævulose, and galactose. These are called monosaccharides.

Another important group of sugars are called disaccharides; these are formed by what is called condensation; that is, two molecules of monosaccharide combine together with the loss of a molecule of water, thus:-

$$
\mathrm{C}_{6} \mathrm{H}_{12} \mathrm{O}_{6}+\mathrm{C}_{6} \mathrm{H}_{12} \mathrm{O}_{6}=\mathrm{C}_{12} \mathrm{H}_{22} \mathrm{O}_{11}+\mathrm{H}_{2} \mathrm{O} \text {. }
$$

The principal members of the disaccharide group are cane sugar, lactose, and maltose.

If more than two molecules of the monosaccharide group undergo a corresponding condensation, we get what are called polysaccharides.

$$
n \mathrm{C}_{6} \mathrm{H}_{12} \mathrm{O}_{6}=\left(\mathrm{C}_{6} \mathrm{H}_{10} \mathrm{O}_{5}\right)_{n}+n \mathrm{H}_{2} \mathrm{O} \text {. }
$$

The principal polysaccharides are starch, glycogen, various dextrins, and cellulose. We may, therefore, arrange the important carbo-

\begin{tabular}{|c|c|c|}
\hline $\begin{array}{l}\text { 1. Monosaccharides, } \\
\mathrm{C}_{6} \mathrm{H}_{12} \mathrm{O}_{6}{ }^{7}\end{array}$ & $\begin{array}{l}\text { 2. Disaccharides, } \\
\mathrm{C}_{12} \mathrm{H}_{22} \mathrm{O}_{11} \text {. }\end{array}$ & $\begin{array}{l}\text { 3. Polysaccharides } \\
\left(\mathrm{C}_{6} \mathrm{H}_{10} \mathrm{O}_{5}\right)_{n}\end{array}$ \\
\hline $\begin{array}{l}\text { + Dextrose. } \\
\text { - Læevulose. } \\
\text { + Galactose. }\end{array}$ & $\begin{array}{l}\text { + Cane sugar. } \\
\text { + Lactose. } \\
\text { + Maltose. }\end{array}$ & $\begin{array}{l}\text { + Starch. } \\
\text { + Glycogen. } \\
\text { + Dextrin. } \\
\text { Cellulose. }\end{array}$ \\
\hline
\end{tabular}
hydrates of the hexose family in a tabular form as follows:-

The + and - signs in the above list indicate that the substances to which they are prefixed are dextro-and lævo-rotatory respectively as regards polarised light. The formulæ given in the table are merely empirical; the quantity $n$ in the starch group is variable and often large. The following are the chief facts in relation to each of the principal carbohydrates.

Dextrose, Glucose, or Grape Sugar.-This carbohydrate is found in many fruits, honey, and in minute quantities in the blood and numerous tissues, organs, and fluids of the body. It is the form of sugar found in large quantities in the blood and urine in the disease known as diabetes.

Dextrose is soluble in hot and cold water and in alcohol. It is crystalline, but not so sweet as cane sugar. When heated with strong potash certain complex acids are formed which have a yellow or 
brown colour. This constitutes Moore's test for sugar. In alkaline solutions dextrose reduces salts of silver, bismuth, mercury, and copper. The reduction of cupric to cuprous salts constitutes Trommer's test, which is performed as follows: put a few drops of copper sulphate into a test-tube, then solution of dextrose, and then strong caustic potash. On adding the potash a precipitate is first formed which dissolves, forming a blue solution. On boiling this, a yellow or red precipitate (cuprous hydrate or oxide) forms.

On boiling a solution of dextrose with an alkaline solution of picric acid, a dark red opaque solution due to reduction to picramic acid is produced.

Another important property of grape sugar is that under the influence of yeast it is converted into ethyl alcohol and carbonic a.cid $\left(\mathrm{C}_{6} \mathrm{H}_{12} \mathrm{O}_{6}=2 \mathrm{C}_{2} \mathrm{H}_{6} \mathrm{O}+2 \mathrm{CO}_{2}\right)$.

Dextrose may be estimated by the fermentation test, by the polarimeter, and by the use of Fehling's solution. The last method is the most important: it rests on the same principles as Trommer's test, and we shall study it in connection with diabetic urine.

Lævulose or Fructose.-When cane sugar is treated with dilute mineral acids it undergoes a process known as inversion-i.e., it takes up water and is converted into equal parts of dextrose and lævulose. The previously dextro-rotatory solution of cane sugar then becomes lævo-rotatory, the lævo-rotatory power of the lævulose being greater than the dextro-rotatory power of the dextrose formed. Hence the term inversion. The same hydrolytic change is produced by certain enzymes, such as the invertase of the intestinal juice and of yeast. Pure lævulose can be crystallised with difficulty. It gives the same general reactions as dextrose.

Galactose is formed by the action of dilute mineral acids or of inverting enzymes on lactose. It resembles dextrose in its action on polarised light, in reducing cupric salts in Trommer's test, and in being directly fermentable with yeast. When oxidised by means of nitric acid it yields an acid called mucic acid $\left(\mathrm{C}_{6} \mathrm{H}_{10} \mathrm{O}_{8}\right)$, which is only slightly soluble in water. Dextrose when treated in this way yields an isomeric acid-i.e., an acid with the same empirical formula, called saccharic acid, which is very soluble in water.

Cane Sugar is generally distributed in the vegetable kingdom, but especially in the juices of the sugar cane, beetroot, mallow, and sugar maple. It is a substance of great importance as a food. It undergoes inversion in the alimentary canal. It is crystalline, and dextro-rotatory. With Trommer's test it gives a blue solution, but no reduction occurs in boiling. After inversion it is, of course, strongly reducing.

Inversion may be accomplished by boiling with dilute mineral acids, or by means of inverting enzymes such as that occurring in the 
intestinal juice. It then takes up water, and is split into equal parts of dextrose and lævulose.

$$
\underset{\text { [Cane sugar.] }}{\mathrm{C}_{12} \mathrm{H}_{22} \mathrm{O}_{11}}+\mathrm{H}_{2} \mathrm{O}=\underset{\text { [Dextrose.] }}{\mathrm{C}_{6} \mathrm{H}_{12} \mathrm{O}_{6}}+\underset{\text { [Levulose.] }}{\mathrm{C}_{6} \mathrm{H}_{12} \mathrm{O}_{6}}
$$

With yeast, cane sugar is first inverted by means of a special enzyme invertase secreted by the yeast cells, and then there is an alcoholic fermentation of the monosaccharides so formed, which is accomplished by another enzyme called zymase.

Lactose, or Milk Sugar, occurs in milk. It is occasionally found in the urine of women in the early days of lactation, or after weaning. It is crystallisable, dextro-rotatory, much less soluble in water than other sugars, and has only a slightly sweet taste. It gives Trommer's test, but when the reducing power is tested quantitatively by Fehling's solution it is found to be a less powerful reducing agent than dextrose, in the proportion of 7 to 10.

When hydrolysed by similar agencies as those mentioned in connection with cane sugar, it takes up water and splits into dextrose and galactose.

$$
\underset{\text { [Lactose.] }}{\mathrm{C}_{12} \mathrm{H}_{22} \mathrm{O}_{11}}+\mathrm{H}_{2} \mathrm{O}=\underset{\text { [Dextrose.] }}{\mathrm{C}_{6} \mathrm{H}_{12} \mathrm{O}_{6}}+\underset{\text { [Galactose.] }}{\mathrm{C}_{6} \mathrm{H}_{12} \mathrm{O}_{6}}
$$

With yeast it is first inverted, and then alcohol is formed. This, however, occurs slowly.

The lactic acid fermentation which occurs when milk turns sour is brought about by enzymes secreted by certain micro-organisms, which are somewhat similar to yeast cells. Bacteria in the intestine bring about the same result. The two stages of the lactic acid fermentation are represented in the following equations :-

$$
\begin{aligned}
& \text { (1.) } \underset{12}{\mathrm{C}_{12} \mathrm{H}_{22} \mathrm{O}_{11}}+\mathrm{H}_{2} \mathrm{O}=\underset{\text { [Lactic acid.] }}{4 \mathrm{C}_{3} \mathrm{H}_{6} \mathrm{O}_{3} \text {. }} \\
& \text { (2.) } \underset{\text { [Lactic acid.] }}{4 \mathrm{C}_{3} \mathrm{H}_{6} \mathrm{O}_{3}}=\underset{\text { [Butyric acid.] }}{2 \mathrm{C}_{4} \mathrm{H}_{8} \mathrm{O}_{2}}+4 \mathrm{CO}_{2}+4 \mathrm{H}_{2} \text {. }
\end{aligned}
$$

Maltose is the chief end-product of the action of malt diastase on starch, and is also formed as an intermediate product in the action of dilute sulphuric acid on the same substance. It is the chief sugar formed from starch by the diastatic enzymes contained in the saliva and pancreatic juice. It can be obtained in the form of acicular crystals, and is strongly dextro-rotatory. It gives Trommer's test; but its reducing power, as measured by Fehling's solution, is one-third less than that of dextrose. With yeast it yields alcohol.

By prolonged boiling with water, or, more readily, by boiling with a dilute mineral acid, or by means of an inverting enzymes such as occurs in the intestinal juice, it is converted into dextrose.

$$
\underset{\text { [Maltose.] }}{\mathrm{C}_{12} \mathrm{H}_{22} \mathrm{O}_{11}}+\mathrm{H}_{2} \mathrm{O}=\underset{\text { [Dextrose.] }}{2 \mathrm{C}_{6} \mathrm{H}_{12} \mathrm{O}_{6}} \text {. }
$$


Phenyl-hydrazine Test.-The three important reducing sugars with which we have to deal in physiology are dextrose, lactose, and maltose. They may be distinguished by their relative reducing powers on Fehling's solution, or by the characters of their osazones. The osazone is formed in each case by adding phenyl hydrazine hydrochloride, and sodium acetate, and boiling the mixture for about half an hour. In each case the osazone is deposited in the form of bright canary-coloured, needle-like crystals, usually in bunches, which differ in their crystalline form, melting-point, and solubilities. Cane sugar does not yield an osazone.

Starch is widely diffused through the vegetable kingdom. It occurs in nature in the form of microscopic grains, varying in size and

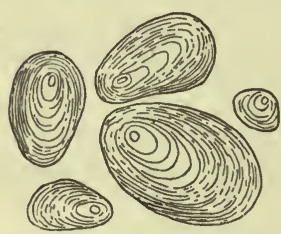

FIG. 312.-Grains of potato starch. appearance, according to their source. Each consists of a central spot, round which more or less concentric envelopes of starch proper or granulose alternate with layers of cellulose. Cellulose has very little digestive value, but starch is a most important food.

Starch is insoluble in cold water: it forms an opalescent solution in boiling water, which if concentrated gelatinises on cooling. Its most characteristic reaction is the blue colour it gives with iodine.

On heating starch with mineral acids, dextrose is formed. By the action of diastatic enzymes, maltose is the chief end-product. In both cases dextrin is an intermediate stage in the process.

Before the formation of dextrin the starch solution loses its opalescence, a substance called soluble starch being formed. This, like native starch, gives a blue colour with iodine. Although the molecular weight of starch is unknown, the formula for soluble starch is probably $5\left(\mathrm{C}_{12} \mathrm{H}_{20} \mathrm{O}_{10}\right)_{20}$. Equations that represent the formation of sugars and dextrins from this are very complex, and are at present only hypothetical.

Dextrin is the name given to the intermediate products in the hydrolysis of starch or glycogen, and two chief varieties are distinguished:-erythro-dextrin, which gives a reddish-brown colour with iodine; and achroo-dextrin, which does not.

It is readily soluble in water, but insoluble in alcohol and ether. It is gummy and amorphous. It does not give Trommer's test, nor does it ferment with yeast. It is dextro-rotatory. By hydrolysing agencies it is converted into glucose.

Glycogen, or animal starch, is found in liver, muscle, and white blood-corpuscles. It is also abundant in embryonic tissues.

Glycogen is a white tasteless powder, soluble in water, but it forms, like starch, an opalescent solution. It is insoluble in alcohol 
and ether. It is dextro-rotatory. With Trommer's test it gives a blue solution, but no reduction occurs on boiling.

With iodine it gives a reddish or port-wine colour, very similar to that given by erythro-dextrin. Dextrin may be distinguished from glycogen by (1) the fact that it gives a clear, not an opalescent, solution with water; and (2) it is not precipitated by basic lead acetate as glycogen is. It is, however, precipitated by basic lead acetate and ammonia. (3) Glycogen is precipitated by 55 per cent. of alcohol; the dextrins require 85 per cent. or more. (4) It is precipitated by saturation with ammonium sulphate; erythro-dextrin is only partially precipitable by this means.

Cellulose.-This is the colourless material of which the cell-walls and woody fibres of plants are composed. By treatment with strong mineral acids it is, like starch, converted into glucose, but with much greater difficulty. The various digestive enzymes have little or no action on cellulose; hence the necessity of boiling starch before it is taken as food. Boiling bursts the cellulose envelopes of the starch grains, and so allows the digestive juices to get at the starch proper. Cellulose is found in a few animals, as in the outer investment of the Tunicates.

Inosite was discovered by Scherer in 1850 as a constituent of muscle, and for a long time was known as muscle sugar. It occurs also in small quantities in other animal organs (liver, kidney, etc.), and in plants it is a fairly constant constituent of roots and leaves, especially growing leaves.

It has the same molecular formula as the simple sugars $\left(\mathrm{C}_{6} \mathrm{H}_{12} \mathrm{O}_{6}\right)$, but it has none of the other properties of these substances. Maquenne ascertained that it has the following lormula-

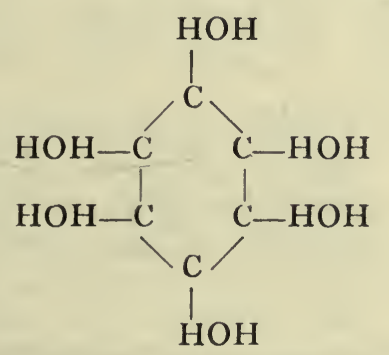

which a mere glance at will show is very different from those of the sugars given on p. 408. For the six carbon atoms, instead of forming an open chain, are linked into a ring, as in the benzene derivatives. It is in fact a reduced hexa-oxybenzene. It probably represents a transition stage between the carbohydrates and the benzene compounds. By a closing-up of the open chain of the carbo- 
hydrate molecule its formation from the latter is theoretically possible. On the other hand, the opening of the inosite ring would give rise to an open chain, and it has indeed been found that lactic acid is formed from inosite by the action of certain bacteria.

\section{The Fats.}

Fat is found in small quantities in many animal tissues. It is, however, found in large quantities in three situations, viz., marrow, adipose tissue, and milk.

The contents of the fat cells of adipose tissue are fluid during life, the normal temperature of the body $\left(37^{\circ}\right.$ C., or $99^{\circ} \mathrm{F}$.) being considerably above the melting-point $\left(25^{\circ} \mathrm{C}\right.$.) of the mixture of. the fats found there. These fats are three in number, and are called palmitin, stearin, and olein. They differ from one another in chemical composition and in certain physical characters, such as melting-point and solubilities. Olein solidifies at $-5^{\circ} \mathrm{C}$., palmitin at $45^{\circ} \mathrm{C}$., and stearin at $53-65^{\circ} \mathrm{C}$. It is thus olein which holds the other two dissolved at the body temperature. Fats are all soluble in hot alcohol, ether, and chloroform, but insoluble in water.

Chemical Constitution of the Fats. - The fats are compounds of fatty acids with glycerin, and may be termed glycerides or glyceric ethers.

The fatty acids form a series of acids derived from the monatomic alcohols by oxidation. Thus, to take ordinary ethyl alcohol, $\mathrm{C}_{2} \mathrm{H}_{6} \mathrm{O}$, the first stage in oxidation is the removal of two atoms of hydrogen to form aldehyde, $\mathrm{C}_{2} \mathrm{H}_{4} \mathrm{O}$; on further oxidation an atom of oxygen is added to form acetic acid, $\mathrm{C}_{2} \mathrm{H}_{4} \mathrm{O}_{2}$. thus:-

A similar acid can be obtained from all the other alcohols,

From methyl alcohol
," ethyl ", propyl ",
," butyl ",
," hexyl ",

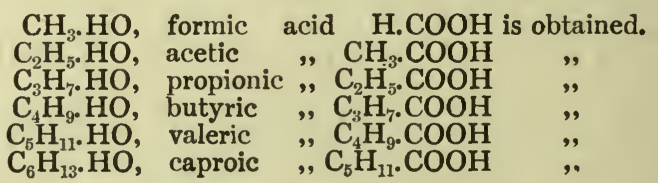

and so on.

The sixteenth term of this series has the formula $\mathrm{C}_{15} \mathrm{H}_{31} . \mathrm{COOH}$, and is called palmitic acid; the eighteenth has the formula $\mathrm{C}_{17} \mathrm{H}_{35} \mathrm{COOH}$, and is called stearic acid. Each acid, as will be seen, consists of a radical, $\mathrm{C}_{n-1} \mathrm{H}_{2 n-1} \mathrm{CO}$, united to hydroxyl $(\mathrm{OH})$.

Oleic acid, however, is not a member of this series, but belongs to a somewhat similar series known as the acrylic series, of which 
the general formula is $\mathrm{C}_{n-1} \mathrm{H}_{2 n-3} . \mathrm{COOH}$. It is the eighteenth term of the series, and its formula is $\mathrm{C}_{17} \mathrm{H}_{33}$. $\mathrm{COOH}$.

The first member of the group of alcohols from which this acrylic series of acids is obtained is called allyl alcohol $\left(\mathrm{CH}_{2}: \mathrm{CH} \cdot \mathrm{CH}_{2} \mathrm{OH}\right)$; the aldehyde of this is acrolein ( $\mathrm{CH}_{2}: \mathrm{CH} . \mathrm{CHO}$ ), and the formula for the acid (acrylic acid) is $\mathrm{CH}_{2}$ : $\mathrm{CH}$. $\mathrm{COOH}$. It will be noticed that two of the carbon atoms are united by two valencies, and these bodies are therefore unsaturated; they are unstable and are prone to undergo by uniting with another element a conversion into bodies in which the carbon atoms are united by only one bond. This accounts for their reducing action, and it is owing to this that the colour reactions with osmic acid and Sudan III. (red coloration) are due. Fat which contains any member of the acrylic series, such as oleic acid, blackens osmic acid, by reducing it to a lower (black) oxide. The fats palmitin and stearin do not give these reactions.

Glycerin or Glycerol is a triatomic alcohol, $\mathrm{C}_{3} \mathrm{H}_{5}(\mathrm{HO})_{3}$-i.e., three atoms of hydroxyl united to a radical glyceryl $\left(\mathrm{C}_{3} \mathrm{H}_{5}\right)$. The hydrogen in the hydroxyl atoms is replaceable by other organic radicals. As an example, take the radical of acetic acid called acetyl $\left(\mathrm{CH}_{3} \cdot \mathrm{CO}\right)$. The following formulæ represent the derivatives that can be obtained by replacing one, two, or all three hydroxyl hydrogen atoms in this way :-

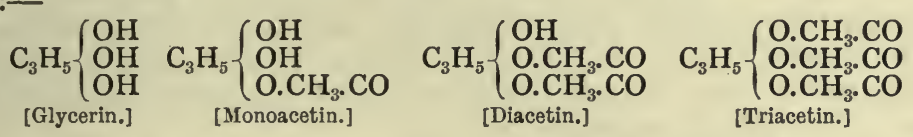

Triacetin is a type of a neutral fat; stearin, palmitin, and olein ought more properly to be called tristearin, tripalmitin, and triolein respectively. Each consists of glycerin in which the three atoms of hydrogen in the hydroxyls are replaced by radicals of the fatty acid. This is represented in the following formulæ:-

Acid.

Palmitic acid $\mathrm{C}_{15} \mathrm{H}_{31} \cdot \mathrm{COOH}$ Stearic acid $\mathrm{C}_{17} \mathrm{H}_{35} \cdot \mathrm{COOH}$ Oleic acid
Radical.

Palmityl $\mathrm{C}_{15} \mathrm{H}_{31}$. CO

Stearyl $\mathrm{C}_{17} \mathrm{H}_{35} \cdot \mathrm{CO}$

Oleyl $\mathrm{C}_{17}^{17} \mathrm{H}_{33}$. $\mathrm{CO}$
Fat.

Palmitin $\mathrm{C}_{3} \mathrm{H}_{5}\left(\mathrm{OC}_{15} \mathrm{H}_{31} \cdot \mathrm{CO}\right)_{3}$

Stearin $\mathrm{C}_{3} \mathrm{H}_{5}\left(\mathrm{OC}_{17} \mathrm{H}_{35} \cdot \mathrm{CO}\right)_{3}$

Decomposition Products of the Fats.-The fats split up into the substances out of which they are built up.

Under the influence of superheated steam, mineral acids, and in the body by means of certain enzymes (for instance, the fat-splitting enzyme, lipase, of the pancreatic juice), a fat combines with water and splits into glycerin and the fatty acid. The following equation represents what occurs in a fat, taking tripalmitin as an example:-

$$
\underset{\text { [Tripalmitin- a fat.] }}{\mathrm{C}_{3} \mathrm{H}_{5}\left(\mathrm{O}_{3} \mathrm{C}_{15} \mathrm{H}_{31} \mathrm{CO}\right)_{3}}+3 \mathrm{H}_{2} \mathrm{O}=\underset{\text { [Glycerin.] }}{\mathrm{C}_{3} \mathrm{H}_{5}(\mathrm{OH})_{3}}+\underset{\substack{\text { [Palmitic acid-2 } \\ \text { fatty acid.] }}}{3 \mathrm{C}_{15} \mathrm{H}_{31} \mathrm{CO} . \mathrm{OH}}
$$

In the process of saponification much the same sort of reaction occurs, the final products being glycerin and a compound of the base 
with the fatty acid which is called a soap. Suppose, for instance, that potassium hydrate is used; we get-

$$
\underset{\text { [Tripalmitin-2 fat.] }}{\mathrm{C}_{3} \mathrm{H}_{5}\left(\mathrm{O}_{3} \mathrm{C}_{15} \mathrm{H}_{31} \mathrm{CO}\right)_{3}}+3 \mathrm{KHO}=\underset{\text { [Glycerin.] }}{\mathrm{C}_{8} \mathrm{H}_{5}(\mathrm{OH})_{3}}+\underset{\substack{\text { [Potassium palmitate- } \\ \text { a soap.] }}}{3 \mathrm{C}_{15} \mathrm{H}_{31} \mathrm{CO} . \mathrm{OK} .}
$$

Emulsification.-Another change that fats undergo in the body is very different from saponification. It is a physical not a chemical change; the fat is broken up into very small globules, such as are seen in the natural emulsion-milk.

\section{The Proteins.}

The proteins are the most important substances that occur in animal and vegetable organisms, and protein metabolism is, as already noted (p. 6), the most characteristic sign of life.

They are highly complex compounds of carbon, hydrogen, oxygen, nitrogen, and sulphur, occurring in a solid viscous condition or in solution in nearly all parts of the body. The different members of the group present great similarities, for instance, in the heaviness of their molecules, and in giving certain colour tests we shall be describing presently; there are, on the other hand, considerable differences between the various proteins.

The proteins in the food form the source of the proteins in the body tissues, but the latter are usually different in composition from the former. The food proteins are in the process of digestion broken up into simpler substances, usually called cleavage products, and it is from these that the body cells reconstruct the proteins peculiar to themselves. As a result of katabolic processes in the body, the proteins are finally again broken down, carbonic acid, water, sulphuric acid (combined as sulphates), urea, and creatinine being the principal final products which are discharged in the urine and other excretions. The substances intermediate between the proteins and these final katabolites will be discussed under urine.

The following figures will show how different the proteins are even in elementary composition. Hoppe-Seyler many years ago gave the variations in percentage composition as follows:-

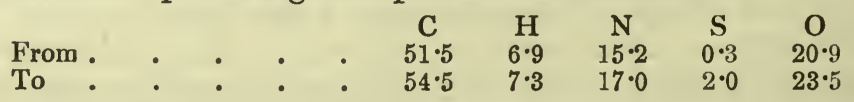

Recent research has since shown that the variations are even greater than those given by Hoppe-Seyler.

Differences are also seen when the cleavage products are separated and estimated. These differ both in kind and in amount, but nearly all of them are substances which are termed amino-acids. Emil Fischer to whom we owe so much of our knowledge in this 
direction, considers that the proteins are linkages of a greater or lesser number of these amino-acids, and there is little doubt that in the future this work will result in an actual synthesis of the protein molecule, and with that will come an accurate knowledge of its constitution.

When the protein molecule is broken down in laboratory processes, or by the digestive enzymes which occur in the alimentary canal, the essential change is due to what is called hydrolysis; that is, the molecule unites with water and then breaks up into smaller molecules. The early cleavage products, which are called proteoses, retain many of the characters of the original protein, and the same is true, though to a less degree, of the peptones, which come next in order of formation. The peptones, in their turn, are decomposed into short linkages of amino-acids, which are called polypeptides, and finally the individual amino-acids are obtained separated from each other.

What we have already learnt about the fatty acids will help us in understanding what is meant by an amino-acid.

If we take acetic acid, which is one of the simplest of the fatty acids, its formula is

\section{$\mathrm{CH}_{3} \cdot \mathrm{COOH}$.}

If one of the three hydrogen atoms in the $\mathrm{CH}_{3}$ group is replaced by $\mathrm{NH}_{2}$, we get a substance which has the formula

$$
\mathrm{CH}_{2} \cdot \mathrm{NH}_{2} \cdot \mathrm{COOH} \text {. }
$$

The combination $\mathrm{NH}_{2}$, which has stepped in, is called the aminogroup, and the new substance now formed is called amino-acetic acid; it is also termed glycine or glycocoll.

We may take another example from another fatty acid. Propionic acid is $\mathrm{C}_{2} \mathrm{H}_{5} . \mathrm{COOH}$; if we replace an atom of hydrogen by the amino-group as before, we obtain $\mathrm{C}_{2} \mathrm{H}_{4} \cdot \mathrm{NH}_{2} \cdot \mathrm{COOH}$, which is amino-propionic acid or alanine.

If instead of propionic we take hydroxy-propionic acid, its aminoderivative (amino-hydroxy-propionic acid) is termed serine.

A fourth amino-acid is similarly obtained by the introduction of the $\mathrm{NH}_{2}$ into valeric acid $\mathrm{C}_{4} \mathrm{H}_{9} \cdot \mathrm{COOH}$. Amino-valeric acid $\mathrm{C}_{4} \mathrm{H}_{8} \cdot \mathrm{NH}_{2}$. COOH is called valine.

Going to the next fatty acid in the series, caproic acid $\mathrm{C}_{5} \mathrm{H}_{11} \cdot \mathrm{COOH}$, we obtain from it in an exactly similar way, $\mathrm{C}_{5} \mathrm{H}_{10} \cdot \mathrm{NH}_{2} \cdot \mathrm{COOH}$, which is amino-caproic acid or leucine.

According to the way in which the amino-group is linked, a large number of isomeric amino-caproic acids, all with the same empirical formula, are theoretically possible. Many of these have been prepared synthetically, and it has been shown that the amino-caproic acid called leucine, formed by hydrolysis from proteins, is the lævo-rotatory variety, and should be more accurately named a-amino-isobutyl- 
acetic acid $\left(\mathrm{CH}_{3}\right)_{2} \mathrm{CH} . \mathrm{CH}_{2}\left(\mathrm{CH} . \mathrm{NH}_{2}\right) \mathrm{COOH}$. It crystallises in spheroidal clumps of crystals, as shown on the left-hand side of fig. 313 .

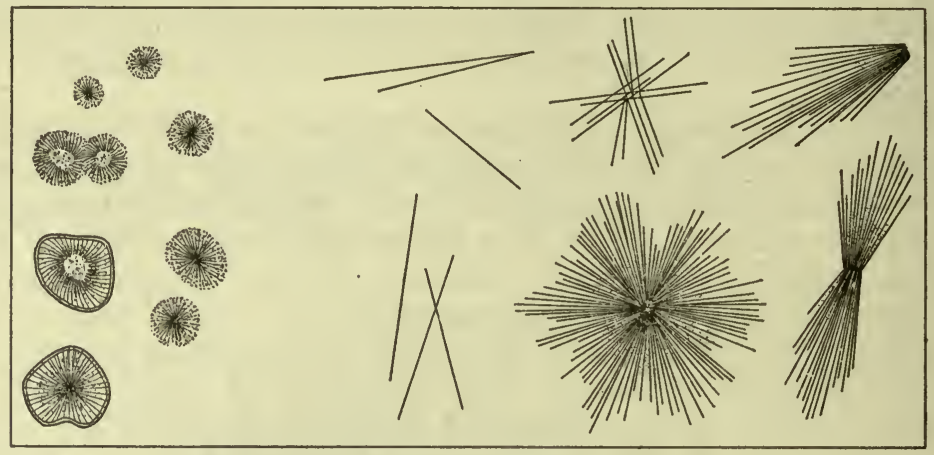

FIG. 313.-Crystals of leucine and tyrosine. $\times 216$.

All the five amino-acids mentioned (glycine, alanine, serine, valine, and leucine) are found among the final products of most proteins.

A second group of amino-acids is obtained from fatty acids, which contain two carboxyl (COOH) groups in their molecules. The most important of the amino-derivatives obtained from these dicarboxylic acids are:-

Amino-succinamic acid (asparagine),

Amino-succinic acid (aspartic acid),

Amino-pyrotartaric acid (glutamic acid).

The third group of amino-acids is a very important one; these are termed the aromatic amino-acids; that is, amino-acids united to the benzene ring, and of these we will mention three, namely, phenylalanine, tyrosine, and a nearly related substance called tryptophane.

Phenyl-alanine is alanine or amino-propionic acid in which an atom of hydrogen is replaced by phenyl $\left(\mathrm{C}_{6} \mathrm{H}_{5}\right)$.

Propionic acid has the formula $\mathrm{C}_{2} \mathrm{H}_{5}$. $\mathrm{COOH}$.

Alanine (amino-propionic acid) is $\mathrm{C}_{2} \mathrm{H}_{4} \mathrm{NH}_{2}$. $\mathrm{COOH}$.

Phenyl-alanine is $\mathrm{C}_{2} \mathrm{H}_{3} \cdot \mathrm{C}_{6} \mathrm{H}_{5} \cdot \mathrm{NH}_{2}$. COOH.

The formula of phenyl-alanine may also be written another way. The graphic formula of benzene $\left(\mathrm{C}_{6} \mathrm{H}_{6}\right)$ is :-<smiles>c1ccccc1</smiles> 
If the $\mathrm{H}$ placed lowermost in the above formula is replaced by $\mathrm{CH}_{2} \mathrm{CH}$. $\mathrm{NH}_{2}$. $\mathrm{COOH}$, we obtain the formula of phenyl-alanine:-

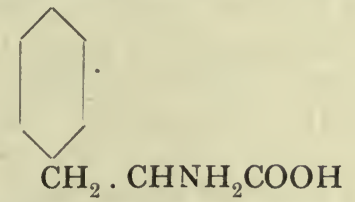

the remainder of the benzene ring, which is unaltered, being represented as usual by a simple hexagon.

Tyrosine is a little more complicated; it is oxyphenyl-alanine; that is, instead of phenyl $\left(\mathrm{C}_{6} \mathrm{H}_{5}\right)$ in the formula of phenyl-alanine, we have now oxyphenyl $\left(\mathrm{C}_{6} \mathrm{H}_{4} \cdot \mathrm{OH}\right)$; this gives us

$$
\mathrm{C}_{2} \mathrm{H}_{3} \cdot\left(\mathrm{C}_{6} \mathrm{H}_{4} \cdot \mathrm{OH}\right) \mathrm{NH}_{2} \cdot \mathrm{COOH}
$$

as the formula for tyrosine written one way, or

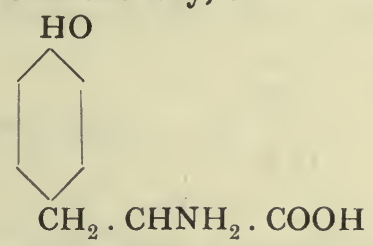

when written in the other way. Tyrosine crystallises in collections of very fine needles (see fig. 313).

Tryptophane is more complex still; it is indole amino-propionic acid: that is, amino-propionic acid united to another ringed derivative called indole. Tryptophane is the portion of the protein molecule which is the parent substance of two evil-smelling products of protein decomposition called indole and scatole or methyl indole. Indole is a combination of the benzene and pyrrol rings as shown below :-

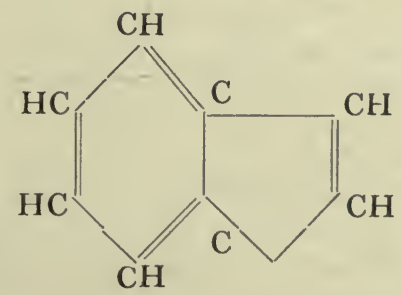

Tryptophane is the radical in the protein molecule which is responsible for the colour test called the Adamkiewicz reaction.

In all the preceding cases, there is only one replacement of an atom of hydrogen by $\mathrm{NH}_{2}$; hence they may be all grouped together as mono-amino-acids.

Passing to the next stage in complexity, we come to another group 
of amino-acids which are called diamino-acids; that is, fatty acids in which two hydrogen atoms are replaced by $\mathrm{NH}_{2}$ groups. Of these we may particularly mention lysine, ornithine, arginine, and histidine.

Lysine is diamino-caproic acid. Caproic acid is $\mathrm{C}_{5} \mathrm{H}_{11}$. $\mathrm{COOH}$. Mono-amino-caproic acid or leucine, we have already learnt, is $\mathrm{C}_{5} \mathrm{H}_{10} \cdot \mathrm{NH}_{2} \cdot \mathrm{COOH}$. Lysine or diamino-caproic acid is $\mathrm{C}_{5} \mathrm{H}_{9}$. $\left(\mathrm{NH}_{2}\right)_{2} \cdot \mathrm{COOH}$.

Ornithine is diamino-valeric acid, and the following formulæ will show its relationship to its parent fatty acid-

$\mathrm{C}_{4} \mathrm{H}_{9} \mathrm{COOH}$ is valeric acid.

$\mathrm{C}_{4} \mathrm{H}_{7}\left(\mathrm{NH}_{2}\right)_{2} \mathrm{COOH}$ is diamino-valeric acid or ornithine.

Arginine is a somewhat more complex substance, which contains the ornithine radical. It belongs to the same group of substances as creatine, another important cleavage product of the protein molecule. Creatine is methyl-guanidine acetic acid, and has the formula

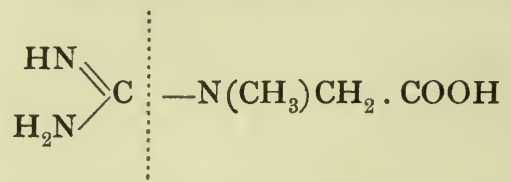

On boiling it with baryta water, it takes up water $\left(\mathrm{H}_{2} \mathrm{O}\right)$ and splits at the dotted line into urea $\left(\mathrm{CO}\left(\mathrm{NH}_{2}\right)_{2}\right)$ and sarcosine, as shown below.

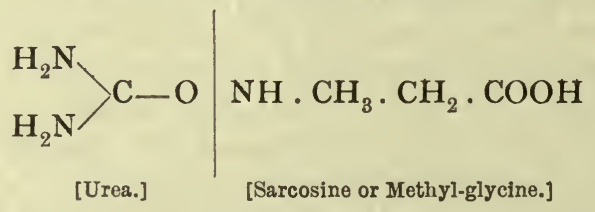

Arginine splits in a similar way, urea being split off on the left, and ornithine instead of sarcosine on the right. Arginine is, therefore, a compound of ornithine with a urea group.

Histidine, though not strictly speaking a diamino-acid, is a diazine derivative (imidazole-amino-propionic acid), and so may be included in the same group.

These substances we have spoken of as acids, but they may also play the part of bases, for the introduction of a second amino-group into the fatty acid molecules confers upon them basic properties. The three substances,

$$
\begin{array}{llll}
\text { Lysine } & \cdot & \cdot & \mathrm{C}_{6} \mathrm{H}_{14} \mathrm{~N}_{2} \mathrm{O}_{2} \\
\text { Arginine } & \cdot & \cdot & \mathrm{C}_{6} \mathrm{H}_{14} \mathrm{~N}_{4} \mathrm{O}_{2} \\
\text { Histidine } & \cdot & \cdot & \mathrm{C}_{6} \mathrm{H}_{9} \mathrm{~N}_{3} \mathrm{O}_{2}
\end{array}
$$

are in fact often called the hexone bases, because each of them contains 6 atoms of carbon, as the above empirical formulæ show. 
Cystine is a complex diamino-acid in which sulphur is present, and in which the greater part of the sulphur of the protein molecule is contained.

In addition to all these numerous amino-acids there are other cleavage products, of which it will be sufficient to mention proline and ammonia. In the case of the nucleo-proteins the nuclein component yields in addition what are known as purine and pyrimidine bases. (See further under nucleic acid, p. 431, also under uric acid, Chapter XXXIX.)

Proline and the purine and pyrimidine bases are all derivatives of rings which remind one of the benzene ring, except that nitrogen is included in the ring formation; such rings are termed heterocyclic. Thus proline (pyrrolidine-carboxylic acid) is a derivative of the pyrrol ring, and its formula is given below. Cytosine is one of the derivatives of the pyrimidine ring, and hypoxanthine (or oxypurine) is given below as an example of the purine bases.

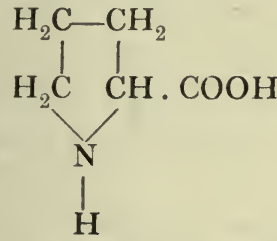

[Proline.]

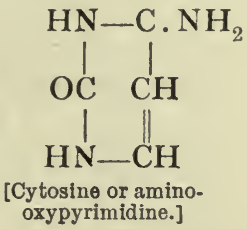

Cytosine or amino
oxypyrimidine.]

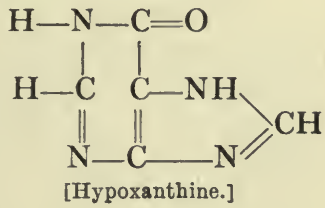

[Hypoxanthine.]

Our list now represents the principal groups of chemical nuclei united together in the protein molecule, and its length makes one realise the complicated nature of that molecule and the difficulties which beset its investigation. We may put the problem another way. In the simple sugars, with six atoms of carbon, there are as many as thirty-six different ways in which the atomic groups may be linked up; the formulæ on p. 408 give only three of these which represent the structure of dextrose, lævulose, and galactose; but the majority of the remainder have also been prepared by chemists. The molecule of albumin has at least 700 carbon atoms, so the possible combinations and permutations must be reckoned by thousands.

The workers in Fischer's laboratory are steadily working through the various known proteins, taking them to pieces and identifying and estimating the fragments. I do not intend to burden the readers of this book with anything more than a sample of their results, and will, therefore, only give in a brief table (see page 422 ) the results obtained with some of the cleavage products of a few proteins. The numbers given are percentages.

Such numbers, of course, are not to be committed to memory, but they are sufficient to convey to the reader the differences between the proteins. There are several blanks left, on account of no accurate 
estimations having yet been made. Where the sign + occurs, the substance in question has been proved to be present, but not yet determined quantitatively. Among the more striking points brought out are:-

1. The absence of glycine from albumins.

2. The high percentage of glycine in gelatin.

3. The absence of tyrosine and tryptophane in gelatin.

4. The high percentage of the sulphur-containing substance (cystine) in keratin.

5. The high percentage of glutamic acid in vegetable proteins.

\begin{tabular}{|c|c|c|c|c|c|c|c|c|c|}
\hline & 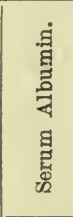 & 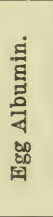 & 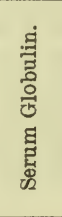 & 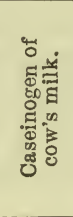 & 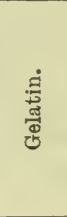 & 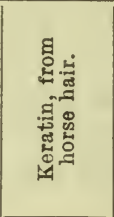 & 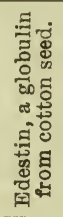 & 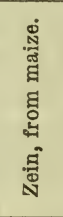 & 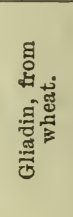 \\
\hline Glycine . & 0 & 0 & $3 \cdot 5$ & 0 & $16 \cdot 5$ & $4 \cdot 7$ & $1 \cdot 2$ & 0 & 0.02 \\
\hline Leucine & 20.0 & $6 \cdot 1$ & $18 \cdot 7$ & 10.5 & $2 \cdot 1$ & $7 \cdot 1$ & $15 \cdot 5$ & $18 \cdot 6$ & $5 \cdot 6$ \\
\hline Glutamic acid & $7 \cdot 7$ & $8 \cdot 0$ & $8 \cdot 5$ & $11 \cdot 0$ & 0.9 & $3 \cdot 7$ & $17 \cdot 2$ & $18 \cdot 3$ & $37 \cdot 3$ \\
\hline Tyrosine . & $2 \cdot 1$ & $1 \cdot 1$ & $2 \cdot 5$ & $4 \cdot 5$ & 0 & $3 \cdot 2$ & $2 \cdot 1$ & $3 \cdot 5$ & $1 \cdot 2$ \\
\hline Arginine . & $\ldots$ & $\ldots$ & $\ldots$ & $4 \cdot 8$ & $7 \cdot 6$ & ... & $11 \cdot 7$ & $1 \cdot 2$ & $3 \cdot 2$ \\
\hline Tryptophane & + & + & + & 1.5 & 0 & & + & 0 & + \\
\hline Cystine . & $2 \cdot 5$ & 0.3 & 0.7 & 0.06 & $\cdots\{$ & $\begin{array}{c}\text { More } \\
\text { than } 10\end{array}$ & 0.2 & ... & $0 \cdot 4$ \\
\hline
\end{tabular}

Emil Fischer, Abderhalden, T. B. Osborne, Levene, van Slyke, and others are attempting to make the list complete, and are devising new and more accurate methods of analysis. Fischer has also discovered the way in which the amino-acids are linked together into groups; and the culmination of his work will be the discovery of the way in which such groups are linked together to form the protein molecule. The last stage has not yet been reached, but it will be interesting to see how the amino-acids are linked together into groups.

The groups are termed peptides or polypeptides; many of these have been made synthetically in his laboratory, and so the synthesis of the protein molecule is foreshadowed.

We may take as our examples of the peptides some of the simplest, and may write the formulæ of a few amino-acids as follows:-

$$
\begin{array}{ll}
\mathrm{NH}_{2} \cdot \mathrm{CH}_{2} \cdot \mathrm{COOH} & \text { Glycine } \\
\mathrm{NH}_{2} \cdot \mathrm{C}_{2} \mathrm{H}_{4} \cdot \mathrm{COOH} & \text { Alanine } \\
\mathrm{NH}_{2} \cdot \mathrm{C}_{5} \mathrm{H}_{10} \cdot \mathrm{COOH} & \text { Leucine }
\end{array}
$$

or in general terms

HNH . R . COOH. 
Two amino-acids are linked together as shown in the following formula :-

\section{HNH.R.CO OH.H $: \mathrm{NH} . \mathrm{R} . \mathrm{COOH}$}

What happens is that the hydroxyl $(\mathrm{OH})$ of the carboxyl $(\mathrm{COOH})$ group of one acid unites with one atom of the hydrogen of the next amino (HNH) group, and water is thus formed, as shown within the dotted lines: this is eliminated and the rest of the chain closes up. In this way we get a dipeptide. The names glycyl, alanyl, leucyl, etc., are given by Fischer to the $\mathrm{NH}_{2} . \mathrm{R}$. CO groups which replace the hydrogen of the next $\mathrm{NH}_{2}$ group. Thus glycyl-glycine, glycylleucine, leucyl-alanine, alanyl-leucine, and numerous other combinations are obtained. If the same operation is repeated we obtain tripeptides (leucyl-glycyl-alanine, alanyl-leucyl-tyrosine, etc.); then come the tetrapeptides, and so on. In the end, by coupling the chains sufficiently often and in appropriate order, Fischer has already obtained substances which give some of the reactions of peptone.

Hausmann's Method.-This is a short and trustworthy procedure, by which an approximate knowledge of the nitrogen distribution in the protein molecule is ascertained.

It is shortly as follows:-The whole nitrogen of the protein is estimated by Kjeldahl's method. A weighed amount is then hydrolysed by means of hydrochloric acid, and then the cleavage products are separated into three classes and the nitrogen estimated in each, as -

1. Ammonia nitrogen. This comprises the nitrogen of the protein molecule which is easily split off as ammonia, and is determined by distilling off the ammonia with magnesia.

2. Diamino-N. The fluid, free from ammonia, is precipitated by phosphotungstic acid, and the nitrogen present in the precipitate determined. This represents the nitrogen of the diamino-acids (lysine, arginine, etc.).

3. Mono-amino- $\mathrm{N}$ is then estimated in the residual fluid.

The method has proved useful for the differentiation of proteins, and interesting deductions as to their food value has been drawn from its results.

Solubilities.-The proteins are insoluble in alcohol and ether. Some are soluble in water,* others insoluble. Many of the latter are soluble in weak saline solutions. Some are insoluble, others soluble in concentrated saline solutions.

All proteins are soluble with the aid of heat in concentrated mineral acids and alkalis. Such treatment, however, decomposes as well as dissolves the protein. Proteins are also soluble in gastric and pancreatic juices; but here, again, they undergo a change, as we have already seen.

Heat Coagulation.-Most native proteins, such as white of egg,

* The proteins are not truly soluble in water; they are in a state of colloidal solution, a condition intermediate between true solution and suspension. Many of their properties are due to this fact. 
are rendered insoluble when their solutions are heated. The temperature of heat coagulation differs in different proteins; thus myosinogen and fibrinogen coagulate at $56^{\circ} \mathrm{C}$., serum albumin and serum globulin at about $75^{\circ} \mathrm{C}$.

The proteins which are coagulated by heat come mainly under two classes: the albumins and the globulins. These differ in solubility; the albumins are soluble in distilled water, the true globulins require salts to hold them in solution.

Indiffusibility.-The proteins (peptones excepted) belong to the class of substances called colloids by Thomas Graham; that is, they

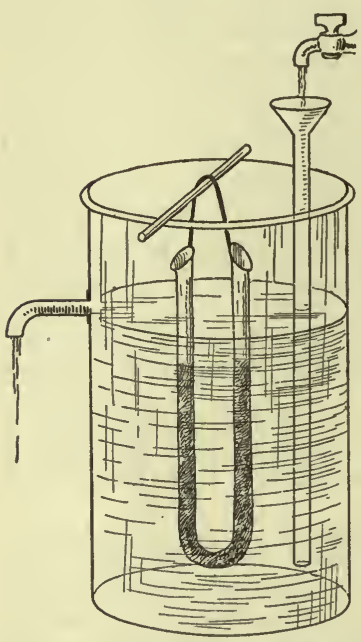

Fra. 314.-Dialyser made of a tube of parchment paper, suspended in a vessel through which water is kept flowing. pass with difficulty, or not at all, through animal membranes. In the construction of dialysers, vegetable parchment is largely used.

Proteins may thus be separated from diffusible (crystalloid) substances such as salts, but the process is a tedious one. If some serum or white of egg is placed in a dialyser (fig. 314) and distilled water outside, the greater amount of the salts passes into the water through the membrane and is replaced by water; the two proteins albumin and globulin remain inside; the globulin is, however, precipitated, as the salts which previously kept it in solution are removed.

Crystallisation.-Hæmoglobin, the red pigment of the blood, is a protein substance and is crystallisable (for further details, see The Blood, Chapter XXIX.). Like other proteins it has an enormously large molecule; though crystalline, it is not crystalloid in Graham's sense of that term. Blood pigment, however, is not the only crystallisable protein. Long ago crystals of protein (globulin or vitellin) were observed in the aleurone grains of many seeds, and in the somewhat similar granules occurring in the egg-yolk of some fishes and amphibians. By appropriate methods these have been separated and recrystallised. Further, egg albumin itself has been crystallised. If a solution of white of egg is diluted with an equal volume of saturated solution of ammonium sulphate, the globulin present is precipitated and is removed by filtration. The filtrate is now allowed to remain some days at the temperature of the air, and as it becomes more concentrated from evaporation, minute spheroidal globules and finally minute needles, either aggregated or separate, make their appearance (Hofmeister). Crystallisation is more rapid if 
a little acetic or sulphuric acid is added (Hopkins). Serum albumin (from some animals) has also been similarly crystallised (Gürber).

Action on Polarised Light.-All proteins are lævo-rotatory, the amount of rotation varying with individual proteins. Several of the conjugated proteins, e.g., hæmoglobin and nucleo-proteins, are dextrorotatory, though their protein components are lævo-rotatory (Gamgee).

Colour Reactions.-The principal colour reactions by which proteins are recognised are the following:-

(1) The xantho-proteic reaction; if nitric acid is added to a solution of a protein such as white of egg, the result is a white precipitate; this and the surrounding liquid become yellow on boiling and are turned orange by ammonia. The preliminary white precipitate is not given by certain proteins such as peptones; but the colours are the same. The colour is due to the formation of nitro-derivatives from the aromatic portion of the protein molecule.

(2) Millon's reaction. Millon's reagent is a mixture of mercuric and mercurous nitrate with excess of nitric acid. This gives a white precipitate which is turned brick-red on boiling. This reaction depends on the presence of the tyrosine radical.

(3) Copper sulphate (Rose's or Piotrowski's) test. A trace of copper sulphate and excess of strong caustic potash give with most proteins a violet solution. Proteoses and peptones, however, give a rose-red colour instead; this same colour is given by the substance called biuret; hence the test is generally called the biuret reaction. This name does not imply that biuret is present in protein; but both protein and biuret give the reaction because they possess a common radical, namely, two $\mathrm{CONH}_{2}$ groups linked to a carbon or nitrogen atom, or to one another. The native proteins give a violet colour, because the red tint of the copper compound with the biuret group is ${ }^{\circ}$ mixed with another copper compound with a blue colour. thus :-

Biuret is formed by heating solid urea ; ammonia passes off and leaves biuret,

$$
\underset{\text { [Ure..] }}{2 \mathrm{CON}_{2} \mathrm{H}_{4}}=\underset{\text { [Biuret.] }}{\mathrm{C}_{2} \mathrm{O}_{2} \mathrm{~N}_{3} \mathrm{H}_{5}}+\underset{\text { [Ammonia.] }}{\mathrm{NH}_{3} .}
$$

(4) Adamkiewicz reaction.* When a solution of protein is mixed with a dilute solution of formaldehyde, and then excess of commercial sulphuric acid is added, an intense violet colour is obtained. This is due to the tryptophane radical.

Precipitants of Proteins.- Solutions of most proteins are precipitated by:-

* In the original test, glacial acetic acid was used, but it is really an impurity in this acid that gives the reaction. Rosenheim was the first to show that this impurity is formaldehyde. The presence of impurities (oxidising agents) in the sulphuric acid is also necessary. 
Strong acids such as nitric acid; picric acid; acetic acid and potassium ferrocyanide; acetic acid and excess of a neutral salt such as sodium sulphate, when these are boiled with the protein solution; salts of the heavy metals such as copper sulphate, mercuric chloride, lead acetate, silver nitrate, etc.; tannin; alcohol; saturation with certain neutral salts such as ammonium sulphate.

It is necessary that the words coagulation and precipitation should in connection with proteins be carefully distinguished. The term coagulation is used when an insoluble protein (coagulated protein) is formed from a soluble one. This may occur:

1. When a protein is heated-heat coagulation;

2. Under the influence of an enzyme; for instance, when a curd is formed in milk by rennet or a clot in shed blood by the fibrin ferment-enzyme coagulation;

3. When an insoluble precipitate is produced by the addition of certain reagents (nitric acid, picric acid, tannin, etc.).

There are, however, other precipitants of proteins in which the precipitate formed is readily soluble in suitable reagents such as saline solutions, and the protein continues to show its typical reactions. This is not coagulation. Such a precipitate is produced by saturation with ammonium sulphate. Certain proteins, called globulins, are more readily precipitated by such means than others. Thus, globulins are precipitated by half-saturation with ammonium sulphate. Full saturation with ammonium sulphate precipitates all proteins but peptone. The globulins are precipitated by certain salts, such as sodium chloride and magnesium sulphate, which do not precipitate the albumins. This method of precipitation is called "salting out."

The precipitation produced by alcohol is peculiar in that after a time it becomes a coagulation. Protein freshly precipitated by alcohol is readily soluble in water or saline media; but after it has been allowed to stand some time under alcohol it becomes more and more insoluble. Albumins and globulins are most readily rendered insoluble by this method; proteoses and peptones are never rendered insoluble by the action of alcohol. This fact is of value in the separation of these proteins from others.

\section{Classification of Proteins.}

The knowledge of the chemistry of the proteins, which is slowly progressing under Emil Fischer's leadership, will, no doubt, in time enable us to give a classification of these substances on a strictly chemical basis. The following classification must therefore be regarded as a provisional one, which, while it retains - the old familiar names as far as possible, yet attempts also to incorporate some of the new ideas. 
The classes of animal proteins, then, beginning with the simplest, are as follows:-

1. Protamines.

2. Histones.

3. Albumins.

4. Globulins.

5. Sclero-proteins.
6. Phospho-proteins.

7. Conjugated proteins.

i. Chromo-proteins.

ii. Gluco-proteins.

iii. Nucleo-proteins.

\section{The Protamines.}

These substances are obtainable from the heads of the spermatozoa of certain fishes, where they occur in combination with nuclein. Kossel's view that they are the simplest proteins in nature has met with general acceptance, and they give such typical protein reactions as the copper sulphate test (Rose's or Piotrowski's reaction). On hydrolytic decomposition they first yield substances of smaller molecular weight analogous to the peptones which are called protones, and then they split up into amino-acids. The number of resulting amino-acids is small as compared with other proteins, hence the hypothesis that they are simple proteins is confirmed. Notable among their decomposition products are the diamino-acids or hexone bases, especially arginine.

The protamines differ in their composition according to their source, and yield these products in different proportions.

Salmine (from the salmon roe) and clupeine (from the herring roe) appear to be identical, and have the empirical formula $\mathrm{C}_{30} \mathrm{H}_{57} \mathrm{~N}_{17} \mathrm{O}_{6}$; its principal decomposition product is arginine, but amino-valeric acid and a small quantity of serine and proline are also found. Sturine (from the sturgeon) yiclds the same products with lysine and histidine in addition. With one exception, the protamines yield no aromatic amino-acids. The exception is cyclopterine (from Cyclopterus lumpus); this substance is thus an important chemical link between the other protamines and the more complex members of the protein family.

\section{The Histones.}

These are substances which have been separated from bloodcorpuscles; globin, the protein constituent of hæmoglobin, is a wellmarked instance. They yield a larger number of amino-compounds than do the protamines, but diamino-acids are relatively abundant. They are coagulable by heat, soluble in dilute acids, and precipitable from such solutions by ammonia. The precipitability by ammonia is a property possessed by no other protein group.

\section{The Albumins.}

These are typical proteins, and yield the majority of the cleavage products already enumerated. 
They enter into colloidal solution in water, in dilute saline solutions, and in saturated solutions of sodium chloride and magnesium sulphate. They are, however, precipitated by saturating their solutions with ammonium sulphate. Their solutions are coagulated by heat, usually at $70-73^{\circ} \mathrm{C}$. Serum albumin, egg albumin, and lact-albumin are instances.

\section{The Globulins.}

The globulins give the same general tests as the albumins; they are coagulated by heat, but differ from the albumins mainly in their solubilities. This difference in solubility may be stated in tabular form as follows :-

\begin{tabular}{|c|c|c|}
\hline Reagent. & Albumin. & Globulin. \\
\hline $\begin{array}{l}\text { Water baline solution } \\
\text { Dilute sal } \\
\text { Saturated solution of magnesium sul- } \\
\text { phate or sodium chloride. } \\
\text { Half-saturated solution of ammonium } \\
\text { sulphate. } \\
\text { Saturated solution of ammonium sul- } \\
\text { phate }\end{array}$ & $\begin{array}{l}\text { soluble } \\
\text { soluble } \\
\text { soluble } \\
\text { soluble } \\
\text { insoluble }\end{array}$ & $\begin{array}{l}\text { insoluble } \\
\text { soluble } \\
\text { insoluble } \\
\text { insoluble } \\
\text { insoluble }\end{array}$ \\
\hline
\end{tabular}

Globulins are more readily salted out than albumins; they may therefore be precipitated, and thus separated from the albumins by saturation with such salts as sodium chloride, or better magnesium sulphate, or by half saturation with ammonium sulphate.

The typical globulins are also insoluble in water, and so may be precipitated by removing the salt which keeps them in solution. This may be accomplished by dialysis (see p. 424). Their temperature of heat-coagulation varies considerably. The following are the commoner globulins:- fibrinogen and serum globulin in blood, egg globulin in white of egg, paramyosinogen in muscle, and crystallin in the crystalline lens. We must also include under the same heading certain proteins which are the result of enzyme coagulation on globulins, such as fibrin (see blood) and myosin (see muscle).

The most striking and real distinction between globulins and albumins is that the former on hydrolysis yield glycine, whereas the albumins do not.

\section{The Sclero-proteins.}

These substances form a heterogeneous group of substances, which are frequently termed albuminoids. The prefix sclero indicates 
the skeletal origin and often insoluble nature of the members of the group. The principal proteins under this head are:-

Collagen, the substance of which the white fibres of connective tissue are composed. Some observers regard it as the anhydride of gelatin. In bone it is often called ossein.

Gelatin.-This substance is produced by boiling collagen with water. It possesses the peculiar property of setting into a jelly when a solution made with hot water cools. On digestion it is like ordinary proteins converted into peptone-like substances, and is readily absorbed. Though it will replace in diet a certain quantity of such proteins, acting as what is called a "protein-sparing" food, it cannot altogether take their place as a food. Animals whose sole nitrogenous food is gelatin waste rapidly. The reason for this is that gelatin contains neither the tyrosine or the tryptophane groups, and so it gives neither Millon's nor the Adamkiewicz reactions. Animals who receive a mixture of gelatin, tyrosine, and tryptophane in their diet thrive better.

Chondrin is the name given to the mixture of gelatin and mucoid which is obtained by boiling cartilage.

Elastin.-This is the substance of which the yellow or elastic fibres of connective tissue are composed. It is a very insoluble material. The sarcolemma of muscular fibres and certain basement membranes are very similar.

Keratin, or horny material, is the substance found in the surface layers of the epidermis, in hairs, nails, hoofs, and horns. It is very insoluble, and chiefly differs from most other proteins in its high percentage of sulphur. A similar substance, called neurokeratin, is found in neuroglia and nerve-fibres. In this connection it is interesting to note that the epidermis and the nervous system are both formed from the same layer of the embryo-the epiblast.

\section{The Phospho-proteins.}

Vitellin (from egg-yolk), caseinogen, the principal protein of milk, and casein, the result of the action of the rennet-enzyme on caseinogen (see milk), are the chief members of this group. Among their decomposition products is a considerable quantity of phosphoric acid. They have been frequently confused with the nucleo-proteins, and the prefix nucleo-so often applied to themis entirely misleading, since they do not yield the products (purine bases, etc.) which are characteristic of nucleo-compounds. The phosphorus is contained within the protein molecule, and not in another molecular group united to the protein, as is the case in the nucleo-proteins. The phospho-proteins are specially valuable for the growth of young and embryonic animals. 


\section{The Conjugated Proteins.}

These are compounds in which the protein molecule is united to other organic materials, which are as a rule also of complex nature. This second constituent of the compound is usually termed a prosthetic group. They may be divided into the following sub-classes:-

i. Chromo-proteins.-These are compounds of protein with a pigment, which usually contains iron. They are typified by hæmoglobin and its allies, which will be fully considered under Blood.

ii. Gluco-proteins.-These are compounds of protein with a carbohydrate group. This class includes the mucins and the mucoids.

The mucins are widely distributed and may occur in epithelial cells, or be shed out by these cells (mucus, mucous glands, goblet cells). The mucins obtained from different sources are alike in being viscid and tenacious, soluble in dilute alkalis such as lime water, and precipitable from solution by acetic acid.

The mucoids differ from the mucins in minor details. The term is applied to the mucin-like substances which form the chief constituent of the ground substance of connective tissues (tendo-mucoid, chondro-mucoid, etc.). Another (ovo-mucoid) is found in white of egg, and others (pseudo-mucin and para-mucin) are occasionally found in dropsical effusions, and in the fluid of ovarian cysts.

The differences between the mucins and mucoids are due either to the nature of the carbohydrate group, or more probably to the nature of the protein to which it is united. The carbohydrate substance, however, is not sugar, but a nitrogenous substance which has a similar reducing power to sugar, and which is called glucosamine $\left(\mathrm{C}_{6} \mathrm{H}_{11} \mathrm{O}_{5} \mathrm{NH}_{2}\right)$, that is, glucose in which $\mathrm{HO}$ is replaced by $\mathrm{NH}_{2}$.

Pavy and others have shown that a small quantity of the same carbohydrate derivative can be split off from various other proteins which we have already placed among the albumins and globulins. It is, however, probable that this must not be considered a prosthetic group, but is more intimately united within the protein molecule.

iii. Nucleo-proteins. - These are compounds of protein with a complex organic acid called nucleic acid, which contains phosphorus. They are found both in the nuclei and cell-protoplasm of cells. In physical character they often simulate mucin.

Nuclein is the name given to the chief constituent of cell-nuclei. It is identical with the chromatin of histologists (see p. 10).

On decomposition it yields an organic acid called nucleic acid, together with a variable but usually small amount of protein. It contains a high percentage $(10-11)$ of phosphorus.

The nuclein obtained from the nuclei or heads of the spermatozoa consists of nucleic acid without any protein admixture. In fishes' 
spermatozoa, however, there is an exception to this rule, for there it is, as we have already seen, united to protamine.

The nuclco-proteins of cell protoplasm are compounds of nucleic acid with a much larger quantity of protein, so that they usually contain only 1 per cent. or less of phosphorus. Some also contain iron, and the normal supply of iron to the body is contained in the nucleo-proteins or hoematogens (Bunge) of plant or animal cells.

Nucleo-proteins may be prepared from cellular structures such as thymus, testis, kidney, etc. , by two principal methods :-

1. Wooldridge's method. - The organ is minced, and soaked in water for twentyfour hours. Dilute acetic acid added to the aqueous extract precipitates the nucleoprotein.

2. Sodium chloride method.-The minced organ is ground up in a mortar with solid sodium chloride; the resulting viscous mass is poured into excess of water, and the nucleo-protein rises in strings to the top of the water.

The solvent usually employed for a nucleo-protein, whichever method it is prepared by, is a 1 per cent. solution of sodium carbonate. The relationship of nucleo-proteins to the coagulation of the blood is described under that heading.

Nucleic acid yields, among its decomposition products, phosphoric acid, various bases of the purine group, and bases also of the pyrimidine group. A carbohydrate radical is also obtained. The following diagrammatic way of representing the decomposition of nucleo-protein puts the matter more clearly :-

Nucleo-Protein

subjected to gastric digestion yields

Protein converted into peptone, which goes into solution.

Protein-converted into acid meta-protein in solution.
Nuclein, which remains as an insoluble residue. If this is dissolved in alkali and hydrochloric acid added, it yields

A precipitate consisting of nucleic acid. If this is heated in a sealed tube with hydrochloric acid, it yields

Phosphoric acid. Carbohydrate. Purine bases. Pyrimidine bases.

Recent research on the nucleic acids obtained from various mammalian organs indicates that they fall into two main classes:-

(1) Nucleic acid proper.-This yields on decomposition-

(a) Phosphoric acid.

(b) A sugar, which is a hexose, but has not yet been identified further; Levene has found that in the nucleic acids of vegetable origin (for instance from yeast) the sugar present is a pentose ( $\dot{d}$-ribose).

(c) Two members of the purine group in the same proportion namely, adenine and guanine. 
(d) Two pyrimidine bases, namely, cytosine (see p. 421), and thymine (methyl-dioxypyrimidine).

The purine bases are specially interesting because of their close relationship to uric acid, and we shall have to deal with them again in our description of that substance. They are all derivatives of an atomic complex, named purine by Fischer, and their relationship to each other is best seen by their formulæ:-

$$
\text { Purine bases } \begin{aligned}
& \text { Purine } \mathrm{C}_{5} \mathrm{H}_{4} \mathrm{~N}_{4} \\
& \text { Hypoxanthine (monoxy-purine) } \mathrm{C}_{5} \mathrm{H}_{4} \mathrm{~N}_{4} \mathrm{O} \\
& \text { Xanthine (dioxy-purine) } \mathrm{C}_{5} \mathrm{H}_{4} \mathrm{~N}_{4} \mathrm{O}_{2} \\
& \text { Adenine (amino-purine) } \mathrm{C}_{5} \mathrm{H}_{3} \mathrm{~N}_{4} . \mathrm{NH}_{2} \\
& \text { Guanine (amino-oxy-purine) } \mathrm{C}_{5} \mathrm{H}_{3} \mathrm{~N}_{4} \mathrm{O} \cdot \mathrm{NH}_{2} \\
& \text { Uric Acid (trioxy-purine) } \mathrm{C}_{5} \mathrm{H}_{4} \mathrm{~N}_{4} \mathrm{O}_{3}
\end{aligned}
$$

The two bases obtained from nucleic acid are the two which contain the $\mathrm{NH}_{2}$ group. If xanthine and hypoxanthine are obtained, they are the secondary effects of oxidative and de-amidising enzymes.

(2) Guanylic acid.-This is a simpler form of nucleic acid found in certain organs (pancreas, liver, etc.), mixed with the nucleic acid proper. It yields on decomposition only three substances, namely:-

(a) Phosphoric acid.

(b) A carbohydrate (probably a pentose).

(c) Guanine, but no adenine.

From his work on the nucleic acid of yeast, Levene finds that it is composed of complexes consisting of phosphoric acid, carbohydrate (ribose), and a base. 'These are termed nucleotides. Guanylic acid, described above, is a mono-nucleotide, but the majority of nucleic acids are poly-nucleotides. When these are broken down by chemical reagents, the first change is the removal of the phosphoric acid, leaving intact the combinations of base and carbohydrate; these latter combinations are called nucleosides; thus-

$$
\begin{aligned}
& \text { Adenine + ribose }=\text { adenosine. } \\
& \text { Guanine + ribose }=\text { guanosine. }
\end{aligned}
$$

These nucleosides may be further split into base and ribose; or they may be de-amidised (i.e., the amino-group is removed) and nucleosides obtained in which hypoxanthine and xanthine are united with the ribose, and these in their turn may be split into base and ribose.

The same cleavages are accomplished in the body by the action of tissueenzymes contained in varying degrees in the different organs and tissues. As these enzymes are specific, the number which may come into successive play in the decompositions which occur in the body is very large. These enzymes are spoken of under the general term nucleases.

\section{Protein-hydrolysis.}

When protein material is subjected to hydrolysis, as it is when heated with mineral acid, or superheated steam, or to the action of such enzymes as pepsin or trypsin in the alimentary canal, it is finally resolved into the numerous amino-acids of which it is built. But before this ultimate stage is reached, it is split into substances of progressively diminishing molecular size, which still retain many of 
the protein characters. The products may be classified in order of formation as follows:-

1. Meta-proteins.

2. Proteoses.

3. Peptones.

4. Polypeptides.

5. Amino-acids.

The polypeptides are linkages of two or more amino-acids, as already explained. Although most of the polypeptides at present known are products of laboratory synthesis, many have been definitely separated from the digestion products of proteins. The proteoses, peptones, and some of the longer polypeptides give the biuret reaction ; the peptones and polypeptides, however, cannot be salted out of solution as the proteoses can : their molecules are smaller than those of the proteoses. We shall study them more fully under digestion.

It is, however, convenient to add here a brief description of the meta-proteins. They are obtained as the first stage of hydrolysis, and also by the action of dilute acids or alkalis on either albumins or globulins. The general properties of the acid meta-protein and alkali meta-protein (formerly called acid-albumin or syntonin and alkali-albumin), which are thereby respectively formed, are as follows:- They are insoluble in pure water, but are soluble in either acid or alkali, and are precipitated by neutralisation unless certain disturbing influences like sodium phosphate are present. They are precipitated as globulins are by saturation with such neutral salts as sodium chloride or magnesium sulphate. They are not coagulated by heat if in solution.

The word albuminate is used for compounds of protein with mineral substances. Thus if a solution of copper sulphate is added to a solution of albumin, a precipitate of copper albuminate is formed. Similarly, by the addition of other salts of the heavy metals, other metallic albuminates are obtainable. The halogens (chlorine, bromine, iodine) also form albuminates in this sense, and may be used for the precipitation of proteins.

It should be noted in conclusion that the foregoing classification of proteins is mainly applicable to those of animal origin.

There are certain vegetable proteins, such as gliadin from the gluten of wheat, hordein from barley, and zein from maize, which stand apart from all other members of the group in being soluble in alcohol.

The vegetable proteins which have been mainly studied are those contained in the seeds of plants. They may provisionally be grouped into four main classes :-

1. Albumins, such as leucosin in wheat.

2. Globulins, such as edestin of hemp and other seeds; most of these are readily crystallisable.

3. Glutelins. These are insoluble in water and saline solutions, and are soluble only in dilute alkali. They are probably not very strongly marked off from the globulins, since it has been shown that the solubility of globulins in dilute saline solutions is also due to a trace of alkali. The best example of this third class is the glutenin of wheat gluten. 
4. Gliadins; the proteins soluble in alcohol just alluded to. They are characterised also by the absence of lysine among their cleavage products, and usually yield a very high percentage of glutamic acid on decomposition. The gluten of wheat flour, which is formed when water is added to it, has been shown to consist of two proteins-one (gliadin) soluble in alcohol, the other (glutenin) soluble in alkali. It is to the former that the gluten of dough owns its cohesiveness; and grains such as rice, which contain no gliadin, cannot in consequence be employed for making bread.

\section{The Polarimeter.}

This instrument is one by means of which the action of various substances on the plane of polarised light can be observed and measured. Most of the carbohydrates are dextro-rotatory. All the proteins are lævo-rotatory (see p. 425).

There are many varieties of the instrument; these can only be properly studied in the laboratory, and all one can do here is to state briefly the principles on which they are constructed.

Suppose one is shooting arrows at a fence made up of narrow vertical palings ; suppose also that the arrows are flat like the laths of a venetian blind. If the arrows are shot vertically they will pass easily through the gaps between the palings, but if they are shot horizontally they will be unable to pass through at all. This rough illustration will help us in understanding what is meant by polarised light. Ordinary light is produced by the undulations of the æther occurring in all directions at right angles to the path of propagation of the wave. Polarised light is produced by undulations in one plane only; we may compare it to our flat arrows.

In a polarimeter, there is at one end of the instrument a Nicol's prism, which is made of Iceland spar. This polarises the light which passes through it; it is called the polariser. At the other end of the instrument is another called the analyser. Between the two is a tube which can be filled with fluid. If the analyser is parallel to the polariser the light will pass through to the eye of the observer. But if the analyser is at right angles to the polariser it is like the flat arrows hitting horizontally the vertical palings of the fence, and there is darkness. At intermediate angles there will be intermediate degrees of illumination.

If the analyser and polariser are parallel and the intermediate tube filled with water, the light will pass as usual, because water has no action on the plane of polarised light. But if the water contains sugar or some "optically active" substance in solution, the plane is twisted in one direction or the other according as the substance is dextro- or lævo-rotatory. The amount of rotation is measured by the number of angles through which the analyser has to be turned in order to obtain the full illumination. This will vary with the length of the tube and the strength of the solution.

\section{The Lipoids.}

This name was first applied by Overton to a heterogeneous group of substances found in the protoplasm of all cells, especially in their outer layer or cell-membrane, which, like the fats, are soluble in such reagents as ether and alcohol. These substances, though present in smaller amount than proteins, appear to be essential constituents of protoplasm, and the labile character of their molecules is a property many of them share in common with the proteins.

The lipoids are found mixed with fat in the ether-alcohol extract of tissues and organs, and they are specially abundant in nervous tissues. They can be separated by what is called selective extraction. For instance, cold acetone will dissolve out only cholesterin; hot acetone then dissolves out a mixture of substances named 
protagon ; protagon may be separated into its constituents (phrenosin and sphingomyelin) by pyridine, and so forth.

The lipoids may be classified in the following way :-

(1) Those which, like the fats, are free from both nitrogen and phosphorus. The most important member of this group is cholesterin.

(2) Those which are free from phosphorus but contain nitrogen. These yield the reducing sugar called galactose when broken up, and may, therefore, be called galactosides.

(3) Those which contain both phosphorus and nitrogen. These are called the phosphatides, and are grouped according to the proportion of nitrogen and phosphorus in their molecules, as follows:-

(a) Mono - amino - mono - phosphatides, $\mathrm{N}: \mathrm{P}=1: 1$. E.g., lecithin and kephalin.

(b) Diamino-mono-phosphatides, $\mathrm{N}: \mathrm{P}=2: 1$. E.g., sphingomyelin.

(c) Mono-amino-diphosphatides, $\mathrm{N}: \mathrm{P}=1: 2$. One of these, named cuorin, he $s$ been separated out from the heart by Erlandsen, and a similar substance is found in egg-yolk.

(d) Diamino-diphosphatides, $\mathrm{N}: \mathrm{P}=2: 2$. One of these was separated from brain by Thudichum, but has not since been examined.

(e) Triamino-mono-phosphatides, $\mathrm{N}: \mathrm{P}=3: 1$. One of these is present in egg-yolk.

Cholesterin or cholesterol is found in small quantities in all forms of protoplasm. It is a specially abundant constituent of nervous tissues, particularly in the white substance of Schwann. It is found in small quantities in the bile, but it may occur there in excess and form the concretions known as gall-stones. It can be readily extracted from the brain by the use of cold acetone. In the brain it occurs in the free state.

It is a monatomic unsaturated alcohol with the empirical formula $\mathrm{C}_{27} \mathrm{H}_{45} \cdot \mathrm{OH}$. Recent research has shown it to belong to the terpene series, which had hitherto only been found as excretory products of plant life. Windaus has shown that it contains five reduced benzene rings linked together, with a double linkage at the end of an open chain:

Cholesterin is now believed to be not merely a waste product of metabolism, but to exert an important protective influence on the body cells against the entrance of certain poisons called toxins. One of the poisons contained in cobra venom dissolves red bloodcorpuscles; the presence of cholesterin in the envelope of the blood-corpuscles to some extent hinders this action, and it has been stated that the administration of cholesterin increases the resistance of the animal. It is certainly the case that with artificial blood-corpuscles, membranous bags containing hæmoglobin, 
the impregnation of the membrane with cholesterin, prevents the solvent action of toxins.

In order that cholesterin and its derivatives may act in this way, it

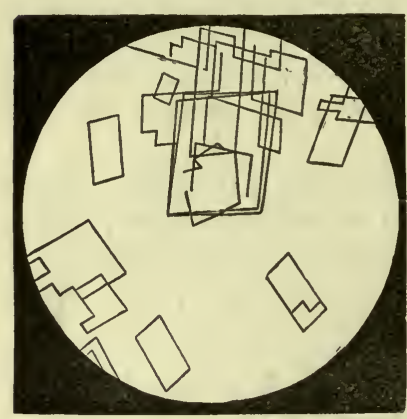

Fia. 315.-Cholesterin crystals. is necessary that the double linkage and the hydroxyl atom just referred to should be intact. The latter would not be the case in an ester, and it is probable that the compounds of cholesterin in the blood previously described as esters by Hürthle are really mixtures of cholesterin and fatty acids.

From alcohol or ether containing water it crystallises in the form of rhombic tables, which contain one molecule of water of crystallisation : these are easily recognised under the microscope(fig. 315). It gives the following colour tests:-

1. Heated with sulphuric acid and water $(5: 1)$, the edges of the erystals turn red.

2. A solution of cholesterin in chloroform, shaken with an equal amount of strong sulphuric acid, turns red, and then purple, the subjacent acid acquiring a green fluorescence. (Salkowski's reaction.)

3. If acetic anhydride is added to a chloroformic solution of cholesterin, and then sulphuric acid, drop by drop, a red coloration, which changes to bluish green, is produced. (Liebermann's reaction.)

A substance called iso-cholesterin is found in the fatty secretion of the skin (sebum); it is largely contained in the preparation called lanoline, made from sheep's wool fat. It differs from cholesterin in being dextro-rotatory instead of lævo-rotatory in solution, and it does not give Salkowski's colour reaction. Cholesterins isomeric with animal cholesterin are also found in many plants; these are termed phyto-cholesterins, or phytosterins for short.

Cholesterin compounds exhibit the physical phenomenon recently studied by Lehmann, namely, the formation of liquid crystals ; this is also shown by several other lipoids. Virchow in 1855 described what he termed "myelin forms"; if brain-substance is mixed with water, where the water touches the brain material, threads are observable shooting out and twisting into fantastic shapes; these are termed "myelin forms," although the word myelin has no definite chemical meaning. It has now been shown that these "myelin forms" are distorted liquid crystals due to the presence of cholesterin and other lipoids. The fat globules seen in the adrenal cortex, and in the liver and other organs during fatty degeneration, are not wholly composed of fat, for the polarisation microscope shows them to be anisotropic, and further investigation has shown them to 
be lipoids in the fluid crystalline condition. Pure cholesterin and pure cholesterin esters do not exhibit the phenomenon; but mixtures of cholesterin and fatty acids do; it has been suggested that in such mixtures the acid is incorporated as "acid of crystallisation," analogous to the "water of crystallisation" in many other crystals.

The Galactosides.-The substance known as protagon can be separated out from the brain by means of warm alcohol; on cooling the extract, protagon is deposited as a white precipitate. This, however, also contains cholesterin, which can be dissolved out by ether. Another method of preparing protagon is to take brain and extract the cholesterin first with cold acetone; then hot acetone is employed to extract the protagon. Protagon is a substance originally described by Couerbe, under the name cérèbrote, but named protagon by Liebreich, who regarded it as a definite compound, and the mother substance of all the other phosphorised and non-phosphorised constituents of the brain. It has now been definitely proved in confirmation of what Thudichum stated in 1874, that protagon is not important quantitatively, and is not a definite chemical unit, but a mixture of phosphorised and non-phosphorised substances in such proportions that it usually contains about 1 per cent. of phosphorus. By treatment with appropriate reagents and recrystallisation, protagon can be separated into its constituents, and those which are free from phosphorus and comprise about 70 per cent. of the original protagon are the galactosides. The known galactosides are two in number, namely, phrenosin (or cerebron) and kerasin. The former is a crystalline product, and the latter of somewhat waxy consistency. They are probably isomerides. They yield on decomposition three substances:-(1) A reducing sugar, galactose. (2) A base termed sphingosine, about which little chemically is yet known. (3) A fatty acid of high molecular weight, termed neuro-stearic acid by Thudichum, but not definitely identified. It is probably an oxy-acid (Thierfelder).

The Phosphatides.-The best known of these is lecithin. This is a very labile substance, but it yields on decomposition four materials, namely-glycerin and phosphoric acid united together as glycero-phosphoric acid, two fatty acid radicals, of which one is usually oleic acid, and an ammonium-like base termed choline. The fatty acid radicals are united to glycerin as in an ordinary fat, the place of the third fatty acid being taken by the radical of phosphoric acid, which in its turn is united in an ester-like manner to the choline. The clinical significance of such substances in cases of degenerative nervous disease has been already alluded to on p. 167 .

Kephalin resembles lecithin in being a mono-amino-monophosphatide. It differs from lecithin in being insoluble in alcohol. On decomposition it yields glycero-phosphoric acid, certain fatty acids 
which are less saturated than oleic acid, and probably belong to the linoleic series. It also yields a base, but it is doubtful if this is identical with choline. Kephalin is the most abundant phosphatide in nerve-fibres, and has also been found in egg-yolk.

Sphingomyelin is the phosphatide obtained from the mixture called protagon. It is the best known of the diamino-monophosphatides. If protagon is dissolved in hot pyridine, and the solution allowed to cool, sphingomyelin is precipitated in an impure form as sphæro-crystals, which rotate the plane of polarised light to the left. Choline, fatty acids, and an alcohol have been found among its cleavage products. It, however, differs from lecithin by containing no glycerin.

\section{Enzymes.}

The word fermentation was first applied to the change of sugar into alcohol and carbonic acid by means of yeast. The evolution of carbonic acid causes frothing and bubbling; hence the term "fermentation." The agent, yeast, which produces this, was called the ferment. Microscopic investigation shows that yeast is composed of minute rapidly-growing unicellular organisms belonging to the fungus group.

The souring of milk, the transformation of urea into ammonium carbonate in decomposing urine, and the formation of vinegar from alcohol are brought about by very similar organisms. The complex changes known as putrefaction, which are produced by the various forms of bacteria (see fig. 316), also come into the same category.

That the change or fermentation is produced by these organisms is shown by the fact that it occurs only when the organisms are present, and stops when they are removed or killed by a high temperature or by antiseptics (carbolic acid, etc).

The "germ theory" of disease explains the infectious diseases by considering that the change in the system is of the nature of fermentation, and, like the others we have mentioned, produced by microbes; the transference of the bacteria or their spores from one person to another constitutes infection. The poisons produced by the growing bacteria appear to be either alkaloidal (ptomaines) or protein in nature. The most virulent poison in existence, namely, snake poison, is a protein of the proteose class.

All these micro-organisms require moisture in which to act. They act best at a temperature of about $40^{\circ} \mathrm{C}$. Their activity is stopped, but the organisms are not destroyed by cold. The organisms are, however, like other living cells, killed by too great heat. Some micro-organisms act without free oxygen; these are called ancërobic ; those that require oxygen are called aërobic.

Another well-known fact concerning micro-organisms is that the substances they produce in time put a stop to their activity; thus. 
in the case of yeast, the alcohol produced, and in the case of bacteria acting on proteins, the phenol, cresol, etc., produced, first stop the growth of, and ultimately kill, these organisms.

For a long time it was uncertain how micro-organisms were able

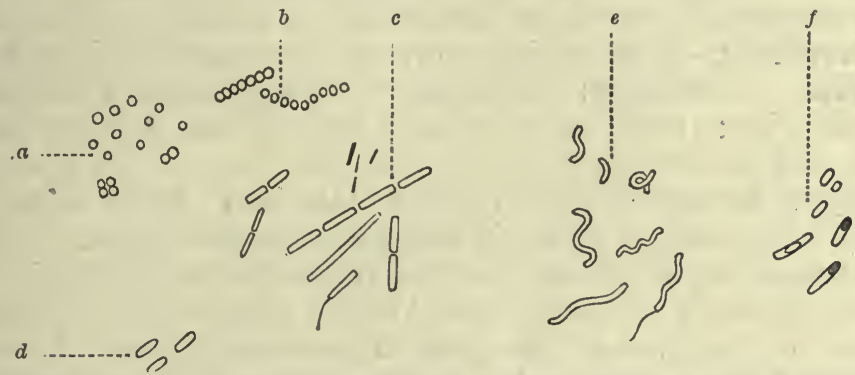

FiG. 316.-Types of micro-organisms : $a$, micrococci arranged singly; in twos, diplococci-if all the micrococci at $a$ were grouped together they would be called staphylococci-and in fours, sarcinæ ; $b$, micrococci in chains, streptococci; $c$ and $d$, bacilli of various kinds (one is represented with a flagellum); $e$, various forms of spirilla; $f$, spores, either free or in bacilli.

to effect these chemical transformations. It is now, however, definitely proved that they do so by producing agents of a chemical nature, which are called enzymes. This was first demonstrated in connection with the invertase of yeast cells, and with the enzyme secreted by the micrococcus ureæ, which converts urea into ammonium carbonate in putrefying urine. For a long time, however, efforts to obtain from yeast cells an enzyme capable of bringing about the alcoholic fermentation were unsuccessful. This is because the enzyme does not leave the yeast cells, but acts intracellularly. Buchner, by crushing the yeast cells, succeeded in obtaining from them the long-sought enzyme (zymase); since then other enzymes have been obtained from other microbes by similar means.

Enzymes are also formed by the cells of the higher organisms, both in animal and vegetable life. Familiar instances of these are ptyalin, the starch-splitting enzyme of saliva, and pepsin, the protein-splitting enzyme of gastric juice. The substance upon which the enzyme acts is spoken of as the substrate.

We may, therefore, place these essential facts concerning enzyme action in the following tabular way:-

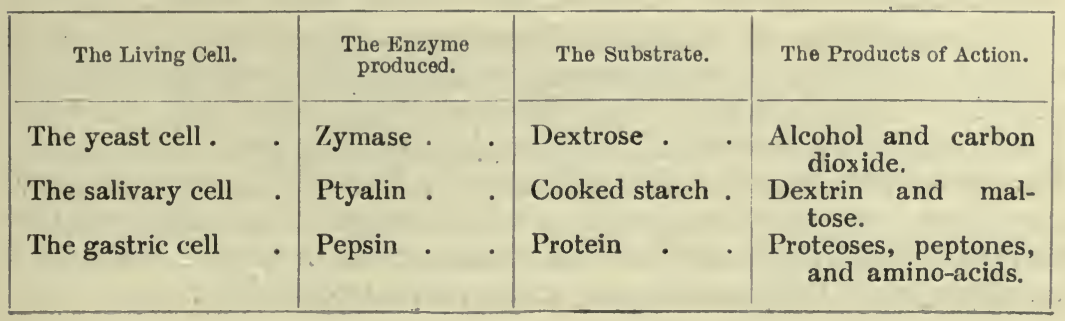


The enzymes which bring about the digestion of food in the alimentary canal may be classified as follows:-

1. Amylolytic-those which convert polysaccharides (starch, glycogen) into sugar with intermediate dextrins. Examples: the diastase of vegetable seeds, and the ptyalin of saliva.

2. Inverting-those which convert disaccharides into monosaccharides. Examples: Invertase of yeast cells; invertase of intestinal juice; these convert cane sugar into equal parts of dextrose and lævulose.

3. Lipolytic-those which split fat into fatty acids and glycerin. An example, lipase, is found in pancreatic juice.

4. Proteolytic or proteoclastic-those which split proteins into proteoses, peptones, polypeptides, and finally amino-acids. Examples: the pepsin of gastric, and the trypsin of pancreatic juice.

5. Peptolytic-those which split proteoses and peptones into polypeptides and amino.acids, e.g., the erepsin of intestinal juice.

But in addition to the digestive enzymes there are others to be mentioned, for instance :-

The enzymes in the foregoing list produce hydrolysis; that is, water is added to the substrate, which then splits into simpler molecules, as for instance in the inversion of cane sugar by invertase.

$$
\underset{\text { [Cane sugar.] }}{\mathrm{C}_{12} \mathrm{H}_{22} \mathrm{O}_{11}}+\underset{\text { [Water.] }}{\mathrm{H}_{2} \mathrm{O}}=\underset{\text { [Dextrose.] }}{\mathrm{C}_{6} \mathrm{H}_{12} \mathrm{O}_{6}}+\underset{\text { [Lævulose.] }}{\mathrm{C}_{6} \mathrm{H}_{12} \mathrm{O}_{6}} \text {. }
$$

Coagulative enzymes - those which convert soluble into insoluble proteins; the best example of this class is thrombin or fibrin-ferment, which comes into play in blood-coagulation, converting the soluble protein in blood-plasma called fibrinogen into fibrin. Rennet or rennin, found in the gastric juice, is another; it converts the soluble caseinogenate of milk into casein.

Oxidases; these are not hydrolytic, but are oxygen carriers and produce oxidation: they are mainly found as intracellular enzymes, and are important in tissue respiration.

Reductases; these are the counterpart of the oxidases, and produce reduction in the tissues.

Deamidases; these remove the amino-group from aminocompounds.

Intracellular or Autolytic Enzymes.-These come into play during cell life, and are important in the metabolic or intracellular chemical changes which occur in protoplasm; they also may be subdivided into proteolytic, peptolytic, lipolytic, etc., according to the substrate upon which they act. After death their activity continues, and so they produce self-digestion or autolysis of the cells in which they are situated, if the tissue or organ is kept at an appropriate temperature and under aseptic conditions. 
The foregoing list is not by any means complete, but includes the most important groups. The individual enzymes will be studied in due course, but for the present we will take general considerations only.

Zymogens.-These are the parent substances or precursors of the enzymes. The granules seen in many secreting cells consist very largely of zymogen, which in the act of secretion is converted into the active enzyme. Thus, pepsin is formed from pepsinogen, trypsin from trypsinogen, thrombin from thrombogen, and so forth.

Activation of Enzymes. Co-enzymes.-Many enzymes contained in secretions are in a condition ready for action. In other cases this is not so, and their action only occurs after they have been rendered energetic by the presence or action of other substances, termed activating agents or co-enzymes.

The Specificity of Enzyme Action.- In most cases the action of an enzyme is extraordinarily limited; thus there are three separate enzymes to hydrolyse the three principal disaccharides, cane sugar, lactose, and maltose, neither of which will act upon either of the other two sugars in the list. Arginase splits arginine into ornithine and urea, but will act upon no other substance. The "lock and key" simile first introduced by Emil Fischer will aid us in understanding this specificity of action. Each lock must have its special key: so the chemical configuration of an enzyme must be related in some way to the configuration of the substrate to enable it to enter and unlock its parts from one another.

The Optimum Temperature of Enzyme Action.-As the temperature rises the velocity of the action increases, until a temperature is reached at which the activity is greatest. Most enzymes act best at $40^{\circ} \mathrm{C}$., but there are exceptions; malt diastase, for instance, acts best at $60^{\circ} \mathrm{C}$. Beyond the optimum temperature a further rise inhibits activity, until a temperature is reached when the enzyme is destroyed. The fatal temperature as a rule is in the neighbourhood of $50^{\circ} \mathrm{C}$.

The effect of a rise of temperature is thus complex, and is of a two-fold nature. In the first place, and between certain limits, the law of Arrhenius is followed, that is, a rise of $10^{\circ}$ doubles or even trebles the velocity of the action of the enzyme, as it does other chemical reactions. But as the temperature rises the velocity of disintegration of the enzyme also rises. The optimum temperature is that at which the enzyme work is best done; this is a temperature at which the accelerating effect is strong enough to finish the reaction quickly, and the retarding effect due to enzyme destruction is not so great as to neutralise the accelerating effect.

The Inexhaustibllity of Enzymes.-A small amount of enzyme will act on an unlimited amount of substrate, provided sufficient 
time is given, and provided also the products of action are removed. The enzyme appears to take a share in intermediate reactions, and there is some evidence that in certain stages it combines with the substrate; but subsequently when the substrate breaks up into simpler materials, the enzyme is liberated unchanged, and so ready to similarly act on a fresh amount of substrate.

Catalytic Action of Enzymes. - The analogy of enzymic action is, in fact, so close to that of inorganic catalysts, that the view at present current regarding it is that the action is a catalytic one. That is to say, the presence of the enzyme induces a chemical reaction to occur rapidly, which in its absence also occurs, but so slowly that any action at all is difficult to discover. To use the technical phrase, its action is to increase the velocity of chemical reactions. It is, for instance, quite conceivable that, if starch and water were mixed together, the starch will in time take up the water and split into its constituent molecules of sugar. But an action of this kind would be so slow, occupying perchance many years, that for practical purposes it does not take place at all. If an inorganic catalyst is added, such as sulphuric acid, and the temperature raised to boiling point, the action takes place in a few minutes; if an organic catalyst, such as the enzyme ptyalin, is added, the velocity of the change is even greater; but what is of more importance for the well-being of the animal, a moderate temperature, namely that of the body, amply suffices. The organic catalysts or enzymes are, however, colloidal in nature (possibly protein), and this explains their destructibility by high temperatures.

Reversibility of Enzyme Action.-On page 329 we have considered the general laws of molecular reactions. The majority of enzymatic reactions are unimolecular, or reactions of the first order; that is to say, one substance only, the substrate, undergoes transformation; the other substance, the enzyme, does not alter in concentration. The law followed in such reactions is therefore the simple logarithmic law. But in these reactions we meet with the peculiarity that it is not quite completed when the reaction ceases. A certain quantity of the substrate never disappears. Thus a small amount of cane sugar remains unchanged whether the hydrolysis is brought about by the action of an acid or of an enzyme. This phenomenon is due to the fact that two reactions are always taking place in opposite directions. Simultaneously with the splitting up the synthetical reaction begins, and synthesis or building up increases in proportion as the splitting of the compound advances. The velocity of the splitting process decreases at the same rate as the velocity of the synthetic process increases. At a certain point, both have the same velocity, and therefore no further change occurs in the mixture when this condition of equilibrium is reached. This rule is expressed 
by writing the chemical equation connected by a double arrow instead of the sign of equation. Two examples follow :-

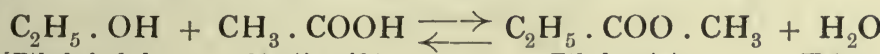

$$
\begin{aligned}
& \text { [Ethyl alcohol.] [Acetic acid.] } \longleftarrow \text { [Ethyl acetate.] [Water.] } \\
& \underset{\text { [Dextrose.] }}{\mathrm{C}_{6} \mathrm{H}_{12} \mathrm{O}_{6}}+\underset{\text { [Lævulose.] }}{\mathrm{C}_{6} \mathrm{H}_{12} \mathrm{O}_{6}} \underset{\text { [Cane sugar.] }}{\mathrm{C}_{12} \mathrm{H}_{22} \mathrm{O}_{11}}+\underset{\text { [Water.] }}{\mathrm{H}_{2} \mathrm{O}}
\end{aligned}
$$

This phenomenon is termed "reversibility" and was first demonstrated by Croft Hill in his experiments with cane sugar and invertase.

In intracellular action this is a factor of importance, for the same enzyme can in the presence of different proportions of the substrate and its cleavage products both tie (in anabolism) and untie (in katabolism) the knot.

It should further be noted that hydrolytic actions are isothermic; that is, the total energy of the products is equal to that of the substance broken up.

The simpler logarithmic law of enzyme action has been demonstrated for the majority of enzymes (invertase, trypsin, erepsin, lipase, etc.). The effect in a given time is directly proportional to the quantity of enzyme present. But there is an exception to this rule in the case of pepsin, as was first pointed out by Schütz in 1885. He found that peptic activity is proportional to the square root of the amount of pepsin prescnt. Thus if a certain quantity of pepsin produced an amount of digestive action which we will call $a$, in order to produce a digestive action equal to $2 \alpha$ in the same time, it would be necessary to employ four times the amount of pepsin; and in order to produce a digestive action equal to $3 a$, it would be necessary to use nine times the amount of pepsin. This rule (Schütz's law) has been often confirmed, and a few years ago Arrhenius explained it on mathematical lines into which we need not enter here.

Anti-enzymes.-Many chemical substances, such as strong acids and alkalis, alcohol, formaldehyde, iodine, potassium cyanide, and salts of the heavy metals, hinder enzyme activity. But the term anti-enzyme is generally limited to substances produced in the metabolism of living organisms. Excess of these organic antienzymes can be readily produced by injecting an enzyme into the blood-stream of an animal. This stimulates the production of an anti-enzyme, so that when the blood-serum is mixed with the original enzyme, its power is inhibited. Anti-enzymes are specific, that is, they inhibit the enzyme which was injected into the blood, and no other. 


\section{CHAPTER XXIX}

\section{THE BLOOD}

ThE blood is the fluid medium by means of which all the tissues of the body are directly or indirectly nourished; by means of it also such of the materials resulting from the metabolism of the tissues which are of no further use in the economy are carried to the excretory organs. It is a somewhat viscid fluid, and in man and in all other vertebrate animals, with the exception of two, ${ }^{*}$ is red in colour. It consists of a yellowish fluid, called plasma or liquor sanguinis, in which are suspended numerous blood-corpuscles, the majority of which are coloured, and it is to their presence that the red colour of the blood is due. In addition to the red corpuscles, there are a smaller number of colourless corpuscles, and some extremely small particles called blood-platelets.

Even when examined in very thin layers, blood is opaque, on account of the different refractive powers possessed by its two constituents, the plasma and the corpuscles. On treatment with ether, water, and other reagents, however, it becomes transparent and assumes a lake colour, in consequence of the colouring matter of the corpuscles having been discharged into the plasma. The average specific gravity of blood at $15^{\circ} \mathrm{C}$. $\left(60^{\circ} \mathrm{F}\right.$.) varies from 1055 to 1062 . A rapid and useful method of estimating the specific gravity of blood was invented by Roy. Drops of blood are taken and allowed to fall into fluids of known specific gravity. When the drop neither rises nor sinks in the fluid it is taken to be of the same specific gravity as that of the standard fluid. The reaction of blood is faintly alkaline to litmus and the taste saltish. Its temperature varies slightly, the average being $37.8^{\circ} \mathrm{C}$. $\left(100^{\circ} \mathrm{F}\right.$.). The blood-stream is warmed by passing through the muscles, and glands, but is somewhat cooled on traversing the capillaries of the skin. Recently drawn blood has a distinct odour, which in many cases is characteristic of the animal from which it has been taken; it may be further developed by adding to blood a mixture of equal parts of sulphuric acid and water.

Quantity of the Blood.-The quantity of blood in an animal is usually estimated in the following manner:-A small quantity

* The amphioxus and the leptocephalus. 
of blood is taken from an animal by venesection; it is defibrinated and measured, and used to make standard solutions of blood. The animal is then rapidly bled to death, and the blood which escapes is collected. The blood-vessels are next washed out with saline solution until the washings are no longer coloured, and these are added to the previously withdrawn blood; lastly, the whole animal is finely minced with saline solution. The fluid obtained from the mincings is carefully filtered and added to the diluted blood previously obtained, and the whole is measured. The next step in the process is the comparison of the colour of the diluted blood with that of standard solutions of blood and water of a known strength, until it is discovered to what standard solution the diluted blood corresponds. As the amount of blood in the corresponding standard solution is known, as well as the total quantity of diluted blood obtained from the animal, it is easy to calculate the absolute amount of blood which the latter contained, and to this is added the small amount which was withdrawn to make the standard solutions. This gives the total amount of blood which the animal contained. It is contrasted with the weight of the animal, previously known. The result of experiments performed in this way showed that the quantity of blood in various animals differs a good deal, but in the dog averages $\frac{1}{12}$ to $\frac{1}{14}$ of the total body-weight. . In smaller animals the proportionate blood volume is greater.

Haldane and Lorrain Smith have invented another method which has the advantage of being applicable to man. The data required are (1) the percentage of hæmoglobin in the blood, and (2) the extent to which the hæmoglobin is saturated by a measured amount of carbonic oxide absorbed into the blood.

The percentage of hæmoglobin is determined colorimetrically by the Gowers or Gowers-Haldane hæmoglobinometer (see p. 470). In the latter instrument the standard 100 per cent. of colour corresponds to a capacity of 18.5 c.c. of oxygen or carbonic oxide per 100 c.c. of blood. The subject whose blood is to be measured breathes a known volume of carbonic oxide, and a few drops of the blood are taken and the saturation of his hæmoglobin is determined colorimetrically. From this result the total capacity of the blood for carbonic oxide is calculated. The "carbonic oxide capacity" is the same as the " oxygen capacity." The volume of the blood is then calculated from the total "oxygen capacity," and the percentage capacity as determined by the hæmoglobinometer. The following is an example:-The subject's blood in a given case has, let us say, the colour of the 100 per cent. standard, and therefore has a capacity of 18.5 c.c. per 100 c.c. blood. He is allowed to breathe 75 c.c. of carbonic oxide, and it is then found that his blood is 15 per cent. saturated with that gas. That is to say, instead of there being 18.5 c.c. of oxygen 
per 100 c.c. of blood, 15 per cent. of this 18.5 c.c. is present in the form of carbonic oxide, 15 per cent. of $18.5=\frac{18.5 \times 15}{100}=2.7$ c.c.

Now if 2.7 c.c. of carbon monoxide per 100 c.c. of blood is the result of breathing 75 c.c. of that gas, the question before us is, How much gas will be necessary to produce the normal figure 18.5 ?

2.7 c.c. per 100 c.c. of blood results from breathing 75 c.c. of $\mathrm{CO}$

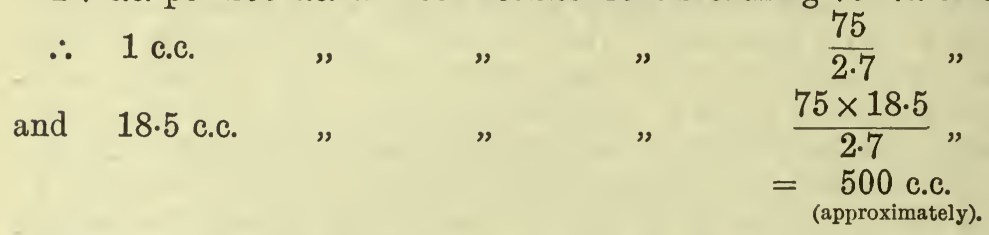

In other words, the total oxygen (or $\mathrm{CO}$ ) capacity of the person's blood is 500 c.c. Sinco 18.5 c.c. of this total is carried by 100 c.c. of blood, the total volume of the person's blood, that is, the amount which will contain 500 c.c. of gas, is $\frac{500 \times 100}{18.5}=2727$ c.c., or nearly three litres. The total weight of the blood is obtained by multiplying the volume by the specific gravity (about 1.055).

Some of the results of this method are as follows:-The mass of the blood in man is about 4.9 per cent. $\left(\frac{1}{20.5}\right)$ of the body-weight. The corresponding ratio of the blood volume is 4.62 c.c. per 100 grammes, or $\frac{1}{21 \cdot 6}$. In pathological conditions the numbers are different; thus in anæmia from hæmorrhage, the volume ratio is 6.5 , in pernicious anæmia 8.6, in chlorosis 10.8 . In other words, in various forms of anæmia the actual volume of the blood is increased, but of course the corpuscular and solid constituents are correspondingly diminished.

\section{Coagulation of the Blood.}

After the blood is shed it rapidly becomes more viscous and then sets into a firm red jelly. The jelly soon contracts and squeezes out a straw-coloured fluid called the serum. With the microscope, filaments or fine threads are seen forming a network throughout the fluid (fig. 317), many radiating from small clumps of blood-platelets. These threads entangle the corpuscles, and so the clot is formed. The threads are composed of a protein substance called fibrin, and the formation of fibrin is the essential act of coagulation. Fibrin is formed from the plasma, and may be obtained free from corpuscles when plasma is allowed to clot, the corpuscles having previously been removed by methods we shall immediately study. It may also 
be obtained from blood by whipping it with a bunch of twigs; the fibrin adheres to the twigs and entangles but fow corpuscles; these may be removed by subsequent washing with water.

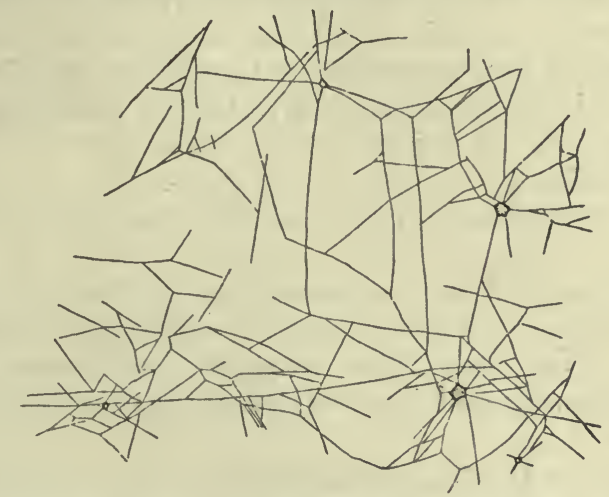

F1G. 317.-Reticulum of fibrin, from a drop of human blood, after treatment with rosanilin. The entangled corpuscles are not seen. (Ranvier.)

Serum is plasma minus the fibrin which it forms. The relation of plasma, serum, and clot can be seen at a glance in the following scheme of the constituents of the blood:-

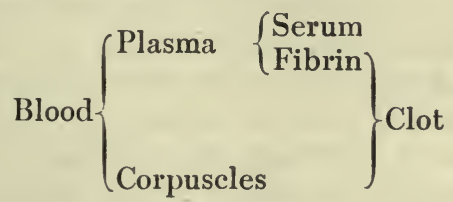

It may be roughly stated that in 100 parts by weight of blood $60-65$ parts consist of plasma and 35-40 of corpuscles.

The buffy coat is seen when blood coagulates slowly, as in horse's blood. The red corpuscles sink more rapidly than the white, and the upper stratum of the clot (buffy coat) consists mainly of fibrin and white corpuscles.

Coagulation is hastened by-

1. A temperature a little over that of the body.

2. Contact with foreign matter.

3. Injury to the vessel walls.

4. Agitation.

5. Addition of calcium salts.

6. Injection of nucleo-protein into the circulation causes intravascular clotting.

Coagulation is hindered or prevented by-

1. A low temperature. In a vessel cooled by ice, coagulation may be prevented for an hour or more. 
2. The addition of a large quantity of neutral salts such as sodium sulphate or magnesium sulphate.

3. Addition of a soluble oxalate, fluoride, or citrate.

4. Injection of commercial peptone (which consists chiefly of proteoses) into the circulation of the living animal.

5. Addition of leech extract to the blood, or injection of leech extract into the cirulation while the animal is alive.

6 . Contact with the living vascular walls.

7. Contact with oil.

The cause of the coagulation of the blood may be briefly stated as follows:-

When blood is within the vessels, one of the constituents of the plasma, a protein of the globulin class, called fibrinogen, exists in a soluble form. When the blood is shed, the fibrinogen molecule is altered in such a way that it gives rise to the comparatively insoluble material fibrin.

The statement has been made that the fibrinogen molecule is split into two parts; one part is a globulin (fibrino-globulin), which remains in solution; the other and larger part is the insoluble substance fibrin. It is, however, doubtful if this really represents what occurs, for recent work seems to show that the fibrinoglobulin is not a product of fibrinogen, but exists in the blood-plasma beforehand. At any rate, whether this is so or not, the fact remains that fibrin is the important product and the only one which need concern us.

The next question is, What causes the transformation of fibrinogen into fibrin? and the answer to that is, that the change is due to the activity of an enzyme which is called fibrin-ferment or thrombin.

This enzyme does not exist in healthy blood contained in healthy blood-vessels, but is formed by the disintegration of the bloodplatelets and colourless corpuscles which occurs when the blood leaves the blood-vessels or comes into contact with foreign matter. Hence the blood does not coagulate during life. But it will be said, disintegration of the blood-corpuscles occurs during life, why, then, does the blood not coagulate? The reason is, that although the formed elements do disintegrate in the living blood, such a phenomenon takes place very slowly and gradually, so that there can never, under normal circumstances, be any massive liberation of fibrin-ferment, and further, that there are agencies at work to neutralise the fibrin-ferment as it is formed. The most noteworthy of these neutralising agencies is the presence in the blood of an antiferment called antithrombin, analogous to the antipepsin and antitrypsin which we shall see are efficacious in preventing the stomach and intestines from undergoing self-digestion.

Nucleo-proteins obtained from most of the cellular organs of the body produce intravascular clotting when injected into the circulation of a living animal. In certain diseased conditions intravascular clotting or thrombosis sometimes occurs, and this, if it 
occurs in the heart and main blood-vessels, is fatal. The condition is doubtless due to the entrance of nucleo-protein into the circulation from disintegrated cells. The relationship of nucleo-protein and thrombin is an unsettled problem; the old view that the two are identical is probably incorrect; it is, however, possible that the nucleo-protein is either identical with thrombokinase, or holds the thrombokinase in loose combination or admixture.

Thrombin is believed to originate chiefly from the blood-platelets and in part from the leucocytes. Birds' blood clots very slowly, and the absence of blood-platelets in this variety of blood will, in part, account for this. Lymph, which contains colourless corpuscles, but no platelets, also clots, so in this case the colourless corpuscles must be the source of the ferment. One should, however, be careful in speaking of the disintegration of leucocytes to remember that the word disintegration does not mean complete breakdown leading to disappearance; the colourless corpuscles do not appreciably diminish in number when the blood clots, but what occurs is a shedding out from the surviving leucocytes of certain products, among which fibrin-ferment is one.

We have now traced fibrin formation, the essential cause of blood-clotting, to the activity of thrombin; it is next necessary to allude to what has been discovered in relation to the origin of thrombin. Like other enzymes, it is preceded by a mother-substance or zymogen. This zymogen is called prothrombin or thrombogen, and there appear to be two necessary agents concerned in the conversion of thrombogen into thrombin; one of these is the action of calcium salts, the other is the presence of an activating agent (analogous to the enterokinase, which activates pancreatic juice) called thrombokinase.

The part played by calcium salts is well illustrated by the fact that coagulation is prevented by the decalcification of the blood. This can be accomplished by the addition of a small amount of a soluble oxalate or fluoride to the blood immediately it is shed. The calcium of the blood plasma is then immediately precipitated as insoluble calcium oxalate or fluoride, and is thus not available for the transformation of thrombogen into thrombin. The addition of the oxalate or fluoride must be rapidly performed, otherwise time will be given for the conversion of thrombogen into thrombin, and thrombin, when formed, will act upon fibrinogen whether the calcium has been removed or not. In other words, calcium is only necessary for the formation of fibrin-ferment, and not for the action of fibrin-ferment on fibrinogen. Fibrin is thus not a compound of calcium and fibrinogen.

The action of a soluble citrate is also, in a certain sense, a decalcifying action, for although calcium citrate is a soluble salt, it 
does not ionise in solution so as to liberate the free calcium ions which are essential for thrombin formation.

Oxalated blood (or oxalated plasma) will clot when the calcium is once more restored by the addition of a small amount of calcium chloride, but such addition to fluoride plasma will not induce clotting; in this case, thrombin itself must be added as well. In some way sodium fluoride interferes with the formation of thrombin, probably by preventing the liberation of thrombokinase from the corpuscular elements of the blood. The latter are certainly very well preserved.

The second activating agent, however, thrombokinase, is not only liberated from the blood-corpuscles, but it is also obtained from many other tissues. If a hæmorrhage takes place under ordinary circumstances the blood as it flows from the wound passes over the muscles and skin that have been cut, and rapidly clots owing to the thrombokinase supplied by those tissues. If blood is obtained by drawing it off through a perfectly clean cannula into a clean vessel without allowing it to touch the tissues, it remains unclotted for a long time; in the case of birds' blood this time may extend to many days; but the addition of a small piece of a tissue such as muscle, or of an extract of such a tissue, produces almost immediate clotting. If a solution of fibrinogen is prepared and calcium added it will not clot; if thrombin, or a fluid such as serum which contains thrombin, is added it will clot. It will not clot if birds' plasma obtained as above is added to it; nor if tissue extract is added to it; but if both are added it will. In other words, the thrombogen of the birds' plasma plus the thrombokinase of the tissue extract have the same effect as thrombin.

The next point to consider is why blood obtained after the previous injection of proteoses (or commercial peptone) into the circulation does not clot. It certainly contains calcium salts, and probably both thrombogen and thrombokinase, for it can be made to clot without the addition of either, for instance by dilution, or the passage of a stream of carbon dioxide through it. There must be something in peptone blood which antagonises the action of thrombin. This something is an excess of antithrombin. Peptone will not hinder blood-coagulation, or only very slightly, if it is added to the blood after it is shed. The antithrombin must therefore have been added to the blood while it was circulating in the body. We can even go further than this, and say what part of the body it is which is concerned in the production of antithrombin. It is the liver; for if the liver is shut off from the circulation, peptone is ineffective in its action. The converse experiment confirms this conclusion, for if a solution of peptone is artificially perfused through an excised surviving liver, a substance is formed which has the power of hindering or preventing the coargulation of shed blood. Peptone blood is very poor in leucocytes; the causo of thcir disappoarance is not clear. 
We are thus justified in two conclusions:-

(1) That the antithrombin (normally present in healthy blood in sufficient quantities to prevent intravascular clotting) is formed in the liver.

(2) That commercial peptone, in virtue of the proteoses it contains, stimulates this action to such an extraordinary degree that the accumulation of antithrombin in the blood becomes so great that the blood does not clot even after it is shed.

We will conclude by considering only one more of the hindrances to coagulation, and that by no means the least interesting. The leech lives by sucking the blood of other animals; from the leech's point of view it is therefore necessary that the blood should flow freely and not clot. The glands at the head end of the leech, often spoken of roughly as its salivary glands, secrete something which hinders the blood from coagulating, and everyone knows by experience, who has been treated by leeches, how difficult it is to prevent a leech-bite from bleeding after the leech has been removed; complete cleansing is necessary to wash away the leech's secretion from the wound. Now if an extract of leeches' heads is made with salt solution and filtered, that fluid will prevent coagulation whether it is injected into the blood-stream or added to shed blood. The substance in question is believed to be antithrombin itself. The purified material obtaincd from leech extract is called hirudin. Blood so obtained can be made to clot by the addition of thrombin, or of such a fluid as serum, that contains thrombin in sufficient amount.

We may summarise this view of the causes of coagulation in the following tabular way:-

From the platelets, and to a lesser degree from the leucocytes, a material is shed out, called-

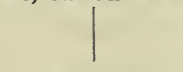

THRONBOG EN.
From the formed elements of the blood, but also from the tissues over which the escaping blood flows, is shed out an activating agent, called-

THROMBOKINASE.
In the blood plasma a protein substancc exists, called -

Fibrinogen.
In the presence of calcium salts, thrombokinase activates thrombogen in such a way that an active enzyme is produced, which is called-

Thrombin or fibrin-ferment acts on fibrinogen in such a way that it is transformed into the insoluble stringy material which is called-

$$
\text { Fibrin. }
$$

Differing hypotheses are held as to the exact rôle played by each of the factors in fibrin-formation, and the views enunciated in the preceding paragraphs are in the 
main those of Morawitz. Howell regards the lack of coagulation seen in birds' blood, and peptone blood, as due to excess of antithrombin, but holds that thrombokinase (or thromboplastin, as he terms it) brings about clotting, not by activating thrombogen, but by neutralising antithrombin. According to him, also, thromboplastin is probably a lipoid of the phosphatide group.

\section{The Plasma and Serum.}

The liquid in which the corpuscles float may be obtained by employing one or other of the methods already described for preventing the blood from coagulating. The corpuscles, being heavy, sink, and the supernatant plasma can then be removed by a pipette or siphon; the separation can be more rapidly effected by the use of a centrifugal machine.

On counteracting the influence which has prevented the blood from coagulating, the plasma then itself coagulates. Thus plasma obtained by the use of cold clots on warming gently; plasma which has been decalcified by the action of a soluble oxalate clots on the addition of a calcium salt; plasma obtained by the use of a strong solution of neutral salt coagulates when this is diluted by the addition of water, the addition of fibrin-ferment being necessary in most cases; where coagulation occurs without the addition of fibrin-ferment no doubt some is present from the partial disintegration of the corpuscles which has already occurred. Pericardial and hydrocele fluids resemble pure plasma very closely in composition. As a rule, however, they contain few or no white corpuscles, and do not clot spontaneously, but after the addition of fibrin-ferment, or of liquids such as serum that contain fibrin-ferment, they always yield fibrin.

Pure plasma may be obtained from horse's veins by what is known as the "living test-tube" experiment. If the jugular vein is ligatured in two places so as to include a quantity of blood within it, then removed from the animal and hung in a cool place, the blood will not clot for many hours. The corpuscles settle, and the supernatant plasma can be removed with a pipette.

The plasma is alkaline, yellowish in tint, and its specific gravity is about 1026 to 1029 . 1000 parts of plasma contain :-

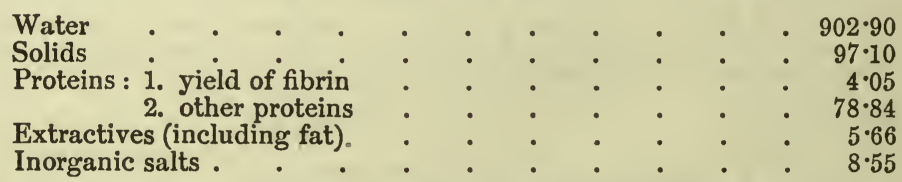

In round numbers, plasma contains 10 per cent. of solids, of which 8 are protein in nature. Fibrinogen, as judged from the yield of fibrin, is the least abundant of the proteins present.

Serum contains the same three classes of constituents-proteins, extractives, and salts. The extractives and salts are the same in 
both liquids. The proteins are different, as is shown in the following table:-

Proteins of Plasma.

Fibrinogen.

Serum globulin.

Serum albumin.
Proteins of Serum.

Serum globulin.

Serum albumin.

Fibrin-ferment + nucleo-protein.

The gases of plasma and serum are small quantities of oxygen, nitrogen, and carbonic acid. The greater part of the oxygen of the blood is combined in the red corpuscles with hæmoglobin; the carbonic acid is chiefly combined as carbonates. The gases of the blood have already been considered under Respiration (see pp. 361$372)$.

We may now study one by one the various constituents of the plasma and serum.

A. Proteins.-Fibrinogen, the mother-substance of fibrin, is a globulin. It differs from serum globulin, and may be separated from it by making use of the fact that half-saturation with sodium chloride precipitates it. It coagulates by heat at the low temperature of $56^{\circ} \mathrm{C}$.

Serum globulin and serum albumin.-These substances exhibit the usual differences already described between albumins and globulins (p. 428). Both are coagulated by heat at a little over $70^{\circ}$ C. They may be separated by dialysis or the use of neutral salts.* The readiest way to separate them is to add to the serum an equal volume of saturated solution of ammonium sulphate. This is equivalent to semi-saturation, and it precipitates the globulin. If magnesium sulphate is used as a precipitant of the globulin it must be added in the form of crystals, and the mixture well shaken to ensure complete saturation.

Fibrin-ferment or Thrombin.-Schmidt's method of preparing it is to take serum and add excess of alcohol. This precipitates all the proteins and the thrombin. After some weeks the alcohol is poured off; the serum globulin and serum albumin have been by this means rendered insoluble in water; an aqueous extract is, however, found to contain fibrin-ferment, which is not so easily coagulated by alcohol as the proteins are.

B. Extractives.-These are non-nitrogenous and nitrogenous. The non-nitrogenous are fats, soaps, cholesterin, and sugar; the nitrogenous are urea (0.02 to 0.04 per cent.), and still smaller quantities of uric acid, creatine, creatinine, xanthine, and hypoxanthine and amino-acids.

* The globulin of the serum precipitated by "salting out" really consists of two proteins, one of which is precipitated by dialysis (euglobulin), and the other is not (pseudo-globulin). 
C. Salts.-The most abundant salt is sodium chloride; it constitutes between 60 and 90 per cent. of the total mineral matter. Potassium chloride is present in much smaller amount. It constitutes about 4 per cent. of the total ash. The other salts are phosphates and sulphates.

Schmidt gives the following table:-

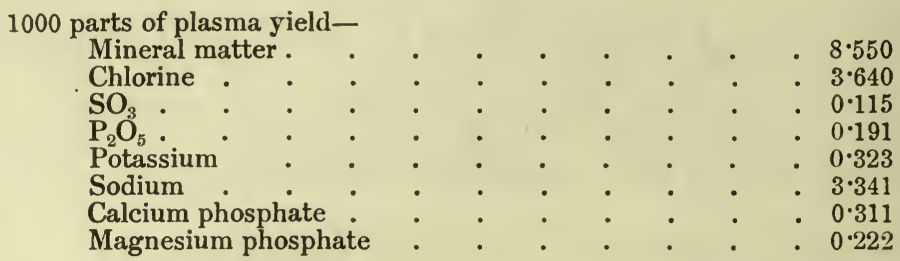

\section{The Blood-Corpuscles.}

Red or Coloured Corpuscles.-Human red blood-corpuscles are circular biconcave discs with rounded edges, $\frac{1}{3200}$ inch in diameter ( $7 \mu$ to $8 \mu$ ) and $\frac{1}{12000}$ inch, or about $2 \mu$, in thickness. When viewed singly they appear of a pale yellowish tinge; the deep red colour which they give to the blood is observable in them only when they are seen en masse.

According to Rollett they are composed of a transparent filmy framework infiltrated in all parts by the red pigment homoglobin. 'This stroma is elastic, so that as the corpuscles circulate, they admit of change in form, and recover their natural shape as soon as they escape from compression. According to this theory, the consistency of the peripheral part of the stroma is greater than that of the central portions; the outer layer thus plays the part of a membrane in the processes of osmosis that occur when water or salt solutions are added to the corpuscles. This view of Rollett has been questioned, particularly by Schäfer, who regards the red corpuscles as composed of a colourless envelope enclosing a solution of hæmoglobin. The presence of a membrane on the exterior of the corpuscle is undoubted, and can be clearly distinguished by a good microscope in the larger corpuscles of amphibia. It is, however, difficult to explain the elasticity of the corpuscles, and the central position of the nucleus in nucleated red corpuscles, unless we also assume that delicate fibres pass across the interior of the corpuscles.

The red corpuscles of other mammals are generally very nearly the size of human red corpuscles. They are smallest in the deer tribe and largest in the elephant. In the camelidæ they are biconvex. In all mammals the corpuscles are non-nucleated, and in all other vertebrates (birds, reptiles, amphibia, and fishes) the corpuscles are oval, biconvex, and nucleated (fig. 319), and larger than in mammals. They are largest of all in certain amphibians (amphiuma, proteus).

A property of the red corpuscles, which is exaggerated in inflammatory blood, is a tendency to adhere together in rolls or columns (rouleaux), like piles of coins. These rolls quickly fasten together 
by their ends, and cluster; so that, when the blood is spread out thinly on a glass they form an irregular network (fig. 318).

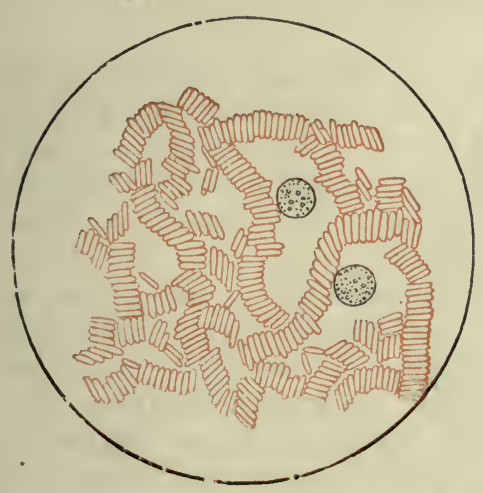

Fic. 318.-Red corpuscles in rouleaux. The white corpuscles are uncoloured.

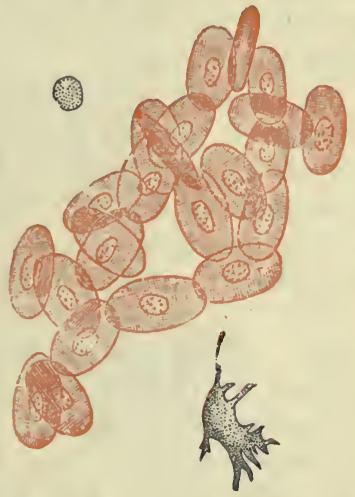

F10. 319.-Corpuscles of the frog. The central mass consists of nucleated coloured corpuscles. The other corpuscles are two varieties of the colourless form.

Action of Reagents.-Considerable light has been thrown on the physical and chemical constitution of red blood-cells by studying the effects produced by mechanical means and by various reagents; the following is a brief summary of these reactions :-

Water. - When water is added gradually to frog's blood, the oval disc-shaped corpuscles become spherical, and gradually discharge their hæmoglobin, a pale, transparent envelope being left behind: human red blood-cells swell, change from a discoidal to a spheroidal form, burst and discharge their pigment, becoming quite transparent and all but invisible. This effect is due to osmosis.

Physiological saline solution causes no effect on the red corpuscles beyond preventing them running into rouleaux. If a stronger salt solution is used, the corpuscles shrink and become crenated (fig. 320), owing to osmosis of water outwards.

Dilute acetic acid causes the nucleus of the red blood-cells in

留㺆

Fio. 320.-Effect of saline solution (crenation). the frog to become more clearly defined; if the action is prolonged, the nucleus becomes strongly granulated, and all the colouring matter seems to be concentrated in it, the surrounding cell-substance and outline of the cell becoming almost invisible ; after a time the cells lose their colour altogether. A similar loss of colour occurs in the red corpuscles of human blood, which, however, from the absence of nuclei, seem to disappear entirely.

Dilute alkalis cause the red blood-cells to dissolve slowly, and finally to disappear. Chloroform, ether, and other reagents that dissolve fats dissolve the lipoid substances (lecithin, etc.) of the membrane which surrounds the corpuscles, and so produce laking of the blood.

Tannic acid. - When a 2 per cent. fresh solution of tannic acid is applied to frog's blood it causes the appearance of a sharply-defined little knob, projecting from the free surface (Roberts' macula): the colouring matter becomes at the same time concentrated in the nucleus, which grows more distinct (fig. 321). A somewhat similar effect is produced on the human red blood-corpuscle, the colouring matter being discharged

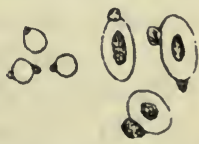

Fia. 321.-Effect of tannin. and coagulated as a little knob of hæmatin on the surface of the corpuscle, 
The Colourless Corpuscles.-The white or colourless corpuscles are masses of nucleated protoplasm; they are nearly spherical when at rest, but owing to their amœboid movements (see p. 11) exhibit considerable changes in outline when they are active, as they are at body temperature.

In health, the proportion of white to red corpuscles is on the average 1 to 500 or 600 , but this varies considerably even in the course of the same day. The number of lymphocytes is greatly increased by a meal. Also, in young persons, after hæmorrhage and during pregnancy, there is a larger proportion of colourless bloodcorpuscles; in old age they are diminished.

Several varieties of colourless corpuscles are found in human blood. They are represented in the accompanying coloured plate, stained by different methods; the column on the left shows their appearances as stained by a mixture of eosin and methylene blue (Jenner's stain). The middle column shows them as stained by Ehrlich's triacid dye (acid fuchsin, methyl-green, and orange G). In the right-hand column, the cells were stained with a mixture of hæmatoxylin and eosin. The following are the varieties shown:-

(a) Lymphocytes. - These are only a little larger than red corpuscles. The nucleus is relatively large, and usually round; the protoplasm around it forms quite a narrow zone. The nucleus, as is the case with all nuclei, is basophile, and stains with such basic dyes as methylene blue. The protoplasm presents no distinct granules and is also basophile. The lymphocytes comprise about 25 per cent. of the total colourless corpuscles.

(b) Large mononuclear leucocytes.-A relatively small oval nucleus lies near the centre of basophile protoplasm, which again presents no definite granulation. Their diameter is $12-20 \mu$, and they form only 1 per cent. of the total colourless corpuscles.

(c) Transitional leucocytes. - The cell-body is somewhat smaller and is mainly basophile. A certain amount of neutrophile granulation may be seen. The strongly basophile nucleus may present all gradations between an oval and lobed condition. In normal blood their number is variable, but, as a rule, they only make up about 2 to 4 per cent. of the total colourless corpuscles. They are called transitional on the hypothesis that they represent an intermediate condition between the large mononuclear leucocytes and the polynuclear leucocytes described under $d$. It is, however, doubtful if this hypothesis is correct, and some histologists think the $b$ and $c$ varieties originate from the endothelial wall.

(d) Polynuclear leucocytes. - These are 9-12 $\mu$ in diameter, and form the main mass of the colourless corpuscles (70 per cent.). They have several nuclei, which are strongly basophile and present many different shapes, and are usually connected by threads of 

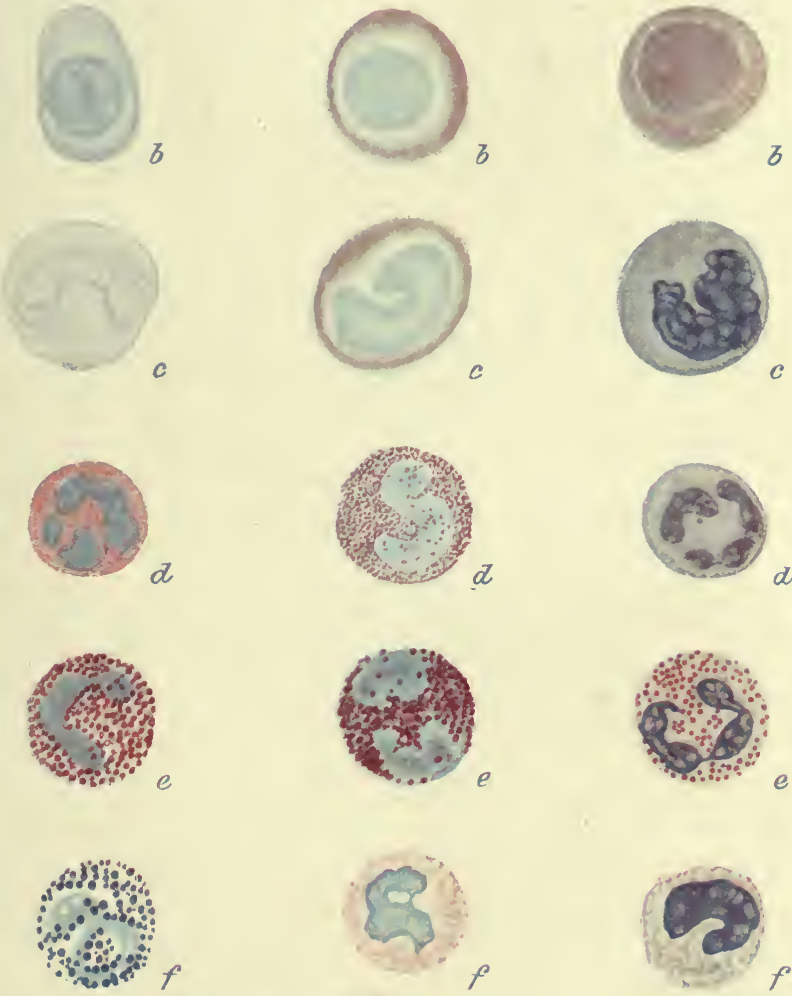

Stained with

Methylene Blue

Stained with

Stained with

Ehrlich's

Hæmatoxylin Tri-acid Dye.

The varieties of eolourless corpuscles in normal human blood, stained by different methods.

$a$, Lymphocyte; $b$, large mono-nuclear hyaline leucocyte; $c$, transition form ; $d$, polynuclear leucocyte ; $e$, eosinophile leucocyte ; $f$, mast-cell. Magnifled about 1000 times. (After Szymonowicz.) 

chromatin. The protoplasm is finely granular, and stains with neutral, and faintly with acid aniline dyes (such as eosin). In certain pathological conditions-for instance, in diabetes-the cellprotoplasm contains excess of glycogen.

(e) Eosinophile leucocytes.-These are usually larger than the preceding (12-15 $\mu$ in diameter). They contain either a single irregular-shaped nucleus, or more often two or three nuclei of unequal size. Their protoplasm contains large distinct granules which have an intense affinity for acid dyes such as eosin, and are therefore termed oxyphile, acidophile, or eosinophile. They are stated to be less actively amœboid than the polynuclear leucocytes.
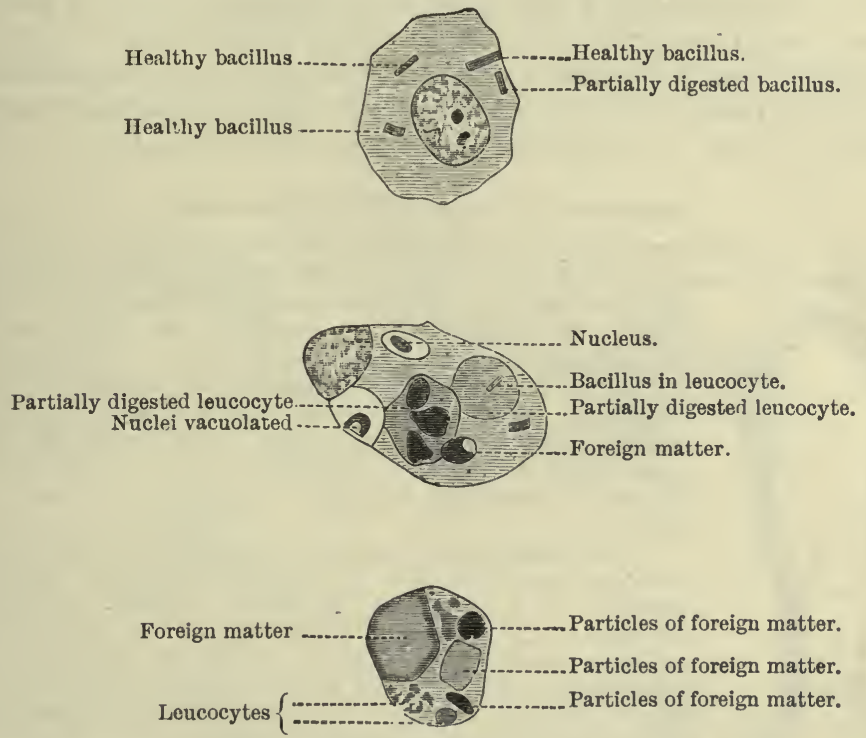

Fio. 322.-Macrophages containing bacilli and other structures undergoing digestion. (Ruffer.)

They comprise from 2 to 4 per cent. of the total colourless corpuscles.

(f) Mast-cells.-These cells we have already seen in the connective tissues (pp. 30-31) and they are very rare in normal blood. Less than 0.5 per cent. is usually present. They measure about $10 \mu$ across; their nucleus is single and irregular in shape. The granules in the protoplasm are much more basophile than the nucleus. (See coloured plate.)

Phagocytosis.-The most important outcome of the amœboid movement of the colourless corpuscles is their power of ingesting foreign particles, such as bacteria, which they engulf and digest. 
This is called phagocytosis (see also p. 298). The polynuclear leucocytes appear to be the most vigorous phagocytes. The drawings in fig. 322 show some stages in this phenomenon; the cells represented there, however, are not normal leucocytes, but certain large amcboid cells found in connective tissues, which congregate specially in inflamed parts.

The Blood-Platelets.-Besides the two principal varieties of blood-corpuscles, a third kind has been described under the name blood-platelets (Blut-plätchen). These are colourless disc-shaped or irregular bodies, much smaller than red corpuscles. Different views are held as to their origin. At first they were regarded as immature red corpuscles; but this view has been discarded. Some state that they are merely a precipitate of nucleo-protein which occurs when the plasma dies or is cooled. There is, however, no doubt that they do occur in living blood, and have been seen to undergo amœboid movement; some observers state that they are nucleated.

\section{Fnumeration of the Blood-Corpuscles.}

Several methods are employed for counting the blood-corpuscles ; most of them depend upon the same principle, i.e., the dilution of a minute volume of blood with

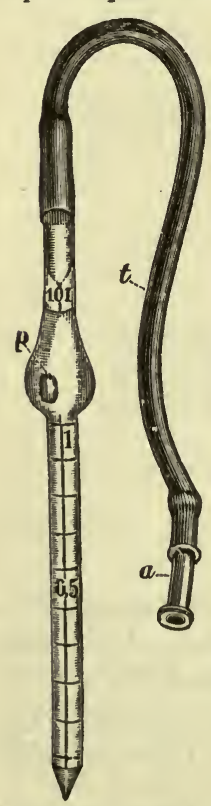

Fia. 323.-ThomaZeiss Hæmacy tometer. a given volume of a colourless saline solution similar in specific gravity to blood-plasma, so that the size and shape of the corpuscles is altered as little as possible. A minute quantity of the well-mixed solution is then taken, examined under the microscope in a cell of known capacity, and the number of corpuscles in a given area of the cell, is counted. Having ascertained the number of corpuscles in the diluted blood, it is easy to calculate the number in a given volume of normal blood.

The hæmacytometer most frequently used at the present time is known as the Thoma-Zeiss hæmacytometer. It consists of a carefully graduated pipette, in which the dilution of the blood is done; this is so formed that the capillary stem has a capacity equalling one-hundredth of the bulb above it. If the blood is drawn up in the capillary tube to the line marked 1 (fig. 323) the saline solution may afterwards be drawn up the stem to the line 101 ; in this way we have 101 parts, of which the blood forms 1 . The blood and the saline solution are well mixed by shaking the pipette, in the bulb of which is contained a small glass bead for the purpose of aiding the mixing. The other part of the instrument consists of a glass slide (fig. 324) upon which is mounted a covered disc, $m$. accurately ruled so as to present one square millimetre divided into 400 squares of one-twentieth of a millimetre each. The micrometer thus made is surrounded by another annular cell, $c$, which has such a height as to make the cell project exactly one-tenth millimetre beyond $m$. If a drop of the diluted blood is placed upon $m$, and $c$ is covered with a perfectly flat cover-glass, the volume of the diluted blood above each of the squares of the micrometer, i.e. above each $\frac{1}{400}$, will be $\frac{1}{4000}$ of a cubic millimetre. An average of ten or more squares is then taken, and this number multiplied by $4000 \times 100$ gives the number of corpuscles in a cubic millimetre of undiluted blood. The average number of corpuscles per cubic millimetre of healthy blood, according to Vierordt 
and Welcker, is 5,000,000 in adult men, and 4,500,000 in women; this corresponds to an average of 11.2 and 12.5 corpuscles respectively per square of the instrument.

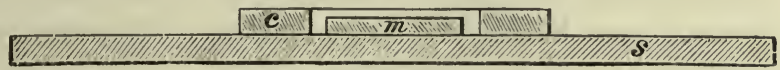

FIG. 324.

The enumeration of the colourless corpuscles depends on the same principle, but the counting has to be carried out over larger areas than the small squares, and the differentiation of the varieties of colourless corpuscles (which is most important from the standpoint of disease) can be accomplished after appropriate staining.

\section{Development of the Blood-Corpuscles.}

Origin of the Red Corpuscles.- Surrounding the early embryo is a circular aroa, called the vascular area, in which the first rudi-

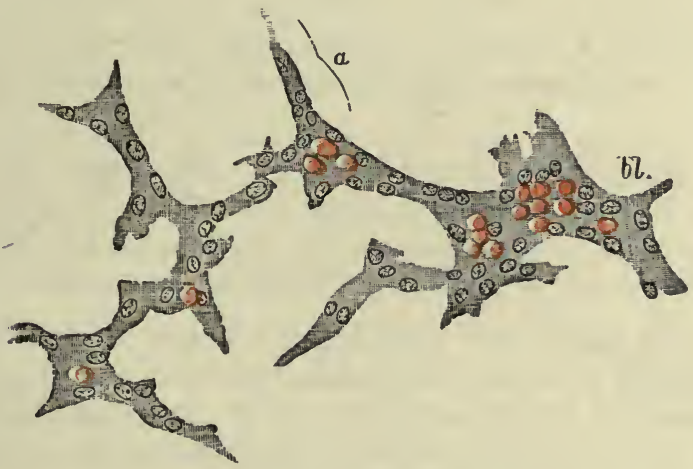

Fia. 325.-Part of the network of developing blood-vessels in the vascular area of a guinea-pig. bl, Blood-corpuscles becoming free in an enlarged and hollowed-out part of the network; $a$, process of protoplasm. (E. A. Schäfer.)

ments of the blood-vessels and blood-corpuscles are developed. Here the nucleated embryonic cells of the mesoblast, from which the bloodvessels and corpuscles are to be formed, send out processes in various directions, and these, joining together, form an irregular meshwork. The nuclei increase in number, and collect chiefly in the larger masses of protoplasm, but partly also in the processes. These nuclei gather around them a certain amount of the protoplasm, and, becoming coloured, form the red blood-corpuscles (fig. 325). The protoplasm of the cells and the branched network in which these corpuscles lie then become hollowed out into a system of canals enclosing fluid, in which the red nucleated corpuscles float. The corpuscles at first are from about $\frac{1}{2500}$ to $\frac{1}{1500}$ of an inch $(10 \mu$ to $16 \mu)$ in diameter, 
mostly spherical, and with granular contents, and a well-marked nucleus.

The corpuscles then strongly resemble the colourless corpuscles of the fully developed blood, but are coloured. They are capable of amœboid movement and multiply by division.

These coloured nucleated cells begin very early in fœtal life to be mingled with coloured non-nucleated corpuscles resembling those of the adult, and at about the fourth or fifth month of embryonic existence are completely replaced by them.

These coloured discs are partly formed in connective-tissue cells in a way similar to that just described, only without the participation of the nuclei in the process, although there is very little doubt that hæmoglobin originates from the hæmatogen (ironcontaining nuclein) of the nuclei in all cases. The fœtal liver, spleen, and thymus are also believed to be seats of formation of the red discs.

Without doubt, the red corpuscles have, like all other parts of the organism, a tolerably definite term of existence, and in a like manner die and waste away when the portion of work allotted to

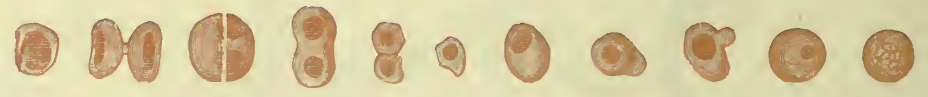

FiG. 326.-Coloured nucleated corpuscles, from the red marrow of the guinea-pig. (E. A. Schäfer.)

them has been performed. Neither the length of their life, however, nor the fashion of their decay, has been yet wholly made out. A certain number of the coloured corpuscles undergo disintegration in the liver and spleen; corpuscles in various degrees of degeneration have been observed in both these organs.

This being so, it is necessary that the red corpuscles should be constantly replenished throughout life. But after the fœtal stage is passed, they originate, not from connective tissues in general, but in one special form of connective tissue, namely, the red marrow of bones. It is possible that in some animals the spleen, which contains cells very similar to those of the marrow, may participate in their formation. In the red marrow, they arise from immature nucleated cells (normoblasts or erythroblasts, fig. 326); the nucleus is not discharged, but is absorbed within the cell, and this is the explanation that some observers give of the biconcave form of the red disc. Sometimes immature nucleated red cells may make their way from the marrow into the circulation; and the free nuclei of these cells are sometimes found in the blood; they never, when once they have entered the blood, develop into discs, and are filtered out of the blood by the spleen. 
Origin of the White Corpuscles.-The lymphocytes are formed in the lymphoid tissue of the lymphatic glands, tonsils, and other parts where this tissue is present. They enter the blood-stream by the thoracic duct, and grow larger, the proportion of protoplasm to nucleus increasing as they become mature. The mononuclear leucocyte is, according to some, a mature lymphocyte; some think it is endothelial in origin (see p. 456); according to others, it, like the polynuclear leucocytes, originates from immature forms in the red marrow, which are called myelocytes. The leucocytes proper, as distinguished from the lymphocytes, do not grow larger in the bloodstream, but rather have a tendency to shrink in size with age.

If immature myelocytes escape from the marrow into the circulating blood, they undergo no further development there, and like the immature nucleated red corpuscles, are filtered off by the spleen. This, of course, is a pathological condition, and leads to the swelling of the spleen, which is such a marked feature in the disease known as splenic leukæmia.

\section{Chemistry of the Blood-Corpuscles.}

The white blood-corpuscles.-Their nucleus consists of nuclein, their cell protoplasm yields proteins belonging to the globulin and nucleo-protein groups. The protoplasm of these cells often contains small quantities of fat and glycogen. tain-

The red blood-corpuscles. - 1000 parts of red corpuscles con-

Water

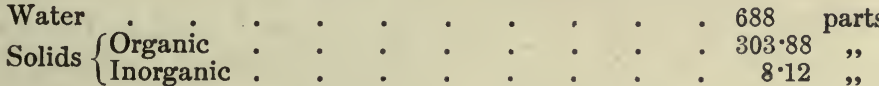

One hundred parts of the dry organic matter contain-

Protein

Phosphatides calculated as lecithin : : : . 86 to 94,

Cholesterin . . . . . . 0 0.1 ,

The protein present appears to be identical with the nucleo-protein of white corpuscles. The mineral matter consists chiefly of chlorides of potassium and sodium, and phosphates of calcium and magnesium. In man potassium chloride is more abundant than sodium chloride; this, however, does not hold good for all animals.

Hæmoglobin and Oxyhæmoglobin.-The pigment is by far the most abundant and important of the constituents of the red corpuscles. It is a conjugated protein, a compound of protein with the iron-containing pigment called hæmatin.

It exists in the blood in two conditions: in arterial blood it is combined loosely with oxygen, is of a bright red colour, and is called 
oxyhæmoglobin; the other condition is the deoxygenated or reduced hæmoglobin (better called simply hæinoglobin). This is found in the blood after asphyxia. It also occurs in all venous blood-that is, blood which is returning to the heart after it has supplied the tissues with oxygen. Venous blood, however, always contains a considerable quantity of oxyhæmoglobin also. Hæmoglobin is the oxygencarrier of the body, and it may be called a respiratory pigment.*

Crystals of oxyhæmoglobin $\dagger$ may be obtained with readiness from the blood of such animals as the rat, guinea-pig, or dog; with difficulty from other animals, such as man, ape, and most of the common mammals. The following methods are the best:-

1. Mix a drop of defibrinated blood of the rat on a slide with a drop of water; put on a cover-glass; in a few minutes the cor-

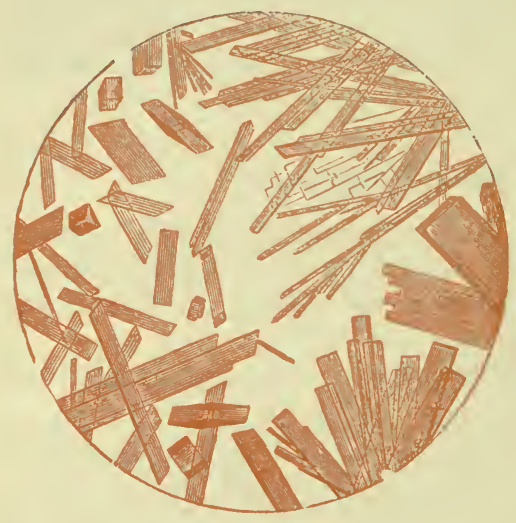

Fia. 327.-Crystals of oxyhæmoglobin-prismatic, from human blood. puscles are rendered colourless, and then the oxyhæmoglobin crystallises out from the solution so formed.

2. Microscopical specimens may also be made by Stein's method, which consists in using Canada balsam instead of water in the foregoing experiment.

3 . On a larger scale, crystals may be obtained by mixing the blood with one-sixteenth of its volume of ether; the corpuscles dissolve, and the blood assumes a laky appearance. After a period varying from a few minutes to days, abundant crystals are deposited.

In nearly all animals the crystals are rhombic prisms (fig. 327); but in the guinea-pig they are rhombic tetrahedra, or four-sided pyramids (fig. 328); in the squirrel and hamster, hexagonal plates (fig. 329).

The crystals contain a varying amount of water of crystallisation; this probably explains their different crystalline form and solubilities. Several observers have analysed hæmoglobin. They find carbon, hydrogen, nitrogen, oxygen, sulphur, and iron. The percentage of

* In the blood of invertebrate animals hæmoglobin is sometimes found, but usually in the plasma, not in special corpuscles. Sometimes it is replaced by other respiratory pigments, such as the green one, chlorocruorin, found in certain worms, and the blue one, hæmocyanin, found in many molluses and crustacea. Chlorocruorin contains iron; hæmocyanin contains copper.

$\dagger$ Crystals of hæmoglobin can also be obtained by carrying out the crystallisation in an atmosphere free from oxygen. 
iron is 0.4 . On adding an acid or alkali to hæmoglobin, it is broken up into two parts - a brown pigment called hoematin, which contains all the iron of the original substance, and a protein called globin.

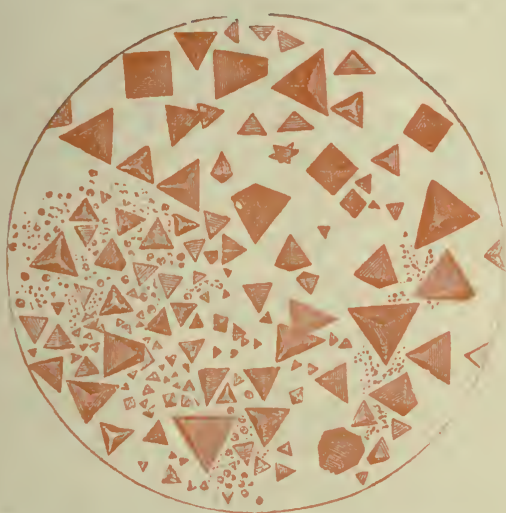

FIG. 323.-Oxyhæmoglobin crystals-tetrahedral, from blood of the guinea-pig.

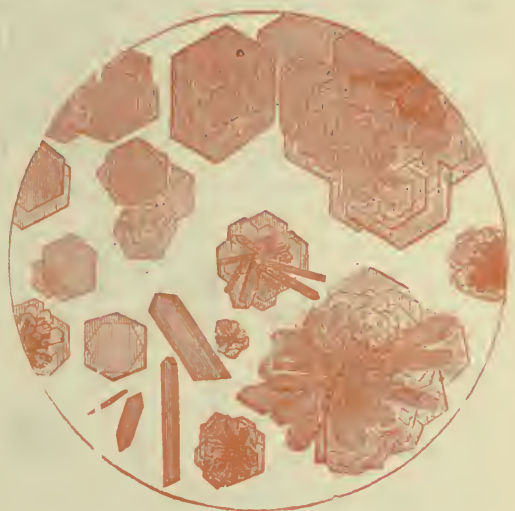

Fı,. 329.- Hexagonal oxyhæmoglobin crystals, from blood of squirrel. (After Funke.)

Hæmatin is not crystallisable; it has the formula $\mathrm{C}_{34} \mathrm{H}_{33} \mathrm{O}_{5} \mathrm{~N}_{4} \mathrm{Fe}$ or $\mathrm{C}_{34} \mathrm{H}_{35} \mathrm{O}_{5} \mathrm{~N}_{4} \mathrm{Fe}$; its constitutional formula is, however, not known. Hæmatin presents different spectroscopic appearances in acid and alkaline solutions (see accompanying plate). On decomposition it yields pyrrol derivatives (see small print, p. 464).

Globin is coagulable by heat, soluble in dilute acids, and precipitable from such solutions by ammonia. It belongs to the class of proteins called histones (see p. 427).

Hæmochromogen is sometimes called reduced hæmatin; it may be formed by adding a reducing agent such as ammonium sulphide to an alkaline solution of hæmatin, and has recently been obtained in crystalline form. Its absorption spectrum, shown on the accompanying plate (No. 8), forms the best spectroscopic test for blood pigment; the suspected pigment is dissolved in potash, and ammonium sulphide added. Very dilute specimens show the absorption bands, especially the one midway between $\mathrm{D}$ and $\mathrm{E}$.

Hæmin is of great importance, as the obtaining of this substance forms the best chemical test for blood. Hæmin crystals may be prepared for microscopical examination by boiling a fragment of dried blood with a drop of glacial acetic acid on a slide; on cooling, triclinic plates and prisms of a dark brown colour, often in star-shaped clusters and with rounded angles (fig. 330), separate out. In the case of an old blood-stain it is necossary to add a crystal of sodium chloride. Fresh blood contains sufficient sodium chloride in itself.

The action of the acetic acid is to split the hæmoglobin into 
hæmatin and globin; a hydroxyl group of the hæmatin is then replaced by chlorine; it is similarly easily replaceable by an atom of bromine or iodine. Nencki and Zaleski have further shown that when prepared in this way, hæmin also contains an acetyl group. It has the empirical formula $\mathrm{C}_{34} \mathrm{H}_{32}$ or ${ }_{34} \mathrm{O}_{4} \mathrm{~N}_{4} \mathrm{FeCl}$.

Hæmatoporphyrin, $\mathrm{C}_{34} \mathrm{H}_{38} \mathrm{O}_{6} \mathrm{~N}_{4}$, is iron-free hæmatin; it may be prepared by mixing blood with strong sulphuric acid; the iron is taken out as ferrous sulphate. It is also found sometimes in nature;

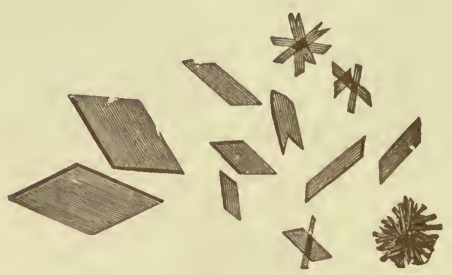

Fig. 330.-Hæmin crystals. (Frey.)

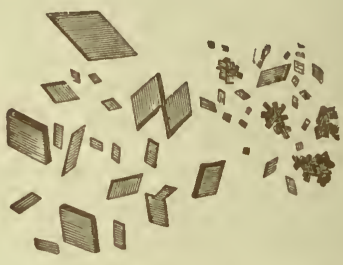

Fig. 331.-Hæmatoidin orystals. (Frey.)

it occurs in certain invertebrate pigments, and may also be found in certain forms of pathological urine. Even normal urine contains traces of it. It presents different spectroscopic appearances according as it is dissolved in acid or alkaline media. The absorption spectrum figured (No. 9) is that of acid hæmatoporphyrin.

If oxyhæmoglobin is treated with dilute acids the result is a formation of hæmatin and globin, but if strong sulphuric acid is employed the iron is removed from the hæmatin and so hæmatoporphyrin is obtained. The stability of the iron in the molecule is due to the presence of oxygen, for with the reduced pigment, hæmatoporphyrin is obtained even when dilute acids are employed. Pure hæmatoporphyrin can once more be converted into hæmatin (that is, the iron can be replaced) by warming a solution in dilute ammonia and adding a little Stokes' fluid, and a few drops of a reducing agent such as hydrazine hydrate. If cuprammonium solution is used instead of Stokes' fluid in this experiment, a copper compound of hæmatoporphyrin is obtained, which is identical with turacin, the bright red copper-containing pigment found in the plumage of the plantain-eating birds. (Laidlaw.)

Hcomopyrrol is a substance obtained by reduction from hæmatoporphyrin. It is dimethyl-ethyl pyrrol, and its formula is :-<smiles>CCCc1c(C)[nH]c(C)c1C</smiles>

There is a near relationship between hæmoglobin and chlorophyll, for the same substance is obtained from phylloporphyrin, $\mathrm{C}_{16} \mathrm{H}_{18} \mathrm{~N}_{2} \mathrm{O}$, a derivative of chlorophyll. Chlorophyll, however, contains no iron.

Hæmatoidin.-This substance is found in the form of yellowish red crystals (fig. 331) in old blood extravasations, and is derived from the hæmoglobin. Its crystalline form and the reaction it gives with 
fuming nitric acid show it to be closely allied to bilirubin, the chief colouring matter of the bile, and on analysis it is found to be identical with it.

Hæmatoidin, like hæmatoporphyrin, is free from iron, but differs from it in showing no spectroscopic bands.

\section{Compounds of Hæmoglobin.}

Hæmoglobin forms at least four compounds with gases :-

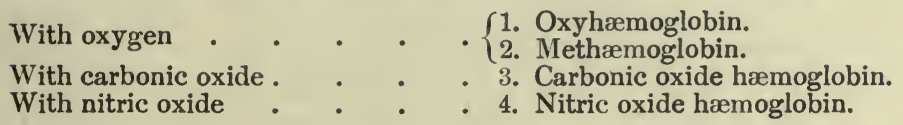

These compounds have similar crystalline forms; they each consist of a molecule of hæmoglobin combined with one of the gas in question (see p. 366). They part with the combined gas somewhat readily; they are arranged in order of stability in the above list, the least stable first.

Oxyhæmoglobin is the compound that exists in arterial blood. Many of its properties have been already mentioned. The oxygen linked to the hæmoglobin, which is removed by the tissues through which the blood circulates, may be called the respiratory oxygen of hæmoglobin. The processes that occur in the lungs and tissues, resulting in the oxygenation and deoxygenation respectively of the hæmoglobin, may be imitated outside the body, using either blood or pure solutions of hæmoglobin. The respiratory oxygen can be removed, for example, in the Torricellian vacuum of a mercurial airpump, or by passing a neutral gas such as hydrogen through the blood, or by the use of reducing agents such as ammonium sulphide and Stokes' reagent.* One gramme of hæmoglobin will combine with 1.34 c.c. of oxygen.

If any of these methods for reducing oxyhæmoglobin is used, the bright red (arterial) colour of oxyhæmoglobin changes to the darker (venous) tint of hæmoglobin. On once more allowing oxygen to come into contact with the hæmoglobin, as by shaking the solution with the air, the bright arterial colour returns.

These colour-changes may be more accurately studied with the spectroscope, and the constant position of the absorption bands seen constitutes an important test for blood pigment. It will be first necessary to describe briefly the instrument used.

The Spectroscope. - When a ray of white light is passed through a prism, it is refracted or bent at each surface of the prism; the

* Stokes' reagent must always be freshly prepared; it is a solution of ferrous sulphate to which a little tartaric acid has been added, and then ammonia till the reaction is alkaline. 
whole ray is, however, not equally bent, but it is split into its constituent colours, which may be allowed to fall on a screen. The band of colours beginning with the red, passing through orange, yellow, green, blue, and ending with violet, is called a spectrum : this is seen in nature in the rainbow.

The spectrum of sunlight is interrupted by numerous dark lines crossing it vertically, called Frauenhofer's lines. These are perfectly constant in position and serve as landmarks in the spectrum. The more prominent are $\mathrm{A}, \mathrm{B}$, and $\mathrm{C}$, in the red; $\mathrm{D}$, in the yellow; $\mathrm{E}, b$, and $\mathrm{F}$, in the green; $\mathrm{G}$ and $\mathrm{H}$, in the violet. These lines are due to certain volatile substances in the solar atmosphere. If the light from burning sodium or its compounds is examined spectroscopically, it will be found to give a bright yellow line, or, rather, two bright yellow lines very close together. Potassium gives two bright red lines and one violet line; and the other elements, when incandescent, give characteristic lines, but none so simple as sodium. If now the flame of a lamp is examined, it will be found to give a continuous spectrum like that of sunlight in the arrangement of its colours, but unlike it in the absence of dark lines; but if the light from the lamp is made to pass through sodium vapour before it reaches the spectroscope, the bright yellow light will be found absent, and in its place a dark line, or, rather, two dark lines very close together, occupying the same position as the two bright lines of the sodium spectrum. The sodium vapour absorbs the same rays as those which it itself produces at a higher temperature. Thus the $\mathrm{D}$ line, as we term it in the solar spectrum, is due to the presence of sodium vapour in the solar atmosphere. The other dark lines are similarly accounted for by other elements.

The large form of spectroscope (fig. 332) consists of a tube A, called the collimator, with a slit at the end S, and a convex lens at the end $\mathrm{L}$. The latter makes the rays of light passing through the slit from the source of light, parallel: they fall on the prism $\mathrm{P}$, and then the spectrum so formed is focussed by the telescope $\mathrm{T}$.

A third tube, not shown in the figure, carries a small transparent scale of wave-lengths, as in accurate observations the position of any point in the spectrum is given in the terms of the corresponding wave-lengths.

If we now interpose between the source of light and the slit $\mathrm{S}$ a piece of coloured glass ( $\mathrm{H}$ in fig. 333), or a solution of a coloured substance contained in a vessel with parallel sides (the hæmatoscope of Herrmann), the spectrum is found to be no longer continuous, but is interrupted by a number of dark shadows, or absorption bands corresponding to the light absorbed by the coloured medium. Thus a solution of oxyhæmoglobin of a certain strength gives two bands 
BLOOD-SPECTRA COMPARED WITH SOLAR SPECTRUM.

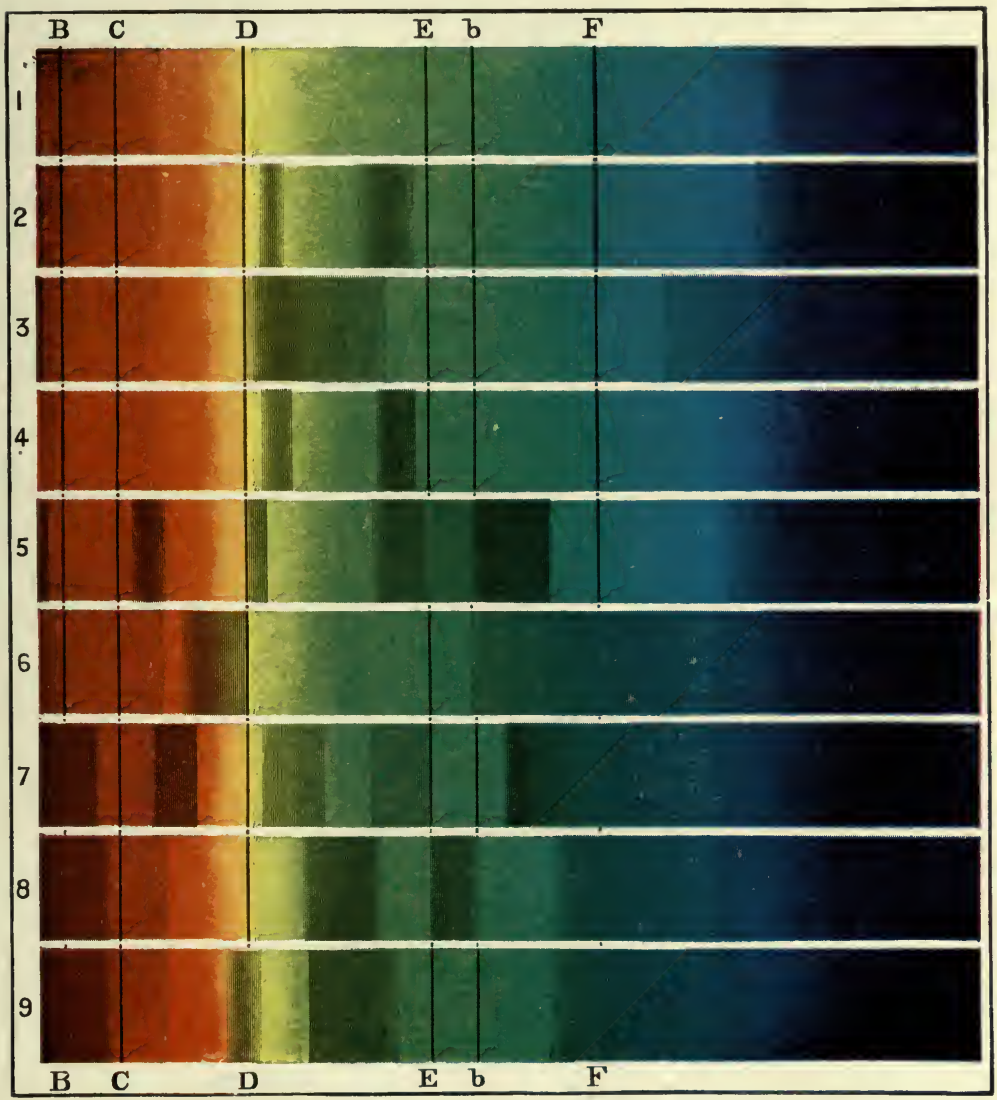

I. Solar spectrum.

2. Spectrum of dilute solution of oxyhæmoglobin.

3. " " hæmoglobin.

4. " " carbonic oxide hæmoglobin.

5. " " acid hæmatin in ethereal solution.

6. " " alkaline hæmatin.

7. " " methæmoglobin.

8. " " hæmochromogen.

9. " , acid hæmatoporphyrin. 

between the $\mathrm{D}$ and $\mathrm{E}$ lines; hæmoglobin gives only one; and other red solutions, though to the naked eye similar to oxyhæmoglobin, will give characteristic bands in other positions.

A convenient form of small spectroscope is the direct vision spectroscope, in which, by an arrangement of alternating prisms of

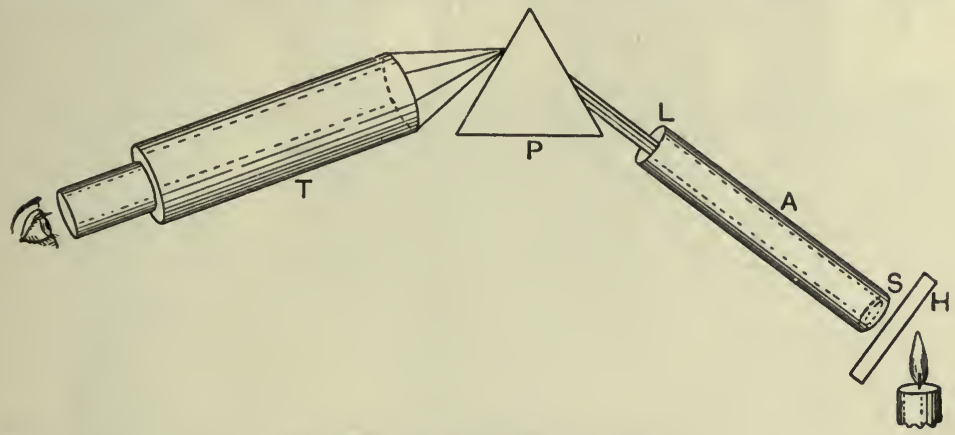

FIG. 332.-Diagram of Spectroscope.

crown and flint glass, the spectrum is observed by the eye in the same line as the tube furnished with the slit-indeed, slit and prisms are both contained in the same tube.

In the examination of the spectrum of small coloured objects a combination of the microscope and direct vision spectroscope, called the micro-spectroscope, is used.

The next figure (fig. 333) illustrates a method of representing absorption spectra diagrammatically. The solution was examined in a layer 1 centimetre thick. The base-line has on it at the proper distances the chief Frauenhofer lines, and along the right-hand edges are percentages of the amount of oxyhæmoglobin present in I, of hæmoglobin in II. The width of the shadings at each level represents the position and amount of absorption corresponding to the percentages.

The characteristic spectrum of oxyhæmoglobin, as it actually appears through the spectroscope, is seen in the accompanying coloured plate (spectrum 2). There are two distinct absorption bands between the $\mathrm{D}$ and $\mathrm{E}$ lines; the one nearest to $\mathrm{D}$ (the $\alpha$ band) is narrower, darker, and has better-defined edges than the other (the $\beta$ band). As will be seen on looking at fig. 333, a solution of oxyhæmoglobin of concentration greater than 0.65 per cent. and less than 0.85 per cent. (examined in a cell of the usual thickness of 1 centimetre) gives one thick band overlapping both $\mathrm{D}$ and $\mathrm{E}$, and a stronger solution only lets the red light through between $\mathrm{C}$ and $\mathrm{D}$. A solution which gives the two characteristic bands must therefore be a dilute one. The one band ( $\gamma$ band) of hæmoglobin (spectrum 3 ) is 
not so well defined as the $\alpha$ or $\beta$ bands. On dilution it fades rapidly ; so that in a solution of such strength that both bands of oxyhæmoglobin
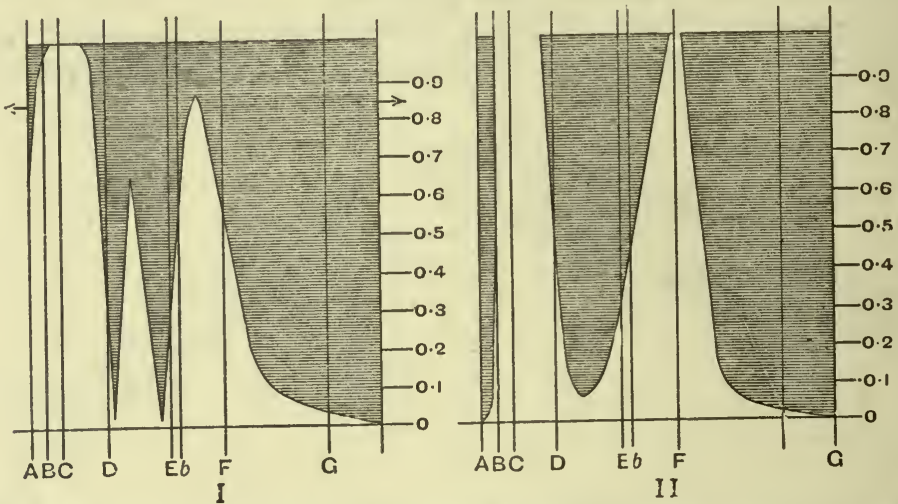

Fia. 333.-Graphic representations of the amount of absorption of light by solution of (1) oxyhæmoglobin, (II) of hæmoglobin, of different strengths. The shading indicates the amount of absorption of the spectrum; the figures on the right border express percentages. (Rollett.)

would be quite distinct, the single band of hæmoglobin has disappeared from view. The oxyhæmoglobin bands can be distinguished in a solution which contains only one part of the pigment to 10,000 of water, and even in more dilute solutions which seem to be colourless the $\alpha$ band is still visible.

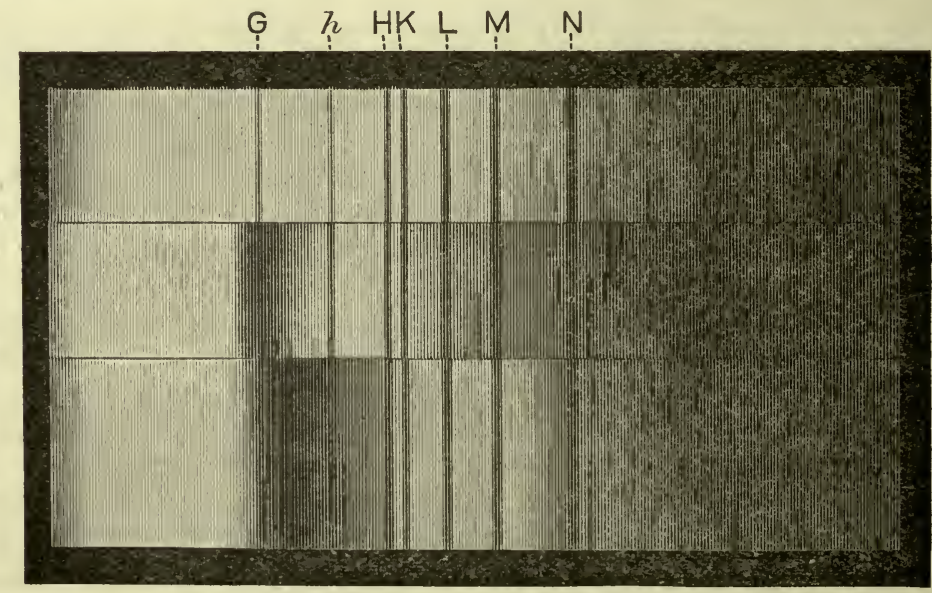

FIG. 334.-The photographic spectrum of hæmoglobin and oxyhæmoglobin. (Gamgee.)

Hæmoglobin and its compounds also show absorption bands in the ultra-violet portion of the spectrum. This portion of the spectrum 
is not visible to the eye, but can be rendered visible by allowing the spectrum to fall on a fluorescent screen, or on a sensitive photographic plate. In order to show absorption bands in this part of the spectrum very dilute solutions of the pigment must be used.

Oxyhæmoglobin shows a band (Soret's band) between the lines G and $\mathrm{H}$. In hæmoglobin, carbonic oxide hæmoglobin, and nitric oxide

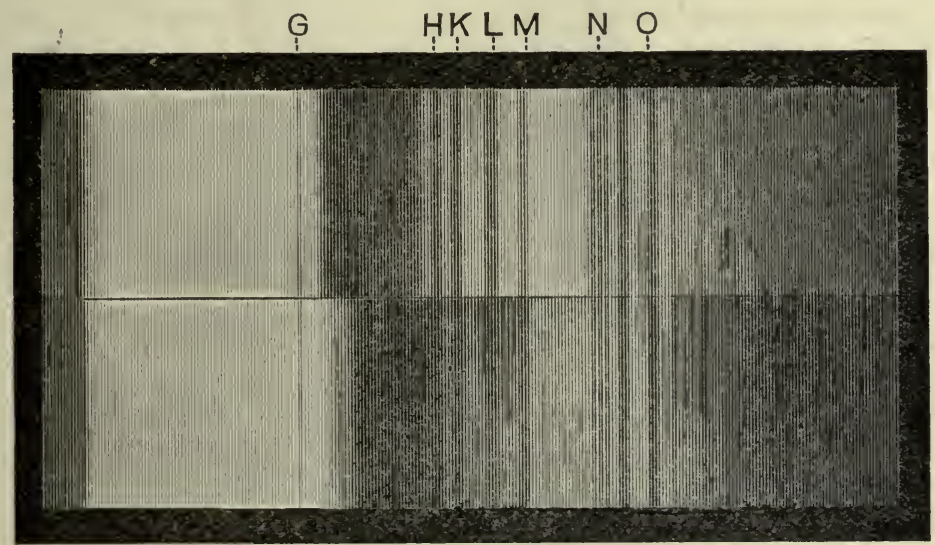

Fro. 335. - The photographic spectrum of oxyhæmoglobin and methæmoglobin. (Gamgee.)

hæmoglobin, this band is rather nearer G. Methæmoglobin and hæmatoporphyrin show similar bands.

We owe most of our knowledge of the "photographic spectrum" to the late Prof. Gamgee, through whose kindness I am enabled to present reproductions of two of his numerous photographs (figs. 334 and 335).

Methæmoglobin.-This may be produced artificially in various ways, as by adding potassium ferricyanide or amyl nitrite to blood, and as it also may occur in certain diseased conditions in the urine, it is of considerable practical importance. It can be crystallised, and is found to contain the same amount of oxygen as oxyhæmoglobin, only combined in a different way. The oxygen is not removable by the air-pump, nor by a stream of neutral gas such as hydrogen. It can, however, by reducing agents like ammonium sulphide, be made to yield hæmoglobin. Methæmoglobin is of a brownish red colour, and gives a characteristic absorption band in the red between the $\mathrm{C}$ and D lines (spectrum 7 in coloured plate). In dilute solutions other bands can be seen.

Potassium ferricyanide is the most convenient reagent for making methæmoglobin. It is, however, necessary to remind the reader that it produces another effect as well, namely, it causes an evolution of gas, if the blood has been previously laked. This gas is oxygen; in fact, all the oxygen combined as oxylumo- 
globin is discharged, and this may be collected and measured as in the method described on p. 364. This discharge of oxygen from oxyhæmoglobin is at first sight puzzling, because, as just stated, methæmoglobin contains the same amount of oxygen that is present in oxyhæmoglobin. What occurs is that after the oxygen is discharged from oxyhæmoglobin, an equal quantity of oxygen, due to the oxidising action of the reagents added, takes its place; this new oxygen, however, is combined in some way different from that which was previously united to the hæmoglobin. (Haldane.)

Carbonic oxide hæmoglobin may be readily prepared by passing a stream of carbonic oxide or coal gas through blood or through a solution of oxyhæmoglobin. It has a peculiar cherry-red colour. Its absorption spectrum is very like that of oxyhæmoglobin, but the two bands are slightly nearer the violet end of the spectrum (spectrum 4 in coloured plate). Reducing agents, such as ammonium sulphide, do not change it; the gas is more firmly combined than the oxygen in hæmoglobin. CO-hæmoglobin forms crystals like those of oxyhæmoglobin. It resists putrefaction for a very long time.

Carbonic oxide is given off during the imperfect combustion of carbon such as occurs in charcoal stoves or during the explosions that occur in coal-mines; it acts as a powerful poison, by combining with the hæmoglobin of the blood, and thus interferes with normal respiratory processes. The bright colour of the blood in both arteries and veins, and its resistance to reducing-agents, are in such cases characteristic.

Nitric Oxide Hæmoglobin.-When ammonia is added to blood, and then a stream of nitric oxide passed through it, this compound is formed. It may be obtained in crystals isomorphous with oxyand CO-hæmoglobin. It also has a similar spectrum. It is even more stable than CO-hæmoglobin; it has no practical interest, but is of theoretical importance as completing the series.

Estimation of Hæmoglobin. - The most exact method is by the estimation of the amount of iron (dry hæmoglobin containing $\bullet 42$ per cent. of iron) in the ash of a given specimen of blood, but as this is a somewhat complicated process, various colorimetric methods have been proposed which, though not so exact, have the advantage of simplicity.

Gowers' Hæmoglobinometer.-The apparatus (fig. 336) consists of two glass tubes of the same size. One contains glyccrin jelly tinted with carmine to a standard colour-viz., that of normal blood diluted 100 times with distilled water. The finger is pricked and 20 cubic millimetres of blood are measured out by the capillary pipette B. This is blown out into the other tube and diluted with distilled water, added drop by drop from the pipette stopper of the bottle $\mathbf{A}$, until the tint of the diluted blood reaches the standard colour. This tube is graduated into 100 parts. If the tint of the diluted blood is the same as the standard when the tube is filled up to the graduation 100, the quantity of oxyhæmoglobin in the blood is normal. If it has to be diluted more largely, the oxyhæmoglobin is in excess; if to a smaller extent, it is less than normal. If the blood has, for instance, to be diluted up to the graduation 50, the amount of hæmoglobin is only half what it ought to be -50 per cent. of the normal - and so for other percentages.

Haldane's Modiflcation of Gowers' Instrument is the one most frequently used now, and gives very accurate results. Instead of tinted gelatin, the standard of comparison is a sealed tube filled with a solution of carbonic oxide hæmoglobin. 
This keeps unchanged for years. A stream of coal gas is passed through the blood to be examined. 'This converts all the hæmoglobin present into carbonic oxide hæmoglobin; this is then diluted with water to match the standard.

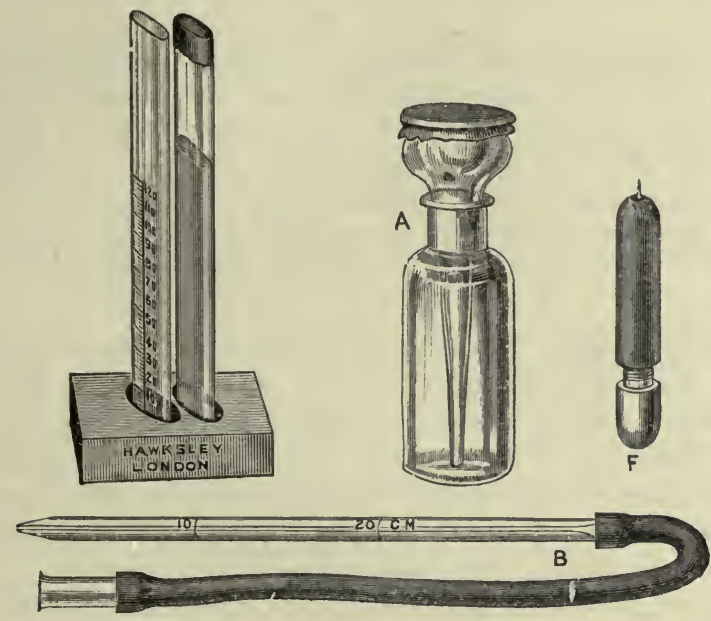

FIG. 336.-Hæmoglobinometer of Dr Gowers.

Von Fleischl's Hæmometer.-'The apparatus (fig. 337) consists of a stand bearing a white reflecting surface (S) and a platform. Under the platform is a slot

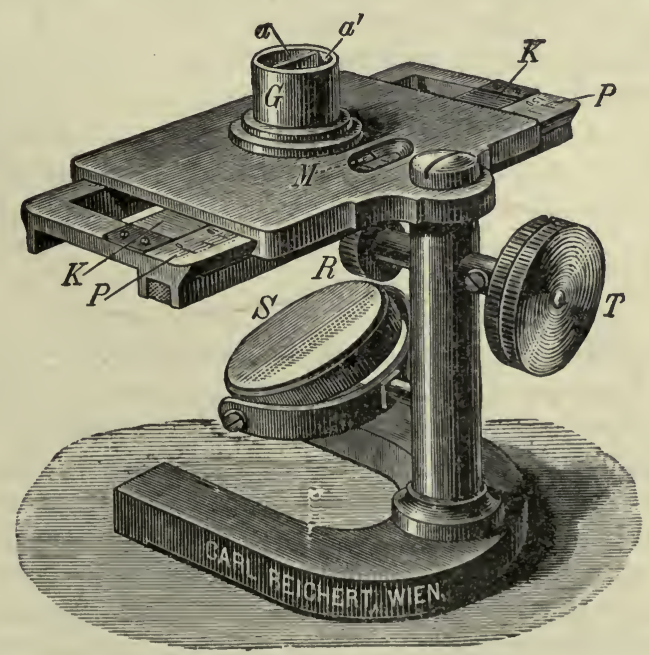

F10. 337.-Von Fleischl's Hæmoglobinometer.

$$
\text { sniq?. }
$$

carrying a glass wedge stained red $(K)$ and moved by a wheel $(R)$. On the platform is a small cylindrical vessel divided vertically into two compartments, $a$ and $\alpha^{\prime}$. 
Fill with a pipette the compartment $a^{\prime}$ over the wedge with distilled water. Fill about a quarter of the other compartment $(a)$ with distilled water.

Prick the finger and fill the short capillary pipette provided with the instrument with blood. Dissolve this in the water in compartment $a$, and fill it up with distilled water.

Having arranged the reflector (S) to throw artificial light vertically through both compartments, look down through them, and move the wedge of glass by the milled head (T) until the colour of the two is identical. Read off the scale, which is so constructed as to give the percentage of hæmoglobin.

Dr George Oliver's IMethod consists in comparing a specimen of blood suitably diluted in a shallow white palette with a number of standard tests very carefully prepared by the use of Lovibond's coloured glasses. These standards are much better matches for blood in various degrees of dilution than in most colorimetric methods. The yellow tint of diluted hæmoglobin is very successfully imitated.

Tests for Blood.-These may be gathered from preceding descriptions. Briefly, they are microscopic, spectroscopic, and chemical. The best chemical test is the formation of hæmin crystals. The old test with tincture of guaiacum and hydrogen peroxide, the blood causing the tincture to become bluish green, is very untrustworthy, as it is also given by many other organic substances. The test, for instance, is given by milk, and is there due to the presence of an enzyme called a peroxidase, which is destroyed by boiling. Boiled blood, however, gives the test as well as fresh blood, and the reaction is due to the presence of the iron-containing radical of hæmoglobin.

In medico-legal cases it is often necessary to ascertain whether or not a red fluid or stain upon clothing is of blood. In any such case it is advisable not to rely upon one test only, but to try every means of detection at one's disposal. To discover whether it is blood or not is by no means a difficult problem, but to distinguish human blood from that of the common mammals is possible only by the "biological" test described at the end of the next section.

\section{Immunity.}

The chemical defences of the body against injury and disease are numerous. The property that the blood possesses of coagulating is a defence against hæmorrhage; the acid of the gastric juice is a great protection against harmful bacteria introduced with food. Bacterial activity in urine is inhibited by the acidity of that secretion.

Far more important and widespread in its effects than any of the foregoing is the bactericidal (i.e. bacteria-killing) action of the blood and lymph; a study of this question has led to many interesting results, especially in connection with the problem of immunity.

It is a familiar fact that one attack of many infective maladies protects us against another attack of the same disease. The person is said to be immune either partially or completely against that 
disease. Vaccination produces in a patient an attack of cowpox or vaccinia. This disease is either closely related to smallpox, or maybe it is smallpox modified and rendered less malignant by passing through the body of a calf. At any rate, an attack of vaccinia renders a person immune to smallpox, or variola, for a certain number of years. Vaccination is an instance of what is called protective inoculation, which is now practised with more or less success in reference to other diseases, such as plague and typhoid fever. The study of immunity has also rendered possible what may be called curative inoculation, or the injection of antitoxic material as a cure for diphtheria, tetanus, snake-poisoning, etc.

The leucocytes or phagocytes destroy bacteria by feeding on them; but the fluid part of the blood is often antagonistic to bacterial life, and this power was first discovered when the effort was made to grow various kinds of bacteria in it; it was looked upon as probable that blood-serum would prove a suitable soil or medium for this purpose. It was found in some instances to have exactly the opposite effect. The chemical characters of the substances which kill the bacteria are not fully known; indeed, the same is true for most of the substances we have to speak of in this connection. Absence of knowledge on this particular point has not, however, prevented important practical discoveries from being made.

So far as is known at present, the substances in question are protein in nature. The bactericidal powers of blood are destroyed by heating it for an hour to $55^{\circ} \mathrm{C}$. Whether the substances are derived from the leucocytes is a disputed point. The substances, whatever be their source or their chemical nature, are called bacterio-lysins.

Closely allied to the bactericidal power of blood, or blood-serum, is its globulicidal power. By this one means that the blood-serum of one animal has the power of dissolving the red blood-corpuscles of another species. If the serum of one animal is injected into the blood-stream of an animal of another species, the result is a destruction of its red corpuscles, which may be so excessive as to lead to the passing of the liberated hæmoglobin into the urine (hæmoglobinuria). The substance or substances in the serum that possess this property are called hocmolysins, and though there is some doubt whether bacterio-lysins and hæmolysins are absolutely identical, there is no doubt that they are closely related substances.

Normal blood possesses a certain amount of substances which are inimical to the life of our bacterial foes. But suppose a person gets run down; every one knows he is then liable to "catch anything." This coincides with a diminution in the bactericidal power of his blood. But even a perfectly healthy person has not an unlimited supply of bacterio-lysin, and if the bacteria are sufficiently numerous he will fall a victim to the disease they produce. Here, however, 
comes in the remarkable part of the defence. In the struggle he will produce more and more bacterio-lysin, and if he gets well it means that the bacteria are finally vanquished, and his blood remains rich in the particular bacterio-lysin he has produced, and so will render him immune for a time to further attacks from that particular species of bacterium. Each bacterium attacked in this way seems to cause the development of a specific anti-substance.

Immunity can more conveniently be produced gradually in animals, and this applies, not only to the bacteria, but in certain cases to the toxins they form. If, for instance, the bacilli which produce diphtheria are grown in a suitable medium, they produce the diphtheria poison, or toxin, much in the same way that yeast-cells will produce alcohol when grown in a solution of sugar. Diphtheria toxin is associated with a proteose, as is also the case with the poison of snake-venom. If a certain small dose called a "lethal dose" is injected into a guinea-pig the result is death. But if the guinea-pig receives a smaller dose it will recover; a fow days after it will stand a rather larger dose; and this may be continued until, after many successive gradually increasing doses, it will finally stand an amount equal to many lethal doses without any ill effects. The gradual introduction of the toxin has called forth the production of an antitoxin. If this is done in the horse instead of the guinea-pig the production of antitoxin is still more marked, and the serum obtained from the blood of an immunised horse may be used for injecting into human beings suffering from diphtheria, and rapidly cures the disease. The two actions of the blood, antitoxic and antibacterial, are frequently associated, but may be entirely distinct.

The antitoxin is also a protein probably of the nature of a globulin; at any rate it is a protein of larger molecular weight than a proteose. This suggests a practical point. In the case of snake-poisoning the poison gets into the blood rapidly owing to the comparative ease with which it diffuses, and so it is quickly carried all over the body. In treatment with the antitoxin or antivenin, speed is everything if life is to be saved; injection of this material under the skin is not much good, for the diffusion into the blood is too slow. It should be injected straight away into a blood-vessel.

There is no doubt that in these cases the antitoxin neutralises the toxin much in the same way that an acid neutralises an alkali. If the toxin and antitoxin are mixed in a test-tube, and time allowed for the interaction to occur, the result is an innocuous mixture. The toxin, however, is merely neutralised, not destroyed; for if the mixture in the test-tube is heated to $68^{\circ} \mathrm{C}$. the antitoxin is coagulated and destroyed, and the toxin remains as poisonous as ever.

Immunity is distinguished into active and passive. Active immunity is produced by the development of protective substances in 
the body; passive immunity by the injection of a protective serum. Of the two the former is the more permanent.

Ricin, the poisonous protein of castor-oil seeds, and abrin, that of the jequirity bean, also produce, when gradually given to animals, an immunity, due to the production of anti-ricin and anti-abrin respectively.

Ehrlich's hypothesis to explain such facts is usually spoken of as the side-chain theory of immunity. He considers that the toxins are capable of uniting with the protoplasm of living cells by possessing groups of atoms like those by which nutritive proteins are united to cells during normal assimilation. He terms these haptophor groups, and the groups to which these are attached in the cells he terms receptor groups. The introduction of a toxin stimulates an excessive production of receptors, which are finally thrown out into the circulation, and the free circulating receptors constitute the antitoxin. The comparison of the process to assimilation is justified by the fact that non-toxic substances like milk or egg-white introduced gradually by successive doses into the blood-stream cause the formation of antisubstances capable of coagulating them.

Up to this point I have spoken only of the blood, but month by month workers are bringing forward evidence to show that other cells of the body may by similar measures be rendered capable of producing a corresponding protective mechanism.

The substances which on injection provolke the appearance of antidotes of this nature are of protein or protein-like nature; they are spoken of as antigens.

One further development of the theory I must mention. At least two different substances are necessary to render a serum bactericidal or globulicidal. The bacterio-lysin or hæmolysin consists of these two substances. One of these is called the immune body, the other the complement. We may illustrate the use of these terms by an example. The repeated injection of the blood of one animal (e.g., the goat) into the blood of another animal (e.g., a sheep) after a time renders the latter animal immune to further injections, and at the same time causes the production of a serum which dissolves readily the red blood-corpuscles of the first animal. The sheep's serum is thus hæmolytic towards goat's blood-corpuscles. This power is destroyed by heating to $56^{\circ} \mathrm{C}$. for half an hour, but returns when the fresh serum of any animal is added. The specific immunising substance formed in the sheep is called the immune body; the enzyme-like substance destroyed by heat is the complement. The latter is not specific, since it is furnished by the blood of non-immunised animals, but it is nevertheless essential for hæmolysis. Ehrlich believes that the immune body has two side groups-one which connects with the receptor of the red corpuscles, and one which unites with the haptophor group of the complement, and thus renders possible the enzyme- 
like action of the complement on the red corpuscles. Various antibacterial serums which have not been the success in treating disease they were expected to be, are probably too poor in complement, though they may contain plenty of the immune body.

To put it another way: the cell-dissolving substances cannot act on their object of attack without an intermediate substance to anchor them on to the object in question. This intermediary substance, known as the immune body or amboceptor, is specific, and varies with the substance to be attacked (red corpuscles, bacterium, toxin, etc.). The complement may be compared to a person who wants to unlock a door; to do this effectively he must be provided with the proper key (amboceptor or immune body).

Quite distinct from the bactericidal, globulicidal, and antitoxic properties of blood is its agglutinating action. This is another result of infection with many kinds of bacteria or their toxins. The blood acquires the property of rendering immobile and clumping together the specific bacteria used in the infection. The test applied to the blood in cases of typhoid fever, and generally called Widal's reaction, depends on this fact. The substances that produce this effect are called agglutinins. They also are probably protein-like in nature, but are more resistant to heat than the lysins. Prolonged heating to over $60^{\circ} \mathrm{C}$. is necessary to destroy their activity.

We thus see that the means of combating our bacterial enemies are various; in some cases they are rendered immobile by agglutinins, and in other cases, killed by bacterio-lysins. In other instances, their toxins are neutralised by antitoxins, and in others again they are directly devoured by phagocytes. Metschnikoff's view, which is shared by many eminent bacteriologists, is that phagocytosis is the supreme method, and the others are merely auxiliaries, or confined to a small number of cases. If a foreign organism is destroyed by the leucocytes, it produces no ill effects when it enters the body of a man or other animal; but if it is not destroyed, it grows and produces a disease, and it is therefore called pathogenic. If the phagocytes can be induced to feed on a pathogenic organism, it is at once rendered non-pathogenic. The recent discovery of opsonins, by Sir A. E. Wright, emphasises this view and shows one means the body possesses of persuading the leucocytes to eat bacteria, which would otherwise be distasteful to them. Washed bacteria from a culture are usually refused by leucocytes; but if the bacteria had been previously soaked in serum, especially if that serum has been obtained from the blood of an animal previously immunised against that special bacterium, then the leucocytes devour them eagerly. Something has either been added to the bacterium to make it tasty, or something removed from it which previously made it distasteful: whichever is the case, the action is 
described as the action of an opsonin (derived from a Greek word which means "to prepare the feast").

We may take the specific case of the tubercle bacillus as an instance where such work is of value. All of us are breathing in these bacilli every day of our lives, but many of us escape tuberculosis because the opsonic power of our blood is sufficiently high to render the bacilli an easy prey to leucocytes. In those to whom the organism is pathogenic, the modern treatment is directed to enhancing nature's cure by increasing the opsonic power of the patient's blood by good food and pure air, or the injection of preparations of the required opsonin.

Lastly, we come to a question which more directly appeals to the physiologist than the preceding, because experiments in relation to immunity have furnished us with what has hitherto been lacking, a means of distinguishing human blood from the blood of other animals.

The discovery was made by Tchistovitch (1899), and his original experiment was as follows:-Rabbits, dogs, goats, and guinea-pigs were inoculated with eel-serum, which is toxic: he thereby obtained from these animals an antitoxic serum. But the serum was not only antitoxic, but produced a precipitate when added to eel-serum, but not when added to the serum of any other animal. In other words, not only has a specific antitoxin been produced, but also a specific precipitin. Numerous observers have since found that this is a general rule throughout the animal kingdom, including man. If, for instance, a rabbit is treated with human blood, the serum ultimately obtained from the rabbit contains a specific precipitin for human blood; that is to say, a precipitate is formed on adding such a rabbit's serum to human blood, but not on adding it to the blood of any other animal. There may be a slight reaction with the blood of allied animals; for instance, with monkey's blood in the case of man. The great value of the test is its delicacy; it will detect the specific blood when it is greatly diluted, after it has been dried for weeks, or even when it is mixed with the blood of other animals.

'The lipoids contained in cells (mainly in the cell-membrane) play some part in the relationship of such cells to toxins. The matter has been mainly studied in relation to red corpuscles, and the hæmolysins (such as snake-venom, saponin, etc.), which attack them. There is some evidence that the cholesterin in the envelope of the red corpuscles is a protective agent (see also p. 435). A few years ago, Preston Kyes stated that lecithin is the amboceptor which anchors the hæmolysin on to the red cells. But more recent research has failed to substantiate this view, and the compounds which Kyes described and called lecithides are impure mixtures of several substances. It is much more probable that the real agent at work in hæmoylsis is a lipolytic or fat-splitting enzyme; this splits up the lecithin of the cell, liberating oleic acid and deoleolecithin (that is, lecithin minus its oleic acid radical), and it is these cleavage products which dissolve out the hæmoglobin and so destroy the corpuscles. 
Anaphylaxis.-The word anaphylaxis dates from 1905 and was coined by Richet; he was studying the action of poisons obtained from the sea anemone, and he found that if a small dose which caused no symptoms in a dog was followed a week or two later by the same small dose, the animal became ill and usually died. This increased susceptibility lasted a considerable time, and he called it anaphylaxis (ana against, phylaxis protection). Since this time it has been found that most antigens produce a similar condition, but the phenomenon was really known before Richet gave it its name. For instance, in vaccination the incubation period is four days; in a second vaccination this period is shortened, and the increased power of the body to respond readily is in this case not harmful but beneficial. Similarly, the tuberculous patient is hypersensitive to tuberculin. Anaphylaxis has been largely studied in the guinea-pig, and it is remarkable how small is the dose of a foreign protein which produces the exaggerated sensitiveness. A millionth part of a cubic centimetre of bone serum is often enough. Very small amounts also will produce a serious condition or even a fatal result when the second dose is given. In face of such small figures it seems hopeless to isolate the toxic principle. The hypersensitive state may be transmitted by the female guinea-pig to her offspring. Death, when it occurs as the result of a second dose, is usually a matter of minutes only; the blood pressure falls enormously, the abdominal viscera are gorged with blood, and hæmorrhages are frequent; the bronchial muscles are also acutely constricted. Numerous theories have been advanced to explain these remarkable facts, and numerous names, such as anaphylactin, sensibiline, etc., have been invented for the supposed toxic material. There is some evidence that the precipitin content of the serum runs parallel with the severity of the symptoms, and that this is the factor to which the difference between the normal and the sensitive animal is due. But how the interaction of precipitin and antigen produces the symptoms is entirely a matter of speculation. 


\section{CHAP'TER XXX}

FOOD

THE chief chemical compounds or proximate principles in food aro:-

1. Proteins

2. Carbohydrates

3. Fats

4. Water

5. Salts

In milk and in eggs, which form the exclusive foods of young animals, all varieties of these proximate principles are present in suitable proportions. Hence they are spoken of as perfect foods. Eggs, though a perfect food for the developing bird, contain too little carbohydrate for a mammal. In most vegetable foods carbohydrates are in excess; while in animal foods, such"as meat, the proteins are predominant. In a suitable diet these should be mixed in proper proportions, which must vary for herbivorous and carnivorous animals.

A healthy and suitable diet must possess the following characters :-

1. It must contain the proper amount and proportion of the various proximate principles.

2. It must be adapted to the climate; to the age and weight of the individual, and to the amount of work done by him.

3. The food must contain not only the necessary amount of proximate principles, but these must be present in a digestible form. As an instance of this, many vegetables (peas, beans, lentils) contain even more protein than beef or mutton, but are not so nutritious, as they are less digestible, much passing off in the fæces unused.

The nutritive value of a diet depends chiefly on the amount of carbon and nitrogen it contains. A man doing a moderate amount of work and taking the usual diet will eliminate, chiefly from the lungs, in the form of carbonic acid, from 250 to 280 grammes of carbon per diem. During the same time he will eliminate, chiefly in the form of urea in the urine, about 15 to 18 grammes of nitrogen. These substances are derived partly from the food and partly from the metabolism of the tissues, various forms of energy-mechanical motion 
and heat being the chief-being simultaneously liberated. During muscular exercise the output of carbon greatly increases; the increased excretion of nitrogen is not noticeable. Taking, then, the state of moderate exercise, it is necessary that the waste should be replaced by fresh material in the form of food; and the proportion of carbon to nitrogen should be the same as in the excretions: 250 to 15 , or 16.6 to 1 . The proportion of carbon to nitrogen in protein is, however, 53 to 15 , or 3.5 to 1 . The extra supply of carbon must come from non-nitrogenous food-viz., fat and carbohydrate.

Voit gives the following daily diet:-

\section{Protein.}

Fat

Carbohydrate

Ranke's diet closely resembles Voit's; it is -

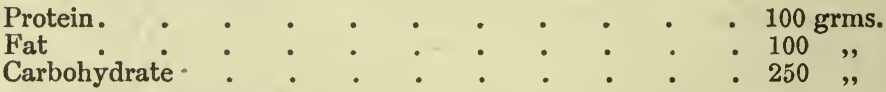

Such typical diets as these must not be considered as more than rough averages of what is necessary for a man in the course of the day. Actual experience shows that in the diets of different nations there are considerable variations from this standard without the production of ill effects. Age, and the amount of work done, also influence the amount of food necessary; growing children, for instance, require a relatively rich diet; thus, milk, the diet of the infant, is proportionally twice as rich in proteins, and half as rich again in fats, as the normal diet given above. During work more food is necessary than during inactivity.

Attention has recently been devoted to the question whether as much protein as 100 to 120 grammes daily is really necessary, and by far the most convincing of the experiments published in favour of a reduction are those carried out by Chittenden cn himself, his colleagues, his students, and on soldiers and athletes, over comparatively long periods of time. The protein intake was reduced to half and sometimes to less than half the quantity hitherto regarded as necessary. The deprivation was followed by no untoward results; bodily equilibrium was maintained; the health remained perfect or improved; the muscular force in athletes was increased; mental acuity was undiminished, and desire for richer food soon disappeared.

It may be freely admitted that the majority of well-to-do people eat too much protein; there are not many who limit themselves even to Voit's minimum, and in those who are prone to digestive and uric acid diseases, one cannot but feel that improvement in body and mind would be the result of more temperate habits.

But if we were all to permanently reduce our diet to the Chittenden level, we might be living perilously near the margin; 
any unusual strain, such as privation or a severe illness, would then find us without any reserve of nutrient energy, and we should probably suffer more severely in consequence. The poor around us have had nolens volens to subsist on a Chittenden diet for years, whereas Chittenden's experiments only lasted months, and nearly all of his subjects have returned now to their previous diet. The underfed condition of the poor is apparent, and is not such as to make others inclined to follow their example. In countries like India, where the vegetarian native population is diluted with the meat-eating white races, it is the former who more readily succumb to the effects of disease. The recent development of the Japanese is by some attributed in part to the fact that they are accustoming themselves to a richer nitrogenous diet than they took in the past.

It is doubtful if the minimum is also the optimum. We take in protein, and rapidly eliminate most of its nitrogen as urea, without building it up first into the body tissues; but some is wanted by the body tissues to repair their waste, and some of the cleavage products of the food-protein are especially necessary for the synthesis of tissue protein; it is in order to obtain a sufficient quantity of these scanty cleavage products that we ingest what at first sight is an excess of the proteins which yield them. But after our study of digestion and excretion, we shall be in a better position to discuss this question more fully, and we shall return to it in the chapter on Metabolism.

Milk.

Milk, which we have already spoken of as a perfect food, is only so for young children. For those who are older, it is so voluminous

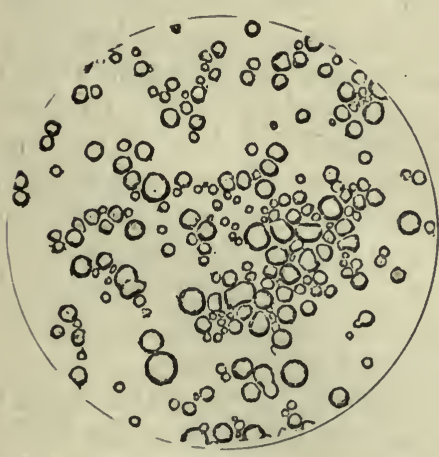

FIa. 338.-Globules of cow's milk. $\times 400$. that unpleasantly large quantities of it would have to be taken in the course of the day to ensure the proper supply of nitrogen and carbon. Moreover, it is relatively too rich in protein and fat. It also contains too littlo iron (Bunge): hence children weaned late become anæmic.

The microscope reveals that it consists of two parts : a clear fluid and a number of minute particles that float in it. These consist of minute oil globules, varying in diameter from 0.0015 to 0.005 millimetre (fig. 338).

The milk secreted during the first few days of lactation is called colostrum. It contains very little casoinogon, but large quantities of albumin and globulin instead. It 
coagulates. like white of egg when boiled. Microscopically, cells from the acini of the mammary gland are seen, which contain fat globules in their interior; they are called colostrum corpuscles.

Reaction and Specific Gravity.- The reaction of fresh cow's milk and of human milk is amphoteric; that is, it turns blue litmus red, and red litmus blue. This is due to the presence of both acid and alkaline salts. All milk readily turns acid or sour as the result of fermentative change, part of its lactose being transformed into lactic acid. The specific gravity of milk is usually ascertained with the hydrometer. That of normal cow's milk varies from 1028 to 1034 . When the milk is skimmed the specific gravity rises, owing to the removal of the light constituent, the fat, to 1033 to 1037 . In all cases the specific gravity of water, with which other substances are compared, is taken as 1000 .

Composition.- Bunge gives the following table, contrasting the milk of woman, and the cow :-

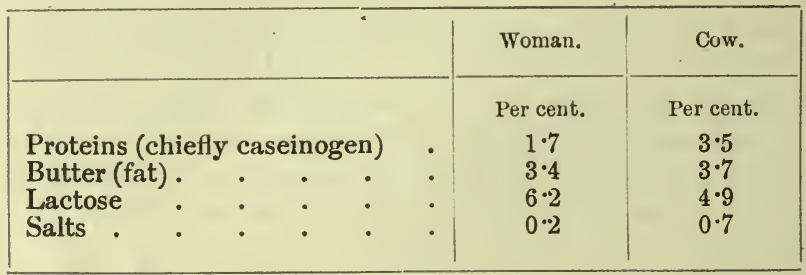

Hence, in feeding infants on cow's milk, it is necessary to dilute it, and add sugar and a little cream to make it approximately equal to natural human milk.

The Proteins of Milk.-The principal protein in milk is called caseinogen; it is precipitable by acids such as acetic acid, and also by saturation with magnesium sulphate, or half saturation with ammonium sulphate, so resembling globulins; it is coagulated by rennet to form casein. Cheese consists of casein with the entangled fat. The other protein in milk is an albumin. It is present in small quantities only; it differs in some of its properties (specific rotation, coagulation temperature, etc.) from serum-albumin; it is called lact-albumin.

The Coagulation of Milk. - Rennet is the agent usually employed for this purpose: it is a enzyme secreted by the stomach, especially in sucking animals, and is generally obtained from the calf.

The curd consists of the casein and entangled fat: the liquid residue called whey contains the sugar, salts, and albumin of the milk. There is also a small quantity of a new protein called whey-protein, which differs from caseinogen by not being convertible into casein; this is produced by the decomposition of the caseinogen molecule during the process of curdling. 
The addition of rennet produces coagulation in milk, provided that a sufficient amount of calcium salts is present. If the calcium salts are precipitated by the addition of potassium oxalate, rennet causes no formation of casein. The process of curdling in milk is a double one; the first action due to rennet is to produce a change in caseinogen; the second action is that of the calcium salt, which precipitates the altered caseinogen as casein. In blood, also, calcium salts are necessary for coagulation, but there they act in a different way, namely, in the production of fibrin-ferment (see p. 449).

Caseinogen is a phospho-protein (see p. 429). In milk it is combined with calcium to form calcium caseinogenate; when acetic acid is added, we therefore get calcium acetate and free caseinogen.

The Fats of Milk. - The chemical composition of the fat of milk (butter) is very like that of adipose tissue. There are, however, smaller quantities of fats derived from fatty acids lower in the series, especially butyrin and caproin. The relation between these varies somewhat, but the proportion is roughly as follows:-Olein, $\frac{3}{7}$; palmitin, $\frac{1}{3}$; stearin, $\frac{1}{6}$; butyrin, caproin, and caprylin, $\frac{1}{14}$. The old statement that each fat globule is surrounded by a film of protein is, according to Ramsden's recent observations, correct. Milk also contains small quantities of lipoids (lecithin, cholesterin, and a yellow fatty pigment or lipochrome).

Milk Sugar, or Lactose.-This is a disaccharide $\left(\mathrm{C}_{12} \mathrm{H}_{22} \mathrm{O}_{11}\right)$. Its properties have already been described in Chap. XXVIII., p. 411.

Souring of Milk.-When milk is allowed to stand, the chief change which it is apt to undergo is a conversion of a part of its lactose into lactic acid. This is due to the action of an enzyme secreted by micro-organisms, and would not occur if the milk were contained in closed sterilised vessels. Equations showing the change produced are given on p. 411. When souring oscurs, the acid formed precipitates a portion of the caseinogen. This must not be confounded with the formation of casein from caseinogen, which is produced by rennet. There are, however, some bacteria which, like rennet, produce true coagulation.

Alcoholic Fermentation of Milk.-When yeast is added to milk, the sugar does not readily undergo the alcoholic fermentation. Other somewhat similar fungoid growths are, however, able to produce the change, as in the preparation of koumiss; the milk sugar is first inverted, that is, dextrose and galactose are formed from it (see p. 411), and it is these sugars from which alcohol and carbonic acid originate.

The Salts of Milk.-The principal salt present is calcium phosphate; a small quantity of magnesium phosphate is also present. The other salts are chiefly chlorides of sodium and potassium.

It is an undoubted fact that the milk provided by Nature for the growing offspring is different in the various classes of the animal 
kingdom. The quantitative variations are often enormous, and it has been shown that the milk best adapted for the nutrition of the young animal is that which comes from its mother, or, at least, from an animal of the same species. The practical application of this rule comes home most to us when dealing with the feeding of children, and it is universally acknowledged that, after all, cows' milk is but a poor substitute for human milk. Cows' milk is, of course, diluted, and sugar and cream added, so as to make it quantitatively like mothers' milk, but even then the question arises whether the essential difference between the two kinds of milk is not deeper than one of mere quantity; and, in particular, the pendulum of scientific opinion has swung backwards and forwards in relation to the question whether the principal protein, called caseinogen, in both is really identical in the two cases. The caseinogen of human milk curdles in small flocculi in the stomach, so contrasting with the heavy curd which cows' milk forms; and even although the curdling of cows' milk be made to occur in smaller fragments by mixing the milk with barley water or lime water, its digestion proceeds with comparative slowness in the child's alimentary canal. These are practical points well known to every clinical observer, and in the past they have been attributed, not so much to fundamental differences in the caseinogen itself, as to accidental concomitant factors; the excess of citric acid in human milk, for instance, and its paucity in calcium salts, have been held responsible for the differences observed in the physical condition of the curd and in its digestibility.

This question is far from settled even to-day, but there are some data now available that point to a qualitative difference between caseinogens. Some of these depend on the application of the "biological test" carried out on the line of immunity experiments, which has been so signally successful in the distinction between the blood-proteins of different species of animals (see p. 477). The differences, however, which lead to the formation of specific precipitins are so slight, that ordinary chemical methods of analysis are, at present, unable to reveal them. But, in the case of milk, there are differences which the chemist can detect. One cannot lay much stress on mere percentage composition, although differences have been noted in that, because we have no guarantee that the proteins investigated were separated from all impurities; there are also small differences in the percentage of mono-amino-acids obtained after hydrolysis; but the present methods of estimating these with accuracy leave much to be desired. A deeper chemical distinction noted is contained in the recent work of Bienenfeld, who finds that human caseinogen contains a carbohydrate complex which is absent from that of the cow. 
A few years ago it was stated that human caseinogen will not curdle with rennet; but this has been shown to be a mistake. The conditions of rennet curdling are somewhat different in the two kinds of milk we are considering, but provided the reaction in the stomach is acid, human milk is curdled by rennet when acted on by gastric juice.

\section{The Mammary Glands.}

The mammary glands are composed of large divisions or lobes, and these are again divisible into lobules; the lobules are composed of the convoluted and dilated subdivisions of the main ducts held together by connective tissue. Covering the general surface of the gland, with the exception of the nipple, is a considerable quantity of fat, itself lobulated by sheaths and processes of areolar tissue (fig. 339)

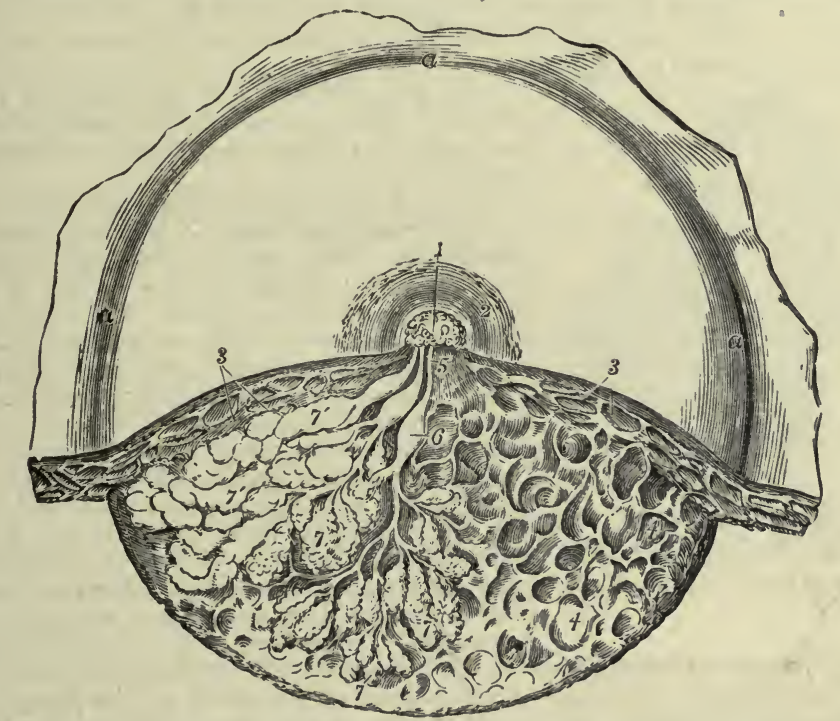

Fia. 339.-Dissection of the lower half of the female mamma, during the period of lactation. 2.-In the left-hand side of the dissected part the glandular lobes are exposed and partially unravelled; and on the right-hand side, the glandular substance has been removed to show the reticular loculi of the connective tissue in which the glandular lobules are placed: 1, upper part of the mamilla or nipple; 2 , areola ; 3 , subcutaneous masses of fat; 4 , reticular loculi of the connective tissue which support the glandular substance and contain the fatty masses; 5 , one of three lactiferous ducts shown passing towards the nipple where they open; 6 , one of the sinus lactei or reservoirs; 7 , some of the glandular lobules which have been unravelled; $7^{\prime}$, others massed together. (Luschka.)

connected both with the skin in front and the gland behind; the same bond of connection extends also from the under surface of the gland to the sheathing connective tissue of the great pectoral muscle on which it lies. The main ducts of the gland, fifteen to twenty in number, called the lactiferous ducts, are formed by the union of the smaller (lobular) ducts, and open by small separate orifices through the nipple. At the points of junction of lobular ducts to form lactiferous ducts, and just before these enter the base of the nipple, the ducts are dilated; and during the period of active secretion by the gland, the dilatations form reservoirs for the milk, which collects in and distends them. The walls of the gland-ducts are formed of areolar with some unstriped muscular tissue, and are lined internally by short columnar and near the nipple by flattened epithelium. 
The nipple is composed of areolar tissue, and contains unstriped muscular fibres. Blood-ressels are also freely supplied to it, so as to give it an erectile structure. On its surface are very sensitive papillæ; and around it is a small area or areola of pink or dark-tinted skin, on which are to be seen small projections formed by minute secreting glands.

Blood-vessels, nerves, and lymphatics are plentifully supplied to the mammary glands; the calibre of the blood-vessels, as well as the size of the glands, varies very greatly under certain conditions, especially those of pregnancy and lactation.

The alveoli of the glands during the secreting periods are found to be lined with short columnar cells (see fig. 340). The edges of the cells towards the lumen may be irregular and jagged, and the remainder of the alveolus is filled up with the materials of the milk. During the intervals between the acts of discharge, the cells of the alveoli elongate towards the lumen, their nuclei divide, and in the part of the cells towards the lumen a collection of oil globules and of other materials takes place.

The next stage is that the cells divide and the part of each towards the lumen containing a nucleus and the materials of the secretion, disintegrates and goes to form the constituents of the milk.

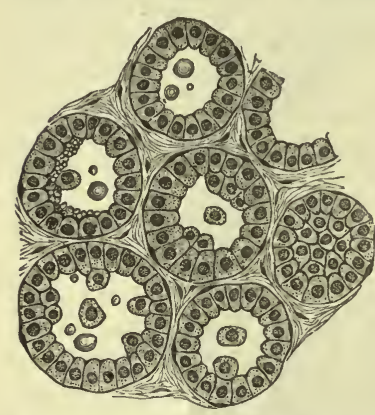

Fig. 340.-Section of mammary gland of bitch, showing acini, lined with epithelial cells of a short columnar form. $\times 200$. (V. D. Harris.)

In the earlier days of lactation, epithelial cells only partially transformed are discharged in the secretion; these are termed colostrum corpuscles.

During pregnancy the mammary glands undergo changes (evolution) which are readily observable. They enlarge, become harder, and more distinctly lobulated; the veins on the surface become more prominent. The areola becomes enlarged and dusky, with projecting papillæ; the nipple, too, becomes more prominent, and milk can be squeezed from the orifices of the ducts. This is a very gradual process, which commences about the time of conception, and progresses steadily during the whole period of gestation. In the gland itself solid columns of cells bud off from the old alveoli to form new alveoli. But these solid columns after a while are converted into tubes by the central cells becoming fatty and being discharged as the colostrum corpuscles above mentioned. After the end of lactation, the mamma gradually returns to its original size (involution). The acini, in the early stages of involution, are lined with cells in all degrees of vacuolation. As involution proceeds, the acini diminish considerably in size, and at length, instead of a mosaic of lining epithelial cells (twenty to thirty in each acinus), we have five or six nuclei (some with no surrounding protoplasm) lying in an irregular heap within the acinus. No secretory nerves of the mammary gland have yet been discovered. It is possible they do not exist, but the normal stimulus to mammary activity is a chemical one formed by the ovary.

\section{Eggs.}

In this country the eggs of hens and ducks are those particularly selected as foods. The chief constituent of the shell is calcium carbonate. The white is composed of a richly albuminous fluid enclosed in a network of firmer and more fibrous material. The amount of solids is 13.3 per cent.; of this, $12 \cdot 2$ is protein in nature (egg-albumin, with smaller quantities of egg-globulin, and of a mucinoid substance called ovo-mucoid), and the remainder is made up of sugar $(0.5$ per 
cent.), traces of fats, lecithin, and cholesterin, and 0.6 per cent. of inorganic salts. The yolk is rich in food materials for the development of the future embryo. In it there are two varieties of yolkspherules, one kind yellow and opaque (due to admixture with fat and a yellow lipochrome), and the other smaller, transparent, and almost colourless; these are protein in nature, consisting of the phospho-protein called vitellin. Small quantities of sugar, lecithin, cholesterin, and inorganic salts are also present.

The nutritive value of eggs is high, as they are so readily digestible; but the more an egg is cooked the more insoluble do its protein constituents become.

\section{Meat.}

This is composed of the muscular and connective (including adipose) tissues of cortain animals. The flesh of some animals is not eaten; in some cases this is a matter of fashion, in others, it is due to an unpleasant taste, such as the flesh of carnivora is said to have; and in other cases (e.g. the horse) because it is more lucrative to use the animal as a beast of burden.

Meat is the most concentrated and most easily assimilable of nitrogenous foods. It is our chief source of nitrogen. Its chief solid constituent is protein, and the principal protein is myosin. In addition to the extractives and salts contained in muscle, there is always a certain percentage of fat, even though all visible adipose tissue is dissected off. The fat-cells are placed between the muscular fibres, and the amount of fat so situated varies in different animals; it is particularly abundant in pork; hence the indigestibility of this form of flesh: the fat prevents the gastric juice from obtaining ready access to the muscular fibres.

The following table gives the chief substances in some of the principal meats used as food:-

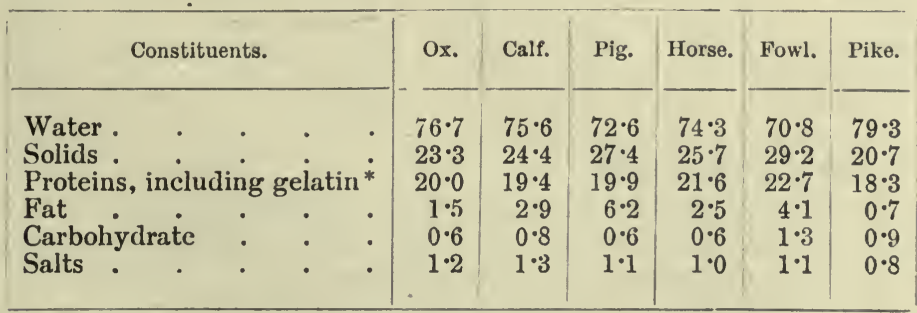

* The flesh of young animals is richer in gelatin than that of old; thus 1060 parts of beef yield 6 , of veal 50 , parts of gelatin.

The large percentage of water in meat should be particularly noted; if a man wished to take his daily supply of 100 grammes of 
protein entirely in the form of meat, it would be necessary for him to consume about 500 grammes (i.e. a little more than $1 \mathrm{lb}$.) of meat.

\section{Flour.}

The best wheat flour is made from the interior of wheat grains, and contains the greater proportion of the starch of the grain and most of the protein. Whole flour is made from the whole grain minus the husk, and thus contains not only the white interior but also the harder and browner outer portion of the grain and the germ or embryo plant. This region contains a somewhat larger proportion of protein. Whole flour contains 1 to 2 per cent. more protein than the best white flour, but it has the disadvantage of being less readily digested. Brown flour contains a certain amount of bran in addition; it is still less digestible, but is useful as a mild laxative, the insoluble cellulose mechanically irritating the intestinal canal as it passes along.

The best flour contains very little sugar. The presence of sugar indicates that germination has commenced in the grains. In the manufacture of malt from barley this is purposely allowed to go on.

When mixed with water, wheat flour forms a sticky, adhesive mass called dough. This is due to the formation of gluten. Gluten is a mixture of two proteins-namely, gliadin, which is soluble in alcohol, and glutenin, which is soluble in alkali (see p. 433). The adhesive character of gluten is due to gliadin; grains which are poor in gliadin (e.g. rice) cannot be used for bread-making.

The following table contrasts the composition of some of the more important vegetable foods :-

\begin{tabular}{|c|c|c|c|c|c|c|c|}
\hline Constituents. & Wheat. & Barley. & Oats. & Rice. & Lentils. & Peas. & Potatoes. \\
\hline Water. & $13 \cdot 6$ & $13 \cdot 8$ & $12 \cdot 4$ & $13 \cdot 1$ & 12.5 & $14 \cdot 8$ & 76.0 \\
\hline Protein & $12 \cdot 4$ & $11 \cdot 1$ & $10 \cdot 4$ & $7 \cdot 9$ & $24 \cdot 8$ & 23.7 & $2 \cdot 0$ \\
\hline Fat. & $1 \cdot 4$ & $2 \cdot 2$ & $5 \cdot 2$ & 0.9 & $1 \cdot 9$ & $1 \cdot 6$ & 0.2 \\
\hline Starch & $67 \cdot 9$ & $64 \cdot 9$ & $57 \cdot 8$ & $76 \cdot 5$ & $54 \cdot 8$ & $49 \cdot 3$ & $20 \cdot 6$ \\
\hline Cellulose : & $2 \cdot 5$ & $5 \cdot 3$ & $11 \cdot 2$ & $0 \cdot 6$ & $3 \cdot 6$ & $7 \cdot 5$ & 0.7 \\
\hline Mineral salts & $1 \cdot 8$ & $2 \cdot 7$ & $3 \cdot 0$ & $1 \cdot 0$ & $2 \cdot 4$ & $3 \cdot 1$ & $1 \cdot 0$ \\
\hline
\end{tabular}

We see from this table-

1. The great quantity of starch always present.

2. The small quantity of fat; that bread is generally eaten with butter is a popular recognition of this fact.

3. Protein, except in potatoes, is pretty abundant, and especially so in the pulses (lentils, peas, etc.). The protein in the pulses is not gluten, but consists mainly of globulins.

In the mineral matters in vegetables, salts of potassium and magnesium are, as a rule, more abundant than those of sodium and calcium. 


\section{Bread.}

Bread is made by cooking the dough of wheat flour mixed with yeast, salt, and flavouring materials. An enzyme in the flour acts at the commencement of the process, when the temperature is kept a little over that of the body, and forms dextrin and sugar from the starch, and then the alcoholic fermentation, due to the action of the yeast, begins. The bubbles of carbonic acid, burrowing passages through the bread, make it light and spongy. This enables the digestive juices subsequently to soak into it readily and affect all parts of it. In the later stages, viz., baking, the temperature is raised, the gas and alcohol are expelled from the bread, the yeast is killed, and a crust forms from the drying of the outer portions of the dough.

White bread contains, in 100 parts, 8 to 10 of protein, 55 of carbohydrates, 1 of fat, 2 of salts, and the rest water.

\section{Cooking of Food.}

The cooking of foods is a development of civilisation, and serves many useful ends:-

1. It destroys all parasites and danger of infection. This relates not only to bacterial growths, but also to larger parasites, such as tapeworms and trichinæ.

2. In the case of vegetable foods it breaks up the starch grains, bursting the cellulose and allowing the digestive juices to eome into contact with the granulose.

3. In the case of animal foods it converts the insoluble collagen of the universally distributed connective tissues into the soluble gelatin. The loosening of the fibres is assisted by the formation of steam between them. By thus loosening the binding material, the more important elements of the food, such as muscular fibres, are rendered accessible to the gastric and other juices. Meat before it is cooked is generally kept a certain length of time to allow rigor mortis to pass off.

Of the two chief methods of cooking, roasting and boiling, the former is the more economical, as by its means the meat is first surrounded with a coat of coagulated protein on its exterior, which keeps in the juices to a great extent, letting little else escape but the dripping (fat). Whereas in boiling, unless both bouillon and bouilli are used, there is considerable waste. Cooking, especially boiling, renders the proteins more insoluble than they are in the raw state; but this is counterbalanced by the other advantages that cooking possesses.

In making beef tea and similar extracts of meat it is necessary that the meat should be placed in cold water, and this is gradually and carefully warmed. In boiling a joint it is usual to put the meat into boiling water at once, so that the outer part is coagulated, and the loss of material minimised. 
An extremely important point in this connection is that beef toa and similar meat extracts should not be regarded as foods. They are valuable as pleasant stimulating drinks for invalids, but they contain very little of the nutritive material of the meat, their chief constituents, next to water, being the salts and extractives of flesh.

Soup contains the extractives of meat, a very small proportion of the myosin, and the principal part of the gelatin. The gelatin is usually increased by adding bones and fibrous tissue to the stock. It is the presence of this substance which causes soup when cold to gelatinise.

\section{Accessories to Food.}

Among these must be placed alcohol, the value of which within moderate limits is not as a food but as a stimulant; condiments (mustard, pepper, ginger, curry powder, etc.), which are stomachic stimulants, the abuse of which is followed by dyspeptic troubles; and tea, coffee, cocoa, and similar drinks. These are stimulants chiefly to the nervous system; tea, coffee, maté (Paraguay), guarana (Brazil), cola nut (Central Africa), bush tea (South Africa), and a few other plants used in various countries all owe their chief property to an alkaloid called theine or caffeine $\left(\mathrm{C}_{8} \mathrm{H}_{10} \mathrm{~N}_{4} \mathrm{O}_{2}\right)$; cocoa to the closely related alkaloid, theobromine $\left(\mathrm{C}_{7} \mathrm{H}_{8} \mathrm{~N}_{4} \mathrm{O}_{2}\right)$; coca to cocaine. These alkaloids are all poisonous, and used in excess, even in the form of infusions of tea and coffee, produce over-excitement, loss of digestive power, and other disorders well known to physicians. Coffee differs from tea in being rich in aromatic matters; tea contains a bitter principle, tannin; to avoid the injurious solution of too much tannin, tea should only be allowed to infuse (draw) for a few minutes. Cocoa is not only a stimulant, but a food in addition; it contains about 50 per cent. of fat, and 12 per cent. of protein. In manufactured cocoa, the amount of fat is reduced to 30 per cent., and the amount of protein rises proportionately to about 20 per cent. The quantity of cocoa usually consumed is too small for these food materials to count very much in the daily supply. The amount of protein in solution (mainly proteose) in a breakfast cup of cocoa is under half a gramme; most of the food stuffs are in suspension, for cocoa is drunk "thick," not as a clear infusion.

Green vegetables are taken as a palatable adjunct to other foods, rather than for their nutritive properties. Their potassium salts are, however, abundant. Cabbage, turnips, and asparagus contain 80 to 92 water, 1 to 2 protein, 2 to 4 carbohydrates, and 1 to 1.5 cellulose per cent. The small amount of nutriment in most green foods accounts for the large meals made by, and the vast capacity of the alimentary canal of, herbivorous animals. 


\section{Unknown but Essential Constituents of Food.}

If an animal is fed upon a mixture of pure protein, fat, and carbohydrate, with a due admixture of salts and water, it does not thrive, but shows evidence of malnutrition, although the quantities given may be theoretically correct. If a growing animal is fed on such a diet it ceases to grow. But if, as Hopkins showed recently, a small amount of a natural food, such as milk, is mixed with the artificial diet just referred to, the animals thrive and grow normally. There is something extra, something which is at present unknown, which is absolutely essential, and quite small amounts of it are usually sufficient.

If this unknown constituent is absent from a man's diet, he undergoes just the same sort of malady, and illnesses so produced, such as scurvy and Beri-beri, are termed "Deficiency diseases."

A good deal of work has recently been done in relation to one of these diseases, namely, Beri-beri (the Kak-ka of Japan). This is prevalent among the natives whose staple article of diet is polished rice, that is, rice grains deprived of their external layer. This disease is characterised by malnutrition of the nerves, and neuritis or inflammation of the nerves is followed by nerve-degeneration and paralysis. It can also be produced in birds by feeding them on polished rice; and in both man and bird can be rapidly cured by adding the polishings of the rice grains. The outer layer of the grain contains the extra something, and Funk and others have recently separated out from the polishings a base which they have termed vitamine, although at present there is no certainty of its chemical composition. At any rate this base is the curative principle, and very small doses will cure the condition.

Vitamine is not confined to rice grains, but is found in many other vegetable and animal foods. The value of whole meal bread, for example, does not depend on the surall extra amount of protein it contains, but probably here also upon vitamine. The amount of vitamine varies considerably. Thus, in pigeons fed upon polished rice, as much as 20 grammes of meat daily must be added to prevent the occurrence of Beri-beri; whereas 3 grammes of egg-yolk are sufficient, and half a gramme of yeast is enough. 


\section{CHAPTER XXXI}

\section{THE ALIMENTARY CANAL; SECRETING GLANDS}

THE alimentary canal consists of a long muscular tube lined by mucous membrane beginning at the mouth, and terminating at the anus. It comprises the mouth, pharynx, œsophagus or gullet, stomach, small intestine and large intestine. Opening into it are numerous glands which pour juices into it; these bring about the digestion of the food as it passes along. Some of the glands, such as the gastric and intestinal glands, are situated in the mucous membrane which lines the canal; others, such as the salivary glands, liver, and pancreas, are situated at a distance from the main canal, and pour their secretion into it by means of side tubes or ducts.

The two important coats in the wall of the canal are:-

(1) The muscular coat. - This consists of two layers; in the outer, the fibres are arranged longitudinally, and in the inner, circularly. In the stomach there is a third coat, in which the fibres have an oblique direction. At the cardiac orifice of the stomach (that is, where the œsophagus enters) and at its pyloric orifice (that is, where the small intestine leaves) the circular fibres are increased in amount to form a sphincter. The muscular fibres are of the plain variety, except in the pharynx and upper part of the œsophagus where they are striated. A nerve plexus (plexus of Aucrbach, fig. 100, p. 79) is situated between the two muscular coats.

(2) The mucous membrane.-This consists of an epithelium on its surface; this is stratified in mouth, pharynx, and œesophagus, but columnar in other parts. Beneath the epithelium is a corium of connective tissue, in which there is a considerable quantity of lymphoid tissue; in the intestine the lymphoid nodules are often spoken of as solitary follicles, except in the lower part of the small intestine (the ileum), where they are congregated together as Peyer's patches. At the back of the mouth, the tonsils are masses of lymphoid nodules covered with mucous membrane. In the deepest part of the mucous membrane is a thin layer of involuntary muscle called the muscularis mucosce. 
These two main coats (muscular and mucous) are connected together by a loose layer of connective tissue known as the submucous coat. In this the larger blood-vessels are situated which give off branches to the other two coats but more abundantly to the mucous membrane. The submucous coat also contains a nerve plexus called the plexus of Meissner. In the stomach and intestines there is a fourth coat, on the exterior, derived from the peritoneum (serous coat).

The secreting glands in the wall of the alimentary canal are:-

(1) A number of simple little mucous glands in the corium of the mucous membrane of the mouth, pharynx, and œsophagus; their ducts open on the surface (see fig. 341).

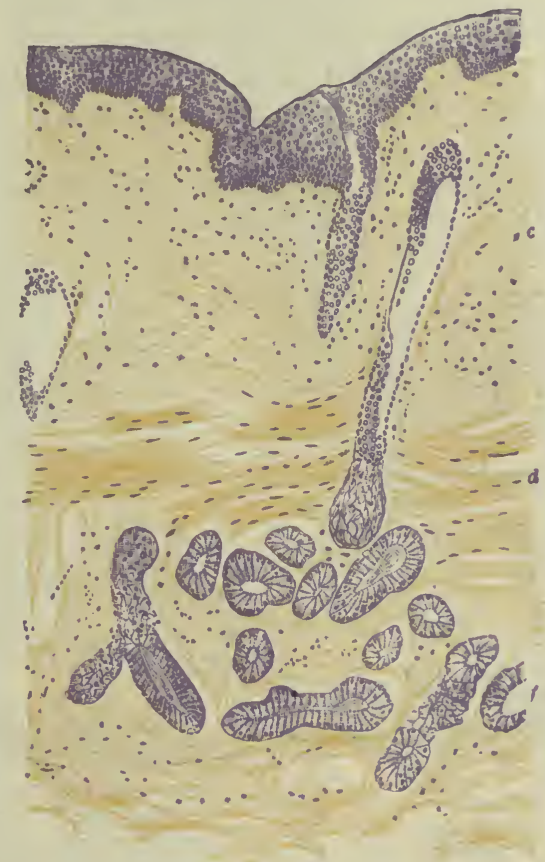

FIG. 341.--Section of the mucous membrane and submucous coat of the osophagus, showing mucous glands.

(2) The gastric glands; these are tubular glands which differ in structure in different regions of the stomach, and which we shall consider at greater length in our description of gastric digestion.

(3) The glands of the small intestine. Throughout the whole of the small intestine there are a large number of simple tubular glands (lined with columnar cells) which open between the villi. They are called the crypts of Lieberkühn. In the first part of the 
small intestine, known as the duodenum, an additional set of glands, called the glands of Brunner, are found. They are imbedded in the submucous coat, and the duct of each gland passes upwards to open on the surface of the mucous membrane. Each gland is a branched and convoluted tube lined with columnar epithelium. Fig. 342 shows these two kinds of glands, and also the villi of the surface.

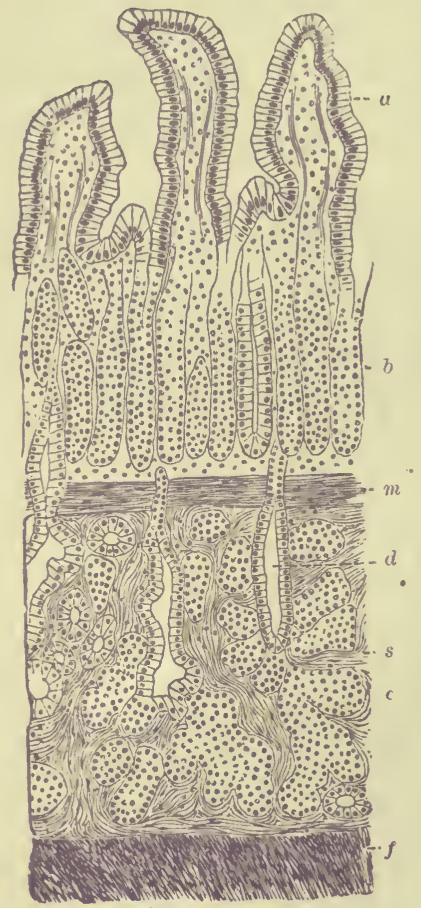

FIG. 342.-Vertical section of duodenum, showing $a$, villi; $b$, crypts of Lieberkühn, and $c$, Brunner's glands in the submucosa $s$, with ducts, $d$; muscularis mucosæ, $m$; and circular muscular coat, $f$. (Schofield.) Figs. 343 and 344 are more bighly magnified views of the villi, which increase the surface of the small intestine mainly for the purpose of absorption. A villus is a small projection made of loose lymphoid tissue, covered with columnar cells; it contains in its interior a plexus of blood-capillaries under the basement membrane, and one or more commencing lymphatic vessels or lacteals situated centrally.

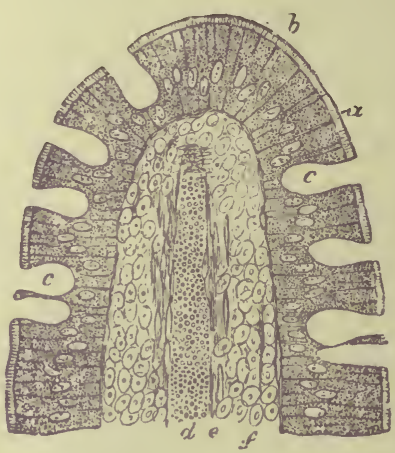

FIG. 343.-Vertical section of a villus of the small intestine of a cat. $a$, Striated border of the epithelium; $b$, columnar epithelium; $c$, goblet cells; $d$, central lymph-vessel; $e$, smooth muscular tibres; $f$, adenoid stroma of tho villus in which lymph corpuscles lie. (Klein.)

(4) Glands of the large intestine. Here there are no villi, but the crypts of Lieberkühn are present and are larger than in the small intestine. Many of the cells lining these tubes are seen breaking down to form goblet cells, and the mucus so furnished is the main substance of importance secreted in this part. of the alimentary canal.

All of the foregoing glands are situated in the wall of the alimentary canal. Those situated at a distance from it, and which 
pour their secretion into it by ducts, are the salivary glands, liver, and pancreas, and will be described in the chapters dealing with those organs.

Before passing on to a study of the digestive secretions on foods, we may consider some general questions relating to secreting organs.

It is the function of gland-cells to produce by the metabolism of their protoplasm certain substances called secretions. These materials are of two kinds, viz., those which are employed for the purpose of

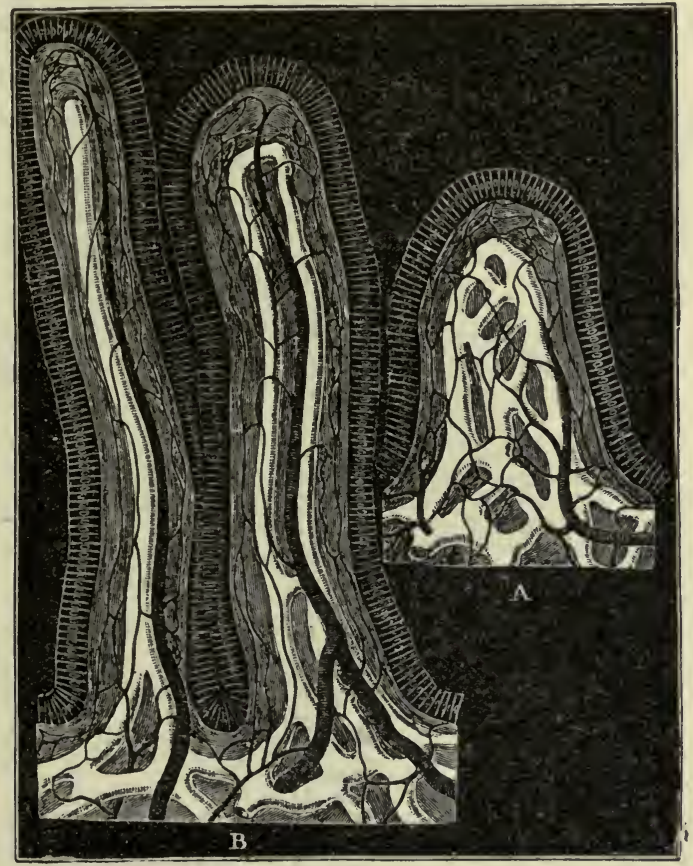

Fia. 344.-A. Villus of sheep. B. Villi of man. (Slightly altered from Teichmann.)

serving some useful office in the economy, and those which are discharged from the body as useless or injurious. In the former case the separated materials are termed secretions; in the latter they are termed excretions.

The circumstances of their formation, and their final destination, are, however, the only particulars in which secretions and excretions can be distinguished; for, in general, the structure of the parts engaged in eliminating excretions is as complex as that of the parts concerned in the formation of secretions. It will, therefore, be sufficient to speak in general terms of the process. 
Every secreting apparatus consists essentially of a layer of secreting cells arranged round a central cavity; they take from the lymph which bathes them the necessary material, and transform it into the secretion which they pour at high pressure into the cavity.

In the case of the glands concerned in the formation of the various digestive juices, the most important material in the secretion is an enzyme or enzymes. In the cells which form the enzyme, it is first present in the shape of a pro-enzyme or zymogen. The transformation of this mother-substance may occur before or during secretion, as is the case for ptyalin, the salivary enzyme; or after
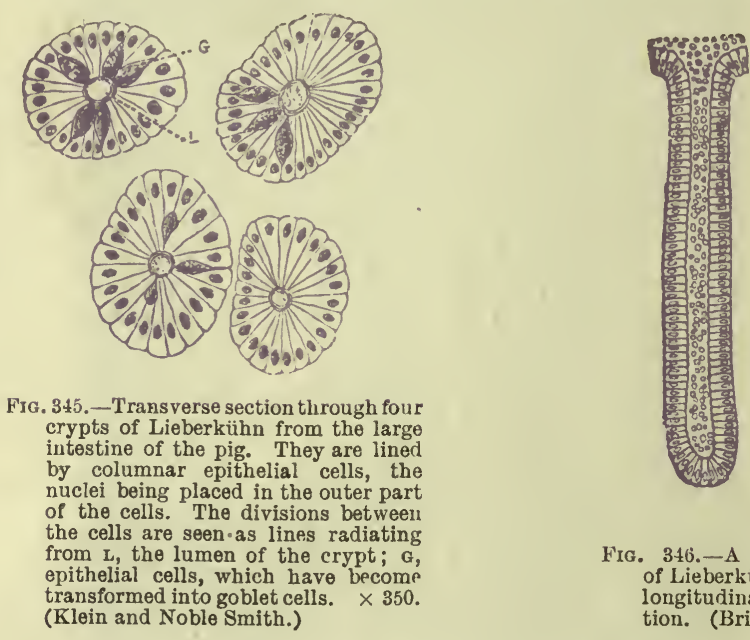

Frg. 346.-A gland of Lieberkiuhn in longitudinal sec. tion. (Brinton.)

secretion, as is the case for trypsin, one of the most important of the pancreatic enzymes.

Secreting glands may be classified as follows :-

1. The simple tubular gland (A, fig. 347), examples of which are furnished by the crypts of Lieberkühn in the intestinal wall. To the same class may be referred the elongated and tortuous sudoriferous or sweat glands.

2. The compound tubular glands (D, fig. 347) form another division. These consist of main gland-tubes, which divide and subdivide.

3. The racemose glands are those in which a number of vesicles or acini are arranged in groups or lobules (c, fig. 347). The Meibomian follicles of the eyelids are examples of this kind of gland. Some glands, like the pancreas, are of a mixed character, combining some of the characters of the tubular with others of the racemose type; these are called tubulo-racemose or tubulo-acinous glands. These 
glands differ from each other only in secondary points of structure, but all have the same essential character in consisting of rounded groups of vesicles containing gland-cells, and opening by a common central cavity into minute ducts, which ducts in the large glands
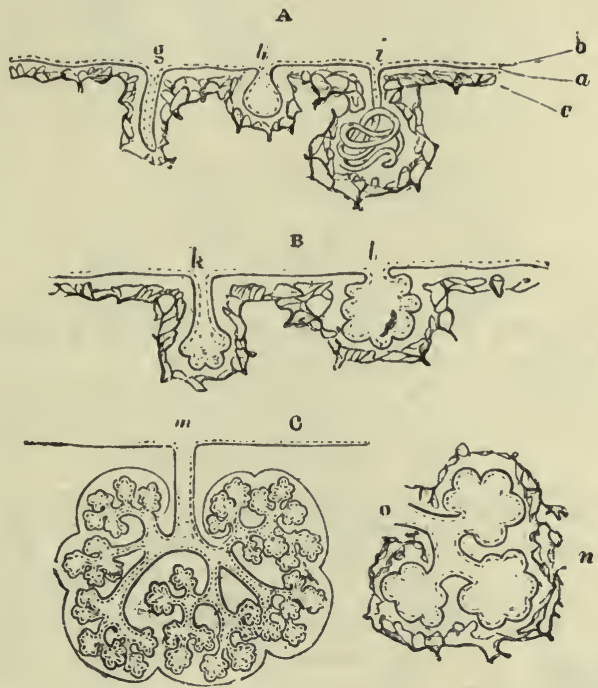

D

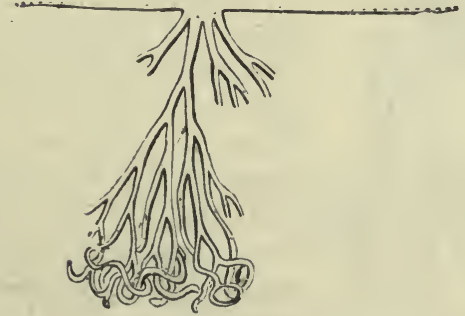

Fie. 347.-Diagram of types of secreting glands. A, Simple glands, viz., $g$, straight tube; $h$, sac; $i$, coiled tube. B, Multilocular crypts; $k$, of tubular form; $l$, saccular. c, Racemose, or saccular compound gland; $m$, entire gland, showing branched duct and lobular structure; $n$, a lobule, detached with 0 , branch of duct proceeding from it. $\mathbf{D}$, Compound tubular gland. (Sharpey.)

converge and unite to form larger and larger tubes, and at length open by one common trunk on a free surface. The larger racemose glands, such as the salivary glands, are called compound racemose glands. 


\section{CHAPTER XXXII}

\section{SALIVA}

THE saliva is formed by three pairs of salivary glands, called the parotid, submaxillary, and sublingual glands.

\section{The Salivary Glands.}

These typical secreting glands are made up of lobules united by connective tissue. Each lobule is made of a group of tubulosaccular alveoli or acini, from which a duct passes; this unites with

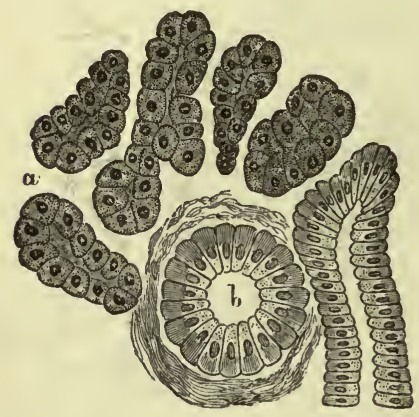

FiG. 318.-From a section through a salivary gland. $a$, Serous or albuminous alveoli; $b$, intralobular duct cut transversely. (Klein and Noble Smith.) the sublingual gland and some of the alveoli in the submaxillary) the cells after treatment with water or dilute acid are clear and swollen (fig. 350); this is the appearance they usually present in sections of the organ. But if examined in their natural state by teasing a portion of the fresh gland in serum, they are seen to be occupied by large granules composed of a substance known as mucigen or mucinogen (fig. 349). When the gland is active, mucigen is transformed into mucin and discharged as 
a clear droplet of that substance into the lumen of the alveolus. Outside these are smaller, highly granular cells containing no
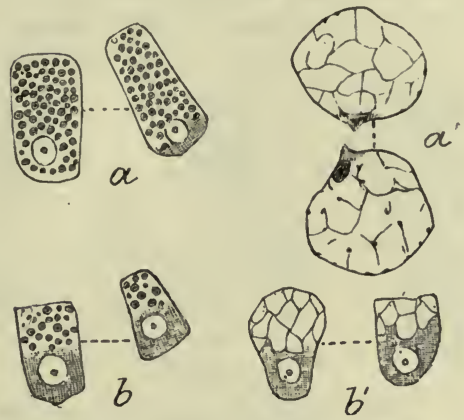

Fia. 349.-Mucous cells from submaxillary gland of dog. $a$, from a resting or loaded gland; $b$, from a gland which has been secreting for some time; $a^{\prime}, b^{\prime}$, similar cells which liave been treated with dilute acid. (Langley.) (From Quain's Anatomy, by permission of Messrs Longmans, Green \& Co.)

mucigen; these marginal cells stain darkly, and generally form crescentic groups (crescents or demilunes of Gianuzzi) next to the basement membrane. They do not secrete mucin, but are albuminous cells. After secretion their granules are lessened. The demilunes are therefore easily seen in the gland before secretion, owing to the contrast they exhibit to the cells loaded with mucin.

In those alveoli which do not secrete mucin, but a watery nonviscid saliva (parotid, and some of the alveoli of the submaxillary), the cells are filled with small granules of albuminous nature. Such alveoli are called serous or

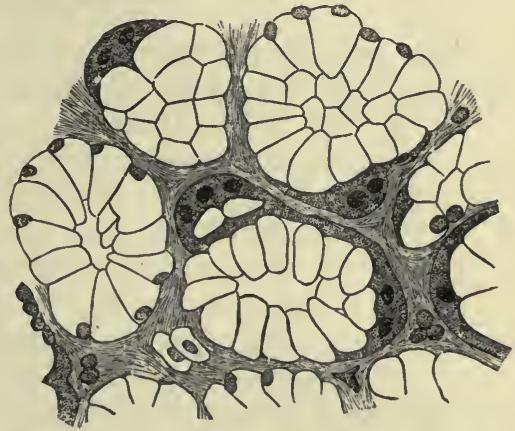

Fig. 350.-Section through a mucous gland hardened in alcohol. The alveoli are lined with mucous cells, and outside these are the demilunes. (Heidenhain.)

albuminous, to distinguish them from the mucous alveoli we have just described.

These yield to the secretion its enzyme, ptyalin. The granular substance within the cell is the mother-substance of the enzyme (zymogen), not the enzyme itself. It is converted into the enzyme in the act of secretion. We shall study the question of zymogens more fully in connection with the gastric glands and the pancreas, where they have been separated from the enzymes by chemical methods. In the case of saliva we may term the zymogen, ptyalinogen provision- 
ally, but it has never been satisfactorily separated chemically from ptyalin.

After secretion, due to the administration of food or of such a drug as pilocarpine, the cells shrink, they stain more readily, their
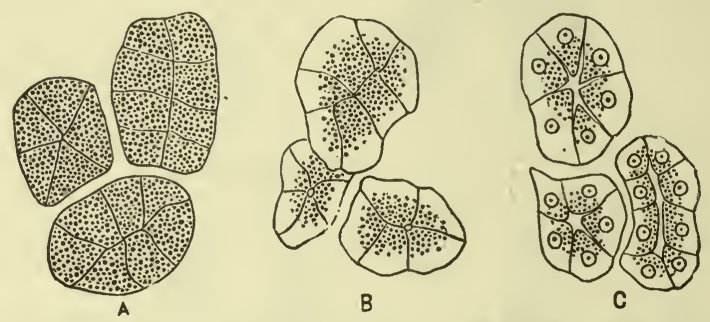

FIG. 351.-Alveoli of parotid gland. A, before secretion; B, in the first stage of secretion; C, after prolonged secretion. (Langley.)

nuclei become more conspicuous, and the outer part of each cell becomes clear and free from granules (fig. 351).

\section{The Secretion of Saliva.}

The process of secretion consists of a number of events which may be divided into two categories:-

1. The transference of water and certain substances dissolved in the water from the blood of the surrounding capillaries to the lumen of the acinus.

2. The modification of the chemical composition of this solution either by the addition to it of substances manufactured by the gland-cells, or by the prevention of substances in the lymph from traversing the gland-cell and reaching the lumen.

In regard to the first of these, we must regard each secreting cell as an organ which pumps the water through its own substance from the lymph space to the lumen. The arrows in the accompanying figure (fig. 352) show the direction in which water passes.

The result of this pumping action is to increase the pressure in the lumen, and diminish that in the lymph space. The reduction of pressure in the lymph space causes water to flow into it through the capillary wall from the blood, and this filtration is further helped by a rise of pressure within the capillary, for during secretion the muscular fibres encircling the arteriole wall relax, and thus the arterial pressure is more fully communicated to the capillary.

The extent to which the pressure in the lumen rises depends upon the ease with which the secreted fluid can pass away along the duct, and if the saliva is made to run up a vertical tube inserted 
into the duct, it may reach a height which is greater than the pressure of the arterial blood; this is an experiment which disposes of the idea that the sole source of the salivary flow is the bloodpressure, for there is in addition to this the pumping action of the secretory cells. Although to aid the imagination we roughly compare the cell to a pump, we have no real knowledge of its mechanism.

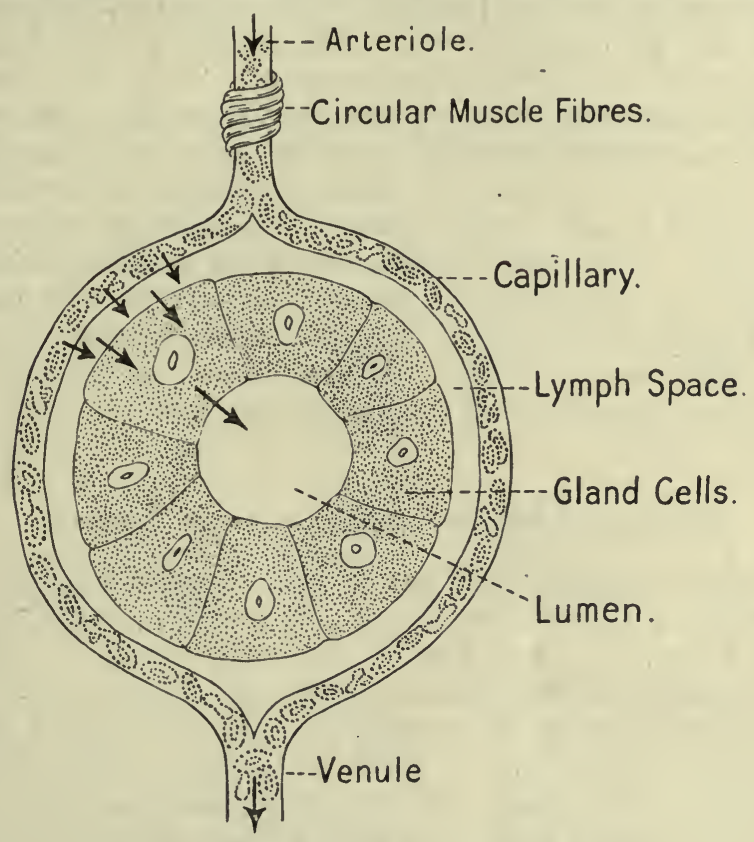

FıG. 352.-Diagram of a secreting acinus.

Whatever may be the actual mechanism, we are nevertheless quite sure that the cells really do active work, and the proofs of this are the following:-

1. Increase of work finds its expression in increase of combustion. By examining the gases of the blood leaving the gland, it is found that the amounts of oxygen used and carbonic acid produced are increased at least fourfold when saliva is being actively produced; for instance, when the submaxillary gland is thrown into action by stimulation of its secretory nerve (chorda tympani).

2. It may be proved mathematically that, whenever any solution of electrolytes is divided into two solutions each differing from the original, positive work must be done. Salivary secretion is a case in point; for the arterial blood is changed into venous blood and saliva, neither of which has the same composition as the arterial blood. 
The loss of water from the arterial blood can be calculated by examining the venous blood, and finding the increase in the concentration of hæmoglobin there; it has been experimentally proved that the amount of water lost from the blood is equal to the quantity of saliva formed in a given time.

We now pass to the second phase of secretion, namely, the alteration in the composition of the fluid. The cell is not only a pumping engine for the movement of water, but is also a factory of organic substances which are thrust into the stream. The two most important of these materials, though neither is of constant occurrence, are mucin, and the enzyme ptyalin. On the other hand, the cell offers an obstruction to the passage of salts; the saliva is therefore poorer (and the lymph in the lymph space correspondingly richer) in salts than the blood. The accumulation of salts in the lymph is an additional factor in attracting (by osmotic pressure) water out of the blood. Osmosis, however, is only a contributory cause of the flow of lymph; it will not account for secretion of saliva; in fact, if saliva and blood were placed in an osmomeler, fluid would pass from the saliva to the blood.

\section{Secretory Nerves of the Salivary Glands.}

The submaxillary gland has a double nerve-supply. (1) The chordu tympani; this is a branch of the seventh cranial nerve, and in part of its course is bound up in the same sheath as the lingual nerve, a branch of the fifth. When the lingual nerve crosses Wharton's duct beneath the tongue, the chorda tympani leaves the lingual, and the preganglionic fibres in it for the submaxillary pass into the hilus of that gland, and end by arborising around a scattered collection of ganglion cells concealed within the substance of the gland. This ganglion is known as Langley's ganglion. From the cells of Langley's ganglion post-ganglionic fibres are distributed to the gland-cells and also to the blood-vessels.

(2) Sympathetic branches are derived from the plexus around the facial artery and accompany the arterial branches which supply the gland. (See fig. 353.)

The chorda tympani is par excellence the secretory nervo of the gland. When it is stimulated, secretion of saliva and dilatation of the arterioles take place invariably. Stimulation of the sympathetic always produces constriction of these blood-vessels, and a secretion of a small quantity of thick viscid saliva may also occur, but often the salivary flow is entirely absent. Recent investigations have shown that the part played by the sympathetic is so inconstant, and the results obtained by different methods of stimulation (e.g., electrical; and the administration of adrenaline) are so different, 
that the many theories formerly advanced of the relative part played by the two nerves must be regarded, as mere matters of speculation.

Section of the chorda tympani produces no immediate result; but after a few days a scanty but continuous secretion of thin watery saliva takes place; this is called paralytic secretion. If the operation is performed on one side, the gland of the opposite side also shows a similar condition, and the thin saliva secreted there is called the antilytic secretion. The meaning of these phenomena is unknown.

Effect of drugs on the gland. Atropine.-After intravenous injection of this alkaloid stimulation of the chorda tympani no longer produces secretion of saliva. Much larger doses are necessary to abolish the vaso dilator effect of chorda stimulation, or the sympathetic flow in those cases where provious stimulation of this nerve evoked a secretion of saliva.

Pilocarpine produces a copious flow of saliva, accompanied by vasodilatation.

Ergotoxine paralyses the effects of sympathetic stimulation, but not those of stimulation of the chorda tympani.

Adrenalıne produces constriction of the blood-vessels. In some animals it evokes a considerable flow of saliva, and when this occurs the constriction of the vessels is followed by dilatation. This favours a view which has been advanced by some observers, that vaso-dilatation is in part produced by the chemical action of the products

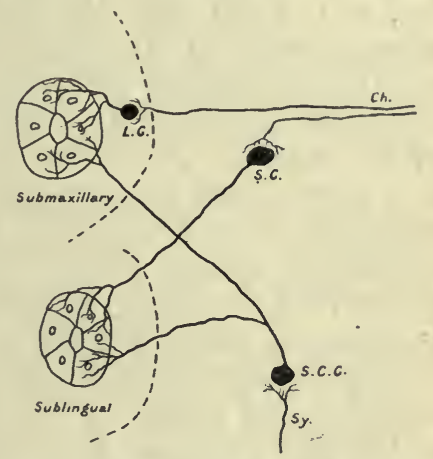

Fia. 353.-Diagram of secretury nerves of submaxillary and sublingual glands. Two fibres of the chorda tympani (Ch.) are shown, one of which supplies the sublingual gland, of which an acinus is shown; the cell-station for this is in $\mathbf{S}$. G., the so-called submaxillary ganglion. The other fibre supplies an acinus of the submaxillary gland; its cell-station is in Langley's ganglion (L. G.), within the substance of the gland. Sy. is a fibre of the sympathetic, which has its cell. station in the superior cervical ganglion, S. C. G. (Atter Dixon.) of activity (carbonic and lactic acids, otc.).

The sublingual gland is innervated by the same nerves as the submaxillary, but the preganglionic fibres of the chorda tympani have their cell-station in the so-called submaxillary ganglion which is situated close to the sublingual gland (see fig. 353). The submaxillary ganglion ought properly to be termed the sublingual ganglion. This has been determined by Langley's nicotine method (seo p. 200).

The parotid gland also receives two sets of nerve-fibres analogous to those we have studied in connection with the submaxillary gland. The principal secrotory nerve-fibres are glosso-pharyngeal in origin; 
the sympathetic is mainly vaso-constrictor, but in some animals it does contain a few secretory fibres also.

Reflex Secretion.-Under ordinary circumstances the secretion of saliva is a reflex action. The principal afferent nerves are those of taste; but the smell or sight of food will also cause "the mouth to water"; and under certain circumstances, as before vomiting, irritation of the stomach has a similar effect. These sensory nerves stimulate a centre in the medulla from which efferent secretory impulses are reflected along the secretory nerves (chorda tympani, etc.) to the glands.

Pawlow has recorded some interesting observations on the salivary glands. He made an external fistula of the submaxillary duct in the $\mathrm{dog}$, and found that the sight of food, the smell of food, or the administration of any kind of food, caused secretion; acid or even sand introduced into the mouth produced a similar effect. By means of similar experiments on the parotid secretion, very different results were obtained. If the dog was shown meat, or the meat was given to it to eat, there was practically no secretion. If, however, the meat was given as a dry powder, a copious secretion followed; dry bread produced a similar effect; in fact, the parotid secretion flows freely if dry food is simply shown to the animal; of course, in all such experiments the dog must be hungry.

Such observations emphasize the psychical element involved in secretion, and point out also the adaptation of the secretory process to the needs of the animal; thus the submaxillary saliva, which is mainly a lubricant in virtue of its mucin, flows whatever the food may be, whereas moist food requiring no watery saliva from the parotid excites the flow of none.

Extirpation of the Salivary Glands.-These may be removed without any harmful effects in the lower animals.

\section{The Saliva.}

The saliva is the first digestive juice to come in contact with the food. The secretions from the different salivary glands are mixed in the mouth; the secretion of the minute mucous glands of the mouth and a certain number of epithelial scales and the so-called "salivary corpuscles" derived from the tonsils are added to it. The liquid is transparent, slightly opalescent, of slimy consistency, and may contain lumps of nearly pure mucin. On standing it becomes cloudy owing to the precipitation of calcium carbonate, the carbonic acid, which held it in solution as bicarbonate, escaping.

Of the three forms of saliva which contribute to the mixture found in the mouth the sublingual is richest in solids ( $2 \cdot 75$ per cent.). The submaxillary saliva comes next $(2 \cdot 1$ to 2.5 per cent.). The 
parotid saliva is poorest in total solids ( 0.3 to 0.5 per cent.), and contains no mucin. Mixed saliva contains in man an average of about 0.5 per cent. of solids : it is alkaline in reaction, due to the salts in it; and has a specific gravity of 1002 to 1006.

The solid constituents dissolved in saliva may be classified thus :

$$
\begin{aligned}
& \text { Organic } \\
& \text { Inorganic } \cdot\left\{\begin{array}{l}
a . \text { Mucin : this may be precipitated by acetic acid. } \\
\text { b. Ptyalin : an amylolytic enzyme. } \\
c . \text { Protein : of the nature of a globulin. } \\
\text { d. Potassium sulphocyanide. }
\end{array}\right. \\
& \text { e. Sodium chloride : the most abundant salt. } \\
& f . \text { Other salts : sodium carbonate, calcium phosphate and } \\
& \text { carbonate; magnesium phosphate; potassium chloride. }
\end{aligned}
$$

The action of saliva is twofold, physical and chemical.

The physical use of saliva consists in moistening the mucous membrane of the mouth, assisting the solution of soluble substances in the food, and in virtue of its mucin, lubricating the bolus of food to facilitate swallowing.

The chemical action of saliva is due to its active principle, ptyalin. This substance belongs to the class of enzymes which are called amylolytic (starch-splitting) or diastatic (resembling diastase, the similar enzyme in germinating barley and other grains).

The starch is first split into dextrin and maltose; the dextrin is subsequently converted into maltose also: this occurs more quickly with erythro-dextrin, which gives a red colour with iodine, than with the other variety of dextrin called achroö-dextrin, which gives no colour with iodine. Brown and Morris give the following equation :-

$$
\begin{aligned}
& 10\left(\underset{\text { [Starch.] }}{\mathrm{C}_{6} \mathrm{H}_{10} \mathrm{O}_{5}}\right) \mathrm{n}+\underset{\text { [Water.] }}{4 \mathrm{nH}_{2} \mathrm{O}} \\
& =\underset{\text { [Maltose.] }}{4 \mathrm{nC}_{12} \mathrm{H}_{22} \mathrm{O}_{11}}+\underset{\text { [Achroö-dextrin.] }}{\left(\mathrm{C}_{6} \mathrm{H}_{10} \mathrm{O}_{5}\right)_{n}}+\underset{\text { [Erythro-dextrin.] }}{\left(\mathrm{C}_{6} \mathrm{H}_{10} \mathrm{O}_{5}\right)_{n}}
\end{aligned}
$$

Ptyalin acts in a similar way, but more slowly, on glycogen : it has no action on cellulose; hence it is inoperative on uncooked starch grains, for in them the cellulose layers are intact.

Ptyalin acts best at about the temperature of the body $\left(35-40^{\circ} \mathrm{C}\right.$.). It acts best in a noutral medium; a small amount of alkali makes but little difference; a very small amount of acid stops its activity. The conversion of starch into sugar by swallowed saliva in the stomach continues for a certain time. It then ceases owing to the hydrochloric acid secreted by the glands of the stomach. The acid which is first poured out neutralises the saliva, and combines with the proteins of the food, but when free acid appears ptyalin is destroyed, and so it cannot resume work when the acid is neutralised in the duodenum. Another amylolytic enzyme contained in pan- 
creatic juice (to bo considered later) continues the digestion of starch in the intestine.

Cannon has shown that salivary digestion continues in the stomach for longer than one supposed. The food lying in the fundus of the stomach undergoes amylolysis for at least two hours, because the absence of peristalsis in this region until quite late stages in digestion prevents admixture with gastric juice, especially in the interior of the swallowed masses. These observations were, however, made on animals in a quiescent horizontal position. It is extremely doubtful if they can be applied to man in a vertical position, especially if he is moving about (see more fully Stomach movements in the chapter entitled the Mechanical Processes of Digestion). 


\section{CHAPTER XXXIII}

\section{THE GASTRIC JUICE}

The juice secreted by the glands in the mucous membrane of the stomach varies in composition in the different regions, but the mixed gastric juice, as it may be termed, is a solution of a proteolytic or proteoclastic enzyme called pepsin in a saline solution, which also contains a little free hydrochloric acid.

The gastric juice can be obtained during the life of an animal by means of a gastric fistula.* Gastric fistulæ have also been made in human beings, either by accidental injury or by surgical operations. The most celebrated case is that of Alexis St Martin, a young Canadian, who received a musket wound in the abdomen in 1822 . Observations made on him by Dr Beaumont formed the startingpoint for our correct knowledge of the physiology of the stomach and its secretion.

Artificial gastric juice is made by mixing weak hydrochloric acid $(0.2$ per cent.) with the glycerin extract of the stomach of a recentlykilled animal. This acts like the normal juice.

When examined with a lens, the internal or free surface of the stomach presents a peculiar honejcomb appearance, produced by shallow polygonal depressions. In the bottom of these little pits, and to some extent between them, minute openings are visible, which are the orifices of the ducts of perpendicularly arranged tubular glands (fig. 354), imbedded side by side in the substance of the mucous membrane.

The glands of the mucous membrane are of three varieties, (a) Cardiac, (b) Fundus, and (c) Pyloric.

(a) Cardiac glands; these are simple tubular glands lined by short columnar granular cells, and are only found quite close to the cardiac orifice.

* A gastric fistula is made by cutting through the abdominal wall so as to expose the stomach. The stomach is then attached to the edges of the abdominal wound, and a small orifice is finally made through the wall of the stomach. When the wound heals there is then a free communication between the stomach and the exterior. 
(b) Fundus glands are found throughout the remainder of the cardiac half and fundus of the stomach. They are arranged in

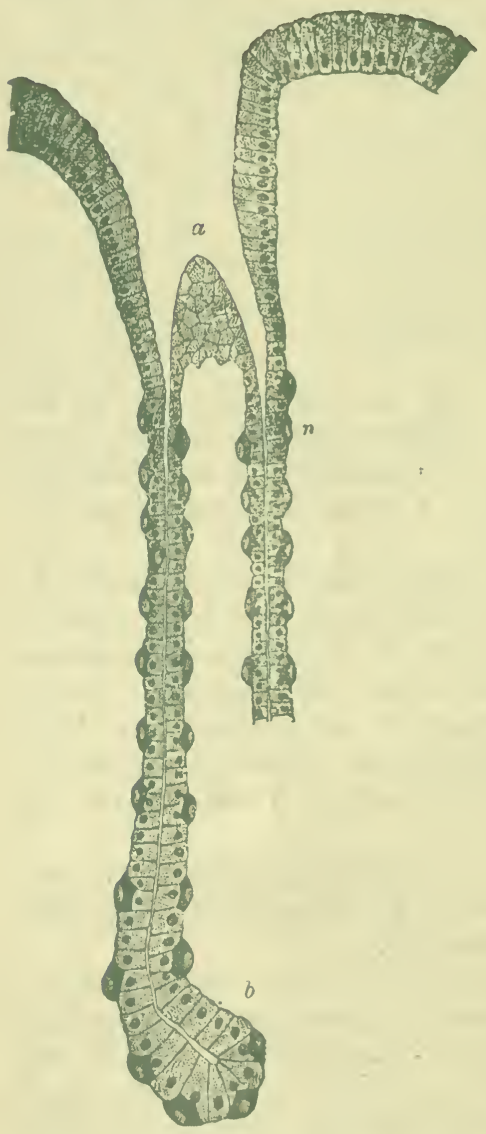

Fig. 354.-From a vertical section through the mucous membrane of the cardiac end of stomach. Two fundus glands are shown with a duct common to both. $a$, Duct with columnar epithelium becoming shorter as the cells are traced downward; $n$, neck of gland tubes, with central and parietal cells $b$, base with curved cæcal extremity-the parietal cells are not so numerous here. (Klein and Noble Smith.) The lumen is large. The ducts are lined with columnar epithelium,
and the tubules with shorter and finely granular cubical cells,
which correspond with the central cells of the fundus glands.
The pyloric glands have no parietal cells. As they approach the The lumen is large. The ducts are lined with columnar epithelium,
and the tubules with shorter and finely granular cubical cells,
which correspond with the central cells of the fundus glands.
The pyloric glands have no parietal cells. As they approach the The lumen is large. The ducts are lined with columnar epithelium,
and the tubules with shorter and finely granular cubical cells,
which correspond with the central cells of the fundus glands.
The pyloric glands have no parietal cells. As they approach the groups of four or five, which are
separated by a fine connective tissue. Several tubules open into one duct, which forms about a third of the whole length of the tube and opens on the surface. The ducts are lined with columnar epithelium. The gland-tubules are lined with coarsely granular polyhedral cells (central cells). Between these cells and the basement membrane of the tubes, are large oval or spherical cells, opaque or gran-

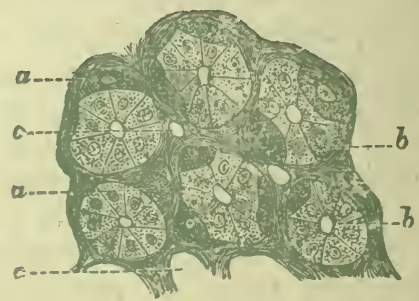

Fig. 355.-Transverse section through lower part of fundus glands of a cat. $a$, Parietal cells; $b$, central cells ; $c$, transverse section of capillaries. (Frey.)

ular in appearance, with clear oval nuclei, bulging out the basement membrane; these cells are called parietal or oxyntic cells. They do not form a continuous layer.

(c) Pyloric Glands. - These glands (fig. 356) have much longer ducts than the fundus glands. Into each duct two or three tubules open by very short and narrow necks, and the body of each tubule is branched, wavy, and convoluted. 
duodenum the pyloric glands become larger, more convoluted and more deeply situated. They are directly continuous with Brunner's glands in the duodenum.

The central cells of the fundus glands and, to a less degree, the cells of the pyloric glands, are loaded with granules. During secretion they discharge their granules, those that remain being chiefly situated near the lumen, leaving in each cell a clear outer zone. These are the cells that secrete the pepsin. Like secreting cells generally, they pump water from the lymph that bathes them into the lumen;

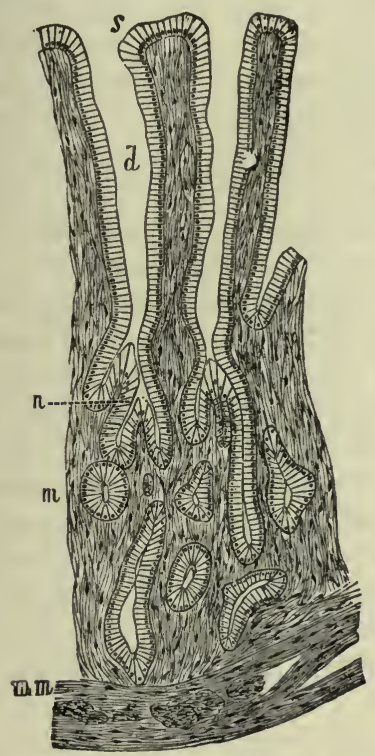

Fig. 356.-Section showing the pyloric glands. $s$, Free sur. face; $d$, ducts of pyloric glands ; $n$, neck of same; $m$, the gland tubules; $\mathrm{mm}$, muscularis mucosæ. (Klein and Noble Smith.)

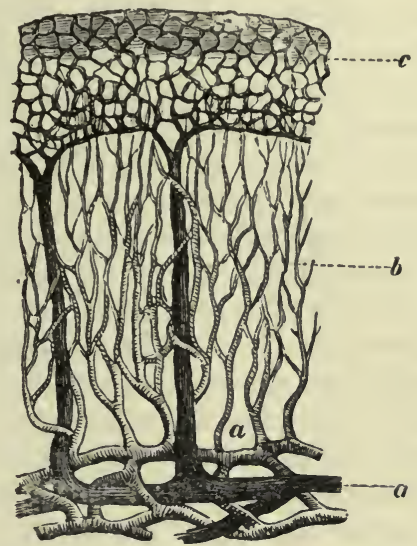

FIG. 357.-Plan of the blood-vessels of the stomach, as they would be seen in a vertical section. $a$, Arteries, passing up from the vessels of submucous coat; $b$, capillaries branching between and around the tubes; $c$, superficial plexus of capillaries occupying the ridges of the mucous membrane; $d$, vein formed by the union of veins which, having collected the blood of the superficial capillary plexus, are seen passing down between the tubes. (Brinton.)

to this certain materials are added which are formed by the protoplasmic activity of the cells. The most important substance in a digestive secretion is the enzyme. In the case of the gastric juice this is pepsin. We can trace an intermediate step in this process by the presence of the granules in the cells. The granules are not, however, composed of pepsin, but of a mother-substance which is readily converted into pepsin. We shall find similar enzyme precursors in the cells of the pancreas, and the term zymogen is applied to these enzyme precursors. The zymogen in the gastric cells is called pepsinogen. The rennet-enzyme that causes the curdling of milk is formed by the same cells. 
The parietal cells undergo merely a change of size during secretion, boing at first somewhat enlarged, and after secretion they are somewhat shrunken. They are also called oxyntic (acid-forming) cells, because they secrete the hydrochloric acid of the juice. Heidenhain succeeded in making in one dog a cul-de-sac of the fundus, in another, of the pyloric region of the stomach; the former secreted a juice containing both acid and pepsin; the latter, parietal cells being absent, secreted a viscid alkaline juice containing pepsin.

The formation of a free acid from the alkaline blood and lymph is an important problem. There is no doubt that it is formed from the chlorides of the blood and lymph, and of the many theories advanced as to its actual mode of formation, none is wholly satisfactory. Some theories are chemical, and explain the formation of the acid by an interaction of the chlorides and phosphates. Others call to their assistance the law of "mass action," and we certainly know that by the action of large quantities of carbonic acid on salts of mineral acids, the latter may be liberated in small quantities. We know further that small quantities of acid ions may be continually formed in the organism by ionisation. But in every case we can only make use of these explanations if we assume that the small quantities of acid are carried away as soon as they are formed, and thus give room for the formation of fresh acid. Even then we are unable to explain the whole process. A specific action of the cells is no doubt exerted, for these reactions can hardly be considered to occur in the blood generally, but rather in the oxyntic cells, which possess the necessary selective powers in reference to the saline constituents of the blood, and the hydrochloric acid, as soon as it is formed, passes into the secretion of the gland in consequence of its high power of diffusion.

\section{Composition of Gastric Juice.}

The following table gives the percentage composition of the gastric juice of man and the dog:-

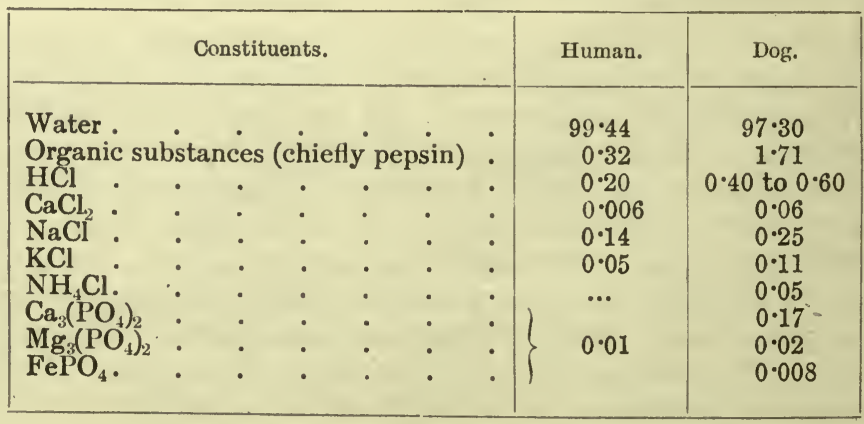


One seos from this how much richer in all constituents the gastric juice of the dog is than that of man. Carnivorous animals have always a more powerful gastric juice than other animals; they have more work for it to do; but the great contrast seen in the table is, no doubt, partly due to the fact that the persons from whom it has been possible to collect gastric juice have been invalids. In the foregoing table one also sees the great preponderance of chlorides over other salts; apportioning the total chlorine to the various metals present, that which remains over must be combined with hydrogen to form the free hydrochloric acid of the juice.

In recent years, the composition and action of the gastric juice has been studied by Pawlow. By an ingenious surgical operation, he succeeded in separating from the stomach of dogs a diverticulum which pours its secretion through an opening in the abdominal wall; the nerves of this small stomach are intact, and the amount of juice that can be collected from it when it is active amounts to several hundred cubic centimetres in a few hours. Pawlow thus obtained a pure gastric juice, which enabled him to study its action and composition. It is clear, colourless, has a specific gravity of 1003-1006, and is foebly dextro-rotatory. It contains 0.4 to 0.6 per cent. of hydrochloric acid. It is strongly proteolytic, and inverts cane sugar. When cooled to $0^{\circ} \mathrm{C}$. it deposits a precipitate of pepsin, and this carries down with it the acid in loose combination, especially in the layers first deposited. Its percentage composition is very similar to that of a protein, only it contains chlorine in addition to the usual elements. The numbers agree closely with those obtained by Kühne, who used ammonium sulphate as the precipitant.

Pepsin stands apart from nearly all other enzymes by requiring an acid medium in order that it may act. A compound of the two substances, called pepsin-hydrochloric acid, is the really active agent. Other acids may take the place of hydrochloric acid, but none act so well. Lactic acid is often found in gastric juice: this is derived by fermentative processes from the food.

The digestive powers of the acids are proportional to their dissociation and the number of $\mathrm{H}$ ions liberated. The anions, however, modify this by having different powers of retarding the action. The greater suitability of hydrochloric over lactic acid, for instance, in gastric digestion is due to the fact that the former acid more readily undergoes dissociation.

\section{The Innervation of the Gastric Glands.}

As long ago as 1852 Bidder and Schmidt showed in a dog with a gastric fistula that the sight of food caused a secretion of gastric juice; and in 1878 Richet observed that in a man with complete occlusion of the gullet the act of mastication caused a copious flow of gastric juice. There could therefore have been no doubt that the 
glands are under the control of the nervous system, but the early attempts to discover the secretory nerves of the stomach were unsuccessful. The Russian physiologist Pawlow solved the problem by the employment of new methods. He experimented on dogs. In the first place he separated off the diverticulum, which we described on the last page, and by careful experiments he showed that the secretion of this small stomach is an exact sample, both as regards composition and rate of formation, of that which occurs in the main stomach, which is still left in continuity with the œsophagus above and the duodenum below.

Another procedure adopted was to divide the œsophagus, and to attach the two cut ends to the opening in the neck. The animal was fed by the lower segment, but any food taken into the mouth, or any saliva secreted there, never reached the stomach, but fell out through the opening of the upper segment. These animals were kept alive for months, and soon accommodated themselves to their new conditions of life. The animals could thus be subjected to, (1) real feeding, (2) sham feeding, by allowing them to eat food which subsequently tumbled out through the neck opening, and (3) psychical feeding, in which the animal was shown the food but was not allowed to eat it. The psychical element is important.

Mechanical excitation of the stomach wall produces no secretion. If water is introduced there is a slight flow, and even if meat is introduced into the main stomach without the knowledge of the dog, the juice formed is scanty and of feeble digestive power.

There is, moreover, no connection between the acts of mastication and swallowing with that of gastric secretion. Sham feeding with stones, butter, salt, pepper, mustard, extract of meat, and acid, though it excited a flow of saliva, produced no effect on the stomach. If, however, meat was used for the sham feeding, an abundant and active secretion occurred in the stomach (that of the small stomach was actually examined) after a latency of about five minutes. The secretion is thus adapted to the kind of food the dog has to digest; the larger the proportion of protein in the diet, the more abundant is the juice, and the richer both in pepsin and acid.

Indeed, if the animal is hungry and shown the meat and not allowed to swallow it, the effect is almost as great. The following striking experiment also shows the importance of the psychical element. Two dogs were taken, and a weighed amount of protein introduced into the main stomach of each without their knowledge; one was then sham fed on meat, and one and a half hours later the amount of protein digested by this dog was five times greater than that which was digested by the other.

In the meat, however, it is not the protein which acts most strongly as the stimulus; egg-white, for instance, is not a stronger 
stimulus than water, but extract of meat is a powerful stimulus; what the exact extractives are that act in this way is not yet known, and Herzen has since shown that dextrin acts even more powerfully. Herzen distinguishes between succagogues (juice-drivers) such as Liebig's extract, and peptogens such as dextrin, which produce not only an increased flow, but a juice rich in pepsin-hydrochloric acid. The products of proteolysis are also peptogenic, so that when once digestion has started, a stimulus for more secretion is provided.

If the vagi are cut (below the origin of the recurrent laryngeal to avoid paralysis of the larynx), and then sham feeding is performed with meat, no secretion is obtained; the vagi therefore contain the secretory fibres. The experiment of stimulating the peripheral end of the cut nerve confirmed this hypothesis. The nerve was cut in the neck four or five days before it was stimulated; in this time degeneration of the cardio-inhibitory fibres took place, so that stoppage of the heart did not occur when the nerve was stimulated; under these circumstances a secretion was obtained with a long latency; the latency is explained by the presence of secreto-inhibitory fibres. Atropine abolishes the action of the vagus. In other animals the spinal cord was cut at the level of the first cervical nerve, and the animal kept alive by artificial respiration; the vagus nerve was then cut, and its peripheral end stimulated; an abundant secretion usually followed. Division of the cord renders an anæsthetic unnecessary, and also prevents the afferent impulses set up by the operation passing to the vagal centres, and thus exciting the inhibitory impulses which pass down the vagus, and tend to prevent secretion under ordinary circumstances.

Pawlow thinks that the sympathetic also contains some secretory fibres, but this has not yet been proved.

\section{Actions of Gastric Juice.}

Gastric juice has the following five actions:-

1. It is antiseptic, owing to the hydrochloric acid present; putrefactive processes do not normally occur in the stomach, and the micro-organisms which produce such processes, many of which are swallowed with the food, are in great measure destroyed, and thus the body is protected from them.

2. It inverts cane sugar into dextrose and lævulose. This also is due to the acid of the juice, and is frequently assisted by inverting enzymes contained in the vegetable food swallowed. The juice has no action on starch.

3. It contains lipase, a fat-splitting enzyme. The protein envelopes of the fat cells are first dissolved by the pepsin-hydrochloric acid, and the solid fats are melted. They are then split in small measure into their constituents, glycerin and fatty acids. This 
action is mainly produced by a regurgitation of the contents of the duodenum mixed with pancreatic juice; but even after the pylorus has been ligatured and regurgitation prevented, the gastric juice itself produces a small amount of fat-splitting, and therefore contains lipase. It is a remarkable fact that the administration of fat in the food increases the regurgitation from the duodenum.

4. It curdles milk. - This is due to the action of the rennet enzyme or rennin. The conditions of this action we have already discussed under milk (see p. 482); but it may here be added that Pawlow has advanced the view that rennin is not a distinct and separate enzyme, but milk-curdling is only one of the activities of pepsin. This hypothesis has been accepted by numerous physiologists; but, on the other hand, there is a number of equally eminent observers who still maintain that pepsin and rennin are two separate enzymes. Whichever view is correct, the curd of casein formed from the caseinogen is subsequently digested as other proteins are.

5. It is proteolytic; this is the most important action of all. The proteins of the food are converted by the pepsin-hydrochloric acid into peptones.

This action is a process of hydrolysis; and peptones may be formed by other hydrolysing agencies, such as superheated steam or heating with dilute mineral acids. The first stage in the process of hydrolysis is that of acid meta-protein, formerly called acid-albumin or syntonin; the next step is the formation of propeptones or proteoses. The word "proteose" includes the albumoses (from albumin), globuloses (from globulin), vitelloses (from vitellin), etc. Similar substances are also formed from gelatin (gelatinoses) and elastin (elastoses). Then peptone (probably a mixture of polypeptides) is produced. The products of digestion of protein may be arranged according to the order in which they are formed, as follows :-

1. Acid meta-protein.

$\int(a)$ Proto-proteose

2. Propeptone $(b)$ Hetero-proteose or proteoses

(c) Deutero- or secondary proteose

3. Peptone.

It has been stated that the prolonged action of gastric juice leads to the further splitting of the peptone into amino-acids. But accurate work has shown that pepsin-hydrochloric acid does not split any of the known polypeptides into their ultimate cleavage products.

1. Acid meta-protein.-The general properties of the metaproteins, the first degradation products in the cleavage of the proteins which occurs during digestion are described on p. 433 . We shall find later that, in pancreatic digestion, an alkali meta-protein is formed instead of the acid modification. 
2. Proteoses.-They are not coagulated by heat; they are procipitated but not coagulated by alcohol: like peptone, they give the pink biuret reaction. They are precipitated by nitric acid, the precipitate being soluble on heating, and reappearing when the liquid cools. This last is a distinctive property of proteoses. They are slightly diffusible.

The primary proteoses are precipitated by saturation with magnesium sulphate or sodium chloride. Deutero-proteose is not; it is, however, precipitated by saturation with ammonium sulphate. Proto- and deutero-proteose are soluble in water; hetero-proteose is not; it requires salt to hold it in solution.

3. Peptones. - They are soluble in water, are not coagulated by heat, and are not precipitated by nitric acid, copper sulphate, ammonium sulphate, and a number of other precipitants of proteins. They are precipitated but not coagulated by alcohol. They are also precipitated by tannin, picric acid, potassio-mercuric iodide, phosphomolybdic acid, and phospho-tungstic acid.

They give the biuret reaction (rose-red solution with a trace of copper sulphate and caustic potash or soda).

Peptone is readily diffusible through animal membranes.

The annexed table will give us at a glance the chief characters of peptones and proteoses in contrast with those of the native proteins. albumins, and globulins.

\begin{tabular}{|c|c|c|c|c|c|c|}
\hline $\begin{array}{c}\text { Varioty } \\
\text { of } \\
\text { proteid. }\end{array}$ & $\begin{array}{l}\text { Action } \\
\text { of } \\
\text { heat. }\end{array}$ & $\begin{array}{l}\text { Action } \\
\text { of } \\
\text { alcohol. }\end{array}$ & $\begin{array}{c}\text { Action } \\
\text { of } \\
\text { nitric acid. }\end{array}$ & $\begin{array}{l}\text { Action of } \\
\text { ammonium } \\
\text { sulphate. }\end{array}$ & $\begin{array}{l}\text { Action of } \\
\text { copper } \\
\text { sulphate } \\
\text { and caustic } \\
\text { potash. }\end{array}$ & $\begin{array}{l}\text { Diffusi- } \\
\text { vility. }\end{array}$ \\
\hline Albumin & Coagulated & $\begin{array}{l}\text { Precipitated, } \\
\text { then coagu- } \\
\text { lated }\end{array}$ & $\begin{array}{l}\text { Precipitated } \\
\text { in the cold; } \\
\text { not readily } \\
\text { soluble on } \\
\text { heating }\end{array}$ & $\begin{array}{l}\text { Precipitated } \\
\text { by complete } \\
\text { saturation }\end{array}$ & $\begin{array}{l}\text { Violet } \\
\text { colour }\end{array}$ & Nil \\
\hline Globulin & Ditto & Ditto & Ditto & $\begin{array}{l}\text { Precipitated } \\
\text { by half satu- } \\
\text { ration; also } \\
\text { precipitated } \\
\text { by } \mathrm{MgSO}_{4}\end{array}$ & Ditto & Ditto \\
\hline Proteoses & $\begin{array}{c}\text { Not } \\
\text { coagulated }\end{array}$ & $\begin{array}{l}\text { Precipitated, } \\
\text { but not co- } \\
\text { agulated }\end{array}$ & $\begin{array}{l}\text { Precipitated } \\
\text { in the cold; } \\
\text { readily sol- } \\
\text { u ble on } \\
\text { heating; the } \\
\text { precipitate } \\
\text { reappears on } \\
\text { cooling* }\end{array}$ & $\begin{array}{l}\text { Precipitated } \\
\text { by } \\
\text { tion }\end{array}$ & $\begin{array}{l}\text { Rose-red } \\
\text { colour } \\
\text { (biuret } \\
\text { leaction) }\end{array}$ & Slight \\
\hline Peptones & $\begin{array}{c}\text { Not } \\
\text { congulated }\end{array}$ & $\begin{array}{l}\text { Precipitated, } \\
\text { but not co- } \\
\text { agulated }\end{array}$ & $\begin{array}{l}\text { Not precipi- } \\
\text { tated }\end{array}$ & Not precipi. & $\begin{array}{l}\text { Rose-red } \\
\text { colour } \\
\text { (binret } \\
\text { reaction }\end{array}$ & Great \\
\hline
\end{tabular}

* In the case of deutero-albumose this reacion only occurs in the presence of excess nf salt. 
The question has been often raised why the stomach does not digest itself during life. The mere fact that the tissues are alkaline and pepsin requires an acid medium in which to act is not an explanation, but only opens up a fresh difficulty as to why the pancreatic juice which is alkaline does not digest the intestinal wall. To say that it is the vital properties of the tissues that enable them to resist digestion only shelves the difficulty and gives no real explanation of the mechanism of defence. Recent studies on the important question of immunity (see p. 474) have furnished us with the key to the problem; just as poisons introduced from without stimulate the cells to produce antitoxins, so harmful substances produced within the body are provided with anti-substances capable of neutralising their effects ; for this reason the blood does not normally clot within the blood-vessels, and Weinland has shown that the gastric epithelium forms an antipepsin, the intestinal epithelium an antitrypsin, and so on. The bodies of parasitic worms that live in the intestine are particularly rich in these anti-bodies.

\section{Mett's Tubes.}

A method which is now generally employed for estimating the proteolytic activity of a digestive juice is one originally introduced by Mett. Pieces of capillary glass-tubing of known length are filled with white of egg. This is set into a solid by heating to $95^{\circ} \mathrm{C}$. They are then placed in the digestive fluid at $36^{\circ} \mathrm{C}$., and the coagulated egg-white is digested. After a given time the tubes are removed; and if the digestive process has not gone too far, only a part of the little column of coagulated protein will have disappeared; the length of the remaining column is easily measured, and the length that has been digested is a measure of the digestive strength of the fluid.

Hamburger has used the same method in investigating the digestive action of juices on gelatin. The tubes are filled with warm gelatin solution, and this jellies on cooling. They are placed as before in the digestive mixture, and the length of the column that disappears can be easily measured. These experiments must, however, be performed at room temperature, for the usual temperature $\left(36^{\circ}-40^{\circ} \mathrm{C}\right.$. $)$ at which artificial digestion is usually carried out would melt the gelatin. He has also used the same method for estimating amylolytic activity, by filling the tubes with thick starch paste.

\section{Colour Tests for Gastric Acids.}

Hydrochloric acid is absent in some diseases of the stomach, notably in cancer; many colour tests for hydrochloric acid have been introduced from time to time, but by far the most characteristic and delicate is the following :-

Töpfer's test. A drop of dimethyl-amido-azo-benzol is spread in a thin film on a white plate. A drop of dilute hydrochloric acid (up to 1 in 10,000) strikes with this in the cold a bright red colour.

Lactic acid is soluble in ether, and is generally detected by making an ethereal extract of the stomach contents, and evaporating the ether. If lactic acid is present in the residue it may be identified by the following way :-

A solution of dilute ferric chloride and carbolic acid is made as follows :-

10 c.c. of a 4-per-cent. solution of carbolic acid.

20 c.c. of distilled water.

1 drop of the liquor ferri perchloridi of the British Pharmacopœia.

On mixing a solution containing a mere trace (up to 1 part in 10,000 ) of lactic acid with this violet solution, it is instantly turned yellow. Larger percentages of other acids (for instance, more than 0.2 per cent. of hydrochloric acid) are necessary to decolorise the test solution, but they do not turn the solution yellow.

Another colour test, that of Hopkins, is performed as follows:-5 c.c. of sulphuric acid and 3 drops of a saturated solution of copper sulphate are added to a few drops of lactic acid dissolved in alcohol. The mixture is placed in boiling water for five minutes, and then cooled; 2 drops of 0.2 per cent. alcoholic solution of thiophene are then added; on replacing the tube in boiling water, a cherry-red colour develops. 


\section{CHAPTER XXXIV}

\section{DIGESTION IN THE INTESTINES}

Here we have to consider the action of pancreatic juice, of bile, and of the succus entericus.

\section{The Pancreas.}

This is a tubulo-racemose gland closely resembling the salivary glands in structure. The principal differences are that the alveoli or acini are more tubular in character; the connective tissue between them is looser, and in it are small groups of epithelium cells (islets of Langerhans) which are supplied by a close network of capillaries (fig. 35y).

The secreting cells of the pancreas are polyhedral. When examined in the fresh condition, or in preparations preserved by osmic acid, their protoplasm is seen to be filled in the inner two-thirds with small granules; but tho outer third is left clear, and stains readily with protoplasmic dyes (fig. 358).

During secretion the granules are discharged; the clear zone consequently becomes wider, and the granular zone narrower.

These granules indicate the

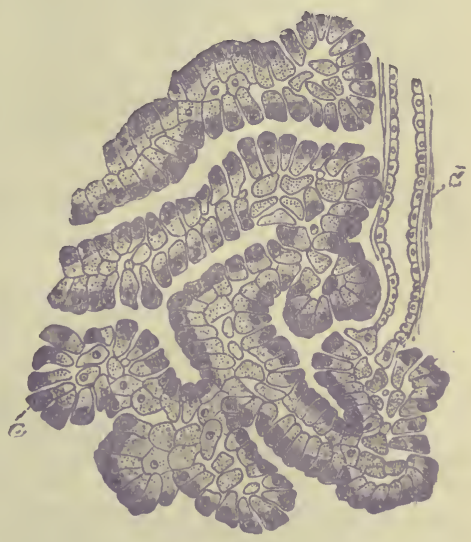

Fic. 35s.- Section of the pancreas of a dog during digestion. $a$, Alveoli lined with cells, the clear outer zone of which is well stained with hæmatoxylin; $d$, duct lined with short cubical cells. $\times 350$. (Klein and Noble Smith.)

\section{presence of a zymogen or more} probably of a mixture of zymogens, the precursors of the enzymes in the juice.

In the centre of the acini, spindle-shaped cells (centro-acinar cells) are often seen; their function and origin are unknown. 


\section{Composition and Action of Pancreatic Juice.}

The pancreatic juice may be obtained by a fistula in animals, a cannula being inserted into the main pancreatic duct; but as in the case of gastric juice, experiments on the pancreatic secretion are frequently performed with an artificial juice made by mixing a weak alkaline solution (1 per cent. sodium carbonate) with an extract cf pancreas which is usually made with glycerin.

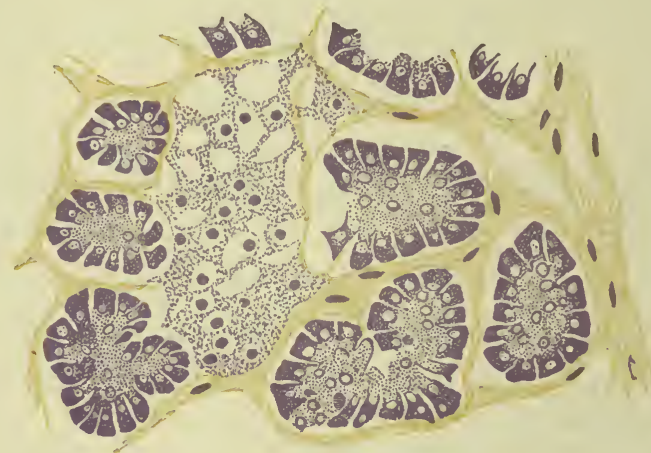

FIG. 359.- Section of the pancreas of armadillo, showing alveoli and an islet of Langerhans in the connective tissue. (V. D. Harris.)

Quantitative analysis of human pancreatic juice gives the following results :-

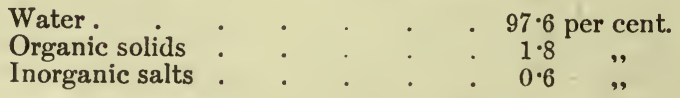

In the dog the amount of solids is much greater.

The organic substances in pancreatic juice are-

(a) Enzymes. These are the most important both quantitatively and functionally. They are four in number:-

i. Trypsin, a proteolytic or proteoclastic enzyme. In the fresh juice, however, this is present in the form of trypsinogen.

ii. Amylopsin, an amylolytic (amyloclastic) enzyme.

iii. Lipase, a fat-splitting or lipolytic (lipoclastic) enzyme.

iv. A milk-curdling enzyme.

(b) A small amount of protein matter, coagulable by heat.

(c) Traces of leucine, tyrosine, xanthine, and soaps.

The inorganic substances in pancreatic juice are-

Sodium chloride, which is the most abundant, and smaller quantities of potassium chloride, and phosphates of sodium, calcium, and 
magnesium. The alkalinity of the juice is due to phesphates and carbonates, especially of sodium.

1. Action of Trypsin.-Trypsin acts like pepsin, but with certain differences, which are as follows :-

(a) It acts in an alkaline, pepsin in an acid medium.

(b) It acts more rapidly than pepsin; deutero-proteoses can be detected as intermediate products in the formation of peptone; the primary proteoses have not been detected.

(c) Alkali-meta-protein is formed in place of the acid-metaprotein of gastric digestion.

(d) It acts more powerfully on certain proteins (such as elastin) which are difficult of digestion in gastric juice. It does not, however, digest collagen.

(e) Acting on solid proteins such as fibrin, it eats them away from the surface to the interior; there is no preliminary swelling as in gastric digestion.

$(f)$ Trypsin acts further than pepsin, and rapidly splits up the proteose and peptone which have left the stomach into simpler substances, the polypeptides. The polypeptides in their turn are resolved into their constituent amino-acids, such as leucine, tyrosine, alanine, aspartic acid, glutamic acid, arginine, tryptophane, and many others. The constitution and properties of these cleavage products are described on pp. 417 to 422 . In addition to these there is a certain amount of ammonia. The red colour which a tryptic digest strikes with chlorine or bromine water is due to the presence of tryptophane (indole-amino-propionic acid).

When once the peptone stage is passed, the products of further. cleavage no longer give the biuret reaction; hence they are frequently termed abiuretic.

A variable fraction of the protein molecule is broken off with comparative ease, so that certain free amino-acids appear in the mixture, at a time when the remainder are still linked together as polypeptides. But ultimately the whole molecule is resolved into amino-acids, either entirely separated or in very short polypeptide linkages.

It will thus be seen that there are two important differences between pepsin and trypsin; one is a difference of degree, trypsin being by far the more powerful and rapid catalyst; the second is a difference of kind, pepsin not being able to cleave polypeptides into amino-acids in the way trypsin can. The preliminary action of pepsin, however, is beneficial, for trypsin cleavage occurs more readily after pepsin has acted on a protein.

2. Action of Amylopsin.-The conversion of starch into maltose is the most rapid of all the actions of the pancreatic juice. Its power in this direction is much greater than that of saliva, and it will act 
even on unboiled starch. The absence of this enzyme in the pancreatic juice of infants is an indication that milk, and not starch, is their natural diet.

3. Action on Fats.-These are split by pancreatic lipase into glycerin and fatty acids. The fatty acids unite with the alkaline bases present to form soaps (saponification, see p. 415). If a glycerin extract of pancreas is filtered, the filtrate has no lipoclastic action; the material deposited on the filter is also inactive, but on mixing it with the inactive filtrate once more, a strongly lipoclastic material is obtained. In this way lipase is separable into two fractions: the material on the filter is inactive lipase; the material in the filtrate is its co-enzyme; the latter is not destroyed by boiling. Bile salts also activate the inactive lipase, and this explains the fact that bile favours fat-splitting.

Pancreatic juice also assists in the emulsification of fats; this it is able to do because it is alkaline, and it is capable of liberating fatty acids, which form soaps with the alkali present; the soap forming a film on the outer surface of each fat globule prevents them running together. Emulsions are much more permanent in the presence of such colloids as gum or protein. The presence of protein in the pancreatic juice renders it therefore specially suitable for the purpose of emulsification.

4. Milk-curdling Enzyme.-The addition of pancreatic extracts or pancreatic juice to milk causes clotting; but this action (which differs in some particulars from the clotting caused by rennet) can hardly ever be called into play, as the milk upon which the juice has to act has been already curdled by the rennin of the stomach.

\section{The so-called Peripheral Reflex Secretion of the Pancreas.}

One of the most effective ways of producing a flow of pancreatic juice is to introduce acid into the duodenum. Popielski and Wertheimer and Le Page showed that this flow still occurs when the nerves supplying the duodenum and pancreas have been cut through. Wertheimer also mentions that the flow can be excited by injection of acid into the jejunum, but not when it is injected into the lower part of the iloum. These authors concluded that the secretion is a local reflex, the centres being situated in the scattered ganglia of the pancreas, or, in the case of the jejunum, in the ganglia of the solar plexus.

This subject has been reinvestigated by Starling and Bayliss, and the results they have obtained are most noteworthy. They consider that the secretion cannot be reflex, since it occurs after extirpation of the solar plexus, and destruction of all nerves passing to an isolated loop of intestine. Moreover, atropine does not paralyse the secretory 
action. It must therefore be due to direct excitation of the pancreatic cells, by a substance. or substances conveyed to the gland from the bowel by the blood-stream. So many of the connections between organs are made by nerves (the telegraphic service of the body), that we are apt to forget the other messenger, the blood, whom we may compare to the postman.

The exciting substance is not acid; injection of 0.4 per cent. of hydrochloric acid into the blood-stream has no influence on the pancreas. The substance in question must be produced in the intestinal mucous membrane under the influence of the acid. This conclusion was confirmed by experiment. If the mucous membrane of the jejunum or duodenum is exposed to the action of 0.4 per cent. hydrochloric acid, a substance is produced which, when injected into the blood-stream in minimal doses, produces a copious secretion of pancreatic juice, and also, but to a less extent, of bile. This substance is termed secretin. It is associated with another substance which lowers arterial blood-pressure. The two substances are not identical, since acid extracts of the lower end of the ileum produce a lowering of blood-pressure, but have no excitatory influence on the pancreas.

Secretin is split off from a precursor, pro-secretin, which is present in relatively large amounts in the duodenal mucous membrane, and gradually diminishes as we descend the intestine. Pro-secretin can be dissolved out of the mucous membrane by normal saline solution. It has no influence on the pancreatic secretion. Secretin can be split off from it by boiling or by treatment with acid.

What secretin is chemically we do not yet know. It is soluble in alcohol and ether. It is not a protein, but probably is an organic substance of low molecular weight. It is, moreover, the same substance in all animals, and not specific to different kinds of animals.

Pawlow by experiments of a similar nature to those which led him to the discovery of the secretory nerves of the gastric mucous membrane, thought he had also discovered the secretory nerves of the pancreas in the vagus, and to a less extent in the splanchnic nerves. His failure to produce this result in some experiments he explained by the concomitant stimulation of secreto-inhibiting fibres. It is quite possible that nerves of this nature exist; but Pawlow's experiments do not prove their existence, because the passage of acid chyme into the duodenum was not excluded, and so he may only have been dealing with a production of secretin, the chemical stimulus to pancreatic activity.

Starling's work on secretin naturally led him and others to seek for other chemical messengers employed in the regulation of the activities of the body, and it has already been established that secretin is by no means a solitary instance of such. The general name given to these agents is that of hormone. The chemical 
substances secreted by such glands as the thyroid and suprarenal must be included under this term, and the part played by carbonic acid in the regulation of breathing (see p. 380) also comes into the same category. In our study of gastric digestion, we have seen the powerful peptogenic action of dextrin, a substance formed during the salivary digestion of starch; Edkins has given the name gastrin to the special hormone which is the result of the action of the salivary products on the gastric mucous membrane. Another example of a hormone is furnished by the material formed in the ovary, and which, passing into the maternal blood-stream, stimulates the mammary gland to action.

Adaptation of the Pancreas. - To a certain degree it cannot be doubted that the pancreas adapts its secretion to the work it has to do. 'Thus, whereas gastric juice has a maximal flow soon after the ingestion of food, the pancreatic flow does not attain its full force until some time later, that is, when it is wanted. The view that this is due to the hormone named secretin, which is not formed until the gastric contents enter the intestine, fully explains the reason for the delay.

But Pawlow went further than this, and stated that the proportion of the various enzymes of the juice was adapted to the proportions of proteins, carbohydrates, and fats in the food taken. Considerable doubt has been cast on these results, because of the failure to confirm one of the most remarkable instances of such adaptation; this is the power of the pancreas to secrete lactase (an enzyme capable of hydrolysing lactose into glucoses). Normal pancreatic juice contains no lactase, but certain observers stated that by feeding an animal on milk, the pancreas could be educated to secrete it. Careful experiments by Plimmer have recently shown this is not really so, and so much more stringent experimental conditions will have to be imposed before the other cases of adaptation can be considered proven.

Internal Secretion of the Pancreas-See Diabetes, next chapter.

\section{The Succus Entericus.}

Succus entericus has been obtained free from other secretions by means of a fistula. Thiry's method is to cut the intestine across in two places; the loop so cut out is still supplied with blood and nerves, as its mesentery is intact; this loop is emptied, one end is sown up, and the other stitched to the abdominal wound, and so a cul-de-sac from which the secretion can be collected is made. The continuity of the remainder of the intestine is restored by fastening together the upper and lower portions of the bowel from which the loop has been removed. Vella's method resembles Thiry's, except that both ends of the loop are sutured to the wound in the abdomen. Fig. 360 illustrates the two methods.

The succus entericus possesses the power of converting disaccharides into monosaccharides. This power it owes to three enzymes. Invertase or sucrase is the enzyme which inverts cane sugar-that is, it converts cane sugar into dextrose and lævulose. The original use of the term " inversion" has been explained on p. 410 . It may be extended to include the similar liydrolysis of other disaccharides, although there 
may be no formation of lævo-rotatory substances. The enzyme in the juice which converts maltose into dextrose is called maltase; and that which acts upon lactose is callod lactase.

Up till a few years ago little or nothing was known regarding the action of the intestinal juice beyond this, but investigations published since that time have altered this state of things, and in the light of these the succus entericus is seen to be a juice of the highest importance.

Pawlow was the first to show that one of its main actions is to reinforce and intensify the action of the pancreatic juice, especially in reference to its proteolytic power. Fresh pancreatic juice has practically no digestive power on proteins. Claude Bernard, the earliest to study the pancreatic secretion, entirely missed its tryptic action. On standing, the juice very slowly acquires proteolytic activity. Vernon has shown that much the same is true for extracts of the pancreas. There is no doubt that what the fresh juice con-
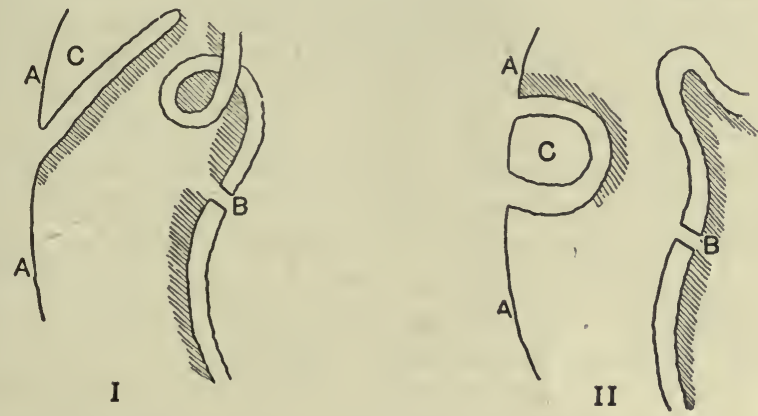

Fıo. 360.-Diagram of intestinal fistula. I., Thiry's method; II., Vella's method. A, Abdominal wall ; B, intestine, with mesentery; C, separated loop of intestine, with attached mesentery.

tains is trypsinogen, and this is slowly transformed into the active enzyme trypsin.

If fresh pancreatic and intestinal juices are mixed together, the result is a powerful proteolytic mixture, though neither juice by itself has any proteolytic activity.

Pawlow speaks of the substance in the intestinal juice which has this action as an "enzyme of enzymes," and has named it enterokinase.

Starling, like Pawlow, worked with dogs, and has confirmed his main results. A valuable contribution to the same subject has also been made by Hamburger. He has had the unusual opportunity of examining human succus entericus. It became necessary in a patient for surgical reasons to isolate a loop of the small intestine, and this loop continued to discharge intestinal juice to the exterior for some 
time after the operation. He found that this juice, like that of the dog, contains a substance which renders pancreatic juice active. $\mathrm{He}$ could not find that it exercised any activating influence on the fat-splitting and amylolytic enzymes of the pancreas, but its action on the tryptic enzyme was most marked. His quantitative experiments do not bear out Pawlow's view that the active substance in the intestinal juice is an enzyme, for it is unable, like an enzyme, to act on an unlimited amount of pancreatic juice. Starling, however, supports Pawlow's view; provided sufficient time is allowed to elapse, it will activate any amount of pancreatic juice.

Delezenne has advanced a hypothesis on the lines of Ehrlich's explanation of the action of hæmolysins (see p. 475). He regards trypsinogen as the amboceptor which enables the enterokinase to become effective.

Starling's subsequent work did not support this view. We may therefore best explain the action of enterokinase as an activating agent, by the fact that it is capable of transforming the zymogen trypsinogen into the effective enzyme trypsin. How it does this is explained by J. Mellanby and Woolley in the following way:-Trypsinogen is a complex consisting of trypsin united by a protein moiety, and so long as the enzymo is combined in this way it is inactive; enterokinase is a proteolytic enzyme which adsorbs and then digests this protein moiety, and thus liberates the trypsin.

The mixture of pancreatic and intestinal juice is extraordinarily powerful. If secretin is administered to a fasting animal, the juice secreted, having no food to act upon, will produce erosion and inflammation of the intestinal wall. (Starling.)

Dixon and Hamill's recent work has made clearer the mechanism of pancreatic secretion. There are in the pancreas three precursors of enzymes, namely, protrypsinogen, proamylopsin, and prolipase. Secretin combines chemically, or at any rate acts chemically, on all three; it liberates amylopsin and lipase from their precursors, and these two active enzymes pass into the pancreatic juice. It liberates trypsinogen from protrypsinogen, and trypsinogen passes into the juice; finally trypsinogen is converted into the active enzyme trypsin by the enterokinase of the succus entericus.

Another discovery in connection with succus entericus has been made by Otto Cohnheim. The juice has no action on native proteins such as fibrin and egg-white, but it acts on proteoses and peptone. It rapidly breaks them up into simpler substances, of which ammonia, leucine, tyrosine, and the hexone bases have been identified. Cohnheim has named the enzyme to which this is due erepsin. Hamburger found that erepsin is also present in the human juice; it is not identical with enterokinase, because erepsin is destroyed by heating the juice to $59^{\circ} \mathrm{C}$. for three hours; enterokinase is not destroyed 
until the temperature is raised to $67^{\circ} \mathrm{C}$. Other observers have confirmed the discovery of erepsin, but have found that it or a similar enzyme is present in most tissues; it is most abundant in the kidney (Vernon).

Cohnheim has investigated the action of erepsin on a large number of proteins; it acts energetically on proteoses, peptone, and protamines: on histone, which occupies an intermediate place between protamines and the other proteins, it has a slight action. On the other native proteins it has no action, with the single exception of caseinogen, which is speedily broken up into simple substances; this opens up the interesting physiological possibility that the suckling infant is able to digest its protein nutriment even if pepsin and trypsin are absent.

The bile, as .we shall find, has little or no digestive action by itself, but combined with pancreatic juice it assists the latter in all its actions. This is true for the digestion of starch and of protein, but most markedly so for the digestion of fat. Occlusion of the bileduct by a gall-stone or by inflammation prevents bile entering the duodenum. Under these circumstances the frees contain a large amount of undigested fat.

The importance of the work of Pawlow, and the other physiologists whose names have been mentioned, arises from the entirely new light thrown upon the digestion process as a whole. We have been too apt to think of the occurrences in the alimentary canal as a series of isolated phenomena. We now see that each step follows in an orderly manner as the result of the previous steps. For example, the acid gastric juice reaches the small intestine, and there produces secretin from its forerunner; the secretin is taken by the blood-stream to the pancreas, where it excites a flow of pancreatic juice; this juice arrives in the duodenum ready to act on starchy substances and on fat. With the assistance of the bile, fatty acid is liberated which in its turn forms more secretin, and so more pancreatic juice. The pancreatic juice, however, cannot act on proteins without enterokinase, which is supplied by the succus entericus; this sets free the trypsin; and trypsin with the assistance of erepsin effectively carries out digestive proteolysis.

\section{Bacterial Action.}

The gastric juice is an antiseptic; the pancreatic juice is not. An alkaline fluid like pancreatic juice is just the most suitable medium for bacteria to flourish in. Even in an artificial digestion the fluid is very soon putrid, unless special precautions to exclude or kill bacteria are taken. It is often difficult to say where pancreatic action ends and bacterial action begins, as many of the bacteria that grow in the intestinal contents (having reached that situation in 
spite of the gastric juice) produce enzymes which act in the same way as the pancreatic juice. Some form sugar from starch, others peptone, and amino-acids from proteins, while others, again, break up fats. There are, however, certain actions that are entirely due to these putrefactive organisms.

i. On carbohydrates. The most frequent fermentation they set up is the lactic acid fermentation : this may go further and result in the formation of carbonic acid, hydrogen, and butyric acid (see p. 411). Cellulose is broken up into carbonic acid and methane. This is the chief cause of the gases in the intestine, the amount of which is increased by vegetable food.

ii. On fats. In addition to acting like lipase, they produce lower acids (valeric, butyric, etc.). The formation of acid products from fats and carbohydrates gives to the intestinal contents an acid reaction. Recent researches show that the contents of the intestine become acid much higher up than was formerly supposed. Organic acids do not, however, hinder pancreatic digestion.

iii. On proteins. Peptones, amino-acids, and ammonia are produced; but the enzymes of these putrefactive organisms have a specially powerful action in liberating substances having an evil odour, such as indole $\left(\mathrm{C}_{8} \mathrm{H}_{7} \mathrm{~N}\right)$, skatole $\left(\mathrm{C}_{9} \mathrm{H}_{9} \mathrm{~N}\right)$, and phenol $\left(\mathrm{C}_{6} \mathrm{H}_{6} \mathrm{O}\right)$. Indole and skatole originate from the trytophane radical of proteins. There are also gaseous products in some cases.

Ammonia-producing organisms flourish best in the lower regions of the small intestine; the ammonia neutralises the organic acids produced higher up, and in the large intestine the contents have in consequence an alkaline reaction.

If excessive, putrefactive processes are harmful ; if within normal limits, they are useful, helping the pancreatic juice, and, further, preventing the entrance into the body of poisonous products. It is possible that, in digestion, poisonous alkaloids are formed. Certainly this is so in one well-known case. Lecithin, a material contained in small quantities in many foods, and in large quantities in egg-yolk and brain, is broken up by the pancreatic juice into glycerophosphoric acid, fatty acids, and an alkaloid called choline. We are, however, protected from the poisonous action of choline by the bacterial enzymes, which break it up into carbonic acid, methane, and ammonia.

Sour milk has been recently extolled not only as a useful food, but as a cure for many dyspeptic disorders. Although its efficacy in this direction has been much exaggerated, its usefulness in certain cases is explicable on the ground that the lactic acid bacillus, which is a harmless one in itself, possesses the power, when it is actively growing, of destroying other micro-organisms of a more harmful kind. 


\title{
CHAPTER XXXV
}

\author{
THE LIVER
}

THE Liver, the largest gland in the body, is an extremely vascular organ, and receives its supply of blood from two sources, viz., from the portal vein and from the hepatic artery, while the blood is returned from it into the vena cava inferior by the hepatic veins.

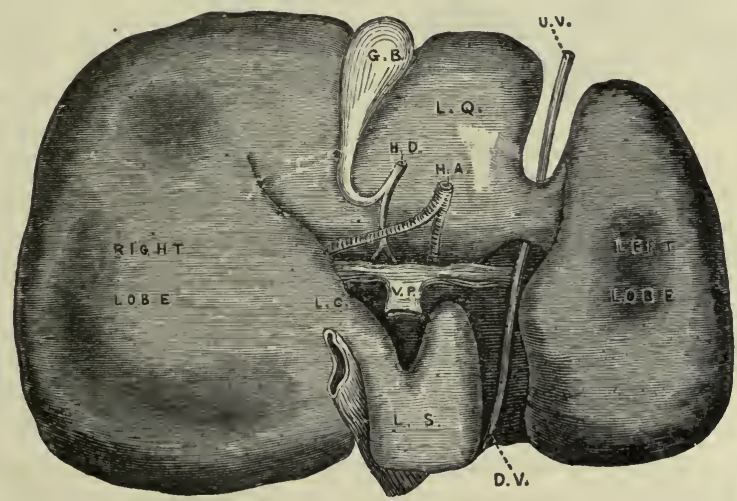

Fig. 361.-The under surface of the liver. G. B., Gall-bladder; H. D., common bile-duct; H. A., hepatic artery ; v. P., portal vein ; L. Q., lobulus quadratus ; L. S., Jobulus spigelii ; L. C., lobulus caudatus; D. v., ductus venosus; U. $\vee$., umbilical vein. (Noble Smith.)

Its secretion, the bile, is conveyed from it by the hepatic duct, either directly into the intestine, or, when digestion is not going on, into the cystic duct, and thence into the gall-bladder, where it accumulates until required.

The liver is in origin a tubular gland, but as development progresses it soon loses all resemblance to the tubular glands found elsewhere. It is made up of small roundish or oval portions called lobules, each of which is about $\frac{1}{20}$ of an inch (about $1 \mathrm{~mm}$.) in diameter, and composed of the liver cells, between which the bloodvessels and bile-vessels ramify. The hepatic cells (fig. 364), which form the glandular or secreting part of the liver, are of a spheroidal 
form, but somewhat polygonal from mutual pressure. Each possesses a nucleus, sometimes two. The cell protoplasm contains numerous fatty particles, as well as a variable amount of glycogen.

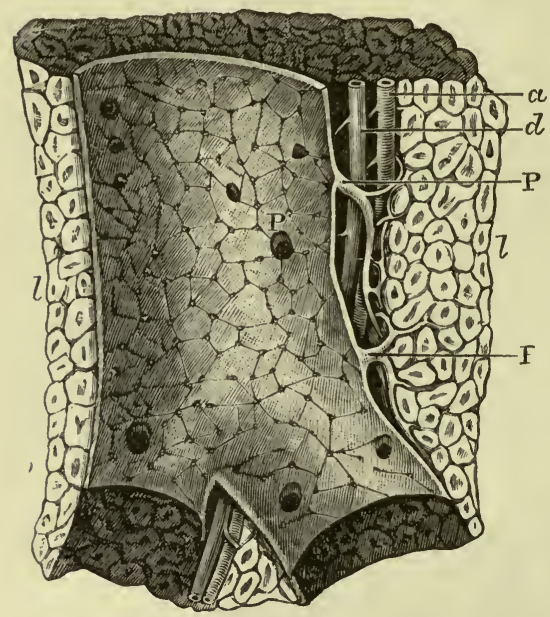

Fro. 362.-Longitudinal section of a portal canal, containing a portal vein, hepatic artery and hepatic duct, from the pig. $P$, Branch of vena portæ, situated in a portal canal amongst the lobules of the liver; $l, l$, and giving off interlobular veins; there are also seen within the large portal vein numerous orifices of interlobular veins arising directly from it; $a$, hepatic artery ; $d$, bile duct. $\times 5$. (Kiernan.)

The portal vein, hepatic artery, and hepatic duct, run in company, and their appearance on longitudinal section is shown in fig. 362. Running together through the substance of the liver, they are contained in small channels called portal canals, their immediate invest. ment being a sheath of areolar tissue continuous with Glisson's capsule.

In its course through the liver the portal vein gives off small branches which divide and subdivide between the lobules surrounding them and limiting them, and from this circumstance called interlobular veins. From these vessels a dense capillary network is prolonged into the substance of the lobule, and this network converges to a single small vein, occupying the centre of the lobule, and hence called intra-lobular. This arrangement is well seen in fig. 363 , which represents a section of a small piece of an injected liver.

The intra-lobular veins discharge their contents into veins called sub-lobular; these by their union, form the main branches of the hepatic veins, which leave the posterior border of the liver to end by two or three principal trunks in the inferior vena cava, just before its passage through the diaphragm.

The so-called capillaries of the liver are really sinusoids (see p. $222)$; they are in direct contact with the liver-cells, and are not surrounded with lymph spaces as in other secreting glands; their endothelial covering is in many places incomplete, and its cells are irregularly branched and more or less isolated from their neighbours. They are called the stellate cells of Kupffer. The result is that the blood comes into direct contact with the liver-cells.

The hepatic artery, the chief function of which is to distribute blood for nutrition to Glisson's capsule, the walls of the ducts and blood-vessels, and other parts of the liver, is distributed in a very 
similar manner to the portal vein, its blood being returned by small

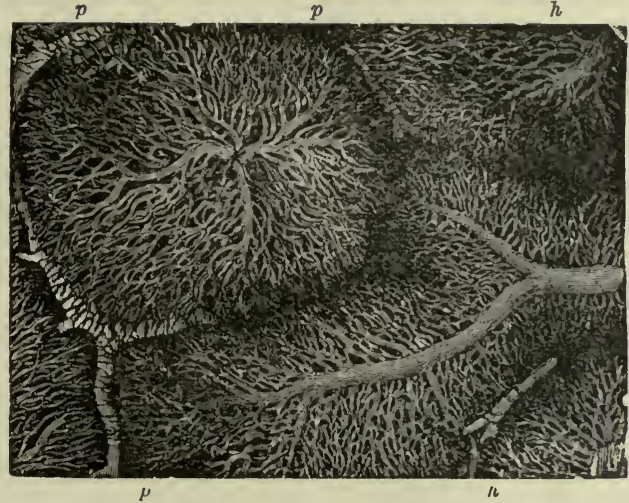

F1G. 363.-Capillary network of the lobules of the rabbit's liver. The figure is taken from a very successful injection of the liver veins, made by Harting: it shows nearly the whole of two lobules, and parts of three others; $p$, interlobular (portal) branclies running in the interlobular spaces; $h$, intralobular (hepatic) veins occupying the centre of the lobules. The interlobular and intralobular vessels are connected by radiating capillaries. $\times 45$. (Kölliker.)

branches which pass into the capillary plexus of the lobules which connects the inter-and intra-lobular veins.

The hepatic duct divides and subdivides in a manner very like that of the portal vein and hepatic artery, the larger branches being lined by columnar, and the smaller by small polygonal epithelium.

The bile capillaries commence between the hepatic cells, and are always bounded by hepatic cells on all sides, and are thus separated from the nearest blood capillary by at least the breadth of one cell (fig. 364).

To demonstrate the inter-cellular network of bile capillaries, Chrzonszezewsky employed a method of natural injection. A saturated aqueous solution of sulphindigotate of soda is introduced into the circulation of dogs and pigs by the jugular vein. The animals are killed an hour and a half afterwards, and the blood-vessels washed free from blood, or injected with gelatin stained with carmine. The bileducts are then seen filled with blue, and

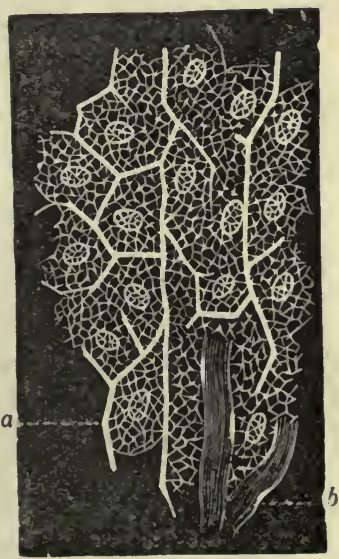

Fig. 364.-Portion of a lobule of liver. $a$, Bile capillaries between liver-cells; $b$, blood capillaries, $\times 350$. (Klein and Noble Smith.) the blood-vessels with red material. If the animals are killed sooner than this, the indigo pigment is found within the hepatic cells, thus demonstrating it was through their agency that the canals were filled. 
Pflüger and Kupffer later discovered that the relation between the hepatic cells and the bile canaliculi is even more intimate, for they demonstrated the existence of vacuoles in the cells communicating by minute intra-cellular channels with the adjoining bile canaliculi (fig. 365).

Intra-cellular canaliculi in the liver-cells are not unique. Recent research by Golgi's method has shown that in the salivary and gastric glands, and in the pancreas, there is a similar condition.
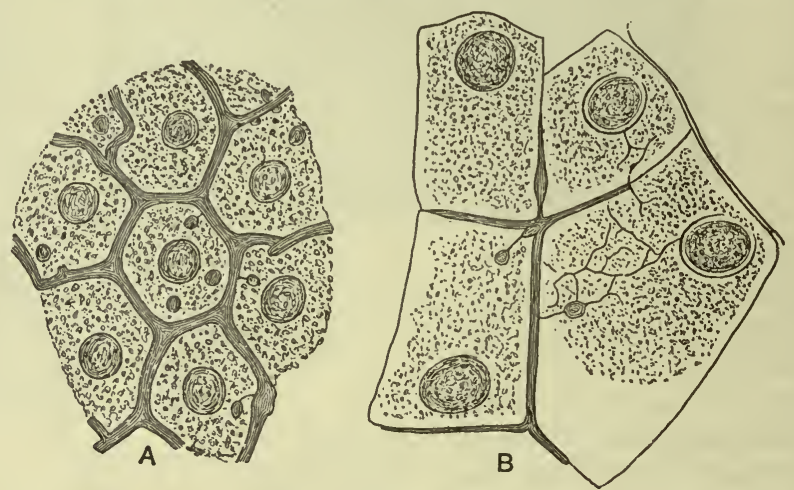

F1G. 365. - Sketches illustrating the mode of commencement of the bile canaliculi within the livercells (Heidenhain, after Kupffer). A, rabbit's liver, injected from hepatic duct with Berlin blue. The inter-cellular canaliculi give off minute twigs which penetrate into the liver-cells, and there terminate in vacuole-like enlargements. B, frog's liver naturally injected with sulph-indigotate of soda. A similar appearance is obtained, but the intra-cellular canaliculi are ramified.

Schäfer has further demonstrated that the liver-cells contain not only the intracellular bile canaliculi, but also intracellular blood canaliculi passing into the cells from the capillaries between them. These are too minute to admit blood-corpuscles. The liver-cells take certain materials from the plasma and elaborate the constituents of the bile, the bile-salts, and the bile pigments. There can be no doubt that these substances are formed by the hepatic cells, for they are not found in the blood nor in any other organ or tissue; and after extirpation of the liver they do not accumulate in the blood. We thus see that in the liver, lymph does not act as a middleman, as it does in the formation of other secretions.

The functions of the liver are connected with the general metabolism of the body, especially in connection with the metabolism of carbohydrates (glycogenic function) and of fats; its relationship to the metabolism of nitrogenous material (formation of urea, uric acid, etc.), we shall discuss with the urine. Another function is the formation of bile, which it will be convenient to take first. 


\section{Bile.}

Bile is the secretion of the liver which is poured into the duodenum: it has been collected in living animals by means of a biliary fistula; the same operation has occasionally been performed in human beings. After death the gall-bladder yields a good supply of bile which is more concentrated than that obtained from a fistula.

Bile is being continuously poured into the intestine, but there is an increased discharge soon after the arrival of food in the duodenum.

Though the chief blood supply of the liver is by a vein (the portal vein), the amount of blood in the liver varies with its needs, being increased during the periods of digestion. This is due to the fact that in the area from which the portal vein collects bloodstomach, intestine, spleen, and pancreas - the arterioles are all dilated, and the capillaries are thus gorged with blood. The peristalsis of the intestine and the pumping action of the spleen are additional factors in driving the blood onwards to the liver.

The bile is secreted from the portal blood at much lower pressure than one finds in glands such as the salivary glands, the blood supply of which is arterial. Herring and Simpson, in experiments performed upon numerous animals, found that the bile pressure averages $30 \mathrm{~mm}$. of mercury, which is about three times the pressure in the portal vein. This fact illustrates the general truth that secretory cells exercise pressure.

Nothing is known of any nervous agency which regulates the flow of bile; the stimulus appears to be of a chemical nature, and the increased flow which occurs soon after the arrival of the chyme in the intestine is chiefly due to the action of secretin, for this material stimulates the liver as well as the pancreas.

The chemical process by which the constituents of the bile are formed is obscure. We, however, know that the biliary pigment is produced by the decomposition of hæmoglobin. Bilirubin is, in fact, identical with the iron-free derivative of hæmoglobin called hæmatoidin, which is found in the form of crystals in old blood-clots such as occur in the brain after cerebral hæmorrhage (see p. 464).

An injection of hæmoglobin into the portal vein or of substances such as water which liberate hæmoglobin from the red blood-corpuscles produces an increase of bile pigment. If the spleen takes any part in the elaboration of bile pigment, it does not proceed so far as to liberate hæmoglobin from the corpuscles. No free hæmoglobin is discoverable in the blood plasma in the splenic vein.

The amount of bile secreted is differently estimated by different observers; the amount secreted daily in man varies from 500 c.c. to a litre (1000 c.c.). 
The constituents of the bile are the bile salts proper (taurocholate and glycocholate of soda), the bile pigments (bilirubin, biliverdin), a mucinoid substance, small quantities of fats, soaps, cholesterin, lecithin, urea, and mineral salts, of which sodium chloride and phosphates of calcium and iron are the most important.

Bile is a yellowish, reddish-brown, or green fluid, according to the relative preponderance of its two chief pigments. It has a musk-like odour, a bitter-sweet taste, and an alkaline reaction.

The specific gravity of human bile from the gall-bladder is 1026 to 1032 ; that from a fistula, 1010 to 1011 . The greater concentration of gall-bladder bile is partly explained by the addition to it from the walls of that cavity of the mucinoid material it secretes.

The amount of solids in gall-bladder bile is from 9 to 14 per cent., in fistula bile from 1.5 to 3 per cent. The following table shows that this low percentage of solids is almost entirely due to want of bile salts. This can be accounted for in the way first suggested by Schiff - that there is normally a bile circulation going on in the body, a large quantity of the bile salts that pass into the intestine being first split up, then reabsorbed and again secreted. Such a circulation would obviously be impossible in cases where all the bile is discharged to the exterior.

The following table gives some analyses of human bile:-

\begin{tabular}{|c|c|c|c|}
\hline Constitueuts. & $\begin{array}{c}\text { Fistula bile } \\
\text { (healthy woman. } \\
\text { Copeman and } \\
\text { Winston). }\end{array}$ & $\begin{array}{l}\text { Fistula bile (case } \\
\text { of cancer. Yoo } \\
\text { and Herroun). }\end{array}$ & $\begin{array}{l}\text { Normal bile } \\
\text { (Frerichs). }\end{array}$ \\
\hline $\begin{array}{l}\text { Sodium glycocholate } \\
\text { Sodium taurocholate } \\
\text { Cholesterin, lecithin, fat } \\
\text { Mucinoid material : } \\
\text { Pigment : } \\
\text { Inorganic salts }\end{array}$ & $\begin{array}{l}0.6280 \\
0.0990 \\
0 \cdot 1725 \\
0.0725 \\
0.4510\end{array}$ & $\begin{array}{l}0.165 \\
0.055 \\
0.038 \\
0.148 \\
0.878\end{array}$ & $\begin{array}{l}9 \cdot 14 \\
1 \cdot 18 \\
2 \cdot 98 \\
0 \cdot 78\end{array}$ \\
\hline $\begin{array}{l}\text { Total solids } \\
\text { Water (by difference) }\end{array}$ & $\begin{array}{r}1 \cdot 4230 \\
98 \cdot 5570\end{array}$ & $\begin{array}{r}1 \cdot 284 \\
98 \cdot 716\end{array}$ & $\begin{array}{l}14 \cdot 08 \\
85 \cdot 92\end{array}$ \\
\hline
\end{tabular}

Bile Mucin.-There has been considerable diversity of opinion as to whether bile mucin is really mucin. The most recent work in Hammarsten's laboratory shows that differences occur in different animals. Thus in the ox there is very little true mucin, but a great amount of nucleo-protein; in human bile, on the other hand, there is very little if any nucleo-protein; the mucinoid material present there is really mucin.

The Bile Salts.-The bile contains the sodium salts of complex 
amino-acids called the bile acids. The two acids most frequently found are glycocholic and taurocholic acids. The former is the more abundant in the bile of man and herbivora; the latter in carnivorous animals, such as the dog. An important difference between the two is that taurocholic acid contains sulphur, and glycocholic acid does not.

Glycocholic acid $\left(\mathrm{C}_{26} \mathrm{H}_{43} \mathrm{NO}_{6}\right)$ is by the action of dilute acids and alkalis, and also in the intestine, hydrolysed and split into glycine or amino-acetic acid and cholalic acid.

$$
\begin{aligned}
& \mathrm{C}_{26} \mathrm{H}_{43} \mathrm{NO}_{6}+\mathrm{H}_{2} \mathrm{O}=\mathrm{CH}_{2} \cdot \mathrm{NH}_{2} \cdot \mathrm{COOH}+\mathrm{C}_{24} \mathrm{H}_{40} \mathrm{O}_{5} \text {. } \\
& \text { [Glycocholic acid.] } \\
& \text { [Glycine.] } \\
& \text { [Cholalic acid.] }
\end{aligned}
$$

The glycocholate of soda has the formula $\mathrm{C}_{26} \mathrm{H}_{42} \mathrm{NaNO}_{6}$.

Taurocholic acid $\left(\mathrm{C}_{26} \mathrm{H}_{45} \mathrm{NO}_{7} \mathrm{~S}\right)$ similarly splits into taurine or amino-isethionic acid and cholalic acid.

$$
\begin{aligned}
& \mathrm{C}_{26} \mathrm{H}_{45} \mathrm{NO}_{7} \mathrm{~S}+\mathrm{H}_{2} \mathrm{O}=\left(\mathrm{CH}_{2}\right)_{2} \cdot \mathrm{NH}_{2} \cdot \mathrm{SO}_{2} \cdot \mathrm{OH}+\mathrm{C}_{24} \mathrm{H}_{40} \mathrm{O}_{5} \\
& \text { [Taurocholic acid.] [Taurine.] [Cholalic acid.] }
\end{aligned}
$$

The taurocholate of soda has the formula $\mathrm{C}_{26} \mathrm{H}_{44} \mathrm{NaNO}_{7} \mathrm{~S}$.

The colour reaction called Pettenkofer's reaction is due to the presence of cholalic acid. Small quantities of cane sugar and strong sulphuric acid are added to the bile. The sulphuric acid acting on sugar forms a small quantity of a substance called furfuraldehyde, in addition to other products. The furfuraldehyde gives a brilliant purple colour with cholalic acid.

The Bile Pigments. - The two chief bile pigments are bilirubin and biliverdin. Bile which contains chiefly the former (such as dog's bile) is of a golden or orange-yellow colour, while the bile of many herbivora, which contains chiefly biliverdin, is either green or bluishgreen." Human bile is generally described as containing chiefly bilirubin; but this is by no means always so. The bile pigments show no absorption bands with the spectroscope.

Bilirubin has the formula $\mathrm{C}_{32} \mathrm{H}_{36} \mathrm{~N}_{4} \mathrm{O}_{6}$ : it is thus an iron-free derivative of hæmoglobin. The iron is apparently stored up in the liver cells, perhaps for future use in the manufacture of new hæmoglobin. The bile contains only a trace of iron.

Biliverdin has the formula $\left(\mathrm{C}_{16} \mathrm{H}_{18} \mathrm{~N}_{2} \mathrm{O}_{4}\right)_{x}$; the value of $x$ is uncertain: it may occur as such in bile; it may be formed by simply exposing red bile to the oxidising action of the atmosphere; or it may be formed as in Gmelin's test by the more vigorous oxidation produced by fuming nitric acid.

Gmelin's test consists in a play of colours-green, blue, red, and finally yellow, produced by the oxidising action of fuming nitric acid (that is, nitric acid containing nitrous acid in solution). The end or yellow product is called choletelin, $\mathrm{C}_{32} \mathrm{H}_{36} \mathrm{~N}_{4} \mathrm{O}_{12}$.

Hydrobilirubin. - If a solution of bilirubin or biliverdin in dilute 
alkali is treated with sodium amalgam or allowed to putrefy, a brownish pigment, which is a reduction product, is formed called hydrobilirubin, $\mathrm{C}_{32} \mathrm{H}_{40} \mathrm{~N}_{4} \mathrm{O}_{7}$. It shows a dark absorption band between $b$ and $F$, and a fainter band in the region of the $\mathrm{D}$ line.

This substance is interesting because a similar substance is formed from the bile pigment by reduction processes in the intestine, and constitutes stercobilin, the pigment of the fæces. Some of this is absorbed and ultimately leaves the body in the urine as one of its pigments called urobilin. A small quantity of urobilin is sometimes found preformed in the bile. The identity of urobilin and stercobilin has been frequently disputed, but the recent work of Garrod and Hopkins has confirmed the old statement that they are the same substance with different names. Hydrobilirubin differs from urobilin in containing more nitrogen ( $9 \cdot 2$ instead of $4 \cdot 1$ per cent.).

Cholesterin.-Small quantities of this substance are found in normal bile. It may occur in excess, and form the concretions known as gall-stones, which are usually more or less tinged with bilirubin. Its properties and reactions are described on p. 435.

The Uses of Bile.-Bile is doubtless, to a certain extent, excretory. Some state that it has a slight action on fats and carbohydrates, but its principal action is as a coadjutor to the pancreatic juice (especially in the digestion of fat). In some animals it has a feeble diastatic power.

Bile is said to be a natural antiseptic, lessening the putrefactive processes in the intestine. This is very doubtful. Though the bile salts are weak antiseptics, the bile itself is readily putrescible, and the power it has of diminishing putrescence in the intestine is due chiefly to the fact that by increasing absorption it lessens the amount of putrescible matter in the bowel.

When the bile meets the chyme the turbidity of the latter is increased owing to the precipitation of unpeptonised protein. This is an action due to the bile salts, and it has been surmised that this conversion of the chyme into a more viscid mass is to hinder somewhat its progress through the intestines; it clings to the intestinal wall, thus allowing absorption to take place. Bile stimulates peristalsis in the large intestine.

Bile is alkaline; it therefore assists the pancreatic juice in neutralising the chyme that leaves the stomach. It assists the absorption of fats (see p. 548). It is also a solvent of fatty acids.

We have seen that fistula bile is poor in solids as compared with normal bile, and that this is explained on the supposition that the normal bile circulation is not occurring - the liver cannot excrete what it does not receive back from the intestine. Schiff was the first to show that if the bile is led back into the duodenum, or even if the animal is fed on bile, the percentage of solids in the bile excreted is 
at once raised. It is on these experiments that the theory of a bile circulation is mainly founded. The bile circulation relates, however, chiefly, if not entirely, to the bile salts: they are found but sparingly in the frces; they are only represented to a slight extent in the urine: hence it is calculated that seven-eighths of them are reabsorbed from the intestine. Small quantities of cholalic acid, taurine, and glycine are found in the fæces; the greater part of these products of the decomposition of the bile salts is taken by the portal vein to the liver, where they are once more synthesised into the bile salts. Some of the taurine is absorbed and excreted as tauro-carbamic acid in the urine. Some of the absorbed glycine may be excreted as urea. The pigment is changed into stercobilin and leaves the body partly in the fæces, but some is absorbed and is finally excreted as urobilin in the urine. The cholesterin in the fæces was formerly supposed to be a bile-residue; but in some animals, especially those which feed on grass, the source of the fæcal cholesterin is the phytosterin (vegetable cholesterin) in the food. In some cases it is reduced and forms a derivative termed coprosterin (Austin Flint's stercorin).

The bile-expelling mechanism must be carefully distinguished from the bile-secreting action of the liver-cells. The bile is forced into the ducts, and ultimately into the duodenum, by the pressure of newly-formed bile pressing on that previously in the ducts, and this is assisted by the contraction of the plain muscular fibres of the larger ducts and gall-bladder, which occurs when the food enters the duodenum. In cases of obstruction, as by a gall-stone, in the ducts, this action becomes excessive, and gives rise to the intense pain known as hepatic colic.

Many cholagogues (bile-drivers), such as calomel, act on the bileexpelling mechanism and increase the peristalsis of the muscular tissue; they do not really cause an increased formation of bile.

Jaundice.-The commonest form of jaundice is produced by obstruction in the bile-ducts preventing the bile entering the intestine. A very small amount of obstruction, for instance, a plug of mucus produced in excess owing to inflammatory processes, will often be sufficient, as the bile is secreted at comparatively low pressure. Under these circumstances, the fæces are whitish or clay coloured, and the bile passing backwards into the lymph,* enters the blood and is thus distributed over the body, causing a yellow tint in the skin and mucous membranes, and colouring the urine.

In some cases of jaundice, however (e.g., produced by various poisons), there is no obvious obstruction; the causes of nonobstructive, or blood-jaundice, form a pathological problem of some interest. Some years ago it was believed that the bile pigment was

* The absorption is by the lymph, because if jaundice is produced in an animal by ligature of the bile duct, it will cease when the thoracic duct is tied, 
actually produced in the blood. But all recent work shows that the liver is the only place where production of bile occurs, and that in all cases of so-called non-obstructive jaundice, the bile is absorbed from the liver. There may be obstruction present in the smaller ducts, or the functions of the liver may be so upset that the bile passes into the lymph even when there is no obstruction.

\section{The Glycogenic Function of the Liver.}

The important fact that the liver normally forms sugar, or a substance readily convertible into it, was discovered by Claude Bernard in the following way: He fed a dog for seven days with food containing a large quantity of sugar and starch; and, as might be expected, found sugar in both the portal and hepatic blood. But when this dog was fed with meat only, to his surprise, sugar was still found in the blood of the hepatic veins. Repeated experiments gave invariably the same result; no sugar was found, under a meat diet, in the portal vein, if care were taken, by applying a ligature on it at the transverse fissure, to prevent reflux of blood from the hepatic venous system. Bernard found sugar also in the substance of the liver. It thus seemed certain that the liver formed sugar, even when, from the absence of carbohydrates in the food, none could have been brought directly to it from the stomach or intestines.

Bernard found, subsequently, that a liver, removed from the body, and from which all sugar had been completely washed away by injecting a stream of water through its blood-vessels, contained sugar in abundance after the lapse of a fow hours. This post-mortem production of sugar was a fact which could only be explained on the supposition that the liver contained a substance readily convertible into sugar; and this theory was proved to be correct by the discovery of a substance in the liver allied to starch, and now termed glycogen or animal starch. We are thus led to the conclusion that glycogen is formed first and stored in the liver cells, and that the sugar, when present, is the result of its transformation.

Source of Glycogen.-Although the greatest amount of glycogen is produced by the liver upon a diet of starch or sugar, a certain quantity is produced upon a protein diet. It must, then, be produced by protoplasmic activity within the cells. The glycogen when stored in the liver cells may readily be demonstrated in sections of liver containing it by its reaction (red colour) with iodine, and moreover, when the hardened sections are soaked in water to dissolve out the glycogen, the protoplasm of the cell may be so vacuolated as to appear little more than a framework. In the liver of a hibernating frog the amount of glycogen stored up in the liver cells is very considerable. 
Average Amount of Glycogen in the Liver of Dogs under various Diets (Pavy).

\begin{tabular}{|c|c|}
\hline $\begin{array}{l}\text { Diet. } \\
\text { Animal food }\end{array}$ & $\begin{array}{l}\text { Amount of } \\
\text { Glycogen in Liver } \\
7 \cdot 19 \text { per cent. }\end{array}$ \\
\hline $\begin{array}{l}\text { Animal food with sugar (about } \frac{1}{4} \text {-lb of sugar daily) } \\
\text { Vegetable diet (potatoes, with bread or barley-meal). }\end{array}$ & $\begin{array}{l}14 \cdot 5 \\
17 \cdot 23\end{array}$ \\
\hline
\end{tabular}

The dependence of the formation of glycogen on the kind of food taken is also well shown by the following results, obtained by the same experimenter :-

Average Quantity of Glycogen found in the Liver of Rabbits after Fasting, and after a Diet of Starch and Sugar respectively.

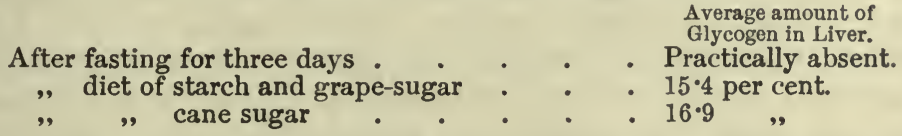

The diet most favourable to the production of a large amount of glycogen is a mixed diet containing a large amount of carbohydrate, but with some protein. It is also possible that fats may form a source of glycogen, in virtue of the glycerin they contain.

Destination of Glycogen.-There are two chief theories as to the destination of the hepatic glycogen. (1) That the glycogen is converted into sugar during life by the agency of an enzyme (liver diastase or glycogenase) found in the liver; and that the sugar is conveyed away by the blood of the hepatic veins, to undergo combustion in the tissues. (2) That the conversion into sugar only occurs after death, and that during life glycogen is transformed into fat.

The first view is that of Claude Bernard, and has been adopted by the majority of physiologists. The second view is that of Pavy: he denies that the liver is a sugar-forming organ, he regards it as a sugar-destroying organ; the sugar is stored as animal starch, but never again leaves the liver as sugar during life. He has been unable to find more sugar in the hepatic blood than in the portal blood. Other observers have found an increase in the sugar of the blood leaving the liver, but the accurate estimation of sugar in a fluid rich in proteins is a matter of great difficulty. Even if the increase is so small as hardly to be detected, it must be remembered that the whole blood of the body passes through the liver about once a minute, so that a very small increase each time would mount up to a large total.

Pavy further denies that the post-mortem formation of sugar from glycogen that occurs in an excised liver is a true picture of what occurs during life, but is due to an enzyme which is only formed after death. During life, he regards the glycogen as a source of other substances, such as fat and protein. It is certainly a fact that increase 
of carbohydrate food leads to the formation of fat in the body and in the liver-cells. In support of the theory that glycogen may also contribute to the formation of proteins, he has shown that many proteins contain a carbohydrate radical.

The prevalent opinion is that the liver-cells may be able to convert part of the store of glycogen into fat; part also of the sugar formed from glycogen may unite with protein to form a glucoprotein; but most of the glycogen leaves the liver as sugar (dextrose), so justifying the name (literally, mother-substance of sugar) given to it by Bernard.

Diabetes.-In certain disorders of metabolism, excess of sugar occurs in the blood, and leaves the body by the urine (glycosuria). Under normal circumstances, the transformation of the hepatic glycogen into sugar is a sufficiently slow process to keep the sugar in the blood at such a low percentage that glycosuria does not occur. Glycosuria takes place when the transformation of glycogen into sugar is excessive, as in puncture diabetes, described below.

"Alimentary glycosuria" is usually a temporary condition, in which either the diet contains too much carbohydrate for the liver to store as glycogen, or else the liver is comparatively inactive and incapable of dealing with the usual carbohydrate supply. This state of things may be remedied by reducing the amount of carbohydrate ingested, or by improving the condition of the liver. The normal "assimilation limit" for dextrose in man given in one dose by the mouth is about 200 grammes.

We must, however, remember that sugar is not poured into the blood to accumulate there, but is removed by the muscular and other tissues which the blood traverses, and is there burnt to serve as a source of energy; if the tissues are unable to utilise the sugar in this way, it accumulates in the blood and overflows into the urine; this is the usual condition in the disease called diabetes mellitus in man; and a similar condition may be produced in animals by removal of the pancreas. Many cases of diabetes mellitus in man are due to disease of the pancreas. In many cases the diabetic condition may be removed by rigid abstention from starchy and saccharine food. In other cases diet makes little or no difference; in this condition the sugar must come from the metabolism of the protein constituents of protoplasm; 40 per cent. or more of the katabolised protein may leave the body as sugar, certain of its cleavage products (for instance, alanine, see p. 615) acting as intermediate substances in sugar formation. This serious condition is analogous to what can be produced artificially by the poison known as phloridzin, and is possibly produced in man by some poison acting in a similar way.

The principal ways in which diabetes may be produced are:-

(1) By diabetic puncture.-Claude Bernard was the first to show 
that injury to the floor of the fourth ventricle in the region of the vasomotor centre leads to glycosuria. In man, also, disease of the bulb is frequently associated with glycosuria. These observations led to the erroneous conclusion that diabetes is always of nervous origin. The diabetes produced in this way cannot be the result of vaso-motor disturbances, but is due to an influence on the glycogenolytic nervous mechanism (see p. 541), and the glycosuria only occurs when the liver has within it a store of glycogen.

(2) By extirpation of the pancreas.-Minkowski and v. Mering in 1889 showed, that complete extirpation of the pancreas produces in animals a diabetic condition, even if no carbohydrate food is contained in the diet. The disease terminates fatally within a few weeks. If the removal is not complete, the intensity of the glycosuria depends upon the amount of pancreatic tissue left behind. One-fourth to a fifth of the gland is usually sufficient to prevent the occurrence of the diabetic state. It does not depend on the connection of the pancreas with the intestine, and this proves that the suppression of pancreatic juice is not the cause of the diabetes. The same conclusion was reached in other experiments in which the gland ducts were occluded by paraffin wax; no glycosuria resulted. Moreover, the effect of pancreatic extirpation, so far as diabetes is concerned, can be prevented by grafting a portion of the pancreas into the abdominal wall, or even under the skin. It is therefore believed that the pancreas forms an internal secretion, in addition to its external secretion or pancreatic juice. The former passes into the blood and plays an essential part in carbohydrate metabolism.

The islets of Langerhans have by many been held responsible for this portion of the duties of the pancreas. The evidence in favour of this view is that in human diabetes the islets are frequently degenerated, atrophied, or even absent; in animals, ligature of the pancreatic ducts leads to atrophy of the pancreatic acini, but not of the islets, and under those conditions no glycosuria occurs.

This view may be accepted with considerable confidence, for the observations of certain investigators that the islets merely represent a stage in the development of the ordinary acini, have been disproved or at any rate discredited.

We have seen that in diabetes, the power of the tissue cells to burn sugar is lessened; nevertheless, other diseases in which diminished oxidation occurs are not necessarily accompanied with glycosuria. The difficulty in diabetes probably lies in an impairment of the capacity of the cells of the body to prepare the sugar for oxidation. In this process the sugar or its derivative glycuronic acid is split into smaller molecules, and ultimately into water and carbon dioxide. If two hydrogen atoms in the $\mathrm{CH}_{2} \mathrm{OH}$ group of dextrose are replaced.. by one of oxygen, we obtain glycuronic acid. 
This oxidation is readily brought about in the body, and glycuronic acid is usually found in diabetic urine; but the further oxidation into water and carbon dioxide is a more difficult task, because it involves the disruption of the linkage of the carbon atoms. Possibly it is here that the internal secretion of the pancreas comes in.

Cohnheim discovered that muscle by itself and that an extract of pancreas by itself have very little effect in destroying sugar at body temperature; but if a mixture is made of surviving muscle, pancreatic extract, and sugar, the last-named substance rapidly disappears. Several observers have confirmed this observation, but others attribute it to bacterial action; according to this view, the pancreas forms a substance which activates the glycolytic (sugardestroying) enzyme of muscular tissue. The activating substance is not an enzyme, because the pancreatic extract does not lose this property when it is boiled.

Levene, like others, finds that sugar disappears when mixed with muscle and pancreatic extract, but he does not regard this as glycolysis in the usual acceptation of the word, namely, combustion into carbonic acid and water. He considers that the sugar disappears because its molecules are condensed into a heavier carbohydrate, and states that the sugar can once more be recovered from the mixture after hydrolysis with acid. If this is confirmed the view above advanced will need revision, and the action of the pancreatic hormone is best explained, as suggested on the next page, as antagonistic to that of adrenaline. Of all the tissue cells examined by Levene, the leucocytes alone were found to possess real glycolytic power, lactic acid being an intermediate stage before the final products (carbonic acid and water) are reached.

(3) By administration of phloridzin. - Many drugs produce temporary glycosuria; some, such as morphine, may act on the diabetic nerve-centre; others, such as anæsthetics and carbon monoxide, may upset the balance between the blood-gases, and thus affect tissue respiration and lead to an accumulation of sugar in the blood. The most potent poison in producing a diabetic state is, however, phloridzin, which is a glucoside, but the sugar passed in the urine is far too great to be accounted for by the small amount of sugar derivable from the drug. Besides that, phloretin, a derivative of phloridzin, free from sugar, produces the same results.

Phloridzin produces diabetes in starved animals, or in those in which any carbohydrate store must have been got rid of by the previous administration of the same drug. Phloridzin-diabetes is therefore analogous to those intense forms of diabetes in man in which the sugar must be derived from protein metabolism. The increase in protein metabolism is signalised by the rise in the output of nitrogen; in these cases the ratio of dextrose to nitrogen in the urine is $3 \cdot 6: 1$. If such a ratio occurs in man on a diet free from carbohydrates, a serious condition is revealed; Graham Lusk calls it the "fatal ratio."

A puzzling feature is the absence of an increase of free sugar in 
the blood; if the phloridzin is directly injected into one renal artery, sugar rapidly appears in the secretion of that kidney; some explain this by supposing that the sugar is formed within the kidney cells from some substance in the blood, possibly protein; whereas others consider that the kidney is rendered so permeable to sugar, that the percentage in the blood is kept at a low figure. This view is supported by Underhill's recent work: he has shown that in dogs and rabbits, if the renal structures are ligatured, or the kidney injured by sodium tartrate, the secretion of urine ceases, and after the administration of phloridzin, the sugar in the blood rises considerably over the normal.

(4) By administration of adrenaline.-This drug also produces a diabetic condition, but in this case there is excess of sugar in the blood also. Recent research indicates that adrenaline produces an increased discharge of sugar from the liver, and that under normal conditions this is regulated by an antagonistic hormone present in the internal secretion of the pancreas.

Glycogenolytic Nerves.-The disappearance of glycogen from the liver cells after the stimulation of the splanchnic nerves can be seen histologically. This is due to a direct influence of the nerves on the liver cells, for the effect is obtained after the circulation is stopped by ligature of the aorta and portal vein.

The most complete work on this subject is that by J. J. R. Macleod, who finds that the glycogenolytic fibres (the action of which is to increase the sugar in the blood at the expense of the hepatic glycogen) are demonstrable with certainty only in the case of the greater splanchnic nerves. If it occurs as the result of vagus stimulation (as Bernard stated), it is due to the asphyxia which is produced; if precautions are taken to prevent asphyxia, no increase of the blood sugar is found. In asphyxia it is increase of carbonic acid, and not loss of oxygen, which produces the glycosuric condition, and the former gas probably acts directly on the liver cells themselves.

\section{The Liver and Fat Metabolism.}

The work of Leathes, Hartley, and others has shown that the liver has an important use in the preparation of fats for their final disintegration into carbonic acid and water. The fat stored in adipose tissue must first be transported into the bloodstream; the lipase found in connective tissue liberates the glycerin and fatty acids, and thus renders such transportation possible. It is first taken to the liver, where it can be easily detected, but not in other organs, and the rupture of the long carbon chains of the fatty acid begins: it is first desaturated and then broken up into lower fatty acids such as caproic and butyric. The unsaturated products 
which are next found in the cells of other organs throughout the body disintegrate, probably where the unsaturated links have been introduced, and the lower acids so formed by successive oxidations break down to molecules of the size of acetic acid, and are lastly completely burnt to carbonic acid and water.

Acidosis.-This condition is seen in diabetes; poisonous acids in the blood produce a state of coma, or deep unconsciousness, which may finally cause death. For a diabetic is not only unable to burn and so utilise carbohydrate, but he fails in a similar way in his utilisation of fat. Butyric acid and $\beta$-hydroxybutyric acid are probably normal intermediate products in fat katabolism, but a healthy man on a normal diet is able still further to oxidise them into carbonic acid and water. But on an abnormal diet, for instance, when carbohydrate food is absent, fat-cleavage largely stops short at the hydroxybutyric acid stage; consequently this and possibly other related fatty acids accumulate and cause acidosis; this condition is increased the more fat is given in the food, and the acidosis of diabetes is similarly increased by fatty food. These poisonous acids were once believed to originate from proteins; if that were so there ought to be an increase of other protein katabolites in the urine, which there is not. The acids decrease the alkalinity and carbonic acid of the blood, and the ammonia of the urine is increased; this indicates an attempt of the body to neutralise the acids.

The hydroxybutyric acid does not pass entirely unchanged into the urine. $\beta$-Hydroxybutyric acid is $\mathrm{CH}_{3}$. $\mathrm{CHOH} . \mathrm{CH}_{2} . \mathrm{COOH}$. By oxidation, the two hydrogen atoms in thick type are removed to form water, and this leaves $\mathrm{CH}_{3} . \mathrm{CO} \cdot \mathrm{CH}_{2}$. $\mathrm{COOH}$, which is acetoacetic acid: when the $\mathrm{COO}$ in thick type is removed we get acetone $\left(\mathrm{CH}_{3} \cdot \mathrm{CO} . \mathrm{CH}_{3}\right)$, which gives the breath and urine of such patients an apple-like smell.

In these changes the liver plays an important part by means of certain enzymes which Dakin has proved to exist. One enzyme, called $\beta$-hydroxybutyrase, is an oxidase; it oxidises the $\beta$-hydroxybutyric into aceto-acetic acid, and its action is increased by the addition of blood or oxyhæmoglobin, which furnishes the necessary oxygen. It probably is active in health as well as in disease, the aceto-acetic acid being finally burnt into carbonic acid and water. The other enzyme which forms acetone is not an oxidative one, and acetone formation probably never occurs in the healthy state.

Fat Synthesis.- So much for the relationship of the liver to fat katabolism; but it appears that the liver is also important in the building up of fats, especially of those complex fats called.phosphatides. It is, however, possible that this is not exclusively the property of liver cells: when once the desaturated acids are supplied by the liver, each organ can make its own phosphatides for itself. 


\section{CHAPTER XXXVI}

\section{THE ABSORPTION OF FOOD}

FooD is digested in order that it may be absorbed. It is absorbed in order that it may be assimilated, that is, become an integral part of the living material of the body. The digested food thus diminishes in quantity as it passes along the alimentary canal, and the fæces contain the undigested or indigestible residue.

In the mouth and œsophagus the thickness of the epithelium and the quick passage of the food through these parts reduce absorption to a minimum. Absorption takes place very slightly in the stomach. The most recent observations show that water is not absorbed in the stomach, but alcohol is absorbed to some extent. Salts also do not seem to be absorbed unless present in great concentrations, such as do not occur in normal diets; sugar is absorbed with difficulty. The small intestine, with its folds and villi to increase its surface, is the great place for absorption.* Absorption begins in the duodenum, and the products of digestion have largely disappeared by the time the intestinal contents reach the ileo-cæcal valve at the commencement of the large intestine; in the large intestine, absorption (mainly of water) occurs also, but to a less extent.

Foods such as water and soluble salts like sodium chloride are absorbed unchanged. The organic foods are, however, considerably changed, colloid materials such as starch and protein being converted respectively into the diffusible materials sugar and amino-acids.

There are two channels of absorption, the blood-vessels (portal tributaries) and the lymphatic vessels or lacteals. In general terms, the proteins and carbohydrates are absorbed by the blood-vessels, and the fats by the lacteals.

Diffusion and osmosis do occur in the intestine, for if a strong solution of salt is introduced into a loop of intestine, there is a flow of water into the loop, owing to the high osmotic pressure of the salt; at the same time some of the salt diffuses into the blood in accordance with the laws of diffusion. But if some of the animal's own serum is introduced into the loop, it also is absorbed, although it has the

* The superficial area of the small intestine, if it was flat, is about 1.5 square metres. This by the presence of the villi is increased to about 42 square metres. 
same osmotic pressure and concentration as the animal's blood. This experiment alone shows us that known physical laws will not completely explain absorption. In fact, absorption is a subject upon which we can speak with little certainty; the energy that controls it is doubtless some form of imbibition, and resides in the living epithelium; for if the epithelium is injured or destroyed by the action of such a poison as sodium fluoride, absorption almost ceases, and what does occur follows the laws of osmosis and diffusion.

A marked feature during absorption is the increased activity of the lymphocytes which lie beneath the epithelium; the number of these cells in the blood increases markedly; it may be even doubled. It has, therefore, been surmised that these cells share in the work of transporting absorbed materials.

Absorption of Carbohydrates. - Though the sugar formed from starch by ptyalin and amylopsin is maltose, that found in the blood is glucose. Under normal circumstances little, if any, is absorbed by the lacteals. The glucose is formed from the maltose by the succus entericus, aided by the action of the epithelial cells through which it passes. Cane sugar and milk sugar are also converted into monosaccharides before absorption.

The carbohydrate food which enters the blood as glucose is taken to the liver, and there stored up in the form of glycogen-a reserve store of carbohydrate material for the future needs of the body. Glycogen, however, is found in animals who take no carbohydrate food. It must, then, be formed by the protoplasmic activity of the liver cells from their protein constituents (see preceding chapter). Monosaccharides (and especially glucose) are the only sugars from which the liver is capable of forming glycogen. If other carbohydrates such as cane sugar or lactose are injected into the blood-stream direct, they are unaltered by the liver, and finally leave the body by the urine.

Absorption of Proteins.-It is possible for the alimentary canal to absorb soluble protein in an unchanged condition. Thus, after taking a large number of eggs, egg-albumin is found in the urine. Patients fed per rectum derive some nourishment from protein food, although proteolytic enzymes are absent from that part of the intestine. But such occurrences are-exceptional; they are merely illustrations of the fact that under unusual conditions certain parts of the body can rise to the occasion and perform unusual feats.

The normal course of events is that the food proteins are broken up into their constituent amino-acids, and it is in this form that they are absorbed. If an animal receives, instead of protein the final cleavage products of pancreatic digestion, it continues to maintain its nitrogenous equilibrium; that is to say, the cells of the body are able to synthesise tissue-proteins from the fragments of the food proteins. 
It is somewhat difficult to find the amino-acids in the blood during absorption, for several reasons: (1) the absorption during any given time is slow, and the products are diluted with the whole volume of the blood; (2) the presence of coagulable proteins in the blood in large quantity renders a search for the amino-acids difficult; and (3) when the amino-acids get into the blood they do not accumulate there, but are rapidly removed by the cells of the tissues. In spite of these difficulties, Leathes, Howell, and, later, Folin have succeeded in demonstrating that during absorption the non-protein (that is, the amino-acid) nitrogen of the blood increases. The hypothesis that proteins are synthesised during absorption in the intestinal wall from amino-acids was for long held by Abderhalden, but in view of these researches, he has finally abandoned it.

We have now a rational explanation of why it is that the organism can construct the proteins peculiar to itself and maintain its chemical individuality, although the food taken varies so widely in composition.

If a man wants to build a house from the bricks of another previously built house, he naturally takes the latter to pieces first, and uses the bricks most suitable for his purpose, and arranges them in a different way to their previous arrangement. The Germans have recently coined the expression Bausteine (or building stones) for the final products of proteolysis with the same underlying idea; these fragments are rearranged by the tissue cells into tissueprotein, which is different architecturally from the food-protein.

Abderhalden has published a very striking experiment in confirmation of this view. He collected the blood of a horse, separated out the various proteins of the plasma, and estimated in each the yield of certain cleavage products (glutamic acid and tyrosine) which resulted from hydrolysis. He then fed the horse so that it formed new blood, but the only protein given was gliadin, a vegetable protein, which is remarkable for its high percentage yield $(37 \cdot 3)$ of glutamic acid. But in the regenerated blood proteins the percentage yield of glutamic acid was not increased at all ; they exactly resembled the proteins previously present.

It is a far cry from the highly specialised organism of the horse to the protoplasm of the simple mould known as Aspergillus niger; nevertheless, the same general rule holds; the protein matter present yields on hydrolysis, glycine, alanine, leucine, glutamic and aspartic acids, but aromatic products, such as tyrosine and phenylalanine, were not discovered. The mould was then cultivated on media of widely varying composition, but the protein formed in the living protoplasm remained constant in composition, and is thus independent of the composition of the nutritive medium.

What, then, if this is the case, would be the fate of food proteins introduced directly into the blood-stream without the intervention of the alimentary digestive processes? If the preliminary cleavage in the gastro-intestinal tract is absolutely necessary, one would anticipate that a foreign food protein (such as edestin from hemp seed, or excelsin from Brazil nuts) administered by intravenous or intraperitoneal injection would not be assimilated, but would be cast out of the body in one or more of the excretions. But Mendel and Rockwood found that they were not eliminated in either urine or bile. In some cases, a proteose 
was found in small quantities in the urine, but the greater part of the protein administered was retained in the body, especially if the injection was slowly performed.

The fact that proteins are retained after this method of administration and apparently used in the body does not really militate against the theory that proteins under normal conditions are more or less completely broken down in the alimentary tract. It is more than probable that cleavage is absolutely necessary for assimilation, and here the enzymes present in the tissue-cells step in; they are capable of taking the place of the pancreatic trypsin and intestinal erepsin and doing their work. The presence of a proteose in urine in some of Mendel and Rockwood's experiments points in this direction, and this view is supported also by Vernon's discovery that every tissue of the body has an ereptic action, and that in some tissues this power is even greater than in the intestinal mucous membrane.

It must not, however, be supposed that all the building stones of the food-protein are utilised in this way. The body is remarkable for its economical use of the tissue-proteins, and quite a small quantity relatively is used up in our daily activities, and so repair is only necessary to the same small extent. We may again get some assistance from our example of the man building a house. When he takes the first house to pieces, there will be a lot of useless bricks and other rubbish, and if the house he wants to build is a smaller one than the one he has destroyed, he will have to discard also many bricks which are not rubbish. So it is with the fragments of the food-protein, which, on usual diets, are more abundant than is necessary for the building of tissue-protein. The excess is carried to the liver, where the amino-group is removed; this is termed deamidation: the nitrogenous moiety of the amino-acid is then converted into urea, which is finally discharged from the body by the kidneys.

It should be further noted that the nitrogen of the protein is split off from it by hydrolysis, not by oxidation, so that the products of breakdown retain almost intact the previous energy of the protein, and the non-nitrogenous residue is then available for calorific processes in the same way that the non-nitrogenous foods (carbohydrates and fat) are.

To continue our analogy of the house-builder, it is generally found that in addition to the building stones provided from the destruction of the previous house, he may want some new bricks altogether. Is this a possibility in the body? Again recent research answers this question in the affirmative, and has shown that the body-cells possess a previously unsuspected power of synthesising amino-acids for themselves. We shall return to this in the chapter on metabolism.

Absorption of Fats. - The fats undergo in the intestine two changes: one a physical change (emulsification), the other a chemical change (saponification). The lymphatic vessels are the great channels 
for fat absorption, and their name lacteals is derived from the milklike appearance of their contents (ehyle) during the absorption of fat.

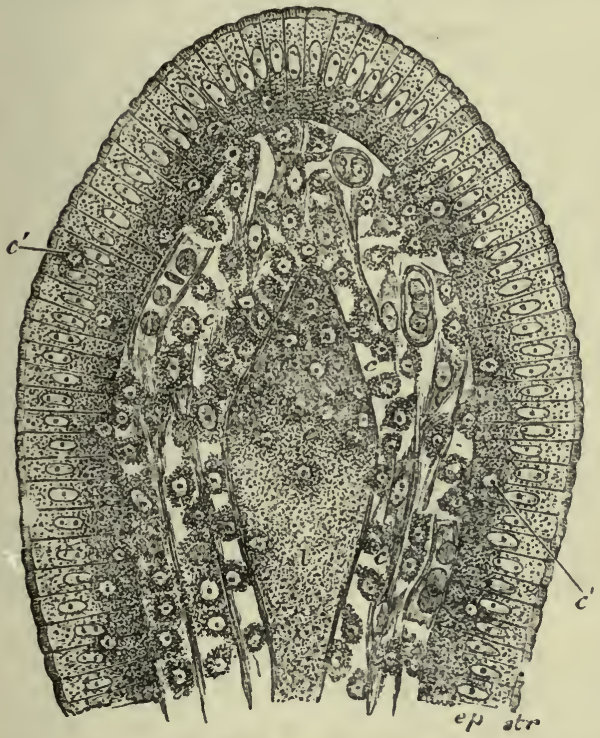

Fra. 366.-Section of the villus of a rat killed during fat absorption. ep, Epithelium; str, striated border; $c$, lymph-cells; $c^{\prime}$, lymph-cells in the epithelium; $l$, central lacteal containing disintegrating lymphcorpuscles. (E. A. Schäfer.)

The course which the minute fat-globules take may be studied by killing animals at varying periods after a meal of fat, and making osmic acid microscopic preparations of the villi. Figs. 366 and 367 illustrate the appearances observed.

The columnar epithelium cells become first filled with fatty globules of varying size, which are generally larger near the free border. The globules pass down the cells, the larger ones breaking up into smaller ones during the journey; they are then transferred to the amœboid cells of the lymphoid tissue beneath: these ultimately penetrate into the central lacteal, where they either disintegrate or discharge their cargo in to the lymph-struam. The globules are by this time divided into immeasurably small ones, the molecular basis of chyle. The chyle enters the blood-stream by the thoracic duct, and after an abundant fatty meal the blood-plasma is quite milky; the fat droplets are so small that they circulate without hindrance through the capillaries. The fat in the blood after a meal is eventually stored up especially in the cells of adipose tissue. It must, however, be borne in mind that the fat of the body is not exclusively derived from the fat of the food, but it may originate from carbohydrate, and according

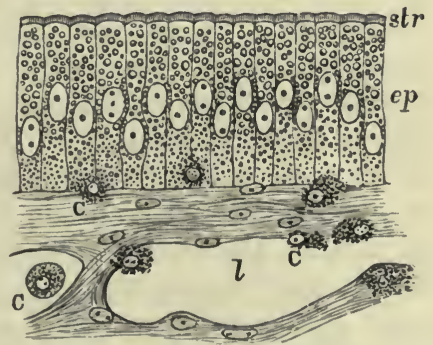

Fio. 367.-Mucous membrane of frog's intestine during fat absorption. $e p$, Epithe. lium; str, striated border; C, lymph corpuscles; $l$, lacteal. (E. A. Schäfer.) to some observers from protein also.

The great difficulty in fat absorption was to explain how the fat first gets into the columnar epithelium: these cells will not take up 
other particles, and it is certain that the epithelial cells do not protrude pseudopodia from their borders (this, however, does occur in the endoderm of some of the lower invertebrates); moreover, fat particles have never been seen in the striated border of the cells.

The difficulty has now been solved. Munk and, later, Moore and Rockwood, showed quite conclusively that in the intestine fat is completely broken down into glycerin and fatty acids; preliminary emulsification is advantageous for the formation of these substances, but is not essential. Fat, therefore, is entirely absorbed as glycerin and fatty acids; the latter, however, in great measure are first converted into soaps, that is, compounds of the fatty acids and alkalis. These soluble cleavage products pass readily through the striated borders of the intestinal epithelial cells; and these cells perform the synthetic act of building them into fat once more, the fat so formed appearing in the form of small globules, surrounding or becoming mixed with the protoplasmic granules that are ordinarily present. Another remarkable fact which Munk made out is that after feeding an animal on fatty acids the chyle contains fat. The necessary glycerin must have been formed by protoplasmic activity during absorption.

Bile aids the digestion of fat, the bile salts acting in the same way as the co-enzyme of pancreatic lipase; bile also is a solvent of fatty acids, and it probably assists fat absorption by reducing the surface tension of the intestinal contents; membrames moistened with bile allow fatty materials to pass through them more readily than would otherwise be the case. In cases of disease in which bile is absent from the intestines, a large proportion of the fat in the food passes into the fæces.

The fæces on an ordinary mixed diet contain comparatively little food residues, and a small quantity is excreted even during starvation. Voit and Hermann showed independently that an intestinal loop which had been emptied and separated from the rest of the bowel contained a few days later, material identical with fæces, and consisting of intestinal juice, desquamated epithelium cells, and bacteria. The increase in the amount of fæces which occurs when food is taken, even when the food is free from cellulose, is due to the mechanical and chemical stimulation which leads to an increase in the succus entericus, and in the shedding of epithelial cells. The fæces contain about 1 per cent. of nitrogen, but this is chiefly contained in the bodies of bacteria, and the disintegrated epithelial cells. Addition of protein to the diet makes practically no difference to the nitrogen in the fæces under normal conditions.

The addition of cellulose to the diet increases the bulk of the fæces, partly because much of the cellulose is excreted unchanged, partly because it stimulates the mucous membrane to secrete more 
succus entericus, and finally because the larger food residue favours the development of bacteria. On an average, from one-third to one-fifth (varying with the diet) of the weight of dried fæces consists of bacteria. The average weight of dried bacteria excreted daily is 8 grammes; this contains 0.8 gramme of nitrogen, or about half the nitrogen of the fæces. Strasburger estimated that about $128,000,000,000,000$ bacteria are evacuated in the fæces of a man every day. The vast majority of these are dead.

When cellulose is absent from the diet, the fæces contain from 65-75 per cent. of water; the dry residue contains about 7 per cent. of nitrogen, and the non-nitrogenous material consists of about equal quantities of ash and substances soluble in ether, with small quantities of stercobilin and other bile residues. The ash contains mainly calcium phosphate, with small amounts of iron and magnesium. The ethereal extract contains cholesterin, lecithin, fatty acids, soaps, and a very small amount of neutral fat. The proteins are chiefly mucin and nucleo-protein, and are derived not from the food, but from the intestinal wall, or are contained in the bacteria; no doubt a large part of the ethereal extract is also supplied by the bacteria.

Cellulose is thus the only important constituent of the food which is unaffected by the digestive juices, although a variable amount, which is largest in herbivorous animals, undergoes bacterial decomposition. The presence of cellulose also interferes with the absorption of proteins, for the digestive juices have difficulty in penetrating the cellulose membranes of vegetable cells. Thus Voit found that 42 per cent. of the nitrogen in the food were lost in the fæces of a vegetarian. This is due solely to the cellulose and not to any difference in the digestibility of animal and vegetable proteins, for if vegetable food is finely subdivided, and then thoroughly cooked and softened, this loss is lessened, and if vegetable protein is entirely freed from cellulose, it is as thoroughly absorbed as animal protein. Fifteen per cent. of the dry substance of green vegetables and brown bread, 20 per cent. of carrots and turnips, and a still larger amount of beans are lost in the fæcal residue.

The intestinal contents travel more rapidly when vegetables are present, for the indigestible cellulose stimulates peristalsis, and therefore a large quantity of water escapes absorption in the colon. Thus on an ordinary mixed diet 35 grammes of dry substance and 100 grammes of water are daily excreted in the fæces, whereas on a vegetable diet the quantities are 75 and 260 grammes respectively. 


\section{CHAPTER XXXVII}

\section{THE MECHANICAL PROCESSES OF DIGESTION}

UNDER this head we shall study the neuro-muscular mechanism of the alimentary canal, which has for its object the onward movement of the food, and its thorough admixture with the digestive juices. We shall therefore have to consider mastication, deglutition, the movements of the stomach and intestines, defæcation, and vomiting.

\section{Mastication.}

The act of mastication is performed by the biting and grinding movement of the lower range of teeth against the upper. The simultaneous movements of the tongue and cheeks assist partly by crushing the softer portions of the food against the hard palate and gums, and thus supplement the action of the teeth, and partly by returning the morsels of food to the teeth again and again, as they are squeezed out from between them, until they have been sufficiently chewed.

The act of mastication is much assisted by the saliva, and the intimate incorporation of this secretion with the food is called insalivation.

Mastication is much more thoroughly performed by some animals than by others. Thus, dogs hardly chew their food at all, but the œsophagus is protected from abrasion by a thick coating of very viscid saliva which lubricates the pieces of rough food.

In vegetable feeders, on the other hand, insalivation is a much more important process. This is especially so in the ruminants; in these animals, the grass, etc., taken, is hurriedly swallowed, and passes into the first compartment of their four-chambered stomach. Later on, it is returned to the mouth in small instalments for thorough mastication and insalivation; this is the act of rumination, or "chewing the cud"; the food is then once more sivallowed, and passes on to the digestive regions of the stomach. 
In man, mastication is also an important process, and in people who have lost their teeth severe dyspepsia is often produced, which can be cured by a new set of teeth.

\section{Deglutition.}

When properly masticated, the food is transmitted in successive portions to the stomach by the act of deglutition or swallowing. This, for the purpose of description, may be divided into three acts. In the first, particles of food collected as a bolus are made to glide between the surface of the tongue and the palatine arch, till they have passed the anterior arch of the fauces; in the second, the morsel is carried through the pharynx; and in the third, it reaches the stomach through the œsophagus. These three acts follow each other rapidly. (1) The first act is voluntary, although it is usually performed unconsciously; the morsel of food when sufficiently masticated, is pressed between the tongue and palate, by the agency of the muscles of the former, in such a manner as to force it back to the entrance of the pharynx. (2) The second act is the most complicated, because the food must go past the posterior orifice of the nose and the upper opening of the larynx without entering them. When it has been brought, by the first act, between the anterior arches of the palate, it is moved onwards by the movement of the tongue backwards, and by the muscles of the anterior arches contracting on it and then behind it. The root of the tongue being retracted, and the larynx being raised with the pharynx and carried forwards under the base of the tongue, the epiglottis is pressed over the upper opening of the larynx, and the morsel glides past it; the closure of the glottis is additionally secured by the simultaneous contraction of its own muscles: so that, even when the epiglottis is destroyed, there is little danger of food passing into the larynx so long as its muscles can act freely. In man, and some other animals, the epiglottis is not drawn as a lid over the larynx during swallowing. At the same time, the raising of the soft palate, so that its posterior edge touches the back part of the pharynx, and the approximation of the sides of the posterior palatine arch, which move quickly inwards like side curtains, close the passage into the upper part of the pharynx and the posterior nares, and form an inclined plane, along the under surface of which the morsel descends; then the pharynx, raised up to receive it, in its turn contracts, and forces it onwards into the œsophagus. The passage of the bolus of food through the three constrictors of the pharynx is the last step in this stage. (3) In the third act, in which the food passes through the œsophagus, every part of that tube, as it receives the morsel and is dilated by it, is stimulated to contract: hence an undulatory or peristaltic contraction of the œsophagus occurs. If we suppose the bolus to be at one particular place in the tube, it acts 
stimulatingly on the circular muscular fibres behind it, and inhibitingly on those in front; the contraction therefore squeezes it into the dilated portion of the tube in front, where the same process is repeated, and this travels along the whole length of the tube. The second and third parts of the act of deglutition are involuntary. The action of these parts is more rapid than peristalsis usually is. This is due to the large amount of striated muscular tissue present. It serves the useful purpose of getting the bolus as quickly as possible past the opening of the respiratory tract.

The swallowing both of solids and liquids is a muscular act, and can, therefore, take place in opposition to the force of gravity. Thus, horses and many other animals habitually drink uphill, and the same feat can be performed by jugglers.

In swallowing liquids the two mylo-hyoid muscles form a diaphragm below the anterior part of the mouth. The stylo-glossi draw the tongue backwards and elevate its base; the two hyo-glossi act with these, pulling the tongue backwards and downwards. The action of these muscles resembles that of a force-pump projecting the mass of fluid down into the csophagus; it reaches the cardiac orifice with great speed, and the pharyngeal and œsophageal muscles do not contract on it at all, but are inhibited during the passage of the fluid through them (Kronecker).

This is proved in a striking way in cases of poisoning by corrosive substances, such as oil of vitriol; the mouth and tongue are scarred and burnt, but the pharynx and ossophagus escape serious injury, so rapidly does the fluid pass along them; the cardiac orifice of the stomach is the next place to show the effects of the corrosive. Kronecker's view has also been confirmed in man by the X-ray method.

There is, however, no hard-and-fast line between the swallowing of solids and fluids: the more liquid the food is, the more does the force-pump action just described manifest itself.

Nervous Mechanism.-The nerves engaged in the reflex act of deglutition are:-sensory, branches of the fifth cranial nerve supplying the soft palate and tongue; glosso-pharyngeal, supplying the tongue and pharynx; the superior laryngeal branch of the vagus, supplying the epiglottis and the glottis; while the motor fibres concerned are:branches of the fifth, supplying part of the digastric and mylo-hyoid muscles, and the muscles of mastication; the bulbar part of the spinal accessory through the pharyngeal plexus, supplying the levator palati, probably by rootlets which are glosso-pharyngeal in origin; the . glosso-pharyngeal and vagus, and possibly the bulbar part of the spinal accessory, supplying the muscles of the pharynx through the pharyngeal plexus; the vagus, in virtue of its spinal accessory roots, supplying the muscles of the larynx through the inferior laryngeal 
branch; and the hypo-glossal, the muscles of the tongue. The nerre-centres by which the muscles are harmonised in their action, are situated in the medulla oblongata.

Stimulation of the vagi gives rise to peristalsis of the cesophagus. The cell-stations of these fibres are in the ganglion trunci vagi. Division of both pneumogastric nerves produces paralysis of the œsophagus and stomach, and firm contraction of the cardiac orifice. These nerves therefore normally supply the cesophagus with motor, and the cardiac sphincter with inhibitory fibres. If food is swallowed after these nerves are divided, it accumulates in the gullet and never reaches the stomach.

In discussing peristalsis on a previous occasion (p. 141), we arrived at the conclusion that it is an inherent property of muscle rather than of nerve; though normally it is controlled and influenced by nervous agency. This nervous control is especially marked in the œesophagus; for if that tube is divided across, leaving the nerve branches intact, a wave of contraction will travel from one end to the other across the cut.

\section{Movements of the Stomach.}

The gastric fluid is assisted in accomplishing its share in digestion by the movements of the stomach. In graminivorous birds, for example, the contraction of the strong muscular gizzard affords a necessary aid to digestion, by grinding and triturating the hard seeds which constitute their food. But in the stomach of man and other Mammalia the movements of the muscular coat are too feeble to exercise any such mechanical force on the food; neither are they needed, for mastication has already done the mechanical work of a gizzard; and it has been demonstrated that substances are digested even when enclosed in perforated tubes, and consequently protected from mechanical influence.

When digestion is not going on, the stomach is uniformly contracted, its orifices not more firmly than the rest of its walls; but, if examined shortly after the introduction of food, it is found closely encircling its contents, and its orifices are firmly closed by sphincters. The cardiac orifice, every time food is swallowed, opens to admit its passage into the stomach, and immediately again closes. The pyloric orifice, during the first part of gastric digestion, is usually so completely closed, that even when the stomach is separated from the intestines, none of its contents escape. But later the pylorus offers less resistance to the passage of substances from the stomach; first it yields to allow the successively digested portions to go through it; and then it allows the transit even of undigested substances. The peristaltic action of the muscular coat, whereby the digested 
portions are gradually moved towards the pylorus, also ensures thorough admixture with the gastric juice.

The contraction of the fibres situated towards the pyloric end of the stomach is more energetic and more decidedly peristaltic than those of the cardiac portion. Thus, it was found in the case of St Martin, that when the bulb of a thermometer was placed about three inches from the pylorus, through the gastric fistula, it was tightly embraced from time to time, and drawn towards the pyloric orifice for a distance of three or four inches. In certain pathological conditions, by a predominant action of strong circular fibres placed between the cardia and pylorus, the two portions, or ends, as they are called, of the stomach, are partially separated from each other by a kind of hour-glass contraction.

A few years ago the subject was taken up by Cannon. He gave an animal food mixed with bismuth subnitrate, and obtained by the Röntgen rays shadow photographs of the stomach, because the bismuth salt renders its contents opaque. His results confirm those of the earlier investigators; the principal peristalsis occurs in the pyloric portion of the stomach. The cardiac portion (including the fundus) presses steadily on its contents, and as they become chymified, urges them onwards towards the pyloric portion; the latter empties itself gradually through the pylorus into the duodenum, and in the later stages of digestion the cardiac part also is constricted in to a tube.

After an ordinary mixed meal it is usually stated that the pylorus usually opens for the first time about half an hour after digestion begins, and some of the acid chyme passes into the duodenum. This, however, is incorrect, as food can be seen by the $\mathrm{X}$-ray method in the duodenum within a few minutes after a meal. The action of this muscular ring is intermittent, and the explanation of its alternate openings and closings may be briefly summed up in the phrase used by Cannon: "the acid control of the pylorus." It is necessary that the food should be retained in the stomach until it is acid; otherwise it would not, on reaching the duodenum, give rise to the formation of secretin, the chemical stimulus for the flow of pancreatic juice, and bile. He has found that acid on the gastric side of the pyloric sphincter opens it, and acid in the duodenum closes it. As soon therefore as the chyme is neutralised by the alkaline juices of the duodenum, there is no longer any hindrance to the action of the acid chyme in the pyloric end of the stomach in opening the door which was temporarily closed by acid on its duodenal side. This action does not occur if a ring is cut through the muscular coat immediately beyond the pylorus, and so the effect from the duodenum is a local reflex action mediated like the movements of the small intestine by the plexus of Auerbach.

The time taken for the complete emptying of the stomach is 
variable; the size of the meal, its digestibility, the general state of the body and mind of the individual, are all factors that influence the rate of the act. The average time, however, is probably somewhere about three hours.

Dr Hertz and his colleagues at Guy's Hospital have recently applied the Röntgen ray method to man with very instructive results. Large doses of bismuth oxychloride (or barium sulphate) can be given to human beings without harm, and by the X-rays the shadow of the opaque food can then be followed, from swallowing onwards to defæcation. The old ideas of the shape and position of the stomach during life, which were derived from examining it in the post-mortem room, are wholly incorrect. In the upright position the pyloric portion is lowermost, and when food is taken it sinks into this lower portion; and above the ordinary semi-fluid contents there is a horizontal upper limit above which is air. In the erect position the stomach, however, is not quite vertical, but is usually slightly inclined tinwards the right; and in the recumbent position this obliquity is increased. The following three figures illustrate the shape and position of the stomach when the man is erect:(a) when the organ is empty; $(b)$ whon it is partly filled; and $(c)$ when it is full.

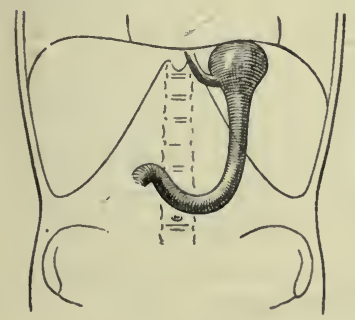

$a$

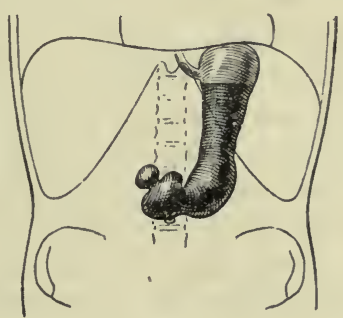

$b$

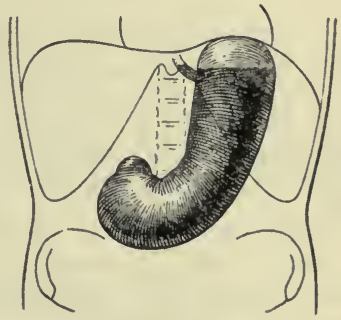

$c$

FiG. 368.- (a) View of the empty stomach in vertical position; $(b)$ stomach as seen soon after a bismuth meal; note the peristaltic waves at the pyloric end; (c) view of filled stomach in vertical position. (After Hertz)

Peristalsis, as in animals, is, during the early stages of gastric digestion, limited to the pyloric portion. But the view that the stomach is separated into two divisions in one of which (the fundus) salivary digestion is in progress, and in other of which gastric digestion proper is progressing, is quite untenable in view of these observations, especially if changes of posture are occurring also.

The fluidity of the food is another factor which is important; the more fluid the food, the more rapidly does it leave the stomach. If water is given to a dog with a duodenal fistula, it flows out of the 
opening almost as rapidly as it is swallowed. It is impossible in man to follow the behaviour of water by X-ray shadows, but Hertz finds that the more fluid his bismuth mixture is, the more rapidly does it issue into the intestine. The rapid relief of thirst which follows the drinking of water, and the superiority of fluid food for the restoration of persons who are faint for want of nourishment, are further facts which point to the rapid arrival of liquids at the absorbing surface of the intestine.

Nervous Mechanism.-The stomach has a double nerve supply, and the fibres terminate in the plexus situated between its muscular coats. The two sets of nerves are :-

(1) The vagus. The cell-stations for these fibres appear to be in the terminal ganglia of the plexus, though possibly some may occur in the ganglion trunci vagi. These nerves are accelerator; when stimulated, the result is increase of peristalsis.

(2) The sympathetic. These leave the spinal cord by the anterior roots of the spinal nerves from the fifth to the eighth thoracic; their cell-stations are in the cœliac ganglion, and the post-ganglionic fibres which arise there pass to the stomach by branches of the splanchnic nerves. The sympathetic fibres are inhibitory; when they are stimulated, peristalsis ceases.

The secretory nerves of the gastric glands are discussed on p. 512 .

\section{VOMITING.}

The act of vomiting is preceded by a feeling of nausea, and the swallowing of a large quantity of saliva. The expulsion of the contents of the stomach, like that of mucus or other matter from the lungs in coughing, is preceded by an inspiration; the glottis is then closed, and immediately afterwards the abdominal muscles strongly act; but here occurs the difference in the two actions. Instead of the vocal cords yielding to the action of the abdominal muscles, they remain tightly closed. Thus the diaphragm, being unable to go up, forms an unyielding surface against which the stomach can be pressed. At the same time the cardiac sphincter being relaxed, and the orifice which it naturally guards being dilated, while the pylorus is closed, and the stomach itself also contracting, the action of the abdominal muscles expels the contents of the organ through the œsophagus, pharynx, and mouth.

It has been frequently stated that the stomach itself is quite passive during vomiting, and that the expulsion of its contents is effected solely by the pressure exerted upon it when the capacity of the abdomen is diminished by the contraction of the diaphragm, and subsequently of the abdominal muscles. The experiments and observations, however, which are supposed to confirm this statement, 
only show that the contraction of the abdominal muscles alone is sufficient to expel matters from an unresisting bag through the œsophagus; and that, under certain circumstances, the stomach, by itself, cannot expel its contents. They by no means show that in ordinary vomiting the stomach is passive, for there are good reasons for believing the contrary. In some cases of violent vomiting the contents of the duodenum are passed by anti-peristalsis into the stomach, and are then vomited. Where there is obstruction to the intestine, as in strangulated hernia, the total contents of the small intestine may be vomited.

Nervous Mechanism.--Some fow persons possess the power of vomiting at will, or the power may be acquired by effort and practice. But normally the action is a reflex one.

The afferent nerves are principally the fifth, and glosso-pharyngeal (as in vomiting produced by tickling the fauces), and the vagus (as in vomiting produced by gastric irritants); but vomiting may occur from stimulation of other sensory nerves, e.g., those from the kidney, uterus, testicle, etc. The medullary centres may also be stimulated by impressions from the cerebrum and cerebellum, producing so-called central vomiting occurring in diseases of those parts.

The efferent (motor) impulses are carried by the vagi to the stomach, by the phrenics to the diaphragm, and by various other spinal nerves to the abdominal muscles.

It is very doubtful whether there is any separate centre for vomiting; the centre for the reflex coincides with those of the nerves mentioned in the medulla oblongata.

Emetics.-Some emetics produce vomiting by irritating the stomach; others, such as tartar emetic, apomorphine, etc., by stimulating the medullary centres.

\section{Movements of the SMall Intestine.}

The intestinal movements, like those of the stomach, take place independently of our volition or consciousness. When, however, they become excessive, as they do under the influence of irritants or the presence of obstruction, they produce pain which is usually intense.

The object of these movements is to force the contents along the tube, and to thoroughly mix them with the digestive juices. But the peristalsis which drives the intestinal contents along does so more slowly than that which occurs in the esophagus; otherwise the mechanism is much the same. There may occur in the small intestine peristaltic waves in the opposite direction (retro-peristalsis) and this probably never occurs in the œsophagus. Retro-peristalsis is most marked when obstruction is present, as in the cases of violent vomiting just referred to. 
Our knowledge of the intestinal movements rests, first, on observations made on the exposed intestines when the abdomen is opened; secondly, they may be studied under more artificial conditions by taking a length of intestine from a freshly killed animal and placing it in a warm bath of oxygenated Ringer's solution; and thirdly, the most valuable method of all is to study the movements in the intact animal by the X-ray method, as in the work of Cannon and of Hertz.

Ludwig was the first to call attention to the fact that peristaltic waves are not the only sort of movements which occur. There is in addition what he termed pendulum or swaying movements. In the exposed intestine the propagation of the peristaltic wave is slow but variable; it may be as small as $1 \mathrm{~cm}$. per minute. In man, as shown by X-ray work, it is more rapid, averaging about an inch per minute. The pendulum movements consist of slight waves of contraction affecting both muscular coats, and these are rapidly propagated at the rate of 2 to $5 \mathrm{cms}$. per second. They cause a movement of the intestine from side to side, and occur at regular intervals of five or six seconds. They are not efficacious in moving the contents onwards, but they bring about a mixing of the contents very thoroughly.

Cannon observed by the X-ray method in dogs and cats that these pendulum movements produce what he calls "segmentation." A dark shadow, due to the bismuth in the food administered, is at one moment of a certain length like a short sausage; it then constricts in the centre, and divides into two; each half divides again; then the two central segments join together, and this repeats itself every few seconds. In man, where the same phenomenon can be seen, Hertz timed the rate, and found it occurred about ten times in a minute and a half. This frequent division and subdivision not only ensures admixture with the juices, but brings every portion in turn in contact with the absorbing mucous membrane, and favours the flow of chyle and blood in their respective vessels.

After a bismuth meal, the shadow appears in the cæcum three and a half to five hours after the food is taken. The average time is four and a half hours. Assuming that it begins to leave the stomach half an hour after a meal, the total journey along the small intestine in man occupies about four hours; the small intestine is $22 \frac{1}{2}$ feet long, so the rate works out at about an inch (a little more than 2 cms.) per minute.

Nervous Mechanism. - The small intestine, like the stomach, has a double nerve supply.

(1) The Vagus. As in the case of the stomach, these fibres are accelerator, and stimulation induces peristaltic movements. If the intestine is contracting peristaltically before the stimulus is applied, 
the movements are inhibited for a brief period, after which they are greatly augmented.

(2) The Sympathetic. These fibres leave the spinal cord by the anterior roots from the sixth thoracic to the first lumbar, pass through the lateral chain, but do not reach their cell-stations until they arrive at the superior mesenteric ganglia: thence they pass as non-medullated, post-ganglionic fibres to the muscular coats. Stimulation of these nerves causes inhibition of any peristaltic movements that may be present. They also contain vaso-motor fibres, and section of these leads to vaso-dilatation and a great increase of very watery succus entericus.

These two sets of nerves (vagus and sympathetic) terminate in the ganglionated plexus of Auerbach, situated between the two muscular layers of the intestinal wall.

Under normal circumstances, the intestinal movements are regulated from the central nervous system via these two channels. Nevertheless, after all the nerves are cut, the movements continue, and may remain normal for months. This independence of control from the central nervous system justifies the use of the term autonomic (see Chapter XVII.). The true peristaltic waves are, however, coördinated reflex actions, the centres for the reflex being situated in the ganglion cells of Auerbach's plexus. The movements entirely cease if the intestine is painted with cocaine, or if nicotine is injected, for under the influence of these drugs the synaptic junctions of the ganglion cells are paralysed. The importance of the integrity of the plexus was also shown by Magnus in his experiments with strips of intestinal muscle; such strips are incapable of spontaneous rhythm if the nerve plexus is not removed with them. Yanasi found that the intestinal muscle of the embryo guinea-pig will contract when directly stimulated, but it is only capable of spontaneous peristalsis after the development of Auerbach's plexus.

In order that peristalsis may attain its object in driving the intestinal contents onwards, it is necessary not only that a wave of contraction should travel along, but a wave of relaxation must also take place in the front of the mass which is urged forwards. This does take place on stimulation; the normal stimulus is the presence of material within the intestine; the intestine is usually quiescent when empty. But, as Starling showed, a pinch applied to any particular spot will cause a wave of contraction behind the point pinched, and a wave of relaxation or inhibition in front of it, which travels downwards.

Peristalsis may be stimulated in many ways, and inhibited in many ways:-

(1) The usual stimulus is doubtless the mechanical one of the 
presence of food-material in the intestine, and especially of indigestible food such as cellulose.

(2) It may be influenced by impulses from the upper part of the alimentary canal; the mere taking of food will stimulate peristalsis even in the large intestine also. This is most marked after abstinence from food, and the usual effect of breakfast as a stimulus for defæcation is a familiar example. The mere taking of a glass of water on rising will in many people have a similar effect.

(3) It may be influenced by sensations and emotions; thus movements are inhibited by pain, by the exposure of the peritoneum to the air, or by handling the gut as in operations. Some emotions, such as anger, will inhibit peristalsis ; others of a more pleasurable kind, leading to what is popularly termed excitement, will increase it and may even lead to diarrhœa. It is increased by muscular exercise, though here no doubt the influence is partly the mechanical one of the abdominal walls pressing about the intestinal loops.

(4) It may be influenced by temperature, but here again we have most knowledge in regard to the large intestine; a cold enema is more efficacious than a warm one; the latter is mainly sedative.

(5) It may be influenced chemically. Drugs given for the relief of diarrhoa or constipation act in various ways; some affect the amount of secretion, and thus increase or decrease the fluidity of the intestinal contents; others act on the muscular tissue or its nerves, and so influence the amount of peristalsis. Organic acids, including the amino-acids, produced during digestion, will increase peristalsis. The bile has a similar action, but only on the large intestine; various oils act in the same way; certain gases do so also, but here again the mechanical effect of distension is a factor to be reckoned with. A vegetable diet stimulates peristalsis, partly for mechanical reasonsthe presence of indigestible cellulose and formation of gas-partly for a chemical reason, namely, the production of organic acids.

The pendulum movements differ from true peristalsis in being myogenic; that is, they are due to the rhythmicality of the muscular fibres themselves, and are propagated from one muscular fibre to another. They are not abolished by cocaine or nicotine. (Starling.)

\section{Movements of tile Large Intestine.}

We have seen that in man the food begins to arrive in the cæecum four and a half hours after it reaches the stomach; when it arrives in the creum it contains 90 per cent. of water, together with a small amount of the unabsorbed products of digestion of proteins, fats, and carbohydrates. During its passage along the large intestine these are absorbed, and most absorption appears to occur in the cæcum; the normal firm consistency of the fæces, which contain 
75 per cent. of water, is not finally attained until they arrive in the pelvic colon, where they are retained until defæcation takes place.

Peristalsis in the colon occurs much more slowly than in the small intestine, and the accompanying diagram gives the time in hours after the taking of a bismuth meal that the shadow appears at various points in man. It reaches the hepatic flexure of the colon about two hours after it appears in the cæcum; another two hours approximately brings it to the splenic flexure (nine hours after the meal). The distance from the cæcum to the splenic flexure is 2 feet; the contents take as long to travel this distance as the contents of the small intestine take to travel 221 feet, that is, from

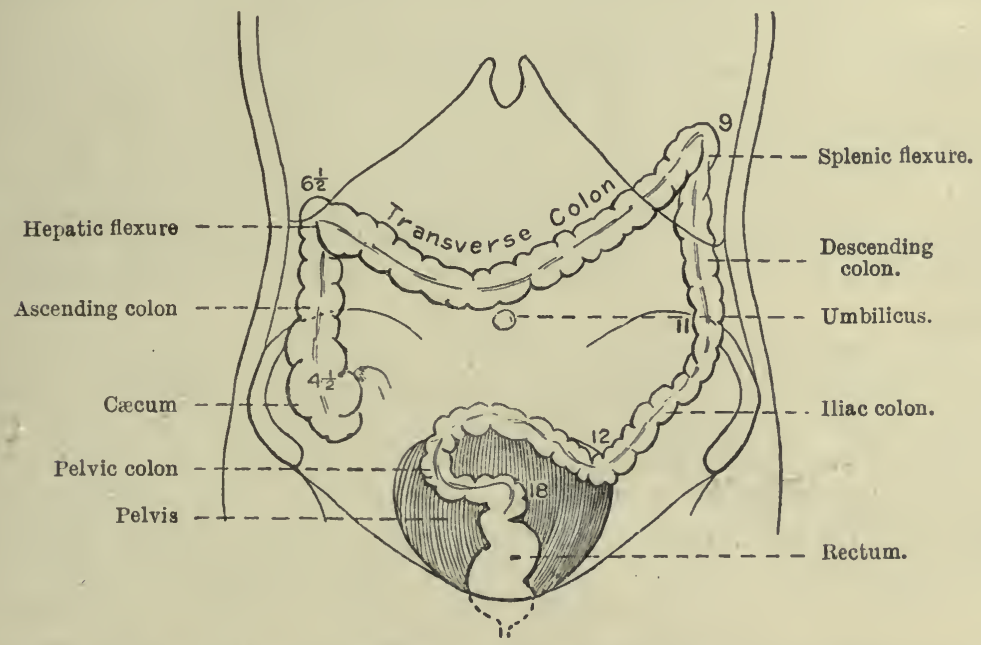

HIG. 369.-Semi-diagrammatic view of the large intestine; the figures give in hours the average times after taking a meal that its débris reaches the various parts. (Hertz.) This diagram shows the transverse colon in a higher position than it occupies when the man is erect, and rather higher than the average even in the horizontal position.

the pylorus to the cæcum. A further two hours is occupied in the journey along the descending colon, and six hours more brings it to the end of the pelvic colon which leads at an angle into the rectum. The total journey from the cæcum to this point occupies thirteen and a half hours. These times were confirmed by auscultation or listening over various parts of the abdomen; the gurgling and splashing sounds made by the arrival of food-material are distinctly audible. These observations were made in the daytime; during sleep the rate of progress may be slower.

Some observers have stated that retro-peristalsis occurs in the colon, especially in its ascending portion. Waves of this kind would 
certainly mix up the cæcal contents very thoroughly. They have, however, only been seen in the exposed intestine of animals, and therefore may be artificially produced. A study of X-ray shadows does not reveal their existence in man. If retro-peristalsis does occur, regurgitation is effectually prevented into the small intestine partly by the ileo-cæcal valve, and mainly by a strong band of circular muscular fibres called the ileo-cæcal sphincter; this is normally kept in a state of tonic contraction by impulses carried by the splanchnic nerve; it is relaxed when this nerve is cut, and then the contents of the two intestines mix freely. (T. R. Elliott.)

Defocation. - The rectum is a short tube about 4 or 5 inches long in man, which is normally empty until immediately before defæcation. In a person of regular habits, a glass of cold water on rising, the stimulus of a cold bath, the taking of breakfast, and the afterbreakfast pipe or cigarette combine to produce peristalsis of the colon, so that a small quantity of freces enters the rectum, and then arises the desire to defæcate. At the end of the rectum is the anal canal, closed by a strong internal sphincter (a thickening of the involuntary circular fibres of the muscular coat), and by the external sphincter, which is a voluntary muscle made of transversely striated fibres.

The "call to defreation" having been thus produced, the act itself is started by the increase in intra-abdominal pressure brought about by the voluntary contraction of the abdominal wall, the diaphragm and the levator ani. The diaphragm is kept down by deep inspirations, followed by closure of the glottis; this depresses the colon, so that the shadow of its transverse portion and the flexures may be lowered as much as 2 inches. The transverse colon may not rise to its normal position until even an hour has elapsed from the act of straining during defæcation. Accompanying the action of these voluntary muscles, the whole colon from the cæecum onwards enters into powerful peristalsis; the contents of the transverse colon are thus forced into the descending colon, from which they are evacuated together with the fæces already present between the splenic flexure and the anus. The entrance of more fæces into the rectum until they reach the anal canal irritates afferent nerves in the wall of the rectum; the nerve impulses so generated pass to a centre or centres in the lumbo-sacral region of the spinal cord, where efferent impulses are set in action upon which depend the reflex acts required to complete the process; these are:-

1. Strong peristalsis of the whole colon.

2. Continued contraction of the abdominal muscles.

3. Relaxation of both the anal sphincters and of the levator ani.

The last traces of fæces are expelled by voluntary contractions of the levator ani. 
If the bowels are opened once a day, the interval between a meal and the evacuation of its residue varies between nine and thirtytwo hours, the time depending on the hours of meals and that of defæcation. Food taken less than nine hours previously would not have reached far enough.

If the call to defæcation is resisted, the desire soon passes away, and may not recur until the next regular period arrives for the opening of the bowels, twenty-four hours later. During this time the rectum contains fæces, there being no retro-peristalsis to carry them back into the colon. This is one of the commonest causes of constipation, for the retained fæces continue to lose water, and get harder, and more difficult to expel.

Nervous Mechanism.-The large intestine resembles the rest of the alimentary canal in having a double nerve supply.

(1) The sympathetic. These fibres leave the cord by the lower lumbar anterior roots; these pass through the lateral chain, and reach their cell-stations in the inferior mesenteric ganglion; the postganglionic fibres arising there, pass by the colonic nerves to the colon, and by the hypogastric nerve to the rectum and internal anal sphincter.

(2) The nervus erigens. This takes the place of the vagus, which forms the second source of nerve supply to the stomach and small intestine. This nerve is excitatory to both coats of the muscular wall, whereas the sympathetic is inhibitory to the internal sphincter.

The fibres which pass to the rectum by the pelvic nerves or nervi erigentes arise from the third sacral nerve, and have their cellstations in the hæmorrhoidal nerve plexus, which is the name given to this portion of the plexus of Auerbach.

The voluntary muscles, namely, the external anal sphincter and the levator ani, are supplied by the fourth sacral nerve, which arises from nerve-cells in the conus terminalis of the spinal cord.

If Starling's experiment of pinching a spot in the large intestine is performed, much the same result follows as in the small intestine; the wave of inhibition which travels downwards is well seen, but the upward wave of contraction is not so marked as in the small intestine. Stimulation of the sympathetic (hypogastric) nerve-fibres produces movements of the colon and rectum, and inhibition of the internal sphincter; that is the main phenomenon in the act of defæcation. If the lower part of the spinal cord is destroyed, defæcation still occurs, but it is an unconscious act, and the reflex is imperfectly executed; the hypogastric part of the mechanism is intact, and probably the reflex contre concerned is, as in the small intestine, in the peripheral ganglia of Auerbach's plexus; but the destruction of the conus terminalis prevents the normal reflexes taking place in which the 
levator ani and external sphincter are concerned, and the paralysis of these voluntary muscles may lead to incontinence of fæces.

We thus see that the lowermost portion of the alimentary canal resembles its uppermost portion (pharynx and œsophagus) in being more under external nervous control than is the small intestine. Autonomy at the rectal and anal portion is for obvious reasons undesirable. 


\title{
CHAPTER XXXVIII
}

\author{
THE URINARY APPARATUS
}

THIS consists of the kidneys; from each a tube called the ureter leads to the bladder, in which the urine is temporarily stored; from the bladder a duct called the urethra leads to the exterior.

The Kidneys are situated in the lumbar region of the abdomen on either side of the spinal column behind the peritoneum. In man each is about 4 inches long, and weighs about $4 \frac{1}{2} \mathrm{oz}$.

Structure.-The kidney is covered by a fibrous capsule, which is slightly attached at its inner surface to the proper substance of the organ by means of very fine bundles of areolar tissue and minute blood - vessels. At the hilus of the kidney it becomes continuous with the external coat of the upper and dilated part of the ureter (fig. 370).

On dividing the kidney into two equal parts by a section carried through its long convex border, it is seen to be composed of two portions, called respectively cortical and medullary; the latter is composed of about a dozen conical bundles of urinary

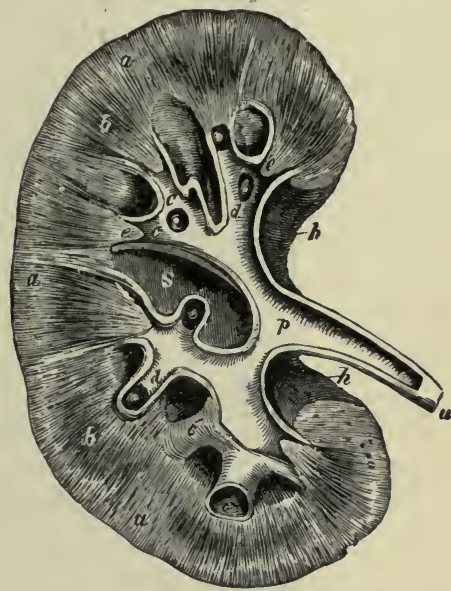

FIG. 370.-Plan of a longitudinal section through the pelvis and substance of the right kidney, $\frac{1}{2}: a$, the cortical substance; $b, b$, broad part of the pyramids of Malpighi; $c, c$, the divisions of the pelvis named calyces, laid open ; $c^{\prime}$, one of those unopened; $d$, summit of the pyramid projecting into calyces; $e$, $e$, section of the narrow part of two pyramids near the calyces; $p$, pelvis or enlarged portion of the ureter within the kidney; $u$, the ureter ; $s$, the sinus; $h$, the hilus.

tubules, each bundle forming what is called a pyramid. The upper part of the ureter is dilated into the pelvis; and this, again, after separating into two or three principal divisions, is finally subdivided into still smaller portions, varying in number from about 8 to 12 , called calyces. Each of these little calyces or cups receives the pointed extremity or papilla of a pyramid. The number of pyramids varies in different animals; in some there is only one.

The kidney is a compound tubular gland, and both its cortical and medullary portions are composed of tubes, the tubuli uriniferi, which, by one extremity, in the cortical portion, commence around 
566

THE URINARY APPARATUS

[CH. XXXVIII.

tufts of capillary blood-vessels, called Malpighian bodies, and, by the other, open through the papilla into the pelvis of the ureter, and

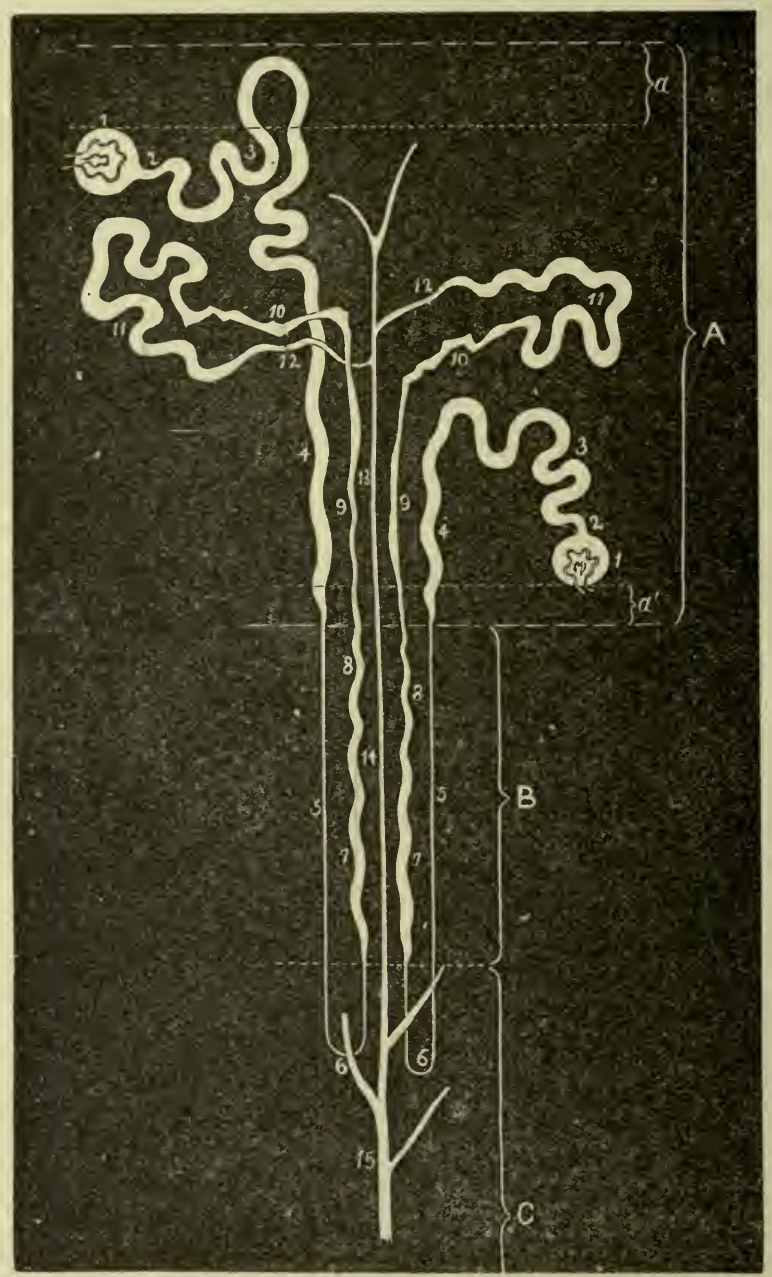

Fig. 371.-A diagram of the uriniferous tubes. A, cortex limited externally by the capsule ; a, subcapsular layer not containing Malpighian corpuscles; $a^{\prime}$, inner stratum of cortex, also without capsule of Malpighian corpusclary layer; C, medullary part next the boundary layer; 1, Bowman's 5, descending limb of Hence's loop; 6 , the loop proper; 7 , thick part of the ascending i mb spiral tubule part of ascending limb; 9 , narrow ascending limb in the medullary ray ; 10, the zigzag tubule; 11 the second convoluted tubule; 12 , the junctional tubule; 13 , the collecting tubule of the medullary ray; 14, the collecting tube of the boundary layer; 15, duct of Bellini. (Klein.)

thus discharge the urine which flows through them. They are bound together by connective tissue. 
In the pyramids the tubes are straight-uniting to form larger tubes as they descend through these from the cortical portion; while in the latter region they are convoluted. But in the boundary zone between cortex and medulla, small collections of straight tubes called medullary rays project into the cortical region.

Each begins in the cortex as a dilatation, called the Capsule of Bowman; this encloses a tuft or glomerulus of capillaries, called a Malpighian corpuscle. The tubule leaves the capsule by a neck, and

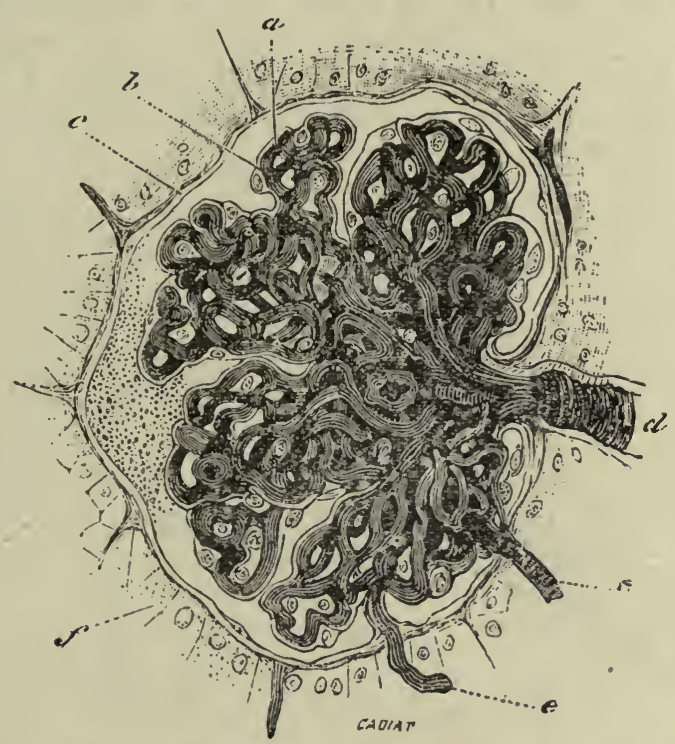

FIo. 372.-Malpighian corpuscle, injected through the reual artery with coloured gelatin; $a$, glomerular vessels; $b, c$, capsule of Buwmau; $d$, afferent vessel of glomerulus; $e$, efferent vessels; $f$, epithelium of tubes. (Cadiat.)

then becomes convoluted (first convolutcd tubule), but soon after becomes nearly straight or slightly spiral (spiral tubule); then rapidly narrowing, it passes down into the medulla as the descending tubule of Henle; this turns round, forming a loop (loop of Henle), and passes up to the cortex again as the ascending tubule of Henle. It then becomes larger and irregularly zigzag (zigzag tubule) and again convoluted (second convoluted tubule). Eventually it narrows into a junctional tubule, which joins a straight or collecting tubule. This passes straight through the medulla, where it joins with others to form one of the ducts of Bellini that open at the apex of the pyramid. These parts are all shown in fig. 371 .

In the capsule, the epithelium is flattened and reflected over the glomerulus, 
In the neck the epithelium is still flattened, but in some animals, such as frogs, where the neck is longer, the epithelium is ciliated.

In the first convoluted and spiral tubules, it is thick, and the cells show a fibrillated structure, except around the nucleus, where the protoplasm is granular. The cells interlock laterally and are difficult to isolate. In the narrow descending tubule of Henle and in the loop itself, the cells are clear and flattened and leave a considerable lumen; in the ascending limb they again become striated and nearly fill the tubule. In the zigzag and second convoluted tubules the fibrillations become even more marked. The junctional tubule has a large lumen, and is lined by clear flattened cells; the collecting tubules and ducts of Bellini are lined by clear cubical or columnar cells (see fig. 373 ).

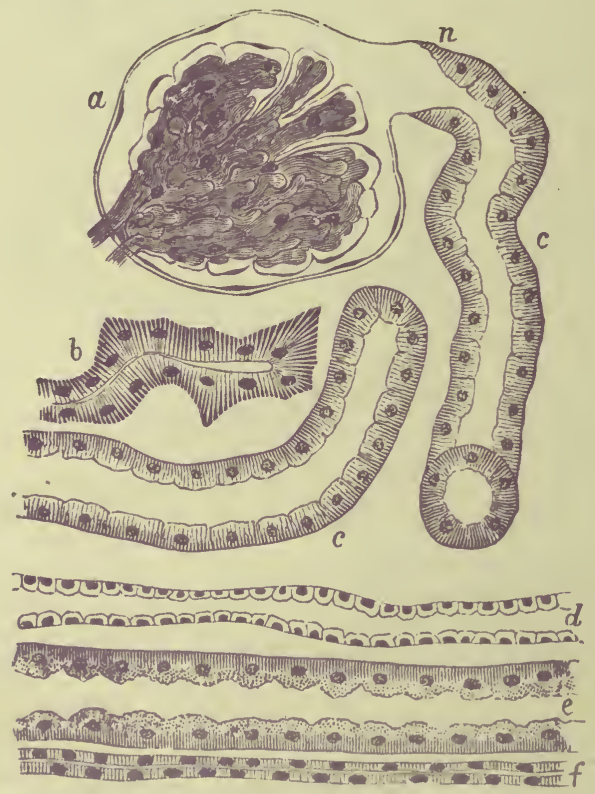

FIG. 373. - From a vertical section through the kidney of a dog-the capsule of which is supposed to be on the right. $a$, The capillaries of the Malpighian corpuscle, which are arranged in lobules; $n$, neck of capsule; $c$, convoluted tubes cut in various directions; $b$, zigzag tubule; $d, e$, and $f$, are straight

tubes in a medullary ray; $d$, collecting tube; $e$, spiral tube; $f$, narrow section of ascending limb.
$\times 380$. (Klein and Noble Smith.)

The extent of the zone of clear cells in the loop of Henle varies a good deal in different animals; a diminution of this part of the tubule lessens the length of the total loop; in most animals there is an admixture of long and short loops, though the proportion of the two varies greatly in different parts of the animal kingdom. 
Blood-vessels of Kidney.-The renal artery enters the kidney at the hilus, and divides into branches that pass towards the cortex,

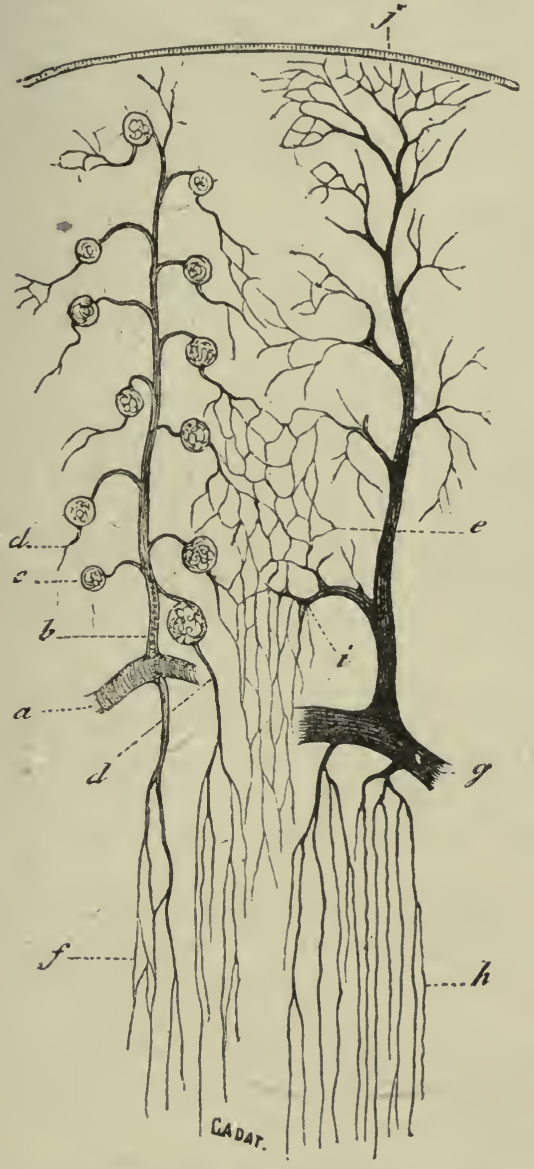

Fif. 374.-Vascular supply of kidney, $a$, Part of arterial $\operatorname{arch} ; b$, interlobular artery; $c$, glomerulus; $d$, efferent vessel passing to the medulla as false arteria recta; $e$, capillaries of cortex; $f$, capillaries of medulla; $g$, venous arch; $h$, straight veins of medulla; $i$, inter. lobular vein; $j$, vena stellula. (Cadiat.) then turn over and form incomplete arches in the region between cortex and medulla. From these arches vessels pass to the surface which are called the interlobular arteries; they give off vessels at right angles,

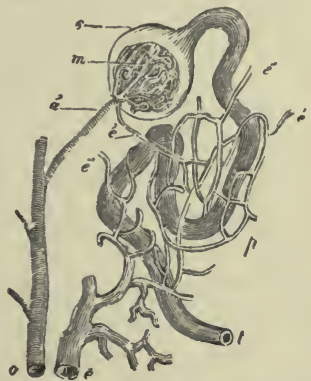

Fig. 375.-Diagram showing the relation of the Malpighian body to the uriniferous ducts and blood-vessels. $a$, One of the interlobular arteries: $a^{\prime}$, afferent artery passing into the glomerulus; $c$, capsule of the Malpighian body, forming the commencement of and continuous with the uriniferous tube; $e^{\prime}, e_{1}^{\prime} e^{\prime}$, efferent vessels which subdivide and form a plexus, $p$, surrounding the tube, and finally terminate in the branch of the renal vein $e$. (After Bowman.)

which are the afferent vessels of the glomeruli; a glomerulus is made up of capillaries as previously stated. From each a smaller vessel (the efferent vessel of the glomerulus) passes out, and like a portal vessel on a small scale, breaks up once more into capillaries which ramify between the convoluted tubules. These unite to form veins (interlobular. veins) which accompany the interlobular arteries; they pass to venous arches, parallel to, but more complete than, the corresponding arterial arches; they ultimately unite to form the renal vein that leaves the hilus. These veins receive also others which have a stellate arrangement near the capsule (vence stellulo). 
The medulla is supplied by pencils of fine straight arterioles which arise from the arterial arches. They are called arterice rectce. The efferent vessels of the glomeruli nearest the medulla may also break up into similar vessels which are called false arterioe rectoe. The veins (venoe rectoe) take a similar course and empty themselves into the venous arches. In the boundary zone groups of vasa recta alternate with groups of tubules, and give it a striated appearanco.

The Ureters.-The duct of each kidney, or ureter, is a tube about the size of a goose-quill, and from twelve to sixteen inches in length, which, continuous above with the pelvis, ends below by perforating obliquely the walls of the bladder, and opening on its internal surface.

It is constructed of three coats: $(a)$ an outer fibrous coat; $(b)$ a middle muscular coat; and (c) a mucous membrane continuous with that of the pelvis above, and of the urinary bladder below; it is composed of areolar tissue lined by transitional epithelium.

The Urinary Bladder is pyriform; its widest part, which is situate above and behind, is termed the frindus; and the narrow constricted portion, by which it becomes continuous with the urethra, is called its cerviic or neck.

It is constructed of four coats,-serous, muscular, areolar or submucous, and mucous. The circular muscular fibres are especially developed around the cervix of the organ and form the sphincter vesicce. The mucous membrane is like that of the ureters. It is provided with mucous glands, which are most numerous near the neck of the bladder.

The bladder is well provided with blood-and lymph-vessels, and with nerves. The latter consist of branches from the sacral and hypogastric plexuses. Ganglion cells are found, here and there, on the course of the nerve-fibres.

The Urethra.--This occupies the centre of the corpus spongiosum in the male. As it passes through the prostate it is lined by transitional, but elsewhere by columnar epithelium, except near the orifice, where the epithelium is stratified like the epidermis, with which it becomes continuous. The female urethra has stratified epithelium throughout. The epithelium rests on a vascular corium, and this is covered by submucous tissue containing an inner longitudinal and an outer circular muscular layer. Outside this a plexus of veins passes insensibly into the surrounding erectile tissue.

Into the urethra open a number of oblique recesses or lacunce, a number of small mucous glands (glands of Littré), two compound racemose glands (Cowper's glands), the glands of the prostate, and the vas deferens. The prostate, which surrounds the commencement of the male urethra, is a muscular and glandular mass. Its glands 
are tubular and lined by columnar epithelium; their secretion dilutes the semen. Very little is known of the function of the prostate; it often enlarges and becomes calcareous in old age, and gives rise to discomfort and difficulty in micturition. Its removal under these circumstances is a most beneficial operation.

\section{The Nerves of the Kidney.}

These are derived from the renal plexus of each side. The renal plexus consists of both medullated and non-medullated nerve-fibres, with collections of ganglion cells. Fibres from the anterior roots of the eleventh, twelfth, and thirteenth dorsal nerves (in the dog) pass into this plexus. They are both vaso-constrictor and vaso-dilator in function. The nerve-cells on the course of the constrictor fibres are situated in the cœliac, mesenteric, and renal ganglia; the nerve-cells on the course of the dilator fibres are placed in the solar plexus and renal ganglia. We have, at present, no knowledge of true secretory nerves to the kidney, and the amount of urine is influenced, to a certain extent at any rate, by the blood-pressure in its capillaries. We shall, a few pages hence, however, see that the amount of urine does not depend wholly on the height of the blood-pressure; and one very striking fact in this relation may be mentioned now,-namely, that if the blood-pressure is increased without allowing the blood to flow, the amount of urine formed is not increased; this can be done by ligaturing the renal vein; the blood-pressure within the kidney then rises enormously, but the flow of urine stops.

\section{The Kidney Oncometer.}

This is an instrument constructed on plethysmographic principles, by means of which the volume of the kidney is registered. The general characters of this instrument are described in the diagrams on p. 310. The special form introduced by Roy for the kidney is shown in fig. 376. Roy's instrument, however, is but seldom used at the present day. An air oncometer, connected with a Marey's tambour or a bellows recorder (like that figured for the spleen on p. 311), is much less complicated, and gives better results.

It is found that the effect on the volume of the organ of dividing or stimulating nerves corresponds to blood-pressure. If a rise of pressure in the renal artery is produced by constriction of the renal arterioles, this is accompanied by a fall of pressure in the renal capillaries and a shrinkage of the kidney. Increase in the volume of the kidney is produced by the opposite circumstances.

The accompanying tracing (fig. 377) shows that in a normal oncometric curve from the kidney there is a rise of volume, due to 
each heart-beat, and larger waves, which accompany respiration. In some cases larger sweeping waves (Mayer curves) are seen as well,

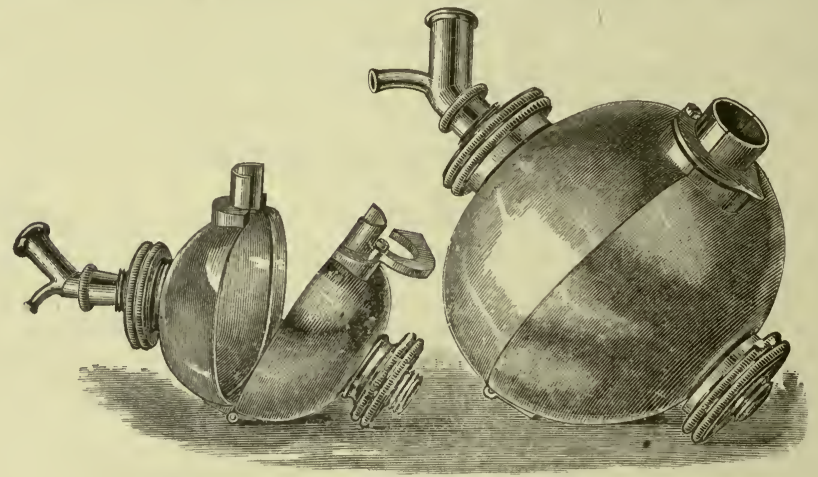

Fig. 376.-Oncometers for kidneys of different sizes.

but they are absent in the tracing reproduced: if, in such a tracing, the kidney curve is compared with the tracing of arterial

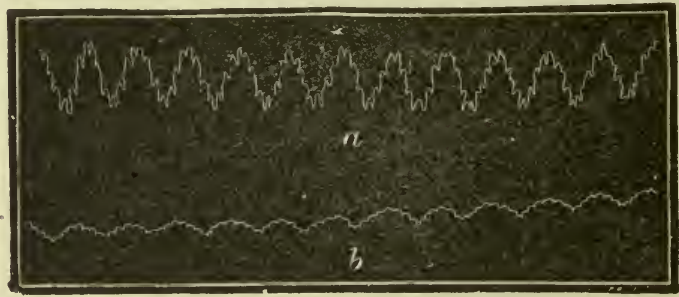

Fio. 377.---Curve taken by renal oncometer compared with that of ordinary blood-pressure. $a$, Bloodpressure curve; $b$, kidney curve. (Roy.)

pressure, it will be seen that the rise of pressure is simultaneous with the fall in kidney volume due to constriction of the renal vessels.

\section{The Functions of the Kidney.}

The function of the kidneys is to separate the urinary constituents from the blood, and by this means the blood is maintained of constant composition. The kidrey cells are remarkably sensitive, so that alterations in the composition of the blood which are too slight to be detected by chemical analysis (such as an increase of water or of chlorides after a meal) are felt by the kidney, and increased secretion (diuresis) occurs. In the case of some urinary constituents, they are practically entirely removed by the kidney; urea is an example of this class. In other cases excess beyond a 
certain percentage is removed from the blood; sodium chloride is an instance of these.

Although the glandular epithelium of the convoluted tubes is par excellence the secreting mechanism of the kidney, much difference of opinion exists as to the part played by each of the several units in the histological complex we have already described, and this is especially the case in relation to that unique structure, the glomerulus. We have seen that the efferent vessel of each glomerulus has a smaller calibre than the afferent vessel, and this produces high pressure in the glomerular capillaries. The efferent vessel, moreover, resembles an arteriole in its abundance of muscular tissue, and this maintains the high intra-glomerular blood-pressure. This arrangement led Ludwig to the conception that the glomerulus is a filter, and the filter theory has formed the basis of much subsequent work, and numerous theories.

It is impossible in a question upon which physiologists are so divided, to make a complete statement of the case which will meet with universal acceptance, and still more difficult is it to build any sure system of medical treatment upon so insecure a foundation; and before we attempt to consider the function of this or that type of cell in the kidney, let us see what can be laid down in relation to the physiology of the kidney as a whole.

We may sum up the process of urinary secretion as follows:Ore fluid, the arterial blood, enters the kidney; two fluids, the venous blood and the urine, leave it. Both of these fluids are different in composition from the arterial blood. The following table gives the approximate values of the principal constituents in the plasma of the arterial blood, and in the urine:-

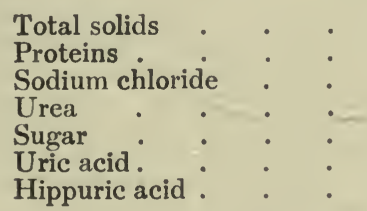

Arterial Blood-plasma.

10 per cent.

$\begin{array}{ccc}7.5 \text { to } 8 & , & , \\ 0.8 & , & , \\ 0.03 & , & , \\ 0.15 & , & , \\ \text { traces } & & \\ 0 & , & ,\end{array}$

Urine.

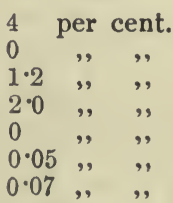

We know that it is not possible to convert any fluid into two others, each of different composition from itself, without an expenditure of energy which must come from somewhere outside the fluids themselves. In the case of the kidney, as in other secreting glands, this energy comes from the cells of the organ.

The secretion of urine is therefore the result of work done by the kidney. The quantity of this work may be measured within certain limits, and the energy transformed by the kidney may be estimated in more than one way. The urine is much more concentrated, as regards 
crystalline constituents, than the plasma from which it was produced. Thus, urine contains about 2 per cent. of urea on an average, plasma 0.03 per cent., and ths same is true in different degrees for other substances. It follows that if urine were placed inside an osmometer and an unlimited supply of plasma outside, water would be sucked into the osmometer until a column of fluid of great height had been established and much work had been performed in raising it. In a specific instance, the blood-plasma had an osmotic pressure equivalent to a 0.92 per cent. solution, and the urine to a 4 per cent. solution, of sodium chloride. From these data, and from the amount of urine secreted, it is possible to make a calculation of the work performed by the kidney. In other words, the energy used by the kidney in secreting the urine cannot be less than what is given by this purely physical consideration.

The maximum energy used up by the kidney may be calculated in quite another way. Estimations have been made of the amount of oxygen used by the kidney in secreting urines of known concentration; this oxygen may be taken as a measure of the amount of kidney material used up. If the amount of metabolism be thus determined, we can arrive at the amount of energy used up by a knowledge of the heat produced by the decomposition of this amount of kidney material.

The kidney cannot be doing more work than its metabolism accounts for. If we suppose the kidney living on protein (and the figures would not differ greatly if we supposed it to be living on carbohydrate), we may start with the following constants : 1 c.c. of oxygen oxidises 1 milligramme of protein, and forms water, carbon dioxide, urea, etc. In doing so, it gives out 4000 small calories (see Chapter XLII.), and this is equivalent to 170,000 gramme-centimetres of work. In a typical experiment during diuresis, the kidney used 4 c.c. of oxygen per minute; this was, therefore, equivalent to 680,000 gramme-centimetres of work, and the energy transformed from potential to kinetic energy by the kidney cannot have been less than this. Let us consider what evidence there is of mechanical work which the organ does as an offset against this; one way in which the work manifests itself is in the concentration of the urine; this fluid is many times more concentrated than the blood-plasma. The degree of concentration can be calculated from a knowledge of the freezing-points of the blood and urine; the greater the concentration of a solution of a crystalline substance, the lower is its freezingpoint (see p. 326). In this way, it was calculated that 14,700 gramme-centimetres of work was done in the case just referred to. If the calculation is made for each salt separately, a much higher figure than this would, however, be obtained.

The practical importance of these considerations to the physician lies in the fact that the expenditure of energy involves combustion, and combustion demands oxygen. For this reason, if for no other, an efficient supply of oxygen, that is, an efficient circulation of blood, is the first condition necessary to a healthy kidney. Renal-trouble is often secondary to cardiac trouble, and may be the result of accumulation of blood in the great veins; in such cases it is obvious that the renal trouble cannot be overcome by treating the kidney, 
but the cardiac difficulty must be dealt with. Again, the physician may endeavour, with a certain measure of success, to decrease the work which falls upon the kidney, by transferring the excretory function as far as possible to the skin. This may be accomplished by stimulating the skin to action by hot air baths, or even more successfully by ordering the patient to a hot dry climate.

The importance of changes in the circulation is still further accentuated by the fact that the arterial pressure appears to exercise a direct effect upon the volume of urine secreted by the kidney. In a general sense, those forms of experimental procedure which increase the volume of the kidney, as measured by the oncometer, increase also the flow of urine. This is illustrated by the data given in the following table:-

\begin{tabular}{|c|c|c|c|c|}
\hline Procedure. & $\begin{array}{l}\text { General blood. } \\
\text { pressure. }\end{array}$ & R^nal vessels. & Kidney volume. & Urinary flow. \\
\hline $\begin{array}{l}\text { Division of spinal cord in } \\
\text { neck }\end{array}$ & $\begin{array}{l}\text { Falls to } \\
40 \mathrm{~mm} \text {. }\end{array}$ & Relaxed & Shrinks & Ceases \\
\hline Stimulation of cord . & Rises & Constricted & Shrinks & Diminished \\
\hline $\begin{array}{l}\text { Stimulation of cord after } \\
\text { section of renal nerves }\end{array}$ & Rises & $\begin{array}{l}\text { Passively } \\
\text { dilated }\end{array}$ & Swells & Increased \\
\hline $\begin{array}{c}\text { Stimulation of renal } \\
\text { nerves }\end{array}$ & Unaffected & Constricted & Shrinks & Diminished \\
\hline $\begin{array}{l}\text { Stimulation of splanchnic } \\
\text { nerve }\end{array}$ & Rises & Constricted & Shrinks & Diminished \\
\hline Plethora . & Rises & Dilated & Swells & Increased \\
\hline Hæmorrhage . & Falls & Constricted & Shrinks & Diminished \\
\hline
\end{tabular}

Results obtained by the oncometer are less easy to interpret in the kidney than in other organs, because of the complexity of its vascular mechanism. For example, constriction of the efferent vessels of the glomeruli might raise the pressure in the glomerular capillaries, and simultaneously lower the pressure in other parts of the kidney, and the net result would be no material alteration in the volume of the whole kidney. The oncometric record is also complicated by the consideration that when diuresis is established an increase in the volume of the kidney may not be wholly of vascular origin, but may be partly due to accumulation of urine.

In spite of these difficulties, we are nevertheless justified in concluding that pronounced changes in the size of the kidney are of 
vascular origin, and they furnish the best index we have of the pressure of the blood in the capillaries. They give no indication of the rate of blood-flow through the organ, which is an altogether different thing.

Finally, from the medical point of view, the activity of the kidney may be invoked by the administration of drugs. Such drugs are called diuretics. They act in different ways, some locally on the kidney, such as caffeine, and certain saline diuretics; others, such as digitalis, act upon the general blood-pressure. It is important, in prescribing these drugs, not to lose sight of the fact that whilst the greatest benefit may attend their action, it is doubtful whether any of those commonly administered through the alimentary canal (the digitalis group excepted) can be regarded as doing their work without throwing a greater or less strain upon some portion of the renal epithelium.

Having in this way considered the kidney as a whole, we must next consider the function of the different types of cell found in the kidney tubule, including the capsule of Bowman in that expression.

A complete statement of the case would involve for each type a description of (1) which of the urinary constituents traverses its protoplasm; (2) the mechanism, whether secretory or physical diffusion or filtration, by which the constituent is propelled; and (3) the direction in which it goes, whether from the blood to the urine, or from the urine to the blood.

Fortunately, however, it is not necessary to discuss the matter quite so exhaustively, for certain possibilities have never been advanced, and so can be put upon one side. For instance, no one has ever suggested that the thick glandular cells of the tubules allow materials to pass through them by physical diffusion; here, undoubtedly, we have to deal with secretory action alone. Again, the direction of flow through the capsule of Bowman is undoubtedly from the blood to the urine, and the passage of water in the opposite direction, if it does occur at all, is limited to certain regions of the tubule itself.

Our problem, therefore, is simplified, and the questions remaining are (1) whether the flow in the glomeruli is due to physical or to physiological (secretory) processes; (2) the evidence of secretory action in the tubules; and (3) the question whether reabsorption of fluid occurs in the tubules. In relation to the first of these points we may ask the preliminary question. Is there any evidence of physical diffusion or filtration in the kidney? or, to put it another way, is there any evidence of a urinary flow without the performance of work on the part of the kidney cells?

The answer to this is as follows: such a flow cannot take place as the result of any agency which alters the composition of the fluid; nevertheless, the appropriate injection into the circulation of 
Ringer's fluid causes a copious flow of urine which is in its composition virtually Ringer's fluid. On physico-chemical grounds such a How would not necessarily demand work on the part of the kidney cells, and as a matter of fact, estimation of the oxygen used during such excretion reveals no measurable rise in the amount of work performed. The general relationship shown in the table on p. 575 , between the pressure of blood in the kidney capillaries and the volume of urine secreted, points also to the possibility that under normal conditions filtration is a factor in urine formation. The cells of Bowman's capsule, thin though they are, can, however, exercise selective action. The simplest phase of this is that massive molecules (protein) do not pass through them in health; this, however, is also true for films of gelatin. In addition to this, these living cells may exercise a more active selection, as is seen in the type of urine excreted by kidneys in which the tubules have undergone necrosis as the result of injection of uranium salts.

We may take next the point mentioned last, namely, that of reabsorption. Carl Ludwig imagined that the urine filtered off at the glomerulus becomes more concentrated as it descends the tubule, and that the work of the cells in the tubule is to reabsorb the water and pass it back into the blood. There is in our opinion no satisfactory evidence that this occurs; still it is not possible to categorically deny its existence; if it does occur it does not take place on the massive scale* which Ludwig's views involve, and is limited to the clear epithelium of the descending limb of Henle's loop. The majority of physiologists do not admit even this, and one piece of evidence which we owe to Brodie appears to me to be quite conclusive; it is this: if the pressure of urine in the ureter is artificially raised by partially blocking it, absorption of water back into the blood ought to be increased; but as a matter of fact this does not take place, but the exact contrary, for the flow of urine provoked by the injection of sodium sulphate is more abundant from the kidney with the partially blocked ureter, than in the other kidney which serves as a control.

This brings us then to our main conclusion concerning the function of the epithelium of the tubular part of the apparatus. Here undoubtedly the main function is secretion, and the obviously secreting nature of the cells is revealed by microscopic examination. The following experiments, among others, support this view:(a) In frogs the glomeruli can be cut out of action by ligaturing the renal artery; the kidney is then supplied only by the renal portal voin, a vessel which goes to the tubules only. If urea is then injected under the skin, a secretion of urine occurs, which though

* Ludwig's theory would involve the reabsorption of nearly 70 litres of water in the day! 
scanty in amount, is peculiarly rich in urea. Urea, therefore, in the frog is secreted by the epithelium of the tubules. In order to obtain this result, the kidney must receive also sufticient oxygen for the maintenance of the functional activity of its cells; as the arterial supply is cut off by ligature of the renal artery, this must be accomplished in some other way, for instance, by keeping the frog during the experiment in an atmosphere of pure oxygen.

(b) The same result is reached in the frog in another way. The renal portal system of the frog's kidney may bo artificially perfused with oxygenated Ringer's solution, the renal arteries as before being ligatured; if certain diuretics are added to the solution (caffeine, urea, phloridzin, sodium sulphate, etc.), these induce secretion which is accompanied by a marked increase in the oxygen consumption of the kidney.

(c) In mammals the arrangement of the blood-vessels is different, and it is not possible to isolate the tubules as in the frog. Nevertheless, the same diuretics cause a copious flow of urine, which is accompanied in all cases by the evidence of increased work on the part of the kidney cells, namely, a large rise in the amount of oxygen used up. In all these cases the urine secreted is unlike the plasma in composition, and varies somewhat with the diuretic employed; for instance, injection of sodium sulphate produces a urine almost devoid of chlorides.

(d) In mammals also it is possible to trace certain substances with the eye. A research of great importance was performed along this line by Heidenhain. By cutting the spinal cord he removed the arterial tone from the whole visceral area, and consequently produced a blood-pressure so low that no fluid came down the tubules. Sulphindigotate of soda is a nitrogenous substance, and it can easily be recognised by its bluo colour. If it is injected into the blood-stream it is excreted by the liver and kidney, and on post-mortem examination of the kidney, it is found in those cells of the tubules which bear the impress of secreting cells, whereas it is absent from the cells of Bowman's capsule.

Numerous hypotheses have been put forward to explain all these results, and the two great historic theories are those of Ludwig and of Bowman. The gist of Ludwig's views will have been gathered from what has just been said; he supposed that the urine filtered through at the glomeruli is a fluid of the same general composition as the blood-plasma, so far as its crystalline constituents are concerned, and that this fluid is gradually turned into urine as it travels along the tubules: by the absorption from it into the blood of water and certain salts. That fluid isotomic with blood-plasma can or does filter through the flat epithelium cells of Bowman's capsule appears to be true, but 
the part of the theory which deals with reabsorption we have seen reasons for not accepting.

The general appearance of the capsular epithelium resembles that lining a lymph space, and some morphologists hold the view that each capsule is in development part of the peritoneum, whilst others regard it as part of the kidney tubule. It is possible that the glomerulus has other functions besides that of a filter. Brodie, for instance, has suggested that its main, if not its only use is that of a driving force to propel the secreted urine along the tubule, the resistance of which is very great; he has invented models which demonstrate that pulsations of the kind alleged to occur in the glomeruli will drive fluid along a tube; he further supports his theory by the observation that the maximum pressure in the ureter as measured by a manometer is usually at least 30 to $44 \mathrm{~mm}$. of mercury less than the arterial blood-pressure, and is probably equal to the pressure in the glomerular capillaries. But these views have not at present been fully accepted by the majority of physiologists.

Bowman's classical theory was formulated more than seventy years ago, that is, long before many of the experiments just described were performed. It accords much more nearly with modern views than that of Ludwig, and was founded mainly as a deduction from anatomical structure, namely, the histological appearances of the epithelial cells which line the tubules, and the double vascular supply which in the frog indicates that the work of the tubule is distinct from that of the glomerulus. He considered that the cells lining the capsule have much the same function as those lining a lymph space, and that it is the glandular epithelium of the tubules which secretes the nitrogenous constituents of the urine. We may accept the last part of the theory as now definitely proven. But whether the glomerular flow is conditioned by physical or by physiological factors is the main point at issue between investigators at present. In the minds of both Ludwig and Bowman the physical factor was uppermost. Heidenhain, and mure recently Brodie, regard the flat glomerular cells as true secreting cells. Our own view is that although active secretion on the part of these cells has not been proved to exist, the possibility cannot be altogether denied, and as will be gathered from what has preceded this, our bias is distinctly in favour of the physical or filtration hypothesis.

\section{Extirpation of the Kidneys.}

Extirpation of one kidney for stone, etc., is a common operation. It is not followed by any untoward result. The remaining kidney enlarges and does the work previously shared between the two. 
Extirpation of both kidneys is fatal; the urea, etc., accumulate in the blood, and the animal dies in a few days; uræmic convulsions do not usually occur in such experiments.

Ligature of both renal arteries amounts to the same thing as extirpation of the kidneys, and leads to the same result. If the ligature is released the kidney after a time again sets to work, but the urine secreted at first is albuminous, owing to the epithelium having been impaired by being deprived for a time of its blood supply.

\section{The Passage of Urine into the Bladder.}

As each portion of urine is secreted it propels that which is already in the uriniferous tubes onwards into the pelvis of the kidney. Thence through the ureter the urine passes into the bladder, into which its rate and mode of entrance has been watched in cases of ectopia vesicce, i.e. of such fissures in the anterior and lower part of the. walls of the abdomen, and of the front wall of the bladder, as expose to view its hinder wall together with the orifices of the ureters. The urine does not enter the bladder at any regular rate, nor is there a synchronism in its movement through the two ureters. During fasting, two or three drops enter the bladder every minute; each drop as it enters first raises up the little papilla through which the ureter opens, and then passes slowly through its orifice, which at once again closes like a sphincter. Its flow is aided by the peristaltic contractions of the ureters, and is increased in deep inspiration, or by straining, and in active exercise, and in fifteen or twenty minutes after a meal. The urine is prevented from regurgitation into the ureters by the mode in which these pass through the walls of the bladder, namely, by their lying for between h.alf and three-quarters of an inch between the muscular and mucous coats before they turn rather abruptly forwards, and open through the latter into the interior of the bladder.

\section{Micturition.}

The desire to void the urine arises from a sense of fullness of the bladder, and the increase of pressure in this viscus, which results from its distension, is probably the most important factor in the causation of the reflex. Mosso states that in the dog's bladder a pressure of $20 \mathrm{cms}$. of water sets the reflex in action.

The afferent impulse so produced finds its way to the sacral region of the cord chiefly through the second and third sacral nerves, and stimulates the so-called vesical centre, which is situated in the grey matter there; the reflex takes place perfectly well in an animal whose spinal cord has been cut across as low as the lower part of the lumbar region. It has therefore been proved that the reflex centre 
must be situated below this point. In such animals there is no cunsciousness of the afferent impulse, and the same is true for the human subject with corresponding injuries to the spinal cord. Such animals or men have also no voluntary control over the act; it occurs in them purely reflexly.

The efferent nerves to the bladder fall into two sets:-(1) i'he nervus erigens; this is undoubtedly the more important of the two. Stimulation of this nerve causes contraction of the bladder, and relaxation of its sphincter, the two necessary acts by which the urine is expelled. (2) The hypogastric nerves; pre-ganglionic fibres leave the cord in the lumbar region, pass thence to the inferior mesenteric ganglion, from the cells of which the post-ganglionic fibres ultimately reach the bladder by the hypogastric nerves. Much difference of opinion has been expressed regarding the action of these nerves, but in most animals they cause constriction of the sphincter, and in some cases relaxation of the bladder walls also. The hypogastric nerves would therefore appear to be the functional antagonists of the nervi erigentes. In many animals the bladder constantly exhibits rhythmic contractions:

In theory, therefore, micturition is a reflex action; but in practice it is a voluntary act, and the voluntary muscles of the abdomen press upon the bladder and assist its emptying. It is only in the cases of cord injury or disease already alluded to that the voluntary factor is absent.

The simplest view to take of voluntary micturition is the following:-The will causes the abdominal muscles to contract, and the increased pressure on the bladder so produced is the signal for the reflex to occur. It is further probable that the mere thought of micturition may influence the sacral vesical centre, and heighten its sensitiveness. This certainly is the case in the neighbouring centre for the erection of the penis; erection can be evoked as a reflex act, yet it is a matter of experience that it also takes place as a result of the emotions."

If urine is voided too frequently, the cause may be.(1) peripheral, as in inflammation of the bladder; here the organ is unduly sensitive to the pressure of fluid; and (2) central, as in cases of fear and excitement; here the sensibility of the vesical centre is heightened. In children where control of the vesical centre is often not fully established while they are young, frequent and involuntary micturition may occur.

Deficiency of power to expel the urine may be due to actual obstruction, from an enlarged prostate or a stricture in the urethra. It may also be due to weakness of the bladder, as in cases where the organ is much distended and its musculature attenuated; this condition is often sccondary to obstruction produced by stricture, or other causes. 


\section{CHAPTER XXXIX}

\section{THE URINE}

Quantity.-A man of average weight and height passes from 1400 to 1600 c.c., or about 50 fluid oz. daily. This contains about 60 grammes $\left(1 \frac{1}{2}\right.$ oz.) of solids.

Colour.-This is some shade of yellow which varies considerably with the concentration of the urine. It is due to a mixture of pigments; of these the most abundant is a yellow one, originally named urochrome by Thudichum, whose investigations have in the main been confirmed and supplemented by the recent work of Dombrowski. It shows no absorption bands, and does not fluoresce with zinc salts as urobilin does. It yields a pyrrol derivative which is not hæmopyrrol, and so urochrome is probably not related to urobilin. It contains 11.1 per cent. of nitrogen and 5 per cent. of sulphur, most of which is easily split off as sulphide by cold alkali. It is probably derived from protein.

Urobilin, which is normally present in small quantities only, has a reddish tint, and like bile pigment is an iron-free substance. It has an absorption band near the $\mathrm{F}$ line. The bile pigment in the intestines is converted into stercobilin, most of which leaves the body with the fæces; some, however, is reabsorbed and is excreted into the urine, and is then called urobilin (see p. 534). A chromogen or mother-substance called urobilinogen, which by oxidation-for instance, standing exposed to the air-is converted into the pigment proper, is more abundant than urobilin itself. In certain diseased conditions the amount of urobilin is considerably increased.

Uroerythrin, the colouring matter of pink urate sediments, appear to be a small but constant constituent of urine, but its origin is unknown. Normal urine contains also a trace of hoematoporphyrin, and the amount is increased in certain diseased states.

Reaction.-The reaction of normal urine is acid; this is not due to free acid, but to acid salts, of which acid sodium phosphate is the most important. The uric and hippuric acids of the urine are combined as urates and hippurates respectively. Under certain circumstances the urine becomes less acid and even alkaline; the most important of these are as follows :- 
1. During digestion. Here there is a formation of free acid in the stomach, and a corresponding liberation of bases in the blood, which, passing into the urine, diminish its acidity, or even render it alkaline. This is called the alkaline tide; the opposite condition, the acid tide, occurs after a fast-for instance, before breakfast.

2. In herbivorous animals and vegetarians. The food here contains excess of alkaline salts of acids such as tartaric, citric, malic, etc. These acids are oxidised into carbonates, which, passing into the urine, give it an alkaline reaction.

Specific Gravity.-The specific gravity varies inversely as the quantity of urine passed under normal conditions from 1015 to 1025. A specific gravity below 1010 should excite suspicion of hydruria; one over 1030, of a febrile condition, or of diabetes, a disease in which it may rise to 1050 . The specific gravity has, however, been known to sink as low as 1002 (after large potations, urina potus), or to rise as high as 1035 (after great sweating) in perfectly healthy persons.

Composition.-The following table gives the average amounts of the urinary constituents passed by a man taking an ordinary diet containing about 100 grammes of protein in the twenty-four hours :-

Total quantity of
Water :
Solids :
Urea
Uric acid
Hippuric acid :
Sodium chloride
Phosphoric acid
Sulphuric acid
Ammonia
Creatinine :
Chlorine :
Potassium :
Sodium : :
Calcium :
Magnesium :

$\begin{array}{cc}1500.00 & \text { grammes. } \\ 1440.00 & , \\ 60.00 & , \\ 35.00 & , \\ 0.75 & , \\ 1.05 & , \\ 16.5 & , \\ 3.5 & , \\ 2.0 & , \\ 0.65 & , \\ 0.9 & , \\ 11 \cdot 0 & , \\ 2.5 & , \\ 5.5 & , \\ 0.26 & , \\ 0.21 & ,\end{array}$

The most abundant constituents of the urine are water, urea, and sodium chloride. In the foregoing table one must not be misled by seeing the names of the acids and metals separated. The acids and the bases are combined to form salts, such as urates, chlorides, sulphates, phosphates, etc.

\section{Urea.}

Urea, or Carbamide, $\mathrm{CO}\left(\mathrm{NH}_{2}\right)_{2}$, is isomeric (that is, has the same empirical, but not the same structural formula) with ammonium cyanate $\left(\mathrm{NH}_{4}\right) \mathrm{CNO}$, from which it was first prepared synthetically by Wöhler in 1828 . Since then it has been prepared synthetically in other ways. Wöhler's observation derives interest from the fact 
that this was the first organic substance which was prepared synthetically by chemists.*

When crystallised out from the urine it is found to be readily soluble both in water and alcohol: it has a saltish taste, and is neutral

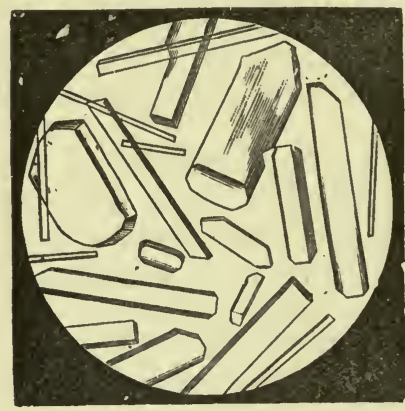

Fia. 378,-Crystals of urea. to litmus paper. The form of its crystals is shown in fig. 378 .

When treated with nitric acid, nitrate of urea $\left(\mathrm{CON}_{2} \mathrm{H}_{4} \cdot \mathrm{HNO}_{3}\right)$ is formed; this crystallises in octahedra, lozenge-shaped tablets or hexagons (fig. 379). When treated with oxalic acid, flat or prismatic crystals of urea oxalate $\left(\mathrm{CON}_{2} \mathrm{H}_{4} \cdot \mathrm{H}_{2} \mathrm{C}_{2} \mathrm{O}_{4}+\mathrm{H}_{2} \mathrm{O}\right)$ are formed (fig. 380).

These crystals may be readily obtained by adding excess of the respective acids to urine which has been concentrated to a third or a quarter of its bulk.

Under the influence of a nicro-organism, the micrococcus ureæ, which grows readily in stale urine, urea takes up water, and is converted into ammonium carbonate $\left[\mathrm{CON}_{2} \mathrm{H}_{4}+2 \mathrm{H}_{2} \mathrm{O}=\right.$ $\left(\mathrm{NH}_{4}\right)_{2} \mathrm{CO}_{3}$ ]. Hence the ammoniacal odour of putrid urine.

By means of nitrous acid, urea is broken up into carbonic acid, water, and nitrogen, $\mathrm{CON}_{2} \mathrm{H}_{4}+2 \mathrm{HNO}_{2}=\mathrm{CO}_{2}+3 \mathrm{H}_{2} \mathrm{O}+2 \mathrm{~N}_{2}$. The

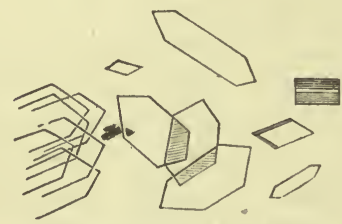

Fia. 379.-Crystals of urea nitrate.

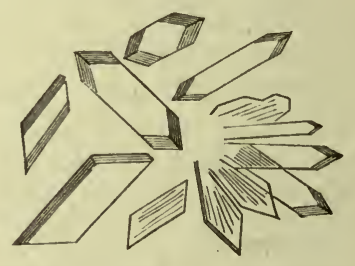

FiG. 380.--Crystals of urea oxalate.

evolution of gas bubbles which takes place on the addition of fuming nitric acid may be used as a test for urea.

Hypobromite of soda decomposes urea in the following way :-

$$
\underset{\text { [Urea.] }}{\mathrm{CON}_{2} \mathrm{H}_{4}}+\underset{\substack{\text { [Sodium } \\ \text { hypobromite.] }}}{3 \mathrm{NaBrO}}=\underset{\substack{\text { [Carbonic [Nitrogen.] } \\ \text { acid.] }}}{\mathrm{CO}_{2}}+\underset{\text { [Water.] }}{2 \mathrm{~N}_{2}}+\underset{\substack{\text { [Sodium } \\ \text { bromide.] }}}{3 \mathrm{NaBr}}
$$

This reaction is important, for on it one of the readiest methods for estimating urea depends. There have been various pieces of

* Meldola has pointed out that the English chemist Henry Hennell prepared alcohol from olefiant gas simultaneously with Wöhler's synthesis of urea. The honour of founding the science of organic chemistry must, therefore, be shared between the two men. 
apparatus invented for rendering the analysis easy; but the one describer. below is the best. If the experiment is performed as directed, nitrogen is the only gas that comes off, the carbonic acid being absorbed by excess of soda. The amount of nitrogen is a measure of the amount of urea.

Dupré's apparatus (fig. 381) consists of a bottle (A) united to a measuring tube by india-rubber tubing. The measuring tube (C) is placed within a cylinder of water (D), and can be raised and lowered at will. Measure 25 c.c. of alkaline solution of sodium hypobromite (made by mixing 2 c.c. of bromine with 23 c.c. of a 40 per cent. solution of caustic soda) into the bottle A. Measure 5 c.c. of urine into a small tube (B), and lower it carefully, so that no urine spills, into the bottle. Close the bottle securely with a stopper perforated by a glass tube; this glass tube (the bulb blown on this tube prevents froth from passing into the rest of the apparatus) is connected to the measuring tube by indiarubber tubing and a T-piece. The third limb of the T-piece is closed by a piece of india-rubber tubing and a pinch-cock, seen at the top of the figure. Open the pinch-cock and lower the measuring tube until the surface of the water with which the outer cylinder is filled is at the zero point of the graduation. Close the pinchcock, and raise the measuring tube to ascertain if the apparatus is air-tight. Then lower it again. Tilt the bottle $A$ so as to upset the urine, and shake well for a minute or so. During this time there is an evolution of gas. Then immerse the bottle in a large beaker containing water of the same temperature as that in the cylinder. After two or three minutes raise the measuring tube until the surfaces of the water inside and outside it are at the same level. Read off the amount of gas (nitrogen) evolved. $35 \cdot 4$ c.c. of nitrogen are yielded by $0^{\cdot} 1$ gramme of urea. From this the quantity of urea in the 5 c.c. of urine and the percentage of urea can be calculated. If the total urea passed in the twenty-four hours is to be ascertained, the twenty-four hours' urine must be carefully measured and thoroughly mixed. A sample is taken from the total for analysis; and then, by a simple sum in proportion, the total amount of urea is ascertained.

Folin's method is the best for accurate work; it depends on the fact that urea is decomposed into ammonia and carbonic acid by boiling with magnesium chloride in the presence of hydrochloric acid. The ammonia is estimated by distilling it into standard acid and subsequent titration.

Kjeldahl's method of estimating nitrogen consists in boiling the material under investigation with strong sulphuric acid. The nitrogen present is by this means converted into ammonia. Excess of soda is then added, and the ammonia distilled over into a known volume of standard acid. The amount of diminution of acidity in the standard enables one to calculate the amount of ammonia, and thence the amount of nitrogen. This is the best method for the estimation of the total nitrogen in the urine.

The quantity of urea is variable, the chief cause of variation being the amount of protein food ingested. In a man in a state of 
nitrogenous equilibrium, taking daily 100 to 120 grammes of protein in his food, the quantity of urea secreted daily is about 33 to 35 grammes (500 grains). The percentage in human urine would then be 2 per cent.; but this also varies, because the concentration of the urine varies considerably in health. In dogs it may be 10 per cent. The excretion of urea is usually at a maximum three hours after a meal, especially after a meal rich in proteins. If, therefore, people adopt the Chittenden diet, which contains about half the quantity of protein which is present in the more usual Voit dietary, their urine will naturally show a nitrogenous output of half of that which is now regarded as normal. In those who adopt such a reduced diet, Folin has shown that the decrease in urinary nitrogen falls mainly on the urea fraction, and in some cases the urea excreted accounted for only 66 per cent. of the total nitrogen. The other nitrogenous katabolites of the urine alter comparatively little under such circumstances, and the creatinine in particular remains remarkably constant in amount.

In our study of protein absorption (p. 545), we have already indicated that the amino-acid fragments of the food-protein are utilised in two ways. A small part is used by the tissue cells for the reconstruction of their protein which has undergone katabolism. In time this will in turn be katabolised, and the waste products discharged as ammonia, creatinine, and a certain amount of urea. This form of metabolism may be termed tissue or endogenous metabolism, and its amount is constant and independent to a great extent of the food. The other and larger part of the cleavage products of the food protein are not made use of thus, but are deamidised, and the nitrogenous portion is converted into urea by the liver, and discharged by the kidney. This part of metabolism may be termed exogenous; it is variable in amount, and depends on the quantity of ingested protein.

That the liver is the organ where urea is made is shown by the following considerations:-

1. After removal of the liver in such animals as frogs, urea formation almost ceases, and ammonia is found in the urine instead.

2. In mammals, the extirpation of the liver is such a severe operation that the animals do not live. But the liver of mammals can be very largely thrown out of gear by connecting the portal vein directly to the inferior vena cava (Eck's fistula). This experiment has been done successfully in dogs; the amount of urea in the urine is lessened, and its place is taken by ammonia.

3 . When degenerative changes occur in the liver, as in cirrhosis of that organ, the urea formed is much lessened, and its place is taken by ammonia. In acute yellow atrophy urea is almost absent in the urine, and, again, there is considerable increase in the ammonia. In this disease amino-acids such as leucine and tyrosine are also found 
in the urine; they arise from the disintegration of the proteins of the liver cells, but they may in part originate in the intestine, and, escaping further decomposition in the degenerated liver, pass as such into the urine.

That the amino-acids are the substances from which the liver forms urea is shown by the fact that if such amino-acids as glycine, leucine, arginine, etc., are administered by the mouth, or injected into the blood-stream, the excretion of urea is correspondingly raised.

The transformation of arginine into urea is a subject on which we have more accurate information than in the case of any other aminoacids, for there is no doubt that the change, which can be brought about in a test-tube, is also accomplished in the organism. If the account of arginine given on p. 420 is referred to, it will be seen to consist of a urea radical and a substance called ornithine. On hydrolysis we therefore get urea and ornithine (diamino-valeric acid), and this in the body is accomplished by a special enzyme called arginase (Kossel and Dakin), which is more abundant in the liver than in any other tissue. The actual yield of urea is, however, greater than one would anticipate, and so it must be supposed that the ornithine in its turn is broken up and urea is the result. If we glance at the formula of ornithine, and compare it with that of certain other amino-acids which are also undoubted urea foreruuners, we have the following:-

$$
\begin{aligned}
& \text { Glycine }: \quad: \quad: \quad: \quad: \begin{array}{l}
\mathrm{C}_{2} \mathrm{H}_{5} \mathrm{NO}_{2} \\
\text { Leucine } \\
\text { Ornithine : }
\end{array} \mathrm{C}_{5} \mathrm{H}_{13} \mathrm{NO}_{2} \\
& \mathrm{C}_{5} \mathrm{H}_{12} \mathrm{~N}_{2} \mathrm{O}_{2}
\end{aligned}
$$

In all cases, the atoms of carbon are more numerous than those of nitrogen. In urea $\left(\mathrm{CON}_{2} \mathrm{H}_{4}\right)$ the reverse is the case. The aminoacids must therefore be split into simpler compounds which unite with one another to form urea. Urea formation is thus, in part, synthetic. These simpler compounds are ammonium salts. Schröder's work, which has been confirmed by subsequent investigators, proves that ammonium carbonate is one of the urea precursors, if not the principal one. The equation which represents the reaction is as follows:-

$$
\underset{\substack{\text { [Ammonium } \\ \text { carbonate.] }}}{\left(\mathrm{NH}_{4}\right)_{2} \mathrm{CO}_{3}}=\underset{\text { [Urea.] }}{\mathrm{CON}_{2} \mathrm{H}_{4}}+2 \mathrm{H}_{2} \mathrm{O} .
$$

Schröder's principal experiment was this: a mixture of blood and ammonium carbonate was injected into the liver by the portal vein; the blood leaving the liver by the hepatic vein was found to contain urea in abundance. This does not occur when the same experiment is performed with any other organ of the body, so that Schröder's experiments also prove the great importance of the liver 
in urea formation. Similar results were obtained by Nencki with ammonium carbamate.

The importance of ammonia is accentuated when we remember that ammonia is one of the products of pancreatic digestion, and probably also of endogenous protein metabolism. The ammonia, whether it is formed directly or through the intermediate stage of amino-acid, will combine with the carbonic acid of the blood to form ammonium carbonate or carbamate, and the following structural formulæ exhibit the close relationship between these substances and urea. The loss of one molecule of water from ammonium carbonate produces ammonium carbamate; the loss of a second molecule of water produces urea-
$\mathrm{O}=\mathrm{C} / \mathrm{O}_{\mathrm{O}} \mathrm{NH}_{4}$
$\mathrm{O}=\mathrm{C} / \mathrm{NH}_{2}$
$\mathrm{O}=\mathrm{C} / \mathrm{NH}_{2}$
[Ammonium carbonate.]
[Ammonium carbamate.]
[Urea or carbamide.]

Urea is absent, or nearly so, from the muscles, and its place there is taken by the substance called creatine. It is, however, doubtful whether creatine is a precursor of urea in the body. The fact that muscular work does not appreciably increase protein katabolism is intelligible, when, in light of recently acquired knowledge, we realise that protein katabolism, in so far as its nitrogen is concerned, is independent of the oxidations which give rise to heat, or to the energy which is converted into work.

Uramia. - The older authors considered that urea was formed in the kidneys, just as they also erroneously thought that carbonic acid was formed in the lungs. Prévost and Dumas were the first to show that after complete extirpation of the kidneys the formation of urea goes on, and that it accumulates in the blood and tissues. Similarly, in those cases of disease in which the kidneys cease work, urea is still formed and accumulates. This condition is called urcomia, and unless the products of nitrogenous breakdown are discharged from the body the patient dies in a condition of coma preceded by convulsions.

This term was originally applied on the erroneous supposition that it is urea or some antecedent of urea which acts as the poison. There is no doubt that the poison is not any constituent of normal urine; if the kidneys of an animal are extirpated, the animal dies in a few days, but there are no uræmic convulsions. In man, also, if the kidneys are healthy, or approximately so, and suppression of urine occurs from the simultaneous blocking of both renal arteries by clot, or of both ureters by stones, again uræmia does not follow. On the other hand, uræmia may occur even while a patient with diseased kidneys is passing a considerable amount of urine. What the poison is that is responsible for the convulsions and coma, is unknown. It is doubtless some abnormal katabolic product, but whether this is produced by the diseased kidney cells, or in some other part of the body, is also unknown.

\section{Ammonia.}

A small quantity of ammonia always slips through into the urine, because a portion of the ammonia-containing blood passes through the kidney before reaching the organs that are capable of converting it into urea. In man the daily amount of ammonia excreted varies between 
0.3 and 1.2 grammes; the average is 0.7 gramme. The ingestion of ammonium carbonate does not increase the amount of ammonia in the urine, but increases the amount of urea, into which substance the ammonium carbonate is easily converted. But if a more stable sålt, such as ammonium chloride, is given, it appears as such in the urine.

Under normal conditions the amount of ammonia depends on the adjustment between the production of acid substances in metabolism and the supply of bases in the food. Ammonia formation is the physiological remedy for deficiency of bases.

When the production of acids is excessive (as in diabetes), or when mineral acids are given by the mouth or injected into the blood-stream, the result is an increase of the physiological remedy, and excess of ammonia passes over into the urine. Under normal circumstances ammonia is kept at a minimum, being finally converted into the less toxic substance urea, which the kidneys easily excrete. The defence of the organism against acids which are very toxic, is an increase of ammonia formation, or, to put it more correctly, less of the ammonia formed is converted into urea.

Under the opposite conditions, namely, excess of alkali, either in food or given as such, the ammonia disappears from the urine, all being converted into urea. Hence the diminution of ammonia in the urine of man on a vegetable diet, and its absence in the urine of herbivorous animals.

Not only is this the case, but if ammonium chloride is given to a herbivorous animal such as a rabbit, the urinary ammonia is but little increased. It reacts with sodium carbonate in the tissues, forming ammonium carbonate (which is excreted as urea) and sodium chloride. Herbivora also suffer much more from, and are more easily killed by, acids than carnivora, their organisation not permitting a ready supply of ammonia to neutralise excess of acids.

\section{Creatine and Creatinine.}

Creatine is an abundant constituent of muscle; its chemical structure is very like that of arginine; it contains a urea radical, and by boiling it with baryta it splits into urea and sarcosine (methyl-glycine), as shown in the following equation:-

$$
\underset{\text { [Creatine.] }}{\mathrm{C}_{4} \mathrm{H}_{3} \mathrm{~N}_{3} \mathrm{O}_{2}}+\underset{\text { [Water.] }}{\mathrm{H}_{2} \mathrm{O}}=\underset{\text { [Urea.] }}{\mathrm{CON}_{2} \mathrm{H}_{4}}+\underset{\text { [Sarcosine.] }}{\mathrm{C}_{3} \mathrm{H}_{7} \mathrm{NO}_{2}} \text {. }
$$

The same decomposition is shown graphically on p. 420 .

Creatine is absent from normal urine, but it is present in the urine during starvation, in acute fevers, in women during involution of the uterus, and in certain other conditions in which there is rapid loss of muscular material.

Its normal fate in the body is unknown; it may be converted into urea as in the foregoing equation, but injection of creatine into 
the blood-stream does not cause any increase in urea formation; the creatine injected is almost wholly excreted unchanged.

It also is not converted into creatinine, although it has been generally assumed that this conversion does occur. The transformation of creatine into creatinine is shown in the following equation:-

$$
\underset{\text { [Creatine.] }}{\mathrm{C}_{4} \mathrm{H}_{9} \mathrm{~N}_{3} \mathrm{O}_{2}}-\underset{\text { [Water.] }}{\mathrm{H}_{2} \mathrm{O}}=\underset{\text { [Creatinine.] }}{\mathrm{C}_{4} \mathrm{H}_{7} \mathrm{~N}_{3} \mathrm{O} .}
$$

Recent researches have entirely failed to substantiate the view that the urinary creatinine originates from the muscular creatine. If creatine (an innocuous neutral substance) were converted by the loss of water in the muscles into creatinine (a strongly basic substance), it would be contrary to all that is known of the chemical changes that occur in the body.

Creatinine is present in the urine; it is, in fact, next to urea the most abundant nitrogenous substance found there. Amid all the inconstancies of urinary composition, it appears to be the substance most constant in amount, diet and exercise having no effect on it. Folin's view, that its amount is a criterion of the extent of endogenous nitrogenous metabolism, has steadily gained ground, and the work of the past few years has shown that the liver and not the muscles is the seat of its formation. Some observers have supposed that certain tissue enzymes, termed creatase and creatinase, are agents in its formation and destruction; others have failed to discover the presence of these enzymes in the liver. On this and on other points there are differences of opinion, but without discussing the pros and cons of minor details, the following viow of Mellanby may be taken as a working hypothesis of the metabolic history of the substances in question. Mellanby took as his starting-point an investigation of the contradictory data relating to the proportion of creatine and creatinine in muscle, and by improved methods showed that creatinine is never present in muscle at all, even after prolonged muscular work. He then studied in the developing bird the amount of creatine at different stages, and found that it is entirely absent in the chick's musculature up to the twelfth day of incubation; after this date the liver and the muscular creatine develop pari passu. After hatching, the liver still continues to grow rapidly, and the creatine percentage in the muscles increases also, although the development in the size of the muscles occurs very slowly. This and other experiments on the injection of creatine and creatinine into the blood-stream finally led Mellanby to the following hypothesis:-Certain products of protein katabolism, the nature of which is uncertain, are carried by the blood to the liver, and from these the liver forms creatinine; this is transported to the muscles and there stored as creatine; when the muscles are saturated with creatine, 
excess of creatinine is then excreted by the kidneys. The small amount of creatinine excreted in diseases of the liver also supports the view that that organ is responsible for creatinine formation.

These viows will no doubt be subjected to the usual tests of criticism and renewed research; they certainly appear to explain some of our previous difficulties, though the ultimate fate of the muscular creatine is still unsolved.

\section{Uric Acid.}

Uric Acid $\left(\mathrm{C}_{5} \mathrm{~N}_{4} \mathrm{H}_{4} \mathrm{O}_{3}\right)$ is, in mammals, the medium by which a very small quantity of nitrogen is excreted from the body. It is, however, in birds and some reptiles the principal nitrogenous constituent of their urine. It is not present in the free state, but is combined with bases to form urates.

It may be obtained from human urine by adding 5 c.c. of hydrochloric acid to 100 c.c. of the urine, and allowing the mixture to stand for twelve to twenty-four hours. The crystals which form are deeply tinged with urinary pigment, and though by repeated solution in caustic soda or potash, and precipitation by hydrochloric acid, they may be obtained fairly free from pigment, pure uric acid is more readily obtained from the solid urine of a serpent or bird, which consists principally of the acid ammonium urate. This is dissolved in soda, and then the addition of hydrochloric acid produces as before the crystallisation of uric acid from the solution.

The pure acid crystallises in colourless rectangular plates or prisms. In striking contrast to urea it is a most insoluble substance, requiring for its solution 1900 parts of hot and 15,000 parts of cold water. The forms which uric acid assumes when precipitated from human urine, either by the addition of hydrochloric acid or in certain pathological processes, are very various, the

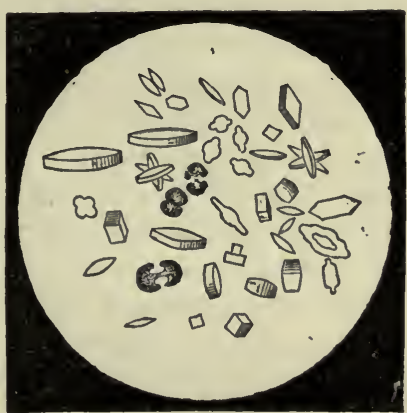

Fra. 382.-Various forms of uric acid crystals.

most frequent being the whetstone shape; there are also bundles of crystals resembling sheaves, barrels, and dumb-bells (see fig. 382).

The murexide test is the principal test for uric acid. The test has received the name on account of the resemblance of the colour to the purple of the ancients, which was obtained from certain snails of the genus Murex. It is performed as follows: place a little uric acid or a urate in a capsule; add a little dilute nitric acid and evaporate to dryness. A yellowish-red residue is left. Add a little ammonia carefully, and the residue turns violet; this is due to the 
formation of purpurate of ammonia. On the addition of potash the colour becomes bluer.

Another reaction that uric acid undergoes (though it is not applicable as a test) is, that on treatment with certain oxidising reagents urea and oxalic acid can be obtained from it. Alloxan $\left(\mathrm{C}_{4} \mathrm{H}_{2} \mathrm{~N}_{2} \mathrm{O}_{4}\right)$ or allantoin $\left(\mathrm{C}_{4} \mathrm{H}_{6} \mathrm{~N}_{4} \mathrm{O}_{3}\right)$ are intermediate products. It is, however, doubtful whether a similar oxidation occurs in the normal metabolic processes of the body.

Uric acid is dibasic, and thus there are two classes of uratesthe normal urates and the acid urates. A normal urate is one in which two atoms of the hydrogen are replaced by two of a monad metal such as sodium; an acid urate is one in which only one atom of hydrogen is thus replaced. The formulæ would be-

$$
\begin{aligned}
\mathrm{C}_{5} \mathrm{H}_{4} \mathrm{~N}_{4} \mathrm{O}_{3} & =\text { uric acid. } \\
\mathrm{C}_{5} \mathrm{H}_{3} \mathrm{NaN}_{4} \mathrm{O}_{3} & =\text { acid sodium urate. } \\
\mathrm{C}_{5} \mathrm{H}_{2} \mathrm{Na}_{2} \mathrm{~N}_{4} \mathrm{O}_{3} & =\text { normal sodium urate. }
\end{aligned}
$$

The acid sodium urate is the chief constituent of the pinkish deposit of urates, which often occurs in urine, and is called the lateritious deposit.

If uric acid is represented by $\mathrm{H}_{2} \mathrm{U}$, the normal urates may be represented by $\mathrm{M}_{2} \mathrm{U}$, and the acid urates by MHU. Bence Jones, and later Sir W. Roberts, considered that the urates actually occurring in urine are what are termed quadriurates $\mathrm{MHU} . \mathrm{H}_{2} \mathrm{U}$. There is no doubt that such compounds do not really exist; they are merely mixtures of acid urate, $\mathrm{MHU}$, and free uric acid, $\mathrm{H}_{2} \mathrm{U}$.

The quantity of uric acid excreted by an adult varies from 7 to 10 grains $(0.5$ to 0.75 gramme) daily.

The best method for estimating uric acid in urine is that of Hopkins (Folin's modification). The urine is made alkaline with ammonia and saturated with ammonium sulphate. This precipitates all the uric acid as ammonium urate; the precipitate is collected on a filter, and washed free from chlorides with a 10 per cent. solution of ammonium sulphate; it is then rinsed into a flask with hot water; when cold, sulphuric acid is added. It is then titrated at $60^{\circ} \mathrm{C}$. with a standard solution of potassium permanganate until a permanent rose colour is obtained. From the amount of the permanganate solution used to obtain the end point, the quantity of uric acid present is calculated. Uric acid may also be determined colorimetrically by the blue colour it strikes with phospho-tungstic acid (Folin and A. B. Macallum, jun.).

Origin of Uric Acid.-Uric acid is not made by the kidneys; when these organs are removed, uric acid continues to be formed, and accumulates in the organs, especially in the liver and the spleen. After extirpation of the liver in birds (in which animals uric acid is such an important katabolite), the formation of uric acid practically ceases, and its place is taken by ammonia and lactic acid, and the 
conclusion is therefore drawn that in these animals, ammonia and lactic acid are normally synthesised in the liver to form uric acid.

But in mammals, this is not the history of uric acid formation; in these animals, including man, uric acid is the end-product of the metabolism of nuclein, from the bases of which it arises by oxidation.

Nuclein, the main constituent of the nuclei of cells (see p. 431), yields, on decomposition, certain products called purine substances, and their close relationship to uric acid is shown by their formulæ:-

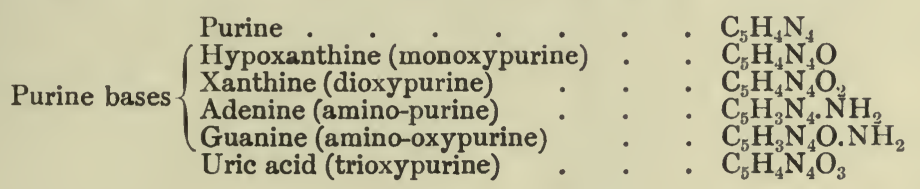

Just as the ordinary protein metabolism is both exogenous and endogenous, so is it the case with nuclein metabolism. There are certain kinds of food (such as liver and sweetbread) which are rich in nuclei, and others, such as meat, which are rich in purine bases (especially hypoxanthine). The increase in uric acid excretion after partaking of such food is exogenous, and those liable to uric acid disorders should avoid such articles of diet. Other forms of diet lead to an increase in uric acid formation by increasing the number of leucocytes in the blood, and there is a consequent increase in the meviabolism of their nuclei. Increase in leucocytes may, however, be present independently of diet, and in the disease known as leucocythomia, this occurs to a marked degree; in such cases uric acid formation increases. Although special attention has been directed to the nuclei of leucocytes because these can readily be examined during life, it must be remembered that the nuclein metabolism of all cells may contribute to uric acid formation. Uric acid, which originates by metabolism, is spoken of as endogenous.

We must next consider the mechanism by which the tissue cells form uric acid from nuclein. This question is not only of interest in itself, but also because it illustrates a general truth concerning the importance of the tissue enzymes. The enzyme of the liver which turns glycogen into sugar is the oldest known example of these; in more recent times, the importance of autolytic enzymes (see pp. 136 and 440) of tissue erepsins (see p. 546) and arginase (see p. 587) has been recognised. In uric acid formation we have the very striking example of the action of a succession of enzymes. These are present to an almost negligible extent in the juices of the alimentary canal, and have been studied in the extracts of different organs; their distribution varies a good deal in different animals, and in the different organs of the same animal; speaking generally, they are most abundant in liver and spleen. The general term 
nuclease is given to the whole group, and a dozen or more have been isolated which deal with different steps in the cleavage of the nucleic acid complex. They are classified into nucleinases which resolve the molecule into mononucleotidases, i.e., compounds of carbohydrate, phosphoric acid, and one base; nucleotidases which liberate phosphoric acid, leaving the carbohydrate still united to the base; nucleosidases which hydrolytically cleave the base and carbohydrate apart; deamidases which remove the amino-group from the purine bases so set free; one of these, called adenase, converts adenine into hypoxanthine, and another, called guanase, converts guanine into xanthine. Finally, oxidases step in, which convert hypoxanthine into xanthine, and xanthine into uric acid. But even that does not bring the list to a conclusion, for in some organs (especially the liver) there is a capacity to destroy uric acid after it is formed, and so we are protected from a too great accumulation of this substance. What exactly happens to the uric acid is not certain, although it is clear that the products of its breakdown (probably allantoin and urea) are not so harmful as uric acid itself. The enzyme responsible for uric acid destruction is called the uricolytic enzyme. The uric acid which ultimately escapes as urates (normally) in the urine is the undestroyed residue.

In gout and allied disorders there may be increased formation of uric acid, or a smaller amount of that formed may be destroyed; the excess may pass into the urine, partly as free uric acid or excess of urates, and so there is a liability to concretions (calculi, gravel, etc.), forming in the kidney or bladder. There is also a tendency to the deposition of urates in certain parts, and the joint cartilages in particular are liable to these concretions. The uric acid diathesis is, however, much too large a subject to treat in a physiological textbook, and medical students, when they come to the study of pathology, will discover that many views are held in relation to it.

\section{Hippuric Acid.}

Hippuric Acid $\left(\mathrm{C}_{9} \mathrm{H}_{9} \mathrm{NO}_{3}\right)$, combined with bases to form hippurates, is present in small quantities in human urine, but in large quantities in the urine of herbivora. This is due to the food of herbivora containing substances belonging to the aromatic groupthe benzoic acid series. If benzoic acid is given to a man, it unites with glycine with the elimination of a molecule of water, and is excreted as hippuric acid-

$$
\mathrm{C}_{6} \mathrm{H}_{5} \cdot \mathrm{COOH}+\underset{\text { [BOOH }}{\left.\right|_{\text {[Glycine.] }} ^{\mathrm{CH}_{2} \cdot \mathrm{NH}_{2}}} \cdot=\underset{\text { COOH acid.] }}{\mathrm{CH}_{2} \mathrm{NH} . \mathrm{CO} . \mathrm{C}_{6} \mathrm{H}_{5}}+\mathrm{H}_{2} \mathrm{O} \text {. }
$$

This is a well-marked instance of synthesis carried out in the 
animal body, and experimental investigation shows that it is accomplished by the living cells of the kidney itself; for if a mixture of glycine, benzoic acid, and blood is injected through the kidney (or mixed with a minced kidney just removed from the body of an animal), their place is found to have been taken by hippuric acid. In the conversion of benzoic into hippuric acid which occurs in herbivora, the necessary glycine comes from the kidney itself.

\section{The Inorganic Constituents of Urine.}

The inorganic or mineral constituents of urine are chiefly chlorides, phosphates, sulphates, and carbonates;

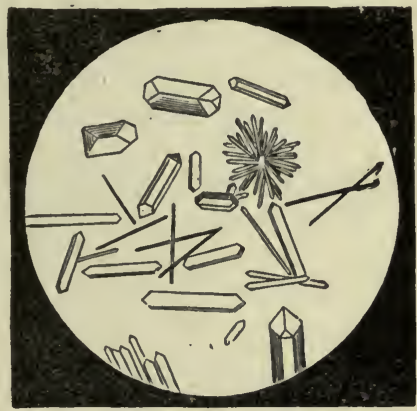

FiG. 383.-Crystals of hippuric acid. the metals with which these are in combination are sodium, potassium, ammonium, calcium, and magnesium. The total amount of these salts varies from 19 to 25 grammes daily. The most abundant is sodium chloride, which averages in amount 10 to 16 grammes per diem. These substances are derived from two sources-first from the food, and secondly as the result of metabolic processes. The chlorides and most of the phosphates come from the food; the sulphates and some of the phosphates, as a result of metabolism.

Chlorides.-The chief chloride is that of sodium. The ingestion of sodium chloride is followed by its appearance in the urine, some on the same day, some on the next day. Some is decomposed to form the hydrochloric acid of the gastric juice. The salt in the body fulfils the useful office of stimulating metabolism and secretion.

Sulphates.-The sulphates in the urine are principally those of potassium and sodium. Only the smallest trace enters the body with the food. Sulphates have an unpleasant bitter taste (for instance, Epsom salts): hence we do not take food that contains them. The sulphates vary in amount from 1.5 to 3 grammes daily.

They are derived from the metabolism of proteins, and the excretion of sulphates, though it occurs earlier than that of urea, runs parallel with it. The sulphates are, therefore, like urea, the result of exogenous protein metabolism. The sulphur of the protein, which is endogenously katabolised, is not converted into ordinary sulphates to any great extent, but reappears in the urine partly as ethereal sulphates, and partly as certain obscuro but not fully oxidised sulphur compounds, and is usually spoken of as neutral sulphur. 
The ethereal sulphates just mentioned form about a tenth of the total sulphates. They are combinations of sulphuric acid with organic radicals, and the greater part of them originate from putrefactive changes in the intestine. The chief of these ethereal sulphates are phenyl sulphate of potassium and indoxyl sulphate of potassium. The latter originates from the indole formed in the intestine, and as it yields indigo when treated with certain reagents it is sometimes called indican. The indican of urine, however, is not the same thing as the indican of plants, which is a glucoside. Both yield indigo, but there the resemblance ceases.

The formation of these sulphates is somewhat important; the aromatic substances liberated by putrefactive processes in the intestine are poisonous, but their conversion into ethereal sulphates renders them harmless.

The equation representing the formation of potassium phenyl-sulphate is as follows :-

$$
\begin{aligned}
& \mathrm{C}_{6} \mathrm{H}_{5} \mathrm{OH}+\mathrm{SO}_{2}<\mathrm{OK}=\mathrm{SO}_{2}\left\langle\mathrm{OKK}_{6} \mathrm{OK}_{6} \mathrm{H}_{5}+\mathrm{H}_{2} \mathrm{O} .\right. \\
& \text { [Phenol.] [Potassium } \begin{array}{c}
\text { [Potassium } \\
\text { hydrogen } \\
\text { phenyl-sulphate.] [Water.] }
\end{array}
\end{aligned}
$$

Indole $\left(\mathrm{C}_{8} \mathrm{H}_{7} \mathrm{~N}\right)$ on absorption is converted into indoxyl :-

$$
\mathrm{C}_{6} \mathrm{H}_{4}<\text { C.OH : } \mathrm{CH}
$$

The equation representing the formation of potassium indoxyl-sulphate is as follows :-

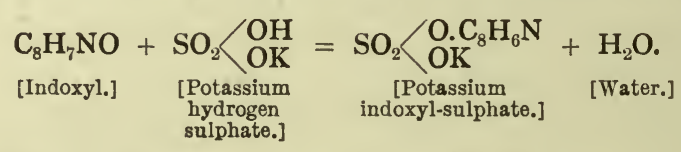

Carbonates.-Carbonates and bicarbonates of sodium, calcium, magnesium, and ammonium are only present in alkaline urine. They arise from the carbonates of the food, or from vegetable acids (malic, tartaric, etc.) in the food. They are, therefore, found in the urine of herbivora and vegetarians, whose urine is thus rendered alkaline. Urine containing carbonates becomes, like saliva, cloudy on standing, the precipitate consisting of calcium carbonate, and also phosphates.

Phosphates.-Two classes of phosphates occur in normal urine:-

(1) Alkaline phosphates - that is, phosphates of sodium (abundant) and potassium (scanty).

(2) Earthy phosphates-that is, phosphates of calcium (abundant) and magnesium (scanty).

The composition of the phosphates in urine is liable to variation. In acid urine the acidity is due to the acid salts. The chief are:-

Sodium dihydrogen phosphate, $\mathrm{NaH}_{2} \mathrm{PO}_{4}$, and calcium dihydrogen phosphate, $\mathrm{Ca}\left(\mathrm{H}_{2} \mathrm{PO}_{4}\right)_{2}$. 
In neutral urine, in addition, disodium hydrogen phosphate, $\mathrm{Na}_{2} \mathrm{HPO}_{4}$, calcium hydrogen phosphate, $\mathrm{CaHPO}_{4}$, and magnesium hydrogen phosphate, $\mathrm{MgHPO}_{4}$, are found. In alkaline urine there may be instead of, or in addition to, the above, the normal phosphates of sodium, calcium, and magnesium $\left[\mathrm{Na}_{3} \mathrm{PO}_{4}, \mathrm{Ca}_{3}\left(\mathrm{PO}_{4}\right)_{2}, \mathrm{Mg}_{3}\left(\mathrm{PO}_{4}\right)_{2}\right]$.

The earthy phosphates are precipitated by rendering the urine alkaline by ammonia. In decomposing urine, ammonia is formed from the urea: this also precipitates the earthy phosphates. The phosphates most frequently found in the white creamy precipitate which occurs in decomposing urine are:-

(1) Triple phosphate or ammoniomagnesium phosphate $\left(\mathrm{NH}_{4} \mathrm{MgPO}_{4}+\right.$ $6 \mathrm{H}_{2} \mathrm{O}$ ). This crystallises in "coffin-lid" crystals (see fig. 384 ) or feathery stars.

(2) Stellar phosphate, or calcium phosphate; this crystallises in star-like clusters of prisms.

As a rule normal urine gives no precipitate when it is boiled; but sometimes neutral, alkaline, and occasionally faintly acid urines give a precipitate of calcium phosphate when boiled: this precipitate is amorphous, and is liable

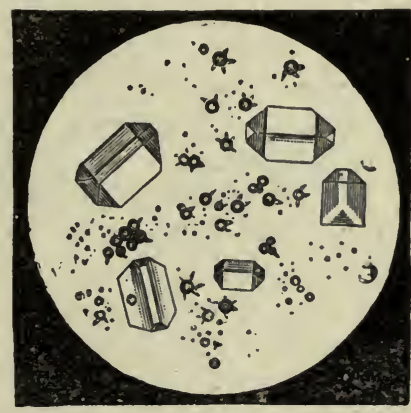

FIG. 384.-Urinary sediment of triple phosphates (large prismatic crystals) and urate of ammonium, from urine which had undergone alkaline fermentation.

to be mistaken for albumin. It may be distinguished readily from albumin, as it is soluble in a few drops of acetic acid, whereas coagulated protein does not dissolve.

The phosphoric acid in the urine chiefly originates from the phosphates of the food, but is partly a decomposition product of the phosphorised organic materials in the body, such as lecithin and nuclein. The amount of $\mathrm{P}_{2} \mathrm{O}_{5}$ in the twenty-four hours' urine varies from 2.5 to 3.5 grammes, of which the earthy phosphates contain about half (1 to $1.5 \mathrm{gr}$.). The urine also contains minute quantities of organic phosphates, for instance, glycero-phosphates.

\section{Tests for the Inorganic Salts of Urine.}

Chlorides. - Acidulate with nitric acid and add silver nitrate; a white precipitate of silver chloride, soluble in ammonia, is produced. The object of acidulating with nitric acid is to prevent phosphates being precipitated by the silver nitrate.

Sulphates. - Acidulate with hydrochloric acid, and add barium chloride. A white precipitate of barium sulphate is produced. Hydrochloric acid is again added first, to prevent precipitation of phosphates.

Phosphates. - i. Add ammonia; a white crystalline precipitate of earthy (that is, calcium and magnesium) phosphates is produced. This becomes more apparent on standing. The alkaline (that is, sodium and potassium) phosphates remain in solution. ii. Mix another portion of urine with half its volume of nitric acid; add ammonium molybdate, and boil. A yellow crystalline precipitate falls. This test is given by both classes of phosphates. 
Quantitative estimation of the salts is accomplished by the use of solutions of standard strength, which are run into the urine till the formation of a precipitate ceases. The standards are made of silver nitrate, barium chloride, and uranium nitrate or acetate for chlorides, sulphates, and phosphates respectively.

\section{Urinary Deposits.}

The different substances that may occur in urinary deposits are formed elements and chemical substances.

The formed or anatomical elements may consist of bloodcorpuscles, pus, mucus, epithelium cells, spermatozoa, casts of the urinary tubules, fungi, and entozoa. All of these, with the exception of a small quantity of mucus, which forms a flocculent cloud in the urine, are pathological, and the microscope is chiefly employed in their detection.

The chemical substances are uric acid, urates, calcium oxalate, calcium carbonate, and phosphates. Rarer forms are leucine, tyrosine, xanthine, and cystine. We shall, however, here only consider the commoner deposits, and for their identification the microscope and chemical tests must both be employed.

Deposit of Uric Acid.- This is a sandy reddish deposit resembling cayenne pepper. It may be recognised by its crystalline form (fig. 382, p. 591) and by the murexide reaction. The presence of these crystals generally indicates an increased formation of uric acid, and, if excessive, may lead to the formation of stones or calculi in the bladder.

Deposit of Urates. - This is much commoner, and may, if the urine is concentrated, occur in normal urine when it cools. It is generally found in the concentrated urine of fevers; and there appears to be a kind of fermentation, called the acid fermentation, which occurs in the urine after it has been passed, and which leads to the same result. The chief constituent of the deposit is the acid sodium urate, the formation of which from the normal sodium urate of the urine may be represented by the equation:-

$$
\underset{\substack{\text { [Normal sodium } \\ \text { urate.] }}}{2 \mathrm{C}_{5} \mathrm{H}_{2} \mathrm{Na}_{2} \mathrm{~N}_{4} \mathrm{O}_{3}}+\underset{\text { [Water.] }}{\mathrm{H}_{2} \mathrm{O}}+\underset{\substack{\text { [Carbonic } \\ \text { acid.] }}}{\mathrm{CO}_{2}}=\underset{\text { [Acid sodium urate.] }}{2 \mathrm{C}_{5} \mathrm{H}_{3} \mathrm{NaN}_{4} \mathrm{O}_{3}}+\underset{\substack{\text { [Sodium } \\ \text { carbonate.] }}}{\mathrm{Na}_{2} \mathrm{CO}_{3}} \text {. }
$$

This deposit may be recognised as follows:-

(1) It has a pinkish colour; the pigment called uro-erythrin is one of the pigments of the urine, but its relationship to the other urinary pigments is not known.

(2) It dissolves upon warming the urine.

(3) Microscopically it is usually amorphous, but crystalline forms similar to those depicted in fig. 384 may occur. Crystals of-calcium oxalate may be mixed with this deposit (see fig. 385).

Deposit of Calcium Oxalate.-This occurs in envelope crystals (octahedra) or dumb-bells. It is insoluble in ammonia, and in acetic 
acid. It is soluble with difficulty in hydrochloric acid. Calcium oxalate calculi are the commonest kind of stones in the kidney.

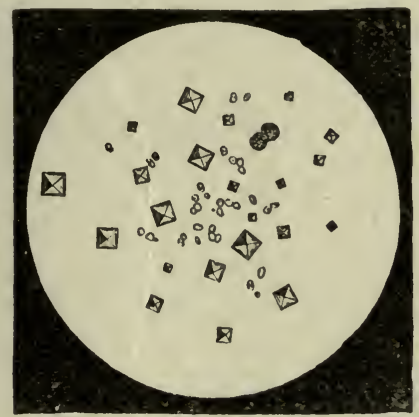

FIG. 335.-Crystals of calcium oxalate.

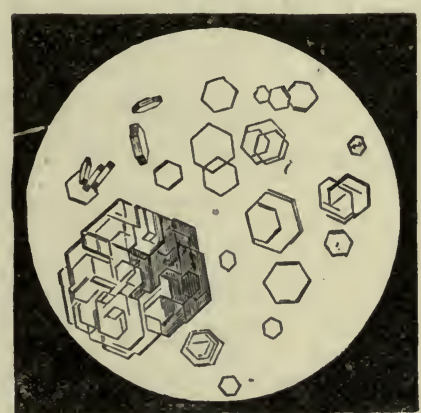

Fra. 386.-Crystals of cystin.

Deposit of Cystine.-Cystine $\left(\mathrm{C}_{6} \mathrm{H}_{12} \mathrm{~N}_{2} \mathrm{~S}_{2} \mathrm{O}_{4}\right)$ is recognised by its colourless six-sided crystals (fig. 386). These are rare: they occur only in acid urine, and they may form concretions or calculi. Cystinuria (cystine in the urine) is hereditary.

Deposit of Phosphates.-These occur in alkaline urine. The urine may be alkaline when passed, due to fermentative changes occurring in the bladder. All urine, however, if exposed to the air (urless the air is perfectly pure, as on the top of a snow mountain), will in time become alkaline, owing to the growth of the micrococcus urece. This forms ammonium carbonate from the urea.

$$
\underset{\text { [Urea.] }}{\mathrm{CON}_{2} \mathrm{H}_{4}}+\underset{\text { [Water.] }}{2 \mathrm{H}_{2} \mathrm{O}}=\underset{\begin{array}{c}
\text { [Ammonium } \\
\text { carbonate.] }
\end{array}}{\left(\mathrm{NH}_{4}\right)_{2} \mathrm{CO}_{3}} .
$$

The ammonia renders the urine alkaline and precipitates the earthy phosphates. The chief forms of phosphates that occur in urinary deposits are-

(1) Calcium phosphate, $\mathrm{Ca}_{3}\left(\mathrm{PO}_{4}\right)_{2}$; amorphous.

(2) Triple or ammonio-magnesium phosphate, $\mathrm{MgNH}_{4} \mathrm{PO}_{4}$; coffinlids and feathery stars (fig. 384).

(3) Crystalline phosphate of calcium, $\mathrm{CaHPO}_{4}$, in rosettes of prisms, in spherules, or in dumb-bells.

(4) Magnesium phosphate, $\mathrm{Mg}_{3}\left(\mathrm{PO}_{4}\right)_{2}+22 \mathrm{H}_{2} \mathrm{O}$, occurs occasionally, and crystallises in long plates.

All these phosphates are dissolved by acids, such as acetic acid, without effervescence.

A solution of ammonium carbonate (1 in 5) eats magnesium phosphate away at the edges; it has no effect on the triple phosphate. A phosphate of calcium $\left(\mathrm{CaHPO}_{4}+2 \mathrm{H}_{2} \mathrm{O}\right)$ may occasionally be 
deposited in acid urine. Pus in urine is apt to be mistaken for phosphates, but can be distinguished by the microscope.

Deposit of calcium carbonate, $\mathrm{CaCO}_{3}$, appears but rarely as whitish balls or biscuit-shaped bodies. It is commoner in the urine of herbivora. It dissolves in acetic or hydrochloric acid, with effervescence.

The following is a summary of the chemical sediments that may occur in urine:-

\section{CHEMICAL SEDIMENTS IN URINE.}

IN AcID Urine.

Uric Acid.-Whetstone, dumb-bell, or sheaf-like aggregations of crystals deeply tinged by pigment.

Urates.-Generally amorphous. The acid urate of sodium and of ammonium may sometimes occur in star-shaped clusters of needles or spheroidal clumps with projecting spines. Tinged brickred. Soluble on warming.

Calcium Oxalate. - Octahedra, socalled envelope crystals. Insoluble in acetic acid.

Cystine.-Hexagonal plates. Rare.

Leucine and Tyrosine.-Rare.

Calcium Phosphate,

$\mathrm{CaHPO}_{4}+2 \mathrm{H}_{2} \mathrm{O}$.- Rare.
In Alkatine Urine.

Phosphates. - Calcium phosphate, $\mathrm{Ca}_{3}\left(\mathrm{PO}_{4}\right)_{2 \circ}$ Amorphous.

Triple phosphate,

$\mathrm{MgNH}_{4} \mathrm{PO}_{4}+6 \mathrm{H}_{2} \mathrm{O}$. Coffin-lids or feathery stars.

Calcium hydrogen phosphate, $\mathrm{CaHPO}_{4}$. Rosettes, spherules, or dumbbells.

Magnesium phosphate, $\mathrm{Mg}_{3}\left(\mathrm{PO}_{4}\right)_{2}+22 \mathrm{H}_{2} \mathrm{O}$. Long plates.

All the preceding are soluble in acetic acid without effervescence.

Calcium Carbonate, $\mathrm{CaCO}_{3}$.-Biscuitshaped crystals. Soluble in acetic acid with effervescence.

Ammonium Urate,

$\mathrm{C}_{5} \mathrm{H}_{2}\left(\mathrm{NH}_{4}\right)_{2} \cdot \mathrm{N}_{4} \mathrm{O}_{3}$. - "Thorn - apple" spherules.

Leucine and Tyrosine.-Very rare.

\section{Pathological URINe.}

Under this head we shall briefly consider only those abnormal constituents which are most frequently met with.

Proteins.-There is no protein in normal urine,* and the most common cause of the appearance of albumin in the urine is disease of the kidney (Bright's disease). The term "albumin" is the one used by clinical observers. Properly speaking, it is a mixture of serum albumin and serum globulin. Of these, serum albumin is usually the more abundant. Globulins, and especially euglobulins, have probably larger molecules, so escape of globulin indicates more serious damage to the renal cells. The best methods of testing for and estimating the protein are the following:-

(a) Boil the top of a long column of urine in a test-tube. If the urine is acid, the albumin is coagulated. If the quantity of albumin is small, the cloudiness produced is readily seen, as the unboiled urine below it is clear. This is insoluble

* This absolute statement is true for all practical purposes. Mörner, however, has stated that a trace of protein (serum albumin plus the protein constituent of mucin) does occur in normal urine; but the trace is negligible, many hundreds of litres of urine having to be used to obtain an appreciable quantity. 
in a few drops of acetic acid, and so may be distinguished from phosphates. If the urine is alkaline, it slould be first rendered acid with a little dilute acetic acid.

(b) Heller's Nitric-acid Test.-Pour some of the urine gently on to the surface of some nitric acid in a test-tube. A ring of white precipitate occurs at the junction of the two liquids. This test is used for small quantities of albumin.

(c) Estimation of Albumin by Esbach's Albuminometer.Esbach's reagent for precipitating the albumin is made by dissolving 10 grammes of picric acid and 20 grammes of citric acid in 800 or 900 c.c. of boiling water, and then adding sufficient water to make up to a litre (1000 c.c.). fig. 387 .

The albuminometer is a test-tube graduated as shown in

Pour the urine into the tube up to the mark $U$; then the reagent up to the mark $R$. Close the tube with a cork, and to ensure complete mixture, tilt it to and fro a dozen times without shaking. Allow the corked tube to stand upright twenty-four hours ; then read off on the scale the height of the coagulum. The figures indicate grammes of dried albumin in a litre of urine. The percentage is obtained by dividing by 10 . Thus, if the coagulum stands at 3 , the amount of albumin is 3 grammes per litre, or $0.3 \mathrm{gr}$. in 100 c.c.

A condition called "peptonuria," or peptone in the urine, is observed in certain pathological states, especially in diseases where there is a formation of pus, and particularly if the pus is decomposed owing to the action of bacterial growths such as staphylococcus; one of the products of disintegration of pus cells appears to be peptone; and this leaves the

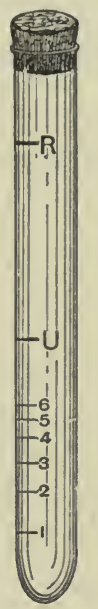

FIG. 387.-Esbach's Albuminometer. body by the urine. The term "peptone," however, is in the strict sense of the word incorrect; the protein present is deutero-proteose. In certain diseases of bone a curious protein passes into the urine. It is called Bence-Jones protein, after its discoverer; it somewhat resembles hetero-proteose in its properties.

Sugar.-Normal urine contains no sugar, or so little that for clinical purposes it may be considered absent. The conditions in which glycosuria occurs are described on p. 538 .

The sugar present is dextrose. Lactose may occur in the urine of nursing mothers. Lævulose, pentoses, and other sugars are found but rarely. Diabetic urine also contains hydroxybutyric acid, and may contain and yield on distillation acetone, and aceto-acetic acid. The methods usually adopted for detecting and estimating the sugar are as follows:-

(a) The urine has generally a high specific gravity.

(b) The presence of sugar is shown by the reduction (yellow precipitate of cuprous oxide) that occurs on boiling with Fehling's solution. Fehling's solution is an alkaline solution of copper sulphate to which Rochelle salt has been added. The Rochelle salt (double tartrate of potash and soda) holds the cupric hydrate in solution. Fehling's solution should always be freshly prepared, as, on standing, an isomeride is formed from the tartaric acid, which reduces the cupric to cuprous oxide. Fehling's solution should, therefore, always be tested by boiling before it is used. If it remains clear on boiling, it is in good condition. 
(c) Quantitative Determination of Sugar in Urine.-Fehling's solution is prepared as follows :- -34.639 grammes of copper sulphate are dissolved in about 200 c.c. of distilled water; 173 grammes of Rochelle salt are dissolved in 600 c.c. of a 14 per cent. solution of caustic soda. The two solutions are mixed and diluted to a litre. Ten c.c. of this solution are equivalent to 0.05 gramme of dextrose. Dilute 10 c.c. of this solution with about 40 c.c. of water, and boil it in a porcelain basin. Run into this from a burette the urine (which should be previously diluted with nine times its volume of distilled water) until the blue colour of the copper solution disappears - that is, till all the cupric hydrate is reduced. The mixture in the basin should be boiled after every addition. The quantity of diluted urine used from the burette contains 0.05 gramme of sugar. Calculate the percentage from this, remembering that the urine has been diluted to ten times its original volume.

Pavy's modification of Fehling's solution is often used. Here ammonia holds the copper in solution, and no precipitate forms on boiling with sugar, as ammonia holds the cuprous oxide in solution. The reduction is complete when the blue colour disappears; 10 c.c. of Pavy's solution=1 c.c. of Fehling's solution= 0.005 gramme of dextrose.

There are several other modifications of the original Fehling method which have been introduced with the purpose of making the end-point clearer. Allihn's method is the most accurate ; the cuprous oxide is collected, and finally reduced to metallic copper, which is then waighed.

Fehling's test is not absolutely trustworthy. Often a normal urine will decolorise Fehling's solution, though seldom a red precipitate is formed. 'This is due to excess of urates and creatinine. Another substance called glycuronic acid $\left(\mathrm{C}_{6} \mathrm{H}_{10} \mathrm{O}_{7}\right)$ is, however, very likely to be confused with sugar by Fehling's test; the cause of its appearance is sometimes the administration of drugs (chloral, camphor, etc.); but sometimes it appears independently of drug treatment.

In the rare and hereditary condition called alcaptonuria, confusion may also arise. Alcapton is a substance which originates from tyrosine by an unusual form of metabolism. It gives the urine a brown tint, which darkens on exposure to the air. It is an aromatic substance, which Baumann and Wolkow and later Garrod identified with homogentisic acid $\left(\mathrm{C}_{6} \mathrm{H}_{3} \cdot(\mathrm{OH})_{2} \mathrm{CH}_{2} \cdot \mathrm{COOH}\right)$.

(d) A good confirmatory test for sugar is the fermentation test, which is performed as follows :-

Half fill a test-tube with the urine and add a little German yeast. Fill up the tube with mercury; invert it in a basin of mercury, and leave it in a warm place for twenty-four hours. The sugar will undergo fermentation : carbonic acid gas accumulates in the tube, and the liquid no longer gives the tests for sugar, or only faintly, but gives those for alcohol instead. The specific gravity falls.

(e) The phenylhydrazine test (p. 412) may also be applied.

Another hereditary abnormality of metabolism is pentosuria; this is apt to be mistaken for diabetes, for pentose reduces Fehling's solution, but it will not ferment with yeast. It is a rare condition, but does not appear to do any harm. The source of the pentose is not the food, for pentosuria continues in these cases when the food contains no pentose. The pentose of the urine, moreover, is a different one from that found in the nucleic acids of the body. Neuberg thinks it arises in some way from galactose, and that it originates from the galactosides of the body.

Bile.-This occurs in jaundice. The urine is dark-brown, greenish, or in extreme cases almost black in colour. The most readily applied test is Gmelin's test for the bile pigments. Excess of urobilin should not be mistaken for bile pigment. Pettenkofer's test for the bile acids seldom succeeds in urine if the test is done in the ordinary way. The best method is to warm a thin film of urine and cane sugar solution in a flat porcelain dish. Then dip a glass rod in strong sulphuric acid, and draw it across the film. Its track is marked by a purplish line. Hay's sulphur test is a good one for bile 
salts. If some flowers of sulphur are sprinkled on the surface of normal urine, it remains floating on the top. If bile salts are present even in small quantities, the fine sulphur particles fall down to the bottom of the vessel in which the urine is contained; this is due to an alteration of surface tension which bile salts produce.

Blood.-When hæmorrhage occurs in any part of the urinary tract, blood appears in the urine. It is found in the acute stage of Bright's disease. If a large quantity is present, the urine is deep red. Microscopic examination then reveals the presence of bloodcorpuscles, and on spectroscopic examination the bands of oxyhæmoglobin are seen.

If only a small quantity of blood is present, the secretionespecially if acid-has a characteristic reddish-brown colour, which physicians term "smoky."

The blood pigment may, under certain circumstances, appear in the urine without the presence of any blood-corpuscles at all. This is produced by a disintegration of the corpuscles occurring in the circulation. The condition so produced is called homoglobinuria; it occurs in several pathological states, as for instance in the tropical disease called "Black-water fever." The pigment is in the condition of methæmoglobin mixed with more or less oxyhæmoglobin, and the spectroscope is the means used for identifying these substances.

Pus occurs in the urine as the result of suppuration in any part of the urinary tract. It forms a white sediment resembling that of phosphates, and, indeed, is always mixed with phosphates. The pus corpuscles may, however, be seen with the microscope; their nuclei are rendered evident by treatment with 1 per cent. acetic acid, and the pus-corpuscles are seen to resemble white blood-corpuscles, which, in fact, they are in origin. They dissolve in glacial acetic acid.

Some of the protein constituents of the pus cells-and the same is true for blood-pass into solution in the urine, so that the urine pipetted off from the surface of the deposit gives the tests for protein.

On the addition of liquor potassæ to the deposit of pus cells, a ropy gelatinous mass is obtained. This is distinctive. Mucus treated in the same way is dissolved.

Amino-acids.-Normal urine contains traces of glycine. Leucine, tyrosine, and other amino-acids may be present after extensive disintegration of tissue protein, such as occurs in acute atrophy of the liver (p. 586). Cystine may occur as a rare anomaly of metabolism (p. 599). Associated with cystinuria one often finds diaminuria, that is, the passage of diamines into the urine; these are known as cadaverine $\left(\mathrm{C}_{5} \mathrm{H}_{14} \mathrm{~N}_{2}\right)$ and putrescine $\left(\mathrm{C}_{4} \mathrm{H}_{12} \mathrm{~N}_{2}\right)$, and are the result of the removal of $\mathrm{CO}_{2}$ from the diamino-acids lysine and ornithine respectively. Homogentisic acid, found in alcaptonuria (see preceding page), is another somewhat similar anomaly; it arises from tyrosine. 


\section{CHAPTER XL}

\section{THE SKIN AND ITS APPENDAGES}

THE skin is composed of two parts, epidermis or cuticle, and dermis or cutis vera.

The Fpidermis is a thick stratified epithelium. The deeper layers are composed of protoplasmic cells, and form the rete mucosum, or Malpighian layer; the surface layers are hard and horny; this horny layer is the thickest part of the epidermis, and is specially thick on the palms and soles, where it is subjected to most friction. It is in the cells of the Malpighian layer that pigment granules are deposited in the coloured races.

Between the horny layer and the Malpighian layer are two intermediate strata, in which the transformation of protoplasm into horny material (keratin) is taking place. In the first of these-that is, the one next to the Malpighian layer-the cells are flattened, and filled with large granules of eleidin, an intermediate substance in the formation of horn. This layer is called the stratum granulosum.

Above this are several layers of clear, more rounded cells, which constitute the stratum lucidum; and above this the horny layer proper, many strata deep, begins. The cells become more and more scaly as they approach the surface, where they lose their nuclei and eventually become detached.

The epidermis grows by a multiplication of the deepest layer of cells; the newly-formed cells push towards the surface those previously formed, in their progress undergoing the transformation into keratin.

The epidermis has no blood-vessels; nerve-fibrils pass into its deepest layers, and ramify between the cells.

The Dermis is composed of dense fibrous tissue, which becomes looser and more reticular in its deeper part, where it passes by insensible degrees into the areolar and adipose tissue of the sub-

- cutaneous region. The denser superficial layer is very vascular, and is covered with minute papilloe; the epidermis is moulded over these, 
and in the palms and soles, where the papillæ are largest and are disposed in rows, their presence is indicated by the well-known ridges on the surface.

The papillæ contain loops of capillaries, and in some cases, especially in the palm of the hand and fingers, they contain tactile corpuscles (which will be more fully described in connection with the sense of touch). Special capillary networks are distributed to the sweat-glands, sebaceous glands, and hair follicles.

The deeper portions of the dermis in the scrotum, penis, and nipple, contain involuntary muscular tissue; there is also a bundle of muscular tissue attached to each hair follicle.

The Nails are thickenings of the stratum lucidum. Each lies in a depression called the bed of the nail, the posterior part of which is overlapped by epidermis, and called the nail-groove. The dermis beneath is beset with longitudinal ridges instead of papillæ; these are very vascular; but in the lunula, the crescent at the base of the nail, there are papillæ, and this part is not so vascular.

The Hairs are epidermal growths, contained in pits called hair follicles. The part within the follicle is called the root of the hair.

The main substance of the hair is composed of pigmented horny fibrous material, in reality long fibrillated cells. It is covered by a layer of scales imbricated upwards (hair cuticle). In many

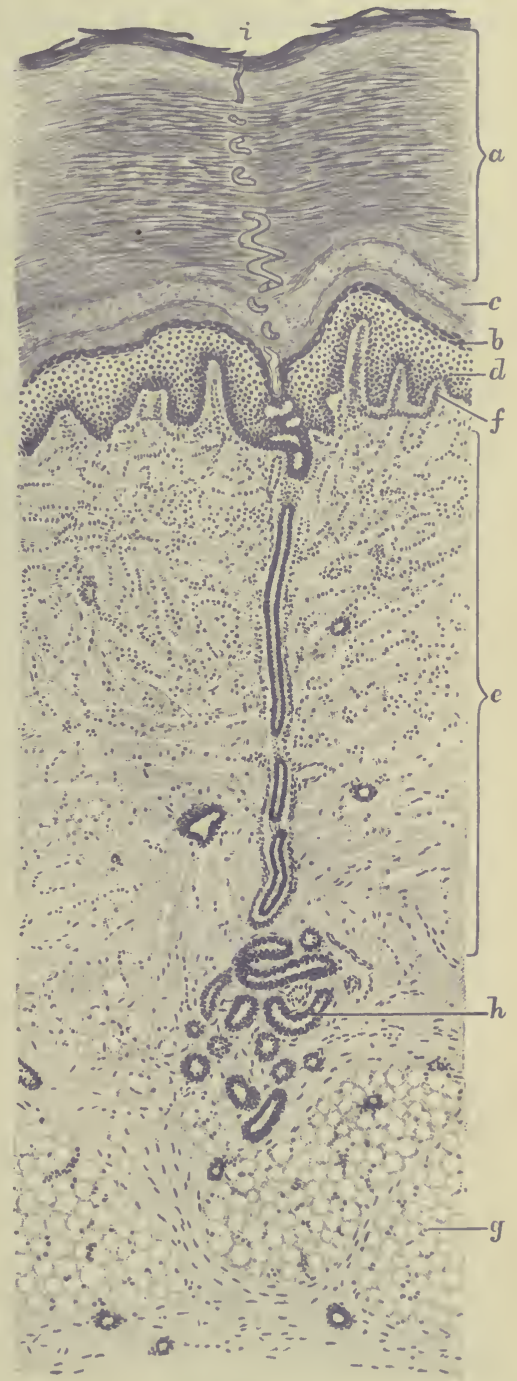

Fia. 388,-Vertical section through the skin of the sole of the foot. $a$, Horny layer; $b$, stratum granulosum; $c$, stratum lucidum; $d$, Malpighian layer; $e$, cutis vera; $f$, papilla of cutis vera; $g$, fat lobule of subcutaneous tissue; $h$, sweat-gland; $i$, orifice of sweat. duct. (Szymonowicz.) 
hairs the centre is occupied by a medulla, formed of rounded cells containing eleidin granules. Minute air-bubbles may be present in both medulla and fibrous layer, and cause the hair to look white by

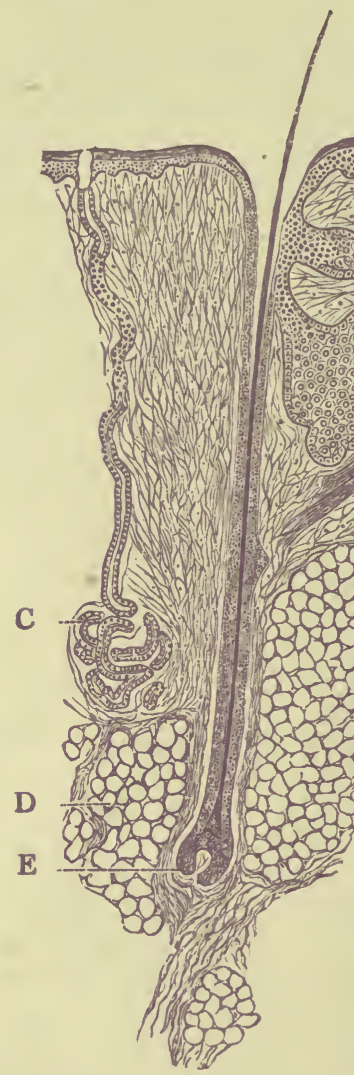

FIG. 389.-Vertical section of skin. A. Sebaceous gland opening into hair follicle. B. Muscu. lar fibres. C. Sudoriferous or sweat-gland. D. Subcutaneous fat. E. Fundus of hair follicle, with hair papilla. (Klein.) reflected light. The grey hair of old age, however, is produced by a loss of pigment.

The root is enlarged at its extremity into a knob, into which projects a vascular papilla from the true skin.

The hair follicle consists of two parts, one continuous with the epidermis, called the root-sheath, the other continuous with the dermis, called the dermic coat. The two are separated by a basement membrane called the hyaline layer of the follicle. The rootsheath consists of an outer layer of cells like the Malpighian layer of the epidermis, with which it is directly continuous (outer root-sheath), and of an inner horny layer (inner root-sheath), continuous with the horny layer of the epidermis. The inner root-sheath consists of three layers, the outermost being composed of long, non-nucleated cells (Henle's layer), the next of squarish nucleated cells (Huxley's layer), and the third is a cuticle of 
scales, imbricated downwards, which fit over the scales of the cuticle of the hair itself.

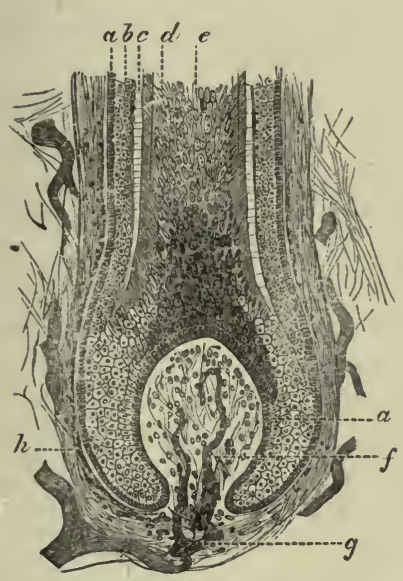

FIG. 390.-Longitudinal section of a hair follicle. $a$ and $b$, External rootsheath; $c$, internal root-sheath ; $d$, tibrous layer of the hair; $e$, medulla; $f$, hair papilla; $g$, blood. vessels of the hair papilla; $h$, aernic coat. (Cadiat.)

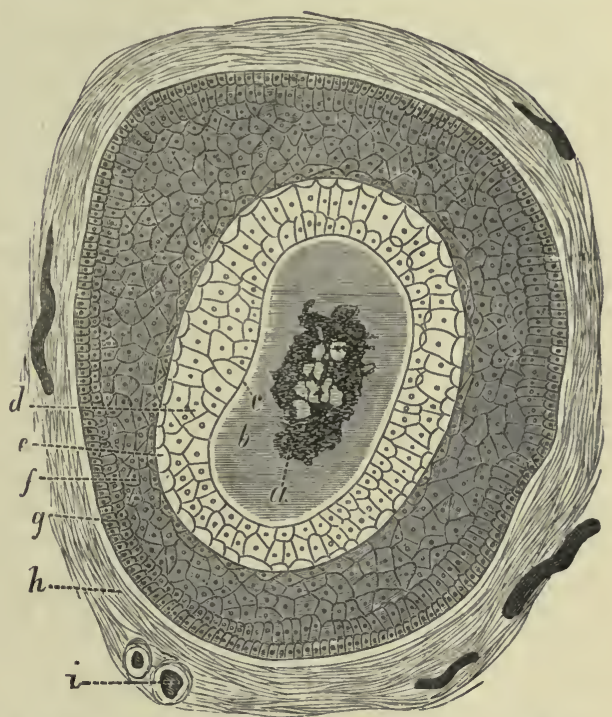

FIG. 391.-Transverse section of a hair and hair follicle made below the opening of the sebaceous gland. $a$, Medulla, or pith of the hair; $b$, fibrous layer; $c$, cuticle; $d$, Huxley's layer; $e$, Henle's layer of internal root-sheath; $f$ and $g$, layers of external rootsheath, outside of $g$ is the basement membrane or hyaline layer; $h$, dermic (fibrous) coat of hair follicle $i$, vessels. (Cadiat.)

A small bundle of plain muscular fibres is attached to each follicle (fig. 389). When it contracts, as under the influence of cold, or of certain emotions such as fear, the hair is erected and the whole skin is roughened ("goose skin"). The nerves supplying these muscles are called pilo-motor nerves. The distribution of these nerves closely follows those of the vaso-constrictor nerves of the skin; their cell-stations are in the lateral sympathetic chain.

The sensitiveness of the hairs or more properly of the hair follicles is subserved by a ring-like plexus of nerve-fibrils around the hair follicle, within the outer sheath,

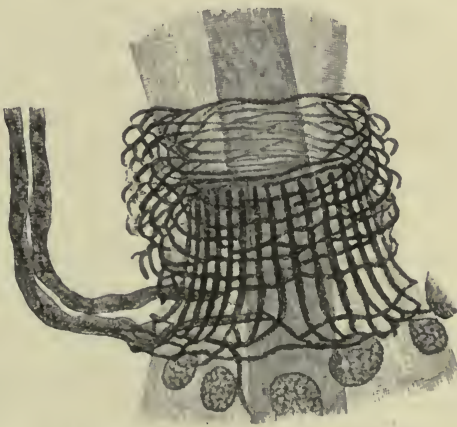

F19. 392.- Senvory nerve ending of hair fol. licle. Gold chlorido preparation. $\times 900$. (Szymonowicz.) just beneath the entrance of the sebaceous gland (see fig. 392). 
The sebaceous glands (fig. 389) are small saccular glands, with ducts opening into the upper portion of the hair follicles. The secreting cells become charged with fatty matter, which is discharged into the lumen of the saccules owing to the disintegration of the cells. The secretion, sebum, contains isocholesterin (see p. 436) in addition to fatty matter. It acts as a lubricant to the hairs.

The sweat-glands (fig. 388) are abundant over the whole human skin, but are most numerous where hairs are absent, on the palms and soles. Each consists of a coiled tube in the deepest part of the dermis, the duct from which passes up through the dermis, and by a corkscrew-like canal through the epidermis to the surface.

The secreting tube is lined by one or two layers of cubical or columnar cells; outside this is a layer of longitudinally arranged muscular fibres, and then a basernent membrane.

The duct is of similar structure, except that there is usually but one layer of cubical cells, and muscular fibres are absent; the passage through the epidermis has no proper wall; it is merely a channel excavated between the epidermal cells.

The ceruminous glands of the ear are modified sweat-glands.

\section{The Functions of the Skin}

Protection.-The skin acts as a protective organ, not only by mechanically covering and so defending internal structures from external violence, but more particularly in virtue of its being an organ of sensation (see later in the chapter on Touch).

Heat Regulation.-See chapter on Temperature.

Respiration.-A small amount of respiratory interchange of gases occurs through the skin, but in thick-skinned animals this is very small. In man, the carbonic acid exhaled by the skin is about $\frac{1}{150}$ to $\frac{1}{200}$ of that which passes from the lungs. But in thin-skinned animals, such as frogs, cutaneous respiration is very important; after the removal of the lungs of a frog, the respiratory interchange through the skin is sufficient to keep the animal alive, the amount of carbonic acid discharged being about half as much as when the lungs are present (Bischoff).

Absorption.-This also is an unimportant function; but the skin will in a small measure absorb oily materials placed in contact with it; thus in some cases infants who will not take cod-liver oil by the mouth, can yet be dosed with it by rubbing it into the skin. Many ointments also are absorbed, and thus general effects are produced by local inunction.

Secretion.-The secretions of the skin are two in number. The sebum is the natural lubricant of the hairs. The secretion of sweat is an important function of the skin, and we will therefore discuss it at greater length. 


\section{The Sweat}

Physiology of the Secretion of Sweat.-We have seen that the sweat-glands are most abundant in man on the palms and soles, and here the greatest amount of perspiration occurs. Different animals vary a good deal in the amount of sweat they secrete, and in the place where the secretion is most abundant. Thus the ox perspires less than the horse and sheep; perspiration is absent from rats, rabbits, and goats; pigs perspire mostly on the snout; dogs and cats on the pads of the feet.

As long as the secretion is small in amount, it is evaporated from the surface at once; this is called insensible perspiration. As soon as the secretion is increased or evaporation prevented, drops appear on the surface of the skin. This is known as sensible perspiration. The relation of these two varies with the temperature of the air; the drier and hotter the air, the greater is the proportion of insensible to sensible perspiration. In round numbers the total amount of sweat secreted by a man is two pounds in the twenty-four hours.

The amount of secretion is influenced by the vaso-motor nerves; an increase in the size of the skin-vessels leads to increased, a constriction of the vessels to diminished, perspiration. There are also special secretory fibres, stimulation of which causes a secretion even when the circulation is suspended, as in a recently amputated limb. These fibres are paralysed by atropine. They are contained in the same nerve-trunks as the vaso-motor nerves, as are also the nervefibres which supply the plain muscular fibres of the sweat-glands which act during the expulsion of the secretion. The secretory nerves for the lower limbs issue from the spinal cord by the last two or three dorsal and first two or four lumbar nerves (in the cat); they have cell-stations in the lower ganglia of the lateral chain, and pass thence to the sciatic nerve. They are controlled by a centre in the upper lumbar region of the cord; those for the upper limbs leave the cord by the sixth, seventh, and eighth anterior thoracic roots, have cell-stations in the ganglion stellatum, and ultimately pass to the ulnar and median nerves; they are controlled by a centre in the cervical enlargement of the cord. The secretory fibres for the head pass in the cervical sympathetic, and in some branches of the fifth cranial nerves. These subsidiary centres are dominated by one in the medulla oblongata (Adamkiewicz). These facts have been obtained by experiments on animals (cat, horse).

The sweat-centres may be excited directly by venous blood, as in asphyxia ; or by over-heated blood (over $45^{\circ} \mathrm{C}$.) ; or by certain drugs (see further); or reflexly by stimulation of afferent nerves such as the crural and peroneal.

Nervous diseases are often accompanied with disordered sweating; 
thus unilateral perspiration is sometimes seen in cases of hemiplegia; degeneration of the anterior nerve-cells of the cord may cause stoppage of the secretion.

The changes that occur in the secreting cells have been investigated by Renaut in the horse. When charged they are clear and swollen, the nucleus being situated near their attached ends; when discharged they are smaller, granular, and their nucleus is more central.

The sweat, like the urine, must be regarded as an excretion, the secreting cells eliminating substances formed elsewhere.

Composition of the Sweat.-Sweat may be obtained in abundant quantities by placing the animal or man in a closed hot-air bath, or from a limb by enclosing it in a vessel made air-tight with an elastic bandage. Thus obtained, it is mixed with epidermal scales and a small quantity of fatty matter from the sebaceous glands. The continual shedding of epidermal scales is in reality an excretion. Keratin, of which they are chiefly composed, is rich in sulphur, and, consequently, this is one means by which sulphur is removed from the body.

The reaction of sweat is acid, and the acidity, as in the urine, is due to acid sodium phosphate. In profuse sweating, however, the secretion usually becomes alkaline or neutral. It has a peculiar and characteristic odour, which varies in different parts of the body, and is due to volatile fatty acids; its taste is saltish, its specific gravity about 1005 .

In round numbers the percentage of solids is 1.2 , of which 0.8 is inorganic matter.

The salts are in kind and relative quantity very like those of the urine; sodium chloride is the most abundant salt. Funke was unable to find any urea, but most other observers agree on the presence of a minute quantity. It appears to become quickly transformed into ammonium carbonate. The protein which is present is probably derived from the epithelial cells of the epidermis, sweat-glands, and sebaceous glands, which are suspended in the excretion; but in the horse there is albuminous matter actually in solution in the sweat.

Abnormal, Unusual, or Pathological Conditions of the Sweat. -Drugs.-Certain drugs (sudorifics) favour sweating, e.g., pilocarpine, Calabar bean, strychnine, picrotoxine, muscarine, nicotine, camphor, ammonia. Others diminish the secretion, e.g., atropine and morphine in large doses.

Large quantities of water, by raising the blood-pressure, increase the perspiration.

Some substances introduced into the body reappear in the sweat, e.g., benzoic, tartaric, and succinic acids readily, quinine and iodine 
with more difficulty. Compounds of arsenic and mercury behave similarly.

Diseases.-Cystine has been found in some cases of cystinuria; dextrose in diabetic patients; bile-pigment in those with jaundice (as evidenced by the staining of the clothes); indigo in a peculiar condition known as chromidrosis; blood or hæmatin derivatives in red sweat; albumin in the sweat of acute rheumatism, which is often very acid; urates and calcium oxalate in gout; lactic acid in puerperal fever, and occasionally in rickets and scrofula.

Kidney Diseases. - The relation of the secretion of the skin to that of the kidneys is a very close one. Thus copious secretions of urine, or watery evacuations from the alimentary canal, coincide with dryness of the skin; abundant perspiration and scanty urine generally go together. In the condition known as urcemia (see p. 588), when the kidneys secrete little or no urine, the percentage of urea rises in the sweat; the sputum and the saliva also contain urea under those circumstances. The clear indication for the physician in such cases is to stimulate the skin to action by hot-air baths and pilocarpine, and the alimentary canal by means of purgatives. In some of these cases the skin secretes urea so abundantly that when the sweat dries on the body, the patient is covered with a coating of urea crystals.

Varnishing the Skin.-By covering the skin of such an animal as a rabbit with an impermeable varnish, the temperature is reduced, a peculiar train of symptoms set up, and ultimately the animal dies. If, however, cooling is prevented by keeping such an animal in warm cotton-wool, it lives longer. Varnishing the human skin does not seem to be dangerous. Many explanations have been offered to explain the peculiar condition observed in animals; retention of the sweat would hardly do it; the blood is not found post-mortem to contain any abnormal substance, nor is it poisonous when transfused into another animal. Cutaneous respiration is so slight in mammals that stoppage of this function cannot be supposed to cause death. The animal, in fact, dies of cold; the normal function of the skin in regulating temperature is interfered with, and it is animals with delicate skins which are most readily affected. 


\section{CHAPTER XLI}

\section{GENERAL METABOLISM}

THE word metabolism has been often employed in the preceding chapters, and, as there explained, it is used to express the sum total of the chemical exchanges that occur in living tissues. The chemical changes have been considered separately under the headings Alimentation, Excretion, Respiration, etc. We have now to put our knowledge together, and consider these subjects in their relation to one another.

The living body is always giving off by the lungs, kidneys, and skin the products of its combustion, and is thus always tending to lose weight. This loss is compensated for by the intake of food and of oxygen. For the material it loses, it receives in exchange fresh substances. If, as in a normal adult, the income is exactly equal to the expenditure, the body-weight remains constant. If, as in a growing child, the income exceeds the expenditure, the body gains weight; and if, as in febrile conditions, or during starvation, the expenditure exceeds the income, the body wastes.

The different parts of the body have very different compositions; still, speaking of the body as a whole, Volkmann and Bischoff state that it contains 64 per cent. of water, 16 of proteins, 14 of fat, 5 of salt, and 1 of carbohydrates. The carbohydrates are thus the smallest constituent of the body; they are the glycogen of the liver and muscles, and small quantities of dextrose in various parts.

The most important, because the most abundant of the tissues of the body, is the muscular tissue. Muscle forms about 42 per cent. of the body-weight,* and contains, in round numbers, 75 per cent. of water and 21 per cent. of proteins; thus about half the protein material and of the water of the body exist in its muscles.

The body, however, does not remain in a stable condition; even while nutrition is occurring, destructive changes are taking place simultaneously; each cell may be considered to be in a state of unstable equilibrium, undergoing anabolic, or constructive processes, on the one hand, and destructive, or katabolic, processes on the other.

* The following is in round numbers the percentage proportion of the different structural elements of the body : skeleton, 16 ; muscles, 42 ; fat, 18 ; viscera, 9 : skin, 8 ; brain, 2 ; blood, 5. 
The two sides of metabolism may be compared by means of a balance-sheet, and the necessary data for the construction of such a comparison are:

(1) The weight of the animal before, during, and after the experiment.

(2) The quantity and composition of its food.

(3) The amount of oxygen absorbed during respiration.

(4) The quantity and composition of urine, fæces, sweat, and expired air.

(5) The amount of work done, and the amount of heat developed. (The subject of animal heat will be considered in the next two chapters.)

Water is determined by subtracting the amount of water ingested as food from the quantity lost by bowels, urine, lungs, and skin. The difference is a measure of the katabolism of hydrogen.

Nitrogen.-The nitrogen is derived from proteins, and appears chiefly in the urine. Smaller quantities are eliminated in sweat and fæces. From the amount of nitrogen so found, the amount of proteins which have undergone katabolism is calculated. Proteins contain, roughly, 16 per cent. of nitrogen; so 1 part of nitrogen is equivalent to 6.3 parts of protein; or 1 gramme of nitrogen to 30 grammes of flesh.

Fat and Carbohydrate. - Subtract the carbon in the katabolised protein (protein contains 54 per cent. of carbon) from the total carbon eliminated by lungs, skin, bowels, and kidneys, and the difference represents fat and carbohydrate which have undergone katabolism.

Balance of Income and Discharge in Health.

In Chapter XXX. tables are given of adequate diets; these will in our balance-sheet represent the source of income; the other side of the balance-sheet, the expenditure, consists of the excretions.

We may select as our example a typical table of this daily exchange of material on an ordinary diet from the work of Pettenkofer and Voit. In the first experiment the man did no work.

\begin{tabular}{|c|c|c|c|c|c|c|c|c|}
\hline \multicolumn{5}{|c|}{ Income. } & \multicolumn{4}{|c|}{ Expenditure. } \\
\hline & & & Nitrogen. & Carbon. & Excretions. & Nitrogen. & Carbon. & Water. \\
\hline \multirow{2}{*}{$\begin{array}{l}\text { Protein } \\
\text { Fat. } \\
\text { Carbohy- } \\
\text { drate } \\
\text { Water }\end{array}$} & \multirow{2}{*}{\multicolumn{2}{|c|}{$\begin{array}{r}137 \mathrm{gr} . \\
117 \text {," } \\
352 \text {," } \\
2016 \text { ", }\end{array}$}} & \multirow{2}{*}{\}$_{\ldots}^{19 \cdot 5}$} & \multirow[t]{2}{*}{$315 \cdot 5$} & \multirow[t]{2}{*}{$\begin{array}{l}\text { Urine } \\
\text { Fæces } \\
\text { Expired } \\
\quad \text { air }\end{array}$} & $\begin{array}{r}17 \cdot 4 \\
2 \cdot 1 \\
\\
\ldots\end{array}$ & $\begin{array}{r}12 \cdot 7 \\
14 \cdot 5 \\
248 \cdot 6\end{array}$ & $\begin{array}{r}1279 \\
83 \\
828\end{array}$ \\
\hline & & & & & & $19 \cdot 5$ & $275 \cdot 8$ & 2190 \\
\hline
\end{tabular}


Here the body was in nitrogenous equilibrium, and it eliminated more water than it took in by 174 grammes, this being derived from oxidation of hydrogen. It stored $39 \cdot 7$ grammes of carbon, which is equivalent to 52 grammes of fat.

The next table gives the results of an experiment on the same man on the same diet, but who did active muscular work during the day :-

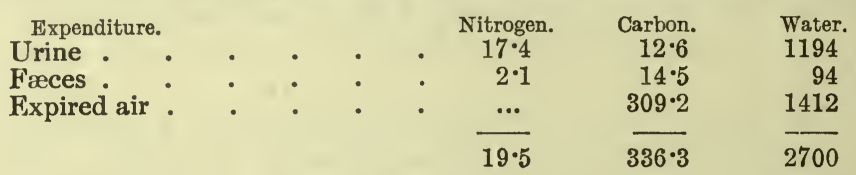

It is important to notice that the discharge of nitrogen was unaltered, while that of both carbon and hydrogen was increased. At one time protein was considered to be the great source of muscular energy; this was first disproved by an historical experiment made by Fick and Wislicenus on themselves in their ascent of the Faulhorn. Nature works in a most economical way in reference to protein waste, and any increase in nitrogenous katabolism which occurs during muscular work is insignificant.

The balance-sheet method of investigation, though one of great usefulness, tells us very little of the details which lead to the end results. We must therefore now proceed to study the details, and although there is a good deal of guesswork, and even ignorance upon some essential points, we may most conveniently consider the question under the three headings of our principal food materials, namely, carbohydrates, fats, and proteins.

\section{Metabolism of Carbohydrates.}

In plants, carbohydrates are synthesised by the agency of chlorophyll from the simple materials carbonic acid and water, which form their chief foods. The first substance formed is probably formic aldehyde, H.COH (which is the simplest carbohydrate known), and this by condensation is converted into sugar, and finally, into starch. We have no clear evidence that a synthesis of this kind ever takes place in animals, but the main source of animal carbohydrate is vegetable carbohydrate. This is taken in the food and converted into glucose; the glucose is taken to the liver and stored as glycogen, and in the liver is once more liberated as glucose, and distributed to the body in this form. The change from glycogen to sugar is the work of an enzyme. Is the change from sugar to glycogen also an enzyme action? And if so, is another enzyme responsible for it, or have we to deal with a case of reversible zymolysis? This is one of many unanswered questions. The other important animal carbohydrate is lactose, a compound of dextrose and galactose. If the food contains galactose as well as dextrose, the 
condensation of these two sugars to form lactose is a comparatively simple problem; there is no doubt that galactose is present in certain foods, for instance it is contained in some vegetables, and it also comes from the sugar of milk. Lactose in the mammary gland is, however, not the only place where galactose is necessary, for the galactosides of nervous tissue (see p. 437) also contain it. But it is not necessary to assume that all the galactose necessary for the formation of milk sugar and of the galactosides comes direct from the galactose of the food. Dextrose, galactose, and lævulose are all isomeric, and the intramolecular rearrangements that would be necessary to convert one into another member of this group do not seem to be beyond the power of the tissue cells, and there is a good deal of evidence that such transformations actually occur in the body. The other carbohydrate constituents of the body are pentoses found in some nucleo-proteins, and glucosamine in the gluco-proteins, but in relation to these our knowledge is highly speculative.

It is further known that the hepatic glycogen may, under certain circumstances, originate from proteins, and that many proteins contain a carbohydrate radical; some, such as mucin, yield a considerable amount, but in the commoner proteins, the amount is in the neighbourhood of 1 per cent. or less. The mucins do not participate to any great degree in metabolism, and so the question arises whether the small amount of carbohydrates in the ordinary proteins is sufficient to account for the amount of glycogen formed. Arithmetic shows it will not; moreover, a protein (such as casein) which contains no carbohydrate radical at all, is equally efficacious as the others in yielding sugar when administered to animals suffering from glycosuria produced by phloridzin. We must therefore search for something in the protein molecule, as the source of the carbohydrate, and leucine, having like sugar 6 atoms of carbon, was naturally the first substance to be examined. But all experiments on the administration of leucine led to negative or nearly negative results. Another amino-acid, alanine (with its compounds tyrosine, phenyl-alanine, and tryptophane), was found to be the much more probable source of the carbohydrate. The relationship of alanine to the carbohydrates is a near one, for if $\mathrm{HO}$ is substituted in its molecule for $\mathrm{NH}_{2}$, we get lactic acid, and it was found that the administration of alanine to rabbits led to glycogen formation in their livers, and to the passage of lactic acid into their urine. Another cleavage product of protein, namely, aspartic acid, may act in a similar way, and this also is intelligible on chemical lines, for if aspartic acid loses carbon dioxide, it is converted into lactic acid, and it is no great step from this to sugar. Similar experiments have been performed with glycine and other amino-acids, but the results, though in part positive, are by no means so clear, nor is the chemical relationship between 
them and carbohydrate so easy to understand. Glycerin is another substance the conversion of which into carbohydrate appears to be possible; glyceric aldehyde is isomeric with lactic acid, so here again we have a feasible explanation.

Turning now to the other side of the picture, what information have we about carbohydrate katabolism? The final products of combustion are carbonic acid and water, but what are the intermediate steps? Just as lactic acid has been assumed to be sometimes a stage in the formation of sugar, so also there is evidence that it is a stage in its breakdown. We know that certain microorganisms possess the power of transforming sugar into lactic acid, and even still further into butyric acid (see formulæ, p. 411), and Buchner has recently asserted that lactic acid is a stage in the formation of alcohol and carbonic acid from sugar by means of yeast. The atoms in lactic acid $\left(\mathrm{C}_{3} \mathrm{H}_{6} \mathrm{O}_{3}\right)$ are in the same proportion as in sugar $\left(\mathrm{C}_{6} \mathrm{H}_{12} \mathrm{O}_{6}\right)$, but of course they are very differently arranged, and the rearrangement involved in the conversion of the one into the other, or vice vers $d$, is differently explained by different chemists. Lactic acid undoubtedly occurs in the body, but whether it all comes from sugar is extremely doubtful. The principal lactic acid found is the dextro-rotatory variety (sarco-lactic acid), whereas that formed in fermentative processes, as in milk, is the optically inactive variety. Now, there is a good deal of evidence that sarco-lactic acid originates from proteins; for instance, in the birds from which Minkowski removed the liver, the giving of protein food increased the lactic acid (which was not synthesised in the absence of the liver into uric acid) of their urine, and we have further seen that alanine and other protein cleavage products are possible parent substances of lactic acid. Still, if we admit that some of the lactic acid is of carbohydrate origin, and the admission is quite justifiable, we must remember that such a breakdown of sugar yields no heat; the calorific value of sugar and lactic acid being equal. The formation of lactic acid involves no transformation of energy; there is no formation of animal heat, or of its equivalent in work, and so the change is merely preliminary to a further change into carbonic acid and water, in which there will be that liberation of energy which it is the main object of carbohydrate breakdown to accomplish. (Glycolysis in the blood and tissues, and the importance of glycuronic acid as an intermediate substance in carbohydrate cleavage, are discussed on p. 540.)

\section{Metabolism of Fat.}

The most conspicuous use of the fat in the body is to act as a reserve fund of fuel. The storage of 100 calories in the form of fat may be effected in the space of about 12 c.c. of tissue, weighing 
about 11 grammes; the storage of the same amount of potential heat as glycogen is never effected in less than ten times that bulk of liver tissue weighing 130 grammes, and rarely in less than double that amount.

Another use of fat, and one concerning which we know less, is to participate in the actual construction of protoplasm, a rôle which is not exclusively, though it is mainly, that of the proteins. In this direction it is probable that the simple fats such as are found in adipose tissue are less concerned than the more complex fats, which contain phosphorus and nitrogen and are known as the phosphatides. Many years ago Hoppe-Seyler pointed out that these lipoids are as universally distributed as are the proteins wherever the phenomena of life are observable, and the more recent work of bio-chemists has fully confirmed the view, that though present usually in small quantities, they are nevertheless indispensable.

We have on pp. 541, 542 seen that the liver is important from both these aspects. It not only prepares the fats for combustion into their final products (carbon dioxide and water), with the simultaneous liberation of heat, but it is also of use in synthesising the more complex fatty compounds, the importance of which has just been alluded to.

Just as the carbohydrate of the food is the usual source of the carbohydrate of the body, so the fat of the food is the usual source of the fat of the body. But, again, fat may arise from something which is not fat in the food.

During absorption, the fatty acid and glycerin components of the fat undergo a temporary separation, but they soon reunite, and the fat which is not needed for immediate use, passes vid the chyle and blood to the cells of adipose tissue, where it is stored. The composition of the body fat depends to some extent on the composition of the fat in the food. The proportion of the olein, stearin, and palmitin in the fat of an animal can be varied by variations in their proportion in the food, and if unusual glycerides or unusual fatty acids (such as linolein from linseed oil, erucic acid from rapeseed oil, or iodised fats) are administered, they will be discoverable in the storage fat of the body.

How the fat which is transported to the adipose tissue by the blood enters the cells of that tissue is probably to be explained by the existence of an enzyme in the cells; this intracellular lipase acts as the pancreatic enzyme acts in the intestine, cleaving fat into its soluble constituents, fatty acid and glycerin; these pass through the cell membrane and are once more re-synthesised in to fat, the action of lipase being a reversible one. When the fat leaves the cells of adipose tissue for utilisation, that is, combustion in the tissues, lipase unties. the knot once more, and so fat is rendered soluble in the 
blood for transportation. There is no loss of energy in this process of fat-hydrolysis, any more than there is in the formation of lactic acid from sugar; or, to use the technical phrase, the reaction is an isothermic one.

Some observers have failed to discover lipase in the cells of the tissues which contain fat. It is probable that here as in the pancreas, lipase consists of two parts, an inactive component and an activator (see p. 520). The failure to discover lipase is therefore probably due to the circumstance that only the inactive portion is present. It has been found that blood or blood-serum has no power to split fats; it, however, does contain the activator of pancreatic lipase. These facts justify the conclusion that the co-enzyme or activator is a hormone produced in the pancreas, and carried by the blood to the fat depôts of the body, enabling the tissue lipase to hydrolyse the fat carried to them by the blood-stream after digestion.

The fat of the body may also arise from carbohydrate food. This is a physiological fact which was first firmly established by Lawes and Gilbert in their classical experiments on the fattening of pigs sixty years ago. The transformation is a monopoly of the living body: chemists were at first inclined to regard the fact as fiction, and they have never been able to repeat it in the laboratory. How the long carbon chains of the fat are linked together from the shorter carbohydrate chains of sugar is at present a riddle. Microorganisms can accomplish the change of lactic acid into such fatty acids as acetic, butyric, and caproic ; boiling with alkali brings about a similar reaction; and the same sort of change must occur in the body with the formation of higher fatty acids. The liver appears to be the place where the change occurs.

May fats also arise from proteins? This is a controversial question. Voit and Pettenkofer said yes, because they were able to fatten dogs on lean meat, but as the amount of fat left in the meat, and the glycogen also present, were not taken into account, their proof can hardly be considered satisfactory. The majority of physiologists to-day either answer the question in the negative, or regard it as unproven one way or the other. They adopt this attitude because the main proof adduced by those who believed in the transformation of protein into fat has been shown to be fallacious. It was stated that in certain pathological conditions, for instance in phosphorus poisoning, a fatty degeneration of cells of certain organs takes place, and the fat which appeared was believed to originate from the protein constituents of the cell-protoplasm. This is now known to be incorrect; every case of so-called fatty degeneration has been shown to be either due to an infiltration of fat transported from elsewhere, or to a transformation of the fat previously present in the protoplasm, although not in the form of droplets, and probably also not in the 
form of the usual glycerides. In many cases, the total fat present in this concealed form (possibly in combination with protein) in the cells of the heart, kidney, and liver may be greater than when with disease it takes the form of droplets.

With regard to the origin of glycerin, there is no doubt that the cells are able to produce it, as was shown by Munk's experiments on chyle, which are referred to on p. 548. There is no necessity to suppose that it originates from protein, for if glycerin can be converted into sugar, there is good ground for believing that the converse also takes place.

On katabolism, the fats yield the same ultimate products as the carbohydrates, namely, carbonic acid and water. A great deal of the oxygen we breathe in is used up in the burning of fats, and the simultaneous liberation of heat and work. It is quite certain that sugar is an important source of muscular energy, but the fats also play the same rôle, and muscles which are perpetually at work, such as the heart and the diaphragm, are particularly rich in fats. No actual lessening of the fat has yet been demonstrated to occur in excised muscles subjected to stimulation, but we have other and more trustworthy evidence that it does take place. During muscular work, the output of carbonic acid is increased, but the respiratory quotiont is almost unaltered; if sugar alone was undergoing combustion, this quotient would rise. Again, if the carbohydrate stores of the body are depleted by inanition, or by giving phloridzin to an animal, muscular work has but little influence on protein katabolism, and therefore the necessary increased combustion must fall on the fat. But how the long carbon chains of the fatty acids are taken to pieces and burnt up is very largely a matter of guesswork; still the recent work of Leathes and Hartley, which is briefly summarised on p. 541, has given us some insight into the way in which the liver accomplishes this object. Much the same problem is presented when we consider the fate of the proteins. We know fairly accurately how the protein nitrogen is disposed of, but the non-nitrogenous residue (which is chiefly lower fatty acid, and which like a fat is used for combustion, and as a source of heat and energy) is no doubt dealt with by the liver, as the fats themselves are. The existence of this non-nitrogenous and fat-like component of protein should make physiologists hesitate before they finally deny the possible conversion of the food-protein and tissueprotein into fat.

The study of acidosis or acidæmia referred to on p. 542 is important, because it sheds light on the phenomena of fat katabolism ; $\beta$-hydroxybutyric acid and acids of similar molecular size are probably normal intermediate products in the process; a healthy man on a properly mixed diet is able to oxidise $\beta$-hydroxybutyric 
acid in his liver into aceto-acetic acid, and then finally burn that acid into carbonic acid and water. The diabetic patient breaks down at this very point; he is able to form a certain amount of aceto-acetic acid, but this passes unchanged into his urine, or if any is changed at all, it is not into carbonic acid and water, but into acetone instead.

Our next question is whether during life fat may be converted into carbohydrate? In plants, the utilisation of fats by the embryo during germination has been studied. When growth begins enzymes are formed which hydrolyse fats, and v. Fürth found in his work on the oil in the seeds of the sunflower and castor-oil plant that the changes which occur consist in an increase in the saponification value and a lowering in the iodine and acetyl values. The increase in the saponification value indicates a fall in the molecular weight of the fatty acids, that is to say, the formation of lower fatty acids from higher ones has occurred; the lowering of the iodine and acetyl values indicates that it is the unsaturated linkages and the hydroxylated carbon atoms that have been the weak places where the cleavage has occurred. But beyond these observations there is nothing known of the metabolism of fats in plants; certainly any evidence that they are transformed into carbohydrates is lacking.

The evidence that the transformation of fats into carbohydrates can occur in animals is also indirect or unsatisfactory. Pflüger argued that in glycosuria following extirpation of the pancreas, or phloridzin administration in dogs, large quantities of fat are converted into sugar. This was an assumption based upon the idea that none of the sugar could have been formed from protein. This view does not accord with the observations and opinions of others who have worked at the subject, and for many years Pflüger fought in his usual strenuous manner for his own view that protein can never be a source of sugar. However, facts were too strong for him, and in almost the last paper he wrote before he died he withdrew it.

We have already seen the chemical difficulties of explaining how sugar can be converted into fat, but it is a fact nevertheless. The difficulty of explaining the converse change is equally great; still in spite of this difficulty and in spite of the unsatisfactory nature of any proofs that this actually occurs during life, it cannot be maintained that the formation of sugar from fatty acids is impossible; for we have already seen that the formation of sugar from certain amino-acids such as alanine, which are of protein origin, has been proved to occur. Amino-acids deprived of their amino-group are fatty acids ; alanine, for instance, minus its amino-group, is propionic acid. The ultimate fate of lower fatty acids derived from fats can hardly be different from that of those which are derived from proteins. 


\section{Metabolism of Protein.}

In our discussion of the origin of urea in the urine, we have mentioned some of the main facts in relation to the metabolism of proteins, and it would be well if the student again reads these pages (pp. 586 to 588) before studying the paragraphs which now follow; for the laws which govern the composition of urine are the effect of more fundamental laws governing protein katabolism.

Liebig was the first to divide foods into flesh-forming and heatforming, that is, into those which repair the tissue waste, and those which are not so intimately assimilated into the protoplasm, but are utilised as sources of energy. The latter function is the one performed by the fats and carbohydrates, and the former is more particularly the duty of the proteins. This idea is reflected in the popular use of the term nutritious; it is used almost synonymously with nitrogenous, and the notion that the non-nitrogenous foods, although they form the greater part of our daily diet, are not nutritious and next door to useless, is a most mischievous one, though it is carefully fostered by the advertisers of patent foods. Both kinds of food are equally necessary, and equally though differently nutritious.

It is now known that the proteins are not only flesh-formers, but also that they play the other rôle in nutrition and act as a source of energy. The complete breakdown into amino-acids which occurs in the gastro-intestinal tract, has in fact a double signification. It enables the cells of the body to construct from the cleavage products the proteins peculiar to themselves, and it further enables the body to easily rid itself of the nitrogenous portions of the food-proteins which are not wanted for the repair of tissue waste. This portion is never really assimilated in the sense that it is built into protoplasm, but it is taken in by the liver cells, which deamidise it and convert the nitrogenous portion into urea. The non-nitrogenous moiety is then utilisable for energy and heat production.

In starvation, the income of the body is limited to oxygen, but if water is given also, an animal will generally live a little over four weeks. During this time the excretion of nitrogenous and carbonaceous waste continues and the body loses weight day by day. The excretion of carbon dioxide continuously falls until death supervenes. The nitrogen of the urine falls also within the first few days, and then remains at a low but constant level to the end of the fourth week. Then for the few days preceding death, its amount again increases. By this date nearly every trace of the fat of the body has disappeared, and so the cells fall back on their more precious protein material and consume it in greater quantity than before. The nitrogen elimination during the weeks when it remains constant 
must be derived from the proteins of the body, for there is none coming in, in the way of food. It might be thought if at this time an amount of protein food containing the same quantity of nitrogen as was being lost by the body, was administered, that the loss of nitrogen from the body would be checked, and that the tissues would seize the opportunity of repairing their waste. But this is not the case; what happens is that the amount of nitrogen lost in the day is almost doubled; and this is an undoubted proof that nearly all the protein in the food is disintegrated and its nitrogen discharged within the twenty-four hours. In order to get nitrogenous equilibrium, it is necessary to give in the day two and a half times as much protein as is lost during starvation in that period of time.* This was one of the earliest proofs adduced that all the food protein is not used in tissue repair, and it led Voit to formulate his celebrated theory of the distinction between "tissue protein" and what he termed "circulating protein." The latter expression was coined because Voit believed that the katabolism of this variety of protein occurred in the blood, or at any rate in the tissue juices. In fact, he cousidered that katabolism occurred only in the "circulating protein," the small amount of "living protein" which dies being dissolved and so added to the "circulating protein" before katabolism occurs. Voit's great opponent was Pflüger, and for many years Pflüger's theory replaced Voit's; this theory states that all protein must first become assimilated; that is, must be built into and become part and parcel of living protoplasm before it undergoes katabolism. Pflüger did good service in emphasising the importance of the cells in metabolic processes, and we certainly do not now believe that respiration or any other metabolic process has its seat in the circulating fluids. But at the same time Voit's theory possesses the correct underlying idea which forms the basis of our present doctrine of metabolism. In every living tissue there exists a framework of what we may call more distinctly living substance, the metabolism of which is constant and does not give rise to massive discharges of energy; in the interstices of this are various kinds of material related in different degrees to this framework; these materials are less eminently living, and the chief part of the energy set free comes directly from the metabolism of some or other of this material. Both the framework and the intercalated material undergo metabolism, and have in different degrees their anabolic and katabolic

* It has recently been stated by Michaud that this amount may be greatly reduced by feeding the animal on proteins which are as near as possible in composition to the tissue proteins of the animal, for instance, by feeding a dog on dog's flesh. Knowing, however, as we do now that during digestion protein material undergoes almost complete cleavage, it is difficult to accept such a view, and many observers have not confirmed the experiments, or at least have shown that the advantage is far less than Michaud found. 
changes; both are concerned in the life of the organism; but one more directly than the other. When we now speal of endogenous protein metabolism we refer to that in the material highly endowed with life; when we apply the term exogenous protein metabolism to the changes by which the liver brings about the conversion of aminoacids from the food into urea, we refer to its action on intercalated material, and no longer use the phrase "circulating protein."

We have already discovered in our study of the urine, that exogenous protein katabolism is mainly represented in the urine by urea and inorganic sulphates; while the final katabolites of endogenous metabolism are substances like creatinine and "neutral sulphur"; but there is no doubt that some urea is formed also: this is seen, for instance, during starvation.

Let us consider a man taking the customary Voit dietary of 16 or 17 grammes of nitrogen in his daily food; probably only a quarter or even less of this is destined for endogenous use, and the protein sufficient to maintain this is indispensable. Would it be possible to dispense entirely with the amount which is exogenously metabolised, and reduce our protein intake to the low level of, say, 4 grammes of nitrogen per diem. The old observations on starving animals we have just referred to shows that this would not be possible; the minimum is not the optimum; and even Chittenden (see p. 480) does not recommend a reduction lower than 7 or 8 daily grammes of nitrogen. If an animal cell is presented only with protein food, it takes and uses it eagerly, even although it may not ultimately build much of it into protoplasm. If substances such as fat, carbohydrate, or the incomplete protein we call gelatin, is presented to it also, the amount of protein necessary is reduced, and so we speak of such foods as being "protein-sparing."

The important character of Chittenden's work has given the faddists on matters of diet an important opportunity of being listened to. There is, for instance, a group of these to whom the very necessary act of chewing has assumed almost the nature of a religious ceremony, and they have sought to convince mankind of its superlative importance. These, however, need not concern us, but there are some even in the scientific world who seem almost to believe that the law of conservation of energy does not apply to the chemical changes in a living animal. They cite instances of people who do a large amount of work, and do it upon what most would regard as an insufficient diet, without detriment or loss of body-weight. If a man only receives food in the day of the energy value say of 1500 large calories, and the heat he produces and the work he does are equivalent to 2000 ; then the additional 500 must have come from his internal resources, and he must have used up some of the material formerly stored in his body. This is as 
certain as is the fact that one and one make two. It is quite conceivable that his body may not have lost weight, but nevertheless fat may have disappeared, and been replaced by an equivalent weight of water, and excess of carbohydrate food which usually is a character of the diets of such people is just the sort of diet likely to cause retention of water in the body.

We have in our mention of the Chittenden diet alluded to several circumstances that should make us pause before we accept his conclusions to the full. Many people eat too much; would it be advisable for us all to eat too little, and is Chittenden's diet too scanty?

No doubt the over-eaters would benefit by eating too little for a time. They would give their overtaxed digestive and secretory organs a necessary rest, and have time to consume some of their accumulated stores of material. It is quite possible that the benefit noticed in some of the subjects of Chittenden's experiments might have been due to such a circumstance as this, or to the regular life they were compelled to live, quite apart from diet altogether. But to eat too little as an ordinary and permanent thing is quite another matter; and it is interesting to be able to record that most of the subjects of Chittenden's experiments have now returned to their previous dietetic habits.

So far as it is possible to read history correctly, man has always, where he can, taken instinctively more protein than Chittenden would allow him, and with few exceptions, the meat-eating nations are those which have risen to the front.

So far as it is possible to draw correct deductions on questions of diet from animals to man, a restricted diet over a long period has proved detrimental. Moreover, a careful study of Chittenden's own analytical figures, such as Benedict has made, shows there was in some cases distiuct impairment of health.

But still the question remains, why an apparently large excess of nitrogen which the body casts out within a few hours should be advisable? The answer to this appears to be, that though most of the cleavage products are dealt with in this way, there are some which are especially precious for tissue reconstruction, and it is for these that we put up with the excess of waste. The large size and activity of the normal liver seem to be for the express purpose of dealing with this waste rapidly.

Nature does not work in minimums: Leathes puts it very well when he says it is not considered unphysiological to take more food than will yield the minimum of fæcal refuse; and he also points out that in the infant, even allowing for its growth, the normal amount of milk provided for it by nature is ten times greater than would appear to be the necessary minimum; and this is probably a safer 
argument than the one so often used when the instinctive habits of past centuries of adults are appealed to.

We may also draw a useful lesson from disease. In the modern treatment of consumption, the open-air cure is combined with a steady process of generous feeding; in certain cases of nervous breakdown, an important part of the "rest cure" is the providing of abundant and appetising meals. One can hardly doubt that much of the benefit noticeable in both classes is due to the "reserve energy" provided, enabling the body more fully to grapple with the malady. "Reserve energy" may be objected to as a vague phrase which, though comforting to those who use it, is nevertheless very difficult to explain. There is a good deal of reason in such an objection, for "reserve force" is difficult to define clearly. We have, for instance, no knowledge of any storage places for protein, in the same way in which the liver and adipose tissue act as storehouses for carbohydrate and fat respectively. But it is an undoubted factor all the same; many people have more of it than others; and this "stamina," as it is sometimes called, is a lucky possession for those who have it. Research on immunity has, however, shown us that this is in part due to the condition of our leucocytes, and the opsonic power of the blood-plasma (see p. 476). It may be that it is in this direction among others, that the abundance of protein food may assist us in repelling disease. Each leucocyte may not require much in the wœy of repair every day, but it is more likely to get this "stitch in time" if there is an abundant supply of repairing material available.

Rubner has called attention to what he terms the specific dynamic action of food-stuffs. Weight for weight, fat yields more heat when burnt than protein does, and outside the body it is more easily combustible than either protein or carbohydrate. Inside the body it is just the reverse; proteins are the most readily burnt of any food material, and fats the least. In other words, proteins (and the same is true for amino-acids) have a specific value in stimulating metabolism, and so leading to an increase of oxidation in the body. Some of the subjects of Chittenden's experiments suffered intensely from the cold in the winter, and this use of protein must not be lost sight of in settling the right amount which we should take in our daily food.

Some attempt has been made to determino which of the protein cleavage products, or Bausteine, to use the German term, are specially valuable in the body, either for the synthesis of tissue protein, or, as Hopkins has suggested, for the formation of the special hormones or chemical messengers of the body, such as adrenaline.

Recent research has established the fact that one of the important building stones for these purposes is phenyl-alanine, and its near 
relative tyrosine is another; for when they are injected into the blood-stream they do not reappear as urea in the urine. We also know that proteins which yield no tyrosine, such as gelatin, are of inferior value as food. Gelatin is also destitute of the tryptophane radical, and tryptophane is specially useful too. Zein, the protein of maize, lacks tryptophane, and if tryptophane is added to a zein diet, animals fed on the mixture thrive better than those whose sole nitrogenous food is zein. Histidine and pyrrolidine have been suggested as being in the same category, but here we must await further information.

\section{Growth and Maintenance. Synthesis in the Body.}

The different members of the protein family have thus unequal powers in repairing the body waste. Still more important is their difference in promoting growth in young animals. Recent work by Osborne, Mendel, and others has shown that it is possible to keep animals alive and in health when they receive their nitrogen in the form of one protein only, mixed of course with the proper supply of non-nitrogenous food. But if these animals are growing, the choice of the protein given is a matter of great importance. Repair processes in the adult are of a different character from those of growth in the young animal; in the adult cell, katabolism and repair do not involve the destruction and resynthesis of entire protein-molecules, such as obviously must be the case when growth is taking place. This work has also brought to light the fact that, in the animal body, there is a power of synthesising comparatively simple food material into more complex substances far in excess of what was previously considered to be the case. Thus lipoids can be built from inorganic phosphorus compounds, and proteins constructed from amino-acids. Some even have asserted that ammonium salts given by the mouth can be utilised for protein construction, but this has not yet been conclusively proved. The body also possesses the power of building up amino-acids which are absent from the food, and of converting one amino-acid into others. This ability is most marked in the adult animal; it is in the young animal that the actual administration in the food of the necessary building stones is indispensable.

Let us take one or two instances which illustrate this. Rats were fed for long periods on food mixtures which contained only a single protein. When gliadin, edestin, or caseinogen, proteins of very different composition were administered, the animals thrived and showed neither loss of weight nor any other metabolic disorder. Glycine is absent from caseinogen, lysine and glycine from gliadin, phosphorus from gliadin and edestin, and purines were absent from 
all threee. The synthetic activities of the animal body, or the possibility of the transmutation of one amino-acid into others are thus clearly brought to mind.

My next example may' be taken from an animal higher in the scale. A food-mixture in which gliadin was the only source of nitrogen was given to a puppy in place of its mother's milk; this produced typical failure in growth. Nevertheless the mother dog thrived on the same diet and actually produced young, and secreted milk in sufficient quantity and quality to induce normal growth in her offspring. No stronger proof could be adduced of a power in the adult body to synthesise "Bausteine" which are absent from the food. The liver cells have been usually credited with the main share in such marvellous synthetic power; but there is a reaction in the minds of physiologists just now against this tendency to pile up hepatic duties. Several pieces of work show that, although the liver doubtless does its due share of the work, other tissues and organs also participate in it.

The only other example I select from the large mass of material at hand illustrates the importance of those unknown but indispensable constituents of a diet which are referred to in our chapter on Foods (p. 491). An animal, or at any rate a growing animal, cannot live on protein alone, even if it is mixed with appropriate mineral salts, and with a proper supply of fat and carbohydrate to supply the necessary energy; these other organic compounds of uncertain nature are absolutely indispensable. F. G. Hopkins was one of the earliest to recognise the importance of what, for want of a better nane, we may term these accessory factors in a dietary, and the effect of quite small quantities of such substances is admirably illustrated in his most recently published work. Groups of young rats were fed on a diet of caseinogen, fats, carbohydrate, and salts, and compared with other rats on the same diet, plus a minute ration of fresh milk. The former soon ceased to grow; the latter grew normally. The consumption of food was practically the same in all the animals, but the milk addendum reduced the food necessary for a given increment in weight to one-half or less. Moreover, cessation of growth occurred before there was any loss of appetite to account for it. What the actual substances are in the milk which thus markedly, although in a secondary way, affect growth is not yet known. We can therefore only surmise that the unknown material (provisionally termed vitamine) may contain some particular "Bausteine" which the animal body is not able to build up for itself; or it may be that the effect is due to a stimulating effect upon the cell-protoplasm which leads to the development of the necessary synthetic power. Funk has suggested that the "vitamines" are derivatives of pyrimidine. 


\section{Inanition or Starvation.}

During starvation the body gradually loses weight; the temperature, after a preliminary rise, sinks; the functions get weaker by degrees, and ultimately death ensues when the body has lost about 50 per cent. of its original weight. Death may be delayed somewhat by artificial warmth, so that the strain on the internal production of heat is not so great. If water is given, life may continue for rather more than a month. The age of the animal influences the time at which death occurs. This statement was originally made by Hippocrates, and has been borne out by the experiments of Martigny and Chossat. Young animals lose weight more quickly, and die after a smaller loss of weight than old ones.

The following table from Ranke's experiment on himself represents by the balance-sheet method the exchange for a period of twenty-four hours, the same time having elapsed since the last meal.

\begin{tabular}{|c|c|c|c|c|c|c|}
\hline \multicolumn{4}{|c|}{ Income, due to disintegration of Tissues. } & \multicolumn{3}{|c|}{$\begin{array}{l}\text { Expenditure, obtained by analysing the } \\
\text { Excretions. }\end{array}$} \\
\hline 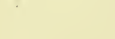 & & Nitrogen. & Carbon. & & Nitrogen. & Carbon. \\
\hline \multirow[t]{2}{*}{$\begin{array}{l}\text { Protein } \\
\text { Fat }\end{array}$} & $\begin{array}{l}50 \mathrm{gr} . \\
200 \text {, }\end{array}$ & $\begin{array}{l}7 \cdot 8 \\
0.0\end{array}$ & $\begin{array}{r}26 \cdot 5 \\
157 \cdot 5\end{array}$ & $\begin{array}{l}\text { Urine } \\
\text { Respiration }\left(\mathrm{CO}_{2}\right)\end{array}$ & $\begin{array}{l}7 \cdot 8 \\
0 \cdot 0\end{array}$ & $\begin{array}{r}3 \cdot 4 \\
180 \cdot 6\end{array}$ \\
\hline & & $7 \cdot 8$ & $184 \cdot 0$ & & $7 \cdot 8$ & $184^{\circ} 0$ \\
\hline
\end{tabular}

The excretion of nitrogen falls quickly at the commencement of starvation, and even on the first day the above table shows us it has sunk to half the normal. This lessening goes on for a fow days, after which it remains constant; about the ond of the fourth week it rises again when the fat of the animal has been used up, and the body makes an increased call on the protein constituents of its protoplasm. With the onset of symptoms of approaching death, which is sometimes accompanied by convulsions, the excretion of nitrogen rapidly falls again. The sulphates and phosphates of the urine show much the same series of changes. The discharge of carbonic acid and the intake of oxygen fall continuously over the whole period.

It is important to note, that wasting does not occur to an equal extent in all the tissues and organs. Those which are most essential to life are fed at the expense of the others; thus the heart loses little or none, and the central nervous system loses at most 3 per cent. of its 
weight. The fat nearly all disappears, at least 97 per cent. of it being used up; muscles lose 30 per cent. of their original weight, and most of the other organs suffer also but in varying degrees. Taking the total loss as 100, Voit gives the loss due to that of individual organs as follows:-

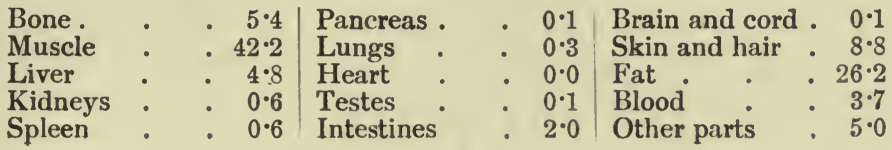




\section{CHAPTER XLII}

\section{THE CONSERVATION OF ENERGY}

THE nutrition of the body has been considered in the preceding pages from the standpoint of a detailed examination of the fate of the various foodstuffs which enter the body from the alimentary canal. Furthermore, by a consideration of the substances which the body excretes, an attempt has been made to arrive at some understanding of the processes of metabolic activity.

The knowledge thus obtained is of more than theoretical interest. It throws much light on one of the most important subjects which confronts the physiologist, namely, the suitability of various substances as articles of diet. This subject we propose to discuss in the present chapter, but before doing so we must lay down two propositions.

(1) A suitable diet must provide at least as much of each chemical element as is excreted from the body.

(2) The daily food must supply a store of potential energy which shall equal the actual energy dissipated in the twenty-four hours.

The first of these propositions is self-evident; the second, which resolves itself into an enquiry as to whether the living body obeys the law of the conservation of energy, has been the subject of much laborious research.

In the cruder investigations of earlier workers (Lavoisier, etc.), a considerable discrepancy appeared between the actual potential energy of the food taken in, and the proven actual energy which is dissipated by the body. More exact methods, especially in the hands of Rubner, have gone far to put the energy changes of living matter on a more intelligible basis, while investigations undertaken during the last two decades, under the auspices of Atwater, Benedict, and their colleagues, have finally established that the law of conservation of energy holds in relation to the animal body.

Among the forms which energy derived from the combustion of any substance, whether within or without the body, may assume, two, namely, mechanical work and heat, demand the attention of the physiologist. The simplest case which can present itself, that in 
which the body does no work, and neither gains nor loses in weight, resolves itself into the following problem: Does the heat given out by the body equal that which would be given out by the complete combustion of the various food substances, minus that given out by the complete combustion of the excreta?

The data necessary for settling such a question are determined by the process known as calorimetry.

The Bomb Calorimeter.-The heat of combustion of any of the food substances or of the excreta is determined by placing a known weight of the substance in question (A, fig. 393) within a bomb (B) immersed in a known volume of water; the water is at air-temperature in a brass vessel (E), enclosed within an ebonite casing $(\mathrm{F})$, which acts as a non-conductor of heat. The bomb is connected with a cylinder of oxygen at high pressure; and the substance $A$ is ignited by an electric spark by means of the wires $\mathrm{D}$. The products of combustion pass out through the spiral tube $\mathrm{C}$, and on their journey give off their heat to the water. If the heat of combustion of gases or volatile liquids is to be determined, a special form of burner is introduced at the opening at the bottom of the bomb. When the combustion is complete the rise of temperature of the water is observed by the thermometer T. During the

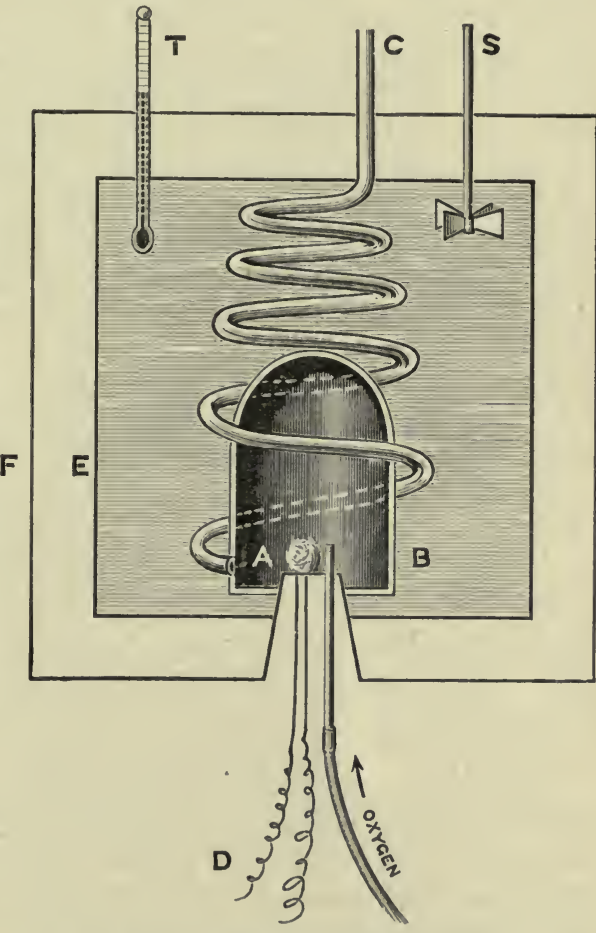

Fia. 393.-Diagram of Bomb Calorimeter. (After Thomsen.)

combustion, the water is kept in movement by the stirrer S, which is worked by a small motor. The rise of temperature multiplied by the weight of the water gives the amount of heat expressed in calories, 1 calorie or heat-unit being the quantity of heat necessary to raise 1 gramme of water $1^{\circ} \mathrm{C}$.

Any given oxidation will always produce the same amount of heat. Thus, if we oxidise a gramme of carbon, a known amount of 
heat is produced, whether the element is free or in a chemical compound. The following figures show the approximate number of heat-units produced by the combustion of 1 gramme of the following substances:-

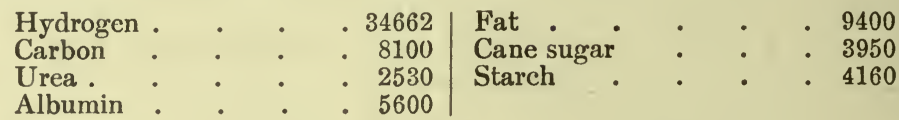

It is, however, most important to remember that the "physiological heat-value" of a food may be different from the "physical heatvalue," i.e., the amount of heat produced by combustion in the body may be different from that produced when the same amount of the same food is burnt in a calorimeter. This is the case with the proteins, because they do not undergo complete combustion in the body, for each gramme of protein yields a third of a gramme of urea, which has a considerable heat-value of its own. Thus albumin, which, by complete combustion, yields 5600 heat-units, has a physiologica] heat-value $=5600$ minus one-third of the heat-value of urea $(2530)$ $=5600-846=4754$. Rubner has shown that this figure must be reduced to nearly 4000 , as some of the imperfectly burnt products of decomposition of proteins escape as uric acid, creatinine, etc., in the urine, and there is a small quantity of similar substances in the fæces. No difference between the physical and physiological heatvalues of fats and carbohydrates exists, provided, of course, that all the fat and carbohydrate in the food is absorbed.

Having obtained in this way the energy value of the food taken in, expressed as units of heat, the next step is to arrive at the heat produced in the animal body. Other manifestations of energy in the body, such as kinetic energy, must also be taken into account, and it is usual to express these also in terms of heat, one calorie being equivalent to 425.5 gramme-metres (see p. 129).

This is also accomplished by calorimetry. From time to time numerous calorimeters designed for this purpose have been introduced, but by far the best is the Atwater-Benedict instrument, and its special value consists in the circumstance that it can be used for making observations on human beings. The method employed will be seen to be based precisely on the same principles as those of the bomb calorimeter. The apparatus is represented diagrammatically in the accompanying drawing (fig. 394).

The Atwater-Benedict Calorimeter consists of a room with nonducting walls. Through this run coils of water-pipes, fitted with metal discs. Only one of these tubes is shown in the figure (A). Any rise of the temperature of the room is at once taken up by the discs and communicated to the water. The whole of the heat production of the individual in the calorimeter is therefore spent 
in raising the temperature of the water. The amount of water which goes through the pipes multiplied by the difference in the temperature of the water as it enters and as it leaves the calorimeter, gives the heat output of the person within it. This is, ascertained by the thermometers $(\mathrm{T}, \mathrm{T})$.

In the case of the bomb calorimeter it is possible to ensure the complete combustion of the substance placed in the bomb. It is not possible to ensure the complete oxidation of the food eaten. For instance, food may be retained and assimilated with a gain of weight to the individual. This is met in the following way. The air of the calorimeter is kept circulating through a series of chambers in which

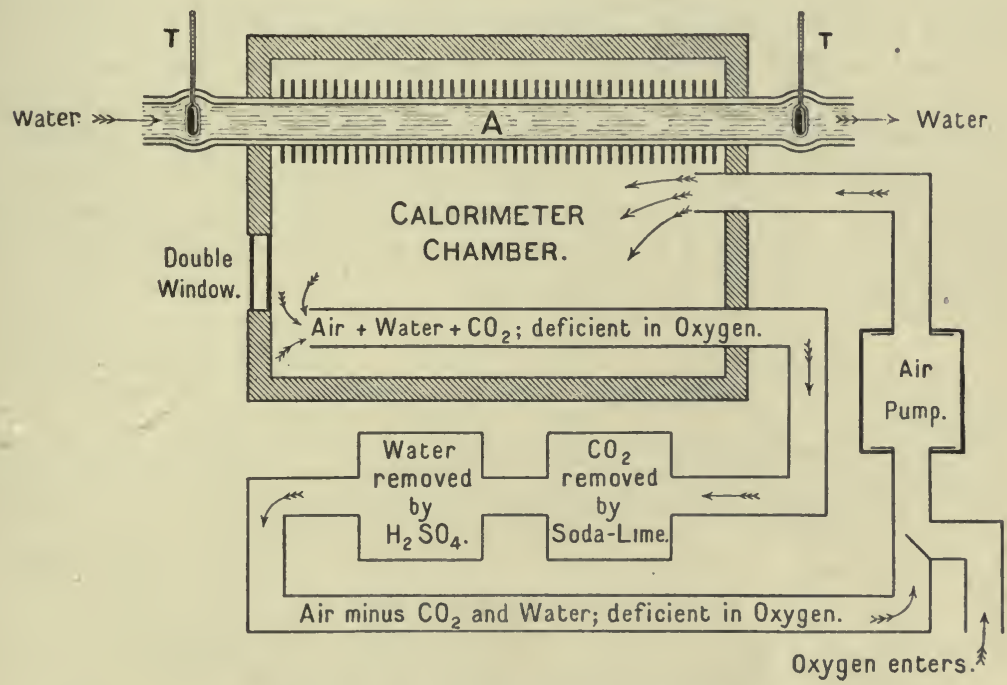

Fı. 394.-The Atwater-Benedict Calorimeter.

The above drawing contains one mistake; the position of the soda-lime and sulphuric acid should be transposed.

the carbon dioxide and the water are absorbed, and subsequently estimated. As the oxygen is used up by the individual, fresh oxygen is admitted in known quantities. The urine and fæces are analysed as well as the air, at the beginning and end of the experiment. The following additional data are therefore forthcoming: (1) the carbon, hydrogen, and nitrogen given out by the body; (2) the oxygen taken in, and from these the amounts of protein, fat, and carbohydrate metabolised in the body, can be calculated and compared with the food ingested (see p. 613).

In the calorimeter is a bicycle, the hind-wheel of which is replaced by a copper disc. The disc may be rotated in the field of 
an electro-magnet by the turning of the pedals, which thus enables the rider to perform a measurable quantity of mechanical work.

The calorimeter is also supplied with a bed, a table, a chair, and a double window, through which food of known weight and composition can be supplied, so that an experiment may continue over two or three days, and the effect of work, sleep, various diets, etc., can be studied.

Of the heat produced in the body, it is estimated by Helmholtz that about 7 per cent. is represented by external mechanical work, and that of the remainder about four-fifths are discharged by radiation, conduction, and evaporation from the skin, and the remaining fifth by the lungs and excreta. This is only an average estimate, subject to much variation, especially in the amount of work done.

The following table exhibits the relation between the production and discharge of energy in twenty-four hours in the human organisn at rest, estimated in calories.* The table conveniently takes the form of a balance-sheet in which production and discharge of heat are compared; to keep the body-temperature normal these must be equal. The basis of the table in the left-hand (income) side is the same as Voit's diet (see p. 480):-

Production of heat.

Metabolism of

Protein $(120$ gr. $) \cdot 120 \times 4000=480,000$

Fat (100 gr.) $\quad .100 \times 9400=940,000$

Carbohydrates

$(=333$ gr. starch $)\} 333 \times 4160=1,385,280$

$2,805,280$

\section{Discharge of heat.}

Warming water in food,

$2 \cdot 6$ kilos $\times 25^{\circ} \mathrm{C} .=65,000$

Warming air in respiration,

16 kilos $\times 25^{\circ} \times 0.24=96,000$

Evaporation in lungs, 630 gr. $\times 582=366,660$

Radiation, evaporation, etc., at surface, plus the thermal equivalent of mechanical work done accounts for the remainder .

$2,277,620$

$2,805,280$

The figures under the heading Production are obtained by multiplying the weight of food by its physiological heat-value. The figures on the other side of the balance-sheet are obtained as follows: The water in the food is reckoned as weighing 2.6 kilos. This is supposed to be at the temperature of the air, taken as $12^{\circ} \mathrm{C}$.; it has to be raised to the temperature of the body, $37^{\circ} \mathrm{C}$., that is, through $25^{\circ} \mathrm{C}$. Hance the weight of water multiplied by 25 gives the number of calories expended in heating it. The weight of air is taken as

* The calorie we are taking is sometimes called the small calorie; by some the word calorie is used to denote the amount of heat necessary to raise 1 kilogramme of water $1^{\circ} \mathrm{C}$. This is called the large calorie. 
weighing 16 kilos; this also has to be raised $25^{\circ} \mathrm{C}$., and so to be multiplied by 25 ; it has further to be multiplied by the relative heat of air (0.24). The 630 grammes of water evaporated in the lungs must be multiplied by the potential or latent heat of steam at $37^{\circ} \mathrm{C}$. (582); the portion of heat lost by radiation, conduction, and evaporation from the skin constitutes about four-fifths of the whole, and is obtained by deducting the three previous amounts from the total. This table does not take into account the small quantities of heat lost with urine and fæces. If the man does external work the amount of energy dissipated is increased, and he would, in consequence, require more to be supplied in the form of food. Very few men in active work get on well with a smaller supply than 3000 large calories $(=3,000,000$ small calories) in their diet. A man, however, at rest is always doing what is called internal work, that is, maintaining the circulation, respiration, etc.

From experiments of this nature, it has been found that the principle of the conservation of energy holds in the living body. The results may be stated as follows:-

1. If an animal is doing no external work, and is neither gaining nor losing substance, the potential energy of the food (expressed as its heat of combustion) will be equal to that of the excreta, plus that given off as heat, plus that of internal work.

2. If an animal is doing external work, and is neither gaining nor losing substance, the potential energy of the food will be equal to the potential energy of the excreta, plus that given off as heat, plus that of the internal work, plus that of the external work.

3. If an animal is doing no external work, but gaining or losing body-substance, the potential energy of the food will equal the potential energy of the excreta, plus that given off as heat, plus that of the internal work, plus that of the gain by the bodysubstance (a loss by the body being regarded as a negative gain).

4. In an animal doing external work, and gaining or losing bodysubstance, the potential energy of the food will equal the potential energy of the excreta, plus that given off as heat, plus that of the internal and external work, plus that of the gain (positive or negative) of the body-substance. 


\section{CHAPTER XLIII}

\section{TEMPERATURE}

SINCE departures from the normal body-temperature are among the fundamental physical signs of disease, and since observations of the temperature of the patient are only less frequent in medical practice than those of the pulse or of the tongue, it is necessary to have as complete an understanding as possible of the principles that regulate the fluctuations of the clinical thermometer.

Animals may be divided into two great classes :-

(1) Warm-blooded or homoiothermal animals, or those which have an almost constant temperature. (Mammals and birds.)

(2) Cold-blooded or poikilothermal animals, or those whose temperature varies with that of the surrounding medium, being always, however, a degree, or a fraction of a degree, above that of the medium. This class includes reptiles, amphibians, fish, embryonic birds and mammals, and probably all invertebrates.

The temperature of a man in health varies but slightly, being between $36.5^{\circ}$ and $37.5^{\circ}$ C. $\left(98^{\circ}\right.$ to $99^{\circ}$ F.). Most mammals have approximately the same temperature: horse, donkey, ox, $37.5^{\circ}$ to $38^{\circ}$; dog, cat, $38.5^{\circ}$ to $39^{\circ}$; sheep, rabbit, $38^{\circ}$ to $39.5^{\circ}$; mouse, $37.5^{\circ}$; rat, $379^{\circ}$. Birds have a higher temperature, about $42^{\circ} \mathrm{C}$. The temperature varies a little in different parts of the body, that of the interior being greater than that of the surface; the blood coming from the liver, where chemical changes are very active, is warmer than that of the general circulation; the blood becomes rather cooler in its passage through the lungs.

The temperature also shows slight diurnal variations, reaching a maximum about 4 or 5 P.M. $\left(37.5^{\circ}\right.$ C. $)$ and a minimum about 3 A.M. $\left(36.8^{\circ}\right.$ C. $)$; that is, at a time when the functions of the body are least active. If, however, the habits of a man are altered, and he sleeps in the day, working during the night, the times of the maximum and minimum temperatures are also inverted. Inanition causes the temperature to fall, and just at the onset of death it may be below $30^{\circ} \mathrm{C}$. Active muscular exercise raises the temperature temporarily by about $0-5^{\circ}$ to $1^{\circ} \mathrm{C}$. 


\section{Heat Production.}

(1) Effect of Changes of External Temperature.-In theory there is a fundamental difference between cold- and warm-blooded animals in their reactions to external temperature. A cold environment, since it lowers the temperature of the poikilothermic creature, reduces the metabolism of all its tissues, and thus reduces its heat production.

The warm-blooded individual reacts in precisely the opposite way. Since his temperature remains constant, his heat production increases, in order to neutralise the effect of his cold surroundings. This has been demonstrated in the case of fasting dogs. An example may be given.

\begin{tabular}{|c|c|c|c|c|}
\hline Temperature of Air. & $13 \cdot 8^{\circ} \mathrm{C}$ & $14 \cdot 7^{\circ} \mathrm{C}$. & $17 \cdot 3^{\circ} \mathrm{C}$ & $18^{\circ} \mathrm{C}$. \\
\hline $\begin{array}{l}\text { Heat production in calories } \\
\text { per kilo per diem }\end{array}$ & $78 \cdot 7$ & $74 \cdot 7$ & $69 \cdot 8$ & $67 \cdot 1$ \\
\hline
\end{tabular}

In practice it is doubtful whether any such exact relation can be discerned in man, as it may be masked by other factors. We have already insisted upon the equality between the respective energy values of the food eaten and of the heat produced, and upon the advantage of an ample diet. In practice it is the amount of food taken which controls the heat production, rather than the reverse. The majority of well-to-do people, whose appetite is stimulated by their palate, maintain a constant body-temperature by regulating the loss rather than the production of heat. In this connection the following figures, derived from observations made upon a dog who was fed upon considerable quantities of meat, may be compared with those obtained when the same animal was fasting.

\begin{tabular}{|c|c|c|c|c|c|}
\hline Temperature of Air. & $7^{\circ} \mathrm{C}$ & $15^{\circ} \mathrm{C}$. & $20^{\circ} \mathrm{C}$. & $25^{\circ} \mathrm{C}$. & $30^{\circ} \mathrm{C}$. \\
\hline 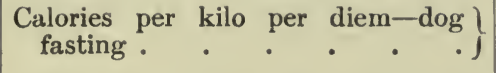 & $86 \cdot 4$ & $63 \cdot 0$ & $55 \cdot 5$ & $54 \cdot 2$ & $56 \cdot 2$ \\
\hline $\left.\begin{array}{l}\text { Calories per kilo per diem-dog } \\
\text { given } 320 \mathrm{~g} . \text { meat }=81 \text { calories per } \\
\text { kilo }\end{array}\right\}$ & $87 \cdot 9$ & $86 \cdot 6$ & $76 \cdot 2$ & $\cdots$ & $83 \cdot 0$ \\
\hline
\end{tabular}

In the fasting dog a lowering of the surrounding temperature, increases heat production in the animal; in the well-fed dog this is hardly noticeable.

On the other hand, it is instructive to note the types of food eaten by the natives of different climates. The Indian, who eats rice gets 
his carbon with less than half the heat production of the Esquimaux, who makes blubber his staple article of diet.

(2) The Seat of Heat Production.-So far as our present knowledge goes, the amount of metabolism in the bones, cartilages, and connective tissues is so small as to form but a trifling part of the whole metabolism of the body. The same is probably true of unstriped muscle. Of the coefficient of oxidation (i.e. the amount of oxygen used up per gramme of tissue per minute) of the central nervous system we have no accurate knowledge. Any discussion, therefore, of the principal seats of chemical action in the body resolves itself into a comparison between the glandular and muscular (skeletal) structures. These present a remarkable contrast. The very vascular nature of the secreting glands (the liver is said to contain one-quarter of all the blood in the body), as well as actual measurements of the oxygen used up by many of them, indicate that they are the seat of very active chemical changes, which, relatively to muscle, is maintained with a considerable degree of constancy. The very function which the digestive glands serve implies at least a certain constancy of rhythm. Be the climate what it may, the daily food must be digested. Quite otherwise is it with the muscles. When they are active they are the seat of metabolism as great as that of the glands, but their metabolism is capable of much more complete suspension during rest. When the muscles are inactive the glandular structures, in spite of their smaller bulk, account for a very appreciable quantity of the whole metabolism of the body-perhaps as much as half. But when the muscles are exercised to any considerable extent, the contribution of the glands becomes an insignificant item in the metabolism of the body. The muscles, then, by reason of their large mass, and of the great variations of which their metabolism is capable, are essentially the regulators of heat production.

Apart from active contraction, the muscles differ at different times in tonus. This difference finds its metabolic expression. Zuntz, by cutting the nerves of the already resting leg of a dog, abolished the muscular tonus and greatly lessened the metabolism. Alterations in tonus probably play a very important part in the production of heat. Our muscles are "braced" in cold and "slack" in warm climates. The latter effect is very strikingly shown by the extreme muscular flabbiness which evinces itself in such a climate as that of the Red Sea. Where the cold is such that increased tonus proves inadequate to meet the demand for heat, a greater degree of muscular activity (shivering) supervenes unless actual exercise is taken.

Heat Loss.

The two channels of loss susceptible of any amount of variation are the lungs and the skin. The more air that passes in and out 
of the lungs, the greater will be the loss in warming the expired air and in evaporating the water of respiration. In such animals as the dog, which perspire but little, respiration is a most important means of regulating the temperature; and in these animals a close connection is observed between the production of heat and the respiratory activity. The panting of a dog when overheated is a familiar instance of this. A dog also, under the same circumstances, puts out its tongue, and loses heat from the evaporation that occurs from its surface. The great regulator, however, is undoubtedly the skin, and this has a double action. In the first place, it regulates the loss of heat by its vaso-motor mechanism; the more blood passing through the skin, the greater will be the loss of heat by conduction, radiation, and evaporation. Conversely, the loss of heat is diminished by anything that lessens the amount of blood in the skin, such as constriction of the cutaneous vessels, or dilatation of the splanchnic vascular area. In the second place, the special nerves of the sweat-glands are called into action. Familiar instances of the action of these two sets of nerves are the reddening of the skin and sweating that occurs after exercise, on a hot day, or in a hot-air or vapour bath, and the pallor of the skin and absence of sensible perspiration on the application of cold to the body.

The production of perspiration has a cooling effect, since the latent heat necessary for the evaporation of the sweat is derived chiefly from the body.

The relative importance of radiation and evaporation depends very much upon the humidity of the atmosphere. Here it is necessary to distinguish between "relative" and "absolute" humidity. The important point is the amount of water which the air can absorb. Now, cold air, even though it is almost dry, is capable of taking up very little aqueous vapour. Warm air may contain a good deal (i.e. the absolute humidity may be greater than that of the cold) and yet may be far from saturated (i.e. its relative humidity may be low). The loss of heat by evaporation is therefore relatively small in cold weather, even though it be dry. The burden of heat regulation then falls upon radiation, and it, to be efficient, demands a warm skin; hence the glow of heat we experience when we take exercise in still, cold weather.

In hot climates radiation becomes less important, and the possibility of heat loss from the skin therefore depends upon evaporation. Evaporation in its turn depends upon the "relative" humidity of the air and upon the existence of winds.

The loss of heat by evaporation is at its maximum in dry hot climates, and is greatly promoted by the wearing of clothes which are relatively porous. In such climates physical "fitness" is promoted by the taking of a considerable amount of out-of-door exercise. 
Quite otherwise is it in climates like that of the coast-line of British East Africa, where the tropical sun is combined with the moisture-laden wind. There the possibilities of heat loss both by radiation and by evaporation are small, and the English official perforce reduces his heat production to a minimum. He lives indoors, takes as little exercise as possible, and the pallor of his countenance contrasts strongly with the high colour which his colleague in India or Egypt exhibits.

\section{Certain Factors which govern the Relation between Heat Production and Heat Loss.}

(1) Size.-The heat production of the body, other things being equal, depends upon the mass of the body; the heat loss, on the surface. The production therefore varies with the cube of the linear dimensions, whilst the loss of heat only varies with the square.

The smaller the animal the greater must be its heat production relatively to its heat loss. The loss of heat is diminished both by the occurrence of fur and by the absence of sweat in the skins of most small animals, and the smaller the animal the greater is its metabolism per gramme (see also p. 399).

The same is, no doubt, true of individuals; but in this, as in other cases, the natural conditions may be much modified by artificial ones, such as clothing.

(2) Age.-Inasmuch as the young are small, active, and growing, their heat production is relatively large; and further, since the extreme constancy of temperature which an adult man has attained is an evolved characteristic, very young children, in common with animals, are subject to changes of body-temperature which would be of much graver import in older people. Warm-blooded animals in the embryonic stage are practically cold-blooded, the regulatory mechanism which keeps the body-temperature constant not being fully developed at this stage.

(3) Constitution.-Different individuals differ greatly in their power of heat loss. Apart from differences in size and in the faculty of perspiration, there remains such differences as those of compactness of shape, and especially in the amount of adipose tissue with which the viscera are protected.

The Influence of the Central Nervous System on Heat Regulation. -The central nervous system controls the loss of heat directly through the vaso-motor and secretory nerves supplying the skin. That the control of heat production is important, is shown by the effect on the body-temperature of cutting the spinal cord, or of the drug curare. Curare cuts off the muscles from the stimuli which would naturally reach them through the motor nerves. Not only 
does the temperature at once fall, but the animal becomes poikilothermic.

The seat of the heat-regulating mechanism in the brain is a matter of much uncertainty. It is possibly in the basal ganglia of the cerebrum, or in this neighbourhood.

Fever.-Diseases may cause the temperature to vary considerably, especially those which we term febrile.

A mere increase in the production of heat does not necessarily cause fever. The administration of food causes increased combustion in the body; but there is no rise of temperature in health, because pari passu with the increased production there is increased loss of heat. Similarly, diminution in the loss of heat, such as occurs on a hot as compared with a cold day, does not produce fever, because the production of heat within the body is correspondingly diminished. A febrile condition may, however, occur if tight-fitting and otherwise unsuitable clothing which interferes with the proper action of the skin is worn in hot weather; this is the frequent cause of "heatstroke" among soldiers in the tropics.

In fever there is increased production of heat, as is shown by metabolic balance-sheets; the intake of food is usually small, and the discharge of carbon, nitrogen, etc., results mainly from tissue disintegration; this is even greater than in ordinary inanition; the tissues are said to be in a "labile" condition, that is, they are easily broken down. Usually the skin is dry, the sweat-glands, like most of the secretory glands, being comparatively inactive, and so the discharge of heat is lessened. The skin, however, may sometimes be bathed in perspiration and yet high fever be present. The essential cause of the high temperature in fever is neither increased formation nor diminished discharge of heat, but an interference with the mechanism which in health operates so as to equalise the two.

The Action of Drugs. - From what has been said, it will be evident that drugs may reduce fever in more than one way: for instance, they may reduce the metabolism of the muscles, e.g. quinine; they may cause increased heat loss by promoting perspiration and vascular dilatation in the skin, e.g. pilocarpine; or they may act on the central heat-regulating mechanism (corpus striatum ?), e.g. phenacetin. 


\section{CHAPTER XLIV}

\section{THE CENTRAL NEIRVOUS SYSTEM}

THE central nervous system is contained within the cranio-spinal cavity, and consists of brain and spinal cord. These two parts are

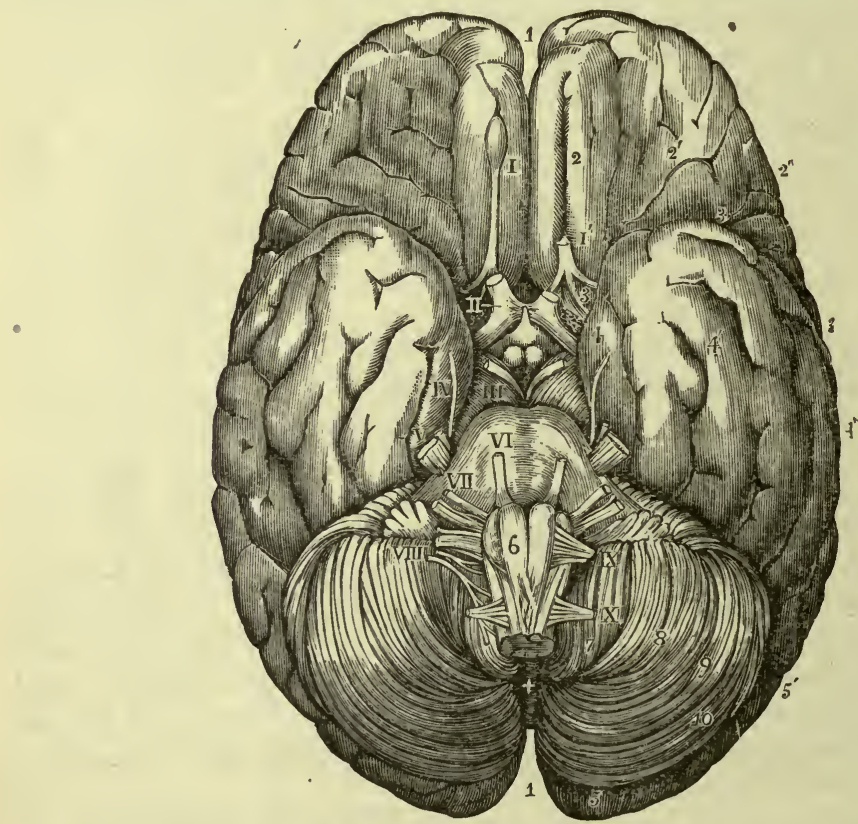

Fro, 395.-Base of the brain. 1, Superior longitudinal fissure; $2,2^{\prime}, 2^{\prime \prime}$, anterior cerebral lobe; 3 , fissure of Sylvius, between anterior and $4,4^{\prime}, 4^{\prime \prime}$, middle cerebral lobe; $5,5^{\prime}$, posterior lobe ; 6 , medulla oblongata; the figure is in the right anterior pyramid; $7,8,9$, 10 , the cerebellum; + , the inferior vermiform process. The figures from I. to IX. are placed against the corresponding cerebral nerves; III. is placed on the right crus cerebri ; VI. and VII. on the pons Varolii ; $\mathbf{X}$. the first cervical or suboccipital nerve. (Allen Thomson.) $\frac{1}{2}$.

continuous with one another, and the line of separation is arbitrarily drawn at the foramen magnum, by which orifice the spinal cord leaves the skull. Both brain and cord are enveloped by three 
connective-tissue membranes, known from without inwards as dura mater, arachnoid, and pia mater respectively.

In Chapter XVI. we have already considered some of the elementary and fundamental problems in relation to the activities of nerve centres, and it would be at this point advisable that the student should refresh his memory on such points by again reading that chapter before he proceeds further.

The next few chapters will deal with that portion of the anatomy of the spinal cord and brain which one must know before it is possible to study profitably the functions of these parts, and we shall start with the spinal cord and reach the cerebrum last.

Before, however, passing on to these details, a few general words are necessary in relation to the construction of the central nervous system in vertebrate animals.

A student's first glance at the human brain, or at such a drawing of it as is given in the accompanying figure (fig. 395), will be sufficient to convince him of its complicated structure. The next figure, however, representing semi-diagrammatically its different parts, will make an enumeration of its subdivisions more intelligible.

At the lowest part of the brain (fig. 396), continuing the spinal cord upwards, is the medulla oblongata or bulb (D). Next comes the

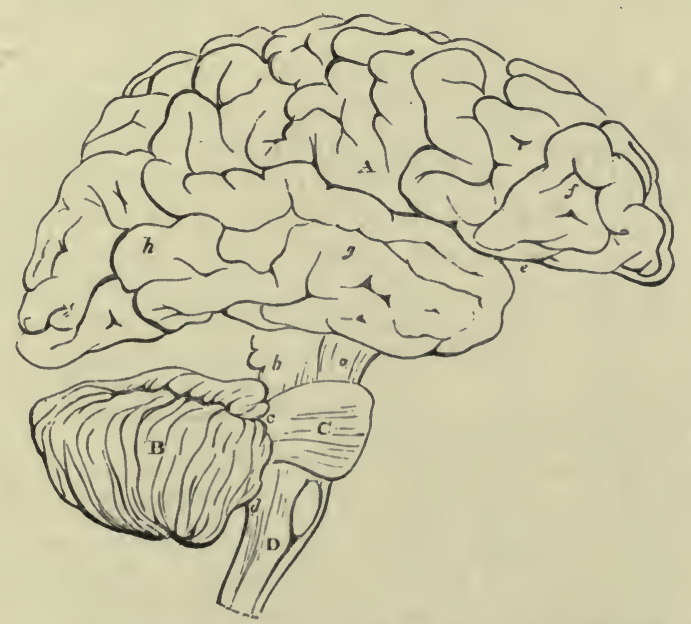

F1G. 396.-Plan in outline of the brain, as seen from the right side. $\frac{1}{2}$. The parts are represented as separated from one another somewhat more than natural, so as to show their connections. A, cerebrum; $f, g, h$, its anterior, middle, and posterior lobes; $e$, fissure of Sylvius; B, cerebellum; $\mathrm{C}$, pons Varolil; $\mathrm{D}$, medulla oblongata; $a$, peduncles of the cerebrum; $b, c, d$, superior, middle, and inferior peduncles of the cerebellum. (From Quain.)

pons Varolii (C), very appropriately called the bridge, because in it are the connections between the bulb and the upper regions of the 
brain, and between the cerebellum or small brain (B), and the rest of the nervous system.

The mid-brain comes next $(a, b)$, and this leads into the peduncles or crura of the cerebrum (A), the largest portion of the brain.

Through the brain runs a cavity filled with cerebro-spinal fluid and lined by ciliated epithelium; this is continuous with the central canal of the spinal cord. In the brain, however, it does not remain a simple canal, but is enlarged at intervals into what are called the ventricles. There is one ventricle in each half or hemisphere of the cerebrum; these are called the lateral ventricles, they open into the third ventricle, which is in the middle line; and then a narrow canal (aqueduct of Sylvius) leads from this through the mid-brain to the fourth ventricle, which is placed on the back of the bulb and pons, which form its floor; its roof is formed partly by the overhanging cerebellum, partly by pia mater. This piece of pia mater is pierced by a hole (Foramen of Magendie), and so the cerebro-spinal fluid in the interior of the cerebro-spinal cavity is continuous with that which bathes the external surface of brain and cord in the sub-arachnoid space. The fourth ventricle leads into the central canal of the spinal cord. Taking origin from the wall of the cerebral ventricles, and running down the central canal of the cord, is a fine thread called Reissner's fibre; the function of this thread is entirely unknown.

Speaking generally, there are two main collections of grey matter-that on the surface, called the cortex, and that in the interior, bordering on the cerebro-spinal cavity, and subdivided into various masses (grey matter of cord, floor of fourth ventricle, corpora striata, optic thalami, etc.), whose closer acquaintance we shall make presently.

But such a complex brain as the human brain does not obtain throughout the vertebrate series. The lower one goes in the scale, the less important and large does the cerebrum become, until in the fishes the cerebral hemispheres are practically absent. It is the large size and convoluted grey cortex of these hemispheres which distinguishes the higher from the lower vertebrates.

A comparative study of the brain in different animals has been most valuable in the elucidation of the functions of its various parts.

It is in fact possible to-day to foretell, if one knows the habits of an animal, what sort of brain it possesses. The converse is also true; given the brain of an animal, one can describe its habits and mode of life very fairly accurately. For instance, animals which rely largely on the sense of smell for their prey will have a large olfactory area; whereas in such animals as the porpoise, which have no sense of smell, the olfactory area of the brain is absent. Animals with keen vision will have a large visual area in their brains ; animals of nocturnal habits, or who live underground in the dark, will have 
a very small one. A highly intellectual man has a more elaborately convoluted cerebrum than a savage.

In spite of these differences, and many more might be mentioned, there is throughout the vertebrate series from fish up to man, the same general plan of construction; and the brain of the human embryo is very much like the adult condition of the brain of the fish.

In the fotus the central nervous system is formed by an infolding of a portion of the surface epiblast. This becomes a tube of nervous matter, which loses all connection with the surface of the body, though later in life this is in a sense re-established by the nerves that grow from the brain and cord to the surface. The anterior end of this tube becomes greatly thickened, to form the brain, its cavity becoming the cerebral ventricles; the rest of the tube becomes the spinal cord. The primitive brain is at first subdivided into three parts, the primary cerebral vesicles; the first and third of these again subdivide, so that there are ultimately fivg divisions, which have received the following names :-

1. Pros-encephalon, telencephalon or forebrain. This is developed into the cerebrum with the corpora striata. It encloses the lateral ventricles.

2. Thalam-encephalon, diencephalon, or twixt brain. This is developed into the parts including the optic thalami, which enclose the third ventricle.

3. Mes-encephalon, or mid-brain, consists of the parts which enclose the aqueduct of Sylvius-namely, the corpora quadrigemina, which form its dorsal, and the crura cerebri, which form its ventral aspect. The corpora quadrigemina in many lower animals instead of being four in number are two, and are called the optic lobes.

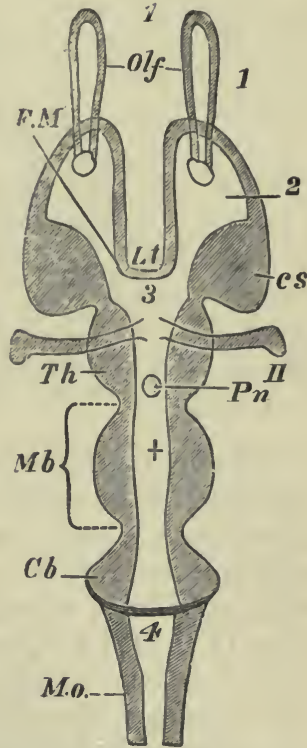

FIG. 397.-Diagrammatic horizontal section of a vertebrate brain. The figures serve both for this and the next diagram. $M b$, mid-brain : what lies in front of this is the fore-, and what lies behind, the hind. brain; $L t$, lamina terminalis ; Olf, olfactory lobes; $H m p$, hemispheres; $T h$. $E$, thalam encephalon; $P n$, pineal gland; $P y$, pltuitary body F.M., foramen of Munro; $c s$, corpus striatum; Th, optic thalamus ; $C C$, crura cerebri : the mass lying above the cana. represents the corpora quad. rigemina ; $\mathrm{Cb}$, cerebellum ; Mi.o., medulla oblongata; $I-I X$, nine pairs of crania nerves; 1 , olfactory ventricle; 2, lateral ventricle; 3 , third ventricle; 4 , fourth ventricle; +, iter a tertio ad quartum ventriculum, or aqueduct of Sylvius.

(Huxley.)

4. Met-encephalon, or hind-brain, which forms the cerebellum and pons.

5. Myel-encephalon, or after-brain, which forms the bulb or medulla oblongata. 
Figs. 397 and 398 represent a diagrammatic view of a vertebrate brain; the attachments of the pineal gland, pituitary body, and olfactory (I) and optic (II) outgrowths are also shown.

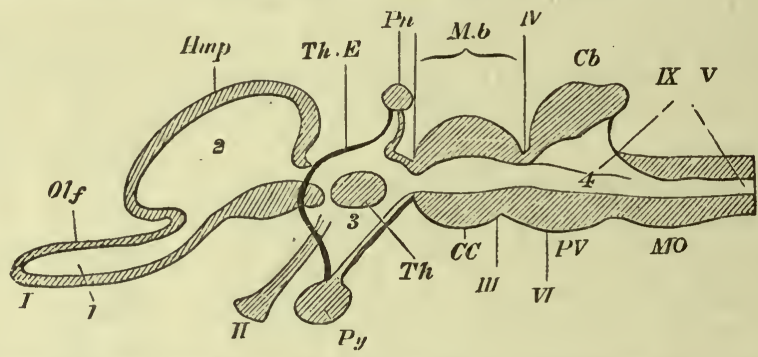

FIG. 398.-Longitudinal and vertical diagrammatic section of a vertebrate brain. I tetters as before. $P V$, pons Varolii. Lamina terminalis is represented by the strong black line joining $P n$ and $P y$. (Huxley.)

These diagrams might serve very well for the brain of an adult selachian fish, such as a shark. The olfactory bulb is, however, very much larger, and the fore-brain smaller. In the shark, smell is the all-important sense; the olfactory nerves, which originate from the olfactory bulb, spread out over an immense area many square feet in size (12 to 13 square feet in a shark 25 feet long). Behind the olfactory bulb is another focus of grey matter, called by Edinger the parolfactory lobe, connected to the fifth nerve, the sensory nerve of the mouth. No doubt the oral sense, as it is termed, is important in the pursuit and capture of prey; it always is in animals who catch food with the mouth. One sees it highly developed in animals with prehensile tongues, and bristles or whiskers on the lips; also in birds, with their sensitive beaks and bills.

Returning, however, to the shark, we find the cerebellum is large, as it is in all powerful swimmers and flyers, but the cerebrum in the strict sense is absent; there are no hemispheres and no grey cortex; the fore-brain consists of little else but the corpora striata.

The cerebral hemispheres are later growths superimposed upon this primitive brain, and in the animal series one notes the progressive development of the cerebrum in relation to function and adaptation to environment.

The primitive brain as exemplified in that of the shark is common to all animals up to man, and is termed by Edinger the Palaencephalon or old brain. The cerebral hemispheres constitute what he terms the Neo-encephalon or new brain.

The neo-encephalon is specially characterised by the possession of a grey cortex, and this is the seat of the psychical or mental processes termed volition and sensation. The first part of the cortex to appear in development is called the archipallium (or old cortex), 
and this is associated with smell, the most important sense in the old primitive brain. The rest of the cortex is termed the neopallium (or new cortex); it subserves the functions of hearing, vision, touch, and the muscular sense, and is also concerned in the origination of those volitions which result in movements initiated and guided by those senses. The progressive development of the neopallium is especially marked in the primates and in man, for in these the more primitive olfactory and oral senses are unimportant in the struggle for existence; in man the receptive olfactory membrane in the nose measures considerably less than a square inch instead of the many square feet it extends over in the shark; but the visual and other faculties enumerated become preponderatingly important as associative memory develops and the brain becomes the organ of mind. The structure of the neopallium is more elaborate than that of the archipallium.

The following table will make the relationship of these parts clear:-

\section{The Vertebrate Brain} consists of

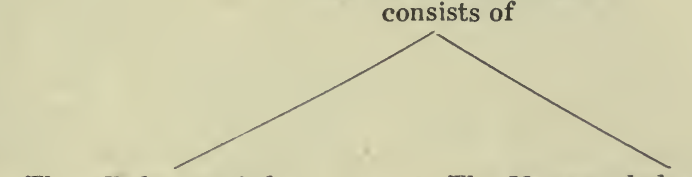

1. The Pala-encephalon, or old primitive brain.

2. The Neo-encephalon, or new brain. This consists of the cerebral cortex (with its efferent and afferent fibres)and may besubdivided into

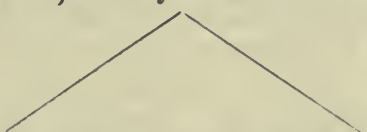

a. The Archipallium, or old cortex. b. The Neopallium, or new cortex. 


\section{CHAPTER XLV}

\section{STRUCTURE OF THE SPINAL CORD}

THE spinal cord is a column of nerve-substance connected above with the brain through the medium of the bulb, and situated in the spinal canal. In transverse section it is approximately circular, but the cord is not of the same size throughout its course. It exhibits two enlargements, one in the cervical, the other in the lumbar region. These are the situations whence the large nerves for the supply of the limbs issue. The cord ends below, about the lower border of the first lumbar vertebra, in a cone-shaped termination (the conus medullaris) from which passes a slender filament of grey substance, the filum terminale, which lies in the midst of the roots of many nerves forming the cauda equina.

It is composed of grey and white matter; the white matter is situated externally, and constitutes its chief portion; the grey matter is in the interior, and is so arranged that in a transverse section of the cord it appears like two crescentic masses (the horns of each of which are called respectively the anterior and posterior cornua) connected together by a narrower portion or isthmus, called the posterior commissure (fig. 399). Passing through the centre of this isthmus in a longitudinal direction is a minute canal; in a transverse section it appears as a hole; this central canal of the spinal cord is continued throughout its entire length, and opens above into the space at the back of the medulla oblongata and pons Varolii, called the fourth ventricle.

The spinal cord consists of two symmetrical halves, separated anteriorly and posteriorly by vertical fissures (the posterior fissure being deeper, but less wide and distinct than the anterior), and united in the middle by nervous matter which is usually described as forming two commissures-an anterior commissure (consisting of medullated nerve-fibres) in front of the posterior commissure, which is the isthmus of grey matter pierced by the central canal, to which we referred in the last paragraph (fig. 399, B). Each half of the spinal cord is marked on the sides (obscurely at the lower part, but dis- 
tinctly above) by two longitudinal furrows, which divide it into three portions, columns, or tracts, an anterior, lateral, and posterior. From the groove between the anterior and lateral columns spring the anterior roots of the spinal nerves (fig. 399, B and $\mathrm{C}, 5$ ); and just in front of the groove between the lateral and posterior column the
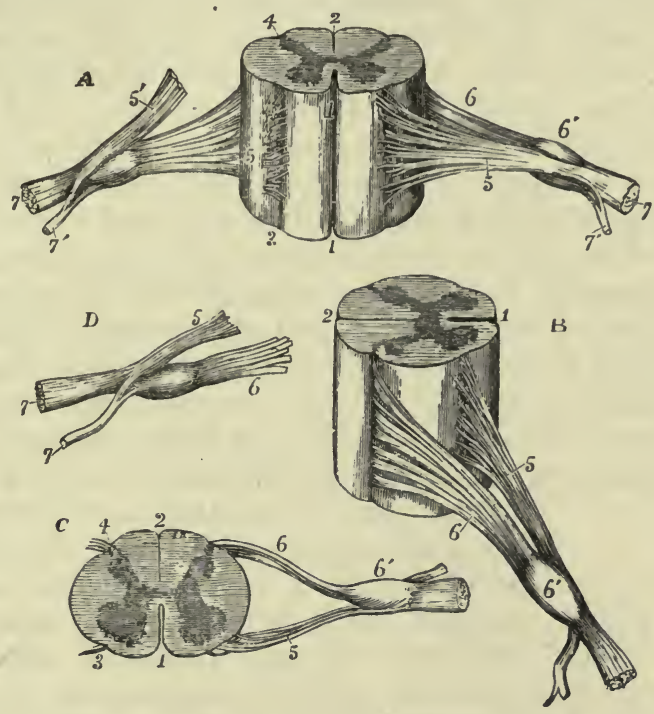

Fia. 399.-Different views of a portion of the spinal cord from the cervical region, with the roots of the nerves (slightly enlarged). In $\mathbf{A}$, the anterior surface of the specimen is shown ; the anterior nerveroot of its right side is divided; in $\mathrm{B}$, a view of the right side is given; in $\mathrm{C}$, the upper surface is shown; in $\mathrm{D}$, the nerve-roots and ganglion are shown from below. 1 , the anterior median fissure; 2, postérior median fissure; 3, anterior lateral depression, from which the anterior nerve-roots are seen to issue; 4, posterior lateral groove, into which the posterior roots are seen to sink; 5 , anterior roots passing the ganglion ; $5^{\prime}$, in $\Lambda$, the anterior root divided; 6 , the posterior roots, the tibres of which pass into the ganglion $6^{\prime} ; 7$, the united or compound nerve; $7^{\prime}$, the posterior primary branch, seen in $A$ and $D$ to be derived in part from the anterior and in part from the posterior root. (Allen Thomson.)

posterior roots enter $(\mathrm{B}, 6)$ : a pair of roots on each side corresponds to each vertebra.

White matter. - The white matter of the cord is made up of medullated nerve-fibres, of different sizes, running mainly in a longitudinal direction, and of a supporting material of two kinds, viz.:-(a) ordinary fibrous connective tissue with elastic fibres, which is connected with septa from the pia mater which pass into the cord to carry the blood-vessels. (b) Neuroglia; the processes of the neuroglia-cells are arranged so as to support the nerve-fibres, which are without the usual neurilemmal nerve-sheaths.

The general rule respecting the size of different parts of the cord is, that each part is in direct proportion to the size and number of nerve-roots given off from it. Thus the cord is very large in the 
middle and lower part of its cervical portion, whence arise the large nerve-roots for the formation of the brachial plaxuses and the supply of the upper extremities; it again enlarges at the lowest part of its dorsal portion and the upper part of its lumbar, at the origins of the large nerves which, after forming the lumbar and sacral plexuses, are distributed to the lower extremities. The chief cause of the greater size at these parts of the spinal cord is increase in the quantity of grey matter; the white part of the cord (especially the lateral columns) becomes gradually and progressively smaller from above downwards, because a certain number of fibres coming down from the brain pass into the spinal grey matter at different levels.

Grey matter.- The grey matter of the cord consists of nervefibres, most of which are very fine and delicate, of nerve-cells with branching processes, and of an extremely delicate network of the primitive fibrillæ of axis-cylinders and of dendrites. This fine plexus is called Gerlach's network, and is mingled with the meshes of neuroglia. The neuroglia of the grey matter resembles that of the white, but instead of everywhere forming a close network to support the nerve-fibres, here and there it is in the form of a more open

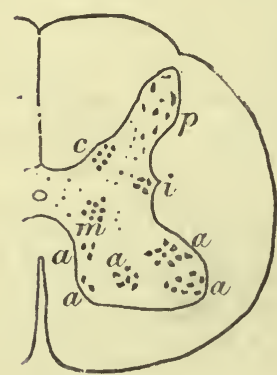

FIG. 400.-Section of half the spinal cord to show the principal groups of cells in the grey matter; $a$, groups of cells in the anterior horn ; c, Clarke's column: $i$, intermedio-lateral group; $m$, middle cell column; $p$, scattered cells of the pos. terior horn. (Diagrammatic after Schäfer.) sponge-work to support the nerve-cells. It is especially developed around the central canal, which is lined with columnar ciliated epithelium, the cells of which at their outer ends terminate in fine processes, which join the neuroglia network surrounding the canal, and form the substantia gelatinosa centralis. It is also developed at the tip of the posterior cornu of grey matter, forming what is known as the substantia gelatinosa lateralis of Rolando, which is much enlarged in the upper cervical region.

Groups of cells in the grey matter.-The multipolar cells of the grey matter are either scattered singly or arranged in definite groups (see fig. 400).

(1) Anterior horn cells.-In the cervical and lumbar enlargements there are several groups of large multipolar cells in the anterior horn; in the thoracic region these are reduced to two, a mesial and a lateral group. The larger groups correspond with segments of the limbs, and in the cervical cord there is one special group from which the phrenic nerve arises for the supply of the diaphragm. The axons pass out by the anterior nerve-roots of the same side, but a few axons pass to the antero-lateral column of the same side, and by the white commissure to that of the opposite side. In birds, a few axons are stated to pass to the posterior roots. 
(2) Posterior vesicular column of Lockhart Clarke; generally known as Clarke's column.- This is a group of large nerve-cells with their long axis vertical. It lies at the base of the posterior horn, and is best marked in the thoracic region. The axons of these cells pass into the cerebellar tracts.

(3) Intermedio-lateral group.- This is seen in the outer part of the grey matter of the lateral horn, and is most distinct in the upper thoracic and lower cervical regions.

(4) The middle cell column lies in the middle of the crescent.

(5) The cells of the posterior horn are usually small; they are numerous, but are not disposed in special groups.

Columns and tracts in the white matter of the spinal cord.-The columns of the white matter which are marked out by the points from which the nerve-roots issue, are called the anterior, the lateral, and the posterior columns; the posterior is further divided by a septum of the pia mater into two almost equal parts, constituting the postero-external column, or column of Burdach, and the posteromedian, or column of Goll (fig. 402). In addition to these columns, however, it has been shown that the white matter can be still further subdivided. These tracts in the white matter perform different functions in the conduction of impulses.

These tracts have been made out by the following methods:-

(a) The embryological method. It has been found by examining the spinal cord at different stages of its development that certain groups of the nerve-fibres put on their myelin sheath at earlier periods than others, and so the different groups of fibres can be easily distinguished. This is also known as the method of Flechsig.

(b) Wallerian or degeneration method.-This method depends upon the fact that if a nerve-fibre is separated from its nerve-cell, it wastes or degenerates. It consists in tracing the course of tracts of degenerated fibres, which result from an injury to any part of the central nervous system. When fibres degenerate below a lesion, the tract is said to be of descending degeneration, and when the fibres degenerate in the opposite direction, the tract is one of ascending degeneration. By the modern methods employed in staining the central nervous system, it has proved comparatively easy to distinguish degenerated parts in sections of the cord and of other portions of the central nervous system. Degenerated fibres have a different staining reaction when the sections are stained by what are called Weigert's and Pal's methods; this consists in subjecting them to a special solution of hæmatoxylin, and then to certain differentiating solutions. The degenerated fibres appear light yellow, whereas the healthy fibres are a deep blue. Marchi's method is even better. After hardening in Müller's fluid, Marchi's solution (a mixture of Müller's fluid and 
osmic acid) stains degenerated fibres black, and leaves the rest of the tissue unstained. Accidents to the central nervous system in man have given us much information upon this subject, but this has of late years been supplemented and largely extended by experiments on animals, particularly upon monkeys; and considerable light has been shed upon the conduction of impulses to and from the nervous system by the study of the results of section of different parts of the central nervous system, and of the spinal nerve-roots.

By these methods the tracts in the white matter have now been mapped out, and the principal ones are shown in the succeeding diagrams.

It will be convenient to begin by considering the result of cutting through the roots of the spinal nerves.

Cutting the anterior roots produces chromatolysis of the cells of the anterior horn from which they originate; this slow atrophy is the result of disuse of the axons which are cut and still remain attached to the cell-bodies. Wallerian degeneration is limited to the motor nervefibres on the distal side of the point of section. The fact that chromatolysis (see p. 193) occurs when the axon of a nerve-cell is cut through, furnishes us with a valuable method of ascertaining from what nerve-cells various tracts originate.

Cutting the posterior roots between the spinal ganglia and the cord leaves the peripheral part of the nerve healthy, and degeneration occurs in the portion of the root which runs into the cord, because the fibres are cut off from the cells of the spinal ganglion from which they grew. These degenerated nerve-fibres may be traced up the cord for a considerable distance. Each posterior root-fibre when it enters the cord bifurcates, the main branch passing upwards, and the shorter branch downwards, so that the degeneration is seen in a small tract called the comma tract (fig. 403) immediately below the point of entrance of the cut posterior root. The upgoing fibre is contained in the posterior column of white matter, and it terminates in the grey matter either in the cord itself at a higher level, or in the medulla oblongata.

Fig. 401 represents in a schematic way the manner in which the fibres of the two roots of a spinal nerve are connected to the grey matter in the cord.

$1,2,3,4$ represent four cells of the anterior horn. Each gives rise to an axis-cylinder process $A$, one of which is shown terminating in its final ramification in the end-plate of a muscular fibre M. Each of these four cells is further surrounded by an arborisation (synapse) derived from the fibres of the pyramidal tract $\mathrm{P}$, which comes down from the brain. The pyramidal fibres really terminate around the cells at the base of the posterior horn; these cells therefore act as intermediate cell-stations on the way to those in the anterior horn. 
These are omitted from the diagram to avoid confusion (se日, however, fig. 185, p. 190).

A fibre of the posterior root is also shown; this originates from the cell $G$ of a spinal ganglion; the process of this cell bifurcates, one branch (B) passing to the periphery, where it ends in an arborescence in the skin (S); the arrow by the side of this branch represents the direction of conduction of the sensory impulses from the skin. An arrow in the opposite direction would indicate the direction of its growth. The other branch (C) passes into the spinal

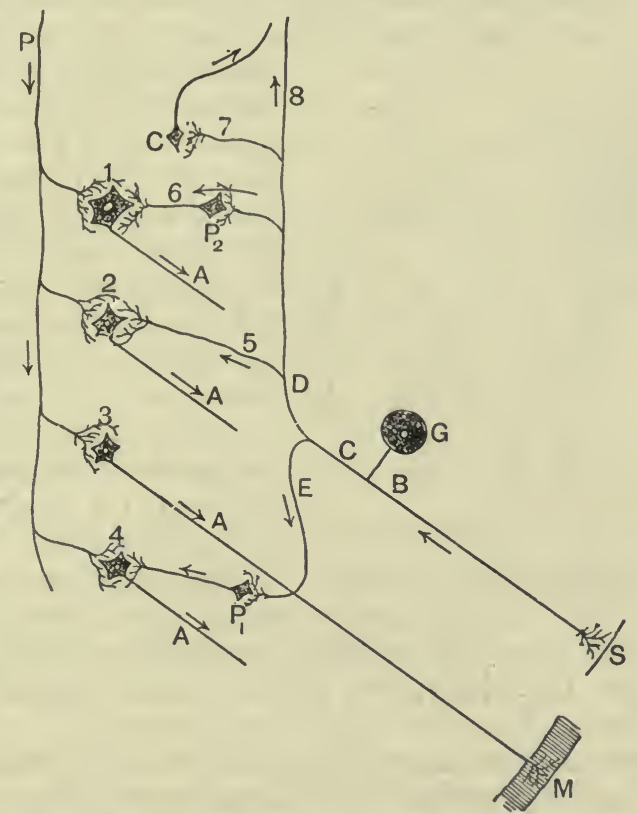

Fig. 401.-Course of nerve-fibres in spinal cord. (After Schäfer.)

cord, where it again bifurcates; the branch $\mathrm{E}$, a short one, passes downwards and ends in an arborisation around one of the small cells $\left(\mathrm{P}_{1}\right)$ of the posterior cornu; from which a new axis-cylinder arises, and terminates around one or more of the multipolar cells (4) of the anterior horn.

The main division $\mathrm{D}$ travels up in the posterior column of the cord, and ends in grey matter at various levels. Some collaterals (5) possibly terminate by arborising directly around the anterior cornual cells, principally of the same side; others (6) do so with an intermediate cell-station in a posterior cornual cell $\left(\mathrm{P}_{2}\right)$; others $(7)$ arborise around the cells of Clarke's column (C) in the thoracic region of the cord, 
and from these cells fresh axis-cylinders carry up the impulse to the cerebellum in what are called the cerebellar tracts, while the main fibre (8) may terminate in any of these ways at a higher level in the cord, or above the cord in the medulla oblongata. A certain number of posterior root-fibres, however, cross the middle line and pursue their way up to the bulb in the ascending tracts of the opposite side of the cord.

In general terms the anterior root-fibres pass out of the grey matter of the anterior horns, and after a short course leave the spinal cord in the anterior spinal nerve-roots. The posterior roots, on the other hand, do not pass to any great extent into the grey matter

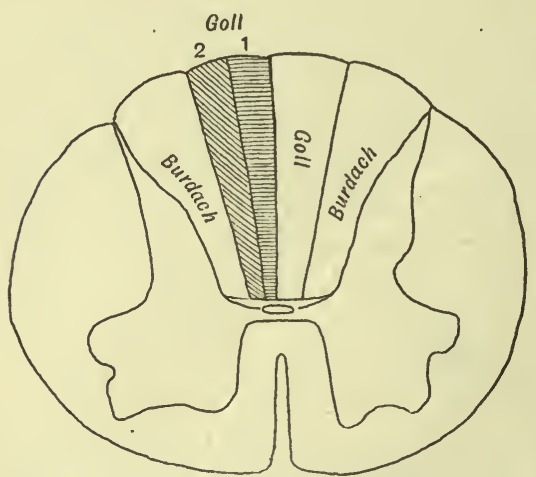

F1a. 402.-Degeneration in column of Goll after section of posterior nerve-roots. immediately, but into the white matter on the inner side of the posterior horn; in other words, they go into the column of Burdach (fig. 402); they pass up in this column, but gradually approach the middle line, and are continued upwards to the medulla in the column of Goll; but as they go up they become less numerous, as some terminate in the grey matter of the cord on the way in the manner described. A few fibres of the posterior root, however, travel for a short distance in a small tract on the outer side of the posterior horn; this is called the tract of Lissauer (fig. 404); the comma tract (fig. 403) has been already explained.

Suppose now one cuts through several posterior roots between the spinal ganglia and the cord, so that the course of degeneration may be more readily traced. Immediately below the points of entrance of these nerve-roots, the comma tract will be found degenerated; immediately above, the degenerated fibres will be found in the column of Burdach; higher up in the cord they will be less numerous, and have approached the middle line; the fibres which enter the cord lowest get ultimately nearest the middle line, so that the greater part of the column of Goll is made up of sensory fibres from the legs; the fibres which enter the cord last, for instance those from the upper limbs and neck, pursue their upward course in the column of Burdach.

The preceding figure (fig. 402) shows the degeneration in a section of the spinal cord, after the division of a number of nerve-roots on one side. The microscopic section is taken high up, so that all the degenerated fibres have passed into the column of Goll on the same 
side; the inner set (1) are shaded differently from the outer set (2), indicating that those nearest the middle line come from the lowest nerve-roots. Those which cross to the opposite side soon after entrance into the cord, are not shown; they will be found forming a scattered degeneration in the ascending tracts of the other side.

We may pass from this to consider the tracts of degeneration that occur when the spinal cord is cut right across in the thoracic region. Some tracts will be found degenerated in the piece of cord below the lesion; these consist of nerve-fibres that are connected with the nerve-cells in the brain; the principal ones are the pyramidal tracts. Other tracts are found degenerated in the piece of cord above the lesion; these consist of nerve-fibres that are connected with the nerve-cells of the spinal ganglia, or with the cells of the spinal cord itself below the lesion, and are passing upwards.

In general terms we may say that the tracts which degenerate downwards are the motor tracts, and those which degenerate upwards are the afferent or sensory channels. We must also take into account groups of association fibres which unite together different regions of the cord; these are generally short tracts in which, therefore, degeneration can only be traced a short distance up or down. The long tracts are those which connect cord or spinal nerves with brain, such as those of Goll and Burdach just mentioned, or the pyramidal tracts the main efferent pathways.

Tracts of Descending Degeneration (fig. 403).

(1.) The crossed pyramidal tract.-This is situated in the lateral column on the outer side of the posterior cornu of grey matter. At the lower part of the spinal cord it extends to the margin, but higher up it becomes displaced from this position by the interpolation of another tract of fibres, to be presently described, viz., the direct cerebellar tract. The crossed pyramidal tract is large, and may touch the grey matter at the tip of the posterior cornu, but is separated from it elsewhere. Its shape on cross-section is somewhat like a lens, but varies in different regions of the cord, and diminishes in size from the cervical region downwards, its fibres passing off as they descend, to arborise around the nerve-cells in the grey matter of the cord. The fibres of which this tract is composed are moderately large, but are mixed with some that are smaller.

(2.) The direct or uncrossed pyramidal tract, or column of Türck.This is situated in the anterior column by the side of the anterior fissure. It ends in the mid or lower thoracic region of the cord.

The two pyramidal tracts come down from the brain; in the medulla oblongata, the greater number of the pyramidal fibres cross over to the other side of the cord, which they descend; hence the term crossed pyramidal tract; a smaller collection of the pyramidal 
fibres goes straight on, on the same side of the cord, and these cross at different levels in the anterior commissure of the cord lower down; hence the disappearance of the direct pyramidal tract in the lower part of the cord. The fact that the crossed pyramidal tract of one side is the fellow of the direct pyramidal tract of the other side, is indicated in the diagram by the direction of shading (see fig. 403).

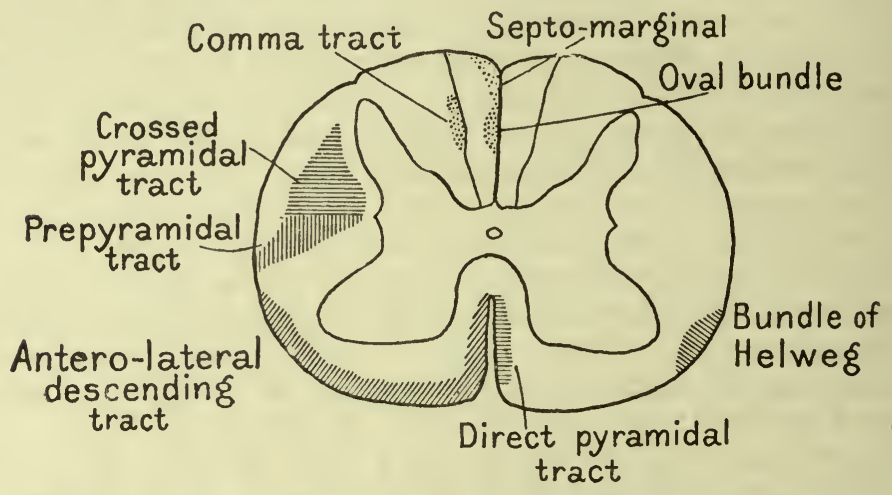

Fig. 403.-Tracts of descending degeneration. For the sake of clearness each is shown on only one side. (After Schäfer.)

Mingled with the fibres of the crossed pyramidal tract are a fow fibres of the pyramid which have not crossed in the medulla oblongata, and are therefore derived from the same side of the cerebrum (uncrossed lateral pyramidal fibres).

The pyramidal fibres are not found at all in vertebrates below the mammals. In the lower mammals they are very few, and in some rodents (rat, mouse, guinea-pig) they are placed in the posterior columns. The direct pyramidal tract is found only in man and the higher apes.

Paralysis resulting from section of the pyramidal tracts is not complete and persistent. There is, therefore, some other path by which volitional impulses arising in the cortex can reach the motor cells of the cord. This alternative path is furnished by the descending fibres of the anterior column, and of the ventral parts of the lateral column, especially in the tract next to be described. Section of this part of the cord produces quite as complete a paralysis as does section of the pyramidal tracts. In vertebrates below mammals this is probably the only path between the higher brain centres and the lower motor centres of the cord (Schäfer).

(3.) Antero-lateral descending tract, or tract of Loewenthal, lies by the side of the anterior median fissure, and extends along the margin of the cord towards the lateral column. These fibres originate from 
the posterior longitudinal bundle of the medulla oblongata; and from other sources to be described later. They end by synapses in the anterior horn.

(4.) The prepyramidal or mubro-spinal tract (Monakow's bundle).This is situated just in front of the crossed pyramidal tract. Its origin is in the cells of the red nucleus in the mid-brain; hence its name, rubro-spinal. Its fibres end by arborisations in the grey matter about the middle of the crescent.

(5.) Bundle of Helweg.-The exact origin and destination of these fibres are not known: they can be traced from the neighbourhood of the olivary body in the medulla oblongata, and pass down in the anterior part of the lateral column in the cervical region.

(6.) Short tracts in the posterior column.-These are (a) the Comma tract; though this degenerates downwards, it is in reality a sensory tract, being composed, as we have already seen, of the branches of the entering posterior root-fibres which pass downwards on entering the cord. It is only found for a comparatively short distance below the actual lesion. (b) Septo-marginal fibres; these are few in number, and are mainly found near the median fissure, where they constitute the oval bundle, and near the posterior surface, where they form the median triangle bundle. These are doubtless short association tracts, and are mixed with others, especially in the ventral part of the posterior column, which have an "ascending" course.

Tracts of Ascending Degeneration (fig. 404).

(1.) Postero-median column, or column of Goll.-This consists of fibres derived from the posterior roots of the sacral, lumbar, and lower

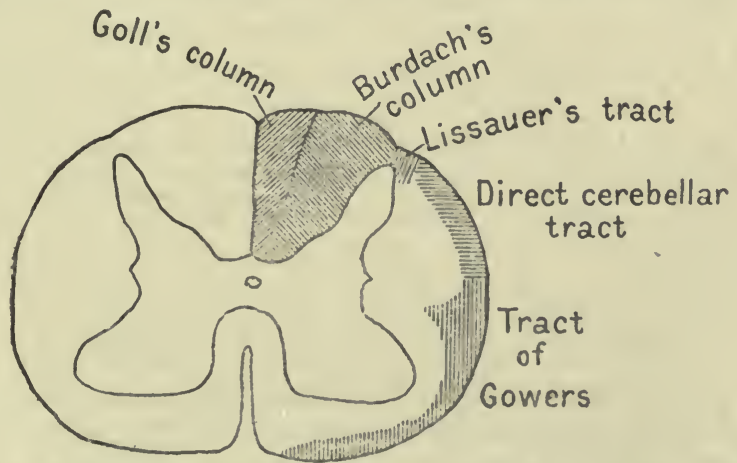

FiG. 404.-Tracts of ascending degeneration, shown on one side of the cord only. (After Schäfer.)

thoracic nerves. These fibres enter the postero-lateral column, and gradually pass towards the mid-line, as already explained. They end in the grey matter of the nucleus gracilis of the bulb. 
(2.) Postero-lateral column, or column of Burdach.-Many of the fibres of this tract, which is also composed of the entering posterior nerve-roots, pass into the grey matter of the cord either immediately on entrance, or in their course upwards. The rest continue upwards to the medulla oblongata, but those from the lower roots pass into the column of Goll, as just stated; those from the upper roots continue to travel upwards in the column of Burdach, and end in the grey matter of the nucleus cuneatus in the medulla oblongata.

(3.) Dorsal or direct cerebellar tract, or tract of Flechsig.-This is found in the cervical and thoracic regions of the cord, and is situated between the crossed pyramidal tract and the margin. It degenerates on injury or section of the cord itself, but not on section of the posterior nerve-roots. In other words, its fibres are endogenous, i.e., arise from cells within the grey matter of the cord; these cells are those of Clarke's column of the same side; the fibres are large ones.

(4.) Ventral cerebellar or antero-lateral ascending tract, or tract of Gowers.-This is situated in front of the crossed pyramidal and direct cerebellar tracts in the lumbar region, while in the thoracic and cervical regions it forms a narrow band at the margin of the cord, curving round even into the anterior column. Its fibres intermingle with those of the antero-lateral descending tract. They originate from the lower cells of Clarke's column of the same side.

Both of these tracts, as their names indicate, go to the cerebellum, and form parts of one and the same system; they connect the cells of Clarke's column of the same side with the cerebellum. In the bulb, the system divides; the greater number of fibres pass to the vermis by the inferior cerebellar peduncle, while a smaller number run up farther, and then return to the vermis by the superior cerebellar peduncle. A certain number of fibres, especially the smaller and more centrally situated ones, end in grey matter in the bulb, pons, and mid-brain.

(5.) Tract of Lissauer, or posterior marginal zone.-This is a small tract of ascending fibres situated at the outer side of the tip of the posterior horn. These are fine fibres from the posterior roots; they subsequently pass into the posterior cornu.

\section{Association fibres in the Spinal Cord.}

Some of the short tracts already alluded to as demonstrable in the spinal cord are bundles of association fibres which connect its different levels together. The main difficulty of investigating them by the degeneration method has arisen from the fact that they are largely intermingled with, and so are hard to distinguish from, the long tracts which connect brain and cord together. In 1853 Pflüger stated that reflex irradiation within the 
spinal cord always takes place in an upward direction, but Sherrington in his work found many exceptions to this rule, and he sought for the paths which are capable of carrying the impulses down the cord by a very ingenious method. The spinal cord of a dog was completely divided across, and the animal was kept alive for a considerable time afterwards; sufficient time was allowed to elapse (roughly about a year) for all traces of the degeneration due to this lesion to have disappeared. The cord is then left, as it were, like a cleaned slate, on which once more a new degeneration can be written without fear of confusion with a previous one. The second degeneration produced by such an operation as hemisection would then affect the intra-spinal fibres only, all the long tracts from brain to cord having been wiped out by the first operation. The complete topography of all these fibres, which are very numerous, has not yet been worked out. The degenerated fibres are scattered throughout the white matter, but are most numerous at the margins of the cord. This is especially true for the longer fibres, and some of them appear to be very long indeed. In the case of the longer fibres there is no evidence of decussation; in the case of the shorter fibres there is some evidence that they in part cross to the other side.

\section{Section of the Spinal Cord.}

Complete transverse section of the spinal cord leads to :-

1. Loss of motion of the parts supplied by the nerves below the section on both sides of the body. The paralysis is not confined to the voluntary muscles, but includes the muscular fibres of the blood-vessels and viscera. Hence there is fall of blood-pressure, paralysis of sphincters, etc., immediately after the operation, but there is considerable recovery of involuntary muscles, as they are supplied by autonomic nerves; any voluntary control over the sphincters is, however, permanently lost.

2. Loss of sensation in the same regions.

3. Degeneration, ascending and descending, on both sides of the cord.

Complete transverse section of the spinal cord may produce immediate death if the operation is performed sufficiently high in the cervical region; for the paralysed muscles will then include those of respiration. The spinal cells from which the phrenic and other respiratory nerves originate are then cut off from the respiratory centre in the bulb above them, and the animal will die of asphyxia. One sees the same thing after severe injury to the upper cervical cord in man, as when he "breaks his neck."

Hemisection. - If the operation performed is not a complete cutting of the spinal cord across transversely, but a cutting of half the cord across, it is termod hemisection, or semi-section. This loads to :- 
1. Loss of motion of the parts supplied by the nerves below the section on the same side of the body as the injury.

2. Loss of sensation in the same region. The loss of sensation is not a very prominent symptom, and is limited to the sense of tactile discrimination and the muscular sense. The animal can still feel

A

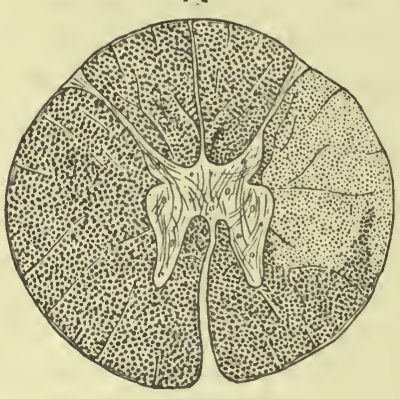

C

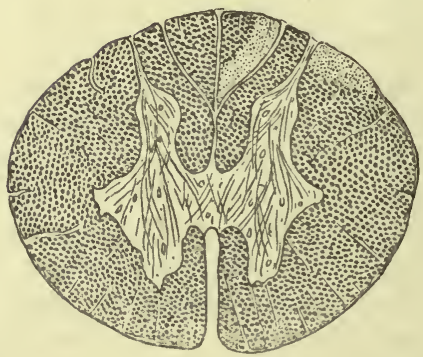

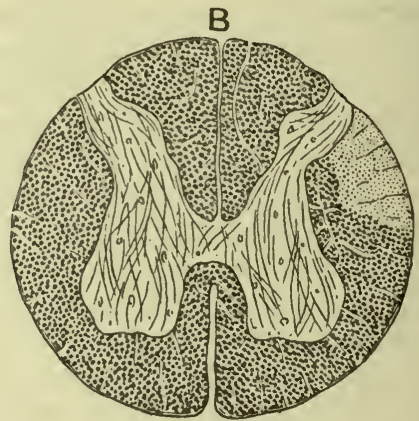

D

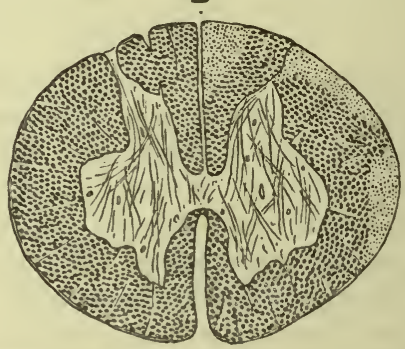

FıG. 405. - The above diagrams are reproductions of photo-micrographs from the spinal cord of a monkey, in which the operation of left hemisection had been performed some weeks previously (Mott). The sections were stained by Weigert's method, by which the grey matter is bleached, while the healthy white matter remains dark blue. The degenerated tracts are also bleached. A is a section of the cord in the thoracic region below the lesion; the crossed pyramidal tract is degenerated. $\mathrm{B}$ is a section lower down in the lumbar enlargement; the degenerated pyramidal tract is now smaller. $\mathrm{C}$ is a section in the thoracic region some little distance above the lesion. The degenerated tracts seen are in the outer part of Goll's column, and in the direct cerebellar tract. D is a section higher up in the cervical region; the degeneration in Goll's column now occupies a median position; the degenerations in the direct cerebellar tract, and in the tract of Gowers, are also well shown. Notice that in all cases the degenerated tracts are on the same side as the injury.

sensations of pain and of heat and cold. This is more fully explained in Chapter XIIX.

3. Degeneration, ascending and descending, largely confined to the same side of the cord as the injury. The most important of these are shown in the accompanying diagrams (fig. 405), the small text beneath which should be carefully studied. 


\section{CHAPTER XLVI}

STRUCTURE OF THE BULB, PONS, AND MID-BRAIN

WE may study the bulb and pons by examining first the anterior or ventral, then the posterior or dorsal aspect, and last of all the interior.

\section{Anterior Aspect.}

The bulb is seen to be shaped, like an inverted truncated cone, larger than the spinal cord, and enlarging as it goes up until it terminates in the still larger pons (fig. $406, p$ ). In the middle line is a groove, which is a continuation upwards of the anterior median fissure of the spinal cord; the columns of the bulb are, speaking roughly, continuations upwards of those of the cord, but there is a considerable rearrangement of the fibres in each. Thus the prominent columns in the middle line, called the pyramids $(\alpha a)$, are composed of the pyramidal fibres, which in the spinal cord are situated principally in the lateral columns of the opposite side (crossed pyramidal tracts). The decussation or crossing of the pyramids (b) occurs at their lower part: a small collection of the pyramidal fibres is, however, continued down the cord in the anterior column of the same side of the cord (direct pyramidal tract): these cross at different levels lower down in the cord.

On the outer side of each pyramid is an oval prominence $(c c)$, which is not represented in the spinal cord at all. These are called the olivary bodies or olives; they "consist of white matter outside, with grey and white matter in their interior.

The restiform bodies at the sides $(d d)$ are the continuation upwards of those fibres from cord and bulb which enter the cerebellum, and the upper part of each restiform body is called the inferior peduncle of the cerebellum.

\section{Posterior Aspect.}

Fig. 407 shows a surface view of the back of the bulb, pons, and mid-brain. Again we recognise some of the parts of the spinal cord 
continued upwards, though generally with new names, and again we see certain new structures.

The posterior median fissure is continued upwards, and on each side of it is the prolongation upwards of the posterior column of

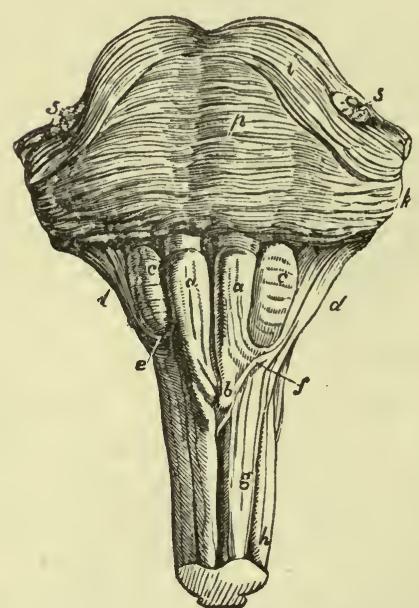

Fia. 406.-Ventral or anterior surface of the pons Varolii, and medulla oblon. gata. $a, a$, Pyramids ; $b$, their decus. sation; $c, c$, olivary bodies; $d, d$, restiform bodies; $e$, arcuate fibres; $f$, fibres passing from the anterior column of the cord to the cerebellum; $g$, anterior column of the spinal cord; $h$, lateral column; $p$, pons Varolii; $i$, its upper fibres; 5,5 , roots of the fifth pair of nerves.

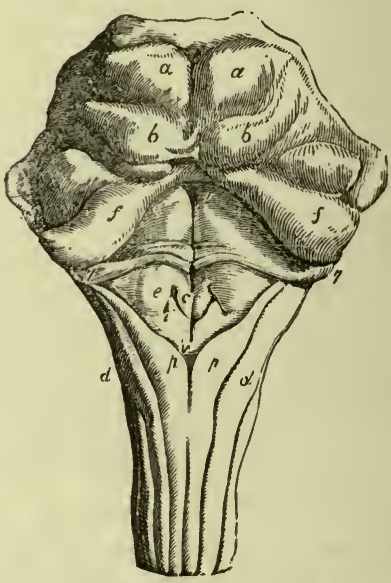

Fig, 407.-Dorsal or posterior surface of the pons Varolii, corpora quad. rigemina, and medulla oblongata. The peduncles of the cerebellum are cut short at the sides. $a, a$, The upper pair of corpora quadri. gemina ; $b, b$, the lower; $f, f$, supe. rior peduncles of the cerebellum; $c$, eminence connected with the nucleus of the hypoglossal nerve: $e$, that of the glosso-pharyngeal nerve; $i$, that of the vagus nerve; $d, d$, restiform bodies; $p, p$, poste. rior columns; $v, v$, groove in the middle of the fourth ventricle, ending below in the calamus scrip. torius; 7,7 , roots of the auditory nerves.

the cord. The column of Goll is now called the Funiculus gracilis, and the column of Burdach the Funiculus cuneatus.

The two funiculi graciles lie at first side by side, but soon diverge and form the two lower boundaries of a diamond-shaped space called the floor of the fourth ventricle; this is made of grey matter; the central canal of the cord gets nearer and nearer to the dorsal surface of the bulb, till at last it opens out on the back of the bulb, and its surrounding grey matter is spread out to form the floor of the fourth ventricle. The two upper boundaries of the diamond-shaped space are made by the superior peduncles of the cerebellum, which contain fibres going up through the mid-brain to the cerebrum. The middle peduncles of the cerebellum are made up of fibres running towards each cerebellar hemisphere from the opposite side of the pons. 
Running down the centre of the floor of the fourth ventricle is a shallow groove; on each side of this is a rounded longitudinal eminonce called the eminentia teres; running across the middle of the floor are a number of fibres (the strice acoustica), which join the auditory nerve.

In the upper part of the diagram, the mid-brain, with the corpora quadrigemina $(a a, b b)$, is shown. Here there is once more a canal which penetrates the substance of the mid-brain, and is called the aqueduct of Sylvius, or the iter a tertio ad quartum ventriculum; it leads, as its name indicates, from the third to the fourth ventricle.

\section{The Internal Structure of the Bulb, Pons, and Mid-Brain.}

The structure of the interior of these parts is complex, and the complexity arises from the circumstance that here we have to deal not only with parts running upwards from cord to brain, or down from brain to cord, but also with a considerable amount of grey matter in which some of the white tracts terminate, or from which new tracts issue. The most important stretch of grey matter is that which appears on the floor of the fourth ventricle, and which is continued upwards around the Sylvian aqueduct, and downwards into the spinal cord; here are situated groups of nerve-cells, which are spoken of as centres, or nuclei. The most important of these are thoss which are connected with the cranial nerves. There are twelve pairs of cranial nerves, and of these the last ten pairs originate from the floor of the fourth ventricle or the neighbouring grey matter.

The following is a list of the cranial nerves:-

1. Olfactory. - This is the nerve of smell.

2. Optic.-This is the nerve of sight.

$\left.\begin{array}{l}\text { 3. Motor oculi } \\ \text { 4. Trochlear }\end{array}\right\}$ These three nerves supply the muscles of the

6. Abducens eyeball.

5. Trigeminal.-This is the great sensory nerve of the face and head. Its smaller motor division supplies the muscles of mastication and a few other muscles also.

7. Facial.-This is mainly the motor nerve of the face muscles.

8. Auditory.-This is divided into two parts, one of which, called the cochlear nerve, is the true nerve of hearing, and is distributed to the cochlea of the internal ear ; the other division, called the vestibular nerve, is distributed to the vestibule and semicircular canals of the internal ear.

9. Glosso-pharyngeal.-This is a mixed nerve; its motor fibres pass to certain of the pharyngeal muscles; its sensory fibres are mainly concerned in the sense of taste. 
10. Vagus or pneumogastric.-This is a nerve with varied efferent and afferent functions; its branches pass to pharynx, larynx, œsophagus, stomach, lungs, heart, intestines, liver and spleen. These functions we have already studied in connection with those organs.

11. Spinal accessory. - The internal branch of this nerve blends with the vagus, and its larger external division supplies the trapezius and the sterno-mastoid muscles.

12. Hypoglossal.-This is the motor nerve to the tongue muscles.

A mere enumeration of the nerves connected to the bulb shows how supremely important this small area of the brain is for carrying on the organic functions of life. It contains centres which regulate deglutition, vomiting, the secretion of saliva, etc., respiration, the heart's movements, and the vaso-motor nerves.

When we further consider that the various centres are connected by groups of association fibres, we at once realise the reason for the complexity of the structures where all this busy traffic takes place.

In the enumeration of the cranial nerves, it will be noticed that many of them are either wholly motor or wholly sensory, and that some of them, like the spinal nerves, have a double function. The motor nerve fibres start as axons from the groups of nerve-cells in the grey matter of this region, just as the motor fibres in the spinal nerves originate from the cells of the spinal grey matter. There is a corresponding resemblance in the origin of the sensory fibres of the cranial and spinal nerves. In the latter, it will be remembered, they originate as outgrowths from the cells of the spinal ganglia, one branch growing to the periphery, and the other to the spinal cord, where it terminates after a more or less extended course by forming synapses with the cells of the grey matter. In the sensory cranial nerves the fibres have a corresponding origin in peripheral ganglia, and those branches which grow towards the bulb terminate by arborising around special groups of cells spoken of as the sensory nuclei.

The following diagram (fig. 408) roughly indicates the position of these nuclei; the motor nuclei are coloured blue, and the sensory red. It must, however, be clearly recognised that while the motor nuclei are true centres of origin, the so-called sensory nuclei are groups of cells around which the entering sensory fibres arborise; these cells do not give origin to the axons of the sensory nerves. After we have studied the internal structure of the bulb we shall be able to return once more to the cranial nerves, in order that we consider their origin and function in greater detail.

But this diagram will give a general idea of the positions of the nuclei. We see that the so-called sensory nuclei (coloured red) are in the minority; they comprise the sensory nucleus of the 
fifth nerve with its long descending root, the nuclei of the eighth nerve (only one of which, VIIIm., is seen in the diagram), and the glosso-pharyngeal and vagal portions of a long strand of nerve-cells

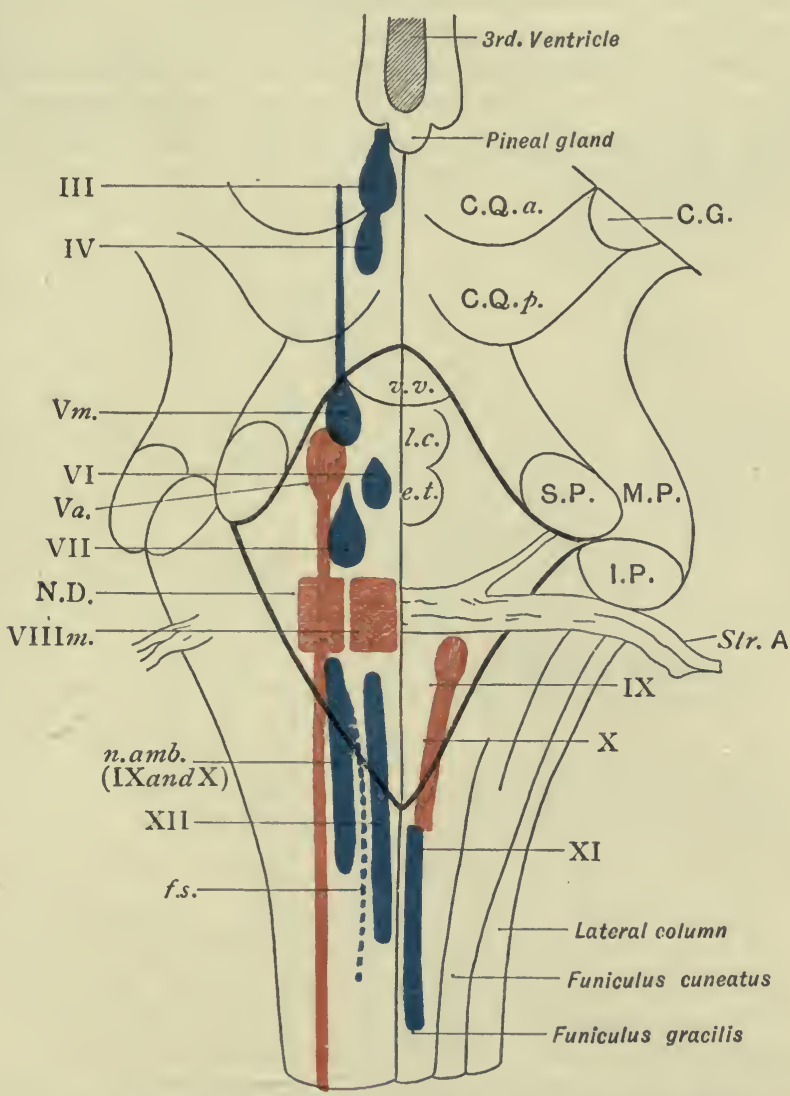

F1G. 408.-Diagram to show the position of the nuclei of the cranial nerves (after Sherrington). The meduila and pons are viewed from the dorsal aspect, the cerebrum and cerebellum haviug been cut away. The nuclei (sensory coloured red, and motor blue) are represented as being seen through transparent material. C.Q. $a_{n}$, anterior corpus quadrigeminum; C.Q. $p_{.,}$, posterior corpus quadri. geminum; C.G., corpus geuiculatum; v.v., value of Vieussens ; l.c., locus coeruleus ; e.t., eminentia teres; str. A., stria acousticæ. S.P., M.P., and I.P., superior middle and inferior cerebellar peduncles respectively cut through. The numerals III. to XII. indicate the nuclei of the respec. tive cranial nerves, all shown on the left side except the accessory-vago-glosso-pharyngeal IX., X., XI., which to avoid confusion is placed on the right side. V $m$. is the motor nucleus of the fifth nerve; $V d$., the sensory nucleus of the same nerve with its long descending root; VIII $m_{0}$, the median nuclens of the auditory nerve; N.D. Nucleus of Deiters; $n$. amb. nucleus ambiguus. The position of the descending root of the ninth and tenth (fasciculus solitarius) is also indicated $(f . s$.$) .$

called the combined nucleus of the ninth, tenth, and eleventh nerves. The remaining nuclei (coloured blue) are efferent, and may be principally arranged into two groups:-(1) the nuclei of the third, 
fourth, sixth, and twelfth nerves, which are close to the middle line; and (2) the motor nucleus of the fifth, the nucleus of the seventh, and the nucleus ambiguus (motor nucleus of the ninth and tenth nerves) which form a line more lateral in position.

It should be added that, except a portion of the optic nerve fibres, a few fibres of the third, and the whole of the fourth nerves, none of the fibres of the cranial nerves cross to the opposite side.

The first two pairs of cranial nerves, the olfactory and the optic, will be studied in connection with smell and vision later on.

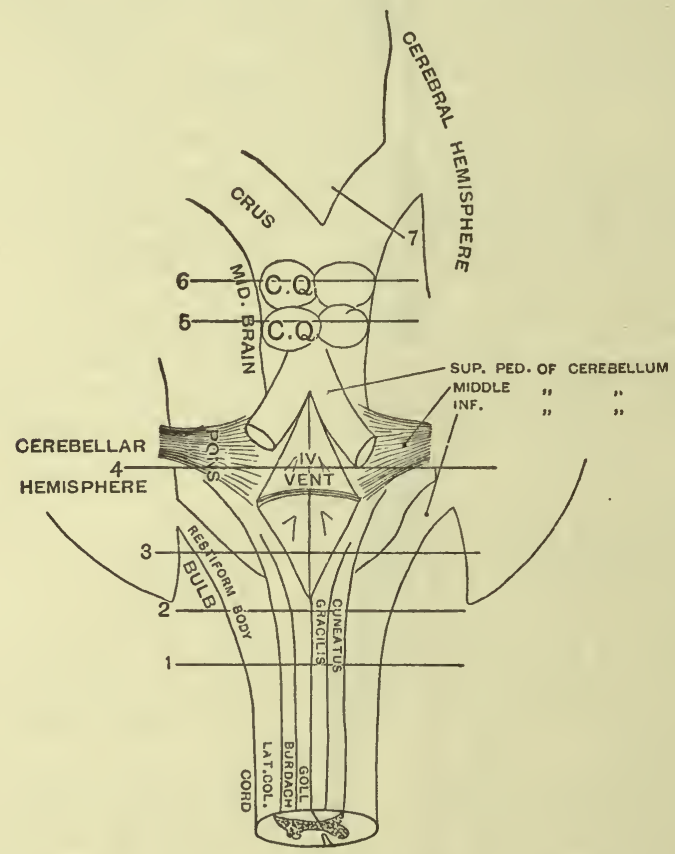

Fig. 409.-Diagrammatic representation of dorsal aspect of medulla, pons, and mid-brain.

We can now pass to the consideration of transverse sections of this part of the central nervous system. We will limit ourselves to seven, the level of which is indicated in the above diagram (fig. 409). The cerebellum has been bisected into two halves and turned outwards, its upper peduncles having been cut through to render the parts more evident. The position of our seven sections is indicated by the transverse lines numbered 1 to 7 .

First section (fig. 410).-This is taken at the lowest level of the bulb, through the region of the decussation of the pyramids. The similarity to the cervical cord will be at once recognised; the 
passage of the pyramidal fibres (P) from the anterior part of the bulb to the crossed pyramidal tract of the opposite side of the cord cuts off the tip of anterior horn (A), which in sections higher up appears as an isolated mass of grey matter, called the lateral nucleus (fig. $411, n l$ ). The $\mathrm{V}$ formed by the two posterior horns is opened out, and thus the grey matter with the central canal is brought nearer to the dorsal aspect of the bulb; the tip of the cornu swells out to form the substantia gelatinosa of Rolando $(\mathrm{R})$, which causes a prominence on the surface called the tubercle of Rolando; $\mathrm{G}$ and $\mathrm{C}$ are the funiculi gracilis and cuneatus respectively, the continuations upwards of the columns of Goll and Burdach.

Many of the fibres of the pyramidal tract terminate in the mid-brain and pons, hence this tract is reduced in size when it reaches the bulb. The pyramidal fibres on their long journey give off collaterals to the cortex cerebri, the basal ganglia of the cerebrum, the substantia nigra of the mid-brain, the nuclei pontis of the pons, and lower down in

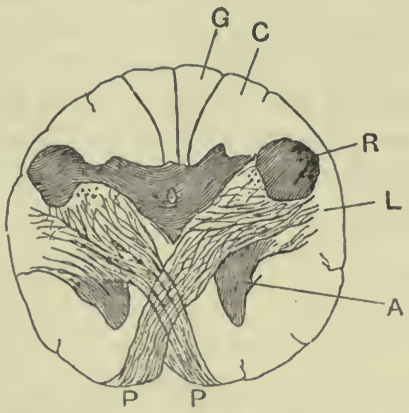

Fic. 410.-Section through the bulb at the level of the decussation of the pyramids. $\mathrm{G}$, funiculus gracilis, continuation of column of Goll; $\mathrm{C}$, funiculus cuneatus, continuation of column of Burdach; $R$, substantia gelatinosa of Rolando, continuation of posterior horn of spinal cord; $\mathbf{L}$, continuation of lateral column of cord; $\mathbf{A}$, remains of part of the anterior horn, separated from the rest of the grey matter by the pyramidal fibres $\vec{P}$, which are crossing from the pyramid of the medulla to the posterior part of the lateral column of the opposite side of the cord.

(After L. Clarke.) the cord to the base of its posterior horn. They, however, do not give off collaterals to the motor nuclei of the cranial nerves on their passage through the bulb (Schäfer). The only collaterals given off in this region are a few to the olivary nuclei.

Second section (fig. 411). - This is taken through the upper part of the decussation. Beginning in the middle line at the top of the diagram, we see first the posterior median fissure (p.m.f.), below which is the grey matter enclosing the central canal (c.c.), and containing the nuclei of the eleventh and twelfth nerves; the funiculus gracilis (f.g.) comes next, and then the funiculus cuneatus (f.c.); these two funiculi have now grey matter in their interior: these masses of grey matter are called respectively nucleus gracilis (n.g.) and nucleus cuneatus (n.c.); the fibres which have ascended the posterior columns of the cord terminate by arborising around the cells of this grey matter; the fibres from the lower part of the body end in the nucleus gracilis, and those from the upper part of the body in the nucleus cuneatus. These nuclei form a most important position of relay in the course of the afferent fibres from cord to brain. The new fibres (the second relay of the sensory spinal path) arising from the cells of these nuclei pass in a number of different directions, and break up the rest of the grey matter into what is called the formatio reticularis (f.r.) 
The nucleus gracilis and nucleus cuneatus are often spoken of as the posterior column nuclei; they do not receive all the ascending branches of the posterior root fibres, for a number of these branches have already entered the grey matter and arborised amongst its cells in the spinal cord itself. The cells of the posterior column nuclei are of moderate size, and their axons pass as internal arcuate fibres into the reticular formation between the two olivary bodies, which is known as the inter-olivary layer. They cross the median raphe dorsal to the pyramids, and then turn upwards towards the upper

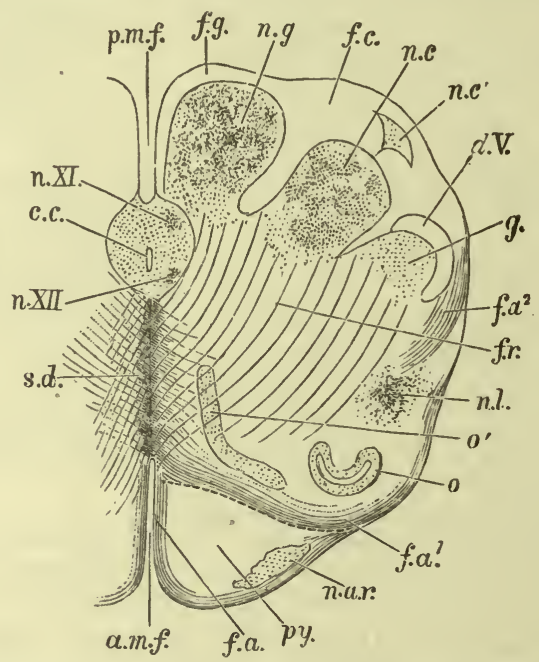

FIG. 411.- Transverse section of the medulla oblongata in the region of the superior decussation. a.m.f., Anterior median fissure; f.a., superficial arcuate fibres; $p y$. , pyramid; n.a.r., nuclei of arcuate fibres; f. $a^{1}$, deep arcuate fibres becoming superficial; 0 , $o^{\prime}$, lower end of olivary nucleus; $n . l$. , nucleus lateralis ; $f . r$., formatio reticularis; $f . a^{2}$, arcuate fibres proceeding from the formatio reticularis; $g$, substantia gelatinosa of Rolando; d.V., descending root of fifth nerve; f.c., funiculus cuneatus; n.c., nucleus cuneatus; n.c.', external cuneate nucleus; n.g., nucleus gracilis; f.g., funiculus gracilis; p.m.f., posterior median fissure; c.c., central canal surrounded by grey matter, in which are $n . X I$. , nucleus of the eleventh and $n . X I I$. , nucleus of the twelfth nerve; s.d., superior decussation (decussation of fillet). (Modified from Schwalbe.)

parts of the brain, and so constitute what is known as the fillet. In the higher parts of the bulb and pons, this tract is reinforced by fibres from the cells of the sensory nuclei of the cranial nerves. The fillet becomes a longitudinal bundle, which passes upwards to the optic thalamus, which forms the next cell-station on the path of the sensory impulses to the cortex.

Other points to be noticed in the section are the substantia gelatinosa of Rolando $(g)$ (representing the tip of the posterior cornu of the cord), now separated from the surface by the descending root of the fifth nerve $(\bar{d} . V$.) ; the lateral nucleus (n.l.) (remains of the 
anterior cornu of the cord); the lower part of the grey matter of the olivary body $\left(o, o^{\prime}\right)$, and most anteriorly the pyramid (py).

Third section.-This (fig. 412) is taken at about the middle of the olivary body, and passes also through the lower part of the floor of the fourth ventricle. The central canal has now opened out into the fourth ventricle, and the grey matter on its floor contains the nuclei of the twelfth and tenth nerves; bundles of the fibres of these nerves course through the substance of the bulb, leaving it at the places indicated in the diagram.

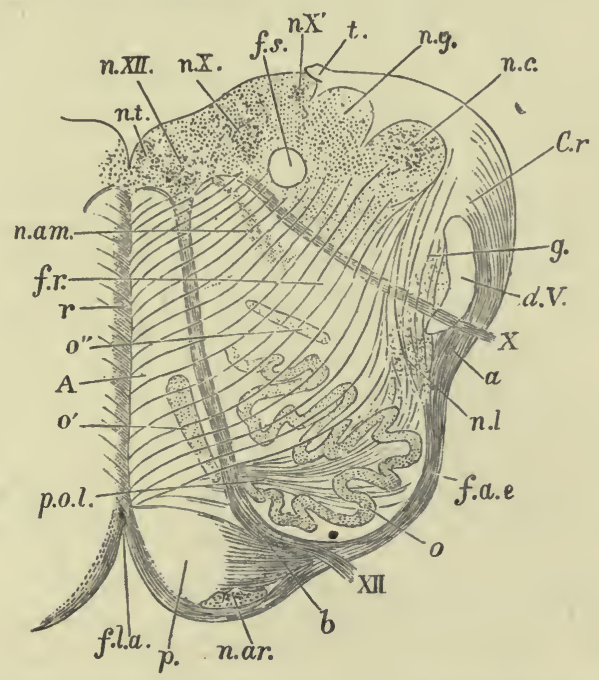

Fic. 412. - Section of the medulla oblongata at about the middle of the olivary body. f.l.a., Anterior median fissure; n.ar., nucleus arcuatus; $p$, pyramid; $X I I$. , bundle of hypoglossal nerve emerging from the surface; at $b$, it is seen coursing between the pyramid and the olivary nucleus, $0 ; f$.a.e., external arcuate fibres; n.l., nucleus lateralis; $a_{0}$, arcuate fibres passing towards restiform body, partly through the substantia gelatinosa, $g_{.,}$, partly superficial to the descending root of the fifth nerve, $d_{.} V_{.} ; X_{\text {. }}$, bundle of vagus root emerging; $f . r_{\text {., }}$ formatio reticularis; $C . r$., corpus restiforme, beginning to be formed, chlefly by arcuate fibres, superficial and deep; n.c., nucleus cuneatus; $n . g$., nucleus gracilis; $t$, attachment of the ligula; f.s., funiculus solitarius ; n.X., n.X.', two parts of the vagus nucleus; $n . X I I .$, hypoglossal nucleus; n.t., nucleus of the funiculus teres; n.am., nucleus ambiguns; $r$., raphe; $A$., continuation of the anterior column of cord; $o^{\prime}, o^{\prime \prime}$, accessory olivary nucleus; p.o.l., pedunculus olivæ. (Modifled from Schwalbe.)

The nucleus gracilis and nucleus cuneatus are pushed into a more lateral position, and higher up are replaced by small masses of grey matter mingled with nerve-fibres (nucleus posterior); the restiform body (C.r.) now forms a well-marked prominence, and the olivary body is well seen with its dentate nucleus; from the open mouth of this corrugated layer of grey matter a large number of fibres issue, and passing through the raphe, course as internal arcuate fibres to the opposite restiform body, and thus to the cerebellum; some pass to the restiform body of the same side; the continuation of the 
direct cerebellar tract of the cord also passes into the restiform body. Its fibres terminate by arborisations round Purkinje's cells in the vermis of the cerebellum. The continuation of the tract of Gowers lies just dorsal to the olivary body. The funiculus solitarius and nucleus ambiguus, also seen in this section, will be considered in our account of the origin of the ninth and tenth cranial nerves.

Fourth section (fig. 413). - This is taken through the middle of the pons, and shows much the same kind of arrangement as in the upper part of the bulb. The general appearance of the section is, however, modified by a number of transversely coursing bundles of

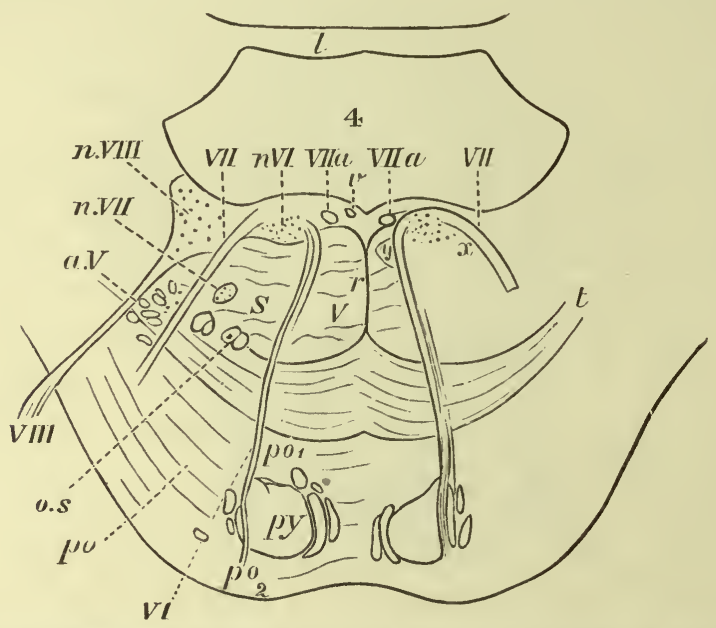

FIa. 413. - Section across the pons, about the middle of the fourth ventricle. $p y$, Pyramidal bundles; po., transverse fibres passing $p_{1}$ behind, and $p_{2}$, in front of $p y ; r_{.}$, raphe; o.s., superior olive; a. $V$., bundles of motor root of $V$. nerve enclosed in a prolongation of the substance of Rolando; $t$, trapezium; VI., the sixth nerve, n.VI., its nucleus ; VII., facial nerve; VII.a., intermediate portion, n.VII., its nucleus; VIII., auditory nerve; n.VIII., Deiters' nucleus, formerly called the lateral nucleus of the auditory. (After Quain.)

fibres, most of which are passing to the cerebellar hemispheres and form the middle cerebellar peduncles. Intermingled with these is a considerable amount of grey matter (nuclei pontis).

From the cells of the nuclei pontis, the fibres of the middle peduncle take origin, and many fibres and collaterals of the pyramidal tract arborise around them. The continuation of the pyramids $(p y)$ is imbedded between these transverse bundles. The pyramidal fibres which terminate in the pons are situated postero-laterally, and are spoken of as cortico-pontine in contradistinction with those of the pyramidal tract proper (cortico-spinal) which pass down through the bulb to the cord.

The pyramidal bundles are separated from the reticular formation 
by deeper transverse fibres, which constitute what is known as the trapezium $(t)$. These fibres belong to a different system, and form part of the central auditory path; some of them connect the auditory nuclei of the two sides together. The larger olivary nucleus is no longer seen, but one or two small collections of grey matter (o.s.) represent it and constitute the superior olivary nucleus. These as well as a collection of nerve-cells in the trapezium (nucleus of the trapezium) are connected with fibres of the trapezium, while some of their axons pass into the adjacent lateral part of the fillet.

The nucleus of Deiters (n.VIII., fig. 413) begins to appear in the upper part of the bulb, and extends into the pons; it lies near the floor of the ventricle, a little mesial to the restiform body. The nerve-fibres connected with its cells pass towards the middle line, and enter the posterior longitudinal bundle, which is more clearly seen in the two next sections (fig. 414). This bundle of fibres connects Deiter's nucleus, the nucleus of the third and sixth nerves, and the anterior horn cells of the spinal cord. The fibres which pass into it from Deiters' nucleus bifurcate, one branch passing upwards to arborise around the cells mainly of the oculo-motor nucleus of the opposite side; the other extends downwards through the bulb into the cord, where they are found in the antero-lateral descending tract of each side. They end by synapses around the anterior horn cells.

This bundle receives in addition to the fibres from Deiters' nucleus, other fibres from the sensory nucleus of the fifth nerve, and from large cells in the reticular formation of mid-brain, pons, and bulb.

The nerves which are connected with the grey matter of this region of the pons are the sixth, seventh, and eighth, as shown in the diagram. The nuclei in connection with the fifth nerve are higher up, where the floor of the ventricle is again narrowing. At last, in the region of the mid-brain, we once more get a canal (Sylvian aqueduct) which corresponds to the central canal of the spinal cord.

Fifth and Sixth sections are taken through the mid-brain, and are drawn on a smaller scale than the others we have been examining; they represent the actual size of the sections obtained from the human subject.

Near the middle is the Sylvian aqueduct, with its lining of ciliated epithelium. In the grey matter which surrounds it are large nervecells from which the fourth nerve, and higher up the third nerve, originate; the fibres of the third nerve are seen issuing from these in fig. 414, B., III. The reticular formation of the pons is continued up into the mid-brain, and is called the tegmentum. It is composed of both longitudinal and transverse bundles of fibres intermingled with grey matter. Its transverse fibres include those of the superior peduncles of the cerebellum which decussate in the middle line (fig. 414 , A., S.C.P.). 
Another important longitudinal bundle in the tegmentum is the fillet. This, we have seen, is the longitudinal continuation of the internal arcuate fibres, which, starting from the cells of the posterior column nuclei of the opposite side, form the second relay on the sensory path; to these fibres others are added which originate from
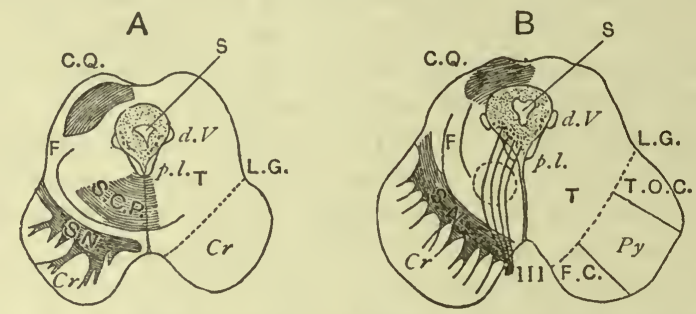

FIG. 414.-Outline of two sections across the mid-brain: A, through the middle of the inferior; $B$, through the middle of the superior corpora quadrigemina, C.Q. Cr., crusta; S.N., substantia nigra - shown only on one side; T, tegmentum; S, Sylvian aqueduct, with its surrounding grey matter; L.G., lateral groove; p.l., posterior longitudinal bundle; $d . V$. , descending root of the fifth nerve; S.C.P., superior cerebellar peduncle; F, fillet; III., third nerve. The dotted circle in B represents the situation of the tegmental nucleus. In $B$ the three divisions of the crusta are indicated on on $\theta$ side. The pyramidal fibres $(P y)$ are in the middle, and the fronto-cerebellar (F.C.) and temporooccipital cerebellar (T.O.C.) at the sides. (After Schäfer.)

other masses of grey matter in bulb and pons. In the mid-brain the fillet splits into three bundles, termed the lateral, the upper, and the mesial fillet.

(1) The lateral fillet is chiefly formed by fibres derived from the accessory auditory, the inferior olivary, and trapezoid nuclei of the opposite side. Some of its fibres terminate by synapses around a new collection of cells (the lateral fillet nucleus); their axons pass inwards towards the raphe. The rest of its fibres can be traced to the grey matter of the inferior corpora quadrigemina.

(2) The upper fillet consists of fibres which go to the superior corpora quadrigemina and partly to the tegmental region of the mid-brain and optic thalamus.

(3) The mesial fillet goes on through the tegmentum of the crus cerebri, and its fibres terminate around the cells of the optic thalamus, and the subthalamic region. From here fresh axons forming a new relay continue the afferent impulses to the cortex of the cerebrum.

The mesial fillet is the important link in this region between the sensory spinal nerves and the part of the brain which is the seat of those processes we call sensations. But most of the fibres which continue the sensory path of the cranial nerves form another less well-defined tract (the central tract of the sensory cranial nerves) which lies dorsal to the fillet, but terminates like it in the subthalamic region and optic thalamus, whence a new relay carries on the impulses to the cortex.

Ventral to the tegmentum is a layer of grey matter, of which the cells are deeply pigmented; hence it is called the substantia nigra (S.N.). This receives many collaterals from the pyramidal bundles.

The white matter on the ventral side of this is known as the 
crusta $(C r)$ or pes. It is here that the pyramidal bundles are situated; these occupy its middle three-fifths $(P y)$. The mesial fifth is occupied by fibres passing from the frontal region of the cerebrum to the pons, and thence to the cerebellum; hence they are called fronto-cerebellar fibres. The fibres occupying the lateral fifth are usually spoken of as temporo-occipital cerebellar fibres, but there is no certainty as yet regarding their origin or functions.

The corpora quadrigemina are formed mainly of grey matter; from each superior corpus a bundle of white fibres passes upwards and forwards to the geniculate bodies, eventually joining the optic tract of the same side. The white layer on the surface of the grey matter of the C. quadrigemina is derived from the optic tract; these fibres come from the retina, and terminate by arborising around the cells of the grey matter of the C. quadrigemina.

The cells of the grey matter of the corpora quadrigemina differ greatly in form and size; the destination of their axons is not precisely known, but some pass ventralwards, cross at the raphe, and constitute the fountain decussation of Meynert; after decussation they form the main mass of the ventral longitudinal bundle; this gives off collaterals to the nuclei of the three nerves that supply the eye muscles, and then runs ventro-laterally to the posterior longitudinal bundle, with which its fibres ultimately $\operatorname{mix}$ in the antero-lateral descending tract of the spinal cord.

Seventh section.-This is through the crus. It is made up of crusta (which contains the motor fibres), tegmentum (which contains

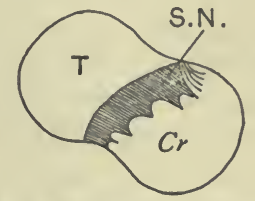

FIG. 415.- Section through crus of cerebrum. Cr, crusta; S.N., substantia nigra ; T, tegmentum. the sensory fibres, especially the bundle called the mesial fillet), and the substantia nigra, the grey matter which separates them.

\section{Origins and Functions of the Cranial Nerves.}

Having now studied the internal construction of these parts, we can take up more fully the origins and functions of the cranial nerves which originate there. The olfactory nerve is connected to the cerebrum, and will be considered with the sense of smell. The optic nerve will be studied with vision, though it is, as we have seen, immediately connected with the mid-brain.

The third, fourth, and sixth nerves supply the muscles of the eye. Gaskell discovered among the rootlets of the third and fourth nerves the vestiges of a degenerated and functionless ganglion, which indicates the previous existence of a separate sensory root. Sherrington has shown that in these three nerves sensory fibres are present which are connected to the sensorial nerve endings (muscle-spindles).

The third nerve (motor oculi) arises in a group of nerve-cells in 
the grey matter on the side of the Sylvian aqueduct underneath the superior corpus quadrigeminum, and close to the middle line. The anterior part of this nucleus is composed of small cells from which small nerve-fibres originate for the ciliary muscle and sphincter of the iris (intrinsic muscles of the eyeball). These fibres correspond to the visceral fibres of a spinal nerve, and, like them, have a cell station, namely, in the ciliary ganglion. The posterior part of the nucleus is composed of larger cells, and these give rise to larger fibres which supply the following extrinsic eye-muscles:-superior rectus, inferior rectus, internal rectus, inferior oblique and levator palpebræ.

The fourth nerve (trochlear) takes origin from the grey matter immediately below the centre of the third, but slightly more lateral in position. It is underneath the inferior corpus quadrigeminum. It supplies the superior oblique muscle of the opposite eyeball.

The sixth nerve (abducens) arises from a centre beneath the eminentia teres in the upper part of the floor of the fourth ventricle near the middle line. It supplies the external rectus.

It is obviously necessary that the eye-muscles should work together harmoniously, that the two eyeballs should also be moved simultaneously and in corresponding directions, and that such movements should take place in accordance with the necessities of vision. This is provided for in the shape of association fibres which link the centres of the eye-muscles together. The principal association tracts are the posterior longitudinal bundle, which connects the nuclei of the third and sixth nerves, and the ventral longitudinal bundle which unites the optic nerves through the intermediation of the cells of the C. quadrigemina, with the nuclei of all these nerves. It should also be remembered that all the fibres of the fourth, and some of those of the third nerve, decussate in the middle line.

The fifth nerve (trigeminal) is a mixed nerve; it leaves the side of the pons in a smaller motor, and a larger sensory division. The former supplies the muscles of mastication, the tensors of the palate and tympanum, the mylo-hyoid, and the anterior belly of the digastric; the sensory division has upon it a ganglion called the Gasserian ganglion; it is the great sensory nerve of the face and head. The motor fibres arise from the motor nucleus (Vm, fig. 408), which lies at the lateral edge of the upper part of the floor of the fourth ventricle, but a certain number of its fibres arise from cells in the lower part of the mid-brain and upper part of the pons; this long stretch of nerve-cells, indicated by the long blue tail in the diagram, is called the accessory or superior motor nucleus of the fifth. The sensory fibres arise from the cells of the Gasserian ganglion, which resemble in structure those of a spinal ganglion; one branch of each passes to the periphery in the skin of the head and face, and 
the other grows centralwards; on reaching the pons these bifurcate, the ascending branches arborise around the principal sensory nucleus of the fifth (Vd, fig. 408), which lies just lateral to the motor nucleus, while the descending branches pass down into the bulb, where they form the descending root of the fifth, and some reach as far down in the spinal cord as the second cervical nerve. Mingled with these descending fibres are numerous nerve-cells, many of which are grouped in clusters (islands of Calleja), and the descending fibres form synapses around them. The new axons arising from the cells of the sensory nuclei pass upwards in three principal tracts:-(1) The greater number cross the raphe and join the mesial fillet; (2) some ascend the fillet of the same side; and (3) others pass into a special ascending bundle which lies near the ventricular floor (the central tract of the cranial sensory nerves).

The seventh nerve (facial) is the great motor nerve of the face muscles. It also supplies the platysma, the stapedius, stylo-hyoid, and posterior belly of the digastric. When it is paralysed, the muscles of the face being all powerless, the countenance acquires on the paralysed side a characteristic, vacant look, from the absence of all expression: the angle of the mouth is lower, and the paralysed half of the mouth looks longer than that on the other side; the eye has an unmeaning stare, owing to the paralysis of the orbicularis palpebrarum. All these peculiarities are exaggerated when at any time the muscles of the opposite side of the face are made active in any expression, or in any of their ordinary functions. In an attempt to blow or whistle, one side of the mouth and cheeks acts properly, but the other side is motionless, or flaps loosely at the impulse of the expired,air; in trying to suck, one side only of the mouth acts; in feeding, on account of paralysis of the buccinator muscle, food lodges between the cheek and gums.

The motor fibres originate from a nucleus in the ventricular floor below that of the fifth and to the outer side of that of the sixth nerve. As they curve over the nucleus of the sixth, they give off a bundle of fine fibres which cross the raphe, but their destination is unknown. The facial nucleus receives collaterals from the sensory tracts in the reticular formation.

The seventh nerve, however, is not wholly motor. The geniculate ganglion on it is of spinal type; the fibres which arise from it pass centrally into the pars intermedia of Wrisberg, which enters the pons between the seventh and eighth nerves; these, like other sensory fibres, divide into ascending and descending branches; the latter have been traced down to the sensory nucleus of the glosso-pharyngeal nerve. The peripheral branches of the geniculate ganglion cells pass into the large superficial petrosal and chorda tympani, the gustatory fibres of which they probably furnish. The secretory fibres of the chorda 
tympani are efferent fibres which reach it from the facial nucleus via the pars intermedia.

The eighth nerve (auditory) runs into the hinder margin of the pons by two roots. One winds round the restiform body dorsal to it, and is known as the dorsal or cochlear division; the other passes ventro-mesially on the other side of the restiform body, and is known as the ventral or vestibular division.

We will take these two parts separately. The fibres of the cochlear nerve take origin from the bipolar nerve-cells of the spiral ganglion of the cochlea; the peripheral axons ramify among the hair cells of the organ of Corti, and the central axons pass towards the pons; as they enter they bifurcate, and some pass to and arborise

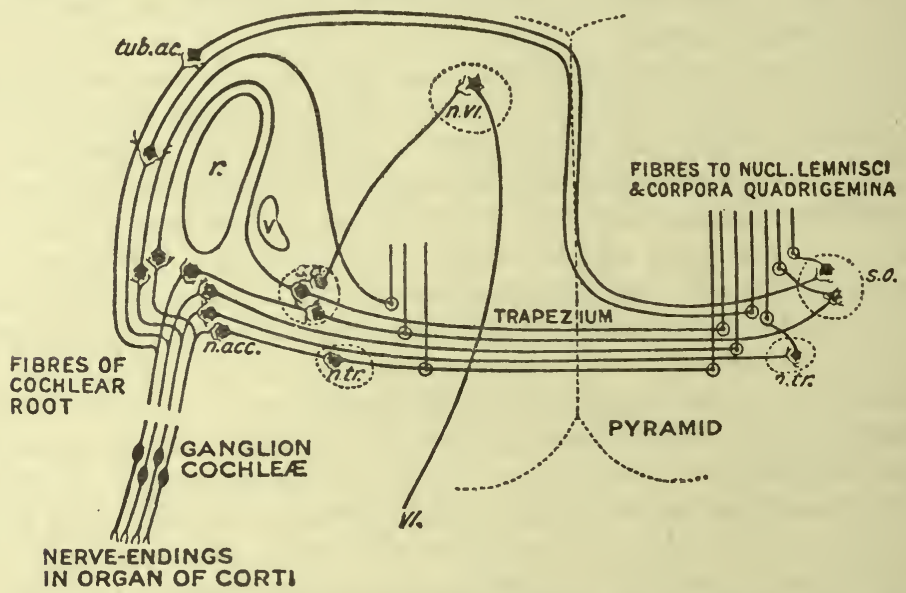

FIG. 416.-Cochlear division of the auditory nerve. $r$, Restiform body; $V$, descending root of the fifth nerve; tub.ac., acoustic tubercle; n.acc., accessory nucleus; s.o., superior olive; n.tr., trapezoid nucleus; $n . V I$., nucleus of the sixth nerve; VI., issuing fibre of sixth nerve. (Schäfer.)

around a collection of nerve-cells situated between the two roots and the restiform body, called the accessory auditory nucleus; the remaining fibres terminate similarly in a collection of cells in the grey matter overlying the restiform body, and extending into the ventricular floor in its widest part. This is called the ganglion of the root, and the mass of grey matter is termed the acoustic tubercle. The auditory path is continued by new axons that arise from these cells. Those from the accessory nucleus enter the trapezium, and pass in it partly to the superior olive and trapezoid nucleus of the same side, but mainly to the corresponding nuclei of the opposite side; some fibres end here, others traverse the nuclei, and merely give off collaterals to them; they then turn upwards in the lateral fillet, and so reach the inferior C. quadrigemina. The fibres which arise in the acoustic 
tubercle pass superficially over the floor of the ventricle, forming the strice acousticae; having crossed the raphe, they join the fibres from the accessory nucleus in their course to the superior olive and fillet. Here again, however, a few fibres pass to the fillet of the same side.

The vestibular nerve arises from the bipolar cells of the ganglion of Scarpa, which is situated in the internal auditory meatus. The peripheral axons ramify among the hair cells of the epithelium in the utricle, saccule, and semi-circular canals. The central axons enter a collection of small nerve-cells between the restiform body and the descending root of the fifth; this is termed the principal nucleus; here

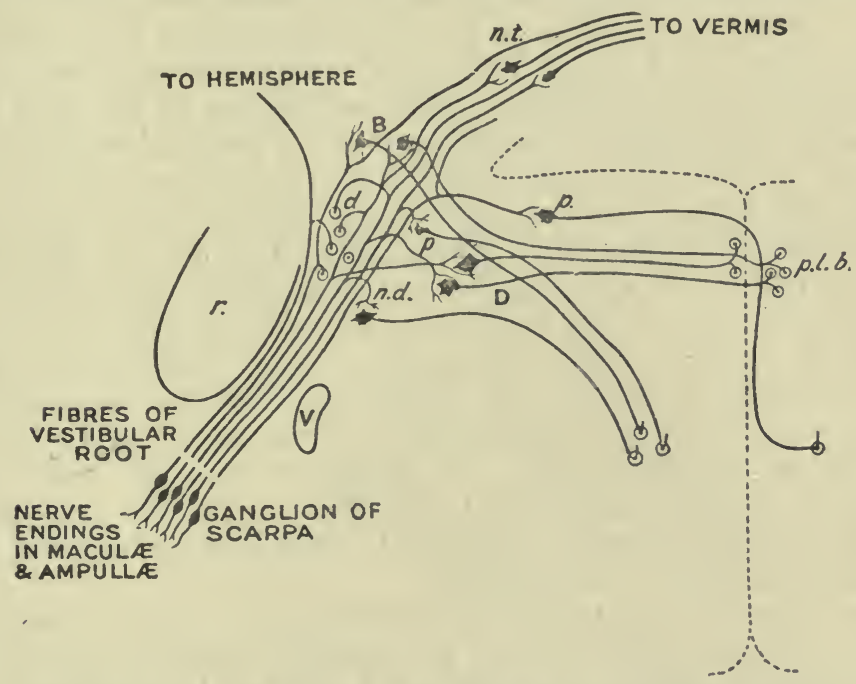

FIG. 417.-Vestibular division of the auditory nerve. $r$, Restiform body; $V$, descending root of the fifth nerve; $d$, fibres of descending vestibular root; n.d., cell of descending vestibular nucleus; $D$, nucleus of Deiters; $\mathrm{B}$, nucleus of Bechterew; n.t., nucleus tectl of cerebellum : p.l.b., posterior longitudinal bundle. (Schäfer.)

they bifurcate; the descending branches run towards the lower part of the bulb, and arborise around the cells of the neighbouring grey matter (descending vestibular nucleus). The ascending branches pass upwards in the restiform body to the cerebellum, in their course giving off many collaterals which form synapses with the large cells of two nuclei near the outer angle of the ventricular floor, and known as the nucleus of Deiters and nucleus of Bechterew respectively. The fibres which arise from Deiters' nucleus pass into the posterior longitudinal bundles of both sides (see p. 674); those which start in Bechterew's nucleus become longitudinal, but their destination is uncertain.

The accompanying diagrams (figs. 416 and 417) will serve to render these complex relationships clearer. 
The ninth nerve (glosso-pharyngeal) gives filaments through its tympanic branch (Jacobsen's nerve) to parts of the middle ear; also, to the carotid plexus, and through the great superficial petrosal nerve to the spheno-palatine (Meckel's) ganglion. After communicating, either within or without the cranium, with the vagus, it leaves the cranium, divides into the two principal divisions indicated by its name, and supplies the mucous membrane of the posterior and lateral walls of the upper part of the pharynx, the Eustachian tube, the arches of the palate, the tonsils and their mucous membrane, and the tongue as far forwards as the foramen cæcum in the middle line, and to near the tip at the sides and inferior part.

It contains motor fibres to the stylo-pharyngeus, the constrictors of the pharynx, and probably to the levator palati and other muscles of the palate, except the tensor, which is supplied by the fifth nerve. The nerve also contains fibres concerned in common sensation, and the sense of taste, and secretory fibres for the parotid gland.

The cells from which the motor fibres originate are situated in a special nucleus, which is a continuation upwards of the nucleus ambiguus (the chief motor nucleus of the tenth or vagus nerve). The sensory fibres arise in the jugular and petrosal ganglia from cells of the spinal ganglion type. When the central axons reach the bulb they bifurcate as usual; the descending branches pass down the funiculus solitarius and terminate in synapses around the cells scattered among its fibres. The ascending branches pass almost horizontally to arborise around the cells of the principal nucleus (IX. in fig. 408). The arrangement, in fact, is very like that of the tenth nerve now to be described.

The tenth nerve (vagus or pneumo-gastric) has so many and important functions that I shall not attempt to describe them here; it would mean rewriting a great deal of what we have already learnt in connection with heart, respiration, digestion, etc. It is sufficient to say that it contains both efferent and afferent fibres. The efferent fibres arise partly from the upper part of the combined nucleus, which lower down gives origin to the spinal accessory nerve (fig. $408, \mathrm{X}$.) but mainly from the nucleus ambiguus, the position of which is shown in fig. 408, coloured blue, and also in transverse section in figs. 412 and 418. The afferent fibres originate from the cells of the ganglion of the trunk and of the root; they enter the bulb and bifurcate; the ascending branches are short and arborise around the cells of the principal nucleus (X. in fig. 408); the descending fibres, together with similar ones derived from the glosso-pharyngeal nerve, and pars intermedia, pass down in the descending root of vagus and glossopharyngeal, which is also known as the funiculus solitarius. These fibres terminate by arborising around the cells of the grey matter that lies along its mesial border (descending nucleus of vagus and 
glosso-pharyngeal). This approaches the middle line as it descends, and finally joins that of the opposite side over the central canal (commissural nucleus).

The eleventh nerve (spinal accessory) is wholly efferent: it arises by two distinct origins - one from a centre in the floor of the fourth ventricle, and connected with the glosso-pharyngeal-vagus-nucleus; the other, from the outer side of the anterior cornu of the spinal cord as low down as the fourth cervical nerve. The fibres from the two origins come together at the jugular foramen, but separate again into two branches, outer and inner. The outer, consisting of large medullated fibres from the spinal origin, supplies the trapezius and

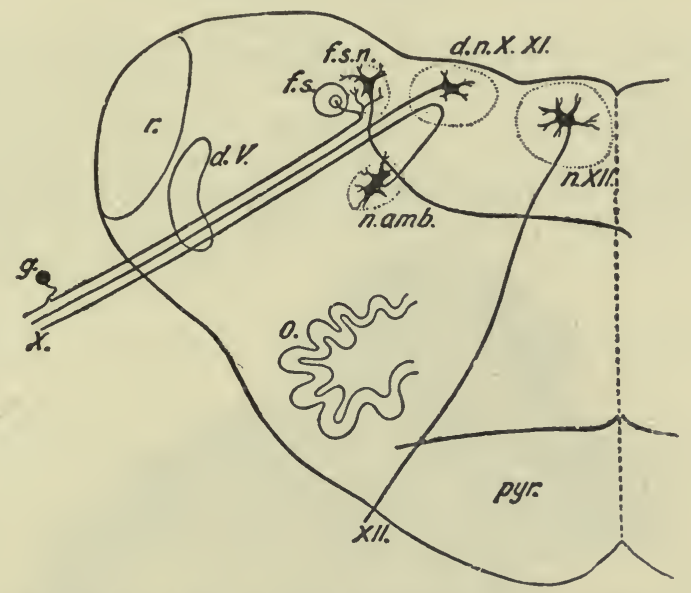

FIa. 418.-The tenth and twelfth nerves. $p y r$, Pyramid ; n.XII., nuclens of hypoglossal ; XII., fibre of hypoglossal; $d . n . X . X I$., combined nucleus of vagus and spinal accessory; n.amb., nucletus ambiguus; f.s., fasciculus solitarius, descending fibres of vagus and glosso-pharyngeal; f.s.n., its nucleus; $X .$, motor fibre of vagus; $g$, ganglion cell in vagus trunk giving rise to a sensory fibre; $d . V$., descending root of the fifth nerve ; $r$, restiform body. (Schäfer.)

sterno-mastoid muscles. The inner branch, consisting of small medullated fibres from the medulla, supplies chiefly viscero-motor and cardio-inhibitory filaments to the vagus. The muscles of the larynx, all of which are supplied by branches of the vagus, derive their motor nerves from the accessory; Vrolik states that in the chimpanzee the internal branch of the accessory does not join the vagus at all, but goes direct to the larynx. The crico-thyroid, however, receives fibres which leave the bulb by glosso-pharyngeal rootlets.

The twelfth nerve (hypoglossal) is also entirely efferent. It supplies the muscles of the tongue. It arises from a large celled and long nucleus in the bulb, close to the middle line (see figs. 408 and 418). 


\section{CHAPTER XLVII}

\section{STRUCTURE OF THE CEREBELLUM}

THE cerebellum is composed of an elongated central portion or lobe, called the vermis or vermiform process, and two hemispheres. Each hemisphere is connected with its fellow by means of the vermiform process.

The cerebellum is composed of white and grey matter, the latter

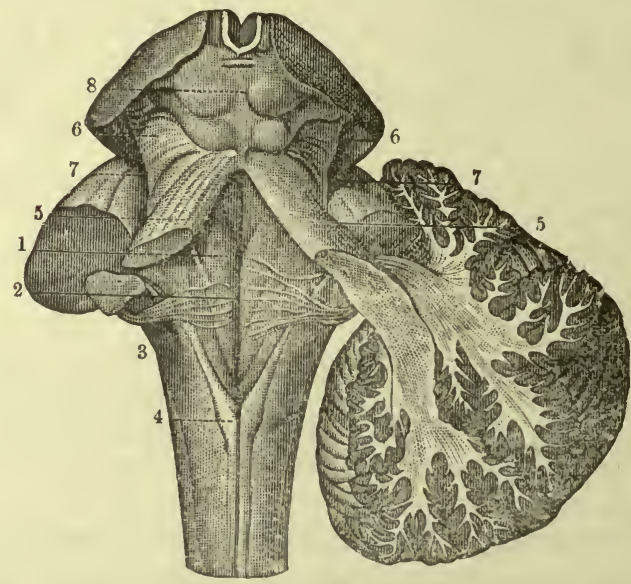

FIG. 419.-Cerebellum in section and fourth ventricle, with the neighbouring parts. 1, median groove of fourth ventricle, ending below in the calamus scriptorius, with the longitudinal eminences formed by the fasciculi teretes, one on each side; 2 , the same groove, at the place where the white streaks of the auditory nerve emerge from it to cross the floor of the ventricle; 3 , inferior peduncle of the cerebellum, formed by the restiform body; 4 , funiculus gracilis; above this is the calamus scrip. torius ; 5 , superior peduncle of cerebellum ; 6,6 , fillet to the side of the crura cerebri; 7,7 , lateral grooves of the crura cerebri; 8, corpora quadrigemina. (From Sappey, after Hirschfeld and Leveillé.)

being external, like that of the cerebrum, and like it, infolded, so that a larger area may be contained in a given space. The convolutions of the grey matter, however, are arranged after a different pattern, as shown in fig. 419. The tree-like arrangement of the white 
matter on section has given rise to the name arbor vitce. Besides the grey substance on the surface, there are, in the centre of the white substance of each hemisphere, small masses of grey matter, the largest of which, called the corpus dentatum (fig. $420, c d$ ), resembles very closely the corpus dentatum of the olivary body in appearance.

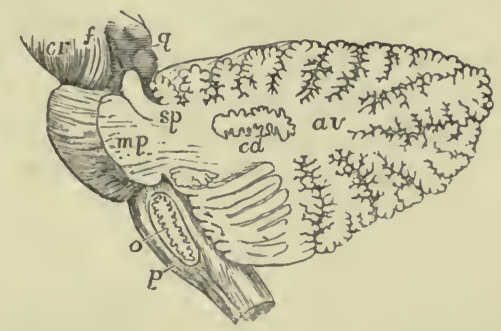

Fia. 420.-Outline sketch of a section of the cerebellum, showing the corpus dentatum. The section has been carried through the left lateral part of the pons, so as to divide the superior peduncle and pass neariy through the middle of the left cerebellar hemisphere. The olivary body has also been divided longitudinally so as to expose in section its corpus dentatum. cr, Crus cerebri; $f$, fillet; $q$, corpora quadrigemina; $s p$, superior peduncle of the csrebellum divided; $m p$, middle peduncle or lateral part of the pons Varolii, with fibres passing from it into the white stem; $u v$, continuation of the white stem radiating towards the arbor vite of the folia; $c d$, corpus dentatum; 0 , olivary body with its corpus dentatum; $p$, pyramid. (Allen Thomson.) 3 .

In a section through the cerebellar cortex the following layers can be seen.

Underneath the pia mater is the external layer of grey matter; it is formed chiefly of fine nerve-fibres with small nerve-cells scattered through it. Into its outer part, processes of pia mater pass vertically; these convey blood-vessels. There are also here numerous long tapering neuroglia-cells. The internal or granular layer of grey matter is made up of a large number of small nerve-cells mixed with a few larger ones, and some neuroglia-cells. Between the two layers is an incomplete stratum of large flask-shaped cells, called the cells of Purkinje. Each of these gives off from its base a process which becomes the axon of one of the medullated fibres of the white matter; the neck of the flask passing in the opposite direction breaks up into dendrites which pass into the external layer of grey matter. By Golgi's method (fig. 421) these dendrons have been shown to spread out in planes transverse to the direction of the lamellæ of the organ.

Each cell of Purkinje is further invested by arborisations of two sets of nerve-fibres. One of these (originating from the fibres of the white matter which are not continuous as axis-cylinders from the cells of Purkinje) forms a basket-work round the dendrons; the other (originating as axis-cylinder processes from the nerve-cells of the external layer) forms a felt-work of fibrils round the body of the cell.

The cells of the internal layer of grey matter are small; their 
dendrites intermingle with those of neighbouring cells; their axons penetrate into the external layer, but their final destination is uncertain. Ramifying among these cells are fibres characterised by possessing bunches of short branches at intervals (moss-fibres of Cajal).

The peduncles of the cerebellum are three in number-superior, middle, and inferior; we have already had occasion to mention them in our study of the bulb, pons, and mid-brain. The course of the fibres has been chiefly studied by the degeneration method.

The inferior peduncle, or restiform body, is composed of ascending fibres which pass into it-(1) from the cerebellar tracts of the

I.

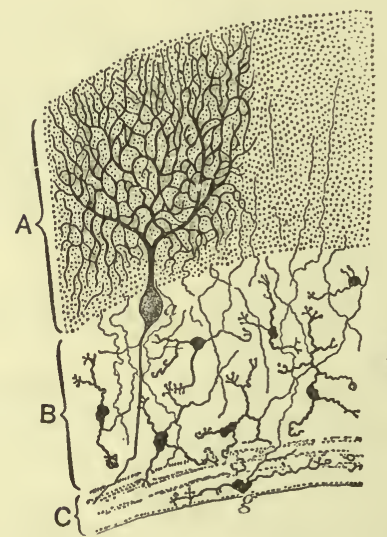

II.

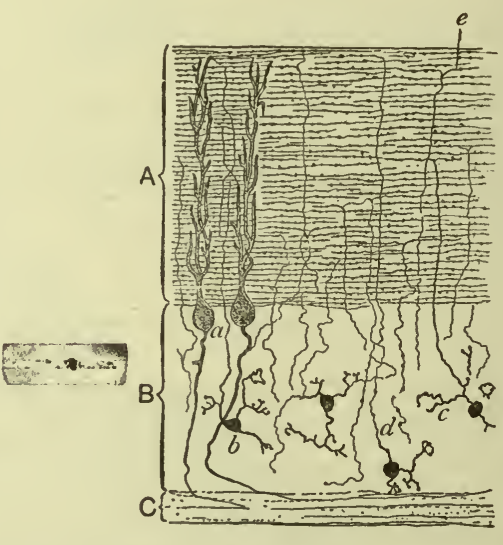

FIg. 421.--Section of cerebellar cortex, stained by Golgi's method; I. taken across the lamina ; II. in the direction of the lamina; $A$, outer or molecular layer; $\mathrm{B}$, inner or granular layer; $\mathrm{C}$, white matter. $a$, Cell of Purkinje; $b$, small cells of inner layer; $c$, dendrons of these cells; $d$, axis. cylinder process of one of these cells becoming longitudinal in the outer layer; $e$, bifurcation of one of these; $g$, a similar cell lying in the white matter. (Ramon y Cajal.)

same side, and (2) from the olivary nucleus of the opposite side; (3) possibly a fow fibres from the nucleus gracilis and nucleus cuneatus also join it; and lastly, (4) it receives numerous fibres from the vestibular nerve, or from the nuclei in which it terminates in the pons. The inferior peduncle is thus mainly a spino-cerebellar path, serving by the cerebellar tracts to unite the same side of the cord with the vermis, and the opposite side of the cord with the cerebellar hemisphere via the opposite olivary nucleus and reticular formation of the bulb.

The middle peduncle is wholly formed of fibres which-originate from the cells of the nuclei pontis: they pass from one side of the pons to the opposite cerebellar hemisphere. This peduncle is the last relay of the cerebro-cerebellar path. 
The superior peduncle: the axons of the cells of Purkinje mainly terminate in the nucleus dentatus, and the other subsidiary masses of grey matter situated in the interior of the cerebellum; from the cells of these nuclei a fresh relay of fibres issues, conveying impulses from the cerebellum to other parts, but mainly to the opposite cerebral hemisphere; these fibres constitute the superior cerebellar peduncle. They cross the middle line, give off numerous collaterals to the red nucleus of the opposite side, and also to the nucleus of the opposite third nerve. The majority terminate in the optic thalamus, whence a fresh relay continues the impulse to the cerebral cortex. This therefore is the cerebello-cerebral path.

After the fibres of the superior peduncle have crossed the middle line, they give off descending branches which run towards the bulb and cord, though whether they reach as far down as the spinal cord is doubtful. There is, however, a cerebello-spinal path via the red nucleus with which the fibres that issue from the cerebellum communicate after crossing, for it is from the red nucleus that the bundle of Monakow arises which crosses the middle line and is seen in the cord as the rubro-spinal or prepyramidal tract; it terminates in the anterior horn of the spinal grey matter. The cerebello-spinal path therefore exhibits a double crossing; the first is that of the superior peduncle to reach the opposite red nucleus, and the second is that of the bundle of Monakow; in this way the cerebellar hemisphere is linked to the same side of the spinal cord.

In addition to all these fibres, the superior peduncle also contains some fibres of the spino-cerebellar tracts, which after ascending the spinal cord, bulb and pons turns round and course back along the superior peduncle into the cerebellum; these fibres are distributed mainly to the lower part of the vermis.

The next figure (fig. 422) shows the principal connections of the cerebellum in a diagrammatic way.

Beginning at the bottom, we see one of the cells of a spinal ganglion (S.G.) sending its peripheral axon to the skin (S.); its central axon enters the spinal cord and ascends its posterior column, to terminate in the posterior column nuclei of the bulb. This is marked "to Bulb." This is the first segment of the sensory path to the cerebrum, but its further course is not shown.

The entering fibre of the posterior root gives off collaterals to the spinal grey matter; some of these pass to cells in the posterior horn (P.H.C.), from which a fresh relay carries on the impulse to anterior horn cells, one of which (A.H.C.) is seen sending its axon via the anterior root, to end in the muscular fibre $\mathrm{M}$.

Other collaterals terminate by synapses around the cells of Clarke's column (c.c.). Two of these cells are shown; this is the 


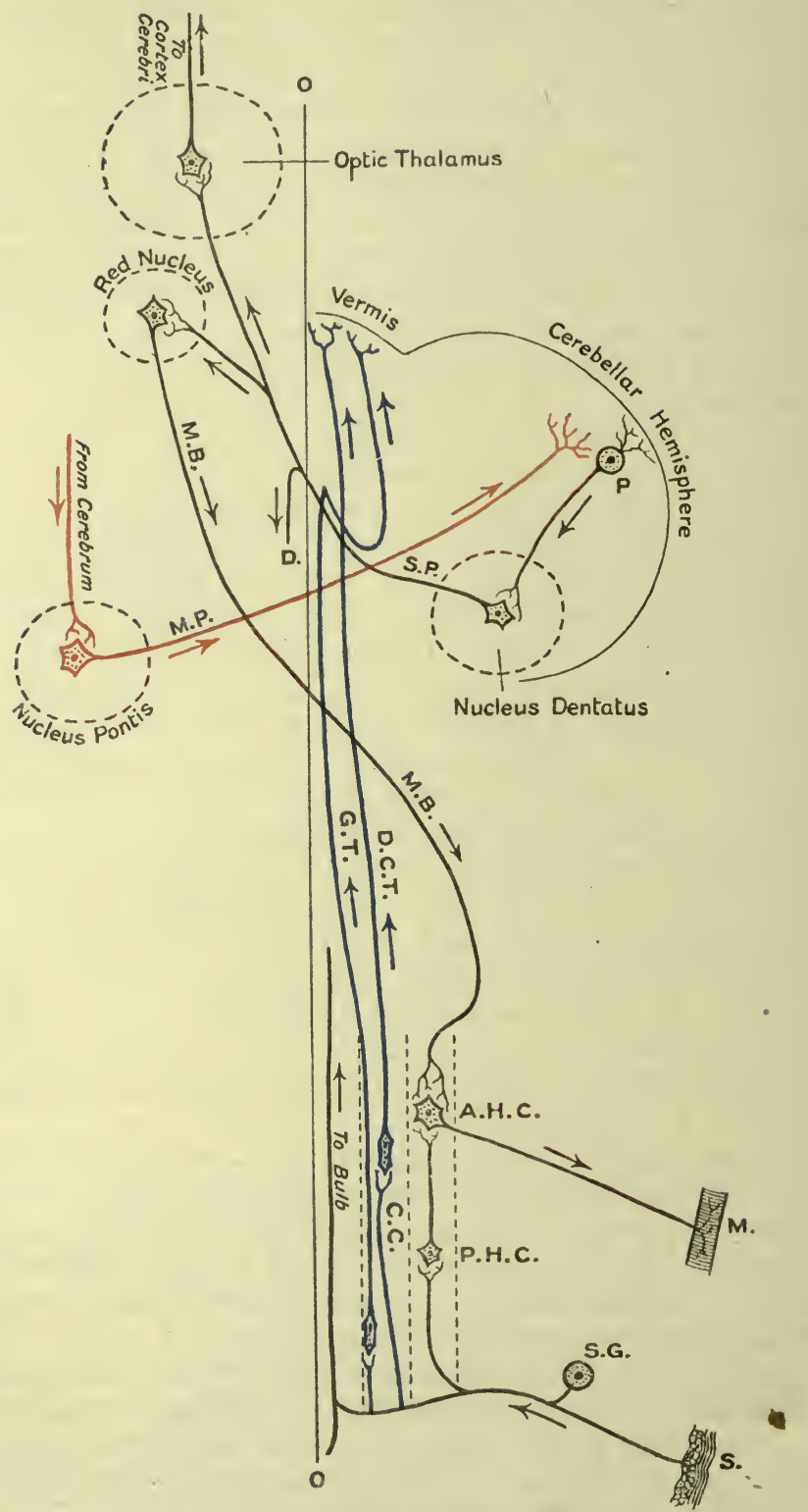

FIG. 422.-The main connections of the cerebellum. 
first cell-station on the cerebellar path. One of these is represented as giving origin to a fibre of the direct cerebellar tract (D.C.T.), which enters the cerebellum by its inferior peduncle. The other cell of Clarke's column is shown giving origin to a fibre of Gowers' tract (G.T.); it is represented as making a sharp turn after having reached its highest point, and enters the cerebellum by its superior peduncle; both of these spino-cerebellar tracts (coloured blue in the diagram) terminate in the cortex of the vermis; but only a small proportion of their fibres take the roundabout path by the superior peduncle (see p. 658).

Coming next to the middle peduncle, we see one of its fibres (M.P.) arising from a cell of the nucleus pontis, and crossing the middle line to terminate in the cortex of the opposite cerebellar hemisphere; entering the nucleus pontis, we see one of the corticopontine fibres from the cerebrum The arrows indicate that this is the path (coloured red in diagram) by which impulses reach the cerebellum from the cortex of the cerebrum. The fibres from the cerebrum to the nucleus pontis come in large measure from the frontal lobe (see next chapter).

The superior peduncle is more complicated. $\mathrm{P}$ is one of the cells of Purkinje in the cortex cerebelli; its axon passes to the nucleus dentatus of the cerebellum; from the cells of the nucleus dentatus fresh axons carry on the impulse to the optic thalamus of the opposite side; one of these fibres (S.P.) is shown. From the optic thalamus a fresh relay continues the impulse to the cortex cerebri. Each fibre of the superior peduncle, after it has crossed the middle line (00), gives off a descending branch (D.), the destination of which is uncertain; it also gives off branches to the red nucleus; from the cells of the red nucleus the fibres of Monakow's bundle (М.в.) continues the impulse down to the anterior horn-cells of the opposite side; owing to the double crossing the cerebellar hemisphere is thus brought into connection with the same side of the spinal cord. 


\section{CHAPTER XLVIII}

\section{STRUCTURE OF THE CEREBRUM}

THE cerebrum consists of two halves, called cerebral hemispheres, separated by a deep longitudinal fissure and connected by a large

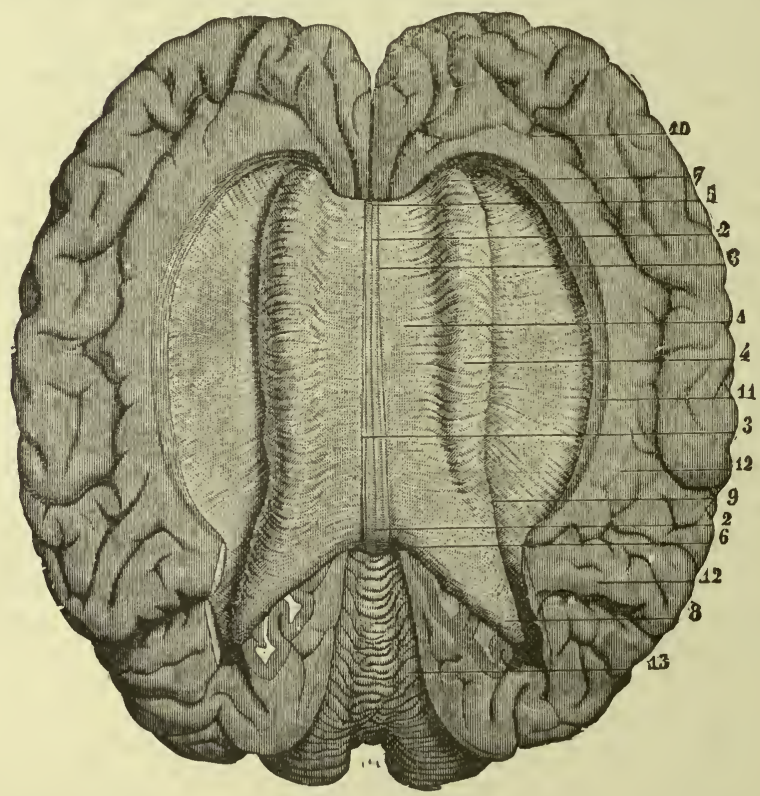

Fra. 423.-View of the Corpus Callosum from above. $\frac{1}{2}$. - The upper surface of the corpus callosum has been fully exposed by separating the cerebral hemispheres and throwing them to the side; the gyrus fornicatus has been detached, and the transverse fibres of the corpus callosum traced for some distance into the cerebral medullary substance. 1, the upper surface of the corpus callosum; 2, median furrow or raphe; 3, longitudinal striæ bounding the furrow; 4 , swelling formed by the transverse bands as they pass into the cerebrum; 5 , anterior extremity or knee of the corpus callosum; 6 , posterior extremity ; 7 , anterior, and 8 , posterior part of the mass of fibres proceeding from the corpus callosum; 9 , margin of the swelling; 10 , anterior part of the convolution of the corpus callosum; 11 , hem or band of union of this convolution; 12 , internal convolutions of the parietal lobe; 13, upper surface of the cerebellum. (Sappey, after Foville.)

band of transverse commissural fibres known as the corpus callosum (fig. 423). The interior of each hemisphere contains a cavity of com- 
plicated shape, called the lateral ventricle; the lateral ventricles open into the third ventricle. Fig. 424 represents a dissected brain in which the greater part of the corpus callosum has been removed; the ventricles are thus exposed.

Each hemisphere is covered with grey matter, which passes down

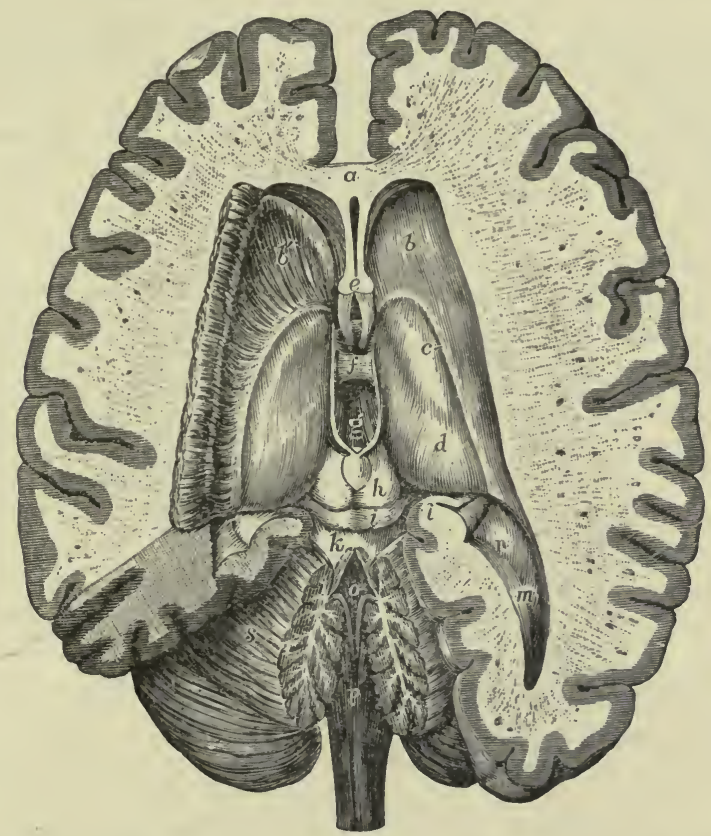

FIa. 424.-Dissection of brain, from above, exposing the lateral, fourth, and fifth ventricles with the surrounding parts. $\frac{1}{2} .-a$, anterior part, or genu of corpus callosum; $b$, corpus striatum; $b^{\prime}$, the corpus striatum of left side, dissected so as to expose its grey substance; $c$, points by a line to the tænia semicircularis; $d$, optic thalamus; $e$, anterior pillars of fornix divided; below they are seen descending in front of the third ventricle, and between them is seen part of the anterior commissure; in front of the letter $e$ is seen the slit-like fifth ventricle, between the two laminæ of the septum lucidum; $f$, soft or middle commissure; $g$ is placed in the posterior part of the third ventricle; immediately behind the latter are the posterior commissure (just visible) and the pineal gland, the two crura of which extend forwards along the inner and upper margins of the optic thalami ; $h$ and $i$, the corpora quadrigemina; $k$, superior crus of cerebellum; close to $k$ is the valve of Vieussens, which has been divided so as to expose the fourth ventricle; $l$, hippocampus maior and corpus fimbriatum, or tænia hippocampi; $m$, hippocampus minor; $n$, eminentla collateralis; $o$, fourth ventricle; $p$, posterior surface of medulla oblongata ; $r$, section of cerebellum ; s, upper part of left hemisphere of cerebellum exposed by the removal of part of the posterior cerebral lobe. (Hirschfeld and Leveillé.)

into the fissures. This surface grey matter is called the cerebral cortex. The amount of this grey matter varies directly with the amount of convolution of the surface. Under it white matter is situated; and at the base there are masses of grey matter; part of these basal ganglia are seen forming part of the wall of the ventricles. The anterior basal ganglion is called the corpus striatum; it is 
divided into two parts, called the lenticular or extra-ventricular nucleus, and the caudate or intra-ventricular nucleus. It has received the latter name because it is seen in the interior of the ventricle. The posterior basal ganglion is called the optic thalamus.

Passing up between the basal ganglia are the white fibres which enter or leave the cerebral hemisphere by the crus; these constitute the internai capsule. This passes in front between the two subdivisions of the corpus striatum, and behind between the optic thalamus and the lenticular nucleus of the corpus striatum.

The relationship of these parts is best seen in a vertical section; such as is represented in the next diagram (fig. 425).

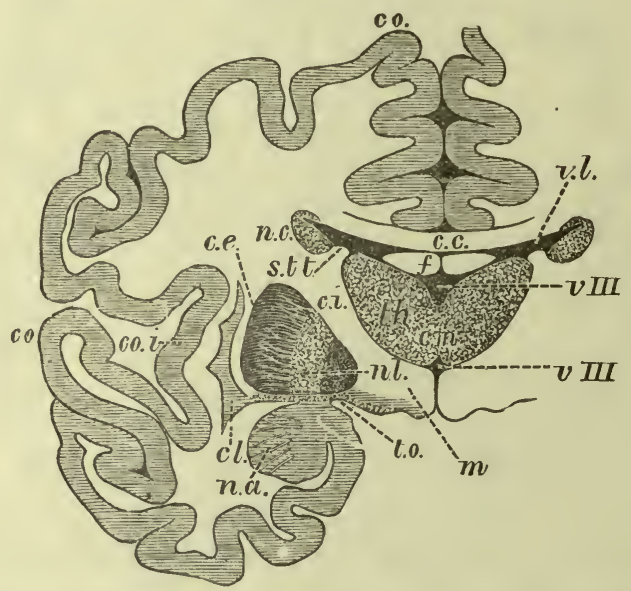

F1G. 425.-Vertical section through the cerebrum and basal ganglia to show the relations of the latter. co., Cerebral convolutions; c.c., corpus callosum; v.l., lateral ventricle; $f$, fornix; $v I I I_{\text {., }}$ third ventricle; n.c., caudate nucleus; th, optic thalamus; n.l., lenticular nucleus; c.i., internal capsule; $c l$. , claustrum; c.e., external capsule; $m$, corpus mammillare; t.o., optic tract; s.t.t., stria terminalis ; n.a., nucleus amygdalæ; $\mathrm{cm}$, soft commissure; co.i., Island of Reil. (Schwalbe.)

One hemisphere is seen, with portions of the other. The surface darkly shaded indicates the grey matter of the cortex, which passes down into the fissures; one very extensive set of convolutions (co.i.), passes deeply into the substance of the hemisphere; this is called the Island of Reil; the lowest stratum of grey matter is separated from this to form a narrow isolated strip of grey matter called the claustrum ( $c l$.$) . In the middle line the great longitudinal fissure$ is seen extending as far as (c.c.) the corpus callosum, the band of white matter that forms the great commissure between the two hemispheres; beneath this are the lateral ventricles which communicate by the foramen of Munro with the third ventricle: the fornix is indicated by the letter $f$. Contributing to the floor of the lateral ventricle, one next sees the optic thalamus $(t h$.$) , and the tail$ 
end of the nucleus caudatus (n.c.); the section being taken somewhat posteriorly. The nucleus lenticularis is marked $n l$.; and the band of white fibres passing up between it and the thalamus is called the internal capsule (c.i.); the narrow piece of white matter between the claustrum and the lenticular nucleus is called the external capsule (c.e.).

For the student of medicine the internal capsule is one of the most important parts of the brain. In it are the continuations of the fibres which we have previously traced as far as the crus cerebri; the motor-fibres of the crusta are continued into the anterior twothirds of its posterior limb (i.e. behind the genu* in fig. 426); the sensory fibres of the tegmentum into the posterior third of this limb. When these fibres get beyond the narrow pass between the basal ganglia, they spread out in a fan-like manner and are distributed to the grey cortex; the motor-fibres come down from the motor area in front of the fissure of Rolando; the sensory fibres go to certain convolutions behind this fissure. The name corona radiata is applied to the fan-like spreading of the fibres; the fibres as they pass through the handle of the fan, or internal capsule, communicate with the nerve-cells of the grey matter of the basal ganglia; the pyramidal fibres on their way down to the medulla and cord from the motor area of the brain send off collaterals or side branches which arborise around the cells of the corpus striatum, and to a less degree around those of the optic thalamus; the axis-cylinder processes of these cells pass out to join the pyramidal tract on its downward course. The sensory fibres on their way up terminate by arborising round the cells of the optic thalamus, and in the subthalamic area. This, in fact, is another cell-station or position of relay : the fibres passing out from the cells of the thalamus continue the impulse up to the cortex.

The importance of the internal capsule is rendered evident when one considers the blood supply of these parts; at the anterior and posterior perforated spots, numerous small blood-vessels enter for the supply of the basal ganglia, and these are liable to become diseased, and if they rupture, a condition called apoplexy is the result; if the hæmorrhage is excessive, death may occur almost immediately; but if the patient recovers, a condition of more or less permanent paralysis remains behind; and a very large amount of paralysis results from a comparatively limited lesion, because so many fibres are congregated together in this narrow isthmus of white matter. If the hæmorrhage is in the anterior part of the posterior limb, motor paralysis of the opposite side of the body (hemiplegia) will be the most marked symptom. If the hæmorrhage occurs in the posterior part, sensory paralysis of the opposite side of the body will be the most marked symptom. If the motor-fibres are affected, degeneration will occur 
in the pyramidal tract, and can be traced through the pes of the crus and mid-brain to the pyramid of the pons and bulb, and then in the crossed pyramidal tract of the opposite side and in the direct pyramidal tract of the same side of the cord.

Fig. 426 represents a horizontal view through the hemisphere. The internal capsule $(c)$ at the point* makes a bend called the genu

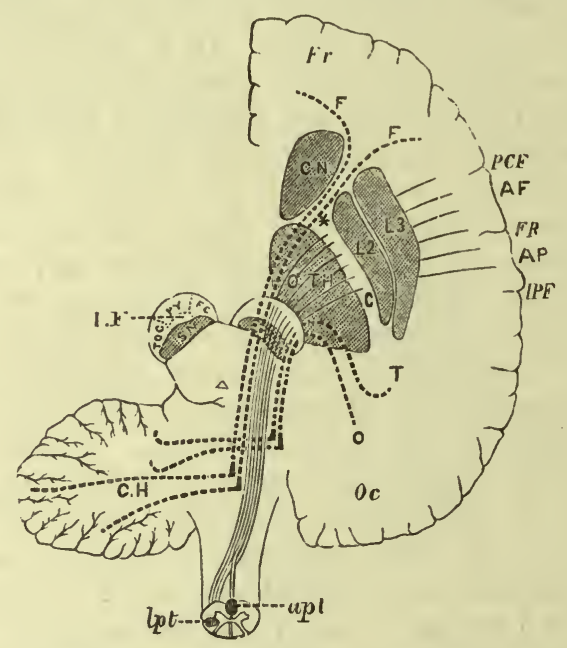

Fig. 426 -Diagram to show the connection of the Frontal and Occipital Lobes with the Cerebellum, etc. The dotted lines passing in the crusta (T.OC.), outside the motor fibres, indicate the connection between the temporo-occipital lobe and the cerebellum. F.C., The fronto-cerebellar fibres, which pass anteriorly to the motor tract in the crusta; I.F., fibres from the caudate nucleus to the pons. Fr., frontal lobe; Oc., occipital lobe; A.F., ascending frontal; AP., ascending parietal convolutions; PCF., precentral fissure in front of the ascending frontal convolution; FR, fissure of Rolando; IPF., intraparietal fissure. A section of the crus is lettered on the left side. s.N., Substantia nigra ; PY., pyramidal motor fibres, which on the right are shown as continuous lines converging to pass through the posterior limb of I.C., internal capsule (the knee or elbow of which is shown thus *) upwards into the hemisphere and downwards through the pons to cross at the medulla in the pyramidal decussation. lpt, Crossed pyramidal tract; apt, direct pyramidal tract. (Gowers.)

or knee, behind which the motor-fibres, and more posteriorly still the sensory-fibres, pass. Some of the connections between cerebrum and the cerebellum are also indicated.

\section{The Convolutions of the Cerebrum.}

The surface of the brain is "marked by a great number of depressions which are called fissures or sulci, and it is this folding of the surface that enables a very large amount of the precious material called the grey matter of the cortex to be packed within the narrow compass of the cranium. In the lowest vertebrates the surface of the brain is smooth, but going higher in the animal scale the fissures make their appearance, reaching their greatest degree of complexity in the higher apes and in man. 
In an early embryonic stage of the human fotus the brain is also smooth, but as development progresses the sulci appear, until the climax is reached in the brain of the adult.

The sulci, which make their appearance first, both in the animal
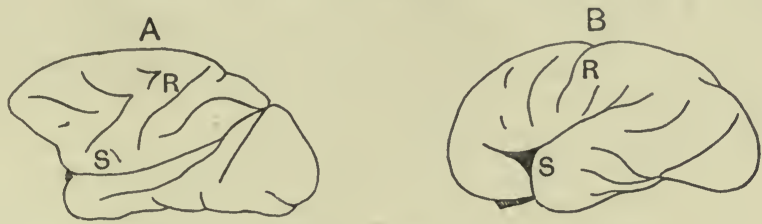

Frg. 427.

A. Cerebral Hemisphere of adult Macacque monkey.

B. Cerebral Hemisphere of child shortly before birth.

The two biains are very much alike, but the growth forwards of the frontal lobes even at this early stage of development of the human brain is quite well seen. $S$, fissure of Sylvius; $R$, fissure of Rolando.

scale and in the development of the human fœtus, are the same. They remain in the adult as the deepest and best-marked sulci; they are called the primary fissures or sulci, and they divide the brain into

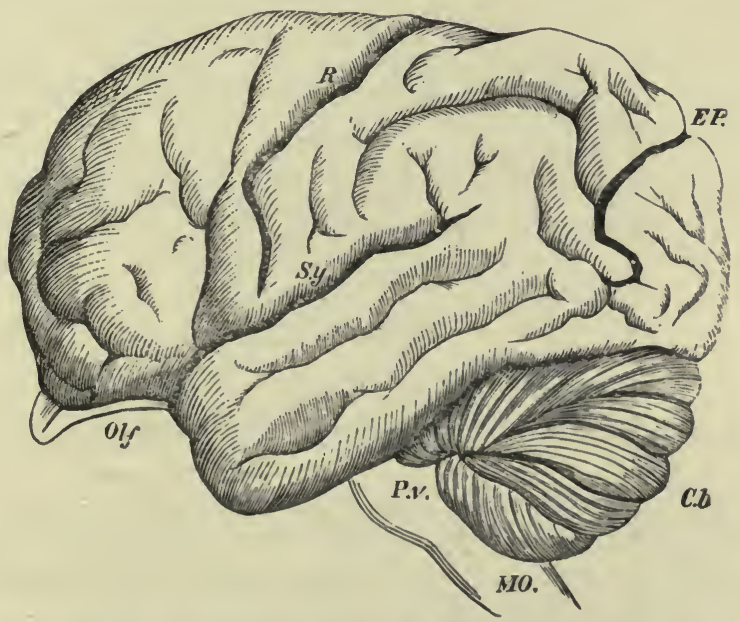

Fig, 428.-Brain of the Orang, I natural size, showing the arrangement of the convolutions. Sy, fissure of Sylvius; $R$, fissure of Rolando; $E P$, external parieto-occipital fissure; Olf, olfactory lobe; $C b$, cerebellum; $P V$, pons Varolii ; $M O$, medulla oblongata. As contrasted with the human brain, the frontal lobe is short and small relatively, the fissure of Sylvius is oblique, the temporo-sphenoldal lobe very prominent, and the external parieto-occipital fissure very well marked. Note also the bend or genu in the Rolandic fissure. This is found in all anthropoid apes.

lobes; the remaining sulci, called the secondary fissurcs or sulci, further subdivide each lobe into convolutions or gyri.

A first glance at an adult human brain reveals what appears to be a hopeless puzzle; this, however, is reduced to order when one 
studies the brain in different stages of development, or compares the brain of man with that of the lower animals. The monkey's brain in particular has given the key to the puzzle, because there the primary fissures are not obscured by the complexity and contoried arrangement of secondary fissures.

The preceding figure (fig. 427), comparing the brain of one of the lower monkeys with that of the child shortly before birth, shows the close family likeness in the two cases.

Fig. 428 gives a representation of the brain of one of the higher monkeys, the orang-outang, where there is an intermediate condition of complexity by which we are led lastly to the human brain.

Let us take first the outer surface of the human hemisphere; the primary fissures are-

1. The fissure of Sylvius ; this divides into two limbs, the posterior of which is the larger, and runs backwards and upwards, and the anterior limb, which, passing into the substance of the hemisphere, forms the Island of Reil.

2. The fissure of Rolando (the central fissure) runs from about

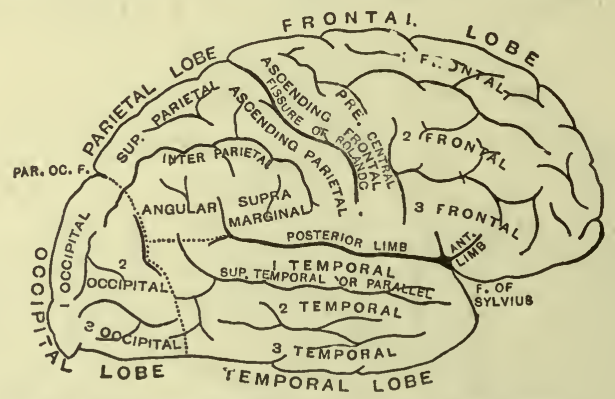

Fro. 429.-Right cerebral hemisphere, outer surface.

the middle of the top of the diagram (fig. 429), downwards and forwards.

3. The external parieto-occipital fissure (PAR. OC. F.) is parallel to the fissure of Rolando, but more posterior and much shorter; in monkeys it is longer (see fig. 428), as it is not interrupted by annectent gyri.

These three fissures divide the brain into five lobes:-

1. The frontal lobe; in front of the fissure of Rolando.

2. The parietal lobe; between the fissure of Rolando and the external parieto-occipital fissure.

3. The occipital lobe; behind the external parieto-occipital fissure.

4. The temporo-sphenoidal lobe; below the fissure of Sylvius.

5. The Island of Reil.

It will be noticed that the names of the lobes correspond to those of the bones of the cranial vault which cover them. There is no 
exact correspondence between the bones and the lobes, but the precise position of the various convolutions in relation to the surface of the skull is a matter of anatomy which, in these days of brain-surgery, is of overwhelming importance to the surgeon. The position of a localised disease in the brain can be determined very accurately, as we shall see later, by the symptoms exhibited by the patient, and it would be obviously inconvenient to the patient if the surgeon was unable to trephine over the exact spot under which the diseased convolution lies, but had to make a number of exploratory holes to find out where he was.

Each lobe is divided into convolutions by secondary fissures.

1. The frontal lobe is divided by the central frontal or precentral sulcus, which runs upwards parallel to the fissure of Rolando, and two transverse frontal sulci, upper and lower, into four convolutions; namely, the ascending frontal convolution, in front of the fissure of Rolando, and three transverse frontal convolutions, upper, middle, and lower, which run outwards and forwards from it.

2. The parietal lobe has one important secondary sulcus, at first running parallel to the fissure of Rolando and then turning back parallel to the margin of the brain. It is called the intra-parietal sulcus. The lobe is thus divided into the ascending parietal convolution behind the fissure of Rolando, the supra-marginal convolution between the intra-parietal sulcus, and the fissure of Sylvius, the angular convolution which turns round the end of the Sylvian fissure, and the superior parietal convolution, or parietal lobule, in front of the external parieto-occipital fissure.

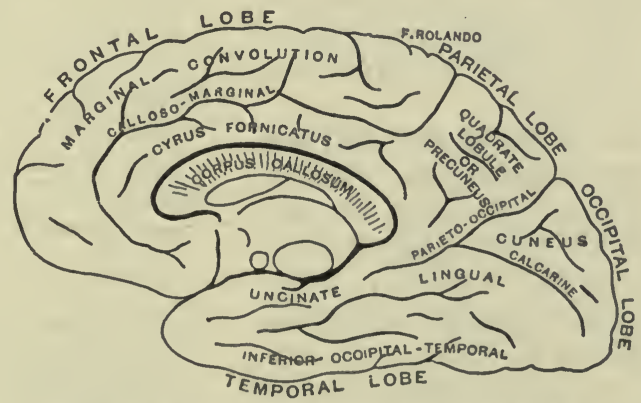

FIG. 430.-Right cerebral hemisphere, mesial surface.

3. The occipital lobe is divided into upper, middle, and lower occipital convolutions by two secondary fissures running across it.

4. The temporal or temporo-sphenoidal lobe is similarly divided into upper, middle, and lower temporal convolutions by two fissures running parallel to the fissure of Sylvius; the upper of these fissures is called the parallel fissure. 
5. The Island of Reil is divided into convolutions by the breaking up of the anterior limb of the Sylvian fissure.

Coming now to the mesial surface of the hemisphere (fig. 430), its subdivisions are made evident by cutting through the corpus callosum, which unites the hemisphere to its fellow. The subdivision into lobes is not so apparent here as on the external surface of the hemisphere, so we may pass at once to the convolutions into which it is broken up by fissures.

In the middle the corpus callosum is seen cut across; above it and parallel to its upper border is a fissure called the calloso-marginal fissure, which turns up and ends on the surface near the upper end of the fissure of Rolando. The convolution above this is called the marginal convolution, and the one below it the callosal convolution or gyrus fornicatus. The deep fissure below the corpus callosum running from its posterior end forwards and downwards is called the dentate fissure; this forms a projection seen in the interior of the lateral ventricle, and called there the hippocampus major; the hippocampal convolution, together with the gyrus fornicatus above the corpus callosum, constitutes the limbic lobe. Bolow the dentate fissure is another called the collateral fissure, above which is the uncinate convolution, and below which is the inferior temporal convolution which we have previously seen on the external surface of the hemisphere (see fig. 429). In the occipital region the internal parietooccipital fissure, which is a continuation of the external parieto-occipital fissure, passes downwards and forwards till it meets the calcarine fissure, which is a primary fissure; these two enclose between them a wedge-shaped piece of brain called the cuneus or cuneate lobule; the square piece above it is called the precuneus or quadrilateral lobule.

The only convolutions now left are those which are placed on the surface of the frontal lobe that rests on the orbital plate of the frontal bone; they are shown in fig. $395,22^{\prime} 2^{\prime \prime}$ (p. 642), and may be seen diagrammatically in fig. 431 , the end of the temporal lobe being cut off to expose the convolutions of the central lobe or Island of Reil.

Along the edge is the continuation

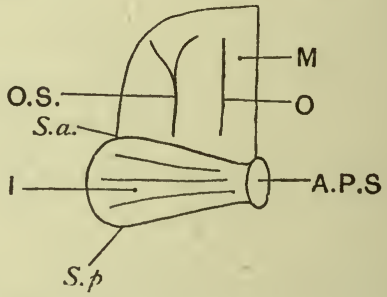

FIG. 431.-Orbital surface of frontal lobe.

M, marginal convolution.

$\mathrm{O}$, olfactory sulcus.

O.S., orbital sulcus.

I, Island of Reil.

S.a., anterior limb of Sylvian fissure.

S.p., posterior limb of Sylvian fissure. A.P.S., anterior perforated spot. of the marginal convolution (M); next comes the olfactory sulcus (0), in which the olfactory tract and bulb lie; then the triradiate orbital sulcus (0.s.), which divides the rest of this surface into three convolutions. 


\section{Histological Structure of the Cerebral Cortex.}

The possibility of differentiating the cerebral cortex in relation to its functions took origin in the work of Bevan Lewis and Henry Clarke in 1878, and the subject has in recent years received attention

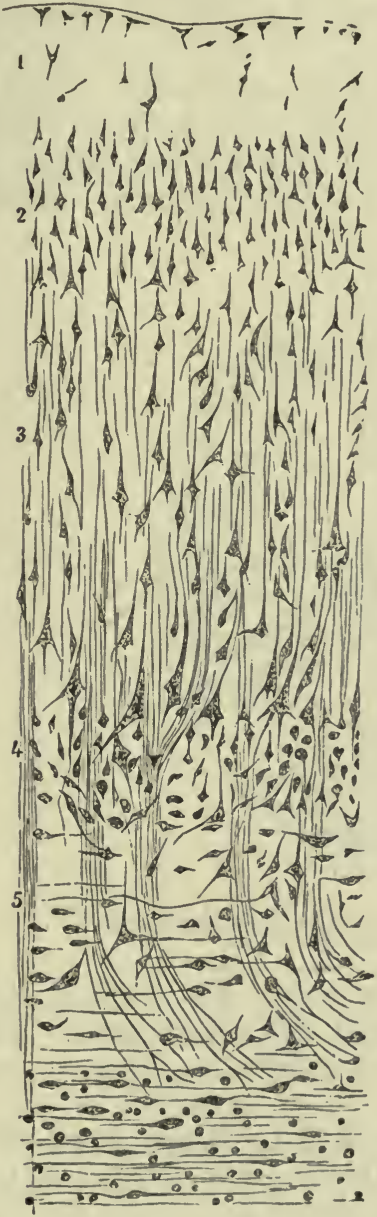

Fic, 432,-The layers of the cortical grey matter of the cerebrum. (Meynert.)

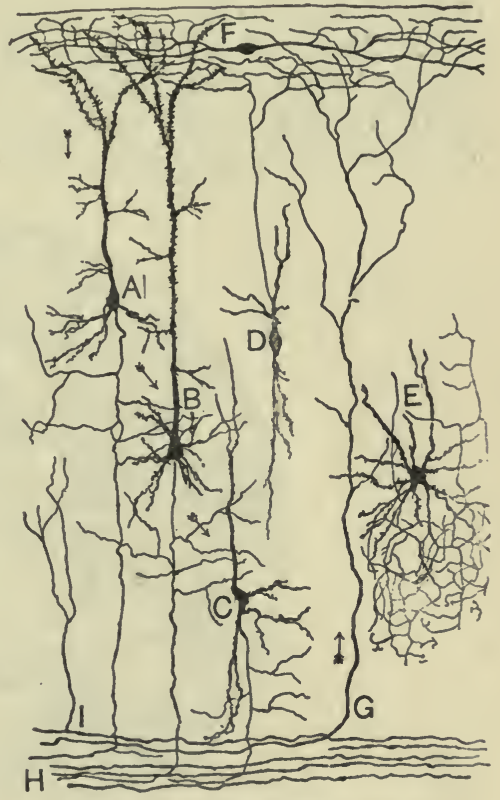

Fic. 433.-Principal types of cells in the cerebral cortex.

A, medium-sized pyramidal cell of the second layer.

B, large pyramidal cell.

C, polymorphic cell.

$D$, cell of which the axis.cylinder process is ascending.

E, neuroglia cell.

$\mathrm{F}$, cell of the first layer, forming an intermediate cell-station between sensory tibres and motor cells. Notice the tangential direction of the nervefibres.

G, sensory flbre from the white matter.

$\mathrm{H}$, white matter.

1, collateral of the white matter. (Ramon y Cajal.)

at the hands of A. W. Campbell, Brodmann, J. S. Bolton, and others. Certain main features have been established, although unanimity on the interpretation of all the facts has not yet been reached. 
The cortex may be divided into five primary laminæ:-

1. The outer fibre layer or superficial lamina.-The fibres are largely derived from the dendrons of the cells of the next layer. The nerve-cells ( $\mathrm{F}$ in fig. 433) intermingled with these are branched, and have several processes which lie horizontally beneath the surface (tangential fibres). There are doubtless association units linking the incoming afferent neurons to those which are motor. Neuroglia cells are also present.

2. The outer cell lamina or layer of small pyramids.-There are several deep, and the largest cells are situated most deeply. Each of these has an apical process running to the surface, where the branches run tangentially. The lateral processes are also branched

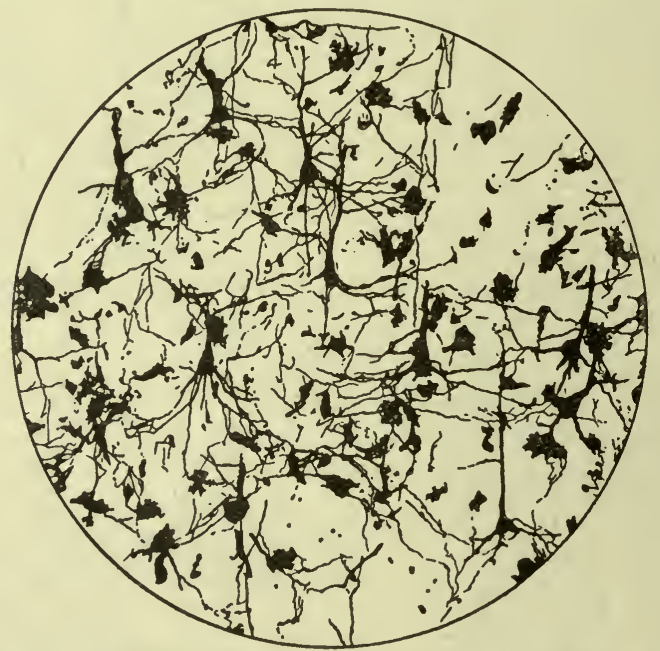

Fra. 434. - Human cerebral cortex : Golgi's method. Low power. (Mott.)

dendrons. The axon originates from the base. The layer of small pyramids increases in depth as we ascend the animal scale. They are believed to be association units subserving the higher mental processes.

3. The middle cell lamina (numbered 4 in figure 432).-This consists of small cells called granules. This layer is a distinguishing mark of sensory areas, and is practically absent in the pre-Rolandic or motor convolutions.

4. The inner fibre layer. - In certain regions of the cortex this contains the giant pyramids or Betz cells, which are characteristic of the motor areas. In the visual cortex the so-called solitary cells of Meynert are present here. 
5. The inner cell lamina or polymorphic layer.-These are small scattered cells, many of a fusiform shape. In the Island of Reil this layer is hypertrophied, and is separated from the rest of the grey matter by a stratum of white fibres; it is known then as the claustrum.

The Golgi method of staining has proved conspicuously useful for studying the shapes and dispositions of the cells (figs. 433, 434, 435).

Nerve-fibres pass in vertical streaks through the deeper layers of the grey matter; some of these are axons conveying impulses downwards, others are sensory in function and carry impulses upwards. Some strands lie parallel to the surface of the cortex; this is specially noticeable in the layers we have numbered 1 and 4 . In

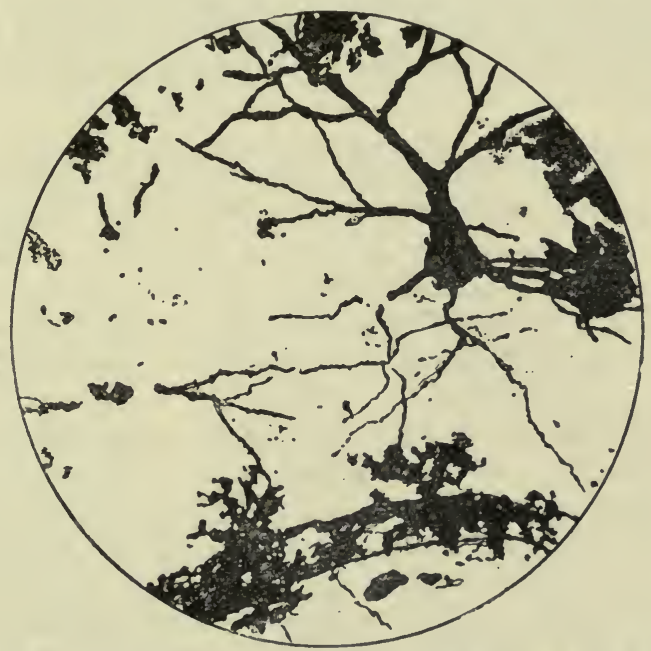

FIG 435.- II :man cerebral cortex, showing a Betz cell or giant pyramid: Golgi's method. High power. (Mott.)

the portion of the occipital lobe which is the visual sphere, the granule layer (No. 3), which is so specially characteristic of sensory function, is of great depth and is divided into two by optic radiations running horizontally, which constitute the white line of Gennari.

Bolton regards the fifth lamina as the fundamental cell layer, the others being formed from it from within outwards, both in embryonic and historical development. Defect of development of the outer layers leads to various forms of amentia (inborn lack of mental development, or idiocy); in dementic (degenerative mental change coming on later in life) there are retrograde changes in the upper layers of cells. The fifth or inner cell layer is probably concerned with the performance of organic and instinctive activities, and there is but 
little difference seen here between man, monkey, and dog. The second layer, on the other hand, is concerned with the psychic or associational functions, and attains its maximum depth in man.

The archipallium (p. 646) is the portion of the cortex which makes it appearance earliest in vertebrates, and is associated with the rhinencephalon or olfactory lobe of the pala-encephalon. In mammals it is reduced to small proportions in comparison with the rest of the cortex, which is termed, on account of its later appearance in historical development, the neopallium. In man, the archipallium is doubled in to form the hippocampus major, which projects into the lateral ventricle; this is continuous externally around the dentate sulcus with the gyrus hippocampi. This part of the cortex is easily distinguishable from the neopallium, being much simpler in structure - the pyramids, for instance, are reduced to a single layer, and the smaller cells nearer the surface are grouped in a characteristic nestlike way.

\section{The White Matter of the Cerebrum.}

The white matter of the cerebrum, like white matter elsewhere, is made up of medullated nerve fibres. According to the direction of the fibres, they may be divided into three principal groups :-

1. Association fibres.-These pass from convolution to convolution, and the principal bundles of these are shown semi-diagrammatically in fig. 436.

2. Commissural fibres.-These pass by the commissures of the brain, of which the most important is the corpus callorum, so as to link the convolutions of one hemisphere with the corresponding convolutions in the opposite hemisphere, where they terminate in arborisations (synapses) around the cells of the grey cortex.

3. Projection fibres.-These are the fibres which run more or less vertically and link the cerebrum to the lower portions of the central nervous system. They may be subdivided into the efferent projection fibres, which convey impulses downwards, and the afferent projection fibres, which convey impulses upwards. They are shown semi-diagrammatically in fig. 437.

The term projection fibre almost explains itself. By means of the efferent projection system the cerebrum is able to project its impulses to the cord, and thence to the muscles at the periphery. By means of the afferent projection system the surface of the body, provided as it is with sense organs, is able to project its impulses to the seat of sensation.

The efferent projection system.- The most important portion of this is the cerebro-spinal motor tract. The cells of the cortex which give rise to these fibres are par excellence the Betz cells, which are found in the fourth layer in the region of the brain, known as the 


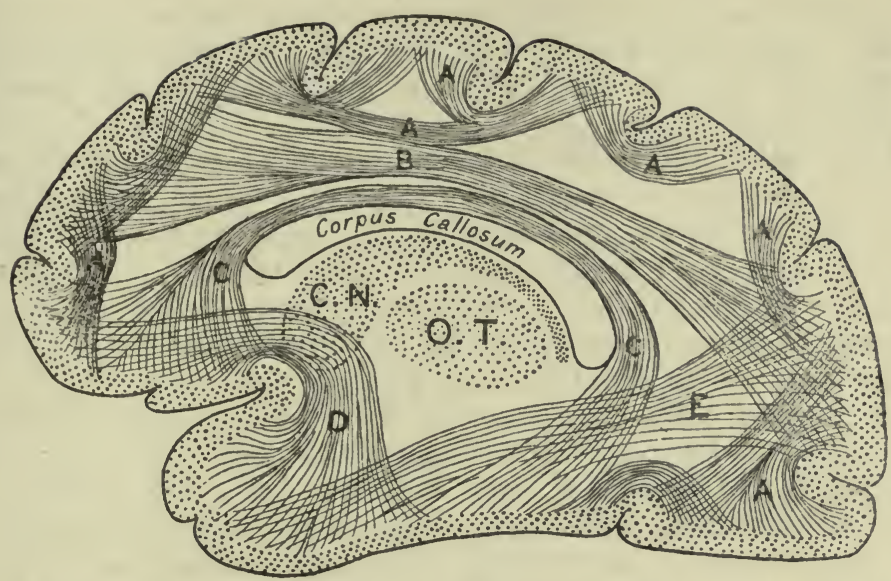

FrG. 436.-Lateral view of a human hemisphere, showing the main bundles of association fibres (Starr). A, A, between adjacent convolutions; B, between frontal and occipital areas; C, between frontal and temporal areas (cingulum); D, between frontal and temporal areas (fasciculus uncinatus); $\mathrm{E}$, between occipital and temporal areas (fasciculus longitudinalis inferior); C.N., caudate nucleus; O.T., optic thalamus.

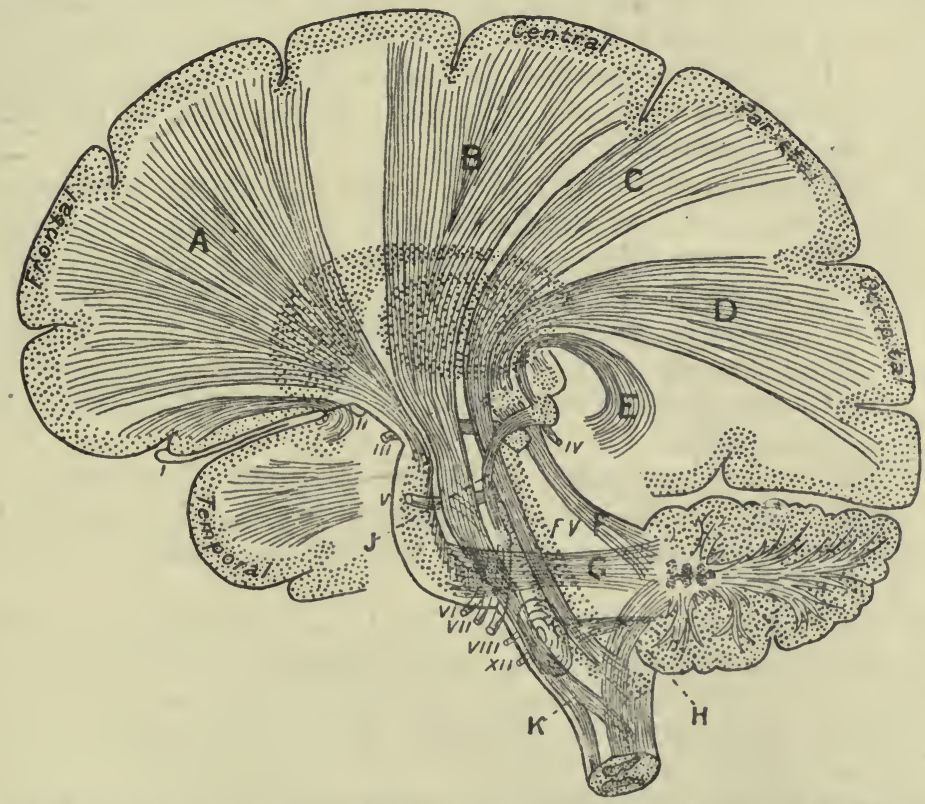

Fia. 437.-Schema of the projection fibres within the brain (Starr). A, tract from the frontal gyri to the pons nuclei and so to the cerebellum; $B$, motor pyramidal tract; $C$, sensory tract for touch; D, visual tract; E, auditory tract; F, G, H, superior, middle, and inferior cerebellar peduncles; J, fibres between the auditory nucleus and the inferior corpus quadrigeminum; $\mathrm{K}$, motor decussation in the bulb; F.V., fourth ventricle. The numerals refer to the cranial nerves. The sensory radia. tions are seen to be massed towards the occipital end of the hemisphere. 
motor area (the convolutions immediately in front of the Rolandic fissure). The axon of each of these giant pyramids originates from its base, and fig. 438 (next page) shows in outline the destination of the fibres.

1 is a cell of the motor or Rolandic area of the cerebral cortex; its axon $(A x)$ passes down in the pyramidal tract, and crosses the middle line (oo) at the pyramidal decussation. It gives off collaterals, one of which (assoc.) is an association fibre passing to terminate in the cortex of a neighbouring convolution; another, labelled commis, is a commissural fibre passing in the corpus callosum to the opposite hemisphere; others pass into basal ganglia.

In the cord, collaterals pass off to end in synapses around cells at the base of the posterior horn, and the main fibre has a similar termination; from each of these posterior horn cells, a short axon passes to end in an arborisation around an anterior cornual cell; only one of these is shown; the motor nerve-fibre passes from this to muscular fibres, to terminate in end-plates there.

The pyramidal cell numbered 2 is taken to illustrate the similar relationships between the cortex and muscles supplied by cranial nerves; its axon is represented as ending in the motor nucleus of the seventh nerve, and the new axon arising there passes to facemuscles. In order to prevent confusion in the diagram, cell 2 is placed in one of the upper convolutions; the face area of the cortex is really below those for the limbs.

The cell numbered 3 illustrates the fact that certain axons never reach the spinal cord, but terminate in the grey matter of the midbrain and pons; such fibres may therefore be called cortico-pontine, in contradistinction to the pyramidal fibres, which are cortico-spinal. From these subsidiary masses of grey matter in the pons and midbrain, new tracts, such as the bundle of Monakow or prepyramidal tract, arise (ponto-spinal fibres), which continue on the impulse to the anterior cornual cells.

No attempt has been made in this diagram to insert autonomic fibres with their accessory cell-stations in ganglia outside the central nervous systems. For these the reader is referred back to Chap. XVII.

The afferent projection system.-This is also indicated in outline in the same diagram (fig. 438). A is a cell of one of the spinal ganglia on the posterior nerve-roots; its peripheral axon terminates in muscular tendons or in skin, and the impulse it conducts is afferent, as shown by the arrow. The central axon passes into the spinal cord, and its impulse ultimately arrives at the cortex of the opposite side through several intermediate cell-stations. The last relay on the sensory path passes from O.T., the optic thalamus, to the cortex, and is linked up to the motor cell (1) by the associa- 
tion unit (4) in the superficial layer of the grey matter. Its intervening course from spinal ganglion to optic thalamus is, to save confusion in the diagram, represented by a dotted line. The diagram on the next page (fig. 439) fills in the details of the sensory path.

Beginning at the lower part of the diagram (fig. 439) on the right-

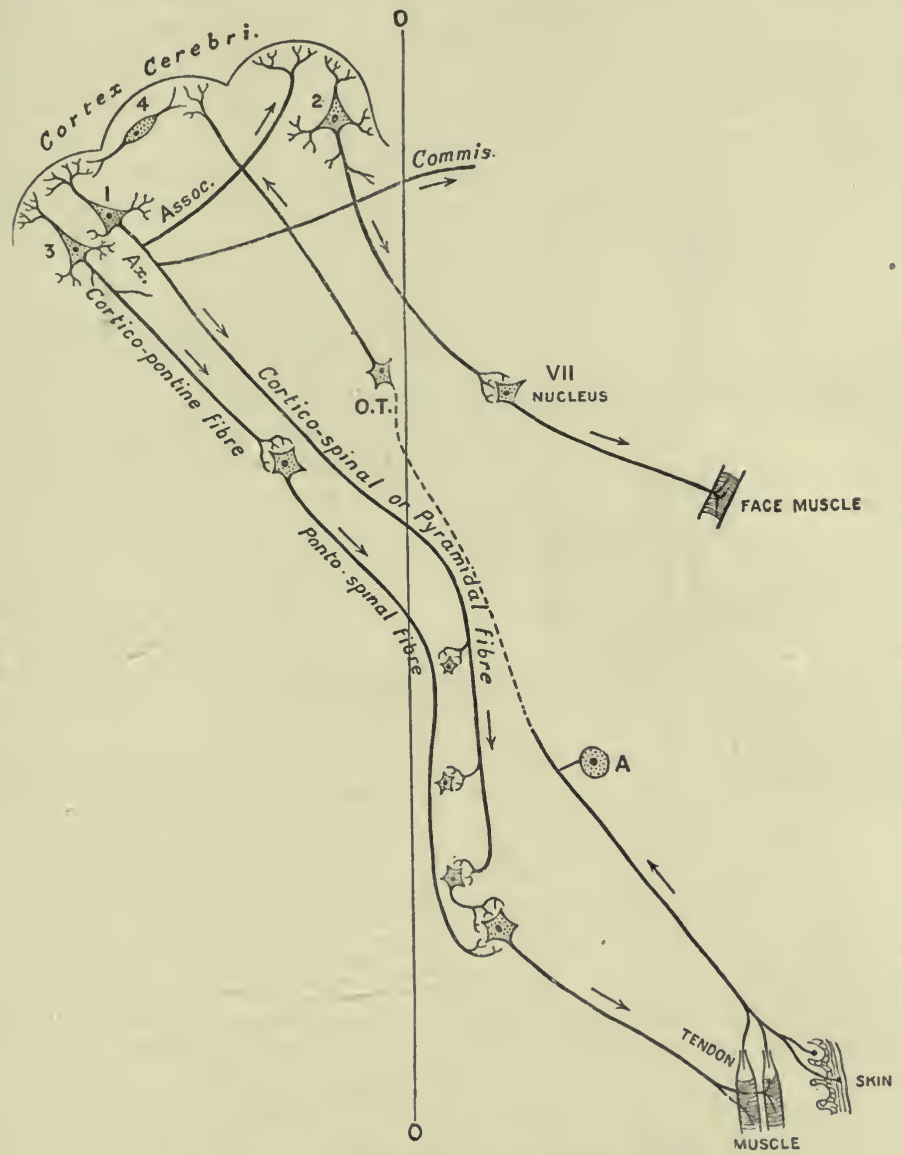

Fig. 438.-Diagram of the principal efferent channels.

hand side, we see one of the cells of a spinal ganglion. Its central axon enters the cord, runs up the posterior column, and terminates in one or other of the dorsal column nuclei (gracilis or cuneatus); the new axons arising there, called arcuate fibres, cross the middle line and ascend as fibres of the main fillet to the optic thalamus; from which, as we have seen, a new relay carries on the impulse to the cortex. 


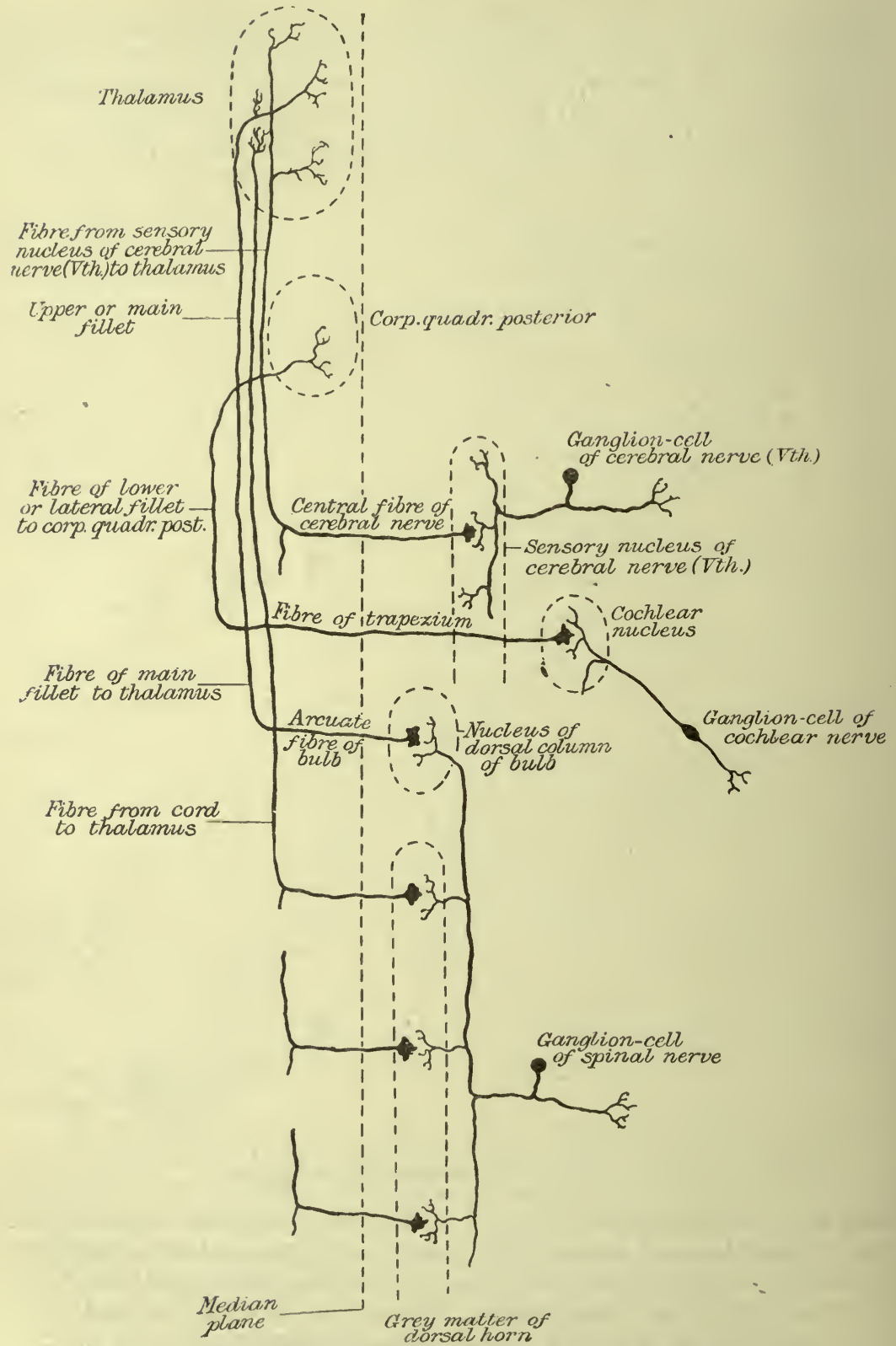

FIG. 439.-Diagram of principal sensory channels. (E. A. Schäfer.) 
In the cord, however, it gives off many collaterals, some of which arborise around anterior horn cells of the same or the opposite side, to form the basis of spinal reflex action. Others are shown crossing the middle line, and they ultimately reach the thalamus by the ascending tracts of the opposite side of the spinal cord and bulb.

The arrangement of the sensory cranial nerves is very similar; a cell of the Gasserian ganglion is seen sending its peripheral axon to the face region in the fifth nerve, and its central axon to arborise around the cells of the sensory nucleus of the fifth nerve in the bulb. From these cells the second relay carries on the impulse to the thalamus.

The arrangement of the cochlear nerve is very similar; the second relay, via trapezium and lateral fillet, carries the impulse, however, to the posterior corpus quadrigeminum instead of to the optic thalamus; a third relay, not shown in the diagram, completes the journey from this mass of grey matter to the cortex.

The connections of the cord and cerebrum to the cerebellum we have previously studied (see fig. 422, p. 684), and so they are not shown in the present diagrams.

Particular attention should be paid to the following point: when an afferent fibre enters the spinal cord, it divides into three main sets of branches. The first set, the shortest, forms synapses with the motor cells of the anterior horn; here we have the anatomical basis of spinal reflex action. The second set passes through an intermediate cell-station in Clarke's column to the cerebellum, the emerging fibres from which also influence the motor discharge of the cortical and anterior horn cells. The third set, the longest, passes through three intermediate cell-stations (the first in the nucleus gracilis or cuneatus, the second in the optic thalamus, the third in the association units in the cortex), and ultimately reaches the pyramidal nerve-cells of the cerebral cortex, the efferent fibres (pyramidal fibres) of which pass to the motor cells of the anterior cornu and influence their discharge. The motor nerve-cells of the anterior horn may thus be influenced by the afferent impulses via three paths or nervous circles. In health, all these nervous circles are in action to produce coördinated muscular impulses. In locomotor ataxy, which is a degeneration of the cells of the ganglia on the posterior roots and their branches, all these nervous circles are deranged, and the result is loss of reflex action, and incoördination of muscular movements. 


\section{CHAPTER XLIX}

FUNCTIONS OF THE SPINAL CORD

THe functions of the spinal cord fall into two categories: functions of the grey matter, which consist in the conversion of afferent into efferent impulses (reflex action); and functions of the white matter, which are those of conduction.

\section{The Cord as an Organ of Conduction.}

The fibres of the spinal cord consist of three main groups; these are-(1) the association tracts, which connect together different segments of the cord and thus bring about coördination of the impulses, which leave it at various levels, in relationship to the impulses which enter the cord either below or above any particular region; (2) the efferent projection fibres, which connect the cord with the different parts of the brain above it. The main motor path, the pyramidal tract, comes into this category; and after our full description of its course, we need not do more here than remind the reader that it originates from the giant pyramids of the motor area of the cortex, and that its fibres cross to the opposite side of the spinal cord, the principal decussation occurring at the lowest level of the bulb; from the grey matter in which it terminates in the cord, the impulse is continued onwards, until in the end it reaches the muscles via the fibres which leave the cord in the anterior nerve-roots; (3) the afferent projection system of fibres; these primarily enter the cord by the posterior spinal nerve-roots. The impulses which these convey enter $(a)$ the spinal grey matter, $(b)$ the cerebellum, and $(c)$ the cerebrum, the seat of consciousness.

It is these sensory tracts which are the most complex, not only on account of the cell-stations in their course, but also on account of the difficulty of determining in animals the different kinds of sensations which are present in health, or which may be absent after injury to various tracts. It is, however, certain that ultimately, so far as the cerebrum is concerned, crossing occurs somewhere, so that 
each hemisphere is related to the opposite half of the body, not only in regard to motion but in regard to sensation also. The main difficulty of investigators has been to determine exactly where the crossing occurs; in man especially, many of the impulses cross shortly after their entry into the cord, and then travel up to the higher centres by paths on the opposite side of the cord to that by which they enter. In such investigations on man, various injuries to the cord have to be carefully studied, and it is such careful study that has led to this conclusion.

For fifty years, physiologists, stimulated by the work of BrownSéquard, have attempted to trace the upward paths of afferent impulses through the spinal cord. By experiments on animals, the secondary ascending degenerations which follow injury can be followed with exactitude, and traced into bulb, cerebrum, cerebellum, or other parts. But even though such animals may be long under observation, they cannot tell us, and we can only with difficulty and ill-success guess, how their sensations are affected by the lesion.

On the other hand, the clinical observer may expend much time and trouble in determining the nature of the loss of sensation, but it is usually impossible to verify the anatomical position and nature of the lesion. In many cases where a microscopical examination has been made, the disease has been of a progressive nature, or the patient has died from complications, which detract from his suitability for an examination of this kind.

Nevertheless, by a combination of the experimental and clinical methods, we have now arrived at some accuracy on these points, and it is impossible to overestimate in this direction the value of the psycho-physical examination of patients which has within the last fow years been made by Head, Rivers, and Sherren.

In order, however, to examine the sensations of a patient, it is necessary to know first how to classify the sensations which are the result of cutaneous stimulation. To do this, we must somewhat anticipate what we shall go into more fully in the chapter on the Cutaneous Senses. It has been proved that, scattered over the external surface of the body, are a number of spots, some which are more susceptible to one form of stimulus than others. The spots are of four kinds, known as touch spots, pain spots, heat spots, and cold spots, and these correspond to the four kinds of sensations which we experience as the result of cutaneous excitation. They correspond to different kinds of end-organs in the skin, and the impulses are carried to the central nervous system by different groups of fibres.

Dr Head found, from an experiment he made by cutting a sensory cutaneous nerve in his own arm, that although the patch of skin supplied by the nerve was entirely destitute of sensation, the underlying parts were still sensitive to pressure and to pain. These deep 
sensations are subserved by norve-fibres which are distributed with the muscular nerves. It is by means of the sensory nerves of muscles, tendons, and joints that we are aware of the position of our limbs, and the extent of muscular contraction.

After a time true cutaneous sensation returned when the severed nerve regenerated, but it was not until many months elapsed that sensation was as sharp and as accurately localised as it was before the nerve had been cut. The first sensations that returned enabled Head to feel pain, to distinguish large differences of temperature, and to localise the position of a touch somewhat inaccurately. Head terms such imperfect sensations protopathic. The ability to localise accurately, to distinguish small differences of temperature and the finer distinctions generally of cutaneous sensations, returned later, and are spoken of as epicritic. We may thus divide the main sensations coming from the periphery of the body into deep and cutaneous; and the cutaneous sensations into protopathic and epicritic. The other classification into sensations of touch, heat, cold, and pain, cuts across the first; thus we may have pain that is of deep or of cutaneous origin; we may have temperature sensations which are both rough or protopathic, and accurate or epicritic; and we may feel pressure and localise it by means of the cutaneous sense proper, or by the stimulation of the sensory nerves in the deeper structures. Whether different nerve-fibres are concerned in the transmission of protopathic and epicritic impulses is a matter of doubt. It is quite possible that the same fibres may be concerned in the transmission of both.

Diseases of the spinal cord in man usually are widespread and affect many tracts; the disorders of muscular paralysis and of sensation thus produced will therefore be complex. The more limited the lesion, the fewer tracts will be affected, and such conditions are therefore more on all fours with these localised lesions or sections of tracts which can be performed on animals. The operation of hemisection in an animal produces paralysis of the same side of the body below the injury. So it is in a man in whom disease has produced an interruption of the pathways on one side only of the cord. But such an animal or man (and the observation is more accurate in man) will not have lost all sensation on the same side; tactile discrimination, and the motorial sense will have largely disappeared, but sensations of pain, of heat, and of cold will still remain, because the tracts which convey such impulses cross over in the cord at varying levels after entering it, and therefore any loss in such sensations will occur on the opposite side to that which is injured.

It was no doubt absence of correct knowledge on this question that led Schiff to imagine that impulses translated by the brain, as sensations of temperature and pain, travelled up by the grey matter, 
and not by the posterior columns. It certainly is the case that in the condition called syringomyelia (a disease of the grey matter of the cord), sensations of heat, cold, and pain are lost, but this is due to the disease cutting through the crossing fibres which convey the impulses in question. Head has pointed out that disease strictly limited to the grey matter does not produce loss of any kind of sensation, except by interfering with those paths which pass through its substance.

We have seen that afferent impulses pass into the cord by peripheral nerves (the primary or peripheral level) in certain combinations from the protopathic, epicritic, and deep systems. In the spinal cord these are sorted out and travel up in new combinations, and it is possible that before they finally reach the cortex a fresh sorting may take place in higher cell-stations before they ultimately arrive at the seat of consciousness. The first rearrangement occurs at the secondary level, that is on entrance into the cord, and a further sorting at the third level, but of this but comparatively little is known at present. We shall here only deal with the rearrangement at the second level, as derived from a study of spinal cord disease.

We may make a rough comparison of what occurs, to what takes place in the correspondence which flows in from all quarters to a busy man, such as a Secretary of State. The letters will come from all quarters, and deal with numerous topics; some will be private letters, some will be advertisements, some will be official, some begging letters, and so forth. This mass of correspondence will be sorted out by minor officials, the advertisements and the begging letters will probably never reach the busy officer of State, and he will therefore not be conscious of their existence unless he examines the waste-paper basket. But the private letters and the official letters will be sorted out into separate bags, whether they come from England or from outlying parts of the Empire, and these ultimately reach his eye. In the same way, the impulses that give rise to pain, whether from cutaneous or deep structures, will all be combined and travel up one path. Those due to heat or to cold, whether protopathic or epicritic, in other paths; those which are tactile or motorial, in another. And so a localised spinal lesion may interrupt all the fibres subserving the sensation of heat without interfering with those which underlie sensations of cold, and so forth.

Tactile, painful, and thermal impulses, and those associated with tactile localisation, cross in their passage through the spinal cord at varying levels soon after entrance. But the sensory impulses which underlie the recognition of passive position and movement, and finer tactile discrimination, do not cross within the limits of the spinal cord; they pass up the posterior column on the side of entrance, and 
so reach the gracile and cuneate nuclei of the bulb, and it is the fibres which arise from the cells of these nuclei which cross in the decussation of the fillet.

The rapidity with which the sensory impulses cross to the opposite side varies greatly. Some, such as those associated with pain, heat, and cold, cross over in the space of five or six spinal segments. With tactile impulses the crossing is evidently less rapid; but until the crossing is completed there will obviously be two channels, one on each side of the cord, open for tactile impulses; and such a double path will be shorter for the impulses which cross more rapidly; and finally, as just stated, the impulses associated with position, movement, and tactile discrimination have only one path in the cord, as the decussation does not take place until the bulb is reached; hence a hemisection of the cord, or of one posterior column, will abolish these forms of sensibility from the parts below the lesion, on the same side of the body as the lesion.

Painful impulses from the skin arriving in the cord from protopathic fibres pass into the second level at the point of entry, and rapidly cross over to the other side. Fibres of the deep system running with the muscular nerves and carrying impulses also of a painful kind from the same part of the body do not necessarily enter the cord by the same posterior roots as those carrying cutaneous painful stimuli. Thus more than one segment of the cord is required before all painful impulses from any one part of the body can be gathered together and recombined. This is the reason why, in a local lesion in the cord, there may be a want of correspondence between the extent of the cutaneous and deep analgesia (loss of sensation to pain).

Up to this point we have only considered the sorting out of those impulses which reach the cerebrum and thus rise into consciousness. In addition to this there is another group of impulses which never rise into consciousness at all, and although these are afferent they are therefore not sensory.

Our previous illustration of the correspondence of a busy man may help us again in understanding this. His clerks sort his letters, and those of a certain kind (circulars and the like) will probably never reach him at all. So it is with afferent impulses; the primary sorting is into sensory and non-sensory; the sensory impulses are again sorted into those of touch, pain, and temperature; the nonsensory impulses are those mainly destined for the cerebellum, and reach it by the cerebellar tracts. These travel up the cord on the side of entry, and reach the same side of the cerebellum. This explains the delay in the crossing of the sensory impulses which subserve the sense of position and movement and tactile discrimination, a crossing which, as we have seen, does not occur until the bulb 
is reached. It is impulses from the joints and muscles which are specially important for the cerebellum to enable it to carry out its functions of equilibration and coördination of muscular movements. These impulses are non-sensory, but they are carried by fibres which originate as collaterals from those which carry the true sensory impulses of the same nature to the cerebrum. This group of fibres therefore remains in the cord on the side of entry, in order to be in the neighbourhood of the cerebellar tracts; the impulses reach the cerebellar tracts with the intermediation of a cell-station in Clarke's column. When the tract conveying what we may term the motorial sensations to the cerebrum has ministered in this way to the needs of the cerebellum, there is nothing to prevent it following the example of the other tracts, so when the spinal cord is passed and the bulb reached, crossing of these fibres occurs in due course.

To sum up-the spinal cord is the seat of the transmutation of most of the impulses of the first or peripheral level into those of the secondary level of the afferent projection system. This recombination takes place on the same side as that by which the impulses enter the cord. The secondary paths for sensory impulses then cross with greater or less rapidity, so that ultimately all except those subserving the sense of position and movement and tactile discrimination have passed to the opposite side within the limit of the spinal cord; and those which do not cross in the cord do so after reaching the nuclei of the posterior columns. At the same time, within the spinal cord afferent impulses become separated into sensory and non-sensory, and the latter are exemplified by those which reach the same side of the cerebellum by the cerebellar tracts.

\section{Reflex Action of the Spinal Cord.}

The reflex actions of the spinal cord may first be studied in the brainless frog. In such a low type of animal, the interdependence of cord and brain is not such a marked feature as it is in the higher animals, and the spinal cord possesses within itself a great power of controlling and coördinating very complex reflex actions. A study of the reactions of the frog's spinal cord, moreover, illustrates most of the fundamental facts in relation to reflex action generally.

After destruction of the brain the shock of the operation renders the animal for a variable time motionless and irresponsive to stimuli, but later on it gradually assumes a position which differs but little from that of a living conscious frog. If thrown into water it will swim; if placed on a slanting board it will crawl up it (Goltz); if stroked on the flanks it will croak (Goltz); if it is laid on its back, and a small piese of blotting-paper moistened with acid be placed on the skin, it will generally succeed in kicking it off; if a 
foot is pinched it will draw the foot away; if left perfectly quiet it remains motionless.

The muscular response that follows an excitation of the surface is purposive and constant, the path along which the impulse is propagated being definite.

Under certain abnormal conditions, however, the propagation of the impulse in the cord is widespread, the normal paths being, as it were, broken down. This is seen in the convulsions that occur on slight excitation in animals or men who have suffered from profuse hæmorrhage, or in the disease called lockjaw or tetanus. Such a condition is easily demonstrable in a brainless frog under the influence of strychnine: after the injection of a few drops of a 1 per cent. solution under the skin, cutaneous excitation no longer produces coordinated responses, but paroxysms of convulsions, in which the frog assumes a characteristic attitude, with arms flexed and legs extended.

Spreading of reflexes.-If one lower limb is excited, it is that limb which responds: if the excitation is a strong one it will spread to the limb of the opposite side, and if stronger still, to the upper limbs also.

Pflüger taught that the direction of irradiation within the spinal cord was always upwards. Sherrington has shown that this is not so, and has discovered many descending paths (see p. 659).

Cumulation of reflexes. - This is well illustrated by Turck's method. If a number of beakers of water are prepared, acidulated with 1, 2, 4 , etc., parts of sulphuric acid per 1000, and the tips of the frog's toes are immersed in the weakest, the frog at first takes no notice of the fact, but in time the cumulation or summation of the sensory impulses causes the animal to withdraw its feet. If this is repeated with the stronger liquids in succession, the time that intervenes before the muscles respond becomes less and less. This method also serves to test reflex irritability when the frog is under the influence of various drugs.

Inhibition of reflexes. - If, instead of the whole brain, the cerebrum only is destroyed, and t? 10 optic lobes are left intact, response to excitation is much slower, the influence of the remaining part of the brain inhibiting the reflex action of the cord. Or if in doing the experiment with acid just described the toes of the other foot are being simultaneously pinched, the response to the acid is delayed.

This influence of the brain on the cord is also illustrated in man, by the fact that a strong effort of the will can control many reflex actions. It is, for instance, possible to subdue the tendency to sneeze; if one accidentally puts one's hand in a flame, the natural reflex is to withdraw it: yet it is well known that Cranmer, when being burnt at the stake, held his hand in the flames till it was consumed.

After the spinal cord has been divided by injury or disease in the thoracic region, the brain can no longer exert this controlling action; 
hence the part of the cord below the injury having it, as it were, all its own way, has its reflex irritability increased.* The increase of reflex irritability is also seen in the disease called lateral sclerosis; here the lateral columns, including the pyramidal tract, become degenerated, and so the path from the brain to the cells of the cord is in great measure destroyed. In these patients the increase of reflex irritability may become a very distressing symptom, slight excitations, like a movement of the bed-clothes, arousing powerful convulsive spasms of the legs.

\section{Reflex Action in Man.}

The reflexes obtainable in man form a most important factor in diagnosis of diseases of the nervous system; each action is effected through an afferent sensory nerve, a system of nerve-cells in the cord termed the reflex centre, and an efferent motor nerve; the whole constitutes what is called the reflex arc. The absence of certain reflexes may determine the position in the spinal cord which is the seat of disease.

Two forms of reflex action must be distinguished:-

1. Superficial reflexes. These are coördinated reflex actions, and are excited by stimulation of the skin.

2. Deep reflexes or tendon reflexes.

Superficial Reflexes.-These are obtained by a gentle stimulation, such as a touch on the skin; the muscles beneath are usually affected, but muscles at a distance may be affected also. Thus a prick near the knee will cause a reflex flexion of the hip.

The most important of these reflexes are:

a. Plantar reflex: withdrawal of the feet when the soles are tickled.

b. Gluteal reflex: a contraction in the gluteus when the skin over it is stimulated.

c. Cremasteric reflex: a retraction of the testicle when the skin on the inner side of the thigh is stimulated.

d. Abdominal reflex: a contraction of the muscles of the abdominal wall when the skin over the side of the abdomen is stroked; the upper part of this reflex is a very definite contraction at the epigastrium, and has been termed the epigastric reflex.

e. A series of similar reflex actions may be obtained in the muscles of the back, the highest being in the muscles of the scapula.

f. In the region of the cranial nerves the most important reflexes are those of the eye-(i) the conjunctival reflex, the movement of the

* In some injuries to the cord produced by crushing, there is a loss of reflexes below the injury. These, however, are not simple transverse lesions; the loss of reflex action is due partly to shock, and partly to extensive injury of the grey matter. 
eyelids when the front of the eyeball is touched; and (ii) the contraction of the pupil on exposure of the eye to light, and its dilatation on stimulation of the skin of the neck.

Tendon Reflexes.-When the muscles are in a state of slight tension, a tap on their tendons will cause them to contract. The

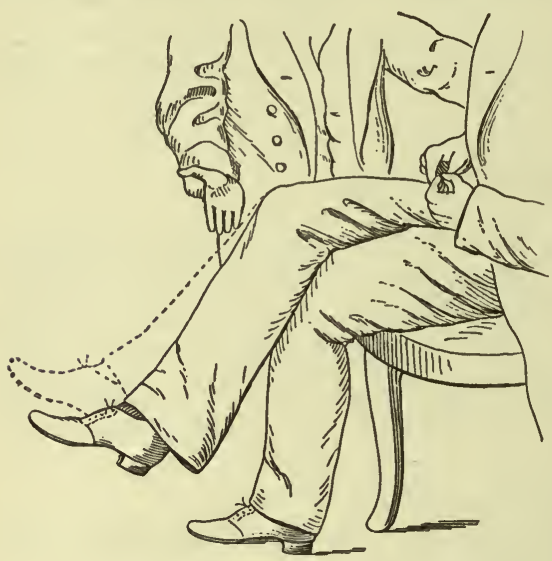

FIG. 410.-The Knee-jerk. (Gowers.) tendon reflexes which are generally examined are the patella tendon reflex or kneejerk, the tendo Achillis reflex or ankle-jerk, and phenomenon known as ankle-clonus.

The knee-jerk. - The quadriceps muscle is slightly stretched by putting one knee over the other; a slight blow on the patella tendon causes a movement of the foot forwards, as indicated in the dotted line of fig. 440. The phenomenon is present in health.

The ankle-jerk is one of great importance, for in such

diseases as locomotor ataxy, in which the tendon reflexes are lost, it usually disappears before the knee-jerk. It is best elicited if the patient kneels with one knee upon a cushioned chair, whilst standing on the other leg by the side of the chair. The calf muscles of the kneeling leg are thus relaxed, and a sharp tap upon the tendo Achillis elicits the jerk.

Ankle-clonus. - This is elicited as depicted in the next figure: the hand is pressed against the sole of the foot, the calf muscles are thus put on the stretch and they contract, and if the pressure is kept up a quick succession or clonic series

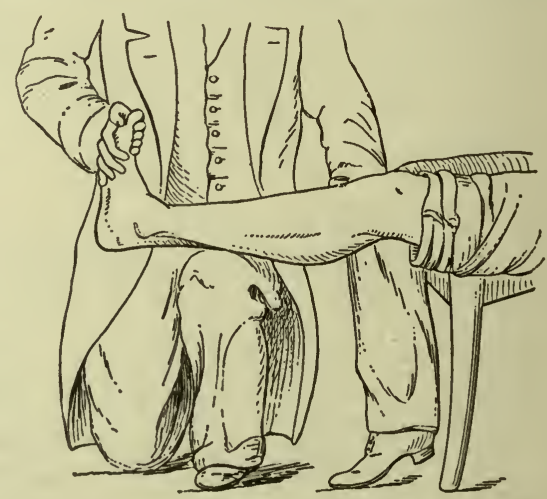

Fig. 441.-Ankle-clonus. (Gowers.) of contractions is obtained. This, however, is not readily obtained in health.

The phenomena depend for their occurrence on the integrity of the reflex arc. Disease or injury to the afferent nerve, efferent 
nerve, or spinal grey matter, abolishes them. Thus they cannot be obtained in locomotor ataxy (damage to the posterior nerve-roots), or in infantile paralysis (damage to the anterior horns of grey matter).

They are excessive in those conditions which increase reflex irritability, such as severance of brain from cord, and in lateral sclerosis, that is, a degenerative condition of the pyramidal tract.

In order that the muscle may respond, it is necessary that it be in an irritable condition; this is accomplished by putting it slightly on the stretch, and so calling forth the condition called tonus, and thus a readiness to contract on slight provocation. Until the last few years, considerable doubt existed as to whether the tendonreflexes were true reflex actions, because it was asserted that the time intervening between the stimulus and the muscular response was too short. Now that we know that nerve-impulses are in man propagated at the rate of 120 metres per second (and not at the rate of 30 metres per second as was formerly supposed), this difficulty has disappeared. Jolly has made careful time measurements, and demonstrated that in the knee-jerk there is sufficient time for the nerve impulse to travel to the spinal cord and back again, but that the time occupied in the cord itself is only about half that which is necessary in the case of ordinary coördinated reflex actions. This is explicable on the assumption that the tendon reflexes are subserved by the collaterals of the entering afferent fibres which go direct to the anterior horn cells, whereas ordinary reflexes are worked through intermediate neurons, and therefore have to pass through additional synapses.

The increased rapidity of a tendon reflex is useful, for a sudden strain on a ligament would rupture some of its fibres or lead to injury of the joint surfaces if too great a time intervened before the muscles could contract and so save the joint.

The exact course of the reflex arc concerned in the knee-jerk has been worked out by Sherrington in the monkey. The nerve-fibres are mainly those which pass (1) to and from the crureus by the anterior crural nerve, and (2) to and from the hamstrings by the sciatic nerve. The fibres which supply the crureus arise from the spinal nerve-roots which in man correspond to the 3rd and 4th lumbar; the hamstring supply is from the 5th lumbar and 1st and 2nd sacral roots.

Lombard's experiments upon the knee-jerk indicate that it is sometimes more readily obtained even in the same person than at other times. It varies with changes in mental activity, and during sleep may be entirely absent. It is increased and diminished by whatever increases or diminishes the relative state of irritability of the nervous system as a whole. 
Closely related to this is the phenomenon known as reinforcement of the knee-jerk, which was first described by Jendrassik in 1883, and has since been studied by numerous observers. The extent of the jerk may be increased if at the time the patella tendon is struck, a strong voluntary contraction, such as clenching the fists or the jaw, is made by the individual. In many normal persons the knee-jerk is difficult to elicit, but in these it may usually be obtained by the reinforcing action just described.

After the reinforcing action has occurred it is followed by an interval in which the knee-jerk is lessened (inhibition or negative reinforcement). Many explanations have been offered of the phenomenon; one is the "overflow" theory, that is, motor impulses from the brain which produce a contraction of hands or jaw will not only affect the lower centres concerned in such movements, but will also overflow to other regions, for instance, those which come into play in the knee-jerk and influence motor irritability there. The "drainage" theory of M'Dougall to be described a few paragraphs ahead may possibly explain reinforcement; the drainage of nervous potential to one part will lessen the resistance of the synaptic junctions and cause a drainage of nervous energy from other parts, and so allow reflex actions to be more readily elicited there.

We have devoted most space to the knee-jerk because this tendon reflex is the one which has been longest known and most worked at. The recent investigations of Babinski have, however, shown that other tendon phenomena may be quite as easily investigated, and in some cases are more valuable aids to diagnosis than the knee-jerk. Amongst these we have already referred to the ankle-jerk. Another phenomenon allied to these is usually called babinski's sign, or the extensor plantar reflex. If the sole of the foot is stroked, the usual response is flexion of the toes, and especially of the big toe. If the pyramidal tract is diseased, extension of the great toe occurs. This is a very delicate reaction, and occurs long before any exaggeration of the knee-jerk takes place. In new-born children the normal response is not obtained because their pyramidal tracts are not then fully developed. The weakness of the test, however, is that in many people it is difficult to elicit the reflex, and minor conditions such as cold feet interfere with it.

Among numerous other reactions there is one which Hertz proposes to call Babinski's second sign; it is this. If a normal person lies down with the legs widely separated and the arms folded, and tries to sit up, both legs rise from the ground to an equal extent. On letting himself fall back shurply both legs again rise equally from the ground. In hemiplegia due to organic disease, as soon as the patient is strong enough to make such an effort, the paralysed leg rises higher than the healthy limb. In hysterical hemiplegia, which often simulates the genuine disease, the paralysed leg remains flat on the ground, both on sitting up or lying down, as this is what an uninitiated person would expect. This has been found of much service in distinguishing the two conditions.

Reciprocal Action of Antagonistic Muscles.-This is an interesting branch of muscle physiology, which we owe to the researches of Sherrington. In brief, he shows that the inhibition of the tonus of a voluntary muscle may be brought about by excitation of its antagonist. 
Movement at a joint in any direction involves the shortening of one set of muscles and the elongation of another (antagonistic) set. The stretching of a muscle produced by the contraction of its antagonist mechanically stimulates the sensory nerve-endings in the muscle-spindles of the muscle that is under extension; in this way a reflex of pure muscular initiation may be started. Experiments show that electrical excitation of the central end of an exclusively muscular nerve produces inhibition of the tonus of its antagonist. For instance, the central end of the severed hamstring nerve is faradised. This nerve contains in the cat 4510 nerve-fibres, and of these about 1810 are sensory in function; * these come from the flexor muscles of the knee, not from the skin. The effect of the stimulation of the nerve on the tonus of the extensor muscles of the knee is seen $(a)$ in elongation of those muscles, $(b)$ in temporary diminution of the knee-jerk. The experiment may be varied as follows: the exposed flexor muscles detached from the knee, and therefore incapablo of mechanically affecting the position of the joint, are stretched or kneaded. This produces a reflex elongation of the extensor muscles of the knee and a temporary diminution of the knee-jerk. The effects are in fact the same as those produced by faradisation of the central ond of the nerve supplying them. It may therefore be that reciprocal innervation, which is a common form of coördination of antagonistic muscles, is secured by a simple reflex mechanism, an important factor in its execution being the tendency for the action of a inuscle to produce its own inhibition reflexly by mechanical stimulation of the sensory apparatus in its antagonist.

We have in our clescription of the anatomical path of the entering posterior roots drawn attention to what may be termed the three "nervous circles" by which an afferent impulse may affect the motor discharge from the anterior horn-cells of the cord; there is the short path by the collaterals of the entering fibre which pass directly to these cells, and there are the two longer paths, via the cerebellum and corebrum respectively. In the execution of a voluntary action all three circles are in activity to produce the coördination and due contraction and elongation of antagonistic muscles which characterise an effective muscular act. Section of the posterior roots produces not only an inability to carry out reflex actions, but also leads to an inability to carry out effectively those more complicated reflex actions which are called voluntary, and in which the brain participates. Locomotor ataxy, or tabes dorsalis, is a slowly progressive disease, the anatomical basis of which is a degeneration of the nerve-units of tho spinal ganglia. It is, therefore, analogous to a physiological

* The number of sensory nerve-fibres is determined by counting the healthy fibres in the nerve after section of the anterior nerve-roots. 
experiment in which the posterior roots are divided, and although some fibres may remain which still allow of the passage of nervous impulses, the action of the three circles is greatly interfered with; the spinal reflex arc is at fault; this is shown by the loss of reflex action, the disappearance of the tendon reflexes, and the want of tonus in antagonistic muscles; the main symptom of the disease is want of muscular coördination, and this is produced not only by the lesion in the spinal cord, but is accentuated by the want of continuity in the other two circles, so that the brain is unable to effectively control the motor discharge from the anterior cornual cells.

$M^{\prime}$ Dougall's "Drainage" theory.-This theory is an attempt to explain the reciprocal action of antagonistic muscles.

The accompanying diagram represents two antagonistic muscles (fig. 442) with

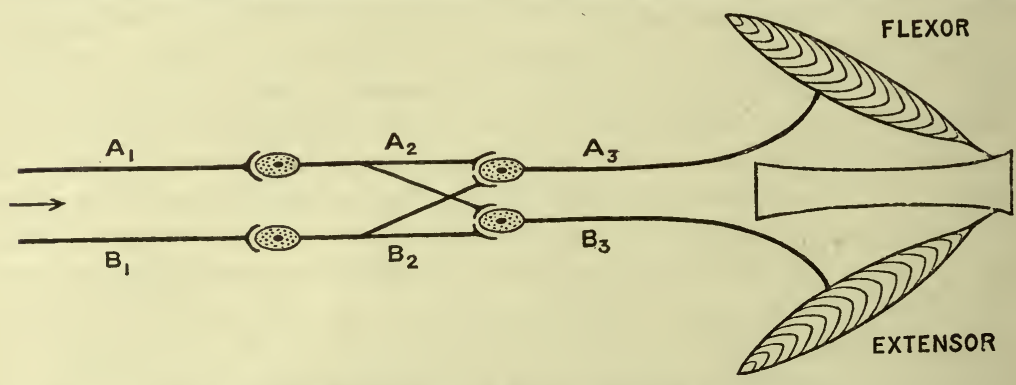

FIG. 442.-Diagram to illustrate M'Dougall's "Drainage" theory.

their nerve supplies. Each is in connection with a reflex arc shown in a simple schematic way, as consisting of three neurons, $A_{1}, A_{2}, A_{3}$, and $B_{1}, B_{2}$, and $B_{3}$ respectivelv.

$A_{1}$ and $B_{1}$ are the afferent neurons;

$A_{2}$ and $B_{2}$ are the association or internuncial neurons within the central nervous system.

$A_{3}$ and $B_{3}$ are the efferent or motor neurons.

When a stimulus is applied to $A_{1}$ it generates nervous energy, and discharges across the synapse to $A_{2}$, and finally to $A_{3}$, and the muscle contracts. The problem then is to imagine such a mode of connection between $\operatorname{arc} A$ and $\operatorname{arc} B$ as will cause arc A during activity to drain off from are $B$ the smaller amount of nerve energy in it which normally keeps the muscle supplied by $\mathrm{B}_{3}$ in a state of tonus; if this is done, the muscle of arc $\mathrm{B}$ will lose its tonicity and become relaxed. It is probable that this connection is by means of a collateral of the internuncial neuron $B_{2}$ crossing, as shown in the figure and taking part with the axon of $A_{2}$ in the formation of the synapse with $A_{3}$. The normal resistance of this synapse is lowered by the stimulation applied to the arc $\mathrm{A}$, and this lowering of resistance is participated in by the part of the synapse to which the crossing collateral from $\mathrm{B}_{2}$ contributes; owing to this lowering of resistance, nervous energy therefore drains over from the arc B, and so the muscle it supplies is relaxed.

The theory may also be applied to explain (1) alternating reflexes, as described under our description of the scratch reflex (p. 712); (2) the reinforcement of the knee-jerk and other phenomena in which reflex arcs are concerned (p. 708); and (3) certain psychological phenomena such as attention.

The Principle of the Common Path (Sherrington).-When an afferent nerve is stimulated, the impulse enters that complex network 
of conducting paths, which is called the central nervous system. So numerous are the potential connections in this labyrinth that the impulse may, under such abnormal conditions as strychnine poisoning, radiate in all directions, and be discharged so as to throw all the muscles of the body into action. But under normal circumstances the irradiation is limited to certain lines, which increase in number with the strength of the entering impulse. The general pattern of the nervous web remains fairly constant, but its details are subject to great variations, and a new stimulus may act like a tap on a kaleidoscope, and throw a new pattern into being.

At the commencement of every reflex arc is a receptive neuron extending from a sensory surface to the brain or cord, and this is a private path exclusively occupied by impulses from its own receptive points on the surface of the body. These impulses pass along certain association tracts or internuncial paths in the central nervous system, and at the termination of the arc we have a final neuron which acts as the conducting link between the central nervous system and the muscle or gland which it supplies. This final neuron does not subserve exclusively impulses generated at one receptive source, but can be used in the conduction of impulses generated at many points of the body's surface. The arm muscles, for instance, can be thrown into play in response to visual, auditory, tactile, and other sensations. The final neuron thus differs from the initial neuron in being public, not private, and may be spoken of as the final common path. Of course, in every reflex action we are not really concerned with individual neurons, but with thousands of them acting in harmony; still, for descriptive purposes, it is well to speak of one set of neurons only as a sample of the rest. An ordinary motor nerve is thus a collection of many final common paths.

Now let us suppose that two stimuli are acting on different parts of the body's surface, each of which would produce impulses that ultimately reach the same final common path together, though they may throw the motor organ into action in rather a different way. Under such circumstances, it is found that the occupation of the public path by one impulse prevents it being simultaneously used by the other; one reflex or the other takes place, but not both of them.

For the investigation of such a problem, the "scratch reflex" of the dog is one that lends itself admirably. This can best be studied in the "spinal" dog, that is in a dog in which cerebral influence is shut off by division of the spinal cord in the lower cervical region. If the skin over a large saddle-shaped area covering the shoulders and back is gently irritated on one side, the hind leg of the same side executes scratching movements, which involve flexor muscles principally ; the rate of scratching is about 4 per second, and each movement is presumably a short tetanus. The best "artificial flea" to 
einploy is a weak faradic current, and it is the nerves at the roots of the hair follicles which are specially susceptible when eliciting

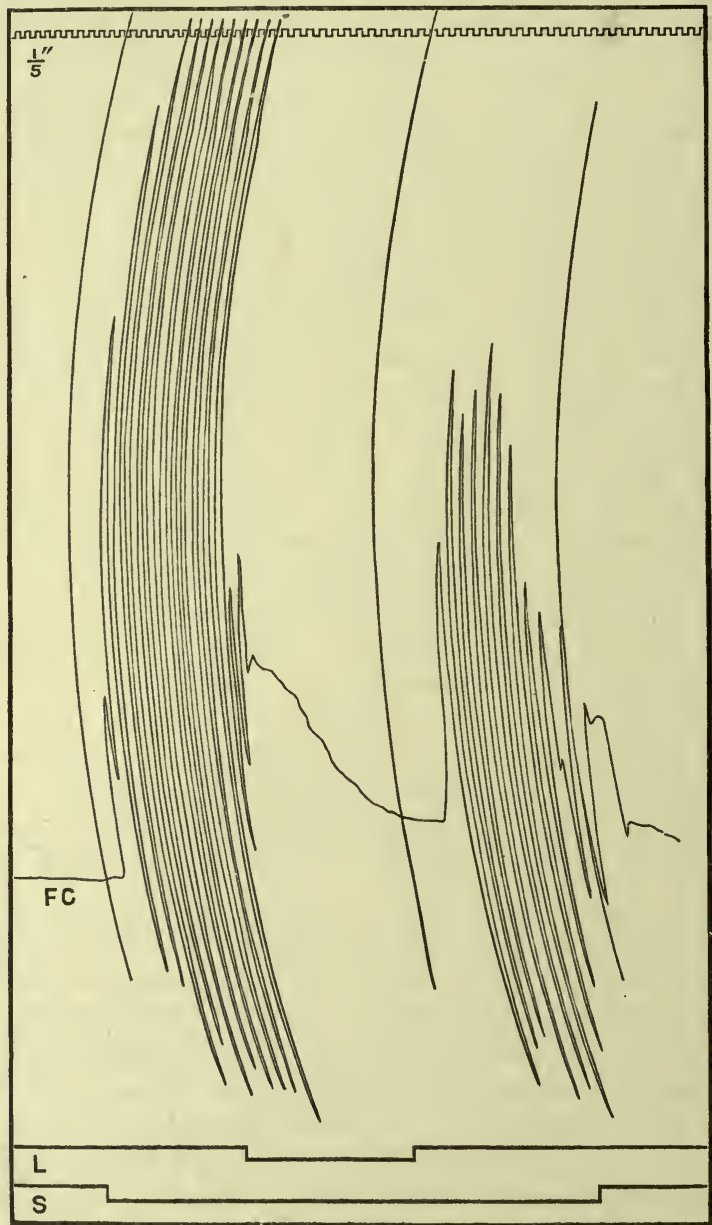

Fig. 443.-The Scratch reflex. Tracing of the flexors of left hip evoked by stimulation of the skin of the shoulder. The depression in the signal line $\mathrm{S}$ indicates the commencement of the stimulation, and its rise the termination. While this was going on, the left foot was stimulated, and the depression of the signal line $L$ indicates the duration of this stimulation; during the stimulation of the foot, and for a short time afterwards, the scratch reflex is inhibited, but the scratch reflex returns so on afterwards. The time is registered in fifths of seconds. To be read from left to right. (Sherrington.)

the reflex. The internuncial paths in the cord are in the lateral part of the lateral column, and division of that region of the cord abolishes the reflex. 
But there is another form of stimulation which also throws the same flexor muscles into action, although in rather a different way, and that is stimulation of the sole of the foot. The foot and leg are withdrawn, and the action is a steady one, and not a succession of rhythmic discharges as in scratching. Both reflexes, however, end in the same final common path; and if while scratching is being elicited by stimulation of the shoulder, the foot is then stimulated simultaneously, scratching immediately ceases; one set of impulses has displaced the other from the final common path. If then one ceases to stimulate the foot, the scratch reflex returns if the irritation of the shoulder is kept up. This is well illustrated by the tracing (fig. 443).

But there is also another way in which the inbibition of reflexes may be produced. The contraction of one set of muscles is usually accompanied by relaxation of its antagonists, and the contraction of the flexors in the scratch reflex may therefore be inhibited by making the antagonistic muscles (the extensors) contract. Further, the scratch reflex is unilateral, but this does not mean that the muscles supplying the other legs are inactive, for they must act in such a way as to support the dog on three legs, while it scratches with the fourth. So if the right shoulder is stimulated, the right hind leg scratches; if the left shoulder is stimulated, the left hind leg scratches; but if both shoulders are stimulated together, only one or the other leg scratches, not the two at once; parts of the final paths are common to both sides, and there is a struggle for their occupation. Some instances of reinforcing action were found; for example, if two points of the skin of one shoulder are stimulated with a very feeble current, neither stimulus alone may be sufficient to evoke the scratch reflex, but the two together may elicit it; in order to attain this result the two points of skin must be fairly close together.

The afferent neurons (private paths) of the body are about five times more numerous than the efferent (final common paths), and in the struggle for the occupation of these public paths by the impulses that enter the central nervous system by the more numerous private paths, three factors are specially concerned:-(1) Strength of stimulus; the stronger the stimulus the better chance the resulting impulse has of getting round to the motor organ. (2) Character of impulse; sensations of painful nature and sexual feelings win the final path easily; it is a matter of common experience that such sensations dominate and even exclude other sensations; a man with bad toothache is not likely to take much notice of anyone who pulls his coat tails. (3) Fatigue; at the end of a long stimulation, a stimulus applied to a fresh reflex arc has a better chance of capturing the common path. 
Reaction Time in Man.-The term reaction time is applied to the time occupied in the central nervous system in that complex response to a pre-arranged stimulus in which the brain as well as the cord comes into play. It is sometimes called the personal equation. It may be most readily measured by the electrical method, and the accompanying diagram (fig. 444) will illustrate one of the numerous arrangements which have been proposed for the purpose.

In the primary circuit two keys $(A$ and $B$ ) are included, and a chronograph (1), arranged to write on a revolving cylinder (fast rate). Another chronograph (2),

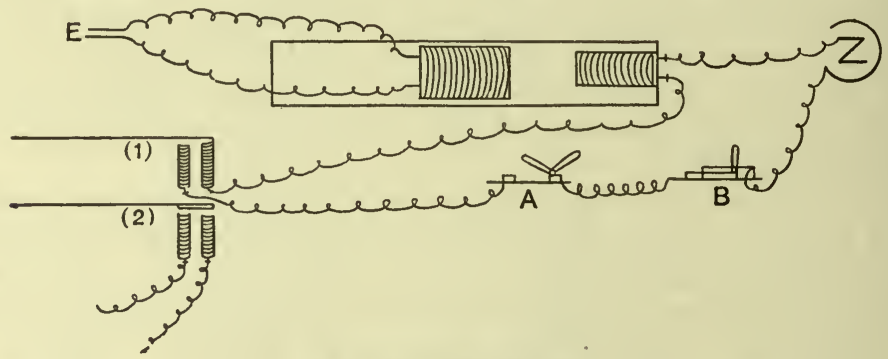

FIG. 444.--Reaction time.

marking 1-100ths of a second, is placed below this. The experiment is performed by two persons $\mathrm{C}$ and $\mathrm{D}$. The key $A$, under the control of $\mathrm{C}$, is opened. The key $B$, under the control of $\mathrm{D}$, is closed. The electrodes $E$ are applied to some part of $D$ 's body. C closes $A$. The primary circuit is made, and the chronograph moves. As soon as D feels the shock he opens $B$, the current is thus broken, and the chronograph lever returns to rest. The time between the two movements of the chronograph (1) is measured by means of the time-tracing written by chronograph (2). From this, the time occupied by transmission along the nerves has to be deducted, and the remainder is the reaction time. It usually varies from 0.15 to 0.2 second, but is increased in :-

The Dilemma.-The primary circuit is arranged as before. The wires from the secondary coil lead to the middle screws of a reverser without cross wires. To each pair of end screws, a pair of electrodes $E$ and $E^{\prime}$ pass; these are applied to different parts of D's body (fig. 445). It is arranged previously that D is to open $B$, when one part is stimulated, but not the other, $\mathrm{C}$ adjusting the reverser unknown to $D$. Under these circumstances the reaction time is longer.

The reaction time in response to various kinds of stimuli, sound, light, pain, etc., varies a good deal; the condition of the subject of the experiment is also an

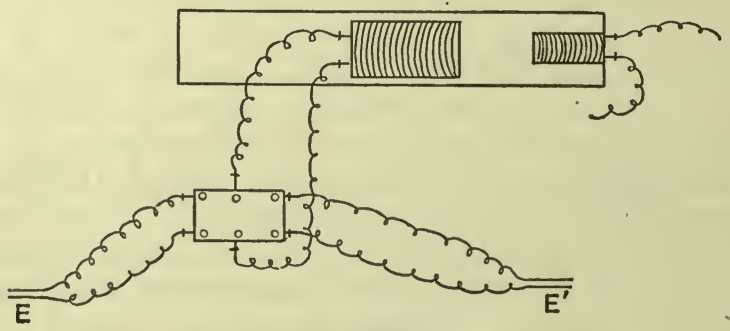

FIG. 445.-The Dilemma.

important factor. This, however, is really a practical branch of psychology, and has rccently been much worked at by students of that science (see also p. 191). 


\section{Spinal Visceral Reflexes.}

The spinal grey matter contains centres which regulate the operation of many involuntary muscles. Some of these centres are :-

The cilio-spinal centre controls the dilatation of the pupil; it is situated in the lower cervical region, reaching as far down as the origin of the first to the third thoracic nerve.

Subsidiary vaso-motor centres. The principal vaso-motor centre is situated in the bulb, and subsidiary centres are scattered through the spinal grey matter (see p. 302).

The same is probably true for all the muscular viscera, but particular study has been directed to those in the pelvis, and centres for micturition, defocation, erection, and parturition are contained in the lumbo-sacral region of the cord. If the spinal cord is cut through above the situation of these centres, the result is in general terms that any influence of the higher (voluntary) centres over these actions is no longer possible. The actions in question are then simply reflex ones occurring unconsciously at certain intervals, and set in movement by the peripheral stimulus (fulness of bladder, or of rectum, etc.). If the portion of the cord where these centres are placed is entirely destroyed, the result is paralysis of the muscles concerned, though in certain cases, even after such a severe injury, some amount of recovery has been noticed, which must be attributed to the peripheral ganglia being able to play the part of reflex centres.

The phenomena of micturition (p. 580), and defæcation (p.562) have, however, already been described at length, and it only remains to add a few words concerning two other reflexes in which the generative organs are concerned.

Uterine Reflexes.-Uterine contractions can be induced by rectal injections, the passage of a foreign body into the uterus, the application of the child to the breast, and by other means. In animals faradisation of the central end of the first sacral nerve produces the same result. The contractions of the uterus are therefore reflex. Several cases have been recorded in which parturition has occurred normally in women who have had the cord divided across completely in the thoracic region; it is thus evident the centre must be a lumbar one. In such cases the uterine contractions technically called " pains" are strong, but pain is, of course, absent. The communication with the lumbar region appears to be principally by the first three lumbar nerves. Similar observations have been made experimentally in animals, and in one of Goltz and Ewald's dogs in which the cord had been removed from the lower thoracic region downwards, pregnancy followed coitus, and terminated with successful parturition. The mammary glands enlarge as usual in such cases, even when, as in Routh's well-known case (where the cord was completely destroyed at the seventh thoracic segment), there can be no nervous communication between the pelvis and the breast.

Erection.-This can be excited in man even immediately after a transverse lesion of the cord; so also can ejaculation, but not so commonly. The evidence in favour of such acts being spinal reflexes is very complete in the case of animals. 


\section{CHAPTER L}

\section{FUNCTIONS OF TIIE CEREBRUM}

THE cerebrum is the seat of those psychical or mental processes which are called volition and feeling; volition is the starting-point in motor activity; feeling or consciousness is the final phase of sensory impressions; the correlation of sensations with one another, and with volitional impulses so generated is what constitutes thought. That the brain is the organ (or anatomical correlate) of mind is to-day a matter of such common knowledge that it is almost superfluous to mention it in a physiological text-book. Yet its functions were entirely unknown or only dimly conjectured by ancient philosophers, and the overwhelming importance of the grey matter on its surface in mental phenomena is a discovery of comparatively recent date.

\section{Effects of Removal of the Cerebrum.}

The functions of any organ may be discovered (in part, at any rate) by removing it; and the brainless frog which we have studied in relation to the functions of the spinal cord is also a useful objectlesson to teach us the uses of the part removed, by observing in what manner the animal differs from one which has its brain intact. If, instead of taking a frog, we take an animal lower in the scale, where the brain is not so fully developed, the effect of removing that organ will be less marked; or if we remove the brain in a more highly developed animal, the simultaneous removal of the brain functions will be naturally more noticeable. We have already seen (Chapter XLIV.) that the development of the cerebral hemispheres. increases in importance as we rise in the animal scale.

If the cerebral hemispheres are removed in a teleostean or bony fish (and in such animals there is only a rudimentary cortex), the animal is to all intents and purposes unaffected; it can distinguish between a worm and a piece of string, and will rise to red wafers in preference to those of another colour. The operation does not 
damage the primary centres of vision (the optic lobes, which correspond to the corpora quadrigemina of the mammal), and in these fishes the eye is the most important sense organ.

A shark, however, subjected to the same operation, is reduced to a condition of complete quiescence; this is due to the circumstance that in this fish the principal sense organ is that of smell, and severance of both olfactory tracts produces the same result as removal of the entire cerebrum. In either case the path between the olfactory bulbs and the centres that control the cord are interrupted.

Going higher in the animal scale to the frog, we find that removal of the hemispheres only does not entirely abolish its apparent spontaneity; it still continues to feed itself, for instance, by catching passing insects. It is not until the optic thalami are removed also that it becomes the purely reflex animal described on p. 709. If the brain and the anterior end of the bulb are removed the lower centres of the cord are set free, and the result is incessant movement provoked by slight stimuli.

A bird treated in the same way remains perfectly motionless, sleepy, and unconscious, unless it is disturbed (see fig. 446). When

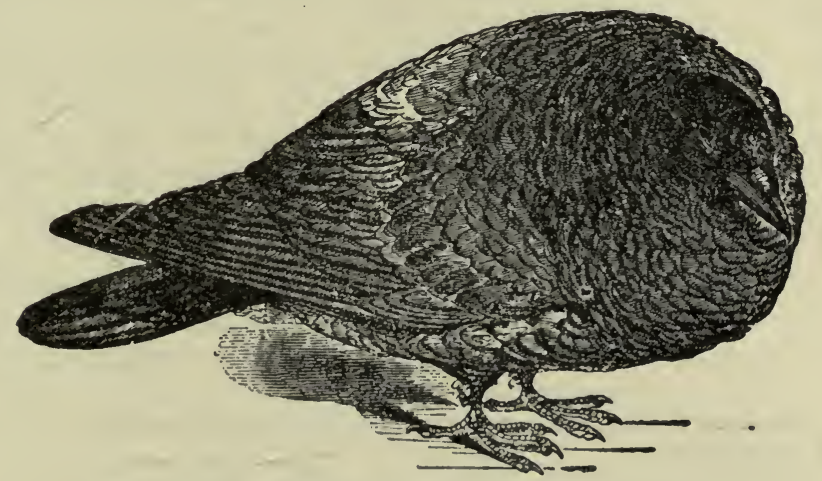

Fig. 446.-Pigeon after removal of the hemispheres. (Dalton.)

disturbed in any way it will move; for instance, when thrown into the air it will fly; but these movements are, as in the frog, purely reflex in character; when the animal is mado to fly its movements are directed by the sense of sight, the optic lobes being still intact, and it will select a perch to settle on in preference to the floor. It will start at a noise; it will not eat voluntarily; it exhibits no emotions such as fear, sexual feeling, or maternal instincts.

In mammals the operation of extirpation of the brain is attended with such severe hæmorrhage that the animal dies very rapidly, but in some few cases where the animals have been kept alive, the 
phenomena they exhibit are similar to those shown by a frog or pigeon. The difficulty of the operation was overcome by Goltz of Strassburg, in dogs, by removing the cerebrum piecemeal. One dog treated in this way lived in good health for eighteen months, when it was killed in order that a thorough examination of the brain might be made. It was then found that not only the hemispheres but the main parts of the optic thalamus and corpus striatum had been removed also. Though it could still carry out coördinated movements, its reactions were entirely reflex, and memory, emotions, feelings, or the capacity to learn were absent.

The higher animal loses just those characters which distinguish it from the lower ones. It is difficult to prophesy what would happen if as extensive operations were carried out in a monkey or a man. But so far as extirpation has been observed, the initial paralysis (which is seen also in the dog) does not disappear so rapidly or so completely. In man, the tendency to recover is least.

If we now compare these effects, it is seen that the results of the operation becomes progressively greater as we ascend the scale. The higher the animal, the more fatal the effects, the immediate disturbance more severe, the return of function slower, and the permanent loss greater. The long life of Goltz's dog was doubtless due to the fact that the removal was accomplished by several operations.

This is anatomically explicable when we remember that the anterior horn cells are influenced chiefly by two sets of impulses, those which enter the cord by the posterior roots, and those which come down from the cerebrum by the pyramidal tracts. In the lower animals the pyramidal pathway is insignificant, and when it is interrupted the disturbance is consequently slight. In animals below the mammals it is absent, and going up the mammalian scale it becomes more and more important, as the following figures show :-

In the mouse the pyramidal fibres constitute 1.14 per cent. of those in the cord.

guinea-pig ,
rabbit
, cat
man

$\begin{array}{cc}, & 3 \cdot 0 \\ , & 5 \cdot 3 \\ , & 7 \cdot 76 \\ , & 11 \cdot 87\end{array}$

", $\quad$ ",

We can therefore quite readily understand that in the apes and in man, a damage to the cortex which causes degeneration of these tracts will cut off many impulses to the anterior cornual cells, and produce a greater or less degree of paralysis.

There are 80,000 fibres in each pyramidal tract of the human cord. They are, moreover, not the only tracts which connect the cerebrum to the spinal cord, and section of these other tracts (see p. 656) may produce quite as marked paralysis. In man, it appears that when the pyramidal tracts are diseased, it is the finer and more delicate movements which are permanently lost (Schäfer.). 


\section{Localisation of Cerebral Functions.}

The different parts of the brain and of its cortex are related to different parts of the body. The right hemisphere, for instance, controls the voluntary movements on the left side of the body, and receives sensory impulses from the left side, and vice versa.

Then in each hemisphere there are certain areas, termed motor areas, which are the starting-points of those volitional impulses which give rise to movements; and other areas primarily concerned in the reception of sensory impulses; these are termed sensory areas. These various areas have been mapped out by means of experiments on animals, and by the observation of disease in man.

Before these facts were ascertained it was usual for physiologists to say that "the brain acts as a whole," and although we do not now attach the same meaning to that phrase as did the physiologists of the past, it still has an underlying substratum of truth. Let us take an example, and imagine the smell of an orange; such an abstract idea of an isolated sensation is impossible; we cannot think of the smell of the orange apart from the other characteristics of the fruit, the smell recalls the taste, the shape, the colour, the act of peeling it, fingering it, cutting it, eating it, and so forth. One sensation due to the activity of one area, such as the olfactory area, calls into play the activity of other sensory areas, and of the motor areas, and of the links between the sensory and motor areas. The brain is acting as a whole because its various parts are called into play simultaneously, though the whole brain is not concerned in each of the component sensations and volitions associated with any particular mental state.

Moreover, the doctrine of cerebral localisation is not accurately expressed by the statement that a cortical centre is one, the stimulation of which produces a definite response, and the extirpation of which abolishes the response. We shall, for instance, immediately see that the stimulation of certain areas in the dog's brain produces certain movements, but Goltz showed that in his dogs, the removal of an entire hemisphere did not cause permanent paralysis of the opposite side of the body.

In the central nervous system there are few or no places where only one set of nerve units are situated, with fibres passing to or from them. Every locality has several connections with other parts, and also fibres passing through it which connect together the parts on all sides of it. Hence in extirpating even a limited area, numerous pathways are interrupted, and the damage is consequently widespread. Much of the disturbance produced at first gradually passes away, and the temporary effects must be distinguished from those which are permanent; the permanent effects have the greater significance of the two. Moreover, it is clear that the relative 
and absolute value of any locality in the central nervous system depends largely on the degree to which centralisation has progressed, and on the amount of connection between the various areas. The closer the connection, the more numerous and intricate the pathways, the greater will be the permanent effects of an extirpation, and the recovery of function the more remote. The lower the animal in the zoological series, or the less the age of the animal, the more imperfectly developed will be the connecting strands, and so the possibility of other parts taking up to some extent the functions of those that are removed will be increased.

The earliest to work in the direction of localisation were Hitzig and Fritsch. The subject was then taken up by Ferrier and Yeo, and later by Schäfer, Horsley, etc., in this country, and by Munk and many others in Germany.

The main point which these researches have brought out is what we have just termed the overwhelming importance of the cortex; it contains the highest cerebral centres. Before Hitzig began his work, the corpus striatum was regarded as the great motor centre, and the optic thalamus as the chief centre of sensation; and the idea that the basal ganglia were so important arose from the examination of the brains of people who had died from, or at least suffered from, cerebral hæmorrhage.

The most common situation for cerebral hæmorrhage is either in the region of the corpus striatum or optic thalamus; it was noticed that motor paralysis was the most marked symptom if the corpus striatum was injured, and sensory paralysis if the optic thalamus was injured. The paralysis, however, is due, not to injury of the basal ganglia, but of the neighbouring internal capsule. The internal capsule consists in front of the motor fibres passing down from the cortex to the cord, and behind of the sensory fibres passing up from the cord to the cortex (see p. 689). Hence, if these fibres are ploughed up by the escaping blood, paralysis naturally is the result. If a hæmorrhage or injury is so limited as to affect the basal ganglia only, and not the fibres that pass between them, the resulting paralysis is slight or absent.

The question will next be asked: What, then, is the function of the basal ganglia? They are what we may term subsidiary centres: the corpus striatum, principally in connection with movement, and the optic thalamus, in connection with sensation, including the sense of vision, as its name indicates.

A subsidiary centre may be compared to a subordinate official in an army. The principal centre may be compared to the commanderin-chief. This highest officer gives a general order for the movement of a body of troops in a certain direction; we may compare this to the principal motor-centre of the cortex sending out an impulse for 
a certain movement in a limb. But the general does not give the order himself to each individual soldier, any more than the cerebral cortex does to each individual muscle; but the order is first given to subordinate officers, who arrange exactly how the movement shall be executed, and their orders are in the end distributed to the individual men, who must move in harmony with their fellows with regard to both time and space. So the subsidiary nerve-centres or positions of relay enable the impulse to be widely distributed by collaterals to numerous muscles which contract in a similar orderly, harmonious, and coördinate manner.

There is just the same sort of thing in the reverse direction in the matter of sensory impulses. Just as a private in the army, when he wishes to communicate with the general, does so through one or several subordinate officers, so the sensory impulse passes through many cell-stations or subsidiary centres on the way to the highest centre, where the mental process called sensation, that is, the appreciation of the impulse, takes place.

There are two great experimental methods used for determining the function of any part of the cerebrum. The first is stimulation; the second is extirpation. These words almost explain themselves; in stimulation a weak interrupted induction current is applied by means of electrodes to the convolution under investigation, and the resulting movement of the muscles of the body, if any occurs, is noticed. In extirpation the piece of brain is removed, and the resulting paralysis, if any, is observed.

It is essential, when the experiment of stimulating the cortex of the brain is being performed, that the animal should be anæsthetised and absolutely unconscious, otherwise voluntary or reflex actions will occur which mask those produced by stimulation. If, however, the animal is too deeply under the influence of a narcotic the brain is inexcitable. In an anæsthetised animal the brain is inactive, and if methylene blue is injected into the blood, the brain is seen to be of a blue colour. If, however, a spot of the cerebral surface is stimulated, that part of the brain is thrown into action, oxygen is used up, and the methylene blue is reduced, and in consequence that area of the brain loses its blue tint. If the animal is so deeply narcotised that the brain does not discharge an impulse, the part stımulated remains blue.

By such means the cortex has been mapped out into what we may term motor areas and sensory areas.

Motor area.-The name Rolandic area which this part of the brain has also received is derived from its anatomical position.

Stimulation of the motor area produces movement of some part of the opposite side of the body; excitation of the same spot is always followed by the same movement in the same animal. In different animals excitation of anatomically corresponding spots produces similar or corresponding results. It is this which has enabled one to apply the results of stimulating areas of the monkey's 
brain to the elucidation of the function of the similar brain of man.

If the stimulation used is too powerful the movement spreads to other parts, and a considerable portion of the body may be thrown into convulsive movements similar to those seen in epilepsy.

Extirpation, or removal, of these areas produces paralysis of the same groups of muscles which are thrown into action by stimulation.

The degeneration tracts after destruction of the motor area are shown in fig. 447. The shaded area in each case represents the injured or degenerated material; $\mathrm{A}$ in the cortex, $\mathrm{B}$ in the anterior part of the posterior limb of the internal capsule, $\mathrm{c}$ in the middle
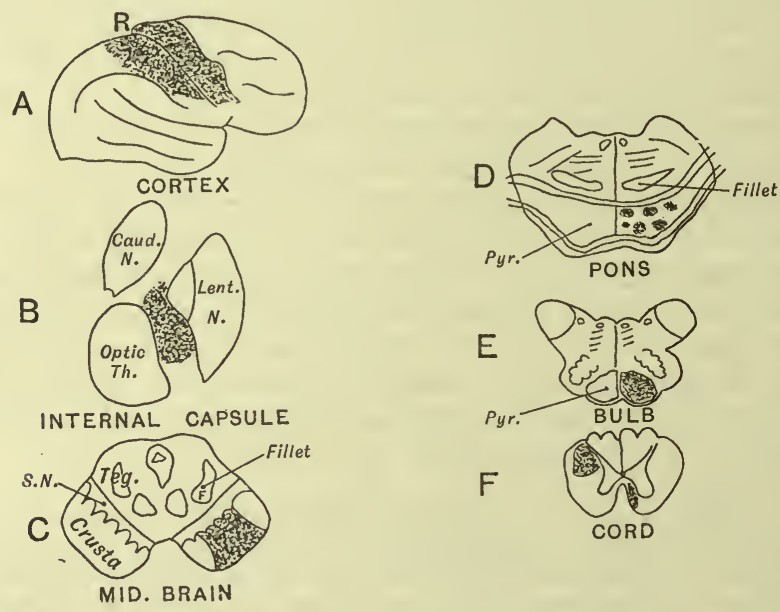

FIG. 447.-Degeneration after destruction of the Rolandic area of the right hemisphere. (After Gowers.)

of the crusta of the mid-brain, $D$ in the pyramidal bundles of the pons, $\mathrm{E}$ in the pyramid of the bulb, and $\mathrm{F}$ in the crossed and direct pyramidal tracts of the cord.

Sensory areas.-Stimulation of these produces no direct movements, but doubtless sets up a sensation called a subjective sensation; that is, one produced in the animal's own brain, and this indirectly leads to movements which are reflex; thus on stimulating the auditory area there is a pricking up of the ears; on stimulating the visual area there is a turning of the head and eyes in the direction of the supposed visual impulse. That such movements are reflex and not direct, is shown by the long period of delay intervening between the stimulation and the movement.

Extirpation of a sensory area leads to loss of the sense in question. 
The rougher experiments performed by nature in the shape of diseases of the brain produce corresponding results.

Some diseases are of the nature of extirpation.

An instance of this is cerebral hæmorrhage. If the hæmorrhage is in the region of the internal capsule, it cuts through fibres to the muscles of the whole of the opposite side of the body, as they are all collected together in a narrow compass, and the condition obtained is called hemiplegia. The varieties of hemiplegia are

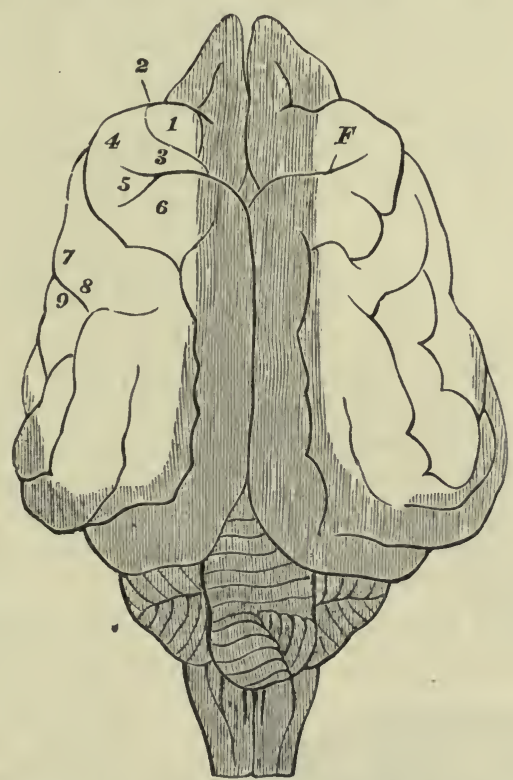

Fia. 448.-Brain of dog, viewed from above. $F$, frontal fissure, sometimes termed crucial sulcus, corresponding to the fissure of Rolando in man. $S$, fissure of Sylvius, around which the fonr longitudinal convolutions are concentrically arranged; 1 , flexion of head on the neck, in the median line; 2 , flexion of head on the neck, with rotation towards the side of the stimulus; 3 , 4, flexion and extension of anterior limb; 5, 6, flexion and extension of posterior limb; 7, 8, 9, contraction of orbicularis oculi, and the facial muscles in general. The unshaded part is that exposed by opening the skull. (Dalton.)

numerous, according as motor or sensory fibres are most affected, and in one variety of hemiplegia, called crossed hemiplegia, the face is paralysed on one side of the body, the limbs on the other; this is due to injury of the tracts in the bulb, above the crossing of the pyramids.

If now the hæmorrhage occurs on the surface of the brain, a much more limited paralysis, called monoplegia, is the result; if the arm area is affected, there will be paralysis of the opposite arm; if the leg area, of the opposite leg; if a sensory area, there. will be loss of the corresponding sense. 
Some diseases, on the other hand, act as the induction currents do in artificial stimulation; they irritate the surface of the brain; such a disease is a tumour growing in the membranes of the brain; if the tumour irritates a piece of the motor area, there will be involuntary movements in the corresponding region of the body; these movements may culminate in the production of epileptiform convulsions commencing in the arm, leg, or other part of the body which corresponds to the brain area irritated. It is these cases of "Jacksonian Epilepsy" which have given the best results in surgery; the movement produced is an indication of the area of the brain which is being irritated, and the surgeon after trephining is able to remove the source of the mischief. If the area of the brain which is irritated is a sensory area, the result produced is a subjective sensation, similar to what we imagine is produced in animals with an electric current.

We may now proceed from these general considerations to particular points, and give maps of the brain to show the areas we have been speaking of.

Fig. 448 is a view of the dog's brain. It is convenient to take this first because it was the starting-point of the experimental work on the subject in the hands of Hitzig and Fritsch. If the text beneath the figure is consulted, it will be seen that the motor areas, mapped out by the method of stimulation, are situated in the neighbourhood of the crucial sulcus, which probably corresponds to the fissure of Rolando in man.

Coming next to the brain of the monkey, figure 449 is reproduced from Ferrier's book. He marked out the surface into a

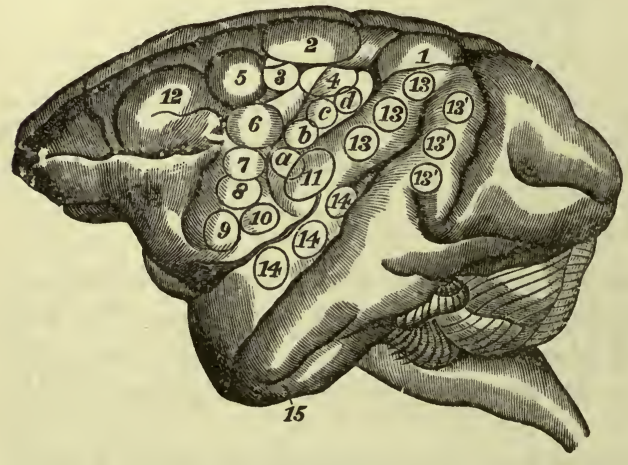

FIg. 449. number of circles, stimulation of each of which produced movements of various sets of muscles, face, arm, and leg, from below upwards; extirpation of these same areas produced the corresponding paralysis. It will be further noticed that these areas are all grouped around the fissure of Rolando, particularly in the ascending frontal convolution.

Much of our knowledge concerning the localisation of the motor area in the human brain has been deduced from experiments on the lower monkeys. Valuable as such knowledge is, infinitely more 
useful knowledge, from the standpoint of the human brain, would be obtained by examining the brains of those monkeys nearest to man, which are known as the anthropoid apes. The difficulty and expense of obtaining such animals has largely deterred investigators from performing such experiments. Horsley and Beevor examined

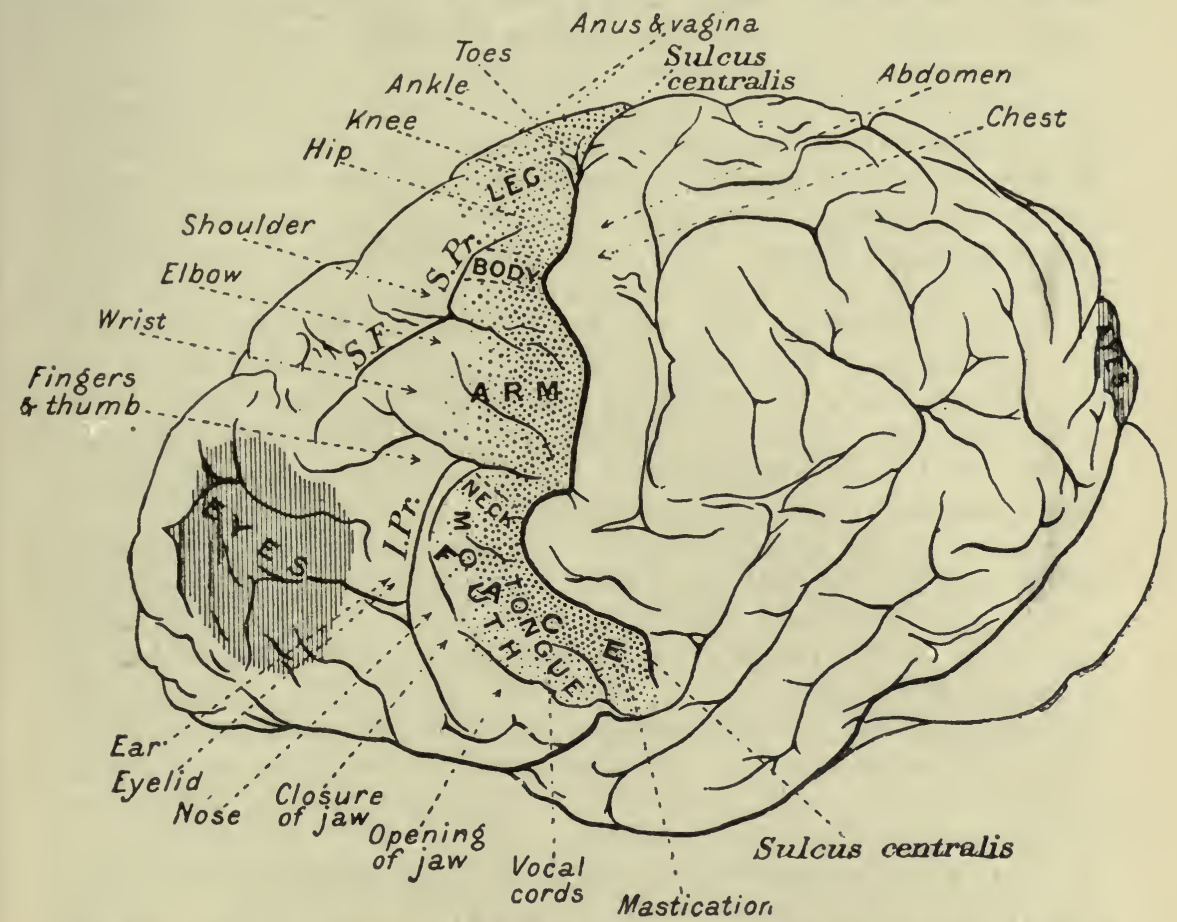

Fro. 450.-Brain of Chimpanzee. Left hemisphere viewed from side and above so as to obtain the configuration of the Rolandic area. The figure involves some foreshortening about both ends of the sulcus centralis or fissure of Rolando. The extent of the s0-called motor area on the free surface of the hemisphere is indicated by black stippling which extends back to the central sulcus. Much of the "motor" area is hidden in sulci ; for instance, it extends into both the central and precentral sulci. The names printed in capitals on the stippled area indicate the main subdivisions of the "motor" area; the names printed small outside the brain indicate by their pointing lines some of the chief subdivisions of the main areas. But there is much overlapping of the areas which it is not possible to indicate in a diagram of this kind. The shaded regions marked "eyes " in the frontal and occipital regions indicate the areas which under faradisation yield conjugate movements of the eyeballs. S.F. =superior frontal sulcus. s.Pr.=superior precentral sulcus. I.Pr. =inferior precentrai sulcus. E(After Sherrington and Grïnbaum.)

the brain of an orang-outang some years ago, and now Sherrington and Grünbaum have made a number of experiments; several specimens of two species of chimpanzee, the orang and the gorilla, have been examined. Their conclusions are of great importance. The above figure (fig. 450) of the chimpanzee's brain shows what has been found; the orang and the gorilla gave practically the same 
results, and no doubt the human brain would give identical results also if it could be examined.

The method used is to expose the brain in an anæsthetised animal, and thoroughly explore it with a weak faradic current, one electrode being placed on the brain, and the other attached to an indifferent part of the animal's body. This allows of finer localisation than is possible with the ordinary double-point electrodes.

The motor area includes continuously the whole length of the ascending frontal, or as it is sometimes called, the precentral convolution. It never extends behind the central sulcus, or, as it is sometimes called, the fissure of Rolando. On the mesial surface it extends but a short distance, and never as far as the calloso-marginal fissure. The motor area extends also into the depth of the Rolandic and other fissures; the part of the excitable area thus hidden equals or may even exceed that on the free surface of the hemisphere. The arrangement of the various regions of the musculature follow the segmental sequence of the cranio-spinal series to a remarkable extent; in fact, the excitable area may be compared to the spinal cord upside down. The accompanying figure indicates this better than any verbal description.

It cannot fail to strike even a superficial observer how large the cortical area is that deals with movements of the head and arm regions when compared with that of the lower limb, and still more with that of the trunk. The trunk itself has a larger mass of muscular tissue, but it is in the head region (which includes the complex movements of the tongue and such structures as the vocal cords) and in the arm and hand that the movements are most varied and most delicate. No doubt this is the explanation of the greater size of their cortical representation.

It is these finer movements which are most affected by a cortical injury, and which exhibit least recovery; in the upper limb, for instance, the shoulder muscles will be the least, and the hand the most paralysed.

In experiments on unilateral extirpation in animals, and in destructive lesions of one side of the brain in man, it is the muscles which act normally unilaterally which are most paralysed. The muscles which normally move bilaterally, e.g., the chest muscles in breathing, the trunk muscles in maintaining an erect position, are comparatively little affected; the spinal centres of such muscles are no doubt connected by commissural fibres, and therefore can be affected from both sides of the brain.

The marginal convolution on the mesial surface of the hemisphere was first investigated by Schäfer and Horsley, in the lower monkeys. They found in these animals that it contained a considerable extension of the "motor" area, including the cortical centres for the trunk muscles. This, at any rate, is not the case for the higher apes, and therefore probably is not true for man. 
It will be noticed in the diagram (fig. 450) that there are two regions of the brain from which eye movements can be elicited; one is in the frontal lobe, the other at the occipital pole. The frontal eye area is the motor centre for conjugate movements of the two eyeballs, and in the lower monkeys is continuous with the rest of the motor area, but in the higher monkeys and man is separated from the Rolandic area by a field of inexcitable cortex. The occipital region from which eye movements can be obtained is the visuo-sensory sphere (see p. 736).

The next illustration is an outline map of the left cerebral hemisphere in man. In it are indicated the various motor and sensory areas, which are largely deduced from experiments on the higher monkeys.

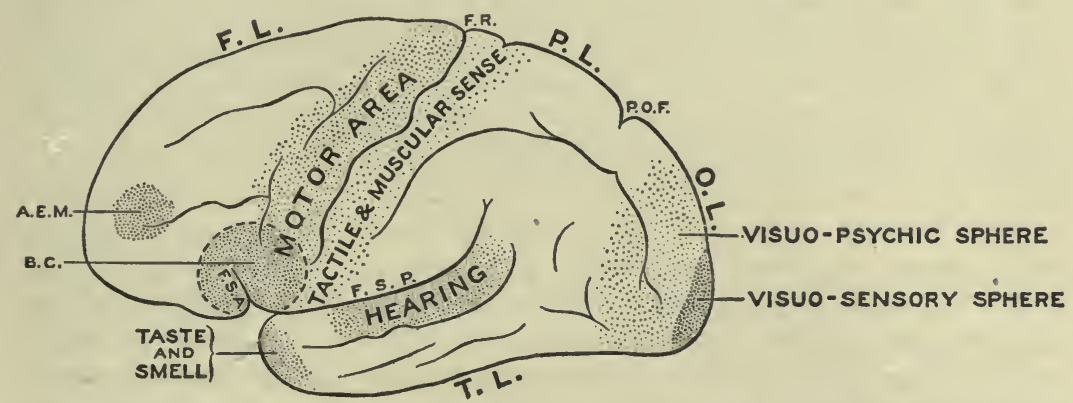

F'IG. 451.-Left cerebral hemisphere, outer surface. The lobes and the principal sulci are indicated by their initial letters; A.E.M., anterior centre for eye movements; B.C., Broca's convolution.

One part of the motor area is peculiar to man, and that is :-

The Speech Centre.-This is surrounded in the diagram by a dotted circle and marked B.C. There are other centres concerned in speech, as we shall see when considering the question of association fibres; but this is the centre for the muscular actions concerned in speech. The discovery of this centre was the earliest feat in the direction of cerebral localisation. It was discovered by a French physician named Broca; he noticed that patients who died after hæmorrhage in the brain, but who previous to death exhibited a curious disorder of speech called aphasia, were found, after death, to have the seat of the hæmorrhage in this convolution. The convolution is generally called Broca's convolution. Experiments on animals are useless in discovering the centre for speech. Sherrington found in the higher apes that faradisation of the Broca area does not evoke vocalisation.

The most curious fact about the speech-centre is that it is unilateral; it is situated only on the left side of the brain, except in left-handed people, where it is on the right. We are thus leftbrained so far as the finer movements of the hand-muscles are con- 
cerned, as in writing, and we are also left-brained in regard to speech, an action intimately associated with writing. There is but little doubt that spoken language originated from gesture language; in fact, one sees this in children learning to speak. In gestures the right hand (and left brain) will take a prominent part; hence the unilateral position of the speech centre receives a rational developmental explanation.

That Broca's area is the chief speech centre has for long been a matter of doubt among physiologists and pathologists. The recent researches of Marie have thrown further doubt upon the hypothesis; for he has shown that in many cases of aphasia Broca's area is uninjured, and that in cases where Broca's area is injured, aphasia is not always present. His main conclusion is that injury to Broca's convolution is only one factor in the causation of aphasia; the other areas related to the speech mechanism are situated more posteriorly, and are called the visual word area and the auditory word area (see further, p. 741), and injury to these alone will produce aphasia, whereas injury to Broca's area alone will not do so; he argues that the cases described in which aphasia followed an injury, apparently limited to Broca's area, had not been thoroughly investigated, and if the other areas had been properly examined injuries would have been found there also. These conclusions will naturally be tested in the future by others who have the opportunity of examining such cases.

The Tactile Area.- Volition and the tactile and muscular senses are associated together so closely physiologically, that anatomically we should expect to find the commencement of the volitional fibres not far removed from the terminations of the sensory fibres, and as a matter of fact, this is actually the case. Some of the sensory fibres possibly pass direct into the ascending frontal convolution, but the vast majority terminate in its neighbour the ascending parietal convolution, which is on the other side of the central or Rolandic fissure. In the early days of brain map-making, the ascending parietal convolution was believed to be a part of the motor area, and this found expression in such diagrams as those of Ferrier (see fig. 449). A cortical injury in man seldom involves the ascending frontal without also involving the ascending parietal, and so loss of sensation and motion usually go together. The more exact methods introduced by Sherrington and Grünbaum have, however, shown that stimulation of the ascending parietal produces no direct movements; secondary movements may be elicited, just as stimulation of the visuo-sensory area provokes secondary movements of the eyes. Extirpation of the ascending parietal, however, leads to no motor paralysis, and no degeneration of the pyramidal tracts. Histological examination of the ascending parietal grey matter shows it, moreover, to possess the structure of a sensory rather than of a motor area. Before this distinction was recognised, the term sensori-motor was used as a comprehensive expression to include the functions of the two convolutions one on each side of the Rolandic fissure. The ascending parietal convolution is the cortical seat of those sensations 
which are tactile discriminatory, and related to position and movement of the muscles. We still await exact information regarding the cortical representation of sensations of pain and temperature.

This conclusion regarding the sensory function of the ascending parietal convolution has received support from a number of carefully observed clinical cases, for Sherrington and Grünbaum's delimitation to a relatively narrow strip of what had previously been considered a widespread motor territory, left for some time uncertain the function of the remainder of the original motor area-Munk's sensori-motor field-lying posterior to the central fissure. The most remarkable confirmatory evidence regarding the sensory functions of what we may call the post-central convolution has been recently afforded by two patients, who voluntarily allowed Dr Cushing of Baltimore the opportunity of experimentally testing the point after operations in which this part of the brain was exposed, and during a time they were in a conscious state.

In both of them characteristic motor responses were obtained from the precentral gyrus (ascending frontal convolution) without any conscious sensation, except that which accompanies forced change of position in the parts moved. On the other hand, stimulation of the post-central gyrus (ascending parietal convolution) produced no movements, but gave definite sensory impressions which were likened by one patient to a sensation of numbness, and by the other to definite tactual impressions.

There is, of course, a close connection between the two convolutions in question, by short association fibres passing from one to the other; and the necessity for sensation in normally provoking the corresponding motor outflow is also illustrated by the following experiment:- If the posterior roots of the spinal nerves are divided there is a loss of sensation, and so the sense of movement cannot reach the brain from the muscles, and consequently the muscles are not called into action; when all the posterior roots coming from a limb in a monkey are cut, the muscles, so far as voluntary movements are concerned, are as effectually paralysed as if the anterior roots of the spinal nerves had been cut. The muscles, however, do not degenerate as they would if the anterior roots had been cut. They merely undergo a small amount of wasting, due to want of use.

The Visual Area.-The lower the animal in the series, the inore readily can its actions be controlled by sensory impulses which have not passed through the cortex cerebri. A decerebrated bony fish can distinguish colours, a frog can catch flies, even a pigeon will select its perch, though it takes no notice of food or of people who try to frighten it. A dog similarly operated on is practically blind, though it will blink at a bright flash of light. In the lower animals the impulses pass in to the primary visual centre in the optic lobes 
which acts as the centre for the reflex; the higher we ascend the animal scale, the path via the cortex becomes more permeable, of greater value or even indispensable, and the reflexes through the lower centres of less importance; not only so, but there are subdivisions of the visual cortical area, which correspond to different regions of the retinæ.

We may in fact speak of the visuo-sensory field in the cortex as the cortical retina upon which the impulses from the actual retina in the eye are projected, in a manner analogous to the way in which the field of vision is projected upon the actual retina.

In the fishes which have no cortex cerebri, the optic lobes, analogous to the C. quadrigemina, are the centres for vision. In some fishes, a small number of the fibres of the optic nerve pass into the geniculate body, which forms a cell-station on the road to the posterior region of the cerebrum, where a primitive cortex begins to appear. On ascending the animal scale, this group of fibres becomes more and more abundant, and this part of the cortex becomes more elaborate in structure. When we reach the monkeys, this part of the brain is cut off from the rest to form a distinct occipital lobe by the parieto-occipital fissure, which is frequently called the Affenspalte (ape's split). In the lower monkeys this lobe is smooth (fig. 427, A, p. 691), but as the great parietal association centres get larger with increase of intelligence, the visuo-sensory area is pushed back, and the lobe thrown into folds. In the highest apes, and in the lower races of mankind, a good deal of the visuo-sensory sphere is still seen on the external cerebral surface; but in the higher races, most is pushed round on to the mesial surface. This calcarine area is also named the striate area, because it is characterised by the white stripe called the line of Gennari.

Some animals have panoramic and others stereoscopic vision. The former (mainly vegetable feeders) have eyes set laterally; each eye receives a different picture, and the decussation of the optic nerves is complete; each eye sends impulses to the opposite hemisphere. Animals with stereoscopic vision have the eyes, as in man, in front, and the optic axes can be converged so that an object is focussed with both eyes. This becomes necessary in carnivora, which have to catch moving prey; the more complex the movements of the fore-limbs, the greater becomes the necessity for fixation of the eyes to guide them. In such animals each visual area corresponds with the same half of both retinæ, that is, with the opposite half of the visual field; the lower half of each area corresponds with the upper half of each half field of vision, and vice versa. The appearance of the macula lutea (with cortical representation in both hemispheres) in the primates is the culminating point in visual development among the mammals. 
A man or an animal who loses both eyes is blind, but in time manages to find his way about. This is not the case when blindness is produced by removal or disease of both occipital lobes; here, the sense of orientation is lost also, for the association of sensory memories and motor impulses is then impossible.

Removal of one occipital lobe will be followed by different results in the two classes of animals just referred to. In those with panoramic vision, the result will be blindness of the opposite eye, because the decussation of the optic nerve is complete at the chiasma. But in animals such as monkeys with stereoscopic vision (in which the only decussating fibres are those which come from the inner halves of the two retinæ) removal of one occipital lobe, or disease of that lobe in man, produces blindness of the same side of each retina, or inability to see the opposite half of the visual field. This is called hemianopsia; the head and eyes are turned to one side, namely, the side of injury (conjugate deviation to the side of the injury). Such an operation does not destroy vision in the central portion (macula lutea) of either retina, because each macula sends impulses to both sides of the brain. Stimulation of one visual area leads to a subjective sensation apparently coming from the same halves of both retinæ, and also excites the solitary cells of Meynert; this produces conjugate deviation of head and eyes towards the opposite side to that stimulated.

These solitary cells are so called because they are few and far between; they are large cells not at all unlike the Betz cells of the motor cortex. Their axons, no doubt, pass in long association tracts to the motor eye centre of the frontal region and to the corpora quadrigemina.

The optic radiations consist of (1) sensory fibres from the optic tracts via the external geniculate bodies; (2) efferent fibres to the centres for eye-movements; and (3) association fibres, which are last developed. The last named link one convolution to others, and the two hemispheres together, and bring about association of ideas of vision in both hemispheres, and with other sensations. A large collection of such fibres runs horizontally through the grey matter. This white stripe is often visible to the naked eye; it is the anatomical mark of the visuo-sensory cortex, and is called the line of Gennari. The visuo-psychic region (fig. 451) has no line of Gennari, but possesses many small and medium-sized pyramidal cells in its outer layers, which play the part of association units, where memory pictures are stored and visual sensations correlated with those from other sense-organs; the higher one ascends the animal scale, the greater becomes the depth of this layer.

The eye centre in the frontal lobe is separated, in the higher apes and man, by inexcitable grey matter from the rest of the 
motor area. No cortical centre is purely motor or purely sensory, and this one, though usually called motor, has its sensory complement probably from the eyeballs and eyelids (5th nerve). The newly developed grey matter between it and the Rolandic region is an area probably concerned in the association of eye movements with equilibration and the maintenance of the erect position; we know, moreover, that the fibres from the frontal lobe to the cerebellum (the centre for equilibration) are very numerous (see fig. 437, A, p. 699).

The Auditory Area is in the posterior part of the upper temporal convolution. This has been definitely proved by clinical observation in man, and supported by experiments on animals, though it is by no means easy to ascertain whether or not an animal is deaf. It is doubtless surrounded, as are the visuo-sensory area and other sense areas, by a psychic or association sphere, and is connected to surrounding parts, and especially to the visual area, by annectent gyri. A good deal of the auditory area is situated in the depth of the Sylvian fissure where the gyri tranversales which cross it are found.

Taste and Smell are closely connected; their cerebral area is the uncinate and hippocampal gyrus, and the tip of the temporal lobe. These parts are relatively more important in animals who rely upon smell and the oral sense for their guidance. This part of the cortex is of simpler structure than the rest, and on account of its early appearance in the animal scale is known as the archipallium (see p. 698).

The Silent Areas. - On referring once more to the maps of the brain, it will be seen that there are many blanks; one of these is in the anterior part of the frontal region. Extirpation or stimulation of this part of the brain in animals produces but little result. The large size of this portion of the brain is very distinctive of the human brain, and it has therefore been supposed that here is the seat of the higher intellectual faculties. Such a question is obviously very difficult to answer by experiments on animals. Both experimental physiology and pathology have localised the sensory areas (and sensations are the materials for intellect) behind the Rolandic fissure, but this does not necessarily mean that the frontal convolutions have nothing to do with intellectual functions. The celebrated American crowbar accident is generally quoted as a proof to the contrary; owing to the premature explosion of a charge of dynamite in one of the American mines a crowbar was sent through the frontal region of the foreman's head, removing the anterior part of his brain. $\mathrm{He}$ is usually stated to have subsequently returned to his work, without any noteworthy symptoms. Recent examination of the records of the case has shown that this is not correct; when he returned to work he was practically useless, having lost just those higher 
functions which are so important in the superintendence of other people. Mott's observations on lunatics show that this region is important for intellectual operations, though not so important as the parietal association area behind the Rolandic area; the greater the intellectual development, the larger and more convoluted does this parietal region become.

The association fibres have been the subject of special study by Flechsig, who has shown that in the development of the brain these are the last to become myelinated; white fibres do not become fully functional until they receive their medullary sheath. This coincides with the well-known fact that association of ideas is the last phase in the psychical development of the child. It has been shown that the frontal convolutions are connected by important association tracts with the more posterior regions of the brain (see fig. 436, p. 699), and there is therefore no difficulty in understanding that the frontal convolutions play the purt of a centre for the association of ideas, or in other words for intellectual operations.

\section{Function and Myelination.}

Flechsig's embryological method has given us most valuable knowledge of the structure and functions of the human brain. The method depends on the fact that various tracts of fibres become myelinated, i.e., acquire their medullary sheath at successive periods of time in development. The myelin sheath appears three or four months after the axis cylinder is formed. The Weigert method of staining renders

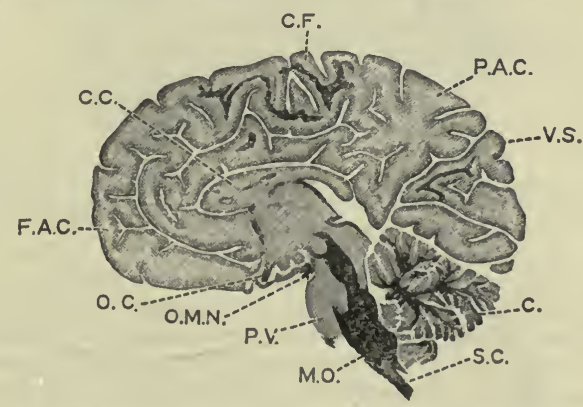

FiG. 452.-Diagram of vertical section through brain of new-born child, drawn from one of Flechsig's photographs. The section was treated by Weigert's method, by which myelinated fibres are deeply stained. Attention is drawn to the deep shading indicating myelination around the central fissure, which corresponds to the sensori-motor area, and also around the calcarine fissure in the visual sphere. The association fibres are not myelinated. The fibres of the pyramidal efferent system have also no myelin. M.O., medulla oblongata ; P.V., pons Varolii ; O.M.N., oculo-motor nerve ; O.C., optic commissure; F.A.C., frontal association centre; C.C., corpus callosum; C.F., central fissure, or fissure of Rolando; P.A.C., posterior association centre; V.S., visual sphere; C., cerebellum; S.C., spinal cord.

the detection of a medullary sheath an easy task. Flechsig's method is in short the complement of the Wallerian method. In the former method the tracts are isolated by the differences in the origin of the myelin sheath; in the latter method, the same object is obtained by observing the degeneration which is most noticeable in the same sheath.

In the central nervous system, the afferent projection fibres are myelinated first; 
the efferent projection fibres and the association fibres are myelinated later. Thus in the human foetus the peripheral nerves and nerve-roots become myelinated in the fifth month of intra-uterine life; of the tracts in the cord, those of Burdach and Goll (exogenous fibres springing from the cells of the spinal ganglia) are the first to be myelinated; next come the tracts of Flechsig (dorsal cerebellar) and of Gowers (ventral cerebellar): these are endogenous fibres springing from cells within the cord. All these tracts are afferent. The pyramidal tracts, the great efferent or motor channels, are not myelinated until after birth. The whole afferent tract is myelinated at birth; these fibres have in utero been exercised in conveying impressions to the afferent reception centres, the stimuli arising from contact of the foetal integuments with the maternal tissues. There is also early myelination around the calcarine fissure in the visual sphere, and in connection with the areas related to other special senses. This is shown in figs. 452 and 453 , where the condition at birth and that some months later are compared.

Ambronn and Held confirm Flechsig in finding that the afferent fibres are myelinated before the efferent, in the central nervous system, but in the case of the nerve-roots this is reversed, the anterior root-fibres being myelinated before the posterior.

Held has also demonstrated the important influence of stimulus on myelination. His experiments were made on cats, dogs, and rabbits, which are born blind. If

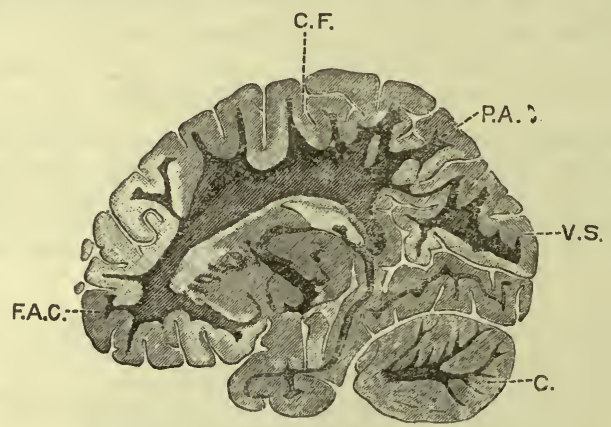

Fia. 453.-Diagram of vertical section of the brain of a child 5 months of age. The greater part of the white matter now shows myelination, thus indicating development of the association centres. The letters have the same meaning as in Fig. 452. (After Flechsig; Weigert method of staining.)

light is admitted to one eye by opening the lid, more obvious myelination is subsequently found in the corresponding optic nerve than in that of the opposite side. This is not due to the irritation caused by forcibly opening the lid, for if the lid be opened and the animal kept in the dark, no difference in the myelination of the two optic nerves is observable. Flechsig also showed that a child born at 8 months had more marked myelination of its optic nerves, a month later, than a child born in the usual way at the ninth month.

The richness of the brain in myelinated fibres increases for many years after birth with the progress of intellectual development. Kaes states this continues up to forty years of age, and that in old age the number diminishes. Myelin appears to be necessary for the functional activity of nerve tracts, and its development progresses pari passu with development of function; the reverse change (atrophy and degeneration) correspondingly accompanies marked disturbances of function.

\section{Association Fibres and Association Centres.}

We know by common experience that any group of muscles can be voluntarily contracted in reply to any form of stimulus, cutaneous, visual, auditory, etc. If, for instance, the wrist is flexed in response to an auditory stimulus, the nerve 
impulses pass first to the auditory area, then by certain fibres to the cerebral cells which control the muscles of the arm. The fibres which connect the two areas are termed association fibres. A diagrammatic view of the principal bundles of association fibres is given in fig. 436, p. 699.

The term "association centres" is given by Flechsig to those portions of the cortex that lie between the sensory centres. The function of these centres is first to furnish pathways between the several centres, and secondly to retain as memories previous sense impressions, so that in action they may modify the impulses sent into them, and by these modifications adjust to an almost infinite degree the form of the final response.

The association centres comprise a very large area of the cortex, and are divided into three:-(1) The great anterior association centre in the frontal lobe; (2) the posterior association centre in the parieto-temporal region; (3) the

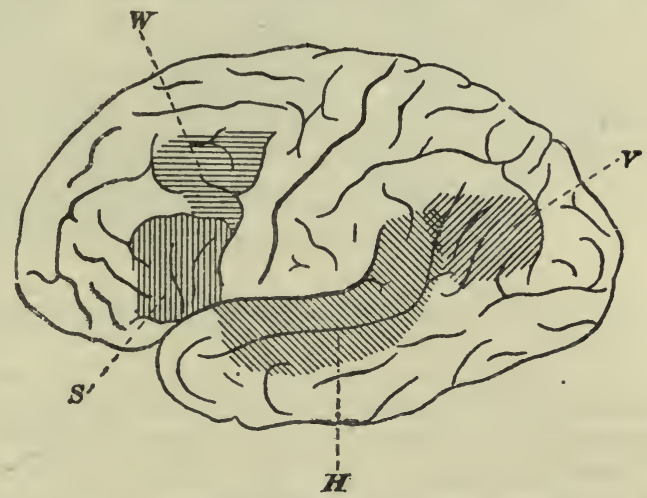

Fie. 454.-I Lateral view of the left cerebral hemisphere of man (after Donaldson). V is the cortical area damage to which produces "word blindness"; it is situated in the angular gyrus, and is called the visual word centre. $H$ is the area in the superior temporal convolution, called the auditory word centre, damage to which produces "word deafness." S is Broca's convolution, damage to which produces loss of audible speech (motor aphasia); this is the motor area for the movements of the tongue, vocal cords, etc., concerned in speaking; Bastian terms it the glosso-kinasthetic area. The area W, called by Bastian the cheiro-kincesthetic area, is the corresponding region concerned in hand movements, damage to which abolishes the power of writing (agraphia).

middle association centre; this is smaller and coincides with the island of Reil. These regions are in fact those in which no evident response follows excitation; they are sometimes called the "latent or silent or inexcitable cortex." The human brain is characterised by the high development of these parts, and as already explained they are doubtless, as Flechsig terms them, the organs of thought.

The importance of the association of ideas, which has for its anatomical basis the association of cortical centres, will be at once grasped when one considers such complex actions as speaking, reading aloud, or writing from dictation. The accompanying diagram (fig. 454) shows the position of the main centres involved, particulars of which will be found in the small text beneath the figure.

In reading aloud, the impressions of the words enter by the eyes, reach that portion of the visual sphere known as the visual word centre, travel across to the auditory word centre by association fibres, where the memory of their sounds is revived; another tract of association fibres connects this to the sensori-motor area in Broca's convolution called by Bastian the glosso-kincesthetic area, whence motor impulses originate which finally reach the muscles concerned in pronouncing the words originally seen.

Writing from dictation is just as complex; the course of the impulses is by the auditory channels to the auditory word centre, then by association tracts to the visual word centre, where the shapes of the letters composing the words are revived; another association tract carries the impulse thence to the sensori-motor 
area connected with the movements of the hand (Bastian's cheiro-kincesthetic area) near the middle region of the Rolandic cortex, and finally the movement of writing is accomplished. The diverse symptoms exhibited by patients suffering from various forms of aphasia can be all explained by more or less extensive damage

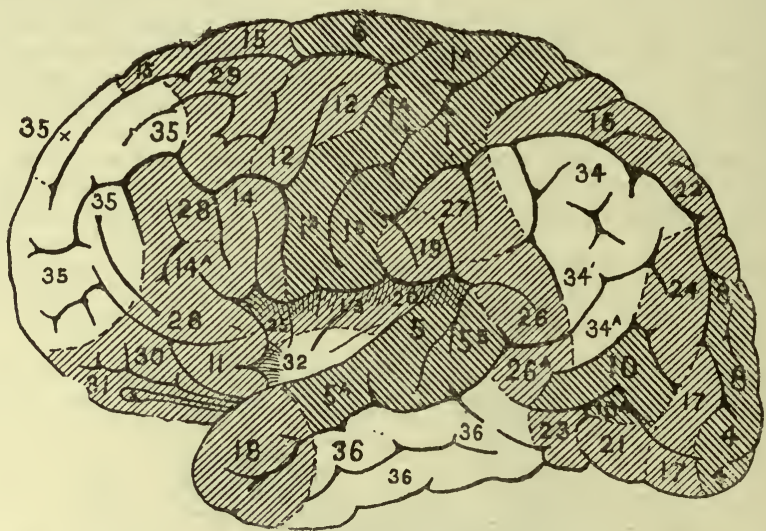

FIG. 455.- Outer surface of human brain, showing Flechsig's developmental zones; primary (1-10) darkly shaded ; intermediate (11-31), less deeply shaded ; terminal (32-36), not shaded. (Flechsig.)

either to the centres themselves or to the association tracts which connect them.

In the cerebral convolutions the fibres become myelinated in a strictly regular sequence; some convolutions have their fibres medullated three months before birth, while in others complete myelination has not occurred six months later.

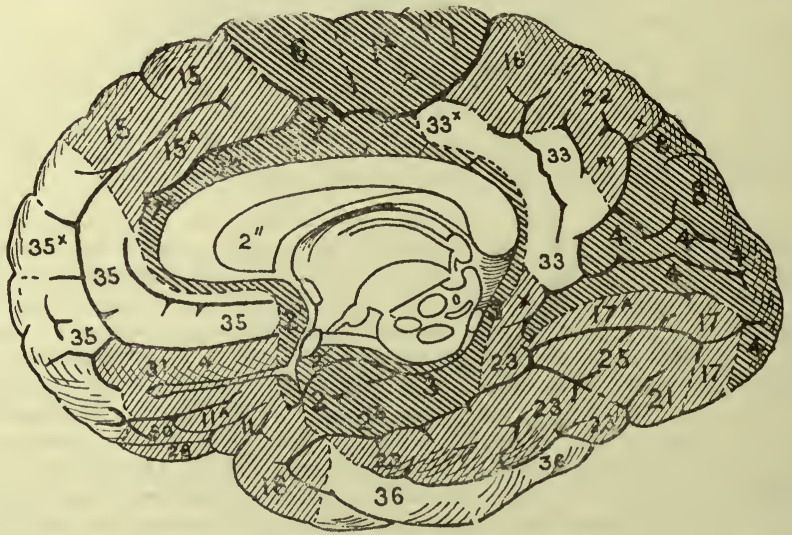

FIG. 456.-Inner surface of same. (Flechsig.)

Fibres of equally great importance become medullated at the same time; those of primary importance first, and so on. In this way, myelogenetic cortical fields can be mapped out, which retain their contours for some time. Thirty-six of such fields were made out by Flechsig, and can be divided chronologically into three groups, primary, intermediate, and terminal. and 456 ).

The primary fields are darkly shaded in the accompanying diagrams (figs. 455 
They are 10 in number, and are those provided with myelinated fibres at birth ; they contain the seats of the cortical representation of all the senses. To No. 1 is assigned the cutaneous and muscular sense; to No. 2 the sense of smell; to No. 4 that of vision; to No. 5 that of hearing. The functions of some of the primary areas had not been determined. The principal efferent projection tracts originate from the primary fields ; thus the pyramidal tract starts from part of No. 1, namely, from the ascending frontal convolution. The sensory fibres connected with the skin and muscles terminate in the ascending parietal convolution. The inferior fornix is connected with Nos. 2 and 3. The inner bundle of the pes springs from $1 \mathrm{~B}, 6,12,14$ and 15 ; the origin of the outer bundle of the pes is doubtful. From the visual area (No. 4). a tract arises which passes mainly into the anterior corpus quadrigeminum; the auditory zone (No. 5), towards which a tract proceeds that leads from the internal corpus geniculatum, sends an outgoing tract into the column of Türck, and thus motor functions of the upper part of the body are possible as a direct result of auditory impressions. In fact, in every case each primordial sensory zone is connected with a well-defined pair of tracts, one proceeding to it (cortico-petal) and the other from it (cortico-fugal). It is thus impossible to speak of a purely motor or a purely sensory area.

The terminal areas (Nos. 31 to 36, unshaded in the diagrams) do not begin to be myelinated until at least a month after birth. These and the majority of the intermediate areas (Nos. 11 to 31 , lightly shaded in the diagrams) show few or no projection fibres even 8 months after birth. They comprise, in fact, the association centres, and are rich in long association fibres.

\section{Electrical Variation in Central Nervous System.}

$\mathrm{Du}$ Bois Reymond found that the spinal cord, like a nerve, exhibits a demarcation current between its longitudinal surface and a cross-section, and that a diminution of this current occurs on excitation (negative variation). Gotch and Horsley investigated the currents of the cord very thoroughly. If the Rolandic area of the cortex is stimulated, and a portion of the thoracic region of the spinal cord is led off to a galvanometer, a persistent negative variation followed by a series of intermittent variations is observed; this exactly corresponds to the tonic spasm followed by clonic contractions which occur in the muscles excited by this means.

The galvanometer in the hands of these observers also proved to be a valuable instrument for determining the paths taken by nervous impulses in the cord. One example will suffice: If the central end of one sciatic nerve is stimulated, the chief electrical variation in the cord is noticed to be obtained when the same side of the cord is led off to the galvanometer, but a certain amount of electrical variation is obtainable from the opposite side of the cord. This coincides with the fact ascertained by other methods, that the main sensory channel is on the same side of the cord as the entering nerves, but that there is a certain amount of decussation below the level of the bulb.

Electromotive changes also occur during activity in the cortex cerebri, but they have not been much studied, and we do not know whether they have their seat in the grey or in the underlying white matter. 


\section{Sleep and Narcosis.}

The conditions that favour sleep are:-

(1) A diminution of the impulses entering the central nervous system by the afferent channels. This is under our voluntary control, as, for instance, in closing the eyes, or retiring to a quiet room.

(2) Fatigue. This diminishes the readiness of the central nervous system to respond to stimuli.

The first two hours of sleep are always the most profound; later on, relatively weak stimuli will cause awakening. Of the parts of the central nervous system, the spinal cord is always less profoundly affected than the brain, but even the brain is never entirely irresponsive, and unless slumber is very profound, dreams are the subjective result of external stimuli. Sensations of sound appear to be the last to disappear as sleep comes on, and the first to be realised on awakening.

Sleep has been attributed by some to changes in the blood-supply of the brain, and ultimately referred to fatigue of the vaso-motor centres. The existence of an effective vaso-motor mechanism in the cerebral blood-vessels themselves is problematical (see p. 313); so that if changes occur in the cerebral blood-pressure or rate of flow, they are mainly secondary to those which are produced in other parts of the body. Plethysmographic records from the arm of a sleeping man show a diminution in its volume every time he is disturbed, even though the disturbance may not be sufficient to awaken him. This is interpreted as meaning a diminution in the blood of the body, and a corresponding increase in the blood-flow through the brain. It is, however, quite possible that the vascular condition is rather the concomitant or consequence of sleep than its cause. Howell among others believes it to be the cause, and attributes the sleepiness that follows a heavy meal to the mechanical effect of a dilatation of the abdominal vessels in producing a diminished bloodflow through the brain; but the sleep that normally comes on at the end of the day, he believes to be produced by cerebral anæmia following dilatation of the blood-vessels of the skin, such dilatation being due to vaso-motor fatigue.

Some of the theories to account for sleep have been chemical. Thus certain observers have considered that sleep is the result of the action of chemical materials produced during waking hours, which have a soporific effect on the brain; according to this theory awakening from sleep is due to the action of certain other-materials produced during rest, which have the opposite effect. Obersteiner has gone so far as to consider that the soporific substances are acid in nature, and others regard them as alkaloidal. These theories 
all rest upon the flimsiest foundations, and none has yet been found to stand experimental tests.

Then there are what we may term histological theories of sleep, and these are equally unsatisfactory. The introduction of the Golgi method opened a fresh field for investigators, and several have sought to find by this method a condition of the neurons produced by narcotics such as opium and chloroform, which is different from that which obtains in the waking state.

Demoor and others found in animals in which deep anæsthesia has occurred, that the dendrites exhibit moniliform swellings, that is, a series of minute thickenings or varicosities. On the strength of this observation, what we may call a biophysical theory of sleep has been formulated; in the waking state, the neighbouring nerve units are in contact with each other; transmission of nerve impulses from neuron to neuron is then possible, and the result is consciousness; during sleep the dendrites are retracted in an amœboid manner; the neurons are therefore separated, and the result is unconsciousness.

Lugaro, on the other hand, takes the precisely contrary view. He was not able to discover moniliform enlargements, and his biophysical hypothesis is that the interlacing of dendrites is much more intimate during sleep than during consciousness. He therefore explains sleep by supposing that the definite and limited relationships between neurons no longer exists, but are lost and rendered ineffective by the universality of the connecting paths. It is not very difficult to explain such divergence of views, for they both depend mainly on observations made by a single method; and the method itself is open to objection. It is one which gives even in the same brain most inconstant results, and is not calculated to show much more than an outline of a few of the cells and their branches.

A more satisfactory histological investigation of the effect of anæsthetics on nerve-cells was carried out by Hamilton Wright.

$\mathrm{He}$ used rabbits and dogs, and subjected them to ether and chloroform narcosis for periods varying from half an hour to nine hours. In both animals he found that the nerve-cells are affected, but in rabbits much more readily. This accords quite well with what is known regarding the susceptibility of rabbits as compared to dogs towards the influence of these narcotising agents. In a rabbit, the nerve-cells, especially of the cerebrum, show changes even after only half an hour's anæsthesia, but in dogs at least four hours' anæsthesia must be employed. By the Golgi method the moniliform enlargements can be seen. These become more numerous, larger, and encroach more and more on the dendritic stems, the longer the anæsthesia is kept up. The accompanying illustrations show the appearances seen (fig. 457). 
Lugaro's failure to find these appearances is doubtless due to his not having maintained the anæsthesia long enough in his dogs.

Wright started his work with a bias in favour of Demoor's biophysical theory, but he soon found that the theory was untenable; the results of his observations have shown him that the action of anæsthetics is biochemical rather than biophysical, and he has been led to this conclusion by the employment of other histological methods, particularly the most sensitive one we possess, namely, the methylene-blue reaction.

Owing to the chemical action of the anæsthetic on the cells, the Nissl bodies have no longer an affinity for methylene-blue, and the cells consequently present what Wright calls a rarefied appearance;

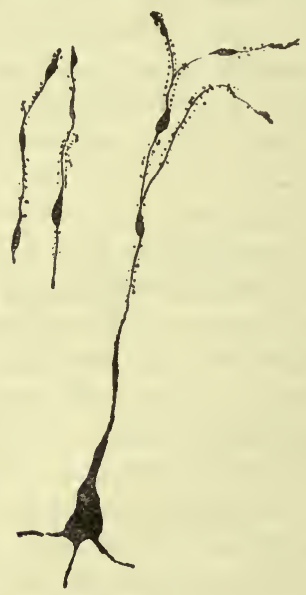

A

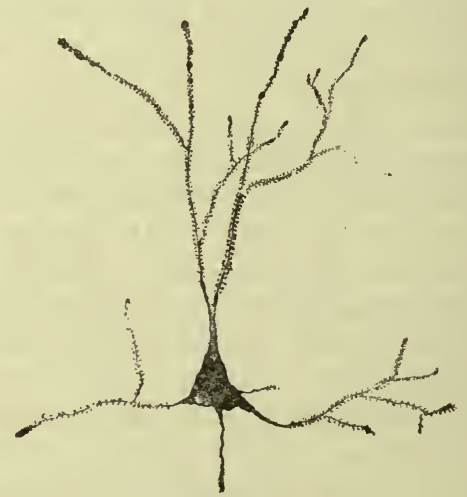

B

FIG. 457.-Moniliform enlargements on dendrites of nerve-cells, rendered evident by Cox's modification of Golgi's method. A, in a cortical cell of a rabbit ; B, in a corresponding cell of a dog's brain, after six hours' anæsthetisation with ether in each case. (Hamilton Wright.)

when this becomes marked the cells appear like the skeletons of healthy cells. In extreme cases the cells look as though they had undergone a degenerative change, and after eight or nine hours' anæsthesia in dogs, even the nucleus and nucleolus lose their affinity for basic dyes. The change, however, is not a real degeneration, and passes off when the drug disappears from the circulation. Even after nine hours' anæsthesia the cells return rapidly to their normal condition, stain normally, moniliform enlargements have disappeared, and no nerve-fibres show a trace of Wallerian degeneration. The pseudo-degenerative change produced by the chemical action of the anæsthetic no doubt interferes with the normal metabolic activity of the cell-body, and this produces effects on the cell-branches. In 
the early stages of Wallerian degeneration, the branch of the nervecell which we call the axis-cylinder presents swellings or varicosities, produced by hydration or some similar chemical change. The moniliform enlargements seen during the temporary pseudo-degenerative effects produced by anæsthetics are comparable to this. These enlargements are therefore not the primary cause of loss of consciousness, but are merely secondary results of changes in the cell-body. When a tree begins to wither the earliest apparent change is noticed in the branches most remote from the centre of nutrition, the root; as the changes in the centre of nutrition become more profound, the larger branches become implicated, but the seat of the mischief is not primarily in the branches. This illustration may serve to render intelligible what is found in nerve-cells and their branches.

Moore and Roaf believe that the substance in the nerve-cells (as well as the other cells of the body) which is affected by chloroform is the protein. They have shown that unstable compounds of protein and chloroform are obtainable; hence the greater solubility of chloroform in blood than in water. They compare the chloroform-protein compound to oxyhæmoglobin, for it undergoes dissociation in the same sort of way. Just as oxyhæmoglobin parts with its oxygen to the tissue-cells, so the chloroform parts company from the bloodprotein, and enters into combination with the cell-protein, limiting its activity and producing quiescence or anæsthesia. When the administration of the chloroform ceases, the tension of chloroform in the blood is no longer maintained, so the combination between the cell-protein and chloroform dissociates, and anæsthesia passes off.

The theory which has met with most favour in relation to anæsthetics, however, is that known as the Meyer-Overton hypothesis; this theory, which has received abundant confirmation by numerous observers, points out that the cells are easily permeable to the volatile anæsthetics owing to the presence of fat and lipoid material in their plasmatic membrane (see p. 330). It can hardly now be doubted that the solubility of the volatile anæsthetics in the lipoids of the membrane (or, what comes to the same thing, the solubility of the lipoids in the anæsthetic) is an important factor in anæsthesia; the anæsthetic thus enters the cell easily, and throws the lipoid constituents of the protoplasm (and perhaps secondarily the protein constituents also) out of gear, the net result being a lessening of the oxidative changes which are essential in active vital processes.

The volatile anæsthetics, and especially chloroform, are dangerous drugs, and although their discovery is one of the greatest blessings to suffering humanity, and in experiments on animals, means should be adopted for preventing the fatalities which are even now too frequent. It is specially needful that anæsthetists should not administer the drug in a haphazard way, but take care that the amount 
of chloroform in the inspired air should never rise over 2 per cent.; smaller percentages may be employed when once the patient is under the influence of the drug. There have been several instruments invented (by Waller and others) by means of which the concentration of the chloroform given can be easily measured.

But the artificial sleep of a deeply-narcotised animal is no criterion of what occurs during normal sleep. The sleep of anæsthesia is a pathological condition due to the action of a poison. The drug reduces the chemico-vital activities of the cells, and is, in a sense, dependent on an increasing condition of exhaustion, which may culminate in death. Natural sleep, on the other hand, is the normal manifestation of one stage in the rhythmical activity of nerve-cells, and though it may be preceded by fatigue or exhaustion, it is accompanied by repair, the constructive side of metabolic activity. This is true for many other organs in addition to the central nervous system; sleep is a time of repose for them also, but the amount of rest varies; the voluntary muscles, except those concerned in breathing, will rest most, but the heart continues to beat, the urine is still being secreted, the processes of digestion go on, so that for such organs activity is only diminished.

It should be recognised by the public that sleep is the period of anabolism, repair and growth, and a large allowance is therefore necessary in growing children. The mistaken Spartan discipline of certain parents and schoolmasters in insisting upon a short period of repose often does incalculable harm both mental and physical to those under their charge. When in the children of the poorer classes, early rising for the purpose of earning a miserable pittance is combined with late hours of retiring to rest, and with the discomforts of crowded bedrooms, and crowded beds which render real rest impossible, the damage done is greater still, and is one cause of physical deterioration. Many children judged to be "defective" are really only suffering from want of sleep.

Loss of sleep is more damaging than starvation. Dogs will recover after being starved for three weeks, but they die from loss of sleep in five days. The body temperature falls, reflexes disappear, and post-mortem the brain is found to contain capillary hæmorrhages, the cord is dry and anæmic, and fatty degeneration is found in most of the tissues.

In man, loss of sleep curiously enough causes a slight rise in weight; the body temperature falls; the excretion of nitrogen and still more so that of phosphoric acid increases; the reactions of the muscular, and later those of the nervous, system diminish in intensity, except that in all cases there is an increase in acuteness of vision. These experiments were made by Patrick and Gilbert on three young men, who voluntarily went without sleep for ninety hours. At the end of the experiment a very small extra amount of sleep beyond the normal caused complete restoration, and all the symptoms, including the increase of weight, disappeared.

The sleep produced by hypnotic suggestion differs from ordinary sleep. But exact knowledge of the phenomena of this kind of sleep is at present lacking. 


\section{CHAPTER LI}

\section{FUNCTIONS OF THE CEREBELLUM}

IN past times there have been several views held as to the functions of the cerebellum. One of the oldest of these was the idea that the cerebellum was associated with the function of generation; another view, first promulgated by Willis, was that the cerebellum contained the centres which regulate the functions of organic life; this arose from the circumstance that diseases of the cerebellum are often associated with nausea and vomiting; it is a familiar fact that in displacements of equilibrium such as occur on board ship in a rough sea, or in the disease called Menière's disease, sickness is a frequent result; it appears from this that the cerebellum does receive certain impulses from the viscera. The third and last of these older theories was that the cerebellum was the centre for sensation. This arose from the fact that certain of the afferent channels of the spinal cord were traced into the cerebellum. The impulses that travel along these, however, though afferent, are not truly sensory, and their reception in the cerebellum is not associated with consciousness.

The true function of the cerebellum was first pointed out by Flourens, who showed that the cerebellum is the great centre for the coördination of muscular movement, and especially for that variety of coördination which is called equilibration - that is, the harmonious adjustment of the working of the muscles which maintain the body in a position of equilibrium.

It must not be supposed from this that the cerebellum is the sole centre for coördination. We have already seen that all the machinery necessary for carrying out very complicated locomotive movements is present in the spinal cord. The higher centres set this machinery going, and the work of arranging what muscles are to act, and in what order, is carried out by the whole of the grey matter from the corpora striata to the end of the spinal cord, including such outgrowths as the corpora quadrigemina and cerebellum. An instance of a complex coördinated movement is seen in what we learnt to call in the last chapter conjugate deviation of head and eyes. The higher cortical centre gives the general word of command to turn the head 
rbic

and eyes to the right: the subsidiary centres or subordinate officials arrange that this is to be accomplished by the external rectus of the right eye supplied by the right sixth nerve, the internal rectus of the left eye supplied by the left third nerve, and numerous muscles of neck and back of both sides supplied by numerous nerves. The relaxation of the antagonistic muscles has also to be provided for. We thus see how the complicated intercrossing of fibres and connections of the centres of the various nerves are brought into play.

The functions of the cerebellum are investigated by the same two methods of experiment (stimulation and extirpation) which are employed in similar researches on the cerebrum. The anatomical connections of the cerebellum with other parts of the cerebro-spinal axis by its three peduncles have been already considered on p. 684 .

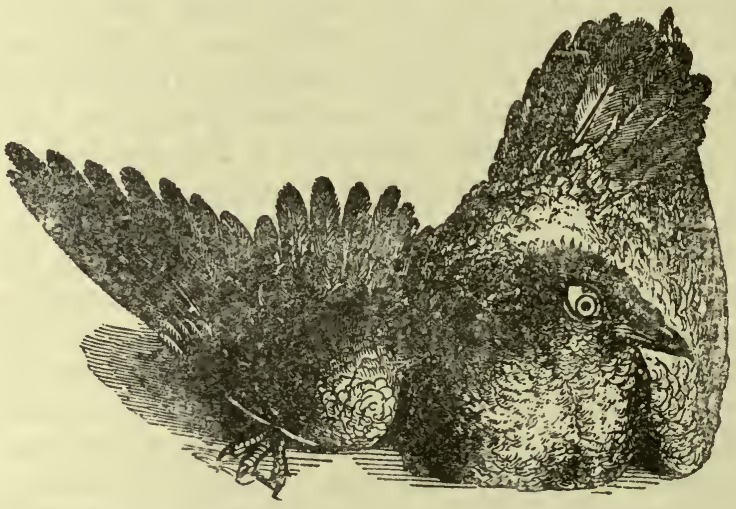

Fia. 458.-Pigeon after removal of the cerebellum. (Dalton.)

In some of the lower animals the vermis is practically the only part of the cerebellum which is present, and it is this part of the cerebellum which is principally concerned in the coördination of the bodily movements. The cerebellar hemispheres are especially connected with the opposite cerebral hemispheres; and just as the different regions of the body have corresponding areas in the cerebrum, so also they are similarly represented in the cerebellum; but it does not appear necessary from the practical standpoint to go here into the scanty and disputed details of cerebellar localisation already discovered.

If the cerebellum is removed in an animal, or if it is the seat of disease in man, the result is a condition of slight muscular weakness ; but the principal symptoms observed are loss of muscular tonus and a condition of incoördination, chiefly evidenced by a staggering gait similar to that seen in a drunken man. It is called cerebellar ataxy.

This condition is well illustrated in the figure (fig. 458); the 
disturbed condition of the animal contrasts very forcibly with the sleepy state produced by removal of the cerebrum (see fig. 446, p. 723).

In order that the cerebellum may duly execute its function of equilibration, it is necessary that it should send out impulses; this it does by fibres that leave its cells and pass out through its peduncles; they pass out to the opposite cerebral hemisphere, and so influence the discharge of the impulses from the cortex of the cerebrum. Impulses also pass out to the cord (see p. 685), but the exact course of some of the descending tracts has still to be worked out.

The cerebellum thus acts upon the muscles of the same side of the body in conjunction with the cerebral hemisphere of the opposite side. The close inter-relation of one cerebral with the opposite cerebellar hemisphere is shown in cases of brain disease, in which atrophy of one cerebellar hemisphere follows that of the opposite cerebral hemisphere (see fig. 459).

In order that the cerebellum may send out impulses in this way, it is necessary that it receive impulses which

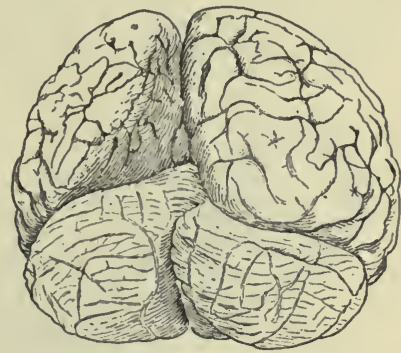

Fif. 459.-This is a reproduction of a photograph of a lunatic's brain lent me by Dr Fricke. One cerebral and the opposite cerebellar hemisphere are atrophied. guide it by keeping it informed of the position of the body in space. These impulses, we have already insisted, though afferent are nonsensory; they travel by paths which at the start, however, are offshoots from those which carry the real sensory impulses to the cerebrum. These afferent impulses originate from or are associated with the impulses which in the cerebrum produce sensations of the four following kinds :-

1. Tactile.

3. Visual.

2. Motorial.

4. Labyrinthine.

1. Tactile impressions. - The importance of the tactile sense is obvious; and in diseases of the afferent tracts, loss of that sense in the lower limbs leads to disturbances of equilibrium; in such cases a man has difficulty in balancing himself while standing with his eyes shut. Sherrington, however, has shown how comparatively unimportant is the loss of tactile sensibility from the feet. A cat, in which the feet have been completely desensitised by division of all their nerves, can stand and walk without obvious inconvenience. It is not until the sensitiveness of the joints, especially in the upper segments of the limb, is interfered with that marked disturbances of balance are noticeable.

2. Motorial impressions.-Another important sense is that which 
enables us to know what we are doing with our muscles. Sensory fibres pass from the muscles, and their tendons to the posterior roots of the spinal nerves, and the impulses ascend the sensory tracts through cord-and brain to reach the ascending parietal convolution. Their offshoots, which carry the non-sensory impulses to the cerebellum, reach it via Clarke's column and the cerebellar tracts. In many cases of locomotor ataxy there is but little loss of tactile sensibility, and the condition of incoördination is then chiefly due to

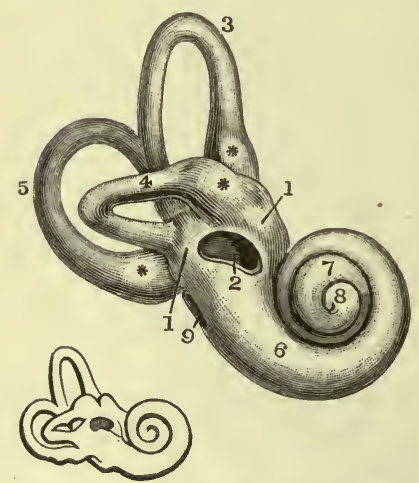

FIG. 460.-Right bony labyrinth, viewed from the outer side. The specimen here represented was prepared by separating piecemeal the looser substance of the petrous bone from the dense walls which immediately en. close the labyrinth. 1, the vestibule; 2 , fenestra ovalis; 3 , superior semicircular canal; 4, horizontal or external canal; 5 , posterior canal; *, ampullæ of the semicircular canals: 6 , first turn of the cochlea; 7 , second turn; 8 , apex; 9 , fenestra rotunda. The smaller figure in outline below shows the natural size. (Sömmering.) the loss of impressions from motorial organs (muscles and joints).

3. Visual impressions. - The use of visual impressions in guiding the nervous centres for the maintenance of equilibrium is seen in those cases of locomotor ataxy where there is loss of equilibrium when the patient closes his eyes. Destruction of the eyes in animals often causes them to spin round and lose their balance. The giddiness experienced by many people on looking at moving water, or after the onset of a squint, or when objects are viewed under unusual circumstances, as in the ascent of a mountain railway, is due to the same thing. The importance of keeping one's eyes open is brought home to one very forcibly when one is walking in a perilous position, as along the edge of a precipice, where an upset of the equilibrium would be attended with serious consequences. Under more ordinary circumstances, the non-sensory visual offshoots which enter the cerebellum are sufficient to maintain equilibrium. In speaking of visual impressions it should be understood that these in themselves are not the actual guide. It is the projection of what is seen in relation to the position of the body (ascertained by the innervation of the head muscles and ocular muscles) that is the chief guide.

4. Labyrinthine impressions.-These are the most important of all; they are the impressions that reach the central nervous system from that part of the internal ear called the labyrinth, and in this case the sensory element is subordinate to the non-sensory. Here, however, we must pause to consider some anatomical facts in connection with the semicircular canals that make up the labyrinth. Fig. 460 is an external view of the internal ear; it is enclosed within 
the petrous portion of the temporal bone; and consists of three parts - the vestibule (1), the three semicircular canals $(3,4,5)$ which open into the vestibule, and the tube, coiled like a snail's shell, called the cochlea $(6,7,8)$. The cochlea is the part of the apparatus which is concerned in the reception of auditory impressions; it is supplied by the cochlear division of the eighth or auditory nerve. The remainder of the internal ear is concerned not in hearing, but in the reception of the impressions we are now studying; it is supplied by the vestibular division of the eighth nerve. Within the vestibule are two chambers made of membrane, called the utricle and the saccule; these communicate with one another and with the canal of the cochlea. Within each bony semicircular canal is a membranous semicircular canal of similar shape. Each canal is filled with a watery fluid called endolymph, and separated from the bony canal by another fluid called perilymph. Each canal has a swelling at one end called the ampulla. The membranous cauals open into the utricle; the horizontal canal by each of its ends; the superior and posterior vertical canals by three openings, these two canals being connected at their non-ampullary ends.

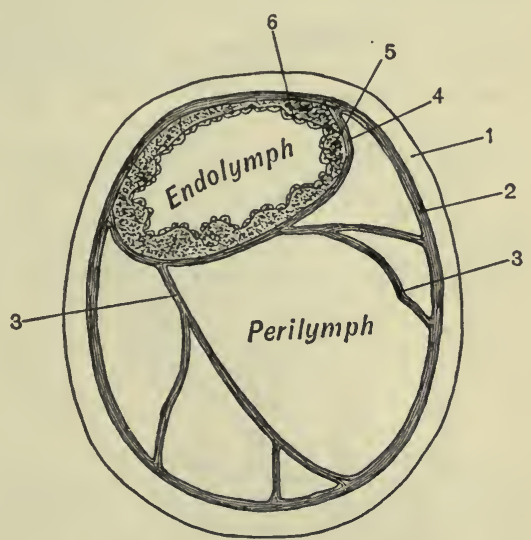

FIG. 461.-Section of human semicircular canal. (After Rüdinger.) 1, Bone; 2, periosteum; 3, 3, fibrous bands connecting the periosteum to 4 , the outer fibrous coat of the membranous canal; 5 , tunica propria; 6 , epithelium.

Fig. 461 shows in transverse section the way in which the membranous is contained within the bony canal; the membranous canal consists of three layers, the outer of which is fibrous and continuous with the periosteum that lines the bony canal; then comes the tunica propria, composed of homogeneous material, and thrown into papillæ except just where the attachment of the membranous to the bony canal is closest; and the innermost layer is a somewhat flattened epithelium.

At the ampulla there is a different appearance; the tunica propria is raised into a hillock called the crista acoustica (see fig. 462); the cells of the epithelium become columnar in shape, and to some of them fibres of the eighth nerve pass, arborising round them; these cells are provided with stiff hairs, which project into what is called the cupula, a mass of mucus-like material containing otoliths or crystals of calcium carbonate. Between the hair-cells are fibre- 
cells which act as supports (fig. 463). When the endolymph in the interior of the canals is thrown into vibration, the hairs of the hair-

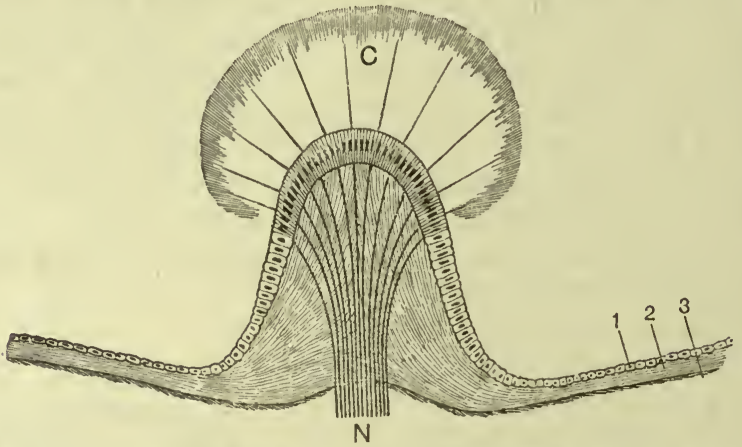

Fic. 462.-Section through the wall of the ampulla of a semicircular canal, passing through the crista acoustica. 1, Epithelium ; 2, tunica propria ; 3, fibrous layer of canal ; N, bundles of nerve-fibres ; C, cupula, into which the hairs of the hair-cells project. (After Schäfer.)

cells are affected, and a nervous impulse is set up in the contiguous nerve-fibres, which carry it to the central nervous system.

The walls of the saccule and

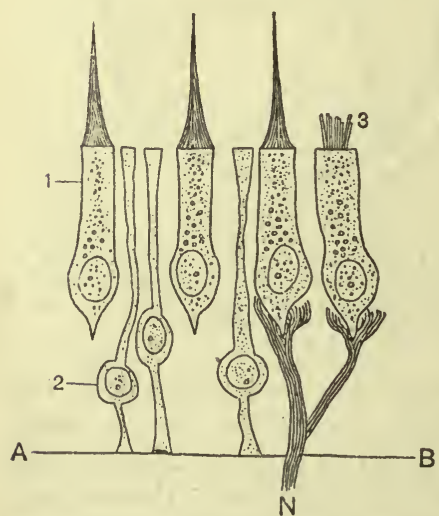

Fra. 463.-1, Hair-cell ; 3, hair-cell, showing the hair broken, and the base of the hair split into its constituent fibrils; 2 , fibre. cell; $N$, bundle of nerve-fibres which have lost their medullary sheath, and terminate by arborising round the base of the hair-cells ; A B, surface of tunica propria. (After Retzius.) utricle are similar in composition, and each has a similar hillock, called a macula, to the hair-cells on which nerve-fibres of the vestibular nerve are distributed.

It will be noticed that the canals of each side are in three planes at right angles to each other, and we learn the movements of our body with regard to the three dimensions of space by means of impressions from the ampullary endings of the vestibular nerve; these impressions are set up by the varying pressure of the endolymph in the ampullæ.

Thus a sudden turning of the head from right to left will cause movement of the endolymph towards, and therefore increased pressure on, the hair-cells connected to the ampullary nerve-endings of the left horizontal canal, and diminished pressure on the corresponding apparatus of the right side. It is probable that resulting from such a movement two impulsos reach the brain, one the effect of increased pressure in one ampulla, the second the effect of decreased pressure in its fellow. It may 
even be that increased pressure on one side of a crista is accompanied by diminished pressure on the opposite face of the same crista.

"One canal can be affected by, and transmit the sensation of rotation about one axis in one direction only; and for complete perception of rotation in any direction about any axis, six canals are required in three pairs, each pair being in the same or parallel planes, and their ampullæ turned opposite ways. Each pair would thus be sensitive to any rotation about a line at right angles to its plane or planes, the one canal being influenced by rotation in one direction, the other by rotation in the opposite direction." (Crum-Brown.)

The two horizontal canals are in the same plane; the posterior vertical of one side is in a plane parallel to that of the superior vertical of the other side (see fig. 464).
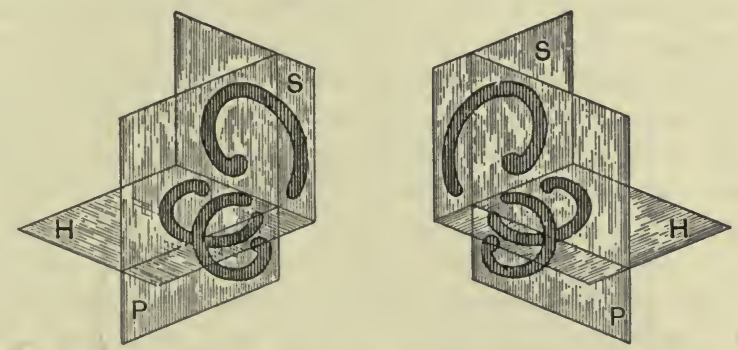

F1G. 464.-Diagram of semicircular canals, to show their positions in three planes at right angles to each other. It will be seen that the two horizontal canals $(\mathrm{H})$ lie in the same plane : and that the superior vertical of one side (S) lies in a plane parallel to that of the posterior vertical (P) of the other. (The student will understand that though in the diagram the canals are entirely separated from one another, that they are really connected, as shown in fig. 460.) (After Ewald.)

When these canals are diseased in man as in Menière's disease, there are disturbances of equilibrium: a feeling of giddiness, which may lead to the patient's falling down, is associated with nausea and vomiting. In animals similar results are produced by injury, and the subject has been chiefly worked out on birds by Flourens, where the canals are large and readily exposed, and more recently in fishes, by Lee.

Thus, if the horizontal canal is divided in a pigeon, the head is thrown into a series of oscillations in a horizontal plane, which are increased by section of the corresponding canal of the opposite side. After section of the vertical canals, the forced movements are in a vertical plane, and the animal tends to turn somersaults.

"When the whole of the canals are destroyed on both sides the disturbances of equilibrium are of the most pronounced character. Goltz describes a pigeon so treated which always kept its head with the occiput touching the breast, the vertex directed downwards, with the right eye looking to the left and the left looking to the right, the head being incessantly swung in a pendulum-like manner. Cyon says it is almost impossible to give an idea of the perpetual 
Inovements to which the animal is subject. It can neither stand, nor lie still, nor fly, nor maintain any fixed attitude. It executes violent somersaults, now forwards, now backwards, rolls round and round, or springs in the air and falls back to recommence anew. It is necessary to envelop the animals in some soft covering to prevent them dashing themselves to pieces by the violence of their movements, and even then not always with success. The extreme agitation is manifest only during the first few days following the operation, and the animal may then be set free without danger; but it is still unable to stand or walk, and tumultuous movements come on from the slightest disturbance. But after the lapse of a fortnight it is able to maintain its upright position. At this stage it resembles an animal painfully learning to stand and walk. In this it relies mainly on its vision, and it is only necessary to cover the eyes with a hood to dispel all the fruits of this new education, and cause the reappearance of all the motor disorders." (Ferrier.)

It is these canals which enable all of us to know in which direction we are being moved, even though our eyes are bandaged, and the feet are not allowed to touch the ground. On being whirled round, such a person knows in which direction he is being moved, and feels that he is moving so long as the rate of rotation varies, but when the whirling stops he seems, especially if he opens his eyes, to be whirling in the opposite direction, probably owing to the rebound of the fluid in the canals. The forced movements just described in animals are due both to the absence of the normal sensations from the canals and to delusive sensations arising from their irritation, and the animal makes efforts to correct the movement which it imagines it is being subjected to.

Artificial stimulation of the canals produces movements of the head and orbits, and giddiness. Similar movements occur during bodily rotation, and giddiness is the result of a rivalry of sensations which afford conflicting ideas of the position of the body relatively to external objects. A certain proportion of deaf mutes lose their sense of direction under water, cannot maintain their equilibrium when their eyes are shut, exhibit no orbital movements when rotated, and never suffer from sea-sickness or giddiness. This proportion is approximately the frequency in which abnormal conditions of the canals have been found post-mortem in deaf mutes.

Section and stimulation of the inferior cerebellar peduncles (the path by which the vestibular fibres reach the cerebellum, see p. 682) cause incoördination, chiefly evidenced by rotatory and circus movements similar to those that occur when the nerve-endings in the semicircular canals are destroyed or stimulated. Stimulation of the cerebellum itself-and this has been done through the skull in man-causes giddiness, and consequent muscular efforts to correct it. The results of stimulation, indeed, are precisely analogous to those of extirpation, only in the reverse direction. Loss of muscular tone which follows extirpation of the canals is probably the result of secondary changes in the brain. 


\section{CHAPTER LII}

\section{THE PHYSIOLOGY OF CONSCIOUS STATES}

THERE are certain considerations, relating to the physiology of conscious states in general, to which it will be well to pay attention, before we pass to a detailed study of the special senses.

It is sometimes argued that states of consciousness are the product of the activity of nerve-cells, just as bile is the product of the activity of the liver-cell, or as contraction results from the activity of the muscle fibre. But this analogy will not bear close investigation. It is, however, true:-

(1) That the different senses are dependent for their manifestation on the integrity of different definitely localisable areas of the cerebral cortex.

(2) That such drugs as alcohol, caffein, and chloroform, which have a known action on living substance, also affect the course of conscious processes.

(3) That disease or malformation of the brain is accompanied by impairment or absence of intelligence.

But because nervous substance is essential for the manifestation of conscious states, one cannot legitimately infer that this substance pro:luces those states. Indeed, by a vast number of philosophers a very different position has been upheld. So far from believing that mind results from the activity of living matter, they have insisted that all matter, living and lifeless, results from the activity of mind. They maintain that, were it not for mental activity, there would be no conception, nay not even existence, of those qualities (e.g., sound, colour, force, weight, hardness) of which our non-mental world of matter is composed.

There is no difficulty in accepting the statement that bile is secreted by the liver; in this case the product is physical, and it is produced by physiological (i.e., presumably, by chemical and physical) conditions. On the other hand, if we state that consciousness is secreted by the brain, we are linking together two sets of phenomena, the psychical and the physiological, between which a connection is inconceivable. 
Consequently, instead of stating that physiological activity is the cause of mental (or psychical) activity, it is more satisfactory to assume that the two activities run parallel with one another, and to recognise that the nature of their relation is unknown. This conception of psycho-physical parullelism affords the physiologist by far the best working hypothesis.

It leaves unanswered the great question whether brain ever acts on mind, or mind on the brain-which of the two is the master or the servant of the other. It merely implies that a change in nerve substance underlies every psychical change; and it bids the physiologist investigate the functions of the nervous system, and determine what structures are called into activity in the development of various conscious states.

We must recognise that, however completely we may one day have mapped out the functions of the various parts of the brain, we shall nevertheless not.have approached a step nearer towards understanding the relation between the data of physiological and psychical activity. If we knew the function of every nerve cell of the body, the gap between the material and the mental would not be a bit less wide. Just as a ray of light cannot see itself, so we cannot expect to understand states of consciousness from a mere study of cerebral function.

It is therefore imperative to avoid confusion between the two aspects involved in this psycho-physical parallelism. The psychical is one language, the physical (i.e. the physiological) is another; and the two vocabularies must be kept distinct from one another. Psychology and physiology stand in the relation of an object and its mirrored reflection. To confound object and image-to speak, for instance, of a sensation (instead of an impulse) being transmitted along a nerve-fibre, is to blur and to confuse two distinct sciences.

The psychologist distinguishes three modes in which consciousness is manifested. These are (1) the cognitive, (2) the affective, and (3) the conative modes. Through the cognitive mode we become aware of the object thought of. Owing to the affective mode, our state of consciousness is toned with pleasure, indifference, or displeasure. The conative mode manifests itself as a striving or "felt tendency" towards an end. In every state of consciousness these three modes are present, but their relative prominence is always different. For example, in perception, in memory, or imagination, the cognitive element is to the fore; in love, sorrow, or doubt, the affective element predominates; while in intense desire, the conative element is most easily recognisable. Into the physiology of affection and conation we shall not enter here. They receive adequate attention in books devoted to physiological and experimental psychology.

But a conscious state implies also a contrast between what is 
outside of ourselves (the object) and our feelings and strivings in connection with it, which are spoken of as subjective. The existence of this "subject-object relation" implies the activity of an Ego, who experiences conscious states, who is cognisant, feels or strives. Indeed no state of consciousness is ever possible, unless experienced by the Ego. In becoming manifest, it blends with the Ego, and is modified or rather determined by the Ego's previous experiences; and in turn it modifies the Ego. Thus the Ego everlastingly moulds and is itself moulded by its own states of consciousness or experiences. Consequently, states of consciousness are not independent units. The mind, like its physiological correlate, the central nervous system, works as a single, unitary entity, despite its complex differentiation (see also p. 725).

From one aspect "states" of consciousness is an inaccurate expression. The essential features of consciousness are its incessant change and its intimate relation to past and future consciousness; whereas the word state implies a period of rest and a certain isolation or independence. Save for this difficulty, it would be possible to regard a given state of consciousness as the cross-section of a stream which is always flowing. The simile may be deemed of value, in so far as it allows us to represent different levels of conscious states. At any moment, there is always part which is in the focus, or full glare of consciousness, and part of which we are dimly conscious or wholly unconscious, but of which we may at any moment become conscious - for example, the ticking of a clock in the room or the pressure of a pipe between the teeth while these lines are being written or read. We may imagine that as the stream of consciousness flows on, different portions come to the surface at different times and under different conditions, while others fall below, often to such a depth that they pass altogether beyond the margin of consciousness.

To speak of a "stream of consciousness" is in one sense correct; but at any moment there are probably innumerable streams, which, under normal circumstances, play the part of a single or unitary stream, owing to that integrative activity which we term the Ego. These various streams at any moment form a pattern, but that pattern is ceaselessly changing, as the streams run hither and thither.

On the physiological side, we see the analogue of these streams in the streams of nervous impulses which are perpetually coursing through the brain. The pattern of these streams is likewise always changing. And we may suppose that some patterns are incompatible with the simultaneous occurrence of certain other patterns. In this way, we may form a physiological conception of the basis of inhibition; the pattern which inhibits and that which is inhibited cannot coexist. This incompatibility has doubtless been developed 
in evolutional history owing to the necessity of adjustment to environment.

We may regard the physiological correlate of consciousness as a state of resistance to the onward passage of the nervous impulse. When the resistance is high, there is consciousness; when it is low, there is none. Thus when any new action (such as skating or bicycling) is being learnt, the resistance is, as we should expect, high. But the more often that act is repeated, the lower becomes the resistance, until ultimately the act becomes a habit and is performed in the complete absence of consciousness far more surely and rapidly than in the earlier stages of learning. It must be borne in mind, however, that this conception of lowered resistance is purely hypothetical. We have no actual evidence as to which part of the neuron it is that offers resistance, although we may conjecture that the resistance occurs at the synapses, when the dendritic processes of one neuron meet those of another.

The hypothesis is at all events valuable in so far as it contradicts an old and erroneous conception that, as an action becomes habitual and no longer accompanied by consciousness, the nervous impulses quit the higher parts of the brain and confine themselves to the subcortical and spinal regions. There can be no doubt that nervous impulses pursue the same course in the brain, whether at one moment consciousness be present, or at another absent.

In the spinal cord, on the other hand, there is no evidence of the presence of consciousness. The acts which are executed by the isolated cord are reflex. In so far as they are unaccompanied by consciousness, they are comparable to habits acquired by training in the higher parts of the nervous system.

Within certain limits, reflex actions can be predicted. If we apply a known stimulus to the afferent portion of a reflex system, we can with fair confidence predict the result of the stimulus on the efferent portions connected therewith. When, on the other hand, the stimulus involves the manifestation of consciousness, prediction is almost impossible; there is so little fixity, the nervous connections are so complex, and the nervous impulse may wander in such a variety of directions, that one cannot forecast with certainty how an individual will behave under the influence of external circumstances.

It is common to speak of the most primitive cognitive experience as sensation. On the physiological side, sensation involves (1) an end-organ in a sensory epithelium, adapted to receive the stimulus; (2) a sensory nerve path transmitting the nerve impulse, which ultimately reaches (3) a sensory centre in the cortex of the brain. But it is very doubtful whether the sensory cortical areas should be regarded as the "seats" of sensation. It is quite conceivable that 
they are merely areas through which the nervous impulses must pass in order that the corresponding sensations may be developed.

In any case, we must recognise that from infancy onwards we never have a pure sensation, that is to say, an experience devoid of meaning and totally dissociated from past experiences - an experience only dependent on end-organ, nerve-fibre and sensory centre. Our experiences come to us for the purpose of adjusting ourselves to the outer world ; consequently they possess such meaning as is necessary for that end. It is true that in infancy our states of consciousness are vague; but they are always related to previous experiences and are motives for action. Thenceforth they gradually become more definite. The various elements which they contain become differentiated, recognised, and separated. What was at first homogeneous is later found to consist of heterogeneous parts.

Consequently it is incorrect to say, as is so often said, that with growing experience sensations are grouped together so as to give rise to the perception of objects. It is true that from our adult perception of an object, e.g. of an orange, certain sensations of colour, taste, smell, etc., may be analysed and separated. But a moment's reflection will convince us that our perception of the orange has never arisen by the converse synthesis or building together of such sensations. From infancy onwards the world appears to us (however vaguely) as composed of objects. The sensations of which we have presently to treat are the artificial products of the analytical activity of the Ego.

Recognising that sensations are not truly immediate experiences, but are very abstract in origin, we may proceed to consider the various characters with which they may be invested. Sensations may differ from one another in modality or in quality. Modally different sensations are derived from different senses, qualitatively different sensations from the same sense. Blue and green are qualitatively different sensations; it is possible to pass by gradual transition from one to the other. Heat and noise are modally different; such gradual transition is impossible.

Now every peripheral end-organ is specially destined to respond to a certain form of stimulus. The end-organs of the ear respond to sound waves: those of the eye to light waves; those of the skin to heat, cold, touch, and pain. That stimulus to which the end-organ is thus fitted to respond, is called its adequate or homologous stimulus. But an end-organ will often respond to other, inadequate, stimuli. For example, when the eyeball is struck, sparks are seen; when a "cold spot" on the skin is stimulated by a hot point, a cold sensation results; when an electric current is applied to the papillæ of the tongue, sensations of taste arise.

Hence it has been argued that the modality of a sensation 
depends not upon the nature of the stimulus, but upon the nature of the sensory apparatus on which the stimulus acts. Johannes Müller expressed this conception in what is known as the law of specific nervous energy. He supposed that every sensory apparatus had its own "specific energy," and that that energy was evoked by any stimulus so long as the stimulus was at all effective. We have, however, no physiological evidence that the nerve impulses passing, say, along the optic fibres, are different in "energy," or in any other character, from those which are transmitted, say, by the auditory fibres. Indeed, the experiments of Langley and others on nervecrossing (p. 161) would seem to indicate that the nervous impulse is an identical process in all nerves. It may be that the "specific energy" of sensations resides in the various sensory centres of the brain. But if that be so, it is important to realise how dependent that "energy" is for its development on the corresponding endorgans. A person whose visual or auditory end-organs have been functionless from birth, can never know what it is to see or hear; he can never think or dream in terms of visual or auditory imagery.

Whether qualitatively different sensations involve separate endorgans, or whether they are the outcome of different kinds of activity in one and the same end-organ, is at present far from certain. Probably there are a few "primary sensations" for each sense organ, and the many different qualities of sensation obtainable are due to various combinations of such elements.

We know, generally speaking, that sensations differ in quality according to the rate of vibration of the stimulus. Sound waves of rapid and slow vibration give rise to sensations of high and low pitch respectively. Light waves of rapid and slow vibration give rise to sensations of blue and red respectively. Differences in intramolecular vibration probably give rise to qualitative differences in olfactory, gustatory, and thermal sensations.

The strength of the stimulus (e.g. the amplitude of vibration) determines a third character in which sensations may differ from one another, namely, in intensity (for instance, the loudness of a sound, or the brightness of a light).

Yet another character of many sensations is extensity, or "spreadoutness." Smell and taste and some other sensations seem to be devoid of extensity. It is best developed in visual and cutaneous sensations, and these possess yet another characteristic, local signature. Every point stimulated on the retina or skin has its local sign, in virtue of which we are able to localise the stimulus at that point and to distinguish the sensation from those produced by the stimulation of neighbouring points. On the basis of extensity and local signature is built up our perception of extension, form, and spatial relations generally. 
The remaining characters ascribable to sensation are protensityon which our perception of duration is based-and affective tone, which give us our experience of pleasure, indifference, or displeasure. But these we will not discuss; they are more suitably studied in works on psychology.

It is of interest to note how intimately the various characters of sensation are bound up with one another. If we attempt experimentally to change one character, it is difficult to avoid simultaneously changing another. For example, when we increase the extensity of a warm sensation by putting more of our arm into hot water, we at once increase the intensity of the sensation. If we increase the area of a very distant colour stimulus, we alter its hue. The hue of a colour is also apparently altered by increasing the intensity of the stimulus. To many people the pitch of a sound appears altered by increasing its loudness.

It is likewise important to remember that the characters of a sensation depend not only on the strength, vibration-rate, duration, etc., of the stimulus, but also upon the condition of the sensory apparatus which is stimulated and upon the temporary condition of neighbouring sensory areas; nay, the characters of a sensation depend upon the state of the nervous system generally, that is to say, upon the total mental state at the moment of application of the stimulus.

The strength of a stimulus must not fall below a certain minimum in order that a sensation may result. Too light a touch, too faint a sound, will produce no effect on consciousness. That strength of stimulus which just suffices to evoke a sensation is called the liminal (from limen, a threshold)* value of the stimulus, or its absolute threshold.

Similarly, the difference between two stimuli must not fall below a certain minimum in order that that difference may be appreciated. If two musical tones are of too nearly identical pitch, if two colours are of too nearly identical hue, the difference may be imperceptible. There is, hence, a liminal value for a stimulus difference. This is known as the differentical threshold of the stimulus.

Weber's law states that the just appreciable difference between two stimuli depends on the ratio of that difference to their magnitudes, and not on the absolute difference between their magnitudes. Fechner, after bringing forward further evidence in favour of the law, endeavoured to deduce from it the conclusion that the strength of a sensation is proportional to the logarithm of its stimulus; in other words, that the stimulus must increase in geometrical proportion for the sensation to increase in arithmetical proportion.

* Strictly speaking, the liminal value is that strength of stimulus which in a series of trials as often just fails as it just succeeds in evoking a sensation. 
Fechner's interpretation of Weber's law is, however, open to serious criticism, into which we cannot enter here.

Weber's law is but an expression of everyday experience. A rushlight will brighten a dark cellar, but its presence is unfelt in sunshine. So, too, if a room be lighted by 100 candles, and if one candle more be brought in, the increased illumination produced by the extra candle would be just perceptible to the eye. But if a room were lighted by 1000 candles, no appreciable difference would result from the introduction of an extra candle. Ten candles would have to be introduced, in order to effect a just noticeable difference. In each case a difference of one-hundredth of the original strength of stimulus is needful to cause a just appreciable difference in the sensation; and this is in accordance with Weber's law.

For light, the fraction is about $\frac{1}{100}$; for noise, it is about $\frac{1}{3}$; for cutaneous pressure, it varies between $\frac{1}{30}$ and $\frac{1}{10}$; for weight, between $\frac{1}{70}$ and $\frac{1}{40}$, according to the part of the body which is under investigation.

A sensation requires an appreciable time for its development. Part of this time is spent at the end-organ on which the stimulus acts, part in conveying the nervous impulse along the sensory nerve to the brain, and part within the brain itself. This latent period varies in length according to the sensation; e.g., it is longer for sight than for sound, and longer for pain than for touch.

A sensation outlasts its stimulus. Indeed, a single stimulus may produce a whole train of after-sensations. These are specially noticeable in the case of visual sensations, which we shall be considering later.

When the sensation and its after-sensations have passed away, the original experience may still be revived, either spontaneously or by an effort of volition. This revival involves what is called the memory image. When, in this way, a tune "comes into the head," we recognise that it is only a reproduction, or a representation, of what we have previously heard.

Occasionally, however, the revived image has all the vividness and distinctness of objective experience, and we believe that it is "real." In other words, we have a hallucination. Hallucinations occur normally in all people; but they are, of course, particularly common in sleep and in conditions of insanity or delirium.

It is still disputed whether the difference between original and revived experiences corresponds to an excitement of distinct regions of the brain. Some physiologists have gone so far as to speak of "memory centres" as existing apart from the sensory centres which are supposed originally to have excited them, and they have considered that the recall of a scene or of a tune is due to the reexcitation of the appropriate memory centres, while the correspond- 
ing sensory centres are quiescent. The balance of evidence, however, is very strongly against this view. It is better to suppose that the physiological processes underlying a sensation and its revived memory image are broadly the same. There is unquestionably some physiological difference corresponding to the difference between sensory experiences and hallucinations on the one hand, and revived experiences on the other. But at present it is impossible to say in what that difference consists.

When, as occurs under certain conditions, an object is adjudged different from what general experience teaches us to be its "real" character, we have an illusion. Thus a line or figure may appear to be longer or shorter than it really is, or to take a direction different from its real direction. Or a weight may appear heavier than another which is really equal to it. Illusions are due partly to peripheral, partly to central factors. Their investigation falls within the province of experimental psychology. 


\section{CHAPTER LIII}

\section{CUTANEOUS SENSATIONS}

THE tactile end-organs are of numerous kinds, but the following are the principal ones:-

Pacinian Corpuscles.-These are named after their discoverer Pacini. They are little oval bodies, situated on some of the cerebrospinal and sympathetic nerves, especially the cutaneous nerves of

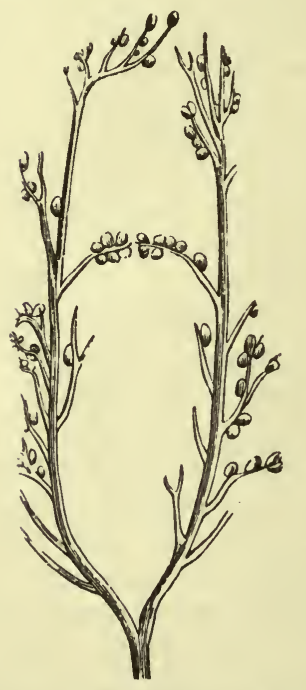
of the finger with Paciuian cor. puscles attached, about the natural size. (Adapted from Henle and Kölliker.)
F1G. 465.-Extremities of a nerve the hands and feet, where they lie deeply placed in the true skin. They also occur on the nerves of the mesentery of some animals such as the cat. They have been observed also in the pancreas, lymphatic glands, and thyroid glands, as well as in the penis. They are about $\frac{1}{12}$ inch long. Each corpuscle is attached by a narrow pedicle to the nerve on which it is situated, and is formed of several concentric sheaths of connective tissue, each layer being lined by endothelium (figs. 466, 467); through its pedicle passes a single nerve-fibre, which loses its medullary sheath and enters a central core, at or near the distal end of which it terminates in an arborisation. Some of these layers are continuous with those of the perineurium, but some are superadded. In some cases two nerve-fibres have been seen entering one Pacinian body, and in others a nerve-fibre after passing through it has been observed to terminate in a second.

The corpuscles of Herbst (fig. 468) are closely allied to Pacinian corpuscles, except that they.are smaller and longer, with a row of nuclei around the central termination of the nerve in the core. They have been found chiefly in the tongues and bills of ducks. 
End-bulbs are found in the conjunctiva (where in man they are spheroidal, but in most animals oblong), in the glans penis and clitoris, in the skin of the lips, in the epineurium of nerve-trunks, and in tendon; each is about $\frac{1}{600}$ inch in diameter, oval or spheroidal,

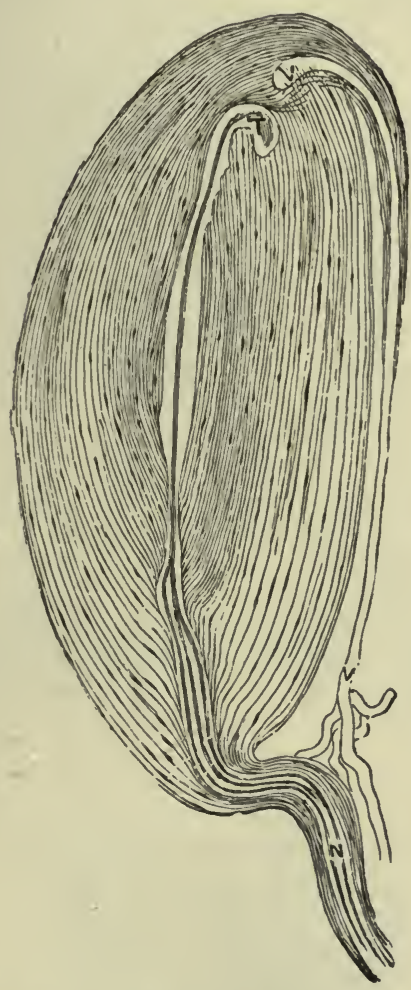

Fra. 466. - Pacinian corpuscle of the cat's mesen. tery. The stalk consists of a nerve-fibre(N) with its thick outer sheath. The peripheral capsules of the Pacinian corpuscle are continuous with the outer sheath of the stalk. The intermediary part becomes much narrower near the entrance of the axis-cylinder into the clear central core. A hook-shaped termination $(\mathrm{T})$ is seen in the upper part. A blood-vessel (V) enters the Pacinian corpuscle, and approaches the end; it possesses a sheath which is the continuation of the peripheral capsules of the Pacinian corpuscle. $\times 100$. (Klein and Noble Smith.)

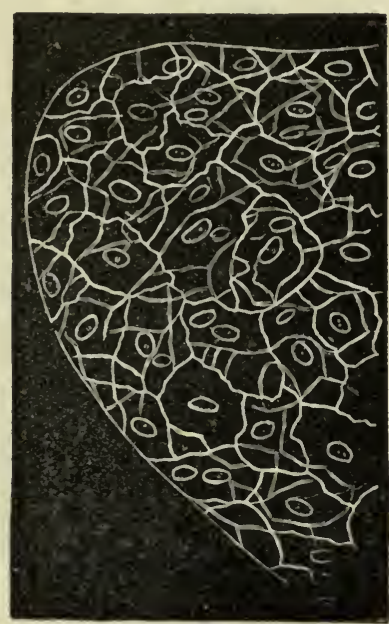

FIG. 467.-Summit of a Pacinian cor. puscle of the human finger showing the endothelial membranes lining the capsules. $\times 220$. (Kloin and Noble Smith.)

and is composed of a medullated nerve-fibre, which terminates among cells of various shapes. Its capsule contains a transparent or striated core, in the centre of which the axis-cylinder terminates (fig. 469).

Touch-corpuscles (Meissner's corpuscles) (figs. 470,472), are found in the papillæ of the skin of the fingers and toes. They are oblong, about $\frac{1}{990}$ inch long, and $\frac{1}{800}$ inch broad, and composed of connective tissue, surrounded by elastic fibres and a capsule of nucleated cells. They do not occur in all the papillæ of the parts where they are found, and, as a rule, in the papillæ in which they are present there are no blood-vessels. 
The peculiar way in which the medullated nerve winds round and round the corpuscle before it enters it is shown in fig. 472. It

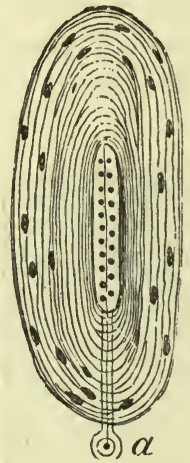

FIG. 468.-A corpuscle of Herbst, from the tongue of a duck. a, Medullated nerve cut away. (Klein.)

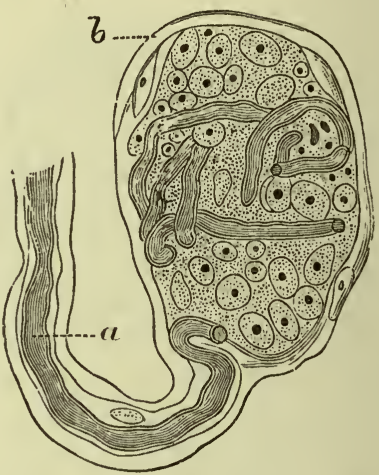

FIG. 469.-End-bulb of Krause. $a$, Medullated nerve-fibre; $b$, capsule of corpuscle.

loses its sheath before it enters into the interior, and then its axiscylinder branches, and the branches after either a straight or convoluted course terminate within the corpuscle.

A

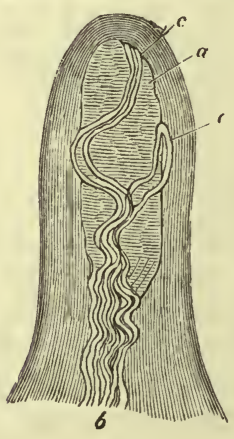

B

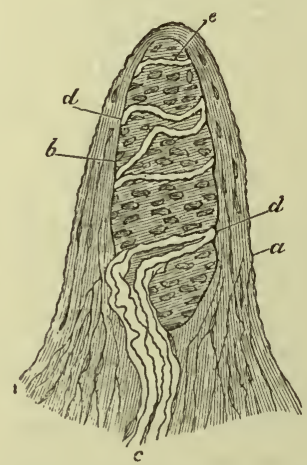

F1G. 470.-Papillæ from the skin of the hand, freed from the cuticle and exhibiting Meissner's corpuscles. A. Simple papilla with four nerve-fibres; $a$, tactile corpuscle; $b$, nerves with winding fibres $c$ and $e$. B. Papilla treated with acetic acid; $a$, cortical layer with cells and fine elastic filaments; $b$, tactile corpuscle with transverse nuclei; $c$, entering nerve; $d$ and $e$, nerve-fibres winding round the corpuscle. $\times 350$. (Kölliker.)

The corpuscles of Grandry (fig. 471) form another variety, and have been noticed in the beaks and tongues of birds. They consist of oval or spherical cells, two or more of which compressed vertically 
are contained within a delicate nucleated sheath. The nerve enters on one side, and, laying aside its medullary sheath, terminates between the cells in flattened expansions.

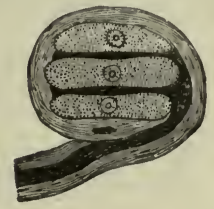

Fig. 471. - A corpuscle of Grandry,. from the tongue of a duck.

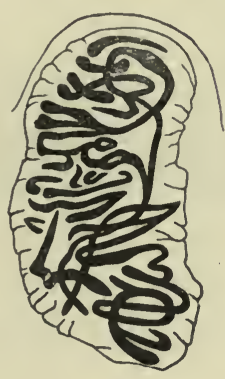

Fxa. 472.-A touch-corpuscle from the skin of the human hand, stained with gold chloride.

Sensory nerve-endings in muscle. - Nerve terminations, sensory in function, are found in tendon. Some of these are endbulbs, and others appear very much like end-plates, as represented in figs. 471 and 472 . The neuro-muscular spindles, one of which is

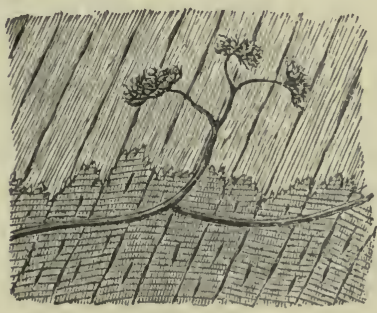

Fig. 473.-Termination of medullated nerve-fibres in tendon near the mus. cular insertion. (Golgi.)

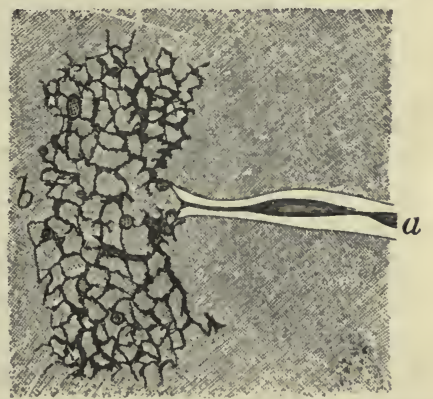

F1G. 474.-One of the reticulated end-plates of fig. 473 , more highly magnified. $a$, Medullated nerve-fibre; $b$, reticulated end-plate. (Golgi.)

shown in the accompanying drawing (fig. 475), are principally found in muscles in the neighbourhood of tendons and aponeuroses.

The principal grounds for believing the neuro-muscular spindles to be sensory are, first, that the nerve-fibres that supply them do not degenerate when the anterior roots of the spinal nerves are cut, and secondly, that they do degenerate when the posterior roots are divided (Sherrington). They also undergo degenerative changes in 
locomotor ataxy, which is a disease of the sensory nerve-units, and remain healthy in infantile paralysis, which is a disease of the motor cells of the anterior horn of the cord (Batten).

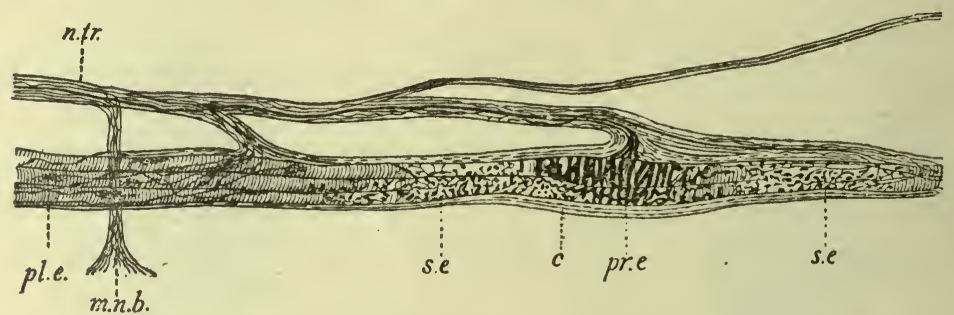

Fic. 475.-Neuro-muscular spindle. c., Capsule; n.tr., nerve trunk; m.n.b., motor nerve bundle; pl.e., plate-ending; pr.e., primary nerve-ending; s.e., secondary ending. (After Ruflini.)

In addition to the special end-organs, sensory fibres may

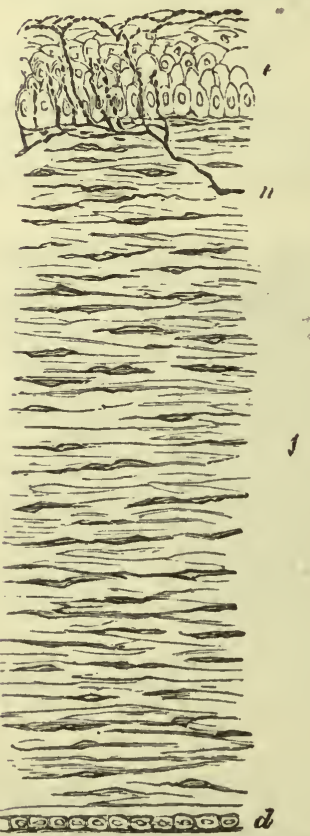

Frg. 476.-Vertical section of rabbit's cornea, stained with gold chloride. The nerves, $n$, terminate in a plexus uuder and within the epithelial layer, $e$. eyes are closed, with the points of a pair
of compasses, and ascertaining how close the points may be brought the subepithelial and the intra-epithelial plexus of the cornea (fig. 476) and around the hair follicles in the skin generally (see fig. 392, p. 607). In some cases the nerve-fibrils within a stratified epithelium ond in crescentic expansions (tactile discs) which are applied to the deeper epithelium cells. These are well seen in the skin of the pig's snout.

\section{Localisation of Tactile Sensations.}

The localisation of a tactile sensation is of two kinds, absolute and relative. We can localise a touch on the arm absolutely by indicating the exact spot which has been touched, or we may localise it relatively to another spot on the arm which is simultaneously or successively touched. Generally speaking, the delicacy of these two kinds of localisation is similarly variable in different parts of the body.

The "local signature" (p. 762) of cutaneous sensations may be easily investigated by touching the skin, while the eyes are closed, with the points of a pair
ing how close the points may be brought terminate in plexuses of fibrils, as in 
to each other, and still be felt as two points. (Weber.) A few results are as follow:-

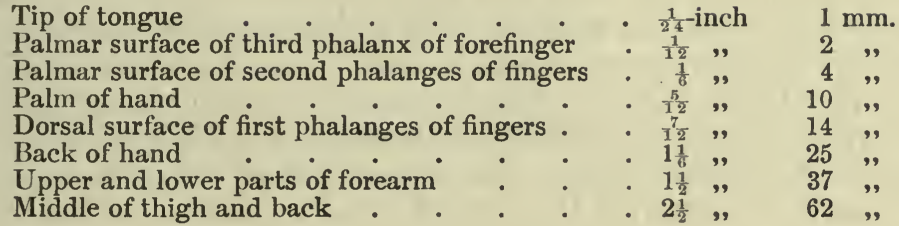

In the case of the limbs, it is found that before they are recognised as two, the points of the compasses have to be further separated when the line joining them is in the long axis of the limb, than when in the transverse direction.

We may thus assume that minute areas of the body surface have each their "local sign," i.e., the sensation arising from stimulation of one area differs in some obscure quality from the sensations arising from stimulation of neighbouring areas, thereby acquiring its own spatial colouring which enables us to identify the area when stimulated. The difference of local sign between two near points may be imperceptible in one region of the body, but fully recognisable in another. Again, the delicacy of the sense of touch may be very much increased by practice. A familiar illustration occurs in the case of the blind, whe, by constant practice, can acquire the power of reading raised letters, the forms of which are almost if not quite undistinguishable by the sense of touch to an ordinary person.

The different delicacy of local signature possessed by different parts may give rise to errors of judgment in estimating the distance between two points where the skin is touched. Thus, if the blunted points of a pair of compasses (maintained at a constant distance apart) are slowly drawn over the skin of the cheek towards the lips, it is almost impossible to resist the conclusion that the distance between the points is gradually increasing. When they reach the lips they seem to be considerably further apart than on the cheek. Then, too, our estimate of the size of a cavity in a tooth is usually exaggerated when based upon sensations derived from the tongue alone. Another curious illusion is the following:-If we close the eyes, and place a marble between the crossed fore and middle fingers, we seem to be touching two marbles. This illusion is due to an error of judgment. The marble is touched by two surfaces which, under ordinary circumstances, could only be touched by two separate marbles; hence, regardless of the fact that the fingers are crossed, the judgment is formed that the two sensations are due to two marbles. 


\section{Varieties of Cutaneous Sensations.}

The surface of the skin is a mosaic of tiny sensorial areas; but these areas are not set edge to edge as in the retina, but separated by relatively wide intervals which are not sensitive to stimuli just above liminal intensity. If the stimuli are made nearly minimal, the individual fields are reduced to small spots. Each of these spots subserves a specific sense, touch, cold, heat or pain, and each doubtless coincides with the site of some special end-organ, placed either singly or in clusters. The "touch spots," "cold spots," "heat spots," and "pain spots" are intercommingled. In some districts one variety predominates, in others another. "Pain spots" are the most and "heat spots" the least numerous. It is a matter of common experience that the sensitiveness of these varieties of cutaneous sensation differs in different parts of the body. The tip of the finger, which is very sensitive to the true tactile sense (sense of pressure or contact), is not nearly so sensitive to alterations of temperature as the forearm or cheek, to which a washerwoman generally holds her iron when forming a judgment of its temperature. Some parts of the skin are more sensitive to pain than others, and in the cornea we have an instance of a surface in which "pain spots" alone are present.

For the more accurate exploration of the skin, cesthesiometers of various kinds have been invented. The sense of pressure may be estimated by the ability of the skin to distinguish different weights placed upon it; there must be no lifting of the weight, or the motorial sense is brought into play. The fraction which by Weber's law represents the differential threshold (see p. 763) varies from $\frac{1}{30}$ to more than $\frac{1}{10}$ in different parts of the body. It does not, however, follow that the acuteness of the pressure sense varies exactly as the ability of accurately localising sensations; for instance, the skin of the forearm is as sensitive to pressure changes as that of the palm; and the tip of the tongue, which is the most discriminative region of the body for locality, is not so for pressure. For pressure stimuli which are near the limen or threshold of sensation, the hair æsthesiometer is much used; this is a hair suitably mounted in a holder; the hair can then be shifted backwards or forwards in the holder, and the amount of pressure it exercises can thus be varied. It is used for the exploration of "touch spots," and these are found most numerously around the hair follicles. The touch spots are more numerous in some parts than in others, but fifteen for each square centimetre of skin is a rough average. To explore "pain spots" a stout hair or needle is used; in the latter case the needle shifts up and down in the holder, and works against a spring which registers the amount of pressure exerted to 
evoke a painful sensation. The sensation evoked by a "pain spot" is unaccompanied by "cold" or "heat" even if a cold or hot needle is used. For the exploration of "heat spots" a small, hollow, metallic pencil is kept warm by a stream of warm water; this is moved over the surface; at the site of the "heat spots" the pencil will feel peculiarly warmer. "Cold spots" can be similarly mapped out by the use of a cold pencil. The accompanying figure (fig. 477) indicates the distribution of cold and heat spots over six squares, each of 1 sq. cm., on the back of the left hand. The black dots

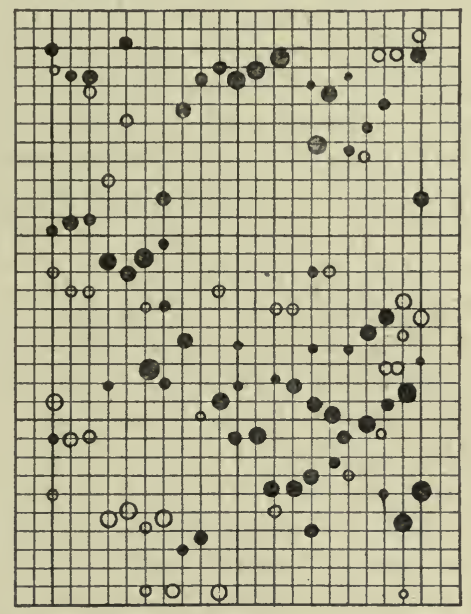

Fig. 477.-Heat and cold spots. (Somewhat enlarged; after Donaldson.)

represent cold spots, their size indicating the strength of the reaction. The open circles represent heat spots.

All these facts clearly indicate that different varieties of sensation are the result of the stimulation of different end-organs, and that the impulses are conveyed to the central nervous system by different groups of nerve-fibres; they moreover form the clearest piece of evidence we have that pain is a distinct kind of sensation.

The question is more difficult to answer, which particular endorgan is concerned with each variety of sensation. There is, however, little doubt that the nerve-fibrils around the hair follicles of the short hairs are the terminations most affected by changes of pressure, and also that Meissner's corpuscles are purely tactual, taking the place of hairs in hairless parts. In the palmar surface of the last phalanx of the index finger, there are 21 Meissner's corpuscles per square centimetre; in other parts of the palm and sole the number varies from 2 to 8 . End-bulbs are believed to 
be the organs for cold; they are most numerous in the conjunctiva and glans penis, where "cold spots" are almost exclusively present. The end-organs in "heat spots" have not been identified with certainty, but they are probably larger organs, and placed more deeply in the skin.

We have spoken of the pressure sense as the true tactile sense; but Meissner pointed out many years ago that the hand immersed in a fluid such as mercury at body-temperature, does not feel the contact of the fluid, although the fluid pressure may be far above the limen; it is, however, equal in all directions; it is therefore clear that the adequate stimulus for touch organs consists in a deformation of the skin surface.

As compared with the sensation obtained from pain spots, touch is quicker both in development and subsidence. Thus vibrations of strings are recognisable as such by the finger, even at a frequency of 1500 vibrations per second. A revolving wheel with toothed edge does not give a sensation of smoothness till the teeth meet the skin at the rate of from 480 to 640 per second.

Head, in his recent study of nerve-regeneration, cut one of the nerves in his own arm, and, in conjunction with Rivers, noted accurately the date and other particulars of return of function. The first sensations return about the eightieth day after the operation; they are termed by him protopathic. Protopathic sensibility depends on definite specific end-organs distributed over the skin as sensory "spots," viz., heat, cold, and pain spots. When this sensibility is alone present, the spaces between these spots are insensitive to cutaneous stimuli; the heat spots only react to temperatures above $37^{\circ} \mathrm{C}$., the cold only to temperature below $26^{\circ} \mathrm{C}$.; the sensation radiates widely, and is often wrongly localised. The tactile sensations of the skin, the intermediate temperature sensations, the power to localise them accurately, the sensibility of the spaces between the spots, and a more refined sensibility to pain, return much later, and this epicritic sensibility was not perfect until many months after the regeneration started. As previously stated (p. 706), it is not known whether protopathic and epicritic impulses are subserved by the same or by different nerve-fibres. Quite apart from these two forms of cutaneous sensation is the deep sensibility of subjacent structures, and the fibres subserving this run mainly with the motor nerves; this form of sensation is not destroyed by division of all the nerves to the skin (see also p. 705).

Adaptation plays a part as important in cutaneous as in other sensations. The same room feels warm to a man who enters it from the street, and cold to another who has been in a conservatory. Hering calls the point of adaptation to temperature " the physiological zero." Thus the temperature of the mouth and the lips may actually differ by several degrees, yet neither of them will feel hot or cold because each is at the physiological zero temperature. Sensations of warmth or cold arise when the 
physiological zero is altered : they persist until a new zero is formed, i.e. until adaptation is complete; according to Rivers and Head, adaptation to temperature is impossible when epicritic sensibility is absent. So, too, heavy weights feel unduly heavy after light weights, and vice versa. When eyeglasses or false teeth are first worn, their contact is well-nigh unbearable; yet later, through adaptation, the discomfort vanishes.

It is very difficult to draw any hard-and-fast line between the cutaneous sensations we have just described, and those which are grouped under the name "common or general sensibility (coencesthesia)." Sensations which are difficult to describe but which are perfectly familiar, such as those accompanying tickling, shivering, shuddering, and the like, are regarded as varieties of "common sensation." Pain may be looked upon as an excessive form of "common sensation," but cutaneous pain is so distinct a sensation that most psychologists agree to place it under a "special" rather than a "common" heading. The term "common sensation" is most frequently employed in reference to sensations from the interior of the body.

Drugs. - Cocaine applied locally depresses all forms of cutaneous sensibility, but especially the true tactile sense ; carbolic acid acts similarly but less strongly. Chloroform produces a temporary burning sensation, and then blunts sensibility, especially to temperature changes. Menthol produces a feeling of local cold because it first causes hyperæsthesia of the end-organs for cold ; this is followed by a depression of the activity of these organs, together with that subserving other forms of cutaneous sensation. 


\section{CHAPTER LIV}

\section{MOTORIAL AND VISCERAL SENSATIONS}

WE shall in the present chapter deal with the motorial or muscular sense, and with sensations from the viscera.

\section{The Motorial or Kinæsthetic Sense.}

By this sense we become aware that movement is taking place in some part of the body. We are especially conscious of willed muscular action, and the sense has thus been confused and identified with the "feeling of innervation," or "sense of effort," which accompanies volitional movements. By some this feeling has been attributed to a direct discharge from the motor to the sensory cells of the cerebral cortex occurring at the very birth of the efferent impulse. No doubt part of the effect involved in movement is of central origin, and this part is the effect inherent in all conative (p. 758) processes, and characterises all forms of mental activity, for instance, reasoning or imagination; but its physiological basis is quite unknown. Most, however, of the sense of effort is unquestionably due to afferent impulses peripherally generated by the accompanying respiratory and other strains.

It is in the estimation of weights that the value of these peripheral sensations can be most clearly seen. When a weight is first handled, the amount of force necessary to lift it is estimated in the light of past experience. As it is being lifted, sensations from the moving limb guide the expenditure of force: a weight which flies up too fast or does not move at once, calls for less or more muscular force. Similarly, the motorial sense is invoked when we estimate the extent to which we have moved our limbs, or to which they have been passively moved by others.

These guiding sensations are not merely of cutaneous origin. Persons whose skin has been rendered insensitive by cocaine, or by certain diseases, yet retain the power of estimating weights and the extent of their movements. In locomotor ataxy the motorial sense may be destroyed while the skin retains its usual sensitiveness to touch. On the other hand, we must remember that it is not at all certain that the muscles are solely or even predominantly the seat of these peripheral sensations; the term "motorial" or "kinæsthetic" is therefore preferable to that of "muscular" sense, by which name 
it is still often called. It is true that sensory end-organs and nerve-fibres occur in muscles and tendons, which presumably transmit impulses upon change of muscular form or of tendinous strain. But we have experimental evidence that the pressure and movement of jointsurfaces are most important factors in the development of kinæsthetic sensations. The "motorial sense" is thus of very complex origin.

\section{Visceral Sensations.}

Epicritic sensibility is a special characteristic of the cutaneous area. Protopathic sensibility is found in other parts also, but in most internal structures of the body it is limited to pain. The œsophagus and anal canal alone seem to be endowed with the temperature sense; the feelings of warmth and cold on swallowing liquids of different temperatures are entirely referable to the upper portion of the alimentary canal. Hertz's recent experiments place this beyond question; immediately the food has passed into the stomach we are unaware of its temperature except by the warming or cooling of the neighbouring portion of the gullet, or the skin overlying the viscera.

Pain is the most widely distributed sense in the body, but in internal organs is not localised accurately, and it is here that the "referred pains" in corresponding skin areas (see p. 205) are useful for diagnostic purposes. Pain, however, is not produced in the viscera by handling or even by cutting or burning: it appears to be assoriated with excessive action, stretching, and with inflammatory conditions which involve the sensitive parietal layer of the peritoneum. Inflammation of the serous membranes is an exceedingly painful condition-for instance, in pleurisy and peritonitis-but this condition, per se, does not apparently cause any referred pain or tenderness in cutaneous areas. In connection with the question of referred pain, we must mention the pathological condition known as allochiria. When the skin sensations in any given area are depressed, stimulation of that area may give rise to sensations which are referred to the corresponding area on the other side of the body; it appears to be a general rule, as Head first pointed out, that the mind projects sensations arising from an area of low sensibility to that area of higher sensibility which is related to it most closely by connections within the central nervous system, and this underlies the causation of referred visceral pains.

There are, however, special kinds of sensation arising from internal viscera which have no counterpart in the sensations of the cutaneous surface. Of these, hunger and thirst are the most familiar.

Hunger when slight is termed appetite, and there is some difference of opinion whether the two are separate sensations, or only differ in degree. Appetite is referred to the stomach, and is a normal sensation, which arises at an interval after a meal, and as is 
well known it is intensified by muscular exertion, especially if the air is cool. It has been suggested that the oxidation processes which occur in the muscles produce some substance or substances which excite the sensory nerve-terminals in the stomach. In diabetes, where oxidation runs an unusual course, carbohydrates escaping oxidation to a large extent, intense appetite may be present in spite of abundant feeding.

Hunger is due to pronounced motor activity of the stomach; this excites the sensory nerve-terminals there (Hertz); these movements, and therefore the sensation of hunger, can be appeased by filling the stomach even with indigestible or non-nutritious material. Carlson has recently shown that the movements are reflexly inhibited when food enters the mouth and is masticated; the nerves of taste act as the afferent channel for the reflex; hence the feeling of hunger passes off long before absorption of food begins. These observations confirm the view that its origin is a local condition set up in the stomach by its condition of emptiness, and that it is not immediately due to any general change in the nutrition of the body as a whole. We must, however, recognise that the gastric sense is a complex one, as is illustrated by the aversion for food felt during monotonous diets or after over-feeding, or in the case of certain articles of diet, but the explanation of these and similar phenomena we do not know.

Thirst is a sensation referred to the pharyngeal region rather than to the stomach, and appears, like hunger, to be a protective signal, locally excited to warn the living organism of the necessity for regularity in the intake of nutriment. Although its intensity increases with the loss of water from the body, it occurs normally long before there is any serious upset of the normal relationship of the water percentage of the organs and tissues, and may be artificially produced by drying of the throat; it is appeased immediately by the administration of fluid, and although fluids reach the absorbing surface of the duodenum sooner than was formerly supposed to be the case (see p. 555), it is unquestionable that the relief of thirst is mainly the result of moistening the local surface, the impulses from which excite the sensation. Very frequently thirst can be relieved by letting the water touch the pharyngeal mucous membrane without its being swallowed. Thirst which is due to prolonged deprivation of water is not a mere local sensation, but is no doubt produced by loss of water in the tissues, generally, exciting widespread sensory terminations therein; the bodily and mental anguish experienced are then of an intense character.

The independence of the two sensations hunger and thirst is well illustrated in many diseases, where a loss of appetite occurs without any corresponding loss of desire for fluid. 


\title{
CH A P T E R L V
}

\author{
TASTE AND SMELL
}

\section{Taste.}

Certain anatomical facts must be studied first in connection with the tongue, the upper surface of which is concerned in the reception of taste stimuli.

The tongue is a muscular organ covered by mucous membrane. The muscles, which form the greater part of the substance of the tongue (intrinsic muscles) are termed linguales; and by these, which are attached to the mucous membrane, its smaller and more delicate movements are performed.

By other muscles (extrinsic muscles), such as the genio-hyoglossus, the styloglossus, etc., the tongue is fixed to the surrounding parts; and by these its larger movements are performed.

Its mucous membrane resembles other mucous membranes in essential points, but contains papilloe, peculiar to itself. The tongue is also beset with mucous glands and lymphoid nodules.

The lingual papilloe are thickly set over the anterior two-thirds of its upper surface, or dorsum (fig. 478), and give to it its characteristic roughness. Three principal varieties may be distinguished, namely, the (1) circumvallate, the (2) fungiform, and the (3) conical and filiform papillæ. They are all formed by a projection of the corium of the mucous membrane, covered by stratified epithelium; they contain special branches of blood-vessels and nerves. The corium in each kind is studded by microscopic papillæ.

(1.) Circumvallate.-These papillæ (fig. 479), eight or ten in number, are situate in a $\mathrm{V}$-shaped line at the base of the tongue $(1,1$, fig. 478). They are circular elevations, from $\frac{1}{20}$ th to $\frac{1}{12}$ th of an inch wide ( 1 to $2 \mathrm{~mm}$.), each with a slight central depression, and surrounded by a circular moat, at the outside of which again is a slightly elevated ring or rampart; their walls contain taste-buds. Into the moat that surrounds the central tower, a few little glands (glands of Ebner) open. These glands form a thin, watery secretion. 
(2.) Fungiform.-The fungiform papillæ (3, fig. 478) are scattered chiefly over the sides and tip, and sparingly over the middle of the

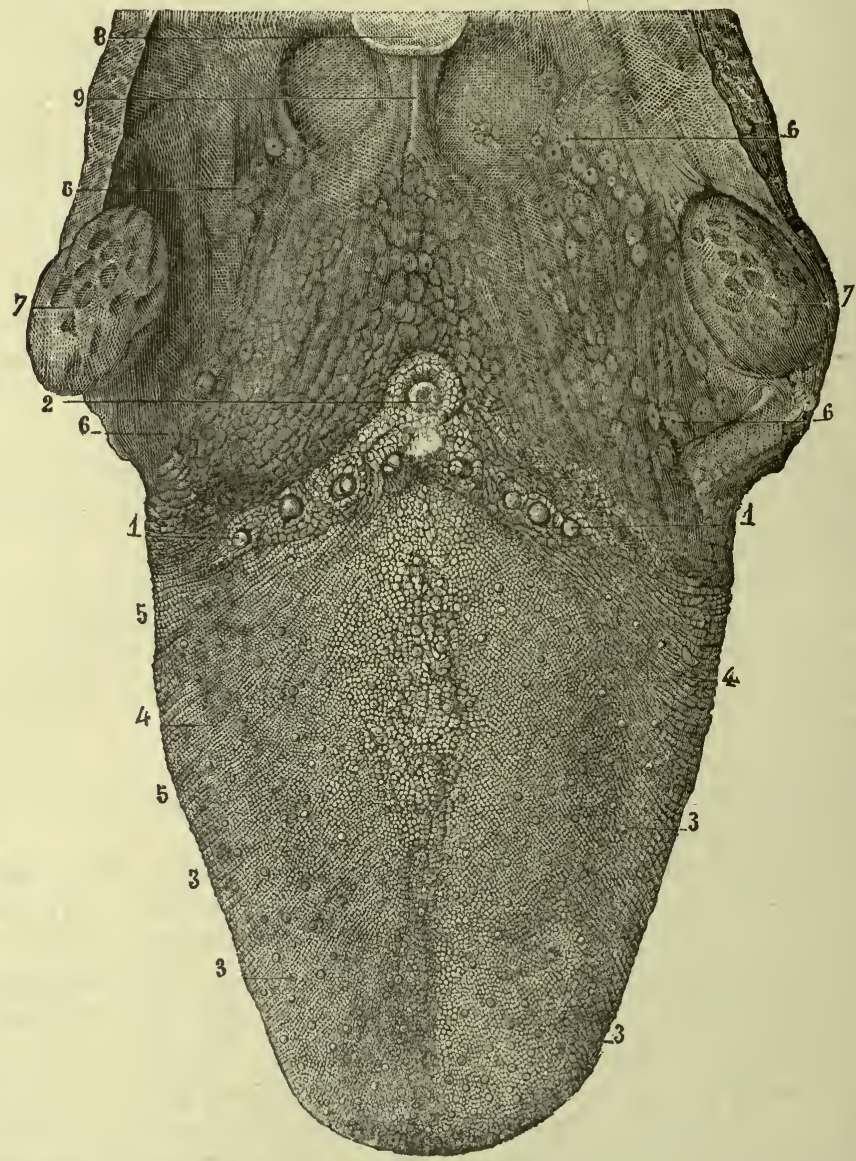

Fig. 478.-Papillar surface of the tongue, with the fauces and tonsils. 1, 1, Circumvallate papillæ in front of 2 , the foramen cæcum ; 3 , fungiform papillæ; 4 , filiform and conical papillæ; 5 , transverse and oblique rugæ ; 6 , mucous glands at the base of the tongue and in the fauces; 7 , tonsils ; 8 , part of the epiglottis; 9 , median glosso-epiglottidean fold (frænum epiglottidis). (From Sappey.)

dorsum, of the tongue; their name is derived from their being shaped like a puff-ball fungus. (See fig. 480.)

(3.) Conical and Filiform.-These, which are the most abundant papillæ, are scattered over the whole upper surface of the tongue, but especially over the middle of the dorsum. They vary in shape, some being conical (simple or compound) and others filiform; they are covered by a thick layer of epithelium, which is either arranged 
over them, in an imbricated manner, or is prolonged from their surface in the form of fine stiff projections (fig. 481). In carnivora they are developed into horny spines. From their structure, it is likely that these papillæ have a mechanical and tactile function, rather than that of taste; the latter sense is seated especially in the other two varieties of papillæ, the circumvallate and the fungiform.

In the circumvallate papillæ of the tongue of man peculiar structures known as taste-buds are found. They are of an oval shape, and consist of a number of closely packed, very narrow and fusiform, cells (gustatory cells). This central core of gustatory cells is enclosed in a single layer of broader fusiform

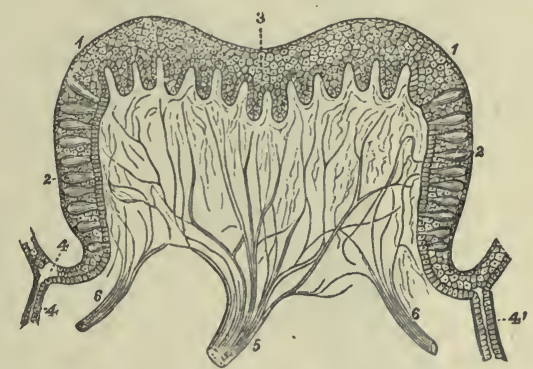

Fig. 479.-Vertical section of a circumvallate papilla of the calf. 1 and 3, Epithelial layers covering it ; 2 , taste-buds ; 4 and $4^{\prime}$, duct of serous gland opening out into the pit in which papilla is situated; 5 and 6 , nerves ramifying within the papilla. (Engelmann.) cells (encasing cells). The gustatory cells terminate in fine stiff spikes which project on the free surface (fig. $482, a$ ).

Taste-buds are also scattered over the posterior third of the tongue, the palate and the pharynx, as low as the posterior (laryngeal)
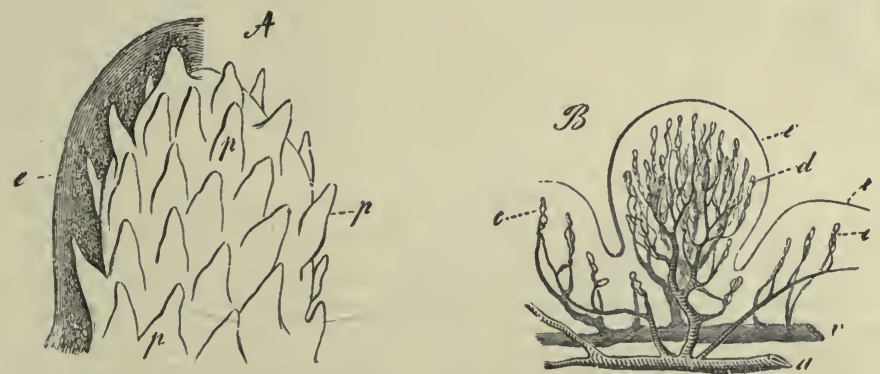

F10. 480.-Surface and section of the fungiform papillæ. A. The surface of a fungiform papilla, partially denuded of its epithelium; $p$, secondary papillæ; $e$, epithelium. B. Section of a fungiform papilla with the blood-vessels injected; $a$, artery; $v$, vein; $c$, capillary loops of sinilar papillæ in the neighbouring structure of the tongue; $d$, capillary loops of the secondary papillæ; $e$, epithelium. (From Kölliker, after Todd and Bowman.)

surface of the epiglottis. The gustatory cells in the interior of the taste-buds are surrounded by arborisations of nerve-fibres.

The arrangement of papillæ, taste-buds, etc., varies a good deal in different animals. The papilla foliata of the rabbit's tongue consists of a number of closely packed papillæ, similar to the circumvallate papillæ of man; this forms a convenient source for the histological demonstration of taste-buds. 
The middle of the dorsum of the tongue is but feebly endowed with the sense of taste; the tip and margins, and especially the

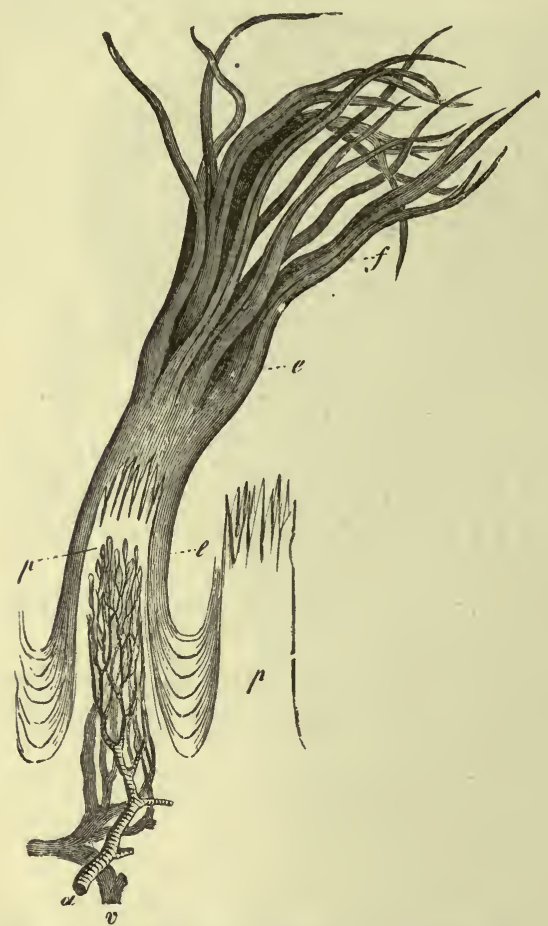

F1G. 481.-Filiform papillæ, one with epithelium, the other without. $\frac{35}{3},-p$, The substance of the papillæ dividing at their upper extremities into secondary papillæ; $a$, artery, and $v$, vein, dividing into capillary loops; $e$, epithelial covering, lamin. ated between the papillæ, but extended into hairlike processes, $f$, from the extremities of the secondary papillæ. (From Kölliker, after Todd and Bowman.) posterior third of the dorsum (i.e., in the region of the tastebuds), possess this faculty. The anterior part of the tongue is supplied by the lingual branch of the fifth

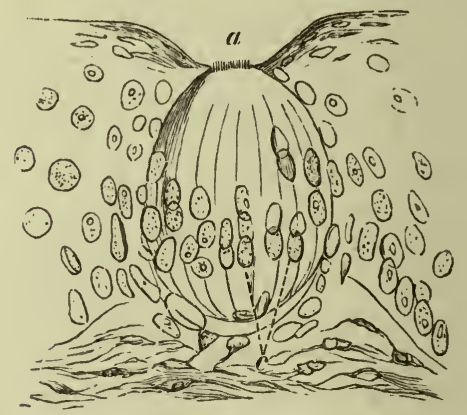

FIG. 482.-Taste-bud from dog's epiglottis (laryngeal surface near the base), precisely similar in structure to those found in the tongue. $a$, Depression in epithelium over bud; below the letter are seen the fine hair-like processes in which the cells terminate; $c$, two nuclei of the axial (gustatory) cells. The more superficial nuclei belong to the superficial (encasing) cells; the converging lines indicate the fusiform shape of the encasing cells. $\times 400$. (Schofield.)

nerve and the chorda tympani, and the posterior third by the glosso - pharyngeal nerve. Considerable discussion has arisen whether there is more than one nerve of

taste. The view generally held is that the glosso-pharyngeal nerve is the nerve of taste, and the lingual the nerve of tactile sensation. Nevertheless, the lingual and the chorda tympani do contain taste-fibres, which probably take origin from the cells of the geniculate ganglion; the central axons of these cells pass by the pars intermedia to the sensory nucleus of the glosso-pharyngeal nerve. Gowers holds that the fifth nerve is the only nerve of taste, and has recorded a case of loss of taste where the fifth nerve alone was the seat of disease; other cases, however, do not support this view. 
Tastes may be classified into-
1. Sweet.
2. Bitter.
3. Acid or Sour.
4. Salt.

Whether alkaline and metallic tastes are elementary, is as yet undecided. All the above affect to a varying extent the nerves of tactile sense as well of those of touch proper, sweet having the least, acids the most marked action upon the latter. Sweet tastes are best appreciated by the tip, acid at the side, and bitter tastes at the back of the tongue.

The substance to be tasted must be dissolved; here there is a striking contrast to the sense of smell; flavours are really odours. In testing the sense of taste in a patient, the tongue should be protruded, and drops of the substance to be tasted applied with a camel's hair brush to the different parts; the subject of the experiment must signify his sensations by signs, for if he withdraws the tongue to speak, the material gets widely spread. The more concentrated the solution, and the larger the surface acted on, the more intense is the taste; some tastes are perceived more rapidly than others, saline tastes the most rapidly of all. The best temperature of the substance to be tasted is from $10^{\circ}$ to $35^{\circ} \mathrm{C}$. Very high or very low temperatures deaden the sense.

Individual papillæ, when thus treated with various solutions, show great diversity: from some only one or two tastes can be evoked, from others all four. The papillæ may also be stimulated electrically.

Cocaine and gymnemic acid, prepared from the leaves of the plant Gymnema sylvestre, act deleteriously, chiefly on the bitter and sweet tastes; cocaine abolishes especially the bitter, gymnemic acid especially the sweet, leaving the salt and acid tastes almost untouched.

It will thus be seen that there are many facts pointing to the conclusion, that the varieties of gustatory like-those of cutaneous sensation are due to the stimulation of different end-organs.

When diluted sweet and salt solutions are simultaneously applied to the tongue, they tend to neutralise one another, but a true indifferent point is difficult or impossible to reach. Sweet and bitter, sweet and acid liquids are antagonistic to a similar but less perfect extent. Contrast-effects of one taste upon another are matters of common observation, but can only be experimentally investigated with difficulty.

\section{Smell.}

The entrance to the nasal cavity is lined with a mucous membrane closely resembling the skin. The greater part of the rest of the cavity is lined with ciliated epithelium; the corium is thick and 
contains numerous mucous glands. The olfactory region in man is limited to a portion of the membrane covering the upper turbinal bone, and the adjacent portion of the nasal septum; it is only 245 square millimetres in area. The cells of the epithelium here are of several kinds :- first, columnar cells not ciliated (fig. 483, st), with the broad end at the surface, and below

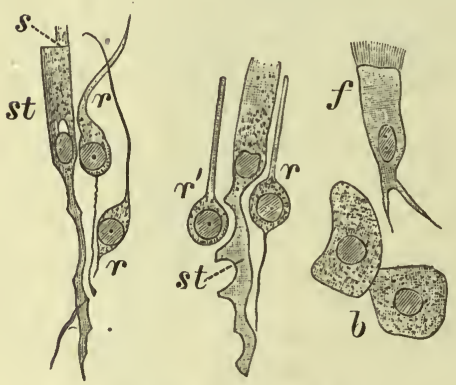

Fig. 483.-Cells from the olfactory region of the rabbit. st, Supporting cells; $r, r^{\prime}$, olfactorial cells ; $f$, ciliated cells ; s, cilia. like processes; $b$, cells from Bowman's glands. (Stöhr.)

it becomes stiff, and in some animals, such as the frog, is provided with hairs. These cells, which are called olfactorial cells, are numerous, and the nuclei of the cells not being on the same level, a comparatively thick nuclear layer is the result. They are in reality bipolar nerve-cells. In the corium are a number of serous glands called Bowman's glands. They open upon the surface by fine ducts passing up between the epithelium cells.

The distribution of the olfactory nerves which penetrate the cribriform plate of the ethmoid bone and pass from this region to the olfactory bulb is shown in fig. 484. The nerve-fibres are the central axons of the bipolar nerve-cells we have termed olfactorial; the columnar cells between these act as supports to them.

The olfactory tract is an outgrowth of the brain, which is originally hollow, and remains so in many animals; in man the cavity is obliterated, and the centre is occupied by neuroglia: outside this the white fibres lie, and a thin superficial layer of neuroglia covers these. The three "roots" of the olfactory tract have been traced to the uncinate gyrus and hippocampal regions of the same side of the brain, which is the portion experimentally found to be associated with the reception of olfactory impulses (see pp. 698 and 738). From the cells of the grey matter here fibres pass by a complex path to the corresponding regions of the opposite side. There is also a communication via the corpora mammillaria with the optic thalamus and tegmentum of the mid-brain. 
- The olfactory bulb has a more complicated structure; above there is first a continuation of the olfactory tract (white fibres enclosing

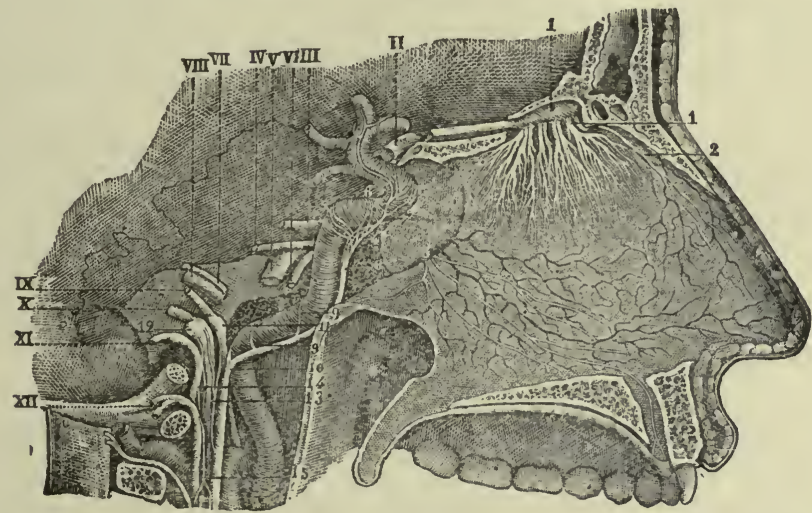

Fig. 484.-Nerves of the septum nasi, seen from the right side. $\frac{2}{3} .-\mathrm{I}$, the olfactory bulb; 1 , the olfactory nerves passing through the foramina of the cribriform plate, and descending to be distributed on the septum; 2 , the internal or septal twig of the nasal branch of the ophthalmic nerve; 3 , naso-palatine nerves. (From Sappey, after Hirschfeld and Leveillé.)

neuroglia); below this four layers are distinguishable; they are shown in the accompanying diagram from Ramon y Cajal's work, the histological method used being Golgi's.

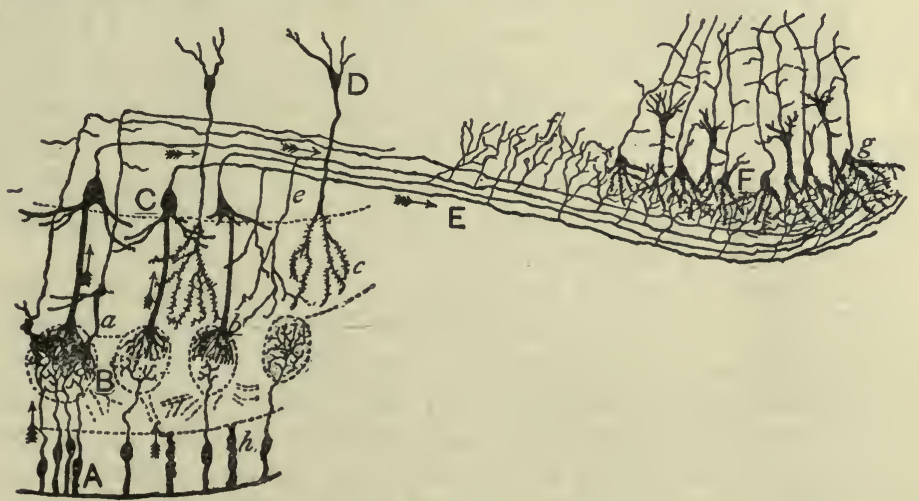

F19. 485.-Nervous mechanism of the olfactory apparatus. A, bipolar cells of the olfactory apparatus (Max Schultze's olfactorial cells); B, olfactory glomeruli; C, mitral cells; D, granule of white layer; $\mathrm{E}$, external root of the olfactory tract; $\mathrm{F}$, grey matter of the sphenoldal region of the cortex ; $a$, small cell of the mitral layer; $b$, basket of a glomerulus ; $c$, spiny basket of a granule ; $e$, collateral of the axis-cylinder process of a mitral cell; $f$, collaterals terminating in the molecular layer of the frontal and sphenoidal convolutions; $g$, superficial triangular cells of the cortex; $h$, supporting epithelium cells of the olfactory mucous membrane. (Ramon y Cajal.)

(1) A layer of white fibres containing numerous small cells, or "granules" (D). 
(2) A layer of large nerve-cells called " mitral cells" (c), with smaller cells $(a)$ mixed with them. The axis-cylinder processes of these cells pass up into the layer above and eventually become fibres of the olfactory tract $\mathrm{E}$, which passes to the grey matter of the base of the brain $F$. They give off numerous collaterals on the way $(e, f)$.

(3) The layer of olfactory glomeruli (B). Each glomerulus is a basket-work of fibrils derived on the one hand from the terminal arborisations of the mitral cells, and on the other from similar arborisations of the non-medullated fibres which form the next layer.

(4) The layer of olfactory nerve-fibres.-These are non-medullated; they continue upwards the bipolar olfactory cells, which are placed among the epithelial cells of the mucous membrane.

Animals may be divided into three classes:- those which, like the porpoise, have no sense of smell (anosmatic); those which possess it in comparatively feeble degree (man, most primates, monotremes, and some cetacea); these are called microsmatic. In man the thickness of the olfactory membrane is only $0.06 \mathrm{~mm}$. Most mammals are in contradistinction macrosmatic, the thickness of the membrane being $0.1 \mathrm{~mm}$. or more, and its area larger.

The mucous membrane must be neither too dry nor too moist; if we have a cold we are unable to smell odours or appreciate flavours (which are really odours). When liquids are poured into the nose, their smell is imperceptible, as they damage the olfactory epichelium, owing to the difference of osmotic pressure. But even if a "normal" saline solution of an odorous substance be substituted, the sense of smell is still lost so long as air-bubbles are carefully excluded from the nasal cavity. It is therefore necessary that odorous substances should be in a gaseous state in order to act upon the olfactory nerve-endings; they are normally conveyed to the olfactory surface by the air currents passing through the nose.

Generally, the odours of homologous series of compounds increase in intensity with increase of molecular weight, but bodies of very low molecular weight are odourless, while vapours of very high molecular weight, which escape and diffuse slowly, have little or no smell. A slight change in chemical constitution may produce marked alteration in the character of the odour of a substance; certain modes of atomic grouping within the molecule appear to be more odoriferous than others. Attempts have been made to discover the elementary sensations of smell, but hitherto with scant success. Many odours have unquestionably a complex physiological effect. For example, when nitrobenzol is held before the nose, it yields first the smell of heliotrope, next the smell of bitter almonds, and finally the smell of benzene; just as if different end-organs became successively fatigued. Some substances have a very different smell according 
to their concentration. Chemical dissociation, too, unquestionably plays a prominent part.

Nevertheless, there are certain observations which indicate the existence of primary sensations of smell. First, some persons are congenitally insensible to one or more odours, but yet smell others quite normally. Hydrocyanic acid, mignonette, violet, vanilla, benzoin, are substances which appear to certain people to have no smell. Secondly, some odorous bodies, when simultaneously given, antagonise one another; others produce a mixed smell. Thirdly, fatigue of the epithelium with one odour will modify or abolish the effect of some smells, but will leave that of others untouched.

The delicacy of the sense of smell is most remarkable even in man. Valentin calculates that even $\frac{3}{100,000,000}$ of a grain of musk can be distinctly smelled. Solutions of camphor afford a good means of testing olfactory acuity. Two tubes of camphor solution are presented to the subject along with two tubes of water, and the former pair is replaced with weaker and weaker solutions until it is indistinguishable from the tubes containing water. Pungent substances, such as ammonia, are unsuited for olfactometrical experiment. They stimulate the endings of the fifth as well as those of the olfactory nerve. 


\section{CHAPTER LVI}

\section{HEARING}

\section{Anatomy of the Ear.}

The Organ of Hearing (fig. 486) is divided into three parts, (1) the external, (2) the middle, and (3) the internal ear.

External Ear.-The external ear consists of the pinna and the external auditory meatus. The central hollow of the former is named the concha. The auditory canal, with a slight arch directed upwards, passes inwards and a little forwards to the membrana tympani, to which it thus serves to convey the vibrating air.

Middle Ear or Tympanum. - The middle ear, or tympanum or drum (3, fig. 486), is separated by the membrana tympani from the external auditory meatus. It is a cavity which communicates posteriorly with the mastoid cells in the mastoid process of the temporal bone; but its only opening to the external air is through the Eustachian tube (4, fig. 486). The walls of the tympanum are osseous, except where apertures in them are closed with membrane, as at the fenestra rotunda, and fenestra ovalis, and at the outer part where the bone is replaced by the membrana tympani. Its cavity is lined with mucous membrane, which is continuous through the Eustachian tube with that of the pharynx. A chain of small bones extends from the membrana tympani to the fenestra ovalis.

The membrana tympani is placed in a slanting direction at the bottom of the external auditory canal, and consists of fibres, some running radially, some circularly; its margin is set in a bony groove; its outer surface is covered with a continuation of the cutaneous lining of the auditory canal, its inner surface with the mucous membrane of the tympanum.

The ossicles are three in number; named malleus, incus, and stapes. The malleus, or hammer-bone, has a long slightly-curved process, called its handle, which is inserted vertically between the 


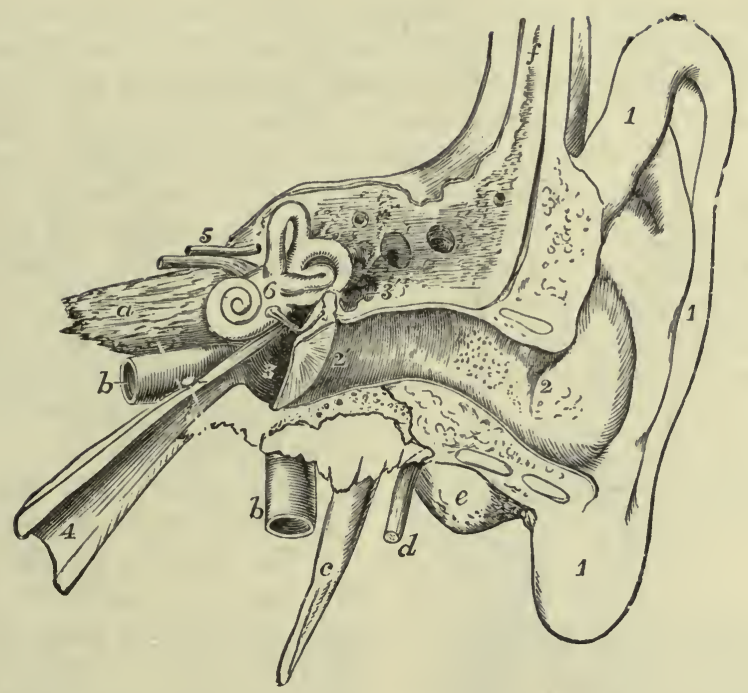

FIG. 486.-Diagrammatic view from before of the parts composing the organ of hearing of the left side. The temporal bone of the left side, with the accompanying soft parts, has been detached from the head, and a section has been carried through it transversely, so as to remove the front of the meatus externus, half the tympanic membrane, the upper and anterior wall of the tympanum and Eustachian tube. The meatus internus has also been opened, and the bony labyrinth exposed by the removal of the surrounding parts of the petrous bone. 1, The pinna and lobe; 2, meatus esternus; $2^{\prime}$, membrana tympani ; 3 , cavity of the tympanum ; $3^{\prime}$, its opening backwards into the mastold cells; between 3 and $3^{\prime}$, the chain of small bones; 4, Eustachian tube; 5, meatus internus, containing the facial (uppermost) and the anditory nerves; 6 , placed on the vestibule of the laby: rinth above the fenestra ovalis; $a$, apex of the petrous bone; $b$, internal carotid artery : $c$, styloid process ; $d$, facial nerve issuing from the stylo-mastoid foramen; $e$, mastold process; $f$, squamous part of the bone covered by integument, etc. (Arnold.)

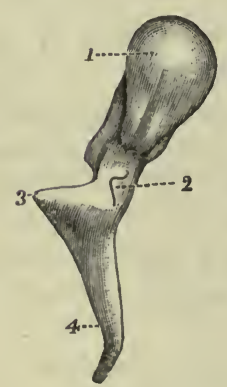

Fro. 487.-The hammerbone or malleus, seen from the front. 1, The head; 2, neck; 3, short process; 4 , handle. (Schwalbe.)

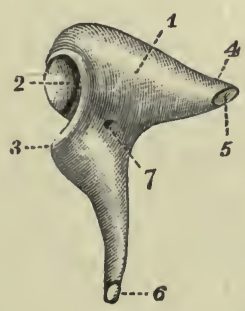

Fia. 488.-The incus, or anvil-bone. 1. Body; 2, ridged articulation for the malleus; 4, processus brevis, with 5, rough articular surface for ligament of incus ; 6 , processus magnus, with articulating surface for stapes; 7 , nutrient foramen. (Schwalbe.)

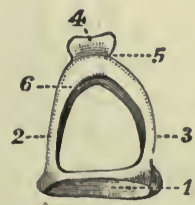

Fig. 489.-The stapes, or stirrup - bone. 1, Base ; 2 and 3 , arch; 4 , head of bone, which articu. lates with orbicular process of the incus : 5, constricted part of neck; 6, one of the crura. (Schwalbe.) 
layers of the membrana tympani. The head of the malleus is irregularly rounded; its neck, or the line of boundary between the head and the handle, supports two processes: a short conical one, and a slender one, processus gracilis, which extends forwards, and is attached to the wall of the cavity at the Glaserian fissure. The incus, or anvil-bone, shaped like a bicuspid molar tooth, is articulated by its broader part, corresponding with the surface of the crown of the tooth, to the malleus. Of its two fang-like processes, one, directed backwards, has a free end attached by ligament to a depression in the mastoid bone; the other, curved downwards, longer and more pointed, articulates by means of a roundish tubercle, formerly

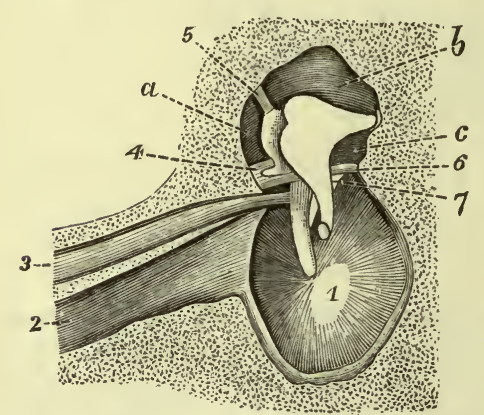

FiG. 490.-Interior view of the tympanum, with membrana tympani and bones in natural position. 1, Membrana tympani ; 2, Eustachian tube; 3 , tensor tympani muscle ; 4 , lig. mallei exter.; 5 , lig. mallei super. ; 6 , chorda-tympani nerve; $a, b$, and $c$, sinuses about ossicles. (Schwalbe.) called os orbiculare, with the stapes, a little bone shaped like a stirrup, of which the base fits into the membrane of the fenestra ovalis.

The muscles of the tympanum are two in number. The tensor tympani arises from the cartilaginous end of the Eustachian tube and the adjoining surface of the sphenoid, and from the sides of the canal in which the muscle lies; the tendon of the muscle bends at nearly a right angle over the end of the processus cochleariformis, and is inserted into the inner part of the handle of the malleus. The stapedius is concealed within a canal in the bone in front of the aqueductus Fallopii. Its tendon is inserted into the neck of the stapes posteriorly.

The Internal Ear.- The proper organ of hearing is formed by the distribution of the auditory nerve, within the internal ear, or labyrinth, a set of cavities within the petrous portion of the temporal bone. The bone which forms the walls of these cavities is denser than that around it, and forms the osseous labyrinth; the membrane within the cavities forms the membranous labyrinth. The membranous labyrinth contains a fluid called endolymph; while outside it, between it and the osseous labyrinth, is a fluid called perilymph. This fluid is not pure lymph, as it contains mucin.

The Osseous Labyrinth consists of three principal parts, namely, the vestibule, the cochlea, and the semicircular canals.

The vestibule is the middle cavity of the labyrinth, and the central chamber of the auditory apparatus. It presents, in its inner wall, several openings for the entrance of the divisions of the auditory nerve; in its outer wall, the fenestra ovalis (2, fig. 491a), 
an opening filled by membrane, in which is inserted the base of the stapes; in its posterior and superior walls, five openings by which the semicircular canals communicate with it: in its anterior wall,

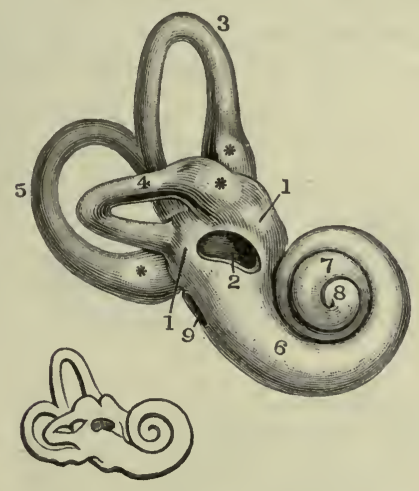

FIG. 491a.-Right bony labyrinth, viewed from the outer side. The specimen here represented is prepared by sepa. rating plecemeal the looser substance of the petrous bone from the dense walls which immediately enclose the labyrinth. 1, The vestibule; 2, fen. estra ovalis; 3, superior semicircular canal; 4, horizontal or external canal; 5, posterior canal; *, ampullæ of the semicircular canals; 5 , first turn of the cochlea; 7, second turn; 8, apex; 9, fenestra rotunda. The smaller figure in outline below shows the natural size. $\frac{21}{1}$. (Sümmering.)

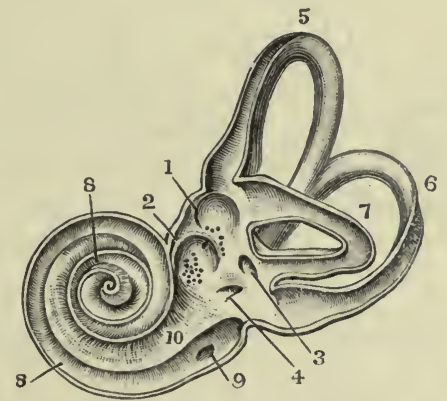

FIG. $491 b$. - View of the interior of the left labyrinth. The bony wall of the laby. rinth is removed superiorly and exter. nally. 1, Fovea hemielliptica; 2, fovea hemispherica; 3 , common opening of the superior and posterior semicircular canals; 4, opening of the aqueduct of the vestibule; 5, the superior; 6 , the posterior, and 7, the external semicir. cular canals; 8, spiral tube of the cochlea (scala tympani); 9, opening of the aqueduct of the cochlea; 10 , placed on the lamina spiralis in the scala ves. tibuli. $\frac{2 \frac{1}{3}}{1}$. (Sömmering.)

an opening leading into the cochlea. The semicircular canals are described in Chapter LI.

The Membranous Labyrinth corresponds in general form with the osseous labyrinth. The vestibule contains two membranous sacs, named the utricle and the saccule (fig. 492); the utricle communicates with the three membranous semicircular canals; the saccule communicates with the utricle and with the canal of the cochlea. The vestibular division of the auditory nerve is distributed to the five spots shown in the diagram, namely, the maculæ of utricle and saccule, and the cristæ of the semicircular canals. The cochlear division of the auditory nerve is distributed to the whole length of the canal of the cochlea.

The Cochlea.-This is shaped like a snail's shell. It is traversed by a central column or modiolus, around which a spiral canal winds with two and a half turns from base to apex. It is seen in vertical section (fig. 493) that this canal is divided partly by bone (the spiral lamina), partly by membrane (the basilar membrane), 
into two spiral staircases or scalæ, the scala tympani and scala vestibuli (fig. 493). The scala vestibuli is separated from the

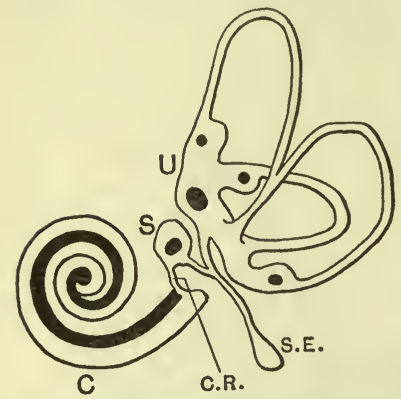

Fia. 492.-Diagram of the right membranous labyrinth. $\mathrm{U}$, utricle, into which the three semicircular canals open ; S, saccule, communicating with the cochlea (C) by C.R., the canalis reuniens, and with the utricle by a canal having on it an enlargement, the saccus endolymphaticus (S.E.). The black shading represents the places of termination of the auditory nerve, namely, in the maculæ of the utricle and saccule; the cristæ in the ampullary ends of the three semicircular canals; and in the whole length of the canal of the cochlea. (After Schäfer.)

and running, and running radially, from the spiral lamina to the spiral ligament, where its other end is again attached to the bone. At the

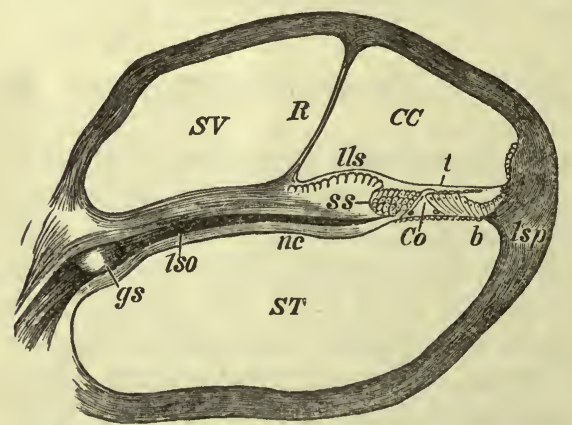

Fig. 494.-Section through one of the coils of the cochlea (diagrammatic). $S T$, scala tympani; $S V$, scala vestibuli; $C C$, canalis cochlea or canalis membranaceus; $R$, membrane of Reissner; lso, lamina spiralis ossea; $l l s$, limbus laminæ spiralis; ss, sulcus spiralis; $n c$, cochlear nerve; $g s$, ganglion spirale; $t$, membrana tectoria (below the membrana tectoria is the lamina reticularis) ; $b$, membrana basilaris ; $C o$, rods of Corti ; $l s p$, ligamentum spirale. (Quain.) its upper wall (separating it from the scala vestibuli) by the apex of the cochlea, the lamina ends in a small hamulus, the inner and concave part of which being detached from the summit of the modiolus, leaves a small aperture named the helicotrema, by which the two scalæ, separated in all the rest of their length, communicate.

Besides the scala vestibuli and scala tympani, there is a third space between them, called scala media or canal of the cochlea (CC, fig. 494). In section it is triangular, its external wall being formed by the wall of the cochlea, 
membrane of Reissner, and its lower wall (separating it from the scala tympani) by the basilar membrane, these two meeting at the outer edge of the bony lamina spiralis. Following the turns of the cochlea to its apex, the scala media there terminates blindly; at the base of the cochlea a narrow passage (canalis reuniens) unites it with the saccule. The scala media (like the rest of the membranous labyrinth) contains endolymph.

Organ of Corti.-Upon the basilar membrane are arranged cells of various shapes. About midway between the outer edge of the lamina spiralis and the outer wall of the cochlea are situated the rods of Corti. Viewed sideways, they are seen to consist of an external and internal pillar, each rising from an expanded foot or base attached to the basilar membrane ( $0, n$, fig. 495$)$. They slant

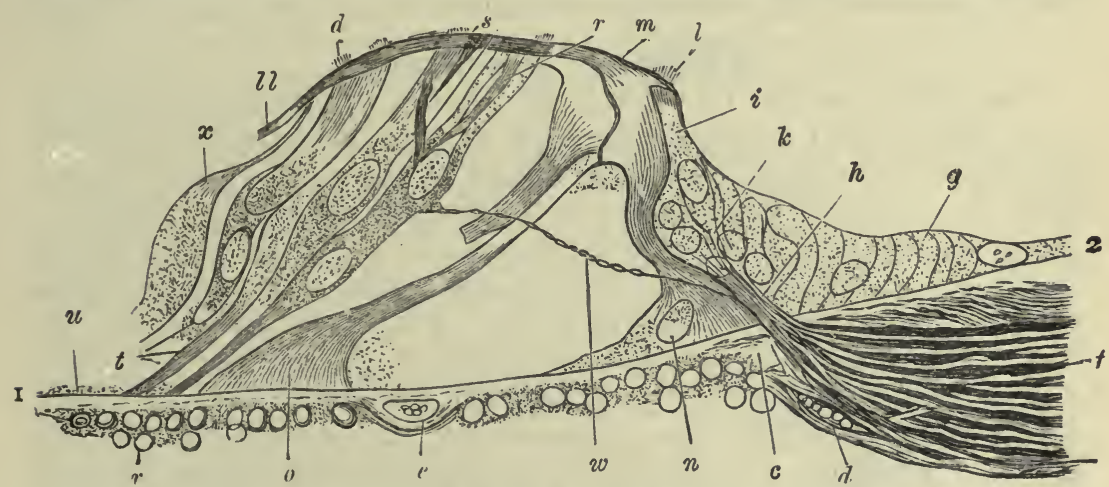

Fig. 495. - Vertical section of the organ of Corti from the dog. 1 to 2, Homogeneous layer of the membrana basilaris; $u$, vestibular layer; $v$, tympanal layer, with nuclei and protoplasm; $a$, prolongation of tympanal periosteum of lamina spiralis ossea; $c$, thickened commencement of the membrana basilaris near the point of perforation of the nerves $h ; d$, blood-vessel (vas spirale); $e$, blood-vessel ; $f$, nerves; $g$, the epithelium of the sulcus spiralis internus; $i$, internal hair-cell, with basal process $k$, surrounded with nuclei and protoplasm (of the granular layer), into which the nerve-fibres radiate; $l$, hairs of the internal hair-cell ; $n$, base or foot of inner pillar of organ of Corti ; $m$, head of the same uniting with the corresponding part of an external pillar, whose under half is missing, while the next pillar beyond, $o$, presents both middle portion and base; $r, s, d$, three external hair-cells ; $t$, bases of two nelghbouring hair or tufted cells ; $x$, supporting cell of Deiters; $w$, nerve-fibre arborising round the first of the external hair-cells; $l \quad l$ to $l$, lamina reticularis. $\times 800$. (Waldeyer.)

inwards towards each other, and each ends in a swelling termed the head; the head of the inner pillar overlies that of the outer. Each pair of pillars forms a pointed roof arching over a space, and by a succession of them a tunnel is formed.

There are about 3000 of these pairs of pillars, in proceeding from the base of the cochlea towards its apex. They are found progressively to increase in length, and become more oblique; in other words, the tunnel becomes wider, but diminishes in height as we approach the apex of the cochlea. Leaning against the rods of Corti are certain other cells called hair-cells, which terminate in small 
hair-like processes. There are several rows of these on the outer and one row on the inner side. Between them are certain supporting cells called cells of Deiters (fig. $495, x$ ). This structure rests upon the basilar membrane; it is roofed in by a fenestrated membrane or lamina reticularis into the fenestræ of which the tops of the various rods and cells are received. When viewed from above, the organ of Corti shows a remarkable resemblance to the keyboard of a piano. The top of the organ is roofed by the membrana tectoria (fig. 494, $t$ ) which extends from the end of the limbus (lls, fig. 494), a connective-tissue structure on the spiral lamina. The spiral ganglion from which the cochlear nerve-fibres originate is situated in the spiral lamina. The peripheral axons of its bipolar cells arborise around the hair-cells of the organ of Corti : the central axons pass down the modiolus, and thence to the pons (see p. 676).

\section{Physiology of Hearing.}

Sounds are caused by vibrations; when a piano-string is struck, it is thrown into a series of rapid regular vibrations; the more rapidly the vibrations occur the higher is the pitch of the musical note; the greater the amplitude of the vibration, the louder or more intense is the tone; if the vibrations are regular and simple (pendular), the tone is pure; if they are regular but compound, the tone is impure, and its quality or timbre is dependent on the rate and amplitude of the simple vibrations of which the compound vibrations are composed. The vibrations are transmitted as waves, and ultimately affect the hair-cells at the extremities of the auditory nerve in the cochlea. The semicircular canals are not concerned in the sense of hearing; their function in connection with equilibration is described in Chapter LI. The external and middle ears are conducting; the internal ear is conducting and receptive. In the external ear the vibrations travel through air; in the middle ear through solid structures-membranes and bones; and in the internal ear through fluid, first through the perilymph on the far side of the fenestra ovalis; and then the vibrations pass through the basilar membrane and membrane of Reissner, and set the endolymph of the canal of the cochlea in motion.

This is the normal way in which the vibrations pass, but the endolymph may be affected in other ways, for instance through the other bones of the head; one can, for example, hear the ticking of one's watch when it is placed between the teeth, even when the ears are stopped. From this fact is derived a valuable practical method of distinguishing in a deaf person what part of the organ of hearing is at fault. The patient may not be able to hear a watch or a tuning-fork when it is held close to the ear; but if he can hear it when it is placed between his teeth, or on his forehead, the malady is localised in either the external or middle ear; if he can hear it in neither situation, it is a much more serious case, for then the internal ear or the nervous mechanism of hearing is at fault. In disease of the middle ear the hearing of low tones is especially affected; high tones appear to be transmissible by boneconduction more readily than low. 
In connection with the external ear there is not much more to be said ; the pinna in many animals is large and acts as a kind of natural ear-trumpet to collect the vibrations of the air; in man this function is to a very great extent lost, and though there are muscles present to move it into appropriate postures, they are not under the control of the will in the majority of people, and are functionless, ancestral vestiges.

In the middle ear, however, there are several points to be considered, namely, the action of the membrana tympani, of the ossicles, of the tympanic muscles, and of the Eustachian tube.

The Membrana Tympani.-This membrane, unlike that of ordinary drums, can take up and vibrate in response to, not only its own fundamental tone, but to an immense range of tones differing from each other by many octaves. This would clearly be impossible if it were an evenly stretched membrane. It is not evenly nor very tightly stretched, but owing to its attachment to the chain of ossicles it is slightly funnel-shaped: the ossicles also damp the continuance of the vibrations.

When the membrane gets too tightly stretched, by increase or decrease of the pressure of the air in the tympanum, then the sense of hearing is dulled. The pressure in the tympanic cavity is kept the same as that of the atmosphere by the Eustachian tube, which leads from the cavity to the pharynx, and so to the external air. The Eustachian tube is not, however, always open; it is opened by the action of the tensor palati during swallowing. Suppose it were closed owing to swelling of its mucous membrane-this often happens in inflammation of the throat-the result would be what is called Eustachian or throat deafness, and this is relieved by passing a catheter so as to open the tube. When the tube is closed, an interchange of gases takes place between the imprisoned air and the blood of the tympanic vessels. In time, as in the aërotonometer (see p. 363), equilibrium is established and the tension of the imprisoned gases becomes equal to that of the blood-gases, not to that of the atmosphere. The membrane is therefore cupped inwards by the atmospheric pressure on its exterior; it is this increased tightening of the membrane that produces deafness. There is also an accumulation of mucus. When one makes a violent expiration, as in sneezing, some air is often forced through the Eustachian tube into the tympanum. The ears feel as though they were bulged out, as indeed the membrana tympani is, and there is again partial deafness, which sensations are at once relieved by swallowing, so as to open the Eustachian tube and thus re-establish equality of pressure.

The ossicles communicate the vibrations of the membrana tympani (to which the handle of the malleus is fixed) to the membrane which closes the fenestra ovalis (to which the foot of the 
stapes is attached). Thus the vibrations are communicated to the fluid of the internal ear, which is situated on the other side of the oval window.

The accompanying diagram will assist us in understanding how this is brought about. The bones all vibrate as if they were one, the slight movements between the individual bones being inappreciable. The utility of there being several bones is seen when the vibrations are excessive; the small amount of "give" at the articulations is really protective and tends to prevent fractures.

The handle of the malleus is inserted between the layers of the tympanic membrane; the processus gracilis (p.g.) has its end A

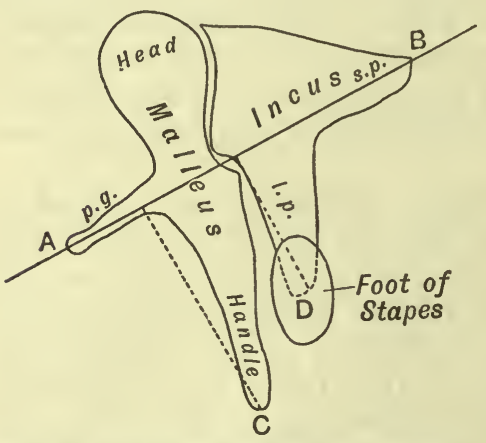

FIG. 496.-Diagrammatic view of ear ossicles. attached to the tympanic wall on the inner aspect of the Glaserian fissure; the end B of the short process (s.p.) of the incus is fastened by a ligament to the opposite wall of the tympanic cavity; the end $\mathrm{D}$ of the long process of the incus articulates with the stirrup, the base of which is turned towards the reader. The handle vibrates with the membrana tympani; and the vibrations of the whole chain take place round the axis of rotation AB. Every time $\mathrm{C}$ comes forwards D comes forwards; but by drawing perpendiculars from $\mathrm{C}$ and $\mathrm{D}$ to the axis of rotation, it is found that $\mathrm{D}$ is about $\frac{2}{3}$ of the distance from the axis that $\mathrm{C}$ is. So in the transmission of the vibrations from membrane to membrane across the bony chain, the amplitude of the vibration is decreased by about $\frac{1}{3}$, and the force is correspondingly increased. This increase of power is augmented by the fact that the tympanic membrane concentrates its power upon an area (the membrane of the oval window) only one-twentieth of its size. The final movement of the stapes is, however, always very small; it varies from $\frac{1}{15}$ to less than $\frac{1}{10000}$ of a millimetre.

The action of the tensor tympani, by pulling in the handle of the malleus, increases the tension of the membrana tympani. It is supplied by the fifth nerve. It is opposed by the strong external ligament of the malleus. The stapedius attached to the neck of the stapes tilts it backwards and diminishes the intra-tympanic airpressure. It is supplied by the seventh nerve.

The next very simple diagram (fig. 497) will explain the use of the fenestra rotunda.

The cochlea is supposed to be uncoiled; the scala vestibuli leads 
from the fenestra ovalis, to the other side of which the stapes is attached; the scala tympani leads to the fenestra rotunda; the two scalæ communicate at the helicotrema, and are separated from the canal of the cochlea by the basilar membrane, and the membrane of Reissner. C.R. is the canalis reuniens leading to the saccule. The cochlea is filled with incompressible fluid in an inexpansible bony case, except where the windows are closed by membranes. Hence every time the membrane of the oval window is bulged in by the stirrup, the membrane of the round window is simultaneously bulged out to the same extent, and vice versa. These changes of pressure are transmitted from one scala to the other directly through the cochlear canal, setting it into vibration, and through the helicotrema.

The range of hearing extends over 10 or 11 octaves; the lowest audible tone having about 20 , the highest about 25,000 , vibrations per second. The range varies in different people, and diminishes from childhood onwards. The upper limit of hearing may be tested

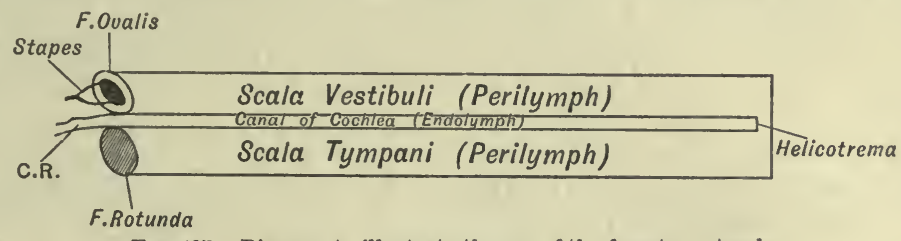

Fia. 497.-Diagram to illustrate the use of the fenestra rotunda.

by minute tuning-forks, metal rods, or by Galton's whistle. Many animals appear to be able to detect high tones which lie beyond the human limit. The lower limit may be determined by very large tuningforks, or by employing very low difference-tones.

Difference-tones are produced when two tones of different pitch, $m$ and $n$, are sounded together. A tone having the pitch $m$ minus $n$ is then heard in addition to the tones $m$ and $n$ : also a summation tone of pitch $m$ plus $n$ may be heard, but with greater difficulty. When $m$ and $n$ are nearly equal, a beating tone, instead of a differencetone, results, having a pitch somewhere intermediate between $m$ and $n$. If the difference between $m$ and $n$ is exceedingly small, this beatingtone alone is heard. The frequency of the beats corresponds to the difference in vibration-rates, $m$ and $n$. Under certain conditions the difference and summation-tones (which are collectively called combination-tones) exist in the air; their presence being demonstrable by their reinforcement before appropriate resonators. More generally, however, they appear to be produced within the ear, i.e., they have merely a subjective origin. The smallest perceptible difference in pitch between two successive tones is about 0.2 vibrations in the middle region of the piano for trained subjects. Practice effects extraordinary improvement, even among the most unmusical. 
There can be little doubt that the cochlea is the organ specially concerned in hearing. It first appears among vertebrata in certain fishes as a very rudimentary structure. If the cochlea is removed from dogs, they become deaf.

There are two classes of theories of hearing, in both of which the basilar membrane of the cochlea plays the essential part.

The one class comprises the many "sound-picture" theories which have been advanced in very various forms by Rutherford, Waller, Hurst, Ewald, and Meyer. The entire basilar membrane is supposed to vibrate either as a telephone plate, or as an elastic membrane, different tones or combinations of tones giving rise to different patterns of vibrations which are communicated to the hair-cells and thence by the auditory nerve-fibres to the brain, where (in Rutherford's theory) the analysis of these patterns is held to take place.

The other is the resonance-theory of Helmholtz, in which the pitch of a tone, or the analysis of a complex sound into its constituent tones, is determined not in the brain but in the cochlea. It depends on the principle of sympathetic vibration. As is well known, if a tone is sung in front of a piano (best with the loud pedal held down), the string of the piano which is attuned to that tone will immediately respond; another tone will elicit response from another string. So in the cochlea the appropriate fibre of the basilar membrane is thrown into vibration when the tone to which it is attuned reaches it. The fibre thus stimulated affects the hair-cells above it, whence the stimulus is conducted to the brain. If two tones are sounded together, the two appropriate fibres respond, and the analysis of the now more complex stimulus is performed in the cochlea. The fibres of the basilar membrane increase in length from the base towards the apex of the cochlea. According to the resonance-theory, the upper part of the organ would thus be affected by low tones, the lower part by high tones.

The first of these two classes of theory makes it difficult or impossible for us to explain our ability to analyse complex chords into their component tones. The full acceptance of the second is difficult in the face of the small difference of length (at most $1: 12$ ) between the shortest and the longest of the basilar fibres. On the other hand, it gains support from the effects of experiment on, and disease of, different portions of the cochlea. For instance, the deafness to high-pitched tones (seen in boiler-makers) is stated to be associated with disease of the lower whorl of the cochlea.

It may be that the fibres of the basilar membrane do not vibrate as Helmholtz supposed, but that the hair-cells themselves are each in some unknown way specially attuned to respond only to one of the many tonal stimuli which may reach them (Myers). 


\section{H A P T E R L V I I}

\section{VOICE AND SPEECH}

THE fundamental tones of the voice are produced by the current of expired air causing the vibration of the vocal cords, two elastic bands contained in a cartilaginous box placed at the top of the windpipe or trachea. This box is called the larynx. The sounds produced here are modified by other parts such as the tongue, teeth, and lips, as will be explained later on.

\section{Anatomy of the Larynx.}

The cartilages of the laryn $x$ are the thyroid, the cricoid, and the two arytenoids. These are the most important for voice production; they are made of hyaline carti-

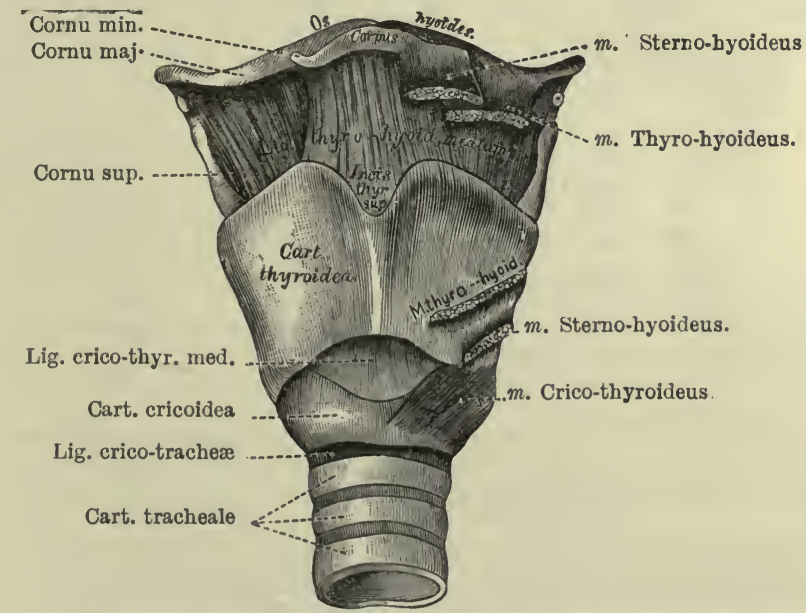

F1d. 498.-The larynx, as seen from the front, showing the cartilages and ligaments. The muscles, with the exception of one crico-thyroid, are cut off short. (Stoerk.)

lage. Then there are the epiglottis, two cornicular, and two cuneiform cartilages. These are made of yellow fibro-cartilage.

The thyroid cartilage (fig. 499, 1 to 4 ) does not form a complete ring around the larynx, but only covers the front portion. It forms the prominence in front of the 
throat known as Adam's apple. The cricoid cartilage (fig. 499, 5, 6), on the other hand, is a complete ring; the back part of the ring is much broader than the front. On the top of this broad portion of the cricoid are the arytenoid cartilages (fig. $499,7)$; the connections between the cricoid below and arytenoid cartilages above are joints with synovial membrane and ligaments, the latter permitting tolerably
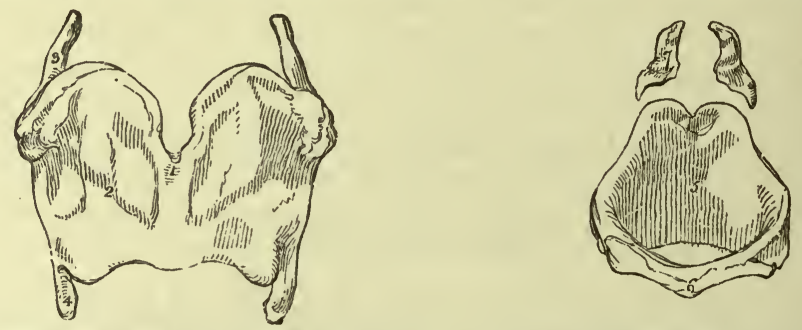

FrG. 499.-Cartilages of the larynx seen from the front. 1 to 4, Thyroid cartilage; 1 , vertical ridge or pomum Adami ; 2, right ala; 3, superior, and 4, inferior cornu of the right side; 5 , 6, cricoid cartilage; 5 , inside of the posterior part ; 6 , anterior narrow part of the ring; 7 , arytenoid cartilages. $\times \frac{3}{4}$.

free motion between them. But although the arytenoid cartilages can move on the cricoid, they accompany the latter in all its movements. The base by means of which each arytenoid cartilage sits on the cricoid is triangular; the anterior angle is often called the vocal process : to it the posterior ends of the true vocal cords are attached. The outer angle is thick, and called the muscular process.

The cornicular cartilages, or cartilages of Santorini, are perched on the top of

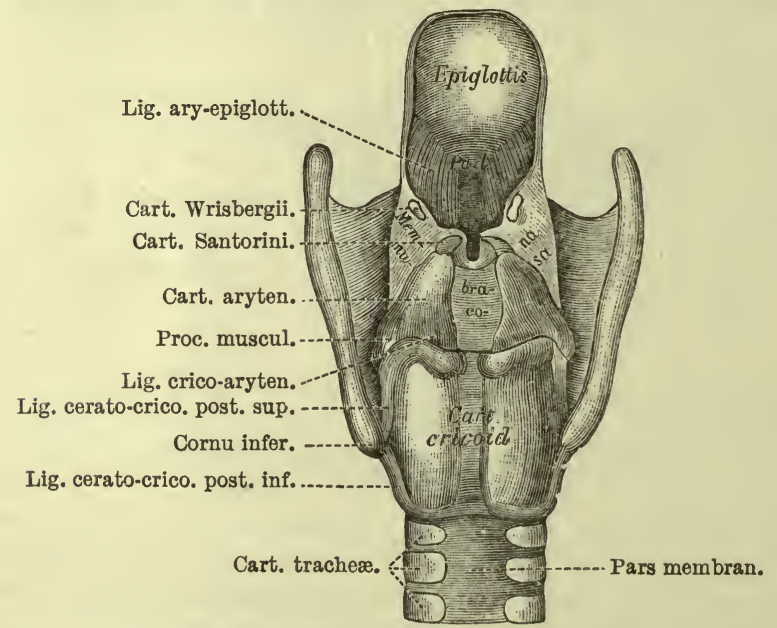

Fig. 500.-The larynx as seen from behind after removal of the muscles. The cartilages and ligaments only remain. (Stoerk.)

the arytenoids ; the cuneiform cartilages, or cartilages of Wrisberg, are in a fold of mucous membrane; the epiglottis looks like a lid to the whole (fig. 500).

The thyroid cartilage is connected with the cricoid, by the crico-thyroid membrane, and also by joints with synovial membranes; the lower cornua of the thyroid clasp the cricoid between them, yet not so tightly but that the thyroid can revolve, within a certain range, around an axis passing transversely through the two joints 
at which the cricoid is clasped. The vocal cords are attached behind to the front portion of the base (vocal process) of the arytenoid cartilages, and in front to the re-entering angle at the back of the thyroid ; it is evident, therefore, that all movements of either of these cartilages must produce an effect on them of some kind or other. Inasmuch, too, as the arytenoid cartilages rest on the top of the back portion of the cricoid cartilage, and are connected with it by capsular and other ligaments, all movements of the cricoid cartilage must move the arytenoid cartilages, and also produce an effect on the vocal cords.

Mucous membrane. - The larynx is lined with a mucous membrane continuous with that of the trachea; this is covered with ciliated epithelium except over the vocal cords and epiglottis, where it is stratified. The vocal cords are thickened bands of elastic tissue in this mucous membrane which run from before back. They are attached behind to the vocal processes of the arytenoid cartilages, and in front to the angle where the two wings of the thyroid meet. The chink between them is called the rima glottidis (see fig. 501). Two ridges of mucous membrane above and parallel to these are called the false vocal cords; between the true and false vocal cord on each side is a recess called the ventricle.

Muscles.-The muscles of the larynx are divided into intrinsic and extrinsic. The intrinsic are named from their attachments to the various cartilages; the extrinsic are those which connect the larynx to other parts, such as the hyoid bone.

The intrinsic muscles of the larynx are as follows:-

1. Crico-thyroid.

2. Posterior crico-arytenoid.

3. Lateral crico-arytenoid.

4. Thyro-arytenoid.

5. Arytenoid.

All these muscles except the arytenoid are in pairs.

Their attachments and actions are as follows :-

1. Crico-thyroid. - This is a short, thick triangular muscle, at-

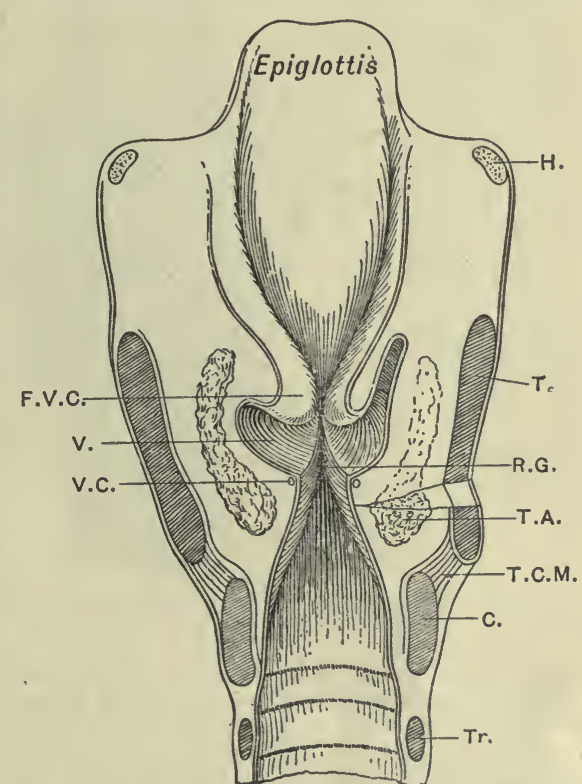

Fig. 501.-Vertical section through the larynx, passing from side to side. H, hyoid bone; T., thyroid cartilage; T.C.M., thyro-cricoid membrane; C., cricoid cartilage; Tr., first ring of trachea; T.A., thyroary tenoid muscle; R.G., rima glottidis ; V.C., vocal cord; V., ventricle; F.V.C., false vocal cord. (After Allen Thomson.) tached below to the cricoid cartilage;

this attachment extends from the middle line backwards. The fibres pass upwards and outwards, diverging slightly to be attached above to the inferior border of the thyroid cartilage, and to the anterior border of its lower cornu.

The thyroid cartilage being fixed by extrinsic muscles, the contraction of this muscle draws upwards the anterior part of the cricoid cartilage, and depresses the posterior part, and with it the arytenoid cartilages, so that the vocal cords are stretched. Paralysis of these muscles therefore causes an inability to produce highpitched tones.

2. Posterior crico-arytenoid.-This arises from the broad depression on the corresponding half of the posterior surface of the cricoid cartilage; its fibres converge upwards and outwards, and are inserted into the outer angle of the base of the arytenoid cartilage behind the attachment of the lateral crico-arytenoid muscle.

These muscles draw the outer angles of the arytenoid cartilages backwards and 
inwards, and thus rotate the anterior or vocal processes outwards, and widen the rima glottidis. They come into action during deep inspiration. If they are paralysed, the lips of the glottis approach the middle line and come in contact during each inspiration, so that dyspnœa is produced.

3. Lateral crico-arytenoid. - This arises from the sloping upper border of the cricoid cartilage, and is inserted into the muscular process of the arytenoid cartilage, and the adjacent part of its anterior surface.

These muscles draw the muscular processes of the arytenoid cartilages forwards

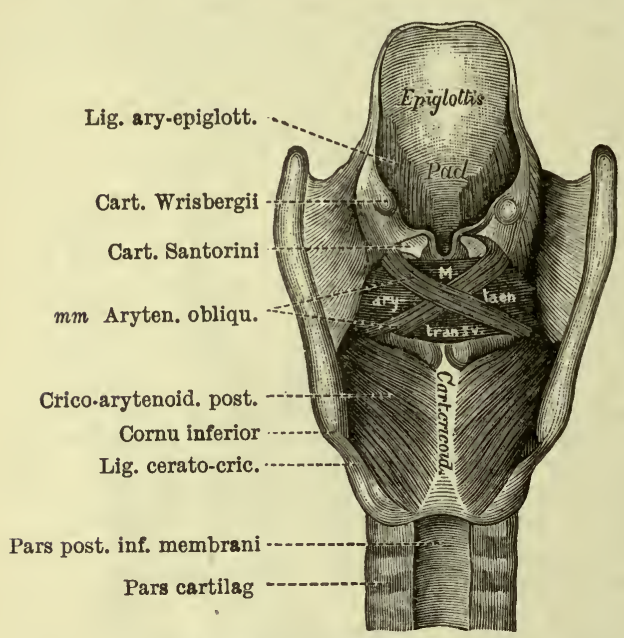

Fic. 502.-The larynx as seen from behind. To show the intrinsic muscles posteriorly. (Stoerk.) and downwards, and thus approximate the vocal cords. They are antagonistic to the posterior crico-arytenoids.

4. Thyro - arytenoid. - This consists of two portions, inner and outer. The inner portion arises in the lower half of the angle formed by the alæ of the thyroid cartilage, and passing backwards is attached behind to the vocal process and to the adjacent parts of the outer surface of the arytenoid cartilage. These fibres are joined internally by short fibres which are attached in front to the vocal cord, and behind to the vocal process. Some oblique fibres pass from the sloping portion of the cricothyroid membrane below the vocal cord, upwards, outwards, and somewhat backwards, to end in the tissue of the false vocal cord. The fibres of the outer portion arise in front from the thyroid cartilage close to the origin of the inner portion and from the crico-thyroid membrane. They pass backwards to be inserted in part into the lateral border and muscular process of the arytenoid cartilage, and in part they pass obliquely upwards towards the arytenoepiglottidean fold, ending in the false vocal cord. The portion of this muscle which extends towards the epiglottis is often described as a separate muscle (thyroepiglottidean); it resembles the crico-arytenoid in that some of its fibres are continuous with those of the arytenoid muscle.

The antero-posterior fibres will tend to draw forward the arytenoid cartilage, and with it the posterior part of the cricoid cartilage, rotating the latter upwards and antagonising the action of the crico-thyroid muscle, the effect being to relax the vocal cords. But if the latter are kept stretched those fibres of the inner portion of the muscle which are inserted into the vocal cord may serve to modify its elasticity, tightening the parts of the cord in front of, and relaxing those behind, its attachment. The vertical fibres of the muscle which extend from the crico-thyroid membrane across the base of the vocal fold and over the ventricle into the false vocal cord, render the free edge of the former more prominent. Then the fibres which are inserted into the muscular process and outer surface of the arytenoid cartilage will tend to draw the arytenoid cartilage forwards and rotate it inwards ; finally, the fibres which pass into the aryteno-epiglottidean fold may assist in depressing the epiglottis.

If these muscles are paralysed, the lips of the glottis are no longer parallel, but are curved with the concavity inwards, and a much stronger blast of air is required for the production of the voice.

5. Arytenoid.- When the mucous membrane is removed from the back of the arytenoid cartilages, a band of transverse fibres is exposed, on the dorsal surface of which are two slender decussating oblique bundles. These are often described as 
separate muscles (arytenoid and aryteno-epiglottidean), but they are intimately blended together. The ventral fibres (arytenoid proper) pass straight across from the outer half of the concave surface on the back of one arytenoid cartilage to the corresponding surface of the other. The dorsal fibres can be followed to the lateral walls of the larynx, the uppermost ones to the cartilage of Santorini, the intermediate ones run with the uppermost fibres of the thyro-arytenoid muscle forming the socalled aryteno-epiglottidean muscle, and the lowest fibres blend at the level of the true vocal cords with the thyro-arytenoid and lateral crico-arytenoid muscles.

The arytenoid muscle draws the arytenoid cartilages together. If it is paralysed, the intercartilaginous part of the glottis remains open, although the membranous lips can still be approximated during vocalisation.

It has been generally supposed that the epiglottis is depressed as a lid over the glottis during swallowing. This may be so in some animals, but in man it is not the case ; the epiglottis projects upwards in close contact with the base of the tongue. The necessary closure of the glottis during swallowing is brought about by the contraction of the arytenoid and thyro-arytenoid muscles; by this means the arytenoid cartilages are drawn towards each other, and also forwards into contact with the posterior surface of the epiglottis (Anderson Stuart). Henle remarked that "the muscles which lie in the space enclosed by the laminæ of the thyroid cartilage and above the cricoid may be regarded in their totality as a kind of sphincter such as is found in its simplest form embracing the entrance of the larynx in reptiles " (Quain's Anatomy).

Nerves.-The larynx is supplied by two branches of the vagus; the superior laryngeal is the sensory nerve ; by its external branch, it supplies one muscle, namely, the crico-thyroid. These fibres, however, probably arise from glosso-pharyngeal rootlets. The rest of the muscles are supplied by the inferior laryngeal nerve, the fibres of which come from the spinal accessory, not the vagus proper.

The laryngoscope is an instrument employed in investigating during life the condition of the pharynx, larynx, and trachea. It consists of a large concave mirror

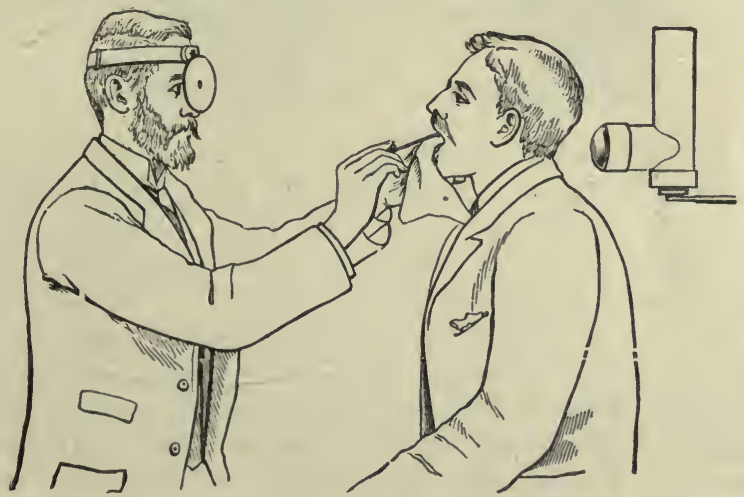

Fra. 503 - To show the position of the operator and patient when using the Laryngoscope.

with perforated centre, and of a smaller mirror fixed in a long handle. The patient is placed in a chair, a good light (argand burner, or electric lamp) is arranged on one side of, and a little above, his head. The operator fixes the large mirror round his head in such a manner, that he looks through the central aperture with one eye. He then seats himself opposite the patient, and so alters the position of the mirror, which is for this purpose provided with a ball-and-socket joint, that a beam of light is reflected on the lips of the patient.

The patient is now directed to throw his head slightly backwards, and to open his mouth; the reflection from the mirror lights up the cavity of the mouth, and by a 
little alteration of the distance between the operator and the patient the point at which the greatest amount of light is reflected by the mirror-in other words, its focal length-is readily discovered. The small mirror fixed in the handle is then warmed, either by holding it over the lamp, or by putting it into a vessel of warm water; this is necessary to prevent the condensation of breath upon its surface. The degree of heat is regulated by applying the back of the mirror to the hand or cheek, when it should feel warm without being painful.

After these preliminaries the patient is directed to put out his tongue, which is held by the left hand gently but firmly against the lower teeth by means of a handkerchief. The warm mirror is passed to the back of the mouth, until it rests upon and slightly raises the base of the uvula, and at the same time the light is directed upon it: an inverted image of the larynx and trachea will be seen in the mirror. If the dorsum of the tongue is alone seen, the handle of the mirror must be slightly lowered until the larynx comes into view; care should be taken, however, not to move the mirror upon the uvula, as it excites retching. The observation should not be prolonged, but should rather be repeated at short intervals.

The structures seen will vary somewhat according to the condition of the parts as to inspiration, expiration, phonation, etc. ; they are (fig. 504) first, and apparently at the posterior part, the base of the tongue, immediately below which is the arcuate outline of the epiglottis, with its cushion or tubercle. Then are seen in the central line the true vocal cords, white and shining in their normal condition. The cords approximate (in the inverted image) posteriorly; between them is left a chink, narrow whilst a high note is being sung, wide during a deep inspiration. On each side of the true vocal cords, and on a higher level, are the pink false vocal cords. Still more externally than the false vocal cords is the aryteno-epiglottidean fold, in which are situated upon each side three small elevations; of these the most external is the cartilage of Wrisberg, the intermediate is the cartilage of Santorini, whilst the summit of the arytenoid cartilage is in front, and somewhat below the preceding, being only seen during deep inspiration. The rings of the trachea, and even the bifurcation of the trachea itself, if the patient be directed to draw a deep breath, may be seen in the interval between the true vocal cords.

\section{Movements of the Vocal Cords.}

In Respiration.-The position of the vocal cords in ordinary tranquil breathing is so adapted by the muscles, that the opening of the glottis is wide and triangular (fig. 504, B). The glottis remains unaltered during ordinary quiet breathing, though in a small proportion of people it becomes a little wider at each inspiration, and a little narrower at each expiration. In the cadaveric position the glottis has about half the width it has during ordinary breathing; during life, therefore, except during vocalisation, the abductors of the vocal cords (posterior crico-arytenoids) are in constant action. (F. Semon.) On making a rapid and deep inspiration the opening is widely dilated (fig. 504, c), and somewhat lozenge-shaped.

In Vocalisation.-At the moment of the emission of a note, the chink is narrowed, the margins of the arytenoid cartilages being brought into contact, and the edges of the vocal cords approximated and made parallel (fig. 504, A); at the same time their tension is much increased. The higher the note produced, the tenser do the cords become; and the range of a voice depends, in the main, on the extent to which the degree of tension of the vocal cords can 
be thus altered. In the production of a high note the vocal cords are brought well within sight. In the utterance of low-pitched tones,
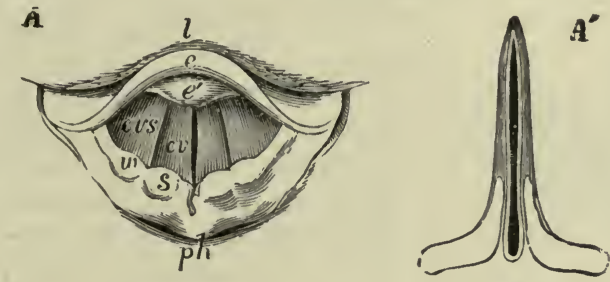

$\mathbf{E}$
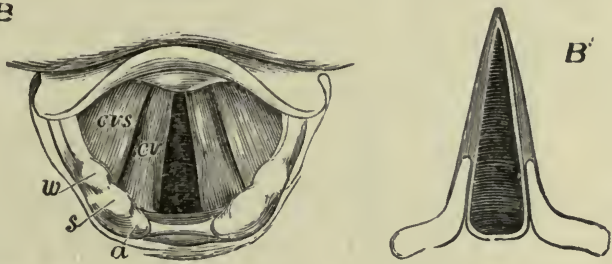

C
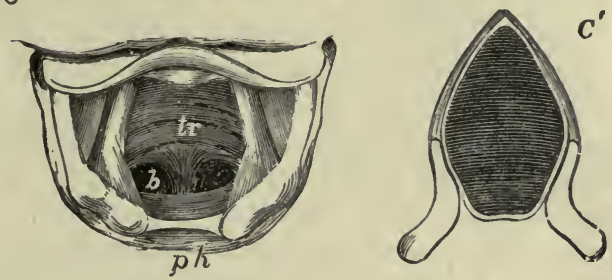

Fiv. 504.-Three laryngoscopic views of the superior aperture of the larynx and surrounding parts. A, the glottis during the emission of a high note in singing; $\mathrm{B}$, in easy and quiet inhalation of air ; $\mathrm{C}$ in the state of widest possible dilatation, as in inhaling a very deep breath. The diagrams $\mathbf{A}^{\prime}, \mathbf{B}^{\prime}$, and $\mathbf{C}^{\prime}$, show in horizontal sections of the glottis the position of the vocal cords and arytenoid cartilages in the three several states represented in the other figures. In all the figures so far as marked, the letters indicate the parts as follows, viz.: $l$, the base of the tongue; $e$, the upper free part of the epiglottis; $e^{\prime}$, the tubercle or cushion of the epiglottis; $p h$, part of the anterior wall of the pharynx behind the larynx; in the margin of the aryteno-epiglottidean fold, $w$, the swelling of the membrane caused by the cartilages of Wrisberg; $s$, that of the cartilages of Santorini ; $a$, the tip or summit of the arytenoid cartilages; $c v$, the true vocal cords or lips of the rima glottidis; $c v s$, the superior or false vocal cords; between them the ventricle of the larynx; in $\mathbf{C}, t r$ is placed on the anterior wall of the receding trachea, and $b$ indicates the commencement of the two bronchi beyond the bifurcation which may be brought into view in this state of extreme dilatation. (Quain, after (zermak.)

on the other hand, the epiglottis is depressed and brought over them, and the arytenoid cartilages look as if they were trying to hide themselves under it (fig. 505).

The approximation of the vocal cords also usually corresponds with the height of the note produced; but the width of the aperture has no influence on the pitch of the note, as long as the vocal cords have the same tension: only with a wide aperture the tone is more difficult to produce and is less perfect, the rushing of the air through the aperture being heard at the same time. 
No true vocal sound is produced at the posterior part of the aperture of the glottis, namely, that which is formed by the space between the arytenoid cartilages.

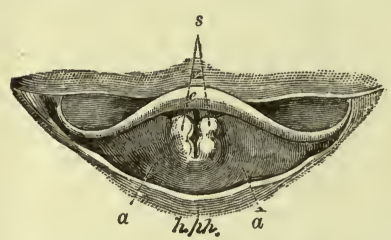

FIG. 505.-View of the upper part of the larynx as seen by means of the laryngoscope during the utterance of a bass note. $e$, Epiglottis ; $s$, tubercles of the cartilages of Santorini; $a$, arytenoid cartilages; $z$, base of the tongue; $p h$, the posterior wall of the pharynx. (Czermak.)

\section{The Voice.}

The human musical instrument is often compared to a reed organ-pipe: certainly the notes produced by such pipes in the vox humana stop of organs is very like the human voice. Here there is not only the vibration of a column of air, but also of a reed, which corresponds to the vocal cords in the air-chamber composed of the trachea and the bronchial system beneath it. The pharynx, mouth, and nasal cavities above the glottis are resonating cavities, which, by alterations in their shape and size, are able to pick out and emphasize certain component parts of the fundamental tones produced in the larynx. The natural voice is often called the chest voice. The falsetto voice is differently explained by different observers; on laryngoscopic examination, the glottis is found to be widely open, so that there is an absence of chest resonance; some have supposed that the attachment of the thyro-arytenoid muscle to the vocal cord renders it capable of acting like the finger on a violin string, part of the cord being allowed to vibrate while the rest is held still. Such a shortening of a vibrating string would produce a higher note than is natural.

Musical sounds differ from one another in three ways :-

1. In pitch.-This depends on the rate of vibration; and in the case of a string, the pitch increases with the tension, and diminishes with the length of the string. The vocal cords of a woman are shorter than those of a man, hence the higher pitched voice of women. The average length of the female cord is 11.5 millimetres; this can be stretched to 14 ; the male cord averages 15.5 , and can be stretched to 19.5 millimetres.

2. In loudness.-This depends on the amplitude of the vibrations, and is increased by the force of the expiratory. blast which sets the cords in motion.

3. In "timbre."-This is the difference of character which distinguishes one voice, or one musical instrument, from another. It is due to admixture of the primary vibrations with secondary vibrations or overtones. If one takes a tracing of a tuning-fork on a revolving cylinder, it writes a simple series of up and down waves corresponding in rate to the note of the fork. Other musical instru- 
ments do not lend themselves to this form of graphic record, but their vibrations can be rendered visible by allowing them to act on a small sensitive gas-flame; this bobs up and down, and if the reflection of this flame is allowed to fall on a series of mirrors, the top of the continuous image formed is seen to present waves. The mirrors are usually arranged on the four lateral sides of a cube which is rapidly rotated (fig. 506). If one sings a note on to the membrane in the side of the gas-chamber with which the flame is in connection, the waves seen are not simple up and down ones, but the primary large waves are complicated by smaller ones on their surface, at twice,

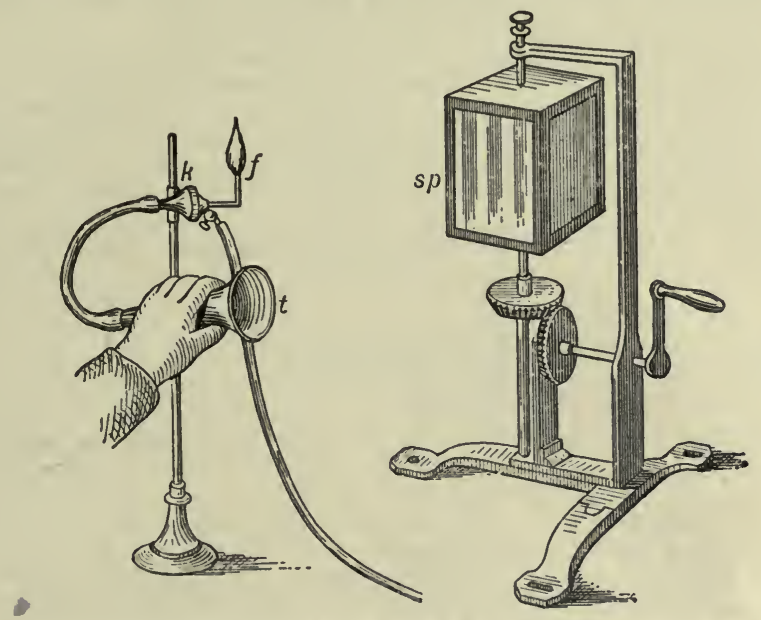

FIG. 506.-König's apparatus for obiaining flame pictures of musical notes.

thrice, etc., the rate of the primary vibration. The richer a voice, the richer the sound of a musical instrument, the more numerous are these overtones or harmonics. The range of the voice is seldom, except in celebrated singers, more than two-and-a-half octaves, and for different voices this is in different parts of the musical scale.

\section{Speech.}

Speech is due to the modification produced in the fundamental laryngeal notes, by the resonating cavities above the vocal cords. By modifying the size and shape of the pharynx, mouth, and nose, certain overtones or harmonics are picked out and exaggerated: this gives us the vowel sounds; the consonants are produced by interruptions, more or less complete, of the outflowing air in different situations. When the larynx is passive, and the resonating cavities alone come into play, then we get whispering. 
The pitch of the Vowels has been estimated musically; $u$ has the lowest pitch, then $o, a$ (as in father), $a$ (as in cane), $i$, and $e$. We may give a few examples of the shape of the resonating cavities in pronouncing vowel sounds, and producing their characteristic timbre : when sounding $a$ (in father) the mouth has the shape of a funnel wide in front; the tongue lies on the floor of the mouth ; the lips are wide open; the soft palate is moderately and the larynx slightly raised.

In pronouncing $u(o o)$, the cavity of the mouth is shaped like a capacious flask with a short narrow neck. The whole resonating cavity is then longest, the lips being protruded as far as possible; the larynx is depressed and the root of the tongue approaches the fauces.

In pronouncing $o$, the neck of the flask is shorter and wider, the lips being nearer the teeth; the larynx is slightly higher than in sounding 00 .

In pronouncing $e$, the flask is a small one with a long narrow neck. The resonating chamber is then shortest as the larynx is raised as much as possible, and the mouth is bounded by the teeth, the lips being retracted; the approach of the tongue near the hard palate makes the long neck of the flask.

The Consonants are produced by a more or less complete closure of certain doors on the course of the outgoing blast. If the closure is complete, and the blast suddenly opens the door, the result is an explosive; if the door is partly closed, and the air rushes with a hiss through it, the result is an aspirate; if the door is nearly closed and its margins are thrown into vibration, the result is a vibrative; if the mouth is closed, and the sound has to find its way out through the nose, the result is a resonant.

These doors are four in number; Bricke called them the articulation positions. They are-

1. Between the lips.

2. Between the tongue and hard palate.

3. Between the tongue and soft palate.

4. Between the vocal cords. plan :-

The following table classifies the principal consonants according to this

Articulation
position.
1
2
3
4

$$
\begin{array}{cc}
\text { Explosives. } & \text { Aspirates. } \\
\text { B, P. } & \text { F, V, W. } \\
\text { T, D. } & \text { S, L, L, Sch, Th. } \\
\text { K, G. } & \text { J, Ch. } \\
\text {... } & \text { H. }
\end{array}
$$

Vibratives.

$\dddot{R}$

Palatal R.

$\mathrm{R}$ of lower Saxon
Resonants.

M.

N.

$\mathrm{Ng}$.

...

The introduction of the phonograph has furnished us with an instrument which it is hoped in the future will enable us to state more accurately than has hitherto been possible, the meaning of the changes in nature and intensity of the complex vibrations which constitute speech. The microscopic study of the tracing on the recording phonographic cylinder, and various methods of obtaining a high magnification of the movements of the recording style have been carried out by $M$ 'Kendrick and others. The subject is, however, not yet sufficiently ripe for definite statements to be made.

\section{Defects of Speech.}

Speech may be absent in certain forms of lunacy, and temporarily in that defect of will called hysteria.

It may be absent owing to congenital defects. Children born deaf are dumb also. This is because we think with remembered sounds, and in a person born deaf the auditory centres are never set into activity. By educating the child by the visual inlet, it can be taught to think with the remembered shapes of the mouth and expressions of the face produced in the act of speaking, and so can itself speak in time.

If a child becomes deaf before it is six or seven years old, there is a liability it will forget the speech it has learnt, and so become dumb.

In congenital hemiplegia there may be speechlessness, especially if the injury is 
due to meningeal hæmorrhage affecting the grey cortex of the left hemisphere. These children generally talk late, the right side of the brain taking on the function of the left.

Disorders of speech and voice occur from affections of the larynx, and of the nerves which supply the larynx. Stammering is a want of coördination between the various muscles employed in the act of speaking.

Perhaps the most interesting of the disorders of speech, however, are those associated with brain disease in adults, and to which the general term Aphasia is given. There may be an entire loss of the power to articulate words, or there may be a mere blurring of the speech. In other cases there is a loss of memory for words, the words spoken being well pronounced, but are not those which the patient wishes to utter. This is often associated with Agraphia, a similar condition in respect to writing. Some writers distinguish between motor aphasia, which is associated with disorganisation of the motor word-centre (Broca's convolution), and sensory aphasia, in which the defect is in the association of ideas of things and the ideas of their names. This is due to injury of the visual word-centre, or the auditory word-centre (see p. 741), or to a severance of the tracts which unite them to one another and to the motor word-centre. It will readily be understood that the actual symptoms will vary greatly according to the position of the lesion. It is generally admitted that injuries to the sensory word-centres are more potent in the production of aphasia than injuries to Broca's convolution. Marie, as already noted on $\mathrm{p}$. 734, has gone so far as to assert that a lesion limited to Broca's convolution will not produce aphasia, but this view has not yet been fully verified.

With regard to the auditory word-centre, impressions for the sounds of words are revived in one of three ways :-

a. Spontaneous or volitional; owing to accumulated traces which constitute memory, a man when he wants to express his thoughts in words remembers the sounds it is necessary to use ; impulses pass to the motor-centre (Broca's convolution), thence to the nerve-centres, nerves, and muscles of the larynx, mouth, chest, etc., and the man speaks.

b. In slight disease of the auditory word-centre, he is unable to do this, but if his mind is set into a certain groove he will speak; thus if the alphabet or a wellknown piece of poetry be started for him he will finish it by himself.

c. Mimetic. In more severe cases, a more powerful stimulus still is needed; he will repeat any words after another person, but forget them immediately afterwards.

With regard to the visual word-centre as tested by writing, there are also three ways of reviving impressions for written words or letters.

(a) Spontaneous or normal.

(b) A train of thought must first be set going ; as, for instance, converting printed words into written characters.

(c) Mimetic; he can only write from a copy.

Some operations, such as reading aloud and writing from dictation, require the combined activity of several centres. This, however, we have previously considered in connection with the subject of association in the brain (see p. 741). 


\section{H A P T E R L V I I I}

\section{THE EYE AND VISION}

THE eyeball is contained in the cavity of the skull called the orbit; here also are vessels and nerves for the supply of the eyeball, muscles to move it, and a quantity of adipose tissue. In the front of the eyeball are the lids and lacrimal apparatus.

The eyelids consist of two movable folds of skin, each of which is kept in shape by a thin plate of fibrous tissue called the tarsus. Along their free edges are inserted a number of curved hairs (eyelashes), which, when the lids are half closed, serve to protect the eye from dust and other foreign bodies: the tactile sensibility of the lids is very delicate. Imbedded in the tarsus are a number of long sebaceous glands (Meibomian), the ducts of which open near the free edge of the lid. In the loose connective tissue in front of the tarsus, the bundles of the orbicularis muscle are situated.

The orbital surface of each lid is lined by a delicate, highly sensitive mucous membrane (conjunctiva), which is continuous with the skin at the free edge of each lid, and after lining the inner surface of the eyelid is reflected on to the eyeball, being somewhat loosely adherent to the sclerotic coat. Its epithelium, which is columnar, is continued over the cornea as its anterior epithelium, where it becomes stratified. At the inner edge of the eye the conjunctiva becomes continuous with the mucous lining of the lacrimal sac and duct, which again is continuous with the mucous membrane of the nose.

The eyelids are closed by the contraction of a sphincter muscle (orbicularis), supplied by the facial nerve; the upper lid is raised by the levator palpebroe superioris, supplied by the third nerve.

The lacrimal gland, composed of lobules made up of acini resembling the serous salivary glands, is lodged in the upper and outer angle of the orbit. Its secretion, which issues from several ducts on the inner surface of the upper lid, under ordinary circumstances just suffices to keep the conjunctiva moist. It passes out through two small openings (puncta lacrimalia) near the inner angle of the eye, 
one in each lower lid, into the lacrimal sac, and thence along the nasal duct into the inferior meatus of the nose. The excessive secretion poured out under the influence of an irritating vapour or painful emotion overflows the lower lid in the form of tears. The secretory nerves are contained in the lacrimal and subcutaneous malar branches of the fifth nerve, and in the cervical sympathetic.

\section{The Eyeball.}

The eyeball (fig. 507) consists of the following structures :-

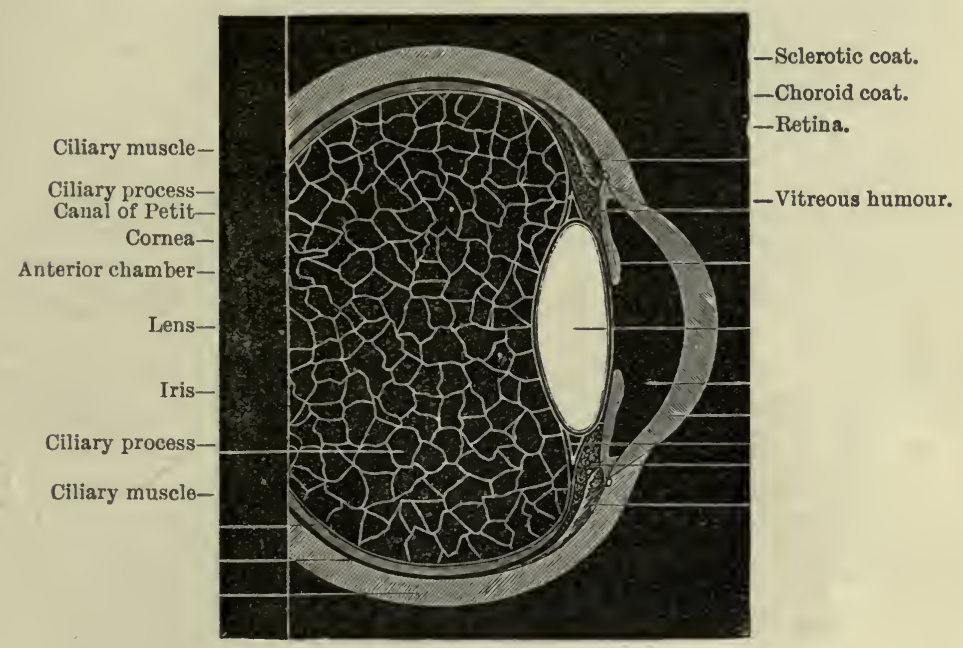

F10. 507.-Section of the anterior four-fifths of the eyeball.

The sclerotic, or outermost coat, is made of connective tissue and envelops about five-sixths of the eyeball: continuous with it, in front, and occupying the remaining sixth, is the transparent cornea (fig. 508). Immediately within the sclerotic is the choroid coat, and within the choroid is the retina. The interior of the eyeball is filled by the aqueous and vitreous humours and the crystalline lens; but, also, there is suspended in the interior a contractile and perforated curtain,-the iris, which is continuous with the choroid; it regulates the admission of light; at the junction of the sclerotic and cornea is the ciliary muscle, the function of which is to adapt the eye for seeing objects at various distances.

The Choroid Coat is the vascular coat of the eyeball, and its connective tissue contains abundance of branched pigment cells. It is separated from the retina by a fine elastic membrane (membrane of Bruch). 
The choroid coat ends in front in what are called the ciliary processes (figs. 509, 510). These consist of from 70 to 80 meridionally arranged radiating plaits, which consist of blood-vessels, fibrous connective tissue, and pigment corpuscles. They are lined by a

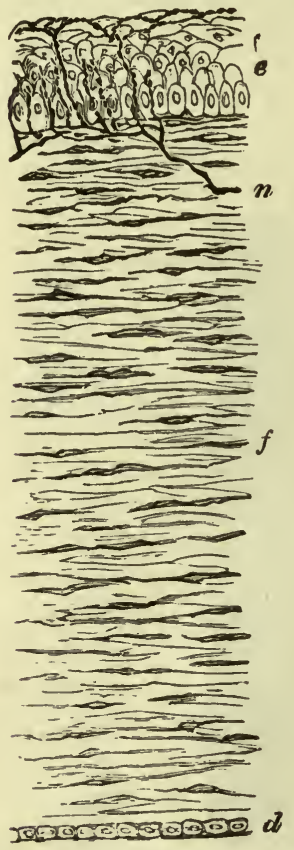

FIa. 508.-Vertical section of rabbit's cornea, stained with gold chloride. $e$, Stratified anterior epithelium. Immediately beneath this is the anterior homogeneous lamina of Bowman. $n$, Nerves forming a delicate sub-epithelial plexus, and sending up fine twigs between the epithelial cells to end in a second plexus on the free surface; $d$, Descemet's membrane; consisting of a fine elastic layer, and a single layer of epithelial cells; the substance of the cornea, $f$, is seen to be fibrillated, and contains many layers of branched corpuscles, arranged parallel to the free surface, and here seen edgewise.

(Schofield.) continuation of the membrane of Bruch. The ciliary processes terminate at the margin of the lens. The ciliary muscle $(13,14$, and 15, fig. 509), takes origin at the corneo-scleral junction. It is a ring of muscle, $3 \mathrm{~mm}$. broad and $8 \mathrm{~mm}$. thick, made up of fibres running in three directions. (a) Meridional fibres near the sclerotic and passing to the choroid; (b) radial fibres inserted into the choroid behind the ciliary processes; and (c) circular fibres (muscle of Müller), more internal; they constitute a sphincter.

The Iris is a continuation of the choroid inwards beyond the ciliary processes. It is a fibro-muscular membrane perforated by a central aperture, the pupil.

Posteriorly is a layer of pigment cells (uvea), which is a continuation forwards of the pigment layer of the retina. The iris proper is made of connective tissue in front with corpuscles which may or may not be pigmented, and behind of similar tissue supporting blood-vessels. The pigment cells are usually well developed here, as are also many nervefibres radiating towards the pupil. Surrounding the pupil is a layer of circular unstriped muscle, the sphincter pupilloe. In some animals there are also musclefibres which radiate from the sphincter in the substance of the iris forming the dilator pupilloe. The iris is covered anteriorly by a layer of epithelium continued upon it from the posterior surface of the cornea.

The Lens is situated behind the iris, being enclosed in a distinct capsule, the posterior layer of which is not so thick as the anterior. It is supported in place by the suspensory ligament, fused to the anterior surface of the capsule. The suspensory ligament is derived from the hyaloid membrane, which encloses the vitreous humour. 
The lens is made up of a series of concentric laminæ (fig. 511), which, when it has been hardened, can be peeled off like the coats of

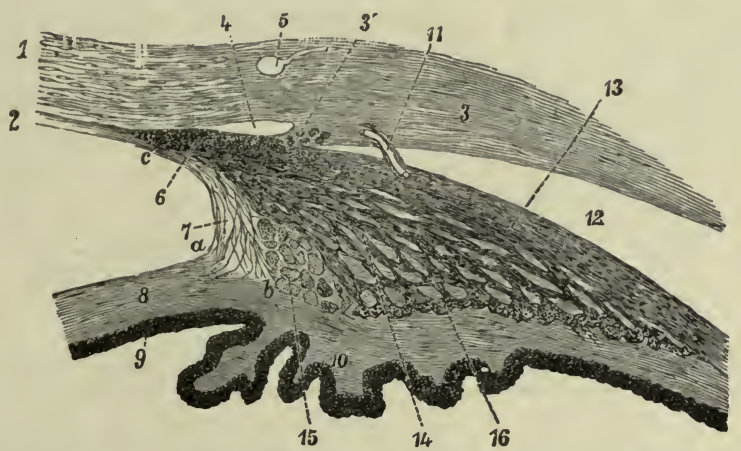

Fig. 509.- Section through the eye carried through the ciliary processes. 1, Cornea; 2, membrane of Descemet; 3, sclerotic; 3', corneo-scleral junction; 4, canal of Schlemm; 5, vein; 6, nucleated network on inner wall of canal of Schlemm; 7, lig. pectinatum iridis, $a b c ; 8$, iris ; 9, pigment of iris (uvea); 10, ciliary processes; 11, ciliary muscle; 12, choroid tissue; 13, meridional, and 14, radiating fibres of ciliary muscle; 15, ring-muscle of Müller; 16 , circular or angular bundles of ciliary muscle. (Schwalbe.)

an onion. The laminæ consist of long ribbon-shaped fibres, which in the course of development have originated from cells. The fibres

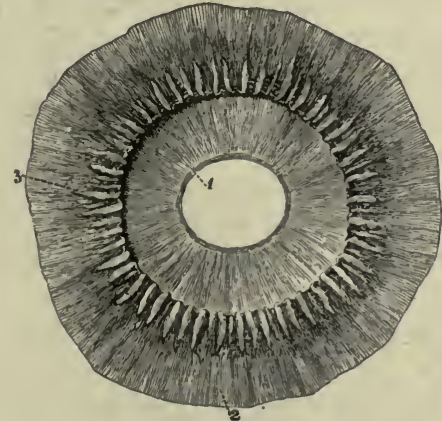

Fig. 510.-Ciliary processes, as seen from behind. 1, Posterior surface of the iris, with the sphincter muscle of the pupil; 2 , anterior part of the chorold coat; 3 , one of the cillary processes, of which about seventy are represented.

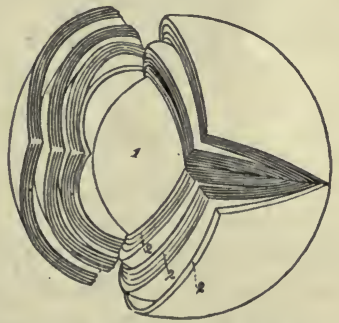

F1G. 511.-Laminated structure of the crystalline lens. Thelaminæ are split up after hardening in alcohol. 1, The denser central part or nucleus; 2 , the successive external layers.

(Arnold.)

are united together by a scanty amount of cement substance. The central portion (nucleus) of the lens is the hardest.

The epithelium of the lens consists of a layer of cubical cells anteriorly, which merge at the equator into the lens fibres. The development of the lens explains this transition. The lens at first 
consists of a closed sac composed of a single layer of epithelium. The cells of the posterior part soon elongate forwards and obliterate the cavity; the anterior cells do not grow, but at the edge they become continuous with the posterior cells, which are gradually developed into fibres (fig. 512). The principal chemical constituent of the lens is a protein of the globulin class called crystallin.

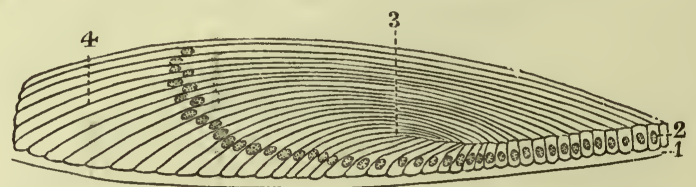

Fig. 512.-Meridional section through the lens of a rabbit. 1, Lens capsule; 2, epithelium of lens; 3 , transition of the epithelium into the fibres; 4 , lens fibres. (Bubuchin.)

Corneo-scleral junction.-At this junction the relation of parts (fig. 509) is so important as to need a short description. In this neighbourhood, the iris and ciliary processes join with the cornea. The proper substance of the cornea and the posterior elastic lamina become continuous with the iris, at the angle of the iris, and the iris sends forwards processes towards the posterior elastic lamina, forming the ligamentum pectinatum iridis, and these join with fibres of the elastic lamina. The epithelial covering of the posterior surface of the cornea is, as we have seen, continuous over the front of the iris. At the iridic angle, the compact inner substance of the cornea is looser, and between the bundles are lymph spaces called the spaces of Fontana. They are but little developed in the human cornea.

The spaces between the bundles of corneal tissue at the angle of the iris are continuous with the larger lymphatic space of the anterior. chamber. Above the angle at the corneo-scleral junction is a canal, which is called the canal of Schlemm. It is a lymphatic channel.

The retina (fig. 513) apparently ends in front, near the outer part of the ciliary processes, in a finely-notched edge,- the ora serrata, but is really represented by the uvea to the very margin of the pupil. The nerve-cells in the retina remind us that the optic, like the olfactory nerve, is not a mere nerve, but an outgrowth of the brain.

In the centre of the retina is a round yellowish elevated spot, about $\frac{1}{24}$ of an inch $(1 \mathrm{~mm}$.) in diameter, having a depression in the centre, called after its discoverer the macula lutea or yellow spot of Scemmering. The depression in its centre is called the fovea centralis. About $\frac{1}{10}$ of an inch $(2.5 \mathrm{~mm}$.) to the inner side of the yellow spot, is the point (optic disc or white spot) at which the optic nerve leaves the eyeball. The optic nerve-fibres are the axons of the 
nerve-cells of the retina; the dendrons of these cells ultimately communicate with the visual nerve-epithelium (rods and cones).

The optic nerve passes backwards to the ventral surface of the brain enclosed in prolongations of the membranes, which cover the brain. This external sheath at the exit of the nerve from the eyeball becomes continuous with the sclerotic, which at this part is perforated by holes to allow of the passage of the optic nervefibres, the perforated part being the lamina cribrosa. The fibres of the nerve themselves are exceedingly fine, and are surrounded by the myelin sheath, but do not possess the ordinary external nerve sheath. In the retina itself they have no myelin sheaths. In the centre of the nerve is a small artery, the arteria centralis retince. The number of fibres in the optic nerve is said to be upwards of 500,000 .

The retina consists of certain elements arranged in ten layers from within outwards (figs. 513, 514, 515).

1. Membrana limitans interna.-This so-called membrane in contact with the vitreous humour is formed by the junction laterally of the bases of the sustentacular or supporting fibres of Muiller, which bear the same relation to the retina as the neuroglia does to the brain. The character of these fibres may be seen in fig. 514 .

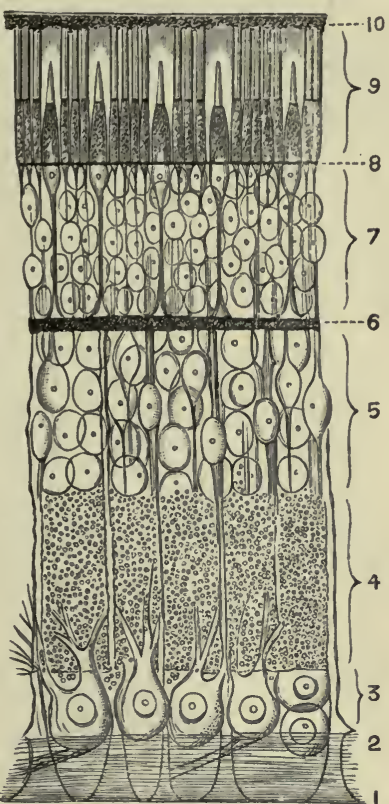

Fro. 513. - Diagrammatic section of husman retina (M. Schultze). 1, Membrana limitans interna; 2, layer of optic nerve-fibres; 3 , layer of optic nerve-cells; 4 , inner synapse or molecular layer; 5 , inner nuclear or bipolar layer; 6 , outer synapse or molecular layer; 7 , outer nuclear layer; 8, membrana limitans ex. terna; 9, layer of rods and cones; 10 , layer of pigment cells.

2. Optic nerve-fibres.-This layer is of very varying thickness in different parts of the retina: it consists of non-medullated fibres which interlace, and most of which are the axons of the large nerve-cells forming the next layer. The fibres are supported by the sustentacular fibres. They are less and less numerous anteriorly, and end at the ora serrata. They all converge towards the optic disc, where they leave the retina as the optic nerve.

3. Layer of ganglion cells.-This consists of large multipolar nervecells with large and round nuclei, forming either a single layer, or in some parts of the retina, especially near the macula lutea, where this layer is very thick, it consists of several strata of nerve-cells. They are arranged with their single axis-cylinder processes inwards. These pass into and are continuous with the layer of optic nerve- 
fibres. Externally the cells send off several branched processes which pass into the next layer.

4. Inner molecular layer.-This presents a finely granulated appearance. It consists of neuroglia traversed by numerous fibrillar processes of the nerve-cells just described, and the minute branchings of the processes of the bipolar cells of the next layer.

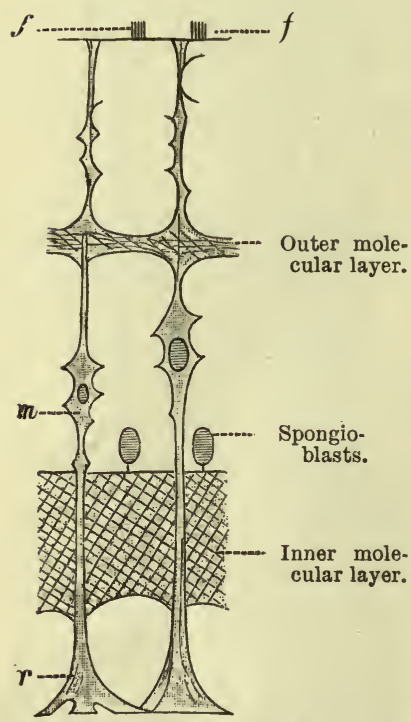

Fig. 514.-Diagram showing the susten. tacular fibres of the retina; $f$, fibrebasket above the external limiting membrane; $m$, nucleus of the fibre; $r$, base of the fibre.

(From M'Kendrick, after Stöhr.)

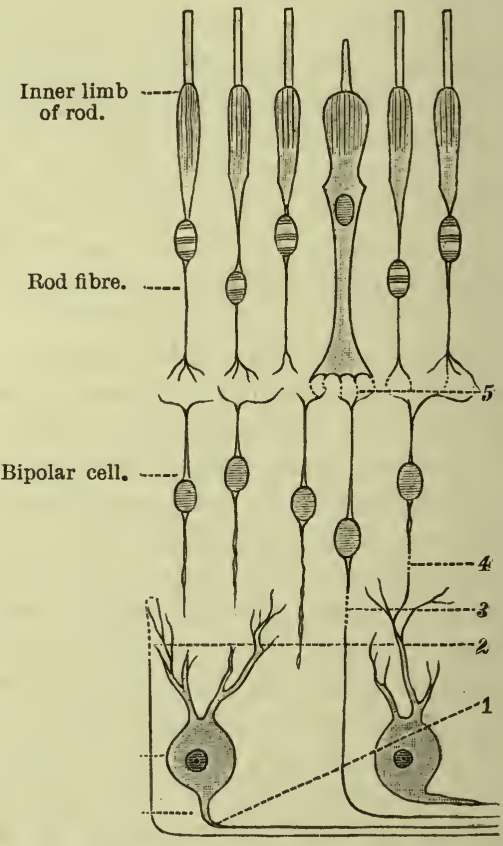

FiG. 515.-Diagram showing the nervous elements of retina. 1, Nerve-fibre of ganglion cell; 2 , processes of ganglion cell going outwards ; 3 , nervefibre passing from bipolar cell in inner nuclear layer ; 4, process of ganglion cell towards bipolar cell ; 5, arborisations of fibres from rods and cones with the branches of bipolar cells. (From M'Kendrick, after Stöhr.)

5. Inner nuclear layer.-This consists chiefly of numerous small round cells, each with a very small quantity of protoplasm surrounding a large ovoid nucleus; they are generally bipolar, giving off one process outwards and another inwards. One process passes inwards to form a synapse with the arborisation of a ganglion cell, the other outwards to similarly arborise with the branchings of the rod and cone fibres. Some cells, called spongioblasts, or amacrine cells, however, only send off one process, which passes inwards (fig. 514). 
The large oval nuclei (fig. 514) belonging to the Müllerian fibres occur in this layer.

6. Outer molecular layer.-This layer closely resembles the inner molecular layer, but is much thinner. It contains the branchings of the rod and cone fibres on the one hand and of the bipolar cells on the other.

7. External nuclear layer.-This layer consists of small cells resembling at first sight those of the internal nuclear layer; they are classed as rod and cone granules, according as they are connected with the rods and cones respectively, and will be described with them. They are lodged in the meshes of a frame-work, which is formed by the breaking up of the Müllerian fibres.

8. Membrana limitans externa.-This is a well-defined membrane, marking the internal limit of the rod and cone layer, and made up of the junction of the sustentacular or Müllerian fibres externally.

9. Layer of rod's and cones.-This layer is the nerve-epithelium of the retina. It consists of two kinds of cells, rods and cones, which are arranged at right angles to the external limiting membrane, and supported by hairlike processes (basket) proceeding from the latter for a short distance (fig. 514).

Each rod (fig. 515) is made up of two parts, very different in structure, called the outer and inner limbs. The outer limb of the rods is about $30 \mu$ long and $2 \mu$ broad, is transparent, and doubly refracting. It is said to be made up of fine superimposed discs. It stains brown with osmic acid but not with hæmatoxylin, and resembles in some ways the myelin sheath of a medullated nerve. It is the part of the rod in which the pigment called visual purple is found. In some animals, a few rods have a greenish pigment instead. The inner limb is about as long but slightly broader than the outer, is longitudinally striated at its outer, and granular at its inner part. It stains with hæmatoxylin, but not with osmic acid. Each rod is connected internally with a rod fibre, very fine, but here and there varicose; in the middle of the fibre is a rod granule, really the nucleus of the rod, striped broadly transversely, and situated about the middle of the external nuclear layer; the internal end of the rod fibre terminates in branchings in the outer molecular layer.

Each cone (fig. 515), like the rods, is made up of two limbs, outer and inner. The outer limb is tapering and not cylindrical like the corresponding part of the rod, and about one-third only of its length. There is, moreover, no visual purple found in the cones. The inner limb of the cone is broader in the centre. It is protoplasmic, and under the influence of light has been seen to execute movements. In birds, reptiles and amphibia, there is often a coloured oil globule present here. Each cone is in connection by its internal end with a cone fibre, which has much the same structure 
as the rod fibre, but is stouter and has its nucleus (cone granule) quite near to the external limiting membraue. Its inner end terminates by branchings in the external molecular layer.

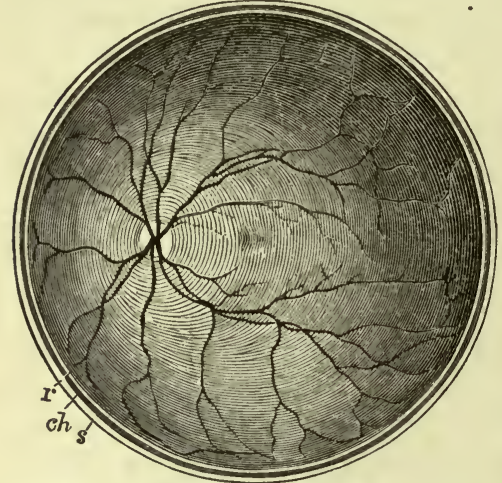

Fis. 516.-The posterior half of the retina of the left eye, viewed from before; $s$, The cut edge of the sclerotic coat; ch, the choroid; $r$, the retina; in the interior at the middle the macula lutea with the depression of the fovea centralis is represented by a slight oval shade; towards the left side the light spot indicates the colliculus or eminence at the entrance of the optic nerve, from the centre of which the arteria centralis is seen spreading its branches into the retina, leaving the part occupied by the macula comparatively free. (After Henle.)

In the rod and cone layer of birds, the cones usually predominate largely in number, whereas in man the rods are by far the more numerous, except in the fovea centralis, where cones only are present. The number of cones has been estimated at $3,000,000$.

10. Pigment-cell layer consists of a single layer of polygonal cells, mostly six-sided, which send down a beard-like fringe to surround the outer ends of the rods. It is this layer which is continuous with the uvea, where, however, the cells become rounded, and arranged two or three deep.

Differences in Structure of different parts.-Towards the centre of the macula lutea all the layers of the retina become greatly thinned out and almost disappear, except the rod and cone layer, and at the fovea centralis the rods disappear, and the cones are long

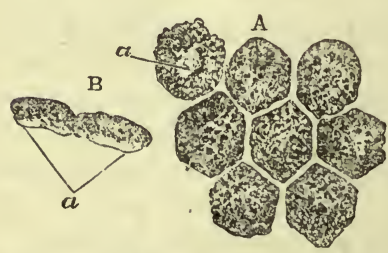

Fro. 517.-Pigment-cells from the retina. A, Cells still cohering, seen on their surface; $a$, nucleus indistinctly seen. In the other cells the nucleus is con. cealed by the pigment granules. $B$, Two cells seen in profile; $a$, the outer or posterior part containing scarcely any pigment. $\times 370$. (Henle.)

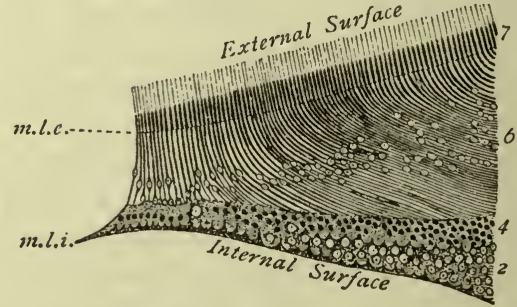

FiG. 518.-Diagram of a section through half the fovea centralis. 2, Ganglionic layer; 4, inner nuclear; 6 , outer nuclear layer, the cone fibres forming the so-called external fibrous layer: 7 , cones; m.l.e., membrana limitans externa; m.l.i., membrana limitans interna. (Schäfer and Golding Bird.)

and narrow. At the margin of the fovea the layers increase in thickness, and in the rest of the macula lutea are thicker than elsewhere. The ganglionic layer is especially thickened, the cells 
being six to eight deep (2, fig. 518). The bipolar inner granules (cone nuclei) are obliquely disposed (figs. 518 and 519) on the course of the cone fibres, and are situated at some distance from the membrana limitans externa, which is cupped towards the fovea (fig. 518). The yellow tint of the macula is due to a diffuse colouring matter in the interstices of the four or five inner layers; it is absent at the centre of the fovea.

It is important to notice what is clearly brought out in fig. 519, that at the fovea each cone is connected to a separate chain of

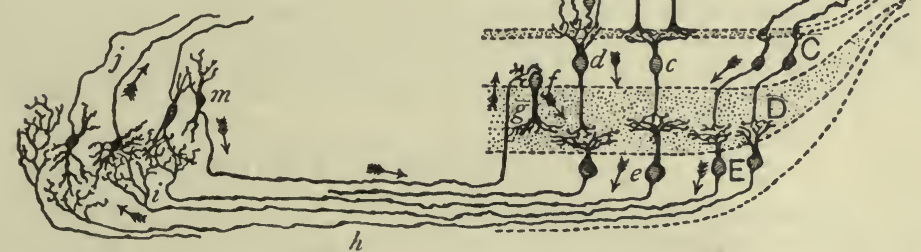

Fig. 519. - Scheme of the retinal elements. A, cones of the fovea centralis; B, granules (nuclei) of these cones; C, synapse between the cones and bipolar cells in external molecular layer; $D$, synapse between the bipolar and ganglion cells in the internal molecular layer; $a$ and $b$, rods and cones in other regions of the retina; $c$, bipolar cell destined for the cones ; $d$, bipolar cell destined for the rods; $\mathrm{E}, e$, ganglion cells; $f$, spongioblast; $g$, efferent fibre (? trophic), originating from the cell $m$, in geniculate body; $h$, optic nerve; $i$, terminal arborisations of optic nerve-fibres in geniculate body; $j$, fibres from the cells of geniculate body on the way to cerebral cortex. (R. y Cajal.)

neurons, whereas in other regions the rods and cones are connected in groups to these chains; this explains the greater sensitiveness of foveal vision.

At the ora serrata the layers are not perfect, and disappear in this order: nerve-fibres and ganglion cells, then the rods, leaving only the inner limbs of the cones, next these cease, then the outer molecular layer, the inner and outer nuclear layers coalescing, and finally the inner molecular layer also is unrepresented.

At the pars-ciliaris retince, the retina consists of a layer of columnar cells, which probably represent the Müllerian fibres. These cells externally are in contact with the pigment layer of the retina, which is continued over the ciliary processes and back of the iris. Nervous structures are absent.

At the exit of the optic nerve the only structures present are nerve-fibres.

The anterior chamber is the space behind the cornea and in front of the iris. It is filled with aqueous humour (dilute lymph).

The vitreous humour, which is a jelly-like connective tissue (see p. 36), is situated behind the crystalline lens. It is enclosed in a membrane called membrana hyaloidea, which in front is continuous 
with the capsule of the lens; round the edge of the lens the canal left is called the Canal of Petit (fig. 507, p. 811), the membrane itself being the Zonule of Zinn. The hyaloid membrane separates the vitreous from the retina.

Blood-vessels of the Eyeball.-The eye is very richly supplied with bloodvessels. In addition to the conjunctival vessels which are. derived from the palpebral and lacrimal arteries, there are at least two other distinct sets of vessels supplying the tunics of the eyeball.

(1) These are the short and long posterior ciliary arteries which pierce the sclerotic in the posterior half of the eyeball, and the anterior ciliary which enter near the insertions of the recti. These vessels anastomose and form a rich choroidal plexus; they also supply the iris and ciliary processes, forming a highly vascular circle round the outer margin of the iris and adjoining portion of the sclerotic. The distinctness of these vessels from those of the conjunctiva is well seen in the difference between the bright red of blood-shot eyes (conjunctival congestion), and the pink zone surrounding the cornea which indicates deep-seated ciliary congestion.

(2) The retinal vessels (fig. 516) are derived from the arteria centralis retina, which enters the eyeball along the centre of the optic nerve. They ramify all over the retina, in its inner layers. They can be seen by ophthalmoscopic examination.

\section{The Eye as an Optical Instrument.}

In a photographic camera images of external objects are thrown upon a screen at the back of a box, the interior of which is painted black. In the eye, the camera is represented by the eyeball with its black pigment, the screen by the layer of rods and cones of the retina, and the lens by the refracting media. In the case of the camera, the screen is enabled to receive clear images of objects at different distances, by an apparatus for focussing. The corresponding contrivance in the eye is called accommodation.

The iris, which allows more or less light to pass into the eye, corresponds with the diaphragms used in the photographic apparatus.

The refractive media are the cornea, aqueous humour, crystalline lens, and vitreous humour. The most refraction or bending of the rays of light occurs where they pass from the air into the cornea; they are again bent slightly in passing through the lens. Alterations in the anterior curvature of the lens lead to accommodation.

We may first consider the refraction through a transparent spherical surface, separating two media of different density.

The rays of light which fall upon the surface exactly perpendicularly do not suffer refraction, but pass through, cutting the optic axis (O A, fig. 520), a line which passes exactly through the centre of the surface, at a certain point, the nodal point (fig. $520, \mathrm{~N}$ ), or centre of curvature. Any rays which do not so strike the curved surface are refracted towards the optic axis. Rays which impinge upon the spherical surface parallel to the optic axis, will meet at a point behind, upon the said axis which is called the chief posterior focus (fig. $520, \mathrm{~F}_{1}$ ); and again there is a point on the optic axis in front of the surface, rays of light from which so strike the surface 
that they are refracted in a line parallel with the axis $d f^{\prime \prime}$; this point (fig. $520, \mathrm{~F}_{2}$ ) is called the chief anterior focus. The optic axis cuts the surface at what is called the principal point.

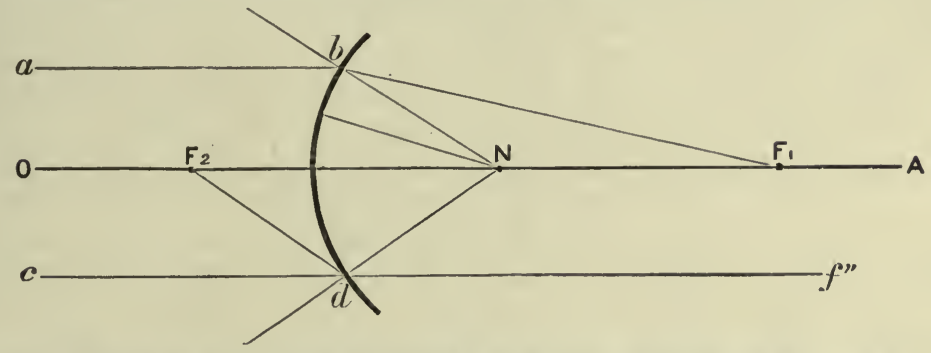

FIG. 520.-Diagram of a simple optical system (after M. Foster). The curved surface, $b, d$, is supposed to separate a less refractive medium towards the left from a more refractive medium towards the right.

It is quite obvious that the eye is a much more complicated optical apparatus than the one described in the figure. It is, however, possible to reduce the refractive surfaces and media to a simpler form when the refractive indices of the different media and the curvature of each surface are known. These data are as follows:-

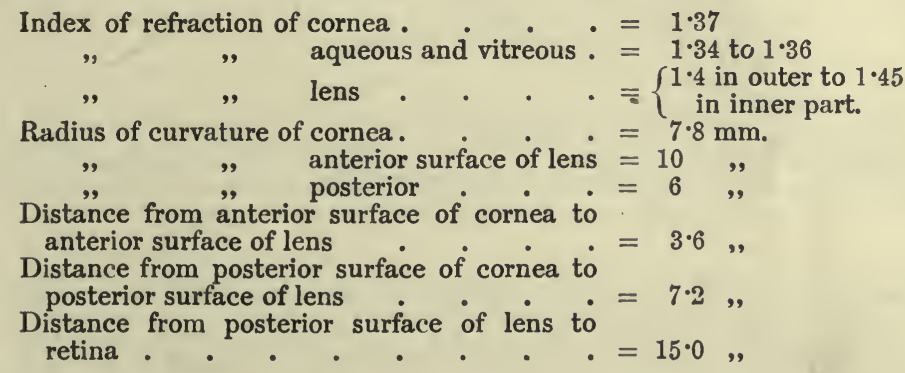

With these data it has been found comparatively easy to reduce by calculation the different surfaces of different curvature into one mean curved surface of known curvature, and the differently refracting media into one mean medium the refractive power of which is known.

The simplest so-called schematic eye formed upon this principle, suggested by Listing as the reduced eye, has the following dimensions :-

From anterior surface of cornea to the principal point $=2.3448 \mathrm{~mm}$.

From the nodal point to the posterior surface of lens $=44764$,

Posterior chief focus lies behind cornea . - . = 22.8237 ",

Anterior chief focus in front of cornea . . . . . =12.8326 ,"

Radius of curvature of ideal surface . - . . . . - 5.1248,

The term index of refraction means the ratio of the sine of the 
angle of incidence to that of the angle of refraction; this is explained in the small text beneath fig. 521.

In this reduced or simplified eye, the principal posterior focus, about $23 \mathrm{~mm}$. behind the spherical surface, would correspond to the

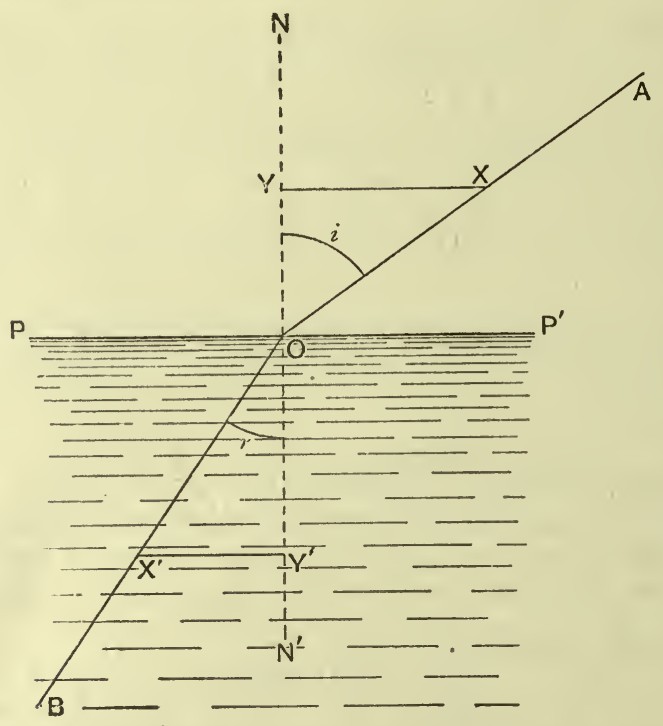

FIG. 521.-If $\mathrm{P}^{\prime}$ is a line which separates two media, the lower one being the denser, and $\mathrm{A} O$ is a ray of light falling on it, it is bent at $O$ towards the normal or perpendicular line $N^{\prime} N^{\prime}$. A $O$ is called the incident ray, and $\mathrm{O} B$ the refracted ray; $\mathrm{A} O \mathrm{~N}$ is called the angle of incidence $(i), \mathrm{N}^{\prime} \mathrm{O} \mathrm{B}$ the angle of refraction $(r)$. . If any distance $O \mathbf{X}$ is measured off along $\mathrm{O} A$, and an equal distance $\mathrm{O} X$, along $\mathrm{O} B$, and perpendiculars drawn to $\mathrm{N} \mathrm{N}^{\prime}$; then $\frac{\mathrm{X} Y}{\mathrm{X}^{\prime} \mathrm{Y}^{\prime}}=$ index of refraction.

position of the retina behind the anterior surface of the cornea. The refracting surface would be situated about midway between the posterior surface of the cornea and the anterior surface of the lens.

The optical axis of the eye is a line drawn through the centres of

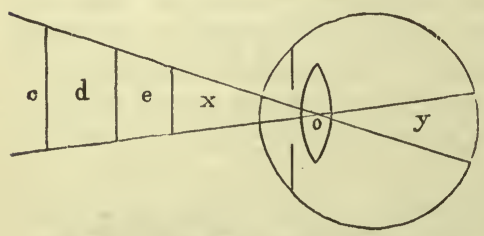

Fra. 522.-Diagram of the optical angle. curvature of the cornea and lens, prolonged backwards to touch the retina between the porus opticus and fovea centralis, and this differs from the visual axis which passes through the nodal point of the reduced eye to the fovea centralis; this forms an angle of $5^{\circ}$ with the optical axis. But for practical purposes the optical axis and the visual axis may be considered to be identical.

The visual or optical angle (fig. 522) is included between the lines 
drawn from the borders of any object to the nodal point; if the lines are prolonged backwards they include an equal angle. It has been shown by Helmholtz that the smallest angular distance between two points which can be appreciated as two distinct points $=50$ seconds, the size of the retinal image being $3.65 \mu$; this is a little more than the diameter of a cone at the fovea centralis which $=3 \mu$, the distance between the centres of two adjacent cones being $=4 \mu$. If the two points are so close together that they subtend a visual angle less than 50 seconds, both images will fall upon one cone, and the two points will therefore appear as one.

Any object, for example, the arrow A B (fig. 523), may be considered as a series of points from each of which a pencil of light diverges to the eye. Take, for instance, the rays diverging from the tip of the arrow A; C C represents the curvature of the schematic or reduced eye; the ray which passes through the centre of the circle

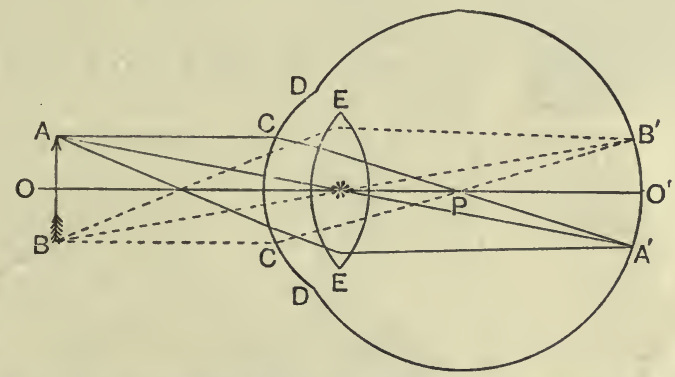

Fia. 523. - Dlagram of the course of the rays of light, to show how an Image is formed upon the retina. The surface $\mathbf{C}$ should be supposed to represent the ideal curvature.

of which $\mathrm{C} \mathrm{C}$ is part is not refracted; this point is represented as an asterisk in fig. 523 ; it is near the posterior surface of the crystalline lens; the ray $\mathrm{A} \mathrm{C}$, which is parallel to the optic axis $\mathrm{O}^{\prime} \mathrm{O}^{\prime}$, is refracted through the principal posterior focus $\mathrm{P}$, and cuts the first ray at the point $A^{\prime}$ on the retina. All the other rays from $A$ meet at the same point. Similarly the other end of the arrow B is focussed at $\mathrm{B}^{\prime}$, and rays from all other points have corresponding focusses.

It will thus be seen that an inverted image of external objects is formed on the retina. The retina is a curved screen, but the images fall only on a small area of the retina under normal circumstances; hence, for practical purposes, this small area may be regarded as flat.

The question then arises, Why is it that objects do not appear to us to be upside down? This cannot be satisfactorily answered without entering into matters which require a previous psychological training. Suffice it to say here that the localisation of objects in space depends not only on the retina, but also on tactile and general 
experience; that the mind localises objects with reference to its own body, and that from the first it knows nothing of the inversion of the retinal image, as its powers of localisation only appear with developing general experience.

\section{ACCOMMODATION}

The power of accommodation is primarily due to an ability to vary the shape of the lens; its front surface becomes more or less convex, according as the distance of the object looked at is near or far. The nearer the object, the more convex, up to a certain limit,

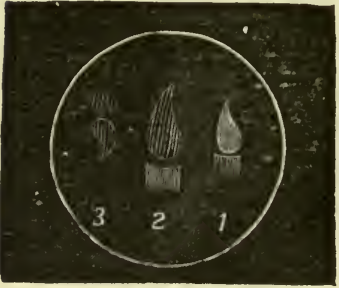

FiG. 524.-Diagram showing three reflections of a candle. 1 , From the anterior surface of cornea; 2 , from the anterior surface of lens; 3 , from the posterior sur. face of lens. the front surface of the lens becomes, and vice vers $d$; the back surface takes no share in the production of the effect required. The posterior surface, which during rest is more convex than the anterior, is thus rendered the less convex of the two during accommodation. The following simple experiment illustrates this point: If a lighted candle be held a little to one side of a person's eye an observer looking at the eye from the other side sees three images of the flame (fig. 524). The first and brightest is (1) a small erect image formed by the anterior convex surface of the cornea; the second (2) is also erect, but larger and less distinct than the preceding, and is formed at the anterior convex surface of the lens; the third (3) is smaller, inverted, and indistinct; it is formed at the posterior surface of the lens, which is concave forwards, and therefore, like all concave mirrors, gives an inverted image. If now the eye under observation is made to look at a near object, the second image becomes smaller, clearer, and approaches the first. If
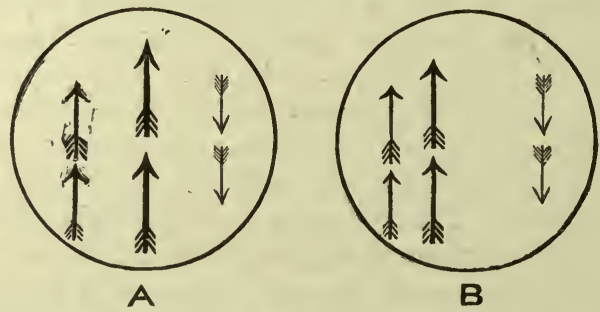

FIG.525.-Diagram of Sanson's images. A, When the eyes are not, and B, when they are focussed for near objects. The fig. to the right in $A$ and $B$ is the inverted image from the posterior surface of the lens.

the eye is now adjusted for a far point, the second image enlarges again, becomes less distinct, and recedes from the first. In both 
cases the first and third images remain unaltered in size, distinctness, and position. This proves that during accommodation for near objects the curvature of the cornea, and of the posterior surface of the lens, remain unaltered, while the anterior surface of the lens becomes more convex and approaches the cornea.

The experiment is more striking when two bright images (represented by arrows in fig. 525) are used; the two images from the front surface of the lens during accommodation not only approach those from the cornea, but also approach one another, and become somewhat smaller. (Sanson's Images.) Helmholtz's Phakoscope

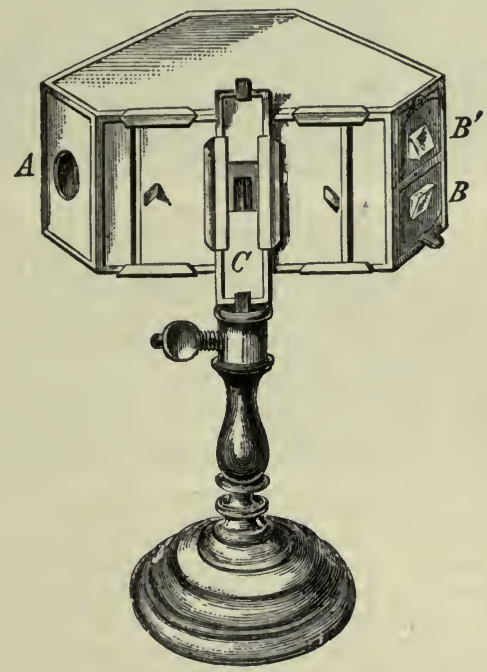

Fig. 526.-Phakoscope of Helmholtz. At $B B^{\prime}$ are two prisms, by which the light of a candle is con. centrated on the eye of the person experimented with, which is looking through a hole in the third angle of the box opposite to the window $C$. $A$ is the aperture for the eye of the observer. The observer notices three double images, represented by arrows, in fig. 525, reflected from the eye under examination when the eye is fixed upon a distant object; the position of the images having been noticed, the eye is made to focus a near object, such as a reed pushed up at $C$; the images from the anterior surface of the lens will be observed to move as described in the text.

(fig. 526) is a box with arrangements for demonstrating this experiment.

Mechanism of Accommodation.-The lens having no inherent power of contraction, its changes of outline must be produced by some power from without; this power is supplied by the ciliary muscle. Its action is to draw forwards the choroid, and by so doing to slacken the tension of the suspensory ligament of the lens which arises from it. The anterior surface of the lens is kept flattened by the action of this ligament. The ciliary muscle during accommodation, by diminishing the tension of this ligament, diminishes to a proportional degree the flattening of which it is the 
cause. On diminution or cessation of the action of the ciliary muscle, the lens returns to its former shape, by virtue of its elasticity (fig. 527). From this it will appear that the eye is usually

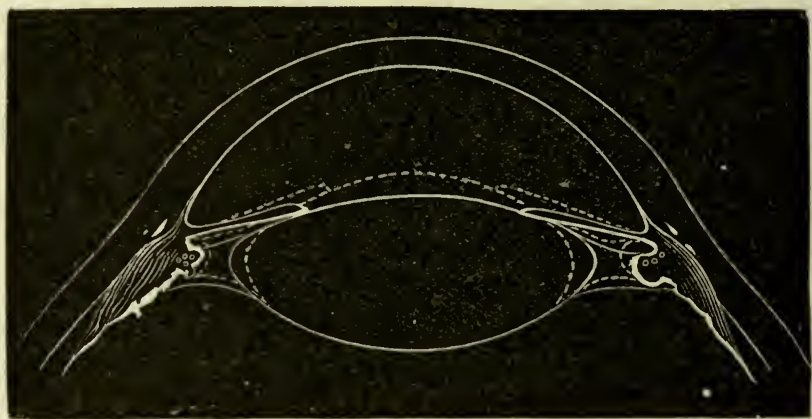

Fia. 527.-Diagram representing by dotted lines the alteration in the shape of the lens on accommodation for near objects. (E. Landolt.)

focussed for distant objects. In viewing near objects the ciliary muscle contracts; the ciliary muscle relaxes on withdrawal of the attention from near to distant objects.

It is possible to calculate the curvature of the lens or cornea in the body, by measuring the size of the image of an object upon it. The radius $(r)$ of curvature of a convex reflecting surface is given by the formula $r=\frac{2 a b}{c} ; a$ is the distance of the object from the surface, $b$ the diameter of the image, and $c$ that of the object. $a$ and $c$ are easily measured; $b$ is measured by Helmholtz's ophthalmometer, the principle of which is as follows :-If a line is looked at through a plate of glass placed obliquely between it and the eye, the line is shifted sideways to either right or left; if the glass plate is then placed obliquely at right angles to its previous position, the line is shifted in the opposite direction. In the ophthalmometer there are two glass plates intersecting each other at an angle; the image of a bright horizontal line upon the lens or cornea is looked at through the junction between the two plates; one plate shifts the image to the right, the other to the left; the angle between the two plates is altered until the line appears as two distinct lines just touching each other. The amount of shifting of each, which must therefore be half the length of the image of the line, can be easily calculated if the thickness of the glass plates, their refractive index, and the angle between them are known. Double this result gives the size of the image on the surface under investigation.

Range of Distinct Vision. Near-point.-In every eye there is a limit to the power of accommodation. If a book be brought nearer and nearer to the eye, the type at last becomes indistinct, and cannot be brought into focus by any effort of accommodation, however strong. This, which is termed the near-point, can be determined by the following experiment (Scheiner). Two small holes are pricked in a card with a pin not more than a twelfth of an inch $(2 \mathrm{~mm}$.) apart; at any rate their distance from each other must not exceed the diameter of the pupil. The card is held close in front of the eye, 
and a small needle viewed through the pin-holes. At a moderate distance it can be clearly focussed, but when brought nearer, beyond a certain point, the image appears double, or at any rate blurred. This point where the needle ceases to appear single is the near-point. Its distance from the eye can of course be readily measured. It is usually about 5 or 6 inches $(13 \mathrm{~cm}$.). In the accompanying figure (fig. 528) the lens $b$ represents the refractive apparatus of the eye; $e$ and $f$ the two pin-holes in the card, $n n$ the retina; $a$ represents the position of the needle. When the needle is at a moderate distance, the two pencils of light coning through $e$ and $f$ are focussed at a single point on the retina $n n$. If the needle is brought nearer than the near-point, the strongest effort of accommodation is not sufficient to focus the two pencils, they meet at a point behind the retina. The effect is the same as if the retina were shifted forward to $\mathrm{mm}$. Two images $h, g$ are formed, one from each hole. It is interesting to note

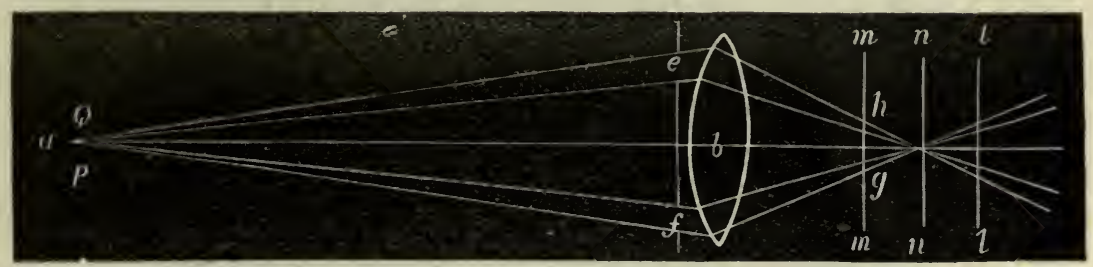

FIG. 528.-Diagram of experiment to ascertain the minimum distance of distinct vision.

that when two images are produced, the lower one $g$ really appears in the position $Q$, while the upper one appears in the position $P$. This may be readily verified by covering the holes in succession.

During accommodation two other changes take place in the eyes: (1) The eyes converge owing to the action of the internal rectus muscle of each eyeball. (2) The pupils contract.

The contraction of all of the muscles which have to do with accommodation, viz., of the ciliary muscle, of the internal recti muscles, and of the sphincter pupillæ, is under the control of the third nerve. It should further be noted that although the act is a voluntary one, the fibres of the ciliary muscle and of the sphincter pupillæ are of the plain variety.

The account of accommodation as given in the preceding pages is true for man and other mammals, birds, and certain reptiles.

Beer has, however, shown that in many animals lower in the scale, the mechanism of accommodation varies a good deal, and is often very different from that just described, consisting, in fact, in a power of altering the distance between the lens and the retina.

In bony fishes, the eye at rest is accommodated for near objects; in focussing for distant objects the lens is drawn nearer to the retina by a special muscle called 
the retractor lentis. In cephalopods the same occurs, but the retractor lentis is absent; here the approach of the lens to the retina is brought about by an alteration of intra-ocular tension. In Amphibia and most snakes, the eye at rest is focussed for distant objects ; in accommodating for near objects the lens, by alteration of intra-ocular tension, is brought forward, that is, the distance between it and the retina is increased. There appear to be not a few animals in all classes which do not possess the power of accommodation at all. Indeed, Barrett states this is so for most mammals.

\section{Defects in the Optical Apparatus}

Under this head we may consider the defects known as (1) Myopia, (2) Hypermetropia, (3) Astigmatism, (4) Spherical Aberration, (5) Chromatic Aberration, and (6) Presbyopia.

The normal (emmetropic) eye is so adjusted that at rest parallel rays are brought exactly to a focus on the retina (1, fig. 529). Hence all objects except near ones (practically ail objects more than twenty feet off) are seen without any effort of accommodation; in other words, the far-point of the normal eye is at an infinite distance. In viewing near objects we are conscious of the effort (the contraction of the ciliary muscle) by which the anterior surface of the lens is rendered more convex, and rays which would otherwise be focussed behind the retina are converged upon the retina (see dotted lines, 2, fig. 529).

1. Myopia (short-sight), (4, fig. 529).- This defect is due to an abnormal elongation of the eyeball. The retina is too far from the lens, and consequently parallel rays are focussed in front of the retina, and, crossing, form little circles on the retina; thus the images of distant objects are blurred and indistinct. The eye is, as it were, permanently adjusted for a near-point. Rays from a point near the eye are exactly focussed on the retina. But those which issue from any object beyond a certain distance ( far-point) cannot be distinctly focussed. This defect is corrected by concave glasses which cause the rays entering the eye to diverge: hence they do not come to a focus so soon. Such glasses, of course, are only needed to give a clear vision of distant objects. For near objects, except in extreme cases, they are not required.

2. Hypermetropia (3, fig. 529).- This is the reverse defect. The eyeball is too short. Parallel rays are focussed behind the retina: an effort of accommodation is required to focus even parallel rays on the retina; and when they are divergent, as in viewing a near object, the accommodation is insufficient to focus them. Thus, in wellmarked cases, distant objects require an effort of accommodation, and near ones a very powerful effort, and the ciliary muscle is, therefore, constantly acting. This defect is obviated by the use of convex glasses, which render the pencils of light more convergent. Such glasses are, of course, especially needed for near objects, as in reading, 
etc. They rest the eye by relieving the ciliary muscle from excessive work.

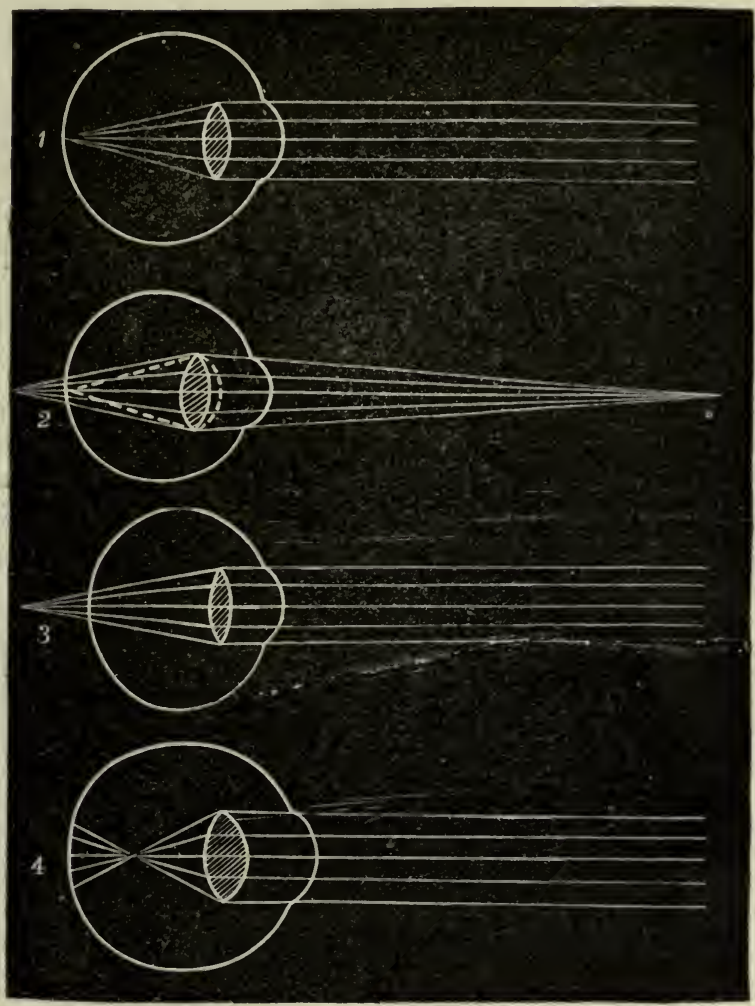

Fili. 529.-Diagram showing-1, normal (emmetropic) eye bringing parallel rays exactly to a focus on the retina; 2, normal eye adapted to a near-point; without accommodation the rays would be focussed behind the retina, but by increasing the curvature of the anterior surface of the lens (shown by a dotted line) the rays are focussed on the retina (as indicated by the meeting of the two dotted lines); 3 , hypermetropic eye ; in this case the axis of the eye is shorter than normal; parallel rays are focussed behind the retina; 4, myopic eye; in this case the axis of the eye is abnormally long; parallel rays are focussed in front of the retina. The figure incorrectly represents the refraction as occurring only in the crystalline lens; the principal refraction really occurs at the anterior surface of the cornea.

3. Astigmatism.-This defect, which was first discovered by Airy, is due to a greater curvature of the eye in one meridian than in others. The eye may be even myopic in one plane, and hypermetropic in others. Thus vertical and horizontal lines crossing each other cannot both be focussed at once; one set stand out clearly, and the others are blurred and indistinct. This defect, which is present in a slight degree in all eyes, is generally seated in the cornea, but occasionally in the lens as well; it may be 
corrected by the use of cylindrical glasses (i.e., curved only in one direction).

4. Spherical Aberration.-The rays of a cone of light from an object situated at the side of the field of vision do not meet all in the same point, owing to their unequal refraction; for the refraction of the rays which pass through the circumference of a lens is greater than that of those traversing its central portion. This defect is known as spherical aberration, and in the camera, telescope, microscope, and other optical instruments, it is remedied by the interposition of a screen with a circular aperture in the path of the rays of light, cutting off all the marginal rays, and only allowing the passage of those near the centre. Such correction is effected in the eye by the iris, which prevents the rays from passing through any part of the refractive apparatus but its centre. The image of an object will be most defined and distinct when the pupil is narrow, the object at the proper distance for vision, and the light abundant; so that, while a sufficient number of rays are admitted, the narrowness of the pupil may prevent the production of indistinctness of the image by spherical aberration.

Distinctness of vision is further secured by the pigment of the outer surface of the retina, the posterior surface of the iris and the ciliary processes, which absorbs most of the light which is reflected within the eye, and prevents its being thrown again upon the retina so as to interfere with the images there formed.

5. Chromatic Aberration.-In the passage of light through an ordinary convex lens, decomposition of each ray into its elementary colours commonly ensues, and a coloured margin appears around the image, owing to the unequal refraction which the elementary colours undergo. In optical instruments this, which is termed chromatic aberration, is corrected by the use of two "or more lenses, differing in shape and density, the second of which continues or increases the refraction of the rays produced by the first, but by recombining the individual parts of each ray into its original white light, corrects any chromatic aberration which may have resulted from the first. It is probable that the unequal refractive power of the transparent media in front of the retina may be the means by which the eye is enabled to guard against the effect of chromatic aberration. The human eye is achromatic, however, only so long as the image is received at its focal distance upon the retina, or so long as the eye is properly accommodated. If these conditions are interfered with, a more or less distinct appearance of colours is produced.

From the insufficient adjustment of the image of a small white object, it appears surrounded by a sort of halo or fringe. This phenomenon is termed Irradiation. It is partly for this reason that 
a white square on a black ground appears larger than a black square of the same size on a white ground. The phenomenon is naturally more marked when the white object is a little out of focus.

6. Defective Accommodation-Presbyopia.-This condition is due to the gradual loss of the power of accommodation which is an early sign of advancing years. In consequence, the person is obliged in reading to hold the book further and further away in order to focus the letters, till at last the letters are held too far for distinct vision. The defect is remedied by weak convex glasses. It is due chiefly to the gradual increase in density of the lens, which is unable to swell out and become convex when near objects are looked at, and also to a weakening of the ciliary muscle, and a general loss of elasticity in the parts concerned in the mechanism.

\section{The Skiascope or Retinoscope.}

The refractive powcr of a lens is expressed in terms of its principal focal distance; if this is 1 metre, it is said to have the refractive power of 1 diopter $(1 \mathrm{D}$.); a lens $2 \mathrm{D}$. has a focal length of $\frac{1}{2}$ a metre, and a lens $\frac{1}{2} \mathrm{D}$. has a focal length of 2 metres, and so on. The lenses necessary for correcting errors of refraction in an eye are best determined by a simple instrument called a retinoscope; this is a small circular plane mirror, perforated by a hole in the centre thrcugh which the observer looks. If one reflects a spot of light from this on to a flat surface, any movement of the mirror produces a movement of the spot of light in the same direction; if the surface selected, however, is the eye of another person, the direction of movement of the illuminated spot on the retina may or may not be the same as that in which the mirror is moved, according as whether the observed eye is normal, hypermetropic, or myopic. If the observed eye is just a metre away from the observer, and is emmetropic, then as the mirror is tilted from side to side the spot moves in the same direction. If a convex lens is placed in a spectacle frame in front of the observed eye, the parallel rays which emerge from the retina are brought to a focus and cross before reaching the eye of the observer. Then the spot will move in the opposite direction to the mirror. A lens of less than $1 \mathrm{D}$. will not, however, accomplish this reversal; a lens of more than $1 \mathrm{D}$. will. So that a lens of $1 \mathrm{D}$. marks the exact point of reversal. If the observed eye is hypermetropic, the movement of the spot of light is also with the mirror, but stronger lenses than $1 \mathrm{D}$. must be introduced to get the point of reversal. If the lens in any particular case necessary for this purpose is $5 \mathrm{D}$., then spectacles of $4 \mathrm{D}$. must be ordered for the patient; for one always has to subtract $1 \mathrm{D}$., since that is required to get reversal with the normal eye. 
When the spot of light moves against the mirror's movements from the first, then the observed eye is myopic, and the myopia is greater than $1 \mathrm{D}$. The "point of reversal" is determined by introducing concave lenses of increasing strength into the spectacle frame, until the spot moves in the same direction as the mirror, and the spectacles ordered must have the value of the lens which accomplishes the reversal plus $1 \mathrm{D}$. to allow as before for the normal eye.

Many people have differences in the refractive error of their two eyes; so each should be tested separately. If the observed eye is astigmatic, the observations are more complicated, and must be made in the different meridians of the eye, and the point of reversal determined in each meridian by means of suitable cylindrical lenses.

\section{FunCtions OF THE IRIS}

The iris has the following two uses:-

1. To act as a diaphragm in order to lessen spherical aberration in the manner just described. This is specially necessary when one wishes to obtain a clearly defined image of an object; the pupil therefore contracts when accommodation for a near object takes place.

2. To regulate the amount of light entering the eye. In a bright light the pupil contracts; in a dim light it enlarges. This may be perfectly well seen in one's own iris by looking at it in a mirror while one alternately turns a gas-light up and down.

The muscular fibres (unstriped in mammals, striped in birds) of the iris are arranged circularly around the margin of the pupil, and radiatingly from its margin. The radiating fibres are best seen in the eyes of birds and otters; some look upon them as elastic in nature, but there is little doubt that they are contractile. Those who believe they are not contractile explain dilatation of the pupil as due to inhibition of the circular fibres. But if the iris is stimulated near its outer margin at three different points simultaneously the pupil assumes a triangular shape, the angles of the triangle corresponding to the points stimulated; this must be due to contraction of three strands of the radiating muscle; inhibition of the circular fibres would occur equally all round.

The iris is supplied by three sets of nerve-fibres contained in the ciliary nerves.

(a) The third nerve via the ciliary ganglion and short ciliary nerves supplies the circular fibres (fig. 530).

(b) The cervical sympathetic supplies the radiating fibres. The cilio-spinal centre which governs them is in the cervical region of the cord (see p. 721). The fibres leave the cord by the anterior root of the second thoracic nerve, pass into the cervical sympathetic, 
and reach the eyeball via the ophthalmic branch of the fifth, and long ciliary nerves (fig. 530).

(c) Fibres of the fifth nerve which are sensory.

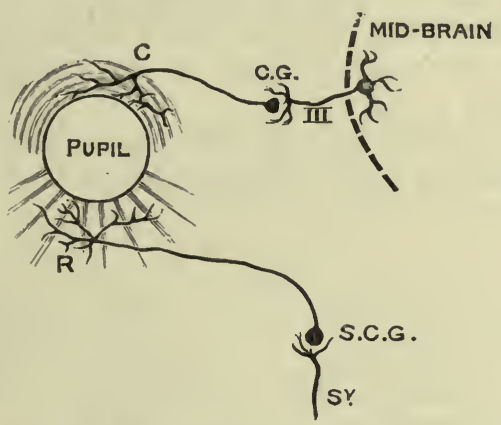

FlG. 530.-Diagram of the motor nerves of the iris. Around the upper half of the pupil the circular fibres (C) only are indicated. These are supplied by the third nerve, one fibre of which (III.) is seen issuing from the mid-brain; the cell-station for these fibres is in the ciliary ganglion (C.G.). Around the lower half of the pupil, the radiating fibres (R) are indicated; these are supplied by the cervical sympathetic (Sy), one fibre of which is shown with its cell-station in the superior cervical ganglion (S.C.G.). (After Dixon.)

The experiments on the motor nerves are those of section and stimulation of the peripheral ends; the usual experiments by which the functions of such nerves are discovered.

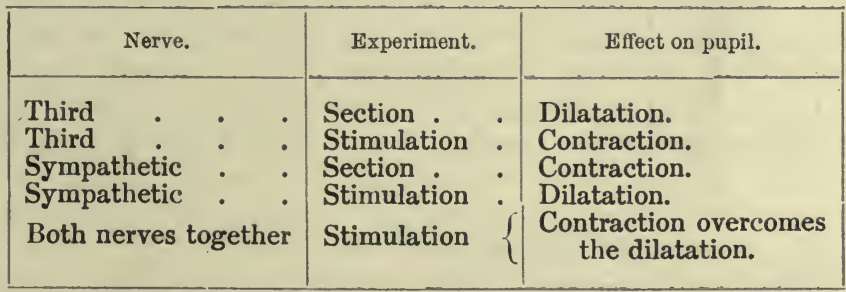

Certain drugs dilate the pupil. These are called mydriatics; atropine is a well-known example. Others cause the pupil to contract. These are called myotics; physostigmine and opium (taken internally) are instances. Different myotics and mydriatics act in different ways, some exerting their activity on the muscular, and others on the nervous structures of the iris.

Reflex actions of the iris. - When the iris contracts under the influence of light, the sensory nerve is the optic, and the motor the third nerve. The central connection of the two nerves in the region of the mid-brain we shall see later on. The iris also contracts on accommodation; and the reflex path concerned in this action is a different one from that concerned in the light reflex, as this reflex 
often remains, in cases of locomotor ataxy, after there is an entire loss of the reflex to light (Argyll-Robertson pupil).

On painful stimulation of any part of the body, there is reflex dilatation of the pupil. This is accompanied by starting of the eyeballs, due to contraction of the plain muscle in the capsule of Tenon, which, like the dilator fibres of the iris, is supplied by the cervical sympathetic nerve.

We may sum up the principal conditions under which the pupil contracts and dilates, in the following table:-

\section{Causes of-}

Contraction of the Pupil.

1. Stimulation of third nerve.

2. Paralysis of cervical sympathetic.

3 . When the eye is exposed to light.

4. When accommodation occurs.

5. Under the local influence of physostigmine.

6. Under the influence of opium.

7. During sleep.

\section{Dilatation of the Pupil.}

1. Paralysis of the third nerve.

2. Stimulation of the cervical sympathetic.

3. In the dark.

4. When the accommodation is relaxed.

5. Under the local influence of atropine. This drug also paralyses the ciliary muscle.

6. In the last stage of asphyxia.

7. In deep chloroform narcosis.

8. Under the influence of certain emotions, such as fear.

9. During pain.

There is a close connection of the centres that govern the activity of the two irides. If one eye is shaded by the hand, its pupil will of course dilate, but the pupil of the other eye will also dilate. The two pupils always contract or dilate together unless the cause is the local injury to the nerves of one side or the local action of drugs.

\section{Functions of the Retina}

The Retina is the nervous coat of the eye; it contains the layer of nerve-epithelium (rods and cones) which is capable of receiving the stimulus of light, and transforming it into a nervous impulse which passes to the brain by the optic nerve.

The bacillary layer, or layer of rods and cones, is at the back of all the other retinal layers, which the light has to penetrate before it can affect this layer. The proofs of the statement that this is the layer of the retina which is capable of stimulation by light are the following:-

(1) The point of exit of the optic nerve from the retina, where the rods and cones are absent, is insensitive to light, and is called the blind spot. This is readily demonstrated by what is known as Mariotte's experiment. If we direct one eye, the other being 
closed, upon a point at such a distance to the side of any object, that the image of the latter must fall upon the retina at the point of entrance of the optic nerve, this image is lost. If, for example, we close the left eye, and look steadily with the right eye at the dot

here represented, while the page is held about six inches from the eye, both dot and cross are visible. On gradually increasing the distance between the page and the eye, still keeping the right eye steadily on the dot, it will be found that suddenly the cross disappears from view, because its image has fallen on the blind spot; on removing the book still farther, it comes in sight again. The question has arisen why we are not normally conscious of a gap in the image. We can only say that owing to the spot being blind from birth onwards we have come to neglect its blindness, and to interpret our experience as if the blind spot always gave rise to the same visual sensations as are evoked by the neighbouring retinal regions.

(2) In the fovea centralis which contains the bacillary layer, but in which the other layers of the retina are thinned down to a minimum, light produces the greatest effect. In the macula lutea, cones occur in large numbers, and in the fovea centralis cones without rods are found, whereas, in the rest of the retina which is not so sensitive to light, there are fewer cones than rods.

(3) If a small lighted candle is moved to and fro at the side of and close to one eye in a darkened room, while the eyes look steadily forward on to a dull background, a remarkable branching figure (Purkinje's figures) is seen floating before the eye, consisting of dark lines on a reddish ground. As the candle moves, the figure moves in the opposite direction, and from its whole appearance there can be no doubt that it is a reversed picture of the retinal vessels projected before the eye.* This remarkable appearance is due to shadows of the retinal vessels cast by the candle; and it is only when they are thrown upon the retina in an unusual slanting direction that they are perceived. The branches of these vessels are distributed in the nerve-fibre and ganglionic layers; and since the light of the candle falls on the retinal vessels from in front, the shadow is cast behind them, and hence those elements of the retina which perceive the shadows must also lie behind the vessels. Here, then, we have a clear proof that the light-perceiving elements are not the inner, but one of the external layers of the retina; further than this, calculation has shown it is the layer of rods and cones. The data for such a calculation are-the dimensions of the eyeball,

* Purkinje's figures can be much more readily seen by simply looking steadily down a microscope, and moving the whole instrument backwards and forwards, or from side to side, while so doing. 
the distance of the screen from the eye, the angle through which the candle is moved, and the displacement of the figure seen.

Duration of Visual Sensations.-The duration of the sensation produced by a luminous impression on the retina is always greater than that of the impression which produces it. However brief the luminous impression, the effect on the retina always lasts for about one-eighth of a second. Thus, supposing an object in motion, say a horse, to be revealed on a dark night by a flash of lightning. The object would be seen apparently for an eighth of a second, but it would not appear in motion; because, although the image remained on the retina for this time, it was really revealed for such an extremely short period (a flash of lightning lasting only a millionth of a second) that no appreciable movement on the part of the object could have taken place in the period during which it was revealed to the retina of the observer. The same fact is proved in a reverse way. The spokes of a rapidly revolving wheel are not seen as distinct objects, because at every point of the field of vision over which the revolving spokes pass, a given impression has not faded before another replaces it. Thus every part of the interior of the wheel appears occupied.

The stimuli which excite the retina are exceedingly slight; for instance, the minimum stimulus in the form of green light is equal in terms of work to that which is done in raising a ten-millionth part of a milligramme to the height of a millimetre, and even some of this is doubtless wasted in the form of heat. The time during which the stimulus acts may be excessively small ; thus light from a rapidly rotating mirror is visible even when it only falls upon the retina for one eight-millionth part of a second. Some physiologists have drawn an analogy between retinal and muscular excitations. There is no complete analogy, but the following points of resemblance may be noted:-

1. The retina like the muscle possesses a store of potential energy, which the stimulus serves to fire off.

2. Fatigue on action, and recovery after rest are noticeable in both.

3. The curve of retinal excitation, like the muscle curve, rises not abruptly but gradually to its full height, and on the cessation of the stimulus takes a measurable time to fall again, the retinal impression outlasting the stimulus by about one-eighth of a second.

4. With comparatively slow intermittent excitation, the phenomenon known as ficker takes place; this may be shown by the slow rotation on Maxwell's machine of a disc painted with alternate black and white sectors. This roughly corresponds with what in a muscle is called incomplete tetanus.

5. When the rate of stimulation is increased, as by increasing the speed of rotation of the disc just alluded to (say to twenty or thirty times a second) the resulting sensation is a smooth one of greyness. This fusion of individual stimuli into a continuous sensation does not by any means correspond to the complete tetanus of muscle, for the resultant sensation has a brightness corresponding not to a summation of the individual fusing sensations, but to a brightness which would ensue if the stimuli were spread evenly over the surface of the disc ('Talbot's Law).

\section{The Ophthalmoscope.}

Every one is perfectly familiar with the fact, that it is quite impossible to see the fundus or back of another person's eye by simply 
looking into it. The interior of the eye forms a perfectly black background.* The same remark applies to the difficulty we experience in seeing into a room from the street through the window unless the room is lighted within. In the case of the eye this fact is partly due to the feebleness of the light reflected from the retina, most of it being absorbed by the retinal pigment; but far more to the fact that every such ray is reflected straight to the source of light (e.g. candle), and cannot, therefore, be seen by the unaided eye without intercepting the incident light from the candle, as well as the reflected rays from the retina. This difficulty is surmounted by the use of the ophthalmoscope.

The ophthalmoscope was invented by Helmholtz; as a mirror for reflecting the light into the eye, he employed a bundle of thin glass plates; this mirror was transparent, and so he was able to look through it in the same direction as that of the rays of the light it reflected. It is almost impossible to over-estimate the boon this instrument has been to mankind; previous to this in the examination of cases of eye disease, the principal evidence on which the surgeon had to rely was that derived from the patient's sensations; now he can look for himself.

The instrument, however, has been greatly modified since Helmholtz's time; the principal modification is the substitution of a concave mirror of silvered glass for the bundle of glass plates; this is mounted on a handle, and is perforated in the centre by a small hole through which the observer can look.

The methods of examining the eye with this instrument are-the direct and the indirect: both methods of investigation should be employed. A drop of a solution of atropine (two grains to the ounce) or of homatropine hydrobromate, should be instilled about twenty minutes before the examination is commenced; the ciliary muscle is thereby paralysed, the power of accommodation is abolished, and the pupil is dilated. This will materially facilitate the examination; but it is quite possible to observe all the details to be presently described without the use of such drugs. The room being now darkened, the observer seats himself in front of the person whose eye he is about to examine, placing himself upon a somewhat higher level. Let us suppose that the right eye of the patient is being examined. A brilliant and steady light is placed close to the left ear of the patient. Taking the mirror in his right hand, and looking through the central hole, the operator directs a beam of light into the eye of the patient. A red glare, known as the reflex, is seen; it is due to the illumination of the retina. The patient is then told to look at the little finger of the observer's right hand as he holds the mirror; to effect this the eye is rotated somewhat inwards, and at the same time the reflex changes from red to a lighter colour, owing to the reflection from the optic disc. 'The

* In some animals (e.g. the cat), the pigment is absent from a portion of the retinal epithelium; this forms the Tapetum lucidum. The use of this is supposed to be to increase the sensitiveness of the retina, the light being reflected back through the layer of rods and cones. It is probably the case that these animals are able to see clearly with less light than we can, hence the popular idea that a cat can see in the dark. In fishes a tapetum lucidum is often present; here the brightness is increased by crystals of guanine. 
observer now approximates the mirror, with his eye to the eye of the patient, taking care to keep the light fixed upon the pupil, so as not to lose the reflex. At a certain point, which varies with different eyes, but is usually reached when

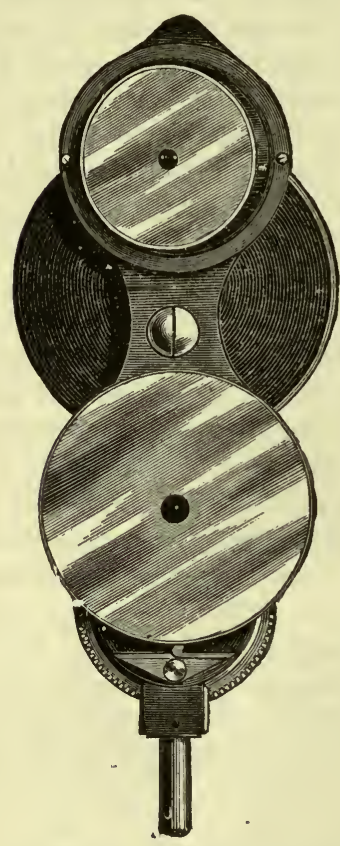

Fig. 531.-The Ophthalmoscope. The small upper mirror is for direct, the larger for indirect, illumination. there is an interval of about two or three inches between the observed and the observing eye, the vessels of the retina become visible. Examine carefully the fundus of the eye, i.e., the red surface-until the optic disc is seen; trace its circular outline, and observe the small central white spot, the porus opticus, or physiological pit: near the centre is the central artery of the retina breaking up upon the disc into branches ; veins also are present, and correspond roughly to the course of the arteries. Trace the vessels over the disc on to the retina. Somewhat to the outer side, and only visible after some practice, is the yellow spot, with the smaller lighter-coloured fovea centralis in its centre. This constitutes the direct method of examination; by it the various details of the fundus are seen as they really exist, and it is this method which should be adopted for ordinary use.

If the observer is myopic or hypermetropic, he.will be unable to employ the direct method of examination until he has remedied his defective vision by the use of proper glasses.

In the indirect method the patient is placed as before, and the operator holds the mirror in his right hand at a distance of twelve to eighteen inches from the patient's right eye. At the same time he rests his left little finger lightly upon the patient's right temple, and holding a convex lens between his thumb and forefinger, two or three inches in front of the patient's eye, directs the light through the lens into the eye. The red reflex, and subsequently the white one, having been gained, the operator slowly moves his mirror, and with it his eye, towards or away from the face of the patient, until the outline of one of the retinal vessels becomes visible, when very slight movements on the part of the operator will suffice to bring into view the details of the fundus above described, but the image will be much smaller and inverted. The appearances seen are depicted in fig. 516 . The lens should be kept fixed at a distance of two or three inches, the mirror alone being moved until the disc becomes visible : should the image of the mirror, however, obscure the disc, the lens may be slightly tilted.

The two next figures show diagrammatically the course of the rays of light.

Fig. 532 represents what occurs when employing the direct method. $S$ is the source of light, and M M the concave mirror with its central aperture, which reflects the rays; these are focussed by the eye $\mathrm{E}$, which is being examined, to a point in the vitreous humour, and this produces a diffuse lighting of the interior of the eyeball. Rays of light issuing from the point $p$ emerge from the eye parallel to one another, and enter the observer's eye $\mathrm{E}^{1}$; they are brought to a focus $p^{1}$ on the retina as the eye is accommodated for distant vision. Similarly the point $m$ and $n$ will give rise to images at $m^{1}$ and $n^{1}$ respectively.

Fig. 533 represents what occurs in examining the eye by the indirect method.

$\mathrm{S}$ is the source of light, $\mathrm{M} M$ the mirror, $\mathrm{E}$ the observed, and $\mathrm{E}^{1}$ the observing eye as before. The rays of light are reflected from the mirror and form an image at $o^{1}$; they then diverge and are again made convergent by the lens $\mathrm{L}$ held in front of the eye by the observer; by this means a second image is focussed just behind 
the crystalline lens of the eye E. They then again diverge and diffusely light up the interior of the eyeball. The rays of light reflected from two points $i$ and $m$ on

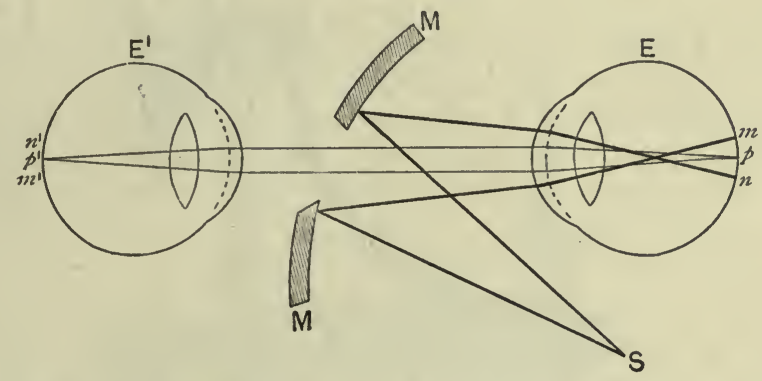

FIn. 532.-The course of the light in examining the eye by the direct method. (T. G. Brodie.)

the retina diverging from the eye are refracted by the glass lens $\mathrm{L}$, and give an inverted real image $i^{1} m^{1}$ larger than the object $i m$. These latter rays then diverge,

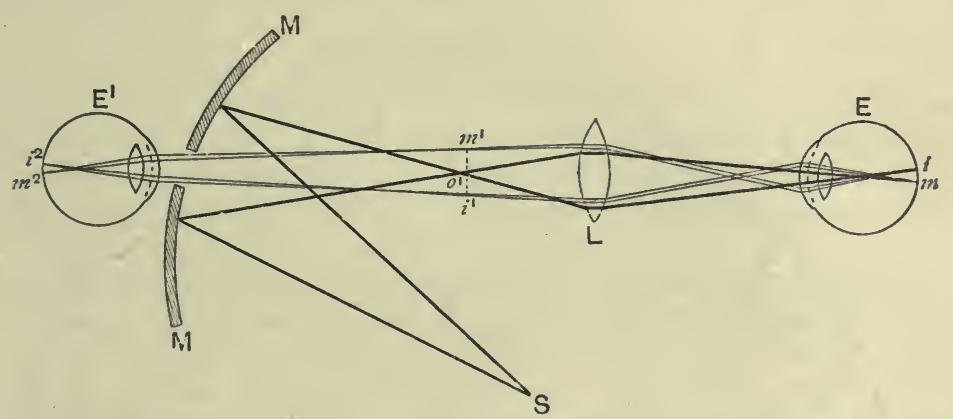

Fic. 533.-The course of the light in examining the eye by the indirect method. (T. G. Brodie.)

and are collected and focussed by the observing eye $\mathrm{E}^{1}$ to give an image $i^{2} m^{2}$ on the retina. (T. G. Brodie.)

\section{The Perimeter.}

This is an instrument for mapping out the field of vision. It consists of a graduated arc, which can be moved into any position, and which when rotated traces out a hollow hemisphere. In the centre of this the eye under examination is placed, the other eye being closed. The examiner then determines on the surface of the hemisphere those points at which the patient just ceases or just begins to see a small object moved along the arc of the circle. These points are plotted out on a chart graduated in degrees, and by connecting them the outline of the field of vision is obtained.

Fig. 534 shows one of the forms of perimeter very generally employed, and fig. 535 represents one of the charts provided with 


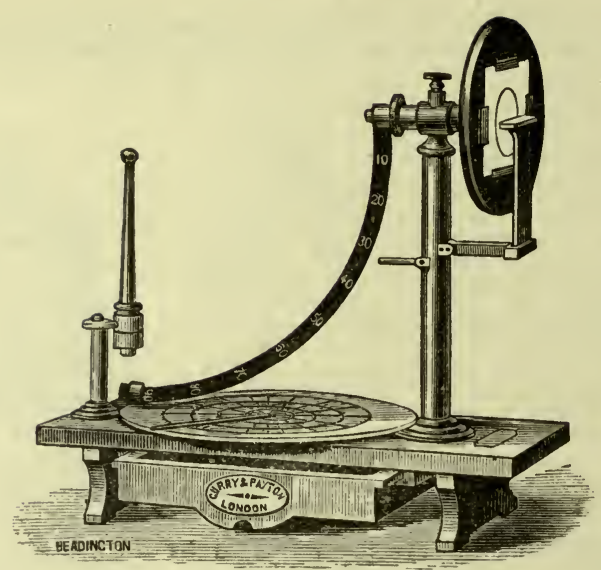

FIG. 534.-Priestley Smith's Perimeter.

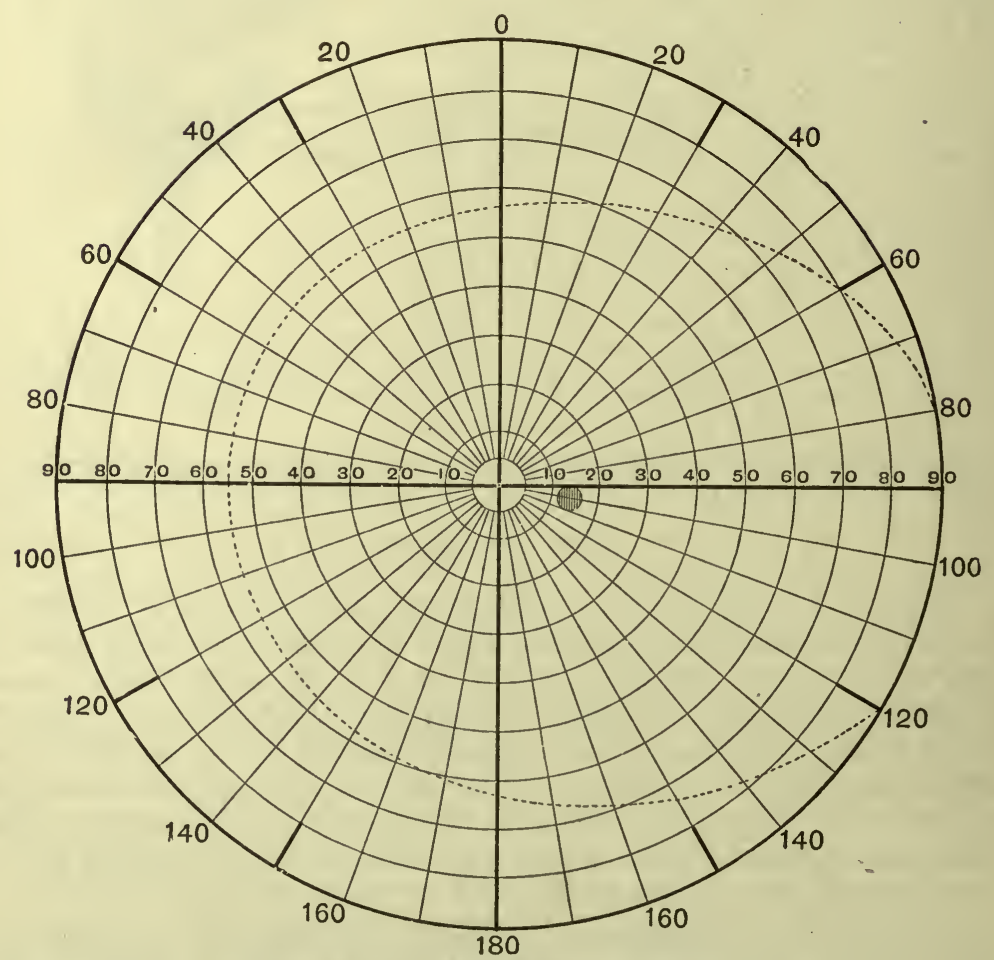

FIG. 535.-Perimeter chart for the right eye. 
the instrument. The blind spot is shown, and the dotted line represents the normal average field of vision for the right eye. It will be seen that the field of vision is most extensive on the outer side; it is less on the inner side because of the presence of the nose.

By the use of the same instrument, it is found that the colour of a coloured object is not distinguishable at the margin, but only towards the centre of the field of vision, but there are differences for different colours; thus a blue or yellow object is seen to be blue or yellow over a wider field than a red or green object.

In disease of the optic nerve, contraction of the field of vision for white and coloured objects is found. This often occurs before any change in the optic nerve is discoverable by the ophthalmoscope.

The yellow spot of one's own eye can be rendered evident by what is called Clerk-Maxwell's experiment:-On looking through a solution of chrome-alum in a bottle with parallel sides, an oval purplish spot is seen in the green colour of the alum. This is due to the pigment of the yellow spot.

\section{Visual Sensations.}

Visual sensations are of two kinds, colour sensations and colourless sensations. Colour sensations differ (1) in hue, for instance, blue, red, yellow ; (2) in saturation, for instance, pale green and full green; this depends upon the degree of admixture with white light; and (3) in intensity, for instance, a weak sensation or a strong sensation. These differences are in part dependent respectively on the length, the purity, and the amplitude of the light-wave; but they are also dependent on the local or general condition of the cerebro-retinal apparatus at the time of stimulation. Colours also differ (4) in brightness or luminosity; this is a purely psychological quality devoid of any known physical counterpart. The brightness of a colour may be measured by determining the shade of grey to which it appears equivalent. Even the most saturated colours (for instance, yellow and blue) have different degrees of brightness. Colourless sensations include the grey series from the deepest black to the most blinding white.

If a ray of sunlight is allowed to pass through a prism, it is decomposed by its passage into rays of different colours, which are called the colours of the spectrum; they are red, orange, yellow, green, blue, indigo, and violet. The red rays are the least turned out of their course by the prism, and the violet the most, whilst the other colours occupy in order places between these two extremes. The differences in the colour of the rays depend upon the rapidity of vibrations producing each, the red rays being the least rapid and the violet the most. In addition to these, there are other rays which are invisible but which have definite properties; those to the 
left of the red are less refrangible, being the calorific rays which act upon the thermometer, and those to the right of the violet, which are called the actinic rays, have a powerful chemical action.

White light may be built from its constituents in several ways, for instance, by a second prism reversing the dispersion produced by the first, or by causing the colours of the spectrum to fall on the retina in rapid succession. The best way to study the effects of compounding successive colour stimuli is by means of a rapidly revolving disc to which two or more coloured sectors are fixed. Each colour is viewed in rapid succession, but owing to the persistence of retinal impressions, the constituent colour stimuli give a single sensation of colour.

A colourless sensation can be produced by the mixture of three colours, or even of two colours in certain hues and proportions. These pairs of colours, of which red and greenish-blue, orange and blue, and violet and yellow are examples, are called complementary.

Thus blue and orange, when rotated on the colour-wheel, produce a colourless sensation; but it is well known that a mixture of blue and orange paint gives green. This is explained on the supposition that the colours used are not pure and that each contains green; the true blue and orange present neutralise each other to produce white, and thus green is the only colour sensation obtained.

Three properly chosen colours will not only produce a colourless sensation, but when combined in appropriate amounts they can be made to yield any other colour sensation. It is on this principle that Thomas Young based his trichromatic theory of colour vision, which was subsequently elaborated by Helmholtz and Clerk-Maxwell. It is known as the Young-Helmholtz theory. The theory selects red, green, and violet as the three primary colour-sensations. These three particular colours are chosen, partly because of their position within the spectrum, partly on account of the phenomena of colourblindness, and for other reasons.

The Young-Helmholtz theory teaches that there are in the retina certain elements (? within the cones) which answer to each of these primary colours, whereas the innumerable intermediate shades of colour are produced by stimulation of the three primary colour terminals in different degrees, the sensation of white being produced when the three elements are equally excited. Thus, if the retina is stimulated by rays of certain wave length, at the red end of the spectrum, the terminals of the other colours, green and violet, are hardly stimulated at all, but the red terminals are strongly stimulated, the resulting sensation being red. The orange rays excite the red terminals considerably, the green rather more, and the violet slightly, the resulting sensation being that of orange, and so on (fig. 536).

Another theory of colour vision (Hering's) supposes that there are six primary colour-sensations, viz.: three antagonistic (complemen- 
tary) pairs, black and whitc, red and green, and yellow and blue.; and that these are produced by the changes either of disintegration or of assimilation taking place in certain substances, which (the theory supposes) exist in the cerebro-retinal apparatus. Each of the substances corresponding to a pair of colours is capable of undergoing two changes, one of disintegration, and the other of construction, with the result of producing one or other colour. For instance, in the white-black substance, when disintegration is in excess of construction or assimilation, the sensation is white, and when assimilation is in excess of disintegration the reverse is the case; and similarly with the

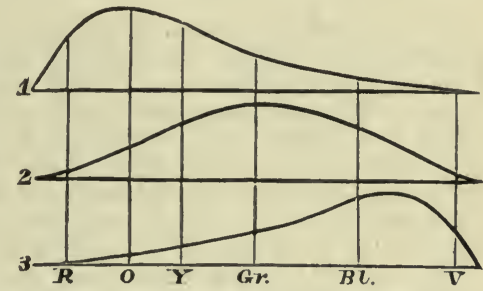

FIG. 536.-Diagram of the three primary colour. sensations. (Young-Helmholtz theory.) 1 is the red; 2, green, and 3 , violet, primary colour-sensation. The lettering indicates the colours of the spectrum. The diagram indicates by the height of the curve to what extent the several primary sensations of colour are excited by vibrations of different wave-lengths. red-green substance, and with the yellow-blue substance. When the repair and disintegration are equal with the first substance, the visual sensation is grey; but in the other pairs, when this is the case, no colour-sensation occurs. The rays of the spectrum to the red end produce changes in the red-zreen substance, with a resulting sensation of red, whilst the (orange) rays further to the right affect both the red-green and the yellow-blue substances; blue rays cause constructive changes in the yellow-blue substances, but none in the red-green, and so on. All colours act on the white-black substance as well as on the red-green or yellow-blue substance.

Neither theory satisfactorily accounts for all the numerous complicated problems presented in the physiology of colour vision. One of these problems is colour-blindness, a by no means uncommon visual defect. Some people are completely colour blind (see further p. 848), but the commonest form is the inability to distinguish between red and green. Helmholtz's explanation of such a condition is, that the elements of the retina which receive the impression of red or green are absent, or very imperfectly developed, and Hering's would be that the red-green substance is absent from the cerebro-retinal apparatus. Other varieties of colour-blindness, in which the other colour-perceiving elements are absent, have been shown to exist occasionally.

Hering's theory appears to meet the difficulty best, for if the red element of Helmholtz were absent, the patient ought not to be able to perceive white sensations, of which red is a constituent part; whereas, according to Hering's theory, the white-black visual substance remains intact. These two theories have been for a long time 
before the scientific world. As facts have accumulated, it has been for some years recognised that many facts could not be reconciled with either; and modifications of one or the other have been from time to time introduced.

C. J. Burch found that by exposing the eye to bright sunlight in the focus of a burning-glass behind transparent coloured screens, it is possible to produce temporary colour blindness. After red light, the observer is for some minutes redblind, scarlet geraniums look black, yellow flowers green, and purple flowers violet. After violet light, violet looks black, purple flowers crimson, and green foliage richer than usual. After light of other colours, corresponding effects are produced. If one eye is made purple-blind, and the other green-blind, all objects are seen in their natural colours, but in exaggerated perspective, due to the difficulty the brain experiences in combining the images from the two eyes.

By using a brightly-illuminated spectrum, and directing the eye to certain of its colours, the eye in time becomes fatigued and blind for that colour, so that it is no longer seen in the spectrum. Thus, after green blindness is induced the red appears to meet the blue, and no green is seen. If, however, the eye is exposed to yellow light, it does not similarly become blind for yellow only, but for red and green too. This supports the Young-Helmholtz theory, that the sensation yellow is one compounded of the red and green sensations. By an exhaustive examination of the different parts of the spectrum, in this way it thus becomes possible to differentiate between the primary colour-sensations and those which are compound. By a study of this kind, Burch concludes that the phenomena of colour vision are in accordance with the Young-Helmholtz theory, with the important addition that there is a fourth primary colour-sensation, namely, blue. He could not discover that colour-sensations are related to each other in the sense indicated by Hering. Each may be exhausted without either weakening or strengthening the others. These observations were confirmed by examining in a similar way the coloursensations of seventy other people, but there are individual differences in the extent to which the colour-sensations overlap.

Testing for Colour-blindness.-The test formerly adopted by the Board of Trade consists in matching skeins of wool from a heap of skeins of different colours (Holmgren's worsteds). It has, however, been shown that the test is not trustworthy, and it has been supplemented by one in which the subject is required to name the colours of lights in a lantern. The Edridge-Green lantern is one of the best to employ; in it the intensity and colour of the light and the order in which the colours are shown can be easily varied. If the colourblind person is made to examine and report on the colours of a spectrum, or in portions of the spectrum exposed to view, the results obtained are more accurate, but this is not so simple as the lantern test.

After-Images.-These are the after-effects of retinal excitation, and are divided into positive and negative. Positive after-images resemble the original image in distribution of brightness and colour. In negative after-images bright parts appear dark, dark parts bright, and coloured parts in the complementary colours.

If a bright white object is looked at, and the eyelids are then closed, a positive after-image is seen which fades gradually, but as it fades it passes through blue, violet or red, to orange; according to 



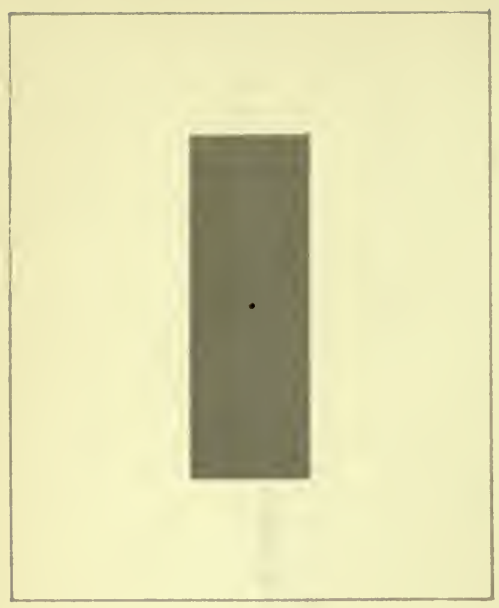

I.

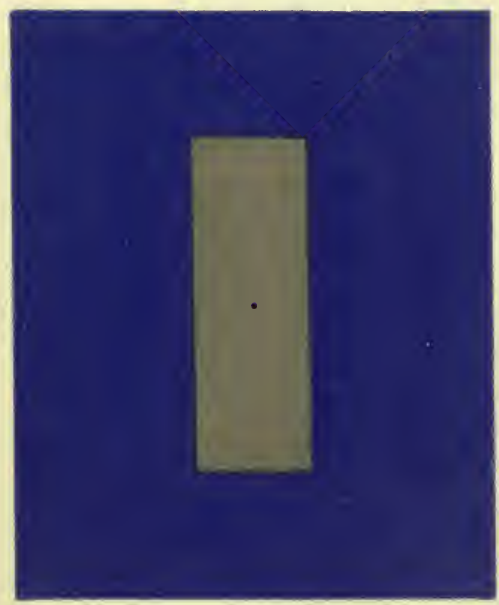

III.

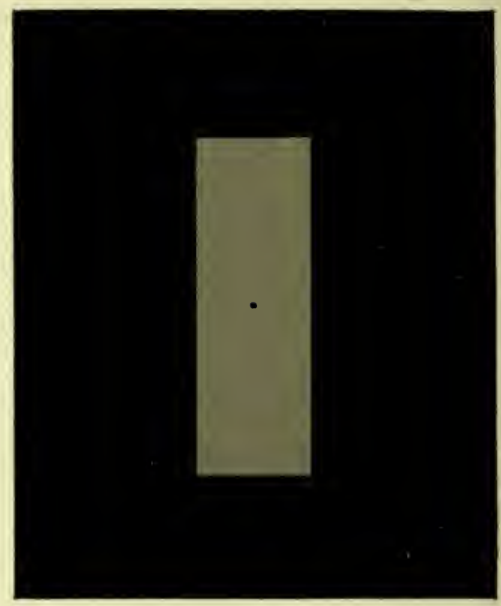

II.

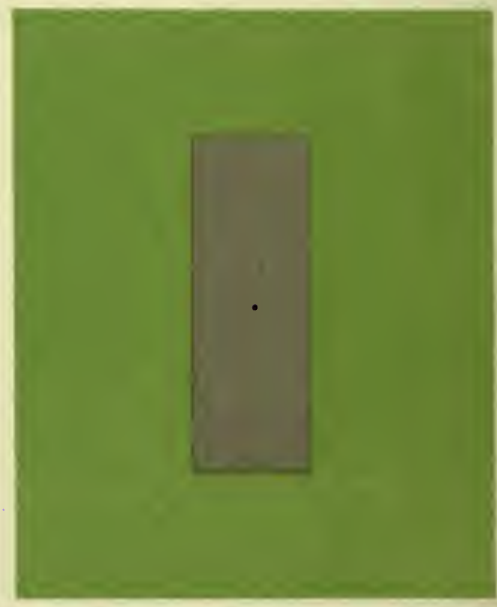

IV.

Plate to illustrate simultaneous and successive contrast.

For explanation see text. 
the Young-Helmholtz theory, this is explained on the hypothesis that the excitation does not decline with equal rapidity in the three colour terminals. A positive after-image is readily obtained by momentarily looking at a bright object, e.g. a window, after waking from sleep. Negative after-images may be seen either by closing the eyes or by turning them to a uniform grey surface after viewing an object steadily. If the object looked at is coloured, the negative after-image seen upon such a background is in its complementary colour; this is explained by the Young-Helmholtz theory, on the supposition that the colour-perceiving element for the colour looked at is the most fatigued, and the terminals for its complementary colour least fatigued. On the Hering theory, one colour produces anabolic or katabolic effects as the case may be; on withdrawing the eye from stimulation by that particular colour, the opposite phase of metabolism takes place and produces the complementary colour.

Simultaneous and Successive Contrast.-Negative after-images are frequently spoken of as phenomena of successive contrast. The phenomena of simultaneous contrast are well illustrated by the four figures of the accompanying Plate. In all these figures the oblong grey strip is actually of the same brightness. This can easily be proved by screening from view the surrounding parts of the figures, which cause the greys to appear different. The grey in I. appears darker than that in II., while the grey in III. appears yellowish and in IV. reddish. If these effects are not sufficiently obvious, they immediately become so when the entire surface is covered over with a sheet of thin tissue paper.

Figs. I. and II. are examples of brightness contrast; Figs. III. and IV. of colour contrast. The effects of these two varieties of simultaneous contrast may be stated thus: a given grey object looks darker when viewed against a bright background than when viewed against a dark background; when the background is coloured, it is tinged with the complementary colour of the former.

Helmholtz attributed the effects of simultaneous contrast to errors of judgment, and not to altered conditions of the retinal apparatus.* But there can be no doubt that simultaneous contrast has as simple a sensory origin as successive contrast (negative afterimages). For if either of the two lower figures of the plate is carefully fixated for about a minute (fixation of the central dot will help to prevent involuntary movements of the eyes), and if the gaze be then transferred to a spot on a sheet of white or grey paper, not only will the outer squares appear in their complementary colour, but also the grey strips will appear tinged, now likewise in a

* By "retina" here and elsewhere we mean "cerebro-retinal apparatus." We have no knowledge of the precise share of retina and brain in the development of visual sensations and after-sensations. 
complementary colour. So, too, if a point midway between Figs. I. and II. is fixated, and the plate held at a sufficient distance for both figures to be simultaneously visible, the after-image of the grey strip of II. will appear darker than that of I.

Seeing that simultaneous contrast persists in after-images, and seeing how generally recognised are its effects (for instance, by the painter, who depicts in blue the shadows cast by an object on the yellow sand), it seems far more probable that the part played by the higher mental processes consists, not, as Helmholtz supposed, in causing the illusion, but in reducing or overcoming it. According to this view, experience educates us in seeing objects in what we know to be their real colour, instead of in the colour which would result from the operation of simultaneous contrast. Some support is lent to this view by the fact that contrast is much enhanced when all irregularities are, as far as possible, eliminated from the surface of the object (here, the grey oblong) in which the contrast colour is induced, or when that object is made to appear, e.g. by covering the whole with tissue paper to combine with the object (the coloured square) which induces the contrast colour, so as to form an apparently single object. On the other hand, colour contrast is very markedly reduced, if the grey object is outlined in pencil on the tissue paper through which it is viewed. Thus, whatever tends to the apparent independence of the object in which the contrasting colour is induced tends to the reduction of the contrast effect.

Insisting on the sensory nature of simultaneous contrast, Hering explained it in the following way. He supposed that excitation of an area of the retina by a stimulus of given colour or brightness simultaneously induces an opposite metabolic process in the same colour apparatus in neighbouring areas of the retina. When, for example, a part of the retina is being stimulated by blue, the anabolic change thus evoked in the yellow-blue apparatus simultaneously is supposed to induce a katabolic change in the same apparatus in the neighbouring retinal area which is being excited by a grey stimulus. Consequently, the grey acquires a yellowish tinge.

Binocular colour-mixture.-By means of the stereoscope, binocular combinations of colour can be obtained. Thus, if one eye is exposed to a red disc, and the corresponding portion of the other eye to a yellow one, the mind usually perceives one disc of an orange tint; but frequently, especially if there be differences of brightness or of form in the two objects, we notice that "rivalry of the fields of vision" occurs, first one then the other disc rising into consciousness. A stereoscopic combination of black and white produces the appearance of metallic lustre; this is very beautifully shown with figures of crystals, one black on a white ground, the other white on a black ground. Probably the combination of black and white is interpreted 
as indicating a polished surface, because a polished surface reflects rays irregularly, so that the two eyes receive stimuli of unequal intensity.

\section{Changes in the Retina during Activity.}

The method by which a ray of light is able to stimulate the endings of the optic nerve in the retina in such a manner that a visual sensation is perceived by the cerebrum, is not yet understood. It is supposed that the change effected by the agency of the light which falls upon the retina is in fact a physico-chemical alteration in the protoplasm, and that this change stimulates the optic nerve-endings. The discovery of a certain temporary reddish-purple pigmentation of the outer limbs of the retinal rods in certain animals (e.g. frogs) which had been killed in the dark, forming the so-called rhodopsin or visual purple, appeared likely to offer some explanation of the matter, especially as it was also found that the pigmentation disappeared when the retina was exposed to light, and reappeared when the light was removed, and also that it underwent distinct changes of colour when other than white light was used. It was also found that if the operation were performed quickly enough, the bleached image of a bright object (optogram) might be fixed on the retina by soaking the retina of an animal which has been killed in the dark, in alum solution.

The rhodopsin is derived in some way from the black pigment (melanin or fuscin) of the polygonal epithelium of the retina, since the colour is not renewed after bleaching, if the retina is detached from its pigment layer.

Certain pigments, not sensitive to light, are contained in the inner segments of the cones. These are oil globules of various colours, red, green, and yellow, called chromophanes, and are found in the retinæ of marsupials (but not other mammals), birds, reptiles, and fishes. Nothing is known about the yellow pigment of the yellow spot.

Another change produced by the action of the light upon the retina is the movement of the pigment cells. On being stimulated by light the granules of pigment in the cells which overlie the outer part of the rod and cone layer of the retina pass down into the processes of the cells, which hang down between the rods: these melanin or fuscin granules are generally rod-shaped, and look almost like crystals. In addition to this, a movement of the cones and possibly of the rods occurs, as has been already mentioned; in the light the cones shorten, and in the dark they lengthen (Engelmann).

Red light has no action on visual purple; the maximum bleaching effect takes place in greenish-yellow light. Now, when the living eye is brought into a condition of "dark adaptation," that is, when 
the retina has become adapted to light of low intensity, the colours of the spectrum alter in brightness; the red end becomes shortened and much darker; the blue end becomes brighter, and the region of maximum brightness is in the green. This change of brightness with change of adaptation, known as Purkinge's phenomenon, is absent in the fovea, where there are no rods. The selective action of the colours of the spectrum on the visual purple is so strikingly similar to the altered conditions of brightness just described, that changes in the visual purple of the rods have been supposed to be the cause of sensations excited by feeble illumination (i.e. in the "dark-adapted" eye), while the cones are affected under more ordinary conditions of illumination. This conclusion gains support from several interesting facts. Visual purple is specially abundant in the retinæ of almost all animals whose habits are nocturnal, or who live underground. Further, if the intensity of a colour stimulus is gradually increased, it at first is too faint to produce any sensation; then it produces a sensation of greyness, and at last the colour itself is seen; the interval between the appearance of the grey or white-black effect and of the true colour effect of the stimulus is spoken of as the "photo-chromatic interval." Red light has no effect on visual purple, and has no photo-chromatic interval (that is, it appears either red or nothing), and according to several observers, there is no such interval at the fovea, where the rods, and therefore visual purple, are absent. Thirdly, a very similar effect has been described by $M$ 'Dougall, when the retina is momentarily stimulated by a coloured light; the sensation arising from the stimulus is followed by a series of "primary responses" or after-sensations; the first members of the series have the same colour as the stimulus, and these are sometimes followed by a series of colourless (grey) sensations; these grey sensations are only present outside the fovea, and under conditions of "dark adaptation" are absent with red and brightest with green stimuli. Here again we are able to differentiate between a visual-purple (rod) effect, and a cone effect, the former, active under conditions of feeble illumination, affected most by green and unaffected by red light, and yielding colourless sensations; the latter being more specially concerned in developing sensations of colour under conditions of adaptation to ordinary light. The fovea centralis thus becomes the region where the colours of objects are best distinguishable, and where with ordinary illumination visual acuity is most marked. In the dark, however, extra-foveal (rod) vision is more sensitive than foveal (cone) vision; astronomers see faint stars more readily in the periphery of the field of vision.

Two abnormal conditions may be described here, for they throw light on these phenomena. In cases of achromatopsia (total colour-blindness) the spectrum is seen as a band of light differing only in brightness; the region of maximum brightness 
is the same as in extra-foveal vision of the normal eye ; in many of these cases there is a central scotoma (blind spot), that is, the rodless fovea is blind; there is reduced acuity of vision as in the "dark-adapted "eye, and photophobia (fear of strong light); nystagmus (oscillating movements of the eye) also occurs, due to absence of an area of distinct vision. We are thus in typical cases of achromatopsia dealing with cases of cone blindness. In nyctalopia (night blindness), on the other hand, we meet the converse condition. Here there is an abnormal slowness of "dark adaptation," and a pathological change known as retinitis pigmentosa is present, suggesting an impaired function of the visual purple. Pilocarpine has been found an effective drug in such cases, and this is also interesting because it hastens the regeneration of visual purple in the extirpated eye.

The electrical variations in the retina under the influence of light were discovered by $M \cdot K e n d r i c k$ and Dewar, and have been recently reinvestigated by Waller. The excised eyeball of a frog is led off by non-polarisable electrodes to a galvanometer. One electrode is placed on the front, the other on the back of the eye. If the eyeball is quite fresh, a current is observed passing through the eyeball from back to front. When light falls on the eye this current is increased; on shutting off the light there is a momentary further increase, and then the current slowly returns back to its previous condition. Waller explains this by supposing that anabolic changes in the eye predominate during stimulation by light. With the onset of darkness, the katabolic changes cease at once, and the anabolic more slowly; hence a further positive variation.

As already stated, the current in a fresh eyeball passes from back to front before the stimulus is applied, but this cannot be regarded as a true current of rest, but as a current due to previous action which very slowly subsides. When this has subsided, the true current of rest is from cornea to fundus, i.e., it is like that of the skin, ingoing - the response to stimulation is like that of the skin, outgoing. Waller has also studied the electrical responses of the eyeball to other methods of stimulation; if electrical currents are employed, and the eyeball is still healthy, the response is always an outgoing current, whatever may be the direction of the electrical current used as the stimulus. These currents of action are no doubt mainly of retinal origin, but later Waller showed that the anterior portions of the eye, especially the crystalline lens, participate in their causation. The response of the eye to non-luminous stimuli lasts some time, and is spoken of as a "blaze current." An analogous response has been seen in skin, plant-tissues, etc.

Gotch has studied the photo-electric changes in the frog's eyeball with the capillary electrometer. He, like Waller, draws attention to the long latent period and sustained character of the response. The photo-electric changes are all monophasic effects, whether produced by illumination, or by shulting off the light. Gotch suggests there are two chemical substances in the retina, one of which reacts to light, the other to darkness. Each reaction is a change of the same type, but for the change to occur markedly, the eye must be previously adapted, i.e., the substances must undergo a phase of metabolism under conditions opposite to those which evoke the reaction effects. Observations with red and green light do not support the view that the photo-chemical changes are of opposite characters, for the photo-electric change is always in the same direction, differing only in period of latency, that for red being the longer.

\section{Movements of the Eyeball}

Protrusion of the eyeballs occurs (1) when the blood-vessels of the orbit are congested; (2) when contraction of the plain muscular fibres of the capsule of Tenon takes place; these are innervated by the cervical sympathetic nerve; and (3) in the disease called exophthalmic goitre.

Retraction occurs (1) when the lids are closed forcibly; (2) when the blood-vessels of the orbit are comparatively empty; (3) when the fat in the orbit is reduced in quantity, as during 
starvation; and (4) on section or paralysis of the cervical sympathetic nerves.

The most important movements, however, are those produced by the six ocular muscles.

The internal rectus turns the eyeball inwards, the external rectus turns it outwards. If the superior rectus acted alone, it would turn the eyeball not only upwards, but owing to the sloping direction of the muscle, the eyeball would be turned inwards also; in turning the eyeball directly upwards, this inward movement is arrested by the outward tendency of the inferior oblique. Similarly, in turning the eyeball directly downwards, the inferior rectus acts in conjunction with the superior oblique. Movements in intermediate directions are produced by other combinations of the muscles.

These muscles are all supplied by the third nerve except the superior oblique, which is supplied by the fourth, and the external rectus by the sixth nerve.

The muscles of the two eyes act simultaneously, so that images of the objects looked at may fall on corresponding points of the two retinæ. The inner side of one retina corresponds to the outer side of the other, so that any movement of one eye inwards must be accompanied by a movement of the other eye outwards. If one eyeball is forcibly fixed by pressing the finger against it so that it cannot follow the movement of the other, the result is double vision (diplopia), because the image of the objects looked at will fall on points of the two retinæ which do not correspond. The same is experienced in a squint, until the subject learns to disregard the image from one eye.

If the external rectus is paralysed, the eye will squint inwards; if this occurs in the right eye the false image will lie on the left side of the yellow spot, and appear in the field of vision to the right of the true image. If the third nerve is paralysed, the case is a more complicated one: owing to the paralysis of the levator palpebræ superioris, the patient will be unable to raise his upper lid (ptosis), and so in order to see will walk with his chin in the air. If the paralysis is on the right side, the eyeball will squint downwards and to the right; the false image will be formed below and to the right of the yellow spot, and the apparent image in the field of vision will consequently appear above and to the left of the true image, and owing to the squint being an oblique one, the false image will slant in a corresponding direction.

\section{Various Positions of the Fyeballs.}

All the movements of the eyeball take place around the point of rotation, which is situated $1.77 \mathrm{~mm}$. behind the centre of the visual axis, or $10.9 \mathrm{~mm}$. behind the front of the cornea. 
The three axes around which the movements occur are:-

1. The visual or antero-posterior axis. (A P, fig. 537.)

2. The transverse axis, which connects the points of rotation of the two eyes. (Tr, fig. 537.)

3. The vertical axis, which passes at right angles to the other two axes through their point of intersection.

The line which connects the fixed point in the outer world at which the eye is looking to the point of rotation is called the visual

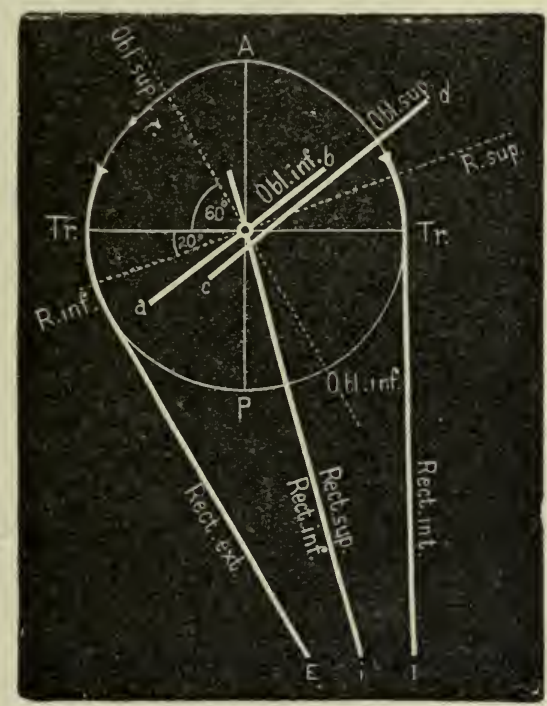

FIG. 537.-Diagram of the axes of rotation to the eye. The thin lines indicate axes of rotation, the thick the position of muscular attachment.

line. The plane which passes through the two visual lines is called the visual plane.

The various positions of the eyeballs are designated primary, secondary, and tertiary.

The primary position occurs when both eyes are parallel, the visual lines being horizontal (as in looking at the horizon).

Secondary positions are of two kinds:-

(1) The visual lines are parallel, but directed either upwards or downwards from the horizontal (as in looking at the sky).

(2) The visual lines are horizontal, but converge towards one another (as in looking at a small object near to and on the same level as the eyes).

Tertiary positions are those in which the visual lines are not horizontal, and converge towards one another (as in looking at the tip of the nose). 
It is possible to conceive positions of the eyeballs in which the visual lines diverge from one another; but such positions do not occur in normal vision in man.

Both eyes are moved simultaneously, even if one of them happens to be blind. They are moved so that the object in the outer world is focussed on the two yellow spots, or other corresponding points of the two retinæ. The images which do not fall on corresponding points are seen double, but these are to a great extent disregarded by the brain, which pays particular attention to those images which fall on corresponding points.

The accompanying diagrams will assist us in understanding what is meant by corresponding or identical points of the two retinæ.

If $\mathrm{R}$ and $\mathrm{L}$ (fig. 538) represent the right and left retinæ respectively, $\mathrm{O}$ and $\mathrm{O}^{\prime}$ the two yellow spots are identical; so are $\mathrm{A}$
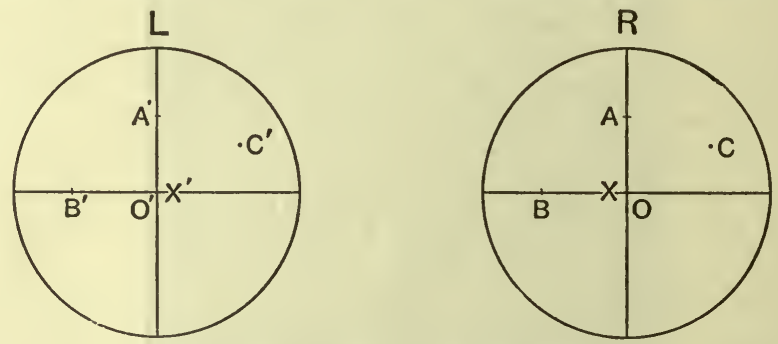

Fia. 538.-Identical points of the retinæ.

and $\mathrm{A}^{\prime}$, both being the same distance above $\mathrm{O}$ and $\mathrm{O}^{\prime}$. But the corresponding point to $\mathrm{B}$ on the inner side of $\mathrm{O}$ in the right retina, is $\mathrm{B}^{\prime}$, a point to the same distance on the outer side of $\mathrm{O}^{\prime}$ in the left retina; similarly $C$ and $C^{\prime}$ are identical. The two blind spots $X$ and $\mathrm{X}^{\prime}$ are not identical.

Fig. 539 shows the same thing in rather a different way; $A$ and $B$ represent horizontal sections through the two retinæ; the points

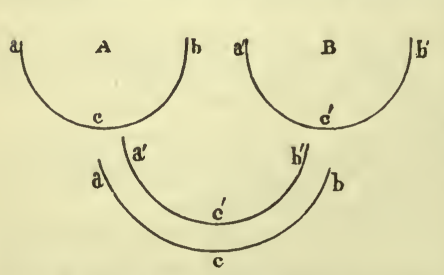
a $a^{\prime}, b b^{\prime}$, and $c c^{\prime}$, being identical. In the lower part of the diagram is shown the way in which the brain combines the images in the two retinæ, one overlapping so as to coincide with the other.

The Horopter is the name given to the surface in the outer world which Fra. 539. - Diagram to show the correspond- contains all the points which fall on
ing parts of both retinæ. the identical points of the retinæ. The shape of the horopter will vary with the position of the eyeballs. In the primary position, and in the first variety of the 
secondary position, the visual lines are parallel; hence the horopter will be a plane at an infinite distance.

In the other variety of the secondary position, and in tertiary positions in which the visual lines converge, as when looking at a near object, the horopter is a circle (fig. 540) which passes through the nodal points of the two eyes, and through the fixed point $(\mathrm{I})$ in the outer world at which the eyes are looking, and which will consequently fall on the two yellow spots $\left(\mathrm{O}\right.$ and $\left.\mathrm{O}^{\prime}\right)$. All other points in this circle (II, III) will fall on identical points of the retinæ. The image of II will fall on $\mathrm{A}$ and $\mathrm{A}^{\prime}$; of III on $\mathrm{B}$ and $\mathrm{B}^{\prime}$; it is a simple mathematical problem to prove that $O A=O^{\prime} A^{\prime}$, and $O B=O^{\prime} B^{\prime}$.

In those animals in which the eyes are lateral in position, and the visual lines diverge, the problem of binocular vision is a very different one (see also p. 736).

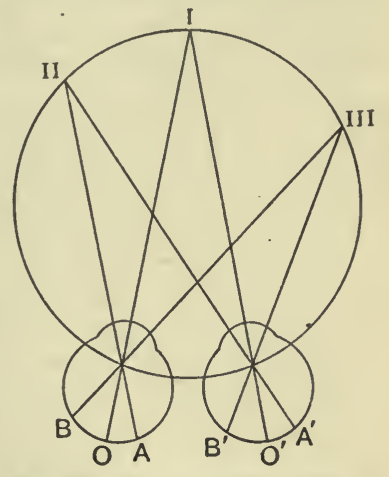

Fra. 540.-The Horopter, when the eyes are convergent.

\section{Nervous Paths in the Optic Nerves.}

The correspondence of the two retinæ and of the movements of the eyeballs is produced by a close connection of the nervous centres controlling these phenomena, and by the arrangement of the nervefibres in the optic nerves. The crossing of the nerve-fibres at the optic chiasma is incomplete, and the next Left Retina. Right Retina diagram (fig. 541) gives a simple idea of the

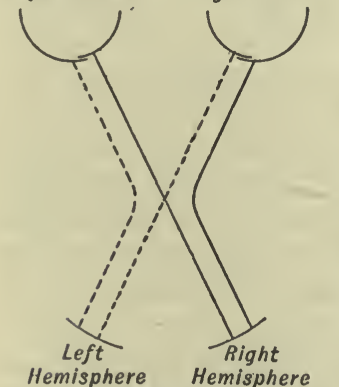

Fia. 541.-Course of fibres at optic chiasma.

\section{way the fibres go.}

It will be seen that it is only the fibres from the inner portions of the retinæ that cross; and that those represented by continuous lines from the right side of the two retinæ ultimately reach the right hemisphere, and those represented by interrupted lines from the left side of the two retinæ ultimately reach the left hemisphere. The two halves of the retinæ are not, however, separated by a hard-and-fast line from one another; this is represented by the two halves being depicted as slightly overlapping, and this comes to the same thing as saying that the central region of each retina is represented in each hemisphere.

The part of the hemisphere concerned in vision is the occipital lobe, and the reader should turn back to our previous consideration 
of this subject in connection with cerebral localisation, the phenomena of hemianopsia and the conjugate deviation of head and eyes (pp. 736,737$)$.

Fig. 542, though diagrammatic, will assist the reader in more fully comprehending the paths of visual impulses, and the central connections of the nerves and nerve-centres concerned in the process. The fibres from the retina to the external geniculate body end there by arborising around its cells, and a fresh relay of fibres from

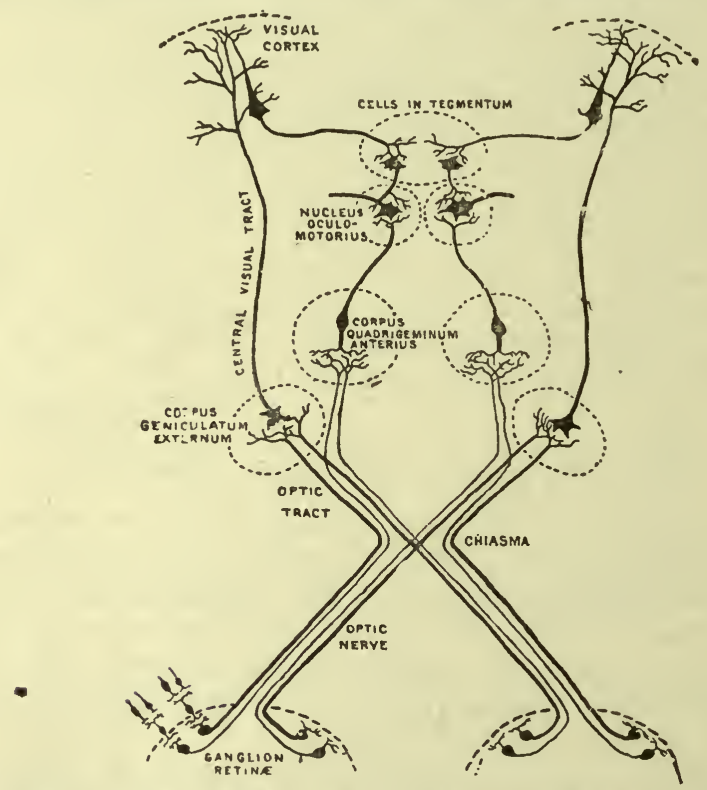

Fiq. 542 -Relations of nerve cells and fibres of visual apparatus. (Schäfer)

these cells passes in the posterior part of the internal capsule to the cortex of the occipital lobe. Those to the anterior corpus quadrigeminum are continued on by a fresh relay to the nuclei of the nerves concerned in eye-movements (represented by the oculo-motor nucleus in the diagram); the axons of the cortical cells pass to the tegmentum, whence a fresh relay continues the impulse to the oculo-motor nucleus.

Sherrington's observations on binocular flicker have shown that there are difficulties in accepting fig. 542 as a complete anatomical basis for the psychological processes involved in binocular vision, although it is probably correct so far as the motor mechanisms involved are concerned.

\section{Visual Judgments.}

The psychical or mental processes which constitute the visual sensation proper have been studied to a far greater degree than is possible in connection with other forms of sensation. 
We have already seen that in spite of the reversion of the image in the retina, the mind sees objects in their proper position; this is explained on p. 823.

We are also not conscious of the blind spot. This is partly due to the fact that those images which fall on the blind spot of one eye are not focussed there in the other eye. But even when one looks at objects with one eye, there is no blank, for the reason explained on p. 835 .

Our estimate of the size of various objects is based partly on the visual angle (p. 822) under which they are seen, but much more on the estimate we form of their distance. Thus a lofty mountain many miles off may be seen under the same visual angle as a small hill near at hand, but we infer that the former is much the larger object because we know it is much farther off than the hill. Our estimate of distance is, however, often erroneous, and consequently the estimate of size also. Thus persons seen walking on the top of a small hill against a clear twilight sky appear unusually large, because we over-estimate their distance, and for similar reasons most objects in a fog appear immensely magnified.

The action of the sense of vision in relation to external objects is, therefore, quite different from that of the sense of touch. The objects of the latter sense are immediately present to it; and our own body, with which they come in contact, is the measure of their size. The part of a table touched by the hand appears as large as the part of the hand receiving an impression from it, for the part of our body in which a sensation is excited, is here the measure by which we judge of the magnitude of the object. In the sense of vision, on the contrary, the imgges of objects are mere fractions of the objects themselves, realised upon the retina, the extent of which remains constantly the same. But the mind, into which the sensations of vision are incorporated, invests the images of objects, together with the whole field of vision in the retina, with very varying dimensions; the relative size of the image in proportion to the whole field of vision, or of the affected parts of the retina to the whole retina, alone remains unaltered.

The estimation of the form of bodies by sight is the result partly of the mere sensation, and partly of the association of ideas. Since the form of the images perceived by the retina depends wholly on the outline of the part of the retina affected, the sensation alone is adequate to the distinction of superficial forms from each other, as of a square from a circle. But the idea of a solid body such as a sphere, or a cube, can only be attained by the action of the mind constructing it from the different superficial images seen in different positions of the eye with regard to the object, and, as shown by Wheatstone and illustrated in the stereoscope, from two different perspective pro- 
jections of the object being presented simultaneously to the mind by the two eyes.

Thus, if a cube is held at a moderate distance before the eyes, and viewed with each eye successively while the head is kept perfectly steady, a (fig. 543) will be the picture presented to the right eye, and $\mathrm{B}$ that seen by the left eye. Wheatstone has shown that on this circumstance depends in a great measure our conviction of the solidity of an object, or of its projection in relief. If different perspective drawings of a solid body, one representing the image seen by the right eye, the other that seen by the left (for example, the drawing of a cube, A, B, fig. 543), be presented to corresponding parts of the two retinæ, as may be readily done by means of the stereoscope, the mind will perceive not merely a single representa-

A

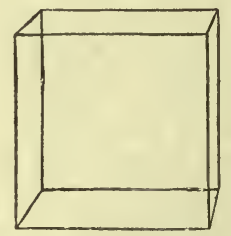

B

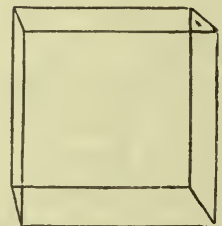

Fig. 543.-Diagrams to illustrate how a judgment of a figure of three dimensions is obtained.

tion of the object, but a body projecting in relief, the exact counterpart of that from which the drawings were made.

By transposing two stereoscopic pictures a reverse effect is produced; the elevated parts appear to be depressed, and vice versa. An instrument contrived with this purpose is termed a pseudoscope. Viewed with this instrument a bust appears as a hollow mask, and as may readily be imagined the effect is most bewildering.

The clearness with which the details of an object are perceived irrespective of accommodation, would appear to depend largely on the number of rods and cones which its retinal image covers. Hence the nearer an object is to the eye (within moderate limits) the more clearly are all its details seen. Further, if we want carefully to examine any object, we always direct the eyes straight to it, so that its image shall fall on the two yellow spots, where an image of a given area will cover a larger number of cones than anywhere else in the retina. Moreover, as previously pointed out, each cone in the macula lutea is connected to a separate chain of neurons.

The importance of binocular vision is very great. If an object is looked at with one eye only, it is impossible to estimate its distance by the sense of vision alone. For instance, if one eye is closed and the other looks at a wire or bar, it is impossible to tell 
whether, if some one drops a small object, it falls in front of or behind the bar.

Visual judgments are not always correct; there are a large number of puzzles and toys which depend on visual illusions. One or two of the best known are represented in the accompanying diagrams.

In fig. 544, A, B, and $\mathrm{C}$ are of the same size; but A looks taller than $\mathrm{B}$, while $\mathrm{C}$ appears to cover a less area than either. The sub-

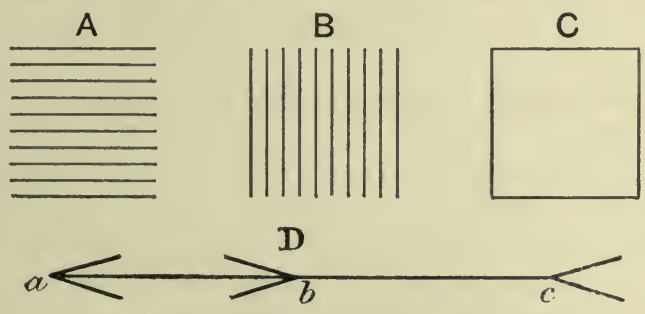

Fra. 544.-Diagrams to illustrate visual fllusions.

division of a space or line increases its apparent size or length. In fig. $544 \mathrm{D}, a b$ is equal to $b c$. Vertical distances also are usually over-estimated. In fig. 545 the long lines are parallel, though

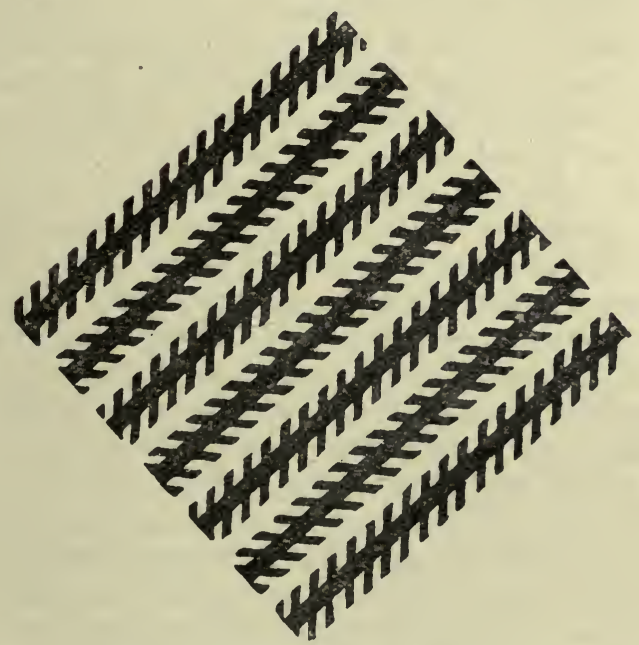

Fıa. 545.-Zöllner's lines

they do not appear so, owing to the influence of the intercrossing lines. 


\title{
CHAPTER LIX
}

\author{
REPRODUCTION, DEVELOPMENT, GROWTH AND DEATH
}

THE history of the fully formed animal from the ovum, and a description of the origin and formation of its tissues and organs, constitutes the portion of biological science known as embryology.

The scientific discussion of embryology must not be limited to man, however important he may be, but must embrace a wide survey of the whole animal kingdom, because the changes which occur in the embryological history of the highest animals forms a compressed, though in many cases a modified picture of the changes which have taken place in their historical development from lower types, during the long and misty ages of the past.

To attempt an adequate discussion of this large subject would mean the writing of a book as long as the present volume. It would lead us far into biological fields, and also into discussions of a philosophical and hypothetical nature which would be quite out of place in a physiological text book. All we can do is to put down the important facts, and specially to dwell on those which have a physiological bearing. Evolution has in the past been specially studied from the anatomical point of view; but it has its physiological counterpart; for as structures increase in complexity, so also does function become correspondingly differentiated and varied.

The great problem of evolution is associated with the immortal name of Darwin. He showed that although the offspring resembles the parents there are always certain slight variations which may be transmitted to future generations, and in their turn these are magnified or admixed with other variations in each succeeding generation. In the struggle for existence those will survive in which the variations are beneficial and helpful, so that in the end the fittest will live on. The piling up of variations which in themselves may be trivial thus leads to the development of new types and species, and this, prolonged over immense periods of time, has led to the evolution of complex from simple forms of life.

The explanation of heredity is a subject on which much difference 858 
of opinion prevails. It does, however, appear to be pretty well accepted that the material of the nucleus of the male and female reproductive elements is of special importance in the transmission of hereditary characters. That the chromosomes of the nucleus are of vital importance is clearly shown by several facts. For instance, they are constant in number not only in the cells of the body but in all individuals of any particular species of animal or plant, though differing in number in different species. The equal halving of each chromosome, which occurs during mitosis, maintains this numerical constancy, except in a certain period of the life history of each individual, and this period occurs in the formation of the reproductive cells (often called gametes). During one step in the karyokinetic cell-division, half of the normal number of chromosomes are thrown out, and the act of fertilisation consists in the fusion of the male and female gametes; each parental nucleus provides half the normal number of chromosomes, and thus the fertilised egg-cell starts with the full complement once more. A large number of biologists regard the chromosomes as the actual bearers of the characters which an organism inherits from its parents, or, at any rate, adopt this view as a working hypothesis. This places the transmission of characters from parent to offspring upon a material foundation. Weismann's view that characters acquired after birth are not transmissible rests mainly upon negative evidence, and so cannot be considered as fully proved.

The development of the two sexes took place fairly early in the history of the animal world, but there are many animals of the simplest kind in which no differentiation of sex occurs. The determination of sex, that is, why a mother should bear at one time a male, at another time a female infant, is a question of great importance, and numerous theories have been advanced to explain it. In certain invertebrate animals there is some evidence that sex is preformed either in the ovum or the spermatozoon, or in both; that is to say, there are male and female ova, and male and female spermatozoa which exhibit slight differences of structure; the question of sex of the offspring will then depend upon which element predominates after union. Others hold that potentialities of producing either sex are present in all ova and all spermatozoa, and that the ultimate sex is determined by some relationship which is at present unknown, but which in all probability operates at the time of fertilisation.

The original Darwinian doctrine, just stated in outline, has since been modified in several directions as research has progressed. The work of Weismann just alluded to is important. But of all the theories which have been grafted upon the original theory, that of Mendel is perhaps of the greatest interest; it certainly appeals to 
the scientific mind, for it is one which is susceptible of proof and demonstration. It may be best illustrated by a concrete example. If two kinds of plant, such as dwarf and tall peas, are crossed, the seeds obtained all produce tall plants; but if the plants of this generation are crossed, the third generation contains 25 per cent. dwarf and 75 per cent. of tall plants. If the dwarfs are crossed, they breed true, that is, they produce dwarf plants only; but if the tall plants are inter-crossed, again one-quarter of the next generation are dwarfs and the remainder tall. Of the 75 tall plants, 25 breed true, and of the remaining 50, one-quarter produce dwarfs which breed true, and the remaining three-quarters are tall plants. In successive generations the same holds : the dwarfs, when self-crossed, always produce dwarfs, and tall plants always produce a progeny in which 25 per cent. are dwarfs and 75 per cent. tall, some of which breed true and the remainder breed a mixture in the same proportions as before.

This is explained by calling certain characters dcminant and others recessive; thus, in the example taken, tallness is dominant and dwarfness is recessive. So in the first generation the progeny all exhibit the dominant character, but in the second only 25 per cent. will be pure dominants (that is, will produce only dominants in successive generations), 25 per cent. will be pure recessives, and 50 per cent. are mixed; and the mixed type will again produce 25 per cent. dominants, 25 per cent. recessives, and 50 per cent. mixed, as before.

This discovery has been found important for breeders and horticulturists, but the law appears to fail sometimes; for instance, when the black and white races intermarry, the result is brown children, and not a definite proportion of blacks and whites.

Looked at from the evolutionary point of view, the whole of the complex animal body is but the temporary dwelling-place of the reproductive cells, and nature provides lavishly, both in animals and plants, for the continuance of the species. These temporary homes, which we call the father and the mother, pass away, but they live on and on in their descendants. In the future we cannot doubt that upward progress will still continue as in the past, and man in time should be in very truth "but little lower than the angels." Some hold that civilisation in some ways places a slight obstacle on the path of progress of the race; numerous works of charity militate against the inexorable law of the survival of the fittest, so that many live to propagate their species who may be weaklings both in body and mind. There are no doubt extreme cases where it is desirable that deteriorated characters should be debarred from introducing into the world a continuance of beings destined for misery and crime. But I myself believe that the present development 
of charitable endeavours is in itself a sign of progress; it indicates a growth of the feelings of compassion and unselfishness which constitute a great asset in the possessions of a race. The laws of evolntion operate in spite of artificial hindrances; this is exemplified in what is termed the law of anticipation; by this is meant that in a heritable disease its effects develop at an earlier and earlier age in successive generations, until at last it causes death at a period before the sexual organs attain maturity, and thus automatically its continued propagation is put a stop to.

In a simple unicellular organism such as the amœba, there is not only no differentiation of sex, but there is also no differentiation between the reproductive element (Weismann's germ plasm) and the remainder of the body (Weismann's somatoplasm). When the amoba propagates itself by dividing into two new amœbæ, the whole animal is concerned in the act of reproduction, and, barring accidents, the new amœbæ may behave in this way indefinitely, and so may be spoken of as immortal. In this sense the only part of the body which is immortal (in the material as opposed to the theological use of the word) in the higher animals is the germ plasm, which lives beyond us to repeat the process an infinite number of times in our descendants.

If now we deal in a more matter-of-fact way with the present instead of the past or the future, the faculty which living organisms have of reproducing themselves furnishes us with a new conception of the life process. The common lot of all is to die, and life in the individual is then extinct; but each individual is only a link in a long chain of lives, and life is thus not something which begins and ends like a straight line; it is rather to be compared to a circle, in which a series of events is repeated over and over again. If we start with the ovum we are led to the fœtus, and then to the child, and the adult who produces or fertilises an ovum once more; and so history goes on repeating itself.

I propose, however, in the following account of some of the details of the long story, to start at a different point in the cycle. We will take first the new-born child, and rapidly run through what occurs after its birth until the state of sexual maturity is reached. Next it will be our duty to consider the structure and physiology of the reproductive organs, and how the ova in one sex, and the spermatozoa in the other sex, originate. Next will follow an account of the union of these reproductive cells, and the production of a fertilised ovum. This will be followed by a brief account of the development and life of the fotus, until we reach the mechanism by which the new-born child is expelled from the uterus into the world, which will bring us back to the point from which we started. 


\section{The New-Born Child and the Changes it Undergoes AFTER BIRTH.}

The new-born child, which in the uterus was obtaining its nutriment and oxygen from its mother's blood, is severed from the organ called the placenta, by means of which this was accomplished, by cutting through the umbilical cord. The want of oxygen is met by the child beginning to breathe, and its nutriment is supplied by its mother's milk, which later on is supplemented and replaced by other articles of diet. Immediately after birth certain changes occur in the circulatory system; the foramen ovale, the opening between the two auricles, begins to close, and so do the ductus arteriosus and the ductus venosus. The now functionless umbilical vessels close also until they are reduced to mere fibrous cords. These changes are completed in a few days, and the circulation then takes the course it traverses for the rest of life.

In addition to this there are changes of a more general kind, the most obvious of which is growth; this is accompanied with the completion in the formation of certain organs and tissues which are in a comparatively immature condition when the child is born. Thus medullation of the fibres in the central nervous system is taking place, and the process of ossification continues until the bony skeleton is perfected. The generative organs reach maturity at the period of life known as puberty.

The rate of growth after birth is not so rapid as it is in utero; and every year the relative increase in size gets less and less. On the average, girls in the earlier years grow more than boys, but at the onset of puberty this relationship is usually reversed. At puberty there is generally an acceleration of the rate of growth in both sexes, but this gradually declines, and finally growth ceases.

Puberty then is the period at which the sexual organs become matured and functional. In girls this occurs on the average at about fourteen or fifteen years of age, and is marked by the onset of menstruation. Menstruation, or the monthly flow, continues until the age of forty-five to fifty, when it ceases either gradually or suddenly, and after this period (the menopause or climacteric) further production of offspring is not possible. The menopause is usually accompanied with great depression and other disturbances of a physical and mental nature.

In boys, puberty is usually a little later developed than in girls, but there is no limit at the other end of life corresponding to the menopause.

In both sexes the onset of puberty is accompanied by the secondary sexual characters becoming pronounced, such as the increase in fullness of the mammary glands in the female, and the 
growth of hair on the face and the increase in size of the larynx which leads to the deepening of the pitch of the voice in the male.

\section{The Male Reproductive Organs}

These consist of the two testes which produce spermatozoa, and the ducts which lead from them.

The testis is enclosed in a serous membrane called the tunica vaginalis, originally a part of the peritoneum, which descends into the

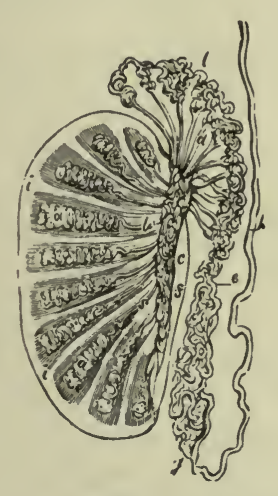

F1G. 546.-Plan of a vertical section of the testicle, showing the arrangement of the ducts. The true length and diameter of the ducts have been disre. garded. $a a$, Tubuli semi. niferi coiled up in the separate lobes; $b$, tubuli recti ; $c$, rete testis ; $d$, vasa efferentia ending in the coni vasculosi ; $l, e, g$, convoluted canal of the epidi. dymis; $h$, vas deferens; $f$, body of Highmore ; $i$, tibrous processes running between the lobes.

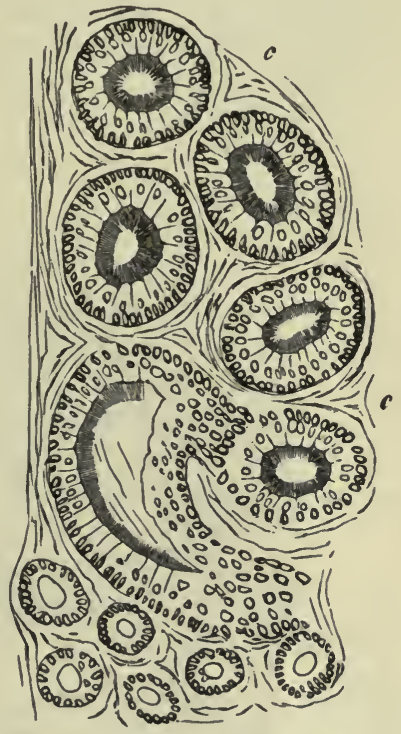

FIG. 547. - Section of the epididymis of a dog.-The tube is cut in several places, both transversely and ob. liquely; it is seen to be lined by a ciliated epithelium, the nuclei of which are well shown. $c$, Connective tissue. (Schofield.)

scrotum before the testis and subsequently gets entirely cut off from the remainder of the peritoneum. There are, however, many animals in which the testes remain permanently in the abdomen. The external covering of the testicle itself is a strong fibrous capsule, called, on account of its white appearance, the tunica albuginea. Passing from its inner surface are a number of septa or trabeculæ, which divide the organ imperfectly into lobules. On the posterior aspect of the organ the capsule is greatly thickened, and forms a mass of fibrous tissue called the Corpus Highmorianum (body of High- 
more) or mediastinum testis. Attached to this is a much convoluted tube, which forms a mass called the epididymis. This receives the ducts of the testis, and is prolonged into a thick-walled tube, the vas deferens, by which the semen passes to the urethra.

Each lobule of the testicle contains several convoluted tubes. Every tube commences near the tunica albuginea, and terminates after joining with others in a straight tubule, which passes into the body of Highmore, where it ends in a network of tubes, the rete testis. From the rete about fifteen efferent ducts (vasa efferentia) arise, which become convoluted to form the coni vasculosi, and then pass into the tube of the epididymis.

The convoluted or seminiferous tubes (fig. 548) have the following

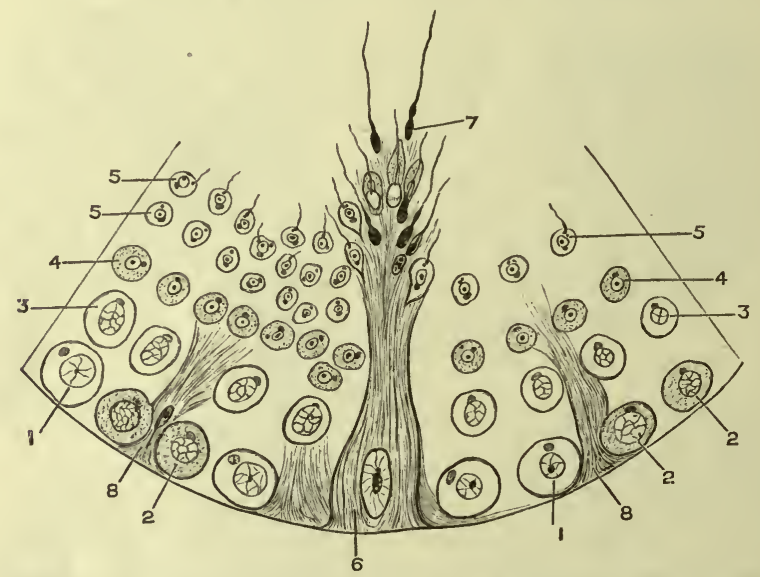

FIG. 548.-Diagram of a portion of a seminal tubule showing development of spermatozoa. 1, Primitive germ cell; 2, spermatogonia; 3 , primary spermatocytes; 4, secondary spermatocytes; 5 , spermatids, some with commencement of axial filament; 6 , a nurse cell with spermatids and spermatozoa in various stages of development; 7 , free spermatozoa in lumen of tube; 8 , portions of nurse cells. (After Waldeyer.)

structure: each consists of (1) an outer boundary of flattened connective-tissue cells intermingled with elastic fibres; (2) a fine membrana propria; (3) a lining epithelium of many layers of germinal cells. Next to the membrana propria is a layer of cells, some of which are primordial germinal cells, others are spermatogonia produced from the primordial germinal cells, but differing from them in structure, and the remainder are supporting or nurse cells (Cells of Sertoli) which provide nutriment for the developing spermatozoa. More internally, between the projecting processes of the nurse cells, are large primary spermatocytes, derived from the division of the spermatogonia. Still nearer the lumen of the tube lie the secondary spcrmatocytes, which are the daughter-cells of the primary spermatocytes; the secondary 
spermatocytes give rise by division to the spermatids which lie next the lumen. The spermatids become imbedded in the inner ends of the nurse cells, where they grow and become converted into

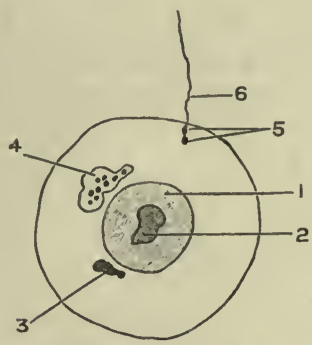

Fig. 549.-A spermatid largely magnified. 1, nucleus; 2 , nucleolus; 3, chromatoid body; 4 , idiosume; 5 , centrosomes ; 6 , commencement of axial filament. (After Meves.)

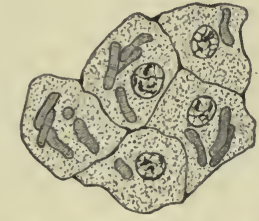

Fre. 550.-Cells of the interstitial tissue of the testis with crystal. loid bodies.

spermatozoa. Every spermatid contains a nucleus, and near the nucleus is another structure called an idiosome, containing a number of microsomes. There are also a coloured or chromatoid body whose function is not known, and two centrosomes (see fig. 549).

The interstitial connective tissue of the testis is loose, and contains numerous lymphatic clefts. Lying in it, accompanying the bloodvessels, are strands of polyhedral epithelial cells, of a yellowish colour (interstitial cells), which frequently contain crystalloid bodies (fig. 550).

The straight tubules consist of basement membrane and lining cubical epithelium only. The tubules of the rete testis are lined by cubical epithelium; the basement membrane is absent. The vasa efferentia, coni vasculosi, and epididymis are lined by columnar cells, some of which are ciliated, whilst others are devoid of cilia, and probably possess secretory functions. There is a good deal of musoular tissue in their walls. The vas deferens consists of a musoular

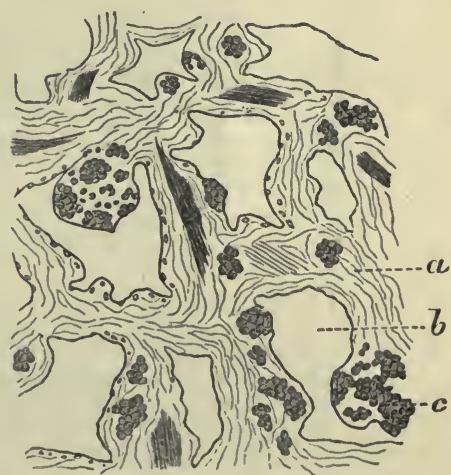

Frg. 551.-Erectile tissue of the human penis. a, Fibrous trabecule with their ordinary capillaries; $b$, section of the venons sinuses $c$, muscular tissue. (Cadiat.) wall (outer layer longitudinal, middle circular, inner longitudinal), lined by a mucous membrane, the inner surface of which is covered by columnar epithelium.

The vesiculoe seminales are outgrow ths of the vasa deferentia. Each 
is a much convoluted, branched, and sacculated tube of structure similar to that of the vas deferens, except that the wall is thinner; their secretion is added to the semen, as is also the secretion of the glands of the prostate.

The penis is composed of cavernous tissue covered by skin. The cavernous tissue is collected into three tracts, the two corpora cavernosa and the corpus spongiosum in the middle line inferiorly.
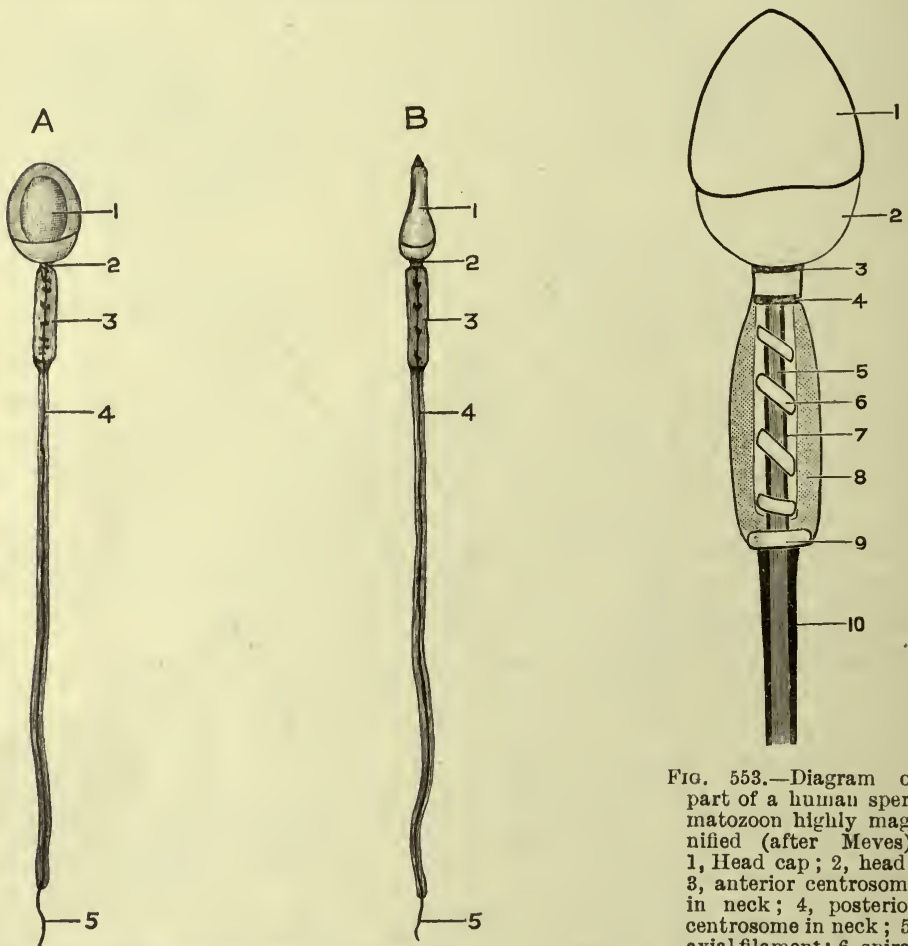

Fig. 553.-Diagram of part of a humau sper. matozoon highly magnified (after Meves).

1, Head cap ; 2 , head ;

3 , anterior centrosome in neck; 4 , posterior centrosome in neck ; 5 , axial filament; 6 , spiral sheath; 7 , sheath of axial filament in body ; 8 ,mitochondrial sheath; 9 , annulus ; 10 , thick sheath of axial filament in tail.

All these are enclosed in a capsule of fibrous and plain muscular tissue; the septa which are continued in from this capsule, form the boundaries of the cavernous venous spaces of the tissue. The arteries run in the septa; the capillaries open into the venous spaces. The arteries are often called helicine, as in injected specimens they form twisted loops projecting into the cavernous spaces (see also pp. 313,314$)$. 
The Spermatozoa, suspended in a richly albuminous fluid, constitute the semen. Each spermatozoon consists of a head, a very short neck, a body, a tail, and an end-piece. The head is of flattened ovoid shape, and in the anterior two-thirds of its extent is surmounted by a head-cap which, sharpened at its extremity, forms a cutting edge. The neck is very short, and contains two centrosomes. The body is about the same length as the head; it is traversed by an axial filament and a spiral fibril wound round the sheath of the axial filament. More externally is a layer called the mitochondrial sheath, which terminates at the junction with the tail on an annular disc. The axial filament is continued through the tail into the endpiece, and in the tail is surrounded by thick sheath. In some animals, newts and salamanders, the tail is surrounded by a spiral membrane, but this is not present in the human spermatozoon. The head of the spermatozoon is formed from the nucleus of the spermatid, the head-cap from the idiosome; the centrosomes of the spermatid pass to the neck, and the cytoplasm of the spermatid is transformed into the parts of the body and tail of the spermatozoon.

\section{The Female Reproductive Organs}

These consist of the two ovaries which produce ova, and the uterus with the Fallopian tubes and vagina which are continuous with it.

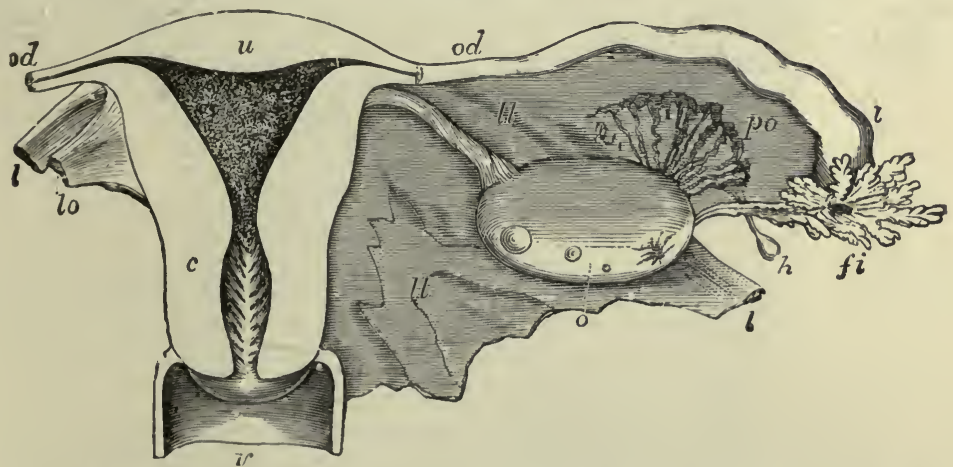

F1a. 554.-Diagrammatic view of the uterus and its appendages, as seen from behind. The uterus and upper part of the vagina have been laid open by removing the posterior wall; the Fallopian tube, round ligament, and ovarian ligament have been cut short, and the broad ligament removed on the left side; $u$, the upper part of the uterus; $c$, the cervix opposite the os internum; the triangular shape of the uterine cavity is shown, and the dilatation of the cervical cavity with the rugæ termed arbor vitæ; $v$, upper part of the vagina ; od, Fallopian tube or oviduct; the narrow communication of its cavity with that of the cornu of the uterus on each side is seen; $l$, round ligament; $l o$, liga. ment of the ovary; 0 , ovary; $i$, wide outer part of the right Fallopian tube; $f$, its fimbriated extremity; po, parovarium; $h$, one of the hydatids frequently found connected with the broad ligament. $\frac{1}{2}$. (Allen Thomson.)

The Ovary is composed of fibrous tissue (stroma) containing, near its attachment to the broad ligament, a number of plain 
muscular fibres. It is covered by a layer of cubical cells, called the germinal epithelium, which, in young animals, is seen dipping

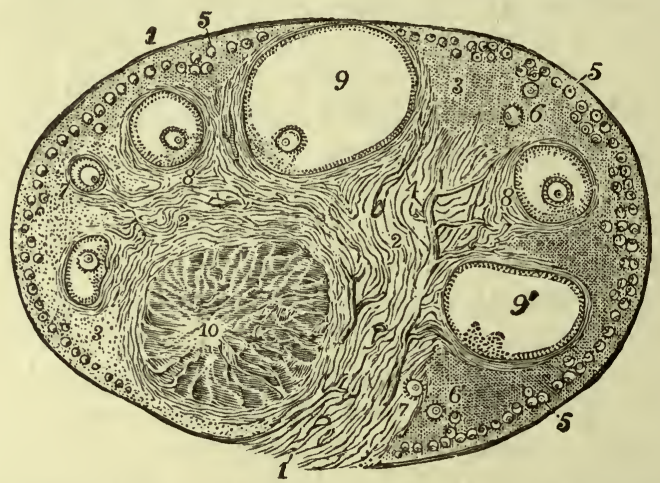

Fia. 555.-View of a section of the ovary of the cat. 1, Outer covering and free border of the ovary; $I^{\prime}$, attached border; 2, the ovarian stroma, presenting a fibrous and vascular structure; 3, granular substance lying external to the fibrous stroma ; 4, blood-vessels ; 5, primary oöcytes in their earliest stages occupying a part of the granular layer near the surface; 6 , oöcytes which have begun to enlarge and to pass more deeply into the ovary; 7 , oöcytes round which the Graafian follicle and tunica granulosa are now formed, and which have passed somewhat deeper into the ovary and are surrounded by the fibrous stroma; 8, more advanced Graafian follicle with the oöcyte imbedded in the layer of cells constituting the proligerous disc; 9 , the most advanced follicle containing the oöcyte, etc.; 9', a follicle from which the oöcyte has accidentally escaped; 10, corpus luteum. (Schrön.)

down, here and there, into the stroma. The stroma contains a number of yellow polyhedral cells similar to the interstitial cells of the testicle.

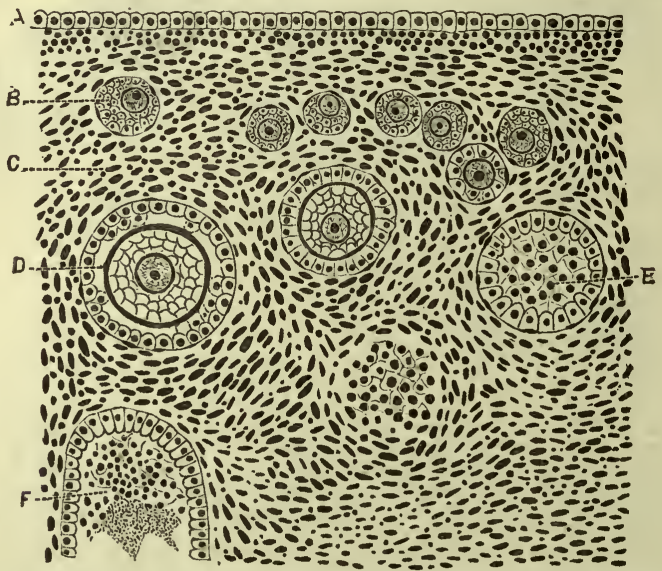

FIG. 556. - Section of the ovary of a cat. A, germinal epithelium; B, immature Graafian follicle ; C, stroma of ovary; D, zona pellucida surrounding the primary oöcyte; E, Graafian follicle showing lining cells; F, follicle from which the oöcyte has fallen out. (V. D. Harris.)

Sections of the ovary show that the stroma is crowded with a number of rounded cells, the oöcytes, derived from primitive germ 
cells, which, in the early stages, were intermingled with the cells of the germinal epithelium. There are also numerous vesicles of different sizes which are called Graafian follicles. The smallest follicles are near the surface, the largest are deeply placed, but as they expand they again approach the surface, and ultimately rupture upon it.

A Graafian follicle has an external wall formed by the stroma; this is lined internally by a layer of cells, derived from the germinal epithelium, which surrounds the oöcyte. At a later stage there are two layers of cells, one lining the cavity, and the other surrounding

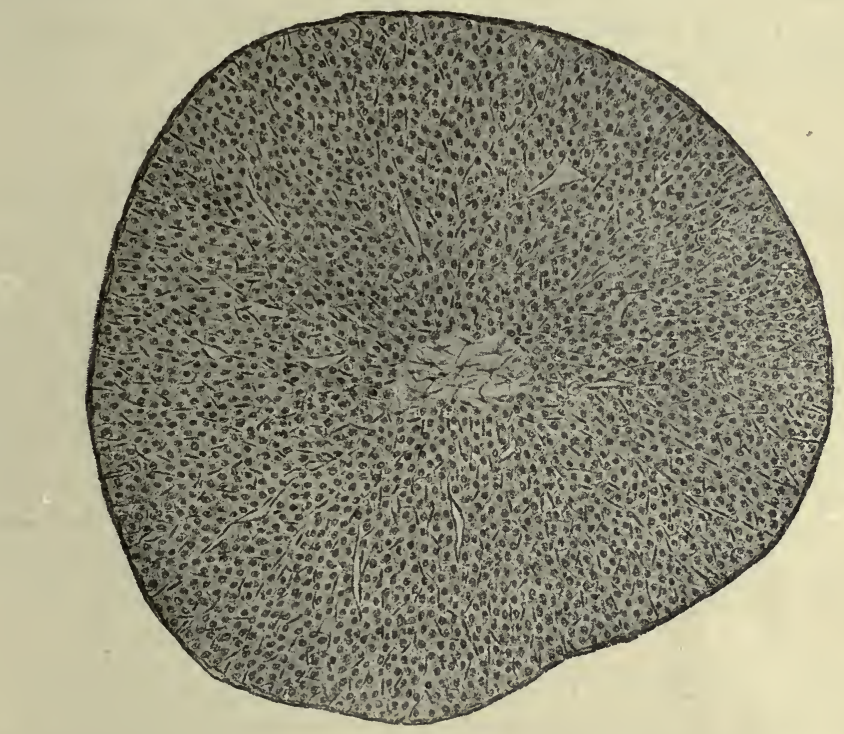

Fia. 557.-Corpus luteum of mouse, showing its formation completed. The central cavity is occupied by jelly-like connective tissue; the converging trabeculæ anastomose with one another so as somewhat to break up the columnar arrangement of the luteal cells. (Sobotta.) (From Schäfer's Text-book of Microscopic Anatomy.)

the oöcyte, but the two are close together. A viscid fluid collects between the two, and as the follicle grows, separates them.

The cells in each layer multiply, and are eventually arranged in several strata. The lining epithelium of the follicle is then called the membrana granulosa, and the heaped mass of cells around the oöcyte, the discus proligerus. The fluid increases in quantity, the follicle becomes tenser, and finally it reaches the surface of the organ and bursts; the oöcyte or ovarian ovum is thus set free; it enters the fringed end of the Fallopian tube and thence passes to the uterus. This process is called ovulation, and in the human female the ripening of an ovum occurs about once every four weeks. 
After the rupture of the Graafian follicle, it is filled up with what is known as a corpus luteum. This is derived from the wall of the follicle, and consists of columns of yellow cells developed from the hypertrophy of the epithelial cells of the membrana granulosa; it often contains at first a blood-clot in its centre. The strands of cells get folded and converge to a central mass of jelly-like connective tissue; between the columns there are septa of connective tissue with blood-vessels (fig. 557). The corpus luteum after a time gradually disappears; but if pregnancy supervenes it becomes larger and more persistent. The following table gives the chief facts in the life-history of the ordinary human corpus luteum, compared with that of pregnancy:-

\section{Ordinary \\ Corpus Luteum.}

\section{Corpus Luteum of Pregnancy.}

At the end of three weeks. One month

Two months.

Six months

Nine months .
Three-quarters of an inch in diameter; central clot reddish; convoluted wall pale.

Smaller ; convoluted wall bright yellow ; clot still reddish.

Reduced to the condition of an insignificant cicatrix.

Absent.
Larger; convoluted wall bright yellow; clot still reddish.

Seven-eighths of an inch in diameter; convoluted wall bright yellow; clot decolorised.

Still as large as at end of second month ; clot fibrinous ; convoluted wall paler.

One half an inch in diameter; central clot converted into a radiating cicatrix ; the external wall tolerably thick and convoluted, but without any bright yellow colour.

The ovarian ovum or primary oöcyte (fig. 558) is a large spheroidal cell surrounded by a transparent striated membrane called the zona

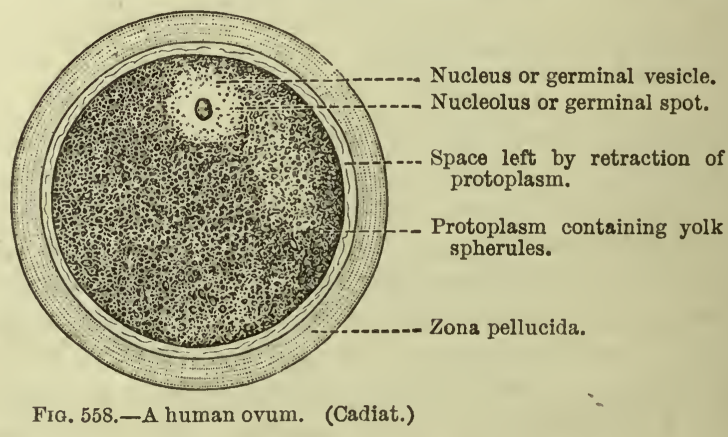

pellucida, or zona striata. The protoplasm is filled with large fatty and albuminous granules (yolk spherules), except in the part 
around the nucleus, which is comparatively free from them. It contains a nucleus, and usually one very well-marked nucleolus. The nucleus and nucleolus are still often called by their old names, germinal vesicle and germinal spot respectively. An attraction sphere, not shown in the figure, is also present, and a fine membrane, the vitelline membrane, immediately invests the protoplasm within the zona pellucida.

The oöcytes are developed from the primitive germ cells which in the earliest stages are interspersed amid other cells of the germinal epithelium. The primitive germ cells divide and produce oögonia; and by the division of the oögonia, primary oöcytes are formed (fig. 559). The oögonia and primary oöcytes sink into the stroma,

A
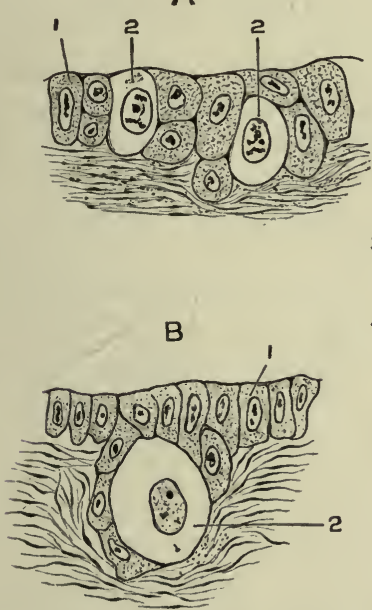

C

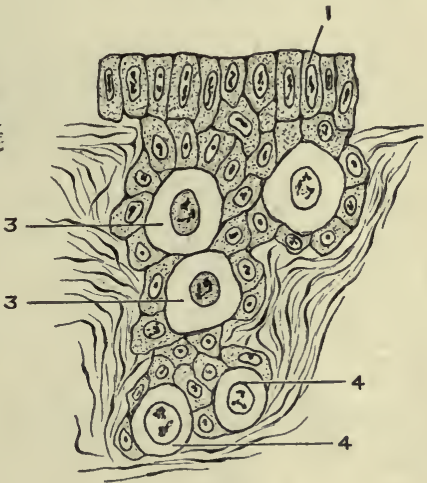

F1G. 559.-Diagram showing mode of development of primary oöcytes from primitive germ cells in mammalian ovary. 1, Germinal epithelium; 2, primitive germ cells; 3, oögonia ; 4, primary oöcytes. In A, two primitive germ cells are seen imbedded in the germinal epithelium. In $\mathrm{B}$, a primitive germ cell has descended into the stroma of the ovary accompanied by cells proliferated from the germinal epithelium which will become the cells of the membrana granulosa. In $\mathrm{C}$, the oögonia derived from primitive germ cells, and primary oöcytes produced by division of the oögonia, are seen. (After Bühler.)

surrounded by cells, produced by the proliferation of the germinal epithelium, which are destined to form the membrana granulosa and the discus proligerus of the Graafian follicles.

The Fallopian Tubes or oviducts which lead to the uterus have externally a serous coat from the peritoneum, then a muscular coat (longitudinal fibres outside, circular inside), and most internally a vascular mucous membrane thrown into longitudinal folds, and covered with ciliated epithelium.

The uterus consists of the same three layers. The muscular 
coat is, however, very thick, and is made up of two strata imperfectly separated by connective tissue and blood-vessels. Of these the thinner outer division is the true muscular coat, the fibres of which are arranged partly longitudinally, partly circularly. The inner division is very thick, its fibres run chiefly in a circular direction; the extremities of the uterine glands extend into its internal surface. It is in fact a much hypertrophied muscularis mucosæ. The mucous membrane is thick and consists of a corium of soft connective tissue, lined with ciliated epithelium; this is continued down into long tubular glands which have, as a rule, a convoluted course. In the cervix the glands are shorter. Near the os uteri the epithelium becomes stratified; stratified epithelium also lines the vagina.

\section{The Physiology of the Reproductive Organs}

The object of the ovary is to produce ova; this is known as ooggenesis. The object of the testis is to produce spermatozoa; this is known as spermatogenesis. The prodigality of nature in providing for the continuance of the species is well illustrated by the fact that at birth the human ovary contains about 70,000 immature oöcytes. Quite a small minority of these attain maturity, and get situated in Graafian follicles: many Graafian follicles, moreover, never burst; after attaining a certain degree of maturity, even during childhood, they atrophy more or less completely. On the average, one Graafian follicle ripens every four weeks, so that in the period between the onset of puberty and the menopause, say from fifteen to forty-five years of age, there is a possibility in the thirty intervening years of the production of about 400 ripe ova. Of these again a very small minority become fertilised. Still more is the lavishness of the provision illustrated in spermatogenesis; it has been calculated that in the semen ejaculated at an act of coitus there are more than two hundred million spermatozoa, and only one of these is needed for the fertilisation of an ovum.

Spermatogenesis.-The spermatozoa result from the division of the original germ cells, and the stages which have already been described in our account of the structure of the testis may bo represented in a diagrammatic form (see fig. 560).

The germ cell divides into spermatogonia which undergo several divisions, two of which are shown in the diagram. Each spermatogonium in the end grows and becomes a primary spermatocyte; it divides into two secondary spermatocytes, and each of these into two spermatids which develop into spermatozoa. In the division of the primary into the secondary spermatocytes, the mitosis is heterotype and the number of chromosomes is reduced to half the normal number. This phenomena is paralleled in the history of the 
oöcyte, and it will be convenient to postpone the histological details until we come to the oöcyte, where we shall see a diagram to represent the process.

The result is that the secondary spermatocyte and its descendants, the spermatids and spermatozoa, have only half the number of chromosomes characteristic of the species. The maturing of a spermatozoon thus occurs within the seminiferous tubes.

Oögenesis.-This occurs in the same general lines as spermatogenesis, but with some rather important differences of detail. In

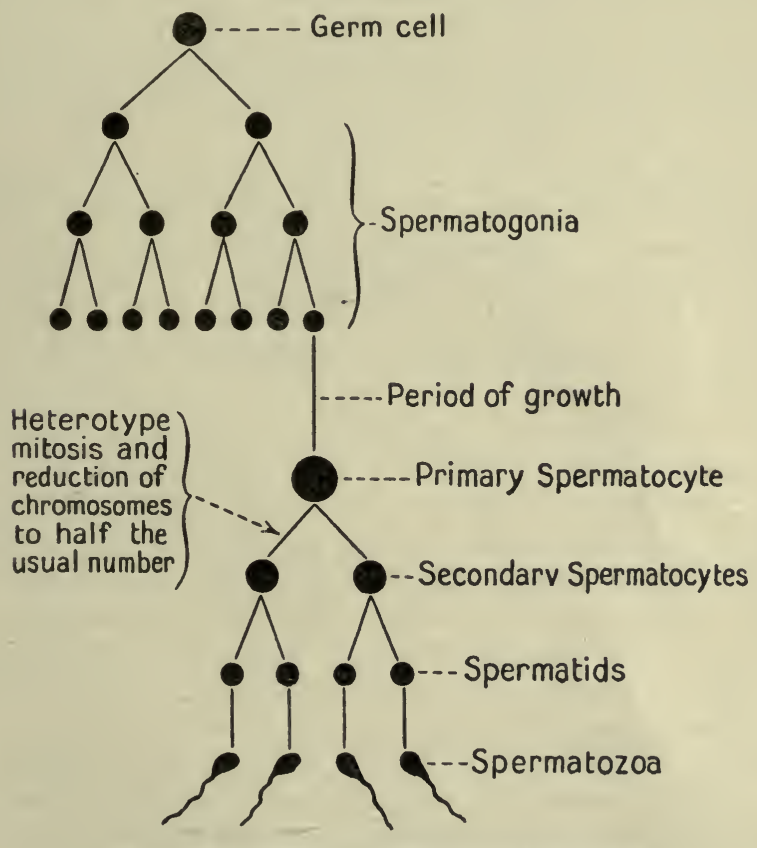

Fia. 560.-Diagram to illustrate spermatogenesis.

the first place, the changes in the ovary occur concurrently with certain changes in the uterus which result in menstruation. A second important difference is that the formation of the mature ovum occurs after the ovarian ovum has left the ovary, and is on its journey along the Fallopian tube to the uterus, previous to fertilisation. A third difference is one of size, the ovum being considerably larger than the spermatozoon.

We will take the phenomenon known as menstruation first. On the average once every four weeks the uterus becomes congested, and its mucous membrane thickened; finally some of the blood- 
vessels of the mucous membrane rupture and the escaping blood together with the secretion of the uterine glands, and some epithelial débris from the surface constitutes the monthly or menstrual flow. The amount of destruction of the mucous membrane varies a good deal, but it usually involves not only the surface epithelium, but extends into the interglandular tissue. The flow lasts for three or four days, and the amount of blood lost may be as much as 300 c.c. After the cessation of the flow, the mucous membrane repairs itself; this takes about a fortnight, and then after a brief period, generally not more than a few days, preparation for the next menstrual epoch begins once more. These phenomena are accompanied with a feeling of malaise, and in some cases with more or less pronounced pain.

Menstruation is absent during pregnancy, and as a rule also during the subsequent period of lactation.

There is no doubt that this uterine phenomenon is related to ovulation (that is, the discharge of ova from the ripe follicles); for menstruation begins at puberty when ovulation starts, and ceases to occur at the menopause when ovulation ceases ; an artificial menopause may be created by removal of the ovaries, and in certain cases it has been claimed that menstruation was resiumed after an ovary had been transplanted back again. This looks as if the relationship is not a nervous one. Moreover the monthly periodicity is the same in both processes. The period of sexual activity varies greatly in different animals; in some of the monkeys the monthly period occurs as in the human female; but in many of the lower animals the times of sexual activity and sexual desire are much more widely separated. In animals such times are known popularly as heat or rut, and technically as the cestrus. It is the pre-cestral period in animals which is homologous with menstruation, while œstrus is the period of sexual desire, when coitus may occur.

Although there is no doubt of the relationship between ovulation and menstruation, there has been much discussion as to which occurs first. But in animals, where the phenomena of the pre-œstral, œstral, and post-œstral periods can be followed more easily, there is no doubt that ovulation occurs, or the ova reach maturity after or at the very end of the uterine flow; that is to say, ovulation takes place during œstrus and not during the pre-œstral period, which is the homologue of menstruation. This has been shown more particularly in the case of the dog, the sheep, and the pig.

Many more or less fantastic theories have in the past been put forward to explain menstruation. There is, however, but little doubt that its object is the preparation of the uterine lining for the reception of an ovum, either because a monthly regeneration maintains it in a condition of irritability which enables it to respond 
promptly to the stimulus of an ovum by the formation of a decidua, or as Pflüger taught, because it provides a raw surface on which the ovurn is easily grafted.

The ovum, after it is liberated from the ovary by the rupture of a Graafian follicle, enters the Fallopian tube, the cilia on the fimbriæ of which are the main instruments for the transportation. It travels along the Fallopian tube, and finally reaches the uterus, and again this is accomplished by ciliary action. During this journey, which probably occupies some days, it becomes mature, it is fertilised, and some of the early steps in further development may also occur.

Maturation of the Ovum.-We have already seen that the germ cells which form the ova are at first imbedded in the germinal

A

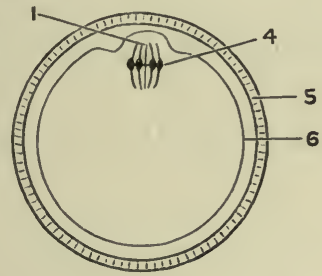

B

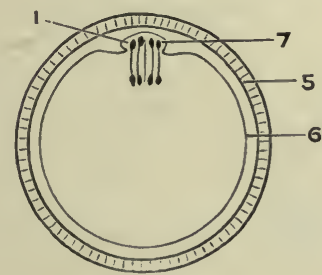

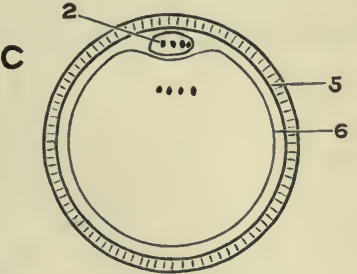

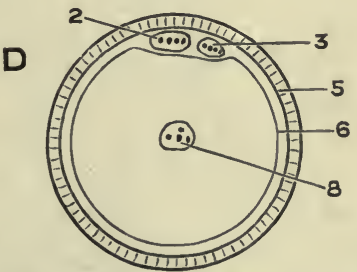

FIG. 561.-Diagram showing the formation of the polar bodies (maturation of the ovum). A, B, and C show stages in the formation of the first polar body by heterotype mitosis. $A$ is a primary oücyte at the commencement of mitosis, when only half the usual number of chromosomes appear. $\mathrm{C}$ is a secondary oöcyte; it has no distinct nucleus, because no resting-stage occurs; after the separation of the first polar body, the chromosomes which remain in the secondary oücyte at once rearrange themselves on a new spindle. D is the mature ovum, with the female pronucleus and the two polar bodies. 1, First polar bud; 2, first polar body; 3 , second polar body; 4 , chromosomes on spindle of primary oöcyte; 5 , zona striata; 6 , vilelline membrane; 7 , daughter chromosomes in first polar bud; 8 , female pronucleus.

epithelium, from which they pass into the stroma of the ovary, and then by division and growth they form oögonia; from the oögonia, primary oöcytes are developed, and these become enclosed in Graafian follicles. The process by which the primary oöcytes become converted into mature ova is known as maturation; this consists essentially of a double mitotic division of the oöcyte, each division producing two unequal parts. The first division produces a secondary oöcyte and the first polar body; the second division, which takes place 
without any resting-stage, results in the formation of the mature ovum and the second polar body. Thus, when the two divisions are completed, the mature ovum and two polar bodies lie inside the zona pellucida.

The unequal division is associated with an eccentric position of the spindle. At each division one end of the spindle projects on the surface with a little surrounding protoplasm, and it is this small nodule which becomes the polar body (see fig. 561).

One of the essential features of this maturation is the halving of the number of chromosomes in the nucleus. When maturation commences in the primary oöcytes, an achromatic spindle is formed in the usual way; but instead of the ordinary number of chromosomes appearing at its equator, only half that number are seen: for example, if eight be the normal number of chromosnmes, only four appear. Further, each chromosome is not a slender V-shaped loop, but a short, thick rod, or ring, or group of four particles. Neither

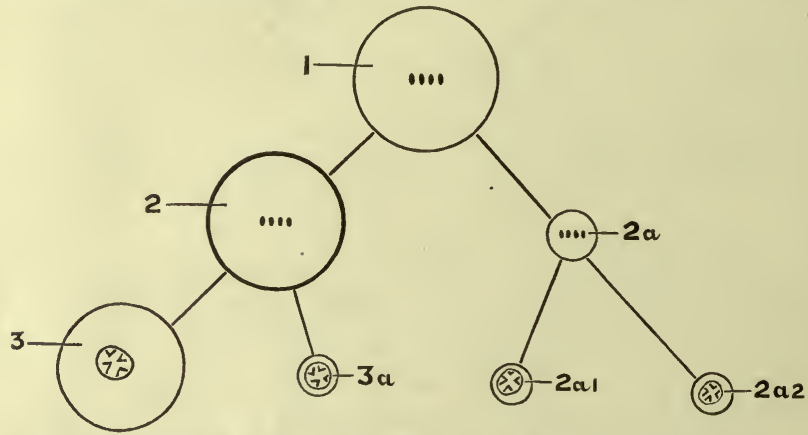

FIG. 562.-Diagram showing the stages in the maturation of the ovum when the first polar body divides. 1, Primary oöcyte; 2 , secondary oöcyte; $2 a$, first polar body; 3 , mature ovum; $3 a$, second polar body; $2 a 1$, and $2 \alpha 2$, daughter cells of the first polar body.

does it split longitudinally in the usual way, but transversely; and at the end of the process the secondary oöcyte and the first polar body both contain four chromosomes. This form of mitosis is known as heterotype, in contradistinction to the ordinary form which is called homotype. The second division which produces the mature ovum and the second polar body is homotype, and the final result is that each of the segments into which the primary oöcyte divides contains half the number of chromosomes present in the parent germinal cell. In some cases, the first polar body divides at the same time that the second polar body is formed; the whole process is represented in the schema in fig. 562. The nucleus of the mature ovum is known as the female pronucleus.

It should be added that the mature ovum is destitute of a entrosome; that is lost with the formation of the polar bodies. 
We are now in a position to construct a diagram similar to that in fig. 560, which represents the history of the male reproductive element. The history of the female element from the original germ cell is shown in fig. 563.

As in the male the germ cell divides and subdivides, and in this way successive generations of oögonia are produced; two of the subdivisions are shown in the diagram. Each oögonium then grows, and becomes a primary oöcyte, which is the ovarian ovum

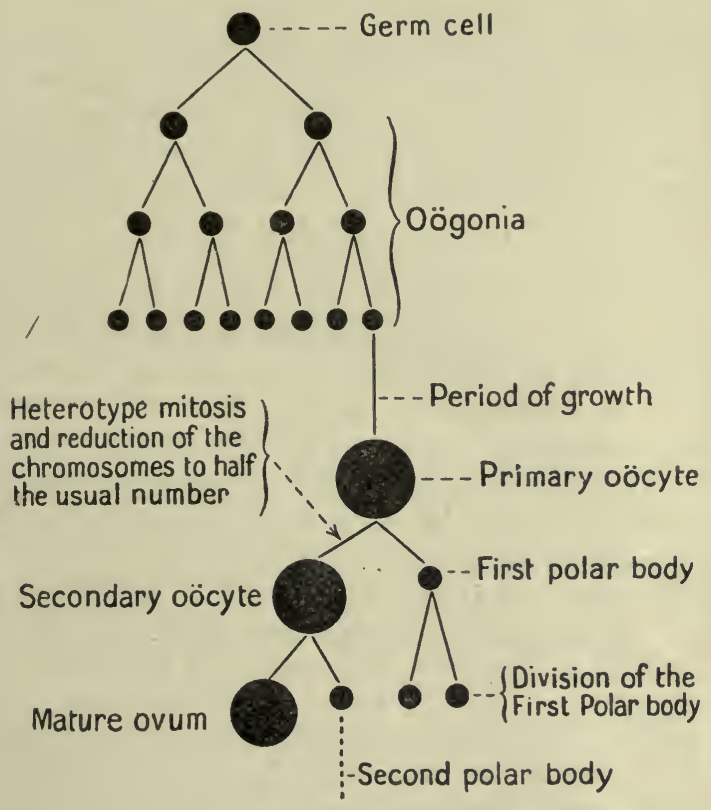

Fı. 563.-Diagram to illustrate oögenesis.

within the Graafian follicle. Its maturation, which occurs outside the ovary, is represented in the lower part of the diagram, which is a repetition of what is also seen in fig. 562 .

Each primary spermatocyte gives rise to four spermatozoa of -equal value. Each primary oöcyte gives rise to four cells, which are of unequal value; one of these is the mature ovum, the other three are the minute polar bodies which may be regarded as abortive ova. The mitosis which results in the halving of the normal number of chromosomes occurs in each sex at the same stage, namely, in the formation of the secondary spermatocyte, and of the secondary oöcyte respectively. 
The meaning of the polar bodies has been the subject of much speculation; it is supposed that the female cell casts out certain constituents in order to make room for the addition to it of material from another individual, namely, the male. Some animals multiply without the intervention of the male sex, or the intervention occurs at long intervals with many intermediate generations; this is known as parthenogenesis. One must therefore suppose that the female cell has within it a male component which can be transmitted to future generations. The ovum of these animals only extrudes one polar globule. It may therefore be that the second polar body contains the hereditary male element which is retained in parthenogenesis, but in animals which multiply sexually this is got rid of prior to the inception of the new male element.

This brings us to the stage in our story when both male and female elements are ripe and ready for union. Logically we should next study how the union is accomplished. But first we must step into a bye-path, and before leaving the ovary and testis enquire whether these organs have any other functions than those we have already discussed. This may best be done under the following new heading.

\section{Internal Secretions of Ovary and Testis}

The operation of castration, that is, the removal of the essential generative organs, naturally leads to a loss of reproductive power, but it has other effects of a more general kind on the organism, which mainly influence what are known as the secondary sexual characters. This effect is believed to be due to the lack of certain internal secretions formed by testis and ovary respectively.

Testis.-Some years ago Brown-Séquard, then an old man of seventy-two, stated that the subcutaneous injection of testicular extracts into himself produced marked rejuvenating effects. More recently Poehl has described the beneficial stimulating effects of a substance prepared from the testis, which he terms spermine. He has assigned to it the formula $\mathrm{C}_{5} \mathrm{H}_{14} \mathrm{~N}_{2}$, but Dixon has shown that it is a mixture of proteins with other organic and inorganic materials. The supposed tonic effects of such injections are regarded with great suspicion, and temporary benefit, if it does occur, is mainly attributable to suggestion.

The principal evidence upon which the assumption rests that the testis forms an internal secretion, is derived from the effects of castration, or from cases in which the testes do not descend into the scrotum. If the operation of castration is performed before puberty, the reproductive apparatus which is left (vesiculæ seminales and prostate, but not the penis) atrophy ; the secondary sexual characters (growth of hair on the face, deepening of the voice, etc.) do not develop; the body remains infantile, but never assumes female characters. The body, however, grows, and in some cases there is overgrowth of the skeletal and adipose tissues.

In animals there is corroborative evidence of the same nature. 
Thus in the cock castration arrests the development of the comb and spurs; in the stag of the antlers. In the eland and in horned cattle where both sexes have horns, their growth is not inhibited by castration, though their shape may be affected (Marshall). In Herdwick sheep, where the males are horned and the females hornless, it has been shown that the presence of the testes is essential, not merely for the initiation but also for the continuance of horn growth. Castration stops further horn growth forthwith, and at every stage of development.

Ligature of the vas deferens leads to atrophy of the seminiferous tubules, whilst the interstitial cells are not affected, and the secondary sexual characters develop as usual. It is on this ground that most investigators agree that the interstitial cells of the testis are the source of the internal secretion. These colls have all the appearances of secreting cells, and their full development coincides with the first appearance of spermatogenesis. Transplantation of a testis in an abnormal position in the body cavity is followed by development of the secondary sexual characters, and the viow is generally held that the internal secretion acts chemically on the parts concerned, rather than through the intermediation of the nervous system.

The Prostate.-As already stated, the removal of the testis leads to atrophy of the prostate, but the converse has been stated to be also true, and that in dogs prostatectomy causes the testes to lose their functional activity. Attempts to repeat these effects, by other observers, have, however, failed, and as Marshall points out, it is unlikely, on phylogenetic grounds, that the functions of the essential reproductive organ should depend upon the presence of an accessory gland of comparatively late appearance in the history of evolution.

The Ovary-Extirpation of the ovaries prevents the onset of puberty and the occurrence of menstruation, but produces no noticeable effects on the general form and appearance of a woman. Ovariotomy after puberty brings menstruation to an end, and there may be slight atrophy of the breasts, uterus, and external genital organs; this, however, does not always occur.

In animals the same operation prevents the occurrence of the œstral cycle, but the "periods" continue to recur if one of the ovaries is grafted into the abdomen.

The ovary does not appear to have such far-reaching effects on general metabolism as the testis possesses; its internal secretion seems to act especially upon the uterus. Marshall's observations on dogs, and Heape's on monkeys, favour the view that the interstitial cells of the ovary are responsible for the production of a hormone which initiates the ostrous cycle, and if this is the case the occurrence of menstruation in the human female is attributable to the action of the same cells. 
The Corpus Luteum.-Special interest attaches to the function of the corpus luteum which is formed in the Graafian follicle after the exit of the ovum. We have already seen that this structure attains a great size if pregnancy ensues. The luteal cells are formed from the lining cells of the follicle and not from the interstitial cells just alluded to. It has been generally assumed that the corpus luteum forms a hormone or hormones, and the functions which have been assigned to these chemical messengers are four in number.

1. To affect the uterus and bring about there the oestrous or menstrual cycle. This view may be dismissed at once; the hypothesis is negatived, first, because it does not explain the occurrence of the first œstrous cycle in the young animal, and secondly, because injection of luteal extracts does not induce uterine changes. The cells concerned in that function are, as already stated, the interstitial ovarian cells.

2. To inhibit the ripening of Graafian follicles during pregnancy (Loeb). This may be an effect, but it is hardly a function.

3. To assist in some way the fixation and early development of the impregnated ovum in the uterus. This view is much favoured by most authorities, and the principal evidence in its favour is that removal of the ovaries, or even destruction of the corpus luteum shortly after pregnancy has begun, brings that process to an end; the same operation later in pregnancy has no effect upon the development of the fœtus or the subsequent act of parturition.

4. To stimulate the growth of the mammary glands during the early months of pregnancy. Prolonged search for the secretory nerves of the milk glands have failed to reveal their presence. Cases have occurred in which severe injuries to the nervous system preclude any possible nervous connection between the generative organs and the mammary glands; nevertheless, the latter grow and ultimately produce milk. Such considerations point to the conclusion that the correlation must be chemical rather than nervous. A few years ago Starling and Miss Lane Claypon thought that the hormone in question must be secreted by the fœtus, because the injection of fœetal extracts in rabbits led to a hypertrophy of the mammary tissue. The hypertrophy, however, is imperfect, and doubt was felt in many quarters whether this could be the whole explanation, because mammary evolution occurs in many virgin animals in which ovulation is not followed by pregnancy; moreover, in many cases enlargement of the breasts occurs at each menstrual epoch. The view is now advanced that a hormone is secreted by the corpus luteum, which is an inciting cause of the early growth of mammary tissue. The close correspondence between the stages in the development of the corpus luteum and of the mammary glands has been clearly brought out 
by the recent work of $O^{\prime}$ Donoghue. One must, however, point out that as a rule there is no corpus luteum in the ovaries during menstruation, or during the pre-œstral period in sows, when the mammary glands show signs of growth and congestion.

The therapeutic use of ovarian extracts appears, from the evidence available, to stand in the same uncertain position as that of testicular extracts.

If the reader refers back to the chapter on the ductless glands he will find that some of these (thymus, pituitary, etc.) influence the formation of the generative organs.

The structure of the mammary glands and the composition of milk have already been treated at length in our chapter on Foods (see pp. 481-486).

\section{Fertilisation}

We are now in a postion to resume the thread of the history of the further development of a new individual. The next step is the union of the male and female gametes, that is to say, of the spermatozoon and the mature ovum.

The act of coitus or copulation is associated in both sexes with much psychical excitement, and with the phenomenon of erection (see p. 313). The spermatozoa are thus deposited at the entrance of the uterus, and by means of the flagellar movement of their tails they make their way against the stream of ciliary movement into the Fallopian tubes, where they are found in a living condition for many days. It is here that they meet the mature ovum; but fertilisation or impregnation only requires the entrance of one spermatozoon into the ovum. By means of its sharp head cap the spermatozoon pierces the zona pellucida, and the head, neck, and possibly part of the body, enter the substance of the ovum, where they undergo transformation, and are converted into a male pronucleus with an attendant attraction sphere and its centrosome. The male pronucleus contains the same number of chromosomes as the female pronucleus, for the mitosis which occurs when the primary spermatocyte divides to form two

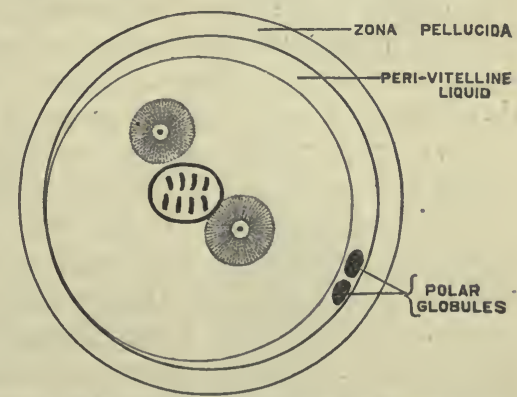

Fia. 564.-The fertilised ova or blastosphere, showing its new nucleus and attraction spheres; the yolk grauules have been omitted. secondary spermatocytes, is a heterotype mitosis, in which only half the usual number of chromosomes appear; and consequently the secondary spermatocytes, and their descendants the spermatids, also contain only half the typical number of chromosomes. These are retained in the spermatozoa, which are produced by modification of the spermatids, and they reappear in the male pronucleus. 
After the male pronucleus has formed in the substance of the mature ovum, it approaches the female pronucleus, and when the two pronuclei fuse, fertilisation is completed. The nucleus which results from the fusion-the first segmentation nucleus-contains the typical number of chromosomes, half being derived from the female and half from the male germinal element. When the fertilisation is completed, the segmentation nucleus is accompanied by two attraction spheres with their centrosomes (see fig. 564); one of these spheres is introduced with the male pronucleus, and the other probably originates from it by division, since the centrosome of the ovum is lost during maturation.

Loeb has suggested that the action of the spermatozoon is essentially chemical, because in certain invertebrate animals (for instance, sea urchins) he has been able to produce artificial parthenogenesis by purely chemical methods. In his latest work, he placed the ova in dilute acetic or formic acid; by this means, a membrane is formed upon the surface of the egg-cell as it is in normal fertilisation; if the ova are then transferred to concentrated sea water for a short time and then placed in ordinary sea water, they segment and produce normal larvæ. He considers that the spermatozoon brings with it enzymes or other chemical substances which excite the ovum in the same way as the chemical reagents mentioned.

The changes by which the fertilised ovum is transformed into the young animal may take place either inside or outside the body of the parent. If they take place inside the parent, as in mammals, including the human subject, the ovum is small, and the nutriment necessary for its growth and development is derived from the surrounding tissues and fluids of the mother. If the development takes place outside the parent's body, as in birds, the egg is larger; it contains a large amount of nutritive material called the yolk, and it may, in addition, be surrounded by sheaths of nutritive substance. Thus, in the hen's egg, the yellow part alone is comparable with the mammalian ovum, and the larger part of that is merely nutritive substance. Upon the yolk is a whitish speck, the cicatricula, which is a small mass of protoplasm, about $\frac{1}{6}$ of an inch in diameter. In the cicatricula lies the nucleus or germinal vesicle, and it is this small mass of protoplasmic substance which divides and grows to produce the chick; the yolk and the surrounding white being used as food.

Ova such as the hen's, in which only a small part, the cicatricula, divides and grows, are called meroblastic. Small ova, with little food yolk, such as the human ovum, divide completely during development, and are called holoblastic, but numerous gradations occur between the two extreme types.

The further development of the individual systems of organs by 
which the embryonic rudiments are converted into the more fully developed condition in which they are found at birth is a subject fully treated in works on anatomy, embryology, and obstetrics, and we shall not go into those matters here. The nutrition of the embryo and the circulation of its blood are, however, matters of physiological moment, so that it will be necessary to refer to the origin of the fœtal membranes, as it is by their means that nutrition is carried on. Let us, however, first briefly take up the early stages in development.

\section{Segmentation}

After fertilisation is completed, the ovum divides into two parts; each of these again divides, and so on till a mulberry-shaped massthe morula -is formed. It consists of a large number of small cells and it is enclosed together with the polar bodies, in the zona pellucida. The polar bodies soon disappear; indeed in many cases they have vanished long before the morula is completed. A cavity soon appears in the morula, which thus becomes converted into a blastula or blastocyst. The cells which form the peripheral wall of the blastula assume a more or less cubical form, whilst those which lie in the interior and form the inner cell mass are irregular in outline, and they are grouped together at one pole of the blastula (see figs. 566, 567). At this period the blastula is unilaminar, except at the region where the inner cell mass is situated; but soon the cells of the inner mass extend round the cavity and the wall of the cyst becomes bilaminar, the outer layer being called epiblast and the inner hypoblast. A little later the mesoblast or third layer of the blastoderm grows and extends between the epiblast and hypoblast over the whole area of the vesicle.

That portion of the mesoblast which lies immediately at the sides of the neural groove becomes partially separated from the rest, and at the same time divided into cuboidal blocks, the protovertebræ or mesoblastic somites (see also fig. 570). The more laterally situated part of the mesoblast

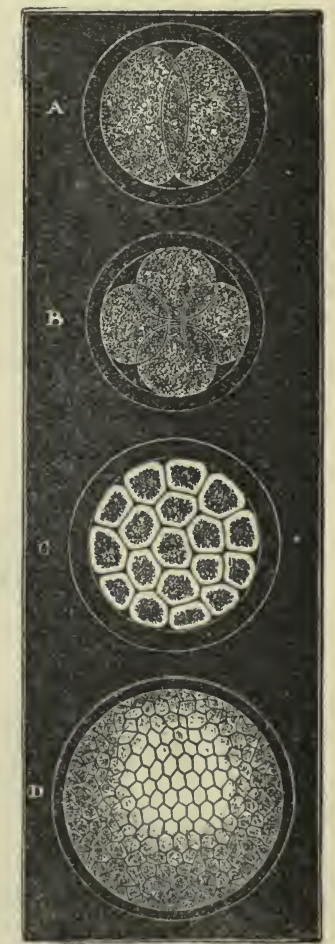

Fia. 565.-Diagrams of the early stages of cleavage of the ovum. (Dalton.) constitutes the lateral plates, and the narrow strand of mesoblastic cells which connects the lateral plate on each side with the 
protovertebral somites is the intermediate cell mass. Soon after its formation the lateral mesoblast is cleft into two layers, and the space which appears between the two layers is called the colom (figs. 568, 569). The outer or somatic layer of the mesoblast adheres to the epiblast; the two together form the somatopleur. The inner or splanchnic layer fuses with the hypoblast to form the splanchnopleur.

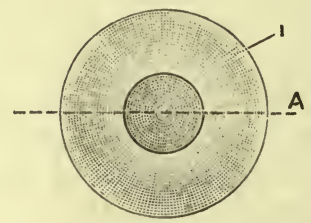

FIG. 563.-Diagram of a surface view of a young mammalian blastula. 1, Germinal area. A, line of section represented in fig. 567.

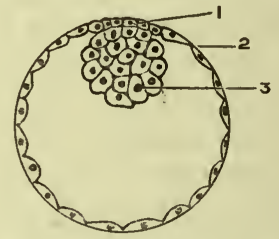

Fin. 567. $\rightarrow$ Diagram of a section of the mammalian blastula shown in fig. 566 along the line A. 1, Germinal area; 2, epiblast; 3 , inner cell mass.

Whilst the mesoblast is extending and cleaving, the neural ridges, which are longitudinal epiblastic upgrowths on each side of the middle line, gradually grow in height, and their free margins turn inwards and fuse together. This fusion commences in the cervical region, and extends forwards and backwards, and when it is completed the neural groove is converted into a closed tube, the neural

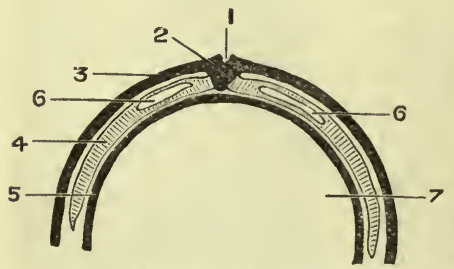

Fig. 568.-Diagram of a transverse section through a mammalian blastoderm after the formation of the three primary layers. 1, Primitive groove; 2, primitive streak; 3 , epiblast; 4, mesoblast; 5 , hypoblast ; 6 , cœlom ; 7 , archenteron.

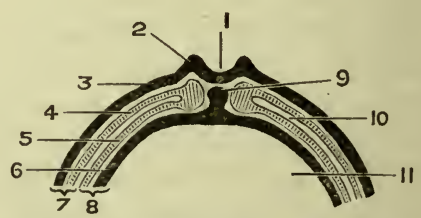

Fia. 569.-Diagram of a transverse section through a mammalian blastoderm, show. ing the neural ridges. 1, Neural groove ; 2 , neural ridge; 3 , epiblast; 4 , sumatic mesoblast; 5 , splanchnic mesoblast; 6 , hypoblast; 7 , somatopleur; 8 , splanch. nopleur ; 9 , notochord ; 10 , cœlom; 11 , archenteron.

canal, which is the central canal of the nervous system. In the embryo at this period there are, therefore, three cavities: (1) The neural canal lined by epiblast; (2) The cœlom or space in the mesoblast; (3) The archenteron within the hypoblast. While these changes are in progress the embryonic area begins to fold off from the rest of the embryo. A sulcus appears all round the margins of the area, and over this sulcus the area bends forwards, backwards, 
and laterally. It looks as if some constricting agent had been placed round the margin of the area, and that afterwards the area above the constriction, and the area below had gone on growing rapidly. In this way, the embryo is clearly separated into two parts, an upper, the fœtus, and a lower, which becomes the appendages of the fœetus.* The anterior part of the folded embryonic area is known as the head fold, the posterior as the tail fold, and the two are connected together on each side by the lateral folds.

The portion of the archenteron enclosed in the fœtus forms the primitive gut. The part contained in the head fold is the fore-gut, that in the tail fold is the hind-gut, and the remainder is the midgut (fig. 572).

The constriction where the body of the fœtus becomes continuous with the remaining structures, is known ultimately as the umbilicus. It remains pervious till birth, and through it the mid-gut is connected with the remainder of the archenteron (which is henceforth called the yolk-sac) by a narrow hypoblastic tube, the vitello-intestinal duct (fig. 571, 10).

The portion of the mesoblastic cavity enclosed in the fœtus is called the body cavity. It gradually differentiates into the pericardial, pleural and peritoneal cavities, which are eventually entirely separated from one another.

Before the neural groove is closed and becomes the neural canal, the hypoblast beneath the middle of the groove becomes thickened to form a longitudinal ridge (fig. 569). This ridge is the notochord or

* Among many anatomists and obstetricians the word ovum is applied to the foctus and its appendages. It should be properly restricted to

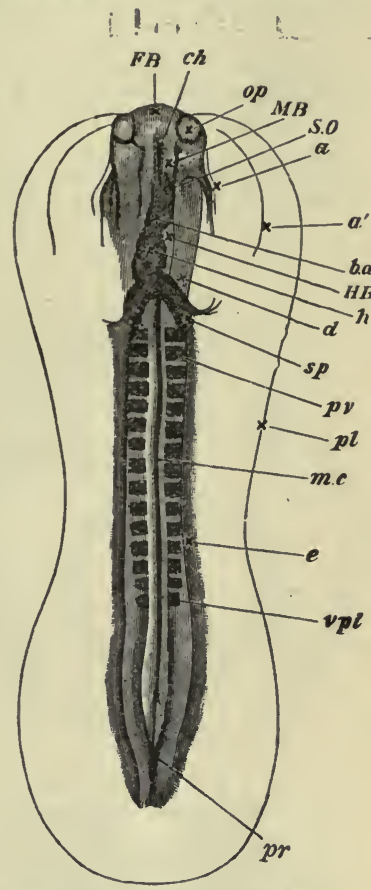

Fis. 570,-Embryo chick (36 hours), viewed from beneath as a trans. parent object (magnified). $p l$, Outline of pellucid srea; $F B$, fore-brain, or first cerebral vesicle: from its sides project $o p$, the optic vesicles; SO, back ward limit of somatopleur fold, "tucked in" under head; $a$, head fold of true amnion; $a^{\prime}$, re. flected layer of amnion, sometimes termed "false amnion"; $s p$, backward limit of splanchnopleur folds, along which run the omphalomesenteric veins uniting to form $h$, the heart, which is continued forwards into $b a$, the bulbus arte. riosus; $d$, the fore-gut, lying behind the heart, and having a wide cres. centic opening between the splanch. nopleur folds; $H B$, hind-brain ; $M B$, mid.brain ; $p v$, protovertebra lying behind the fore-gut; $m c$, line of junction of medullary folds and of notochord; $c h$, front end of noto. chord ; $v p l$, vertebral plates; $p r$, the primitive groove at its candal end. (Foster and Balfour.) the female gamete. After development has commenced the term embryo should be employed, and that word includes the embryo proper which we call the foctus, and its appendages. 
primitive skeletal axis. It soon separates from the remainder of the hypoblast, and forms a round cord, which lies at first immediately beneath the neural groove, and afterwards beneath the neural tube, extending from the anterior end of the primitive gut, which lies beneath that region of the neural tube which afterwards becomes the mid-brain, to the caudal end of the embryo (figs. 571, 572). The notochord is subsequently obliterated by the formation of the vertebræ from the protovertebral somites.

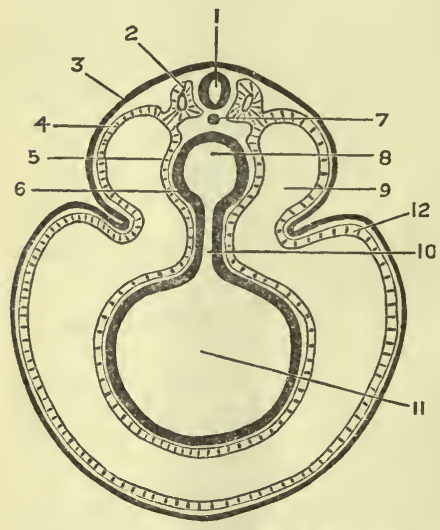

FiG. 571.-Diagram of a transverse section through a mammalian embryo at the period when the folding off of the fotus has commenced. 1, Neural tube; 2, protovertebral somite; 3 , epiblast; 4 , somatic mesoblast; 5 , splanchnic mesoblast: 6 , hypoblast; 7 , notochord; 8 , primitive alimentary canal ; 9 , colom 10, vitello-intestinal duct; 11 , yolk-sac; 12 , lateral fold of amnion.

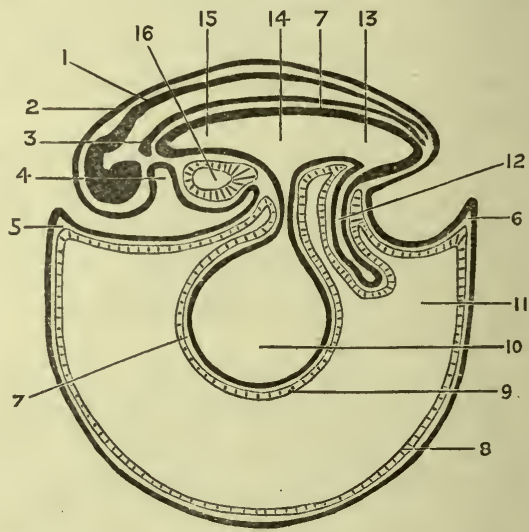

Fia. 572.-Diagram of a longitudinal section of a mammalian embryo at the period when the folding off of the foetus has commenced. 1, Neural tube ; 2, epiblast ; 3 , notochord ; 4, stomadoeal space ; 5 , head fold of amnion; 6 , tail fold of amnion; 7 , hypoblast; 8 , somatic mesoblast; 9 , splanchnic mesoblast ; 10 , yolk-sac ; 11, colom ; 12, allantois ; 13 , hind-gut; 14 , mid-gut; 15 , fore-gut; 16 , pericardium.

The fœtus contains parts of all the layers of the blastoderm, and from each of these certain organs are formed as indicated in the following list:-

1. From Epiblast.- $a$. The epidermis and its appendages.

b. The nervous system, both central and peripheral.

c. The epithelial structures of the sense-organs.

$d$. The epithelium of the mouth, and the enamel of the teeth.

e. The epithelium of the nasal passages.

$f$. The epithelium of the glands opening on the skin and into the vestibule of the mouth, and nasal passages.

$g$. The muscular fibres of the sweat-glands.

2. From Mesoblast. - $a$. The skeleton and all the connective tissues of the body.

$b$. All the muscles of the body (except those of the sweat-glands).

c. The vascular system, including the lymphatics, serous membranes, and spleen. 
d. The urinary and generative organs, except the epithelium of the bladder and urethra.

The Somatic mesoblast forms the osseous, fibrous, and muscular tissues of the body-wall, including the true skin.

The Splanchnic mesoblast forms the fibrous and muscular wall of the alimentary canal, the vascular system, and the urino-genital organs.

3. From Hypoblast. - $a$. The epithelium of the alimentary canal from the inner sides of the teeth to the anus, and that of all the glands (including liver and pancreas) which open into this part of the alimentary tube.

b. The epithelium of the respiratory cavity.

c. The epithelium of the Eustachian tube and tympanum.

$d$. The epithelium lining the vesicles of the thyroid.

e. The epithelial nests of the thymus.

$f$. The epithelium of the bladder and urethra.

\section{The Decidua and the Foetal Membranes}

When the uterus is ready for the reception of an embryo it is lined by a greatly hypertrophied mucous membrane; this is called the decidua, because, after the delivery of the child, a portion of it comes away from the uterus with the other membranes.

The ovum has been fertilised in the Fallopian tube, and the embryo, by the time it reaches the uterine cavity, has usually reached the stage of a morula or blastula. It rapidly eats its way into the substance of the decidua which closes over it, obliterating the opening through which it passed, and thus the embryo becomes imbedded in the membrane, which thereupon becomes separable into three parts. 1 . The part between the embryo and the muscular wall of the uterus, the decidua basalis or serotina. 2. The part between the embryo and the uterine cavity, the decidua capsularis or reflexa. 3. The remaining part is called the decidua vera.

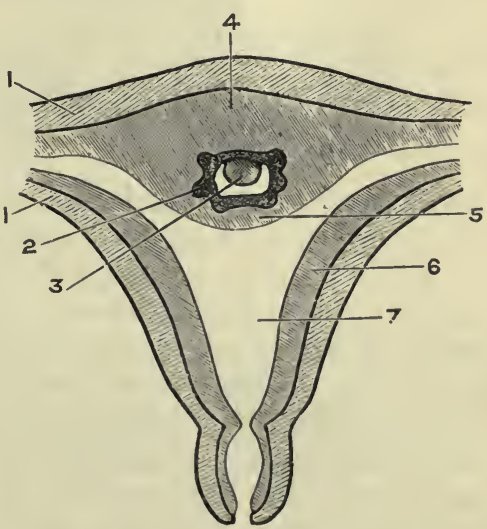

Fro. 573.-Diagram representing the relation of the developing embryo to the decidua at a very early stage. 1, Uterine muscle; 2 , epiblast of embryo; 3, inner cell mass of embryo; 4 , decidua basalis; 5 , decidua capsularis; 6 , decidua vera $; 7$, cavity of uterus.

Between the decidua capsularis and the decidua basalis lies the embryo, which speedily becomes differentiated into the fœtus and 
its membranes. The outermost of the fœtal membranes is the chorion; this is covered with vascular villi, which dip into the decidua capsularis and basalis. Inside the chorion is the amnion, a closed sac, which surrounds the embryo and is attached to its ventral wall at the umbilicus. The amnion is filled with fluid, the amniotic fluid in which the foetus floats, and it forms a sheath for the umbilical cord by which after a certain time, the fotus is attached to the inner surface of the chorion, or outer embryonic membrane. The umbilical cord

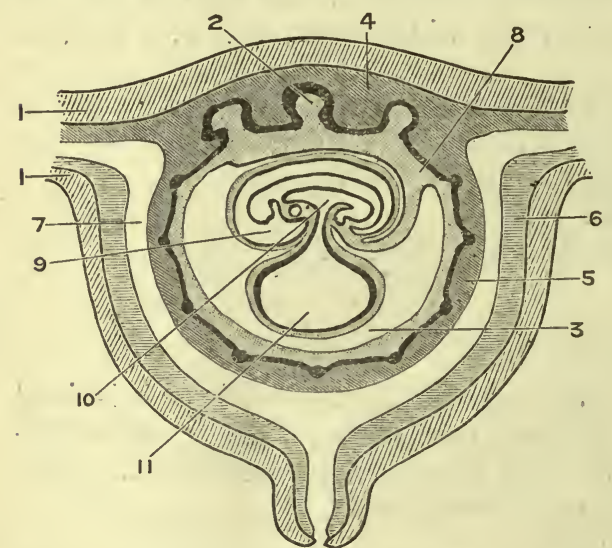

FIG. 574.-Diagram representing a later stage of development than that shown in fig. 573. 1, Uterine muscle; 2 , villi of chorion of embryo; 3 , colom; 4 , decidua basalis ; 5 , decidua capsularis ; 6 , decidua vera $; 7$, cavity of uterus ; 8 , allantois $; 9$, amnion cavity $; 10$, primitive intestine; 11, yolk-sac. contains not only the bloodvessels which pass between a specialised portion of the chorion, which forms the fœtal part of the placenta, and the fotus, but also the remains of the yolk-sac, and the duct by which it is connected with the intestine of the fœtus. In animals which develop outside the mother's body (for instance, birds) the yolli-sac is much larger and is the great source of nutriment during growth.

As the embryo grows, the decidua capsularis is expanded over its surface, and as the growth continues the uterine cavity is gradually obliterated, and the decidua capsularis is forced into contact with the decidua vera, with which it fuses.

As the decidua is merely thickened mucous membrane, it naturally contains glands which become enlarged as the decidua thickens. It was believed, at one time, that the villi of the chorion entered the glands, but this is now known to be incorrect. The villi enter the interglandular substance, and, in the human subject, the glands of the decidua capsularis eventually disappear entirely. In the decidua basalis and the decidua vera the superficial portions of the glands also disappear; their deep portions remain in an almost unchanged condition, and furnish the epithelium for the regeneration of the glands and the lining of the uterine cavity after parturition. The intermediate parts of the glands in the decidua vera and the decidua basalis become very much enlarged, and form a stratum of the decidua called the spongy layer, and ultimately this layer is converted into a series of clefts, and it is along the line of these clefts that the decidua is separated at birth. 
In some mammals in which the connection between the chorion and the decidua is less intimate than in the human subject, the glands persist to a greater or less extent, and secrete a fluid called uterine milk, which is absorbed by the chorion.

The portion of the decidua which undergoes the greatest change is the decidua basalis. In it a number of large blood spaces are formed, and these are separated into masses or cotyledons by fibrous strands.

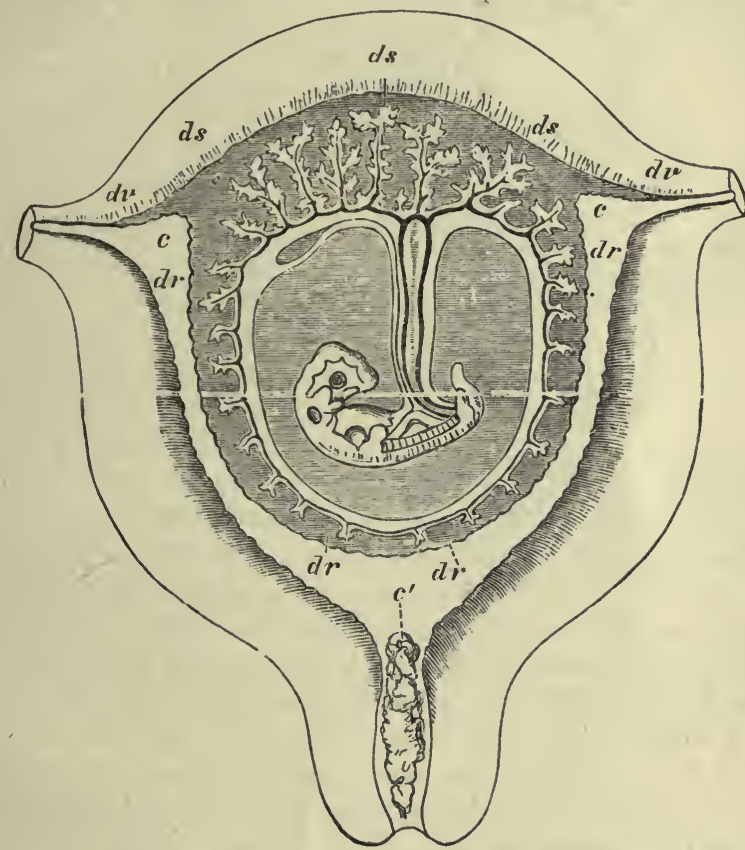

Fra. 575.-Diagrammatic view of a vertical transverse section of the uterus at the seventh or eighth week of pregnancy. $c, c, c^{\prime}$, Cavity of uterus, which becomes the cavity of the decidua, opening at $c$, $c$, the cornua, into the Fallopian tubes, and at $c$ into the cavity of the cervix, which is closed by a plug of mucus; $d v$, decidua vera; $d r$, decidua reflexa, with the sparser villi imbedded in its substance; $d s$, decidua basalis or serotina, involving the more developed chorionic villi of the commencing placenta. The foetus is seen lying in the amniotic sac; passing up from the umbilicus is seen the umbilical cord and its vessels passing to their distribution in the villi of the chorion; also the pedicle of the yolk-sac, which lies in the cavity between the amnion and chorion. (Allen Thomson.)

The cotyledons are penetrated by chorionic villi, and it is this conjunction of chorionic villi and decidua basalis which produces the placenta. The blood-vessels of the chorionic villi are formed by the mesoblastic covering of the allantois, another fœtal outgrowth. Its origin from the hind-gut is shown in fig. 572 .

The placenta is the organ of fœtal nutrition and excretion, and at full term it is seven or eight inches across and weighs nearly a pound. Its blood sinuses are filled with maternal blood, which is carried to 
them by the uterine arteries and away from them by the uterine veins. Into these blood-filled spaces the vascular fotal villi project; hence

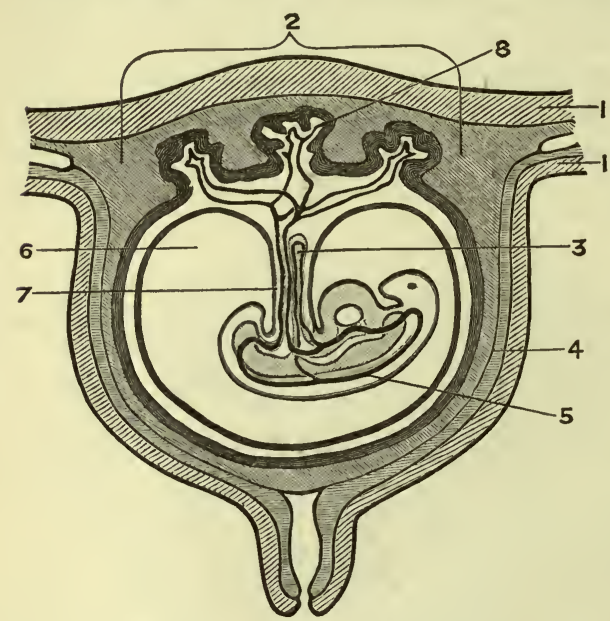

FIG. 576.-Diagram representing a later stage of development of membranes and placenta than that shown in fig. 574. 1, Uterine muscle; 2, placenta ; 3, yolk-sac : 4 , fused decidua vera and capsularis ; 5 , primitive bloodvessel of embryo; 6 , amnion cavity (outer surface of amnion is fused with inner surface of chorion); 7 , umbilical cord ; 8 , fœtal villus in placenta. it is easy for exchanges to take place between the fœtal and the maternal blood, though the two blood-streams never mix together. Oxygen and nutriment pass from the maternal blood through the coverings of the fotal vessels into the fœtal blood, and carbonic acid, urea, and other waste products pass in the contrary direction. The fœtal blood is carried to the placenta by the umbilical arteries, which are the terminal branches of the aorta of the fœtus; these pass to the placenta by the umbilical cord, and the blood is returned, through the cord, by the umbilical vein.

The amniotic fluid consists of water containing small quantities of protein, urea, and salts. It is an exudation from the fotal and the maternal blood, and the urea in it comes from the fotal urine which is poured into the amniotic cavity in the later part of pregnancy. Its function is mainly mechanical; it supports the embryo on all sides, and protects it from blows and other injuries to the abdomen of the mother, and from sudden irregular contractions of the abdominal walls.

Intra-vitam Staining.-Within the last few years Goldmann of Freiburg has made observations in which he has injected animals (rats and mice) with certain blue solutions of which pyrrhol-blue may be taken as an example. The animal suffers from no ill effects, the only outward change being that a white rat becomes a blue rat. When the animal is subsequently killed, the stain is found embodied in the granules of specific cells throughout the body. Although it circulates in the blood, no blood corpuscle takes it up, nor has it any effect on the vascular lining. In the skin it is found in the fixed connective tissue cells, but chiefly in free phagocytic cells in the lower layers of the cutis and subcutis. But these migratory cells appear also in every internal organ (except the nervous system), and always in connection with interstitial fibrous tissue; they 
occur in muscles, glands, tendons, and especially in serous membranes. On account of their affinity for pyrrhol-blue they were originally termed pyrrhol cells, and it seems probable that they originate in the bone marrow.

By means of such intra-vitam stains one can further differentiate the Kupffer-cell of the liver, the reticulum cell of lymph glands and spleen, the interstitial cell of the testis, the follicular cell in the maturing follicles of the ovary, the cortical cell of the suprarenal, the epithelial covering of the choroid plexuses, and the cells which line the convoluted tubules of the kidney.

When pregnancy occurs in the stained animal, the appearance and behaviour of the placenta are most striking; the blue colour disappears from the skin and is concentrated in the uterus, and in time the latter, forming a centre of attraction for the dye, ultimately dispossesses all the remaining tissues of their blue. In the uterus it is the free cells of the decidua basalis where the stain is mainly found. In quite early stages the stained cells penetrate into the primitive placenta and cast off their stained granules, which are snatched up by fœtal cells in the way nutritive material is. But when once the placenta has attained maturity, the dye is found only in the fotal cells which form the layer which separates the maternal and fœtal tissues. The footus itself remains perfectly colourless, the stain not being able to penetrate this protective barrier. Further research has shown another important point, for the same cells which vigorously absorb the vital stain store also glycogen, fat, and hæmoglobin temporarily before these substances pass into the foetal circulation. The avidity of such cells for the dye is thus connected with their functional activity in relation to really nutritive material; the importance of vital staining in embryological research is therefore apparent.

Equally important are its applications to pathological research, but into this aspect of the question it will be beyond our purpose to pass.

\section{The Feetal Circulation}

We shall not enter into the complex manner in which the heart and blood-vessels of the fœetus develop from the embryonic rudiments; but when these are fully formed the circulation of the blood is found to differ considerably from that which occurs after birth. It will be convenient to begin its description by tracing the course of the blood, which, after being carried to the placenta by the two umbilical arteries, has returned, oxygenated and replenished, to the fœetus by the umbilical vein.

It is at first conveyed to the under surface of the liver, and there the stream is divided, - a part of the blood passing straight on to the 
inferior vena cava, through a venous canal called the ductus venosus, while the remainder passes into the portal vein, and reaches the inferior vena cava after circulating through the liver. Whether, however, by the direct route through the ductus venosus or by the roundabout way through the liver,-all the blood which is returned

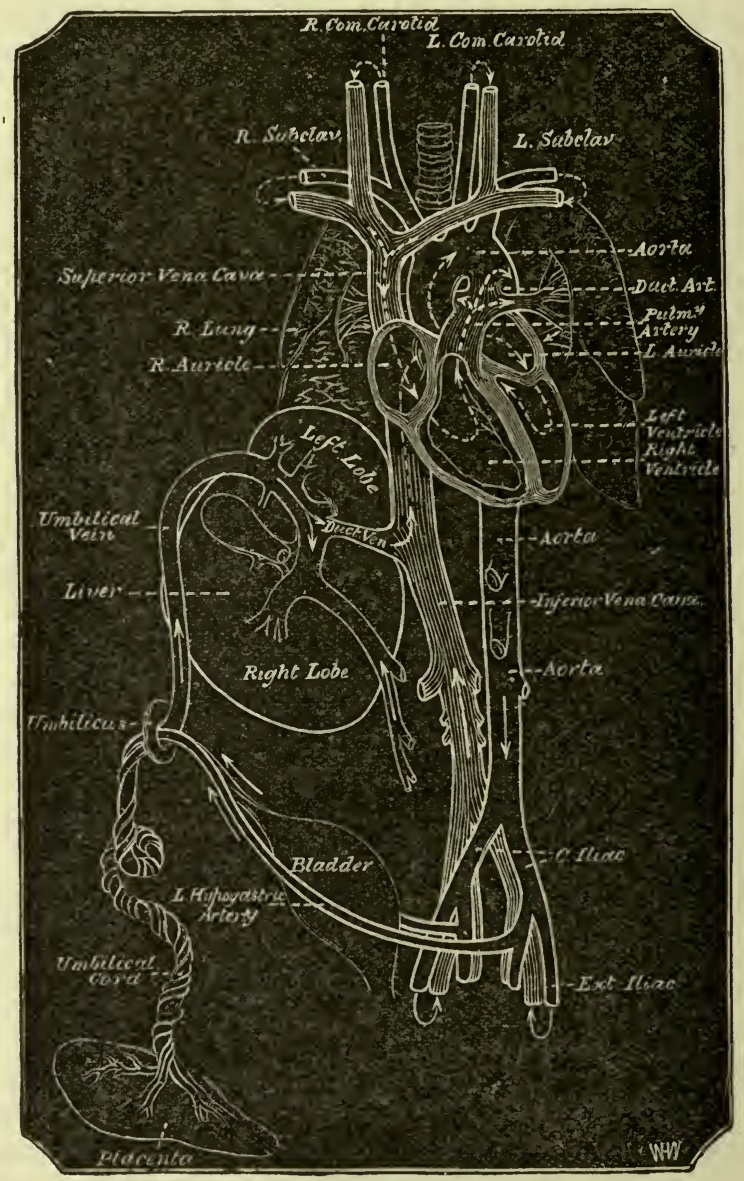

FIG. 577.-Diagram of the Fœtal Circulation.

from the placenta by the umbilical vein reaches the inferior vena cava at last, and is carried by it (together with the blood-from the lower part of the body and lower limbs) to the right auricle of the heart, into which cavity is also pouring the blood that has circulated in the head and neck and arms, and has been brought to the auricle by the 
superior vena cava. It might be naturally expected that the two streams of blood would be mingled in the right auricle, but such is not the case, or only to a slight extent. The blood from the superior vena cava - the less pure fluid of the two-passes almost exclusively into the right ventricle, through the auriculo-ventricular opening, just as it does in the adult; while the blood of the inferior vena cava is directed by the fold of the lining membrane of the heart, called the Eustachian valve, through the foramen ovale into the left auricle, whence it passes into the left ventricle, and out of this into the aorta, and thence to all the body, but chiefly to the head and neck. The blood of the superior vena cava, which, as before said, passes into the right ventricle, is sent out from there in small amount through the pulmonary artery to the lungs, and thence to the left auricle, by the pulmonary veins, as in the adult. The greater part, however, does not go to the lungs, but instead, passes through a canal, the ductus arteriosus, leading from the pulmonary artery into the aorta just below the origin of the three great vessels which supply the upper parts of the body; and there meeting that part of the blood of the inferior vena cava which has not gone into these large vessels, it is distributed with it to the trunk and other parts-a portion passing out by way of the two umbilical arteries to the placenta. From the placenta it is returned by the umbilical vein to the under surface of the liver, from which the description started.

\section{Parturition}

During pregnancy the uterus and its contents increase in size, and we have already alluded to the changes in its mucous membrane or decidua, and the formation of the placenta; the principal factor in the distension of the uterus is the accumulation of the amniotic fluid. The muscular wall of the uterus also hypertrophies; this is in part due to the formation of new muscular fibres, and in part of the increase in size of the pre-existing muscular fibres. The muscular wall is one of immense strength.

The fœtus "comes to term" in the human subject on the tenth menstrual epoch after conception; this averages about 280 days after the last menstruation. Delivery is the result of uterine contractions or "labour pains"; the liquor amnii is thus forced downward and presses the membrane formed by the fused amnion and chorion through the cervix of the uterus which is gradually distended. When the distension is sufficient the membrane ruptures, and the amniotic fluid escapes. The os is then fully distended, and the fœtal head enters the pelvis; the pains become more frequent and energetic, and the voluntary muscles of the abdomen are brought into play, so that ultimately the new-born child is expelled to the 
exterior. The process usually lasts some hours, but the time is much prolonged (ten, twenty, or even more hours) in the birth of a first child. The child is still connected with the placenta by the umbilical cord, which is about 20 inches long, and this connection should not be severed for a few minutes in order that as much blood as possible may be aspirated from the fœtal part of the placenta into the child as breathing commences.

After the child is expelled, the contractions of the uterine walls recommence after a lapse of twenty to thirty minutes, and the placenta is separated and forced out. The separation extends through the decidua along the line of the stratum spongiosum, and the fused chorion, amnion and decidua turned inside out, follow the placenta to which they are attached, constituting, with the placenta, the after-birth.

After the umbilical cord is tied and separated, the umbilical arteries inside the child become filled with blood-clot, and ultimately they are converted into fibrous cords, the so-called obliterated hypogastric arteries, and at the same time the allantois is also converted into a fibrous strand, the urachus, which extends from the apex of the bladder to the umbilicus.

The hæmorrhage from the uterus which accompanies and follows the after-birth may be profuse at first, but under normal circumstances is soon checked by the firm contraction of the uterine walls.

Although it has been shown that delivery may occur when all nerves connecting the uterus with the central nervous system are cut through (see p. 721), the contractions of the organ are normally influenced reflexly through the nervous system. Stimulation of various sensory nerves will produce contractions of the pregnant uterus, and premature delivery may occur as the result of mental and physical disturbances.

The determining factor which produces the labour pains at a particular date has been much discussed; some suppose it may be maternal in origin, such as a degenerative condition set up in the placenta or decidua, whereas others consider the initial impulse may come from the foetus, which secretes certain products that stimulate uterine contraction.

After delivery, the uterus undergoes reduction in size at a fairly rapid rate. This has been attributed to fatty degeneration, but of this there is but little evidence. The theory at present most in vogue to explain "involution of the uterus" is that the process is one of autolysis due to the action of intracellular digestive enzymes. Whilst it is occurring, the urine of the mother contains a good deal of creatine, a substance which is normally absent from that secretion. It has been supposed that this substance originates from the rapid destruction of the uterinomusele. : It has, however, been shown that creatine occurs after delivery even if the uterus is amputated, so that the muscular creatine cannot then be the source of the urinary 
creatine; there is some evidence that the creatine is associated in some way with the metabolism of the mammary gland.

The atrophy or involution of the uterus which occurs at the menopause appears also to be produced in the same way, and it has been suggested, with some reason, that the symptoms exhibited at that period of life may be in part explained as due to the absorption of the products of the autolysis of the uterine tissue.

\section{DraTII}

We have now completed the task we set ourselves, and having arrived at the new-born child have reached the point in the life cycle from which we set out. The bearers and transmitters of the germ plasm, its hosts, the parents, pass away in due course, making room for their successors who live, repeat the process, and likewise die in their turn.

It is not altogether inappropriate to conclude a book which deals with life, by a few sentences on Death, which forms the final chapter for each individual. As the prime of life is past, signs of old age begin to appear, the eyes become feeble, the hair becomes grey, the cartilages calcify, the muscles become weaker, digestion gets feebler, and metabolism in every way more and more imperfect. If this continues, life is ultimately terminated by natural death, in which the functions get weaker and weaker and finally cease. Death from old age is, however, comparatively rare; the common cause of death is accident, in which term we include disease. In the activity of youth many a disease is vanquished, but as the powers of resistance diminish with increasing years, some ailment usually upsetting more particularly some important organ will ultimately find the body unable to repel its attack.

\section{"That ends this strange, eventful history."}

\section{COMPLIMENTS}

OF

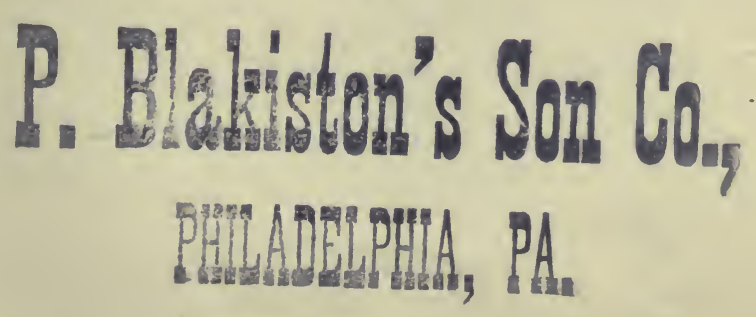





\section{N D E X}

A.

Abderhalden's feeding experiments, 545

Abdominal muscles, action in respiration, 357

Abdominal reflex, 711

Abducens nerve, 663,674

centre, 674

Aberration,

chromatic, 830

spherical, $i b$.

A biuretic products, 519

Abrin, 475

Absorption

of carbohydrates, 544

fats, 546, 547

food, 543 et seq.

proteins, 544

by the skin, 608

Acapnia, 402

Accelerator nerves, 144

uriuæ, 580

Accessory auditory nucleus, 676

Accommodation of eye, 820,824 et seq.

defects of, 828,831

mechanism of, 825

Acotonæmia, 549

Acetyl, 415

Achromatic spindle, 16, 876

Achromatin, 10

Achromatopsia, 848

Achroo-dextrin, 412, 505

Acidxmia, 542, 619

Acids in gastric juice, 511

Acld-albumin, 433

Acidosis, 542, 619

Acini of secreting glands, 496,498

Acoustic tubercle, 670

Acrolein, 415

Acroniegaly, 343

Acrylic series, 414

Adamantoblasts, 58,59

Adamkiewicz reaction, 425,429

Adam's apple, 800

Adaptation in sensations, 774

Addison's disease, 341, 342

Adenase, 594

Adenine, 432, 593

Adenoid or lymphoid tissue, 35

Adipose tissue, 28, 33-35. See Fat cells, 34,35

development, 33

situations of, $i b$.

structure, $i b$.

uses, 84

vessels and nerves, 34,35

Adrenaline, 342

administration of, 541

effect of, on salivary gland, 503

897

\section{Alimentary Canal.}

Adsorption, $328 n$.

Aërobic, 438

Aërotonometer, 363

Esthesiometers, 77 .

Affective mode in consciousness, $75 \mathrm{~S}$

tone, 763

Affenspalte, 736

Afferent fibres when entering the spinal cord, 703

Afferent nerves, 73,144 et seq.

projection system, 700

root-cells, 194

After-birth, 894

After-images, 844

After-sensations, 764

Agglutinin, 470

Agraphia, 809

Air,

atmospheric, composition of, 392

breathing, 358

changes by breathing, 392

complemental, 359

quantity breathed, $i b$.

reserve, $i b$.

residual, $i b$.

tidal, $i b$.

transmission of sonorous vibrations through, 794

undulations of, conducted by external ear, 795

Air-calorimeters, 631, 633

Air-sacs, 351, 352

Air-tubes. See Broncht.

Airy's discovery of astigmatism, 829

Alanine, 417, 422

Albumin, 424, 427, 433, 601

absence of glycine from, 422

acid, 433

alkali, 433

character of, 515

egg, 433

of blood, 454

Albuminates, 433

Albuminolds, 428, 429

Albuminomieter, Esbach's, 601

Albuminous alveoli, 499

Albuminous substances. See Proteins.

action of gastric fluid on, 519

Albumins, 424, 427

Alcapton, 602

Alcaptonuria, 602

Alcohol as an accessory to food, 490

Alcohols, monatomic, 408

Aldehyde, 407

Aldoses, 407

Alimentary canal, 492 et seq.

glycosuria, 538

nerves of, 563 
Alkall-albumin, 433 properties of, $i b$. Allantoin, 592

Allantois, development of, 886

Allochiria, 777

Alloxan, 592

Allyl alcohol, 415

Alveolar air apparatus, 373-375

Alveoli, 498.500

Amacrine cells, 816

Amino-acids, 416, 433, 545, 603

Amino-caproic acid, 417

Amino-oxypyrimidine, 421

Ammonia, 421, 588, 589

cyanate of, isomeric with urea, 583

Amnion, 886, 888, 890

Amniotic cavity, 888

fluid, 888,890

Amœbæ, 5, 11, 867

Amœboid movements, 11 et seq., 86

colls, 5,13

colourless corpuscles, 14,455

cornea-cells, 813

protoplasm, 11

Tradescantia, 12, 13

Amyloids or starches, 412

action of pancreas and intestinal glands, 519 of saliva on, 513

Amylolytic enzymes, 440

Amylopsin, action of, 519

Anabolic nerve groups, 203

Anabolic phenomena, 612

Anacrotic pulse, 293

Anaërobic, 438

Anaphylaxis, 478

Anderson on the auto-genetic theory, 150, 151

Anelectrotonus, 171, 173

Angina pectoris, 312

Angio-neuroses, 311

Angular convolution, 693

Angulus opticus seu visorius, 823

Animal cell, structure of, 7 et seq.

Animal hest. See Heat and Temperature.

Antons, 322

Ankle-clonus, 712

Annulus of Vieussens, 196, 251, 302

Anosmatic animals, 786

Antagonistic muscles, reciprocal action of, 714

Antero-lateral ascending tract, 658

Antero-lateral descending tract, 650

Antidromic, 303

Anti-enzymes, 443

Antllytic secretion, 503

Antithrombin, 448, 450, 451

Antitoxin, 474, 475

Anvil bone, 789

Aphasia, 733, 809

Apnœea, origin of, 379

Apoplexy, 689

Aqueduct of Sylvius, $644,645,663,671$

Aqueductus Fallopil, 790

Aqueous humour, 811

Arbor vitæ, 681

Archenteron, 884 et seq.

Archipallium, 646, 647,698

Arcuate fibres, 668

Area cheiro-kinæsthetic, 741

germinal, or embryonic, 883

glosso-kinæsthetic, 741

Areas, of Cohnheim, 65

intermedlate, 742,743

primary, $i b$.

terminal, $i b$.

Areolæ, 29, 48, 49

Areolar tissue, 28 et seq.
Arginase, 587

Arginine, 420, 422

Argyll-Robertson pupil, 834

Arteria centralis retinæ, 815,820

Arteriæ rectæ, 570

Arterial blood-pressure, 277, 280

venous blood, difference between, 371

Arterial tension in asphyxia, 391

Arteries, 216 et seq.

bronchial, 353

circulation in, velocity of, 281

coronary, 237

development of, 891

distribution, 216

elasticity, 265

interlobular, 569

muscularity, 266

nerves of, 218

nervous system, Influence of, 311

pressure of blood in asphyxia, 391

pulse, 290 et seq.

renal, ligature of, 580

rhythmic contraction, 266

structure, 217 et seq.

umbllical, 894

velocity of blood-flow in, 281

Arterioles, 264, 278

Articulate sounds, classification of, 808

vowels and consonants, $i b$.

Articulation positions, 808

Artifacts, 8

Artificial respiration, 383,387

Aryteno-epiglottidean fold, 805

Arytenoid cartilages, 797,805

effect of approximation, 800

movements of, $i b$.

Arytenold muscle, $799,801,802$

Ascaris, ovum of, 11, 17

Ascending tubule of Henle, 567

Aspergillus niger, 545

Asphyxia, 305, 389 et seq.

causes of death in, 390

conditions of the vascular system in, 389 , 390

symptoms, 389

tracings of, 391

Assimilation, power of, 6

Association centres, 740,741

fibres, 698,740

tracts, 704

Astigmatism, 829

Atmospheric air. See Alr.

composition of, 392

pressure in relation to respiration, 385

Atropine, effect of, on heart, 253

on salivary gland, 503

Attraction sphere, $7,10,16$

Atwater-Benedict calorimeter, 633

Auditory area, 738

Auditory nerve, 663,676

diagrams of, 676,677

origin, 676

word centre, 809

Auerbach's plexus, $79,554,563$

Auricles of heart. See Heart.

Auricular diastole, 232, 233

Auricular systole, $i b$.

Auriculo-ventricular bundle, 213, 257

valves. See Heart valves.

Autogenetic nerve theory, 150

Auto-intoxication theory of the ductless glands, 332

Autolysis, 136, 440

Autolytic enzymes, 440

Autonomic nervous system, 196 et seq. 
AvogADRo's LAW.

Avogadro's law, 326, 392

Axipetal conduction, law of, 191

Axis-cylinder of nerve-fibre, 74,185

Axons. See Nerves.

\section{B.}

13abinski's sign, 714

Bacilli, types of, 438

Bacterial action in intestinal digestion, 525

Bacterio-lysin, 473, 475

Bacterium lactis, 414

Barcroft's tonometer, 366

Barnard's cardlometer, 248

Basal ganglia, 687,726

Basllar membrane of ear, 792, 793

Basophlle cells, 457

Batteries and keys, 87 et seq.

Daniell cell, 87,88

Bausteine, 545, 627

Bayliss, mammalian heart, 245

observations on vaso-dilator nerves of dogs, 303

on adsorption, $328 n$.

on pancreatic secretion, 520

Bechterew, nucleus of, 677

Beckmann's differential thermometer, 326

Beef-tea, the making of, 489

Beer's experiments on accommodation of the eye, 827

Bellini's ducts, 567, 568

Bell's experiments on spinal nerve roots, 156

Bence-Jones' protein, 601

Beri-beri, 491

Bernard's discovery in the liver, 331, 536.539 experiment on independent muscular irrita. billty, 83

on pancreatic secretion, 523

Bert's experiments on crossing of nerves, 161

Bethe's autogenetic theory, 150

Betz cells, 697

Bezold's ganglion, 255

Bicuspid valve, 213

Bidder's ganglion, 255

Biedermann's flutd, $83 n$.

Bllaminar blastoderm, 883

Bile, 525, 527, 531 et seq., 602

absorption by lymph, 536

analyses of human, 532

canaliculi, 530

captllaries, $i b$.

characters of, 531

constituents of, 532

digestive propertles, 525

doubtful antiseptic power, 533

expelling mechanism, 535

human, analyses of, 532

influence of, on fat absorption, 548

mixture with chyme, 534

mucin, 532

pigments, 533

process of secretion, 53

quantity secreted, $i b$.

salts, 532

secretion and flow, 531, 532

specific gravity, 532

uses, 533

Bilirubin, 465, 533

Blliverdin, 533

Bimolecular reactions, 329

Binocular colour-mixture, 846

Binocular vision, 856,857

13iot's respiration, 405

Blpolar nerve-cells, 155, 182 et seq., 786
BLOOD-CORPUSCLES, RED.

Birth, changes after, 894

Biuret reaction, 425

"Black-water fever," 603

Bladder, epithelium of, 22

Bladder, urinary. See Urinary bladder.

Blastocyst, blastoderm, blastula, bilaminar, $\$ 83$ et seq.

Blastosphere, 881

"Blaze current," 849

Blind spot, 834,849

Blocking, 257

Blood, 28, 29, 60, 61, 444 et seq.

agglutinating action of, 476

amino-acids in, 545

artertal and venous, difference between, 215, 371

bactericidal power of, 473

buffy coat, 447

carbonic acid in, 371,375

circulation of, 227 et seq.

in the foetus, 891

local pecullarities, 312

schema of, 229

coagulation, $61,137,446$ et seq.

colour, 61,444

colouring matter, 444

relation to that of bile, 465

corpuscles or cells of, $6,7,60,444$. See Blood. corpuscles.

red, 454

white or colourless, 456

crystals, 462 et seq.

extractive matters, 454

fatty matters, 454

fibrin, $61,137,447$

separation of, 448

flow, velocity of, 281

gases of, 361 et seq., 396 et seq.

globulicidal power of, 473

hæmoglobin. See Hæmoglobin.

lymph, relation to, 821

nitrogen in, 362, 363, 371

odour or halitus of, 444

oxalated, 450

oxygen in, $362,365,372$

oxyhæmoglobin. See Oxyhæmoglobin.

photographic spectrum of, 462,463

plasma, $371,444,452$

proteins of, 452

quantity, 444

Haldane's and Lorrain Smith's experiments, 445

reaction, 444, 455

salts, 454

serum of, 446, 452

specific gravity, 444

splenic, 334,335

structural composition, 454

taste, 444

temperature, $i b$.

tests for, 472

transfusion of, 819

in the urine, 603

venous, 61

Blood-corpuscles, red, 61, 297, 454

action of reagents on, 455 et seq

chemistry of, 461

composition of, 461

development of, 459 et seq.

disintegration and removal, 334

emigration of, 298

formation in the spleen, 460

methods of counting, 458

origin of, 459

rouleaux, 454

specific gravity, 457 
Blood-Corpuscles, RED.

Blood-corpuscles, red-continued stroma, 454

tendency to adhere, $i b$.

vertebrate, various, ib.

Blood-corpuscles, white, 11, 61, 298, 299, 315, 449, 456

amœbold movements of, 457

chemistry of, 461

composition of, 456

formation in spleen, 334

origin of, 461

varioties, 456

Blood-crystals, 462 et seq.

Blood-flow, velocity of, 281

Blood-gas analysis, apparatus fur, 364

Blood-platelets, 444, 448, 458

Blood-pressure, 266 et seq.

arterial, 277, 280

in capillaries, 276

in veins, 275

action of respiratory movements on, 300

influence of cardiac nerves on, 281

measurement in $\operatorname{man}, 277$

schema to illustrate, 267 et seq.

Blood-vessels,

circulation in, 263 et seq.

effect of gravity, 279

time of complete, 289

elasticity of, 265

of eyeball, 820

nutrient, 42

of kidney, 568

of muscle, 71

of stomach, 509

influence of nervous system on, 300

Body-cavity, 884

Body, growth and maintenance of, 626

synthesis in the, ib.

Body, the chemical composition of, 406 et seq.

Bohr on mountain sickness, 400

Boiler-makers' disease, 798

Bomb calorimeter, 631

Bone, 40 et seq.

canaliculi, 42

cancellous, 40

cells or corpuscles, 43

chemical composition, 40

compact, 40

lamellæ of, 44

development, 45 et seq.

growth, 50

Haversian canals, 42

lacunæ, 42,43

marrow, 41

medullary canal, 41

microscopic structure, 42

ossification in cartilage, 46 et seq.

ossification in membrane, 45

periosteum and nutrient blood-vessels, 42

structure, 40 et seg.

Bowman,

on muscle, 65

Bowman's capsule, $566,567,576,579$

glands, 784

lamina, 812

renal epithelium, 576, 577

Boyle-Mariotte's law for gases, 325

Brain. See Bulb, Cerebellum, Cerebrum, Pons, etc.

capillarles of, 312

child's, $691,730,739$

chimpanzee's, 731

eircuiation of blood in, 312 et seq.

convolutions, 690 et seq.

diagrams of, $642,643,645,646,665$ et seq.

dog's, 729

extirpation of, in mammals, 723
Calleja, Islands of.

Brain-continued

fissures, 698

in fotus, 645,691

grey matter, 181

lobes, 691 et seq.

lunatic's, 751

monkey's, 691

motor areas, 731,733

orang's, 691,731

primitive, 645

quantity of blood in, 312,313

sensori-motor area, 734

sensory areas, 733

structure, 643

ventricles, 644

vertebrate (section), 643

vestcles, 645

white matter, 18

Bread as food, 489

Breathing. See Respiration.

Bright's disease, 600,603

Broca's convolution, 733, 734, 809

Brodie, on splenic nerve, 133

curves of extensibility, 110

his bellows-recorder, 134, 311

on heat rigor, 139

rate of blood-flow through an organ, 396

Bronchi, arrangement and structure of, 347

Bronchial arteries and veins, 353

Brown's staminal hairs of Tradescantia, 13

Brown-Séquard, suprarenal capsules, 341 on testis, 878

Brownian movement, 81

Bruch, membrane of, 811,812

Briicke on the self-steering action of the heart, 238

Brunner's glands, 494, 509

Brunton, after Gaskell, tracing of actiuns of vagus on the heart, 250

Buchanan, heart beats, 244

Buffy coat, formation of, 447

Bulb, pons and mid-brain, 661 et seq.

anterior aspect, 661

diagrams of, 662,665 et seq.

internal structure, 663 et seq.

posterior aspect, 661

Bundle of Helweg, 656, 657

of $\mathrm{His}, 213,257$

of Monakow, 657, 683, 685

Burch's experiments on colour vision, 844

Burdach's column, $651,657,658,662$

Bütschli on spongioplasm, 7

Butyric acid, 411, 526

\section{C.}

Cachexia strumipriva, 338

Cæcum, the, 560

Caffeine, 490

Caisson disease, 403

Cajal, formation of nerve axons, 153

law of axipetal conduction, 191

Calamus scriptorius, 680

Calcarine area, 736

Calcarine fissure, 694

Calcification of bone, 47

Calcium carbonate, 40,54

in urine, 600

fluoride, 40,54

metabolism, 340

oxalate in urine, 599,600

phosphate, $40,54,600$

rigor, 261

Calcium salts, the action of, 449

Calleja, islands of, 675 
Callosal Convolution.

Callosal convolution, 694

Calloso-marginal fissure, $i b$.

Calorimeters, 631,633

Calyces of the kidneys, 561

Canal, alimentary. See Stomach, Intestines, etc.

external auditory, 753

function of, $i b$.

spiral, of cochlea, 791

Canal of Schlemm, 813, 814

of Petit, 820

Canalicull of bile, 530

of bone, 43

Canalis cochleæ, 791, 792

Canals, semicircular, of ear, 790

Cancellous tissue of bone, 40

Cane sugar, 410

Cannon, on salivary digestion, 506

shadow photographs of the stomach, showing peristaltic movements, 554

Capacity of chest, vital, 359

Captllaries, 61, 214, 222 et seq.

bile, 529

circulation in, 281, 297

velocity of, 28

diameter, 222

form, $i b$.

influence on circulation, 297

network of, 222,223

number, 223

passage of corpnscles through walls of, 298

pressure in, 276 et seq.

resistance to flow of blood in, 297

still layer in, $i b$.

size, 222

structure of, $i b$.

Capillary flow, 297

Capsule of Bowman, 566, 567

external and internal, 689,690

of Glisson, 528

Carbamide. See Urea.

Carbohydrates, 406 et seq. absorption of, 544

metabolism of, 614

Carbonates in urine, 596

Carbonic acid in atmosphere, 375,376

in blood, 371,875

effect of, 373

increase in breathed air, 392

influence of, on nerve, 158, 159

in lungs, 875

Carbonic oxide hæmoglobin, 445, 465, 470

Carbon dioxide, 439

Carbon monoxide, polsonous action of, 403

Carboxyhæmoglobin, 403

Cardiac cycle, 232

Cardiac glands, 507

Cardiac muscle, 70 rhythm and conduction in, 254

Cardiac nerves, influence on blood-pressure, 281

Cardiac orifice of stomach, action of, 551 sphincter of, 554,556 relaxation in vomiting, $i b$.

Cardiac sympathetic, 251,281

Cardiogram from human heart, 240

Cardiograph, 238 et seq.

Cardio-inhibitory, 249

Cardiometer, Barnard's, 248

Roy's, $i b$.

Cardiophonogram, 249

Carotid gland, 345

Cartilage, 87 et seq.

articular, 33

cellular, 40,48

chondrin obtained from, 38

\section{Cerebehulum}

Cartilage-continued.

classification, 37

costal, 38

development, 39

elastic, 37,39

fibrous, 37. See Fibro-cartilage.

hyaline, $i b$.

matrix, $i b$.

ossification in, 46

perichondrium of, 47

Santorini's, 800, 802, 805

structure, 37

temporary, 38

varieties, 37

Wrisberg's, 800, 805

Gartilages of larynx, 799

Casein, 429, 479, 482. See Milk.

Caseinogen, 429, 479, 4S0, 482, 483

Catalysts, 328, 441, 442

Cauda equina, 648

Caudato nuclens, 688

Cavity of reserve, 60

Cell division, 15

Cells, 5 et seq.

amoboid, 5

blood. See Blood-corpuscles.

bone, 43

cartilage, 48 et seq.

central, 508, 509

characteristics of, 11

cillated, 24

connective tissue $\theta, 30$

contents, 8

definition of, 5

division, 15

epithelium, 22. See Epithelinm.

gustatory, 781

hepatic, 528

nerve, 182

olfactorial, 786

parietal, 510

pigment, 30,82

structure, 7 et seq.

varieties, 15 et seq.

vegetable, 5, 12

distinctions from animal cells, 5 et seq.

Cells of Deiters, 794

of Kupffer, 528

of Purkinje, 187, 681

of Sertoli, 864

Cellular cartilage, 40. See Cartilage.

Cellulose, 413, 549

Cement of teeth, 56,59

Central cells, 508, 509

Centres, nervous, etc. See Nerve-centres. of ossification, 45

Centrifugal machine, 452

nerve-fibres, 143

Centripetal nerve-flbres, 144

Centro-acinar cells, 517

Centrosome, 7, 10, 11, 16, 865

Cerobellar ataxy, 750

Cerebellar cortex, section of, 682

Cerebellum, 680 el seq.

analysis of, 165

connections of, 683

effects of removal or disease, 750,751

equilibration, 751

functions of, 749 et seq.

grey matter, 165, 186, 644, 680

impulses, 751,752

peduncles of, 682

sections of, $680,681,684$

semicircular canals, 752,754

extirpation of, 750,756

sensory impulses, 751 
Cerebeluum.

Cerebellum-continued stimulation, 750,756 structure, 680

Cerebral cortex, 186, 644, 696, 697, 725

grey matter of, 687

histological structure, 695 et seq. pyramidal cells, 695

Cerebral hemispheres. See Cerebrum.

Cerebral nerves, origin of, 663 et seq.

See under names of nerves.

Cerebral vesicles, primary, 645

Cerebro-spinal axis, 181

Cerebro-spinal fluid, 168, 644

Cerebro-spinal nervous system, 181

See Brain, Spinal cord, etc.

Cérébrote, 437

Cerebrum, 686 et seq.

convolutions of, $690 \mathrm{et}$ seq.

crura of, 644

degeneration tracts after injury of Rolandic area, 728

effects of injury, $i b$.

removal, 721

external capsule, 689

extirpation, 727,728

functions of, 721 et seq.

early notions, 721

grey matter, 687,690

hemispheres, $686,691-693,704,731,733,736,741$, 742

internal capsule, 688,689

localisation of functions, 726

motor areas, $i b$.

relation to speech, 808

sensory areas, 728

extirpation, $i b$.

stimulation, 727

structure, 686 et seq.

white matter, 698

Ceruminous glands of ear, 603

Cervix of urinary bladder, 570

Chambers of the eye, 819

Chauveau's dromograph, 287

Chauveau's apparatus for intracardiac pressure, 241

Cheiro-kinæsthetic area, 742

Chemical composition of the human body, 406 et seq.

Chest, expansion in inspiration, 354

vertical dismeter of, $i b$.

vital capacity of, 359

Chest-voice, 806

"Chewing the cud," 550

Cheyne-Stokes' respiration, 404, 405

Child, new-born, and changes after birth, 862 tt seq.

Chimpanzee's brain, 731

Chittenden diet, 480, 481, 623

important character of his work, 623

Chlorides in urine, 595

Chloroform, action on cardiac mechanism, 253

Cholagogues, 535

Cholalio acld, 533

Cholesterin or cholesterol, 9, 75, 166, 435, 436, 534

Chollne, 166

Chondrin, 38, 429

Chorda secretion, 502

Chorda tympani, 502, 504

Chordæ tendtneæ. See Heart.

Chorion, 887 et $\mathrm{seq}$

Chorionic epiblast, 883,884

Chorionle villi, 888,889

Chorold coat of eye, 811

Choroid gland, 168

Chromatic aberration, 830

\section{CoHNheim's EXPERIMENT.}

Chromatin, 10

Chromatolysis, 193

Chromatoplasm, 192

Chromogen, 341

Chromophanes, 847

Chromophilic material, 192

Chromoplasm, 10, 11

Chromo-proteins, 427,430

Chromosomes, 16 et seq, 876

Chrzonszezewski's method of natural injection, 529

Chyle, 226, 316, 547

molecular basis of, 316

Chyme, 554

Cilia, 23, 24

Cillary epithelium, 24

function of, $i b$.

Ciliary motion, 25, 82

nature of, 25

Clliary muscles, 812, 813

action of, in adaptation to distances, 825

Clltary processes, 812,813

Cilio-spinal centre, 721

Circular layer of heart, 212

"Circulating protein," 622

Circulation of blood, 60,227 et seq

action of heart, 213

in blood-vessels, 263 et seq.

in brain, 312

capillaries, 297

course of, 214 et seq.

effect of gravity on, 279

effect of respiration on, 385

erectile structures, 313

in fœetus, 891, 892

influence of respiration on, 385

of gravity, 279

peculiarities of, in different parts, 312

portal, 215

pulmonary, $i b$.

renal, $i b$.

systemic, 3

in veins, 61 velocity of, 281

Circulatory system, 208 et seq.

Circumvallate papillæ of the tongue, 779

Clarke's column, 650,651

Clasmatocytes, 31

Claustrum, 688

Cleavage products, $18,416,417$

Clerk-Maxwell's experiment, 841

Clitoris, 313

Clot or coagulum of blood. See Coagulation.

Clupeine, 427

Coagulation of blood, $60,137,446$ et seq. conditions affecting, 447

theories of, 448

of millk, 482

Coagulative enzymes, 426,439

Cocaine, 490

Coccygeal gland, 345

Cochlea of the ear, 753, 791 et seq.

theories in connection with, 767,768

Cochlear division of auditory nerve, diagram of, 676

Cochlear nerve, 663

Coefficlent of oxidation, 395, 397, 398

Colom, 884

Co-enzymes, 441

Cognitive mode in consciousness, 758

Cohnhelm, areas of, 65

Cohnheim's experiment on passage of bloodcorpuscles, 298

on diabetes, 540

on erepsin, 525

with succus entericus, 524 
Coitus.

Coitus, 881

Collagen, 29, 40, 429

Collateral fissure, 694

Colloidal solution, $328,423 n$.

Colloids, 323, 424

Colon, the, 561

Colostrum, 486 corpuscles, $i b$.

Colour-blindness, 843

testing for, 844

Colour-perception, 841

Colour reactions of proteins, 425

Colour sensations, 841

Burch's experiments, 844

theories of, 842

Colours, optical phenomena, 841 et seq.

Columnæ carneæ, 211

Columnar epithelium, 21, 22, 529

Coma, diabetic, 405

Combination-tones, 797

Comma tract, 654,656

Commissural fibres, 648, 698 nucleus, 679

Common path, principle of, 717

Common sensations, 775

Compleinent, the, 475

Complemental air, $\mathbf{3 5 9}$

Complementary colours, 842

Compound tubular glands, 496, 497 racemose glands, 497

Conative modes in consciousness, 758

Concha, 788

Condiments as accessories to food, 490

Conducting paths in cord, 704 et seq.

Conduction, law of axipetal, 191

Conductivity, 172

Cones and rods, 817

movement of, 847

Conical and filiform papillæ of tongue, 779, 780

Coni vasculosi, 864, 865

Conjugate deviation of head and eyes, 737

Conjugated proteins, 430

Conjunctiva, 810

Conjunctival reflex, 711

Connective tissues, 28 et seq.

classification, $i b$.

corpuscles, 30

elastic, 32

fibrous, $i b$.

general structure of, 28

jelly-like, 86

retiform, 35

varietles, 30,31

Consclous states, physiology of, 757 et seq.

Conservation of energy, la w of, 630

Consonants, 808

Contractile substance, 63

Contractility of muscle, 81 et seq.

Contraction, heat, 165

Contraction of heart, 128, 232

Contraction of pupil, 827

Contraction, Pfluger's law of, 174,175

Contrast of colour, 845

Conus medullaris, 648

Convergence of eyes, 827

Convoluted tubes, 864

Convolutions, cerebral, 690 et seq.

Cooking, effect of, 489

Coürdination of muscular movements, 107

Copper sulphate, or Plotrowski's test, 425

Copulation, 881

Cord, spinal. See Spinal cord.

Cornea, 811, 813

corpuscles, 812

Corneo-scleral junction, 813,814
Cvetic Duct.

Coronary arteries, 237

Coroua radiata, 689

Corpora cavernosa, 313, 866

quadrigemina, $663,673,681$

Corpus Arantil, 214

callosum, 686

dentatum

of cerebellum, 681

of olivary body, $i b$.

Highmorianum, 863

luteum, 870,880

of human female, $i b$.

spongiosum, 313, 866

striatum, 687

Corpuscles of blood, 444. See Blood-corpuscles.

Corpuscles, Malpighian, 334

Corpuscles, of Grandry, 768, 769

of Hassall, 336, 337

of Herbst, 766

of Melssner, 767, 774, 775

of Pacini, 766, 767

Cortex, 181, 342, 644, 682,695

Corti on rotation of cell sap, 13

Corti, organ of, 793

Cortical retina, 736

Cortico-pontine fibres, 670,700

Cortico-spinal fibres, $670,70 \mathrm{C}$

Corti's rods, 793

Cotyledons, 889

Coughing, mechanism of, 381

Cowper's glands, 570

Cranial nerves, 664 et seq.

nuclei of, $664,665,667,670$

origin and functions of, 673 et seq.

Creatine, 166, 420, 589-591, 894

Creatinine, 590

Cremasteric reflex, 711

Crescents of Gianuzzi, 499

Cretinism, cause of, 338

Crico-ary tenoid muscles, $800-803$

Cricoid cartilage, 799

Crico-thyroid muscle, 800

Crista acoustica, 753

Crossed hemiplegia, 729

Crossed pyramidal tract, 655

Crosses of Ranvier, 75

Crowbar accident, 738

Crucial sulcus, 730

Crura cerebelli, 664

cerebri, 644, 673, 689

grey matter of, 645

Crusta, 673

petrosa, 56,59

Crypts of Lieberkuhn, 493

Crystallin, 814

Crystalline lens, 811, 813

Crystallisable proteins, 424

Crystalloids, 424

Cubical epithelium, 21

Cuneus or cuneate lobule, 694

Cuorin, 435

Cupula, 753

Curative inoculation, 473

Curdling ferments, 482

Currents of action, 121

constant, 88

demarcation, 123

induced, 89

of rest, 121

Cutaneous sensations, 766 et seq.

varieties of, 772

Cuticle. See Epidermis, Epithelium.

Cutis vera, 604

Cybulski's hæmatachometer, 286

Cyclopterine, 427

Cystic duct, 527 


\section{Cystink in Urink.}

Cystine in urine, 598

Cystine, 421, 422, 599

Cytosine, 421

D.

Dalton-Henry law on gases in the blood, 362

Daniell's battery, 88

Dark-adaptation of eye, 848

Darwin, 858

Deaf-mutes and equilibrium, 756

Deamidases, 440

Deamidation, 546

Death, 895

Decidua, 887

basalis, or serotina, $887-889$

capsularis or reflexa, 887

development of, 888

menstrualis, 873,874

reflexa, or capsularis, 887.889

vera, 887,888

Decussation of fibres in medulla oblongata, 668 , 669

of optic nerves, 853

Deep sensibility, 774

Defæcation, mechanism of, 562,721 centre, 721

influence of spinal cord on, 563

Deficiency diseases, 491

Degeneration method, 148, 156, 178, 307

tracts of descending, 655 ascending, 657

Deglutition. See Swallowing.

Deiters, cells of, 794 nucleus, $670,671,677$

Delezenne on trypsinogen, 524

Demarcation current, 123

Demilunes of Gianuzzi, 499

Demoor's sleep theory, 746

Dental germ, 56 papilla, 56,58 periosteum, 59

Dentate fissure, 694

Dentate nucleus, 669

Dentine, 28, 54

formation of, 57

structure, 54

Depressor nerve, 249, 305

Dermis, 604

Descemet's membrane, 812,813

Descending tubule of Henle, 567

Development, 858 et seq.

adipose tissue, 34

blood-corpuscles, 459

blood-vessels, 459

bone, 40 et seq.

cartilage, 39

decidua, 887

embryo, diagrams of, $885,887,888$

fertilisation, 881

foetal circulation, 891

fotal membranes, 887

impregnation, 881

nerve-tibres, 83

ovary, 879

ovum, 875

segmentation, 883

toeth, 58

Dextrin, 409, 412

Dextrose, 407, 409, 411, 439, 440 in urine, 601

tests for determining, 409, 410, 601, 602

Diabetes, $409,538,539,542,778$

artificial production in animals, 538, 539

Diabetic coma, 405

Diabetic puncture, 538
EAR.

Dialyser, a, 424

Dialysis, 324,453

Diamino-acids, 421

Diamino-diphosphatides, 435

Diamino-monophosphatides, $i b$.

Diapedesis of blood-corpuscles, 298

Diaphragm. See Inspiration, etc.

Diastase of liver, 537

Diastole of heart, 238

Diastolic pressure, 296

Diastolic sound, 236

Dicrotic pulse, 294

Diet, 613 et seq.

a healthy, 480

Chittenden's, 480, 481

nutritive value, 479 et seq.

Ranke's, 480

tables, 480, 482, 613 et seq.

Voit's, 480

Difference-tones, 797

Diffusion and osmosis distinguished, 323

Digestion,

in the intestines, 517 et seq.

mechanical processes, 550 et seq.

See Gastric fluid, Food, Stomach

Dilator pupillæ, 812

Dilemma, 720

Dipeptides, 423

Diphasic variation, 123, 124

Diphtheria toxin, 474

Diplococcl, 439

Diplopia, 850

Direct cerebellar tract, 658

pyramidal tract, $i b$.

Disaccharides, 409

Discus proligerus, 869

Disease, "germ theory " of, 438

Distributing nerve-cells, 194, 195

Disuse atrophy, 148, 194

Diuretics, $\mathbf{5 7 6}$

Dixon on spermine, 878

Doble's line, 65

Dog, "scratch" reflex of, 719

Dog's brain, 729

nerves, 162,303

spleen, 335

submaxillary gland, 499

Dorsal cord, 165

Double vision, 850

"Drainage" theory, M'Dougall's, 716, 717

Dromograph, Chauvean's, 287

Drugs, action of, 775

on the eye, 833

on the heart, 253

on perspiration, 611

Ductless glands, 331 et seq. theories of secretion, 331

Ducts of Bellini, 566, 567

Ductus arteriosus, 893 closure of, 894 venosus, 892

closure of, 894

Dudgeon's sphygmograph, 292

Dupré's urea apparatus, 585

Dynamometer, 114

Dyne, 267

Dyspnœa, 389

E.

Ear, 788 et seq.

bones or ossicles of, 788

function of, 796

development, 878

external, 788

function of, 795 
EAR.

Ear-continued

internal or labyrinth, 790, 791

function of, 796

middle, 788

function of, 795

Ectopia vesicæ, 580

Edridge-Green lantern, 844

Efferent channels, diagram of, 701

Efferent nerves, 73, 143

nerve-cells, 194

projection system, 698

Egrgs as food, 486

Ehrlich's side-chain theory, 475

Einthoven's string galvanometer, 121, 245

nervous factor in respiration, 376, 377

third heart sound, 246

Elastic cartilage, 37,39

fibres, 37

tissue, 32

Elastin, 30, 429

Electrical currents of retina, 849

nerves, 144

phenomena of muscle, 116 et seq., 176

variation in central nervous system, 743 in glands, 497

Electricity,

in muscle, 116 et seq., 176

nerve, 176

Electrocardiogram, 243 et seq.

Electrodes, non-polarisable, 118, 119

Electrometer, Lippmann's capillary, 120, 121, 124

record, 124-126

Electrotonus, 169 et seq.

Eleidin, 604

Elementary substances in the human body, 406

Embryo, 858 et seq., $885 n$. See Development.

Embryo chick, diagram of, 885

Emb-yological method, 651

Embryonic area, 883

Emetics, 557

Eminentia teres, 663

Emmetropic eye, 828

Emulsification, 416, 520

Enamel of teeth, 55

formation of, 58

Enamel organ, $i b$

Enchylema, 7

End-bulbs, 767

Endocardium, 209

Endogenons fibres, 740

Endogenous protein metabolism, 622

End-plates, motorial, 70, 78

Endolymph, 753, 793

Endoneurium, 80, 81

Endothelial cells, 226, 321

layer, 218

membrane, 222

Endothelium, 21, 208, 222

Energy, law of conservation of, $630 \mathrm{et}$ seq.

Enterokinase, 523,524

Enzyme action,

coagulation, 426

inexhaustibility of, 441

law of, 441

optimum temperature of, 441

reversibility of, 442

specificity of, 441

Enzymes, 426, 438 et seq., 448

activation of, 441

catalytic action of, 442

Inexhaustibility of, 441

Eosinophile cells, 457

Epiblast, 19, 883 et seq.

organs formed from, 886

Epicardium, 208

\section{EYEBALL.}

Epicritic sensibility, 707, 774, 777

Epidermis, 22, 604, 886

Eptdidymis, 864, 865

Epigastric reflex, 711

Eplglottis, 551, 800, 801

Epineurium, 76, 77

Epithelium, 20 et seq., 570, 604, 886, 887

cells of, 784

chemistry of, 27

ciliated, $21,23,24,81,348$

columnar, $21,22,23$

compound, 21

cubical, $i b$.

goblet-shaped, 21, 22

nutrition of, 26 .

pavement, 21

renal, 574

simple, 2

spheroldal, 23

stratified, 22, 23, 26

transitional, 21, 22

Erectile structures, circulation in, 313

tissue of penis, 865

Erection, 313, 314, 721

cause of, 314

influence of muscular tissue in, 314

Erepsin, 440, 524

Erg, 267

Ergograph, Mosso's, 132

Ergotoxin, effect of on salivary glands, 503

Erythroblasts, 41,460

Erythro-dextrin, 412, 505

Esbach's albuminometer, 601

Euglobulins, 600

Eustachian tube, $678,789,790,795$

function of, 795

valve, 209, 893

Ewald's induction coil, 92

Ewald, "sound picture" theories of, 798

Excitabllity of nerves, 172

of tissues, 81

Exhaustion in asphyxia, 389

Exogenous fibres, 741

Exogenous protein metabolism, 622

Exostosis, 60

Expiration, 355 et seq.

force of expiratory act, 360

influence on circulation, 355,360

mechanism of, 355

muscles concerned in, 356

relative duration of, 357

External auditory meatus, 789

External capsule, 689

External respiration, 392

Extractives, 166, 453

Extraventricular nucleus, 688

Eye, 810 et seq.

accommodation, $820,824,827,831$

action of drugs on pupll, 833

adaptation of vision at different distances, 824 et seq.

blood-vessels, 820

causes of dilatation and contraction of pupil, 834

chambers of, 820

focus, 820,821

optical apparatus of, 820,821

defects in, 828

principal point, 821

refractive media of, 820

resemblance to camera, $t b$.

Eyeball, 811

blood-vessels of, 820

electrical currents of, 845

muscles influencing movement, 849

point of rotation, 850 
EYeball.

Eyeball-continued transverse and visual axis, 851 various positions of, 850

Eyelids, development of, 810

Eyes, simultaneous action in vision, 850

\section{F.}

Facial nerve, 663, 675 effects of paralysis of, 675 origin, $i b$.

relation of, to expression, $i b$.

Fæces, composition of, 548 quantity passed, 549

Fallopian tubes, 24, 25, 871 .

False vocal cords, 801,805

Falsetto voice, 806

Faradisation, 101, 104

Far-point, 828

Fat. See Adipose tissue. action of bile on, 535

of pancreatic secretion, 523

metabolism of, 541,616

situations, where found, 33, 34 synthesis, 542

uses of, 34

Fatigue, 744

in nerves, 132

Fats,

absorption of, 546

acids of, 414

action of pancreatic juice on, 520

chemical constitution, 414

decomposition products, 415

emulsification, 416

metabolism of, 616

of milk, 483

saponification, 415

Fatty acids, 414

Fehling's solution, 601, 602

Female generative organs, 867 pronucleus, 876

Fenestrated membrane of Henle, 217

Fenestra ovalis, 788, 791

rotunda, 788,791

action of, 797

Fermentation, 438

Ferments, 440. See also Enzymes Blood, Milk, Digestive juices.

Fertilisation, 881

Fibres of Müller, 815,816

of Romak, 77

of Sharpey, 44

Fibrils of muscle, 65

of nerve, 74

Flbrin, 60, 61, 446

ferment, $440,449,453$

formation, 447,451

reticulum of, 447

Fibrinogen, 60, 315, 448, 451, 453

Fibroblasts, 30

Fibro-cartilage, 37

classification, $i b$.

development, 39

white, $37,38,40$

yellow, $37,39,40,41$

Fibrous tissue, 28 et seq.

white, 29

yellow, 30

Fick on work of muscles, 115

Fick's spring kymograph, 275, 276

Fifth cranlal nerve, 663,674

Fillform paplllæ of tongue, 779 et seq.

Fillet, 668,672

Filtration, 319, 320, 324

Filum terminale, 648
Galactosides.

Fischer's laboratory, the work in, 416, 421 theory of dropsy, 321

Fishes, circulatory system in, 230

Fistula, intestinal, 523

Flechsig's embryological method, 740

Fleischl's hæmoglobinometer, 471

Fleming's chromatin, 10

Flesh of animals, 479

Flicker, 836, 854

Flour as food, 488

Flourens' experiment, 749

Fluids, swallowing, 552

Fluids, tension of gases in, 362 et seq.

Fluoride of calcium, 40

Focal distance, 824

Fotal circulation, 891-893

Fotal membranes, 887

development of, $i b$.

Fotus, 885 n., 886, 893

circulation in, 891

communication with mother, 890

Folin's method of estimating urea, 585

Follicles, Graafian. See Graafian follicles.

Follicles, Meibomian, 496, 810

Fontana, spaces of, 814

Food, 479 et seq.

absorption of, 543 et seq.

accessories to, 490

chemical compounds of, 479

constituents, unknown but essential, 491

cooking, 489

digestibility of articles of, 479

value dependent on, $i b$.

fluidity of, 555

heat-value of, 635

of man, 479

proximate princlples in, $i b$.

vegetable, $i b ., 489$

Foramen ovale, 893

of Magendie, 644

of Munro, 688

Fore-gut, 886

Formaldehyde, 425

Formatio reticularis, 667

Formic acid, 414

Formix, 688

Fossa ovalis, 209

Fovea centralis, $814,818,841$

Fovea hemielliptica et hemispherica, 791

Franck's cannula, 273

Frogs,

circulatory system in, 230,231

corpuscles of, 454

gracilis of, 160

heart, $230,231,251,259$

mucous membrane, 547

nerves, 251

reflexes in, 709

Fromann's lines, 75

Frontal lobe, 690, 692

Fundus glands, 508

Fundus of eye, 836

of urinary bladder, 570

Fungiform papillæ of the tongue, 779,780

Funiculus cuneatus, 662,665

Funiculus gracilis, 662,665

Funiculus solitarius, 670,678

Furfuraldehyde, 533

Fürth, on muscle proteins, 138, 139

Fuscin granules, 847

G.

Galactose, 410

Galactosides, 166, 437 
Gali,-HLADDER.

Gall-bladder, 532

structure, $i b$.

Galvanism, 122

Galvanometer, 117 et seq., 245

the string, 121, 245

Gametes, 859

Gamgee, photographic spectrum of hæmoglobln and its derivatives, 468,469

Ganglia, 180, 196 et seq. See Nerve-centres. sympathetic, functions of, 500,502

Ganglion cells, layer of, 815

Ganglion of Scarpa, 677

Ganglion spirale, 794

trunci vagi, 249

Gases,

extraction from blood, 364

of the blood, 361 et seq.

in the lungs, 372

of plasma and serum, 453

tension, 362

Gaskell's heart-block, 257

Gastric fistula, 507

Gastric glands, 493

Gastric juice, 507 et seq. aclds in, 510

test for, 510,516

action on bacteria, 525

action on food, 513

artificial, 507

composition of, 510,511

pepsin of, 511

secretion of, 509

Gastrin, 522

Gay-Lussac's law for gases, 325

Gehuchten, van, law of axipetal conduction, 191

Gelatin, 29, 40, 54, 429

Generative organs of the female, 867 of the male, 863

Gennari, line of, $697,736,737$

Genu of internal capsule, 690

Gerlach's network, 650

Germ plasm, Weismanns', 861

Germ theory, 438

Germinal area, 883

epithelium, 863, 871

spot, 18, 19, 871 vesicle, $i b$.

Giant cells, 41

Gianuzzi's crescents or demilunes, 499

Gibson, third heart sound, 246

Gilbert's experiments, 748

Gland cells, function of, 496

Glands. See names of different.

Gliadin, 434

Glisson's capsule, 528

Globin, 427,463

Globulins, 138, 165, 424, 427, 433, 600

character of, 515

distinctions from albumin, 428

Glomerull, 567, 569, 573

olfactory, 786

Glosso-kinæsthetic area, 741

Glosso-pharyngeal nerve, 663,678

communications of, 678

functions, $i b$.

motor fllsments, $i b$.

a nerve of common sensation and of taste, $i b$.

Glottls, movements of, 551, 805

Gluco-proteins, 427,430

Glucosamine, 430

Glucose. See Dextrose.

in llver, 537

test for, 412

Glutamic acld, 422

Gluteal reflex, 711

Glutelins, 433
HEMODYNAMOMETER.

Gluten, 488

Glutenin, $i b$.

Glycerides, 414

Glycerin or Glycerol, 415

origin of, 619

Glycine, 417, 422

Glycocholic acid, 533

Glycogen, 8, 407, 412, 413, 537, 53s

characters, 412

destination of, 537

preparation, $i b$.

quantity formed, $i b$.

source of, 536

variation with diet, 537

Glycogenolytic nerves, 541

Glycolysis, 540

Glycosurla, 538, 540

Glycuronlc acid and sugar, 540

Gmelin's test, 533,602

Goblet cells, 21, 22

Goldman's intra-vitam staining, 890

Golgi's method, 186, 697, 746

Goll's column, $651,654,657,660,662$

Goltz, experiments on the cerebrum, '/25

"Goose skin," 607

Gotch, experiments on heart, 248

on nerves, 159,176

Gowers' hæmoglobinometer, 470, 471

tract, 657

Gowers-Haldane hæmoglobinometer, 470

Graafian follicles, 869 et seq.

formation and development of, ib. et seq.

relation of ovum to, 870

rupture of, changes following, 870 et seq.

Gradlent, pressure, 285, 386

Gramme-molecular solutions, 323

Grandry, corpuscles of, 768, 769

Granular layers of retina, 816

Grape-sugar. See Dextrose.

Graphic method, 87

Gravity, influence of, on circulation, 279

Grey matter of cerebellum, 180, 644, 680

of cerebrum, 181, 186, 688

of crura cerebri, 645

of medulla oblongata, $180,667,668$

of spinal cord, 181,650

Grossmann, on the course of the Inhibitory fibres in mammals, 249,252

Ground substance of conuective tissue, 31

Growth of bone, 50

Guanase, 594

Guanine, 432

Guanylic acid, $i b$.

Gullet. See CEsophagus,

Gustatory cells, 781

Gyrus fornicatus, 694

H.

Hæmacy tometers, 458

Hæmadromometer, Volkmann's, 282

Hæmatachometer, Cybulski's, 286

Vierordt's, 287

Hæmatin, 463

Hæmatoblasts, 334

Hæmatogens of cells, 431

IIæmatoidin, 464

Hæmatoporphyrin, 464, 582

Hæmatoscope, Herrmann's, 466

Hæmatoxylin, 10

Hæmautograph, 295

Hæmin, 463, 464

Hæmochromogen, 463

Hæmodromometer, 282

Hæmodynamometer, 272 
H

Hæmoglobin, 61, 370, 424, 454

analysis of, 461

and carbon monoxide, 403

compounds of, 465

crystallisable, 424

crystals of, and how to obtain them, 462

dissociation curve of, 367,370

distribution, 461

estimation of, 470

increase in the blood at high altitudes, 402

photographic spectrum of, $46 \mathrm{~S}, 469$

reaction with oxygen, 367

solution of, 367,368

Hæmoglobinometers, 470, 471

Hæmoglobinuria, 603

Hæmolymph glands, 336

Hæmolysins, 475

Hæmopyrrol, 464

Hair-cells, 754, 794

Hatr-follicles, 605, 606

Hairs, 605

structure of, $i b$.

Haldane's apparatus for estimating the carbonic acid and aqueous vapour given off by an animal, 393

carbonic oxtde method of estimating oxygen tension of arterial blood, 374,375

Haldane and Priestley's method in dealing with respiration, 375

Haldane's measurement of air breathed, 358

Haldane's modification of Gowers' hæmoglobinometer, 445

Hales' investigations on blood-pressure, 271

Hallucination, 764

Hamburger's experiments with Succus entericus, 523

Hammer bone, 788, 789

Hamulus, 792

Hannover's stratum intermedium, 58

Haptophor groups, 475

Hardy, microscoplc structure of cells, 8

Harvey on circulation of the blood, 227, 234

Hassall, concentric corpuscles of, 336,337

Hausmann's method of analysing proteins, 423

Haversian canals, 42, 43

lamellæ, 44

Head's experiments, 378, 379, 705

Hearing, anatomy of organ of, 788 et seq.

influence of external ear on, 794 of middle ear, $i b$.

physiology of, $i b$.

range of, 797

See Sound, Vibrations, etc.

Heart, 208 et seq.

action of,

accelerated, 253

force of, 247

frequency, $i b$.

inhibited, 253

isolated, 260

self-steering, 238

auricles of, 209.212

block, 246, 257

chambers, 209

capacity of, 212

chordæ tendineæ of, 213, 234, 236

columnæ carneæ of, 212

conduction in the, 254

course of blood in, 214

cycle, 232

endocardium, 209

excised mammalian, 261

force, 245

frog's, $230,231,259$

nerves of, 251
Hertz on the Process of Dianstion.

Heart-continued

ganglia of, 255

gaseous exchanges during inhibition, 254

influence of drugs, 253

of sympathetic nerve, 251

innervation, 249

intracardiac pressure, 240

investing sac, 208

muscular fibres of, 68

musculi paplllares, 214, 234

nervous system, influence on, 249

output of, 247

pericardium, 208

physiology, 232 et seq.

plethysmograph, 260

reflex inhibition, 253

situation, 208

size and weight, 212

sounds of, $235,245,246$

causes, 236

structure of, 212

valves, 210,213

auriculo-ventricular, 210-212, 234, 236

function of, 234

semilunar, $210,212,214,235$

function of, 235

structure, 209 et seg.

ventricles, their action, 209-212

work of, 247

Heat, animal. See Temperature.

influence of nervous system, 640

of various circumstances on, 637

losses by radiation, etc., $638-641$

regulation of, 640

value of foods, 634

variations of, 636

Heat and cold spots, 773

Heat coagulation, 423, 424, 426

Heat contraction, 164

Heat production, 637, 638, 640

Heat-rigor of muscle, 139

Heat-value of food, 634

Heidenhain's researches, $320,578,579$

Held, experiments on myelination, 740

Helicine arteries, 866

Helicotrema, 792

Heller's nitric-acid test, 601

Helmholtz's induction coil, 91,92

myograph, 87, 92, 104, 108

phakoscope, 825

resonance theory, 798

Helweg's bundle, 656, 657

Hemianopsia, 737

Hemiplegia, 729

Hemisection of spinal cord, 659

Hemispheres, cerebral. See Cerebrum.

Henle, fenestrated membrane of, 219

layer of, 606

on muscles of the larynx, 803

sheath of, 77

tubule of, 567

Henry-Dalton law for gases, 326

Hensen's line or disc, 65,67

Hepatic artery, 527

Hepatic cells, 527

collc, 535

duct, 527

glycogen, 615

veins, 527,529

Herbst, corpuscles of, 766

Hering's experiments on blood circulation, 289 290

Hering's theory of colour, 842, 843

Herrmann's current of rest, 122, 123

hæmatoscope, 466

Hertz on the process of digestion, 555 
Herzen on Succagogurs.

Herzen on succagogues, 513

Heterocyclic rings, 421

Heterotype mitosis, 876

Hexatomic alcohols, 408

Hexone bases, 420

Hexoses, 408

Hill (Croft) on inverting enzymes, 443

Hill (Leonard) on the circulation of blood in the brain, 313 et seq.

Hilus, the, $317,318,332,565$

Hippocampal convolution, 689

Hippocampus major, 694

Hippuric acid, 594

Hirudin, 451

His, bundle of, 257

His on growth of nerve-fibres, 148

Histidine, 420

Histone, 427

Homoiothermal animals, 636

Homotype mitosis, 876

Hope's experiments on heart sounds, 237

Hopkins' test for lactic acid, 516

for uric acid, 592

on foods, 491,627

Hoppe-Seyler on proteins, 416

Hormone, 521, 880

Horopter, 852

Hunger, 778

Hurst, "sound-picture" theories of, 798

Hiirthle's manometer, 242, 276

Huxley's layer, 606

Hyaline cartilage, 38, 40, 45

Hyaloplasm, $7,14,68,83$

Hydrobilirubin, 534

Hydro-kinetic force, 284 -static force, 284

Hydrolysis, 417, 514

Hypermetropia, 828

Hyperpnca, 389

Hypertonic solutions, 327

Hypoblast, 19, 883

Hypogastric nerves, 581

Hypoglossal nerve, 664, 679

distribution, 679

origin, $i b$.

Hypopituitarism, 345

Hypotonic solutions, 327

Hypoxanthine, 166, 421, 432

Hysteria, 808

\section{I.}

Idiosome, 865

Illusion, 765

Image, formation on retina, 824

Immunity, 472 et seq.

Impregnation of ovum, 881

Inanition or starvation, 628

Incoördination, 750,756

Incus, or anvil-bone, 789

Indican, 596

Indiffusibility of proteins, 424

Indigo, $i b$.

Induction coll, 89 et seq. current, 89

Infantile paralysis, 770

softness of head, 46

Infundibulum, 351

Inhibition, vagus, 253

Inhibitory nerves, 144

Inoculation, curative, 473 protective, $i b$.

Inogen, 138

Inorganic compounds in body, 406 salts in nerve, 166

salts in protoplasm, 9
ISOMETRIC CoNThaotion.

Inosite, 166, 413

Insalivation, 550

Inspiratton, 354,378

elastic resistance overcome by, $i b$.

expansion of chest in, $i b$.

extraordinary, $i b$.

force employed in, 251, 258

mechanism of, 354 et seq.

tracings of diaphragm, 379

Instruments for demonstrating muscular action, 87 et seq.

Intercellular material, 4, 29

passage, 351

Intercentral nerves, 145

Intercostal muscles, action in inspiration, 355 et seq.

action in expiration, 355

Intercrossing tibres of Sharpey, 44, 45

Interglobular layer, 55

Interglobular spaces, 57

Interlobular arteries, 569

veins, $i b$.

Intermediary nerve-cells, 194, 195

Intermediate areas, 742,743

Intermittent pulse, 290

Internal capsule, 688

importance of, $i b$.

respiration, 392

Internal ear, 790

Internal secretion theory of the ductless glands, 331,332

Interstitial cells, 44, 865

Intestinal fistula, diagram of, 523

Intestinal juice, 518 et seq.

Intestines, 492, 557 et seq.

digestion in, 517 et seq.

duration of, 563

large, 560

coats of, 493

glands, 494

structure, $i b$.

view of, 561

movements, 560

mucous membrane of, 494

nervous mechanism, 558, 563

small,

coats of, 493

glands, 493, 494

movements of, 557

structure, 493, 494

Intracardiac nerves, 252 pressure, 240

Intracellular enzymes, 440

Intraventricular nucleus, 688

Intra-vitam staining, 890

Inversion, 410,522

Invertase, 522

Inverting enzy mes, 440

Involuntary muscles, 62 (see 140 et seq.)

structure of, 62

Iodo-thyrin, 339

Ionic reactions, 328

Iris, 812, 813

angle of, 814

functions, 832

motor nerves, diagram of, 833

reflex actlons, $i b$.

Irradiation, 830

Irritabllity of tissues, 81 et seq.

Island of Reil, 688,692

Islands of Calleja, 675

Islets of Langerhans, 517, 539

Iso-cholesterin, 436

Isolated heart, 260

Iso-maltose, 411

Isometric contraction, 115 
Isotonic Contraction.

Isotonic contraction, 115 solutions, 326

Ivory, 54

\section{J.}

Jacksonlan eptlepsy, 730

Jacobsen's nerve, 678

Jaundice, 535

Jecorin, 341

Jelly-like connective tissue, 28, 36

Jugular ganglia, 249

Juice, gastric, 507

\section{K.}

Kaiser's views on muscular contraction, 115

Karyokinesis, 16 et seq. phases of, 17

Katabollc nerve groups, 203

Katabollc phenomena, 612

Katabolism, 618, 619

Katelectrotonus, 171, 173

Kations, 322

Kennedy, experiment on nerve crossing, 161

Kent's bridge, 257

Kephalin, 166, 435, 438

Kerasin, 438

Keratin, 23, 27, 429, 436, 604

Ketone, 407

Ketoses, 408

Key, Du Bois Reymond's, 88

Kidney oncometer, the, 571

Kidneys, 561 et seq.

blood-vessels of, how distributed, 569

calyces, 565

captllaries of, 568

diseases of, effect on the skin, 611

extirpation of, 579

function, 572. See Urine.

hilus of, 565

Malpighian corpuscles of, 566, 567

nerves, 571

oncometer, $i b$.

pelvis of, 565

plan of, $i b$.

structure, $i b$.

tubules of, 566 et seq., 569

vascular supply of, 569

weight, 565

Kinæsthetic sense, 776

Kinetoplasm, 192

Kjeldahl's method of estimating nitrogen, 585, 586

Knee-jerk, 712

Könlg's apparatus for obtaining flame-pictures of musical notes, 807

Kossel on protamines, 428

Krause's membrane, 65,67

Krogh's tonometer, 363, 373

Kühne's gracilis experiment, 160

muscle plasma experiment, 138

Kupffer's stellate cells, 528

Kyes on lecithin, 477

Kymograph, Fick's spring, 275, 276

diagrams of mercurial, 272,274

Ludwig's, 272, 273

tracings, 275, 277

Kymoscope, Anderson Stuart's, 271

\section{L.}

Labyrinth of the ear. See Ear.

Labyrinthine impresslons, 752

Lacrimal gland, 810

Lact-albumin, 482
LIVER.

Lacteals, 225, 316,

Lactic acid, 166

fermentation, 411

tests for, 516

Lactiferous ducts, 485

Lactose, 407, 411, 483, 601

Lacunæ, 43, 570

Lævulose, 408-410

Lamellæ of compact bone, 44

Lamellar cells, 30

Lamina of cortex, 696, 697

cribrosa, 815

spiralis, 792

terminalis, 646

Langerhans, islets of, 517,539

Langley on the autogenetic theory, 150, 151

Langley's experiment on vagus and cervical symp thetic nerve, 161 ganglion, 502,504

nicotine method, 200

Lanoline, 436

Large intestine. See Intestines.

Laryngoscope, 803

Larynx, 346, 347, 799 et seq.

anatomy of, 799

cartllages of, $i b$.

diagrams of, 805

mucous membrane, 801

muscles of, 801 et seq.

nerves of, 803

ventricle of, 801,805

vocal cords, 801

movements of, 804

Lateral sclerosis, 711

Lateral ventricle, 687

Lateritious deposit, 592

Lawes' and Gilberts' experiments, 618

Layer, papillary, 212

circular, $i b$

spiral, 213

Lecithin, 9, 166, 435, 437, 477, 526

Lee's experiments, 755

Lens, crystalline, 812-814

Lenticular nucleus, 688

Le Page's investigations into pancreatic secre. tion, 520

Leucine, 417, 418, 422

Leucocytes. See Blood-corpuscles (white).

Leucocy thæmia, 334, 593

Leucosin, 433

Levene on nucleic ac:d of yeast, 432

Lewis on hæmolymph glands, 336

Leydig's theory of protoplasmic structure, 7

Lleberkühn's glands, 493, 494, 496, 497

Liebermann's reaction, 436

Ligamentum pectinatum iridis, 813,814

Limbic lobe, 694

Line of Gennari, 697, 736, 737

Lipase, 440, 513, 518, 617

Lipoids, 9, 165, 166, 43 t

Lipolytic ferments, 440

Lippmann's capillary electrometer, 120, 121

Liquor sanguints, or plasma, 60,444

Lissauer, tract of, 657

Listing's reduced eye, 821

Litmus, 165

Littré, glands of, 570

Liver, 527 et seq.

bile, 528

blood-vessels, 528,529

capillarles, 529

colls of, 528

cirrhosis of, 586

circulation in, 528

diastase, 537

extirpation in mammals, 539 
LIVER.

Liver-continued

fat metabolism, 541 et seq.

glycogenic function of, 536

lobules of, $527-529$

secretion of. See Bile.

structure, 528

sugar formed by, 537,538

supply of blood to, 527

under-surface of, $i b$.

"Living test-tube" experiment, 452

Local sign, 762

Localisation of tactile sensations, 770

Locke's solution, 262

Locomotor ataxy, 715

Loeb on classification of ions, 323

on spermatozoon, 882

Loewenthal's tract, 656

Loop of Henle, 567

Lortet on the carotid flow, 289

Loudness of voice, 806

Ludwig's graphic method, 87

Lud wig's ky mograph, 272, 273

Ludwig on the lymph flow, 319

on function of kidneys, 573,577

on swaying movements of small intestine, 557

Stromuhr, 282

Lugaro's sleep theory, 745

Lunatic's brain, 751

Lungs, 346 et $\mathrm{seq}$.

air-sacs of, 351 et seq.

area of surface of, 352

blood-supply, 353

capillaries of, 352

changes of air in, 372

circulation in, 352

coverings of, 350

diffusion of gases within, 372

lobes of, 351

lobuies of, $i b$.

lymphatics, 353

muscular tissue, 352

nerves, 353

nutrition of, 352

position of, 346

structure, 349

Lunula, 605

Lymph, 26, 32, 61, 70, 224, 315 et seq.

composition of, 315

current of, 318

filtration theory, 319,320

formation of, 26,319

path, 317

relation to blood, 319

Lymph capillaries, 224

origin of, 226

structure, $i b$.

Lymph-hearts, structure and action of, 318

relation to spinal cord, $i b$.

Lymphagogues, 320

Lymphatic glands, 36,316 et seq.

Lymphatic vessels, 208, 223 et seq.

of arteries and veins, 221

communtcation with blood-vessels, 224

structure of, 221

Lymphocytes, 315,456

Lymphold or retiform tissue, 28, 35 See Adenoid tissue.

Lysine, 420

M.

Macallum's reagent, 27,166

Macdonald on nerves, 164,166

M'Dougall's "drainage " theory, 715, 716

MacEwen on bone regeneration, 50

Macleod on the nerves of the liver, 541
Membrana.

MacMunn, use of the term myo-hæmatin, 138

Macrophages, 457

Macrosmatic animals, 786

Macula, 754

lutea, 737, 814, 815, 818

Magendie, experiments on spinal nerve-roots, 156

foramen of, 644

Magnesium phosphate, 40, 54

Male organs of generation, 863

pronucleus, 881

sexual functions, 863

Malleus or hammer bone, 789,790

Malpighian bodies or corpuscles of kidney, 566 , 567, 569. See KIdney.

corpuscles of spleen, 36, 334

Malpighian discovery of capillaries, 228

Malpighian layer, 604

Maltase, 441

Maltose, 411, 439, 441

Mammal, nerves of, 248

Mammalian heart, excised, 261

Mammalian ovum, $884,886,887$

Mammary glends, 485

evolution, 486

involution, $i b$.

lactation, $i b$.

structure, 485

Mannite, 408

Mannose, $i b$.

Manometer, Hürthle's, 242, 276

Martin's, 391

Marchi reaction, 168, 169

Marchl's method, 651

Marey's sphygmograph, 291

tambour, 105, 107, 240

Marrow, 41

Martin's sphygmometer, 295, 296

Mast cells, 30

Mastication, 550

Mastoid cells, 788

Maturation of the ovum, 875

Maximal pulsation, 296

Maxwell's experiments on nerve impulse, 163

May, Page, reaction of degeneration, 179

Mayer's waves, 305

Mayo's experiments on cranial nerves, 156

Meat as food, 487

Meatus of ear, 789

Meckel's ganglion, 678

Mediastinum testis, 864

Medulla oblongata, 180, 643 et seq.

decussation of fibres, 666.668

diagrams of, 668,669

dorsal aspect, 666

fibres of, how distributed, 201, 662

pyramids, anterior, 661

postertor, $i b$.

structure of, 662

Medullary cavity, 41

segments, 75

sheath, 75 et seq.

substance, 340

Meibomian follicles, 496, 810

Melssner's corpuscles, 768, 774, 775

plexus, 493

Melanin granules, 847

Mellanby on creatine, 590

Membrana,

dectdua, 857

granulosa, 869

development into corpus luteum, 870

hyaloidea, 819

limitans externa, $817 \cdot 819$

interna, 815

propria or basement membrane. See Basementmembrane. 
Membrana.

Meinbraua_continued

tectoria, 792, 794

action of, 797

tympani, 788, 789, 792, 795

Membrane, vitelline, 871

Membranes of the brain and spinal cord, 180

Membranes, mucous. See Mucous membranes. semipermeable, 325

Membranous labyrinth, 792, 793. See Ear.

Memory image, 764

Mendel and Rockwood's experiments, 546

Menière's disease, 755

Meningeal streak, 312

Menopause, 862, 874

Menstruation, 862, 873, 874

coincident with discharge of ova, 862

corpus luteum and, $i b$.

Mercurial air-pumps, 364

Mercurial kymograph, 272, 274

Mesencephalon, 645

Mesial fillet, 672

Mesoblast, 19, 29, 41, 75, 883 et seq. organs formed from, 887

Mesoblastic somites, 883

Metabollc balance-sheets, 613 et seq

Metabolism, 6, 541

general, 612 et seg.

of fat, 616 et seq.

of protein, 621 et seq.

Metakinesis, 17, 18

Meta-proteins, 433

Metencephalon, 645

Methæmoglobin, 465 photographic spectrum of, 469

Metschnikoff on inflammation, 298 on phagocytosis, 476

Mett's tubes, 516

Meyer, anæsthetics, 167

Meyer, "sound-picture" theories of, 798

Meyer-Overton on narcotic effect on cells, 330

Meynert's fountain decussation, 673

Micrococci, 439

Micrococcus ureæ, 599

Micro-organisms, types of, 439

Microsmatic animals, 786

Micro-spectroseope, 467

Micturition, 580 centre, 580, 721

Mid-brain, 644

anterior aspect, 661

posterior aspect, ib.

structure of, 661 et seq.

Middle ear. See Tympanum.

Mid-gut, 886

Milk, as food, 481,482

alcoholic fermentation of, 483

chemical composition, 472

coagulation of, $i b$.

fats of, 483

chemical composition, $i b$.

globules of cow's milk, 481

proteins of, 482

reaction and specific gravity, ib.

salts of, 483

secretion of, 48

souring of, 483

Milk-curdling ferment, 520

Milk-sugar, 411, 483

properties of, 411

Milk-teeth, 50 et seq

Millon's reagent and test, 425

Mitochondrial sheath, 866

Mitosis, 15, 876

Mitral cells, 785

Mitral valve, 211, 214

Modiolus, 791

\section{Musclk.}

Molars. See Teeth.

Molecular layers, 816, 817

reactions, 329

Momentum, 267

Monakow's bundle, 657, 683, 685

Monaster stage of karyokinesis, 16, 18

Monatomic alcohols, 408

Monkey's brain, 730

spinal cord, 660

Mono-amino diphosphatides, 435

Mono-amino-mono phosphatides, 435

Monophasic variation, 125

Monoplegia, 729

Monosaccharides, 409

Monro-Kellie doctrine, 313

Moore and Rockwood's experiments on fat absorption, 548

Moore's test for sugar, 410

Morula, 883

Mosso's ergograph, 132

experiments on the effects of fattgue, 133

experiments on micturition, 580

Motor areas of cerebrum, 728

impulses, transmission in cord, 709

nerve-fibres, 73

Motor nerves, 70

of the iris, 833

Motor oculi nerve, 663,673

origin of, 663

Motorial impressions, 751

sense, 776

Mountain sickness, 400

Movements of protoplasm, 11, 12, 82

peristaltic, of involuntary muscle, 140 of stomach, 555

Mucic acid, 410

Mucigen or Mucinogen, 21, 27, 499

Mucin, 21, 27, 430, 505

Mucolds, 36, 430

Mucous membrane, 493

gland-cells of, $i b$.

of intestines, $i b$.

of larynx, 801

of stomach, 493

Müller's fibres, 815, 816

Miiller's fluid, 168

Muller's law of specific nerve energy, 762

Müller's muscle, \&12, 813

Multipolar nerve-cells, 183 et seq.

Munk's experiments on fat absorption, 548

Munro, foramen of, 645,688

Murexide test, 591

Muscarine, action of, on the heart, 253

Muscle, 62 et seq.

blood-vessels of, 70

cardiac, 71,255

changes in form, when it contracts, 86 et seq.

chemical changes in, 131

composition of, 136

clot, 137

columns, 65

contractility, 68

curves, 93, 96, 99, 101, 114

development, 72

dynamometer, 114

elasticity, 108 et seq.

electrical phenomena of, 116 et seq., 176

extensibility of, 116 et seq.

fatigue, effect of, $97,98,132$

curves, 96

Hensen's line, 65, 67

involuntary, 62, 79 (see 140 et seq.)

irritability, 83

evidence of, $i b$.

lever system of, 106 


\section{MUSCL.}

Muscle-continued

nerves of, 70

plain, 71

plasma, 137, 138

proteins, 139

reciprocal action of antagonistic, 714

red, 70

response to stimuli, 84 et seq.

rigor, 136,139

sarcolemma, 63,64

sensory nerve-endings in, 769

serum, 138

shape, changes in, 104

skeletal, 63

sound, developed in contraction of, 104

spindle, 70 . See Neuro-muscular spindle.

stimull, 85, 101

striated, structure of, 66 et seq.

tetanus, 103 et seq.

negative variation of, 123

thermal changes in, 129

tonus, 113, 142

twitch, 101 (see 123)

voluntary, 62,140 et seq.

wave, 100,123

work of, 113

Muscular action, conditions of, 114

Muscular coat of allmentary canal, 492

Muscular contraction, 84, 94, 96 et seq.

effect of two successive stimuli, 101 of more than two stimuli, $i b$.

voluntary tetanus, 104

Muscular fibres

development, 72

plain, 62 et seq.

transversely striated, $i b$.

voluntary, 72

Muscular force, 111

Irritability, 81

tissue, 62 et seq. composition of, 13 ?

Muscular sound, 236

Muscularis mucosæ, 348

Musculi papillares, 213, 214, 234

Musical sounds, 806

Mydriatics, 833

Myelencephalon, 645

Myelination, 739

Myelocytes, 461

Myeloplaxes, 41

Myogen-fibrin, 139

Myogenic, 560

Myoglobulin, 138

Myohæmatin, $i b$.

Myograph, 87, 92

Helmholtz's, 92

pendulum, 94

spring, $i b$.

transmission, 105

Myopta, or short-sight, 828

Myorectes, 65

Myoprotein, 139

Myosin, 136 et seq.

Myosinogen, 137 et seq.

Myotics, 833

Myxœdema, 33.

N.

Nalls, 605

Narcosis, 744 et seq.

Nasal cavities in relation to smell, 783 et seq.

Nasmyth's membrane, 59

Near point, 826
Nerves.

Negative variation, 121

Neoencephalon, 646, 647

Neopallium, 647

Nerve-cells, classlfication of, 194

structure of, 182 et seq.

Nerve-centres, 180 et seq. See Corebellum, Cerebrum, etc.

ano-spinal, 564

cillo-spinal, 721

defæcation, 562

deglutition, 551

erection, 721

micturition, 580,721

parturition, 721

secretion of saliva, 500

speech, 733

vaso-motor, 300,716

Nerve-corpuscles, 176

bipolar, 183

unipolar, $i b$.

Nerve-fibres, cardio-inhlbitory, 249

in spinal cord, 653

intercentral, 145

Nerve-impulse, nature of, 163

velocity of, 159

Nerves, 73 et seq.

accelerator, 144

action of stimuli on, 81

afferent, 73, 144, 204, 557

analyses of, 166

axis-cylinder of, 74

axons, 152, 188, 198

colls, 79,181

centrifugal, 143

centripetal, 144

cerebro-spinal, 181

changes in, during activity, 158

classification, 143

conductivity of, 159

cranial, 180, 181, 663 et seq.

crosses of Ranvier, 75

crossing of, 161

degeneration, 146, 178, 307

chemistry of, 165

reaction of, 178

direction of a nerve impulse, 160

efferent, $73,143,557$

electrical, 144

stimulation of, 176

excitability of, 159

fibres, 74 et seq., 143 et seq.

development of, 79

functions of, 146

funicull of, 76

grey matter, 73

hypogastric, $5 \mathrm{~S}$

Inhibitory, 144

Irritability of, 81

laws of conduction, 144 et seq., 159

medullary sheath, 74

medullated, 74

motor, 143

termination of, 78

nodes of Ranvier, 75

non-medullated, 77

olfactory, $180,663,786$

physiology of, 143 et seq.

pilo-motor, 607

plexuses of, 79

reflex actions, 145, 188

secretory, 144

section of, 146,500

shocks, method of slow interrupted, 307

size of, 77

spinal. See Spinal nerves.

stimulation of cut, 146 
Nerves.

Nerves-continued

structure, 74

sympathetic, influence on heart, 249

taste, 781

temperature, infiuence of, 308

terminations of,

in corpuscles of Golgi, 769

in corpuscles of Grandry, 768

in corpuscles of Herbst, 766

in end-bulbs, 767

in motorial end-plates, 78

in networks or plexuses, 770

in Pacinian corpuscles, 766

in touch-corpuscles, 767

trophic, 144

Nervous circles, 715

Nervous system,

autonomic, 196 et seq.

central, 640, 642 et seq.

cerebro-spinal, 181

electrical variation in central, 743

influence on the heart, 389

sympathetic, 249

vaso-motor, 300 et seq.

Nervous tissues, chemistry of, 165 et seq. chemical changes during activity, 167 chemical changes in degenerative conditious, 167

potassium salts in, 166

Nervus erigens, 581

Neurilemma, $74,153,181$

Neuroglia, 181, 649

Neurokeratin, $75,165,182,429$

Neuro-muscular spindles, $70,769,770$

Neuron, 189

Neutral sulphur, 595

New-born child and changes after birth, 862 et seq.

Nicol's prism, 68, 434

Nicotine, action of, 200

Nipple, ths, 485

Nissl's granules, 165, 184, 192, 194 significance of, 192

Nitric oxide hæmoglobin, 465, 470

Nitrogen in the blood, 362

eliminated in the form of urea, 479

Nodal point, 820

Nodes of Ranvier, 75

Noll, nerve degeneration, 167

Normoblasts, 460

Nose. See Smell.

Notochord, $884,885,886$

Nuclear layers, 816,817

Nuclear membrane, 9 sap or matrix, 9,10

Nuclease, 432, 594

Nucleic acid, 431,432

Nuclein, 8, 10, 430

Nuclei pontis, 664

Nucleoli, 9, 10, 12, 15

Nucleo-proteins, 8, 165, 427, 430, 431

Nucleotides, 432

Nucleus of animal cell, 5, 7, 9 et seq. chomical composition, 10 division, 15

of crantal nerves, diagram of, 665 staining of, 10 structure, ib.

Nucleus ambiguus, $669,670,678$

Nucleus cuneatus, 667, 669 gracilis, ib.

Nucleus of Bechterew, 677 of Deiters, 670, 671, 677

Nutrition, effect on respiration, 392

Nyctalopia, 849

Nystagmus, ib.

\section{Ovo-MUCOID}

O.

Occipital convolutions, 693

Occipital lobe, $690,692,693$

O'Donoghue on corpus luteum, 881

Odontoblasts, $53,55,57$

Odontogen, 57

Odours, 786. See Smell.

Ersted's electro-maguetism, 117

Esophagus, 492, 493

(Estrus, 874

Oleaginous principles, 414

Oleic acid, $i b$.

Olein, 35, 414

Olfactory bulb, 784

cells, $i b$.

glomeruli, 786

nerves, $663,785,786$

tract, 784

"roots" of, $i b$.

Olivary body, 661, 668, 671, 681

Oliver's method of estimating hæmoglobin, 472

Oncograph, Roy's, 310

Oncometer, 309, 571

Roy's, 310, 571, 572

Oöcytes, 868 et seq.

Oögenesis, 873, 877

Oögonia, 871

Ophthalmometer, Helmholtz's, 826

Ophthalmoscope, 836 et seq.

Opsonins, 476

Optic dise, 814

Optic nerve, 663,815

decussation of fibres in, 853

fibres, 815

nervous paths in, 853

Optic radiations, 737

Optic thalamus, 689

Optical angle, 822,823

apparatus of eye, 820 defects in, 828

axis, 822

defects in, 828

Optogram, 847

Ora serrata of retina, 814, 815, 819

Orang's brain, 691

Orbicularis muscle, 810

Orbital sulcus, 691

Organ of Corti, 794

Organic compounds in body, 406 et seq.

Ornithine, 417, 583

Osazone, 412

Osmosis, 323, 324-6

distinguished from diffusion, 424

Osmotic pressure, method of estimating, 322 et seq.

calculation of, 326

determination of, 326

of proteins, 327

phenomena, 322 et seq.

physiological applications, 326

Os orbiculare, 790

Osseous labyrinth, 790. See Ear.

Ossicles of the ear, 788, 795

action of, 796

Ossification, stages of, 45 et seq.

Osteoblasts, 45 et seq.

Ostooclasts, 49,50

Osteogen, 45

Ovarian ovum, 870

Ovariotomy, 879

Ovary, 867 et seq., 878, 879

Graafian follicles in, 869,870

internal secretions of, 878

Oviduct, or Fallopian tube, 867

Ovo-mucoid, 430 
Ovulation.

Ovulation, 869,874

Ovum, 18, 19, $870,885 n$.

action of seminal fluid on, 875 et seq

changes in ovary, 875

previous to fecundation, 875

cleaving of yolk, 883

development, 882

diagrams of, 868,870 et seq.

fertillsed, 881

formation of, 870

germinal vesicle and spot of, 1S, 19, 870, 871

impregnation of, 881

maturation, 875 et seq.

segmentation, 883

structure of, 870

in mammals, 871

subsequent to cleavage, 883 et seq

Oxidases, 440, 594

Oxidation, measurement of coefficlent of, 396,397

Oxygen in the blood, 365 et seq., 399 et seq.

pressure of, where fatal, 400

want, 399

Oxyhæmoglobin, 61, 216, 372, 402, 461, 462, 465, 469

crystals of, 462

spectrum of, 468,469

Oxyntic cells, 508,510

\section{P.}

Pacinlan corpuscles, 766, 767

Pain, 777

Pain spots, 772, 774

Pala-encephalon, 646, 647

Palmitic acid, 414

Palmitin, 34, 414

Pancreas, 517

adantation of, 522

extirpation of, 539

diabetic condition produced in animals by, 539

so-called peripheral reflex, secretion of, 520

structure, 517

Pancreatic juice, 518

action on fats, 520

composition and action, 518

ferments in, $i b$.

Papillæ,

of the kidney, 565

of skin, distribution of, 604

of tongue, 779 et seq.

Papillary layer of heart, 212

Paradoxical contraction, 17

Paralytic secretion, 503

Paramyosinogen, 138, 142, 165

Paraplasm, 8

Parathyroids, 339

Parietal cells, 508, 510

Parietal layer of pericardium, 208

Parietal lobe, 692, 693

Parieto-occipital fissure, 692, 694

Parotid gland, 503

alveoli of, 500

Pars clliaris retinæ, 819

Pars intermedia, 343

of Wrisberg, 675

Parthenogenesis, 878

Parturition, 893-895

Par vagum. See Pneumogastric nerve.

Pathological conditions of nervous system, 811

Pathological urine, 600

Pathogenic organism, 476

Patrick's experiments, 748

Pavy's views as to the liver being a sugar. forming organ, 537
Pinna.

Pawlow's method for obtaining pure gastric juice, $511,512,522$

his observations on the salivary glands, 504 on the secretory nerves of the pancreas, 521 on the succus entericus, 523,525

Peduncles of the cerebellum, 643

Peduncles, superior cerebellar, 658

Pelvts of the kidney, 565

Pendulum myograph, 94, 95

Penis, structure, 866

Pentosuria, 602

Pepsin, 439, 440, 510

Pepsinogen, 509

Pepsin-hydrochlortc acid, 511

Peptides, 422

Peptogens, 513

Peptolytic enzymes, 440

Peptones, 417, 433, 450, 601

characters of, 515

Peptonuria, 601

Perforated spots, 689

Perforating fibres of Sharpey, 44

Pericardium, 208

Perichondrium of cartilage, 47

Perilymph, or fluid of labyrinth of ear, 753,790

Perimeter, 839

Perineurium, 76, 77

Periosteum, 42, 45, 50, 59, 60

Peripheral resistance, 264 et seq., 297

Peristalsis, 83, 140, 555, 559, 561

Peristaltic movements of intestines, 83,557

of involuntary muscle, 140 (see 553)

of stomach, 553 et seq.

Peritoneum, 209

Permanent teeth. See Teeth.

Personal equation, 720

Persplration, cutaneous, 609

insensible and sensible, $i b$.

ordinary constituents of, 610

Pes, 673

Petit's canal, 820

Pettenkofer's reaction, 533

Peyer's patches, 36

Pfliuger's law of contraction, 174, 178

on hepatic cells, 530

on menstruation, 875

on proteins, 622

Phagocytes, $457,473,476$

Phakoscope, Helmholtz's, 825

Pharynx, 492, 805

Phenyl alanine, 418

Phenyl.hydrazine test, 412,602

Phloridzin-diabetes, 540

Phosphates in urine, 596, 599, 600

Phosphatides, 166, 167, 438

Phospho-proteins, 427, 429

Photo-chromatic interval, 848

Photo-hæmatochometer, 286

Photophobla, 849

Phrenograph, 357

Physical chemistry, 322 et seq.

Physiological applications of physical chemistry, 327

methods, 1 it seq.

pit, 838

rheoscope, 127

zero, 774

Pia mater, 182,644

Picric acid test, 602

Pigment cells, 30

of retina, 82,818

movement of, 847

Pilocarpine, effect of, on salivary gland, 503

Pilo-motor nerves, 607

Pineal gland, 345, 665

Pinna, 789 
PIOTROWSKI's REACTION.

Piotrowski's reaction, 425

Piper's experiments on muscle contraction, 106

Pitch of voice, 794, 806, 807

Pitot's tube, 286

Pituitary body, 343 et seq.

effects of removal, 345

Placenta, maternal, 889

fotal, $i b$.

Plantar reflex, 711

Plasma cell, 29

Plasma of blood, 60, 444, 449, 452 et seq. gases of, 453

Plasmatic membrane, 330

Plethysmograph, 308

Schäfer's, 260

Pleura, 209, 349

Plexus, terminal, 770

of Auerbach, 79

of Meissner, 493

Pneumogastric nerve, 249 et seq., 664, 678

distribution of, 678

functions, 664,678

influence on

deglutition, 553

gastric secretion, 512

heart, 249

muscles of stomach, 556

pancreatic secretion, 520

vomiting, 556

mixed function of, 678

origin, $i b$.

Poehl on testis, 878

Poggendorf's rheochord, 170

Pohl's commutator, 169

Poikilothermal animals, 636

Poiseuille's hæmodynamometer, 272

Polarimeter, 434

Polygonal epithelium, 529

Polymorphic layer, 697

Polynuclear leucocytes, 456

Polypeptides, 417, 422, 433

Polysaccharides, 409

Pons Varolli, 642, 643, 646, 662

grey matter in, 644

Popielski's investigations into pancreatic secretion, 520

Portal canals, 528

circulation, 215

vein, 527, 528. See Liver.

Postero-lateral column, 658

Post-ganglionic fibres, 198 et seq., 249, 303

Potato starch, grains of, 412

Precipitants of proteins, 426

Precipitin, 477

Precuneus or quadrilateral lobule, 694

Preganglionic fibres, 198 et seq., 302

Pregnancy,

corpus luteum of, 870

Prepyramidal tract, 657

Presbyopla, 831

Pressor nerves, 305

Pressure gradient, 285, 386

Pressure head, 286

Pressure-measurers, 267

Pressure, positive and negative, 271

Primary areas, 742

Primary areolæ, 48

Primary responses of eye, 848

Primitive nerve-sheath, or Schwann's sheath, 78

Primitive gut, 886

Projection fibres, 698,740

Projection systems, 698, 700

Proline, 421

Pronucleus, female, 876,882

male, 876,881 , 882

Propeptone, 514
PUS IN THE URINE.

Prosecretin, 521

Prosencephalon, 645

Prostate gland, 570, 879

Prosthetic group, 430

Protagon, 437

Protamines, 427

Protective inoculation, 473

Protein-hydrolysis, 432

Protein metabolism, 6, 416, 621

Proteins, 6, 8, 10, 165, 416 et seq., 453

absorption of, 544

action on polarised light, 425

of blood, 453

Bence-Jones', 601

classification, 426 et seq.

cleavage products, 416

coagulated, 426

colloidal solution, 424

colour reactions, 425

composition, 416

conjugated, 427, 430

crystallisation, 424

enzy me coagulation, 440

heat coagulation, 425

in food, 479 et seq.

indiffusibility of, 425

metabolism of, 6,621 et seq.

osmotic pressure of, 327

of plasma, 453

precipitants of, $417,425,426$

of serum, 453

simple, 416

solubilities, 423,424

in urine, 600

Protensity, 763

Proteoclastic enzymes, 440

Proteoly tic enzymes, 440, 514

Proteoses, 417, 433, 601

characters of, 515

Prothrombin, 449

Protones, 427

Protopathic sensations, $706,774,777$

Protoplasm, 5, 7, 10

chemical structure, 8

irritabillity, 13

movements, 8, 11 et seg.

Proto-vertebræ, 884

Protrusion of eyeball, 849

Przibram, Hans, theory of classification by muscle proteins, 139

Pseudopodia, 11, 14

Pseudoscope, 856

Pseudo-stoniata, 222

Psycho-physical parallelism, 758

Ptosis, 850

Ptyalin, 439, 499, 505

Ptyalinogen, 499

Puberty, 862

Pulmonary artery, 215

Pulmonary circulation, pressure in, 281

Pulsation, maximal, 296

Pulse, anacrotic, 293

arterial, 290 et seq.

dicrotic, 293, 294

intermittent, 290

velocity, $i b$.

venous, 299

volume, 309

water hammer, 291

wave, 295

Pupll of the eye, 833, 834

Purine bases, 421, 432, 593

Purkinje's cells, 187, 681

fibres, $71,72,213,256,257$

figures, 835

Pus in the urine, 603 
Pyloric Glands.

Pyloric glands, 507, 508

orifice, 553,554

Pyramidal tracts, 655

Pyramids of medulla oblongata, 661 of kidney. See Kidney.

Pyrimidine bases, 421

Pyrrhol blue, 890

Pyrrolldine derivatives, 421

\section{R.}

Racemose glands, 496

Rami communicantes, 196

Ranke's diet, 394, 628

Ranvier's clasmatocytes, 31

Ranvier's crosses, 75

Ranvier's nodes, $i b$.

Raynaud's disease, 312

Reaction time in man, 720 velocity, 328

Reactions of proteins, colour, 425

Receptive groups, 475

substances, 164

Recurrent sensibility, 156

Rectum, the, 562

Red marrow, 41

Reduced eye, 821

Reductases, 440

Reflex arc, 711

actions, 145, 188, 709 et seq.

inhibition of, 253,719

in frog, 709

in man, 145,711

superficlal, 711

tendon, 712

of dog, 719

of nerves, 145

of spinal cord, 709 et seq.

"scratch," 719

Reflex secretion, 504

Reflexes,

cumulation of, 710

inhibition of, $i b$.

spinal visceral, 721

spreading of, 710

uterine, 722

Refraction, laws of, 821

Rofractive media of eye, $i b$.

Reid, osmotic pressure, 328

Reil, island of, $688,692,694$

Reissner's fibre, 644

Reissner's membrane, 792, 794

Relaxation of heart, 232

Remak, tibres of, 77

ganglion of, 255

Renal circulation, 215, 580

epithelium, 577

oncometer, 572

plexus, 571

Rennet or rennin, $440,483,514$

Reproductive organs, 6,858 et seq. development of, 858 et seq.

female, 867 et seq.

male, 863 et seq.

physiology of, 872 et seq.

Requisites of diet, 613

Reserve air, 359

"Reserve force," 625

Residual air, 359

Resistance, peripheral, 264

Resonance theory, 798

Respiration, 346 et seg.

abdominal type, 355

adaptation to high altitudes, 401

siteration in atmospheric pressure, 362

artificial, 383

\section{RHEOCHORD.}

Respiration-continued

breathing or tidal air, 359

cause and regulation of, 375 et seq.

chemical cruse of, 380

chemistry of, $i b$.

Cheyne-Stokes, 404, 405

effect on circulation, 385

effect on nutrition, 392

gases in relation to, 361 et seq.

at high pressure, 402

influence of nervous system, 376

intensity of, 399

mechanism of, 354 et seq.

movements, 356

nervous factor in, 376

of vocal cords in, 804

quantity of air changed, 358

record of, 356

tissue, 397

Respirations, number of, in healthy persen, 359

Respiratory acts, special, 381

apparatus, 346

capacity of chest, 359

centre, 375

methods of recording, 356

muscles, 354 et seq.

muscular force of, 360

nerve-centre, 376

rate, 360

relation to pulse-rate, $i b$.

rhythm, 388

sounds, 357

"Rest cure," 625

Restlform bodies, 661, 669

Rete mucosum, 604

Rete testis, 864, 865

Roticulam, 7, 18

of the thymus, 337

Retlform tissue, 28, 35

Rotina, 811, 814

blind spot, 834,849

blood-vessels, 820

changes in, during activity, 847

duration of impression on, 836

of after-images, 844

electrical variations in, 849

elements of, schems, 819

excitation of, 836

focal distance of, 824

fovea centralis, 835

functions of, 834

identical points of, 852

Image on, how formed distinctly, 823

layers, 815 et seq.

meaning of term, 845

nervous elements of, 816

ora serrata, 814

pigment-cells, 82, 818

movement of, 847

in relation to single vision, 850

structure of, 818

visual purple, 847

Retinitis pigmentosa, 849

Retinoscope, 831

Retraction of eyeball, 849

Retractor lentis muscle, 828

Reverstbility, 329

Reymond, Du Bois,

currents in muscle prism, 121-123, 125, 126

electrical variation in spinal cord, 743

induction coil, 90

key, 88,89

non-polarisable electrodes, 119

spring myograph, 94

Rheochord, 174

Poggendorf's, 170 
Rheoscope, Physiological.

Rheoscope, physiological, 127

Rheoscopic frog, 127

Rhodopsin or visual purple, 847

Rhythm in cardfac muscle, 255

Rhythmical contraction and dilatation, 299

Rhythmicality of movement, 83, 140

Ricin, 475

Rigor mortis, 136 et seq. affects all classes of muscles, 136 phenomena and causes of, $i b$.

physical basis of, 131

Rima glottidis, 801,805

Ringer's investigations on drug action, 260 fluid, 5 T7 solution, 260

Ritter's tetanus, 176

Rocci, Riva, his sphygmometer, 295

Rods and cones, 817

Rolandic area, $687,689,722,723,727,733$

injury of, 723

Rolando, fissure of, $692-694,729,732$ substantia gelatinosa of, 667,668 tubercle of, 667

Rollett's view of the red corpuscles, 454

Rose's test, 425

Rösel on protoplasmic movement, 13

Rotation, 13 method for estimating specific gravity of blood, 444

Roy's cardiometer, 248

oncograph, 310

oncometer, $i b$.

Rubner, on action of food stuffs, 625

law of conservation of energy, 630

Rumination, 550

Rut, 894

Rutherford's " sound-picture" theories, 798

\section{S.}

Saccharic acid, 410

Saccharoses, 409

St Martin, Alexis, case of, 507, 554

Saccule, 791

Salathé, effect of gravity on the circulation, 279

Saliva, 499, 504 et seg.

action of, 505

composition, 504

process of secretion, $i b$.

reflex secretion, $i b$.

secretion following stimulation of nerves, 500 et seq.

Salivary glands, 498 et seq.

digestion, 506

extirpation of, 504

influence of nervous system, 502, 503

effect of drugs on, 503

secretory nerves of, 502

structure, 498

Salkowski's reaction, 436

Salmine, 427

Salts in the blood, 454

Sanderson's cardiograph, 239

on electrical change in muscles, 125

Sanson's images, 824, 825

Santorini's cartilages, 800,802

Saponification, 415,520

Sarcinæ, 439

Sarcolemma, 63, 64, 137

Sarcomeres, 67,68

Sarcoplasm, 65, 67, 72

Sarcostyles, 65 et seq.

\section{SRNSORY IMPRESSIONS.}

Sarcous elements, 67 et seq.

Sartorius, record of injured, 130

Scala media, 792

Scala typani, 791,797

Scala vestibuli, 791,797

Scarpa, ganglion of, 677

Schäfer, heart plethysmograph, 260

observations on liver cells, 530

method of artificial respiration, 383, 384

protoplasmic structure, 7,14

researches on the structure of a sarcostyle, 67

views regarding the function of the Rolandic area, 731

white blood corpuscles, 8

Scheiner's experiment, 826

Schematic eye, 821

Schenk on muscular contraction, 115

Schlemm, canal of, 813,814

Schmidt's method of preparing fibrin ferment, 453

Schröder's experiment, 587

Schwann, white substance of, 74

Sclero-proteins, 427.429

Sclerotic, $807,808,809,811.813$

Scotoma, 849

Scratch reflex, 717,718

Sebaceous glands, 608

Sebum, 608

Secondary contraction, 128

Secretin, 521

Secreting glands, 81, 492 et seq.

classification of, 496

diagram of, 497

Secreting membranes. See Mucous and Serous membranes.

Secretion, internal, 331

of kidney, 571

pancreas, 520

saliva, 500

paralytic, 503

reflex, 504,520

suprarenal, 340

thyroid, 337

Secretory nerves, 144

diagram of, 503

of pancreas, 521

of salivary glands, 502

effect of section of, 503

of sweat glands, 610

Segmentation of cells, 883

in chick, 885

nucleus, 876

ovum, 883

Semen, 866, 867

spermatozoa, ib.

Semicircular canals of ear, 794

diagrams of, 754,755

structure, 753 et seq.

Semilunar valves. See Heart valves.

Semipermeable membranes, 324

Sensation, 760,764

cutaneous, 766 et seq.

difference in quality, 762

extensity, $i b$.

hallucination, 764

memory image, $i b$.

nerves of, 144

tactile, 751

thresholds, 763

Weber's law, $i b$

Sensibility, recurrent, 156

Sensory areas in cerebral cortex, 728

Sensory channels, diagram of, 702

Sensory impresions, conduction of, by spinal cord, 144

in brain, 734 et seq. 
Sensory Nerve-Endings in Muscle.

Sensory nerve-endings in muscle, 769 ; in skin, 766 Sensory nerves, 70

Septum nasi, nerves of, 785

Serine, 417

Serous coat, 493

Serous membranes, 208

Sertoli, cells of, 864

Serum,

albumin, 315,453

of blood, 60,452 et seq.

globulin, 315,453

Seventh cerebral nerve, 663,673

Sex, determination of, 859

Sexual organs in the female, $86 \tau$ et seq.

in the male, 863 et seq.

Sharpey on bone formation, 50

fibres of, 44

Sherrington,

experiments on motor area, 731

on course of reflex in knee jerk, 712

observations on binocular flicker, 854

principal of the common path, 716,717

reciprocal action of antagonistic muscles, 714, 715

Short sight, 828

Side-chain theory, 475

Sight. See Vision.

Silent areas, 738

Silver nitrate reaction of cementing substance, 27

Simple tubular glands, 496

Sino-auricular node, 213,256

Sinus, coronary, 209

Sinuses of Valsalva, 214, 237

Sinusoids, 222, 528

Sixth cerebral nerve, 663, 671

Skein, 15, 16, 18

Skeletal muscles, 63

Skiascot 8,831

Skin, 604

absorption by, 608

dermis, 604

diseases, 611

epidermis of, 604

functions of, 608

nerve-endings in, 760

papillæ of, 604

respiration, 608

rete mucosum of, 604

sebaceous glands of, $60 \mathrm{~S}$

secretions, $i b$.

sections of, 605 et seq.

8 weat, 609 et seq.

sweat-glands, 496, 608

varnishing the, 611

Sleep, 744 et ser.

Smell, sense of, 738,782 et scq.

anatomy of regions, 784

connection with taste, 738

dellcacy of sense of, 787

tests for, varies in different animals, 786

Smith, Lorrain, experiments on quantity of the blood, 445

Smith's perimeter, Priestly, 840

Sneezing, mechanism of, 382

Snoring, mechanism of, $i b$.

Soap, 416

Sobbing, 382

Sodium chloride method, 431

Solltary glands. See Peyer's patches.

Solubilities, 423

Solutions, gramme-molecular, 323

Somatic mesoblast, $872,884,887$

Somatoplasm, "l eismann's, 861

Somatopleur, 884

Somites, mesoblastic, 884
Spirilla, Various Forms of.

Sonorous vibrations, how communicated in ear, 794 et seq.

in air and in water, 795. See Sound.

Sound,

conduction by ear, 794

heart, 235,245

production of, 806

Soup, value as food, 490

Sour milk as cure for dyspeptic disorders, 527

Spaces of Fontana, 814

Speaking, mechanism of, 807

Special senses, 766 et seq.

Specific nerve energy, law of, 762

Spectroscope, 465 et seq.

Speech, 807 et seq.

centre, 733

defects of, 808

Spermatids, 864, 865, 873

Spermatocytes, $i b$.

Spermatogonia, $i b$.

Spermatozoa, 19, 866, 867, 881

form and structure of, 866,867

Spheno-palatine ganglion, 678

Spherical aberration, 830

correction of, $i b$.

Sphincter anl. See Defæcation.

pupillæ, 812

vesicæ, 570

Sphingomyelin, 166, 435, 438

Sphygmographs, 291, 292

tracings, 291 et seq.

Sphygmometers, 295

Spinal accessory nerve, 664

functions of, 679

origin, $i b$.

Spinal cord, 648 et seq.

association fibres in, 658

canal of, 648

columns of, 649

commissures of, 648

conduction of impressions by, 704 et seq.

course of fibres in, 653

diagrams of, $649,650,660$

fissures and furrow' of, 648

functions of, 704 et seq.

of columns, 654

grey matter, 181,650

cells in, 650

hemisection, 659

injuries of, 704, 709

membranes of, 643

nerves of, 653

reflex action of, 709 et seq.

inhibition of, 710

in frog, 709

in $\operatorname{man}, 711$

superficial, $i b$.

reflex irritability of, 712

regions of, 659

section of, $i b$.

special centres in, 720

structure of, 648 et seq.

tracts, $651,655,703$

transverso section of, 659

white matter, 167, 649

tracts in, 651

Splnal nerves, 155

functions of roots of, 155,649

origin of, 155 et seq.

Spinal visceral reflexes, 721

Spiral canal of the ear, 791

Spiral ganglion, 794

Spiral layer, 213

Spiral ligament of ear, 792

Spirem, 15, 18

Spirilla, various forms of, 439 
SPIROMETER.

Spirometer, 359

Splanchnic mesoblast, 884,887

Splanchnic nerves, 342

Splanchnopleur, 884

Spleen, 332

apparatus for splenic curves, 134, 313

functions, 334

Influence of nervous system upon, 335

Malpighian corpuscles of, 334

pulp, 333

structure of, 332

trabeculæ of, $i b$.

Spongioblasts, 816

Spongioplasm, 7, 8, 14, 68, 83

Spot, germinal, 871

Spring myograph, 94

Staircase phenomenon, 97, 141, 259

Stannius' experiment, 258

Stapedius muscle, 790,796

Stapes or stirrup bone, 789,790

Staphylococcl, 439

Starch, 412, 537

Starling mammalian heart, 245

on pancreatic secretion, $520-525$

Starvation, 621,628

effects of, $i b$.

Stearic acid, 414

Stearin, 34, 414

Stellate cells of Kupffer, 528

Stercobilin, 534

Stethographs, 357

Stewart's

experiments on the circulation of the blood, 289,290

on muscle proteins, 142

on the output of the heart, 247

Stimulants as accessories to food, 490

Stimulation fatigue, 134

Stimuli, varietles of, $13,14,81,84,85$

Stirrup bone, 789, 790

Stokes' reagent, 465

Stolnikow, measurement of the heart's output, 247

Stomach,

blood-vessels, 509

digestion in, 525,563

glands, 508

movements, 553

influence of nervous system on, 556

mucous membrane, 507

nervous mechanism of, $556,557,563$

secretion of. See Gastric julce.

time taken to empty, 554

view of empty, 555

Stomata, 226

Stratum granulosum, 604

intermedium of Hannover, 58

lucldum, 604

Streptococci, 439

Strlæ acousticæ, 663,677

Striated muscle, 66 et seq. See Muscle.

Striate area, 736

Stroma, 454, 868

Stromuhr, Ludwig's, 282

Tigerstedt's, 284

Structure of cells, $8 \mathrm{et} \mathrm{seq}$

Stuart's kymoscope, 271

Sturine, 427

Stylo-pharyngeus, 678

Subendothelial layer, 218

Sublingual gland, 503

Submaxillary gland of dog, 499

Submaxillary and sublingual glands, 499, 500

Submaxillary ganglion, 502

Submucous coat, 493

Substantia gelatimosa of Rolando, $650,667,668$ nigra, 672,673
TRMPRRATURE.

Substrate, 439

Subthalamic area, 689

Succagogues, 513

Succus entericus, 522 et seq.

functions of, $i b$.

Sucroses, 409

Sugar. See Dextrose, Lactose, etc.

Sulci, 690 et seq., 730, 731

Sulphates in urine, 595

Summation-tones, 797

Superficial reflexes, 711

Superior laryngeal nerve, effects of stimulation of cut, 378

olivary nuclous, 671

parietal convolution, 693

Supra-renal capsules, 340

cortex, 342

function, 341

structure, 340

Surface tension, 330

Sustentacular fibres of Miiller, 815, 816

Swallowing, 551

centre, $i b$.

fluids, 552

nerves engaged, $i b$.

Sweat, 609 et $s \in q$.

Sweat-glands. See Skin.

Sylvius, aqueduct of, $644,645,663,671$

Sylvius, fissure of, $642,643,692-694$

Sympathetic nerve, 251

Sympathetic secretion, 502

Syntonin, 434

Syringomyelia, 707

Systemic circulation, 215. See Circulation.

Systole of heart, 232

Systolic pressure, 296

Systolic sound, 235

\section{T.}

Tabes dorsalls, 715

Tache cérebrale, 311

Tactile area, 734

discs, 770

end-organs, 766 et seq.

impressions, 751

sensibllity, $734,770,771$

varlations in, 772

Talbot's law, 836

Tapetum lucidum, $837 n$.

Tarsus, 810

Taste, sense of, 738,779 et seq.

classification of, 783

connection with smell, 738

delicacy of, 783

nerves of, 781

Taste-buds, 781, 782

Taurlne, 533

Taurocholic acld, $i b$.

Taxis, positive and negative, $14 n$.

Tchistovitch's discovery of distinguishing human and other blood, 477

Teeth, 50 et seq.

development, 56 eruption, times of, 51

incisor, 51

structure, 52 et seq.

temporary and permanent, 50 et seq.

Tegmental nucleus, 671

Tegmentum, $i b$.

Telencephalon, 645

Temperature, 368, 636 et seq.

average of body, 636

changes of, effects, 636 et seq. 
Temperature.

Temperature-continued

effect on muscular contraction, 99

extensibility, influence on, 112

of cold-blooded and warm-blooded animals, 636

in disease, 641

loss of, $638 \cdot 641$

maintenance of, 636

of Mammalia, birds, etc., $i b$.

sensation of variation of, 772 . See Heat.

Temporo-occipital cerebellar fibres, 673

Temporo-sphenoidal lobe, 692, 693

Tendon-reflexes, 711,712

T'ension of gases in fluids, 365

Tensor palati muscle, 795

tympani muscle, 790,796

action of, 795

Terminal areas, 743

Terminal ganglia, 249

Testlcle, 863, 864

structure, $i b$.

Testls, 863, 878

internal secretions of, 878

Tetanus, composition of, 103

injured sartorius during, 126

Ritter's, 176

voluntary, 104, 142

Tetany, 340

Thalamencephalon, 645

Thalamt opticl, 688

Theine, 490

Theobromine, $i b$.

Thermopile, 130

Thirst, 778

Thoma-Zeiss hremacytometer, 458

'Thomson's galvanometer, 118

Thoracic duct, 61, 224

innervation of, 319

Thresholds of the stimulus, 763

Throat deafness, 795 ventricle, 801

Thrombin, 440,448, 449, 453

Throm bogen, 449-45]

Thrombokinase, $i b$.

Thudichum on protagon, 437

Thymus gland, 336 effects of removal, 337

function, $i b$.

structure, 336

Thyro-arytenoid muscle, 801, 802

Thyro-epiglottidean muscle, 802

Thyroid cartllage, 799

Thyroid gland, 837

function, 342

structure, 337

Thyro-lodin, 839

effect of intravenous injection of, on blood. pressure, 339

Tigerstedt, measurement of the heart's output, 247,290

Stromuhr, 284

Timbre of voice, 794, 806

Tissue-erepsin, 546, 593

Tissue-respiration, 394 et $s e q$.

Tongue, 779

action in deglutition, 548

epithelium of, 780

muscles of, 779

paplliæ of, $i b$.

parts most sensitive to taste, 781

structure of, 779

Tonometer, Barcroft's, 366

Krogh's, 366, 373

Tonus, 113, 142, 713

Töpfer's test, 516

Touch, 766 et seq.

muscular sense, 776

\section{URETRRS.}

Touch-continued

sense of locality, 770

of pressure, 771

of temperature, $i b$.

tactile end-organs, 766

Touch-corpuscles, 767

Toxin, 474

Trabeculæ, 317, 332

Trachea, 847

Tract of Flechsig, 658

of Gowers, 657,658

of Lissauer, $i b$.

of Loewenthal, 656

Tracts in the spinal cord, 651, 655 et seq.

Tradescantia, cells of, 12,13

Transfusion of blood, 319

Transmission myograph, 105, 159

Transverse axis of eyeball, 851

Trapezium, the, 671

Traube-Hering curves, 304, 305

Triacetin, 415

Triamino-mono phosphatides, 4:5

Tricuspid valve, 210,213

Trigeminal nerve, 663,674

function, 674

origin of, $i b$.

Trimethylamine, 166

Trochlear nerve, 663, 674

origin of, 674

Trommer's test, 412

Trophic nerves, 144

Trypsin, action of , 518, 519

Trypsinogen, 523, 524

Tryptophane, 419, 422

Tubercle of Rolando, 667

Tubular glands, 496,497

Tubull seminifert, 864, 865

urinifert, 566 et seq., 569

Tubulo - racemose or tubulo - acinous glands, 496

Tunica adventitia, 217

Tunica albuginea of testicle, 863 dartos, 72

propria, 753

vaginalis, 863

Türck's column, 655

method of cumulation of reflexes, 710

Tympanum or middle ear, 788

diagram of, 780

membrane of, 788,790

muscles of, 790

structure, 788

Tyrosine, 418, 419, 422

U.

Umbilical arteries, 890

cord, 888,890

vesicle, 887

Uncinate convolution, 694

Unicellular organisms, 5

Unilaminar blastoderm, 883

Unimolecular reactions, 329

Uræmia, 588, 611

Urate of sodium, 598

Urates, deposit of, 598,600

Urea, 166, 583

apparatus for estimating quantity, 585

chemical composition of, 588

formation of, by liver, 528,586

isomeric with ammonium cyanate, 583

quantity, 586

Ureters, 565, 570 


\section{URETHRA.}

Urethra, 565, 570

Uric acid, 166, 432, 591

condition in which it exists in urine, 593 crystals, various forms of, 591 deposit of, 598

forms in which it is deposited, 591 origin of, 583,593

presence in the spleen, 335

presence in the urine, 600 proportionate quantity of, 592 iests, 592

Uricolytic enzyme, 594

Urina potus, 583

Urinary apparatus, 565 et seq.

Urinary bladder, 570

nerves, $i b$.

structure, $i b$.

Urinary deposits, 598 et seg.

Urine, 582 et seg.

analysis of, 583

bile in, 602

blood in, 603

chemical sediments in, 600

colour, 582

composition, 583

cystine in, 599

expulsion, 580

flow into bladder, 579

hippuric actd in, 594

inorganic constituents, 595

mineral salts in, $i b$.

pathological, 600

phosphates in, 597, 599

physical characters, 582

pigments, $i b$.

proteins, 600

pus in, 601,603

quantily, 582

varies with blood-pressure, 572

reaction of, 582

in different animals, 583

made alkaline by diet, $i b$.

saline matter, 583,588

solids, 582

specific gravity of, 583

variations of, $i b$.

sugar in, 601,602

tests for estimating, 602

tests for inorganic salts of, 597

urates, 598

urea, 583

uric acid in, 591

Uriniferous tubes, diagram of, 566

Urobilin, 534, 582

Urochrom $\theta, 582$

Uro-erythrin, 582, 594

Uterine muscle, 890 reflexes, 721

Uterus, 867, 871

atrophy or involution of, 895

development in pregnancy, 893

diagram of, $867,887-890$

structure, 871

Utricle, 791

Uvea, 812

\section{V.}

Vaccination, 473

Vagina, 867

Vago-sympathetic of frog, 251

Vagus,

electrical variations in, 377,378

escape, 251

nerve. See Pneumogastric.
Viscrral Layer of Prricardium.

Valentine's experiments on velocity of blood flow, 281

Valine, 417

Valsalva's experiment, 388

Valves of heart, 213. See Heart.

Vas deferens, 864, 865, 879

Vasa efferentia of testicle, 864, 865

Vasa vasorum, 218

Vascular system, in asphyxia, 389

Vaso-constrictor nerves, 300,302

Vaso-dilator nerves, 300,303

Vaso-motor nerves, 264,300

distribution of, 302

effect of section, $307 \mathrm{et} \mathrm{seq}$.

experiments on, 306,307

influence upon blood-pressure, 306

stimulation fatigue, 135

Vaso-motor nerve-centres, 301, 303, 305, 721

nervous system, 300 et seq.

reflex action, 305

Vegetables as food, 479,490

Vegetable cells, 5

protoplasmic movement in, 12,13

Veins, 208, 214, 218 et seq.

circulation in, 299 et seq. velocity of, 281

distribution, 218

interlobular, 569

pressure in, 278

pulmonary, 219

rhythmical action in, 299

structure of, 219

valves of, 219 et seq.

Velocity head, 286

pulse, 284,300

Velocity of blood in arteries, 281

in capillaries, $i b$.

in veins, $i b$.

of circulation, $i b$.

of enzyme action, 516,520

of nervous impulse, 159

Vena cava, 210

Venæ rectæ, 570

Venæ stellulæ, 569

Venous flow, 299

Ventilation, 379,384

Ventral cerebellar tract, 658

Ventricles of the brain, 644

Ventricles of heart. See Heart.

Ventricular diastole, 233,231

systole, $i b$.

Veratrine, effect of, on muscular contraction, 99

Vernon, heat rigor experiment, 139

on tissue-erepsin, 546

pancreatic secretion, 523

Vertical axis of eyeball, 851

Verworn, Max, strychnine and fatigue, 135

Vesical centre, 580

Vesicle,

germinal, 18

Vesiculæ seminales, 865

Vestibular nerve, 663

Vestibule of osseous internal ear, 753,790

Vibrations, conveyance of, to auditory nerve, 796 et seq.

Vierordt's experiments on blood-circulation, 289

Vierordt's hæmatachometer, 287

Vieussen's annulus, 196, 251, 302

Vill in chorion, function of, 888

of intestines, 494, 495

Vincent, Swale, muscle proteins, 142

Visceral layer of pericardium, 208

mesoblast, 884

pain, 777

sensations, 777 
Vision.

Vision, 810

angle of, 822

at different distances, adaptation of eye to, 824 et seq.

correction of aberration, 830 et seq.

defects of, 828 et seq.

distinctness, how secured, 856 et seq.

duration of sensation in, 836

estimation of the size and form of objects, 854 . 856

focal distance of, 824

range, 827

relation of nerve-cells and fibres, 854

single, with two eyes, $850 \mathrm{et}$ seq.

Visual area, 735

apparatus, relations of nerve-cells and fibres, 854

axis, 822,851

impressions, 752

judgments, 854 et seq.

plane, 851

purple, 817,847

sensations, 836, 841 et seq

word-centre, 741,809

Visuo-psychic region, 737

Visuo-sensory cortex, $i b$.

Vital action, 327

force, 2

Vitamine, 491

Vitellin, 429, 487

membrane, 871

Vitello-intestinal duct, 886

Vitreous humour, 811,819

Vocal cords, 801

action in respiratory actions, 804

approximation of, effect on helght of note, 805

vibrations of, cause volce, 806

Voice, 7£9 et seq., 806

range of, 806

Volt's diet, $480,618,629$

on "circulating protein," 622

Volkmann's hæmadromometer, 282

experiment on lymph hearts, 318,319

Volta on galvanism, 116

Voluntary muscle, 63 et seq., 140 et seq.

nerves of, 70

Voluntary tetanus, $10 t$

Vomiting, 556, 557

action of stomach in, ib.

centre, $i b$.

nerve actions in, $i b$.

voluntary and acquired, $i b$.

Vorticellæ, 82

Vowels and consonants, 808

Vulpian's experiments on nerve regeneration, 149,151

\section{W.}

Wagner's hammer, 90

Wallerian degeneration method, 146, 149, 151, 156, 651,652

Waller, dynamograph, 132

electrocardiogram, 245

fatigue theory, 133

on the electrical currents of the eyeball, 849

on the passage of blood-corpuscles, 297, 298

\section{ZYMOLYS1S}

Waller-continued

on work of heart, 247

"sound-plcture" theories of, 798

variation in nerve action, 159

Wander cells, 31

Water-hammer pulse, 291

Wave of blood causing the pulse, $i b$.

velocity of, $i b$.

Weber-Fechner law, 763

Weber's experiment on velocity of blood-flow, 281

on heart-beats, 249

Weber's paradox, 112, 114

Weigert's method, 651,739

Waismann's germ plasm, 859,861

somatoplasm, 861

Wertheimer's investigations into pancreatic secre tion, 520

Wharton's jelly, 36

Whey protein, 482

White corpuscles. See Blood-corpuscles, white; and Lymph-corpuscles.

emigration of, 298

White fibro-cartllage, 37,38

fibrous tissue, 29,80

spot, 814

Widal's reaction, 476

Wooldridge's method of preparing tissue-fibrino. gen, 431

Word.centres, 809

Worms, circulatory system in, 230

Wright, Hamilton, sleep theory, 746

Wright's opsonins, 476

Wrisberg, cartilages of, 800,805

pars intermedia of, 675

X.

Xanthine, 166, 432

Xantho-proteic reaction, 425

Y.

Yawning, mechanism of, 382

Yeast plant, cells of, in process of budding, 5, 439

Yellow elastic fibre, 29, 30

fibro-cartllage, 37,40

marrow, 41

spot of Sömmering, 814

Yoo's experiment on gaseous exchanges in the heart, 254

Yolk-sacs, 888 et seq.

Yolk-spherules, 870

Young-Helmholtz theory, 842 et seq.

\section{Z.}

Yona fasciculata, 340

Zona pellucida, or striata, $19,870,881$

Zonule of Zinn, 820

Zuntz, on mountain sickness, 400

Zymase, 439

Zymogen, 441, 449, 499, 509

Zymolysis, reversible, 614 




\section{DATE DUE SLIP}

UNIVERSITY OF CALIFORNIA MEDICAL SCHOOL LIBRARY

THIS BOOK IS DUE ON THE LAST DATE STAMPED BELOW

AUG 261924

PEC \& 1928

NOV 121931

OCT 121945

OCT 251945 


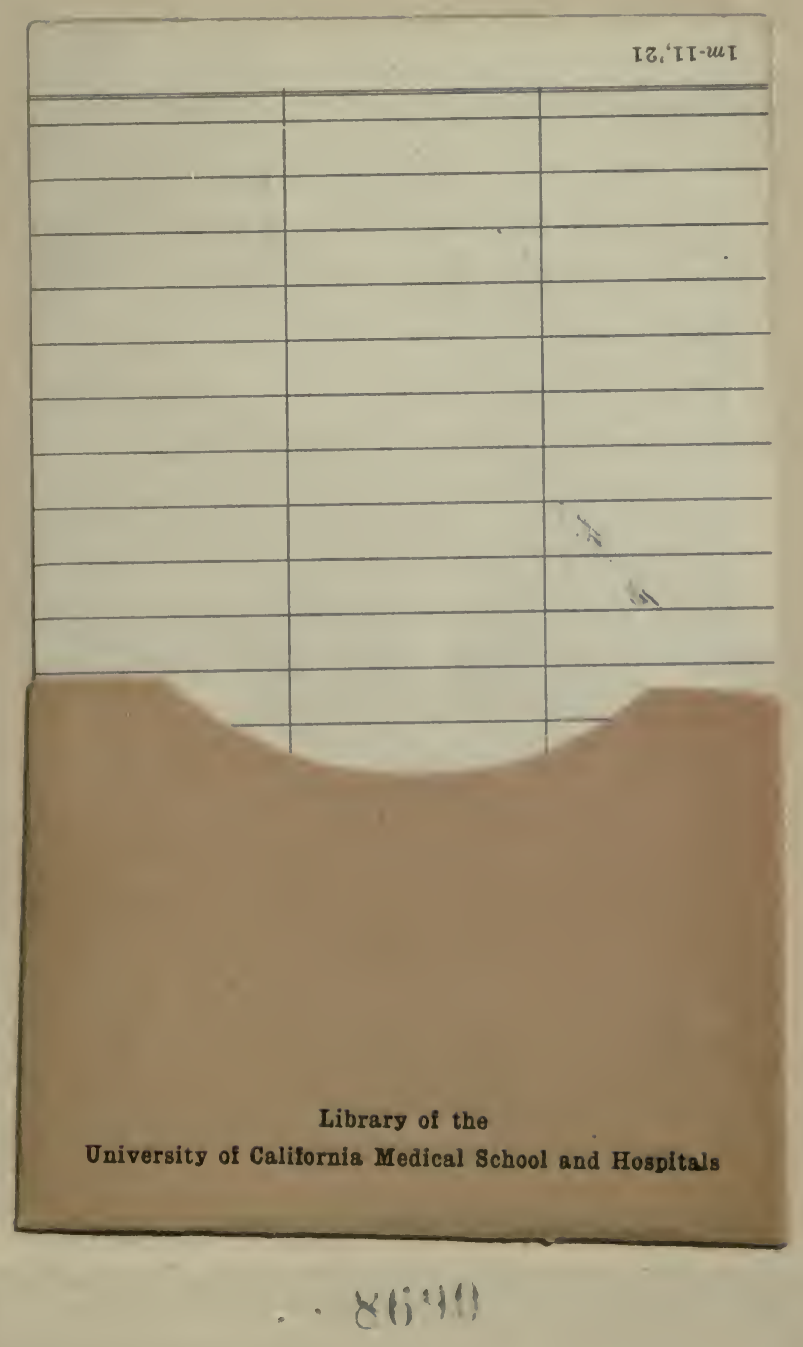




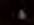

